\title{
ZOOTAXA
}

1418

\section{Checklist of catfishes, recent and fossil (Osteichthyes: Siluriformes), and catalogue of siluriform primary types}

CARL J. FERRARIS, JR.

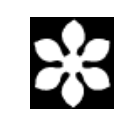

Magnolia Press

Auckland, New Zealand 
CARL J. FERRARIS, JR.

Checklist of catfishes, recent and fossil (Osteichthyes: Siluriformes), and catalogue of siluriform primary types

(Zootaxa 1418)

628 pp.; $30 \mathrm{~cm}$.

8 March 2007

ISBN 978-1-86977-058-7 (hardback)

ISBN 978-1-86977-059-4 (Online edition)

\section{FIRST PUBLISHED IN 2007 BY}

Magnolia Press

P.O. Box 41-383

Auckland 1346

New Zealand

e-mail: zootaxa@mapress.com

http://www.mapress.com/zootaxa/

(C) 2007 Magnolia Press

All rights reserved.

No part of this publication may be reproduced, stored, transmitted or disseminated, in any form, or by any means, without prior written permission from the publisher, to whom all requests to reproduce copyright material should be directed in writing.

This authorization does not extend to any other kind of copying, by any means, in any form, and for any purpose other than private research use.

ISSN 1175-5326 (Print edition)

ISSN 1175-5334 (Online edition) 


\title{
Checklist of catfishes, recent and fossil (Osteichthyes: Siluriformes), and catalogue of siluriform primary types
}

\author{
CARL J. FERRARIS, JR. \\ 2944 NE Couch Street, Portland, Oregon, 97232, U.S.A. E-mail: carlferraris@comcast.net \\ Research Associate, National Museum of Natural History, Smithsonian Institution, Washington \\ Research Associate, American Museum of Natural History, New York \\ Research Associate and Honorary Fellow, California Academy of Sciences, San Francisco
}

\section{Table of contents}

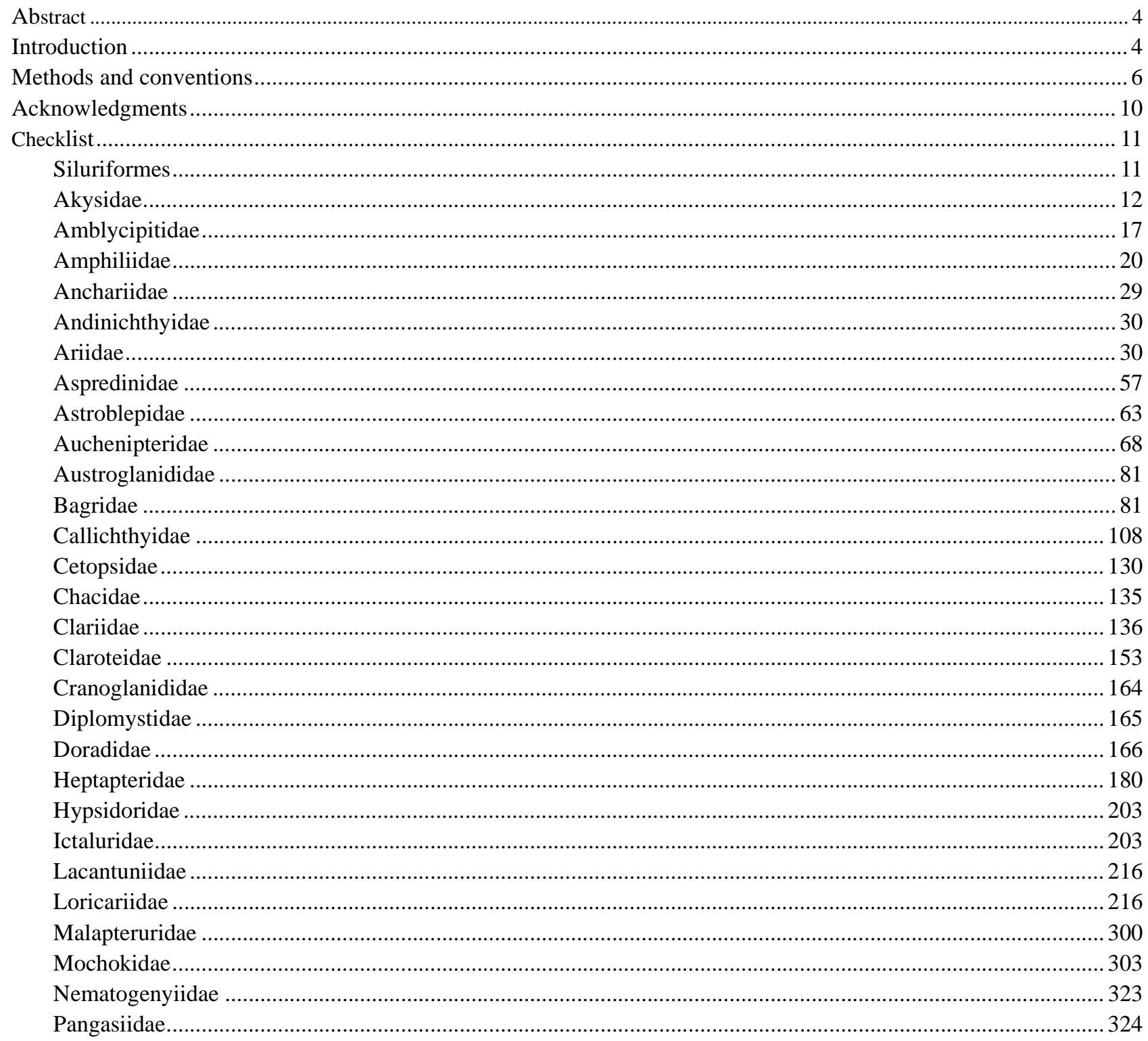


Pimelodidae

Plotosidae

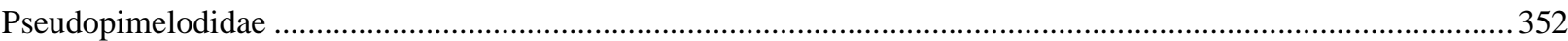

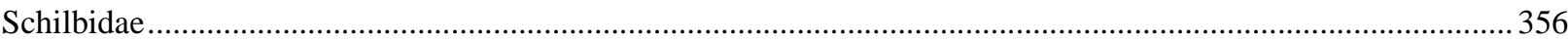

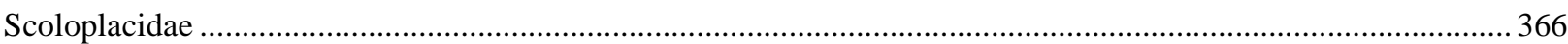

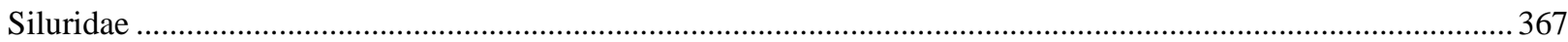

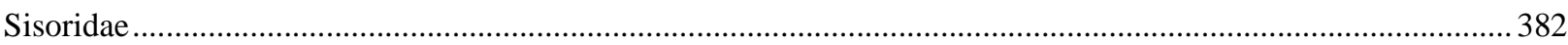

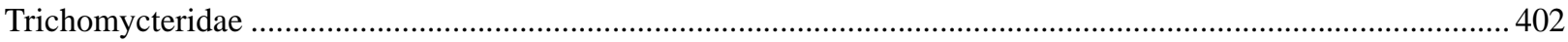

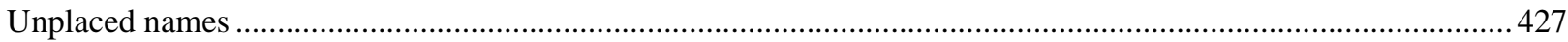

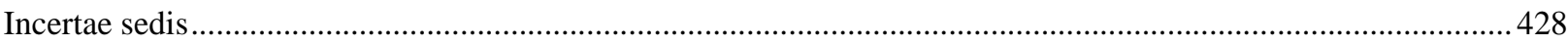

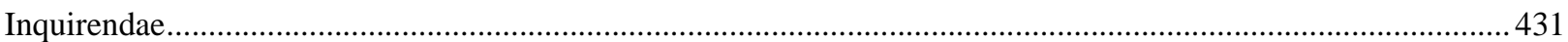

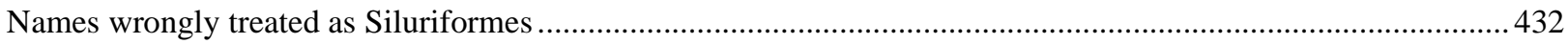

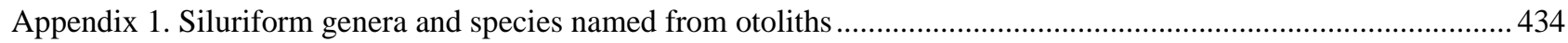

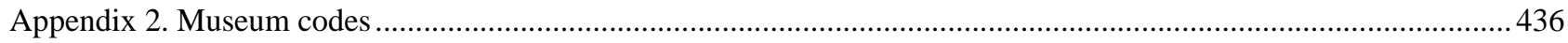

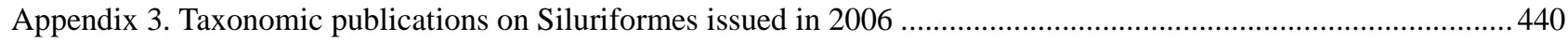

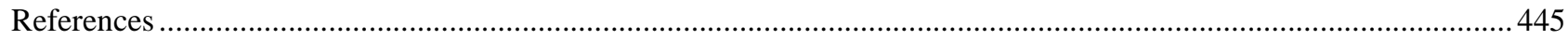

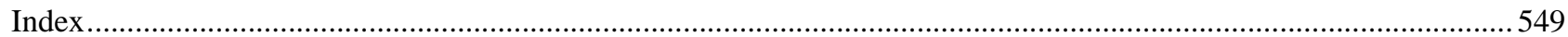

\begin{abstract}
A checklist of Recent and fossil catfishes (Order Siluriformes) is presented, summarizing taxonomic literature published through 2005. From 4624 nominal species group names and 810 genus group names, 3093 species are recognized as valid, and are distributed among 478 genera and 36 families. Distributional summaries are provided for each species, and nomenclatural synonymies, including relevant information on all name-bearing types, are included for all taxa. One new name is proposed herein: Clariallabes teugelsi, as a replacement for Clarias (Allabenchelys) dumerili longibarbis David \& Poll, 1937, which is preoccupied by Clarias longibarbis Worthington, 1933, but has been treated as a valid species of Clariallabes by Teugels. Acrochordonichthys melanogaster Bleeker, 1854, is designated as type species of Acrochordonichthys Bleeker, 1857, inasmuch as no earlier valid designation has been found. A new genus Pseudobagarius, is proposed for the "pseudobagarius group" of species formerly placed in Akysis. The status of 228 species group names remains unresolved and 31 names based on otoliths ascribed to catfishes are listed but not placed into the checklist. The current emphasis given to catfish taxonomy at present is likely to result in a dramatic increase in the total number of valid taxa as well as major changes in the membership of some of the higher level taxa recognized here.
\end{abstract}

Key words: Nematognathi, taxonomy, nomenclature, biodiversity, freshwater fishes

\title{
Introduction
}

Osteichthyian fishes of the Order Siluriformes, known by the English common name of catfishes, form a well diagnosed natural group of primarily freshwater fishes. Most catfishes are apparently tolerant of only fresh water but the salinity tolerance of several species extends to, or is even limited to, estuarine or even oceanic levels of salinity. Thus, while most catfishes are recorded from inland waters, the distribution of the order also includes coastal regions of continents and nearby islands. Catfishes often have large, heavy bones that lend themselves to fossilization and, comparatively large otoliths. As such, a large number of species of catfishes have been named from complete or partial skeletal fossils or even from only otoliths.

A checklist of named Recent and fossil catfishes is presented herein. The checklist is thought to be complete through the end of 2005 for available names proposed for catfishes and literature that treats taxonomic issues involving catfish taxa. From 4620 nominal species group names and 805 genus group names, 3088 species are recognized as valid, and are distributed among 477 genera and 36 families (Table 1). In addition, 228 species group names are of uncertain validity and are in need of further study. Of the valid taxa, 19 genera and 72 species are based exclusively on fossil remains. Additionally, 2 genera and 31 species names that are based exclusively on otoliths are listed herein but are not included in the above mentioned totals. 
TABLE 1. Numbers of valid genera and species of catfishes (Order Siluriformes) and unplaced species-group names, arranged by family. Fossil taxa included in generic and species totals and also listed separately. Otolith based names are not included in any of the summary statistics.

\begin{tabular}{|c|c|c|c|c|c|}
\hline & Genera & Species & Species inquirendae & Fossil genera & Fossil species \\
\hline Akysidae & 5 & 47 & 0 & 0 & 0 \\
\hline Amblycipitidae & 3 & 27 & 3 & 0 & 0 \\
\hline Amphiliidae & 12 & 66 & 0 & 0 & 0 \\
\hline Anchariidae & 2 & 5 & 0 & 0 & 0 \\
\hline Andinichthyidae & 3 & 3 & 0 & 3 & 3 \\
\hline Ariidae & 26 & 133 & 56 & 0 & 13 \\
\hline Aspredinidae & 12 & 36 & 0 & 0 & 0 \\
\hline Astroblepidae & 1 & 54 & 0 & 0 & 0 \\
\hline Auchenipteridae & 20 & 94 & 12 & 0 & 0 \\
\hline Austroglanididae & 1 & 3 & 0 & 0 & 0 \\
\hline Bagridae & 23 & 189 & 33 & 4 & 11 \\
\hline Callichthyidae & 8 & 194 & 0 & 0 & 1 \\
\hline Cetopsidae & 5 & 41 & 0 & 0 & 0 \\
\hline Chacidae & 1 & 3 & 0 & 0 & 0 \\
\hline Clariidae & 17 & 113 & 12 & 0 & 2 \\
\hline Claroteidae & 17 & 83 & 6 & 1 & 3 \\
\hline Cranoglanididae & 1 & 3 & 0 & 0 & 0 \\
\hline Diplomystidae & 2 & 6 & 0 & 0 & 0 \\
\hline Doradidae & 30 & 77 & 4 & 0 & 0 \\
\hline Heptapteridae & 24 & 189 & 3 & 0 & 0 \\
\hline Hypsidoridae & 1 & 2 & 0 & 1 & 2 \\
\hline Ictaluridae & 7 & 64 & 5 & 1 & 14 \\
\hline Lacantuniidae & 1 & 1 & 0 & 0 & 0 \\
\hline Loricariidae & 96 & 716 & 3 & 0 & 0 \\
\hline Malapteruridae & 2 & 19 & 0 & 0 & 0 \\
\hline Mochokidae & 10 & 188 & 3 & 0 & 0 \\
\hline Nematogenyiidae & 1 & 2 & 0 & 0 & 1 \\
\hline Pangasiidae & 5 & 30 & 2 & 1 & 2 \\
\hline Pimelodidae & 29 & 93 & 16 & 0 & 4 \\
\hline Plotosidae & 10 & 35 & 2 & 0 & 0 \\
\hline Pseudopimelodidae & 5 & 29 & 1 & 0 & 0 \\
\hline Schilbidae & 14 & 62 & 1 & 0 & 0 \\
\hline Scoloplacidae & 1 & 4 & 0 & 0 & 0 \\
\hline Siluridae & 12 & 94 & 28 & 0 & 3 \\
\hline Sisoridae & 22 & 168 & 8 & 0 & 1 \\
\hline Trichomycteridae & 41 & 207 & 1 & 0 & 0 \\
\hline Incertae sedis & 8 & 13 & 29 & 7 & 10 \\
\hline Total valid taxa & 478 & 3093 & & 18 & 70 \\
\hline Total species inquirendae & & & 228 & & \\
\hline Otolith-based names & & & & 2 & 31 \\
\hline
\end{tabular}


The primary objective of compiling this checklist is to provide catfish taxonomists with a framework for future studies by summarizing published information on the taxonomy and nomenclature to date, while at the same time highlighting problematic names and taxa that deserve special attention. Valid species that are not placed in genera or, in some cases, families are indicative of broader problems with current taxonomy of the group into which theses taxa belong. Species that have been variously placed in one of two or three genera suggests that one or more of the genera are likely not natural and, therefore, a situation that is in clear need of further study. Catfish taxonomy has long suffered from having many monogeneric, or even monotypic, families. These taxa may indicate highly distinctive taxa but say nothing about the relationship of these "families" within catfishes. Although there is no real distinction between recognizing monotypic families and treating the included species as Incertae sedis in the Siluriformes, named families that have been treated in recent times continue to be listed as such here. Names that have not been treated in recent years as either valid taxa or synonyms, and therefore placed as herein species inquirendae, have in some cases simply been overlooked and likely to be easily associated with a species after study. Others suffer from poor published descriptions, with or without an unambiguous type specimen, and may not be readily linked with any species. Such names may need to be eliminated as available names in the future, but it is too early to do so at this time. In most cases, such names appear to be part of particularly problematic families that need more attention, which may shed light on the appropriate disposition of at least some of those names.

By their very nature, checklists can only represent the state of the taxonomy of the included group for a brief period of time and are out of date soon thereafter. As such, checklists represent a snapshot of a continually evolving attempt to document the biological diversity of a group of organisms. The ephemeral nature of checklists is especially true for this work because of the relatively large amount of taxonomic research that is currently being conducted on catfishes. This is due, at least in part, to the ongoing All Catfish Species Inventory (ASCI), which has focused attention on catfishes and encouraged the publication of reports on the discovery of previously unnamed species as well as revisionary studies. As an example of this emphasis on catfish taxonomy, for 2005 alone 86 publications are listed herein, which include the descriptions of more than 100 newly-named species of catfishes and eight revisionary studies. It is likely that comparable numbers of papers and newly discovered catfish taxa will be published over the next several years, which translates to an approximate increase of up to10\% in number of recognized species during the next few years. The impact of ASCI on the rate of publication of catfish taxonomic studies is likely to extend years into the future, which suggests that a future catfish checklist will be larger and, quite possibly, substantially different in arrangement than the one presented here. Nonetheless, publication of this checklist at this time is considered appropriate to bring to attention taxa in special need of study, and all of the names that need to be treated in these studies, so that future checklists will have fewer such unresolved problems.

\section{Methods and conventions}

Valid taxa. Taxa are hierarchically arranged in alphabetical order at the level of families, genera and species, with valid names highlighted in bold font. Intermediate levels (i. e., subfamilies, tribes, subgenera) and subspecies are not used. Taxa generally accepted in the literature as valid subspecies are listed as valid species here. Names treated variably as subspecies and as synonyms of a species are placed as synonyms here.

After each valid name is the author and year of publication of the information necessary to make the name available. Additional information that facilitates linking the name to the publication in which is was made available will be found in the appropriate entry in the synonymy that follows each valid name.

Species that are treated in recent literature as valid, but of uncertain generic assignment are listed at the beginning of the Family to which they belong, under the heading Incertae sedis, with the originally proposed scientific name. These species are considered valid, but not in the genera in which they were described and, 
possibly, not in any of the listed genera. Valid catfish genera of uncertain family placement, and any species associated with that generic name, are listed in chronological order after the last family account under the heading of Siluriformes Incertae sedis.

A number of available species names and generic names could not be placed either as a valid name or as a synonym of another name. Often, these names were overlooked in previous taxonomic studies, or proposed with descriptions that were not sufficiently precise to allow the assignment the name to a particular species and the type or types are either missing or otherwise unavailable for study. Generally, such names have not been treated in recent literature or treated as a name of uncertain status. Herein, these names are included at the end of the taxon to which their assignment could be confidently made, under the heading Species inquirenda (Species inquirendae, pl.) for species names and Genus inquirendum (Genera inquirendae, pl.) for generic names. Names of uncertain species status that also cannot be assigned to any genus are listed at the end of the family account, and names not assignable to any family are listed at the end of the checklist as Siluriformes Inquirendae immediately after Siluriformes Incertae sedis.

Valid names are corrected, as necessary, to conform to the spelling conventions specified in The International Code of Zoological Nomenclature (ICZN, 1999). That is, species-group names that were originally proposed with an initial capital letter and any name that included a diacritic mark have been corrected to a lowercase initial letter or appropriately modified spelling, respectively. Names based on fossils, both valid and junior synonyms, are preceded by a dagger $(\dagger)$.

For each species, a statement of the geographical distribution is provided. Generally, the statement refers to one or more river basins, but lakes, or land masses are sometimes listed as the best summary of the distribution. Distribution statements are generally based on recently-published accounts of the most comprehensive recorded distribution but in some cases reports from two or more sources were cited. For some species, the distribution is based on personal observation or just the locality of the type(s). Geographical names are in English and the spellings are the preferred spelling in Webster's Geographical Dictionary.

Family names treated here as valid are those in current use by practicing catfish taxonomists. In some cases, those names appear not to correspond with the oldest available name for the family, as shown by the synonymies presented under each valid family name. It was decided not to strictly adopt the senior synonyms for families at this time, inasmuch as the names listed here (derived from Ferraris \& de Pinna, 1999) might not represent the earliest usage. Family-group names have in some cases been casually proposed in early literature and subsequent usage usually did not include an indication of the source of the names. Thus, is it still possible that names adopted here as valid are indeed older than some that appear in the list of synonyms to have been proposed earlier.

Synonymies. In the synonymies, data are presented in the following sequence. For species group names: Species name; Author(s) and date of publication of the name; Citation to source publication of the name, if different from that of the name's author; Page(s) in which the name is made available; Figure(s) of the name's type(s) in the publication; Type locality; Information on the identity of the primary type(s), including, when appropriate, citation to the restriction of the primary type to a single specimen; Expanded description of the species by the name's author; and Published illustration(s) of the primary type specimen (or a syntype) in subsequent literature.

For generic names: Genus or subgenus name; Author(s) and date of publication of the name; Citation to source publication of the name, if different from that of the name's author; Page(s) in which the name is made available; Type species; Source for type species' designation; Gender of name; and, for names originally proposed as a subgenus, the generic name within which the subgenus was originally proposed.

In synonymies, the spelling of a name is reported as it appears in the original description, including diacritic marks and upper case initial letters in species group names. If more than one spelling is found in the original description, the variant spellings are noted and the valid spelling is listed at the head of the synonymy. 
A citation is provided for the first reviser action that provided the basis for the decision on which spelling is treated as valid.

The date of publication of names listed herein represents the best available information of the year that the publication containing the new name, or that portion of the publication in which elements necessary to make the new name available, was actually issued. When necessary, the cited date is followed by the range of dates within which the publication was issued, to facilitate bibliographic access to the publication in question.

The type locality is that which was reported by the author of the name, either in the account of the species or elsewhere in the publication. In some species accounts, the type locality is erroneously reported, place names are misspelled or the locality information is brief or ambiguous. Sometimes, additional information that clarifies a vaguely-expressed type locality is added in square brackets. All stated localities of a syntype series are listed and, when appropriate, the locality of the selected lectotype is noted. When a neotype was selected to replace a missing primary type, the stated type locality is that of the neotype and not the original type(s). If the originally-cited type locality was found to be incorrect, the corrected type locality is reported in brackets with supporting documentation.

Page numbers provided for each proposed new name represent the page of the publication in which the name is first proposed as new and for which information is provided that makes the name available. In a few cases, two page numbers are listed, because the information needed to make the name available is divided between those two pages. In some $19^{\text {th }}$ and early $20^{\text {th }}$ Century journals, offprint of articles were issued with pagination that differs from that of the article in the journal. In general, the page number cited herein is that found in the journal and not that of the offprint. However, if an offprint was issued in advance of the journal and does not include the pagination of the journal, the listed page number is that of the offprint. In a few cases, cited publications were issued without pagination. New names described in such publications are listed with a page number enclosed in square brackets, with the assumption that the article containing the new name began on page 1. Cuvier \& Valenciennes' (1828-1850) Histoire naturelle des Poissons was issued in two, apparently simultaneous, editions. One edition, called the Regular Edition, consists of 22 volumes in octavo format. The other, called the Strasbourg Deluxe Edition, consists of the same number of volumes, but in a slightly larger, royal octavo, format. Although the contents of each volume the two editions are apparently identical, the pagination of the volumes differs, with fewer pages in the Strasbourg deluxe edition. Although the existence of the two editions is well known (e. g., Dean, 1916: 287), most authors have access to only one of the editions (usually the Regular Edition, which is more widely available), which has resulted in a certain amount of confusion about the correct page of a new name. Herein, the page number of the Regular Edition is listed, with the pagination of the Deluxe edition in square brackets.

Museum codes that are listed for primary type specimens used here are primarily those found in Eschmeyer (1998). A few additional codes were added, based on information provided by the author of a publication in which a new code was used. A list of the codes used herein, with the name and city and country of the institution, is provided in Appendix 2, which precedes the list of References. Museum names for which no code was created, or for which a published code conflicts with one used in Eschmeyer (1998), are written out in full.

Only primary type specimens are reported here, including literature citations to specimens that must be treated as part of a syntype series. When reported in the original description, the number of syntypes that comprise the type series is listed in after the word 'Syntypes' and before the colon (:). If the number of syntypes included within a single catalog number is known, that number if listed in parentheses immediately following the catalog number. In some cases, only a single syntype has been identified, and the actual number of syntypes is not known. That specimen is identified as a syntype (singular) in order to assure that it is not treated as a lectotype designation. For holotypes or syntypes that have not been found, or for which there is some question of the validity of the listed specimen (s), the size or range of sizes of the specimen(s) reported in the original description is listed, as stated by the author of the name, to assist in the verification process. Catalog or 
register numbers follow the numbering system used by the museum, whenever possible. The numbering system generally follows that which is listed in a museum's on-line catalog. For example, MNHN now uses a prefix consisting of either a small letter "a" or "b", or a four digit number that represents the year of accession (or 0000 for unknown year), followed by a unique four-digit number for each prefix, separated from the prefix by a dash. Other published versions of the catalog numbers for this museum's holdings variously replace the dash by a period, using capital letters rather than small letters in the prefix, and dispensing with the " 0000 " prefix or any leading zeros in the four-digit number. The Stanford University collection code (SU) is sometimes replaced by CAS-SU, indicating that the material is currently at the California Academy of Sciences. In addition, the Stanford specimen catalog numbers are sometimes replaced by an equivalent catalog number of the CAS numbering system, which was generated by adding 100,000 to the SU catalog number, and replacing SU with CAS. For example, SU 1040 may be seen as written CAS-SU 1040, or CAS 101040. Herein, the original numbering system, with the SU museum code, is followed.

When catalog numbers of types were found to differ from those reported in recent literature, the discrepancy was noted in order to indicate that the numbers reported herein were not the result of typographical errors. One class of error that requires explanation relates to a portion of the Bleeker collection that was registered at the Natural History Museum in London. That collection was registered with the prefix of BMNH 1863.12.4.XXX, but the jar labels for those specimens often listed the prefix as BMNH 1863.12.11.XXX. These incorrect registration numbers were the basis for several published reports of the types, and possible types, of Bleeker catfish names. Herein, published reports of the incorrect number are noted in the remarks of the species account to prevent future use of those same numbers.

The following terms are used in cases where the type specimen(s) are not listed: No types known.- apparently types were never saved, because the descriptions may have been made from specimens that were subsequently discarded, or from illustrations for which the underlying specimens were not retained; Whereabouts unknown.- type specimens were apparently preserved and deposited in a collection but the current location is not known. This does not preclude the possibility that the specimen is in the stated collection (if recorded), but it has not been recognized as a type; At [Museum code].- there is good reason to believe that the specimen was deposited in the collections of the museum listed, but the specimen has not been recognized as a type, or the catalog number of the specimen was not yet found.

Within the synonymies, names are arranged chronologically. Multiple synonyms proposed in a single publication are listed alphabetically by name. If two or more junior synonyms were published in the same year by different authors, the synonyms are ordered alphabetically by author, except when one name is the valid name, in which case that name is listed first.

Type localities for species names are localities as written in the original descriptions, when possible. Type localities from publications written in alphabets other than Roman were written in a transliterated form. When the publication containing an original description was not examined, the type locality was taken from that listed in the Catalog of Fishes (Eschmeyer et al., 1998).

Classification. By their nature, checklists can only provide a single classification of the included taxonomic diversity. This becomes problematic in situations where recently published literature includes competing ideas on the phylogeny and/or classification of one or more taxonomic groups. This was indeed the case for several groups of catfishes and I was forced to make decisions on how best to summarize the diverse conclusions on catfish taxonomy. In some cases, the classification that was grounded on a hypothesis of phylogenetic relationships was used over another that lacked such grounding. However in other cases, none of the recently proposed classifications were based on a phylogenetic study or, alternatively, more than one phylogeneticallybased study disagreed on some aspects of the resulting classification. When left with no single classification to adopt, I attempted to integrate available phylogenetic information together with historically accepted studies to provide a classification that appears to be best supported. Studies that were used to form the final classi- 
fication are cited immediately after the synonymy of the taxon, with comments listed in a remarks section. Undoubtedly, this classification will not please all catfish taxonomists and even those whose studies I drew upon for my classification may feel that their results were misinterpreted. It must be recognized by anyone who uses this checklist that the classification represents my attempt to interpret recent taxonomic literature and may not accurately reflect the ideas of the specialists of the various catfish groups.

Relevant literature. Following the family and Generic synonymies, literature citations are listed that were consulted in making the taxonomic decisions used herein, and were the source of many of the listed distributional summaries. The literature citations are subdivided into categories that indicate the nature of the underlying studies, using the following categories: Taxonomic summary. - listing of valid taxa with nomenclatural summary, without diagnoses or characters for identification; Checklist.— listing of valid taxa without nomenclatural summary, without diagnoses or descriptions; Identification guide- diagnoses of taxa, usually species, generally with illustrations; Revision.- taxonomic summary of all included taxa, with descriptions of all valid species; Review.- Regionally based taxonomic summary, generally with nomenclatural summary and redescriptions of included taxa; Fauna.- regionally based summary, with diagnoses, but usually not with complete nomenclatural summary or redescriptions; Phylogeny.- character based interpretation of phylogenetic history; and Monophyly.- characters presented that demonstrate the monophyly of the group, but interrelationships within the group were not discussed.

References. Literature citations are arranged alphabetically by author(s) and then chronologically by year. When multiple publications were issued within a single year by a single author, or combination of authors, the publications are arranged alphabetically by journal title and, if needed, by pagination within a journal, followed by books or chapters in books, rather than attempting to arrange them by more precise publication date. These publications are noted by lower case letters following the year of publication in the text as well as the References section. In some cases, the use of this convention results in the listing of a publication annotated with a lower letter of the alphabet than a paper published earlier. The difficulty in determining the precise date of publication, or even the relative dates of publication of two papers issued in the same year, made it unnecessarily complicated to attempt to arrange publications chronologically within a year. When considered relevant to the resolution of a issue of priority, more precise dates of publication are cited in brackets at the end of citations. More precise publication dates may be obtained in some cases by consulting the bibliography in Eschmeyer (1998).

Otolith-based names. Names that were proposed based only on otoliths for species that were included in catfish genera, or otherwise hypothesized to be included in the Siluriformes, are listed in a separate section of this publication. The names are listed in chronological order, and no attempt was made to distinguish between valid names and junior synonyms or to place the names into the classification, In most cases, names based on otoliths have been interpreted as belonging to catfishes of the family Ariidae.

\section{Acknowledgments}

Completion of this checklist required access to an immense amount of literature. Examining this literature was accomplished by traveling to institutions in several continents, obtaining copies of publications from colleagues and, for more recent publications, accessing on-line sources. These various activities were accomplished with the assistance of several institutions, colleagues and friends, and without this assistance I cannot imagine how I could have been completed this project.

The following institutional and personal libraries were consulted during research visits: The Natural His- 
tory Library, the Cullman Library, the Vertebrate Paleontology Library, the Division of Fishes Library, and the personal library of Dr. Richard Vari, at the National Museum of Natural History, Smithsonian Institution, Washington; The Research Library, the Dean Memorial Library, the Vertebrate Zoology Library and the personal library of Dr. John Maisey at the American Museum of Natural History, New York; The Vertebrate Zoology Library and the personal library of Dr. Sven Kullander, at the Swedish Museum of Natural History, Stockholm; The main library at The Natural History Museum, London; the Maillard Library and the Ichthyology Department Library at the California Academy of Sciences, San Francisco.

Remote access to the Zoological Record and electronic access to journals was arranged by the staffs of the American Museum of Natural History library and the Maillard library of the California Academy of Sciences (CAS). Interlibrary loans were arranged through the CAS library, with the generous assistance of Annie Malley, Larry Currie and, especially, Patty Shea-Diner. Richard Vari, Heok Hee Ng and John Friel located, and provided me with, copies of publications that I was otherwise unable to find. Years ago, Dr. James Atz generously gave me much of his reprint library that dealt with catfish taxonomy and, similarly, the late Mel Rosen gave me relevant portions of the library of her husband, the late Dr. Donn Rosen.

Dr. Maurice Kottelat generously shared with me information on Southeast Asian catfish nomenclature and taxonomy that he has been compiling. Dr. Ralf Britz examined specimens and literature at the Natural History Museum, London, that was otherwise unavailable to me. Todd Stevenson assisted in preparing the Literature cited, with financial support from the All Catfishes Species Inventory. Portions of this checklist were reviewed by Drs. John Lundberg, John Friel, and Mark Sabaj. The assistance of all of the people named above was greatly appreciated, inasmuch as each contributed valuable insights that improved the quality of the checklist.

In preparing this checklist, I drew heavily on the information and interpretations of several previous publications, most notably the Catalog of Fishes (Eschmeyer, 1998), CLOFFA (Daget et al., 1986), and CLOFFSCA (Reis et al., 2003). The efforts of the authors and editors of these publications, as well as numerous others that cannot all be listed here, to sort out the taxonomic and nomenclatural issues the relate to catfishes is gratefully acknowledged.

Financial support for trips to the visited institutions was provided by the FishBase Project Sweden, the Neotropical Lowlands Project of the National Museum of Natural History, and the All Catfish Species Inventory. Richard \& Ann Vari, Carol Prince \& Jens Vindum, Diana Shih \& Benoit Jadoul, and Ricardo Castro all generously provided me with accommodations during one or more visits to institutions near their homes. Publication support for this checklist was provided by the All Catfish Species Inventory, which received a generous grant from the United States National Sciences Foundation (DEB-0315963).

And, finally, I thank my wife, Susana Ferraris, for tolerating the piles of books, papers, and computer hardware that have been scattered across the house over the past few years, as well as for her patience at the lack of progress on the many household projects that were delayed while this checklist was being prepared.

\section{SILURIFORMES Cuvier, 1816}

Taxonomic overview: Burgess (1989), Teugels (1996), Teugels (2003a), Nelson (2006).

Fauna: Eigenmann (1920b, 1922b, 1927, western South America); Herre (1926, Philippines); Hora \& Gupta (1941, Malay peninsula); Kottelat et al., (1993, western Indonesia); Kottelat (2001a, Vietnam); Kottelat (2001b, Laos); Rainboth (1996, Cambodian Mekong); Skelton (1993, 2001, southern Africa); Nichols (1943, China); Chu et al. (1999, China); Fowler (1951a, 1954, Brazil); Le Bail et al. (2000, French Guiana); Jayaram (1978a, 1981, 1999, southern Asia); Tan \& Ng (2000, central Sumatra), Page \& Burr (1991, North America), Miller et al. (2005, Mexico).

Review: Day (1877a, India); Misra (1976, India and South Asia); Jayaram (1977d, various families); Menon (1999, India); Gayet \& Meunier (2003, fossil taxa).

Phylogeny: Chardon (1968), Mo (1991), Arratia (1992), de Pinna (1998a), Diogo (2003a, 2004 a, b); Hardman (2005); 
de Pinna (1996, several Asian families); Howes (1983b, loricarioid families), Arratia \& Huaquin (1995, loricarioid families); Peng et al. (2005, Chinese species).

Checklist: Kottelat (1985, Cambodia); Kottelat (1989a, Indochina); Mirza (1980, Pakistan and Azad Kashmir); Gosline (1942, 1945, Neotropics); Reis et al. (2003, Neotropics).

\section{AKYSIDAE Gill, 1861}

Akyses Gill, 1861c: 52. Type genus: Akysis Bleeker, 1858.

Parakysidae Roberts, 1989a: 141. Type genus: Parakysis Herre, 1940.

Acrochordonichthyini de Pinna, 1996: 61. Type genus: Acrochordonichthys Bleeker, 1857.

Monophyly and phylogenetic position: de Pinna (1996).

Identification guide: Jayaram (1977d), South Asia.

5 genera, 47 species; no named fossil taxa.

\section{ACROCHORDONICHTHYS Bleeker, 1857}

Acrochordonichthys Bleeker, 1857b: 473. Type species: Pimelodus melanogaster Bleeker, 1854. Type by present designation. Gender: Masculine.

Sosia Vaillant, 1902: 81. Type species: Sosia chamaeleon Vaillant, 1902. Type by monotypy. Gender: Feminine.

Revision: $\mathrm{Ng} \& \mathrm{Ng}$ (2001).

Remarks: Earlier statements (e. g., Eschmeyer, 1990, 1998; Ng \& Ng, 2001) indicating that the type of Acrochordonichthys as A. platycephalus Bleeker, 1858, are in error. They were based on a type designation in Bleeker (1862-63) which was of a species that was not among the available named species when Acrochordonichthys was proposed. Apparently, no valid designation of a type species have been made before now.

Acrochordonichthys chamaeleon (Vaillant, 1902)

Sosia chamaeleon Vaillant, 1902: 82, figs. 19-21. Type locality: Borneo: Raoen/Mandai. Lectotype: RMNH 7849, designated by $\mathrm{Ng} \& \mathrm{Ng}$ (2001: 406).

Distribution: Kapuas River basin, western Borneo (Ng \& Ng, 2001).

Acrochordonichthys falcifer $\mathrm{Ng} \& \mathrm{Ng}, 2001$

Acrochordonichthys falcifer $\mathrm{Ng} \& \mathrm{Ng}$, 2001: 408, figs. 5-6. Type locality: Borneo: Sabah, Sungai Segama. Holotype: ZRC 43321.

Distribution: Kinabatangan and Segama River basins, northeastern Borneo (Ng \& Ng, 2001).

Acrochordonichthys guttatus $\mathrm{Ng} \& \mathrm{Ng}, 2001$

Acrochordonichthys guttatus Ng \& Ng, 2001: 394, figs. 6-8, 10. Type locality: Borneo: Kalimantan Tengah, Barito River drainage, Sungai Barito at Muara Laung. Holotype: MZB 9301.

Distribution: Barito River basin, southern Borneo ( $\mathrm{Ng} \& \mathrm{Ng}, 2001)$.

Acrochordonichthys gyrinus Vidthayanon \& Ng, 2003

Acrochordonichthys gyrinus Vidthayanon \& Ng, 2003: 2, fig. 1. Type locality: Thailand: Phitsanulok Province, Yom River at Primpiram; the Inland fishery Station of Phitsanulok Province. Holotype: NIFI 2645.

Distribution: Yom River, Chao Phraya basin, Thailand (Vidthayanon \& Ng, 2003).

Acrochordonichthys ischnosoma Bleeker, 1858

Acrochordonichthys ischnosoma Bleeker, 1858b: 232. Type locality: Java (Parongkalong provinciae Preanger), in flumine Tjitarum. Holotype (115 mm TL): BMNH 1863.12.4.151, illustrated in Bleeker (1862-63: pl. 84 [=Silur. pl. 36], fig. 4), and $\mathrm{Ng} \& \mathrm{Ng}$ (2001: fig. 4).

Distribution: Sumatra and Java (Ng \& Tan, 2002).

Remarks: Redescribed in Ng \& Tan (2002). Catalog number of holotype of Acrochordonichthys ischnosoma incorrectly reported as BMNH 1863.12.11.151 in Eschmeyer et al. (1998) and $\mathrm{Ng} \& \mathrm{Ng}$ (2001).

Acrochordonichthys mahakamensis $\mathrm{Ng} \& \mathrm{Ng}, 2001$

Acrochordonichthys mahakamensis Ng \& Ng, 2001: 400, figs. 6-7, 11. Type locality: Borneo: Kalimantan Timur, Mahakam River, boulders upstream of Melak ( $\left.0^{\circ} 12^{\prime} \mathrm{S}, 115^{\circ} 47^{\prime} \mathrm{E}\right)$. Holotype: MZB 5952.

Distribution: Mahakam River, eastern Borneo (Ng \& Ng, 2001). 
Acrochordonichthys pachyderma Vaillant, 1902

Acrochordonichthys pachyderma Vaillant, 1902: 66, figs. 11-13. Type locality: Bloeoe, Bornéo central. Holotype: RMNH 7843 (1).

Distribution: Kapuas, Mahakam and Kinabatangan River basins, Borneo (Ng \& Ng, 2001).

Remarks: RMNH 7560, from the Bo River, is incorrectly listed as a syntype in $\mathrm{Ng} \& \mathrm{Ng}$ (2001) and Eschmeyer $e t$ al. (1998).

Acrochordonichthys rugosus (Bleeker, 1847)

Pimelodus rugosus Bleeker, 1847b: 11 (and 1847a: 168). Type locality: Java. Possible syntypes: BMNH 1863.12.4.150 (1), BMNH 1863.12.4.161 (1), RMNH 6883 (2).

Pimelodus melanogaster Bleeker, 1854a: 89. Type locality: Sumatra orientalis, provinc. Palembang, ubi confluunt flumina Lamatang et Enim. Holotype (110 mm TL): possibly BMNH 1863.12.4.160 (see Ng \& Ng: 2001: 413); holotype illustrated in Bleeker (1862-63: 67, pl. 84 [= Silur. 36], fig. 3) as Acrochordonichthys melanogaster.

Pimelodus pleurostigma Bleeker, 1854b: 442. Type locality: Batavia, in fluviis. Syntypes (8, 48-76 mm TL): BMNH 1863.12.4.161 (1), NMV 45993 (1), RMNH 4188 (1), RMNH 6879 (4).

Pimelodus zonatus Bleeker, 1854b: 444. Type locality: Batavia, in fluviis. Syntypes (9, 54-80 mm TL): BMNH 1863.12.4.175 (1), NMV 45992 (1), RMNH 6880 (5). One syntype illustrated in Bleeker (1862-63: 69, pl. 84 [=Silur. 36], fig. 5), as Acrochordonichthys zonatus.

Acrochordonichthys platycephalus Bleeker, 1858b: 224. Type locality: Sumatra (Palembang) in fluviis. Holotype (97 mm TL): BMNH 1863.12.4.152; illustrated in Bleeker (1862-63: pl. 84 [= Silur. 36], fig. 2).

Sosia chamaeleon pallida Vaillant, 1902: 87, figs. 22-24. Type locality: Kapoeas, la plupart du haut du Fleuve (Sous-bassins du Mandaiet du Sibau), Bornéo central. Lectotype: RMNH 7848, designated by $\mathrm{Ng} \& \mathrm{Ng}$ (2001: 406). Originally Sosia chamaeleon var. pallida.

Acrochordonichthys Büttikoferi Popta, 1904: 188. Type locality: le Bongan et le Bo, Bornéo central. Syntypes: RMNH 7557 (1), RMNH 7558 (2); described in more detail, with an illustration of one of the syntypes, in Popta (1906: pl. 3, figs. 12a, 12b).

Acrochordonichthys obscurus Popta, 1904: 187. Type locality: le Bongan, Bornéo central. Holotype: RMNH 7556; described in more detail, with an illustration of the holotype, in Popta (1906: 58, pl. 3, figs. 11a, 11b).

Acrochordonichthys varius Popta, 1904: 189. Type locality: le Bo, Bornéo central. Syntypes: RMNH 7559 (2); described in more detail, with an illustration of one of the syntypes, in Popta (1906: 63, pl. 4, figs. 13a, 13b).

Distribution: Solo, Ciliwung, Citarum River basins, Java; Barito, Kapuas, Mahakam and Rajang River basins, Borneo; Bernam, Terengganu, Mae Nam Sungai Kolok and Pattani River basins, peninsular Malaysia and Thailand; Musi and Tulangbawan River basins, Sumatra ( $\mathrm{Ng} \& \mathrm{Ng}$, 2001).

Remarks: See comments in $\mathrm{Ng} \& \mathrm{Ng}$ (2001: 413) regarding the difficulty in identifying types of species named by Bleeker. Register number of BMNH types of Pimelodus rugosus, P. pleurostigma, P. melanogaster, P. zonatus, and A. platycephalus incorrectly reported in Eschmeyer et al. (1998) and $\mathrm{Ng} \& \mathrm{Ng}$ (2001).

Acrochordonichthys septentrionalis $\mathrm{Ng} \& \mathrm{Ng}, 2001$

Acrochordonichthys septentrionalis $\mathrm{Ng} \& \mathrm{Ng}$, 2001: 401, figs. 6, 12. Type locality: Thailand: Kanchanaburi Province, Kwae Noi River. Holotype: NIFI 3128.

Distribution: Mae Khlong River basin, Thailand, and Pahang River basin, Malaysia (Ng \& Ng, 2001).

Acrochordonichthys strigosus $\mathrm{Ng} \& \mathrm{Ng}, 2001$

Acrochordonichthys strigosus Ng \& Ng, 2001: 403, figs. 5-6, 8, 13. Type locality: Borneo: Kalimantan Barat, Sungai Sibau, Mouth of Sungai Putan to $3 \mathrm{~km}$ downstream (102'28.0"N, 11259'58.6"E). Holotype: MZB 9333.

Distribution: Kapuas River basin, western Borneo (Ng \& Ng, 2001).

AKYSIS Bleeker, 1858

Akysis Bleeker, 1858b: 204, 234 (and 1858d: 419). Type species: Pimelodus variegatus Bleeker, 1846. Type by monotypy. Gender: Masculine.

Reviews: Ng \& Kottelat (1998c, Indochina, with key to species); Ng (1999e, Myanmar).

Akysis brachybarbatus Chen, 1981

Akysis brachybarbatus Chen, in He \& Chen, 1981: 210, fig. 2. Type locality: Nanrui River, Mekong River system, 
Manglian Co., Yunnan, China. Holotype: KIZ 787162.

Distribution: Lancangjiang [= Mekong River] tributaries, China (Chu et al., 1999).

Akysis clavulus $\mathrm{Ng} \&$ Freyhof, 2003

Akysis clavulus Ng \& Freyhof, 2003: 312, figs. 1-2. Type locality: Vietnam: Khanh Hoa province: Song Duc My about $10 \mathrm{~km}$ west of Ninh Hoa; 12³2.22'N, 10901.38'E. Holotype: ZFMK 27159.

Distribution: Cai and Duc My rivers, central Vietnam (Ng \& Freyhof, 2003).

Akysis clinatus Ng \& Rainboth, 2005

Akysis clinatus Ng \& Rainboth, 2005: 34, fig. 1. Type locality: Cambodia: Stung Chhay River, Tok Kong village on road from Phnom Penh to Skhanoukville, 1056'37.8"N 11347'47.4"E. Holotype: ZRC 47288.

Distribution: Stung Chhay River, a Gulf of Thailand draining basin, Cambodia (Ng \& Rainboth, 2005: 35).

Akysis ephippifer $\mathrm{Ng} \&$ Kottelat, 1998

Akysis ephippifer Ng \& Kottelat, 1998c: 1078, fig. 11. Type locality: Cambodia: Stung Santréa, 40 km from Pomnac. Holotype: MNHN 1977-0034.

Distribution: Xe Kong basin, Lower Mekong drainage, Laos and Cambodia (Ng \& Kottelat, 1998c).

Akysis fuliginatus $\mathrm{Ng} \&$ Rainboth, 2005

Akysis fuliginatus Ng \& Rainboth, 2005: 35, fig. 4. Type locality: Cambodia: Stung Treng province, Mekong River on W edge of Kaoh Han, 16 km NE of Stung Treng, 1338'N 106³'E. Holotype: UMMZ 241338.

Distribution: Lower Mekong River basin, Cambodia (Ng \& Rainboth, 2005: 37).

Akysis hendricksoni Alfred, 1966

Akysis hendricksoni Alfred, 1966: 467, fig. 1. Type locality: Terengganu River at Kuala Brang, Terengganu, Malaya. Holotype: ZRC 1414.

Distribution: Sungai Golok, Sungai, Terengganu, Sabak Bernam, and Endau basins, peninsular Malaysia and Thailand ( $\mathrm{Ng} \& \mathrm{Tan}, 1999)$.

Akysis heterurus $\mathrm{Ng}, 1996$

Akysis heterurus Ng, 1996: 4, figs. 1-2. Type locality: Sumatra: Jambi, Sg. Alai at 19.5 km Muara Bungo-Muara Tebo road. Holotype: ZRC 34194.

Distribution: Batang Hari River basin, eastern Sumatra (Ng, 1996).

Akysis maculipinnis Fowler, 1934

Akysis maculipinnis Fowler, 1934a: 97, fig. 46. Type locality: Chantaboon, Southeast Siam. Holotype: ANSP 59366.

Distribution: Rivers of southeastern Thailand (Ng \& Kottelat, 1998c).

Akysis microps Ng \& Tan, 1999

Akysis microps Ng \& Tan, 1999: 359, fig. 5. Type locality: Malaysia: Johor, Sungai Kahang and tributary ca. 44.4 $\mathrm{km}$ from Mersing turnoff to Kluang just before side road to Endau Rompin Taman Negara, km 96 from Mersing to Batu Pahat (2³.93'N, 103³1.58'E). Holotype: ZRC 42596.

Distribution: Endau River basin, southeastern Malay Peninsula (Ng \& Tan, 1999).

Akysis pictus Günther, 1883

Akysis pictus Günther, 1883: 138. Type locality: Tenasserim [Burma]. Syntypes: BMNH 1880.12.1.25-26 (2); illustrated in $\mathrm{Ng}$ (1999e: 542, fig. 1).

Distribution: Southeastern Myanmar, in former Tenasserim district; known only from types (Ng, 1999e).

Remarks: Redescribed in Ng (1999e).

Akysis prashadi Hora, 1936

Akysis variegatus variegatus Prashad \& Mukerji, 1929: 180, pl. 8 (figs. 1-2). Type locality: South end of Indawgyi Lake, along sw. shore, Myitkyina Dist., Upper Burma. Holotype: ZSI F10873/1. Preoccupied in Akysis by Pimelodus variegatus Bleeker, 1846; replaced by Akysis prashadi Hora, 1936.

Akysis prashadi Hora, 1936a: 200, figs. 1, 2 (b-c). Type locality: South end of Indawgyi Lake, along sw. shore, Myitkyina Dist., Upper Burma. Holotype: ZSI F10873/1.

Distribution: Indawgyi Lake and northern Irrawaddy River basin, Myanmar (Ng, 1999e).

Remarks: Redescribed in Ng (1999e).

Akysis recavus $\mathrm{Ng} \&$ Kottelat, 1998

Akysis recavus $\mathrm{Ng} \&$ Kottelat, 1998c: 1090, fig. 16. Type locality: Thailand: Western Chao Phraya basin. Holotype: 
ZRC 40716.

Distribution: Western Chao Phraya basin, Thailand (Ng \& Kottelat, 1998c).

Akysis variegatus (Beeker, 1846)

Pimelodus variegatus Bleeker, 1846: 177. Type locality: Batavia. Syntypes: BMNH 1863.12.4.187 (1), possibly

NMV 46004 (1), RMNH 6881 (16).

Distribution: Java.

Remarks: Register number of syntype of Akysis variegatus incorrectly reported as BMNH 1863.12.11.187 in

Eschmeyer et al. (1998).

Akysis varius $\mathrm{Ng} \&$ Kottelat, 1998

Akysis varius Ng \& Kottelat, 1998c: 1093, fig. 18. Type locality: Xe Bangfai, ca 3 km upriver of Pan Pakphanang,

Khammouan Prov., Laos. Holotype: ZRC 41015.

Distribution: Mekong River basin, Laos, Cambodia and Thailand (Kottelat, 2001).

Akysis vespa $\mathrm{Ng} \&$ Kottelat, 2004

Akysis vespa $\mathrm{Ng}$ \& Kottelat, 2004: 194, fig. 1. Type locality: Myanmar: Kayin State, stream “Chon Son" between

Kyondaw and Phadaw, about $20 \mathrm{~km}$ NW of Payathouzu (at border with Thailand), $15^{\circ} 25^{\prime} \mathrm{N}, 9^{\circ} 15^{\prime} \mathrm{E}$. Holotype:

ZRC 46423.

Distribution: Ataran River basin, Myanmar (Ng \& Kottelat, 2004).

BREITENSTEINIA Steindachner, 1881

Breitensteinia Steindachner, 1881a: 46. Type species: Breitensteinia insignis Steindachner, 1881. Type by monotypy.

Gender: Feminine. Also reported as new in Steindachner (1881e: 213).

Revision: Ng \& Siebert (1998).

Breitensteinia cessator $\mathrm{Ng} \&$ Siebert, 1998

Breitensteinia cessator Ng \& Siebert, 1998: 647, fig. 1. Type locality: Borneo: Kapuas mainstream $58 \mathrm{~km}$ NE of Sintang and $1 \mathrm{~km}$ downstream from Sebruang, $\left(0^{\circ} 25.5^{\prime} \mathrm{N}, 111^{\circ} 52.5^{\prime} \mathrm{E}\right)$. Holotype: MZB 3693.

Distribution: Batang Hari and Tulangbawan basins, Sumatra, and Kapuas River basin, western Borneo (Ng \& Siebert, 1998).

Breitensteinia hypselurus $\mathrm{Ng} \&$ Seibert, 1998

Breitensteinia hypselurus Ng \& Seibert, 1998: 650, figs. 3, 4a. Type locality: Borneo: Sanggau. Holotype: RMNH 16048.

Distribution: Kapuas River basin, western Borneo (Ng \& Siebert, 1998).

Breitensteinia insignis Steindachner, 1881

Breitensteinia insignis Steindachner, 1881a: 46. Type locality: Teweh [= Moara Teweh, Barito River, Borneo]. Holotype: NMW 55042, illustrated in Steindachner (1881f: pl. 1, fig. 2) and described in more detail in Steindachner (1881e: 213).

Distribution: Barito River basin, southern Borneo (Ng \& Siebert, 1998).

PARAKYSIS Herre, 1940

Parakysis Herre, 1940: 11. Type species: Parakysis verrucosa Herre, 1940. Type by original designation. Gender: Masculine. Name treated by Herre as feminine, but subsequently treated as masculine ( $\mathrm{Ng} \& \mathrm{Lim}, 1995)$, presumably following the gender of Akysis.

Revision: $\mathrm{Ng} \&$ Lim (1995).

Parakysis anomalopteryx Roberts, 1989

Parakysis anomalopteryx Roberts, 1989a: 142. Type locality: Kapuas basin, Sungai Seriang, a forested tributary of the Sungai Palin, $37 \mathrm{~km}$ W of Putussibau, Western Borneo (Kalimantan Barat, Indonesia). Holotype: MZB 3702 .

Distribution: Kapuas River basin, western Borneo (Ng \& Lim, 1995).

Parakysis grandis $\mathrm{Ng} \& \mathrm{Lim}, 1995$

Parakysis grandis Ng \& Lim, 1995: 260, fig. 7. Type locality: Sumatra: Riau Province, small stream with grassy 
banks near Pangkalankasai, 43 km. south of Rengat. Holotype: ZRC 39111.

Distribution: Kapuas and Kuching River basins, Borneo; Deli, Indragiri, and Batang Hari basins, Sumatra (Ng \& Lim, 1995).

Parakysis longirostris Ng \& Lim, 1995

Parakysis longirostris Ng \& Lim, 1995: 262, fig. 8. Type locality: Singapore: Nee Soon swamp forest. Holotype: ZRC 34491.

Distribution: Singapore, peninsular Malaysia and Riau Archipelago (Ng \& Lim, 1995; Ng \& Tan, 1999).

Parakysis notialis Ng \& Kottelat, 2003

Parakysis notialis Ng \& Kottelat, 2003: 48, figs. 1, 2a, 3a. Type locality: Borneo: Kalimantan Selatan, Barito River drainage, area of Tamiyang Layang, 201'S, 11507'E. Holotype: MZB 5994.

Distribution: Barito River basin, southern Borneo (Ng \& Kottelat, 2003).

Parakysis verrucosus Herre, 1940

Parakysis verrucosa Herre, 1940: 12, pl. 6. Type locality: Mawai District, Johore, Malaysia. Holotype: SU 33009.

Distribution: Peninsular Malaysia and Riau Archipelago (Ng \& Lim, 1995).

\section{PSEUDOBAGARIUS new genus}

Type species: Akysis pseudobagarius Roberts, 1989. Gender: Masculine.

Diagnosis: Akysidae with the following combination of characters: snout extending well anterior of margin of lower jaw, rendering mouth subterminal; narial openings relatively large and closely set; and caudal fin deeply forked.

Remarks: This genus corresponds to the "pseudobagarius group" of species of the genus Akysis that was first recognized by $\mathrm{Ng} \&$ Kottelat (1998).

Pseudobagarius alfredi (Ng \& Kottelat, 1998)

Akysis alfredi $\mathrm{Ng} \&$ Kottelat, 1998c: 1063, fig. 5. Type locality: Malaysia: Pahang, Tasek Bera, approx. 300 m E of Fort Iskandar. Holotype: ZRC 40714.

Distribution: Tasek Bera, southern Malay Peninsula (Ng \& Kottelat, 1998c).

Pseudobagarius baramensis (Fowler, 1905)

Akysis baramensis Fowler, 1905: 472, fig. 4. Type locality: Baram river, Borneo. Holotype: ANSP 114887.

Distribution: Baram River basin, Borneo (Fowler, 1905).

Pseudobagarius filifer (Ng \& Rainboth, 2005)

Akysis filifer Ng \& Rainboth, 2005: 37, fig. 5. Type locality: Cambodia: Kandal, Tonle Sap, fishing lot 8, 22 miles

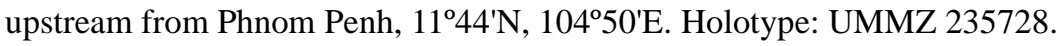

Distribution: Tonlé Sap drainage, Mekong River basin, Cambodia (Ng \& Rainboth, 2005: 39).

Pseudobagarius fuscus (Ng \& Kottelat, 1996)

Akysis fuscus Ng \& Kottelat, 1996: 20, fig. 1. Type locality: Borneo: Kalimantan Barat, Danau Sentarum area, Sungai Hulu Leboyan at Keluwin. Holotype: MZB 5934.

Distribution: Kapuas River basin, western Borneo (Ng \& Kottelat, 1996).

Pseudobagarius hardmani (Ng \& Sabaj, 2005)

Akysis hardmani Ng \& Sabaj, 2005: 218, fig. 3. Type locality: Thailand: Nakhon Sawan, Mae Nam Chao Phraya and tributaries in the vicinity of Phayuka Khiri. Holotype: ANSP 178858.

Distribution: Chao Phraya River basin, Thailand (Ng \& Sabaj, 2005).

Pseudobagarius inermis (Ng \& Kottelat, 2000)

Akysis inermis Ng \& Kottelat, 2000b: 9, figs. 1, 2a. Type locality: Laos: Attapeu province: unnamed creek entering Xe Kaman from the north at proposed Xe Kaman dam site, 1457'40"N, 10709'16"E. Holotype: ZRC 45401.

Distribution: Xe Banghiang and Xe Kong basins, Mekong River drainage, southern Laos (Ng \& Kottelat, 2000b).

Pseudobagarius leucorhynchus (Fowler, 1934)

Akysis leucorhynchus Fowler, 1934a: 97, figs. 44-45. Type locality: Chieng Mai, North Siam. Holotype: ANSP 59346.

Distribution: Chao Phraya basin, and rivers of southern peninsular Thailand (Ng \& Kottelat, 1998c).

Pseudobagarius macronemus (Bleeker, 1860)

Akysis macronema Bleeker, 1860: 11. Type locality: Lahat [Sumatra]. Syntypes (4: 36-44 mm TL): BMNH 
1863.12.4.188 (1), RMNH 6729 (2); one syntype illustrated in Bleeker (1862-63: 65, pl. 84 [= Silur. 36], fig. 1). Distribution: Sumatra (Tan \& Ng, 2000) .

Remarks: Redescribed in Tan \& Ng (2000) from specimens from the Batang Hari basin of central Sumatra. Register number of holotype of Akysis macronema incorrectly reported as BMNH 1863.12.11.188 in Eschmeyer et al. (1998).

Pseudobagarius meridionalis (Ng \& Siebert, 2004)

Akysis meridionalis Ng \& Siebert, 2004: 2, figs. 1, 2a. Type locality: Indonesia, Kalimantan Tengah (Borneo); Barito

River drainage, Sungai Laung at Dessa Maruwei (0²1.986'S, 11444.103'E). Holotype: MZB 6102.

Distribution: Barito River basin, Borneo (Ng \& Siebert, 2004).

Pseudobagarius nitidus (Ng \& Rainboth, 2005)

Akysis nitidus Ng \& Rainboth, 2005: 40, fig. 8. Type locality: Laos: Champasak province, Mekong River at Ban

Hang Khone, just downstream from Khone falls. Holotype: UMMZ 235400.

Distribution: Mekong River basin, Laos (Ng \& Rainboth, 2005).

Pseudobagarius pseudobagarius (Roberts, 1989)

Akysis pseudobagarius Roberts, 1989a: 138, fig. 107. Type locality: Bar in mouth of Sungai Melawi at Sintang,

Western Borneo (Kalimantan Barat, Indonesia). Holotype: MZB 3691.

Distribution: Moesi and Kapuas Rivers, Western Borneo (Roberts, 1989a).

Pseudobagarius similis (Ng \& Kottelat, 1998)

Akysis similis Ng \& Kottelat, 1998c: 1074, figs. 4e, 9, 13c. Type locality: Vietnam: Kihn Thuy Cai canal, $10 \mathrm{~km}$ E of

Vinh Long, at mouth of canal into Mekong. Holotype: UMMZ 214902.

Distribution: Mekong River mouth, southern Vietnam (Ng \& Kottelat, 1998c).

Remarks: Coloration of adults and ontogenetic change from juvenile color pattern discussed in $\mathrm{Ng} \& \mathrm{Rainboth}$ (2005: 41).

Pseudobagarius sinensis (He, 1981)

Akysis sinensis He, in He \& Chen, 1981: 209, fig. 1. Type locality: Lancang Jiang, Xishuangbanna Daizu Zizhizhou,

Yunnan Prov., China. Syntypes: IHASW 638237 (1), IHASW 638283 (1), KIZ 735007 (1), KIZ 735008 (1).

Also as Akysis sinesis once in English summary (p. 213); first reviser apparently Eschmeyer et al. (1998).

Distribution: Lancangjiang [= Mekong River], Yunnan, China (Chu et al., 1999).

\section{AMBLYCIPITIDAE Day, 1873}

Amblycepinae Day, 1873a: cclxviii. Type genus: Amblyceps Blyth, 1858.

Identification guide: Jayaram (1977), South Asia.

Phylogeny and key to genera: Chen \& Lundberg (1995).

Review: Choo (1987), Korea.

3 genera, 27 species; no named fossil taxa.

AMBLYCEPS Blyth, 1858

Amblyceps Blyth, 1858: 281. Type species: Amblyceps caecutiens Blyth, 1858. Type by monotypy. Gender: Neuter. Branchiosteus Gill, 1861c: 52. Olyra laticeps M'Clelland, 1842. Type by original designation. Gender: Masculine.

Review: Hora (1933) south Asia; Ng \& Kottelat (2000a), Indochinese species.

Remarks: Ng \& Kottelat (2000a: 346) list Amblyceps inermis as a nomen nudem published first in M'Clelland (1842). Amblyceps deyi is listed in Tamang (1993: 7), as a name only and is therefore not available.

Amblyceps apangi Nath \& Dey, 1989

Amblyceps apangi Nath \& Dey, 1989: 2, fig. 2. Type locality: Dikrong, Arunachal Pradesh, India. Holotype: F/GUZ 1215.

Distribution: Rivers draining Arunachal Pradesh, India (Nath \& Dey, 1989).

Amblyceps arnunachalensis Nath \& Dey, 1989

Amblyceps arnunachalensis Nath \& Dey, 1989: 3, fig. 3. Type locality: Dikrong, Arunachal Pradesh, India. Holo- 
type: F/GUZ 1213.

Distribution: Rivers draining Arunachal Pradesh, India (Nath \& Dey, 1989).

Amblyceps caecutiens Blyth, 1858

Amblyceps cacutiens Blyth, 1858: 282. Type locality: Maulmein and Pegu [Myanmar]. Syntypes: Whereabouts unknown. Originally: Amb. cacutiens.

Akysis kurzii Day, 1872: 703. Type locality: Pegu Yomas, Burma. Holotype: Whereabouts unknown.

Distribution: Bago River basin, Myanmar (Ng \& Kottelat, 2000a).

Amblyceps carinatum $\mathrm{Ng}, 2005$

Amblyceps carinatum Ng, 2005d: 243, fig. 1. Type locality: Myanmar: Kachin, Irrawaddy drainage, hillstreams at Tonpan village, on road from Myitkyina to Tanai. Holotype: UMMZ 245588.

Distribution: Middle Irrawaddy River basin, Myanmar (Ng, 2005d).

Amblyceps foratum $\mathrm{Ng} \&$ Kottelat, 2000

Amblyceps foratum Ng \& Kottelat, 2000a: 338, fig. 4. Type locality: Malaysia: Terengganu, Sungai Brang just outside Sekayu Waterfall Park; 457'51"N, 10257'46"E. Holotype: ZRC 43718.

Distribution: Northern peninsular Malaysia, southern peninsular Thailand, southeastern Thailand, and coastal Cambodia (Ng \& Kottelat, 2000a).

Amblyceps laticeps (M'Clelland, 1842)

Olyra laticeps M'Clelland, 1842: 588, pl. 21 (fig. 2). Type locality: Kasyah mountains. Holotype: apparently not preserved.

Distribution: South Asia, but distribution not well documented.

Remarks: Inclusion in Amblyceps follows Vinciguerra (1890), Hora (1936a), and Ng \& Kottelat (2000a).

Amblyceps macropterus $\mathrm{Ng}, 2001$

Amblyceps macropterus Ng, 2001b: 202, fig. 1. Type locality: Pakistan: Baluchistan Province: Las Bela District: Hab River at Hinidan. Holotype: UMMZ 187411.

Distribution: Hab River, southwestern Pakistan; known only from the holotype (Ng, 2001b).

Amblyceps mangois (Hamilton, 1822)

Pimelodus mangois Hamilton, 1822: 199, 379. Type locality: Tanks of Northern Behar [India]. No types known. Previously unpublished Hamilton illustration of a type reproduced in Hora (1929: pl. 22, fig. 4).

Distribution: South Asia; precise distribution unknown inasmuch as literature accounts of this species may refer to almost any of the species in this genus.

Amblyceps mucronatum $\mathrm{Ng} \&$ Kottelat, 2000

Amblyceps mucronatum Ng \& Kottelat, 2000a: 340, fig. 6. Type locality: Thailand: Loei Prov.: Mekong River basin, Huang River tributary at $17^{\circ} 16^{\prime} 0.9^{\prime \prime} \mathrm{N}, 101^{\circ} 08^{\prime} 29.2^{\prime \prime E}$. Holotype: ZRC 41323.

Distribution: Mekong River basin, Thailand, and Salween River basin, Thailand and Myanmar (Ng \& Kottelat, 2000a).

Amblyceps murraystuarti Chaudhuri, 1919

Amblyceps murray-stuarti Chaudhuri, 1919: 272, pl. 22 (figs. 1, 1a, 1b). Type locality: A mountain stream in the Putao Plains in the northern Frontier of Upper Burma. Holotype: ZSI F9736/1.

Distribution: Upper Irrawaddy River basin, Myanmar (Ng \& Kottelat, 2000a).

Amblyceps platycephalus $\mathrm{Ng} \&$ Kottelat, 2000

Amblyceps platycephalus Ng \& Kottelat, 2000a: 343, fig. 8. Type locality: Thailand: Mae Hong Son Province: Nam Mae Pai basin, Nam Mae Sa-Nga at Ban Yang Top Sole, immediately downstream of Nam Tok Pa Sua, $19^{\circ} 29^{\prime} 0 " N$, 9757'50"E. Holotype: ZRC 42745.

Distribution: Salween River, Thailand, known only from the holotype (Ng \& Kottelat, 2000a).

Amblyceps serratum $\mathrm{Ng} \&$ Kottelat, 2000

Amblyceps serratum Ng \& Kottelat, 2000a: 344, fig. 9. Type locality: Cambodia: Stung Treng province: shallow channel across S end of Kaoh Han, 14 km NE of Stung Treng (133' N, 10603'E). Holotype: UMMZ 234708.

Distribution: Mekong River drainage of central and southern Laos and Cambodia (Ng \& Kottelat, 2000a).

Amblyceps tenuispinis Blyth, 1860

Amblyceps tenuispinis Blyth, 1860: 153. Type locality: Gházipur [India]. Holotype: Whereabouts unknown. 
Distribution: Gházipur, India.

Amblyceps variegatum $\mathrm{Ng} \&$ Kottelat, 2000

Amblyceps variegatum Ng \& Kottelat, 2000a: 345, fig. 10. Type locality: Thailand: Kanchanaburi province, Mae Nam Khwae Noi basin, Nam Khung, upstream of Ban Huai Pak Khung, riffles between second ford and reservoir (14³7'20"N, 98³1'24"E). Holotype: ZRC 43719.

Distribution: Mae Khlong River basin, Thailand (Ng \& Kottelat, 2000a).

\section{Species inquirendae, Amblyceps}

Pimelodus indicus M'Clelland \& Griffith, in M'Clelland, 1842: 584. Type locality: Loodianah. Holotype: Whereabouts unknown, possibly at BMNH.

LIOBAGRUS Hilgendorf, 1878

Liobagrus Hilgendorf, 1878: 155. Type species: Liobagrus reinii Hilgendorf, 1878. Type by monotypy. Gender: Masculine.

Neobagrus Bellotti, 1892: 100. Type species: Neobagrus fuscus Bellotti, 1892. Type by monotypy. Gender: Masculine.

Remarks: Systematic position discussed in Jayaram (1965b).

Review: Choo (1987), Korea.

Key: Regan (1908g).

Liobagrus andersoni Regan, 1908

Liobagrus andersoni Regan, 1908g: 61, pl. 3 (fig. 4). Type locality: Kimhoa, 65 mi. north-east of Seoul, Corea. Syntypes: BMNH 1907.12.10.63-64 (2), BMNH 1907.12.10.65 (1, skeleton).

Distribution: Chongiu, Kwangiu, Soungchon, Kosong, and Ulchin, Korea (Mori, 1952).

Liobagrus anguillicauda Nichols, 1926

Liobagrus anguillicauda Nichols, 1926b: 1, fig. 1. Type locality: Chungan Hsien, nw. Fukien Prov., China. Holotype: AMNH 8474.

Distribution: Streams of southeast China (Chu et al., 1999).

Liobagrus formosanus Regan, 1908

Liobagrus formosanus Regan, 1908e: 360. Type locality: Lake Candidius, Formosa. Holotype: BMNH 1909.4.28.23.

Distribution: Taiwan (Chu et al., 1999).

Liobagrus kingi Tchang, 1935

Liobagrus kingi Tchang, 1935a: 95, fig. 1. Type locality: Tsin-ning, Yunnan, China. Holotype: at ASIZB [= ZMFMIB 11673].

Distribution: Upper Changjiang [= Yangtze River] (Chu et al., 1999).

Liobagrus marginatoides (Wu, 1930)

Amblyceps marginatoides Wu, 1930a: 256, fig. 2. Type locality: Sichuan, China. Syntypes (2): Whereabouts unknown.

Distribution: Upper Changjiang [= Yangtze River] (Chu et al., 1999).

Liobagrus marginatus (Günther, 1892)

Amblyceps marginatus Günther, 1892: 245, pl. 2 (fig. a). Type locality: Mountain streams running into Min River, Szechwan [Sichuan], China. Holotype: BMNH 1891.6.13.10.

Distribution: Upper Changjiang [= Yangtze River] (Chu et al., 1999).

Liobagrus mediadiposalis Mori, 1936

Liobagrus mediadiposalis Mori, 1936: 673, pl. 24 (fig. 3). Type locality: River Rakuto at Bun-kei, Chosen [Korea]. Holotype (130 mm TL): At Preparatory Department of Keijo Imperial University.

Distribution: Rivers of Natong, Kokson, Yongsan, Kim and Tchongchin, Korea (Mori, 1952).

Liobagrus nantoensis Oshima, 1919

Liobagrus nantoënsis Oshima, 1919: 183, pl. 48 (fig. 3). Type locality: Dainansho, Nanto, Taiwan. Lectotype: 
FMNH 59080, designated by Eschmeyer (in Eschmeyer et al., 1998: 1149).

Distribution: Taiwan (Chu et al., 1999).

Liobagrus nigricauda Regan, 1904

Liobagrus nigricauda Regan, 1904a: 193. Type locality: Sea of Tien [Tien Chih], Yunnan Fu, China, elev. $6000 \mathrm{ft}$.

Syntypes: BMNH 1904.1.26.42-43 (2).

Distribution: Changjiang [= Yangtze River] basin (Chu et al., 1999).

Liobagrus obesus Son, Kim \& Choo, 1987

Liobagrus obesus Son, Kim \& Choo, 1987: 22, figs. 1, 5b. Type locality: Kīm-river, Cho'gang-ri, Simch'ŏn-myŏn,

Yŏngdong, Ch'ungch'ŏngbug-do province, Korea. Holotype: BCUE 2101.

Distribution: Kum River basin, South Korea (Son et al., 1987).

Liobagrus reinii Hilgendorf, 1878

Liobagrus reinii Hilgendorf, 1878: 155. Type locality: Japan. No types known.

Liobagrus sugubii Regan, 1908b: 152. Type locality: Lake Biwa, Japan. Syntypes: BMNH 1907.12.23.56-59 (4).

Distribution: Honshu, Skikoku and Kyushu Islands, Japan (Matsuura et al., 2000).

Liobagrus styani Regan, 1908

Liobagrus styani Regan, 1908b: 152. Type locality: South Hupeh, China. Syntypes (2): BMNH 1902.5.30.2-3 (2, dry), BMNH 1902.5.30.4 (1, skeleton).

Distribution: Middle and lower Changjiang [= Yangtze River] (Chu et al., 1999).

\section{Species inquirenda, Liobagrus}

Neobagrus fuscus Bellotti, 1892: 101, unnumbered figure. Type locality: Jokohama [Japan]. MSNM (destroyed: Eschmeyer et al., 1998).

XIURENBAGRUS Chen \& Lundberg, 1995

Xiurenbagrus Chen \& Lundberg, 1995: 781. Type species: Liobagrus xiurenensis Yue, 1981. Type by original designation. Gender: Masculine.

Xiurenbagrus gigas Zhao, Lan \& Zhang, 2004

Xiurenbagrus gigas Zhao, Lan \& Zhang, 2004: 228, figs. 1-2. Type locality: Zhujiang River basin: Hongshuihe River, Anyang Town (23ำ $17^{\prime}$, 106 $29^{\prime}$ E), Du'an County, Guangxi, P.R. China. Holotype: ASIZB 72944.

Distribution: Hongshuihe River, Pearl [= Zhujiang] River basin, China (Zhao et al., 2004).

Xiurenbagrus xiurenensis (Yue, 1981)

Liobagrus xiurenensis Yue, in Zheng, 1981: 185, fig. 152. Type locality: Xiuren River, Pearl River drainage, Xiuren, Lipu, Guangxi, China, 24²8'N, 110²4'E. Holotype: ASIZB 751087.

Distribution: Zhujiang [= Pearl River] (Chu et al., 1999).

\section{AMPHILIIDAE Regan, 1911}

Amphiliidae Regan, 1911: 565. Type genus: Amphilius Günther, 1864.

Doumeinae Regan, 1911: 554. Type genus: Doumea Sauvage, 1879.

Leptoglaninae [sic, Leptoglanidinae] Roberts, 2003: 87. Type genus: Leptoglanis Boulenger, 1902.

Remarks: The name Amphiliidae takes precedence over the simultaneously-established Doumeinae by virtue of the higher rank initially given to the former, following Art. 24 of the Code. Roberts (2003) and Diogo (2003b) independently proposed the new subfamily name Leptoglanidinae (incorrectly formed as Leptoglaninae by Roberts) for groups of nearly identical membership. Roberts's name was published earlier (March 14, vs. May [31]) and therefore has priority. He et al. (1999) questioned the monophyly of the Amphiliidae as used herein and, instead, restricted the name to include only Amphilius and Paramphilus and included all other taxa in the Doumeidae.

Revision: Roberts (2003, Leptoglanidinae). 
Phylogeny: Harry (1953); He et al. (1999); Diogo (2003b).

Taxonomic summary: Skelton \& Teugels (1986); Skelton (1992, west Africa).

Key to genera: Poll \& Gosse (1994).

12 genera, 66 species; no named fossil taxa.

AMPHILIUS Günther, 1864

Amphilius Günther, 1864: 115, 134. Type species: Pimelodus platychir Günther, 1864. Type by monotypy. Originally proposed as a section of Pimelodus. Gender: Masculine.

Anoplopterus Pfeffer, 1889: 15. Type species: Anoplopterus uranoscopus Pfeffer, 1889. Type by monotypy. Gender: Masculine.

Chimarrhoglanis Vaillant, 1897: 81. Type species: Chimarrhoglanis leroyi Vaillant, 1897. Type by monotypy. Gender: Masculine.

Review: Skelton (1984, east and southern Africa).

Amphilius atesuensis Boulenger, 1904

Amphilius atesuensis Boulenger, 1904a: 17. Type locality: Atesu River, Gold Coast. Syntypes (4): BMNH 1903.4.24.74-77 (4).

Amphilius pictus Nichols \& La Monte, 1933a: 1, fig. 1. Type locality: Stream near Kaleata, Liberia. Holotype: AMNH 12313.

Distribution: Gbin River, Guinea, and Pra River, from Sierra Leone to Ghana (Skelton \& Teugels, 1986).

Amphilius baudoni Pellegrin, 1928

Amphilius Baudoni Pellegrin, 1928c: 453, unnumbered figure. Type locality: rivière Passa (affluent de l'Ogoôué),

Gabon. Syntypes (3): MNHN 1928-0224 (1), MNHN 1928-0225 (1), MNHN 1928-0226 (1). MRAC 20649 listed as a syntype in Eschmeyer et al. (1998), but not included in original description.

Amphilius baudoni uniformis Pellegrin, 1930: 200. Type locality: Riv. Koumbi (Ht. Ogôoué) ... Sangha. Syntypes (6): MNHN 1929-0238 (1), MNHN 1929-0239 (5). Originally as Amphilius baudoni var. uniformis.

Distribution: Ogowe River system and Sangha River, Congo basin (Skelton \& Teugels, 1986).

Amphilius brevis Boulenger, 1902

Amphilius brevis Boulenger, 1902g: 268, pl. 29 (fig. 3). Type locality: Lindi River, which flows into the Congo at the Stanley Falls. Syntypes (2): BMNH 1902.4.14.21 (1), IRSNB 11 (1).

Amphilius nigricaudatus Pellegrin, 1909a: 67. Type locality: Ngomo (Ogôoué). Syntypes (3): MNHN 1909-0014 (1), MNHN 1909-0015 (1), MNHN 1909-0016 (1). Also described as new in Pellegrin (1909d: 47).

Amphilius nigricaudatus multipunctata Pellegrin, 1909a: 67. Type locality: Ngomo (Ogôoué). Holotype: MNHN 1909-0013. Originally as Amphilius nigricaudatus var. multipunctata. Also described as new in Pellegrin (1909d: 48, fig. 2).

Distribution: Congo River system and Leome, Kouliou, and Ogowe rivers (Skelton \& Teugels, 1986).

Amphilius cryptobullatus Skelton, 1986

Amphilius cryptobullatus Skelton, 1986: 279, figs. 15-23. Type locality: Luongo River, Upper Zaire system, above and at Route 74 crossing, $10^{\circ} 11^{\prime}$ S, 29 43 'E, Zambia. Holotype: ROM 46800.

Distribution: Luongo River, upper Congo River basin, Zambia (Skelton, 1986).

Amphilius jacksonii Boulenger, 1912

Amphilius jacksonii Boulenger, 1912a: 602. Type locality: the Hima River, eastern foot-hills of Ruwenzori, 3500 feet, flowing into Lake George (Ruisamba), Nile System. Holotype: BMNH 1912.10.15.47; illustrated in Boulenger (1916: 307, fig. 182), and Seegers (1996: 191, fig. 133).

Distribution: Widely distributed in central and eastern Africa (Seegers, 1996).

Remarks: Species redescribed in Walsh et al. (2000).

Amphilius kakrimensis Teugels, Skelton \& Lévêque, 1987

Amphilius kakrimensis Teugels, Skelton \& Lévêque, 1987: 94, figs. 1-2. Type locality: a small tributary of the Kakrima River, Konkoure basin, at Kasseri (10¹6'N, 12²8'W), Guinea. Holotype: MNHN 1986-0599.

Distribution: Konkoure River basin, Guinea (Teugels et al., 1987).

Amphilius kivuensis Pellegrin, 1933 
Amphilius kivuensis Pellegrin, 1933b: 172. Type locality: Boulira, Kitembo (ouest du Kivu), ... Kilembo (ouest du Kivu, altitude 2000 mètres). Syntypes (8): MNHN 1932-0038 (5), MNHN 1933-0009 (1), MNHN $1933-0010$ (1), MNHN 1933-0011 (1).

Distribution: Rivers to west of Lake Kivu; Ruzizi River and rivers entering Lake Tanganyika (Skelton \& Teugels, 1986).

Amphilius lamani Lönnberg \& Rendahl, 1920

Amphilius lamani Lönnberg \& Rendahl, 1920: 171, figs. 2-3. Type locality: Kingoyi, Lower Congo. Syntypes: NRM 9910 (2).

Distribution: Lower Congo; known only from type locality (Skelton \& Teugels, 1986).

Amphilius lampei Pietschmann, 1913

Amphilius lampei Pietschmann, 1913b: 190, pl. 2 (fig. 1). Type locality: Harrar, Abessynien [Ethiopia]. Holotype: at Weisbaden Museums.

Distribution: Webi Shebeli tributaries, near Harrar, Ethiopia (Skelton \& Teugels, 1986).

Amphilius laticaudatus Skelton, 1984

Amphilius laticaudatus Skelton, 1984: 61, fig. Type locality: Buzi River, at bridge on Inchope to Lourenço Marques road (195 $55^{\prime}$ S, 34²15'E). Holotype: AMG P5815.

Distribution: Buzi River system, Moçambique (Skelton, 1993).

Amphilius lentiginosus Trewavas, 1936

Amphilius lentiginosus Trewavas, 1936: 71, pl. 2 (figs. 10-11). Type locality: a brook at Cuito, Mt. Moco, Luimbale, Cuvo River system, Angola. Syntypes: BMNH 1935.3.20.189-190 (2).

Distribution: Cuvo River system, Luachimo River and Tchimenji River, Angola (Skelton \& Teugels, 1986).

Amphilius longirostris (Boulenger, 1901)

Anoplopterus longirostris Boulenger, 1901f: 447. Type locality: Hills in the Bulu country, some 75 miles in the interior from Batanga, at an elevation of between 1500-2000 feet, Cameroon. Holotype: BMNH 1901.11.12.133.

Distribution: Cameroon and Equatorial Guinea, also tributaries of Ngoko, Congo River system, in Cameroon (Skelton \& Teugels, 1986).

Remarks: Specimen listed as holotype of Anoplopterus longirostris is entered in BMNH register with locality only as Efulen, and Boulenger (1911: 260) indicated that the locality stated in original description was near Efulen.

Amphilius maesii Boulenger, 1919

Amphilius Maesii Boulenger, 1919: 160. Type locality: Oshwe [Congo River system]. Syntypes (7): BMNH 1919.8.28.14-16 (3), MRAC 3180-83 (4).

Distribution: Congo River basin (Skelton \& Teugels, 1986).

Amphilius natalensis Boulenger, 1917

Amphilius natalensis Boulenger, 1917a: 432. Type locality: Krantz Kloof, Natal. Syntypes (2): BMNH 1917.7.21.1 (1).

Distribution: Ruo River, Malawi; Zambezi system of Zimbabwe, south to Umkomaas River (Natal, South Africa) (Skelton, 1993).

Remarks: Redescribed in Barnard (1943).

Amphilius opisthophthalmus Boulenger, 1919

Amphilius opisthophthalmus Boulenger, 1919: 159. Type locality: Oshwe, Lac Léopold II. Syntypes (7): BMNH 1919.8.28.17-18 (2), MRAC 3191-94 (4).

Distribution: Oshwe on the Lukenie River (Lake Mai-Ndombe) (Skelton \& Teugels, 1986).

Amphilius platychir (Günther, 1864)

Pimelodus platychir Günther, 1864: 134. Type locality: Sierra Leone. Syntypes (4): BMNH 2001.12.5.574-577 (4), MNHN 1898-0121 (1).

Amphilius grammatophorus Pellegrin, 1913a: 237, fig. 1. Type locality: Rivière Kikoulo, Guinée française. Syntypes: MNHN 1913-0231 (1), MNHN 1913-0232 (1), MNHN 1913-0233 (1), MNHN 1913-0234 (1), MNHN 1913-0235 (1).

Amphilius grammatophorus brevipinna Pellegrin, 1935: 465. Type locality: Banamanan (cercle de Kissidougou), ... Friguiagbé (près Kindia); Guinée française. Syntypes (2): MNHN 1935-0194 (1), MNHN 1935-0195 (1). Orig- 
inally as Amphilius grammatophorus var. brevipinna. nov. var.

Amphilius grammatophorus inaequalis Pellegrin, 1935: 464. Type locality: Friguiagbé (près Kindia) Guinée française. Syntypes (6): MNHN 1935-0191 (1), MNHN 1935-0192 (1), MNHN 1935-0193 (4). Originally as Amphilius grammatophorus var. inaequalis nov.var.

Amphilius grammatophorus marmoratus Daget, 1963: 584. Type locality: Kana, alt. 600 m, Mont Nimba. Holotype: MNHN 1960-0119.

Distribution: Sierra Leone, Guinea, Côte d'Ivoire (Skelton \& Teugels, 1986).

Amphilius pulcher Pellegrin, 1929

Amphilius pulcher Pellegrin, 1929c: 640, fig. 1. Type locality: rivière Louessé (fleuve Kouilou) [Gabon]. Syntypes (3): MNHN 1929-0224 (3). MRAC 20650 listed as a syntype in Eschmeyer et al. (1998), but not listed in description.

Amphilius pulcher ephippiata Pellegrin, 1930: 200. Type locality: Sebé (haut Ogoôué). Syntypes: MNHN 19290237 (9). Originally as Amphilius pulcher var. ephippiata nov. var.

Distribution: Ogowe and Nyanga River systems, Gabon (Skelton \& Teugels, 1986).

Amphilius rheophilus Daget, 1959

Amphilius rheophilus Daget, 1959: 692, fig. 3. Type locality: Gambie à Mpantié (parc national du Niokolo-Koba); Gambie à Banharé. Syntypes (3): MNHN 1959-0147 (2), MNHN 1960-0486 (1).

Distribution: Gambia River system (Skelton \& Teugels, 1986).

Amphilius uranoscopus (Pfeffer, 1889)

Anoplopterus uranoscopus Pfeffer, 1889: 16; illustrated in Pfeffer (1896: 31, fig. 14). Type locality: Bad bei Ushonda (Ungúu), ... Bäche bei Mhonda. Lectotype: ZMH 11944, selected by, and illustrated in, Seegers (1996: 192, fig. 137).

Chimarrhoglanis Leroyi Vaillant, 1897: 82. Type locality: Zanguebar, torrent de Mrogoro, par 600 mètres d'altitude. Holotype: MNHN 1897-0003.

Amphilius grandis Boulenger, 1905c: 63, pl. 7 (figs. 3, 3a). Type locality: Chania River of Tetse, Tana system, in cold water at an altitude of 7000 feet. Syntypes (3): BMNH 1904.12.23.50-52 (3).

Amphilius Hargeri Boulenger, 1907c: 488. Type locality: Mlangi, British Central Africa. Holotype: BMNH 1907.10.14.8.

Amphilius krefftii Boulenger, 1911a: 356, fig. 276. Type locality: Usambara, in rivulet running down from Anani Hills to Sigi River. Syntypes: BMNH 1909.10.19.26-27 (2).

Amphilius oxyrhinus Boulenger, 1912c: 675, pl. 80. Type locality: Eusso Mara, a swift mountain stream, tributary of Eusso Nyiro, British East Africa. Holotype: BMNH 1912.3.22.120.

Amphilius brevidorsalis Pellegrin, 1919a: 399. Type locality: Rivière Revue (Mozambique). Holotype: MNHN 1919-0488.

Amphilius platychir cubangoensis Pellegrin, 1936a: 56. Type locality: Cubango près de Vila da Ponte [Angola]. Syntypes (9): MHNG 856.86 (1), MNHN 1936-0101 (1), MNHN 1936-0102 (1), MNHN 1936-0103 (3), MRAC 138769, NMBA 5213 (1). Originally as Amphilius platychir var. cubangoensis.

Distribution: Okavango and Zambezi basins, eastern coastal Rivers south to the Mkuze basin, Natal, and throughout east and Central Africa (Skelton, 1993); Lake Rukwa basin (Seegers, 1996).

Remarks: The name Amphilius transvaaliensis that was published in the caption to figure 1 in van der Horst, (1931: 247) fails to qualify as an available name, following the provisions in Art. 13.1.1 of the Code. In addition, there is uncertainty about whether the name was intended to be published, inasmuch as copies of this paper at CAS and $\mathrm{CU}$ have the figure caption crossed out and replaced with the name Amphilius grandis.

Amphilius zairensis Skelton, 1986

Amphilius zairensis Skelton, 1986: 266, fig. 1. Type locality: River Lufu, about two km from confluence with Zaire

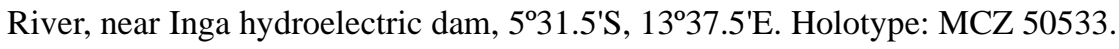

Distribution: Lufu River, Congo River basin (Skelton, 1986).

ANDERSONIA Boulenger, 1900

Andersonia Boulenger, 1900d: 528. Type species: Andersonia leptura Boulenger, 1900. Type by monotypy. Gender: 
Feminine.

Slatinia Werner, 1906: 326. Type species: Slatinia mongallensis Werner, 1906. Type by monotypy. Gender: Feminine.

Andersonia leptura Boulenger, 1900

Andersonia leptura Boulenger, 1900d: 529. Type locality: a pond near Koshek, Soudan. Holotype: BMNH 1907.12.2.2545.

Slatinia mongallensis Werner, 1906: 327. Type locality: Bahar-el-Gebel, bei Mongalla. Holotype: NMW 79741.

Andersonia brevior Boulenger, 1918: 426. Type locality: Shari River, Ubanghi-Shari Colony, French Equatorial Africa. Holotype: BMNH 1918.11.12.13.

Andersonia pellegrini Boulenger, 1918: 427. Type locality: Shari River, Ubanghi-Shari Colony, French Equatorial Africa. Holotype: BMNH 1918.11.12.13.

Distribution: Omo, Niger and Upper Nile Rivers, Lake Chad basin (Skelton \& Teugels, 1986).

Remarks: The name "Andersonia brevior sp. n." appears in the introduction of Boulenger (1918: 426) for a species described on the next page as A. pellegrini. Harry (1953) appears to be the first to have commented on the two names for the species and, therein, he treated A. pellegrini as valid.

BELONOGLANIS Boulenger, 1902

Belonoglanis Boulenger, 1902d: 50. Type species: Belonoglanis tenuis Boulenger, 1902. Type by monotypy. Gender: Masculine.

Belonoglanis brieni Poll, 1959

Belonoglanis brieni Poll, 1959: 107, pl. 22 (fig. 3). Type locality: Stanley Pool, Rivière N'Djili. Holotype: MRAC 118539.

Distribution: Congo River basin (Skelton \& Teugels, 1986).

Belonoglanis tenuis Boulenger, 1902

Belonoglanis tenuis Boulenger, 1902d: 50, pl. 14 (fig. 4). Type locality: Ubangi à Banzyville. Holotype: MRAC 1289.

Belonoglanis nudipectus Lönnberg \& Rendahl, 1920: 175, fig. 6. Type locality: Lower Congo. Holotype: NRM 16118.

Belonoglanis curvirostris Pellegrin, 1922a: 74. Type locality: Bangui (Oubanghi). Syntypes: MNHN 1921-0428 (1), MNHN 1921-0429 (1).

Distribution: Congo River basin (Skelton \& Teugels, 1986).

DOLICHAMPHILIUS Roberts, 2003

Dolichamphilius Roberts, 2003: 100. Type species: Leptoglanis brieni Poll, 1959. Type by original designation. Gender: Masculine.

Dolichamphilius brieni (Poll, 1959)

Leptoglanis brieni Poll, 1959: 96, pl. 24 (fig. 2). Type locality: Stanley Pool, passe devant le refuge Jipo. Holotype: MRAC 118503.

Distribution: Stanley Pool, Congo River (Roberts, 2003).

Dolichamphilius longiceps Roberts, 2003

Dolichamphilius longiceps Roberts, 2003: 100. Type locality: Republic du Congo (Kinshasa): Chutes Wagenia, Kisangani. Holotype: MRAC 90-47-P-704-708.

Distribution: Chutes Wagenia on the Lualaba River, near Kisangani (Roberts, 2003).

DOUMEA Sauvage, 1879

Doumea Sauvage, 1879: 96.Type species: Doumea typica Sauvage, 1879. Type by original designation. Gender: Feminine.

Doumea alula Nichols \& Griscom, 1917

Doumea alula Nichols \& Griscom, 1917: 722, fig. 27. Type locality: Stanleyville, Congo. Holotype: AMNH 6531. Distribution: Congo River basin (Skelton \& Teugels, 1986). 
Doumea angolensis Boulenger, 1906

Doumea angolensis Boulenger, 1906e: 347. Type locality: the interior of Benguella, at an altitude of 4000-5000 feet, Angola. Holotype: BMNH 1906.10.8.12.

Distribution: Quanza River system and the interior of Benguella, Angola (Skelton \& Teugels, 1986).

Doumea chappuisi Pellegrin, 1933

Doumea chappuisi Pellegrin, 1933a: 114, fig. on p. 115. Type locality: Danané (Côte d'Ivoire). Syntypes (8): MNHN 1932-0302 (1), NMBA 4281-87 (7).

Distribution: Guinea, Liberia and Côte d'Ivoire; Cavally, Nuon, St. Paul, and Koumba River systems (Skelton \& Teugels, 1986).

Doumea thysi Skelton, 1989

Doumea thysi Skelton, 1989: 6, fig. 3. Type locality: Cross River, 27 miles from Mamfe towards Eyomojak, Cameroon, 546'N, 903'E. Holotype: MRAC 76-32-P-2260.

Distribution: Cross River basin, Cameroon (Skelton, 1989).

Doumea typica Sauvage, 1879

Doumea typica Sauvage, 1879: 97. Type locality: Doumé [rivière Ogôoué]. Holotype: MNHN a-0965. Described in more detail and illustrated in Sauvage (1880c: 41, pl. 3, fig. 1).

Distribution: Congo River basin and coastal rivers from Cameroon to Congo (Skelton \& Teugels, 1986).

LEPTOGLANIS Boulenger, 1902

Leptoglanis Boulenger, 1902d: 42. Type species: Leptoglanis xenognathus Boulenger, 1902. Type by monotypy. Gender: Masculine.

Remarks: Roberts (2003) removed most species from Leptoglanis and placed them, instead, into Zairichthys.

Leptoglanis xenognathus Boulenger, 1902

Leptoglanis xenognathus Boulenger, 1902d: 43, pl. 14 (fig. 1). Type locality: l’Ubangi à Banzyville. Holotype: MRAC 1287.

Distribution: Congo basin.

PARAMPHILIUS Pellegrin, 1907

Paramphilius Pellegrin, 1907a: 23. Type species: Paramphilius trichomycteroides Pellegrin, 1907. Type by original designation. Gender: Masculine.

Paramphilius firestonei Schultz, 1942

Paramphilius firestonei Schultz, 1942: 328, 333, pl. 36 (fig. 3). Type locality: Bromley, Harbel [Liberia]. Holotype: USNM 118811.

Distribution: St. Paul, Du and Borlor Rivers, Liberia (Skelton \& Teugels, 1986).

Paramphilius goodi Harry, 1953

Paramphilius goodi Harry, 1953: 203, figs. 1-2. Type locality: Lolodorf, Bikui River, Lokunje River system, Cameroon, West Africa. Holotype: SU 16970.

Distribution: Lokunje River basin, Cameroon (Skelton \& Teugels, 1986).

Paramphilius teugelsi Skelton, 1989

Paramphilius teugelsi Skelton, 1989: 2, fig. Type locality: ruisseau affluent de Mamou (Little Scarcies River sys-

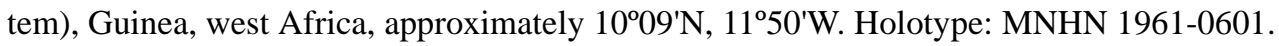

Distribution: Little Scarcies River basin, Guinea (Skelton, 1989).

Paramphilius trichomycteroides Pellegrin, 1907

Paramphilius trichomycteroides Pellegrin, 1907a: 24. Type locality: Ditinn (Fouta-Djalon). Holotype: MNHN 19060343.

Distribution: Senegal River headwaters, near Ditinn and Mamou, Guinea (Skelton \& Teugels, 1986).

Remarks: Holotype catalog number of Paramphilius trichomycteroides incorrectly reported as MNHN 1906-0243 in Eschmeyer et al. (1998). 
PHRACTURA Boulenger, 1900

Peltura Perugia, 1892: 972. Type species: Peltura bovei Perugia, 1892. Type by monotypy. Preoccupied by Peltura Agassiz, 1846, in trilobites; replaced by Phractura Boulenger, 1900. Gender: Feminine.

Phractura Boulenger, 1900d: 527. Type species: Peltura bovei Perugia, 1892. Type by being a replacement name. Replacement for Peltura Perugia, 1892, preoccupied by Peltura Agassiz, 1846. Gender: Feminine.

Paraphractura Boulenger, 1902d: 47. Type species: Paraphractura tenuicauda Boulenger, 1902. Type by monotypy. Gender: Feminine.

Phractura ansorgii Boulenger, 1902

Phractura ansorgii Boulenger, 1902h: 623, pl. 37 (fig. 1). Type locality: Agberi [Nigeria]. Holotype: BMNH 1901.12.18.7.

Distribution: Lower Niger River (Skelton \& Teugels, 1986).

Phractura bovei (Perugia, 1892)

Peltura Bovei Perugia, 1892: 972, unnumbered figures. Type locality: Congo. Holotype: MSNG 8839.

Distribution: Lower Congo River (Skelton \& Teugels, 1986).

Phractura brevicauda Boulenger, 1911

Phractura brevicauda Boulenger, 1911a: 502, fig. 374. Type locality: Trib. of Lobi R., S. W. of Efulen, and Kribi R., South Cameroon; Abanga R., Ogowe. Syntypes: BMNH 1904.7.1.119-126 (8), BMNH 1908.5.30.176-177 (2), BMNH 1908.5.25.118 (1).

Distribution: Lower Congo River (Skelton \& Teugels, 1986).

Remarks: Illustrated specimen, from the Lobi River, may not be considered a holotype, even though labeled as "type" in figure caption inasmuch as the term type is also applied in the text to the single specimen from the Abanga River.

Phractura clauseni Daget \& Stauch, 1963

Phractura clauseni Daget \& Stauch, 1963: 95, fig. 2. Type locality: Haute Comoé à Samago Iri. Holotype: MNHN 1960-0148.

Distribution: Tributaries of the Comoe and Black Volta Rivers, Upper Volta, Ogun River, Nigeria, and Upper Benoue River, Cameroon (Skelton \& Teugels, 1986).

Phractura fasciata Boulenger, 1920

Phractura fasciata Boulenger, 1920a: 34, fig. 18. Type locality: Stanley-Falls. Syntypes (4): BMNH 1919.9.10.284285 (2), MRAC 7241-42 (2).

Distribution: Congo River basin (Skelton \& Teugels, 1986).

Phractura gladysae Pellegrin, 1931

Phractura Gladysae Pellegrin, 1931a: 209. Type locality: Haute-Louessé. Syntypes (3): MNHN 1930-0251(1), MNHN 1930-0252 (1), MNHN 1930-0253 (1).

Distribution: Kouilou River system; known only from type locality (Skelton \& Teugels, 1986).

Phractura intermedia Boulenger, 1911

Phractura intermedia Boulenger, 1911a: 503, fig. 375. Type locality: Ja R., at Bitye, Libi R. affl. of Ja R., Kribi R, and Nong R.[South Cameroon]. Syntypes: BMNH 1903.7.28.114-116 (3), BMNH 1903.7.28.209-210 (2), BMNH 1904.7.1.165-167 (3), BMNH 1904.7.1.213 (1), BMNH 1904.10.26.49-52 (4), BMNH 1907.5.22.187 (1), BMNH 1909.4.29.103-105 (3), BMNH 1909.12.3.28 (1).

Distribution: Coastal Rivers of Cameroon, from Nyong to Kouilou (Skelton \& Teugels, 1986).

Phractura lindica Boulenger, 1902

Phractura lindica Boulenger, 1902g: 268, pl. 28 (figs. 3, 3a, 3b). Type locality: Lindi River, which flows into the Congo at the Stanley Falls. Syntypes (4): BMNH 1902.4.14.25 (1), IRSNB 12 (2 or 3), MSNM 39 (1).

Phractura lukugae Boulenger, 1920c: 402, fig. 9. Type locality: Kabeke, a village 30 miles south of Tumbwe, on the Niemba River, near the west coast of Lake Tanganyika. Holotype: BMNH 1919.7.24.32.

Distribution: Congo River basin (Skelton \& Teugels, 1986).

Phractura longicauda Boulenger, 1903

Phractura longicauda Boulenger, 1903c: 439. Type locality: Kribi River, southern Cameroon. Holotype: BMNH 1903.7.28.117. 
Distribution: Coastal rivers of South Cameroon and Equatorial Guinea, and Congo River basin (Skelton \& Teugels, 1986).

Phractura macrura Poll, 1967

Phractura macrura Poll, 1967: 269, fig. 129. Type locality: Caungula, riv. Cassange, (807'S, 1809'E), affl. riv. Uamba, Angola. Holotype: MD 6272.

Distribution: Uamba and Cuilo Rivers, southern tributaries of Kasai, Congo River basin, Angola (Skelton \& Teugels, 1986).

Phractura scaphyrhynchura (Vaillant, 1886)

Doumea scaphyrhynchura Vaillant, in Rivière, 1886: 18. Type locality: Ogôoué à Diélé. Holotype: MNHN 18860424.

Distribution: Ogowe and Congo River basins (Skelton \& Teugels, 1986).

Remarks: Pellegrin (1900: 181), lists Phractura scaphyrhynchura as a new species (nov. sp.), but cites Vaillant's description in the text.

Phractura tenuicauda (Boulenger, 1902)

Paraphractura tenuicauda Boulenger, 1902d: 48, pl. 14 (fig. 2). Type locality: l'Ubangi à Banzyville. Syntypes (plusieurs): BMNH 1901.12.26.48-49 (2), MRAC 1297-1299 (3).

Distribution: Congo River basin (Skelton \& Teugels, 1986).

PSAMMPHILETRIA Roberts, 2003

Psammphiletria Roberts, 2003: 101. Type species: Psammphiletria nasuta Roberts, 2003. Type by original designation. Gender: Feminine.

Psammphiletria delicata Roberts, 2003

Psammphiletria delicata Roberts, 2003: 102, fig. 17 e. Type locality: Stanley Pool. Holotype: MRAC 118455.

Distribution: Stanley Pool, Congo River (Roberts, 2003).

Psammphiletria nasuta Roberts, 2003

Psammphiletria nasuta Roberts, 2003: 101, fig. 17 a-c. Type locality: Republique Centrafricaine: Central African Republic, sandy riffle in R. Ubangui near Banda, $72 \mathrm{~km}$ upriver from Bangui. Holotype: CAS 92624.

Distribution: Ubangui River above Bangui (Roberts, 2003).

\section{TETRACAMPHILIUS Roberts, 2003}

Tetracamphilius Roberts, 2003: 102. Type species: Tetracamphilius pectinatus Roberts, 2003. Type by original designation. Gender: Masculine.

Tetracamphilius angustifrons (Boulenger, 1902)

Anoplopterus angustifrons Boulenger, 1902d: 42, pl. 10 (fig. 4). Type locality: Banzyville [Ubangi River]. Syntypes (plusieurs): BMNH 1901.12.26.39-40 (2), MRAC 1313 (1).

Distribution: Ubangi River, Congo Basin (Skelton \& Teugels, 1986).

Tetracamphilius clandestinus Roberts, 2003

Tetracamphilius clandestinus Roberts, 2003: 106, fig. 20 b. Type locality: Republique Centrafricaine: riffles in shallow sandy area of Ubangui near Banda. 2 km upstream from Bangui. Holotype: CAS 92653.

Distribution: Ubangui River above Bangui (Roberts, 2003).

Tetracamphilius notatus (Nichols \& Griscom, 1917)

Amphilius notatus Nichols \& Griscom, 1917: 715, fig. 24. Type locality: Faradje, Congo. Holotype: AMNH 6711.

Distribution: Faradje, Uele River, Congo River basin (Skelton \& Teugels, 1986).

Tetracamphilius pectinatus Roberts, 2003

Tetracamphilius pectinatus Roberts, 2003: 104, fig. 18. Type locality: Republique du Congo (Kinshasa): Congo basin, tributary of R. Luala, $29 \mathrm{~km}$ N of Kibunzi (8 km S of turnoff to Kubunzi, Buozi dist.), 45ํ․ $6.5^{\prime} \mathrm{S}, 1^{\circ} 48^{\prime} \mathrm{E}$. Holotype: CAS 92627.

Distribution: Ubangui River above Bangui; Luala River tributary; and Luala River of Southern Congo (Roberts, 2003). 
TRACHYGLANIS Boulenger, 1902

Trachyglanis Boulenger, 1902d: 48. Type species: Trachyglanis minutus Boulenger, 1902. Type by monotypy. Gender: Masculine.

Trachyglanis ineac (Poll, 1954)

Phractura ineac Poll, 1954: 62, fig. 5. Type locality: riv. Loweo, environs de Yangambi (Stanleyville). Holotype: MRAC 77460.

Distribution: Congo River basin (Yangambi, Tohuapa) (Skelton \& Teugels, 1986).

Trachyglanis intermedius Pellegrin, 1928

Trachyglanis intermedius Pellegrin, 1928b: 110. Type locality: Kasai, Congo belge. Holotype: NMBA 3755.

Distribution: Kasai River, Congo River basin (Skelton \& Teugels, 1986).

Trachyglanis minutus Boulenger, 1902

Trachyglanis minutus Boulenger, 1902d: 49, pl. 14 (fig. 3). Type locality: l’Ubangi à Banzyville. Syntypes (4):

BMNH 1901.12.26.50-51 (2), MRAC 1291-1291A (2).

Distribution: Congo River basin (Skelton \& Teugels, 1986).

Trachyglanis sanghensis Pellegrin, 1925

Trachyglanis sanghensis Pellegrin, 1925: 103. Type locality: Ouesso (Sangha). Holotype: MNHN 1925-0145.

Distribution: Sanga River, Congo River basin (Skelton \& Teugels, 1986).

ZAIREICHTHYS Roberts, 1967

Zaireichthys Roberts, 1967: 124. Type species: Zaireichthys zonatus Roberts, 1967. Type by original designation. Gender: Masculine.

Revision: Roberts (2003).

Zaireichthys brevis (Boulenger, 1915)

Leptoglanis brevis Boulenger, 1915: 169. Type locality: Rivière Lubumbashi, à Elisabethville. Syntypes (2): BMNH 1920.5.26.93 (1), MRAC 12702 (1).

Distribution: Congo River basin.

Remarks: Placed by Roberts (2003: 98) in the synonymy of Zairichthys rotundiceps, but comments therein indicate only that the types are "closely similar, possibly conspecific [with Z. rotundiceps]." Therefore, Zaireichthys brevis is retained as valid herein.

Zaireichthys camerunensis (Daget \& Stauch, 1963)

Leptoglanis camerunensis Daget \& Stauch, 1963: 94, fig. 1. Type locality: Bénoué à Lakdo, Cameroun. Syntypes (7): MNHN 1962-1272 (6); one specimen lost.

Distribution: Upper Benue River, Cameroon and Upper Niger River, Guinea (Roberts, 2003).

Zaireichthys dorae (Poll, 1967)

Leptoglanis dorae Poll, 1967: 211, fig. 95. Type locality: rapides de la Luachimo, dans des flaques d'eau résiduelle, à l'occasion de la mise à sec del la partie d'aval du barrage, Angola. Holotype: MRAC 161646.

Distribution: Luachimo, Congo Basin, Angola (Roberts, 2003).

Zaireichthys flavomaculatus (Pellegrin, 1926)

Leptoglanis flavomaculatus Pellegrin, 1926: 204. Type locality: Kamaiembi. Holotype: MRAC 19721.

Distribution: Lulua River, southern Congo; known only from holotype (Roberts, 2003).

Zaireichthys heterurus Roberts, 2003

Zaireichthys heterurus Roberts, 2003: 96. Type locality: Republic du Congo (Kinshasa): R. Avokoko, affluent rive droit fleuve Zaire, km 21 route Kisangani-Wanie Rukula. Holotype: MRAC 87-42-P-1140.

Distribution: Eastern Congo River basin (Roberts, 2003).

Zaireichthys mandevillei (Poll, 1959)

Leptoglanis mandevillei Poll, 1959: 98, pl. 25 (fig. 2). Type locality: Stanley Pool (Congo R., near Kinshasa). Holotype: MRAC 118532.

Distribution: Stanley Pool, Congo River, Ubangui and Lualaba (Roberts, 2003).

Zaireichthys rotundiceps (Hilgendorf, 1905)

Gephyroglanis rotundiceps Hilgendorf, 1905: 412. Type locality: Im Bubu bei Irangi. Lectotype: ZMB 16392, des- 
ignated by, and illustrated in, Seegers (1996: 196, fig. 141).

Distribution: Cunene, Okavango and Zambezi, Pungwe, Buse and Save basins, and Lake Malawi basin (Skelton, 1993); Lake Rukwa basin (Seegers, 1996).

Zaireichthys wamiensis (Seegers, 1989)

Leptoglanis wamiensis Seegers, 1989: 285, unnumbered figure. Type locality: Kisangata -Bach bei Mvumi, 32 kilometer SW Kidete, am Wege nach Kimamba/Kilosa im Wami-Einzug NW Morogoro, Tanzania. Holotype: ZFMK 15899.

Distribution: Wami River basin, Tanzania (Seegers, 1989).

Zaireichthys zonatus Roberts, 1967

Zaireichthys zonatus Roberts, 1967: 124, figs. 3-4. Type locality: Side channel of the lower rapids of the Congo River, below Stanley Pool, at Kinsuka village, within Leopoldville city limits. Holotype: SU 64126.

Distribution: Congo River basin (Roberts, 2003).

\section{ANCHARIIDAE Glaw \& Vences, 1994}

Anchariidae Glaw \& Vences, 1994: 380. Type genus: Ancharius Steindachner, 1880.

Revision, with key to species: Ng \& Sparks (2005).

2 genera, 5 species; no named fossil taxa.

ANCHARIUS Steindachner, 1880

Ancharius Steindachner, 1880a: 158. Type species: Ancharius fuscus Steindachner, 1880. Type by monotypy. Gender: Masculine. Also proposed as new in Steindachner (1880c: 251).

Ancharius fuscus Steindachner, 1880

Ancharius fuscus Steindachner, 1880a: 158. Type locality: Tohizona auf Madagascar. Holotype: NMW 48093. Species illustrated and described in more detail in Steindachner (1880c: 251, pl. 3, fig. 3; pl. 4).

Distribution: Widely distributed along eastern-draining rivers of Madagascar (Ng \& Sparks, 2005).

Ancharius griseus $\mathrm{Ng} \&$ Sparks, 2005

Ancharius griseus Ng \& Sparks, 2005: 308, fig. 5. Type locality: Madagascar: Toliara Province, Ihazofotsy River

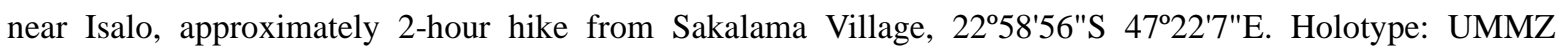
238041

Distribution: Onilahy River basin, western Madagascar (Ng \& Sparks, 2005).

GOGO Ng \& Sparks, 2005

Gogo Ng \& Sparks, 2005: 311. Type species: Gogo ornatus Ng \& Sparks, 2005. Type by original designation. Gender: Masculine.

Gogo arcuatus $\mathrm{Ng} \&$ Sparks, 2005

Gogo arcuatus $\mathrm{Ng} \&$ Sparks, 2005: 313, fig. 10. Type locality: Madagascar: Fianarantsoa province, Andriambondro River, $16 \mathrm{~km}$ N of Karianga at town of Mahevelo (tributary of Rienana River) $22^{\circ} 21^{\prime} 47^{\prime \prime} \mathrm{S}, 47^{\circ} 22^{\prime} 5^{\prime \prime} \mathrm{E}$. Holotype: UMMZ 238042.

Distribution: Sandrananta River basin, Madagascar (Ng \& Sparks, 2005).

Gogo brevibarbis (Boulenger, 1911)

Ancharius brevibarbis Boulenger, 1911a: 380, fig. 294. Type locality: Ambohimango, Madagascar. Holotype: BMNH 1896.10.9.40. Holotype illustrated in Ng \& Sparks (2005: 313, fig. 8).

Distribution: Apparently in Mananjary River basin, Madagascar (Ng \& Sparks, 2005); known only from holotype.

Gogo ornatus $\mathrm{Ng} \&$ Sparks, 2005

Gogo ornatus Ng \& Sparks, 2005: 316, fig. 12. Type locality: Madagascar: Toamasina province, Mangoro River, $200 \mathrm{~km}$ upstream of ferry crossing on road to Marolambo. Holotype: AMNH 235213.

Distribution: Mangoro River basin, Madagascar (Ng \& Sparks, 2005). 


\section{$\dagger$ ANDINICHTHYIDAE Gayet, 1988}

$\dagger$ Andinichthyidae Gayet, 1988: 833. Type genus: $\dagger$ Andinichthys Gayet, 1988.

Diagnosis: Gayet \& Meunier (2003).

Review: Arratia \& Gayet (1995).

3 genera, 3 species; 3 named fossil taxa.

$\dagger$ ANDINICHTHYS Gayet, 1988

$\dagger$ Andinichthys Gayet, 1988: 833. Type species: †Andinichthys bolivianensis Gayet, 1988. Type by original designation. Gender: Masculine.

$\dagger$ Andinichthys bolivianensis Gayet, 1988

$\dagger$ Andinichthys bolivianensis Gayet, 1988: 834, fig. on p. 834. Type locality: Maastrichtien de la Formation El Molino, Tiupampa (Bolivie). Holotype: MNHN Institut de Paléontologie YPFB-PAL 6178 (partial neurocranium).

Distribution: El Molino Formation, Tiupampa, Bolivia; Maastrichtian (Gayet, 1988).

$\dagger$ HOFFSTETTERICHTHYS Gayet, 1990

$\dagger$ Hoffstetterichthys Gayet, 1990: 871. Type species: $†$ Hoffstetterichthys pucai Gayet, 1990. Type by original designation. Gender: Masculine.

Remarks: Placement in †Andinichthyidae follows Gayet (1991).

$\dagger$ Hoffstetterichthys pucai Gayet, 1990

$\dagger$ Hoffstetterichthys pucai Gayet, 1990: 871, figs. 2, 3. Type locality: Maastrichtien de Tiupampa, Province de Mizqué, Département de Cochabamba (Bolivie centrale). Holotype: MHNC 6252 (partial neurocranium).

\section{$\dagger$ INCAICHTHYS Gayet, 1990}

$\dagger$ Incaichthys Gayet, 1990: 868. Type species: $\dagger$ Incaichthys suarezi Gayet, 1990. Type by original designation. Gender: Masculine.

Remarks: Placement in $\dagger$ Andinichthyidae follows Gayet (1991).

$\dagger$ Incaichthys suarezi Gayet, 1990

$\dagger$ Incaichthys suarezi Gayet, 1990: 868, figs 1, 4. Type locality: Maastrichtien de Tiupampa, Province de Mizqué, Département de Cochabamba (Bolivie centrale). Holotype: MHNC 6253 (partial neurocranium).

\section{ARIIDAE Bleeker, 1862}

Hemipimelodinae Gill, 1861c: 46. Type genus: Hemipimelodus Bleeker, 1857.

Arii Bleeker, 1862 (in Bleeker, 1862-63): 7, 25. Type genus: Arius Valenciennes, 1840.

Batrachocephalinae Gill, 1893:132. Type genus: Batrachocephalus Bleeker, 1846.

Doiichthyidae Weber, 1913: 532. Type genus: Doiichthys Weber, 1913.

Bagreidae Schultz, 1944c: 182. Type genus: Bagre Oken, 1817.

Remarks: The classification of species within the Ariidae is arguably the most poorly resolved of any catfish family. In recent years, there have been three independent attempts to examine the phylogeny of the family (Marceniuk, 2003; Kailola, 2004; and Betancur et al., 2004) and produce classifications that reflect the resulting phylogenetic hypotheses. The phylogenies and their resulting classifications differ markedly from each other, which makes it quite difficult to summarize the current understanding of the taxonomy herein. Thus, many of the generic assignments and synonymies listed here must be considered as tentative. One theme that is repeated in the various studies on the Ariidae is that the genus Arius was previously a taxonomic wastebasket and should instead be restricted to a group of Indo-Pacific species. Thus, species that had been treated as valid in Arius from the Americas, or western Africa, are now placed in other genera or, when no clear alternative was indicated, as Incertae sedis, herein. The family-level name Osteogeneiosinae was proposed by Fowler (1951b: 3) in a manner that made it available when published but is now not available (see Ferraris \& de Pinna, 1999, for details). The name Arius cous, which appeared in Heckel 
(1843) and also in Hyrtl (1859) appears to have been based on Silurus cous Linnaeus (now Glyptothorax cous) and is not an available name even though it has been treated as such.

Taxonomic summaries: Taylor (1986a, 1986b, Africa); Taylor (1990, eastern tropical Atlantic); Marceniuk \& Ferraris (2003, Neotropics).

Reviews: Taylor \& Menezes (1978, western central Atlantic) and Acero (2002, western central Atlantic); Jayaram \& Dhanze (1979, South Asia); Jayaram (1983, western Indian Ocean); Kailola (1990, freshwaters of northern New Guinea); Daget (1992, West Africa); Kailola \& Bussing (1995, Eastern Pacific); Kailola (1999, Western Pacific).

Phylogeny: Betancur-R. (2003), Betancur-R. et al. (2004, Neotropics); Kailola (2004, Indo-Pacific).

Evolution: Jayaram \& Dhanze (1985, South Asia).

Identification guide: Jayaram (1982, South Asia).

Biogeography: Cione et al. (1996, southern South America).

Keys: Kailola (2004: 123, genera); Marceniuk (2005a, Brazil).

26 genera, 133 species; 13 fossil species, not including otolith-based taxa.

\section{Incertae sedis}

Arius acutirostris Day, 1877

Arius acutirostris Day, 1877 (in Day, 1875-78): 459, pl. 107 (fig. 1). Type locality: Salwein River at Moulmein in Burma. Possible syntypes: BMNH 1870.6.14.34 (1), NMW 44358 (1), NMW 48327 (1), ZMB 2735 (1), ZSI F.454 (1), ZSI F.500 (1), ZSI A.569 (13).

Distribution: Salween River, Myanmar (Jayaram, 1982).

Arius africana Günther, 1867

Arius falcarius africana Günther, in Playfair \& Günther, 1867: 114. Type locality: Pangani River, East Africa. Syntypes: BMNH 1865.9.21.16 (1), BMNH 1867.3.7.385-386 (2, skins), BMNH 1867.3.7.499 (1). Originally as Arius falcarius var. africana.

Distribution: Pangani River and estuaries in Madagascar (Taylor, 1986a).

Arius brunellii Zolezzi, 1939

Arius brunellii Zolezzi, 1939: 168, fig. 1. Type locality: Giuba, Somalia. Syntypes (4): Whereabouts unknown.

Distribution: Juba River, Somalia (Taylor, 1986).

Remarks: Known only from the original description.

Arius gigas Boulenger, 1911

Arius gigas Boulenger, 1911a: 386, fig. 298. Type locality: Lokoja, Upper Niger. Holotype: BMNH 1904.1.20.41.

Distribution: Volta, Niger, and other rivers of Western Africa (Taylor, 1986).

Hemiarius Harmandi Sauvage, 1880

Hemiarius Harmandi Sauvage, 1880b: 230. Isle de Phu-Quoc (golfe de Siam). Holotype: MNHN a-2390.

? Arius brevirostris Steindachner, 1901: 447. Baram-Flusse, Borneo. Holotype: at NMW.

Distribution: Gulf of Thailand and, possibly, Borneo.

Remarks: Redescribed in Kailola (1999) but not assigned to any valid genus in Kailola (2004).

Arius Heudelotii Valenciennes, 1840

Arius Heudelotii Valenciennes, in Cuvier \& Valenciennes, 1840b: 73 (54 of Strasbourg deluxe edition), pl. 428. Type locality: haut Sénégal. Syntypes: MNHN b-0689 (2).

Arius mercatoris Poll, 1949: 201, fig. 8. Type locality: Atlantic, $12^{\circ} 34^{\prime} \mathrm{N}, 1^{\circ} 09^{\prime} \mathrm{W}, 8-10 \mathrm{fm}$. Holotype: IRSNB 89.

Distribution: West coast of Africa, from Mauritania to Gabon and, possibly, to Angola; in marine waters and estuaries (Taylor, 1986).

Arius latiscutatus Günther, 1864

Arius latiscutatus Günther, 1864: 151, unnumbered figure. Type locality: Fernando Po and West Africa [restricted to Fernando Po by Lectotype designation]. Lectotype: BMNH 1864.2.26.1 (skin), designated by Taylor (1990: 231).

Arius lagoensis Pfaff, 1933: 298, figs. 9-10. Type locality: Lagos [Nigeria]. Holotype: ZMUC P 29625.

Distribution: Along the western coast of Africa, from Dakar to Angola, and Bioko; sometimes in freshwaters (Tay- 
lor, 1986).

Hemipimelodus macrorhynchus Weber, 1913

Hemipimelodus macrorhynchus Weber, 1913: 549, fig. 27. Type locality: Lorentz-Fluss, van Weelskamp; LorentzFluss, "Bivak" Insel; Lorentz-Fluss, Alkmaar [New Guinea]. Syntypes: ZMA 111085 (2), ZMA 111086 (1); dorsal view of head illustrated in Desoutter (1977: 14, fig. 2).

Hemipimelodus aaldereni Hardenberg, 1936b: 367. Type locality: Middle course of Digulriver, southern New Guinea. Holotype: ZMA 110782.

Distribution: Southern Papua (formerly Irian Jaya), New Guinea (Allen, 1996).

Remarks: Treated as valid in Hemipimelodus by Desoutter (1977), but Kailola (2004) considered the species to be more closely related to species of Plicofollis and/or Genidens.

Arius parkii Günther, 1864

Arius parkii Günther, 1864: 154, unnumbered figure. Type locality: Lagos and River Niger [restricted to Lagos, by lectotype designation] Nigeria. Lectotype: BMNH 1865.7.4.4, designated by Taylor (1986a: 156).

Arius Capellonis Steindachner, 1867b: 64. Type locality: Monrovia in West-Afrika. Holotype: at NMW. [Description translated into English in Steindachner (1867g: 442).] Illustrated and described in more detail in Steindachner (1867e: 523, pl. 2, figs. 1-3).

Arius granulatus Peters, 1868a: 454. Type locality: Lagos, Nigeria. Holotype: ZMB 6780.

Distribution: Western Africa, from Angola to Cape Blanco and occasionally further north; in coastal waters and estuaries, sometimes in river mouths (Taylor, 1986).

Arius phrygiatus Valenciennes, 1840

Arius phrygiatus Valenciennes, in Cuvier \& Valenciennes, 1840b: 79 (59 of Strasbourg deluxe edition). Type locality: Cayenne [interpreted by Boeseman (1972) as having come from Suriname]. Holotype: RMNH 3038.

Arius Dieperinki Bleeker, 1862a: 375. Type locality: Surinama. Holotype: RMNH 3038; holotype illustrated in Bleeker (1864a: 51, pl. 10, pl. 12, fig. 3).

Distribution: Atlantic coastal rivers and estuaries of South America from Guyana to the mouth of the Amazon River (Marceniuk \& Ferraris, 2003).

Remarks: Boeseman (1972) treated RMNH 3038 as the holotype of A. phrygiatus.

Arius rugispinis Valenciennes, $1840 \mathrm{~b}$

Arius rugispinis Valenciennes, in Cuvier \& Valenciennes, 1840b: 77 (58 of Strasbourg deluxe edition). Type locality: Cayenne. Syntypes (3): MNHN a-9403 (1).

Tachysurus atroplumbeus Fowler, 1931: 394, fig. 2. Type locality: Vessigny Beach, Brighton, Trinidad. Holotype: ANSP 53316.

Distribution: Atlantic coastal rivers of South America from Venezuela to mouth of Amazon River (Marceniuk \& Ferraris, 2003).

$\dagger$ Felichthys stauroforus Lynn \& Melland, 1939

$\dagger$ Felichthys stauroforus Lynn \& Melland, 1939: 14. Type locality: Calvert Formation, Maryland. Holotype: USNM 15476 (partial neurocranium).

Distribution: Calvert Formation, Zone 12, Miocene (Lundberg, 1975, as $\dagger$ Arius stauroforus).

AMISSIDENS Kailola, 2004

Amissidens Kailola, 2004: 125. Type species: Arius hainesi Kailola, 2000. Type by original designation. Gender: Masculine.

Amissidens hainesi (Kailola, 2000)

Arius hainesi Kailola, 2000: 139, figs. 9-11. Type locality: Ludmilla Creek (12²7'S, 13046'E), Darwin, Northern Territory, Australia. Holotype: NTM S.11507-001.

Distribution: Southern coast of New Guinea and Northern Australia, between Darwin and southern Gulf of Carpentaria (Kailola, 2000).

ARIOPSIS Gill, 1861

Ariopsis Gill, 1861a: 56. Type species: Arius milberti Valenciennes, 1840. Type by monotypy. Gender: Feminine. 
Remarks: Kailola (2004) treated this genus as valid with two distinct geographic components, one distributed in tropical America and the the other in the Australia-New Guinea region. Herein, the two geographic components are treated as separate genera; the American species placed in Ariopsis and the Australia-New Guinean species in Neoarius.

Ariopsis assimilis (Günther, 1864)

Arius assimilis Günther, 1864: 146. Type locality: Lake of Yzabal, Guatemala. Holotype: BMNH 1864.1.26.98.

Distribution: Atlantic draining rivers, from Quintana Roo, Mexico, to Honduras (Miller et al., 2005).

Ariopsis bonillai (Miles, 1945)

Galeichthys bonillai Miles, 1945: 454, figs. 4-4a. Type locality: Río Magdalena, Honda, Colombia. Holotype: at Sec. Caza y Pesca, Min. Nat. Econ. Bogotá.

Distribution: Caribbean draining rivers of Colombia, including Magdalena River (Marceniuk \& Ferraris, 2003).

Ariopsis felis (Linnaeus, 1766)

Silurus Felis Linnaeus, 1766: 503. Type locality: Carolina [now: Charleston Harbor, South Carolina, U.S.A. by neotype designation]. Neotype: BMNH 1985.11.11.1. Name placed on Official List in ICZN Opinion 1547, with neotype designated.

Arius Milberti Valenciennes, in Cuvier \& Valenciennes, 1840b: 74 (56 of Strasbourg deluxe edition). Type locality: New-York ... Charlestown. Syntypes: MNHN b-0593 (3).

Arius equestris Baird \& Girard, 1854: 26. Type locality: Indianola, Texas. Holotype: USNM 836; holotype illustrated in Girard (1859a: 32, pl. 15).

Galeichthys guentheri Regan, 1907, in Regan, 1906-08: 124, pl. 16 (fig. 1), 19 (fig. 3). Type locality: Gulf of Mexico. Syntypes: BMNH 1855.9.19.1105-1106 (2).

Distribution: Western North Atlantic and Gulf of Mexico.

Ariopsis guatemalensis (Günther, 1864)

Arius guatemalensis Günther, 1864: 145, unnumbered figures. Type locality: Guatemala [and] Chiapan. Syntypes (2): BMNH 1853.1.11.6 (1).

Arius carulescens Günther, 1864: 149, fig. on p. 149. Type locality: Huamuchal, Guatemala. Syntypes: BMNH 1864.1.26.208-209 (2).

Galeichthys azureus Jordan \& Williams, in Jordan, 1895: 398, pl. 27. Type locality: Estuary at Mazatlán, Sinaloa, w. Mexico. Holotype: SU 11575.

Distribution: Pacific coast and coastal rivers of North and Central America, from Gulf of California, Mexico to Honduras (Miller et al., 2005).

Ariopsis lentiginosa (Eigenmann \& Eigenmann, 1888)

Tachisurus lentiginosus Eigenmann \& Eigenmann, 1888b: 139. Type locality: Panama. Syntypes (2, $39 \mathrm{~cm})$ : MCZ 4970 (2).

Galeichthys xenauchen Gilbert, in Jordan \& Evermann, 1898: 2777. Type locality: Panama. Holotype: SU 5821; holotype figured in Gilbert \& Starks (1904: pl. 4, fig. 7).

Distribution: Pacific coast in Panama (Kailola \& Bussing, 1995).

Ariopsis seemanni (Günther, 1864)

Arius seemanni Günther, 1864: 147, fig. Type locality: Central America. Holotype: BMNH 1855.9.19.1107.

Tachisurus jordani Eigenmann \& Eigenmann, 1888b: 142. Type locality: Panama. Syntypes (2, 25-26 cm): MCZ 4945 (2).

Galeichthys gilberti Jordan \& Williams in Jordan, 1895: 395, pl. 26. Type locality: Upper part of astillero at Maz atlán, Sinaloa, w. Mexico. Holotype: USNM 29213.

Galeichthys eigenmanni Gilbert \& Starks, 1904: 21, pl. 4 (fig. 8). Type locality: Panama. Holotype: SU 6986.

Galeichthys simonsi Starks, 1906: 764, fig. 1. Type locality: Callao, Peru. Holotype: USNM 53466.

Distribution: Pacific draining rivers and estuaries of the Americas, from Mexico to Peru (Miller et al., 2005).

Species inquirenda, Ariopsis

Hexanematichthys surinamensis Bleeker, 1862a: 380. Type locality: Surinama. Holotype: RMNH 3055; holotype illus- 
trated in Bleeker (1864: 55, pl. 6, fig. 2; pl. 7, fig. 1).

Remarks: Treated as valid in Kailola (2004), but of uncertain status in Marceniuk \& Ferraris (2003).

ARIUS Valenciennes, 1840

Arius Valenciennes, in Cuvier \& Valenciennes, 1840b: 53 (40 of Strasbourg deluxe edition). Type species: Pimelodus arius Hamilton, 1822. Type by absolute tautonymy. Gender: Masculine.

Ariodes Müller \& Troschel, 1849: 9. Type species: Bagrus (Ariodes) arenarius Müller \& Troschel, 1849. Type by subsequent designation, by Bleeker (1862-63: 8). Gender: Masculine. Originally proposed as a subgenus of Bagrus.

Pseudarius Bleeker, 1862 (in Bleeker, 1862-63): 8. Type species: Pimelodus arius Hamilton, 1822. Type by original designation. Gender: Masculine.

Arius arenarius (Müller \& Troschel, 1849)

Bagrus (Ariodes) arenarius Müller \& Troschel, 1849: 9. Type locality: China. Holotype or Syntype: ZMB 3001.

Arius fangi Chaux, in Chaux \& Fang, 1949a: 194, fig. 1. Type locality: Tonkin. Holotype: MNHN 1966-0726.

Distribution: Southern China, in coastal waters.

Remarks: The synonymy here follows the proposal in Kottelat (2001a: 56).

Arius arius (Hamilton, 1822)

Pimelodus arius Hamilton, 1822: 170, 376. Type locality: estuaries of Bengal. No types known.

Arius falcarius Richardson, 1845: 134, pl. 62 (figs. 7-9). Type locality: Canton, China. Syntypes (2): BMNH (apparently lost: Whitehead, 1970: 210). BMNH has two specimens identified as Arius falcarius from the Reeves collection. One specimen preserved in alcohol, $129 \mathrm{~mm}$ SL (BMNH 2005.9.12.2) and the second, a mounted, left one-half specimen, $289 \mathrm{~mm}$ SL (BMNH 2005.9.12.1). One or both are likely to be part of the type series.

Arius cochinchinensis Günther, 1864: 170, unnumbered figures. Type locality: Cochinchina. Holotype (skin): at BMNH, but not found in 2005.

Arius boakeii Turner, 1867: 82. Type locality: Ceylon. Syntypes: BMNH 1866.7.11.1 (1), possibly NMSZ 1987.030 (1).

Arius Buchanani Day, 1877 (in Day, 1875-78): 463, pl. 105 (fig. 6). Type locality: Hooghly at Calcutta and Burma [Irrawaddi]. Possible syntypes: ZSI 456 (1), ZSI 1302 (1, apparently lost).

Distribution: Bay of Bengal, South China Sea and, possibly, coast of Sumatra (Kailola, 1999); Pakistan, west coast of India, and Chilka Lake (Jayaram, 1982).

Remarks: See Wang et al. (2005a) for comments on the validity of this species.

? Arius bicolor (Fowler, 1935)

Hemipimelodus bicolor Fowler, 1935b: 100, fig. 23. Type locality: Bangkok, Siam. Holotype: ANSP 60777.

Hemipimelodus atripinnis Fowler, 1937: 145, fig. 34. Type locality: Bangkok, Siam. Holotype: ANSP 67906.

Distribution: Lower courses of Mekong and Chao Phraya River basins (Rainboth, 1996).

Remarks: Treated as valid in Hemipimelodus by Desoutter (1977) but as a likely synonym of Arius maculatus by Kailola (1999).

Arius dispar Herre, 1926

Arius dispar Herre, 1926: 405, pl. 1 (fig. 6). Type locality: Paco market, Manila, Philippines. Holotype: BSMP (destroyed).

Distribution: Philippines, Taiwan and, possibly, northern Borneo (Kailola, 1999).

Arius festinus $\mathrm{Ng} \&$ Sparks, 2003

Arius festinus Ng \& Sparks, 2003: 5, fig. 4. Type locality: Madagascar, Amboaboa River, near its confluence with the Mangarahara River (left bank tributary of the Sofia River), 15 $5^{\circ} 50^{\prime} 01^{\prime \prime S}, 48^{\circ} 42^{\prime} 52^{\prime \prime E}$. Holotype: UMMZ 239806.

Distribution: Amboaboa River, Madagascar (Ng \& Sparks, 2003).

Arius gagora (Hamilton, 1822)

Pimelodus gagora Hamilton, 1822: 167, 376, pl. 10 (fig. 54). Type locality: estuaries of Bengal. No types known.

Distribution: Bay of Bengal, from India to Thailand; in tidal portions of rivers and along the coast (Kailola, 1999).

Arius leptonotacanthus Bleeker, 1849 
Arius leptonotacanthus Bleeker, 1849: 11. Type locality: In Freto Madurae prope Kammal et Surabaya. Holotype (221 mm TL): BMNH 1863.12.4.114. Holotype illustrated in Bleeker (1862-63: 32, pl. 54 [=Silur. pl. 6], fig. 2).

Distribution: Western Central Pacific, along Gulf of Thailand and Indonesia; in coastal waters and estuaries (Kailola, 1999).

Remarks: Register number of holotype of Arius leptonotacanthus listed as BMNH 1863.12.4.144 in Eschmeyer et al. (1998).

Arius maculatus (Thunberg, 1792)

Silurus maculatus Thunberg, 1792: 31, pl. 1 (fig. 2). Type locality: China, Japan. No types known.

Silurus ocellatus Bloch \& Schneider, 1801: 379. Type locality: Japonia. No types known. Unneeded new name for Silurus maculatus Thunberg, 1792.

Pimelodus thunberg La Cepède, 1803: 691, 692. Type locality: China, Japan. No types known. Unneeded new name for Silurus maculatus Thunberg, 1792.

Bagrus gagorides Valenciennes, in Cuvier \& Valenciennes, 1840a: 441 (327 of Strasbourg deluxe edition). Type locality: [Ganges River]. Holotype: MNHN a-9330 (mounted).

Arius gagoroides Bleeker, 1846a: 168. Type locality: Batavia. Syntypes (size and number not stated): SMNS 10567 (2). [See also Bleeker, 1847c.]

Distribution: Western central Pacific; in inshore coastal waters, estuaries, and lower reaches of rivers (Kailola, 1999).

Arius madagascariensis Vaillant, 1894

Arius madagascariensis Vaillant, 1894a: 77. Type locality: Fl. Moroundava [Madagascar]. Holotype: MNHN 18940002.

Distribution: Western side of Madagascar, in rivers and lakes (Taylor, 1986b; Ng \& Sparks, 2003).

Remarks: Redescribed in Ng \& Sparks (2003).

Arius malabaricus Day, 1877

Arius Malabaricus Day, 1877 (in Day, 1875-78): 464, pl. 107 (fig. 4). Type locality: Canara [India]. Holotype: ZSI 1305.

Distribution: Peninsular India.

Remarks: See Jayaram \& Dhanze (1981a) for comments on validity.

Arius manillensis Valenciennes, 1840

Arius Manillensis Valenciennes, in Cuvier \& Valenciennes, 1840b: 93 (69 of Strasbourg deluxe edition). Type locality: Manille. Holotype (14 pouces): at MNHN.

Pseudarius philippinus Sauvage, 1880a: 226. Type locality: Ile de Luçon, Philippines. Holotype: MNHN a-2615.

Distribution: Philippines, in coastal waters, estuaries, and freshwater (Kailola, 1999).

Arius microcephalus Bleeker, 1855

Arius microcephalus Bleeker, 1855b: 423. Type locality: Bandjermasin, in fluviis [Borneo]. Syntypes (2, 140- 213 mm TL): BMNH 1863.12.4.149 (1, 108 mm SL, 132 mm TL), RMNH 6902 (1). One syntype illustrated in Bleeker (1862-63:37, pl. 51 [Silur. pl. 3], fig. 1, as Pseudarius microcephalus).

? Arius sciurus Smith, 1931: 30. Type locality: Tapi River near Bandon, Peninsular Siam. Holotype: USNM 90310.

Distribution: Borneo and eastern side of Malay peninsula; in lower portions of rivers and in brackish waters (Kailola, 1999).

Remarks: Register number of syntype of Arius microcephalus incorrectly reported as BMNH 1863.12.11.149 in Eschmeyer et al. (1998).

Arius oetik Bleeker, 1846

Arius oetik Bleeker, 1846a: 166. Type locality: Batavia. Syntype (size and number not stated): Possibly BMNH 1863.12.4.111 (1), NMV 45987 (1). Bleeker (1847: 32) changed the spelling to Arius utik, which has been used frequently in literature. [See also Bleeker, 1847c.]

Distribution: Gulf of Thailand and in waters around much of Malaysia and Indonesia; in coastal waters and estuaries (Kailola, 1999).

Arius subrostratus Valenciennes, in Cuvier \& Valenciennes, 1840b

Arius subrostratus Valenciennes, in Cuvier \& Valenciennes, 1840b: 62 (46 of Strasbourg deluxe edition). Type local- 
ity: Malabar. Holotype: MNHN 0000-1190.

Distribution: South Asia and eastward to western central (Jayaram, 1982).

Arius sumatranus (Bennett, 1830)

Bagrus sumatranus Bennett, 1830: 691. Type locality: Sumatra. Syntype: BMNH 1855.12.26.485 (1).

Distribution: Eastern Bay of Bengal to western central Pacific; in coastal waters and estuaries (Kailola, 1999).

Remarks: Treated by Kailola (1999) as a likely synonym of Arius venosus Valenciennes, 1840, but later treated as valid (Kailola, 2004). The distribution limits stated here represent the entire distribution of A. venosus, which may not be that of $A$. sumatranus.

Arius uncinatus Ng \& Sparks, 2003

Arius uncinatus Ng \& Sparks, 2003: 12, fig. 7. Type locality: Madagascar, Lake Andrapongy, near Antsohihy. Holotype: MHNG 2622.81

Distribution: Lake Andrapongy, Ankofia River basin, Madagascar (Ng \& Sparks, 2003).

Arius venosus Valenciennes, 1840

Arius venosus Valenciennes, in Cuvier \& Valenciennes, 1840b: 69 (52 of Strasbourg deluxe edition). Type locality: Rangoon; Manille. Syntypes: MNHN 0000-1205 (2).

Bagrus Schlegelii Bleeker, 1846a: 153. Type locality: Batavia.Type(s) (size and number not stated): possibly AMS B.8123. [See also Bleeker, 1847c.]

? Arius micruropterygius Bleeker, 1847a: 165. Type locality: Batavia. Type(s): Whereabouts unknown.

Distribution: Eastern Bay of Bengal to western central Pacific; in coastal waters and estuaries (Kailola, 1999).

\section{Species inquirendae, Arius}

Arius macracanthus Günther, 1864: 167, unnumbered figure. Type locality: Siam. Holotype: BMNH 1862.11.1.222.

Arius jella Day, 1877 (in Day, 1875-78): 467, pl. 106 (fig. 3). Type locality: Coromandel Coast of India; Madras. Syntype: ZSI 1304 (1, figured specimen).

ASPISTOR Jordan \& Evermann, 1898

Aspistor Jordan \& Evermann, 1898: 2763. Type species: Arius luniscutis Valenciennes, 1840. Type by original designation. Gender: Masculine.

Aspistor hardenbergi (Kailola, 2000)

Arius hardenbergi Kailola, 2000: 137, figs. 7-8. Type locality: Manimeri River (206'S, $\left.133^{\circ} 45^{\prime} \mathrm{E}\right)$, Bintuni Bay, Papua (Irian Jaya). Holotype: WAM P.29966-001.

Distribution: Southern coast of New Guinea, from Vogelkop Peninsula to mouth of Fly River (Kailola, 2000).

Aspistor luniscutis (Valenciennes, 1840)

Arius luniscutis Valenciennes in Cuvier \& Valenciennes, 1840b: 109 (82 of Strasbourg deluxe edition). Type locality: Brésil. Syntypes: MNHN a-8980 (2), MNHN b-0595 (1).

Arius physacanthus Vaillant, 1899: 155. Type locality: le Mahury [French Guiana]. Holotype: MNHN 1899-0058; holotype illustrated in Vaillant (1900: 128, fig. 1).

Arius bonneti Puyo, 1936: 107, fig. 17 (middle). Type locality: not stated [Cayenne River, French Guiana]. Holotype: MNHN 1936-0162. Preoccupied by $\dagger$ Arius bonneti Priem, 1904.

Arius clavispinosus Puyo, 1936: 103, fig. 16 (left). Type locality: île de Cayenne (rivière de Cayenne) [French Guiana]. Holotype: MNHN 1936-0163.

Arius despaxi Puyo, 1936: 110, fig. 17 (right). Type locality: [Cayenne River, French Guiana]. Holotype: MNHN 1936-0161.

Distribution: Atlantic coastal rivers of South America from French Guiana to southern Brazil (Marceniuk \& Ferraris, 2003).

Aspistor parkeri (Traill, 1832)

Silurus parkeri Traill, 1832: 377, pl. 6 (fig. 1). Type locality: Guiana. Holotype: Whereabouts unknown.

Distribution: Atlantic draining coastal rivers of South America from Guyana to northern Brazil (Marceniuk \& Ferraris, 2003). 
Aspistor quadriscutis (Valenciennes, 1840)

Arius quadriscutis Valenciennes, in Cuvier \& Valenciennes, 1840b: 111 (83 of Strasbourg deluxe edition). Type locality: Cayenne ou de la Mana ... Cayenne. Syntypes: MNHN a-9402 (1), MNHN b-0596 (1), MNHN b-0613 (1), RMNH 3049 (1), RMNH 3050 (1).

Distribution: Atlantic coastal rivers of South America from Guyana to northeastern Brazil (Marceniuk \& Ferraris, 2003).

\section{BAGRE Cloquet, 1816}

Bagre Cloquet, 1816: 52. Type species: Silurus bagre Linnaeus, 1766. Type by absolute tautonymy. Gender: Masculine. Placed on Official List (ICZN, Opinion 1402).

Bagre Oken, 1817: 1183. Silurus bagre Linnaeus, 1766. Type by absolute tautonymy. Gender: Masculine. Apparently proposed independently of Bagre Cloquet.

Glanis Spix \& Agassiz, 1829: 46. Type species: Silurus bagre Linnaeus, 1766. Type by subsequent designation by Kottelat (1988: 78). Gender: Masculine. Not preoccupied by Glanis in Rafinesque, 1818, which is not an available name.

Stearopterus Minding, 1832: 116. Type species: Stearopterus bagre Minding, 1832 [= Silurus bagre Linnaeus, 1766]. Type by monotypy. Gender: Masculine.

Breviceps Swainson, 1838: 328, 343. Type species: Silurus bagre of Bloch, 1794. Type by monotypy. Gender: Neuter. Preoccupied by Breviceps Merrem, 1820 (in Amphibia); replaced by Felichthys Swainson, 1839.

Felichthys Swainson, 1839: 305. Type species: Silurus bagre of Bloch, 1794. Type by being a replacement name. Gender: Masculine. Replacement for Breviceps Swainson, 1838.

Ailurichthys Baird \& Girard, 1854: 26. Type species: Silurus marinus Mitchill, 1815. Type by subsequent designation by Jordan \& Evermann (1896: 116). Gender: Masculine. Spelled Aelurichthys in Gill (1863: 172) and elsewhere, but clearly attributed to Baird \& Girard, so treated here as an unintended misspelling and not an available name.

Mystus Gray, 1854: 155. Type species: Mystus carolinensis Gronow, 1854. Type by subsequent designation by Jordan \& Evermann (1896: 116). Gender: Masculine. Preoccupied by Mystus Scopoli, 1777.

Paradiplomystes Bleeker, 1862 (in Bleeker, 1862-63): 8. Type species: Pimelodes coruscans Lichtenstein, 1819. Type by original designation. Gender: Masculine.

Anemanotus Fowler, 1944c: 171. Type species: Aelurichthys panamensis Gill, 1863. Type by original designation. Gender: Masculine. Originally proposed as a subgenus of Ailurichthys.

Taxonomic overview: Acero et al. (2005).

Remarks: See Gill (1891b) for comments on the validity of Felichthys.

Bagre bagre (Linnaeus, 1766)

Silurus Bagre Linnaeus, 1766: 505. Type locality: America meridionali. On several literature accounts, including Gronovius (1763: no. 382). Placed on Official List as type species of Bagre (ICZN, Opinion 1402).

? Pimelodes coruscans Lichtenstein, 1819: 58. Type locality: Brasilien. Holotype: Whereabouts unknown; apparently not ZMB 2978 as listed by Paepke (1999).

Felichthys filamentosus Swainson, 1839: 392. Type locality: Estuaries of rivers near Pernambuco, Brazil. No types known.

Galeichthys Gronovii Valenciennes, in Cuvier \& Valenciennes, 1840b: 40 (30 of Strasbourg deluxe edition). Unneeded new name for Silurus bagre Linnaeus.

Bagrus macronemus Ranzani, 1842: 334, pl. 28. Type locality: Fiume Brasiliano detto di S. Francesco. Holotype: MZUB 930. Name appears first in Ranzani (1841: 65) as a nomen nudum.

Mystus carolinensis Gronow in Gray, 1854: 156. Type locality: fluminibus majoribus Americes Meridionalis. No types known; based on several literature sources.

Distribution: Caribbean and Atlantic coastal rivers of South America, from Colombia to the mouth of the Amazon River (Marceniuk \& Ferraris, 2003).

Bagre marinus (Mitchill, 1815)

Silurus marinus Mitchill, 1815: 433. Type locality: New York. No types known. 
Galeichthys Blochii Valenciennes, in Cuvier \& Valenciennes, 1840b: 44 (33 of Strasbourg deluxe edition). Type locality: Surinam; Bahia. Syntypes: at MNHN (2), and ZMB 2989 (Paepke, 1999: 46). Description based on specimens and reference to Bloch (1794: pl. 365).

Galeichthys Parrae Valenciennes, in Cuvier \& Valenciennes, 1840b: 33 (25 of Strasbourg deluxe edition). Type locality: New York. No types known. Unneeded new name for Silurus marinus Mitchill.

Galeichthys bahiensis Castelnau, 1855: 37, pl. 18 (fig. 1). Type locality: Bahia [Bahia]. Holotype: MNHN b-0692.

Elurichthys longispinis Günther, 1864: 178. Type locality: South America; Mexico. Syntypes (3): BMNH 1976.2.18.1 (1), BMNH 1976.2.18.2 (1).

Distribution: Coast of Gulf of Mexico, western margin of Caribbean and northern margin of South America: sometimes in rivers and estuaries (Marceniuk \& Ferraris, 2003).

Bagre panamensis (Gill, 1863)

Aelurichthys panamensis Gill, 1863: 172. Type locality: Western coast of Central America. Holotype: at USNM (not found, see Jordan \& Gilbert, 1882; Ferraris \& Vari, 1992).

Elurichthys nuchalis Günther, 1864: 179, fig. Type locality: Pacific coast of Panama. Holotype: BMNH 1864.1.26.344; holotype illustrated in Günther (1868c: pl. 81, fig. 2).

Aelurichthys scutatus Regan, 1907, in Regan, 1906-08: 116, pl. 15 (fig. 2), 19 (fig. 2). Type locality: Panama or Ecuador. Syntype: BMNH 1903.5.15.328.

Aelurichthys isthmensis Regan, 1907, in Regan, 1906-08: 117, pl. 15 (fig. 1), 19 (fig. 1). Type locality: Colón, Panama. Syntypes: BMNH 1877.5.31.7-8

Distribution: West coast of Americas, from off Santa Ana River in southern California, USA, to Peru. Only rarely found north of southern Baja California (Marceniuk \& Ferraris, 2003).

Bagre pinnimaculatus (Steindachner, 1876)

? Galeichthys Eydouxii Valenciennes, in Cuvier \& Valenciennes, 1840b: 43 (32 of Strasbourg deluxe edition). Type locality: la rivière de Guayaquil. Holotype: MNHN 0000-1572. Name spelled Eidouxii on p. 43 and Eydouxii in table of contents (p. xviii). First reviser apparently Eschmeyer, Ferraris, Hoang \& Long (1998).

Aelurichthys pinnimaculatus Steindachner, 1876: 565, pl. 8. Type locality: Panama, Altata, Westküste von CostaRica. Syntypes: MCZ 4941 (1), MCZ 23746 (3), MCZ 23747 (1), NMW 47895 (1), NMW 47897 (1), NMW 47898 (1), NMW 47899 (1), NMW 50577 (1), USNM 123011 (2).

Distribution: Pacific draining rivers of North, Central and South America, from the Gulf of California to Ecuador (Marceniuk \& Ferraris, 2003).

Remarks: Species treated as valid in Kailola \& Bussing (1995: 878), but with Galeichthys eydouxii as a possible senior synonym. Therefore, the name G. eydouxii is tentatively placed here.

\section{BATRACHOCEPHALUS Bleeker, 1846}

Batrachocephalus Bleeker, 1846a:176. Type species: Batrachocephalus ageneiosus Bleeker, 1846. Type by monotypy. Gender: Masculine.

Batrachocephalus mino (Hamilton, 1822)

Ageneiosus mino Hamilton, 1822: 159, 375. Type locality: Upper part of [Ganges] estuaries. No types known.

Batrachocephalus ageneiosus Bleeker, 1846a: 176. Type locality: Batavia. Type(s) (size and number not stated): Whereabouts unknown [See also Bleeker, 1847c.]

Batrachocephalus micropogon Bleeker, 1858b: 118. Type locality: Java (Batavia, Surabaja), in mari et aquis fluviomarinis. Syntypes (10: 180-248 mm TL): AMS B.7964, BMNH 1862.2.4.18, also on Ageneiosus mino Hamilton and Batrachocephalus ageneiosus Bleeker). One syntype illustrated in Bleeker (1862-63: pl. 17).

Distribution: Bay of Bengal, and parts of the western central Pacific, in coastal waters, estuaries, and lower reaches of rivers (Kailola, 1999); also Pakistan (Jayaram, 1982).

\section{BRUSTIARIUS Herre, 1935}

Brustiarius Herre, 1935: 388. Type species: Arius nox Herre, 1935. Type by original designation. Gender: Masculine. Originally proposed as a subgenus of Arius.

Brustiarius nox (Herre, 1935) 
Arius nox Herre, 1935: 388. Type locality: Nyaurangai, Sepik River, New Guinea. Holotype: FMNH 17195.

Distribution: Sepik River, New Guinea (Herre, 1935).

Brustiarius solidus (Herre, 1935)

Arius solidus Herre, 1935: 385. Type locality: Timbunke, Sepik River, New Guinea. Holotype: FMNH 17201.

Arius kanganamanensis Herre, 1935: 387, fig. 32. Type locality: Kanganaman, Sepik River, New Guinea. Holotype: FMNH 17194.

Hemipimelodus bernhardi Nichols, 1940: 3. Type locality: Bernhard Camp, Idenburg River, Netherland New Guinea. Holotype: AMNH 15039.

Arius microstomus Nichols, 1940: 2. Type locality: Bernhard Camp, Idenburg River, Netherland New Guinea. Holotype: AMNH 15041.

Distribution: Northern Papua (formerly Irian Jaya) (Allen, 1996) and Sepik River basin.

Remarks: Kailola (1990) and Allen \& Coates (1990) treat Arius kangamanensis as a junior synonym of A. solidus. As such, the earlier of these two publications serves as first reviser in establishing priority but, since the results are identical, the question of which of these papers was published first was not pursued at this time.

CATHOROPS Jordan \& Gilbert, 1882

Cathorops Jordan \& Gilbert, 1882: 39, 54. Type species: Arius hypophthalmus Steindachner, 1876. Type by monotypy. Gender: Masculine. Originally proposed as a subgenus of Arius. Name dates to key on p. 39, which is part of a signature dated as 25 Sept 1882.

Cathorops agassizii (Eigenmann \& Eigenmann, 1888)

Tachisurus agassizii Eigenmann \& Eigenmann, 1888b: 145. Type locality: Rio Grande do Sul [Brazil]. Holotype: MCZ 7670.

Arius pleurops Boulenger, 1897a: 296. Type locality: Magoarisinho, Cape Magoary [Marajo I., Brazil]. Holotype: BMNH 1897.7.17.7 (plus eggs and embryos in mouth).

Distribution: Northern coast of South America including Guyana and the north and northeast Brazil (Marceniuk \& Ferraris, 2003).

Cathorops aguadulce (Meek, 1904)

Galeichthys aguadulce Meek, 1904: 9, pl. 4. Type locality: Río Tesechocán, Pérez, Vera Cruz basin of the Rio Papaloapam [Mexico]. Holotype: FMNH 4678.

Distribution: Atlantic draining rivers from Panuco River basin, Mexico to Izabal Lake, Guatemala (Miller et al., 2005).

Cathorops arenatus (Valenciennes, 1840)

Arius arenatus Valenciennes in Cuvier \& Valenciennes, 1840b: 106 (79 of Strasbourg deluxe edition). Type locality: Cayenne [interpreted by Boeseman (1972) as having come from Suriname]. Holotype: RMNH 3099.

Arius fissus Valenciennes in Cuvier \& Valenciennes, 1840b: 107 (79 of Strasbourg deluxe edition). Type locality: Cayenne [interpreted by Boeseman (1972) as having come from Suriname]. Holotype: RMNH 3036.

Distribution: Atlantic coastal rivers of South America from Guyana to northeastern Brazil (Marceniuk \& Ferraris, 2003).

Remarks: Marceniuk \& Ferraris (2003: 449) acted as first reviser in giving precedence to Arius arenatus over A. fissus.

Cathorops dasycephalus (Günther, 1864)

Arius dasycephalus Günther, 1864: 157, fig. on p. 157. Type locality: Oahu, Sandwich Islands [in Error]. Holotype: BMNH 1855.9.19.1100.

Tachisurus longicephalus Eigenmann \& Eigenmann, 1888b: 143. Type locality: Gulf of Panama, Panama. Holotype: MCZ 4972. Name spelled Tachisurus longicehpalus in account heading, T. longicephalus in introduction ( $\mathrm{p}$. 119); the latter spelling is considered valid.

Distribution: Pacific coast of South and Central America, from Costa Rica to Ecuador (Marceniuk \& Ferraris, 2003).

Cathorops fuerthii (Steindachner, 1876)

Arius Fürthii Steindachner, 1876: 579. Type locality: Panama. Syntypes (4): possibly MCZ 4943 (2), MCZ 4973 (3), MCZ 7691 (1), NMW 50568 (2). 
Tachysurus evermanni Gilbert \& Starks, 1904: 32, pl. 5 (fig. 10). Type locality: Panama Bay. Holotype: SU 6706.

Distribution: Pacific draining rivers of the Americas from Mexico to Ecuador (Miller et al., 2005).

Cathorops hypophthalmus (Steindachner, 1876)

Arius hypophthalmus Steindachner, 1876: 581, pl. 10. Type locality: Panama. Holotype: at NMW.

Tachisurus gulosus Eigenmann \& Eigenmann, 1888b: 146. Type locality: Panama. Syntypes (2): MCZ 4974 (2).

Distribution: Pacific draining rivers of Panama, in brackish and fresh water (Marceniuk \& Ferraris, 2003).

Cathorops mapale Betancur-R. \& Acero P., 2005

Cathorops mapale Betancur-R. \& Acero P., 2005: 47, figs. 1, 2. Type locality: Ciénaga Grande de Santa Marta (CGSM), Madgalena, CO (1059'N, 74²17'W). Holotype: INVEMAR-PEC 5333.

Distribution: Coastal Caribbean region of Colombia (Betancur-R. \& Acero P., 2005).

Cathorops melanopus (Günther, 1864)

Arius melanopus Günther, 1864: 172. Type locality: Rio Motagua. Syntypes (apparently only 2): BMNH 1865.4.29.51-53 (3).

Distribution: Motagua River basin of Guatemala (Marceniuk \& Ferraris, 2003).

Cathorops multiradiatus (Günther, 1864)

Bagrus arioides Kner, 1863: 227, fig. 15. Type locality: Rio Bayano im Staate Panama, Südseite. Holotype (6"): at NMW. Also in Kner \& Steindachner (1864: 47). Preoccupied by Bagrus arioides Valenciennes, 1840; replaced by Arius multiradiatus Günther, 1864.

Arius multiradiatus Günther, 1864: 173. Type locality: Rio Bayano im Staate Panama, Südseite. Holotype: at NMW. Replacement for Bagrus arioides Kner, 1863.

Tachysurus emmelane Gilbert, in Jordan \& Evermann, 1898: 2785. Type locality: Panama. Holotype: SU 5818; holotype illustrated in Gilbert \& Starks (1904: pl. 6).

Tachysurus equatorialis Starks, 1906: 766, fig. 3. Type locality: Guayaquil, Ecuador. Holotype: USNM 53470.

Distribution: Rivers and bays of Pacific coast of Central America, from Guatemala to Panama (Marceniuk \& Ferraris, 2003).

Remarks: Kailola \& Bussing (1995: 881) tentatively placed the species in Cathorops, which is followed herein.

Cathorops spixii (Spix \& Agassiz, 1829)

Pimelodus spixii Spix \& Agassiz, 1829: 19, pl. 7 (fig. 1). Type locality: Brasilia aequatoriali. Whereabouts unknown (Kottelat, 1988).

Pimelodus albidus Spix \& Agassiz, 1829: pl. 7 (fig. 1). Type locality: [Not stated]. Whereabouts unknown (Kottelat, 1988). Name available from plate caption.

Distribution: Atlantic and Caribbean rivers and estuaries of northern South America from Colombia to Brazil (Marceniuk \& Ferraris, 2003), but considered restricted to the Atlantic coast by Betancur-R. \& Acero P. (2005).

Remarks: Taylor \& Menezes (1978) indicate that two or more species may be included here. Agassiz (1831, in Spix \& Agassiz, 1829-1831: conspectus) serves as first reviser in treating Pimelodus spixii as valid over Pimelodus albidus.

Cathorops steindachneri (Gilbert \& Starks, 1904)

Tachysurus steindachneri Gilbert \& Starks, 1904: 29, pl. 5 (fig. 9). Type locality: Panama Bay. Holotype: SU 7026.

Arius taylori Hildebrand, 1925: 250, fig. 10. Type locality: Río Lempa, San Marcos, El Salvador. Holotype: USNM 87224.

Distribution: Pacific draining rivers and estuaries of Central America (Marceniuk \& Ferraris, 2003).

Remarks: Kailola \& Bussing (1995: 882) tentatively placed the species in Cathorops.

Cathorops tuyra (Meek \& Hildebrand, 1923)

Arius tuyra Meek \& Hildebrand, 1923: 128, pl. 5. Type locality: Río Tuyra, mouth of Río Yape, Darien, Panama. Holotype: USNM 79413.

Distribution: Pacific drainage rivers and estuaries of South and Central America, from Panama to Peru (Marceniuk \& Ferraris, 2003).

Remarks: Placed by Kailola \& Bussing (1995: 883) tentatively in Cathorops. 
Arius variolosus Valenciennes in Cuvier \& Valenciennes, 1840b: 107 (80 of Strasbourg deluxe edition). Type locality: Cayenne. Holotype: MNHN 0000-4169.

Arius puncticulatus Valenciennes in Cuvier \& Valenciennes, 1840b: 108 (81 of Strasbourg deluxe edition). Type locality: Buénos-Ayres. Holotype (6 pouces): at MNHN.

Arius nuchalis Günther, 1864: 171, unnumbered figure. Type locality: British Guiana. Syntypes (6): BMNH 2005.5.17.1-2 (2), possibly BMNH 1975.1.17.3-5 (3), but original registration information gives locality as West Indies and having come from "old collection" which does not agree with information provided in Günther.

Arius laticeps Günther, 1864: 171, unnumbered figures. Type locality: British Guiana; Trinidad. Syntypes: BMNH 1863.6.18.8 (1), BMNH 1976.2.25.1 (1, $137 \mathrm{~mm}$ TL).

Tachysurus liropus Bristol, in Gilbert, 1897: 438. Type locality: San Juan Lagoon, near mouth of Rio Ahome, Sonora, Mexico. Syntypes (6): SU 324 (3), USNM 47584 (2).

\section{CEPHALOCASSIS Bleeker, 1857}

Cephalocassis Bleeker, 1857b: 473. Type species: Arius melanochir Bleeker, 1852. Type by monotypy. Also published in Bleeker (1858b: 62, 98). Gender: Feminine.

Hemipimelodus Bleeker, 1857b: 473. Type species: Pimelodus borneënsis Bleeker, 1851. Type by monotypy. Gender: Masculine.

Remarks: Genus revised by Desoutter (1977) as Hemipimelodus. Kailola (2004) apparently acted as first reviser in giving precedence to Cephalocassis over Hemipimelodus, inasmuch as no previous synonymy of the two generic names has been found.

Key: Desoutter (1977, as Hemipimelodus).

Cephalocassis borneensis (Bleeker, 1851)

Pimelodus borneënsis Bleeker, 1851e: 430. Type locality: Sambas, in fluviis. Holotype (110 mm TL): RMNH 6906; dorsal view of head illustrated in Desoutter (1977: 23, fig. 5).

Hemipimelodus macrocephalus Bleeker, 1858b: 239. Type locality: Borneo (Bandjarmasin), in fluviis. Syntypes (3: 125-140 mm TL): BMNH 1863.12.4.116 (1, 109 mm SL, 124 mm TL), RMNH 27618 (1). One syntype illustrated in Bleeker (1862-63: pl. 82 [= Silur. pl. 34], fig. 2).

Hemipimelodus siamensis Sauvage, 1878: 234. Type locality: Laos. Holotype: MNHN 0000-9649.

Distribution: Mekong and Chao Phraya River basins, and Sundaland (Kottelat, 2001b).

Remarks: BMNH 1863.12.4.70 (143 mm ST, $\sim 170 \mathrm{~mm} \mathrm{TL}$ ), listed as type of Pimelodus borneensis, is too large to be the type.

Cephalocassis jatia (Hamilton, 1822)

Pimelodus jatius Hamilton, 1822: 171, 376. Type locality: estuaries of Bengal. No types known.

Distribution: Rivers and estuaries of South Asia, extending well above tidal range (Jayaram, 1982); Myanmar.

Cephalocassis manillensis (Valenciennes, 1840)

Pimelodus Manillensis Valenciennes, in Cuvier \& Valenciennes, 1840b: 192 (142 in Strasbourg deluxe edition). Type locality: Manille. Holotype: MNHN 0000-1209; dorsal view of head illustrated in Desoutter (1977: 16, fig. 3).

Distribution: Philippines.

Remarks: Inclusion in Cephalocassis is tentative, based on the treatment in Desoutter (1977) as a valid species of Hemipimelodus, and the uncertain status of the species in Kailola (2004).

\section{Cephalocassis melanochir (Bleeker, 1852)}

Arius melanochir Bleeker, 1852d: 590. Type locality: Palembang, in fluviis. Holotype (302 mm TL): RMNH 6892.

? Arius doriae Vinciguerra, 1881: 174, unnumbered fig. Type locality: Sarawak state, Borneo, East Malaysia. Syntypes: MSNG 8135 (2), RMNH 10889 (1).

Distribution: Sumatra and Borneo.

Remarks: Synonymy based on Kailola (2004). 


\section{Species inquirenda, Cephalocassis}

Hemipimelodus intermedius Vinciguerra, 1881: 178, unnumbered figure. Type locality: [Sarawak, Borneo]. Syntypes: MSNG 14529 (2). Treated as valid in Hemipimelodus (a synonym of Cephalocassis herein) by Desoutter (1977) but as a possible synonym of Cephalocassis borneensis by Kailola (2004).

\section{CINETODUS Ogilby, 1898}

Cinetodus Ogilby, 1898a: 32. Type species: Arius froggatti Ramsay \& Ogilby, 1886. Type by original designation. Gender: Masculine.

Pachyula Ogilby, 1898a: 33. Type species: Hemipimelodus crassilabris Ramsay \& Ogilby, 1886. Type by original designation. Gender: Feminine.

Tetranesodon Weber, 1913: 545. Type species: Tetranesodon conorhynchus Weber, 1913. Type by monotypy. Gender: Masculine.

Septobranchus Hardenberg, 1941: 223. Type species: Septobranchus johannae Hardenberg, 1941. Type by monotypy. Gender: Masculine.

Cinetodus carinatus (Weber, 1913)

Arius (Hemiarius) carinatus Weber, 1913: 537, figs. 13-14. Type locality: Lorentz-Fluss; bei Van Weelskamp; Van der Sande-Fluss, Nebenfluss des Lorentz-Flusses [New Guinea]. Syntypes: AMNH 9265 (2), ZMA 109295 (1), ZMA 111109 (18), ZMA 111110 (1), ZMA 111111 (1), ZMA 111112 (1).

Distribution: Southern Papua (formerly Irian Jaya), New Guinea (Allen, 1996).

Cinetodus conorhynchus (Weber, 1913)

Tetranesodon conorhynchus Weber, 1913: 546, fig. 24. Type locality: Lorentz-Fluss, v. Weelskamp [New Guinea]. Holotype: ZMA 111084.

Distribution: Southern Papua (formerly Irian Jaya), New Guinea (Allen, 1996).

Cinetodus crassilabris (Ramsay \& Ogilby, 1886)

Hemipimelodus crassilabris Ramsay \& Ogilby, 1886: 18. Type locality: Strickland River, New Guinea. Holotype: AMS B.9961; dorsal view of head illustrated in Desoutter (1977: 18, fig. 4).

Distribution: New Guinea (Allen, 1991).

Cinetodus froggatti (Ramsay \& Ogilby, 1886)

Arius froggatti Ramsay \& Ogilby, 1886: 14. Type locality: Strickland River, New Guinea. Holotype: AMS B.9936.

Septobranchus johannae Hardenberg, 1941: 223, fig. 3. Type locality: Merauke, South New Guinea [Papua, Indonesia]. Holotype (about $30 \mathrm{~cm}$ ): Whereabouts unknown.

Distribution: Southern New Guinea and Roper River system, Northern Australia; in coastal waters, estuaries, and into fresh water (Kailola, in Allen, 1989).

\section{COCHLEFELIS Whitley, 1941}

Cochlefelis Whitley, 1941b: 8. Type species: Arius spatula Ramsay \& Ogilby, 1886. Type by original designation. Gender: Feminine.

Remarks: Elevated from a subgenus of Arius by Kailola (2004).

Cochlefelis burmanica (Day, 1870)

Arius burmanicus Day, 1870c: 618. Type locality: Irrawaddi, Bassein district, and Salwein in the Tenasserim provinces, Burma. Possible syntypes: AMS B.7520 (1), BMNH 1870.6.14.43-44 (2), RMNH 8787 (1), ZMB 2754 (1), ZSI A.568 (8), ZSI B.292 (1, lost), ZSI 456 (1); ZSI 456 illustrated in Day (1875-78: pl. 105) (Jayaram, 1982).

Distribution: Myanmar (Jayaram, 1982).

Remarks: Placement in Cochlefelis first proposed in Kailola (2004).

Cochlefelis danielsi (Regan, 1908)

Arius (Hemiarius) danielsi Regan, 1908c: 154. Type locality: Fly River, British New Guinea. Holotype: BMNH 1905.8.15.21.

Distribution: Southern New Guinea, in river deltas and mangroves (Kailola, 1999). 
Cochlefelis spatula (Ramsay \& Ogilby, 1886)

Arius spatula Ramsay \& Ogilby, 1886: 15. Type locality: Strickland River, New Guinea. Holotype: AMS B.9937.

Distribution: Southern Papua (formerly Irian Jaya), New Guinea (Allen, 1996).

CRYPTARIUS Kailola, 2004

Cryptarius Kailola, 2004: 134. Type species: Arius truncatus Valenciennes, 1840. Type by original designation. Gender: Masculine.

Cryptarius daugueti (Chevey, 1932)

Hemipimelodus Daugueti Chevey, 1932: 41, pl. 13. Type locality: Phnompenh et Snoctrou, au seuil du Grand Lac. Type(s): at Inst. Océanogr. Indochine, Nhatrang, Viet Nam.

Distribution: Large Rivers of South East Asia.

Remarks: Kailola (2004: 135) tentatively assigned this species to Cryptarius.

Cryptarius truncatus (Valenciennes, 1840)

Arius truncatus Valenciennes, in Cuvier \& Valenciennes, 1840b: 64 (48 of Strasbourg deluxe edition). Type locality: Java. Holotype: MNHN b-0590.

Hemipimelodus cochlearis Fowler, 1935b: 101, fig. 25. Type locality: Paknam, Siam. Holotype: ANSP 60767.

Distribution: Western central Pacific; in estuaries and lower portions of rivers (Kailola, 1999); including Chao Phraya and Mekong (Rainboth, 1996).

GALEICHTHYS Valenciennes, 1840

Galeichthys Valenciennes in Cuvier \& Valenciennes, 1840b: 28 (21 of Strasbourg deluxe edition). Type species: Galeichthys feliceps Valenciennes, 1840. Type by subsequent designation by Bleeker (1862-63). Gender: Masculine.

Galeichthys ater Castelnau, 1861

Galeichthys ater Castelnau, 1861: 62. Type locality: les mers du Cap [South Africa]. Syntypes (2, $55 \mathrm{~cm} \mathrm{long):}$ Whereabouts unknown.

Distribution: Cape of Good Hope, Southern Africa (Taylor, 1986).

Galeichthys feliceps Valenciennes, 1840

Galeichthys feliceps Valenciennes, in Cuvier \& Valenciennes, 1840b: 29 (22 of Strasbourg deluxe edition), pl. 424. Type locality: Aux environs du Cap [South Africa]. Syntypes: MNHN a-9361 (1, dry), MNHN a-9362 (1, dry), MNHN a-9363 (1, dry), MNHN a-9364 (1, dry), MNHN a-9365 (1, dry).

Bagrus capensis Smith, 1840, in Smith 1838-49: pl. 8. Type locality: Cape of Good Hope, South Africa. Possible holotype: BMNH 1857.6.13.147 (1, skin). Name available from plate caption.

Pimelodus Peronii Valenciennes, in Cuvier \& Valenciennes, 1840b: 161 (100 of Strasbourg deluxe edition). Type locality: la mer des Indes. Holotype: MNHN 0000-1207.

Galeichthys ocellatus Gilchrist \& Thompson, 1916: 60, figure on p. 61. Type locality: Zwartkops River, Algoa Bay [South Africa]. Holotype: at PEM.

Distribution: Widely distributed in southern Africa; in freshwater, estuaries, and coastal environments (Taylor, 1986).

Remarks: Kailola (1986b) treated Pimelodus peronii as a valid species in Arius, but synonymy herein follows Kailola (2004).

Galeichthys peruvianus Lütken, 1874

Galeichthys peruvianus Lütken, 1874b: 205. Type locality: Callao. Syntypes: ZMUC P 29628 (1), ZMUC P 29629 (1).

Distribution: Pacific coast of South America, from Ecuador to Peru (Marceniuk \& Ferraris, 2003).

GENIDENS Castelnau, 1855

Genidens Castelnau, 1855: 33. Type species: Bagrus genidens Cuv. Val. [= Pimelodus genidens Cuvier, 1829]. Type by absolute tautonymy. Gender: Masculine.

Guiritinga Bleeker, 1858b: 62, 67. Type species: Pimelodus commersonii La Cepède, 1803. Type by monotypy. 
Gender: Feminine.

Genidens barbus (La Cepède, 1803)

Pimelodus barbus La Cepède, 1803: 94, 102. Type locality: Amérique méridionale. No types known.

Pimelodus Commersonii La Cepède, 1803: 95, 103, pl. 3 (fig. 1). Type locality: [No locality stated]. No types known.

Bagrus barbatus Quoy \& Gaimard, 1824: 230, pl. 49 (figs. 1-2). Type locality: Rio de la Plata, South America. Type(s): at MNHN.

Pimelodus versicolor Castelnau, 1855: 35, pl. 16 (fig. 3). Type locality: rio Araguay, dans la province de Goyaz (Brésil). Syntypes: MNHN 0000-1206 (2).

Tachisurus upsulonophorus Eigenmann \& Eigenmann, 1889b: 31. Type locality: Rio Grande do Sul [Brazil]. Holotype: MCZ 23750.

Silurus 16-radiatus Larrañaga, 1923: 386. Type locality: Uruguay. No types known. Apparently an alternate name for Silurus marinus Larrañaga.

Silurus marinus Larrañaga, 1923: 376. Type locality: Uruguay. No types known. Apparently an alternate name for Silurus 16-radiatus Larrañaga. Preoccupied by Silurus marinus Mitchill, 1815.

Distribution: Atlantic coast of South America from the La Plata River to eastern Brazil (Marceniuk, 2005).

Remarks: Redescribed in Marceniuk (2005b).

Genidens genidens (Cuvier, 1829)

Pimelodus genidens Cuvier, 1829: 294. Type locality: Not stated. Syntypes: MNHN 0000-1213 (2), MNHN b-0678 (1). Species described in more detail, as Bagrus genidens, in Cuvier \& Valenciennes (1840a: 453 (335 of Strasbourg deluxe edition), pl. 419), with locality given as Rio-Janéiro.

Genidens granulosus Castelnau, 1855: 34, pl. 16 (fig. 1). Type locality: la rivière d'Araguay, dans la province brésilienne de Goyaz. Holotype: MNHN 0000-1197.

Genidens cuvieri Castelnau, 1855: 34. Type locality: la Plata. Syntypes: MNHN 0000-1213 (2), MNHN b-0678 (1). Unneeded replacement name for "Bagrus genidens Cuv. Val.” [= Pimelodus genidens Cuvier].

Genidens valenciennesii Bleeker, 1858b: 68. Type locality: Rio-Janéiro. Syntypes: MNHN 0000-1213 (2), MNHN b-0678 (1). Unneeded replacement name for “Bagrus genidens CV.” [= Pimelodus genidens Cuvier].

Distribution: Atlantic draining rivers of southern South America (Marceniuk \& Ferraris, 2003).

Genidens machadoi (Miranda Ribeiro, 1918)

Tachysurus machadoi Miranda Ribeiro, 1918b: 108. Type locality: Macaé, Rio de Janeiro, Brasil. Holotype: MNRJ 661.

Distribution: Atlantic coastal rivers of southern South America, from Rio de Janeiro to Argentina and, possibly, Chile (Marceniuk \& Ferraris, 2003).

Remarks: Redescribed in Marceniuk (2005b). Possible inclusion of this species in the fauna of Chile is based on report by Sielfeld (1979).

Genidens planifrons (Higuchi, Reis \& Araújo, 1982)

Netuma planifrons Higuchi, Reis \& Araújo, 1982: 12, fig. 1. Type locality: Lagoa dos Patos, RS, Brazil. Holotype: MZUSP 14828.

Distribution: Laguna dos Patos drainage of Southern Brazil and nearby Atlantic Ocean (Marceniuk \& Ferraris, 2003).

\section{HEMIARIUS Bleeker, 1862}

Hemiarius Bleeker, 1862 (in Bleeker, 1862-63): 7, 29. Type species: Cephalocassis stormii Bleeker, 1858. Type by original designation. Gender: Masculine.

Hemiarius dioctes (Kailola, 2000)

Arius dioctes Kailola, 2000: 128, figs. 1-2. Type locality: Norman River at Karumba (17²9'S, $\left.140^{\circ} 50^{\prime} \mathrm{E}\right)$, Queensland, Australia. Holotype: CSIRO C.3798.

Distribution: Southern coast of New Guinea and rivers from Kamora River to Otokwa River, and eastward to Gulf of Papua rivers; northern coast and rivers of Australia from Adelaide to the Gulf of Carpentaria (Kailola, 2000).

Hemiarius insidiator (Kailola, 2000) 
Arius insidiator Kailola, 2000: 131, figs. 3-4. Type locality: Mouth of Wildman River, (12²6'S, $\left.132^{\circ} 09^{\prime} \mathrm{E}\right)$, Northern Territory, Australia. Holotype: NTM S.11189-001.

Distribution: Southern coast of New Guinea, along the Gulf of Papua, and northern coast of Australia, east of Darwin (Kailola, 2000).

Hemiarius sona (Hamilton, 1822)

Pimelodus sona Hamilton, 1822: 172, 376. Type locality: estuaries of Bengal. No types known. Unpublished Hamilton illustration reproduced in Hora (1929: pl. 19, fig. 7).

Distribution: South Asia and southern portion of Malay Peninsula; in coastal waters, estuaries, and lower portions of rivers (Jayaram, 1982); also Mekong River delta and possibly into Cambodia (Rainboth, 1996).

Hemiarius stormii (Bleeker, 1858)

Cephalocassis Stormii Bleeker, 1858b: 246. Type locality: Sumatra (Palembang), in flumine Mussi. Syntypes (2: 415-520 mm TL): possibly BMNH 1863.12.4.65 (1, $276 \mathrm{~mm} \mathrm{L,} 315 \mathrm{~mm}$ TL), but apparently too short. Illustrated in Bleeker (1862-63: pl. 100 [= Silur. pl. 52], as Hemiarius Stormi).

Distribution: Rivers of Sumatra and Borneo (Ng, 2003e).

Hemiarius verrucosus $(\mathrm{Ng}, 2003)$

Arius verrucosus Ng, 2003e: 3, fig. 1. Type locality: Laos, Champasak, Mekong River at Ban Hang Khone, just downstream from Khone Falls. Holotype: UMMZ 235408.

Distribution: Middle and lower Mekong River basin (Ng, 2003e).

\section{Species inquirenda, Hemiarius}

Hexanematichthys leptocassis Bleeker, 1861: 65. Type locality: Pinang. No types known (see Bleeker, 1862-63: 30, for details).

HEXANEMATICHTHYS Bleeker, 1858

Hexanematichthys Bleeker, 1858b: 61, 126. Type species: Bagrus sondaicus Valenciennes, 1840. Type by monotypy. Gender: Masculine.

Hexanematichthys mastersi (Ogilby, 1898)

Arius mastersi Ogilby, 1898a: 34. Type locality: Port Darwin, Northern Australia. Holotype: AMS I.25690-001.

Arius sagoroides Hardenberg, 1941: 221, fig. 2. Type locality: Merauke, ... mouth of the Oetakwa River, New Guinea [Papua, Indonesia]. Syntypes (3, 24-32 cm): Whereabouts unknown.

Tachysurus (Pararius) godfreyi Whitley, 1941b: 11, fig. 8 (nos. 3-4). Type locality: Port Darwin, Northern Territory, Australia. Holotype: AMS I.5270.

Distribution: Sahul Shelf (Kailola, 2004).

Hexanematichthys sagor (Hamilton, 1822)

Pimelodus sagor Hamilton, 1822: 169, 376. Type locality: estuaries of Bengal. No types known.

Bagrus doroides Valenciennes, in Cuvier \& Valenciennes, 1840a: 448 (331 of Strasbourg deluxe edition), pl. 418.

Type locality: Pondichéry ... Bengale. Syntypes: MNHN a-8668 (1), MNHN a-9349 (1).

Bagrus Javensis Valenciennes, in Cuvier \& Valenciennes, 1840a: 445 (330 of Strasbourg deluxe edition). Type locality: Java. Holotype (6 pouces): Whereabouts unknown.

Bagrus sondaicus Valenciennes, in Cuvier \& Valenciennes, 1840a: 444 (329 of Strasbourg deluxe edition). Type locality: dans le détroit de Sonde. Holotype: MNHN b-0604.

Hexanematichthys sundaicus Bleeker, 1858c: 2. Type locality: Strait of Sonde. Holotype: MNHN b-0604. Unjustified emendation of Bagrus sondaicus Valenciennes, 1840.

Distribution: Sunda shelf (Kailola, 2004); also Pakistan and west coast of India (Jayaram, 1982).

\section{KETENGUS Bleeker, 1847}

Ketengus Bleeker, 1847a:167. Type species: Ketengus typus Bleeker, 1847. Type by original designation. Gender: Masculine.

Ketengus typus Bleeker, 1847 
Ketengus typus Bleeker, 1847b: 9. Type locality: Banjermassing, in fluviis. Syntype: BMNH 1863.12.4.112 (1, 191 $\mathrm{mm}$ SL).

Pimelodus pectinidens Cantor, 1849: 1243. Type locality: Pinang. Holotype (4 2/8 inches): BMNH 1860.3.19.499, one-half skin only.

Distribution: Eastern Bay of Bengal and Malay Peninsula; in nearshore coastal waters, estuaries, and lower reaches of rivers (Kailola, 1999).

Remarks: Redescribed in Jayaram (1972b).

NEDYSTOMA Ogilby, 1898

Nedystoma Ogilby, 1898a: 32. Hemipimelodus dayi Ramsay \& Ogilby, 1886. Type by original designation. Gender: Neuter.

Doiichthys Weber, 1913: 532. Type species: Doiichthys novaeguineae Weber, 1913. Type by monotypy. Gender: Masculine.

Remarks: Kailola (2004) considered Doiichthys novaeguineae to be the sister group to Nedystoma dayi and, on that basis, placed the two formerly-monotypic genera into synonymy.

Nedystoma dayi (Ramsay \& Ogilby, 1886)

Hemipimelodus dayi Ramsay \& Ogilby, 1886: 16. Type locality: Strickland River, New Guinea. Holotype: AMS B.9938.

Distribution: Southern Papua (formerly Irian Jaya), New Guinea (Allen, 1996).

Nedystoma novaeguineae (Weber, 1913)

Doiichthys novae-guineae Weber, 1913: 534, fig. 12. Type locality: "Varen"-Fluss, einem Nebenfluss des LorentzFlusses [New Guinea]. Syntypes: AMNH 9482 (1), ZMA 104122 (5).

Distribution: Southern New Guinea, in estuaries and lower reaches of rivers (Kailola, 1999).

NEMAPTERYX Ogilby, 1908

Nemapteryx Ogilby, 1908: 3, 10. Type species: Arius stirlingi Ogilby, 1898. Type by original designation. Gender: Feminine.

Nemapteryx armiger (De Vis, 1884)

Arius armiger De Vis, 1884: 454. Type locality: New Britain [in error]. Syntypes: QM I.3088 (1), QM I.3039 (1).

Arius stirlingi Ogilby, 1898b: 281. Type locality: Estuary of the Adelaide River, Northern Territory [Australia]. Holotype: SAMA F1095.

Distribution: Southern New Guinea and northern Australia; in coastal waters, estuaries, and lower reaches of rivers (Kailola, 1999).

Remarks: See Kailola (1983, 2004) for synonymy.

Nemapteryx augusta (Roberts, 1978)

Arius augustus Roberts, 1978: 36, figs. 14, 20d. Type locality: Mouth of Binge River, a large strongly flowing tributary of the Middle Fly, $675 \mathrm{~km}$ upriver from Toro Pass, 6³2.5'S, 14055.0'E; Fly River, Papua New Guinea. Holotype: AMS I.27090-001.

Distribution: Middle reaches of the Fly River (Roberts, 1978).

? Nemapteryx bleekeri (Popta, 1900)

Arius bleekeri Popta, 1900: 71. Type locality: Malay Archipelago. Syntypes: RMNH 6825 (4).

Distribution: Malay Archipelago.

Remarks: Validity and placement in Nemapteryx considered tentative by Kailola (2004).

Nemapteryx caelata (Valenciennes, 1840)

Arius calatus Valenciennes, in Cuvier \& Valenciennes, 1840b: 66 (49 of Strasbourg deluxe edition). Type locality: Batavia ... Bombay. Syntypes: MNHN b-0589 (1), MNHN b-0614 (2).

Distribution: Widely distributed from Pakistan to Borneo (Jayaram, 1982).

Remarks: Generally treated as valid, but Kailola (1999: 1859; 2004: 139) indicated that it might, instead, be a synonym of Nemapteryx nenga (Hamilton, 1822).

Nemapteryx macronotacantha (Bleeker, 1846) 
Arius macronotacanthus Bleeker, 1846a: 159. Type locality: Batavia. Holotype (size not stated): possibly RMNH 6901 (but see BMNH 1863.12.4.59). [See also Bleeker, 1847c.]

? Arius parvipinnis Day, 1877 (in Day, 1875-78): 460, pl. 113 (fig. 1). Coromandel coast of India. Syntypes: Whereabouts unknown.

Distribution: Bay of Bengal and western central Pacific; in coastal waters and estuaries (Kailola, 1999).

Nemapteryx nenga (Hamilton, 1822)

Pimelodus nenga Hamilton, 1822: 171, 376. Type locality: estuaries of Bengal. No types known.

Distribution: Eastern Arabian Sea, Bay of Bengal, and western central Pacific; in nearshore turbid waters and lower reaches of rivers (Kailola, 1999).

NEOARIUS Castelnau, 1878a

Neoarius Castelnau, 1878a: 237. Type species: Arius curtisii Castelnau, 1878. Type by monotypy. Gender: Masculine.

Remarks: Treated as a synonym of Ariopsis in Kailola (2004); treated herein as valid for the Australia-New Guinea species of Kailola's Ariopsis.

Neoarius berneyi (Whitley, 1941)

Tachysurus (Pararius) berneyi Whitley, 1941b: 9, fig. 8 (no. 5). Type locality: pools of the Flinders River, near Hughenden and Richmond, Queensland (freshwater), Australia. Holotype: AMS I.13076.

Arius cleptolepis Roberts, 1978: 37, figs. 15, 20e. Type locality: Elevala River, a major lowland tributary of the Upper Fly, $17 \mathrm{~km}$ E of Kiunga, $859 \mathrm{~km}$ upriver from Toro Pass, 605.7'S, 141 $27.7^{\prime} \mathrm{E}$; Fly River, Papua New Guinea. Holotype: AMS I.27092-001.

Distribution: Northern Australia and central-southern New Guinea (Kailola, in Allen, 1989).

Neoarius coatesi (Kailola, 1990)

Arius coatesi Kailola, 1990: 18, figs. 5. Type locality: Market at Angoram, Sepik River, Papua New Guinea. Holotype: AMS I.25405-001.

Distribution: Sepik River basin, Papua New Guinea (Kailola, 1990).

Neoarius graeffei (Kner \& Steindachner, 1867)

Arius Graeffei Kner \& Steindachner, 1867: 383, pl. 4 (fig. 12). Type locality: Samoa-Inseln [in error]. Holotype: NMW 67152.

Arius australis Günther, 1867d: 103, figure on p. 103. Type locality: Hunter River, New South Wales, near Ash Island [Australia]. Syntypes: BMNH 1866.2.13.4 (1), BMNH 1866.6.19.7-8 (2).

Arius curtisii Castelnau, 1878a: 236. Type locality: Moreton Bay, Australia. Syntypes (several, to 15 inches): MNHN b-0693 (1).

Distribution: Southern New Guinea and northern Australia, in rivers and along coast (Kailola, 1999).

Remarks: Synonymy follows Kailola (1983 and 2004).

Neoarius latirostris (Macleay, 1883)

Arius latirostris Macleay, 1883: 277, unnumbered figure. Type locality: Goldie River, New Guinea. Syntypes: AMS I.9072 (1), AMS I.9073 (1), AMS I.9074 (1), AMS I.9127 (1), AMS I.13398 (1).

Arius acrocephalus Weber, 1913: 543, figs. 20-21. Type locality: Van der Sande-Fluss (Bibis-Fluss), ein Nebenfluss des Lorentz-Flusses; Lorentz-Fluss bei Regen-Insel; Lorentz-Fluss bei Alkmaar [New Guinea]. Syntypes: AMNH 9514 (1), ZMA 111087 (1), ZMA 111088 (2), ZMA 111089 (1), ZMA 111090 (1).

Arius digulensis Hardenberg, 1936b: 369. Type locality: Middle course of Digulriver [southern New Guinea]. Holotype: ZMA 110781.

Distribution: Southern Papua (formerly Irian Jaya) (Allen, 1996), Goldie River, Papua New Guinea.

Neoarius leptaspis (Bleeker, 1862)

Hexanematichthys leptaspis Bleeker, 1862 (in Bleeker, 1862-63): 27, pl. 65. Type locality: Nova Guinea austrooccidentalis. Holotype: RMNH 3060.

Distribution: Southern New Guinea and northern Australia, in coastal waters and rivers (Kailola, 1999).

Neoarius midgleyi (Kailola \& Pierce, 1988)

Arius midgleyi Kailola \& Pierce, 1988: 75, figs. 1-2, 5-6. Type locality: Wickham Gorge, Victoria River, Northern 
Territory, Australia. Holotype: AMS I.20858-006.

Distribution: Victoria, Katherine, Daly, Ord, remaining Kimberley and northern River systems, Northern Australia (Kailola, 2000).

Neoarius paucus (Kailola, 2000)

Arius paucus Kailola, 2000: 141. Type locality: Flinders River near Maxwellton (2047'S, $\left.142^{\circ} 43^{\prime} \mathrm{E}\right)$, Queensland, Australia. Holotype: QM I.12910.

Distribution: Roper and Flinders River systems, and other rivers draining into Gulf of Carpentaria, Australia (Kailola, 2000).

Neoarius pectoralis (Kailola, 2000)

Arius pectoralis Kailola, 2000: 133, figs. 5-6. Type locality: Chapman River, Queensland, 1456'S, 141 ${ }^{\circ} 38^{\prime} \mathrm{E}$, Australia. Holotype: AMS I.27415-001.

Distribution: Southern coast of New Guinea and into estuaries and tidal rivers, and northern Australia from Darwin to the Chapman River, Queensland (Kailola, 2000).

Neoarius taylori (Roberts, 1978)

Hemipimelodus taylori Roberts, 1978: 40, figs. 19, 20h. Type locality: Mainstream of Plamer and lower end of small tributary about $1 \mathrm{~km}$ up the Palmer from Thompson Junction (mouth of Wai Mungi), 646'48"S, 141'36'36"E; Fly River, Papua New Guinea. Holotype: AMS I.27087-001. Previously considered preoccupied in Arius by Arius taylori Hildebrand, 1925; replaced by Arius robertsi Kailola, 1990 [see Remarks].

Arius robertsi Kailola, 1990: 24. Type locality: Mainstream of Plamer and lower end of small tributary about $1 \mathrm{~km}$ up the Palmer from Thompson Junction (mouth of Wai Mungi), 646'48"S, 141³6'36"E; Fly River, Papua New Guinea. Holotype: AMS I.27087-001. Replacement for Hemipimelodus taylori Roberts, 1978, when preoccupied in Arius by Arius taylori Hildebrand, 1925..

Distribution: Fly River, Papua New Guinea (Roberts, 1978).

Remarks: Arius robertsi Kailola, 1990, was proposed as a replacement for Hemipimelodus taylori Roberts, 1978, which was then considered to be preoccupied in Arius by Arius taylori Hildebrand, 1925. Kailola (2004) subsequently placed A. taylori Hildebrand in Cathorops, as a junior synonym of C. steindachneri (Gilbert \& Starks), and H. taylori Roberts in Ariopsis, as a synonym of Ariopsis robertsi Kailola. In adopting the interpretation of Kailola (2004), Hemipimelodus taylori Roberts is no longer treated as congeneric with Arius taylori and, following Art. 59.4 of the Code (ICZN, 1999) H. taylori must be reinstated as valid, with Arius robertsi as an objective junior synonym.

Neoarius utarus (Kailola, 1990)

Arius utarus Kailola, 1990: 12, figs. 4. Type locality: Murik Lakes, New Guinea. Holotype: AMS I.25406-001.

Distribution: Northern New Guinea; in freshwater and estuaries (Kailola, 1990).

Neoarius velutinus (Weber, 1907)

Hemipimelodus velutinus Weber, 1907: 225. Type locality: Sentani-see, Nord-Neu-Guinea; Mündung des Tami, Nord-Neu-Guinea; Fluss Tawarin, Nord-Neu-Guinea. Syntypes (31): AMNH 9478 (1), FMNH 52392 (1), NMW 8222 (1), NMW 8223 (1), RMNH 8001 (1), ZMA 112654 (3), ZMA 112655 (6), ZMA 112656 (12).

Hemipimelodus papillifer Herre, 1935: 390. Type locality: Timbunke, a village on the Sepik River, about 120 miles from the sea, New Guinea. Holotype: FMNH 17211.

Distribution: Northern Papua (formerly Irian Jaya) (Allen, 1996) and Sepik River basin.

NETUMA Bleeker, 1858

Netuma Bleeker, 1858b: 62, 93. Type species: Bagrus netuma Valenciennes, 1840. Type by absolute tautonymy. Gender: Feminine.

Pararius Whitley, 1940b: 409. Type species: Arius proximus Ogilby, 1898. Type by original designation. Gender: Masculine. Originally proposed as a subgenus of Tachysurus.

Remarks: The names Catastoma (in Cuvier \& Valenciennes, 1840b: 60), and Sarcogenys (in Bleeker, 1858b: 96), were not published in a manner that made them available for purposes of zoological nomenclature and are therefore not listed above as available names; Kailola (2004) considered both to refer to alternate names for Netuma Bleeker. 
Netuma bilineata (Valenciennes, 1840)

Bagrus bilineatus Valenciennes, in Cuvier \& Valenciennes, 1840a: 434 (322 of Strasbourg deluxe edition). Type locality: Pondichéry ... Rangoon. Syntype: MNHN a-9344 (1, mounted).

Bagrus rhodonotus Bleeker, 1846a: 157. Type locality: Batavia. Syntypes (size and number not stated): RMNH 15865 (5), SMNS 10568 (1). [See also Bleeker, 1847c.]

Netuma osakae Jordan \& Kasawa, in Jordan \& Hubbs, 1925: 157, pl. 9 (fig. 1). Type locality: Fish market, Osaka, Japan. Holotype: FMNH 59388.

Arius dayi Dmitrenko, 1974: 39, figs. 1-2. Type locality: Masir Strait, Gulf of Oman, 19²7.8'N, 58²8.3'E. Holotype: Zool. Mus. Ukranian Acad. Kiev Pi 2/1-2.

Distribution: Arabian Sea, Bay of Bengal, Western Central Pacific and northern coast of Australia; from estuaries out to continental shelf (Kailola, 1999).

Remarks: See Kailola (1986a) for discussion of validity of this species. See Wang et al. (2005b) for comments on the distribution of this species.

Netuma proxima (Ogilby, 1898)

Arius proximus Ogilby, 1898b: 280. Type locality: Port Darwin, Northern Australia. Holotype: AMS I.25691-001.

Arius arafurensis Hardenberg, 1948: 409, fig. on p. 409. Type locality: Fish market of Dobo (Aru Islands), IndoAustralian Archipelago. Holotype (about 320 mm SL): Whereabouts unknown.

Distribution: Southern New Guinea, northern Australia and Aru Islands; in nearshore coastal waters and estuaries (Kailola, 1999).

Netuma thalassina (Rüppell, 1837)

Bagrus thalassinus Rüppell, 1837, in Rüppell, 1835-38: 75, pl. 20 (fig. 2). Type locality: Massawa, Eritrea, Red Sea. Syntypes SMF 544 (1), SMF 5414 (1, dry), SMF 2627 (1, dry), SMF 5740 (1, skeleton).

Bagrus laevigatus Valenciennes, in Cuvier \& Valenciennes, 1840a: 439 (325 of Strasbourg deluxe edition). Type locality: la Mer Rouge. Holotype: MNHN b-0710.

Arius nasutus Valenciennes, in Cuvier \& Valenciennes, 1840b: 60 (45 of Strasbourg deluxe edition). Type locality: Java ... Malabar ... la Mer Rouge. Syntypes: MNHN a-9407 (1), MNHN a-9408 (1, dry), MNHN a-9409 (1, dry), RMNH D2390 (1).

Bagrus netuma Valenciennes, in Cuvier \& Valenciennes, 1840a: 438 (324 of Strasbourg deluxe edition), pl. 417. Type locality: Pondichéry. Syntype: MNHN a-9345 (1).

Bagrus carchariorhijnchos Bleeker, 1846b: 291. Type locality: Batavia, Java. Holotype: RMNH 6885.

Arius serratus Day, 1877 (in Day, 1875-78): 462, pl. 105 (fig. 3). Type locality: Sind [Pakistan]. Holotype: ZSI 467. Ariodes aeneus Sauvage, 1883c: 160. Type locality: Ile Rafflès. Syntypes: MNHN a-5155 (2).

Netuma thalassina jacksonensis Whitley, 1941b: 12, fig. 8 (nos. 1-2). Type locality: Port Jackson, New South Wales [Australia]. Holotype: AMS I.10095.

Distribution: Widely distributed through Red Sea, Indian Ocean and western central Pacific; in coastal waters out to continental shelf margin.

Remarks: See Kailola (1986a) and Wang (2005b) for discussion of validity. See Jayaram \& Dhanze (1978: 203) and Kailola (1999: 1842, 1865) for comments on status of Arius serratus.

\section{Species inquirenda, Netuma}

Galeichthys stanneus Richardson, 1846: 284. Type locality: Canton, China. No types known. Appeared as ? Galeichthys stanneus. Based solely on illustration by Reeves which was reproduced in Whitehead (1970: 210, pl. 20a).

NOTARIUS Gill, 1863

Notarius Gill, 1863: 171. Type species: Arius grandicassis Valenciennes, 1840. Type by monotypy. Gender: Masculine.

Remarks: The limits of Notarius are not clearly diagnosed. Kailola (2004) included some of the species listed here in Aspistor.

Notarius biffi Betancur \& Acero, 2004 
Notarius biffi Betancur \& Acero, 2004: 3, figs. 1-3. Type locality: Bahía La Unión, El Salvador (SV) (13²0'42"N, 87²9'07"W. Holotype: STRI 6674.

Distribution: Tropical Eastern Pacific from Costa Rica to El Salvador (Betancur \& Acero, 2004).

Notarius cookei (Acero \& Betancur, 2002)

Arius cookei Acero \& Betancur, 2002a: 134, fig. 1. Type locality: 7 km from the mouth of Rio Santa Maria, Paris, Herrera, Panama ( $\left.8^{\circ} 9^{\prime} \mathrm{N}, 80^{\circ} 33^{\prime} \mathrm{W}\right)$. Holotype: INVEMAR-PEC 3752.

Distribution: Pacific coastal rivers and estuaries of Central and South America, from Costa Rica to Colombia (Acero \& Betancur, 2002).

Notarius grandicassis (Valenciennes, 1840)

Arius grandicassis Valenciennes, in Cuvier \& Valenciennes, 1840b: 54 (41 of Strasbourg deluxe edition), pl. 427. Type locality: la Guyane. Holotype: MNHN a-4608.

Arius parmocassis Valenciennes, in Cuvier \& Valenciennes, 1840b: 57 (43 of Strasbourg deluxe edition). Type locality: Bahia. Syntypes: MHNG (several), MNHN a-8966.

Arius stricticassis Valenciennes, in Cuvier \& Valenciennes, 1840b: 58 (44 of Strasbourg deluxe edition). Type locality: Cayenne. Syntypes: MNHN a-9406 (1), RMNH 3034 (1).

Arius vandeli Puyo, 1936: 123, fig. 21g. Type locality: [French Guiana]. Holotype: MNHN 1936-0160.

Distribution: Rivers and estuaries of northern South America from Gulf of Venezuela to the mouth of the Amazon River (Marceniuk \& Ferraris, 2003).

Notarius insculptus (Jordan \& Gilbert, 1882)

Arius elatturus Jordan \& Gilbert, 1882: 45. Type locality: Panama. Syntypes (3): USNM 29408 (1, missing), USNM 30995 (1 of 2).

Arius insculptus Jordan \& Gilbert, 1882: 41. Type locality: Panama. Syntypes: USNM 29415 (1), USNM 30977 (2). Betancur \& Acero (2004) referred to USNM 29415 as a lectotype, but no previous lectotype designation has been found, and indications of a lectotype in that publication do not meet the requirements of the Code for lectotype designation. Because no previously published lectotype designation was found, the specimens listed here are considered as syntypes.

Netuma insularum Greene, in Gilbert, 1897: 439. Type locality: Galapagos Archipelago [later corrected to: Gulf of Panama]. Holotype: USNM 47577.

Distribution: Pacific coast of Central America, from Costa Rica to Panama (Marceniuk \& Ferraris, 2003).

Remarks: Jordan \& Gilbert (1882) were reported to have designated a holotype for Arius elatturus by Ferraris \& Vari (1992), Eschmeyer et al. (1998), Marceniuk \& Ferraris (2003). However, a closer reading of the paper shows that the specimen subsequently cited as holotype (USNM 29408) was the basis for the species description but otherwise not explicitly designated as the type. Therefore, it must instead be considered to be a syntype as one of three "specimens at the United States National Museum" representing the new species. Betancur \& Acero (2004: 7) appear to be have acted as first reviser in treating Arius insculptus as a valid species in Notarius, with Arius elatturus as a synonym.

Notarius kessleri (Steindachner, 1876)

Arius Kessleri Steindachner, 1876: 574, pl. 5. Type locality: Panama, Altata. Syntypes: MCZ 4944 (6), NMW 48112 (1), NMW 48247 (1), NMW 48248 (1), NMW 48249 (2).

Distribution: Eastern tropical Pacific Ocean (Marceniuk \& Ferraris, 2003).

Notarius neogranatensis (Acero \& Betancur, 2002)

Arius neogranatensis Acero \& Bentancur, 2002b: 7, fig. 1. Type locality: mouth of Río Tinajones medio, San Antero, Córdoba, Colombia (9 25'N 75 48'W). Holotype: INVEMAR-PEC 3754.

Distribution: Caribbean coast of Colombia and nearby rivers mouths (Acero \& Betancur, 2002).

Notarius osculus (Jordan \& Gilbert, 1882)

Arius osculus Jordan \& Gilbert, 1882: 46. Type locality: Panama. Holotype: USNM 29476.

Distribution: Pacific coast of Central America, from Costa Rica and Panama (Marceniuk \& Ferraris, 2003).

Remarks: Placed by Kailola \& Bussing (1995: 871) tentatively in Arius.

Notarius planiceps (Steindachner, 1876)

Arius planiceps Steindachner, 1876: 576, pl. 4. Type locality: Panama, Altata. Syntypes: NMW 48194 (1), NMW 
48195 (1), NMW 48196 (1), NMW 48197 (1), NMW 48198 (1), NMW 48199 (2), ZMUC P 29627 (1).

Distribution: Pacific Ocean draining rivers and estuaries of North and Central America from Mexico to Panama (Marceniuk \& Ferraris, 2003).

\section{OSTEOGENEIOSUS Bleeker, 1846}

Osteogeneiosus Bleeker, 1846a:173. Type species: Arius militaris Valenciennes, 1840 (= Silurus militaris Linnaeus, 1758). Type by subsequent designation, by Bleeker (1862-63: 8). Gender: Masculine.

Osteogeneiosus militaris (Linnaeus, 1758)

Silurus militaris Linnaeus, 1758: 305. Type locality: Asia. No types known.

? Osteogeneiosus Blochii Bleeker, 1846a: 175. Type locality: Batavia. Type(s) (size and number not stated): Whereabouts unknown [See also Bleeker, 1847: 50.]

? Osteogeneiosus gracilis Bleeker, 1846a: 175. Type locality: Batavia. Type(s) (size and number not stated): Whereabouts unknown [See also Bleeker, 1847:50.]

? Osteogeneiosus ingluvies Bleeker, 1846a: 174. Type locality: Batavia. Type(s) (size and number not stated): Whereabouts unknown [See also Bleeker, 1847: 50.]

? Osteogeneiosus longiceps Bleeker, 1846a: 174. Type locality: Batavia. Type(s) (size and number not stated): Whereabouts unknown [See also Bleeker, 1847: 50.]

? Osteogeneiosus macrocephalus Bleeker, 1846a: 173. Type locality: Batavia. Syntypes (size and number not stated): possibly BMNH 1863.12.4.51 (1, 214 mm SL, 252 mm TL), NMV 45847 (1).

? Osteogeneiosus valenciennesi Bleeker, 1846a: 175. Type locality: Batavia. Syntypes (size and number not stated): possibly BMNH 1863.12.4.53 (1245 mm SL, 289 mm TL), NMV 46593 (1), NMV 46594 (1).

? Osteogeneiosus Cantoris Bleeker, 1854c: 120, pl. 2 (fig. 4). Type locality: Calcutta, in flumine Hooghly. Syntype (2, 199 - 225 mm): RMNH 6907 (1).

? Osteogeniosus sthenocephalus Day, 1877 (in Day, 1875-78): 469, pl. 108 (fig. 3). Type locality: Moulmein [Burma]. Holotype: ZSI 1263. Name spelled Osteogeniosus stenocephalus on plate caption; first reviser not yet found.

Distribution: Bay of Bengal and western central Pacific, in inshore waters, river mouths, and coastal lake systems (Kailola, 1999); also Pakistan and west coast of India (Jayaram, 1982).

Remarks: At present, there is only one recognized valid species in Osteogeneiosus. On that basis, all of the other names are tentatively placed here as synonyms of $O$. militaris.

\section{PLICOFOLLIS Kailola, 2004}

Plicofollis Kailola, 2004: 141. Type species: Arius argyropleuron Valenciennes, 1840. Type by original designation. Gender: Masculine.

Plicofollis argyropleuron (Valenciennes, 1840)

Arius argyropleuron Valenciennes, in Cuvier \& Valenciennes, 1840b: 104 (78 of Strasbourg deluxe edition). Type locality: Java. Holotype: RMNH 3041.

Arius acutus Bleeker, 1846a: 167. Type locality: Batavia. Syntypes (size and number not stated): possibly BMNH 1863.12.4.67 (1), RMNH 15975 (2), SMNS 10566 (4).

Arius Hamiltonis Bleeker, 1846b: 291. Type locality: [Batavia]. Syntypes (size and number not stated): RMNH 6895 (at least part of series).

Arius macrocephalus Bleeker, 1846a: 167. Type locality: Batavia. Syntypes (size and number not stated): BMNH 1863.12.4.78 (1), RMNH 6896 (1). One syntype illustrated in Bleeker (1862-63: 39, pl. 58 [Silur. pl. 10], as Ariodes macrocephalus.

Arius layardi Günther, 1866: 474, pl. 15. Type locality: Ceylon. Holotype: BMNH 1866.7.13.13.

Tachysurus broadbenti Ogilby, 1908: 8. Type locality: Cape York, Queensland, Australia. Syntypes (3): QM I.9745 (1), QM I.9746 (1).

Hemipimelodus colcloughi Ogilby, 1910: 7. Type locality: Croker Island, N.T., Australia. Holotype: QM I.1538.

Distribution: Bay of Bengal, western Pacific Ocean, southern coast of New Guinea and northern coast of Australia; in coastal waters and in lower portions of estuaries (Kailola, 1999). 
Remarks: Synonymy from Kailola (1999).

Plicofollis dussumieri (Valenciennes, 1840)

Arius Dussumieri Valenciennes, in Cuvier \& Valenciennes, 1840b: 84 (63 of Strasbourg deluxe edition). Type locality: la côte de Malabar. Holotype: MNHN a-9401.

Arius kirkii Günther, 1864: 163, unnumbered figure. Type locality: Shupanga, Zambezi [River]. Holotype: BMNH 1864.1.9.54 (skin).

Arius goniaspis Bleeker, 1858a: 44. Type locality: Priaman [Sumatra, Indonesia]. Syntypes (2): BMNH 1863.12.4.159 (1, $71 \mathrm{~mm} \mathrm{SL,} 84 \mathrm{~mm}$ TL).

Distribution: East coast of Africa, Madagascar, Arabian Sea, Bay of Bengal, and eastern Sumatra; possibly along eastern part of Malay peninsula; in coastal waters and estuaries (Taylor, 1986; Kailola, 1999).

Remarks: Register number of holotype of Arius goniaspis incorrectly reported as BMNH 1863.12.11.159 in Eschmeyer et al. (1998).

Plicofollis nella (Valenciennes, 1840)

Pimelodus nella Valenciennes, in Cuvier \& Valenciennes, 1840b: 162 (121 of Strasbourg deluxe edition). Type locality:[Vizagapatam]. Holotype: not saved, based on Russell (1803: pl. 170). Originally as Pimelodus ? nella.

Arius leiotetocephalus Bleeker, 1846b: 292. Type locality: Batavia. Syntypes: RMNH 5277 (1), RMNH 16011 (5), SMNS 817 (2).

Bagrus (Ariodes) Meyenii Müller \& Troschel, 1849: 9. Type locality: unbekannt. Holotype: ZMB 3002.

Distribution: Bay of Bengal, western central Pacific and northern coast of Australia.

Plicofollis platystomus (Day, 1877)

Arius platystomus Day, 1877 (in Day, 1875-78): 464, pl. 107 (fig. 3). Type locality: Canara [India]. Holotype: ZSI 1306.

Distribution: Kanara, Malabar and Konkan coasts, India, and Hoogly River mouth (Jayaram, 1982).

Plicofollis polystaphylodon (Bleeker, 1846)

Arius polystaphylodon Bleeker, 1846a: 172. Type locality: Batavia. Holotype or Syntype (size and number not stated): BMNH 1863.12.4.98 (1, $121 \mathrm{~mm} \mathrm{SL).}$

Distribution: Western central Pacific, eastward to northern New Guinea; in turbid coastal waters, and river mouths (Kailola, 1999).

Plicofollis tenuispinis (Day, 1877)

Arius tenuispinis Day, 1877 (in Day, 1875-78): 466, pl. 107 (fig. 5). Type locality: Bombay, and perhaps Ceylon. Holotype: ZSI F482.

Distribution: Coast of India and eastern Africa (Jayaram, 1982).

Remarks: Redescribed in Talwar (1976), with illustration of oral tooth patterns.

Plicofollis tonggol (Bleeker, 1846)

Arius crossocheilos Bleeker, 1846a: 172. Type locality: Batavia. Syntypes (size and number not stated): RMNH 6894 (1 or more of 3).

Arius tonggol Bleeker, 1846b: 292. Batavia. Syntypes: BMNH 1863.12.4.56 (1, 183 mm SL, 246 mm TL), RMNH 6894.

Distribution: Western Central Pacific, and Bay of Bengal; in estuaries (Kailola, 1999).

Remarks: The names Arius crossocheilos and A. tonggol were first made available in separate publications, but in the same issue of Natuur-en Geneeskundig Archif voor Neerlandsch Indië. As such, they must be treated as having been published simultaneously and, therefore, in need of first reviser action to establish precedence. The earliest first reviser action I found was Bleeker (1862 [in Bleeker, 1862-63]: 39) in which Ariodes tonggol is treated as valid with Arius crossocheilos a synonym. First included in Plicofollis by Kailola (2004), but as Plicofollis crossocheilos.

\section{Species inquirenda, Plicofollis}

Arius magatensis Herre, 1926: 396, pl. 1 (fig. 1). Type locality: Magat River at Bagabag, Nueva Vizcaya Province, Philippines. Syntypes (6): BSMP (destroyed). 
POTAMARIUS Hubbs \& Miller, 1960

Potamarius Hubbs \& Miller, 1960: 101. Type species: Conorhynchos nelsoni Evermann \& Goldsborough, 1902. Type by original designation. Gender: Masculine.

Potamarius grandoculis (Steindachner, 1877)

Arius grandoculis Steindachner, 1877b: 644, pl. 11. Type locality: Rio doce (nach Salmin), südöstlichen Brasilien. Holotype: NMW 48272. Name spelled Arius granducolis in plate caption.

Distribution: Doce and Paraíba do Sul rivers of Brazil, at and near their mouths (Marceniuk \& Ferraris, 2003).

Potamarius izabalensis Hubbs \& Miller, 1960

Potamarius izabalensis Hubbs \& Miller, 1960: 104, figs. 2. Type locality: Lago de Izabal, Guatemala, 1 mi. west of El Paraíso. Holotype: USNM 134347.

Distribution: Central America: Izabal Lake and, possibly, Polochic River, Guatemala (Marceniuk \& Ferraris, 2003).

Potamarius nelsoni (Evermann \& Goldsborough, 1902)

Conorhynchos nelsoni Evermann \& Goldsborough, 1902: 140, fig. 1. Type locality: Río Usumacinta, Montecristo, Yucatán, Mexico. Holotype: USNM 50001.

Distribution: Usumacinta River basin, Mexico and Guatemala (Miller et al., 2005).

SCIADES Müller \& Troschel, 1849

Sciades Müller \& Troschel, 1849: 6. Type species: Bagrus (Sciades) emphysetus Müller \& Troschel, 1849. Type by subsequent designation by Bleeker (1862-63: 8). Gender: Masculine. Originally proposed as a subgenus of Bagrus.

Sciadeichthys Bleeker, 1858b: 62, 66. Type species: Bagrus (Sciades) emphysetus Müller \& Troschel, 1849. Type by monotypy. Gender: Masculine.

Selenaspis Bleeker, 1858b: 62. Type species: Silurus herzbergii Bloch, 1794. Type by subsequent designation, apparently first by Jordan \& Evermann (1896: 119). Gender: Feminine.

Leptarius Gill, 1863: 170. Type species: Leptarius dowii Gill, 1863. Type by monotypy. Gender: Masculine.

Sciadeops Fowler, 1944c: 211. Type species: Sciades troschelii Gill, 1863. Type by original designation. Gender: Masculine. Originally proposed as a subgenus of Sciades.

Sciades couma (Valenciennes, 1840)

Bagrus couma Valenciennes, in Cuvier \& Valenciennes, 1840a: 459 (340 of Strasbourg deluxe edition). Type locality: Cayenne. Syntypes: MNHN a-9338 (1), MNHN a-9339 (1).

Sciadeichthys (Selenaspis) walrechti Boeseman, 1954: 60, pl. 3. Type locality: Surinam, probably neighbourhood of Paramaribo. Holotype: RMNH 2141.

Distribution: Atlantic draining rivers of South America, from the Gulf of Paria to the mouth of the Amazon (Marceniuk \& Ferraris, 2003).

Sciades dowii (Gill, 1863)

Leptarius dowii Gill, 1863: 171. Type locality: Western coast of Central America. Holotype: at USNM (not found).

Arius alatus Steindachner, 1876: 569, pl. 6. Type locality: Panama. Syntype: MCZ 7725.

Distribution: Pacific draining rivers and estuaries of South and Central America, from Panama to Ecuador (Marceniuk \& Ferraris, 2003).

Sciades herzbergii (Bloch, 1794)

Silurus Herzbergii Bloch, 1794: 33, pl. 367. Type locality: Surinam. Syntypes: ZMB 2962 (1), ZMB 8785 (1, skin).

Pimelodus argenteus La Cepède, 1803: 94, 102. Type locality: Surinam. Syntypes: ZMB 2962 (1), ZMB 8785 (1). Unneeded new name for Silurus herzbergii Bloch.

Bagrus mesops Valenciennes, in Cuvier \& Valenciennes, 1840a: 456 (338 of Strasbourg deluxe edition). Type locality: [Puerto Rico]. Holotype: MNHN a-9347.

Bagrus pemecus Valenciennes in Cuvier \& Valenciennes, 1840a: 456 (338 of Strasbourg deluxe edition). Type locality: Cayenne. Holotype: MNHN a-9348 (dry).

Bagrus (Bagrus) coelestinus Müller \& Troschel, 1849: 7. Type locality: Guiana. Syntypes: ZMB 2970 (1), ZMB $2971(1)$. 
Hexanematichthys hymenorrhinos Bleeker, 1862a: 377. Type locality: [Not stated]. Holotype: RMNH 3056; holotype illustrated in Bleeker (1864: 57, pl. 11, fig. 2; pl. 13, fig. 4), with type locality stated as Guatemala.

Netuma dubia Bleeker, 1862a: 382. Type locality: Surinama. Syntypes: RMNH 3051(1), RMNH 3052 (1); syntype illustrated in Bleeker (1864: 63, pl. 15, fig. 2; pl. 13, fig. 5).

Distribution: Caribbean and Atlantic draining rivers and estuaries of South America from Colombia to Brazil (Marceniuk \& Ferraris, 2003).

Sciades passany (Valenciennes, 1840)

Bagrus passany Valenciennes, in Cuvier \& Valenciennes, 1840a: 458 (340 of Strasbourg deluxe edition). Type locality: Cayenne. Holotype: MNHN a-9399.

Distribution: Atlantic draining coastal rivers from Guyana to the mouth of the Amazon River (Marceniuk \& Ferraris, 2003).

Sciades platypogon (Günther, 1864)

Arius platypogon Günther, 1864: 147, unnumbered figures. Type locality: Guatemala, San Jose. Syntypes: BMNH 1864.1.26.316-319 (4).

Netuma mazatlana Gilbert, 1904: 255, pl. 25. Type locality: Mazatlán, Sinaloa, Mexico. Holotype: SU 7138.

Distribution: Pacific coast of the Americas, from Mexico to Ecuador (Marceniuk \& Ferraris, 2003).

Sciades proops (Valenciennes, 1840)

Bagrus proops Valenciennes, in Cuvier \& Valenciennes, 1840a: 457 (339 of Strasbourg deluxe edition). Type locality: Cayenne ou de Surinam ... Porto-Rico. Syntypes: MNHN a-9331 (1), MNHN a-9332 (1), MNHN a-9333 (1), MNHN b-0245 (1), MNHN b-0618 (2).

Distribution: Caribbean and Atlantic draining rivers and estuaries of South America and some Caribbean Islands from Colombia to Brazil (Marceniuk \& Ferraris, 2003).

Sciades troschelii Gill, 1863

Sciades troschelii Gill, 1863: 171. Type locality: Western coast of Central America. Holotype: USNM (not found).

Arius Brandtii Steindachner, 1876: 571, pl. 3. Type locality: Altata, Panama. Syntypes: possibly MCZ 4964 (1), NMW 48107 (3), NMW 48110 (1).

Distribution: Pacific coast of the Americas, from Mexico to Peru (Marceniuk \& Ferraris, 2003).

\section{Species inquirenda, Sciades}

Bagrus (Sciades) emphysetus Müller \& Troschel, 1849: 8. Type locality: Surinam. Holotype: ZMB 2990.

\section{Species inquirendae, Ariidae}

Silurus ascita Linnaeus, 1758: 306. Type locality: India. Syntypes: NRM 72 (4). Inclusion in the Ariidae follows Fernholm \& Wheeler (1983: 222).

Pimelodus gambensis Bowdich, 1825: 234, fig. 50. Type locality: Gambia River, west Africa. No types known.

Arius nigricans Valenciennes, 1835, in Valenciennes, 1835-47: pl. 3 (fig. 3). Type locality: [No locality stated, apparently La Plata River, Montevideo, Uruguay]. Holotype: MNHN 0000-0176. Name available from plate; described in Cuvier \& Valenciennes (1840b: 83, 62 of Strasbourg deluxe edition).

Bagrus albicans Valenciennes, in Cuvier \& Valenciennes, 1840a: 461 (342 of Strasbourg deluxe edition), pl. 420. Type locality: Cayenne. Holotype: MNHN a-9350.

Bagrus arioides Valenciennes, in Cuvier \& Valenciennes, 1840a: 440 (326 of Strasbourg deluxe edition). Type locality: Bengal. Holotype (dix pouces): at MNHN.

Arius Belangerii Valenciennes, in Cuvier \& Valenciennes, 1840b: 71 (53 of Strasbourg deluxe edition). Type locality: Bombay. Holotype (6 pouces): at MNHN.

Arius granosus Valenciennes, in Cuvier \& Valenciennes, 1840b: 69 (51 in Strasbourg deluxe edition). Type locality: Pondichéry. Holotype: MNHN b-0681.

Bagrus flavescens Valenciennes, in Cuvier \& Valenciennes, 1840a: 462 (342 of Strasbourg deluxe edition). Type locality: Cayenne. Holotype: MNHN a-8566 (mounted). 
Arius molliceps Valenciennes, in Cuvier \& Valenciennes, 1840b: 108 (80 of Strasbourg deluxe edition). Type locality: on en ignore l'origine. Holotype (5 1/2 pouces): at MNHN.

Arius rostratus Valenciennes, in Cuvier \& Valenciennes, 1840b: 63 (47 of Strasbourg deluxe edition). Type locality: dans les environs d'Alipey. Type(s): at MNHN.

Arius sinensis Valenciennes, in Cuvier \& Valenciennes, 1840b: 72 (54 of Strasbourg deluxe edition). Type locality: Touraine. Holotype (4 pouces): at MNHN.

Bagrus Temminckii Valenciennes, in Cuvier \& Valenciennes, 1840a: 463 (343 of Strasbourg deluxe edition). Type locality: Cayenne [apparently in error for Suriname]. Holotype: at RMNH (apparently lost). As Bagrus temminckii in contents, p. xxii, and Bagrus temminckianus on p. 463. Günther (1864: 151) used temminckii as valid while noting that the name was spelled temminckianus in Cuvier \& Valenciennes (1840a: 463) thereby acting as first reviser. Type locality corrected by Boeseman (1972: 297).

Bagrus trachipomus Valenciennes, in Cuvier \& Valenciennes, 1840a: 442 (328 of Strasbourg deluxe edition). Type locality: [Bengal]. Holotype: MNHN a-9346 (mounted).

Arius aequibarbis Valenciennes, in Cuvier \& Valenciennes, 1840b: 68 (51 of Strasbourg deluxe edition). Type locality: Rangoon; Bengal. Syntypes (2): MNHN 0000-1200 (2), MNHN b-0682 (1).

Bagrus venaticus Richardson, 1845, in Richardson, 1844-48: 33. Type locality: North-west coast of Australia. Holotype: apparently destroyed (see Günther, 1864: 174).

Bagrus vertagus Richardson, 1845, in Richardson, 1844-48: 33. Type locality: North-west coast of Australia. Holotype: apparently destroyed (see Günther, 1864: 174).

Arius caelatoides Bleeker, 1846a: 159. Type locality: Batavia. Type(s) (size and number not stated): Whereabouts unknown.

Arius chondropterygius Bleeker, 1846a: 162. Type locality: Batavia. Type(s) (size and number not stated): Whereabouts unknown.

Arius chondropterygioides Bleeker, 1846a: 170. Type locality: Batavia. Type(s) (size and number not stated): Whereabouts unknown.

Arius clijpeaster Bleeker, 1846a: 161. Type locality: Batavia. Type(s) (size and number not stated): Whereabouts unknown.

Arius clypeastroides Bleeker, 1846a: 161. Type locality: Batavia. Type(s) (size and number not stated): Whereabouts unknown.

Arius Heckelii Bleeker, 1846a: 169. Type locality: Batavia. Type(s) (size and number not stated): Whereabouts unknown.

Arius laeviceps Bleeker, 1846a: 165. Type locality: Batavia. Type(s) (size and number not stated): Whereabouts unknown.

Arius macruropterygius Bleeker, 1846a: 164. Type locality: Batavia. Type(s) (size and number not stated): Whereabouts unknown.

Arius manjong Bleeker, 1846a: 163. Type locality: Batavia. Type(s) (size and number not stated): Whereabouts unknown.

Arius microgastropterygius Bleeker, 1846a: 160. Type locality: Batavia. Type(s (size and number not stated): Whereabouts unknown.

Arius micronotacanthus Bleeker, 1846a: 162. Type locality: Batavia. Type(s) (size and number not stated): Whereabouts unknown.

Arius viviparus Bleeker, 1846a: 171. Type locality: Batavia. Type(s) (size and number not stated): Whereabouts unknown.

Arius angulatus Bleeker, 1846a: 170. Type locality: Batavia. Type(s) (size and number not stated): Whereabouts unknown.

Arius pidada Bleeker, 1846a: 169. Type locality: Batavia. Syntypes (size and number not stated): possibly AMS B.7965 (1), BMNH 1863.12.4.57 (1), NMV 45948 (1). Type series apparently includes at least two species. In the absence of a lectotype, the position of this name is not clear.

Pimelodus mong Richardson, 1846: 286. Type locality: Canton, China. No types known. Based solely on illustration by Reeves (reproduced in Whitehead (1970: 210, pl. 20b).

Bagrus crinalis Richardson, 1846: 282. Type locality: Canton, China. No types known. Originally as ? Bagrus crinalis. 
Based solely on illustration by Reeves, reproduced in Whitehead (1970: 210, pl. 19c).

Arius melanopterygius Bleeker, 1849: 10. Type locality: In Freto Madurae prope Kammal et Surabaya [Java]. Holotype (310 mm TL): Whereabouts unknown.

Arius borneënsis Bleeker, 1851c: 67. Type locality: Bandjarmassing. Syntypes (2, 101, 170 mm): Whereabouts unknown, perhaps at RMNH.

Arius schlegeli Bleeker, 1863d: 146. Type locality: Amoy, China. Syntypes (2): possibly AMS B.8123 (1).

Arius andamanensis Day, 1871: 699. Type locality: Andaman Is. Possible syntypes: AMS B.7931 (1), ZSI F307 (1).

Type series is apparently mixed (Kailola, 1999: 1842, 1865) and, in the absence of a lectotype, position of this name is not clear.

Arius argenteus Lütken, 1874b: 211. Type locality: Canton, China. Holotype: ZMUC P 29624.

Arius festae Boulenger, 1898c: 5. Type locality: Narrangal, Equateur occidental. Holotype: MZUT 1479.

Arius (Hemiarius) nudidens Weber, 1913: 538, fig. 15. Type locality: Lorentz-Fluss [New Guinea]. Syntypes (4): ZMA 111507 (1), ZMA 111508 (1), ZMA 111509 (1).

Netuma aulometopon Fowler, 1915a: 204, fig. 1. Type locality: Dutch Guiana. Holotype: ANSP 8372.

Arius satparanus Chaudhuri, 1916: 430, figs. 6-8. Type locality: Channel between Satpara and Barnikuda, Chilka Lake, Orissa, India. Holotype: ZSI F8784/1; illustrated in Jayaram \& Dhanze (1981b: fig. 1).

Remarks: Possibly a synonym of Arius tenuispinis (Jayaram \& Dhanze, 1981b), or Arius argyropleurodon (Valenciennes) (Kailola, 1999).

Hexanematichthys henni Fisher \& Eigenmann, in Eigenmann, 1922: 30, fig. 9. Type locality: Colimes, Rio Daule, Ecuador. Holotype: CAS 60620. Authorship based on footnote on p. 27.

Netuma hassleriana Borodin, 1934: 33, fig. b. Type locality: Panama. Holotype: MCZ 33213.

Hemipimelodus sundanensis Hardenberg, 1948: 411. Type locality: Kota Agung (Sunda Strait), Sumatra. Syntypes (2, 270-280 mm): Whereabouts unknown.

\section{Unconfirmed fossil Ariidae}

$\dagger$ Arius bartonensis Woodward, 1887: 306, fig. 3. Type locality: High Cliff, Barton Hampshire, England. Upper Eocene. Holotype: BMNH 40280 (dorsal fin spine). Originally: Arius (?) bartonensis.

$\dagger$ Arius egertoni belgicus Leriche, 1905. (no additional information; name from Weiler, 1956: 187).

$\dagger$ Arius Bonneti Priem 1904: 44 (figs. 3-8). Type locality: Bartonien superieur de Marines. Types: dans la collection Bonnet (dorsal and pectoral fin spines); also illustrated in Priem (1908: 123, figs. 63, 64, pl. 3, figs. 5-6).

$\dagger$ Arius Dutemplei Leriche, 1900: 181, pl. 1, figs 13-15. Type locality: Cuis, Chavot [ Marne, Lower Eocene]. Types: at laboratoire de Géologie de l'Université de Lille (dorsal fin spine, pectoral fin spine).

Distribution: several Paris Basin localities, lowermost Eocene (Gaudant, 1981) (from Gayet \& Meunier, 2003).

$\dagger$ Silurus Egertoni Dixon, in Dixon \& Egerton, 1850: 112, 204, pl. 11, figs. 11-13. Bracklesham Bay, Sussex, Middle Eocene. Holotype: BMNH 25612 (pectoral arch and spine).

Remarks: Redescribed as $\dagger$ Arius egertoni in Woodward (1887: 304, figs. 1-2).

$\dagger$ Arius Fraasi Peyer, 1928: 17, pl. 6, fig. 2. Type locality: Unterste Nokkattam-Stufe, Eocän, Cairo. Holotype: at Naturalienkabinett Stuttgart.

$\dagger$ Arius gigas [no additional information; from Gayet \& Meunier (2003)].

Distribution: Chad, Mio-Pliocene (Brunet et al., 2000).

Remarks: Gayet \& Meunier (2003) placed this species in the Ariidae, but without any additional information. It is unclear whether the use of this name in Brunet et al. (2000) and Gayet \& Meunier (2003) was intended to identify fossils with the Recent species Arius gigas Boulenger or this name was proposed independently of, and therefore a homonym of, that name.

$\dagger$ Arius heward-belli White, 1926: 56, pl. 13, figs. 4-12. Type locality: Ameki, Nigeria, Eocene. Holotype: National Collection [Nigeria] or BMNH (pectoral spine tip).

$\dagger$ Arius kitsoni White, 1926: 54, pl. 13, figs. 1-2. Type locality: Ameki, Nigeria, Eocene. Holotype: National Collection [Nigeria] or BMNH (proximal half of right pectoral fin spine).

$\dagger$ Arius kutchensis Raghavendra Rao, 1956: 181, figs. 1-2, pl. 28. Type locality: Gypseous shale beds about two miles 
west of Nareda, Western Kutch, India; Lower Eocene. Holotype: (skull) Geological Survey of India, Calcutta 17539. Remarks: See Sahni \& Mishra (1975: 11, pl. 2, figs. 2-4) for emended diagnosis.

$\dagger$ Arius nucleus Weinfurter, 1952. Type locality: [Austria, Miocene]. [No other information]

$\dagger$ Arius russi White, 1926: 58, pl. 13, fig. 3. Type locality: Oshosun, Nigeria, Eocene. Holotype: National Collection [Nigeria] or BMNH (proximal end of pectoral fin spine).

$\dagger$ Tachysurus landanensis [No other information, taken from Gayet \& Meunier (2003)].

Distribution: Zaire, Eocene (Gayet \& Meunier, 2003).

Remarks: Placement in Ariidae supported by Gayet \& Meunier (2003).

\section{ASPREDINIDAE Adams, 1854}

Aspredinidae Adams, in Adams et al., 1854: 107. Type genus: Aspredo Scopoli, 1777. Bunocephalini Bleeker, 1858b: xi, 328. Type genus: Bunocephalus Kner, 1855.

Platystacinae Eigenmann \& Eigenmann, 1890: 9, 13, 20. Type genus: Platystacus Bloch, 1794.

Hoplomizoninae [sic Hoplomyzontinae] Fernández-Yépez, 1950b:113. Type genus: Hoplomyzon Myers, 1942.

Taxonomy summary: Gill (1891a); Friel (2003); Myers (1960); Mees (1987b, Aspredininae); Mees (1988b Bunocephalinae).

Keys: Mees (1988b, Aspredininae).

Revision: Stewart (1985b, Hoplomyzontinae).

Phylogeny: Friel (1994); Diogo et al. (2001).

12 genera, 36 species; no named fossil taxa.

ACANTHOBUNOCEPHALUS Friel, 1995

Acanthobunocephalus Friel, 1995: 90. Type species: Acanthobunocephalus nicoi Friel, 1995. Type by original designation. Gender: Masculine.

Acanthobunocephalus nicoi Friel, 1995

Acanthobunocephalus nicoi Friel, 1995: 90, fig. 1. Type locality: Río Sipapo, $200 \mathrm{~m}$ from Salto Remo, 4³4'N, $67^{\circ} 18^{\prime} \mathrm{W}$, Venezuela. Holotype: MCNG 29000.

Distribution: Upper Orinoco River basin of Venezuela, including Sipapo, Ramoni and Casiquiare rivers (Friel, 2003).

AMARALIA Fowler, 1954

Amaralia Fowler, 1954: 40. Type species: Bunocephalus hypsiurus Kner, 1855. Type by original designation. Gender: Feminine.

Amaralia hypsiura (Kner, 1855)

Bunocephalus hypsiurus Kner, 1855a: 98, pl. 1 (fig. 1). Type locality: Rio Branco [Brazil]. Syntypes: NMW 47626 (2).

Distribution: Throughout the Amazon River basin (Friel, 2003).

ASPREDINICHTHYS Bleeker, 1858

Aspredinichthys Bleeker, 1858b: 328, 329. Type species: Aspredo tibicen Valenciennes, 1840. Type by monotypy. Gender: Masculine.

Chamaigenes Eigenmann, 1910: 380. Type species: Aspredo filamentosus Valenciennes, 1840. Type by original designation. Gender: Masculine.

Revison: Mees (1987b).

Aspredinichthys filamentosus (Valenciennes, 1840)

Aspredo filamentosus Valenciennes, in Cuvier \& Valenciennes, 1840b: 437 (324 of Strasbourg deluxe edition), pl. 450. Type locality: Cayenne. Syntypes: MNHN 0000-4401 (4), MNHN b-0594 (2).

Distribution: Lower portions of rivers and in coastal waters of northern South America from Venezuela to northern Brazil (Friel, 2003). 
Aspredinichthys tibicen (Valenciennes, 1840)

Aspredo tibicen Valenciennes, in Cuvier \& Valenciennes, 1840b: 438 (325 of Strasbourg deluxe edition). Type locality: Surinam. Holotype: RMNH 3111.

Distribution: Lower portions of rivers and in coastal waters of northern South America from Venezuela to northern Brazil (Friel, 2003).

ASPREDO Scopoli, 1777

Aspredo Scopoli, 1777: 453. Type species: Silurus aspredo Linnaeus, 1758. Type by absolute tautonymy. Gender: Masculine.

Aspredo aspredo (Linnaeus, 1758)

Silurus aspredo Linnaeus, 1758: 304. Type locality: in Americae fluviis. Syntype: ZMUU Linnaean Coll. 48 (1); also based on descriptions in Gronovius (1754: 8), which apparently is based on the same Linnaean specimen, and on Klein (1775).

Platystacus laevis Bloch, 1794: 58. Type locality: [No locality stated]. Syntypes: ZMB 3153 (3), and several literature sources, including Silurus aspredo Linnaeus.

Aspredo sicuephorus Valenciennes, in Cuvier \& Valenciennes, 1840b: 439 (326 of Strasbourg deluxe edition). Type locality: La Mana [French Guiana]. Holotype: MNHN 0000-6362.

Aspredo batrachus Gronow, in Gray, 1854: 137. Type locality: fluminibus Americes Meridionalis. Syntype or holotype: ZMUU Linnaean Coll. 48 (1); inasmuch as Gronovius (1754: 8) based the description on an earlier Linnaean description.

Aspredo sicyephorus Günther, 1864: 269. Type locality: Mana [French Guiana]. Holotype: MNHN 0000-6362. Unjustified emendation of Aspredo sicuephorus Valenciennes, 1840.

Distribution: Lower portions of rivers from Venezuela to northern Brazil (Friel, 2003).

\section{BUNOCEPHALUS Kner, 1855}

Aspredo Swainson, 1838: 332. Type species: Aspredo gronovii Swainson, 1838. Type by monotypy. Gender: Masculine. Preoccupied by Aspredo Scopoli, 1777; apparently not replaced.

Bunocephalus Kner, 1855a: 95. Type species: Platystacus verrucosus Bloch, 1794. Type by subsequent designation by Bleeker (1862-63: 19). Gender: Masculine.

Platystacus Bleeker, 1858b: 329. Type species: Bunocephalichthys verrucosus Bloch, 1794. Type by subsequent designation by Jordan (1919a: 279). Gender: Masculine.

Dysichthys Cope, 1874b: 133. Type species: Dysichthys coracoideus Cope, 1874. Type by monotypy. Gender: Masculine.

Agmus Eigenmann, 1910: 379. Type species: Bunocephalus scabriceps Eigenmann \& Eigenmann, 1889. Type by original designation. Gender: Masculine.

Remarks: Ferraris (1991) clarified type species of Bunocephalus, but see Mees (1997) for alternate opinion. Many of the species listed here were placed in Dysichthys by Mees (1988b, 1989), but Dysichthys was placed in the synonymy of Bunocephalus in Friel (2003).

Review: Mees (1989, as Dysichthys, in part).

Bunocephalus aleuropsis Cope, 1870

Bunocephalus aleuropsis Cope, 1870b: 568. Type locality: Pebas, Eastern Ecuador [now Peru]. Syntypes: ANSP 8286-8288 (3); one syntype illustrated in Mees (1989: 206, fig. 4).

Bunocephalus melas Cope, 1874b: 132. Type locality: Nauta. Holotype: ANSP 21235 (in pieces).

Distribution: Amazon and Orinoco River basins (Friel, 2003).

Bunocephalus amaurus Eigenmann, 1912

Bunocephalus amaurus Eigenmann, 1912b: 126, fig. 24; pl. 2 (fig. 2). Type locality: Konawaruk, British Guiana. Holotype: FMNH 53121.

Bunocephalus amaurus aloikae Hoedeman, 1961: 130. Type locality: Litany R., Aloiké village, French Guiana. Holotype: ZMA 102229.

Bunocephalus amaurus sipaliwini Hoedeman, 1961: 130, fig. 1. Type locality: Sipaliwini, Suriname, $20 \mathrm{~km}$ from 
frontier with Brazil. Holotype: ZMA 102228.

Distribution: Coastal rivers of northern South America between Orinoco to Amazon mouths (Friel, 2003).

Remarks: Redescribed in Mees (1989: 216) as a subspecies of Dysichthys coracoideus.

Bunocephalus amazonicus (Mees, 1989)

Dysichthys amazonicus Mees, 1989: 241, fig. 21. Type locality: Bolivia: creek near Todos Santos, upper course of R.

Mamoré, Cochabamba. Holotype: ZMA 109246 (37 mm SL specimen).

Distribution: Upper and middle Amazon River basin (Friel, 2003).

Bunocephalus bifidus Eigenmann, 1942

Bunocephalus bifidus Eigenmann, in Eigenmann \& Allen, 1942: 86, pl. 2 (fig. 2). Type locality: creek, Yurimaguas [Huallaga River, Peru]. Holotype: CAS 35105.

Distribution: Amazon River basin of Bolivia, Brazil, Ecuador, and Peru (Friel, 2003).

Remarks: Name appeared several times (Pearson, 1924: 9; Pearson 1937: 111; Fowler, 1940c: 94) as a nomen nudum prior to being made available in Eigenmann \& Allen (1942).

Bunocephalus chamaizelus Eigenmann, 1912

Bunocephalus chamaizelus Eigenmann, 1912b: 127, fig. 24; pl. 2 (fig. 1). Type locality: Erukin, British Guiana. Holotype: FMNH 53122.

Distribution: Essequibo River basin (Friel, 2003).

Bunocephalus colombianus Eigenmann, 1912

Bunocephalus colombianus Eigenmann, 1912a: 10. Type locality: Raspadura [Colombia]. Holotype: FMNH 56038.

Distribution: Atrato and Magdalena River basins (Friel, 2003); also Cauca, San Jorge, Sinu and Patía basins, Colombia (Maldonado-Ocampo et al., 2005).

Bunocephalus coracoideus (Cope, 1874)

Dysichthys coracoideus Cope, 1874b: 133. Type locality: Nauta [Peru]. Syntypes: ANSP 21212-21215 (4).

Bunocephalus bicolor Steindachner, 1882b: 176. Type locality: Aus dem Huallaga [Peru]. Holotype: MTD F345.

Species illustrated and described in more detail in Steindachner (1882c: 8, pl. 2, figs. 1, 1a, 1b).

Bunocephalus haggini Eigenmann \& Allen, 1942: 86, pl. 2 (fig. 1). Type locality: Amazon at Iquitos [Peru]. Holotype: CAS 35107.

Distribution: Amazon River basin of Bolivia, Brazil, and Peru (Friel, 2003).

Bunocephalus doriae Boulenger, 1902

Bunocephalus doriae Boulenger, 1902b: 286. Type locality: Villa Rica, Paraguay; and Posadas, Río Parana [Argentina]. Syntypes: BMNH 1902.2.10.22-23 (2), MSNG 14274 (1), MSNG 14275 (3).

Bunocephalus retropinnis Eigenmann, in Eigenmann \& Allen, 1942: 85, pl. 2 (fig. 3). Type locality: Cacequi, Brazil. Holotype: CAS 35246.

Distribution: Paraguay-Paraná and Uruguay River basins, of Argentina, Brazil, Paraguay and Uruguay (Friel, 2003).

Bunocephalus iheringii Boulenger, 1891

Bunocephalus iheringii Boulenger, 1891: 235, pl. 26 (fig. 2). Type locality: Not stated [apparently from Rio Grande do Sul, Brazil]. Syntypes: BMNH 1891.3.16.77-78 (2).

Bunocephalus salathei Myers, 1927: 125. Type locality: Brazil: Morro Ajudo, about $100 \mathrm{~km}$ from Rio de Janeiro. Holotype: MCZ 31583.

Bunocephalus minutus Güntert, 1942: 28, figs. 1-2. Type locality: Paraguay, Dept. Villetta. Syntypes: NMBA 529698 (3), NMBA 5299-5300 (2).

Bunocephalus carvalhoi Miranda Ribeiro, 1944a: 1, figure on p. 2. Type locality: Magé, Estado do Rio de Janeiro [Brazil]. Holotype: MNRJ 1060.

Distribution: Atlantic, Paraguay and Uruguay River basins of Brazil and Paraguay (Friel, 2003).

Bunocephalus knerii Steindachner, 1882

Bunocephalus Knerii Steindachner, 1882b: 176. Type locality: Canelos [Ecuador]. Syntype: NMW 47628. Species illustrated and described in more detail in Steindachner (1882c: 9, pl. 2, figs. 2, 2a, 2b).

Distribution: Upper Amazon River basin of Colombia, Ecuador, and Peru (Friel, 2003).

Bunocephalus larai Ihering, 1930

Bunocephalus larai Ihering, 1930: 101, pl. 13 (figs. 3-3a). Type locality: Rio Piracicaba, junto ao Salto; ao 20 klm 
abaixo (ilha das Flechas) [São Paulo, Brazil]. Syntypes: Whereabouts unknown.

Distribution: Paraná River basin of Brazil (Friel, 2003).

Bunocephalus quadriradiatus (Mees, 1989)

Dysichthys quadriradiatus Mees, 1989: 244, figs. 23, 24. Type locality: Peru: Samiria, Chinguito [= Cocha Shinguita or Shirguita]. Holotype: MHNG 2157.21.

Distribution: Upper Amazon River basin of Peru (Friel, 2003).

Bunocephalus rugosus Eigenmann \& Kennedy, 1903

Bunocephalus rugosus Eigenmann \& Kennedy, 1903: 498. Type locality: Laguna near Arroyo Chagalalina [Paraguay]. Holotype: IU 9819 (whereabouts unknown).

Dysichthys australe Eigenmann \& Ward, in Eigenmann, McAtee \& Ward, 1907: 113, pl. 31. Type locality: Corumba [Paraguay]. Holotype: CAS 35240.

Distribution: Paraguay-Paraná River basin of Brazil and Paraguay (Friel, 2003).

Bunocephalus verrucosus (Walbaum, 1792)

Silurus verrucosus Walbaum, 1792: 574. Type locality: [No locality stated]. No types known. Based on Gronovius (1754, pl. 5, fig. 3).

Platystacus verrucosus Bloch, 1794: 63, pl. 373 (fig. 3). Type locality: Wahrscheinlich gerhört er in Surinam zu Hause [= Suriname]. On Gronovius (1754: 5, pl. 5, fig. 3; and 1763: 102, no. 325).

Aspredo gronovii Swainson, 1838: 332, fig. 80. Type locality: [No locality stated]. No types known.

Aspredo verrucosa Gronow, in Gray, 1854: 137. Type locality: America Meridionali. No types known; based on Gronovius (1763: no. 325, pl. 5, fig. 3.).

Bunocephalichthys gronovii Bleeker, 1858b: 329. Type locality: Am. Merid. No types known; based on Gronovius (1754: 153, pl. 5, fig. 3).

Bunocephalus scabriceps Eigenmann \& Eigenmann, 1889b: 49. Type locality: Jutahy [Brazil]. Lectotype: MCZ 7967; lectotype illustrated and designated in figure caption of Eigenmann (1912: pl. 4) as 42 mm specimen.

Agmus lyriformis Eigenmann, 1912b: 128, fig. 25; pl. 3. Type locality: Gluck Island [Rupununi River], British Guiana. Holotype: FMNH 53120.

Distribution: Rivers of the Guianas and throughout the Amazon River basin (Friel, 2003).

\section{DUPOUYICHTHYS Schultz, 1944}

Dupouyichthys Schultz, 1944c: 244. Type species: Dupouyichthys sapito Schultz, 1944. Type by original designation. Gender: Masculine.

Dupouyichthys sapito Schultz, 1944

Dupouyichthys sapito Schultz, 1944c: 245, pl. 4 (fig. d); fig. 4b. Type locality: Río Motatán, at the bridge 22 km north of Motatán, Venezuela. Holotype: USNM 121072.

Distribution: Magdalena River and Lake Maracaibo basins (Friel, 2003).

Remarks: Redescribed in Mojica et al. (2000).

ERNSTICHTHYS Fernández-Yépez, 1953

Ernstichthys Fernández-Yépez, 1953: 3, 4. Type species: Ernstichthys anduzei Fernández-Yépez, 1953. Type by original designation. Gender: Masculine.

Ernstichthys anduzei Fernández-Yépez, 1953

Ernstichthys anduzei Fernández-Yépez, 1953: 5, fig. 1. Type locality: Río Salinas, northeast of El Baúl, Estado Cojedes, Venezuela. Holotype: MHNLS 28.

Distribution: Orinoco River basin of Venezuela (Friel, 2003).

Ernstichthys intonsus Stewart, 1985

Ernstichthys intonsus Stewart, 1985b: 16, figs. 1f, 3, 9. Type locality: Río Napo at Añangu, Napo Prov, 0³0.8'S, 76²4.0'W, Ecuador. Holotype: FMNH 94603.

Distribution: Napo River basin of Ecuador (Friel, 2003).

Ernstichthys megistus (Orcés, 1961)

Hoplomyzon megistus Orcés, 1961: 3, figs. 1-2. Type locality: Small river off of the Río Chicherota, Río Bobonaza 
system, Prov. Pastaza, e. Ecuador. Holotype: Author's personal coll. 4305 (current whereabouts unknown).

Distribution: Bobonaza and Marañon River basins of Ecuador and Peru (Friel, 2003).

HOPLOMYZON Myers, 1942

Hoplomyzon Myers, 1942: 94. Type species: Hoplomyzon atrizona Myers, 1942. Type by original designation. Gender: Masculine.

Hoplomyzon atrizona Myers, 1942

Hoplomyzon atrizona Myers, 1942: 95, fig. 3. Type locality: Brook trib. to Río Zulia, at Estacion Tachira, 60 km north of San Cristobal, Venezuela, elev. ca. 150 m. Holotype: SU 36494.

Hoplomyzon atrizona petroleus Schultz, 1944c: 248, pl. 4 (fig. C); fig. 4a. Type locality: Río Motatán, 4 km above Motatán, Maracaibo basin, Venezuela. Holotype: USNM 121070.

Distribution: Lake Maracaibo basin (Friel, 2003).

Hoplomyzon papillatus Stewart, 1985

Hoplomyzon papillatus Stewart, 1985b: 8, figs. 1b, 2b, 4b, 5. Type locality: Río Aguarico, 1 km upstream from confluence with Río Shushufindi, Napo Prov., 0¹7'S, 76²4.5'W, Ecuador. Holotype: FMNH 94908.

Distribution: Napo and Portuguesa River basins of Ecuador and Venezuela, respectively (Friel, 2003).

Hoplomyzon sexpapilostoma Taphorn \& Marrero, 1990

Hoplomyzon sexpapilostoma Taphorn \& Marrero, 1990: 4, figs. 1-4. Type locality: Río Masparro at site of Masparro Dam, Barinas, Venezuela, 850'40"N, 7006'00"W. Holotype: MCNG 18669.

Distribution: Orinoco River basin of Venezuela (Friel, 2003).

MICROMYZON Friel \& Lundberg, 1996

Micromyzon Friel \& Lundberg, 1996: 642. Type species: Micromyzon akamai Friel \& Lundberg, 1996. Type by original designation. Gender: Masculine.

Micromyzon akamai Friel \& Lundberg, 1996

Micromyzon akamai Friel \& Lundberg, 1996: 643, fig. 1. Type locality: Rio Tocantins, above confluence with Rio Pará, 202'S, 49¹7'W, Pará, Brazil, 10-14 m. Holotype: MZUSP 48550.

Distribution: Lower Amazon River basin (Friel, 2003).

PLATYSTACUS Bloch, 1794

Platystacus Bloch, 1794: 52. Type species: Platystacus cotylephorus Bloch, 1794. Type by subsequent designation by Bleeker (1862-63: 19). Gender: Masculine.

Platysomatos Bloch, 1797: 115. Type species: Platystacus cotylephorus Bloch, 1794. Type by being a replacement name. Unneeded new name for Platystacus Bloch, 1794. Gender: Masculine. See Harry (1953) for comments on this name.

Cotylephorus Swainson, 1838: 354. Type species: Cotylephorus blochii Swainson, 1838. Type by monotypy. Gender: Masculine.

Platystacus cotylephorus Bloch, 1794

Platystacus cotylephorus Bloch, 1794: 54, pl. 372. Type locality: Ostindischen Gewässer [in error; certainly from South America]. Syntypes: ZMB 3157 (2), and several specimens associated with literature sources.

Silurus hexadactylus La Cepède, 1803: 58, 82. Type locality: [No locality stated]. Holotype: MNHN b-0029.

Cotylephorus Blochii Swainson, 1838: 354, fig. 90. Type locality: Ostindischen Gewässer [in error; certainly from South America]. Syntypes: ZMB 3157. Unneeded new name for Platystacus cotylephorus Bloch, 1794, apparently to avoid tautonymy.

Aspredo sexcirrhis Valenciennes, in Cuvier \& Valenciennes, 1840b: 441 (326 of Strasbourg deluxe edition). Type locality: Surinam. Syntypes: MNHN a-9313 (1), MNHN b-0029 (1).

Aspredo spectrum Gronow, in Gray, 1854: 137. Type locality: in fluminibus Americes Meridionalis. Types: Whereabouts unknown; based on Gronovius (1763: no. 324), and Seba (1734-65: pl. 29, fig. 9).

Platystacus nematophorus Bleeker, 1862a: 371. Type locality: Surinama. Holotype: RMNH 3105; holotype illustrated in Bleeker (1864: 96, pl. 1, fig. 1). 
Distribution: Coastal waters and lower portions of rivers of northern South America, from Venezuela to northern Brazil (Friel, 2003).

PTEROBUNOCEPHALUS Fowler, 1943

Pterobunocephalus Fowler, 1943c: 1. Type species: Bunocephalus albifasciatus Fowler, 1943. Type by original designation. Gender: Masculine.

Petacara Böhlke, 1959: 2. Type species: Bunocephalus dolichurus Delsman, 1941. Type by original designation. Gender: Neuter.

Pterobunocephalus depressus (Haseman, 1911)

Bunocephalus depressus Haseman, 1911a: 319, pl. 48 (fig. 1), pl. 49. Type locality: Río Machupo near San Joaquín, Bolivia. Holotype: FMNH 54338.

Bunocephalus albifasciatus Fowler, 1943c: 2, fig. 1. Type locality: Todos Santos, Bolivia. Holotype: ANSP 69193. Distribution: Amazon, Orinoco and Paraguay River basins (Friel, 2003).

Pterobunocephalus dolichurus (Delsman, 1941)

Bunocephalus dolichurus Delsman, 1941: 77, fig. 12. Type locality: Trombetas-river, near Obidos [Brazil]. Holotype: IRSNB 359.

Distribution: Amazon River basin of Brazil and Peru (Friel, 2003).

XYLIPHIUS Eigenmann, 1912

Xyliphius Eigenmann, 1912a: 10. Type species: Xyliphius magdalenae Eigenmann, 1912. Type by monotypy. Gender: Masculine. Spelled two ways in original description: Xyliphius and Xiliphius, first reviser not yet identified, but may not have been formalized until noted by Eschmeyer \& Bailey, in Eschmeyer (1990).

Xyliphius barbatus Alonso de Arámburu \& Arámburu, 1962

Xyliphius barbatus Alonso de Arámburu \& Arámburu, 1962: 219, figs. 1-2. Type locality: río Paraná en Rosario, provincia de Santa Fe, Argentina. Holotype: MLP 6798.

Xyliphius labrosus Risso \& Risso, 1964a: 11. Apparently an unneeded new name for Xyliphius barbatus Alonso de Arámburu \& Arámburu, 1962.

Distribution: Paraguay-Paraná River basin, Argentina (Friel, 2003).

Xyliphius kryptos Taphorn \& Lilystrom, 1983

Xiliphius kryptos Taphorn \& Lilystrom, 1983: 43, fig. 1. Type locality: Cerca del Puente de la carretera 6 sobre el Río Aricuaisá, Estado Zulia, Venezuela. Holotype: MCNG 1224.

Distribution: Lake Maracaibo basin (Friel, 2003).

Xyliphius lepturus Orcés, 1962

Xyliphius lepturus Orcés, 1962: 50, figs. 1-2. Type locality: Cerca de Desembocadura del rio Pucayacu en el Bobonaza [Ecuador]. Holotype: Author's personal collection 1307 (current whereabouts unknown).

Distribution: Upper Amazon and Orinoco River basins of Colombia, Ecuador and Venezuela (Friel, 2003).

Xyliphius lombarderoi Risso \& Risso, 1964

Xyliphius lombarderoi Risso \& Risso, 1964a: 12, pl. 1 (figs. 1-2). Type locality: el Riacho Barranqueras [Paraná, Argentina]. Holotype: Authors' personal collection (current whereabouts unknown).

Distribution: Paraná River basin, Argentina (Friel, 2003).

Xyliphius magdalenae Eigenmann, 1912

Xyliphius magdalenae Eigenmann, 1912a: 10. Type locality: Girardot, Colombia. Holotype: FMNH 56039; holotype illustrated in Eigenmann (1922: 27, pl. 1, fig. 1).

Distribution: Magdalena River basin, Colombia (Friel, 2003; Maldonado-Ocampo et al., 2005).

Xyliphius melanopterus Orcés, 1962

Xyliphius melanopterus Orcés, 1962: 52, figs. 3-4. Type locality: el bajo Pucayacu no lejos de su desembocadura en el Bobonaza [Ecuador]. Holotype: Author's personal coll. 2021 (current whereabouts unknown).

Distribution: Upper Amazon and Orinoco River basins (Friel, 2003). 


\section{ASTROBLEPIDAE Bleeker, 1862}

Argeini Bleeker, 1862 (in Bleeker, 1862-63): 15. Type genus: Arges Valenciennes, 1840.

Astroblepiformes Bleeker, 1862 (in Bleeker, 1862-63): 15. Type genus: Astroblepus Humboldt, 1805.

Cyclopidae Eigenmann, 1910: 416. Type genus: Cyclopium Swainson, 1838.

Remarks: The name Astroblepiformes takes precedence over the simultaneously published Argeini, because it was proposed at a higher taxonomic level (subfamily vs. stirps).

Taxonomic summary: Schaefer (2003b).

1 genus, 54 species; no named fossil taxa.

ASTROBLEPUS Humboldt, 1805

Astroblepus Humboldt, 1805a: 19. Type species: Astroblepus grixalvii Humboldt, 1805. Type by monotypy. Gender: Masculine.

Cyclopium Swainson, 1838: 355. Type species: Cyclopium humboldtii Swainson, 1839. Type by subsequent monotypy. Gender: Neuter. Genus first proposed without species; species first added in Swainson (1839: 305).

Arges Valenciennes, in Cuvier \& Valenciennes, 1840b: 333 (247 in Strasbourg deluxe edition). Type species: Arges sabalo Valenciennes, 1840. Type by subsequent designation by Bleeker (1862-63: 15). Gender: Masculine.

Brontes Valenciennes, in Cuvier \& Valenciennes, 1840b: 341 (254 in Strasbourg deluxe edition). Type species: Brontes prenadilla Valenciennes, 1840. Type by monotypy. Gender: Masculine. Preoccupied by Brontes Fabricius, 1801, in Coleoptera; replaced by Strephon Gistel, 1848.

Strephon Gistel, 1848: viii. Type species: Brontes prenadilla Valenciennes, 1840. Type by being a replacement name. Gender: Masculine. Replacement for Brontes Valenciennes, 1840, preoccupied by Brontes Fabricius, 1801 , in Coleoptera.

Stygogenes Günther, 1864: 223. Type species: Cyclopium humboldtii Swainson, 1839. Type by subsequent designation of Jordan (1919b: 332). Gender: Masculine.

Astroblepus boulengeri (Regan, 1904)

Arges boulengeri Regan, 1904b: 310. Type locality: Canelos, Ecuador. Syntypes: BMNH 1890.6.13.12-13.

Distribution: Upper Amazon River basin of Ecuador (Schaefer, 2003b).

Astroblepus brachycephalus (Günther, 1859)

Arges brachycephalus Günther, 1859: 92. Type locality: Andes of western Ecuador. Syntypes: BMNH 1858.7.25.37-40 (4).

Distribution: Pacific draining rivers of Ecuador (Schaefer, 2003b).

Astroblepus caquetae Fowler, 1943

Astroblepus caquetae Fowler, 1943a: 246, fig. 23-25. Type locality: Florencia, Río Ortueguasa, Colombia. Holotype: ANSP 70506.

Distribution: Orteguaza River, upper Amazon River basin of Colombian Andes (Schaefer, 2003b).

Astroblepus chapmani (Eigenmann, 1912)

Cyclopium chapmani Eigenmann, 1912a: 13. Type locality: Boquia, Colombia. Holotype: FMNH 56071; holotype illustrated in Eigenmann (1922: 51, pl. 6, fig.2).

Distribution: Andean streams of Cauca, Magdalena and San Juan River basins, Colombia (Maldonado-Ocampo et al., 2005).

Astroblepus chimborazoi (Fowler, 1915)

Cyclopium chimborazoi Fowler, 1915a: 241, fig. 11. Type locality: Junction of Chanchan and Chiguancay rivers. Province of Chimborazo, Ecuador. Holotype: ANSP 43523.

Distribution: Pacific draining rivers of Ecuador (Schaefer, 2003b).

Astroblepus chotae (Regan, 1904)

Arges chotae Regan, 1904b: 313, pl. 21 (fig. 5). Type locality: Chota Valley, northern Ecuador. Holotype: BMNH 1898.11.4.22.

Distribution: Pacific draining rivers of Colombia, Ecuador, and Peru; Magdalena and Orinoco River basins (Schaefer, 2003b). 
Astroblepus cirratus (Regan, 1912)

Arges cirratus Regan, 1912b: 670. Type locality: Río Condoto, Río San Juan, S.W. Colombia. Holotype: BMNH 1912.3.2.7.

Distribution: San Juan River basin, Colombia (Maldonado-Ocampo et al., 2005).

Astroblepus cyclopus (Humboldt, 1805)

Pimelodus cyclopum Humboldt, 1805b: 24, pl. 6. Type locality: Subterranean waters in Andes of Quito (basin of Río Esmeraldas), Ecuador. Type(s): Whereabouts unknown.

Cyclopium Humboldtii Swainson, 1839: 305. Unneeded new name for Pimelodus cyclopum Humboldt, 1805 (but listed as Pimelodus cyclopium).

Stygogenes humboldtii Günther, 1864: 223. Type locality: Azores [in error]. Syntypes (4): BMNH 1855.6.26.14.

Distribution: Esmeraldas River and other Pacific coastal drainages of Ecuador (Schaefer, 2003b); Andean streams of Colombia (Maldonado-Ocampo et al., 2005).

Astroblepus eigenmanni (Regan, 1904)

Arges eigenmanni Regan, 1904b: 312, pl. 21 (fig. 3). Type locality: Cayambe and Machachi, Ecuador. Syntypes: BMNH 1892.5.14.1-8 (8).

Distribution: Pacific coastal drainages of Ecuador (Schaefer, 2003b).

Astroblepus festae (Boulenger, 1898)

Arges festae Boulenger, 1898c: 9. Type locality: Vallée de Gualaquiza, Equateur Oriental. Syntypes: BMNH 1898.11.4.23-29; MZUT 1486, MZUT 1497.

Distribution: South America: Upper Marañon River of southern Ecuador (Schaefer, 2003b).

Astroblepus fissidens (Regan, 1904)

Arges fissidens Regan, 1904b: 316. Type locality: Andes of Ecuador. Syntypes: BMNH 1887.1.7.2, BMNH 1889.2.1.4397.

Distribution: Andes of Ecuador (Schaefer, 2003b).

Astroblepus formosus Fowler, 1945

Astroblepus formosus Fowler, 1945c: 10, fig. 1-2. Type locality: Acobamba, near Tarma, at 2900 meters elevation, Río Ucayali basin, Peru, Holotype: ANSP 71647.

Distribution: Upper Ucayali River basin, Peru (Schaefer, 2003b).

Astroblepus frenatus Eigenmann, 1918

Astroblepus frenatus Eigenmann, 1918b: 676. Type locality: Quebrada de San Joaquín, Santander, Colombia. Holotype: FMNH 58384.

Distribution: Streams of Magdalena, Catatumbo and Orinoco river basins, Colombia (Maldonado-Ocampo et al., 2005).

Astroblepus grixalvii Humboldt, 1805

Astroblepus grixalvii Humboldt, 1805a: 19, pl. 7. Type locality: Río Palacé, near Popayan, Colombia. Type(s): Whereabouts unknown.

Distribution: Cauca and Magdalena River basins, Colombia (Maldonado-Ocampo et al., 2005).

Astroblepus guentheri (Boulenger, 1887)

Stygogenes guentheri Boulenger, 1887b: 348. Type locality: New Granada. Syntypes: BMNH 1880.2.26.18-25 (8).

Distribution: Andean streams of the Cauca and Magdalena River basins, Colombia (Maldonado-Ocampo et al., 2005).

Astroblepus heterodon (Regan, 1908)

Arges heterodon Regan, 1908f: 799, pl. 49 (fig. 2, 2a, b). Type locality: Jimenez, western Colombia. Holotype: BMNH 1908.5.29.80.

Distribution: Pacific draining rivers of Colombia (Schaefer, 2003b).

Astroblepus homodon (Regan, 1904)

Arges homodon Regan, 1904b: 309, pl. 21 (fig. 1). Type locality: Villeta, Colombia, 3500 ft. Holotype: BMNH 1902.5.15.27.

Distribution: Andean streams of the Cauca and Magdalena River basins, Colombia (Maldonado-Ocampo et al., 2005). 
Astroblepus jurubidae Fowler, 1944

Astroblepus jurubidae Fowler, 1944b: 237, fig. 10-13. Type locality: clear brook, tributary to Río Jurubidá [northwestern Colombia]. Holotype: ANSP 71431.

Distribution: Pacific draining rivers of Colombia (Schaefer, 2003b).

Astroblepus labialis Pearson, 1937

Astroblepus labialis Pearson, 1937: 95, pl. 13 (fig. 4). Type locality: Balsas, Peru. Syntypes: CAS 64617 (7).

Distribution: Marañon River basin, Peru (Schaefer, 2003b).

Astroblepus latidens Eigenmann, 1918

Astroblepus latidens Eigenmann, 1918b: 674. Type locality: Piperel, Colombia. Holotype: FMNH 58366; holotype illustrated in Eigenmann (1922: 228, pl. 6, fig. 3).

Distribution: Upper Meta River basin, Colombia (Schaefer, 2003b); and Suarez River basin, Colombia (MaldonadoOcampo et al., 2005).

Astroblepus longiceps Pearson, 1924

Astroblepus longiceps Pearson, 1924: 15, pl. 2 (fig. 5). Type locality: Río Colorado, Lower Bopi, Bolivia. Holotype: CAS 64618.

Distribution: Upper Madeira River basin of Bolivia and Peru (Schaefer, 2003b).

Astroblepus longifilis (Steindachner, 1882)

Arges longifilis Steindachner, 1882b: 177. Type locality: Río Huambo und de Tortora [Peru]. Syntypes (4): at NMW. Species described in more detail and illustrated in Steindachner (1882c: 19, pl. 5, figs. 3, 3a, 3b).

Distribution: Upper Huallaga River basin, Peru (Schaefer, 2003b); Magdalena and Cauaca River basins, Colombia (Maldonado-Ocampo et al., 2005).

Astroblepus mancoi Eigenmann, 1928

Astroblepus mancoi Eigenmann, in Myers, 1928: 85. Type locality: Río Comberciato, Urubamba, Peru, 1800 feet. Syntypes: CAS 64620 (5), MCZ 30969 (11), MCZ 30970 (3).

Distribution: Upper Ucayali River basin, Peru (Schaefer, 2003b).

Astroblepus mariae (Fowler, 1919)

Cyclopium mariae Fowler, 1919a: 125, pl. 8. Type locality: Brook near the small village of Choachi, 25 kilometers east of Bogota and at $1800 \mathrm{~m}$. of altitude, Colombia. Holotype: ANSP 49368.

Distribution: Upper Meta River basin, Colombia (Schaefer, 2003b).

Astroblepus marmoratus (Regan, 1904)

Arges marmoratus Regan, 1904b: 314, pl. 21 (fig. 6). Type locality: St. Augustin, Andes of Colombia, 5000 ft. Syntypes: BMNH 1899.8.21.10-11 (2).

Distribution: Upper Orinoco River basin, Colombia (Schaefer, 2003b).

Astroblepus micrescens Eigenmann, 1918

Astroblepus grixalvii micrescens Eigenmann, 1918b: 677. Type locality: Quebrada de Agua Larga, western slope of the eastern Andes, north of Bogotá, Colombia. Holotype: FMNH 58376; holotype illustrated in Eigenmann (1922: 54, pl. 7, fig. 4).

Distribution: Upper Orinoco River basin (Schaefer, 2003b); widely distributed in Colombian Andes (MaldonadoOcampo et al., 2005).

Astroblepus mindoensis (Regan, 1916)

Cyclopium mindoense Regan, 1916: 80. Type locality: Mindo, western Ecuador. Holotype: BMNH 1916.8.1.1.

Distribution: Pacific coastal drainage of Ecuador (Schaefer, 2003b).

Astroblepus nicefori Myers, 1932

Astroblepus nicéfori Myers, 1932: 137. Type locality: Sonsón, Antioquia, Colombia. Holotype: SU 24796.

Distribution: Magdalena River basin (Schaefer, 2003b); Cauca River basin, Colombia (Maldonado-Ocampo et al., 2005).

Astroblepus orientalis (Boulenger, 1903)

Arges orientalis Boulenger, 1903a: 601. Type locality: Albirregas and Milla rivers above Merida, Venezuela, 3500 m. altitude. Syntypes: BMNH 1903.6.30.81-89, USNM 133142.

Distribution: Lake Maracaibo drainage (Schaefer, 2003b). 
Astroblepus peruanus (Steindachner, 1876)

Arges peruanus Steindachner, 1876: 601, pl. 9 (fig. 3-6). Type locality: Amable Maria, Peru. Syntypes: NMW 16974 (3).

Distribution: Upper Ucayali River basin, Peru (Schaefer, 2003b).

Astroblepus phelpsi Schultz, 1944

Astroblepus phelpsi Schultz, 1944c: 283, pl. 7 (fig. d). Type locality: Río Cobre above its mouth near La Grita, tributary of Río Quinta, latter tributary to Río La Grita, Catatumbo system, Venezuela. Holotype: USNM 121126.

Distribution: Lake Maracaibo drainage (Schaefer, 2003b).

Astroblepus pholeter Collette, 1962

Astroblepus pholeter Collette, 1962: 311, fig. 1-2. Type locality: Cave in Latas, 4 km north of Archidona, Napo Prov., eastern Ecuador. Holotype: USNM 196623.

Distribution: Napo River basin, Ecuador (Schaefer, 2003b).

Astroblepus pirrensis (Meek \& Hildebrand, 1913)

Cyclopium pirrense Meek \& Hildebrand, 1913: 83. Type locality: Río Cana, Cana, Panama. Holotype: FMNH 7586.

Distribution: Cana River basin, Panama (Schaefer, 2003b).

Astroblepus praeliorum Allen, 1942

Astroblepus praeliorum Allen, in Eigenmann \& Allen, 1942: 169, pl. 13 (fig. 6-7). Type locality: Tarma; Palca; Oroya; Huancachupa Creek, Húanuco, Peru. Syntypes: CAS 7509 (4), CAS 16114 (82), CAS 16115 (2), CAS 47068 (10), CAS 47069 (1).

Distribution: Upper Marañon River basin, Peru (Schaefer, 2003b).

Astroblepus prenadillus (Valenciennes, 1840)

Brontes prenadilla Valenciennes, in Cuvier \& Valenciennes, 1840b: 343 (255 of the Strasbourg deluxe edition), pl. 444. Type locality: des ruisseaux qui descendent du Cotopaxi [Ecuador], à 5000 mètres au-dessus dy niveau e l'Ocean. Syntypes (2): MNHN a-4227 (1), MNHN a-5164 (1).

Distribution: Andes of Ecuador (Schaefer, 2003b).

Astroblepus regani (Pellegrin, 1909)

Arges regani Pellegrin, 1909c: 518. Type locality: [Río Cariyacu, Ecuador, elev. 3100 m]. Syntypes: MNHN 19030108 (1), MNHN 1903-0109 (2), MNHN 1903-0110 (3).

Distribution: Mira River basin and Pacific coastal drainage of Ecuador (Schaefer, 2003b).

Astroblepus rengifoi Dahl, 1960

Astroblepus rengifoi Dahl, 1960a: 459, unnumbered figure. Type locality: small brook, tributary to the Atrato River on the left side, approx. $6 \mathrm{ks}$. above the town El Carmen de Atrato, Colombia. Holotype: at ICNMHN, current whereabouts unknown.

Distribution: Atrato River basin, Colombia (Schaefer, 2003b).

Astroblepus retropinnus (Regan, 1908)

Arges retropinna Regan, 1908f: 800, pl. 48 (fig. 2). Type locality: Jimenez, western Colombia. Syntypes: BMNH 1908.5.29.81-82 (2).

Distribution: Dagua River basin and Pacific coastal drainage of Colombia (Schaefer, 2003b).

Astroblepus riberae Cardona \& Guerao, 1994

Astroblepus riberae Cardona \& Guerao, 1994: 21, fig. Type locality: Cueva de Ninabamba, Peru, $06.33^{\circ} \mathrm{S}, 78.51^{\circ} \mathrm{W}$. Holotype: UAB unnumbered.

Distribution: Reque River basin and Pacific coastal drainage, Peru (Schaefer, 2003b).

Astroblepus rosei Eigenmann, 1922

Astroblepus rosei Eigenmann, 1922b: 55. Type locality: Río Jequetepeque, Llallán, Peru. Syntypes: CAS 64649 (11), FMNH 58640 (1).

Distribution: Pacific coastal drainages of Cajamarca, Peru (Schaefer, 2003b); widely distributed in Colombian Andes (Maldonado-Ocampo et al., 2005).

Remarks: Eigenmann (1922: 55) stated that the type [= holotype] was a specimen deposited in the Indiana University collection, but eleven specimens were deposited there (now CAS 64649), and no indication was given in the publication as to which was the type. Therefore, all eleven, plus one specimen deposited in the Carnegie 
Museum collection (now FMNH 58640), must be considered syntypes.

Astroblepus sabalo (Valenciennes, 1840)

Arges sabalo Valenciennes, in Cuvier \& Valenciennes, 1840b: 335 (249 in Strasbourg deluxe edition), pl. 445. Type locality: le haut Pérou. Holotype: MNHN a-4226.

Distribution: Upper Ucayali River basin, Peru (Schaefer, 2003b).

Astroblepus santanderensis Eigenmann, 1918

Astroblepus cyclopus santanderensis Eigenmann, 1918b: 675. Type locality: Quebrada de Guapota, Colombia. Lectotype: FMNH 58433, designated by Henn (1928: 82).

Distribution: Magdalena River basin, Colombia (Schaefer, 2003b); Suarez River basin, Colombia (MaldonadoOcampo et al., 2005).

Astroblepus simonsii (Regan, 1904)

Arges simonsii Regan, 1904b: 317, pl. 21 (fig. 9). Type locality: Huaras, Peru, elev. 10700 ft. Syntypes: BMNH 1900.6.20.51-55 (5).

Distribution: Santa River basin and Pacific coastal drainages of Peru (Schaefer, 2003b).

Astroblepus stuebeli (Wandolleck, 1916)

Arges stübeli Wandolleck, 1916: 158, fig. 1-4. Type locality: Lake Titicaca. Syntypes (40): MTD F977, MTD F4639-87.

Distribution: Lake Titicaca basin (Schaefer, 2003b).

Astroblepus supramollis Pearson, 1937

Astroblepus supramollis Pearson, 1937: 94, pl. 13 (fig. 3). Type locality: Balsas, Peru. Syntypes: CAS 47012 (389), CAS 47015 (36), CAS 47022 (31).

Distribution: Marañon River basin, Peru (Schaefer, 2003b).

Astroblepus taczanowskii (Boulenger, 1890)

Arges taczanowskii Boulenger, 1890b: 451, pl. 41 (fig. 1). Type locality: Amable Maria, Peruvian Andes. Holotype: BMNH 1875.10.14.19.

Distribution: Upper Ucayali River basin, Peru (Schaefer, 2003b).

Astroblepus theresiae (Steindachner, 1907)

Arges theresiae Steindachner, 1907c: 228. Type locality: Hohen Anden von Cayendelet. Syntypes: NMW 48091 (10), NMW 92 (7).

Distribution: Pacific coastal drainages of Ecuador (Schaefer, 2003b).

Astroblepus trifasciatus (Eigenmann, 1912)

Cyclopium trifasciatum Eigenmann, 1912a: 14. Type locality: Caldas, Colombia. Holotype: FMNH 56076; holotype illustrated in Eigenmann (1922: 52, fig. 15; pl. 6, fig. 6).

Distribution: Dagua River basin and Pacific coastal drainages of Colombia (Schaefer, 2003b); also Magdalena and Cauca River basins (Maldonado-Ocampo et al., 2005).

Astroblepus ubidiai (Pellegrin, 1931)

Cyclopium ubidiai Pellegrin, 1931b: 114. Type locality: [Lac St. Paul, near Otavalo, Imbabura Prov.,] Equateur, elev. 2600 m. Syntypes: MNHN 1930-0172 (1), MNHN 1930-0173 (1).

Distribution: Mira River basin and Pacific coastal drainages of Ecuador (Schaefer, 2003b; Vélez-Espino, 2003).

Astroblepus unifasciatus (Eigenmann, 1912)

Cyclopium unifasciatum Eigenmann, 1912a: 15. Type locality: Caldas, Colombia. Holotype: FMNH 56079; holotype illustrated in Eigenmann (1922: 52, pl. 7, fig. 2).

Distribution: Dagua River basin and Pacific coastal drainages of Colombia (Schaefer, 2003b); also Magdalena and Cauca River basins (Maldonado-Ocampo et al., 2005).

Astroblepus vaillanti (Regan, 1904)

Arges vaillanti Regan, 1904b: 312. Type locality: Huamani Peak, Ecuador, elev. 10700 ft. Syntypes: BMNH 1897.2.16.2-4 (3).

Distribution: Pacific coastal drainages of Ecuador (Schaefer, 2003b).

Astroblepus vanceae (Eigenmann, 1913)

Cyclopium vanceae Eigenmann, 1913: 421, pl. 32 (fig. 2). Type locality: Small stream in highlands, southeast of 
Tarma, Peru. Holotype: FMNH 56066.

Distribution: Upper Ucayali River basin, Peru (Schaefer, 2003b).

Astroblepus ventralis (Eigenmann, 1912)

Cyclopium ventrale Eigenmann, 1912a: 15. Type locality: Caldas, Colombia. Holotype: FMNH 56074.

Distribution: Dagua River basin and Pacific coastal drainage of Colombia (Schaefer, 2003b).

Astroblepus whymperi (Boulenger, 1890)

Arges whymperi Boulenger, 1890b: 451, pl. 41 (fig. 2). Type locality: Milligalli, Andes of Ecuador. Holotype:

BMNH 1887.1.7.1.

Distribution: Esmeraldas River basin and other Pacific coastal drainages of Ecuador (Schaefer, 2003b).

\section{AUCHENIPTERIDAE Bleeker, 1862}

Euanemini Bleeker, 1858b: 49, 189. Type genus: Euanemus Müller \& Troschel, 1842.

Trachelyopterini Bleeker, 1858b: 49, 250, 257. Type genus: Trachelyopterus Valenciennes, 1840.

Ageneiosi Bleeker, 1862 (in Bleeker, 1862-63): 14. Type genus: Ageneiosus La Cepède, 1803.

Astrophysi [= Asterophysi] Bleeker, 1862 (in Bleeker, 1862-63): 7. Type genus: Asterophysus Kner, 1857.

Auchenipterini Bleeker, 1862 (in Bleeker, 1862-63): 14. Type genus: Auchenipterus Valenciennes, 1840.

Centromochli Bleeker, 1862 (in Bleeker, 1862-63): 7. Type genus: Centromochlus Kner, 1857.

Pseudauchenipterini Bleeker, 1862 (in Bleeker, 1862-63): 6. Type genus: Pseudauchenipterus Bleeker, 1862.

Taxonomic summary: Britski (1972), Soares-Porto (1998, Centromochlinae), Ferraris (2003d).

Reviews: Mees (1974, Guianas).

Phylogeny: Ferraris (1988b), Curran (1989), Soares-Porto (1998, Centromochlinae), Royero (1999), Diogo et al. (2003c).

20 genera, 94 species; no named fossil taxa.

AGENEIOSUS La Cepède, 1803

Ageneiosus La Cepède, 1803: 132. Type species: Ageneiosus armatus La Cepède, 1803. Type by subsequent designation by Eigenmann \& Eigenmann (1890: 299). Gender: Masculine. Earlier type designation by Bleeker (1862-63: 14) of "Ageneiosus militaris Blkr (nec Val.) = Silurus militaris Bl." not based on one of the originally included species and therefore not valid.

Ceratorhynchus Spix \& Agassiz, 1829: 10. Type species: Silurus militaris [= ? Silurus militaris of Bloch, nec Linnaeus]. Type by monotypy. Gender: Masculine.

Agenius Agassiz, 1846: 11. Type species: Ageneiosus armatus La Cepède, 1803. Gender: Masculine. Unjustified emendation of Ageneiosus, therefore taking the same type species.

Davalla Bleeker, 1858b: 58, 64. Type species: Davalla schomburgkii Bleeker, 1858. Type by monotypy. Gender: Feminine.

Pseudageneiosus Bleeker, 1862 (in Bleeker, 1862-63): 14. Type species: Ageneiosus brevifilis Valenciennes, 1840. Type by original designation. Gender: Masculine.

Tympanopleura Eigenmann, 1912b: 203. Type species: Tympanopleura piperata Eigenmann, 1912. Type by original designation. Gender: Feminine.

Remarks: Genus revised, with key to species, in Walsh (1990).

Ageneiosus atronasus Eigenmann \& Eigenmann, 1888

Ageneiosus atronasus Eigenmann \& Eigenmann, 1888b: 149. Type locality: Exact locality unknown. Holotype: MCZ 27270.

Ageneiosus melanopogon Miranda Ribeiro, 1917: 51. Type locality: Fluvio Solimões [Brazil]. Holotype: Whereabouts unknown.

Tympanopleura nigricollis Eigenmann \& Allen, 1942: 139, pl. 5 (figs. 2-3), 6 (fig. 3). Type locality: Iquitos [Peru]. Holotype: CAS 57940.

Distribution: Middle and upper Amazon River basin (Walsh, 1990). 
Ageneiosus brevis Steindachner, 1881

Ageneiosus brevis Steindachner, 1881d: 16. Type locality: Amazonenstrom bei Coary und aus dem Hyavary [Brazil]. Syntypes (4): NMW 47801 (2).

Ageneiosus rondoni Miranda Ribeiro, 1914: 12, pl. 1 (fig. 3). Type locality: Rio Negro (Manáos) [Brazil]. Lectotype: MNRJ 962A, designated by Miranda Ribeiro (1953: 394).

Ageneiosus madeirensis Fisher, 1917: 426, pl. 42. Type locality: San Joaquín, Bolivia. Holotype: FMNH 58143.

Tympanopleura alta Eigenmann \& Myers, in Myers, 1928: 85. Type locality: Iquitos, Rio Marañon, Peru. Holotype: CAS 58258.

Distribution: Upper Amazon River basin (Walsh, 1990).

Ageneiosus inermis (Linnaeus, 1766)

Silurus inermis Linnaeus, 1766: 503. Type locality: Surínami. Type (s): Whereabouts unknown.

Ageneiosus armatus La Cepède, 1803: 132, 133. Type locality: les eaux de Surinam, et peut-etre dans celles des grandes Indes. Types: On Bloch (1794: 19, pl. 362); Silurus militaris Linné; and others.

Ageneiosus brevifilis Valenciennes, in Cuvier \& Valenciennes, 1840b: 242 (180 in Strasbourg deluxe edition). Type locality: Cayenne [but interpreted as having come from Suriname by Boeseman (1972: 303)]. Holotype: RMNH 2975 (larger of 2).

Hypothalmus dawalla Jardine, in Schomburgk, 1841: 191, pl. 9. Type locality: Junction of Rupununi and Essequibo [Guyana], and Fort St. Joaquim, in the Rio Bronco. No types known. Name spelled Hypothalmus davalla on plate; H. dawalla in text; first reviser not found.

Davalla Schomburgkii Bleeker, 1858b: 64. Type locality: Junction of Rupununi and Essequibo [Guyana], and Fort St. Joaquim, in the Rio Bronco. No types known. Unneeded replacement for Hypothalmus dawalla Jardine, 1841.

Ageniosus axillaris Günther, 1864: 431. Type locality: Surinam. Holotype: BMNH 1864.6.2.2.

Ageneiosus sebae Günther, 1864: 192. Type locality: Suriname. Syntypes: On specimen(s) described as Ageneiosus inermis in Cuvier \& Valenciennes (1840b: 240 (178 in Strasbourg deluxe edition), pl. 440) and specimen illustrated in Seba (1734-65: pl. 29, fig. 8).

? Ageneiosus (Pseudageneiosus) therezinae Steindachner, 1909b: 341. Type locality: Rio Parnahyba und Rio Puty bei Therezina [Brazil]. Syntypes: NMW 47840-41 (2 of "several”).

Ageneiosus ogilviei Fowler, 1914: 266, fig. 15. Type locality: Rupununi River, British Guiana ... in the highlands of British Guiana, approximately secured in North Latitude $2^{\circ}$ to $3^{\circ}$, and West Longitude $50^{\circ} 20^{\prime}$. Holotype: ANSP 39343.

Ageneiosus gabardinii Risso \& Risso, 1964b: 12, pl. 3 (fig. 1). Type locality: Río Paraná, a la altura de la Ciudad de Corrientes [Argentina]. Holotype: Mus. Cienc. Nat. del Chaco 3, VI-63-2.

Distribution: Widely distributed in South America, east of the Andes (Walsh, 1990).

Ageneiosus magoi Castillo \& Brull, 1989

Ageneiosus magoi Castillo \& Brull, 1989: 73, fig. 1-2. Type locality: Los esteros de Camaguán, km 270, Estado Guárico [Venezuela]. Holotype: MBUCV V-15666.

Distribution: Orinoco basin of Venezuela (Ferraris, 2003d).

Ageneiosus marmoratus Eigenmann, 1912

Ageneiosus marmoratus Eigenmann, 1912b: 206, pl. 22 (fig. 1). Type locality: Creek below Potaro Landing, British Guiana. Holotype: FMNH 53245.

? Ageneiosus barranquerensis Risso \& Risso, 1964b: 11, pl. 2. Type locality: frente a la desembocadura del Río Negro, Riacho Barranqueras [Argentina]. Holotype: Mus. Cien. Nat. del Chaco 11, III-63.

Distribution: Widely distributed in South America east of the Andes mountains (Walsh, 1990).

Ageneiosus militaris Valenciennes, 1836

Ageniosus militaris Valenciennes, 1836, in Valenciennes, 1835-47: pl. 4 (fig. 1). Type locality: [not stated]. Holotype: possibly MNHN b-0690 (1), MNHN b-0691 (1), or MNHN b-3167 (1). Name available from plate (illustrated specimen is holotype, if identifiable); later described in Cuvier \& Valenciennes (1840b: 233; 173 in Strasbourg deluxe edition), with type locality as: La Plata.

Ageneiosus valenciennesi Bleeker, 1864a: 82. Type locality: La Plata. Holotype: Possibly MNHN b-0690, MNHN 
b-0691, or MNHN b-3167. New name for Ageneiosus militaris Valenciennes.

Silurus 38-radiatus Larrañaga, 1923: 386. Type locality: Uruguay. No types known.

Silurus imberbis Larrañaga, 1923: 376. Type locality: Uruguay. No types known. Either based on, or preoccupied by,

Silurus imberbis Gmelin, 1789.

Ageneiosus uruguayensis Devincenzi, 1933: 3, pl. 1. Type locality: Río Uruguay frente a Paysandú [Uruguay].

Holotype: MHNM CI 317.

Ageneiosus marquesi Risso \& Risso, 1964b: 20, pl. 6 (fig. 1). Type locality: Río Paraná, frente al tanque de obras

Sanitarias de la ciudad de Corrientes, Argentina. Holotype: Mus. Cienc. Nat. del Chaco 12-V-64-1.

Distribution: La Plata River basin (Walsh, 1990).

Ageneiosus pardalis Lütken, 1874

Ageneiosus pardalis Lütken, 1874b: 190. Type locality: Caraccas [apparently in error, S. Walsh (1990, and pers. comm.)]. Holotype: ZMUC P 29664.

Ageneiosus caucanus Steindachner, 1880b: 61, pl. 6 (figs. 1, 1a). Type locality: [Río] Cauca [Colombia]. Syntypes: NMW 47811 (2).

Ageneiosus virgo Posada, 1909: 295. Type locality: [Río] Magdalena [Colombia]. Type(s): Whereabouts unknown.

Ageneiosus freiei Schultz, 1944c: 240, pl. 4 (fig. b). Type locality: Río Agua Caliente, 2 to 3 km above Lago Maracaibo [Venezuela]. Holotype: USNM 121260.

Distribution: Lake Maracaibo basin and Magdalena, Cauca, San Juan, and Tuira River basins (Walsh, 1990).

Ageneiosus piperatus (Eigenmann, 1912)

Tympanopleura piperata Eigenmann, 1912b: 203, pl. 20 (fig. 3). Type locality: Crab Falls [Essequibo River], British Guiana. Holotype: FMNH 53243.

Distribution: Essequibo and upper Negro rivers near confluence of Branco River (Walsh, 1990).

Ageneiosus polystictus Steindachner, 1915

Ageneisus polystictus Steindachner, 1915b: 217. Type locality: [not stated]. Holotype: NMW 47839. Illustrated and described in more detail in Steindachner (1915e: 84, pl. 7, figs. 1-3), as Agenciosus polystictus, with origin of specimen reported as "Mündung des Rio Negro."

Distribution: Negro River and lower Branco River, Brazil (Walsh, 1990).

Ageneiosus ucayalensis Castelnau, 1855

Ageneiosus ucayalensis Castelnau, 1855: 49, pl. 17 (fig. 2). Type locality: un lac, près de l'Ucayale, et qui communiquait avec cette rivière [Peru]. Holotype: MNHN b-0611.

? Ageneiosus dentatus Kner, 1857: 441. Type locality: Surinam. Syntypes: NMW 47823 (6).

? Ageniosus porphyreus Cope, 1867: 404. Type locality: Surinam. Lectotype: ANSP 8389, designated by Fowler (1915a: 224).

? Ageneiosus parnaguensis Steindachner, 1910c: 399, figured on p. 400. Type locality: see von Lake Parnaguá in brasílienischen Staate Piauhy [Brazil]. Syntypes: NMW 47832 (1), NMW 47837 (1), NMW 47838 (2).

? Ageneiosus guianensis Eigenmann, 1912b: 204, pl. 21 (fig. 2). Type locality: Wismar, British Guiana. Holotype: FMNH 53247.

Distribution: Amazon and Orinoco River basins; Guianas, and upper Paraná River basin (Walsh, 1990).

Ageneiosus vittatus Steindachner, 1908

Ageneiosus vittatus Steindachner, 1908b: 64. Type locality: Rio Purus [Brazil]. Holotype: NMW 47853.

Distribution: Middle Orinoco and upper Amazon River basins (Walsh, 1990).

ASTEROPHYSUS Kner, 1857

Asterophysus Kner, 1857: 402. Type species: Asterophysus batrachus Kner, 1857. Type by monotypy. Gender: Masculine.

Astrophysus Bleeker, 1858b: 356, 358. Type species: Asterophysus batrachus Kner, 1857. Type by being a replacement name. Gender: Masculine. Unjustified emendation of Asterophysus Kner, 1857.

Asterophysus batrachus Kner, 1857

Asterophysus batrachus Kner, 1857: 403, pl. 5 (fig. 13). Type locality: Marabitanos [Brazil]. Syntypes: NMW 47515-16 (2). 
Distribution: Orinoco and Negro River basins, Brazil and Venezuela (Ferraris, 2003d).

AUCHENIPTERICHTHYS Bleeker, 1862

Auchenipterichthys Bleeker, 1862 (in Bleeker, 1862-63): 7. Type species: Auchenipterus thoracatus Kner, 1857.

Type by original designation. Gender: Masculine.

Revision: Ferraris et al. (2005).

Auchenipterichthys coracoideus (Eigenmann \& Allen, 1942)

Trachycorystes coracoideus Eigenmann \& Allen, 1942: 120. Type locality: Iquitos [Peru]. Syntypes: CAS 63746 (3).

Distribution: Amazon River basin and Araguaia River of the Tocantins basin (Ferraris et al., 2005).

Auchenipterichthys longimanus (Günther, 1864)

Auchenipterus longimanus Günther, 1864: 195. Type locality: River Capin, Para [Brazil]. Syntypes: BMNH 1849.11.8.11 (3).

Distribution: Lower and middle Amazon, Tocantins, and Orinoco river basins (Ferraris et al., 2005).

Remarks: Syntype register number misreported in Eschmeyer et al. (1998) as BMNH 1849.121.8.? BMNH 1849.11.8.? and Ferraris (2003d) as BMNH 1849.121.8.

Auchenipterichthys punctatus (Valenciennes, 1840)

Auchenipterus punctatus Valenciennes, in Cuvier \& Valenciennes, 1840b: 219 (163 in Strasbourg deluxe edition). Type locality: Probablement du Brésil. Holotype: MNHN b-0216; illustrated in Royero \& Hureau (1996: fig. 3). Auchenipterichthys dantei Soares-Porto, 1994: 282, fig. 3. Type locality: Brazil, Amazonas, Paricatuba, Rio Negro ( $\left.3^{\circ} 07^{\prime} \mathrm{S}, 60^{\circ} 26^{\prime} \mathrm{W}\right)$. Holotype: MZUSP 43332.

Distribution: Upper Orinoco River and Upper Amazon River basins, including the Negro River (Ferraris et al., 2005).

Auchenipterichthys thoracatus (Kner, 1857)

Auchenipterus thoracatus Kner, 1857: 425, pl. 7 (fig. 22). Type locality: Rio Guaporé. Syntypes: NMW 47454 (2).

Auchenipterus thoracicus Günther, 1864: 194. Type locality: Rio Guaporé. Syntypes: NMW 47454 (2). Unjustified emendation of Auchenipterus thoracatus Kner, 1857.

Distribution: Upper Madeira River basin (Ferraris et al., 2005).

Remarks: Syntype register number incorrectly reported as NMW 47452 in Eschmeyer et al. (1998) and Ferraris (2003d).

AUCHENIPTERUS Valenciennes, 1840

Auchenipterus Valenciennes, in Cuvier \& Valenciennes, 1840b: 207 (154 in Strasbourg deluxe edition). Type species: Hypophthalmus nuchalis Spix \& Agassiz, 1829. Type by subsequent designation by Bleeker (1862-63: 15). Gender: Masculine.

Euanemus Müller \& Troschel, in Müller, 1842a: 203. Type species: Euanemus colymbetes Müller \& Troschel, 1842. Type by monotypy. Gender: Masculine. Also as new in Müller (1842b: 320). Priority of publication not established.

Auchenopterus Agassiz, 1846: 40. Type species: Hypophthalmus nuchalis Spix \& Agassiz, 1829. Type by being a replacement name. Gender: Masculine. Unjustified emendation of Auchenipterus Valenciennes, 1840.

Ceratocheilus Miranda Ribeiro, 1918d: 644. Type species: Ceratocheilus osteomystax Miranda Ribeiro, 1918. Type by monotypy. Gender: Masculine. Preoccupied by Ceratocheilus Wesché, 1810, in Diptera, replaced by Osteomystax Whitley, 1940.

Osteomystax Whitley, 1940a: 242. Type species: Ceratocheilus osteomystax Miranda Ribeiro, 1918. Type by being a replacement name. Gender: Masculine. Replacement for Ceratocheilus Miranda Ribeiro, 1918, preoccupied by Ceratocheilus Wesché, 1810, in Diptera.

Remarks: Revised, with a key to species, in Ferraris \& Vari (1999).

Auchenipterus ambyiacus Fowler, 1915

Auchenipterus ambyiacus Fowler, 1915a: 222, fig. 6. Type locality: Ambyiacu River, Ecuador. Holotype: ANSP 21484. 
Distribution: Upper and middle Amazon and Orinoco River basins, coastal rivers of Guyana (Ferraris \& Vari, 1999). Auchenipterus brachyurus (Cope, 1878)

Euanemus brachyurus Cope, 1878: 676. Type locality: Peruvian Amazon. Holotype: ANSP 21552.

Distribution: Upper Amazon River basin (Ferraris \& Vari, 1999).

Auchenipterus brevior Eigenmann, 1912

Auchenipterus brevior Eigenmann, 1912b: 202. Type locality: Tumatumari, British Guiana. Holotype: FMNH 53249.

Distribution: Potaro River basin, Guyana (Ferraris \& Vari, 1999).

Auchenipterus britskii Ferraris \& Vari, 1999

Auchenipterus britskii Ferraris \& Vari, 1999: 414, figs. 10-11. Type locality: Lago Janauacá, vicinity of Rio Solimões (approx. 328'S, 60²17'W), Amazonas, Brazil. Holotype: MZUSP 48358.

Distribution: Middle Amazon River, Brazil (Ferraris \& Vari, 1999).

Auchenipterus demerarae Eigenmann, 1912

Auchenipterus demerarae Eigenmann, 1912b: 202, pl. 21 (fig. 1). Type locality: Wismar, British Guiana. Holotype: FMNH 53248; holotype illustrated in Ferraris \& Vari (1999: fig. 12).

Distribution: Demerara, Rupununi, Essequibo and Cuyuni River basins (Ferraris \& Vari, 1999).

Auchenipterus dentatus Valenciennes, 1840

Auchenipterus dentatus Valenciennes in Cuvier \& Valenciennes, 1840b: 210 (156 in Strasbourg deluxe edition). Type locality: envoyé de Cayenne [apparently originating from vicinity of Paramaribo, Suriname]. Holotype: MNHN b-0592.

Euanemus colymbetes Müller \& Troschel, in Müller, 1842: 203. Type locality: Suriname. Lectotype: ZMB 3095, designated by Ferraris \& Vari (1999). Also indicated as new, with one syntype illustrated, in Müller \& Troschel (1849: 11, pl. 1, fig. 2).

Distribution: Northern coastal rivers of South America: Corantijn, Suriname, Arataye, Sinnamary and Oyapock rivers (Ferraris \& Vari, 1999).

Auchenipterus fordicei Eigenmann \& Eigenmann, 1888

Auchenipterus fordicei Eigenmann \& Eigenmann, 1888b: 151. Type locality: Coary [Brazil]. Holotype: MCZ 7289.

Distribution: Middle Amazon River basin, Brazil (Ferraris \& Vari, 1999).

Auchenipterus menezesi Ferraris \& Vari, 1999

Auchenipterus menezesi Ferraris \& Vari, 1999: 426, fig. 17. Type locality: Lago de Viana, Rio Pindaré, Mirim basin, Maranhão, Brazil. Holotype: MZUSP 43591.

Distribution: Parnaíba and Pindaré-Mirim River basins of Maranhão and Piaui States (Ferraris \& Vari, 1999).

Auchenipterus nigripinnis (Boulenger, 1895)

Euanemus nigripinnis Boulenger, 1895b: 524. Type locality: Paraguay. Lectotype: BMNH 1895.5.17.37, designated by Ferraris \& Vari (1999); one syntype illustrated in Boulenger (1896c: pl. 4, figs. 2, 2a).

Auchenipterus paysanduanus Devincenzi, 1933: 2. Type locality: Río Uruguay, frente a Paysandú, Uruguay. Holotype: MNHM 4889; illustrated in Ferraris \& Vari (1999: fig. 20).

Distribution: La Plata River basin (Ferraris \& Vari, 1999).

Auchenipterus nuchalis (Spix \& Agassiz, 1829)

Hypophthalmus nuchalis Spix \& Agassiz, 1829: 17, pl. 17. Type locality: Brasilia aequatoriali [now: Rio Capim, near São Domingos do Capim, Brazil, by neotype designation]. Neotype: MZUSP 47945, illustrated in, and designated by, Ferraris \& Vari (1999: fig. 21).

Distribution: Lower Amazon and Tocantins rivers northward to Marowijne River; possibly also in Rupununi River, Guyana, and Negro River, Brazil (Ferraris \& Vari, 1999).

Auchenipterus osteomystax (Miranda Ribeiro, 1918)

Ceratocheilus osteomystax Miranda Ribeiro, 1918d: 644, unnumbered pl. Type locality: Santa Rita das Antas, Rio Vermelho, Goyaz [Brazil]. Holotype: MZUSP 2317.

Distribution: La Plata River basin, Tocantins River, and several rivers of lower Amazon River basin (Ferraris \& Vari, 1999). 


\section{CENTROMOCHLUS Kner, 1857}

Centromochlus Kner, 1857: 430. Type species: Centromochlus megalops Kner, 1857. Type by subsequent designation by Bleeker (1862-63: 7). Gender: Masculine.

Remarks: Included species follows Soares-Porto (1998), except for continued recognition of Gelanoglanis.

Centromochlus altae Fowler, 1945

Centromochlus altae Fowler, 1945a: 109, figs. 7-9. Type locality: Morelia, Río Caquetá basin, Colombia. Holotype: ANSP 71700.

Distribution: Caquetá River basin, Colombia (Ferraris, 2003d).

Centromochlus concolor (Mees, 1974)

Tatia concolor Mees, 1974: 84, fig. 22. Type locality: Headwaters of Coppename Rivier [sic] ( $\left.3^{\circ} 49^{\prime} \mathrm{N}, 5^{\circ} 57^{\prime} \mathrm{W}\right)$, Suriname. Holotype: ZMA 106210.

Distribution: Coppename River basin, Suriname (Ferraris, 2003d).

Centromochlus existimatus Mees, 1974

Centromochlus existimatus Mees, 1974: 50, fig. 8 (right). Type locality: Harbour of Manaos [Brazil]. Holotype: ANSP 73154.

Distribution: Amazon River basin (Ferraris, 2003d).

Centromochlus heckelii (De Filippi, 1853)

Auchenipterus Heckelii De Filippi, 1853: 167. Type locality: Rio Napo. Syntype: ZMUT 233.

Centromochlus Steindachneri Gill, 1870: 95. Type locality: Marañon River, upper Amazon and Napo Rivers.

Type(s): at USNM, but not found (Ferraris \& Vari, 1992).

Distribution: Amazon and Orinoco River basins (Ferraris, 2003d).

Centromochlus macracanthus Soares-Porto, 2000

Centromochlus macracanthus Soares-Porto, 2000: 282, fig. 1. Type locality: Brazil: Amazonas: São Pedro, rio Negro. Holotype: MZUSP 30620.

Distribution: Upper Negro River basin, Brazil (Soares-Porto, 2000).

Centromochlus perugiae Steindachner, 1882

Centromochlus Perugiae Steindachner, 1882b: 178. Type locality: Canelos [Ecuador]. Syntypes: NMW 47358 (3),

SMF 5355 (1). Species illustrated and described in more detail in Steindachner (1882c: 29, pl. 7, figs. 2, 2a).

Distribution: Upper Amazon River basin (Ferraris, 2003d).

Remarks: Wrongly listed as Tatia perugiae by Ferraris (2003d).

Centromochlus punctatus (Mees, 1974)

Tatia punctata Mees, 1974: 88, fig. 24. Type locality: Creeks between Kabel and Lombé [Suriname]. Holotype: RMNH 26495.

Distribution: Coastal rivers of northern South America, above Amazon River mouth (Ferraris, 2003d).

Centromochlus reticulatus (Mees, 1974)

Tatia reticulata Mees, 1974: 90, fig. 25. Type locality: Karanambo, Rupununi, British Guiana. Holotype: BMNH 1972.7.27.702.

Distribution: South America: Rupununi River basin, Guyana (Ferraris, 2003d).

Centromochlus romani (Mees, 1988)

Tatia romani Mees, 1988a: 412, fig. 3. Type locality: Río Guarapiche, affluent of the Río San Juan, near Maturín, Monagas [Venezuela]. Holotype: RMNH 30490.

Distribution: San Juan River, Orinoco Basin, Venezuela (Ferraris, 2003d).

Centromochlus schultzi Rössel, 1962

Centromochlus schultzi Rössel, 1962a: 27, fig. 1. Type locality: Brasilien, oberer Rio Xingu. Holotype: SMF 5462.

Distribution: Upper Xingu and Tocantins River basins (Ferraris, 2003d).

\section{Species inquirenda, Centromochlus}

Centromochlus megalops Kner, 1857: 430, pl. 8 (fig. 24). Type locality: Bogota. Syntypes: NMW 47359 (1), NMW 47360 (1). 
ENTOMOCORUS Eigenmann, 1917

Entomocorus Eigenmann, 1917a: 403. Type species: Entomocorus benjamini Eigenmann, 1917. Type by monotypy. Gender: Masculine.

Entomocorus benjamini Eigenmann, 1917

Entomocorus benjamini Eigenmann, 1917a: 403, fig. 3, pl. 41. Type locality: San Joaquin [Bolivia]. Holotype: FMNH 58109.

Distribution: Upper Madeira River basin, Bolivia (Ferraris, 2003d).

Entomocorus gameroi Mago-Leccia, 1984

Entomocorus gameroi Mago-Leccia, 1984: 217, figs. 1-6. Type locality: Boca del Río Apurito en el Río Apuré, cerca de San Fernando de Apuré, Guárico State, Venezuela. Holotype: MBUCV V-13808.

Distribution: Apuré River basin, Orinoco River drainage, Venezuela (Ferraris, 2003d).

Entomocorus melaphareus Akama \& Ferraris, 2003

Entomocorus melaphareus Akama \& Ferraris, 2003: 78, figs, 1-2. Type locality: Brazil, Pará, Monte Alegre, Costa do Curuá, left bank of Amazon River, 2¹3'46.2"S, 5401'27.6"W. Holotype: MZUSP 76413.

Distribution: Lower and Middle Amazon River (Akama \& Ferraris, 2003).

\section{EPAPTERUS Cope, 1878}

Epapterus Cope, 1878: 677. Type species: Epapterus dispilurus Cope, 1878. Type by monotypy. Gender: Masculine. Revision, with a key to species: Vari \& Ferraris (1998).

Epapterus blohmi Vari, Jewett, Taphorn \& Gilbert, 1984

Epapterus blohmi Vari, Jewett, Taphorn \& Gilbert, 1984: 463, fig. 1. Type locality: Laguna Los Guácimos, a broad shallow lagoon located on Fundo Pecuario Masaguaral, Guarico State, Venezuela, $8^{\circ} 34^{\prime} \mathrm{N}, 6^{\circ} 35^{\prime} \mathrm{W}$. Holotype: MBUCV V-14100.

Distribution: Orinoco River basin and Tuy River of the Caribbean coast of Venezuela (Vari \& Ferraris, 1998).

Epapterus dispilurus Cope, 1878

Epapterus dispilurus Cope, 1878: 677. Type locality: Peruvian Amazon. Lectotype: ANSP 21353, designated by Fowler (1941b: 468, fig. 26) in caption to illustration.

Euanemus longipinnis Steindachner, 1881d: 17. Type locality: Hyavary. Lectotype: NMW 46682.1 (93 m SL), designated by Vari \& Ferraris (1998: 992).

Epapterus chaquensis Risso \& Risso, 1962: 5, figs. 1-3. Type locality: La laguna concida como "del Golf," en la proximidades de la ciudad de Resistencia, Provincia del Chaco, Argentina. Holotype: Univ. Nac. del Nordeste [Argentina] 17/VII/961-1.

Distribution: Central and western parts of the Amazon River basin along and south of the main channel of the Amazon River, and Paraguay River basin in Paraguay, northern Argentina and southern Brazil (Vari \& Ferraris, 1998).

GELANOGLANIS Böhlke, 1980

Gelanoglanis Böhlke, 1980: 150. Type species: Gelanoglanis stroudi Böhlke, 1980. Type by original designation. Gender: Masculine.

Gelanoglanis nanonocticolus Soares-Porto, Walsh, Nico \& Netto, 1999

Gelanoglanis nanonocticolus Soares-Porto, Walsh, Nico \& Netto, 1999: 65, fig. 1. Type locality: Venezuela: Amazonas State: Río Asisa 22-30 km upstream from confluence with Río Paru, Río Ventuari drainage, Orinoco River basin (approximately 4³3'N, 6554'W). Holotype: MCNG 22690.

Distribution: Asisa River basin in Orinoco drainage, Venezuela, and Negro River basin, Brazil (Soares-Porto et al., 1999).

Gelanoglanis stroudi Böhlke, 1980

Gelanoglanis stroudi Böhlke, 1980: 152, fig. 1. Type locality: Colombia, Depto. Meta, Río Metica, ca. 22 km (air) SW of Puerto Lopez and 3 km SE of Hacienda Mozambique, 356'42"N, 7302'23"W. Holotype: ANSP 142937.

Distribution: Meta, Apure and Masparro River basins, Colombia and Venezuela (Provenzano, 1997). 
GLANIDIUM Lütken, 1874

Glanidium Lütken, 1874c: 31. Type species: Glanidium albescens Reinhardt, 1874. Type by monotypy. Gender: Neuter.

Gephyromochlus Hoedeman, 1961: 135. Type species: Centromochlus (Gephyromochlus) leopardus Hoedeman, 1961. Type by monotypy. Gender: Masculine.

Glanidium albescens Reinhardt, 1874

Glanidium albescens Reinhardt, in Lütken, 1874c: 31. Type locality: in flumine Rio das Velhas cum affluentibus [Brazil]. Syntypes: MNHN 0000-9571 (1), NMW 46572 (1), ZMB 9176 (1), ZMUC P 29670-29675 (1 each).

Distribution: Das Velhas River basin, Brazil (Ferraris, 2003d).

Glanidium bockmanni Sarmento-Soares \& Buckup, 2005

Glanidium bockmanni Sarmento-Soares \& Buckup, 2005: 847, fig. 1. Type locality: Brazil, Minas Gerais state, Rio São Francisco, downstream from Três Marias Dam. Holotype: MZUSP 82803.

Distribution: São Francisco River basin, Brazil (Sarmento-Soares \& Buckup, 2005).

Glanidium catharinensis Miranda Ribeiro, 1962

Glanidium catharinensis Miranda Ribeiro, 1962a: 3, fig. 2. Type locality: Rio do Braço do Norte, São Ludgero, Municipio de Tubarão, Estado de Santa Catarina [Brazil]. Holotype: MNRJ 5169.

Distribution: Tubarão River basin in Santa Catarina State, Brazil (Ferraris, 2003d).

Glanidium cesarpintoi Ihering, 1928

Glanidium cesarpintoi Ihering, 1928b: 46, fig. 1. Type locality: Cachoeira de Emas (Pirassununga), Rio Mogyguassú, Est. de S. Paulo [Brazil]. Syntypes (3): whereabouts unknown.

Distribution: Mogi-guassú River basin, São Paulo State, Brazil (Ferraris, 2003d).

Glanidium leopardum (Hoedeman, 1961)

Centromochlus (Gephyromochlus) leopardus Hoedeman, 1961: 135, fig. 3. Type locality: Litany River, village Aloiké, sta. 295 [French Guiana]. Holotype: ZMA 102233.

Distribution: Coastal rivers of the Guianas (Ferraris, 2003d).

Glanidium melanopterum Miranda Ribeiro, 1918

Glanidium melanopterum Miranda Ribeiro, 1918d: 643. Type locality: Piquete, S. Paulo [Brazil]. Lectotype: MZUSP 345, designated in Britski (1969).

Distribution: Piquete, São Paulo, Brazil (Ferraris, 2003d).

Glanidium ribeiroi Haseman, 1911

Glanidium ribeiroi Haseman, 1911b: 381, pl. 78. Type locality: Porto União da Victoria, Paraná, Brazil, from Rio Iguassú. Holotype: FMNH 54253.

Distribution: Iguaçu River basin (Ferraris, 2003d).

LIOSOMADORAS Fowler, 1940

Liosomadoras Fowler, 1940a: 226. Type species: Liosomadoras morrowi Fowler, 1940. Type by original designation. Gender: Masculine.

Liosomadoras morrowi Fowler, 1940

Liosomadoras morrowi Fowler, 1940a: 226, figs. 11-14. Type locality: Ucayali River basin, Contamana, Peru. Holotype: ANSP 68646.

Distribution: Ucayali River basin, Peru (Ferraris, 2003d).

Liosomadoras oncinus (Jardine, 1841)

Arius oncinus Jardine, in Schomburgk, 1841: 173, pl. 4. Type locality: Padauiri River. No types known. Name spelled Arius oncina on figure caption, A. oncinus in text. Mees (1978a) served as first reviser in treating Liosomadoras oncinus as valid.

Distribution: Negro and Branco River basins, Brazil (Ferraris, 2003d); Venezuela (Taphorn et al., 1997); Orinoco River basin (Royero, 1999).

PSEUDAUCHENIPTERUS Bleeker, 1862

Pseudauchenipterus Bleeker, 1862 (in Bleeker, 1862-63): 6. Type species: Silurus nodosus Bloch, 1794. Type by 
original designation. Gender: Masculine.

Silvaichthys Fernández-Yépez, 1973: [3]. Type species: Silvaichthys aguilerae Fernández-Yépez, 1973. Type by original designation. Gender: Masculine.

Pseudauchenipterus affinis (Steindachner, 1877)

Auchenipterus (Pseudauchenipterus) affinis Steindachner, 1877b: 651. Type locality: Rio S. Matheus; Rio Mucury bei Porto Allegre [Brazil]. Syntypes: NMW 47384 (2), NMW 47385 (2), NMW 47386 (2), NMW 47387 (2).

Distribution: Mucuri and São Mateus River basins, Brazil (Ferraris, 2003d).

Pseudauchenipterus flavescens (Eigenmann \& Eigenmann, 1888)

Felichthys flavescens Eigenmann \& Eigenmann, 1888b: 152. Type locality: Rio San Francisco [Brazil]. Holotype: MCZ 7362.

Distribution: São Francisco River basin, Brazil (Ferraris, 2003d).

Pseudauchenipterus jequitinhonhae (Steindachner, 1877)

Auchenipterus (Pseudauchenipterus) Jequitinhonhae Steindachner, 1877b: 647, pl. 6 (fig. 1). Type locality: Rio Jequitinhonha [Brazil]. Syntypes: NMW 19785-92 (8).

Distribution: Jequitinhonha River basin, Brazil (Ferraris, 2003d).

Pseudauchenipterus nodosus (Bloch, 1794)

Silurus nodosus Bloch, 1794: 35, pl. 368 (fig. 1). Type locality: Tranquebar [in error]. Lectotype: ZMB 3096, designated by, and illustrated in, Paepke (1999: 47, pl. 26, fig. 1).

Auchenipterus furcatus Valenciennes, in Cuvier \& Valenciennes, 1840b: 211 (157 in Strasbourg deluxe edition). Type locality: Cayenne ou de Surinam. Syntypes: MNHN b-0213 (1), MNHN 0000-3049 (1), MNHN a-8855 (1); MNHN b-0213 illustrated in Royero \& Hureau (1996: fig. 1).

Pseudauchenipterus guppyi Regan, 1906c: 387, pl. 23. Type locality: Caroni River [Trinidad]. Syntypes: BMNH 1906.6.23.49-50 (2).

Pseudauchenipterus nigrolineatus Fowler, 1911: 434, fig. 5. Type locality: Pedernales, Venezuela. Holotype: ANSP 37876.

Silvaichthys aguilerae Fernández-Yépez, 1973: [4], unnumbered figure. Type locality: Gulfo de Paria, noreste de Venezuela, frente a IRAPA, Estado Suene. Syntypes (4): originally as "Holotipo macho AFY 731.201, Holotipo hembra AFY 731.202, y los paratipos ... AFY 731.203 (2)”; whereabouts unknown, not at ANSP as indicated in the description.

Distribution: Lower reaches of rivers and into estuaries from Venezuela and southern Trinidad to Brazil, south at least to Bahia (Ferraris, 2003d).

PSEUDEPAPTERUS Steindachner, 1915

Pseudepapterus Steindachner, 1915a: 199. Type species: Auchenipterus (Pseudepapterus) hasemani Steindachner, 1915. Type by monotypy. Gender: Masculine. Originally proposed as a subgenus of Auchenipterus.

Revision, with a key to species: Ferraris \& Vari (2000).

Pseudepapterus cucuhyensis Böhlke, 1951

Pseudepapterus cucuhyensis Böhlke, 1951b: 38, fig. 1. Type locality: Sandbank at Cucuhy on the Columbian [sic] border, Rio Negro, Brazil. Holotype: SU 16788.

Distribution: Amazon and Negro River basins (Ferraris \& Vari, 2000).

Pseudepapterus gracilis Ferraris \& Vari, 2000

Pseudepapterus gracilis Ferraris \& Vari, 2000: 109, fig. 5. Type locality: Venezuela, Bolivar, Caño Chuapo, approximately 20 minutes downstream from Jabillal (on opposite bank) along Rio Caura, $7^{\circ} 07^{\prime} \mathrm{N}, 65^{\circ} 00^{\prime} \mathrm{W}$. Holotype: ANSP 139502.

Distribution: Caura River basin, Venezuela; known only from holotype (Ferraris \& Vari, 2000).

Pseudepapterus hasemani (Steindachner, 1915)

Auchenipterus (Pseudepapterus) hasemani Steindachner, 1915a: 199. Type locality: Pará (Belem) [Brazil]. Holotype: NMW 47397. Described in more detail in Steindachner (1915e: 82).

Distribution: Amazon River and many of its major tributary rivers, and Tocantins River basin (Ferraris \& Vari, 2000). 
PSEUDOTATIA Mees, 1974

Pseudotatia Mees, 1974: 105. Type species: Pseudotatia parva Mees, 1974. Type by original designation. Gender: Feminine.

Pseudotatia parva Mees, 1974

Pseudotatia parva Mees, 1974: 105, fig. 29. Type locality: Joazeiro, Brazil. Holotype: FMNH 70580.

Distribution: São Francisco River basin, Brazil (Ferraris, 2003d).

TATIA Miranda Ribeiro, 1911

Tatia Miranda Ribeiro, 1911: 360. Type species: Centromochlus intermedius Steindachner, 1877. Type by subsequent designation by Jordan (1920: 545). Gender: Feminine.

Tatia aulopygia (Kner, 1857)

Centromochlus aulopygius Kner, 1857: 432, pl. 8 (fig. 25). Type locality: Rio Guaporé [Brazil]. Syntypes: NMW 47329 (2), NMW 47330 (2), NMW 47331 (3), NMW 47332 (3), NMW 47333 (5) NMW 47388 (1).

Distribution: Guaporé River basin (Ferraris, 2003d).

Tatia boemia Koch \& Reis, 1996

Tatia boemia Koch \& Reis, 1996: 86, fig. 2. Type locality: Rio Pelotas (tributary of the Rio Uruguai) approx. $27^{\circ} 47^{\prime} \mathrm{S}, 51^{\circ} 11^{\prime} \mathrm{W}$, Rio Grande do Sul, Brazil. Holotype: MCP 17438.

Distribution: Upper Uruguay River basin (Ferraris, 2003d).

Tatia brunnea Mees, 1974

Tatia brunnea Mees, 1974: 84, fig. 21. Type locality: Compagnie Kreek [Suriname]. Holotype: RMNH 26196.

Distribution: Suriname and Marowijne River basins (Ferraris, 2003d).

Tatia creutzbergi (Boeseman, 1953)

Centromochlus creutzbergi Boeseman, 1953: 7, fig. 1c. Type locality: Djaikreek, Surinam. Holotype: RMNH 19440.

Distribution: Suriname coastal rivers and Cudajas (Ferraris, 2003d).

Tatia dunni (Fowler, 1945)

Centromochlus dunni Fowler, 1945a: 111, figs. 11-13. Type locality: Morelia, Río Caquetá drainage, Colombia. Holotype: ANSP 71705.

Distribution: Caquetá River basin, Colombia (Ferraris, 2003d).

Tatia galaxias Mees, 1974

Tatia galaxias Mees, 1974: 86, fig. 23. Type locality: Caño de Quiribana into Río Orinoco, Venezuela. Holotype: CAS 6567.

Distribution: Middle Orinoco River basin (Ferraris, 2003d).

Tatia gyrina (Eigenmann \& Allen, 1942)

Centromochlus gyrinus Eigenmann \& Allen, 1942: 118, pl. 5 (fig. 4). Type locality: brook near Rio Itaya, Iquitos [Peru]. Holotype: CAS 36979.

Distribution: Upper Amazon River basin (Ferraris, 2003d).

Tatia intermedia (Steindachner, 1877)

Centromochlus intermedius Steindachner, 1877b: 664. Type locality: Marabitanos; Pará [Brazil]. Syntypes (2): NMW 47355 (1).

Distribution: Amazon River basin and rivers of Guianas (Ferraris, 2003d).

Remarks: Steindachner (1881d: 4) indicated that the original description was based on two specimens.

Tatia musaica Royero, 1992

Tatia musaica Royero, 1992: 301, fig. 1. Type locality: Río Atuana, Caño Cabeza de Manteco, approximadamente a $12 \mathrm{kms}$ debajo del raudel Pereza, en el pidemonte del tepui Autana, Territorio Federal, Amazonas, Venezuela, $4^{\circ} 47^{\prime} \mathrm{N}, 67^{\circ} 23^{\prime} \mathrm{W}$. Holotype: MBUCV V-15663.

Distribution: Atabapo and Autana River basins in upper Orinoco River drainage, Venezuela (Ferraris, 2003d).

Tatia neivai (Ihering, 1930)

Glanidium neivai Ihering, 1930: 99, pl. 13 (fig. 1). Type locality: Piracicaba, Rio Piracicaba, Est. de S. Paulo. Holotype: Whereabouts unknown.

Distribution: Piracicaba River basin in São Paulo State (Ferraris, 2003d). 
Tatia simplex Mees, 1974

Tatia simplex Mees, 1974: 90, fig. 26. Type locality: Xaventina house beach, Rio das Mortes, Mato Grosso, Brazil. Holotype: BMNH 1971.7.29.5.

Distribution: Das Mortes River in Mato Grosso State, Brazil (Ferraris, 2003d).

Tatia strigata Soares-Porto, 1995

Tatia strigata Soares-Porto, 1995: 202, fig. 1. Type locality: Brazil, Amazonas, Municipio de Maués, Igarapé Limãozinho. Holotype: MZUSP 44065.

Distribution: Amazon River basin and upper reaches of the Orinoco River (Ferraris, 2003d).

\section{TETRANEMATICHTHYS Bleeker, 1858}

Tetranematichthys Bleeker, 1858b: 357, 359. Type species: Ageneiosus quadrifilis Kner, 1857. Type by monotypy. Gender: Masculine.

Tetranematichthys quadrifilis (Kner, 1857)

Ageneiosus quadrifilis Kner, 1857: 442, pl. 9 (fig. 29). Type locality: Rio Guaporé. Holotype: NMW 43343.

Distribution: Upper Guaporé River; reports of specimens from other localities in the Amazon and Orinoco river basins refer to a currently unnamed species.

TOCANTINSIA Mees, 1974

Tocantinsia Mees, 1974: 108. Type species: Tocantinsia depressa Mees, 1974. Type by original designation. Gender: Feminine.

Tocantinsia piresi (Miranda Ribeiro, 1920)

Glanidium piresi Miranda Ribeiro, 1920: 14, pls. 15-17. Type locality: Rio S. Manoel [Mato Grosso, Brazil]. MNRJ 264.

Tocantinsia depressa Mees, 1974: 108, fig. 30. Type locality: Tocantins near Porto National, Goyaz, Brazil. Holotype: CAS 6573.

Distribution: Upper Tocantins River basin (Ferraris, 2003d).

Remarks: Synonymy follows Mees (1984).

\section{TRACHELYICHTHYS Mees, 1974}

Trachelyichthys Mees, 1974: 111. Type species: Trachelyichthys decaradiatus Mees, 1974. Type by original designation. Gender: Masculine.

Trachelyichthys decaradiatus Mees, 1974

Trachelyichthys decaradiatus Mees, 1974: 112, fig. 31. Type locality: Karanambo area, Rupununi, British Guiana. Holotype: BMNH 1971.7.29.30.

Distribution: Rupununi River basin, Guyana (Ferraris, 2003d).

Trachelyichthys exilis Greenfield \& Glodek, 1977

Trachelyichthys exilis Greenfield \& Glodek, 1977: 49, fig. 2. Type locality: Río Mamón, a tributary of Río Nanay, approximately $25 \mathrm{~km}$ west of Iquitos, $3^{\circ} 42^{\prime} \mathrm{S}, 73^{\circ} 16^{\prime} \mathrm{W}$, Peru. Holotype: FMNH 80476.

Distribution: Nanay River basin in upper Amazon River drainage (Ferraris, 2003d).

TRACHELYOPTERICHTHYS Bleeker, 1862

Trachelyopterichthys Bleeker, 1862 (in Bleeker, 1862-63): 16. Type species: Trachelyopterus taeniatus Kner, 1857.

Type by original designation. Gender: Masculine.

Trachelyopterichthys anduzei Ferraris \& Fernandez, 1987

Trachelyopterichthys anduzei Ferraris \& Fernandez, 1987: 257, fig. 1. Type locality: Laguna de Carida at the mouth of Caño Carida, Río Orinoco, Terr. Federal Amazonas, Venezuela. Holotype: MBUCV V-14627.

Distribution: Upper Orinoco River basin (Ferraris, 2003d).

Trachelyopterichthys taeniatus (Kner, 1857)

Trachelyopterus taeniatus Kner, 1857: 434, pl. 8 (fig. 26). Type locality: Rio Guaporé [Brazil]. Holotype: NMW 43346. 
Distribution: Upper Amazon River basin (Ferraris, 2003d).

TRACHELYOPTERUS Valenciennes, 1840

Trachelyopterus Valenciennes, in Cuvier and Valenciennes, 1840b: 220 (163 in Strasbourg deluxe edition). Type species: Trachelyopterus coriaceus Valenciennes, 1840. Type by monotypy. Gender: Masculine.

Parauchenipterus Bleeker, 1862 (in Bleeker, 1862-63): 7. Type species: Silurus galeatus Linnaeus, 1766. Type by original designation. Gender: Masculine.

Review: Bertoletti et al. (1992), southeastern Brazil.

Trachelyopterus albicrux (Berg, 1901)

Trachycorystes albicrux Berg, 1901: 303. Type locality: Río de la Plata, cerca de la embocadura del Río Santiago, Buenos Aires. Holotype: MACN 5161.

Distribution: La Plata River basin (Ferraris, 2003d).

Trachelyopterus amblops (Meek \& Hildebrand, 1913)

Felichthys amblops Meek \& Hildebrand, 1913: 77. Type locality: Río Tuyra, Marriganti, Panama. Holotype: FMNH 7576.

Distribution: Tuira River basin, Panama (Ferraris, 2003d).

Trachelyopterus ceratophysus (Kner, 1857)

Auchenipterus ceratophysus Kner, 1857: 427, pl. 7 (fig. 23). Type locality: Matogrosso am Rio Guaporé, Rio Branco, und Rio Negro. Syntypes: NMW 47390-93 (4), NMW 50585 (1).

Distribution: Guaporé, Branco and Negro River basins (Ferraris, 2003d).

Trachelyopterus coriaceus Valenciennes, 1840

Trachelyopterus coriaceus Valenciennes, in Cuvier \& Valenciennes, 1840b: 221 (164 in Strasbourg deluxe edition), pl. 438. Type locality: [Unknown, apparently from vicinity of Cayenne, French Guiana]. Holotype: MNHN b0242.

Trachelyopterus coriaceus maculosus Eigenmann \& Eigenmann, 1888b: 157. Type locality: Porto do Moz [Pará, Brazil]. Holotype: MCZ 7337.

Distribution: Coastal rivers of Guianas and Brazil and Amazon River basin (Ferraris, 2003d).

Trachelyopterus fisheri (Eigenmann, 1916)

Trachycorystes fisheri Eigenmann, 1916: 82. Type locality: Río Sucio [Colombia]. Holotype: FMNH 57695; illustrated in Eigenmann (1922: 48, pl. 5, fig. 1).

Distribution: Sucio River basin, Colombia (Ferraris, 2003d).

Trachelyopterus galeatus (Linnaeus, 1766)

Silurus galeatus Linnaeus, 1766: 503. Type locality: in America australi. Syntypes: On Seba (1734-65: 85, pl. 19, fig. 7) and at least one additional specimen which has not been located.

Auchenipterus maculosus Valenciennes, 1840b: 216 (161 in Strasbourg deluxe edition). Type locality: Cayenne. Syntypes: MNHN a-8856 (1), ?RMNH D3007; MNHN a-8856 illustrated in Royero \& Hureau (1996: fig. 2).

Parauchenipterus paseae Regan, 1906c: 387, pl. 24. Type locality: Caroni River, Trinidad. Holotype: BMNH 1906.6.23.48.

Distribution: Widespread in northern South America (Ferraris, 2003d).

Trachelyopterus insignis (Steindachner, 1878)

Auchenipterus insignis Steindachner, 1878a: 89. Type locality: Magdalenen-Stromes [Colombia]. Syntypes: at NMW. Illustrated and described in more detail in Steindachner (1879d: 35, pl. 3, figs. 2, 2a).

Auchenipterus Magdalenae Steindachner, 1878a: 89. Type locality: Magdalenen-Stromes [Colombia]. Syntypes: NMW 47409-12 (4). Illustrated and described in more detail in Steindachner (1879d: 36, pl. 4, figs. 1, 1a).

Distribution: Lower Magdalena River basin, Colombia (Ferraris, 2003d).

Remarks: The name Auchenipterus insignis given precedence over A. magdalenae by first reviser action of Miles (1947: 73). Redescribed, as Trachycorystes magdalenae, in Schmidt (1928).

Trachelyopterus lacustris (Lütken, 1874)

Auchenipterus lacustris Lütken, 1874c: 30. Type locality: in flumine Rio das Velhas et in lacu Lagoa Santa. Syntypes: MNHN 0000-9572 (1), NMW 47402 (1), ZMB 9179 (1), ZMUC P 29665 (1), ZMUC P 29666 (1), 
ZMUC P 29667 (1), ZMUC P 29668 (1).

Distribution: Das Velhas River and Lagoa Santa basins (Ferraris, 2003d).

Trachelyopterus leopardinus (Borodin, 1927)

Trachycorystes leopardinus Borodin, 1927b: 3, fig. 2. Type locality: Rio S. Francisco, Minas Geraes [sic] Province, Brazil. Holotype: AMNH 8637.

Distribution: São Francisco River basin, Brazil (Ferraris, 2003d).

Trachelyopterus lucenai Bertoletti, da Silva \& Pereira, 1995

Trachelyopterus lucenai Bertoletti, da Silva \& Pereira, 1995: 71, fig. 2-3. Type locality: Rio Jacuí at saco do Quilombo, ilha das Flores, Porto Alegre, Rio Grande do Sul, Brazil (approximately $29^{\circ} 58^{\prime} \mathrm{S}, 5^{\circ} 15^{\prime} \mathrm{W}$ ). Holotype: MCP 17174.

Distribution: Lowland tributaries of Uruguay River and Laguna dos Patos basins, Brazil (Bertoletti et al., 1995).

Trachelyopterus peloichthys (Schultz, 1944)

Trachycorystes insignis peloichthys Schultz, 1944c: 236, pl. 4 (fig. a). Type locality: Río Agua Caliente, 2 to 3 km above Lake Maracaibo, Venezuela. Holotype: USNM 121281.

Distribution: Maracaibo River basin (Ferraris, 2003d).

Trachelyopterus striatulus (Steindachner, 1877)

Auchenipterus (Pseudauchenipterus) striatulus Steindachner, 1877b: 656, pl. 5. Type locality: Rio Parahyba, Rio Doce, Rio Mucury. Syntypes: MCZ 25506 (1), NMW 10852 (1), NMW 47445 (5), NMW 47446 (2), NMW 47447 (1), SMNS 1955 (1).

Distribution: Coastal rivers in southeastern Brazil (Ferraris, 2003d).

Trachelyopterus teaguei (Devincenzi, 1942)

Trachycorystes teaguei Devincenzi in Devincenzi \& Teague, 1942: 37, pl. 7 (figs. 1-2). Type locality: Río Queguay, Uruguay. Holotype: MHNM CI 350.

Distribution: Queguay River basin in lower Uruguay River drainage (Ferraris, 2003d).

\section{Species inquirendae, Trachelyopterus}

Auchenipterus immaculatus Valenciennes, 1840b: 218 (162 in Strasbourg deluxe edition). Type locality: Cayenne. Holotype: RMNH 3008. Comments on type locality in Boeseman (1972: 302).

Auchenipterus robustus Günther, 1864: 197. Type locality: Demerara [Guyana]. Holotype: BMNH 2005.9.12.3.

Auchenipterus glaber Steindachner, 1877b: 655, footnote. Type locality: Demerara [Guyana]. Holotype: at NMW.

Auchenipterus isacanthus Cope, 1878: 677. Type locality: Peruvian Amazon. Syntypes: ANSP 21444-45 (2).

Auchenipterus brevibarbis Cope, 1878: 676. Type locality: Peruvian Amazon. Holotype: ANSP 21519.

Trachycorystes analis Eigenmann \& Eigenmann, 1888b: 156. Type locality: Arary? [possibly = Lago Arari, Pará, Brazil]. Holotype: MCZ 7354.

Trachycorystes insignis badeli Dahl, 1955: 13, fig. on p. 14. Type locality: Cereté [Sinu River, Colombia]. Holotype: Whereabouts unknown (possibly at ICNMHN).

Trachycorystes jokeannae Hoedeman, 1961: 138. Type locality: French Guiana, Ile de Cayenne, ruisseaux du Rorota. Holotype: ZMA 102371.

TRACHYCORYSTES Bleeker, 1858

Trachycorystes Bleeker, 1858b: 200, 208. Type species: Auchenipterus trachycorystes Valenciennes, 1840. Type by absolute tautonymy. Gender: Masculine.

Trachycorystes cratensis Miranda Ribeiro, 1937

Trachycorystes cratensis Miranda Ribeiro, 1937: 55. Type locality: Rio Granjeiro, Crato, Ceará [Brazil]. Holotype: MNRJ 947.

Distribution: Granjeiro River basin in Ceará State, Brazil (Ferraris, 2003d).

Trachycorystes trachycorystes (Valenciennes, 1840)

Auchenipterus trachycorystes Valenciennes, in Cuvier \& Valenciennes, 1840b: 214 (159 in Strasbourg deluxe edition), pl. 437. Type locality: Brésil. Holotype: MNHN a-9422; illustrated in Mees (1974: pl. 1). 
Trachycorystes typus Bleeker, 1862 (in Bleeker, 1862-63): 6. Type locality: Bresil. Holotype: MNHN a-9422. Replacement for Auchenipterus trachycorystes Valenciennes, 1840, presumably to avoid tautonymy, therefore taking the same type.

Auchenipterus obscurus Günther, 1863: 442. Type locality: the Essequibo, British Guiana. Syntypes: BMNH 1864.1.21.13-14 (2); one syntype illustrated in Eigenmann (1912: pl. 17, fig. 2).

Distribution: Amazon River basin, Essequibo River basin, Guyana, and Orinoco River basin, Venezuela (Ferraris, 2003d).

\section{Species inquirendae, Auchenipteridae}

Arius obesus Jardine, in Schomburgk, 1841: 174. Type locality: Rio Branco. No types known.

Pimelodus carulescens La Cepède, 1803: 95, 103. Type locality: Cayenne. No types known. Name spelled Pimelodus coerulescens on p. 95; Pimelodus caerulescens on 103.

Trachycorystes porosus Eigenmann \& Eigenmann, 1888b: 154. Type locality: Brazil. Syntypes (2, 13-15 cm): MCZ 7351 (2).

\section{AUSTROGLANIDIDAE Mo, 1991}

Austroglanididae Mo, 1991: 160. Type genus: Austroglanis Skelton, Risch \& de Vos, 1984.

1 genus, 3 species; no named fossil taxa.

AUSTROGLANIS Skelton, Risch \& de Vos, 1984

Austroglanis Skelton, Risch \& de Vos, 1984: 361. Type species: Gephyroglanis sclateri Boulenger, 1901. Type by original designation. Gender: Masculine.

Austroglanis barnardi (Skelton, 1981)

Gephyroglanis barnardi Skelton, 1981: 220, figs. 2, 4-6, 8-21. Type locality: Noordhoeks River at roadbridge, tributary of Olifants River, Cape Province, S. Africa, 3243'15"S, 1903'59"E. Holotype: AMG P7647(a).

Distribution: Streams in Clanwilliam-Olifants River system, South Africa (Skelton, 1993).

Austroglanis gilli (Barnard, 1943)

Gephyroglanis gilli Barnard, 1943: 227, fig. 23 c-g. Type locality: Olifants River system, Clanwilliam Division: in an irrigation furrow on the Jan Diesels River in Bosch Kloof, Clanwilliam; Boontjes River, Citrusdal; upper reaches of Olifants River at the farm "Keerom" and in a side tributary of the farm "Noordhoek." Lectotype: SAM 29231 [now at AMG], designated by Skelton (1981: 267).

Distribution: Streams in Clanwilliam-Olifants River system (Skelton, 1993).

Austroglanis sclateri (Boulenger, 1901)

Gephyroglanis sclateri Boulenger, 1901g: 228, pl. 13. Type locality: Vaal River, South Africa. Syntypes: BMNH 1901.2.11.23 (1), SAM 4708 [now at AMG] (1).

Distribution: Orange-Vaal River basin, in major tributaries and main river channel (Skelton, 1993).

\section{BAGRIDAE Bleeker, 1858}

Bagrichthyoidei Bleeker, 1858b: 49, 54. Type genus: Bagrus Bosc, 1816.

Bagrichthyes Bleeker, 1862 (in Bleeker, 1862-63): 8, 48. Type genus: Bagrichthys Bleeker, 1857.

Ritae Bleeker, 1862 (in Bleeker, 1862-63): 8. Type genus: Rita Bleeker, 1854.

Olyrinae Gill, 1893: 132. Type genus: Olyra M'Clelland, 1842.

Porcinae Fowler, 1915a: 219. Type genus: Porcus Geoffroy St. Hilaire, 1809. Based on a generic name that was placed on the Official Index of Rejected and Invalid Generic Names in Zoology, Opinion 1402 (Melville \& Smith, 1987).

Batasinae Tilak, 1967: 431. Type genus: Batasio Blyth, 1860.

Pelteobagrini Jayaram, 1966b: 1071. Type genus: Pelteobagrus Bleeker, 1865. 
Bagroidinae Jayaram, 1968b: 371. Type genus: Bagroides Bleeker, 1851.

Taxonomic summaries: Risch (1986, Africa); Chu et al. (1999, China).

Reviews: Jayaram (1955a, genera); Jayaram (1966c, various Asian genera); Jayaram (1966b, African genera); Jayaram (1968b, Japanese, Chinese, Malayan and Indonesian genera); Jayaram (1978a, Chinese species of "Mystus"); Risch (1992b, West Africa); Desoutter (1975, Cambodia); Lee \& Kim (1990, Korea).

Identification guides: Jayaram (1977d, 1979 South Asia).

Phylogeny: Jayaram (1976); Jayaram \& Singh (1982); Mo (1991); Peng et al. (2002, East Asian species); Ng (2003j).

23 genera, 189 species; including 4 fossil genera and 11 fossil species.

\section{Incertae sedis}

\section{Pseudobagrus kyphus Mai, 1978}

Pseudobagrus kyphus Mai, 1978: 261, fig. 119. Type locality: [Ky Phu stream in Dai tu district, Thai Nguyen Province, Northern Vietnam]. Holotype: at DVZUT.

Distribution: Vu Quang, northern Vietnam (Kottelat, 2001a), Guangxi Zhuangzu Autonomous Region, China (Watanabe et al., 2002).

Remarks: Currently treated as valid as Pseudobagrus kyphus or Pelteobagrus kyphus. Redescribed in Watanabe, et al. (2002).

Pseudobagrus nitidus Sauvage \& Dabry de Thiersant, 1874

Pseudobagrus nitidus Sauvage \& Dabry de Thiersant, 1874: 5. Type locality: Yang-tze-kiang, China. Type(s) (130 $\mathrm{mm})$ : at MNHN.

Distribution: from Heilongjiang [Amur River] to Minjiang [Yangtze River tributary] (Chu et al., 1999).

Remarks: Currently treated as valid as Pelteobagrus nitidus or Leiocassis nitidus.

$\dagger$ Brachyspondylus saropterix Marck, 1876a: 411, pl. 23, fig. 2. Type locality: Sumatra; Tertiary. Holotype: at Royal Geol. Mus. Dresden (Posterior end of fish, without Weberian complex, pectoral girdle or head).

Distribution: Sumatra (Gayet \& Otero, 1999).

Remarks: Treated as valid in the Bagridae S.L. by Gayet \& Meunier (2003), but the generic name $\dagger$ Brachyspondylus does not have a siluriform taxon as its type; therefore this species is without a valid generic name. Sanders (1934: 28) placed the species into Aoria, but that name is preoccupied in Insecta.

\section{BAGRICHTHYS Bleeker, 1857}

Bagrichthys Bleeker, 1857b: 473. Type species: Bagrus hypselopterus Bleeker, 1852. Type by monotypy. Gender: Masculine.

Pseudobagrichthys Bleeker, 1862 (in Bleeker, 1862-63): 9, 49. Type species: Bagroides macropterus Bleeker, 1853.

Type by original designation. Gender: Masculine.

Key: Linder \& Ng (2002a).

Bagrichthys hypselopterus (Bleeker, 1852)

Bagrus hypselopterus Bleeker, 1852d: 588. Type locality: Palembang, in fluviis. Holotype (260 mm TL): possibly RMNH 6877.

Distribution: Sumatra and Borneo (Roberts, 1989a).

Bagrichthys macracanthus (Bleeker, 1854)

Bagrö̈des macracanthus Bleeker, 1854a: 88. Type locality: Sumatra orientalis, ubi confluunt flumina Lamatang et Emin. Holotype (240 mm TL): RMNH 5216 (1 of 27); illustrated in Bleeker (1862-63: 50, pl. 67 [=Silur. pl. 19], fig. 1) as Pseudobagrichthys macracanthus.

Distribution: Mekong and Chao Phraya River basins and Sundaland (Kottelat, 2001b).

Bagrichthys macropterus (Bleeker, 1853)

Bagrö̈des macropterus Bleeker, 1853b: 515. Type locality: Moara kompeh, in fluviis [Sumatra]. Holotype (256 mm TL): Whereabouts unknown.

Distribution: Sumatra.

Remarks: As Bagroides macropterus in some recent literature. 


\section{Bagrichthys majusculus $\mathrm{Ng}, 2002$}

Bagrichthys majusculus Ng, 2002a: 50, fig. 1. Type locality: Thailand: Ubon Ratchathani Province, Khong Chiam district, Mun River at Ban Dan, 3 km upstream from Mekong River confluence. Holotype: UMMZ 214916.

Distribution: Mekong and Chao Phraya River basins (Ng, 2002a).

Bagrichthys micranodus Roberts, 1989

Bagrichthys micranodus Roberts, 1989a: 115, fig. 89. Type locality: Kapuas mainstream 58 km NE of Sintang and 1 km downstream from Sebruang, Western Borneo (Kalimantan Barat, Indonesia). Holotype: MZB 3578.

Distribution: Kapuas River, Western Borneo (Roberts, 1989a).

\section{Bagrichthys obscurus $\mathrm{Ng}, 1999$}

Bagrichthys obscurus Ng, 1999f: 546, figs. 1-2. Type locality: Thailand: Roi Et Province, Lam Chi, $1.5 \mathrm{~km}$ below highway 23 bridge, 4 km W. of Selaphum. Holotype: USNM 317511.

Distribution: Mekong, Bang Pakong and Chao Phraya River basins (Ng, 1999f).

Bagrichthys vaillantii (Popta, 1906)

Leiocassis macropterus Vaillant, 1902: 58, figs. 6-7. Type locality: Tepoe, bords du Mahakam, Bornéo central. Holotype: Whereabouts unknown. Considered to have been preoccupied in Bagroides by Bagroides macropterus Bleeker, 1853; replaced by Bagroides vaillantii Popta, 1906.

Bagroides Vaillantii Popta, 1906: 228. Type locality: Tepoe, bords du Mahakam, Bornéo central. Holotype: Whereabouts unknown. Replacement name for Leiocassis macropterus Vaillant, 1902, considered preoccupied in Bagroides by Bagroides macropterus Bleeker, 1853.

Distribution: Mahakam River basin, eastern Borneo (Ng, 2000).

Remarks: Redescribed in Ng (2000: 327).

BAGROIDES Bleeker, 1851

Bagrö̈des Bleeker, 1851d: 204. Type species: Bagroides melapterus Bleeker, 1851. Type by monotypy. Gender: Masculine.

Bagroides melapterus Bleeker, 1851

Bagrö̈des melapterus Bleeker, 1851d: 204. Type locality: Bandjarmassing, in fluviis. Syntypes (2, $142,200 \mathrm{~mm}$ TL): BMNH 1863.12.4.71 (1), possibly NMV 46011 (1).

Bagroides melanopterus Bleeker, 1852b: 413. Type locality: Bandjarmassing, in fluviis. Syntypes: BMNH 1863.12.4.71 (1), possibly NMV 46011 (1). Unjustified emendation, or incorrect subsequent spelling, for Bagroides melapterus Bleeker, 1851.

Distribution: Sumatra and Borneo (Roberts, 1989a).

\section{BAGRUS Bosc, 1816}

Porcus Geoffroy Saint-Hilaire, 1809: pl. 15. Type species: Silurus bajad Forsskål, 1775. Type by monotypy. Gender: Masculine. Name placed on Official Index of Rejected and Invalid Generic Names In Zoology (ICZN, Opinion 1402).

Bagrus Bosc, 1816: 147. Type species: Silurus bajad Forsskål, 1775. Type by subsequent designation by Bailey \& Stewart (1983: 168). Gender: Masculine. Placed on Official List (ICZN, Opinion 1402).

Remarks: The names Porcus and Bagrus were each proposed as new independently in several publications; see Bailey \& Stewart (1983) for details. See also Jayaram (1956a) for additional comments on the names. An earlier type designation of Bagrus may date to Desmarest as Bagrus bayad (see Whitley, 1939: 224), but that action may have been overridden by ICZN Opinion 1402, which listed Silurus bajad Forsskål as type.

Key (as genus Porcus): Jayaram (1966b).

\section{Bagrus bajad (Forsskål, 1775)}

Silurus bajad Forsskål, 1775: 66, xiii. Type locality: in Nilo. No types known. Placed on Official List (ICZN, Opinion 1402) as type species of Bagrus.

Porcus bayad Geoffroy Saint-Hilaire, 1809: pl. 15 (figs. 1-2). Type locality: Nil. Misspelling or unjustified emendation of Silurus bajad Forsskål.

Bagrus bayad macropterus Pfaff, 1933: 287, fig. 4. Type locality: Kabara. Holotype (47 cm): ZMUC P 28667. Orig- 
inally as Bagrus bayad var. macropterus var. $\mathrm{n}$.

Distribution: Lakes Chad, Mobutu and Turkana; Nile, Niger, and Senegal rivers (Risch, 1986).

Bagrus caeruleus Roberts \& Stewart, 1976

Bagrus caeruleus Roberts \& Stewart, 1976: 275. Type locality: Near Inga hydroelectric dam. Lat. 5³1.5'S, Long. $13^{\circ} 37.5^{\prime} \mathrm{E}$; lower Zaïre or Congo River. Holotype: MCZ 50582.

Distribution: Lower Congo River; known only from type series (Risch, 1986).

Bagrus degeni Boulenger, 1906

Bagrus Degeni Boulenger, 1906b: 438. Type locality: Entebbe, Lake Victoria. Syntypes (7): BMNH 1906.5.30.172 (1), BMNH 1906.5.30.173-177 (5).

Distribution: Lake Victoria (Risch, 1986).

Bagrus docmak (Forsskål, 1775)

Silurus docmak Forsskål, 1775: 65, xiii. Type locality: in Nilo inferiori ad Deltam. No types known.

Bagrus koenigi Pietschmann, 1932: 92, fig. 1. Type locality: In Oberägypten im Nil. Holotype: at ZFMK.

Bagrus docmac niger Daget, 1954: 244, fig. 89. Type locality: Bamako [Niger River]. Syntypes: MNHN 1954-0004 (1), MNHN 1960-0465 (2), MNHN 1960-0466 (1).

Distribution: Nile, Niger, Senegal and Volta rivers; Lack Chad basin, East African rift lakes, except Lake Kivu (Risch, 1986).

Bagrus filamentosus Pellegrin, 1924

Bagrus filamentosus Pellegrin, 1924a: 462. Type locality: Cercle de Ségou (du Niger). Holotype: MNHN 19240215.

Distribution: Niger River system (Risch, 1986).

Bagrus lubosicus Lönnberg, 1924

Bagrus lubosicus Lönnberg, 1924: 619. Type locality: Lubosi river, a tributary to Luala River, Lower Congo. Holotype: NRM 9939.

Distribution: Lower Congo River system (Risch, 1986).

Bagrus meridionalis Günther, 1894

Bagrus meridionalis Günther, 1894: 626. Type locality: Upper Shire River [British Central Africa]. Holotype (22 1/2 inches): BMNH 1893.11.15.87 (skin).

Distribution: Shire River and Lake Malawi (Risch, 1986).

Bagrus orientalis Boulenger, 1902

Bagrus orientalis Boulenger, 1902d: 37. Type locality: du Pangani, Côte de Zanzibar. Syntypes (2): BMNH 1865.9.21.17 (1), BMNH 1867.3.7.488 (1). Name made available in key.

Distribution: Pangani River, Tanzania (Risch, 1986).

$\dagger$ Bagrus shuwaiensis Forey \& Young, 1999

$\dagger$ Bagrus shuwaiensis Forey \& Young, 1999: 125, figs. 12.6-12.16. Type locality: Late Miocene, Bahnunah Formations, west side of Shuwaihat, Abu Dhabi. Holotype: AUH 678 (posterior portion of cranium).

Distribution: Bahnunah Formations, west side of Shuwaihat, Abu Dhabi.

Remarks: Considered to be Bagridae S.L. by Gayet \& Meunier (2003).

Bagrus ubangensis Boulenger, 1902

Bagrus ubangensis Boulenger, 1902d: 38, pl. 11 (fig. 1). Type locality: l'Ubangi à Banzyville. Holotype: MRAC 1295.

Distribution: Congo River system (Risch, 1986).

Bagrus urostigma Vinciguerra, 1895

Bagrus urostigma Vinciguerra, 1895: 36. Type locality: Ganana, Giuba [Somali]. Syntypes (4): MSNG 14598 (1, dry), MSNG 14599 (1), MSNG 14600 (1).

Distribution: Giuba River, Somalia (Risch, 1986).

BATASIO Blyth, 1860

Batasio Blyth, 1860: 149. Type species: Batasio buchanani Blyth, 1860. Type by original designation. Type designated as "B. Buchanani, nobis; Pimelodus batasio, B.H." Gender: Masculine. 
Macronoides Hora, 1921a: 179. Type species: Possibly Batasio affinis Blyth, 1860. Type by subsequent designation by Jordan (1923: 148). Gender: Masculine. Originally proposed as a subgenus of Macrones.

Revision: Hora \& Law (1941).

Review: Ng \& Kottelat (2001, Indochinese species)

Remarks: Systematic position reviewed in Tilak (1967) and Jayaram (1977b). Type species for Macronoides taken from Misra (1976: 215) although no type designation has been found; type species was not designated in Hora \& Law (1941).

Batasio affinis Blyth, 1860

Batasio affinis Blyth, 1860: 150. Type locality: Tenasserim [Myanmar]. Holotype: ZSI F7880/1 [? = A.549].

Macrones Blythii Day, 1877 (in Day, 1875-78): 445. Type locality: Tenasserim provinces. Holotype: ZSI F7880/1 [? = A.549]. Proposed as a replacement for Batasio affinis Blyth, 1860 when thought to be preoccupied in Macrones by Bagrus affinis Jerdon, 1849 [see Ng \& Kottelat (2001: 500) for discussion].

? Leiocassis fluviatilis Day, 1888: 805. Type locality: The Anin, a stream rising near Weywoon, Wagroo, in the Tenasserim district [Myanmar]. Syntypes (4): Whereabouts unknown.

Distribution: Eastern Myanmar (Ng \& Kottelat, 2001).

Batasio batasio (Hamilton, 1822)

Pimelodus batasio Hamilton, 1822: 179, 377. Type locality: River Tista [Ganges River]. No types known. Previously unpublished Hamilton illustration of a type specimen reproduced in Hora (1929: pl. 22, fig. 3).

Batasio Buchanani Blyth, 1860: 150. Type locality: River Tista [Ganges River]. No types known. Unneeded replacement name for Pimelodus batasio Hamilton, 1822.

Distribution: Ganges River basin, India.

Remarks: Hamilton's plate (pl. 23, fig. 60), labeled Pimelodus batasius and referred to in the account of Pimelodus batasio, has been shown by Day (1871) and Hora (1929: 182) to fit instead the description of Pimelodus carcio Hamilton.

Batasio dayi (Vinciguerra, 1890)

Macrones Dayi Vinciguerra, 1890: 230, pl. 7 (fig. 3). Type locality: Meetan; M. ${ }^{\text {ti }}$ a Est di Toungoo (Carin Biapò), Birmania [Myanmar]. Syntypes (6): BMNH 1893.2.16.8 (1), MSNG 14586 (1), NMW 45184 (1), RMNH 10225 (1).

Distribution: Salween and Irrawaddy River basins, Myanmar (Ng \& Kottelat, 2001).

Batasio elongatus $\mathrm{Ng}, 2004$

Batasio elongatus Ng, 2004c: 68, fig. 1. Type locality: Myanmar, Rakhine state: Baw Di Chaung, drainage, Baw Di Chaung, at Baw Di bridge, 20 miles from Gwa on road to Ngathaingchaung (17 $\left.34^{\prime} 15^{\prime \prime} \mathrm{N} 94^{\circ} 43^{\prime} 47^{\prime \prime E}\right)$. Holotype: NRM 40748.

Distribution: Baw Di Chaung and Kanamae Chaung basins, western Rakihine Yoma, Myanmar (Ng, 2004c).

Batasio havmolleri (Smith, 1931)

Mystus havmolleri Smith, 1931: 24, fig. 12. Type locality: Klong Thalerng near Ronpibun, Peninsular Siam. Holotype: USNM 90304.

Mystus stigmaturus Fowler, 1934a: 94, figs. 41-42. Type locality: Nakon Sritamarat, Peninsular Siam. Holotype: ANSP 59338.

Distribution: Malay Peninsula, south of isthmus of Kra and north of Perak River basin and Terengganu River (Ng \& Kottelat, 2001).

Batasio macronotus Ng \& Edds, 2004

Batasio macronotus Ng \& Edds, 2004: 296, fig. 1. Type locality: Nepal: Saptari/Sunsari, Kosi River drainage, purchased at Kosi barrage, 26³1'30"N 8656'00"E. Holotype: KU 34546.

Distribution: Kosi River basin, eastern Nepal (Ng \& Edds, 2004).

Batasio merianiensis (Chaudhuri, 1913)

Macrones merianiensis Chaudhuri, 1913: 253, pl. 9 (figs. 1, 1a, 1b). Type locality: Pond at Mariani junction, Assam [India]. Holotype: ZSI F7781/1.

Distribution: Assam, India.

Batasio pakistanicus Mirza \& Jan, 1989 
Batasio pakistanicus Mirza \& Jan, 1989: 283, figs. 1, 2. Type locality: Jinnah Barrage (River Indus) [Pakistan]. Holotype: GCM 17-F.

Distribution: Punjab, Indus River basin, Pakistan (Mirza, 2003).

Remarks: Treated as a synonym of Batasio batasio by Jayaram (1999).

Batasio sharavatiensis Bhatt \& Jayaram, 2004

Batasio sharavatiensis Bhatt \& Jayaram, 2004: 1339. Type locality: River Sharavati $\left(14^{\circ} 14^{\prime} \mathrm{N}, 7^{\circ} 49^{\prime} \mathrm{E}\right)$, Joginmatha, Uttara Kannada, Karnataka [India]. Holotype: ZSI/SRS F 6419.

Distribution: Sharavati River, Uttara Kannada, Karnataka State, India (Bhatt \& Jayaram, 2004).

Batasio tengana (Hamilton, 1822)

Pimelodus tengana Hamilton, 1822: 176, 377, pl. 39 (fig. 58). Type locality: Brahmaputra River. No types known.

Distribution: Brahmaputra River, India and Bangladesh.

Batasio tigrinus $\mathrm{Ng} \&$ Kottelat, 2001

Batasio tigrinus Ng \& Kottelat, 2001: 504, fig. 6. Type locality: Thailand: Kanchanaburi Province, Mae Nam Khwae Noi basin, Huai Lia, km 49 on road from Thon Pha Phun to Sangkhla (1504'25"N, 98 33'51"E). Holotype: ZRC 40624.

Distribution: Mae Nam Khwae Noi basin, Mae Khlong drainage, western Thailand (Ng \& Kottelat, 2001).

Batasio travancoria Hora \& Law, 1941

Batasio travancoria Hora \& Law, 1941: 40, pl. 2 (figs. 7-9); fig. 3. Type locality: Peruntenaruvi, a tributary of the Pamba River at Edakadathy [Travancore, India]. Holotype: ZSI F13449/1.

Distribution: Pamba, Kallada and Chittar River basins, Anaimalai Hill Range, Kerala, India (Jayaram, 1999).

\section{CHANDRAMARA Jayaram, 1973}

Chandramara Jayaram, 1973a: 816. Type species: Pimelodus chandramara Hamilton, 1822. Type by original designation. Gender: Masculine.

Chandramara chandramara (Hamilton, 1822)

Pimelodus chandramara Hamilton, 1822: 162, 375. Type locality: Atreyi River [India]. No types known. Previously unpublished Hamilton illustration of a type specimen reproduced in Hora (1929: pl. 21, fig. 6).

Ageniosus (Silonia) diaphina Swainson 1839: 306. Type locality: Atreyi River [India]. No types known. On "Ham. P. 162" [= Hamilton (1822: 162)]. Unneeded new name for Pimelodus chandramara Hamilton, 1822.

Distribution: Base of eastern part of Himalaya, and Sarda River basin (Tilak, 1987).

Remarks: Also treated in recent literature as valid as Rama chandramara.

COREOBAGRUS Mori, 1936

Coreobagrus Mori, 1936: 672, 675. Type species: Coreobagrus brevicorpus Mori, 1936. Type by monotypy. Gender: Masculine.

Remarks: Species diagnosed in Watanabe et al. (1992).

Coreobagrus brevicorpus Mori, 1936

Coreobagrus brevicorpus Mori, 1936: 672, pl. 24 (fig. 2). Type locality: River Rakuto at Ei-yo [but, Bun-kei elsewhere in text], Chosen [Korea]. Holotype: At Preparatory Department of Keijo Imperial University.

Distribution: Naktong River and Kim River, Korea (Mori, 1952).

Remarks: Treated in some recent literature as valid in Pseudobagrus.

Coreobagrus ichikawai Okada \& Kubota, 1957

Coreobagrus ichikawai Okada \& Kubota, 1957: 144, unnumbered figure. Type locality: Miya River, Mie Prefecture, Japan. Holotype: FRLM 6230.

Coreobagrus okadai Jayaram, 1966a: 30, fig. 1. Type locality: Upper region of Suzuka River, Mie Prefecture, Honshu, Japan. Holotype: at ZSI.

Distribution: In the vicinity of Ise and Mikawa bays, Honshu, Japan (Katsuura et al., 2000).

Remarks: Treated in some recent literature as valid as Pseudobagrus ichikawai. See Watanabe (1998) for intraspecific variation, and Watanabe \& Ito (1999) for comments on distribution. 
$\dagger$ EOMACRONES White, 1937

$\dagger$ Macronoides White, 1934: 54. Type species: $†$ Macronoides wilsoni White, 1934. Type by original designation. Gender: Masculine. Preoccupied by Macronoides Hora, 1921, in Recent fishes. Replaced by $†$ Eomacrones White, 1937.

$\dagger$ Eomacrones White, 1937: 144. Type species: $†$ Macronoides wilsoni White, 1934. Type by being a replacement name. Gender: Masculine. Replacement for $\dagger$ Macronoides White, 1934, preoccupied by Macronoides Hora, 1921.

Remarks: Considered to be Bagridae (sensu lato) by Gayet \& Meunier (2003).

$\dagger$ Eomacrones wilsoni (White, 1934)

$\dagger$ Macronoides wilsoni White, 1934: 54, fig. 13. Type locality: Gada, Nigeria, in scree; Eocene. Holotype: at National Collection [Nigeria] or BMNH (incomplete skull).

$\dagger$ GOBIBAGRUS Sytchevskaya, 1986

$\dagger$ Gobibagrus Sytchevskaya, 1986: 90. Type species: †Gobibagrus hoerdzanicus Sytchevskaya, 1986. Type by original designation. Gender: Masculine.

$\dagger$ Eobagrus Sytchevskaya, 1986: 92. Type species: $†$ Gobibagrus hoerdzanicus Sytchevskaya, 1986. Type by original designation. Gender: Masculine. Apparently a lapsus calami for Gobibagrus.

Remarks: Type species cited as †Gobibagrus hoerdzanicus, but species account lists name as $\dagger$ Eobagrus hoerdzanicus. Assigned to the family Bagridae by Sytchevskaya, 1986.

$\dagger$ Gobibagrus hoerdzanicus Sytchevskaya, 1986

$\dagger$ Eobagrus hoerdzanicus Sytchevskaya, 1986: 92, pl. 15, figs. 6-10. Type locality: [Mongolia, Lower Oligocene]. Holotype: ПИН N 3874/408 (pectoral spine)

Distribution: Mongolia, upper Miocene (Gayet \& Meunier, 2003: 501).

Remarks: Name as $\dagger$ Eobagrus hoerdzanicus in heading of species account, but $\dagger$ Gobibagrus hoerdzanicus on plate caption and as type species in genus account.

\section{HEMIBAGRUS Bleeker, 1862}

Hemibagrus Bleeker, 1862 (in Bleeker, 1862-63): 9. Type species: Bagrus nemurus Valenciennes, 1840. Type by original designation. Gender: Masculine. Placed on Official List (Opinion 2011; ICZN, 2002).

Macropterobagrus Nichols, 1925: 1. Type species: Hemibagrus macropterus Bleeker, 1870. Type by monotypy. Gender: Masculine. Originally proposed as a subgenus of Hemibagrus.

Brachymystus Fowler, 1937: 148. Type species: Bagrus nemurus Valenciennes, 1840. Type by original designation. Gender: Masculine. Originally proposed as a subgenus of Mystus.

Review: Ng \& Rainboth (1999, central Indochina); Ng \& Ferraris (2000, southern Asia).

Hemibagrus baramensis (Regan, 1906)

Macrones baramensis Regan, 1906b: 68. Type locality: Baram River, Sarawak state, Borneo, East Malaysia. Holotype: BMNH 1895.7.2.50.

Distribution: Baram River, Borneo (Regan, 1906b).

Hemibagrus bongan (Popta, 1904)

Macrones bongan Popta, 1904: 182. Type locality: le Bongan, Bornéo central. Syntypes: RMNH 7548 (1), RMNH 7549 (1); described in more detail, with an illustration of one of the syntypes, in Popta (1906: 35, pl. 2, fig. 5).

Distribution: Kapuas, Rajang and Baram River drainages, northern Borneo (Ng \& Rachmatika, 1999).

Hemibagrus caveatus Ng, Wirjoatmodjo \& Hadiaty, 2001

Hemibagrus caveatus Ng, Wirjoatmodjo \& Hadiaty, 2001b: 359, fig. 1. Type locality: Sumatra: Aceh Province, Sungai Soraya, a tributary of Sungai Alas [Indonesia]. Holotype: MZB 8714.

Distribution: Alas River basin, northern Sumatra (Ng, et al., 2001b).

Hemibagrus centralus Mai, 1978

Hemibagrus centralus Mai, 1978: 253, fig. 115 [not 116]. Type locality: Northern Vietnam. Syntypes: at DVZUT. Distribution: Northern Vietnam (Kottelat, 2001b).

Hemibagrus chrysops Ng \& Dodson, 1999 
Hemibagrus chrysops Ng \& Dodson, 1999: 48, figs. 1, 2a, 3c-d. Type locality: Borneo: Sarawak, Serian wet market, from Sadong River. Holotype: ZRC 42653.

Distribution: Sadong and Rajang River basins, northern Borneo (Ng \& Dodson, 1999).

Hemibagrus filamentus (Fang \& Chaux, 1949)

Macrones (Hemibagrus) filamentus Fang \& Chaux, in Chaux \& Fang, 1949a: 200, fig. 4. Type locality: Cambodge. Holotype: MNHN 1966-0728.

Distribution: Middle and lower Mekong River basin of Laos, Thailand, and Cambodia (Ng \& Rainboth, 1999).

Hemibagrus fortis (Popta, 1904)

Macrones fortis Popta, 1904: 185. Type locality: le Bo, Bornéo central. Syntypes: RMNH 7551 (4); described in more detail, with an illustration of one of the syntypes, in Popta (1906: 44, pl. 2, figs. 8a, 8b).

Macrones bo Popta, 1904: 183. Type locality: le Bo, Bornéo, central. Holotype: RMNH 7549; described in more detail, with illustration of holotype, in Popta (1906: 38, pl. 2, fig. 6).

Macrones howong Popta, 1904: 181. Type locality: le Howong, Bornéo central. Holotype: RMNH 7547; described in more detail, with illusration of holotype, in Popta (1906: 32, pl. 1, fig. 4).

Macrones kajan Popta, 1904: 184. Type locality: le Kajan, Bornéo central. Holotype: RMNH 7550; described in more detail, with illustration of holotype, in Popta (1906: 41, pl. 2, fig. 7). [Possibly synonym of Hemibagrus nemurus].

Macrones fortis capitulum Popta, 1906: 48, pl. 3 (fig. 9). Type locality: le Bo, Bornéo central. Holotype: RMNH 7552. Originally as Macrones fortis var. capitulum. [First mentioned in Popta (1904: 186) as Macrones fortis var.]

Distribution: Borneo.

Remarks: Tan \& Ng (2000: 271) acted as first reviser and gave precedence to Macrones fortis over M. howong, M. kajan and $M$. bo.

Hemibagrus furcatus $\mathrm{Ng}$, Martin-Smith \& $\mathrm{Ng}, 2000$

Hemibagrus furcatus $\mathrm{Ng}$, Martin-Smith \& Ng, 2000: 66, figs. 1, 2a, 3. Type locality: Sungai Segama, Danum Valley Conservation Area, Lahad Datu dist., Sabah, Borneo. Holotype: SBM uncataloged.

Distribution: Segama River basin, eastern Borneo (Ng, et al., 2000).

Hemibagrus gracilis $\mathrm{Ng} \& \mathrm{Ng}, 1995$

Hemibagrus gracilis Ng \& Ng, 1995: 136, figs. 1-3, 4a. Type locality: Peninsular Malaysia: Johor, Ulu Endau, Sungei Jasin. Holotype: ZRC 21484.

Distribution: Upper reaches of Sungei Endau and Sungei Kinchin, Endau-Rompin region, Peninsular Malaysia (Ng $\& \mathrm{Ng}, 1995)$.

Hemibagrus guttatus (La Cepède, 1803)

Pimelodus guttatus La Cepède, 1803: 96, 111, pl. 5 (fig. 1). Type locality: [China]. No types known; description based on illustration [reproduced in Kottelat et al. (1994)].

Macrones elongatus Günther, 1864: 77. Type locality: Singapore [probably in error, possibly China; see Kottelat et al. (1998: 567)]. Holotype: BMNH 1855.9.19.1099; illustrated in Jayaram (1978b: 223, fig. 2, and Kottelat et al. (1998: 567, fig. 1).

Macrones chinensis Steindachner, 1883: 196. Type locality: Kanton, China. Holotype: at NMW. Species illustrated and described in more detail in Steindachner (1884: 1111, pl. 8). Holotype also illustrated in Jayaram (1978b: 223, fig. 3).

Distribution: Qiantangjiang, Jiulongjiang, Hanjiang, Zhujiang, Yuanjiang, China (Chu et al., 1999); Nam Xam and Nam Ma Basins, Laos, and Red River (Kottelat, 2001b).

Remarks: Synonymy follows Kottelat et al. (1998).

Hemibagrus hainanensis (Tchang, 1935)

Leiocassis hainanensis Tchang, 1935b: 175, fig. 2. Type locality: Hainan I., China. Holotype: ASIZB [= ZMFMIB 12904].

Distribution: Hainan Island, China.

Hemibagrus hoevenii (Bleeker, 1846)

Bagrus Hoevenii Bleeker, 1846a: 154. Type locality: Batavia [now Sungai Muar at Kampung Bukit Kebong, Johor, 
Malaysia, by neotype designation]. Neotype: ZRC 37472, proposed in Kottelat et al. (1994: 332) and designated by ICZN (1996; Opinion 1840). Neotype illustrated by Kottelat \& Lim (1995: fig. 1).

Distribution: Kapuas and Baram River basins, Borneo, Musi and Batang Hari River basins, Sumatra, and Johor, Paahang, and Muar River basins, western Malaysia (Kottelat \& Lim, 1995).

Remarks: Redescribed in Kottelat \& Lim (1995).

Hemibagrus hongus Mai, 1978

Hemibagrus elongatus hongus Mai, 1978: 252. Type locality: [Hông River and Lam River] Vietnam. Syntypes: at DVZUT.

Distribution: Northern Vietnam (Kottelat, 2001a).

Hemibagrus imbrifer Ng \& Ferraris, 2000

Hemibagrus imbrifer Ng \& Ferraris, 2000: 126, fig. 1. Type locality: Mae Nam Moei at Ban Wan Le, 16²17'24"N, 9842'21"E, Salween basin, Tak Prov., Thailand. Holotype: ZRC 45406.

Distribution: Salween River basin (Ng \& Ferraris, 2000).

Hemibagrus johorensis (Herre, 1940)

? Macrones bleekeri Volz, 1903a: 556. Type locality: Brackish water at Banjuasin, Palembang, Sumatra. Holotype: NMBE 1020756. Preoccupied by Macrones bleekeri Day, 1877. Species illustrated and described in more detail in Volz (1903b: 387, pl. 26 [not 25 as stated], fig. 3).

Mystus johorensis Herre, 1940: 13, pls. 7-8. Type locality: Sungai Kayu, 16 miles north of Kota Tinggi, Johore [Malaysia]. Holotype: SU 33026.

Distribution: Peninsular Malaysia and Sumatra.

Hemibagrus macropterus Bleeker, 1870

Hemibagrus macropterus Bleeker, 1870: 257, unnumbered pl. (bottom fig.). Type locality: [Yangtze River, China]. Holotype: Whereabouts unknown. Often placed in Mystus in Chinese literature.

Distribution: From Changjiang [Yangtze River] to Zhujiang [=Pearl River], China (Chu et al., 1999).

$\dagger$ Hemibagrus major Roberts \& Jumnongthai, 1999

$\dagger$ Hemibagrus major Roberts \& Jumnongthai, 1999: 168, fig. 14 (tooth only). Type locality: Thailand, North-central, Phetchabun Province, Ban Nong Pla; Miocene. Holotype: Department of Natural Resources, Thailand (DNR) TF 5011 (incomplete specimen).

Distribution: North-central, Phetchabun Province, Ban Nong Pla, Thailand; Miocene; known only from type.

Hemibagrus maydelli (Rössel, 1964)

Mystus (Mystus) maydelli Rössel, 1964: 149, fig. 1. Type locality: Indien, Wadgaon, Bhima-Fluss. Holotype: ZMH H 2180.

Mystus krishnensis Ramakrishniah, 1988: 139, figs. 1-2. Type locality: Nagarjunasagar reservoir, India. Holotype: ZSI FF2532.

Distribution: Middle reaches of Krishna River, peninsular India (Ng \& Ferraris, 2000).

Hemibagrus menoda (Hamilton, 1822)

Pimelodus menoda Hamilton, 1822: 203, 379.Type locality: Kosi, Mahananda, and other rivers in north of Behar and Bengal [now: Bangladesh, Suarma (Meghna) drainage, Sharighat bazaar, 22 miles NE of Sylhet on SylhetShillong hwy, by neotype designation]. Neotype: UMMZ 208726, illustrated in, and designated by, Ng \& Ferraris (2000:136, fig. 5). Plate 1 of Hamilton (1822), labelled Mugil corsula, and replaced by Pimelodus in corrigenda, appears to refer to this species.

Bagrus corsula Valenciennes, in Cuvier \& Valenciennes, 1840a: 408 (302 in Strasbourg deluxe edition). Type locality: Bengale [now: Bangladesh, Suarma (Meghna) drainage, Sharighat bazaar, 22 miles NE of Sylhet on SylhetShillong hwy by neotype designation]. Neotype: UMMZ 208726, illustrated in, and designated by, Ng \& Ferraris (2000:132, fig. 5). Based on illustration in Hamilton (1822: pl. 1) originally labelled as Mugil corsula, but changed in corrigenda to Pimelodus, which apparently was interpreted by Valenciennes to mean Pimelodus corsula. However, there in no justification for ascribing the name P. corsula to Hamilton, so its first use is that of Valenciennes.

Bagrus trachacanthus Valenciennes, in Cuvier \& Valenciennes, 1840a: 419 (311 of Strasbourg deluxe edition). Type locality: Bengale. Syntype: MNHN 0000-1191 (1). 
Macrones corsula Day, 1869: 307. Type locality: Mahanuddi River at Cuttack, India. Syntypes (3): Whereabouts unknown. Apparently intended as a new name, based in part on Hamilton (1822: pl. 1, fig. 72).

Distribution: Ganges, Brahmaputra, Mahanadi and Godavari River basins, Bangladesh and India (Ng \& Ferraris, 2000).

Hemibagrus microphthalmus (Day, 1877)

Macrones microphthalmus Day, 1877 (in Day, 1875-78): 446, pl. 100 (fig. 4). Type locality: Burma, along the valley of the Irrawaddi. Possible syntypes: AMS B.7918 (1), ZSI 2592 (1).

Distribution: Salween River, Thailand; Irrawaddy and Sittang Rivers, Myanmar; and Manipur River, India (Ng \& Ferraris, 2000).

Remarks: Roberts \& Jumnongthai (1999: 176) and Ng \& Rainboth (1999: 576) cite ZSI 2592 as the holotype of the species, although Day indicated that multiple specimens were examined.

Hemibagrus nemurus (Valenciennes, 1840)

Bagrus nemurus Valenciennes, in Cuvier \& Valenciennes, 1840a: 423 (314 of Strasbourg deluxe edition). Type locality: Java. Holotype: Possibly RMNH 269 (not found) or at MNHN. Placed on Official List (Opinion 2011) (ICZN, 2002).

Bagrus Sieboldii Bleeker, 1846a: 155. Type locality: Batavia. Syntypes (size and number not stated): RMNH 15881 (5), SMNS 10572 (1). Placed on Official List (Opinion 2011) (ICZN, 2002).

Mystus pahangensis Herre, 1940: 14, pl. 9. Type locality: Sungai Garam, near Karak, Pahang [Malaysia]. Holotype: SU 33025.

Distribution: Java and likely in river basins of neighboring regions.

Remarks: This name has been applied to populations that have been subsequently determined to belong to distinct species, but the geographic limits of Hemibagrus nemurus have not been clearly stated. Ng \& Rainboth (1999: 560) treat the name Macrones luridus in Tirant (1911) as a nomen nudum and possibly intended for this species.

Hemibagrus olyroides (Roberts, 1989)

Mystus olyroides Roberts, 1989a: 124, fig. 95. Type locality: Sintang Market (reportedly caught in Sungai Kebian), Western Borneo (Kalimantan Barat, Indonesia). Holotype: MZB 3625.

Distribution: Kapuas River, western Borneo (Roberts, 1989a).

Hemibagrus peguensis (Boulenger, 1894)

Macrones peguensis Boulenger, 1894b: 196. Type locality: Sittang River near Toungoo [Burma]. Syntypes: BMNH 1894.5.21.25-26 (2).

Distribution: Irrawaddy, Sittang and Pegu River basins, Myanmar (Ng \& Ferraris, 2000).

Hemibagrus planiceps (Valenciennes, 1840)

Bagrus planiceps Valenciennes, in Cuvier \& Valenciennes, 1840a: 421 (311 of Strasbourg deluxe edition). Type locality: Java. Lectotype: RMNH 2939, designated by Ng et al. (1999: 38). Roberts (1993: 30) citation of MNHN b-0615 as holotype is incorrect (M. Kottelat, pers. comm.). Placed on Official List (Opinion 2011) (ICZN, 2002).

Bagrus anisurus Valenciennes, in Cuvier \& Valenciennes, 1840a: 422 (313 of Strasbourg deluxe edition). Type locality: Java. Lectotype: RMNH 2956; designated by Kottelat (1999: 272). Previously unpublished illustration of lectotype reproduced in Roberts (1993: 30, fig. 66), but incorrectly listing the specimen as holotype (M. Kottelat, pers. comm.).

Bagrus flavus Bleeker, 1846a: 156. Type locality: Batavia. Holotype or syntype (size and number not stated): RMNH 15897 (1 of 4), SMNS 10570 (1). Placed on Official List (Opinion 2011) (ICZN, 2002).

Distribution: Java.

Remarks: Bleeker (1858e: 417) acted as first reviser in giving precedence to Hemibagrus planiceps over Bagrus anisurus.

Hemibagrus pluriradiatus (Vaillant, 1892)

Macrones pluriradiatus Vaillant, 1892a: 126. Type locality: Tonkin. Holotype: MNHN 1892-0048; illustrated in Vaillant (1904: 462, pl. 23, fig. 2) and Jayaram (1978b: 223, fig. 1).

Distribution: Yuianjiang, Hainan Island, China (Chu et al., 1999); Nam Xam basin, Laos, Red River, Viet Nam and China (Kottelat, 2001a, b). 
Remarks: Also treated as valid in Mystus in some recent literature.

Hemibagrus punctatus (Jerdon, 1849)

Bagrus punctatus Jerdon, 1849: 339. Type locality: Cavery and its principal tributaries, southern India. No types known.

Distribution: Cauvery River basin to Bhavani River in Tamil Nada; also rivers in Mysore, Western Ghats and adjoining hills, peninsular India (Jayaram \& Anuradha, 2003).

Hemibagrus sabanus (Inger \& Chin, 1959)

Mystus sabanus Inger \& Chin, 1959: 294. Type locality: Kinabatangan River at Deramakot, Kinabatangan District, North Borneo. Holotype: FMNH 68088.

Distribution: Kinabatangan River, Borneo, Malaysia (Inger \& Chin, 1959).

Hemibagrus spilopterus $\mathrm{Ng} \&$ Rainboth, 1999

Hemibagrus spilopterus Ng \& Rainboth, 1999: 562, figs. 5, 7a, 8a. Type locality: Cambodia: Kandal, Bassac River at village of Prek Chey on Vietnamese border (1057'N, 10506'E). Holotype: UMMZ 232611.

Distribution: Lower Mekong River basin, Cambodia (Ng \& Rainboth, 1999).

Hemibagrus variegatus $\mathrm{Ng} \&$ Ferraris, 2000

Hemibagrus variegatus Ng \& Ferraris, 2000: 139, fig. 12. Type locality: Tenasserim River, Myanmar. Holotype: BMNH 1992.11.16.11.

Distribution: Tenasserim River basin, Myanmar (Ng \& Ferraris, 2000).

Hemibagrus velox Tan \& Ng, 2000

Hemibagrus velox Tan \& Ng, 2000: 272, figs. 2-4. Type locality: Sumatra Barat: Sungai Dareh, Pulau Punjung market, Sumatra. Holotype: MZB 9305.

Distribution: Rivers of central Sumatra (Tan \& Ng, 2000).

Hemibagrus vietnamicus Mai, 1978

Hemibagrus vietnamicus Mai, 1978: 252, fig. 116 [not 115]. Type locality: [Mountain streams in North Vietnam]. Syntypes: at DVZUT. Spelled Hemibagrus vietnammicus in index (p. 337). Eschmeyer et al. (1998: 1760) acted as first reviser in selecting vietnamicus as the correct spelling.

Distribution: Nam Ma and Nam Xam, Laos, and Northern Vietnam (Kottelat, 2001b).

Hemibagrus wyckii (Bleeker, 1858)

Bagrus Wyckii Bleeker, 1858b: 150, 156. Type locality: Java, in flumine Tjitarum, provinciae Preanger, prope vicum Parongkalong. Syntypes (2: 320-440 mm TL): BMNH 1863.12.4.88 (1, 247 mm SL, 306 mm TL), RMNH 6897 (1). Illustrated in Bleeker (1862-63: 57, pl. 72 [= Silur. pl. 24]).

Distribution: Mekong and Chao Phraya River basins, and Sundaland (Kottelat, 2001b).

Hemibagrus wyckioides (Fang \& Chaux, 1949)

Macrones (Hemibagrus) Wyckioides Fang \& Chaux, in Chaux \& Fang, 1949a: 199, fig. 3. Type locality: Cambodge. Holotype: MNHN 1966-0727.

Mystus aubentoni Desoutter 1975: 449, figs. 2-3. Type locality: Stung Treng [Cambodia.] Holotype: MNHN 19740039.

Distribution: Mekong River basin, Cambodia, Laos, Thailand and China (Kottelat, 2001b).

\section{Species inquirenda, Hemibagrus}

Aoria Amemiyai Kimura, 1934: 166, pl. 5 (fig. 2). Type locality: Howchwan, Szechwan Province, China. Holotype: at Laboratory of Biological Department of the Shanghai Science Institute, Shanghai. As Aoria amemiyae on pp. 12 and 166, Aoria amemiyai on plate 5; Kottelat et al. (1998: 570) act as first revisers in selecting amemiyai as valid. Treated as a possible synonym of Hemibagrus macropterus by Kottelat et al. (1998: 570).

HEMILEIOCASSIS Ng \& Lim, 2000

Hemileiocassis Ng \& Lim, 2000: 192. Type species: Hemileiocassis panjang Ng \& Lim, 2000. Type by original designation. Gender: Feminine.

Hemileiocassis panjang $\mathrm{Ng} \& \mathrm{Lim}, 2000$ 
Hemileiocassis panjang Ng \& Lim, 2000: 192, figs. 1, 2a, 3. Type locality: Indonesia: Java, Bogor. Holotype: ZMA 121529.

Distribution: Western Java (Ng \& Lim, 2000).

HYALOBAGRUS Ng \& Kottelat, 1998

Hyalobagrus Ng \& Kottelat, 1998a: 336. Type species: Pseudobagrus ornatus Duncker, 1904. Type by original designation. Gender: Masculine.

Hyalobagrus flavus $\mathrm{Ng} \&$ Kottelat, 1998

Hyalobagrus flavus Ng \& Kottelat, 1998a: 340, fig. 6. Type locality: Indonesia: Sumatra: Jambi: Danau Arang Arang in the vicinity of Muara Kompeh, 1³7'32.0"S, 10347'19.0"E. Holotype: MZB 9302.

Distribution: Batang Hari drainage, Sumatra and Mentaya River basin, southern Borneo (Ng \& Kottelat, 1998a).

Hyalobagrus leiacanthus $\mathrm{Ng} \&$ Kottelat, 1998

Hyalobagrus leiacanthus Ng \& Kottelat, 1998a: 341, fig. 9. Type locality: Indonesia: Borneo: Kalimantan Tengah: Sungai Barito at Muara Laung, 0³5'34.6"S, 11444'12.3"E. Holotype: MZB 9304.

Distribution: Kapuas and Barito River basins, central Borneo (Ng \& Kottelat, 1998a).

Hyalobagrus ornatus (Duncker, 1904)

Pseudobagrus ornatus Duncker, 1904: 173, pl. 2 (figs. 13, 13a-c). Type locality: Muar Fluß bei Tubing Tinggi, malayischen Halbinsel. Lectotype: ZMH H69, designated by Ladiges et al. (1958: 160); lectotype illustrated by Jayaram (1968b: pl. 1) and Ng \& Kottelat (1998a: fig. 10).

Distribution: Muar River drainage, southern Malay Peninsula (Ng \& Kottelat, 1998a).

LEIOCASSIS Bleeker, 1857

Leiocassis Bleeker, 1857b: 416. Type species: Bagrus micropogon Bleeker, 1852. Type by monotypy. Gender: Feminine.

Liocassis Günther, 1864: 86. Type species: Bagrus micropogon Bleeker, 1852. Type by monotypy. Gender: Feminine. Unjustified emendation of Leiocassis Bleeker, 1857.

Rhinobagrus Bleeker, 1865: 7. Type species: Rhinobagrus dumerili Bleeker, 1865. Type by monotypy. Gender: Masculine.

Nasocassis Nichols, 1925: 1. Type species: Liocassis longirostris Günther, 1864. Type by original designation. Originally proposed as a subgenus of Leiocassis. Gender: Feminine.

Dermocassis Nichols, 1925: 1. Type species: Bagrus ussuriensis Dybowski, 1872. Type by original designation. Originally proposed as a subgenus of Leiocassis. Gender: Feminine.

Leiocassis aculeatus $\mathrm{Ng} \&$ Hadiaty, 2005

Leiocassis aculeatus Ng \& Hadiaty, 2005: 84, fig. 1. Type locality: Sumatra: Aceh province: Sungai Soraya, tributary of Sungai Alas. Holotype: MZB 8715.

Distribution: Alas River basin, northern Sumatra Ng \& Hadiaty (2005).

Leiocassis crassilabris Günther, 1864

Liocassis crassilabris Günther, 1864: 88. Type locality: China. Holotype: BMNH 1864.7.9.9.

Leiocassis crassilabris macrops Nichols, 1926a: 2, fig. 2. Type locality: Min R., near Yenping (Nanping), Fukien Prov., China. Holotype: AMNH 8445.

Distribution: from Huanghe [= Yellow River] to Zhujiang [= Pearl River] (Chu et al., 1999).

Leiocassis crassirostris Regan, 1913

Liocassis crassirostris Regan, 1913b: 552. Type locality: Kiatiang-fu, Sze Chuen, China. Syntypes (2): BMNH 1891.6.13.23.

Distribution: Sichuan, China.

Leiocassis herzensteini (Berg, 1907)

Macrones herzensteini Berg, 1907: 421. Type locality: [Onon River mouth, Russia]. Syntypes: ZIN 7092.

Distribution: Onon River basin, Russia.

Leiocassis longibarbus Cui, 1990

Leiocassis longibarbus Cui, 1990: 153, fig. 156. Type locality: Kinsha River system, Yunnan, China. Holotype: KIZ 
818719.

Distribution: Jinsha River basin [= upper Yangtze River], China (Cui, 1990).

Leiocassis longirostris Günther, 1864

Liocassis longirostris Günther, 1864: 87, unnumbered figure. Type locality: Japan [possibly in error for China]. Holotype: BMNH 1862.11.1.1.

Rhinobagrus dumerili Bleeker, 1865: 7. Type locality: China. Holotype: Whereabouts unknown.

Distribution: from Yalujiang [= Yalu River] to Minjiang [Yangtze River tributary], China (Chu et al., 1999); Seoul, Pyongyang Kim River, Korea (Mori, 1952).

Remarks: Lee \& Kim (1990: 133) state that Günther's name appeared first; Rendahl (1927) apparently came to the opposite conclusion and treated Leiocassis dumerili as valid. Weber \& de Beaufort list the Bleeker paper as having been published in 1865, which is followed here, which results in Leiocassis longirostris as the valid name.

Leiocassis micropogon (Bleeker, 1852)

Bagrus micropogon Bleeker, 1852a: 94. Type locality: Blitong, in flumine Tjirutjup [Malaysia]. Holotype (79 mm TL): possibly in RMNH 6873 (2).

Leiocassis chaseni de Beaufort, 1933: 34. Type locality: Ulu Jelai, Pahang, Pahang basin, Malaysia. Holotype: ZRC 290.

Distribution: Malay Peninsula, Sumatra and Borneo (Roberts, 1989a).

Leiocassis poecilopterus (Valenciennes, 1840)

Bagrus poecilopterus Valenciennes, in Cuvier \& Valenciennes, 1840a: 431 (320 of Strasbourg deluxe edition). Type locality: rivière de Hèbak à Java. Holotype: RMNH 3004.

Leiocassis regani Jayaram, 1965a: 9. Type locality: North Borneo: Sadong. Holotype: USNM 35732.

Distribution: Java, Borneo and possibly elsewhere in Southeast Asia.

Leiocassis saravacensis Boulenger, 1894

Liocassis saravacensis Boulenger, 1894a: 246. Type locality: Senah, Sarawak state, Borneo, East Malaysia. Syntypes: BMNH 1893.3.6.168-169 (2).

Distribution: Borneo.

Leiocassis tenuifurcatus Nichols, 1931

Leiocassis tenuifurcatus Nichols, 1931: 1. Type locality: Chungan Hsien, northwestern Fukien Province, China. Holotype: AMNH 9681; holotype illustrated in Nichols (1943: 46, fig. 9).

Distribution: Fukien Province, China.

\section{Species inquirendae, Leiocassis}

Liocassis baramensis Regan, 1906b: 67. Type locality: Baram R., Sarawak state, Borneo, East Malaysia. Holotype: BMNH 1898.11.14.1. Treated in recent literature as a synonym of either Leiocassis micropogon or Leiocassis poecilopterus.

Liocassis doriae Regan, 1913b: 551. Type locality: Borneo. Holotype: BMNH 1868.1.28.30. Treated in recent literature as valid or as a synonym of Leiocassis micropogon or Leiocassis poecilopterus.

Liocassis Hosii Regan, 1906b: 67. Type locality: Sibu, Sarawak state, Borneo, East Malaysia. Syntypes (6): BMNH 1906.10.29.18-22 (5). Treated as a synonym of Leiocassis micropogon or Leiocassis poecilopterus.

Liocassis merabensis Regan, 1913b: 550. Type locality: Merabeh, North Borneo. Syntypes: BMNH 1893.3.6.170-171

(2). Treated as a synonym of Leiocassis micropogon or Leiocassis poecilopterus.

\section{MYSTUS Scopoli, 1777}

Mystus Scopoli, 1777: 451. Type species: Appeared first without species. Type widely listed as Bagrus halepensis

Valenciennes, 1840, with type by subsequent designation by Jordan \& Evermann (1917: 21) [see Remarks].

Gender: Masculine.

Aspidobagrus Bleeker, 1862 (in Bleeker, 1862-63): 9. Type species: Bagrus gulio [= Pimelodus gulio Hamilton, 1822]. Type by original designation. Gender: Masculine.

Hypselobagrus Bleeker, 1862 (in Bleeker, 1862-63): 10. Type species: Bagrus macronemus Bleeker, 1846. Type by 
original designation. Gender: Masculine.

Heterobagrus Bleeker, 1864b: 355. Type species: Heterobagrus bocourti Bleeker, 1864. Type by original designation. Gender: Masculine.

Prajadhipokia Fowler, 1934b: 338. Type species: Prajadhipokia rex Fowler, 1934. Type by original designation. Gender: Feminine.

Remarks: Heterobagrus is treated in some literature as valid with Prajadhipokia as a synonym. Roberts (1994: 243) indicated that Jordan \& Evermann's (1917: 21) selection of Bagrus halepensis as type of Mystus Gronovius (a name that was ruled to be unavailable in ICZN Opinion 261 (ICZN, 1954: 281) also applied to Mystus Scopoli. See Jayaram \& Anuradha (1984) for summary of nomenclatural history of Mystus.

Revision: Jayaram \& Anuradha (2003, senso lato, including Hemibagrus).

Review: Jayaram (1954, southern Asia); Roberts (1994, selected species, as Mystus sensu stricto).

Mystus alasensis Ng \& Hadiaty, 2005

Mystus alasensis Ng \& Hadiaty, 2005: 86, fig. 3. Type locality: Sumatra: Aceh province: Sungai Lembang, Gunung Leuser National Park. Holotype: MZB 8704.

Distribution: Alas River basin, northern Sumatra (Ng \& Hadiaty, 2005).

Mystus albolineatus Roberts, 1994

Mystus albolineatus Roberts, 1994: 245, fig. 1. Type locality: Prachinburi market, Bangpakong basin [Thailand]. Holotype: CAS 79030.

Distribution: Mekong, Bangpakong and Chao Phraya River basins (Roberts, 1994; Kottelat, 2001b).

Mystus armatus (Day, 1865)

Macrones armatus Day, 1865b: 289, fig. Type locality: Rivers and occasionally in backwaters, Cochin, India. Possible syntypes: BMNH 1865.7.17.21 (1), BMNH 1975.9.30.13 (1, dry), NMW 45165 (1), ZSI F1084 (1, lost).

Distribution: Malabar and Wayanad hills, Kerala and Manipur, India, and Mergui District, Myanmar (Jayaram \& Anuradha, 2003).

Mystus armiger $\mathrm{Ng}, 2004$

Mystus armiger Ng, 2004b: 883, fig. 1. Type locality: Malaysia: Kelantan, Kelantan River at Kampung Kuala Besar, $200 \mathrm{~m}$ from the sea $\left(6^{\circ} 12^{\prime} \mathrm{N}, 102^{\circ} 12^{\prime} \mathrm{E}\right)$. Holotype: CAS 218896.

Distribution: Kelantan River, Malaysia (Ng, 2004b).

Mystus atrifasciatus Fowler, 1937

Mystus atrifasciatus Fowler, 1937: 146, figs. 35-37. Type locality: Pitsanulok, Siam. Holotype: ANSP 67907.

Distribution: Mekong, Meklong and Chao Phraya River basins (Roberts, 1993; Kottelat, 2001b).

Mystus bimaculatus (Volz, 1904)

Macrones bimaculatus Volz, 1904: 466. Type locality: Sengi Si Russu and Kwantang River, near Djapura (Indragari), Sumatra. Syntypes (5): MHNG 683.28 (1), NMW 45166 (1), NMW 45167 (1).

Distribution: Sumatra (Volz, 1904).

Mystus bleekeri (Day, 1877)

Macrones Bleekeri Day, 1877 (in Day, 1875-78): 451, pl. 101 (fig. 1). Type locality: Sind, Jumna, upper waters of the Ganges, and Burma [locality of lectotype not stated]. Lectotype: ZSI 1076, designated by Sharma \& Dutt (1983: 334).

Distribution: Ganges, Indus and Mahanadi River basins (Roberts, 1994).

Remarks: Lectotype designation by Roberts (1994: 246) of RMNH 3024 is invalid because of the prior designation by Sharma \& Dutt (1983: 334). This name may be a senior synonym of Mystus rufescens (Vinciguerra), if the lectotype was selected from the Burmese specimens of Day.

Mystus bocourti (Bleeker, 1864)

Heterobagrus Bocourti Bleeker, 1864b: 355, pl. Type locality: Siam. Holotype: MNHN 0000-1553.

Prajadhipokia rex Fowler, 1934b: 339, figs. 3-4. Type locality: Bangkok, Siam. Holotype: ANSP 60179.

Distribution: Mekong and Chao Phraya River basins (Roberts, 1994; Kottelat, 2001b).

Mystus castaneus $\mathrm{Ng}, 2002$

Mystus castaneus Ng, 2002d: 163, fig. 2. Type locality: Borneo: Sarawak, Serian market, from Sungai Sadong. Holotype: ZRC 41848. 
Distribution: Central Sumatra, Borneo and the Malay Peninsula (Ng, 2002d).

Mystus cavasius (Hamilton, 1822)

Pimelodus cavasius Hamilton, 1822: 203, 379, pl. 11 (fig. 67). Type locality: larger freshwater rivers of the Gangetic provinces. No types known.

Mystus mukherjii Ganguly \& Datta, 1975: 293, figs. 1-2. Type locality: Subarnarekha River, below water falls at Hundru, Ranchi District, Bihar, India. Holotype: at Fish. Lab., Dept. Zool., Univ. Calcutta.

Distribution: Ganges, Brahmaputra, Mahanadi, Subarnarekhar and Godavari river basins, India, Bangladesh and Nepal (Chakrabarty \& Ng, 2005).

Remarks: See Dutt et al. (1982) for comments on the validity of this species.

$\dagger$ Mystus dalungshanensis Li \& Wang, 1979

$\dagger$ Mystus dalungshanensis Li \& Wang, 1979: 48, fig. 13. Type locality: China: Guangdong, Sanshui Basin and adjacent districts; Early-Tertiary. Holotype: H-74116-1 (repository not stated).

Distribution: Guangdong, China; Eocene (Chang \& Zhou, 1993)

Remarks: Apparently also described as new in Wang et al. (1981: 21, English 73).

Mystus falcarius Chakrabarty \& Ng, 2005

Mystus falcarius Chakrabarty \& Ng, 2005: 13, fig. 7. Type locality: Myanmar: Kachin State, Myitkyina market. Holotype: CAS 89001.

Distribution: Irrawaddy, Salween and coastal river basins, Myanmar (Chakrabarty \& Ng, 2005).

Mystus gulio (Hamilton, 1822)

Pimelodus gulio Hamilton, 1822: 201, 379, pl. 23 (fig. 66). Type locality: Higher parts of the Gangetic estuaries, where the water is not very salt. No types known.

Bagrus abbreviatus Valenciennes, in Cuvier \& Valenciennes, 1840a: 420 (311 of Strasbourg deluxe edition). Type locality: Java. Holotype: RMNH 2942; previously unpublished illustration of holotype reproduced in Roberts (1993: 28, fig. 62).

Bagrus albilabris Valenciennes, in Cuvier \& Valenciennes, 1840a: 416 (308 of Strasbourg deluxe edition). Type locality: Calcutta ... Bengal ... Ceilan ... Pondichéry. Syntypes: MNHN 0000-4172 (1), MNHN 0000-4336 (2), MNHN a-8967 (6), MNHN a-9009 (1).

Bagrus Birmannus Valenciennes, in Cuvier \& Valenciennes, 1840a: 419 (310 of Strasbourg deluxe edition). Type locality: dans l'Irawadi. Holotype: MNHN 0000-0577.

Bagrus fuscus Valenciennes, in Cuvier \& Valenciennes, 1840a: 417 (309 of Strasbourg deluxe edition). Type locality: Environs de Cananor. Holotype: MNHN 0000-0590.

Bagrus gulioides Bleeker, 1846a: 152. Type locality: Batavia. Syntypes (size and number not stated): RMNH 6862 (some of 23).

Bagrus melas Bleeker, 1846a: 152. Type locality: Java. Type(s) (size and number not stated): Whereabouts unknown.

Bagrus rhodopterygius Bleeker, 1846a: 153. Type locality: Batavia. Type(s) (size and number not stated): Whereabouts unknown.

Bagrus Schlegelii Bleeker, 1846a: 153. Type locality: Batavia. Type(s) (size and number not stated): Whereabouts unknown.

Distribution: Estuaries and lower portions of rivers of India, Bangladesh and Myanmar; and Java (Roberts, 1993).

Remarks: Synonymy of Bagrus birmannus based on examination of holotype; synonymy of Bagrus abbreviatus follows Roberts (1993: 28). The name Silurus porosus in Hora (1933: 133), which has often been treated as a synonym of Mystus gulio, is not available, as it was not treated as a valid species therein.

Mystus horai Jayaram, 1954

Mystus (Mystus) vittatus horai Jayaram, 1954: 536, pl. 19; text fig. 4. Type locality: River Indus, Kalabagh [Pakistan]. Syntypes (4): lost (Jayaram \& Anuradha, 2003).

Distribution: Indus River (Jayaram \& Anuradha, 2003; Mirza, 2003).

Mystus impluviatus $\mathrm{Ng}, 2003$

Mystus impluviatus Ng, 2003a: 373, fig. 1. Type locality: Borneo: Kalimantan Timur, small river on road $12.2 \mathrm{~km}$ from Sebulu crossroads towards forest and $59 \mathrm{~km}$ from Air Putih crossorads $\left(0^{\circ} 16^{\prime} \mathrm{S}, 117^{\circ} 0^{\prime} \mathrm{E}\right)$. Holotype: CAS 
97049.

Distribution: Mahakam River basin, eastern Borneo (Ng, 2003a).

Mystus leucophasis (Blyth, 1860)

Bagrus leucophasis Blyth, 1860: 148. Type locality: Sitang and other Burma rivers. Possible type: AMS B.7925 (1).

Distribution: Irrawaddy and Sittang River basins, Myanmar (pers. obs.).

Mystus malabaricus (Jerdon, 1849)

Bagrus Malabaricus Jerdon, 1849: 338. Type locality: Mountain streams of Malabar [southern India]. No types known.

Hara Malabarica Day, 1865a: 184, pl. 13 (fig. 3). Type locality: Mountain streams of Malabar, India. Possible types: at ZSI and NMW 45228 (1). Neotype designation by Grant (1999: 170) not valid; absence of types and nomenclatural need not clearly demonstrated. Apparently a synonym, and therefore a junior homonym, of Bagrus malabaricus Jerdon, 1849.

Mystus canarensis Grant, 1999: 170. Type locality: Mountain streams of Malabar, India. Replacement name for Hara malabarica Day, 1865, preoccupied in Mystus by Bagrus malabaricus Jerdon, 1849.

Distribution: Malabar Coast, Wayanad hills and Travancore hill ranges, India (Jayaram \& Anuradha, 2003).

Remarks: Synonymy of Hara malabarica follows Jayaram \& Anuradha (2003).

Mystus montanus (Jerdon, 1849)

Bagrus montanus Jerdon, 1849: 337. Type locality: River at Manantoddy in Wynaad, southern India. No types known.

Macrones montanus dibrugarensis Chaudhuri, 1913: 254, pl. 9 (figs. 2, 2a, 2b). Type locality: Dibrugarh [India]. Holotype: ZSI F7795/1. Originally Macrones montanus var. dibrugarensis.

Distribution: Peninsular India, especially in the western Ghats (Jayaram \& Anuradha, 2003).

Mystus multiradiatus Roberts, 1992

Mystus multiradiatus Roberts, 1992: 82, fig. 3b. Type locality: Prachinburi market [Thailand]. Holotype: CAS 76119.

Distribution: Mekong and Chao Phraya River basins (Kottelat, 2001b).

Mystus mysticetus Roberts, 1992

Mystus mysticetus Roberts, 1992: 84, fig. 3c. Type locality: Nakorn Phanom market [Thailand]. Holotype: CAS 76121.

Distribution: Mekong and Chao Phraya River basins (Kottelat, 2001b).

Mystus nigriceps (Valenciennes, 1840)

Bagrus nigriceps Valenciennes, in Cuvier \& Valenciennes, 1840a: 412 (305 of Strasbourg deluxe edition). Type locality: Java. Holotype: RMNH 2948; previously unpublished illustration of holotype reproduced in Roberts (1993: 29, fig. 64). Neotype (RMNH 3009) designation by Roberts (1993: 29) apparently invalid, following the reported rediscovery of the holotype by $\mathrm{Ng}(2002 \mathrm{~d}$ : 166$)$.

Bagrus micracanthus Bleeker, 1846a: 151. Type locality: Batavia. Syntypes (size and number not stated): possibly AMS B.7574 (1), AMS B.7979 (1), BMNH 1863.12.4.77 (1, 105 mm SL, 134 mm TL), NMV 46463 (1), RMNH 5216. Neotype (RMNH 15857) designation by Roberts (1989a) regarded by Ng (2002d: 166) as unnecessary and therefore invalid.

Distribution: Java and southern Sumatra (Ng, 2002d).

Mystus oculatus (Valenciennes, 1840)

Bagrus oculatus Valenciennes, in Cuvier \& Valenciennes, 1840a: 424 (314 of Strasbourg deluxe edition). Type locality: la côte de Malabar. Holotype: MNHN 0000-1195.

Distribution: Nilgiris, Western Ghats and hills of Karnataka, Kerala and Tamil Nadu, India (Jayaram \& Anuradha, 2003).

Mystus pelusius (Solander, 1794)

Silurus pelusius Solander, in Russell, 1794: 210, pl. 7 (fig. 1). Type locality: Aleppo. No types known.

Bagrus Halepensis Valenciennes, in Cuvier \& Valenciennes, 1840a: 413 (306 of Strasbourg deluxe edition). Type locality: la riviere de l'Alep. Syntype: BMNH 1955.6.25.1 (1, 6 inches) Description based on illustrations in Russell (1794: pl. 13, fig. 1) and Gronovius (1763: pl. 8a, fig. 6); BMNH specimen from collection of Russell. 
Macrones aleppensis Günther, 1864: 75. Type locality: Aleppo. No types known, based on illustrations in Russell (1794: pl. 13, fig. 1) and Gronovius (1763: pl. 8a, fig. 6). Unjustified emendation of Bagrus Halepensis Valenciennes, 1840.

Macrones colvillii Günther, 1874: 36, pl. 8. Type locality: Tigris at Baghdad. Syntypes: BMNH 1874.4.28.6-8 (3), BMNH 1875.1.14.19-21 (3).

Mystus misrai Anuradha, 1986: 292, figs. 1-2. Type locality: Lake Antioche, Syria. Holotype: MHNG 603.95.

Distribution: Euphrates River basin (Roberts, 1994); Tigris River basin (Coad, 1995).

Remarks: Redescribed in Anuradha \& Jayaram (1985).

Mystus pulcher (Chaudhuri, 1911)

Macrones pulcher Chaudhuri, 1911: 20, pl. 1 (fig. 4). Type locality: district of Bhamo close to Yunnan border [Upper Burma]. Syntypes: ZSI F4716/1 to F4719/1 (4).

Distribution: Irrawaddy basin and Sittang River, Myanmar.

Mystus punctifer Ng, Wirjoatmodjo \& Hadiaty, 2001

Mystus punctifer Ng, Wirjoatmodjo \& Hadiaty, 2001a: 356, figs. 1, 2a. Type locality: Sumatra: Aceh, trail in Suag

Belimbing research station, Gunung Leuser National Park [Indonesia]. Holotype: MZB 8703.

Distribution: Alas River basin, northern Sumatra (Ng et al., 2001a).

Mystus rhegma Fowler, 1935

Mystus rhegma Fowler, 1935b: 102, fig. 27. Type locality: Bangkok, Siam. Holotype: ANSP 61748.

Distribution: Mekong, Meklong and Chao Phraya River basins (Roberts, 1994; Kottelat, 2001b).

Mystus rufescens (Vinciguerra, 1890)

Macrones rufescens Vinciguerra, 1890: 226, pl. 7 (fig. 2). Type locality: Meetan, Birmania [Myanmar]. Holotype: MSNG 14585.

Distribution: Irrawaddy, Sittang and lower Salween Rivers, Myanmar (Roberts, 1994).

Mystus seengtee (Sykes, 1839)

Pimelodus Seengtee Sykes, 1839: 164. Type locality: Deccan, India. Holotype (6 inches): possibly BMNH 1857.6.13.154 (1, dry). Also described as new in Sykes (1840: 61); illustrated and described in more detail in Sykes (1841: 374, pl. 66, fig. 2). Illustration of type reproduced in Chakrabarty \& $\mathrm{Ng}(2005: 10$, fig. 6).

Distribution: Krishna and Cauvery river basins, India (Chakrabarty \& Ng, 2005).

Remarks: See Chakrabarty \& Ng (2005: 18) for discussion of the status of the holotype of the species.

Mystus singaringan (Bleeker, 1846)

Bagrus heterurus Bleeker, 1846a: 151. Type locality: Batavia [now: Western Java, by neotype designation]. Neotype: RMNH 2951, designated by Roberts (1994: 252).

Bagrus macronemus Bleeker, 1846a: 150. Type locality: Batavia [now: Western Java by neotype designation]. Neotype: RMNH 2951, designated by Roberts (1994: 253), but Eschmeyer et al. (1998) indicated that specimens cataloged as SMNS 10571 may be extant syntypes. Preoccupied by Bagrus macronemus Ranzani, 1842 (now in the Ariidae, as a synonym of Bagre bagre); apparently not replaced.

Bagrus singaringan Bleeker, 1846a: 150. Type locality: Batavia [now Western Java, by neotype designation]. Neotype: RMNH 2951, designated by Roberts (1994: 252).

Distribution: Mekong and Chao Phraya River basins, and Sundaland (Kottelat, 2001b).

Remarks: Roberts (1994: 252) serves as first reviser selecting Mystus singaringan over Bagrus heterurus; earlier first reviser action of Bleeker (1862-63: 58), in which Hypselobagrus macronema is used as valid, with Bagrus heterurus and B. singaringan as synonyms, is invalid because of the homonymy of Bagrus macronemus Ranzani.

$\dagger$ Mystus spinipectoralis Li \& Wang, 1979

$\dagger$ Mystus spinipectoralis Li \& Wang, 1979: 48, fig. 14. Type locality: China: Guangdong, Sanshui Basin and adjacent districts; Early-Tertiary. Holotype: H.74112 (repository not stated).

Distribution: China, Eocene (Chang \& Zhou, 1993).

Remarks: Apparently also described as new in Wang et al. (1981: 25, English 74).

Mystus tengara (Hamilton, 1822)

Pimelodus tengara Hamilton, 1822: 183, 377, pl. 3 (fig. 61). Type locality: in the ponds of India. No types known. 
Distribution: Widely distributed in northern India, as well as Bangladesh and Pakistan (Jayaram, 1999).

Mystus vittatus (Bloch, 1794)

Silurus vittatus Bloch, 1794: 50, pl. 371 (fig. 2). Type locality: Tranquebar. Holotype: ZMB 2939.

Pimelodus Batasius Hamilton, 1822: pl. 23 (fig. 60). Type locality: Not stated [Ponds of northern parts of Bengal]. No type known. Name available from caption to plate, which corresponds to the text of Pimelodus carcio.

Pimelodus carcio Hamilton, 1822: 181, 377, pl. 23 (fig. 60). Type locality: Ponds of northern parts of Bengal. No types known.

Bagrus Keletius Valenciennes, in Cuvier \& Valenciennes, 1840a: 411 (305 of Strasbourg deluxe edition). Type locality: Pondichéry. Lectotype: MNHN a-9011, designated by Ng (2002d: 166).

Distribution: Inland and coastal rivers of Pakistan, Nepal India, Sri Lanka, and Bangladesh (Roberts, 1993); published records from Myanmar apparently in error.

Remarks: Day (1871) and Hora (1929: 182) discuss the confusion surrounding the identity of the fish illustrated in Hamilton (1822: pl. 23, fig. 60) as Pimelodus batasius. Bagrus Keletius Valenciennes, (1840a: 411) was treated by Ng (2002d: 165) as a synonym of either Mystus armatus (Day, 1865) or Mystus vittatus (Bloch, 1794).

Mystus wolffii (Bleeker, 1851)

Bagrus Wolffii Bleeker, 1851d: 205. Type locality: Bandjarmassing, in fluviis. Holotype (131 mm TL): possibly AMS B.7774 (1), BMNH 1863.12.4.94 (1, 102 mm SL, 129 mm TL), NMV 46225 (1), ZMB 4794 (1).

Distribution: Large rivers from Thailand to Indonesia, in the tidal zone and brackish water (Rainboth, 1996).

Remarks: Holotype listed as BMNH 1863.12.4.94 by Desoutter (1975: 447).

\section{Species inquirenda, Mystus}

Pimelodus anisurus M'Clelland, 1842: 583. Type locality: Loodianah and Cabool River at Jullalabad [Afghanistan]. Holotype: Whereabouts unknown, possibly at BMNH. Treated in recent literature as a possible synonym of Mystus tengara Hamilton, 1822, but treated by Günther (1864: 190) as a species of Amblyceps.

NANOBAGRUS Mo, 1991

Nanobagrus Mo, 1991: 125, 143. Type species: Akysis armatus Vaillant, 1902. Type by original designation. Gender: Masculine.

Nanobagrus armatus (Vaillant, 1902)

Akysis armatus Vaillant, 1902: 64, fig. 10. Type locality: Tepoe, bords du Mahakam, Bornéo central. Holotype: RMNH 7844.

Distribution: Mahakam and Kapuas River basins, Borneo (Ng \& Tan, 1999).

Nanobagrus nebulosus Ng \& Tan, 1999

Nanobagrus nebulosus Ng \& Tan, 1999: 357, fig. 3a, 4a. Type locality: Malaysia: Johor, Sungai Kahang and tributary ca. $44.4 \mathrm{~km}$ from Mersing turnoff to Kluang just before side road to Endau Rompin Taman Negara, km 96 from Mersing to Batu Pahat (2³.93'N, 103³1.58'E). Holotype: ZRC 42600.

Distribution: Endau and Sedili River drainages, southeastern Malay Peninsula (Ng \& Tan, 1999).

Nanobagrus stellatus Tan \& Ng, 2000

Nanobagrus stellatus Tan \& Ng, 2000: 278, fig. 5a. Type locality: Jambi: Sg. Alai (from aquarium collectors), Sumatra. Holotype: MZB 9306.

Distribution: Batang Hari basin, Sumatra (Tan \& Ng, 2000).

$\dagger$ NIGERIUM White, 1934

$\dagger$ Nigerium White, 1934: 49, 52. Type species: $†$ Nigerium wurnoense White, 1934. Type by original designation. Gender: Neuter.

Remarks: Considered to be Bagridae S.L. by Gayet \& Meunier (2003).

$\dagger$ Nigerium gadense White, 1934

$\dagger$ Nigerium gadense White, 1934: 52, pl. 7 (fig. 2). Type locality: Gada, Nigeria, in scree; Eocene. Holotype: at National Collection [Nigeria] or BMNH (incomplete skull). 
Distribution: Gada, Nigeria: Eocene.

$\dagger$ Nigerium wurnoense White, 1934

$\dagger$ Nigerium wurnoense White, 1934: 49, pl. 6. Type locality: Reptile bed at Wurno, Nigeria: Eocene. Holotype: at National Collection [Nigeria] or BMNH (incomplete skull).

Distribution: Wurno, Nigeria: Eocene.

$\dagger$ NKONDOBAGRUS Van Neer, 1994

$\dagger$ Nkondobabrus Van Neer, 1994: 108. Type species: $†$ Nkondobagrus longisrostris Van Neer, 1994. Type by monotypy. Gender: Masculine.

$\dagger$ Nkondobagrus longisrostris Van Neer, 1994

$\dagger$ Nkondobagrus longisrostris Van Neer, 1994: 108, pl. 4. Type locality: Nkondo-Kaiso area, Nkondo Formation, Albertine Rift Valley, Upper Miocene, Uganda. Holotype: apparently at MRAC, NK 2637 '89 (anterior portion of neurocranium, with part of vomerine tooth patch).

Distribution: Uganda, upper Miocene (Van Neer, 1994; Gayet \& Meunier, 2003: 498).

Remarks: Considered to be Bagridae S.L. by Gayet \& Meunier (2003).

OLYRA M'Clelland, 1842

Olyra M'Clelland, 1842: 588. Type species: Olyra longicaudata M'Clelland, 1842. Type by subsequent designation, by Gill (1861c: 51). Gender: Feminine.

Review: Hora (1936a).

Olyra burmanica Day, 1872

Olyra burmanica Day, 1872: 711. Type locality: Pegu Yomas, Burma. Lectotype: AMS B.7560; designated by Jayaram (1980: 19).

Distribution: Pegu Yomas; known only from type (Jayaram, 1980).

Olyra horae (Prashad \& Mukerji, 1929)

Amblyceps horae Prashad \& Mukerji, 1929: 173, pl. 7 (fig. 1). Type locality: Indawgyi Lake along its w. shore near Loimon village, Myitkyina District, Upper Burma. Holotype: ZSI F10854/1.

Distribution: Meghalaya, India, and Indawgyi Lake, Myanmar (Jayaram, 1980).

Remarks: Name appears as Olyra horai in recent literature, which reflects an attempt to correct the suffix to the name to the masculine form. However, the original spelling is also an acceptable form and the subsequent spelling should be regarded as a misspelling.

Olyra kempi Chaudhuri, 1912

Olyra kempi Chaudhuri, 1912: 443, pl. 41 (figs. 4, 4a, 4b). Type locality: Mangaldai (Assam-Bhutan Frontier) [North India]. Syntypes (5): ZSI F5387/1 (1).

Distribution: Mangaldai, Assam, India (Jayaram, 1980).

Remarks: Treated in some literature as a synonym of Olyra longicaudatus.

Olyra longicaudata M'Clelland, 1842

Olyra longicaudatus M'Clelland, 1842: 588, pl. 21 (fig. 1). Type locality: Kaysah Mountains. Holotype: not saved (M'Clelland, 1842: 572). Name spelled Olyra longicaudata on p. 574.

Distribution: Base of Darjelling Himalaya, Assam, India; Tenassarim, Myanmar (Jayaram, 1980).

\section{Species inquirendae, Olyra}

Heptapterus Collettii Steindachner, 1881b: 98. Type locality: La Plata [in error]. Syntypes: NMW 46191 (2). Illustrated and described in more detail in Steindachner (1881d: 7, pl. 5, fig. 1). Shown to be a species of Olyra in Bockmann \& de Pinna (2004).

Olyra elongata Günther, 1883: 140. Type locality: Tenasserim [Burma]. Syntypes: BMNH 1880.12.1.64-67 (7 ?).

PELTEOBAGRUS Bleeker, 1865

Pelteobagrus Bleeker, 1865: 9. Type species: Silurus calvarius Basilewsky, 1855. Type by monotypy. Gender: Mas- 
culine.

Remarks: Mo (1991) noted that Pelteobagrus may not be monophyletic if species placed in Pseudobagrus and Coreobagrus were excluded.

Pelteobagrus argentivittatus (Regan, 1905)

Macrones argentivittatus Regan, 1905c: 390, pl. 5 (fig. 2). Type locality: Canton, Cungkun, China. Lectotype: MHNG 677.99, designated by Jayaram (1974).

Distribution: from Heilongjiang [Amur River] to Zhujiang [= Pearl River] (Chu et al., 1999).

Pelteobagrus brashnikowi (Berg, 1907)

Macrones (Leiocassis) brashnikowi Berg, 1907: 421. Type locality: [Amur River mouth, Russia]. Syntypes (5): ZIN 13964 (2), ZIN 13965 (2), ZIN 13966 (1), but only two specimens still at ZIN (Naseka \& Bogutskaya (2004: 286).

Distribution: Amur River basin (Berg, 1907).

Remarks: Also treated in recent literature as valid in either Pelteobagrus brashnikowi or Leiocassis brashnikowi; inclusion of species in Pelteobagrus follows Naseka \& Bogutskaya (2004).

Pelteobagrus eupogon (Boulenger, 1892)

Pseudobagrus eupogon Boulenger, 1892a: 247. Type locality: Shanghai, China. Holotype: BMNH 1891.1.31.19.

Distribution: Changjiang [= Yangtze River] basin (Chu et al., 1999).

Pelteobagrus fulvidraco (Richardson, 1846)

Pimelodus fulvi-draco Richardson, 1846: 286. Type locality: Canton, China. No types known; based on illustration by Reeves, reproduced in Whitehead (1970: pl. 1b). Originally as: Pimelodus ? fulvi-draco.

Silurus calvarius Basilewsky, 1855: 241, pl. 9 (fig. 1). Type locality: in aquis fluentibus et stagnantibus atque in sinu Tschiliense [China]. Type(s): Whereabouts unknown.

Distribution: Widely throughout China, except the western plateau and Xinjiang (Chu et al., 1999); Nam Ma Basin, Laos, and northern and central Vietnam (Kottelat, 2001b).

Pelteobagrus intermedius (Nichols \& Pope, 1927)

Pseudobagrus intermedius Nichols \& Pope, 1927: 331, fig. 5. Type locality: Nodoa, Hainan [China]. Holotype: AMNH 8360. Also treated as valid in Pseudobagrus.

Aoria henryi Herre, 1932: 432. Type locality: Canton wholesale fish market, China. Holotype: SU 25724. Treated in recent literature as either a synonym of Pelteobagrus intermedius or as valid as Pseudobagrus henryi.

Distribution: Hainan Island, from Minjiang to Zhujiang (Chu et al., 1999); Vietnam (Kottelat, pers. comm).

Pelteobagrus mica (Gromov, 1970)

Mystus mica Gromov, 1970: 400, unnumbered figure. Type locality: Lake Ommi, middle Amur basin, Russia. Holotype: ZIN 39434.

Distribution: Amur River basin, Russia (Gromov, 1970).

Remarks: Inclusion of species in Pelteobagrus follows Naseka \& Bogutskaya (2004).

Pelteobagrus microps (Rendahl, 1932)

Leiocassis microps Rendahl, 1932: 93. Type locality: Chungking, China. Holotype: NRM 10163.

Distribution: Chungking, China.

Pelteobagrus nudiceps (Sauvage, 1883)

Pseudobagrus nudiceps Sauvage, 1883a: 145. Type locality: le Lac Biwako, Japon. Holotype: MNHN a-6525. Also described as new (from Siam) in Sauvage (1883b). The later description has been treated as a Lapsus calami by Smith (1934: 296; 1945: 6) and Kottelat (1984b: 811).

Distribution: Lake Biwa; central and western Honshu, eastern Shikoku, and northwestern Kyushu islands, Japan (Matsuura et al., 2000). Reported from the Lower Pliocene of Mie Prefecture, Japan, in Watanabe \& Okuyama (1994).

Remarks: Occasionally treated as valid as Pseudobagrus nudiceps.

Pelteobagrus ussuriensis (Dybowski, 1872)

Bagrus ussuriensis Dybowski, 1872: 210. Type locality: Ussuri and Sungari rivers, Amur R. basin, and Khanka Lake, Russia. Syntype(s): ZMB 7954 (1?).

Pseudobagrus emarginatus Sowerby, 1921: 1. Type locality: Mouth of the Hun Kiang, in the Yalu River on the 
South Manchurian Border. Holotype: USNM 76716.

Distribution: from Heilongjiang [= Amur River] to Zhujiang [= Pearl River], China (Chu et al., 1999); Pyeuktong, Engpyen, Taedong River, Han River, Korea (Mori, 1952).

Remarks: Treated in recent literature as valid as either Leiocassis ussuriensis and Pseudobagrus ussuriensis; inclusion of species in Pelteobagrus follows Naseka \& Bogutskaya (2004).

Pelteobagrus vachellii (Richardson, 1846)

Bagrus vachellii Richardson, 1846: 284. Type locality: Chikiang, China. Neotype: BMNH 1855.3.27.9, designated by Jayaram (1968b: 300). Originally as "? Bagrus vachellii."

Pseudobagrus chinensis Wu, 1930b: 53, fig. 4. Type locality: Sze-Chwan, China. Holotype: Whereabouts unknown. Name apparently not treated since original description.

Pseudobagrus fangi Wu 1930c: 84, fig. 8. Type locality: Kiating [Szechwan, China]. Holotype: Whereabouts unknown.

Pseudobagrus wui Miao, 1934: 210, fig. 42. Type locality: Chinkiang, southern Kiangsu, China. Holotype: Mus. Biol. Lab. Sci. Soc. China 12495.

Distribution: from Huanghe [= Yellow River] to Zhujiang [= Pearl River], China (Chu et al., 1999); Seoul, Suwon, Taeque, Taedong River, Korea (Mori, 1952).

Pelteobagrus virgatus (Oshima, 1926)

Aoria virgatus Oshima, 1926: 4. Type locality: Kachek River, about 40 mi. above Kachek, Hainan Island, China. Holotype: Whereabouts unknown.

Distribution: Hainan Island, Yuanjiang (Chu et al., 1999).

Remarks: Treated as valid in recent literature as Pelteobagrus virgatus, Pseudobagrus virgatus or Leiocassis virgatus. The name Pelteobagrus virgatus vinhensis in Nguyen (1982: 26), is a nomen nudum and is not available.

PSEUDOBAGRUS Bleeker, 1859

Pseudobagrus Bleeker, 1859: 257. Type species: Bagrus aurantiacus Temminck \& Schlegel, 1846. Type by monotypy. Gender: Masculine.

Fluvidraco Jordan \& Fowler, 1903: 904. Type species: Pseudobagrus ransonnetii Steindachner, 1887. Type by original designation. Gender: Masculine.

Pseudobagrus adiposalis Oshima, 1919

Pseudobagrus adiposalis Oshima, 1919: 181, pl. 48 (fig. 2). Type locality: Tamusui R. near Shinten, Taiwan. Lectotype: FMNH 59079; designated by Eschmeyer (in Eschmeyer et al., 1998: 40).

Distribution: Lingjiang, Oujiang [= Wu River], Xiangjiang, Zhujiang [Pearl River], China (Chu et al., 1999); Taiwan.

Pseudobagrus albomarginatus (Rendahl, 1928)

Leiocassis albomarginatus Rendahl, 1928: 170. Type locality: Tang-tu-hsien, Anhui, China. Syntypes: NRM 10017 (5).

Distribution: Anhui and Guangdong, China.

Pseudobagrus analis (Nichols, 1930)

Leiocassis (Dermocassis) analis Nichols, 1930: 4, fig. 3. Type locality: Hokou (Chaoshan), ne. Kiangsi Prov., China. Holotype: AMNH 9680.

Distribution: middle and lower Changjiang [= Yangtze River], China (Chu et al., 1999).

Pseudobagrus aurantiacus (Temminck \& Schlegel, 1846)

Bagrus aurantiacus Temminck \& Schlegel, 1846, in Temminck \& Schlegel, 1843-50: 227, pl. 104 (fig. 2). Type locality: Japan. Lectotype: RMNH 2952a, designated by Boeseman (1947: 169).

Pseudobagrus ransonnettii Steindachner, in Steindachner \& Döderlein, 1887: 287. Type locality: Osaka, Japan. Syntypes (2): at NMW.

Distribution: Western Kyushu and Iki Islands, Japan (Matsuura et al., 2000).

Remarks: Redescribed in Watanabe \& Maeda (1995).

Pseudobagrus brevianalis Regan, 1908

Pseudobagrus brevianalis Regan, 1908f: 151. Type locality: Lake Candidius, Formosa. Syntypes: BMNH 
1908.5.27.23-28 (6).

Distribution: Taiwan (Chu et al., 1999).

Pseudobagrus brevicaudatus (Wu, 1930)

Leiocassis brevicaudatus Wu, 1930c: 81, fig. 7. Type locality: Chungking, Szechwan, China. Holotype: Whereabouts unknown.

Leiocassis lui Tchang \& Shih, 1934: 434. Type locality: valey of Lower Kialingkiang, from Chungking to Hochuan of Szechwan province [China]. Holotype: ZMFMIB 9720.

Distribution: Changjiang [= Yangtze River] basin, China (Chu et al., 1999).

Remarks: See Zhang \& Wang (1996) for taxonomic discussion.

Pseudobagrus gracilis Li, Chen \& Chan, 2005

Pseudobagrus gracilis Li, Chen \& Chan, 2005: 50, fig. 1. Type locality: China: Guangxi Province, Guilin City, Zhujiang basin, Lijiang drainage, 2955'23 N, 118²8'12 E. Holotype: SCNU 0410002.

Distribution: Beijiang and Xijiang [= West River] River basins, southern China (Li et al., 2005).

$\dagger$ Pseudobagrus ikiensis Watanabe \& Uyeno, 1999

$\dagger$ Pseudobagrus ikiensis Watanabe \& Uyeno, 1999: 398, figs. 2-3 Type locality: Chojabaru Formation (Ika Group) Middle Miocene, diatomite at Hachiman, Ashibe, Iki Island, Nagasaki Prefecture, Japan. Holotype: GSJ F 7735 (complete skeleton).

Distribution: Nagasaki Prefecture, Japan; Middle Miocene.

Pseudobagrus koreanus Uchida, 1990

Pseudobagrus koreanus Uchida, in Lee \& Kim, 1990: 124, figs. 3b, 6. Type locality: Namwôn-shi, Sômjin R., Korea. Holotype: BKNU 1111.

Distribution: Sômjin River, Korea (Uchida, in Lee \& Kim, 1990).

Pseudobagrus medianalis (Regan, 1904)

Macrones medianalis Regan, 1904a: 194. Type locality: Sea of Tien [Tien Chih], Yunnan Fu, China, elev. $6000 \mathrm{ft}$. Syntypes: BMNH 1904.1.26.44-46 (3).

Distribution: Dianchi Lake, China (Chu et al., 1999).

Pseudobagrus omeihensis (Nichols, 1941)

Leiocassis omeihensis Nichols, 1941: 1. Type locality: Omeihsien, Szechwan Prov., China, elev. 1600 ft. Holotype: AMNH 15217.

Distribution: Emei, Sichuan, China (Chu et al., 1999).

Pseudobagrus ondon Shaw, 1930

Pseudobagrus ondon Shaw, 1930: 111, figs. 1-2. Type locality: Small river at Shing-Tsong, Chekiang [Zhekjiang], China. Holotype: ASIZB [= ZMFMIB 4449].

Distribution: Luohe, Oujiang, Linghiang, Caojiang, Huaihe, Hanshui, China (Chu et al., 1999).

Pseudobagrus pratti (Günther, 1892)

Macrones pratti Günther, 1892: 245, pl. 1 (fig. b). Type locality: Kia-tiang-fu, Szechwan [Sichuan], Prov., China, elev. 1070 ft. Holotype: BMNH 1891.6.13.25.

Liocassis emarginatus Regan, 1913b: 553. Type locality: Kia-tiang-fu, Sze Chuen [China]. Syntypes (4): BMNH 1891.6.13.21 (1?).

Distribution: Changjiang [= Yangtze River] basin, China (Chu et al., 1999).

Remarks: Synonymy follows Watanabe (1995).

Pseudobagrus taeniatus (Günther, 1873)

Macrones (Liocassis) taeniatus Günther, 1873b: 245. Type locality: Shanghai, China. Holotype: BMNH 1873.7.30.73

Distribution: from Changjiang [= Yangtze River] to Minjiang, China (Chu et al., 1999).

Pseudobagrus taiwanensis Oshima, 1919

Pseudobagrus taiwanensis Oshima, 1919: 180, pl. 48 (fig. 1). Type locality: Tozen R. near Taichu, Taiwan. Lectotype: FMNH 59078; designated by Eschmeyer (in Eschmeyer et al., 1998: 1646).

Distribution: Taiwan (Chu et al., 1999).

Pseudobagrus tenuis (Günther, 1873) 
Macrones (Pseudobagrus) tenuis Günther, 1873b: 244. Type locality: Shanghai, China. Holotype: BMNH 1873.7.30.72.

Distribution: from Changjiang [= Yangtze River] to Minjiang, China (Chu et al., 1999).

Pseudobagrus tokiensis Döderlein, 1887

Pseudobagrus tokiensis Döderlein, in Steindachner \& Döderlein, 1887: 288. Type locality: River near Tokyo, Japan. Possible syntypes: BMNH 1891.5.26.19-20 (2), MZS 498 (7), MZS 643 (1), ZMB 12246 (2).

Distribution: northern portions of Honshu, Japan (Matsuura et al., 2000).

Remarks: Redescribed in Watanabe \& Maeda (1995).

Pseudobagrus trilineatus (Zheng, 1979)

Leiocassis trilineatus Zheng, 1979: 182, fig. 1. Type locality: Mountain stream in Luofu Shan, Boluo Xian, Guangdong Province, China. Holotype: JNU 76VII Luofu Shan 029.

Distribution: Dongjiang basin, China (Chu et al., 1999).

Pseudobagrus truncatus (Regan, 1913)

Liocassis truncatus Regan, 1913b: 553. Type locality: Kia-tiang-fu [China]. Syntypes (5): BMNH 1891.6.13.24.

Distribution: Changjiang [= Yangtze River] basin, China (Chu et al., 1999).

\section{Species inquirenda, Pseudobagrus}

Pseudobagrus changi Miao, 1934: 213, fig. 43. Type locality: Chinkiang, southern Kiangsu, China. Holotype: Mus. Biol. Lab. Sci. Soc. China 12494. [name not in recent use.]

PSEUDOMYSTUS Jayaram, 1968

Pseudomystus Jayaram, 1968b: 359. Bagrus stenomus Valenciennes, 1840. Type by original designation. Originally proposed as a subgenus of Leiocassis. Gender: Masculine.

Remarks: Treated in some recent literature as a synonym of Leiocassis.

Pseudomystus bomboides Kottelat, 2000

Pseudomystus bomboides Kottelat, 2000a: 77, fig. 69. Type locality: Laos: Vientiane Province, Mekong basin, confluence of Nam Leuk and Nam Ngong, 18²2'04"N, 10305'27"E. Holotype: ZRC 45426.

Distribution: Mekong River basin and southern Thailand (Kottelat, 2001b).

Pseudomystus breviceps (Regan, 1913)

Liocassis breviceps Regan, 1913b: 551. Type locality: Deli, Sumatra. Syntypes: BMNH 1889.11.12.64-65 (2).

Distribution: Sumatra, known only from types.

Remarks: BMNH 1889.11.12.66 was incorrectly listed as holotype by Ng \& Rachmatika, (1999: 178).

Pseudomystus carnosus Ng \& Lim, 2005

Pseudomystus carnosus Ng \& Lim, 2005: 7, fig. 5. Type locality: Sumatra: Sukadana. Holotype: RMNH 15859.

Distribution: Way Seputih River basin, southern Sumatra (Ng \& Lim, 2005).

Pseudomystus flavipinnis Ng \& Rachmatika, 1999

Pseudomystus flavipinnis Ng \& Rachmatika, 1999: 175, fig. 5. Type locality: Borneo: Kalimantan Barat, Sungai Embaloh at Benua Marinus. Holotype: MZB 6593.

Distribution: Kapuas River basin (Ng \& Rachmatika, 1999).

Pseudomystus fumosus $\mathrm{Ng} \& \mathrm{Lim}, 2005$

Pseudomystus fumosus Ng \& Lim, 2005: 11, fig. 6. Type locality: Malaysia: Pahang, Taman Negara, Kuala Tahan. Holotype: ZRC 3229.

Distribution: Pahang River basin, peninsular Malaysia (Ng \& Lim, 2005).

Pseudomystus fuscus (Popta, 1904)

Leiocassis fuscus Popta, 1904: 186. Type locality: le Mahakam supérior, Bornéo central. Holotype: RMNH 7555; described in more detail, with illustration of holotype, in Popta (1906: 53, pl. 3, fig. 10).

Distribution: Upper Mahakam River basin, Borneo, and Malay peninsula.

Pseudomystus inornatus (Boulenger, 1894)

Liocassis inornatus Boulenger, 1894a: 245. Type locality: Senah, Sarawak state, Borneo, East Malaysia. Holotype: 
BMNH 1893.3.6.179.

Distribution: Sungai Penyalin, Indonesia and Sarawak (Ng \& Rachmatika, 1999).

Pseudomystus leiacanthus (Weber \& de Beaufort, 1912)

Leiocassis leiacanthus Weber \& de Beaufort, 1912: 536, pl. 2 (figs. 1-1a). Type locality: Sumatra (Kwantan river,

Ringat and Taluk). Syntypes: ZMA 112671 (1), ZMA 112672 (1); syntype illustration reproduced in Weber \& de Beaufort (1913: fig. 148).

Distribution: Sumatra; Malay Peninsula (Mohsin \& Ambak, 1983).

Pseudomystus mahakamensis (Vaillant, 1902)

Leiocassis mahakamensis Vaillant, 1902: 55, figs. 4-5. Type locality: Tepoe, bords du Mahakam, Bornéo central. Syntypes: RMNH 7838 (2).

Distribution: Mahakam River basin, Borneo.

Pseudomystus moeschii (Boulenger, 1890)

Liocassis moeschii Boulenger, 1890a: 39. Type locality: Deli River, Sumatra. Lectotype: BMNH 1889.11.12.66; lectotype designated by $\mathrm{Ng} \& \operatorname{Lim}$ (2005: 14).

Distribution: Deli and Banjuasin Rivers, Sumatra (Ng \& Lim, 2005).

Remarks: Species redescribed in Ng \& Lim (2005).

Pseudomystus myersi (Roberts, 1989)

Leiocassis myersi Roberts, 1989a: 117, fig. 92. Type locality: Small forest stream flowing into Kapuas mainstream NE of Gunung Setunggul, $53 \mathrm{~km} \mathrm{NW}$ of Sintang, Western Borneo (Kalimantan Barat, Indonesia). Holotype: MZB 3588.

Distribution: Kapuas River, western Borneo (Roberts, 1989a).

Pseudomystus robustus (Inger \& Chin, 1959)

Leiocassis robustus Inger \& Chin, 1959: 290, fig. 48. Type locality: Kinabatangan River at Deramakot, Kinabatangan District, North Borneo. Holotype: FMNH 68001

Distribution: Kinabatangan River, Borneo, Malaysia (Inger \& Chin, 1959).

Pseudomystus rugosus (Regan, 1913)

Liocassis rugosus Regan, 1913b: 552. Type locality: Poeh, Sarawak. Holotype: BMNH 1893.3.6.172.

Distribution: Sarawak.

Pseudomystus siamensis (Regan, 1913)

Liocassis siamensis Regan, 1913b: 550. Type locality: Bangpakong River, Siam. Holotype: BMNH 1897.10.8.126.

Leiocassis albicollaris Fowler, 1934b: 337, fig. 2. Type locality: Bangkok, Siam. Holotype: ANSP 60178.

Leiocassis bicolor Fowler, 1934a: 95, fig. 43. Type locality: Chieng Mai, North Siam. Holotype: ANSP 59284.

Distribution: Mekong and Chao Phraya River basins (Kottelat, 2001b), and peninsular Thailand.

Pseudomystus sobrinus Ng \& Freyof, 2005

Pseudomystus sobrinus $\mathrm{Ng} \&$ Freyof, 2005: 745, figs. 1, 2a. Type locality: Vietnam, Phu Yen province, stream about $15 \mathrm{~km}$ south of Tuy Hoa, 1253'05"N, 109²3'70"E. Holotype: ZFMK 27167.

Distribution: Song Ba and nearby rivers, southern Vietnam (Ng \& Freyhof, 2005).

Pseudomystus stenogrammus Ng \& Siebert, 2005

Pseudomystus stenogrammus Ng \& Siebert, 2005: 2, figs, 1, 3a [with captions identifying the species as Pseudomystus ornatus]. Type locality: Borneo: Kalimantan Tengah, Sungai Laung at Dessa Marwei. Holotype: MZB 6103. Distribution: Barito River basin, southern Borneo (Ng \& Siebert, 2005).

Remarks: The name Pseudomystus ornatus, which appears in the figure caption of the description of this species is clearly a lapsus calami for Pseudomystus stenogrammus and is thus a nomen nudum.

Pseudomystus stenomus (Valenciennes, 1840)

Bagrus stenomus Valenciennes, in Cuvier \& Valenciennes, 1840a: 415 (307 of Strasbourg deluxe edition). Type locality: Java. Holotype: RMNH 2986; previously unpublished illustration of holotype reproduced in Roberts (1993: fig. 61).

Leiocassis Ellenriederii Bleeker, 1860: 10. Type locality: Lahat, Palembang. Syntypes (3, 105-110 mm TL): Whereabouts unknown.

Distribution: South East Thailand, Kampuchea, Malay Peninsula, Sumatra, Java, Borneo. 
Pseudomystus vaillanti (Regan, 1913)

Liocassis vaillanti Regan, 1913b: 549. Type locality: Embouchure du Raoun, Kapuas basin, Borneo. Holotype: RMNH 7840. Based on specimen identified in Vaillant (1902; fig. 8, 9) as Leiocassis moeschii; Vaillant's illustration reproduced in Roberts (1989a: fig, 93).

Distribution: Kapuas River, known only from holotype (Roberts, 1989a).

$\boldsymbol{R A M A}$ Bleeker, 1858

Rama Bleeker, 1858b: 201, 205, 208. Type species: Rama buchanani Bleeker, 1858 (= Pimelodus rama Hamilton, 1822). Type by monotypy. Gender: Feminine.

Rama rama (Hamilton, 1822)

Pimelodus rama Hamilton, 1822: 176, 377, pl. 3 (fig. 55). Type locality: Brahmaputra River. No types known.

Rama buchanani Bleeker, 1858b: 201, 205, 208. Type locality: Brahmaputra River. No types known. Unneeded replacement for Pimelodus rama Hamilton, 1822, apparently to avoid tautonymy.

Distribution: Brahmaputra River basin, India (Jayaram, 1999).

Remarks: Treated in some recent literature as synonym of Chandramara chandramara (Hamilton).

RITA Bleeker, 1854

Rita Bleeker, 1854c: 122. Type species: Rita buchanani Bleeker, 1854. Type by monotypy. Gender: Feminine.

Gogrius Day, 1867: 563. Type species: Gogrius sykesii Day, 1867. Type by monotypy. Gender: Masculine.

Key to species: Ferraris (1999b), Ng (2004i).

Rita chrysea Day, 1877

Rita chrysea Day, 1877 (in Day, 1875-78): 455, pl. 104 (fig. 1). Type locality: Orissa. Holotype: ZSI F498.

Distribution: Mahanadi River system, Orissa, Madhya Pradesh, India (Jayaram, 1999).

Remarks: See Jayaram (1960) for interpopulational variation.

Rita gogra (Sykes, 1839)

Phractocephalus gogra Sykes, 1839: 164. Type locality: Deccan, India. Type(s): Whereabouts unknown. Also proposed as new in Sykes (1840: 61); illustrated and described in more detail in Sykes (1841: 374, pl. 66, fig. 1).

Arius pavimentatus Valenciennes, in Cuvier \& Valenciennes, 1840b: 94 (70 of Strasbourg deluxe edition). Type locality: trouvé dans la collection de feu Jacquemont [= vicinity of Bombay, India]. Holotype: MNHN b-0684; illustrated in Jacquemont (1835-1844: pl. 17, fig. 2).

Gogrius sykesii Day, 1867: 563. Type locality: Kurnool and the Deccan, India. Possible syntypes: ZSI 1200 (1), ZSI 3107 (1, lost), and types of "Pimelodus gogra, Sykes." Unndeeded new name for Phractocephalus gogra Sykes, 1839, apparently to avoid tautonymy.

Distribution: Rivers of Deccan, India, up to Krishna River basin (Jayaram, 1999).

$\dagger$ Rita grandiscutata Lydekker, 1886

$\dagger$ Rita grandiscutata Lydekker, 1886: 251, pl. 37, fig. 3. Type locality: Punjab, Siwalik Formation Lower Pliocene. Holotype: at Indian Museum, Calcutta E. 157 (large basal bone of dorsal spine).

? † Chrysichthys (?) theobaldi Lydekker, 1886: 249, pl. 37, fig. 4. Type locality: Punjab, Siwalik Formation, Lower Pliocene. Holotype: Indian Museum, Calcutta E. 158 (hind part of cranium).

Distribution: Punjab, Siwalik Formation Lower Pliocene.

Remarks: $\dagger$ Chrysichthys theobaldi was assigned to Rita by Jayaram (1955a: 121). Because the two names listed here were applied to different parts of specimens that have been assigned to the same genus and both were found in the same locality, I am tentatively placing the two names into synonymy and treat $\dagger$ Rita grandiscutata as the valid name of the species.

Rita kuturnee (Sykes, 1839)

Phractocephalus kuturnee Sykes, 1839: 164. Type locality: Deccan, India. Type(s): Whereabouts unknown. Also described as new in Sykes (1840: 61); illustrated and described in more detail in Sykes (1841: 372, pl. 65, fig. 3).

Arius hastatus Valenciennes, in Cuvier \& Valenciennes, 1840b: 97 (72 of Strasbourg deluxe edition). Type locality: [India]. Holotype: MNHN b-0591. Illustrated in Jacquemont (1835-1844: pl. 18, fig. 2). 
Distribution: Peninsular India.

Rita macracanthus $\mathrm{Ng}, 2004$

Rita macracanthus Ng, 2004i: 2, fig. 1. Type locality: Pakistan: Indus River at Attock. Holotype: UMMZ 237502.

Distribution: Indus River basin (Ng, 2004i).

Rita rita (Hamilton, 1822)

Pimelodus rita Hamilton, 1822: 165, 376, pl. 24 (fig. 53). Type locality: estuaries of Bengal. No types known.

Arius ritoides Valenciennes, in Cuvier \& Valenciennes, 1840b: 92 (69 of Strasbourg deluxe edition). Type locality:

Bengale. Holotype: MNHN b-0683.

Rita Buchanani Bleeker, 1854c: 123, pl. 3 (fig. 1). Type locality: Hooghly, Calcutta. No types known. Unneeded new name for Pimelodus rita Hamilton, 1822, apparently to avoid Stricklandian tautonymy.

Distribution: Rivers of northern India, Pakistan, Bangladesh and Nepal (Jayaram, 1999).

Rita sacerdotum Anderson, 1879

Rita sacerdotum Anderson, 1879: 864, pl. 79 (fig. 3). Type locality: Irrawaddy River at location of pagoda of Thingadow, 3rd Defile of Irrawaddy River, Burma. Holotype: BMNH 1875.8.4.7 (stuffed).

Distribution: Irrawaddy River basin, Myanmar (Ferraris, 1999b).

Remarks: Redescribed in Ferraris (1999b).

\section{Species inquirenda, Rita}

Arius cruciger Owen, 1853: 26. Type locality: Ganges. Type (s): Whereabouts unknown.

SPERATA Holly, 1939

Macrones Duméril, 1856: 484. Type species: Bagrus lamarrii Valenciennes, 1840. Type by monotypy. Gender: Masculine. Preoccupied by Macrones Newman, 1841, in Coleoptera; replaced by Aoria Jordan, 1917, and by Sperata Holly, 1939.

Aoria Jordan, 1919b: 341. Type species: Bagrus lamarrii Valenciennes, 1840. Type by being a replacement name. Gender: Feminine. Replacement for Macrones, Duméril, 1856; preoccupied by Macrones Newman, 1841, in Coleoptera. Aoria preoccupied by Aoria Baly, 1863, in Coleoptera; replaced by Aorichthys Wu, 1939, and by Macronichthys White \& Moy-Thomas, 1940.

Sperata Holly, 1939: 143. Type species: Bagrus lamarrii Valenciennes, 1840. Type by being a replacement name. Gender: Feminine. Holly stated that the name was intended as a replacement for Macrones, but incorrectly listed Macrones vittata Bloch as type.

Aorichthys Wu, 1939: 131. Type species: Bagrus lamarrii Valenciennes, 1840. Type by being a replacement name. Gender: Masculine. Replacement for Aoria Jordan, 1919, preoccupied by Aoria Baly, 1863, in Coleoptera.

Macronichthys White \& Moy-Thomas, 1940: 505. Type species: Bagrus lamarrii Valenciennes, 1840. Type by being a replacement name. Gender: Masculine. Replacement for Aoria Jordan, 1919, preoccupied by Aoria Baly, 1863, in Coleoptera.

Osteobagrus Jayaram, 1954: 529, 547. Type species: Pimelodus aor Hamilton, 1822. Type by original designation. Gender: Masculine. Originally proposed as a subgenus of Mystus.

Remarks: Ferraris \& Runge (1999: 400) concluded that Sperata has priority over Aorichthys. See also Jayaram (1968a, 1971, 1973b).

Revision: Ferraris \& Runge (1999).

Sperata acicularis Ferraris \& Runge, 1999

Sperata acicularis Ferraris \& Runge, 1999: 403, figs. 2a, 3, 4. Type locality: Myanmar, Yangon Division, South Oak-ka-lar-pa market (eastern Yangon). Holotype: CAS 209024.

Distribution: Irrawaddy, Bago and Tenasserim River systems, Myanmar (Ferraris \& Runge, 1999).

Sperata aor (Hamilton, 1822)

Pimelodus aor Hamilton, 1822: 205, 379, pl. 20 (fig. 68). Type locality: Rivers of Bengal, and upper parts of the Gangetic estuaries. No types known. [Illustration of type reproduced in Ferraris \& Runge (1999: fig. 1)].

Bagrus aorides Jerdon, 1849: 336. Type locality: Cauvery River at Errode, southern India. No types known. 
Distribution: Ganges River system and peninsular India south to Cauvery River basin (Ferraris \& Runge, 1999).

Sperata aorella (Blyth, 1858)

Bagrus aorellus Blyth, 1858: 283. Type locality: Calcutta bazar. No types known.

Distribution: Ganges River, India and Bangladesh (Ferraris \& Runge, 1999).

Sperata sarwari (Mirza, Nawaz \& Javed, 1992)

Aorichthys aor sarwari Mirza, Nawaz \& Javed, 1992: 211, fig. 1. Type locality: Ravi River at Head Balloki, Kasur Dist., Pakistan. Holotype: GCM F-19.

Distribution: Indus River basin (Mirza, 2003).

Sperata seenghala (Sykes, 1839)

Platystoma seenghala Sykes, 1839: 164. Type locality: Deccan, India. Type(s): at BMNH. Also proposed as new in Sykes (1840: 61); illustrated and described in more detail in Sykes (1841: 371, pl. 65, fig. 2) [illustration reproduced in Ferraris \& Runge (1999: fig. 6)].

Bagrus Lamarrii Valenciennes, in Cuvier \& Valenciennes, 1840a: 407 (302 of Strasbourg deluxe edition), pl. 415. Type locality: [Ganges]. Holotype: MNHN a-9343 (mounted).[Illustration of holotype reproduced in Ferraris \& Runge (1999: fig. 7)].

Bagrus aorinus Valenciennes, 1840, in Jacquemont, 1835-44: pl. 17 (fig. 1-1a). Type locality: India. Holotype: Whereabouts unknown. Name available from plate caption. [Illustration of holotype reproduced in Ferraris \& Runge (1999: fig. 8).]

Distribution: Ganges River system and peninsular India at least as far south as the Krishna River (Ferraris \& Runge, 1999).

\section{Genus inquirendum, Bagridae}

Adelopeltis Dabry de Thiersant, 1872: 189, 190. Type species: Apparently not designated previously; Adelopeltis angusticeps Dabry de Thiersant, 1872, designated herein. Gender: Feminine.

\section{Species inquirendae, Bagridae}

Bagrus affinis Jerdon, 1849: 338. Type locality: Neighborhood of Madras, southern India. No types known.

Bagrus agricolus Jerdon, 1849: 339. Type locality: Ditches and inundated paddy fields in the Wynaad, southern India. No types known.

Adelopeltis angusticeps Dabry de Thiersant, 1872: 189, pl. 47 (fig. 3). Type locality: Yang-tsee-kiang [China]. Type (s): Whereabouts unknown.

Pimelodus bilineatus Cuvier, 1829: 293. Type locality: Vishakhapatnam [Vizagapatam], India. Available from the following reference to a published illustration: "Pimel. bilineatus, Deddi-Jallah., Russel, 169 ” [= Russell (1803: pl. 169)].

Pseudobagrus eupogoides Wu, 1930b: 51, fig. 3. Type locality: Sze-Chwan, China. Holotype: Whereabouts unknown. Name not treated in recent literature.

Pseudobagrus fui Miao, 1934: 217, fig. 45. Type locality: Chinkiang, southern Kiangsu, China. Holotype: Mus. Biol. Lab. Sci. Soc. China 13170.

Liocassus hirsutus Herre, 1934: 285. Type locality: Market at Wuchow, Kwangsi, China. Holotype: SU 13884. Name not treated in recent literature.

Aoria hoi Pellegrin \& Fang, 1940: 341, fig. 2. Type locality: Foochow, China. Holotype: MNHN 1939-0033.

Leiocassis hwanghoensis Mori, 1933: 167, figs. 3-4. Type locality: Hwang-ho near Tsi-nan, Shantung, China. Holotype: Whereabouts unknown.

Leiocassis kaifenensis Tchang, 1934: 41. Type locality: Kaifeng, Henan, China. Holotype: ASIZB [= ZMFMIB 8625]. Not treated since description.

Adelopeltis laticeps Dabry de Thiersant, 1872: 190, pl. 48 (fig. 5). Type locality: Yang-tsee-kiang [China]. No types known. Not treated in recent literature.

Bagrus limbatus Richardson 1846: 283.Type locality: Canton. Holotype: at Cambridge Philosophical Inst. Sometimes 
incorrectly considered as Plotosus limbatus Valenciennes, but the description in Richardson is clearly that of a bagrid.

Liocassis naso Garman, 1912: 123. Type locality: Hupei: Ichang, China. Holotype: MCZ 29847. Name not treated in recent literature.

Bagrus (Bagrus) ramentosus Müller \& Troschel, 1849: 7. Type locality: America [probably Java]. Holotype: ZMB 2949.

Treated as a synonym of Pseudobagrus aurantiacus or as valid as Pelteobagrus ramentosus.

Aoria rendahli Pellegrin \& Fang, 1940: 340, fig. 1. Type locality: Ou Tchang, Hou Pé [Hubei], China. Holotype: MNHN 1939-0032. Name not treated in recent literature.

Leiocassis similis Nichols, 1926a: 1, fig. 1. Type locality: Min R., near Yenping (Nanping), Fukien Prov., China. Holotype: AMNH 8444.

Bagrus sinensis Brandt, in Mack, 1861: 195. Possibly name only and not available; from Sungatschi.[Not seen]

Leiocassis sinyanensis Fu, 1935: 178, fig. 1. Type locality: Szewangshan, Sinyang [Xinyang], s. Honan [Henan], China. Holotype: Whereabouts unknown.

Hemibagrus taphrophilus Sauvage \& Dabry de Thiersant, 1874: 6. Type locality: Canaux du Tschuang occidental, China. Type(s) (100 mm): at MNHN.

Liocassis torosilabris Sauvage \& Dabry de Thiersant, 1874: 7. Type locality: Yang-tsee-kiang, China. Holotype: MNHN 0000-5936.

Pseudobagrus wangi Miao, 1934: 215, fig. 44. Type locality: Chinkiang, southern Kiangsu, China. Holotype: Mus. Biol. Lab. Sci. Soc. China 13173. Treated as valid in Pelteobagrus by Jayaram (1968b: 306).

Pseudobagrus wittenburgii Popta, 1911: 335, unnumbered figure. Type locality: Amur at Blagoveshchensk, Russia. Syntypes: SMNS 4390 (2).

$\dagger$ Aoria lacus [No other information, taken from Gayet \& Meunier (2003)].

Distribution: Eocene; Hunan, southcentral China (Chang \& Zhou, 1993).

Remarks: Considered to be Bagridae S.L. by Gayet \& Meunier (2003).

\section{CALLICHTHYIDAE Bonaparte, 1838}

Callichthini Bonaparte, 1838: 131. Type genus: Callichthys Scopoli, 1777.

Aspidoradidi Hoedeman, 1952b: 4. Type genus: Aspidoras Ihering, 1907.

Cascaduridi Hoedeman, 1952b: 3. Type genus: Cascadura Ellis, 1913.

Corydoradinae Hoedeman, 1952b: 4. Type genus: Corydoras La Cepède, 1803.

Dianemidi Hoedeman, 1952b: 4. Type genus: Dianema Cope, 1871.

Hoplosterninae Miranda Ribeiro, 1959: 1. Type genus: Hoplosternum Gill, 1858.

Taxonomic summary: Isbrücker (2001b, Corydoradinae), Reis (2003).

Phylogeny: Reis (1998a); Britto (2003, Corydoradinae); Shimabukuro-Dias et al. (2004).

Biogeography: Reis (1998b).

8 genera, 194 species, one fossil species.

\section{ASPIDORAS}

Aspidoras Ihering, 1907: 30. Type species: Aspidoras rochai Ihering, 1907. Type by original designation. Gender: Masculine.

Revision: Nijssen \& Isbrücker (1976a).

Aspidoras albater Nijssen \& Isbrücker, 1976

Aspidoras albater Nijssen \& Isbrücker, 1976a: 115, fig. 6. Type locality: Brazil, Est. Goiás, Rio Tocantinzinha near São João da Aliança, 14²46'S, 47³0'W, Rio Tocantins system. Holotype: MZUSP 12991.

Distribution: Upper Tocantins River basin, Brazil (Reis, 2003).

Aspidoras belenos Britto, 1998

Aspidoras belenos Britto, 1998: 361, fig. 1. Type locality: Brazil: Estado do Mato Grosso, creek at Primavera do Leste- Paranatinga road, $82 \mathrm{~km}$ N from Primavera do Leste, rio das Mortes basin, $15^{\circ} 03^{\prime} \mathrm{S}, 52^{\circ} 03^{\prime} \mathrm{W}$. Holotype: 
MNRJ 12433.

Distribution: Das Mortes River basin, Araguaia River drainage, Brazil (Reis, 2003).

Aspidoras brunneus Nijssen \& Isbrücker, 1976

Aspidoras brunneus Nijssen \& Isbrücker, 1976a: 116, fig. 7. Type locality: Brazil, Est. Mato Grosso, Serra do Roncador, km 125 of the road Chavantina-Casximba. Holotype: ZMA 113588.

Distribution: Upper Araguaia River basin, Brazil (Reis, 2003).

Aspidoras carvalhoi Nijssen \& Isbrücker, 1976

Aspidoras carvalhoi Nijssen \& Isbrücker, 1976a: 117, fig. 8. Type locality: Brazil, Est. Ceará, Açude Canabrava, Guaramiranga. Holotype: MNRJ 5230.

Distribution: Ceará State, Brazil (Reis, 2003).

Aspidoras depinnai Britto, 2000

Aspidoras depinnai Britto, 2000: 1049, fig. 1. Type locality: Brazil: Pernambuco State, creek at Amaraji-Primavera road, rio Ipojuca basin, $08^{\circ} 21^{\prime} \mathrm{S}, 35^{\circ} 26^{\prime} \mathrm{W}$. Holotype: MZUSP 56214.

Distribution: Ipojuca River basin, Brazil (Reis, 2003).

Aspidoras eurycephalus Nijssen \& Isbrücker, 1976

Aspidoras eurycephalus Nijssen \& Isbrücker, 1976a: 118, fig. 9. Type locality: Brazil, Est. Goiás, Rio Tocantins system, Corrego Vermelho into Rio das Almas, tributary of Rio Maranhão, about $15^{\circ} \mathrm{S}, 49^{\circ} 30^{\prime} \mathrm{W}$. Holotype: CAS 16010 .

Distribution: Upper Tocantins River basin, Brazil (Reis, 2003).

Aspidoras fuscoguttatus Nijssen \& Isbrücker, 1976

Aspidoras fuscoguttatus Nijssen \& Isbrücker, 1976a: 118, fig. 10. Type locality: Brazil, Est. Mato Grosso, Rio Paraná system, Córrego Corguinho, estrada da Três Lagoas $\left(20^{\circ} 46^{\prime} \mathrm{S}, 51^{\circ} 43^{\prime} \mathrm{W}\right)$ - Conceição do Taboado. Holotype: MZUSP 8573.

Distribution: Upper Paraná River basin, Brazil (Reis, 2003).

Aspidoras lakoi Miranda Ribeiro, 1949

Aspidoras lakoi Miranda Ribeiro, 1949a: 143, fig. 1. Type locality: Pequeno, córrego na floresta do Grotão, Fazenda da Cachoeira, Município de Passos, Estado de Minas Gerais [Brazil]. Holotype: MNRJ 5292.

Distribution: Upper Paraná River basin, Brazil (Reis, 2003).

Aspidoras maculosus Nijssen \& Isbrücker, 1976

Aspidoras maculosus Nijssen \& Isbrücker, 1976a: 119, fig. 11. Type locality: Brazil, Est. Bahia, Rio Paiaia, into headwaters of Rio Itapicurú, small, rocky, rapid stream from Serra Jacobina between Bom Fim and Jacobina, about $11^{\circ} \mathrm{S}, 40^{\circ} 30^{\prime} \mathrm{W}$. Holotype: FMNH 54810.

Distribution: Itapicurú River basin, Brazil (Reis, 2003).

Aspidoras menezesi Nijssen \& Isbrücker, 1976

Aspidoras menezesi Nijssen \& Isbrücker, 1976a: 120, fig. 12. Type locality: Brazil, Est. Ceará, Rio Granjeiro at Crato, $7^{\circ} 10^{\prime} \mathrm{S}, 39^{\circ} 25^{\prime} \mathrm{W}$, tributary on left bank of Rio Salgado. Holotype: UMMZ 147336.

Distribution: Coastal rivers in Ceará State, Brazil (Reis, 2003).

Aspidoras microgalaeus Britto, 1998

Aspidoras microgalaeus Britto, 1998: 364, fig. 8. Type locality: Brazil: Estado do Mato Grosso, small tributary of rio Culuene, $\mathrm{km} 86$ of Paranatinga-Canarana road, rio Xingú basin, $14^{\circ} 00^{\prime} \mathrm{S}, 54^{\circ} 38^{\prime} \mathrm{W}$. Holotype: MZUSP 51209.

Distribution: Xingu River basin, Brazil (Reis, 2003).

Aspidoras pauciradiatus (Weitzman \& Nijssen, 1970)

Corydoras pauciradiatus Weitzman \& Nijssen, 1970: 129, fig. 5. Type locality: Brazil, Rio Araguaia, near Aruanã, Est. Goiás (1458'S, 51ํํ'W). Holotype: USNM 191625.

Distribution: Upper Araguaia River basin, Brazil (Reis, 2003).

Aspidoras poecilus Nijssen \& Isbrücker, 1976

Aspidoras poecilus Nijssen \& Isbrücker, 1976a: 121, fig. 13. Type locality: Brazil, Est. Mato Grosso, creek upstream of village Porori, left bank of Rio Xingu, upper Rio Xingu. Holotype: IRSNB 560.

Distribution: Upper Xingu, Araguaia and Tocantins River basins, Brazil (Reis, 2003). 
Aspidoras psammatides Britto, Lima \& Santos, 2005

Aspidoras psammatides Britto, Lima \& Santos, 2005: 474, fig. 1. Type locality: Brazil, Estado da Bahia, município de Lençóis, rio Caldeirão, 12³9'33"S 41²2'12"W. Holotype. MNRJ 28407.

Distribution: Upper Paraguaçu River basin, eastern Brazil (Britto et al., 2005).

Aspidoras raimundi (Steindachner, 1907)

Corydoras raimundi Steindachner, 1907a: 84. Type locality: in dem Bächchen, welches bei Victoria in den Rio Parnahyba mündet [Brazil]. Lectotype: NMW 61110; designated by Nijssen \& Isbrücker (1976a: 111).

Distribution: Parnaíba River basin, Brazil (Reis, 2003).

Aspidoras rochai Ihering, 1907

Aspidoras rochai Ihering, 1907: 30. Type locality: Fortaleza, capital of the state of Ceará [Brazil]. Lectotype: MZUSP 2195; designated by Britski (1969: 206).

Distribution: Coastal rivers in Ceará State, Brazil (Reis, 2003).

Aspidoras spilotus Nijssen \& Isbrücker, 1976

Aspidoras spilotus Nijssen \& Isbrücker, 1976a: 123, fig. 14. Type locality: Brazil, Est. Ceará, Riacho dos Macacos, tributary of Rio Acaráu. Holotype: ZMA 113590.

Distribution: Coastal rivers in Ceará State, Brazil (Reis, 2003).

Aspidoras taurus Lima \& Britto, 2001

Aspidoras taurus Lima \& Britto, 2001: 1011, fig. 1. Type locality: Brazil: Mato Grosso State, município de Alto Garças, Fazenda Cabeceira do Itiquira, close to highway BR-364, rio Itiquira, rio Paraguay system, $16^{\circ} 56^{\prime} \mathrm{S}$ $53^{\circ} 32^{\prime}$ W. Holotype: MZUSP 57154.

Distribution: Upper Paraguay River basin, Brazil (R. Reis, pers. commun., 2004).

Aspidoras velites Britto, Lima \& Moreira, 2002

Aspidoras velites Britto, Lima \& Moreira, 2002: 728, fig. 1. Type locality: Brazil. Estado do Mato Grosso. Município de Alto Araguaia, córrego Boiadeiro, km 487.08 of Ferronorte railroad, 17²0'01"S, 5314'53"W. Holotype: MZUSP 74447.

Distribution: Small tributaries of upper Araguaia River in Mato Grosso State, Brazil (Reis, 2003).

Aspidoras virgulatus Nijssen \& Isbrücker, 1980

Aspidoras virgulatus Nijssen \& Isbrücker, 1980c: 133, fig. 1. Type locality: Brazil: Est. Espírito Santo. Holotype: MNRJ 5371.

Distribution: Coastal rivers in Espírito Santo State, Brazil (Reis, 2003).

\section{CALLICHTHYS}

Calichthys Scopoli, 1777: 451. Type species: Silurus callichthys Linnaeus, 1758. Gender: Masculine. Original spelling not used subsequently and spelling in prevailing usage, Callichthys, is adopted here following ICZN (1999: Art. 33.3.1).

Callichthys Meuschen, 1778: 39. Type species: Silurus callichthys Linnaeus, 1758. Gender: Masculine.

Callichthys Linck, 1790: 32. Type species: Silurus callichthys Linnaeus, 1758. Gender: Masculine.

Cataphractus Bloch, 1794: 80. Type species: Silurus callichthys Linnaeus, 1758. Type by subsequent designation. Gender: Masculine.

Phylogeny: Reis (1997).

Key to species: Lehmann \& Reis (2004).

Remarks: Callichthys was first published by Gronow (1763: 127) in a work that was subsequently placed on the ICZN Official Index of Rejected and Invalid Generic Names in Zoology, Opinion 261 (Melville \& Smith, 1987), which makes the name unavailable from that publication.

Callichthys callichthys (Linnaeus, 1758)

Silurus callichthys Linnaeus, 1758: 307. Type locality: in Americae rivulis. Syntypes: On Callichthys of Gronovius, which may be based on BMNH 1853.11.12.194 (1, left half skin), and specimens from the Linnaean collection: NRM 7015 (2), NRM 7018 (1), ZMUU 55 (1).

Callichthys asper Quoy \& Gaimard, 1824: 232. Type locality: ruissean qui coule devant la ferme de Santa-Anna, sur le chemin qui conduit de Rio de Janeiro à la colonie suisse [Brazil]. Syntypes: MNHN 0000-1951 (2). 
Cataphractus depressus Swainson, 1839: 304. Type locality: [Not stated]. Type(s): Based on Bloch (1794: pl. 377) [= Cataphractus callichthys (Linnaeus)].

Callichthys laviceps Valenciennes, in Cuvier \& Valenciennes, 1840b: 309 (229 in the Strasbourg deluxe edition). Type locality: La Mana, .... Brésil. Syntypes: MNHN 0000-1947 (1), MNHN 0000-4261 (1).

Callichthys calatus Valenciennes, in Cuvier \& Valenciennes, 1840b: 308 (229 in the Strasbourg deluxe edition). Type locality: Bahia, .... Rio-Janéiro. Syntypes: MNHN 0000-4258 (2), MNHN 0000-4259 (1).

Callichthys loricatus Gronow, in Gray, 1854: 157. Type locality: Americes Meridionalis rivulis. Syntypes: BMNH 1853.11.12.194 (1) and several literature sources.

Callichthys kneri Gill, 1858: 394. Type locality: Trinidad. Type(s): Whereabouts unknown, apparently not at USNM.

Callichthys tamoata Bleeker, 1864a: 22. Type locality: Surinama. Syntypes (10, 114- $250 \mathrm{~mm})$ : at RMNH, and many literature sources.

Callichthys affinis Günther, 1864: 226. Type locality: Rio Grande [Brazil]. Syntypes: BMNH 1861.5.7.1-4 (4).

Callichthys arcifer Hensel, 1868: 373. Type locality: Rio de Janeiro. Holotype: ZMB 7433.

Callichthys hemiphractus Hensel, 1868: 374. Type locality: Costa da Serra [Rio Grande do Sul, Brazil]. Holotype: ZMB 7434.

Callichthys callichthys bolteni Hoedeman, 1952b: 9. Type locality: Surinam. Holotype: ZMA 100303a.

Callichthys callichthys demararae Hoedeman, 1952b: 10. Type locality: Demarara, British Guiana. Holotype: ZMA 100304.

Distribution: Most Cis-Andean South American river drainages north of Buenos Aires (Reis, 2003).

Callichthys fabricioi Román-Valencia, Lehmann-A \& Muñoz, 1999

Callichthys fabricioi Román-Valencia, Lehmann-A \& Muñoz, 1999: 54, fig. 1. Type locality: Colombia, Departamento del Cauca, Municipio de Buenos Aires, Zanjón Bagazal afluente del Río Cauca 980 meters (0303'59"N y 76³3'55"W). Holotype: IUQ 295.

Distribution: Cauca River basin, Colombia (Reis, 2003).

Callichthys oibaensis Ardilia Rodríguez, 2005

Callichthys oibaensis Ardilia Rodríguez, 2005: 32, figs. 1-6. Type locality: [Colombia, Vereda Las Monjas, río Oibita, trib. of río Suárez]. Holotype: CAR 251.

Distribution: Suárez River basin, Colombia.

Callichthys serralabium Lehmann \& Reis, 2004

Callichthys serralabium Lehmann \& Reis, 2004: 339, fig. 1. Type locality: Venezuela: Estado Amazonas: La Esmeralda: Laguna Tonina, $1 \mathrm{~km}$ upstream from La Esmeralda, upper Orinoco (030' $\left.00^{\prime \prime} \mathrm{N}, 065^{\circ} 32^{\prime} 00^{\prime \prime} \mathrm{W}\right)$. Holotype: MBUCV V-30980.

Distribution: Upper Orinoco and upper Negro River basins, Venezuela and Brazil (Lehmann \& Reis, 2004: 341 ).

\section{CORYDORAS}

Corydoras La Cepède, 1803: 147. Type species: Corydoras geoffroy La Cepède, 1803. Type by monotypy. Gender: Masculine.

Cordorinus Rafinesque, 1815: 89. Type species: Corydoras geoffroy La Cepède, 1803. Type by being a replacement name. Gender: Masculine. Unjustified emendation of Corydoras La Cepède.

Hoplisoma Swainson, 1838: 336. Type species: Cataphractus punctatus Bloch, 1794. Type by original designation. Gender: Neuter.

Brochis Cope, 1871b: 112. Type species: Brochis coeruleus Cope, 1872. Type by subsequent designation by Cope (1872a). Gender: Feminine. Appeared first without species, more fully described in Cope (1872a) with the description of two new species; type designated by Cope (description of Brochis dipterus includes reference to B. coeruleus as type).

Chaenothorax Cope, 1878: 679. Type species: Chaenothorax bicarinatus Cope, 1878. Type by original designation. Gender: Masculine.

Gastrodermus Cope, 1878: 681. Type species: Corydoras elegans Steindachner, 1877. Type by subsequent designation. Gender: Masculine. Type apparently first designated by Gosline (1940b: 10). 
Osteogaster Cope, 1894: 102. Type species: Corydoras eques Steindachner, 1877. Type by original designation. Gender: Feminine.

Microcorydoras Myers, 1953: 270. Type species: Corydoras hastatus Eigenmann \& Eigenmann, 1888. Type by original designation. Gender: Masculine.

Review: Nijssen \& Isbrücker (1983b, Colombia); Castro (1987, Colombia); Nijssen \& Isbrücker (1986b, Peru and Ecuador).

Taxonomic summary: Nijssen \& Isbrücker (1970), species formerly placed in Brochis; Nijssen \& Isbrücker (1980b), Isbrücker (2001b).

Remarks: Brochis Cope was recently placed in synonymy of Corydoras by Britto (2003), while Scleromystax was removed and treated as a valid genus.

Corydoras acrensis Nijssen, 1972

Corydoras acrensis Nijssen, 1972: 416, fig. 1. Type locality: Brazil, Est. Acre, Furo do Lago São Francisco, tributary at left bank of Rio Juruá, upstream of confluence with Mao at Cruzeiro do Sul. Holotype: IRSNB 505.

Distribution: Juruá River basin, Brazil (Reis, 2003).

Corydoras acutus Cope, 1872

Corydoras acutus Cope, 1872a: 281. Type locality: Ambyiacu River [now: Shansho Caño, Loreto, Peru, by neotype designation]. Neotype: SU 33307, designated by Nijssen \& Isbrücker (1980b: 215); original syntypes ANSP 8292-8293 lost.

Distribution: Amazon River basin in northern Peru and Ecuador (Reis, 2003).

Corydoras adolfoi Burgess, 1982

Corydoras adolfoi Burgess, 1982: 15, fig. 1. Type locality: Small tributary of the upper Rio Negro on the equator near São Gabriel da Cachoeira, Brazil. Holotype: MZUSP 26641.

Distribution: Negro River basin, Brazil (Reis, 2003).

Corydoras aeneus (Gill, 1858)

Hoplosoma aeneum Gill, 1858: 403. Type locality: Island of Trinidad, West Indies. Lectotype: USNM 1116, designated by Nijssen \& Isbrücker (1980b: 208).

Corydoras microps Eigenmann \& Kennedy, 1903: 506. Type locality: From a small lagoon, half dry, near the Rio Branco (Mato Grosso, Brazil). Holotype: CAS 20662.

Corydoras venezuelanus Ihering, 1911: 383. Type locality: Río Cabriales, Valencia, Est. Carabobo, Venezuela. Lectotype: MZUSP 146, designated by Britski (1969: 208).

Corydoras macrosteus Regan, 1912a: 219. Type locality: Rio Piracicaba, San Paulo, Brazil. Lectotype: BMNH 1905.6.9.12, designated by Nijssen \& Isbrücker (1980b: 208).

Corydoras schultzei Holly, 1940: 111. Type locality: Aquarium specimen (said to be from very small water-courses of the Amazon).

Distribution: Widely distributed in South America on the eastern side of the Andes, from Colombia and Trinidad to La Plata River basin (Reis, 2003).

Remarks: Isbrücker (2001b) treated all of the synonyms listed above as valid, or "doubtfully valid" species, but did not comment on the distribution of these species.

Corydoras agassizii Steindachner, 1877

Corydoras Agassizii Steindachner, 1877a: 138, pl. 12 (fig. 2). Type locality: Amazonenstrom bei Tabatinga. Lectotype: NMW 61112, designated by Nijssen \& Isbrücker (1980b: 195).

Distribution: Amazon River basin near border between Peru and Brazil (Reis, 2003).

Corydoras albolineatus Knaack, 2004

Corydoras albolineatus Knaack, 2004: 73, figs. 13-17. Type locality: Bolivien, Departamento Santa Cruz, Provincia J.M. Velasco, Raum Piso firme, System río Itenez, río Paragua (Mündungsgebiet, NW-Ufer), 13³1.989'S, 6150.295'W. Holotype: MTD F 28470.

Distribution: Itenez River basin, Bolivia (Knaack, 2004).

Corydoras amandajanea Sands, 1995

Corydoras amandajanea Sands, 1995: 10, fig. 1. Type locality: Rio Miuã system of streams, possibly the Uarinabe stream (Miuã system) 10-12 kilometers away from its confluence of the upper Negro River, Brazil. Holotype: 
LIVCM 1994.4.21.

Distribution: Upper Negro River basin, Brazil (Reis, 2003).

Corydoras amapaensis Nijssen, 1972

Corydoras amapaensis Nijssen, 1972: 417, figs. 2, 3. Type locality: Brazil, Est. Amapa, Cachoera Creek at right bank of Río Amapari, 4 km downstream of Casa do 7. Holotype: IRSNB 476.

Distribution: Oyapock and Amapari rivers in Amapá State in Brazil and French Guiana (Reis, 2003).

Corydoras ambiacus Cope, 1872

Corydoras ambiacus Cope, 1872a: 280. Type locality: Ambyiacu River. Holotype: ANSP 8291.

Corydoras grafi Holly, 1940: 108. Type locality: Aquarium specimen (said to be from very small water-courses of the Amazon). Holotype: Münchner Tierpark AG 92/139 (reportedly lost, but possibly NMW 84287).

Corydoras melanistius longirostris Hoedeman, 1952a: 17, fig. 13. Type locality: Amazon area. No types known.

Distribution: Upper Amazon River basin of Brazil, Colombia, and Peru (Reis, 2003).

Corydoras amphibelus Cope, 1872

Corydoras amphibelus Cope, 1872a: 282. Type locality: Ambyiacu River. Holotype: ANSP 8290.

Distribution: Upper Amazon River basin in Peru (Reis, 2003).

Corydoras approuaguensis Nijssen \& Isbrücker, 1983

Corydoras approuaguensis Nijssen \& Isbrücker, 1983c: 73, fig. 1. Type locality: fleuve Approuague. Holotype: ZMA 119098.

Distribution: Approuague River basin, French Guiana (Reis, 2003).

Corydoras araguaiaensis Sands, 1990

Corydoras araguaiaensis Sands, 1990: [1], unnumbered figure. Type locality: Rio Araguaia, Brazil. Holotype: at RMNH.

Distribution: Araguaia River basin, Brazil (Reis, 2003).

Corydoras arcuatus Elwin, 1939

Corydoras arcuatus Elwin, 1939: 126, pl. 3. Type locality: Amazon River (aquarium specimen). Holotype: BMNH 1939.3.3.1.

Distribution: Upper Amazon River basin (Reis, 2003).

Corydoras areio Knaack, 2000

Corydoras areio Knaack, 2000b: 47, unnumbered figure. Type locality: Brasilien, Mato Grosso (östlich von Cuiaba), in Fließgewässern des Córr. Areio-Systems, in der Umgebung von Vila Nova, an der Straße 373, ca. 8.5 kilometers und ca.13.5 km von der Ortschaft aus entfernt in Richtung Poxoreo. Holotype: ZMB 33113.

Distribution: Upper Paraguay River basin, Brazil (Reis, 2003).

Corydoras armatus (Günther, 1868)

Callichthys armatus Günther, 1868a: 476. Type locality: Xeberos and Huallaga [Peru]. Lectotype: BMNH 1867.6.13.51, designated by Nijssen \& Isbrücker (1980b: 194). Illustrated and described in more detail in Günther (1868b: 231, fig. 1).

Distribution: Upper Amazon River basin, Peru (Reis, 2003).

Corydoras atropersonatus Weitzman \& Nijssen, 1970

Corydoras atropersonatus Weitzman \& Nijssen, 1970: 123, fig. 2. Type locality: Ecuador, Río Conambo at mouth of Río Shione (about $2^{\circ} 00^{\prime} \mathrm{S}, 76^{\circ} 30^{\prime} \mathrm{W}$ ), Río Tigre system, Est. Pastaza. Holotype: USNM 204359.

Distribution: Upper Amazon River basin, Ecuador and Peru (Reis, 2003).

Corydoras aurofrenatus Eigenmann \& Kennedy, 1903

Corydoras aurofrenatus Eigenmann \& Kennedy, 1903: 507. Type locality: Aguada, near Arroyo Trementina [Paraguay]. Holotype: CAS 20661.

Distribution: Paraguay River basin, Paraguay (Reis, 2003).

Corydoras axelrodi Rössel, 1962

Corydoras axelrodi Rössel, 1962c: 335, figs. 1, 2. Type locality: Columbien, Rio Meta. Holotype: SMF 5700.

Distribution: Meta River basin, Colombia (Reis, 2003).

Corydoras baderi Geisler, 1969

Corydoras baderi Geisler, 1969: 354, fig. 1. Type locality: Brasilien, Staat Para, Rio Paru de Oeste und Bäche bei 
der Missionsstation Tirió ( $\left.2^{\circ} 12^{\prime} \mathrm{N}, 55^{\circ} 59^{\prime} \mathrm{W}\right)$. Holotype: SMF 9651.

Corydoras oelemariensis Nijssen, 1970: 29, fig. 14. Type locality: Creek at left bank of Oelemari River, near airstrip, Marowijne, Suriname. Holotype: IRSNB 472.

Distribution: Pará State in Brazil and Maroni River in Suriname (Reis, 2003).

Corydoras bicolor Nijssen \& Isbrücker, 1967

Corydoras bicolor Nijssen \& Isbrücker, 1967: 36, pl. 3 (fig. 3). Type locality: Sipaliwini River. Holotype: ZMA 104627.

Distribution: Coastal rivers of Suriname (Reis, 2003).

Corydoras bifasciatus Nijssen, 1972

Corydoras bifasciatus Nijssen, 1972: 420, fig. 4. Type locality: Brazil, Est. Para, creek at left bank of Río Cururu, tributary of upper Río Tapajós near Maloca do Conzalo (about 745'S, 5800'W). Holotype: IRSNB 485.

Distribution: Lower Amazon River basin, Brazil (Reis, 2003).

Corydoras bilineatus Knaack, 2002

Corydoras bilineatus Knaack, 2002a: 52, unnumbered figure. Type locality: Santa Cruz, Santiesteban, Chané, Restgewässer eines großen Überflutungs-Nährungsgebietes zwischen den Flüssen Rio Piray und Rio Yapacarie und dem Rio Grande o Guapay mit abfluss (Regenzeit) in den Arroyo Chanè. Holotype: MTD F 25925.

Distribution: Madeira River basin, Bolivia (Reis, 2003).

Corydoras blochi Nijssen, 1971

Corydoras blochi Nijssen, 1971: 92, fig. 2. Type locality: Guyana, district Essequibo, Moco Creek near Lehtem, tributary of Rio Tacutú, Rio Branco system. Holotype: FMNH 75951.

Distribution: Venezuela, Guyana, and northern Brazil (Reis, 2003).

Corydoras boehlkei Nijssen \& Isbrücker, 1982

Corydoras boehlkei Nijssen \& Isbrücker, 1982: 139, fig. 1. Type locality: Venezuela, Edo Bolivar, Río Cuchime (Cusimo), about 20 miles upstream from junction of Río Caura - Río Erebato (Entre Rios) at 5 $5^{\circ} 45^{\prime} \mathrm{N}, 64^{\circ} 24^{\prime} \mathrm{W}$. Holotype: ANSP 148097.

Distribution: Caura River basin in the Orinoco River drainage, Venezuela (Reis, 2003).

Corydoras boesemani Nijssen \& Isbrücker, 1967

Corydoras boesemani Nijssen \& Isbrücker, 1967: 37, pl. 4 (fig. 1). Type locality: Little tributaries of Gran-Rio between Ligolio and Awaradam Falls [Suriname]. Holotype: RMNH 25316.

Distribution: Coastal rivers in Suriname (Reis, 2003).

Corydoras bondi Gosline, 1940

Corydoras bondi Gosline, 1940b: 20. Type locality: Rio Yuruari, 3 km east of El Callao, Venezuela. Holotype: SU 35065.

Distribution: Rio Yuruari in Venezuela, Corantijn and Rupununi River basins, Guianas (Reis, 2003).

Corydoras breei Isbrücker \& Nijssen, 1992

Corydoras breei Isbrücker \& Nijssen, 1992: 10, fig. 1. Type locality: Surinam, Corantijn river system, Kabalebo River, Avanavero Falls. Holotype: RMNH 31912.

Distribution: Corantijn River basin, Suriname (Reis, 2003).

Corydoras brevirostris Fraser-Brunner, 1947

Corydoras melanistius brevirostris Fraser-Brunner, 1947: 244. Type locality: aquarium specimen [said to be from the Orinoco]. Holotype: BMNH 1946.10.10.1.

Distribution: Orinoco River basin, Venezuela, and coastal drainages in Suriname (Reis, 2003).

Corydoras britskii (Nijssen \& Isbrücker, 1983)

Brochis britskii Nijssen \& Isbrücker, 1983a: 179, fig. 1. Type locality: Brazil, Est. Mato Grosso, Lagoas Marginais, rodovia Transpantaneira, Poconé (16 $\left.15^{\circ} \mathrm{S}, 56^{\circ} 37^{\prime} \mathrm{W}\right)$. Holotype: MZUSP 26811.

Distribution: Upper Paraguay River basin, Brazil (Reis, 2003).

Corydoras burgessi Axelrod, 1987

Corydoras burgessi Axelrod, 1987: 22, figures on pp. 22-23. Type locality: reportedly from the Rio Unini, tributary of the Rio Negro, Amazonas, Brazil. Holotype: MZUSP 37692.

Distribution: Upper Negro River basin, Brazil (Reis, 2003). 
Corydoras carlae Nijssen \& Isbrücker, 1983

Corydoras carlae Nijssen \& Isbrücker, 1983c: 76, fig. 5. Type locality: Petit Arroyo, affluent rive gauche du Rio Iguazu, route no. 101 entre Porto Iguazu et Bernardino de Irigoyen, 50 km de Puerto Iguazu, bassin du Paraná. Holotype: IRSNB 688.

Distribution: Lower Iguazu River basin, Argentina (Reis, 2003).

Corydoras caudimaculatus Rössel, 1961

Corydoras caudimaculatus Rössel, 1961: 49, pl. 5 (fig. 1). Type locality: Brasilien, oberer Rio Guaporé (=Itenez). Holotype: SMF 5291.

Distribution: Guaporé River basin, Brazil (Reis, 2003).

Corydoras cervinus Rössel, 1962

Corydoras cervinus Rössel, 1962b: 31, fig. 1. Type locality: Brasilien, oberer Rio Guaporé (=Itenez). Holotype: SMF 5460.

Distribution: Guaporé River basin, Brazil (Reis, 2003).

Corydoras cochui Myers \& Weitzman, 1954

Corydoras cochui Myers \& Weitzman, 1954: 93. Type locality: Santa Maria Nova, Rio Araguaya, State of Goyaz, Brazil. Holotype: SU 47656.

Distribution: Upper Araguaia River basin, Brazil (Reis, 2003).

Remarks: Redescribed in Weitzman (1956).

Corydoras concolor Weitzman, 1961

Corydoras concolor Weitzman, 1961: 105, fig. 1. Type locality: Las Mangas, in a tributary to the Río Parguaza, western part of the State of Bolivar, Venezuela, $6^{\circ} 20^{\prime} \mathrm{N}, 67^{\circ} 10^{\prime} \mathrm{W}$. Holotype: SU 54131.

Distribution: Orinoco River basin, Venezuela (Reis, 2003).

Corydoras condiscipulus Nijssen \& Isbrücker, 1980

Corydoras condiscipulus Nijssen \& Isbrücker, 1980d: 494, fig. 1. Type locality: French Guiana, Cumuri Creek at left bank of Oyapock River, upstream of first rapids of Grande Roche, southwest of village Oiapoque. Holotype: IRSNB 617.

Distribution: Oyapock River basin, Brazil and French Guiana (Reis, 2003).

Corydoras copei Nijssen \& Isbrücker, 1986

Corydoras copei Nijssen \& Isbrücker, 1986a: 70, fig. 11. Type locality: Loreto, cours inférieur du Río Huytoyacu, près du village Nuevo Progresso, affluent de la rive droite du Rio Pastaza, environ 40 km N. du Lago Rimachi (4'13'S, 76³8'W). Holotype: ZMA 119305.

Distribution: Pastaza River basin in Loreto, Peru (Reis, 2003).

Corydoras coppenamensis Nijssen, 1970

Corydoras bondi coppenamensis Nijssen, 1970: 19, fig. 8. Type locality: Creek at left bank of Coppename River $\left(3^{\circ} 52^{\prime} \mathrm{N}, 56^{\circ} 55^{\prime} \mathrm{W}\right)$ [Suriname]. Holotype: ZMA 105877.

Distribution: Coppename River basin, Suriname (Reis, 2003).

Corydoras coriatae Burgess, 1997

Corydoras coriatae Burgess, 1997: 140, unnumbered figure. Type locality: Río Aguaytia, a branch of the Río Ucayali, in jungles of Peru west of the city of Pucallpa. Holotype: MUSM 10701.

Distribution: Ucayali River basin, Peru (Reis, 2003).

Corydoras crimmeni Grant, 1997

Corydoras crimmeni Grant, 1997: 44, fig. 3. Type locality: Aquarium specimen, said to be from Brazil, Roraima State, near the city of Boa Vista, possibly from the Rio Branco. Holotype: MZUSP 52490.

Distribution: Negro River basin, Brazil (Reis, 2003).

Corydoras cruziensis Knaack, 2002

Corydoras cruziensis Knaack, 2002c: 63, unnumbered figure. Type locality: kleinerer Fließgewässer in Santa Cruz, Guarayos, A. Puente [Bolivia]. Holotype: MTD F 25930.

Distribution: Madeira River basin in Santa Cruz and Beni Departments of Bolivia (Reis, 2003).

Corydoras crypticus Sands, 1995

Corydoras crypticus Sands, 1995: 11, fig. 4. Type locality: Rio Miuã system of streams, possibly the Uarinabe 
stream, 10-12 kilometers away from its confluence of the upper Negro River, Brazil. Holotype: LIVCM 1994.4.29.

Distribution: Upper Negro River basin, Brazil (Reis, 2003).

Corydoras davidsandsi Black, 1987

Corydoras davidsandsi Black, 1987: 74, figure on p. 74. Type locality: Reportedly from the Rio Unini, Brazil. Holotype: MZUSP 38632.

Distribution: Negro River basin, Brazil (Reis, 2003).

Corydoras delphax Nijssen \& Isbrücker, 1983

Corydoras delphax Nijssen \& Isbrücker, 1983b: 55, fig. 3. Type locality: Guainía, Río Inírida system, Caño Bacón,

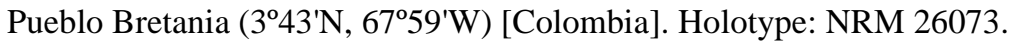

Distribution: Inírida River and upper Orinoco River basin, Colombia (Reis, 2003).

Corydoras difluviatilis Britto \& Castro, 2002

Corydoras difluviatilis Britto \& Castro, 2002: 1007, fig. 1. Type locality: Brazil: São Paulo, Município de Santa Rita do Passa Quatro, Parque Estadual de Vassununga, Glepa Pé-do-Gigante, córrego Paulicéia, tributary of rio Pardo, rio Paraná system, $21^{\circ} 38^{\prime} \mathrm{S}, 47^{\circ} 38^{\prime} \mathrm{W}$. Holotype: MZUSP 75268.

Distribution: Upper Paraná and Grande River basins, Paraná River drainage, and Jequitaí, Preto, and upper Da Prata rivers, São Francisco drainage, Brazil (Reis, 2003).

Corydoras diphyes Axenrot \& Kullander, 2003

Corydoras diphyes Axenrot \& Kullander, 2003: 258, fig. 5. Type locality: Paraguay: Departamento Caaquazú: río Paraná drainage, small sream (arroyo) at Estancia María Belén, $8 \mathrm{~km}$ from Colonel Patricio Colman, 2540'13"S, 555'52"W. Holotype: MNHNP uncat (ex NRM 43483).

Distribution: Monday River and Acaray River, Paraná River basin, Paraguay (Axenrot \& Kullander, 2003).

Corydoras duplicareus Sands, 1995

Corydoras duplicareus Sands, 1995: 12, fig. 6. Type locality: Rio Poranga, 8 kilometers north of the Nobua oba, prior to where it joins a small tributary of the upper Negro River, Brazil. Holotype: LIVCM 1994.4.36.

Distribution: Upper Negro River basin, Brazil (Reis, 2003).

Corydoras ehrhardti Steindachner, 1910

Corydoras ehrhardti Steindachner, 1910a: 60. Type locality: Flußgebiet des Jaraguá [Santa Catarina, Brazil]. Lectotype: NMW 61104, designated by Nijssen \& Isbrücker (1980b: 205).

Corydoras meridionalis Ihering, 1911: 380. Type locality: Colonia Hansa, Est. Santa Catharina [Brazil]. Type(s): Whereabouts unknown.

Distribution: Coastal rivers in Santa Catarina and Paraná States and Iguaçu River basin, Brazil (Reis, 2003).

Corydoras elegans Steindachner, 1877

Corydoras elegans Steindachner, 1877a: 141. Type locality: Teffé [Brazil]. Lectotype: NMW 46729, designated by Nijssen \& Isbrücker (1980b: 213).

Corydoras pestai Holly, 1940: 107. Type locality: in kleinen und kleinsten Wasserläufen der Nähe des Amazonenstromes. Holotype: Münchner Tierpark A.G. 81/1938 (destroyed).

Distribution: Upper Amazon River basin (Reis, 2003).

Remarks: Corydoras pestai Holly, 1940, treated as valid in Isbrücker (2001b: 232).

Corydoras ellisae Gosline, 1940

Corydoras ellisae Gosline, 1940b: 17. Type locality: Arroyo Poná, Sapucay, Paraguay. Holotype: FMNH 57361.

Distribution: Paraguay River basin, Paraguay (Reis, 2003).

Corydoras ephippifer Nijssen, 1972

Corydoras ephippifer Nijssen, 1972: 422, fig. 5. Type locality: Brazil, Est. Amapa, Cachoera Creek at right bank of Río Amapari, 4 kilometers downstream of Casa do 7. Holotype: IRSNM 507.

Distribution: Amapá State, Brazil (Reis, 2003).

Corydoras eques Steindachner, 1877

Corydoras eques Steindachner, 1877a: 140, pl. 12 (fig. 3). Type locality: Amazonenstrom bei Teffé, Cudajas Brazil [restricted to Cudajas by lectotype designation]. Lectotype: NMW 61111, designated by Nijssen \& Isbrücker (1980b: 208). 
Distribution: Amazon River basin, Brazil (Reis, 2003).

Corydoras esperanzae Castro, 1987

Corydoras esperanzae Castro, 1987: 34, fig. 2. Type locality: Caño Orocue at Orocue, Río Meta, Intendencia de Casanare (4º $\left.49^{\prime} \mathrm{N}, 7^{\circ} 20^{\prime} \mathrm{W}\right)$ [Colombia]. Holotype: UBJTL MM275.

Distribution: Meta River basin, Colombia (Reis, 2003).

Corydoras evelynae Rössel, 1963

Corydoras evelynae Rössel, 1963: 360, fig. 4. Type locality: Brazilien, oberer Rio Solimoes. Holotype: SMF 6107.

Distribution: Amazon River basin, Brazil (Reis, 2003).

Corydoras filamentosus Nijssen \& Isbrücker, 1983

Corydoras filamentosus Nijssen \& Isbrücker, 1983c: 77, fig. 6. Type locality: Bassin de la rivière Corantijn, affluent du Sisa Creek, versant nord, environ $700 \mathrm{~m}$ en aval du croisement de Amatopo avec Camp Geologie Road, $03^{\circ} 42^{\prime} \mathrm{N}, 57^{\circ} 42^{\prime} \mathrm{O}$ [Suriname]. Holotype: USNM 225536.

Distribution: Corantijn River basin, Suriname (Reis, 2003).

Corydoras flaveolus Ihering, 1911

Corydoras flaveolus Ihering, 1911: 386. Type locality: Pequenos riachos affluentes do rio Piracicaba, acima do Salto, Est. S. Paulo [Brazil]. Lectotype: MZUSP 424, designated by Britski (1969: 207).

Distribution: Upper Paraná River basin, Brazil (Reis, 2003).

Corydoras fowleri Böhlke, 1950

Corydoras fowleri Böhlke, 1950: 26. Type locality: Chancho Caño, near Pebas (3¹0'S, 7146'W), Peruvian Amazon, Peru. Holotype: SU 16115.

Distribution: Western Amazon River basin (Reis, 2003).

Corydoras garbei Ihering, 1911

Corydoras garbei Ihering, 1911: 382. Type locality: Rio São Francisco, Est. da Bahia [Brazil]. Lectotype: MZUSP 711, designated by Britski (1969: 207) for C. garbei Ihering, not C. garbei of Miranda Ribeiro (1937) [see Remarks].

Distribution: São Francisco River basin, Brazil (Reis, 2003).

Remarks: An account of Corydoras garbei appeared in Miranda Ribeiro, (1937: 54) that has been treated by some as an independent proposal of a new name for this species. Miranda Ribeiro (1953: 394) designated a lectotype for this name and Britski (1969: 207) regarded the name as a homonym and synonym of Corydoras garbei Ihering, 1911. However, it appears that the account was a repeat of the earlier description of the species and, on that basis, treated by Reis (2003) as a lapsus and not a new name proposal.

Corydoras geoffroy La Cepède, 1803

Corydoras geoffroy La Cepède, 1803: 147. Type locality: Suriname, district Brokopondo, Suriname River system, Marchall Creek, east of the road Paranam-Afobaka, $1.5 \mathrm{~km}$ North of Marchall village, by Neotype designation. Neotype: ZMA 106017, designated by Isbrücker (2000: 17).

Corydoras octocirrus Nijssen, 1970: 26, fig. 12. Type locality: Marchall Creek, East of the road Paranam-Afobaka, $1.5 \mathrm{~km}$ North of Marchall village, Suriname River system, Suriname. Holotype: ZMA 106017.

Distribution: Coastal rivers of Suriname and French Guiana (Reis, 2003).

Remarks: Isbrücker (1999a, 2000) discussed the taxonomic history of this species.

Corydoras geryi Nijssen \& Isbrücker, 1983

Corydoras bolivianus Nijssen \& Isbrücker, 1983c: 75, fig. 3. Type locality: Plaine inondée, à quelques kilòmetres de Trinidad (1446'S, 6550'O) Bassin du Rio Mamoré [Beni Prov., Bolivia]. Holotype: MNHN 1983-0528.

Corydoras geryi Nijssen \& Isbrücker, 1983c: 78, fig. 7. Type locality: Plaine inondée, à quelques kilomètres de Trinidad (1446'S, 6550'O), bassin du Rio Mamoré [Beni Prov., Bolivia]. Holotype: MNHN 1983-0529.

Distribution: Mamoré River basin, Bolivia (Reis, 2003).

Remarks: Isbrücker (1999b: 58) acted as first reviser in placing Corydoras bolivianus in the synonymy of C. geryi.

Corydoras gomezi Castro, 1986

Corydoras gomezi Castro, 1986b: 34, fig. 1. Type locality: Lakes of the Isla de Mocagua, Rio Amazonas/Solimões near Leticia, Comisaría del Amazonas (354'S, 70¹4'W) [Colombia]. Holotype: UBJTL MM536.

Distribution: Amazon River basin near the border of Colombia and Brazil (Reis, 2003). 
Corydoras gossei Nijssen, 1972

Corydoras gossei Nijssen, 1972: 424, fig. 6. Type locality: Brazil, Est. Rondonia, creek near Guajará Mirim (1050'S, 65²1'W), Río Mamoré system. Holotype: IRSNB 487.

Distribution: Mamoré River basin, Brazil (Reis, 2003).

Corydoras gracilis Nijssen \& Isbrücker, 1976

Corydoras gracilis Nijssen \& Isbrücker, 1976c: 91, fig. 1 (unnumbered). Type locality: Brazil, Est. Pará, tributary of Rio Tapajós, 66.5 kilometers west of Itaituba, 4¹5'S, 5556'W. Holotype: ZMA 114687. Type locality corrected in Nijssen \& Isbrücker (1980b: 214) to Rio Jauna (= R. Juma) at Trans-Amazonica highway, about 609'S, $59^{\circ} 55^{\prime} \mathrm{W}$, tributary of Rio Aripuaña, Amazonas, Brazil.

Distribution: Middle and lower Amazon River basin, Brazil (Reis, 2003).

Corydoras griseus Holly, 1940

Corydoras griseus Holly, 1940: 110. Type locality: in kleinen und kleinsten Wasserläufen der Nähe des Amazonenstromes [apparently in error, see Remarks]. Holotype: Münchner Tierpark A.G. 72/1938 (lost).

Corydoras griseus de weyeri Meinken, 1957: 5, unnumbered figure. Type locality: British-Guiana. Lectotype: ZMH H1186, designated by, and illustrated in, Nijssen \& Isbrücker (1967: 35, pl. 3, fig. 2).

Distribution: Amazon River basin, Brazil, and Guyana (Reis, 2003).

Remarks: Nijssen \& Isbrücker (1980: 210) indicated that the stated type locality of Corydoras griseus was incorrect and the correct locality was: Guyana, Essequibo, Portaro River, Kuribong trail.

Corydoras guapore Knaack, 1961

Corydoras guapore Knaack, 1961: 135, fig. 32. Type locality: Brasilien, im Hauptstrom des oberen Rio Guaporé. Holotype: ZMB 21406.

Distribution: Guaporé River basin, Brazil (Reis, 2003).

Corydoras guianensis Nijssen, 1970

Corydoras guianensis Nijssen, 1970: 21, fig. 9. Type locality: Creek at right of Nickerie River, 12 kilometers W.S.W of Stondansie Fall [Nickerie, Suriname]. Holotype: ZMA 105933.

Distribution: Coastal rivers of Suriname and French Guiana (Reis, 2003).

Corydoras habrosus Weitzman, 1960

Corydoras habrosus Weitzman, 1960a: 141, fig. 1. Type locality: Río Salinas, a branch of Río Pao Viejo, El Baúl, State of Cojedes, Venezuela [8 $\left.{ }^{\circ} 59^{\prime} \mathrm{N}, 68^{\circ} 16^{\prime} \mathrm{W}\right]$. Holotype: SU 52351.

Distribution: Upper Orinoco River basin, Colombia and Venezuela (Reis, 2003).

Corydoras haraldschultzi Knaack, 1962

Corydoras haraldschultzi Knaack, 1962: 129, fig. 1. Type locality: Brasilien, Rio Guaporé. Holotype: SMF 5475.

Distribution: Brazil and Bolivia (Reis, 2003).

Corydoras hastatus Eigenmann \& Eigenmann, 1888

Corydoras hastatus Eigenmann \& Eigenmann, 1888b: 166. Type locality: Villa Bella [= Parintins, $2^{\circ} 38^{\prime} \mathrm{S}, 56^{\circ} 45^{\prime} \mathrm{W}$, Amazonas, Brazil]. Lectotype: MCZ 7747, designated by Nijssen \& Isbrücker (1980b: 213).

Corydoras australe Eigenmann \& Ward, in Eigenmann, McAtee \& Ward, 1907: 123. Type locality: Corumba [1900'S, 57²5'W, Rio Paraguay system, Mato Grosso, Brazil]. Holotype: CAS 77305.

Distribution: Amazon and Paraguay River basins (Reis, 2003).

Corydoras heteromorphus Nijssen, 1970

Corydoras heteromorphus Nijssen, 1970: 22, fig. 10. Type locality: Creek at right bank of Coppename River (3'52'30"N, 56 53'W), Saramacca, Suriname. Holotype: ZMA 105880.

Distribution: Coppename and Nickerie River basins, Suriname (Reis, 2003).

Corydoras imitator Nijssen \& Isbrücker, 1983

Corydoras imitator Nijssen \& Isbrücker, 1983c: 79, fig. 8. Type locality: haut Rio Negro, localite exact inconnue [Brazil]. Holotype: MZUSP 27894.

Distribution: Upper Negro River basin, Brazil (Reis, 2003)

Corydoras incolicana Burgess 1993

Corydoras incolicana Burgess, 1993: 157, unnumbered figure. Type locality: Rio Içana, upper Rio Negro, Brazil. Holotype: MZUSP 45717. 
Distribution: Upper Negro River basin, Brazil (Reis, 2003).

Corydoras isbrueckeri Knaack, 2004

Corydoras isbrueckeri Knaack, 2004: 88, figs. 30-36. Type locality: Bolivien, Departamento Santa Cruz, Provincia J.M. Velasco, System río Itenez, Nebengewässer des río Paragua (ein namenloser arroyo), 13³4.384'S, 6203.067'W. Holotype: MTD F 28474.

Distribution: Itenez River basin, Bolivia (Knaack, 2004).

Corydoras julii Steindachner, 1906

Corydoras julii Steindachner, 1906: 480. Type locality: im Parahim, dem Ausflusse der Sess von Parnagua und an der Ausmündung des Baches bei Victoria. Lectotype: NMW 61105, designated by Nijssen \& Isbrücker (1980b: 195).

Distribution: Lower Amazon River and coastal rivers in northeastern Brazil (Reis, 2003).

Corydoras kanei Grant, 1997

Corydoras kanei Grant, 1997: 41, fig. 1. Type locality: Aquarium specimens said to be from Brazil, Roraima State near Boa Vista, possibly from the Rio Branco. Holotype: MZUSP 52489.

Distribution: Negro River basin, Brazil (Reis, 2003).

Corydoras lacerdai Hieronimus, 1995

Corydoras lacerdai Hieronimus, 1995: 110, fig. 5. Type locality: Brasilien, Rio Ribeira da Terra Firme, zwischen den Städten Canavieiras und Ilheus, im Südesten des Bundesstaates Brasilien. Holotype: MZUSP 47682.

Distribution: Ribeira da Terra Firme River, Bahia State, Brazil (Reis, 2003).

Corydoras lamberti Nijssen \& Isbrücker, 1986

Corydoras lamberti Nijssen \& Isbrücker, 1986a: 71, fig. 16. Type locality: Loreto, cours inférieur du Rio Huytoyacu, près du village Nuevo Progresso, affluent de la rive droite du Rio Pastaza, 40 kilometers environ au N. du Lago Rimachi (4²13'S, 76³3'W), Peru. Holotype: ZMA 119335.

Distribution: Pastaza River basin, Peru (Reis, 2003).

Corydoras latus Pearson, 1924

Corydoras latus Pearson, 1924: 19, pl. 3 (fig. 1). Type locality: Lagoons, Lake Rogoagua [Bolivia]. Holotype: CAS 36452.

Distribution: Beni River basin, Bolivia (Reis, 2003).

Corydoras leopardus Myers, 1933

Corydoras leopardus Myers, 1933: 188. Type locality: Brazil [considered by Reis (2003) as likely to have come from a Brazilian coastal river south of the Amazon]. Lectotype: USNM 93305, designated by Nijssen \& Isbrücker (1980b: 197).

Corydoras funnelli Fraser-Brunner, 1947: 241, fig. 1. Type locality: Amazon ? (imported aquarium specimen). Holotype: USNM 102222.

Distribution: Amazon River basin (Reis, 2003).

Corydoras leucomelas Eigenmann \& Allen, 1942

Corydoras leucomelas Eigenmann \& Allen, 1942: 178, pl. 12 (fig. 4). Type locality: Yarinacocha [Peru]. Holotype: CAS 36561.

Corydoras caquetae Fowler, 1943a: 248, figs. 26-27. Type locality: Florencia, Rio Orteguasa, Colombia. Holotype: ANSP 70509.

Distribution: Upper Amazon River basin (Reis, 2003).

Corydoras loretoensis Nijssen \& Isbrücker, 1986

Corydoras loretoensis Nijssen \& Isbrücker, 1986a: 68, fig. 4. Type locality: Loreto, Prov. Maynas, Río Nanay, plage sableuge, rive droite, environ 15 minutes en amont de Santa Clara, Peru. Holotype: NRM 26079.

Distribution: Upper Amazon River basin, Peru (Reis, 2003).

Corydoras loxozonus Nijssen \& Isbrücker, 1983

Corydoras loxozonus Nijssen \& Isbrücker, 1983b: 67, fig. 12. Type locality: Meta, Lomalinda near Río Ariari, tributary to Río Guaviare, S.E. of Villavicencio [Colombia]. Holotype: ANSP 150170.

Distribution: Meta River basin, Colombia (Reis, 2003).

Corydoras maculifer Nijssen \& Isbrücker, 1971 
Corydoras maculifer Nijssen \& Isbrücker, 1971: 183, fig. 1. Type locality: Brazil, Est. Mato Grosso, Sangadina stream, 2 kilometers W of Chavantina $\left(14^{\circ} 45^{\prime} \mathrm{S}, 52^{\circ} 20^{\prime} \mathrm{W}\right)$, tributary of the Rio das Mortes. Holotype: BMNH 1970.10.30.3.

Distribution: Upper Araguaia River basin, Brazil (Reis, 2003).

Corydoras mamore Knaack, 2002

Corydoras mamoré Knaack, 2002d: 16, unnumbered figure. Type locality: [Bolivia]. Holotype: MTD F 26741.

Distribution: Bolivia (Knaack, 2002d).

Corydoras melanistius Regan, 1912

Corydoras melanistius Regan, 1912a: 216. Type locality: Essequibo [Guyana]. Lectotype: BMNH 1864.1.21.86, designated by, and illustrated in, Nijssen \& Isbrücker (1967: 32, pl. 2, fig. 1).

Corydoras wotroi Nijssen \& Isbrücker, 1967: 44, pl. 5 (fig. 3). Type locality: Outlet of Kleine Saramacca along and between sand bank [Suriname]. Holotype: RMNH 25331.

Distribution: Coastal rivers of the Guianas (Reis, 2003).

Corydoras melanotaenia Regan, 1912

Corydoras melanotaenia Regan, 1912a: 217. Type locality: Honda [Río Meta system, Colombia]. Lectotype: BMNH 1909.7.23.41, designated by Nijssen \& Isbrücker (1980b: 207).

Distribution: Meta River basin, Colombia (Reis, 2003).

Corydoras melini Lönnberg \& Rendahl, 1930

Corydoras melini Lönnberg \& Rendahl, 1930: 1, fig. 1. Type locality: Jauareté an dem Ausfluss des Rio Papuri in den Rio Uaupés [Brazil]. Lectotype: NRM 11091, designated by Nijssen \& Isbrücker (1980b: 209).

Distribution: Upper Negro and Meta River basins (Reis, 2003).

Corydoras metae Eigenmann, 1914

Corydoras metae Eigenmann, 1914b: 230. Type locality: Barrigona, Rio Meta, Colombia. Holotype: CAS 36447; holotype illustrated in Eigenmann (1916: pl. 14; 1922b: 227, pl. 8, fig. 5).

Distribution: Meta River basin, Colombia (Reis, 2003)

Corydoras micracanthus Regan, 1912

Corydoras micracanthus Regan, 1912a: 211. Type locality: Salta, Argentina. Lectotype: BMNH 1897.1.27.8, designated by Nijssen \& Isbrücker (1980b: 206).

Distribution: Western tributaries of Paraná River in Salta Province, Argentina (Reis, 2003).

Corydoras multimaculatus Steindachner, 1907

Corydoras multimaculatus Steindachner, 1907d: 291. Type locality: Nebenarme des Rio Preto bei Sa. Rita im Staate Bahia [Brazil]. Lectotype: NMW 46783, designated by Nijssen \& Isbrücker (1980b: 196).

Distribution: Preto River basin in Bahia State, Brazil (Reis, 2003).

Corydoras multiradiatus (Orcés, 1960)

Chaenothorax multiradiatus Orcés, 1960: 3, fig. 1. Type locality: afluente occidental del río Lagartococha, cerca del poblado de Garza-Cocha, sistema del alto Napo. Holotype: USNM 200739.

Distribution: Western Amazon River basin, Ecuador and Peru (Reis, 2003), as Brochis multiradiatus.

Corydoras nanus Nijssen \& Isbrücker, 1967

Corydoras nanus Nijssen \& Isbrücker, 1967: 41, pl. 5 (fig. 1). Type locality: Little tributaries of Gran-Rio between Ligolio and Awaradam Falls [Brokopondo, Suriname]. Holotype: RMNH 25333.

Distribution: Suriname and Maroni River basins in Suriname and Iracoubo River basin in French Guiana (Reis, 2003).

Corydoras napoensis Nijssen \& Isbrücker, 1986

Corydoras napoensis Nijssen \& Isbrücker, 1986a: 73, fig. 25. Type locality: Napo, Lagartococha, affluent septentrional du Rio Aguarico, entre l'embouchure de la rivière $\left(0^{\circ} 39^{\prime} \mathrm{S}, 75^{\circ} 16^{\prime} \mathrm{W}\right)$ et le village de Garzacocha $\left(0^{\circ} 28^{\prime} \mathrm{S}\right.$, $\left.75^{\circ} 21^{\prime} W\right)$, bassin du Rio Napo [Ecuador]. Holotype: ZMA 119226.

Distribution: Western Amazon River basin, eastern Ecuador and Peru (Reis, 2003).

Corydoras narcissus Nijssen \& Isbrücker, 1980

Corydoras narcissus Nijssen \& Isbrücker, 1980d: 497, fig. 2. Type locality: Brazil, Est. Amazonas, Rio Purus system, creek into Rio Ipixuna, $7^{\circ} 31^{\prime} \mathrm{S}, 63^{\circ} 16^{\prime} \mathrm{W}, 30 \mathrm{~km}$ west of Humaitá. Holotype: ZMA 115178. 
Distribution: Purus River basin, middle Amazon River system, Brazil, (Reis, 2003).

Corydoras nattereri Steindachner, 1877

Corydoras Nattereri Steindachner, 1877a: 143, pl. 11 (fig. 1). Type locality: Affluent of Rio Parahyba, 3 mi. up, Rio de Janeiro, Brazil, by neotype designation. Neotype: MCZ 8229, designated by Nijssen \& Isbrücker (1980a: 3).

Corydoras juquiaae Ihering, 1907: 36. Type locality: Juquiá River, Poço Grande, State of São Paulo [Brazil]. Lectotype: CAS 16016, designated by Ellis (1913: 403), subsequent designation by Britski (1969: 207) of MZUSP 1984 for Corydoras juquiae is invalid.

Corydoras nattereri triseriatus Ihering, 1911: 386. Type locality: Rio Doce, Est. Espírito Santo [Brazil]. Lectotype: MZUSP 342, designated by Britski (1969: 207).

Distribution: Coastal rivers in southeastern Brazil from Espirito Santo to Paraná, Brazil (Reis, 2003).

Corydoras negro Knaack, 2004

Corydoras negro Knaack, 2004: 81, figs. 22-29. Type locality: Bolivien, Departamento Santa Cruz, Provincia Nuflo de Chavez, Raum Urubicha, System río Blanco, río Negro (Hauptfluss), Restgewässer, 15³1.502'S, 6256.208'W. Holotype: MTD F 28472.

Distribution: Blanco River basin, Bolivia (Knaack, 2004).

Corydoras nijsseni Sands, 1989

Corydoras elegans nijsseni Sands, 1989: 45. Type locality: Tributary of the Rio Negro, Brazil. Holotype: reportedly deposited at RMNH; but reported as four syntypes in the on-line Catalog of Fishes, as RMNH 31625 and 31626.

Distribution: Negro River basin, Brazil (Reis, 2003).

Corydoras noelkempffi Knaack, 2004

Corydoras noelkempffi Knaack, 2004: 94, figs. 37-41. Type locality: Bolivien, Departamento Santa Cruz, Provincia J.M. Velasco, System río Itenez, río San Martin, 15²3.265'S, 61²9.758'W. Holotype: MTD F 28576.

Distribution: Itenez River basin, Bolivia (Knaack, 2004).

Corydoras oiapoquensis Nijssen, 1972

Corydoras oiapoquensis Nijssen, 1972: 425, fig. 7. Type locality: French Guiana, Cumuri Creek at left bank of Rio Oiapoque (= Oyapock River), upstream of first rapids of Grande Roche, southwest of village Oiapoque (Brazil, 035' $\left.\mathrm{N}, 51^{\circ} 46^{\prime} \mathrm{W}\right)$. Holotype: IRSNB 489.

Distribution: Oyapock River basin, Brazil and French Guiana (Reis, 2003).

Corydoras ornatus Nijssen \& Isbrücker, 1976

Corydoras ornatus Nijssen \& Isbrücker, 1976b: 126, fig. 1. Type locality: Brazil, Est. Pará, Rio Tapajós, 80 km E of Jacareacanga, $06^{\circ} 09^{\prime} \mathrm{S}, 58^{\circ} 15^{\prime} \mathrm{W}$. Holotype: ZMA 114690.

Distribution: Lower Tapajós River basin, Brazil (Reis, 2003).

Corydoras orphnopterus Weitzman \& Nijssen, 1970

Corydoras orphnopterus Weitzman \& Nijssen, 1970: 125, fig. 3. Type locality: Ecuador, Lower Río Bobonaza between Montalvo (2 $\left.{ }^{\circ} 06^{\prime} \mathrm{S}, 7^{\circ} 59^{\prime} \mathrm{W}\right)$ and Chicherota, Rio Pastaza system, Est. Pastaza. Holotype: USNM 204361.

Distribution: Pastaza River basin, Ecuador (Reis, 2003).

Corydoras osteocarus Böhlke, 1951

Corydoras osteocarus Böhlke, 1951a: 824. Type locality: San Fernando de Atabapo, where Rio Atabapo empties into the Orinoco, Venezuela (at approximately $04^{\circ} 03^{\prime}$ North Latitude, 67 $45^{\prime}$ West Longitude). Holotype: SU 16333.

Distribution: Orinoco River basin and coastal rivers of Suriname (Reis, 2003).

Corydoras ourastigma Nijssen, 1972

Corydoras ourastigma Nijssen, 1972: 428, fig. 8. Type locality: Brazil, Est. Acre, Rio Iquiri (= Rio Ituxi) at road Rio Branco village - Abuna, 47 kilometers from Rio Branco village (about 1000'S, 67 $30^{\prime} \mathrm{W}$ ), Purus River system. Holotype: IRSNB 498.

Distribution: Purus River basin, middle Amazon system, Brazil (Reis, 2003).

Corydoras oxyrhynchus Nijssen \& Isbrücker, 1967

Corydoras oxyrhynchus Nijssen \& Isbrücker, 1967: 42, pl. 4 (fig. 3). Type locality: Gojo Creek above Posoegroenoe, tributary of Saramacca River. Holotype: RMNH 25329. 
Distribution: Saramacca River basin, Suriname (Reis, 2003).

Corydoras paleatus (Jenyns, 1842)

Callichthys paleatus Jenyns, 1842 (in Jenyns, 1840-42): 113. Type locality: South America. Lectotype: BMNH 1917.7.14.18, designated by Nijssen \& Isbrücker (1980b: 204).

Corydoras maculatus Steindachner, 1879a: 32. Type locality: La Plata [Argentina]. No types known.

Corydoras marmoratus Steindachner, 1879e: 26, pl. 5 (fig. 1). Type locality: Montevideo [Uruguay] und ... La Plata innerhalb der Provinz Buenos-Ayres [Argentina]. Lectotype: NMW 5538, designated by Nijssen \& Isbrücker (1980b: 204).

Corydoras microcephalus Regan, 1912a: 211. Type locality: La Plata. Lectotype: BMNH 1890.3.12.4, designated by Nijssen \& Isbrücker (1980b: 204).

Silurus quadricostatus Larrañaga, 1923: 376. Type locality: Uruguay. No types known.

Silurus 7-radiatus Larrañaga, 1923: 385. Type locality: Uruguay. No types known.

Distribution: Lower Paraná River basin and coastal rivers in Uruguay and Brazil (Reis, 2003).

Remarks: The name Corydoras punctatus argentina first appeared in Steindachner (1879a: 26) as a name only and in synonymy of Corydoras marmoratus; as such it is unavailable from that publication and the name has apparently not been made available subsequently. Isbrücker (2001b) treated Corydoras maculatus, C. marmoratus and $C$. microcephalus as doubtfully valid species, but with no additional information.

Corydoras panda Nijssen \& Isbrücker, 1971

Corydoras panda Nijssen \& Isbrücker, 1971: 186, fig. 2. Type locality: Peru, Est. Huanuco, Aquas Amarillas, tributary of Río Pachitea, Ucayali river system. Holotype: BMNH 1969.7.15.8.

Distribution: Upper Amazon River basin, Peru (Reis, 2003).

Corydoras pantanalensis Knaack, 2001

Corydoras pantanalensis Knaack, 2001: 59, unnumbered figure. Type locality: Bolivien, Prov. Santa Cruz, Restgewässer im nördlichen Pantanal, Überschwemmungsgebiet im Raum San Lorenzo, Flusssystem Rio Cussis und Rio Las Petas. Koordinaten des Fundortes: 1606' 525"S, 5955'620"W [sic]. Holotype: MTD F24774.

Distribution: Cussis and Las Petas River basins, Bolivia (Reis, 2003).

Corydoras paragua Knaack, 2004

Corydoras paragua Knaack, 2004: 65, figs. 6-12. Type locality: Bolivien, Departamento Santa Cruz, Provincia J.M. Velasco, río Itenez system, río Paragua,, Río Paragua, nordwestliches Ufer im Mündungsgebiet. ... 13³1.842'S, 61 $90.015^{\prime}$ W. Holotype: MTD F 28468.

Distribution: Itenez River basin, Bolivia (Knaack, 2004).

Corydoras parallelus Burgess 1993

Corydoras parallelus Burgess, 1993: 154, unnumbered figure. Type locality: Rio Içana, upper Rio Negro, Brazil. Holotype: MZUSP 45716.

Distribution: Upper Negro River basin, Brazil (Reis, 2003).

Corydoras pastazensis Weitzman, 1963

Corydoras pastazensis Weitzman, 1963: 59, fig. 1. Type locality: Chicherata, near the mouth of the Río Bobonaza, a tributary of the Río Pastaza, itself a tributary of the Río Maranon, Pastaza Province, Ecuador, about $02^{\circ} 33^{\prime} \mathrm{S}$ latitude and 76² $40^{\prime} \mathrm{W}$ longitude. Holotype: USNM 177216.

Corydoras pastazensis orcesi Weitzman \& Nijssen, 1970: 120, fig. 1. Type locality: Ecuador, Río Conambo near village of Conambo ( $\left.1^{\circ} 55^{\prime} \mathrm{S}, 76^{\circ} 51^{\prime} \mathrm{W}\right)$, Río Tigre system, Est. Pastaza. Holotype: USNM 204358.

Distribution: Pastaza River basin, Ecuador (Reis, 2003).

Corydoras paucerna Knaack, 2004

Corydoras paucerna Knaack, 2004: 57, figs. 1-5. Type locality: Bolivien, Departamento Santa Cruz, Provincia J.M. Velasco, río Itenez system, río Paragua, Nebengewässer (Oberlauf) eines namenlossen arroyos (Restgewässer): 1334.384'S, 6203.067'W. Holotype: MTD F 28466.

Distribution: Itenez River basin, Bolivia (Knaack, 2004).

Corydoras pinheiroi Dinkelmeyer, 1995

Corydoras pinheiroi Dinkelmeyer, 1995: 60, unnumbered figure. Type locality: Brasilien, Est. Rondonia, in einem Nebenfluss des Rio Ribeiro bei der Ortschaft Guajará-Mirim. Holotype: MZUSP 48099. 
Distribution: Western Amazon River basin in Rondônia State, Brazil (Reis, 2003).

Corydoras polystictus Regan, 1912

Corydoras polystictus Regan, 1912a: 216. Type locality: Descalvados, Matto Grosso [Brazil]. Lectotype: BMNH 1895.5.17.62, designated by Nijssen \& Isbrücker (1980b: 197).

Corydoras virescens Miranda Ribeiro, 1912: 16. Type locality: Rio Paraguay, perto de Cáceres [Brazil]. Holotype: MNRJ 3806.

Distribution: Paraguay River basin, Brazil (Reis, 2003).

Remarks: Publication of the name Corydoras polystictus apparently predates that of $C$. virescens; see Eschmeyer (1998) for reported publication dates of relevant descriptions.

Corydoras potaroensis Myers, 1927

Corydoras potaroensis Myers, 1927: 126. Type locality: Br. Guiana: Creek below Potaro Landing. Lectotype: CAS 24754, designated by Nijssen \& Isbrücker (1980b: 208).

Distribution: Potaro River basin, Guyana (Reis, 2003).

Corydoras pulcher Isbrücker \& Nijssen, 1973

Corydoras pulcher Isbrücker \& Nijssen, 1973: 2, fig. 1. Type locality: Brazil, Est. Amazonas, Rio Purus, north of Lábrea (7²0'S, 64²6'W), Rio Amazonas system. Holotype: SMF 9231.

Distribution: Purus River basin, middle Amazon system, Brazil (Reis, 2003).

Corydoras punctatus (Bloch, 1794)

Cataphractus punctatus Bloch, 1794: 90, pl. 377 (fig. 2). Type locality: Flüssen Surinams. Lectotype: ZMB 3149, designated by Nijssen \& Isbrücker (1975: 63). Earlier neotype designation by Nijssen \& Isbrücker (1967: 28) (RMNH 25301) invalidated by rediscovery of syntype series. Placed on Official list (ICZN Opinion 1169).

Distribution: Suriname River basin in Suriname and Iracoubo River basin in French Guiana (Reis, 2003).

Remarks: Isbrücker $(1999,2000)$ discussed the taxonomic history of this species.

Corydoras pygmaeus Knaack, 1966

Corydoras pygmaeus Knaack, 1966a: 168, first unnumbered figure. Type locality: einem Nebenfluß des Rio Madeira (Brasilien). Holotype: ZMB 21620. Also described as new in Knaack (1966b: 364).

Distribution: Madeira River basin (Reis, 2003).

Corydoras rabauti La Monte, 1941

Corydoras rabauti La Monte, 1941: 5. Type locality: A tributary of the Amazon River [Brazil]. Holotype: AMNH 15644.

Corydoras myersi Miranda Ribeiro, 1942: 427, fig. 1. Type locality: Rio Javari, Município de Banjamin Constant, Estado do Amazonas, Brasil. Holotype: MNRJ 3626.

Distribution: Upper Amazon, Solimões, and Negro River basins (Reis, 2003).

Corydoras reticulatus Fraser-Brunner, 1938

Corydoras reticulatus Fraser-Brunner, 1938: 208, fig. 2 (unnumbered). Type locality: Monte Alegre River, Amazon [Brazil] (200'S, 5404'W), by neotype designation. Neotype: BMNH 1926.10.27.333.

Distribution: Lower Amazon River basin, Brazil (Reis, 2003).

Remarks: Original two syntypes are considered to be lost and Fraser-Brunner (1947: 245) redescribed species with a "holotype" which Weitzman (1960b) designated as neotype (see Nijssen \& Isbrücker, 1980: 197; Reis, 2003).

$\dagger$ Corydoras revelatus Cockerell, 1925

$\dagger$ Corydoras revelatus Cockerell, 1925: 398. Type locality: Sunchal, Province of Jujuy, Argentina; Tertiary. Holotype: At University of Colorado.

Distribution: Maiz Gordo Formation, Sunchal, Jujuy Province, and Salta, Argentina; late Paleocene (Reis, 1998b: 357).

Remarks: Redescribed in Bardack (1961).

Corydoras reynoldsi Myers \& Weitzman, 1960

Corydoras reynoldsi Myers \& Weitzman, 1960: 105, fig. 2. Type locality: Tributary to the Río Orteguaza, opposite the town and air base known as Tres Esquinas, Caquetà Province, Colombia. Holotype: SU 52349.

Distribution: Upper Caquetá River basin, Colombia (Reis, 2003).

Remarks: The caption for figure 2 lists SU 50702 as the holotype of Corydoras reynoldsi. Apparently the holotype 
was removed from this lot and assigned a new number, and the inclusion of the original catalog number in the figure caption is a lapsus calami.

Corydoras robineae Burgess, 1983

Corydoras robineae Burgess, 1983: 42, unnumbered figure. Type locality: Rio Aiuana, a southern tributary of the middle Rio Negro, Amazonas, Brazil. Holotype: MZUSP 27175.

Distribution: Upper Negro River basin, Brazil (Reis, 2003).

Corydoras robustus Nijssen \& Isbrücker, 1980

Corydoras robustus Nijssen \& Isbrücker, 1980d: 499, fig. 3. Type locality: Brazil, Est. Amazonas, Rio Purus system, creek into Rio Ipixuna, 07³1'S, 631' W, 30 km west of Humaitá. Holotype: ZMA 115179.

Distribution: Purus River basin, middle Amazon system, Brazil (Reis, 2003).

Corydoras sanchesi Nijssen \& Isbrücker, 1967

Corydoras sanchesi Nijssen \& Isbrücker, 1967: 43, pl. 5 (fig. 2). Type locality: Gojo Creek above Posoegroenoe, tributary of Saramacca River [Suriname]. Holotype: RMNH 25319.

Distribution: Saramacca River basin, Suriname (Reis, 2003).

Corydoras saramaccensis Nijssen, 1970

Corydoras saramaccensis Nijssen, 1970: 38, fig. 21. Type locality: Creek at right bank of Kleine Saramacca River, 11 km E.S.E. from junction with Saramacca River, Brokopondo, Suriname. Holotype: ZMA 106018.

Distribution: Saramacca River basin, Suriname (Reis, 2003).

Corydoras sarareensis Dinkelmeyer, 1995

Corydoras saraareensis Dinkelmeyer, 1995: 61, unnumbered figure. Type locality: Brasilien, Est. Mato Grosso, Rio Sararé, rechter Nebenfluß des Rio Guaporé. Holotype: MZUSP 48100.

Distribution: Guaporé River basin, Brazil (Reis, 2003).

Corydoras schwartzi Rössel, 1963

Corydoras schwartzi Rössel, 1963: 359, fig. 1. Type locality: Brasilien, Mündungsgebiet des Rio Purus. Holotype: SMF 6425.

Distribution: Purus River basin, middle Amazon system, Brazil (Reis, 2003).

Corydoras semiaquilus Weitzman, 1964

Corydoras semiaquilus Weitzman, 1964: 121, fig. 5. Type locality: Igarapé Preto, a small jungle rivulet at the headwaters of the black water creeks, which empty in the upper Solimões, State of Amazonas, Brazil. Holotype: SU 55939.

Distribution: Western Amazon River basin (Reis, 2003).

Corydoras septentrionalis Gosline, 1940

Corydoras septentrionalis Gosline, 1940b: 16. Type locality: Río Piña, north of Maturín, Venezuela. Holotype: SU 35055.

Corydoras cortesi Castro, 1987: 31, fig. 1. Type locality: Río Arauca near to Arauca, Intendencia de Arauca ( $7^{\circ} 03^{\prime} \mathrm{N}$, $\left.70^{\circ} 46^{\prime} \mathrm{W}\right)$ [Colombia]. Holotype: UBJTL MM671.

Distribution: Orinoco River basin (Reis, 2003).

Corydoras serratus Sands, 1995

Corydoras serratus Sands, 1995: 14, fig. 7. Type locality: Rio Poranga, 8 kilometers north of the Nobua oba, where the two streams join a small tributary of the upper Negro river, Brazil. Holotype: LIVCM 1994.4.38.

Distribution: Upper Negro River basin, Brazil (Reis, 2003).

Corydoras seussi Dinkelmeyer, 1996

Corydoras seussi Dinkelmeyer, 1996: 25. Type locality: Brasilien, Est. Rondonia, kleiner Nebenfluß des Rio Pacaás Novos, eines rechtsseitigen Zuflusses des Rio Mamoré nahe bei Guajara-Mirim. Holotype: MZUSP 49322.

Distribution: Mamoré River basin, Brazil (Reis, 2003).

Corydoras similis Hieronimus, 1991

Corydoras similis Hieronimus, 1991: 39, fig. 1. Type locality: Brasilien, in der Nähe der Stadt Ariqumes in einem Zufluß zum Rio Madeira, Bundesstaat Rondonia. Holotype: ZFMK 16400.

Distribution: Madeira River basin, Brazil (Reis, 2003).

Corydoras simulatus Weitzman \& Nijssen, 1970 
Corydoras simulatus Weitzman \& Nijssen, 1970: 126, fig. 4. Type locality: Colombia, Río Ocoá near Puerto López $\left(04^{\circ} 06^{\prime} \mathrm{N}, 72^{\circ} 57^{\prime} \mathrm{W}\right)$, Río Meta system, Est. Meta. Holotype: USNM 197615.

Distribution: Upper Meta River, Colombia (Reis, 2003).

Corydoras sipaliwini Hoedeman, 1965

Corydoras punctatus sipaliwini Hoedeman, 1965: 87, fig. 46. Type locality: Sipalawini River [Suriname]. Lectotype: ZMA 104.278, designated by Isbrücker (2000).

Distribution: Coastal river basins of Guyana and Suriname (Reis, 2003).

Corydoras sodalis Nijssen \& Isbrücker, 1986

Corydoras sodalis Nijssen \& Isbrücker, 1986a: 68, fig. 6. Type locality: Loreto, Rio Yavari, Cano de Guavariba dans le Lago Matamata $\left(04^{\circ} 12^{\prime} \mathrm{S}, 70^{\circ} 17^{\prime} \mathrm{W}\right)$, Peru. Holotype: NRM 13713.

Distribution: Amazon River of Brazil and Peru (Reis, 2003).

Corydoras solox Nijssen \& Isbrücker, 1983

Corydoras solox Nijssen \& Isbrücker, 1983c: 80, fig. 9. Type locality: bassin du Rio Oiapoque, $60 \mathrm{~km} \mathrm{~S} \mathrm{du} \mathrm{village}$

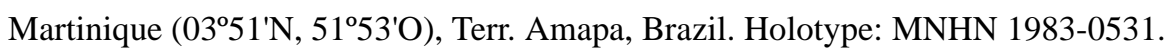

Distribution: Oyapock River basin and smaller coastal drainages in French Guiana (Reis, 2003).

Corydoras spectabilis Knaack, 1999

Corydoras spectabilis Knaack, 1999c: 74, unnumbered figure. Type locality: Brasilien, Mato Grosso, Oberlauf Rio Guaporé im Raum Vila Bela. Holotype: ZMB 33150.

Distribution: Guaporé River basin, Brazil (Reis, 2003).

Remarks: Apparently also published as new in Knaack (1999e).

Corydoras spilurus Norman, 1926

Corydoras spilurus Norman, 1926b: 95. Type locality: Iponcin Creek, into Approuague River, French Guiana. Lectotype: BMNH 1926.3.2.738, designated by, and illustrated in, Nijssen \& Isbrücker (1967: 33, pl. 2, fig. 2).

Distribution: Approuague River basin, French Guiana, and Suriname River basin, Suriname (Reis, 2003).

Corydoras splendens (Castelnau, 1855)

Callichthys splendens Castelnau, 1855: 39, pl. 18 (fig. 3). Type locality: Rio Tocantins [Brazil]. Holotype: MNHN 0000-4291.

Callichthys taiosh Castelnau, 1855: 39, pl. 19 (fig. 1). Type locality: Chiquitos [Bolivia]. Holotype: possibly MNHN 0000-4290. Castelnau indicated that he saw only a drawing of this species, but the specimen listed here was taken by a Mr. Weddell, from the correct locality and appears to be the specimen upon which the illustration was prepared.

Brochis coeruleus Cope, 1872a: 277, pl. 9 (fig. 3). Type locality: Tributaries of the Ambyiacu. Lectotype: ANSP 8231, designated by Nijssen \& Isbrücker (1970: 157-158).

Brochis dipterus Cope, 1872a: 278. Type locality: Ambyiacu River. Holotype: ANSP 117172.

Corydoras semiscutatus Cope, 1872a: 280, pl. 6 (fig. 1). Type locality: Ambyiacu River. Holotype: ANSP 8289.

Chaenothorax bicarinatus Cope, 1878: 679. Type locality: Peruvian Amazon. Holotype: ANSP 21447.

Chaenothorax eigenmanni Ellis, 1913: 393, pl. 26 (fig. 2). Type locality: Cáceres [Mato Grosso, Brazil]. Holotype: FMNH 54880.

Distribution: Amazon River basin, Brazil, Colombia, Ecuador, and Peru, as Brochis splendens (Reis, 2003).

Remarks: Callichthys splendens was apparently first given precedence over $C$. taiosh in Nijssen and Isbrücker (1970: 157).

Corydoras steindachneri Isbrücker \& Nijssen, 1973

Corydoras steindachneri Isbrücker \& Nijssen, 1973: 4, fig. 3. Type locality: Brazil, Est. Paraná, Paranaguá (25³2'S, 4836'W). Holotype: NMW 1504.

Distribution: Coastal rivers in Paraná State, Brazil (Reis, 2003).

Corydoras stenocephalus Eigenmann \& Allen, 1942

Corydoras stenocephalus Eigenmann \& Allen, 1942: 177, pl. 12 (fig. 2). Type locality: Yarinococha [Peru]. Holotype: CAS 36386.

Distribution: Eastern Ecuador and Ucayali River basin, Peru (Reis, 2003).

Corydoras sterbai Knaack, 1962 
Corydoras sterbai Knaack, 1962: 131, pl. 12 (fig. 2). Type locality: Brasilien. Holotype: SMF 5476.

Distribution: Central Brazil and Bolivia (Reis, 2003).

Corydoras surinamensis Nijssen, 1970

Corydoras schwartzi surinamensis Nijssen, 1970: 39, fig. 22. Type locality: Creek at right bank of Coppename River (0352'30"N, 5653'00"W), Saramacca, Suriname. Holotype: ZMA 105876.

Distribution: Coppename River basin, Suriname (Reis, 2003).

Corydoras sychri Weitzman, 1960

Corydoras sychri Weitzman, 1960a: 151, fig. 7. Type locality: Tropical South America. Holotype: SU 51295.

Distribution: Nanay River basin, Peru (Reis, 2003).

Corydoras treitlii Steindachner, 1906

Corydoras treitlii Steindachner, 1906: 478. Type locality: Mündungstelle eines kleinen Baches gefangen, der bei dem Städtchen Victoria in der Parnahyba fällt [Brazil]. Lectotype: NMW 61103, designated by Weitzman (1964: 116).

Distribution: Coastal drainages in northeastern Brazil (Reis, 2003).

Corydoras trilineatus Cope, 1872

Corydoras trilineatus Cope, 1872a: 281, pl. 6 (fig. 2). Type locality: Ambyiacu River [Peru]. Lectotype: ANSP 8294, designated by Nijssen \& Isbrücker (1980b: 194).

Corydoras episcopi Eigenmann \& Allen, 1942: 177, pl. 12 (fig. 3). Type locality: Rio Morona. Holotype: CAS 36448.

Corydoras dubius Nijssen \& Isbrücker, 1967: 38, pl. 4 (fig. 2). Type locality: Suriname, surroundings of Paramaribo. Holotype: ZMA 104623.

Distribution: Middle and upper Amazon River basin and coastal drainages of Suriname (Reis, 2003).

Corydoras tukano Britto \& Lima, 2003

Corydoras tukano Britto \& Lima, 2003: 84, figs. 1, 2. Type locality: Brazil, estado do Amazonas, rio Tiquie, comunidade de Caruru, beaches in pool below the fall, 0¹6'28.9"N, 6954'53.6"W (UTM 19N0398191/0030366). Holotype: MZUSP 82100.

Distribution: Tiquié River basin, Negro River drainage, Brazil (Britto \& Lima, 2003).

Corydoras undulatus Regan, 1912

Corydoras undulatus Regan, 1912a: 217. Type locality: La Plata. Holotype: BMNH 1912.7.10.5.

Distribution: Lower Paraná River basin and coastal rivers in southern Brazil (Reis, 2003).

Corydoras virginiae Burgess, 1993

Corydoras virginiae Burgess, 1993: 152, unnumbered figure. Type locality: Rio Ucayali, Peru. Holotype: MZUSP 45715.

Distribution: Ucayali River basin, Peru (Reis, 2003).

Corydoras vittatus Nijssen, 1971

Corydoras blochi vittatus Nijssen, 1971: 97, fig. 7. Type locality: Brazil, Est. Maranhão, tributary of Rio Itapicurú at Caxias (4º53'S, 4320'W). Holotype: ZMA 109990.

Distribution: Itapicurú River basin in northeastern Brazil (Reis, 2003).

Corydoras weitzmani Nijssen, 1971

Corydoras weitzmani Nijssen, 1971: 91, fig. 1. Type locality: Peru, Est. Cuzco, at Cuzco (13³2'S, 7157'W), Vilcanota river system. Holotype: FMNH 69934.

Distribution: Western Amazon River basin, Peru (Reis, 2003).

Corydoras xinguensis Nijssen, 1972

Corydoras xinguensis Nijssen, 1972: 429, fig. 9. Type locality: Brazil, Est. Mato Grosso, Suia Missú Creek, tributary of upper Río Xingu near Posto Diauarum (about $11^{\circ} 15^{\prime} \mathrm{S}, 5^{\circ} 00^{\prime} \mathrm{W}$ ). Holotype: IRSNB 500.

Distribution: Upper Xingu River basin, Brazil (Reis, 2003).

Corydoras zygatus Eigenmann \& Allen, 1942

Corydoras zygatus Eigenmann \& Allen, 1942: 175, pl. 12 (fig. 1). Type locality: Creek, Yurimaguas [Peru]. Holotype: CAS 24756.

Distribution: Western Amazon River basin (Reis, 2003). 


\section{DIANEMA}

Dianema Cope, 1871b: 112. Type species: Dianema longibarbis Cope, 1872. Type by subsequent monotypy by Cope (1872). Gender: Neuter. Appeared first with brief description and no species; a single species assigned to this genus by Cope (1872a: 276).

Decapogon Eigenmann \& Eigenmann, 1888b: 165. Type species: Callichthys adspersus Steindachner, 1877. Type by monotypy. Gender: Masculine.

Phylogeny: Reis (1997).

Dianema longibarbis Cope, 1872

Dianema longibarbis Cope, 1872a: 276, pl. 7 (fig. 1). Type locality: Ambyiacu River, Peru. Syntypes: ANSP 8285 (1), ANSP 21540 (1).

Callichthys adspersus Steindachner, 1877a: 135, pl. 11 (fig. 2). Type locality: Stromgebiete des Amazonenflusses von Santarem bis Tabatinga ... im Xingu bei Porto do Moz, im Amazonenstrome bei Cudajas und bei Tabatinga [Brazil]. Syntypes: MCZ 8223 (8), MCZ 8245 + 8265 (8), MCZ 18266 (1), NMW 47667 (2), NMW 47668 (2), NMW 47669 (2), NMW 47672 (2), NMW 47673 (2), USNM 41518 (1).

Decapogon verissimi Miranda Ribeiro, 1911: 154, pl. 36 (fig. 1). Type locality: Pará [Brazil]. Lectotype: MNRJ 774a, designated by Miranda Ribeiro (1953: 395).

Distribution: Amazon River basin (Reis, 2003).

Dianema urostriatum (Miranda Ribeiro, 1912)

Decapogon urostriatum Miranda Ribeiro, 1912: 15. Type locality: Manáos [Brazil]. Lectotype: MNRJ 1067a, designated by Miranda Ribeiro (1953: 395). Name spelled Decapogon urotriatum in heading of account, but corrected to urostriatum in attached printed corrigendum.

Distribution: Amazon River basin, Brazil (Reis, 2003).

\section{HOPLOSTERNUM}

Hoplosternum Gill, 1858: 395. Type species: Callichthys laevigatus Valenciennes, 1836. Type by original designation. Gender: Neuter.

Cascadura Ellis, 1913: 387. Type species: Cascadura maculocephala Ellis, 1913. Type by monotypy. Gender: Feminine.

Cataphractops Fowler, 1915a: 231. Type species: Callichthys melampterus Cope, 1872. Type by original designation. Gender: Masculine. Originally proposed as a subgenus of Hoplosternum.

Ellisichthys Miranda Ribeiro, 1920: 11. Type species: Cascadura maculocephala Ellis, 1913. Type by being a replacement name. Gender: Masculine. Proposed as a replacement for Cascadura Ellis, 1913, considered to be an inappropriate choice for a scientific name.

Diasternum Franz, 2001: 17. Type species: Hoplosternum punctatum Meek \& Hildebrand, 1916. Type by original designation. Gender: Neuter.

Revision: Reis (1997).

Remarks: Lundberg (1997) reports a fossil identified as Hoplosternum sp. from the middle Miocene in the La Venta formation, Magdalena River basin, Colombia.

Hoplosternum littorale (Hancock, 1828)

Callichthys littoralis Hancock, 1828: 244, pl. 32 (fig. 1). Type locality: Demerara [Guyana]. BMNH 1996.7.11.1.

Callichthys laevigatus Valenciennes, 1836, in Valenciennes, 1835-47: pl. 5 (fig. 2). Type locality: l'Amerique depuis Corrientes jusque dans les eaux douces des isles des Antilles, car nous l'avons de la Trinité. Holotype: possibly MNHN 0000-4271 (2), MNHN 0000-4272 (2), or MNHN 0000-4274 (1). Name available from plate, with distribution in Valenciennes (1847: 7); description in Valenciennes (1840b: 314), with location given as Buénos-Ayres. Illustrated specimen must be treated as a holotype if it can be recognized among the listed specimens.

Callichthys subulatus Valenciennes, in Cuvier \& Valenciennes, 1840b: 311 (231 in the Strasbourg deluxe edition). Type locality: Cayenne, ... Buénos-Ayres. Syntypes: MNHN 0000-4268 (1), MNHN 0000-4269 (2), MNHN 0000-4270 (1). 
Callichthys albidus Valenciennes, in Cuvier \& Valenciennes, 1840b: 316 (235 in the Strasbourg deluxe edition). Type locality: Cayenne. Syntypes: MNHN 0000-4276 (1), MNHN 0000-4277 (1).

Callichthys chiquitos Castelnau, 1855: 38, pl. 18 (fig. 2). Type locality: la province de Chiquitos [Bolivia]. Holotype: MNHN 0000-4285.

Hoplosternum stevardii Gill, 1858: 401. Type locality: Island of Trinidad, West Indies. Syntypes (2): USNM 5944 (1).

Callichthys melampterus Cope, 1872a: 275, pl. 14 (fig. 4). Type locality: Rio Ambyiacu, Peru. Lectotype: ANSP 8318, designated by Reis (1997: 303).

Hoplosternum schreineri Miranda Ribeiro, 1911: 150, pl. 35 (fig. 3). Type locality: Amazonas, Brazil. Lectotype: MNRJ 1051a, designated by Miranda Ribeiro (1953: 396).

Cascadura maculocephala Ellis, 1913: 387, pl. 25 (fig. 3). Type locality: Uruguayana [Rio Grande do Sul, Brazil]. Holotype: FMNH 54878.

Hoplosternum shirui Fowler, 1940a: 232, figs. 18-20. Type locality: Ucayali River basin, Contamana, Peru. Holotype: ANSP 68648.

Hoplosternum littorale daillyi Hoedeman, 1952b: 7. Type locality: Suriname (near Paramaribo). Holotype: ZMA $100277 \mathrm{a}$.

Hoplosternum thoracatum cayennae Hoedeman, 1961: 130. Type locality: French Guiana: Ile de Cayenne. Holotype: ZMA 102238a.

Distribution: Most cis-Andean South American river drainages north of Buenos Aires (Reis, 2003).

Hoplosternum magdalenae Eigenmann, 1913

Hoplosternum magdalenae Eigenmann, in Ellis, 1913: 412. Type locality: Soplaviento, Colombia. Holotype: FMNH 56281; holotype illustrated in Eigenmann (1922b: 65, pl. 8, figs. 3, 4). Also described as new in Eigenmann (1913: 30).

Distribution: Magdalena and Sinu River basins, Colombia (Reis, 2003).

Hoplosternum punctatum Meek \& Hildebrand, 1916

Hoplosternum punctatum Meek \& Hildebrand, 1916: 264, pl. 14. Type locality: Rio Marte Arnade, 6 miles east of Panama City, Panama. Holotype: FMNH 8943.

Distribution: Pacific coastal drainages of Panama and Atrato River basin, Colombia (Reis, 2003).

\section{LEPTHOPLOSTERNUM}

Lepthoplosternum Reis, 1997: 315. Type species: Callichthys pectoralis Boulenger, 1895. Type by original designation. Gender: Neuter.

Revision: Reis (1997).

Key: Reis \& Kaefer (2005).

Lepthoplosternum altamazonicum Reis, 1997

Lepthoplosternum altamazonicum Reis, 1997: 318, fig. 14. Type locality: Peru: Pucallpa: Río Ucayali. Holotype: MHNG 2551.01.

Distribution: Upper Amazon River basin, Peru (Reis, 2003).

Lepthoplosternum beni Reis, 1997

Lepthoplosternum beni Reis, 1997: 319, fig. 15. Type locality: Peru: Madre de Dios: Tambopata Reserve (12¹5'S, 69¹7'W). Holotype: USNM 329424.

Distribution: Madeira River basin of Bolivia and Peru (Reis, 2003).

Lepthoplosternum pectorale (Boulenger, 1895)

Callichthys pectoralis Boulenger, 1895b: 525. Type locality: Monte Sociedad, Paraguayan Chaco. Syntypes: BMNH 1895.5.17.57-61; syntype illustrated in Boulenger (1896c: pl. 4, fig. 4).

Distribution: Paraguay River basin (Reis, 2003).

Lepthoplosternum stellatum Reis \& Kaefer, 2005

Lepthoplosternum stellatum Reis \& Kaefer, 2005: 725, fig. 2. Type locality: Brazil, Amazonas, Tefé, Igarapé Remartimento, tributary of Lago Tefé, $1.5 \mathrm{~km}$ downstream from bridge on Tefé-Agrovila road, 03²4'28"S, 6444'10"W. Holotype: MCP 35599. 
Distribution: Tefé Lake tributary, Upper Amazon River basin, Brazil (Reis \& Kaefer, 2005).

Lepthoplosternum tordilho Reis, 1997

Lepthoplosternum tordilho Reis, 1997: 321, fig. 16. Type locality: Brazil: Rio Grande do Sul: Marginal lagoon near sanga do Junco, on dust road between hwy BR-116 and Barra do Ribeiro. Holotype: MCP 16310.

Distribution: Northern tributaries of Laguna dos Patos in southern Brazil (Reis, 2003).

Lepthoplosternum ucamara Reis \& Kaefer, 2005

Lepthoplosternum ucamara Reis \& Kaefer, 2005: 728, fig. 4. Type locality: Peru, Loreto, Pacay-Samiria National Reserve, Río Ucayali drainage, small creek into cocha Zapote, tributary to Rio Pacaya, 05²0'16"S, 74²9'10"W. Holotype: MUSM 23000.

Distribution: Ucayali River, Peru and Japurá River mouth, Brazil (Reis \& Kaefer, 2005).

\section{MEGALECHIS}

Megalechis Reis, 1997: 310. Type species: Callichthys thoracatus Valenciennes, 1840. Type by original designation. Gender: Feminine.

Revision: Reis (1997).

Remarks: Nomenclature of species in this genus corrected by Reis et al. (2005).

Megalechis picta (Müller \& Troschel, 1848)

Callichthys pictus Müller \& Troschel, 1848: 630. Type locality: Britisch-Guiana. Syntype: ZMB 3151 (1).

Callichthys sulcatus Kner, 1855a: 110. Type locality: Rio branco und Marabitanas [Brazil]. Lectotype: NMW 4775, designated by (Reis, 1997: 311). Originally Cal. sulcatus.

Hoplosternum oronocoi Fowler, 1915a: 229, fig. 8. Type locality: La Pedrita, Cano Uracoa, Venezuela. Holotype: ANSP 37895.

Distribution: Amazon, Orinoco and upper Essequibo River basins, and coastal rivers of northern Brazil (Reis et al., 2005: 681).

Megalechis thoracata (Valenciennes, 1840)

Callichthys thoracatus Valenciennes, in Cuvier \& Valenciennes, 1840b: 309 (230 in the Strasbourg deluxe edition), pl. 443. Type locality: la Mana [French Guiana]. Holotype: MNHN 0000-4266.

Callichthys longifilis Valenciennes, in Cuvier \& Valenciennes, 1840b: 317 (235 in the Strasbourg deluxe edition). Type locality: Cayenne. Holotype: MNHN 0000-4284.

Callychthys personatus Ranzani, 1841: 113. Type locality: [Unknown, apparently Brazil]. Holotype: MZUB 934. Illustrated and described in more detail in Ranzani (1842: 322, pl. 24, fig.1).

Callichthys exaratus Müller \& Troschel, 1848: 630. Type locality: Britisch-Guiana. Type (s): Whereabouts unknown.

Hoplosternum thoracatum surinamensis Hoedeman, 1952b: 5. Type locality: Surinam. Holotype: ZMA 100292.

Distribution: Amazon, Orinoco and Paraguay River basins, and coastal rivers of the Guianas and northern Brazil (Reis et al., 2005).

\section{SCLEROMYSTAX Günther, 1864}

Scleromystax Günther, 1864: 225, 229. Type species: Callichthys barbatus Quoy \& Gaimard, 1824. Type by monotypy. Gender: Masculine. Described in key (p. 225) as a subgenus of Callichthys, with species on p. 229.

Remarks: Recently removed from the synonymy of Corydoras by Britto (2003).

Scleromystax barbatus (Quoy \& Gaimard, 1824)

Callichthys barbatus Quoy \& Gaimard, 1824: 234. Type locality: ...petit ruisseaux qui aurosent, près de Rio de Janeiro, la ferme de Mandioca; [now: Fazenda da Japuhyba near Angra dos Reis, Rio de Janeiro, Brazil, 2259'S, $44^{\circ} 17^{\prime} \mathrm{W}$, by neotype designation]. Neotype: CAS 16006, designated by Nijssen \& Isbrücker (1980b: 202).

Corydoras kronei Miranda Ribeiro, 1907a: 189, figure on p. 189. Type locality: buracos do leito dos Rios Una da Aldeia e Despraiado [Iporanga, Brazil]. Lectotype: MNRJ 918A, designated by Nijssen \& Isbrücker (1980b: 202).

Corydoras eigenmanni Ihering, 1907: 34. Type locality: Riverlets by Cubatão and Raiz da Serra, State of São Paulo. Lectotype: MZUSP 153, designated by Britski (1969: 206). 
Distribution: Coastal drainages from Rio de Janeiro to Santa Catarina States, Brazil (Reis, 2003).

Scleromystax macropterus (Regan, 1913)

Corydoras macropterus Regan, 1913a: 232. Type locality: Paranagua, Brazil. Lectotype: BMNH 1913.1.1.11, designated by Nijssen \& Isbrücker (1980b: 206).

Corydoras bertoni Eigenmann, in Eigenmann \& Allen, 1942: 179. Type locality: Puerto Bertoni, Alto Paraná, Paraguay. Holotype (32 mm FL): IU 15441 (apparently not found at CAS).

Distribution: Coastal rivers of Brazil from São Paulo to Santa Catarina States, and some tributaries of the upper Paraná River (Reis, 2003).

Remarks: See Buckup \& Malabarba (1990) for comments on the type locality of Corydoras macropterus.

Scleromystax prionotos (Nijssen \& Isbrücker, 1980)

Corydoras prionotos Nijssen \& Isbrücker, 1980a: 5, figs. 6-7. Type locality: Linhares, 19²2'S, 4004'W, Lagoa Juparanã, Rio Doce system [Brazil]. Holotype: MNRJ 10537.

Distribution: Coastal rivers in southeastern Brazil from Espirito Santo to São Paulo, Brazil (Reis, 2003).

Scleromystax salmacis Britto \& Reis, 2005

Scleromystax salmacis Britto \& Reis, 2005: 482, figs. 1-2. Type locality: Brazil, Estado de Santa Catarina, Praia Grande, arroio Molha Coco in Vila Rosa nearly 5.5 km northwest from Praia Grande, 29²10'14"S 4958'53"W. Holotype: MCP 38388.

Distribution: Mampituba, Araranguá and Ratones river basins, Santa Catarina State, Brazil (Britto \& Reis, 2005).

\section{CETOPSIDAE Bleeker, 1858}

Cetopsini Bleeker, 1858b: 49, 250, 257. Type genus: Cetopsis Spix \& Agassiz, 1829.

Helogenidae Regan, 1911: 573. Type genus: Helogenes Günther, 1863.

Taxonomic summary: Vari \& Ferraris (2003).

Revision: Vari et al. (2005), Cetopsinae; Vari \& Ortega (1986), Helogeninae.

Monophyly: de Pinna \& Vari (1995).

5 genera, 41 species; no named fossil taxa.

CETOPSIDIUM Vari, Ferraris \& de Pinna, 2005

Cetopsidium Vari, Ferraris \& de Pinna, 2005: 133. Type species: Pseudocetopsis orientale Vari, Ferraris \& Keith, 2003. Type by original designation. Gender: Neuter.

Cetopsidium ferreirai Vari, Ferraris \& de Pinna, 2005

Cetopsidium ferreirai Vari, Ferraris \& de Pinna, 2005: 133, fig. 1. Type locality: Brazil. Pará: Rio Trombetas basin, Rio Mapuera, last cachoeira before Rio Trombetas (approximately 1º5'S, 5701'W). Holotype: INPA 10194.

Distribution: Trombetas River basin, Brazil (Vari et al., 2005: 135).

Cetopsidium minutum (Eigenmann, 1912)

Hemicetopsis minutus Eigenmann, 1912b: 211, pl. 23 (fig. 2). Type locality: Amatuk Cataract, British Guiana. Holotype: FMNH 53262.

Distribution: Potaro and Middle Essequibo Rivers, Guyana (Vari et al., 2005: 137).

Cetopsidium morenoi (Fernández-Yépez, 1972)

Hemicetopsis morenoi Fernández-Yépez, 1972b: 19. Type locality: Río Aguaro, 1 km. abajo del paso Garcerito al Oeste franco de Santa Rita, Estado Guárico, Venezuela. Holotype: author's personal collection, whereabouts unknown.

Distribution: Central and western tributaries of Orinoco River basin, Colombia and Venezuela (Vari et al., 2005: 141).

Cetopsidium orientale (Vari, Ferraris \& Keith, 2003)

Pseudocetopsis orientale Vari, Ferraris \& Keith, 2003: 693, fig. 1. Type locality: Suriname, Brokopondo District, Mindrineti Kreek, close to mouth of Maykaboeka Kreek, Saramacca River basin, on Gros Rosevel Mining concession (507'08.8"N, 55¹6'59.4"W). Holotype: MHNG 2621-040. 
Distribution: Suriname and French Guiana (Vari et al., 2005: 144).

Cetopsidium pemon Vari, Ferraris \& de Pinna, 2005

Cetopsidium pemon Vari, Ferraris \& de Pinna, 2005: 144, fig. 8-9. Type locality: Venezuela. Bolivar: Río Caroni, at mouth of Río Claro (7º54'45"N, 6302'35"W). Holotype: MCNG 19475.

Distribution: Caroni, Caura and Meta river basins, Venezuela and Colombia, Branco River, Brazil (Vari et al., 2005 : 146).

Cetopsidium roae Vari, Ferraris \& de Pinna, 2005

Cetopsidium roae Vari, Ferraris \& de Pinna, 2005: 147, fig. 10. Type locality: Guyana. Rupununi River, Karanambo $\left(3^{\circ} 45^{\prime} \mathrm{N}, 58^{\circ} 18^{\prime} \mathrm{W}\right)$. Holotype: BMNH 1972.7.27.629.

Distribution: Rupununi region, Guyana (Vari et al., 2005: 149).

\section{CETOPSIS Spix \& Agassiz, 1829}

Cetopsis Spix \& Agassiz, 1829: 11. Type species: Silurus coecutiens Lichtenstein, 1819. Type by subsequent designation by Bleeker (1862-63: 16). Gender: Feminine.

Hemicetopsis Bleeker, 1862c: 403. Type species: Cetopsis candiru Spix \& Agassiz, 1829. Type by monotypy. Gender: Feminine.

Pseudocetopsis Bleeker, 1862c: 403. Type species: Cetopsis gobioides Kner, 1857. Type by monotypy. Gender: Feminine.

Bathycetopsis Lundberg \& Rapp Py-Daniel, 1994: 382. Type species: Bathycetopsis oliveirai Lundberg \& Rapp PyDaniel, 1994. Type by original designation. Gender: Feminine.

Cetopsis amphiloxa (Eigenmann, 1914)

Hemicetopsis amphiloxus Eigenmann, in Eigenmann, Henn \& Wilson, 1914: 14. Type locality: Creek near San Lorenzo, [Río] Patía basin, Colombia. Holotype: FMNH 56519; holotype illustrated in Eigenmann (1922b: 57, pl. 3, fig. 2) and Vari et al. (2005: 151, fig. 11).

Distribution: Patía, upper San Juan and Atrato River basins, Pacific versant, western Colombia, and rivers of northwestern Ecuador (Vari et al., 2005: 152).

Cetopsis arcana Vari, Ferraris \& de Pinna, 2005

Cetopsis arcana Vari, Ferraris \& de Pinna, 2005: 153, fig. 13. Type locality: Brazil. Goiás: São Domingos, Parque Estadual de Terra Ronca, Boca da Lapa de Bezerra (13³2'43"S, 46²2'35"W). Holotype: MZUSP 58637.

Distribution: Middle and upper Tocantins River basin (Vari et al., 2005:155).

Cetopsis baudoensis (Dahl, 1960)

Pseudocetopsis baudoênsis Dahl, 1960a: 452, figured on p. 453. Type locality: Quitasol, Baudó, western Colombia. Holotype: ICNMHN 118.

Distribution: Baudó River basin, Pacific versant, Colombia (Vari et al., 2005:157).

Cetopsis caiapo Vari, Ferraris \& de Pinna, 2005

Cetopsis caiapo Vari, Ferraris \& de Pinna, 2005: 157, fig. 15. Type locality: Brazil: Goiás: Município de Minaçu/ Cavalcante, Rio Tocantins, at location of the future dam of the Usina Hidroeléletrica Serra da Mesa (13⒌'S, 48 $19^{\prime}$ W). Holotype: MNRJ 13236.

Distribution: Tocantins River basin, Brazil (Vari et al., 2005:159).

Cetopsis candiru Spix \& Agassiz, 1829

Cetopsis candiru Spix \& Agassiz, 1829: 13, pl. 10 (fig. 1). Type locality: Brasiliae aequatoralis fluviis. Lectotype: MHNG 210.05, designated by Vari et al. (2005: 160); MNHG syntype illustrated in Mahnert (1985: 12, fig. 4). Name first appeared in Cuvier (1829) as a nomen nudum.

Cetopsis Spixii Swainson, 1839: 308. Type locality: Brasiliae aequatoralis fluviis. Syntypes: MHNG 210.05 (1), MHNN 735-7 (3). Unneeded new name for Cetopsis candiru Spix \& Agassiz, 1829.

Distribution: Amazon River basin (Vari et al., 2005: 160).

Cetopsis coecutiens (Lichtenstein, 1819)

Silurus coecutiens Lichtenstein, 1819: 61. Type locality: Brasilien. Holotype: ZMB 3093, apparently no longer extant (Vari et al., 2005: 164).

Silurus cacutiens Günther, 1864: 199. Type locality: Brazil. Holotype: ZMB 3093, apparently no longer extant (Vari 
et al., 2005: 164). Unjustified emendation of Silurus coecutiens Lichtenstein, 1819.

Distribution: Amazon, Tocantins and Orinoco River basins (Vari et al., 2005: 164).

Cetopsis fimbriata Vari, Ferraris \& de Pinna, 2005

Cetopsis fimbriata Vari, Ferraris \& de Pinna, 2005: 165, fig. 20. Type locality: Colombia. Choco: Creek of upper Río Nercua (approximately $7^{\circ} 01^{\prime} \mathrm{N}, 77^{\circ} 30^{\prime} \mathrm{W}$ ), large tributary of Río Truando. Holotype: USNM 305348.

Distribution: Nercua River, a tributary of the Truando River which flows into the Atrato basin of the Caribbean Sea versant of northwestern Colombia (Vari et al., 2005: 167).

Cetopsis gobioides Kner, 1857

Cetopsis gobioides Kner, 1857: 407, pl. 6 (fig. 16). Type locality: Irisanga [Brazil]. Syntypes: NMW 47378 (2).

Cetopsis chalmersi Norman, 1926a: 116. Type locality: Rio das Velhas about 32 miles north of Bello (=Belo) Horizonte, Brazil. Lectotype: BMNH 1925.10.1.4, designated by Vari et al. (2005: 171).

Distribution: Upper São Francisco, Paraná and Uruguay River basins and Juquiá River of coastal Brazil (Vari et al., 2005: 170).

Remarks: Silurus pygmaeus Natterer is an unavailable manuscript name published in the account of Cetopsis gobioides Kner (1857).

Cetopsis jurubidae (Fowler, 1944)

Pseudocetopsis jurubidae Fowler, 1944b: 235, figs. 7-9. Type locality: Clear waters of Rio Jurubidá, Nuquí [northwestern Colombia]. Holotype: ANSP 71430. Holotype illustrated in Vari et al. (2005: 173, figs. 23-24).

Distribution: Jurubidá River basin, Pacific versant, Colombia (Vari et al., 2005: 174).

Cetopsis montana Vari, Ferraris \& de Pinna, 2005

Cetopsis montana Vari, Ferraris \& de Pinna, 2005: 174, fig. 26. Type locality: Peru. Amazonas: 100 m upriver from Caterpiza (approximately $3^{\circ} 55^{\prime} \mathrm{S}, 7^{\circ} 42^{\prime} \mathrm{W}$ ). Holotype: LACM 41989-1.

Distribution: Amazon River basin of northeastern Peru and southeastern Ecuador (Vari et al., 2005: 150).

Cetopsis motatanensis (Schultz, 1944)

Pseudocetopsis plumbeus motatanensis Schultz, 1944c: 255, pl. 5 (fig. b). Type locality: Río Motatán, 4 km. above Motatán, Venezuela. Holotype: USNM 121265.

Distribution: Lake Maracaibo basin of Venezuela and Colombia (Vari et al., 2005: 178).

Cetopsis oliveirai (Lundberg \& Rapp Py-Daniel, 1994)

Bathycetopsis oliveirai Lundberg \& Rapp Py-Daniel, 1994: 383, figs. 1, 3-4. Type locality: Brazil, Amazonas State, Rio Solimões north of Ilha da Marchantaria, approximately $15 \mathrm{~km}$ upstream of the mouth of Rio Negro at Manaus, approximately $15 \mathrm{~m}$ depth, 3ำ' $15^{\prime}, 60^{\circ} 00^{\prime} \mathrm{W}$. Holotype: INPA 4439.

Distribution: Middle and upper Amazon River basin, Brazil and Peru (Vari et al., 2005: 180).

Cetopsis orinoco (Schultz, 1944)

Pseudocetopsis plumbeus orinoco Schultz, 1944c: 253, pl. 5 (fig. a). Type locality: Río Torbes, 1 km. above Táriba, Orinoco system, Venezuela. Holotype: USNM 121263.

Distribution: Orinoco River basin and Aroa and Yaracuy river basins of coastal Venezuela (Vari et al., 2005: 183).

Cetopsis othonops (Eigenmann, 1912)

Hemicetopsis othonops Eigenmann, 1912a: 17. Type locality: Girardot, Colombia. Holotype: FMNH 56040; holotype illustrated in Eigenmann (1922b: 56, pl. 3, fig. 1).

Distribution: Magdalena and Sinu River basins (Vari et al., 2005); also Cauca, San Jorge, Apulo and Catatumbo basins, Colombia (Maldonado Campo et al., 2005).

Cetopsis parma Oliviera, Vari \& Ferraris, 2001

Cetopsis parma Oliviera, Vari \& Ferraris, 2001: 575, fig. 1. Type locality: Peru. Departamento de Ucayali, Provincia Coronel Portillo. Río Tambo, Río Ucayali basin, Pucallpa, Atalaya ( $\left.8^{\circ} 23^{\prime} \mathrm{S}, 74^{\circ} 32^{\prime} \mathrm{W}\right)$. Holotype: MUSM 2266; illustrated in Vari et al. (2005: 187, fig. 35).

Distribution: Upper Amazon River basin (Vari et al., 2005: 188).

Cetopsis pearsoni Vari, Ferraris \& de Pinna, 2005

Cetopsis pearsoni Vari, Ferraris \& de Pinna, 2005: 189, fig. 36. Type locality: Peru. Puno: Provincia de Sandia, Río Candamo, Zona Reserva Tambopata (13²8.97'S, 6950.08'W), 358 m elevation. Holotype: MUSM 11093.

Distribution: Upper Madeira River basin, Peru and Bolivia (Vari et al., 2005: 190). 
Cetopsis plumbea (Steindachner, 1882)

Cetopsis plumbeus Steindachner, 1882b: 178. Type locality: Canelos [Ecuador]. Lectotype: NMW 47381-1 (46 mm SL), designated by Vari et al. (2005: 194). Species illustrated and described in more detail in Steindachner (1882c: 31, pl. 6, fig. 3).

Cetopsis macroteronema Boulenger, 1898c: 8. Type locality: Rio Zamora, Equateur oriental. Syntypes: BMNH 1898.11.4.11 (1), ZMUT 1548 (1)

Distribution: Upper Amazon River basin, Ecuador and Peru (Vari et al., 2005: 193).

Cetopsis sandrae Vari, Ferraris \& de Pinna, 2005

Cetopsis sandrae Vari, Ferraris \& de Pinna, 2005: 195, figs. 39-40. Type locality: Brazil. Mato Grosso: Nova Mutum, unnamed Corrego, Fazenda Buriti, approximately $1.5 \mathrm{~km} \mathrm{~S}$ of Fazenda (1351'52"S, 56¹1'36"W). Holotype: MZUSP 61091.

Distribution: Arinos River, upper Tapajós River basin, Brazil (Vari et al., 2005: 150).

Cetopsis sarcodes Vari, Ferraris \& de Pinna, 2005

Cetopsis sarcodes Vari, Ferraris \& de Pinna, 2005: 197, fig. 42. Type locality: Brazil. Goiás: Município de Minaçu/ Cavalcante, Rio Tocantins between site of future dam of Usina Hidroeléctrica Cana Brava and Ilha do Orgulho and Porto do Garimpo. Holotype: MNRJ 13024.

Distribution: Tocantins River basin, Brazil (Vari et al., 2005:199).

Cetopsis starnesi Vari, Ferraris \& de Pinna, 2005

Cetopsis starnesi Vari, Ferraris \& de Pinna, 2005: 199, fig. 43. Type locality: Bolivia. Tarija: Argentinian-Bolivian border, Río Bermejo, 4-5 km S of Pueblo Salado, approximately 30 air km S of Bermejo $\left(22^{\circ} 27^{\prime} \mathrm{S}, 64^{\circ} 32^{\prime} \mathrm{W}\right)$. Holotype: USNM 314309.

Distribution: Northwestern part of La Plata River basin and upper Madeira River basin, Bolivia (Vari et al., 2005: 201).

Cetopsis umbrosa Vari, Ferraris \& de Pinna, 2005

Cetopsis umbrosa Vari, Ferraris \& de Pinna, 2005: 201, fig. 44. Type locality: Colombia. Meta: Río Negro, downstream from main Villavicencio to Puerto Lopez highway at La Balsa $\left(04^{\circ} 04^{\prime} \mathrm{N}, 7^{\circ} 04^{\prime} \mathrm{W}\right)$. Holotype: ANSP 137559.

Distribution: Orinoco River basin, Colombia (Vari et al., 2005: 203).

DENTICETOPSIS Ferraris, 1996

Denticetopsis Ferraris, 1996: 162. Type species: Denticetopsis sauli Ferraris, 1996. Type by original designation. Gender: Feminine.

Denticetopsis epa Vari, Ferraris \& de Pinna, 2005

Denticetopsis epa Vari, Ferraris \& de Pinna, 2005: 206, fig. 45. Type locality: Brazil. Pará: Igarapé do Limão, Rio Tocantins, Baião (2\%41'S, 4941'W). Holotype: MZUSP 24060.

Distribution: Lower Tocantins River basin, Brazil (Vari et al., 2005: 208).

Denticetopsis iwokrama Vari, Ferraris \& de Pinna, 2005

Denticetopsis iwokrama Vari, Ferraris \& de Pinna, 2005: 208, fig. 47. Type locality: Guyana. Essequibo: Siparuni River at Tumble Down Creek, Essequibo River basin (448'39"N, 5851'11"W). Holotype: ANSP 177215.

Distribution: Siparuni River basin, Guyana (Vari et al., 2005: 210).

Denticetopsis macilenta (Eigenmann, 1912)

Hemicetopsis macilentus Eigenmann, 1912b: 211, pl. 23 (fig. 1). Type locality: Creek below Potaro Landing, British Guiana. Holotype: FMNH 53260; illustrated in Vari et al. (2005: 211, figs. 48-49).

Distribution: Potaro River, Essequibo River basin, Guyana (Vari et al., 2005: 212).

Denticetopsis praecox (Ferraris \& Brown, 1991)

Pseudocetopsis praecox Ferraris \& Brown, 1991: 162, fig. 1. Type locality: Neblina base camp, Río Mawarinuma of the Río Baria drainage, Territorio Federal Amazonas, southern Venezuela, 055'N, 66º' $10^{\prime} \mathrm{W}$, elevation $120 \mathrm{~m}$. Holotype: AMNH 74446.

Distribution: Baria River, Upper Negro River basin, Venezuela (Vari et al., 2005: 215).

Denticetopsis royeroi Ferraris, 1996 
Denticetopsis royeroi Ferraris, 1996: 167, figs. 4, 12. Type locality: Venezuela: Estado Amazonas, Caño Chola at crossing of San Carlos de Río Negro to Solano Road, $1^{\circ} 58^{\prime} \mathrm{N}, 67^{\circ} 00^{\prime} \mathrm{W}$. Holotype: MBUCV V-26785, illustrated in Vari et al. (2005: 216, fig. 51).

Distribution: Upper Negro River basin, Venezuela; known only from holotype (Vari et al., 2005: 216).

Denticetopsis sauli Ferraris, 1996

Denticetopsis sauli Ferraris, 1996: 164, figs. 3, 11. Type locality: Venezuela, Estado Amazonas, Outflow stream from series of morichales, ca. $5.0 \mathrm{~km}$ from mouth of Río Pamoni, $2^{\circ} 48^{\prime} \mathrm{N}, 65^{\circ} 53^{\prime} \mathrm{W}$. Holotype: MBUCV V20300.

Distribution: Around mouth of Pamoni River, Venezuela; known only from type series (Vari et al., 2005: 218).

Denticetopsis seducta Vari, Ferraris \& de Pinna, 2005

Denticetopsis seducta Vari, Ferraris \& de Pinna, 2005: 218, fig. 53. Type locality: Peru. Loreto: Caño "Sabalito," small creek on terra firma approximately $25 \mathrm{mi}(=\mathrm{km}) \mathrm{S}$ of Iquitos (4'14'45"S, $\left.73^{\circ} 24^{\prime} 57^{\prime \prime} \mathrm{W}\right)$. Holotype: ANSP 178466.

Distribution: Western Amazon River basin, and possibly southwestern Orinoco River basin (Vari et al., 2005: 221).

\section{HELOGENES Günther, 1863}

Helogenes Günther, 1863: 443. Type species: Helogenes marmoratus Günther, 1863. Type by monotypy. Gender: Masculine.

Leyvaichthys Dahl, 1960b: 302. Type species: Leyvaichthys castaneus Dahl, 1960. Type by original designation. Gender: Masculine. Spelled Leyvaichths in one place, precedence established by first-reviser action of Eschmeyer \& Bailey (in Eschmeyer, 1990: 221).

Revision: Vari \& Ortega (1986).

Helogenes castaneus (Dahl, 1960)

Leyvaichthys castaneus Dahl, 1960b: 303. Type locality: Small pond in a brook tributary to the Guayabero River, approx. one kilometer from the mouth of the brook, and 1500 meters from the expedition Camp $\mathrm{N}^{\circ} 1$, situated on the left bank of the Guayabero about 4 kilometers from Camp Thompson, Colombia. Holotype: Whereabouts unknown.

Distribution: Upper Orinoco, Guaviare and Meta River basins, Colombia (Vari \& Ferraris, 2003).

Helogenes gouldingi Vari \& Ortega, 1986

Helogenes gouldingi Vari \& Ortega, 1986: 7, fig. 3. Type locality: Brazil. Amazonas: Igarapé, 15 km from Humaitá along Rio Madeira ( $\left.7^{\circ} 31^{\prime} \mathrm{S}, 63^{\circ} 02^{\prime} \mathrm{W}\right)$. Holotype: MZUSP 28854.

Distribution: Madeira River basin, Brazil (Vari \& Ferraris, 2003).

Helogenes marmoratus Günther, 1863

Helogenes marmoratus Günther, 1863: 443. Type locality: Essequibo River, Guiana. Lectotype: BMNH 1864.1.21.83, designated by Vari \& Ortega (1986: 16).

Helogenes amazonae Delsman, 1941: 80. Type locality: Manaos [=Manaus, Amazonas, Brazil]. Holotype: IRSNB 545.

Helogenes unidorsalis Glodek \& Carter, 1978: 77, fig. 1. Type locality: Eastern Ecuador, Rio Bobonaza between Sarayacu and Montalvo. Holotype: FMNH 80463.

Distribution: Atlantic drainages of Guianas, upper Orinoco and Negro systems and upper Amazon River basin (Vari \& Ferraris, 2003).

Helogenes uruyensis Fernández-Yépez, 1967

Helogenes marmoratus uruyensis Fernández-Yépez, 1967: 166, pls. 2, 3 (fig. 2). Type locality: Río Uruyén, Venezuela. Holotype: AFY 56613 (whereabouts unknown).

Distribution: Uruyén River basin, Venezuela (Vari \& Ferraris, 2003).

PARACETOPSIS Bleeker, 1862

Paracetopsis Bleeker, 1862 (in Bleeker, 1862-63): 16. Type species: Paracetopsis bleekeri Bleeker, 1862. Type by original designation. Gender: Feminine.

Paracetopsis Eigenmann \& Bean, 1907: 615. Type species: Cetopsis occidentalis Steindachner, 1880. Type by orig- 
inal designation. Gender: Feminine. Preoccupied by Paracetopsis Bleeker, 1862.

Cetopsogiton Eigenmann \& Bean, in Eigenmann, 1910: 398. Type species: Cetopsis occidentalis Steindachner, 1880. Type by being a replacement name. Gender: Masculine. Replacement for Paracetopsis Eigenmann \& Bean, 1907, preoccupied by Paracetopsis Bleeker, 1862.

Paracetopsis atahualpa Vari, Ferraris \& de Pinna, 2005

Paracetopsis atahualpa Vari, Ferraris \& de Pinna, 2005: 223, fig. 54. Type locality: Peru. Tumbes: Río Tumbes, Cabo Inga ( $\left.3^{\circ} 58^{\prime} 00^{\prime \prime} \mathrm{S}, 80^{\circ} 24^{\prime} 59^{\prime \prime} \mathrm{W}\right)$. Holotype: MUSM 11560.

Distribution: Tumbes River basin, northwestern Peru and upper Zarumilla River basin, southwestern Ecuador (Vari et al., 2005: 224).

Paracetopsis bleekeri Bleeker, 1862

Paracetopsis bleekeri Bleeker, 1862 (in Bleeker, 1862-63): 16. Type locality: Not stated. Neotype: NMW 47377, designated by Vari et al. (2005: 227).

Cetopsis ventralis Gill, 1870: 95. Type locality: Maranon or upper Amazon, and Napo Rivers [in error, actually rivers of southwestern Ecuador]. Holotype: USNM 8307.

Cetopsis occidentalis Steindachner, 1880b: 99, pl. 8 (figs. 2, 2a). Type locality: Guayaquil [Ecuador]. Holotype: NMW 47377.

Distribution: Guayas and Santa Rosa river basins, Pacific Ocean versant of southwestern Ecuador (Vari et al., 2005: 227).

Paracetopsis esmeraldas Vari, Ferraris \& de Pinna, 2005

Paracetopsis esmeraldas Vari, Ferraris \& de Pinna, 2005: 228, fig. 57. Type locality: Ecuador. Esmeraldas: Río Blanco, $4 \mathrm{~km}$ above junction with Río Quininde $\left(0^{\circ} 20^{\prime} \mathrm{N}, 79^{\circ} 28^{\prime} \mathrm{W}\right.$; Río Esmeraldas basin). Holotype: MCZ 48768 .

Distribution: Pacific Ocean versant rivers of northwestern Ecuador (Vari et al., 2005: 230).

\section{CHACIDAE Bleeker, 1858}

Chacini Bleeker, 1858b: 49, 310, 322. Type genus: Chaca Gray, 1831.

Revision: Roberts (1982a).

Identification guide: Jayaram \& Majumdar (1964), Jayaram (1980), South Asia.

Phylogeny: Brown \& Ferraris (1988).

1 genus, 3 species; no named fossil taxa.

\section{CHACA Gray, 1831}

Chaca Gray, 1831: 9. Type species: Chaca hamiltonii Gray, 1831. Type by monotypy. Gender: Feminine.

Chaca Cuvier \&Valenciennes, in Valenciennes, 1832b: 386. Type species: Chaca lophioides Cuvier \& Valenciennes, 1832. Type by monotypy. Gender: Feminine. Preoccupied by Chaca Gray, 1831.

Brachystacus Van der Hoeven, 1849: 280. Type species: Platystacus chaca Hamilton, 1822. Type by monotypy. Gender: Masculine.

Chaca bankanensis Bleeker, 1852

Chaca bankanensis Bleeker, 1852c: 455. Type locality: Banka, in fluviis. Holotype (68 mm TL): RMNH 5405.

Chaca bankae Giebel, 1857: 325. Type locality: Bangka. Either an emendation of, or misspelling for, Chaca bankanensis Bleeker, 1852.

Distribution: Southern Malay Peninsula, Sumatra and Borneo (Roberts, 1982a).

Remarks: BMNH 1863.12.4.117 (113 mm SL, $131 \mathrm{~mm} \mathrm{TL}$ ), is listed as a type of Chaca bankanensis, but is too large to be the holotype.

Chaca burmensis Brown \& Ferraris, 1988

Chaca burmensis Brown \& Ferraris, 1988: 3, fig. 1. Type locality: Sittang River, Burma. Holotype: BMNH 1891.11.30.144.

Distribution: Lower Irrawaddy and Sittang River basins, Myanmar. 
Chaca chaca (Hamilton, 1822)

Platystacus chaca Hamilton, 1822: 140, 374, pl. 28 (fig. 43). Type locality: rivers and ponds of the northeastern parts of Bengal. No types known.

Chaca hamiltonii Gray, 1831: 9. Type locality: rivers and ponds of the northeastern parts of Bengal. New name for Platystacus chaca Hamilton, 1822, to avoid tautonymy.

Chaca lophioides Cuvier \& Valenciennes, in Valenciennes, 1832b: 386, pl. 4 (fig. 2). Type locality: Bengal. New name for Platystacus chaca Hamilton, 1822, to avoid tautonymy. Also described in Cuvier \& Valenciennes (1840b: 445 [p. 330 in Strasbourg deluxe edition], pl. 451).

Chaca buchanani Günther, 1864: 29. Type locality: Ganges. No types known. New name for Platystacus chaca Hamilton, 1822, to avoid tautonymy.

Distribution: Ganges-Brahmaputra River system, India and Bangladesh (Roberts, 1982a).

\section{CLARIIDAE Bonaparte, 1846}

Clariini Bonaparte, 1846: 5. Type genus: Clarias Scopoli, 1777.

Heterobranchoidei Bleeker, 1858b: 33, 37, 41, 333. Type genus: Heterobranchus Geoffroy St. Hilaire, 1809.

Heteropneustidae Hora, 1936a: 209. Type genus: Heteropneustes Müller, 1840.

Taxonomic summary: Teugels (1986b, Africa).

Review: Jackson (1959, Nyasaland); Skelton \& Teugels (1991, southern Africa); Teugels (1992a, west Africa).

Key to genera: Poll \& Gosse (1994, Africa).

Identification guide: Jayaram (1980, South Asia).

Phylogeny: Teugels \& Adriens (2003); Diogo et al. (2003a), Agnèse \& Teugels (2005, African species); Jansen et al. (2005, anguilliform species).

16 genera, 113 species; including 2 fossil species.

BATHYCLARIAS Jackson, 1959

Bathyclarias Jackson, 1959: 112. Type species: Clarias longibarbis Worthington, 1933. Type by original designation. Gender: Masculine.

Remarks: Removed from the synonymy of Dinotopterus by Anseaume \& Teugels (1999).

Species flock evolution: Agnèse \& Teugels (2001a).

Bathyclarias euryodon Jackson, 1959

Bathyclarias euryodon Jackson, 1959: 120, fig. 4. Type locality: Nkata Bay, Lake Nyasa. Holotype: BMNH 1960.2.29.13

Distribution: Lake Malawi (Teugels, 1986b, as Dinotopterus euryodon).

Bathyclarias ilesi Jackson, 1959

Bathyclarias ilesi Jackson, 1959: 116, fig. 2. Type locality: Nkata Bay, Lake Nyasa. Holotype: BMNH 1960.2.29.8.

Distribution: Lake Malawi (Teugels, 1986b, as Dinotopterus ilesi).

Bathyclarias longibarbis (Worthington, 1933)

Clarias longibarbis Worthington, 1933b: 309, fig. 10. Type locality: Lake Nyasa. Holotype: BMNH 1932.11.15.592.

Distribution: Lake Malawi (Teugels, 1986b, as Dinotopterus longibarbis).

Bathyclarias rotundifrons Jackson, 1959

Bathyclarias rotundifrons Jackson, 1959: 118, fig. 3. Type locality: Lake Nyasa. Holotype: BMNH 1960.2.29.11.

Distribution: Lake Malawi (Teugels, 1986b, as Dinotopterus rotundifrons).

Bathyclarias worthingtoni Jackson, 1959

Bathyclarias worthingtoni Jackson, 1959: 123, fig. 6. Type locality: [Nkata Bay] Lake Nyasa. Holotype: BMNH 1960.2.29.18.

Distribution: Lake Malawi (Teugels, 1986b, as Dinotopterus worthingtoni). 
CHANNALLABES Günther, 1873

Channallabes Günther, 1873a: 143. Type species: Gymnallabes apus Günther, 1873. Type by monotypy. Gender: Feminine. Originally proposed as a subgenus of Gymnallabes.

Channallabes apus (Günther, 1873)

Gymnallabes apus Günther, 1873a: 142. Type locality: Interior of Ambriz, Angola. Holotype: BMNH 1873.7.28.16. Distribution: Congo River basin (Teugels, 1986b).

\section{CLARIALLABES Boulenger, 1900}

Clariallabes Boulenger, 1900a: 136. Type species: Clarias melas Boulenger, 1887. Type by original designation. Gender: Feminine.

Allabenchelys Boulenger, 1902e: 234. Type species: Allabenchelys longicauda Boulenger, 1902. Type by monotypy. Gender: Feminine.

Clariallabes attemsi (Holly, 1927)

Allabenchelys attemsi Holly, 1927b: 202, fig. 2. Type locality: Kamerun. Holotype: NMW 7897.

Distribution: Cameroon, known only from an indefinite type locality (Teugels, 1986b).

Clariallabes brevibarbis Pellegrin, 1913

Clariallabes brevibarbis Pellegrin, 1913b: 272. Type locality: Ngomo (Ogôoué). Holotype: MNHN 1913-0265.

Distribution: Ogowe River, Gabon (Teugels, 1986b).

Clariallabes centralis (Poll \& Lambert, 1958)

Clarias centralis Poll \& Lambert, 1958: 334, figure on p. 335. Type locality: Kunungu (Congo). Holotype: MRAC 29659.

Distribution: Central Congo River system (Teugels, 1986b).

\section{Clariallabes heterocephalus Poll, 1967}

Clariallabes heterocephalus Poll, 1967: 233, fig. 108. Type locality: Poste de Cuílo, Xá-Ua, riv. Luíta, (802'S, $\left.19^{\circ} 25^{\prime} \mathrm{E}\right)$, Angola. Holotype: MRAC 162082.

Distribution: Luita River, Angola (Teugels, 1986b).

Clariallabes laticeps (Steindachner, 1911)

Allabenchelys laticeps Steindachner, 1911d: 532. Type locality: Mawambi, Ituri-Flüss. Syntypes (9): NMW 7649 (1), NMW 47988 (1), NMW 47989 (1), NMW 479890 (1), NMW 47991 (1).

Distribution: Ituri River, Aruwimi Basin, Congo River system; known only from type locality (Teugels, $1986 \mathrm{~b})$.

Clariallabes longicauda (Boulenger, 1902)

Allabenchelys longicauda Boulenger, 1902e: 234, pl. 22 (figs. 1-1a). Type locality: Ja River, flowing into the Sanga, an affluent of the right bank of the Congo, French Congo, 250 miles from the coast. Holotype: BMNH 1902.11.12.129.

Allabenchelys brevior Boulenger, 1903c: 439. Type locality: Ja River, southern Cameroon. Syntypes (2): BMNH 1903.7.28.194-195 (2).

Distribution: Central Congo River system (Teugels, 1986b).

Clariallabes manyangae (Boulenger, 1919)

Allabenchelys manyangae Boulenger, 1919: 159. Type locality: Kinkenda (Manyanga), région des Cataractes du Bas-Congo. Syntypes (3): BMNH 1919.8.28.12 (1), MRAC 1327 (1), MRAC 1329 (1).

Distribution: Manyanga, Kinkenda; known only from type locality (Teugels, 1986b).

Clariallabes melas (Boulenger, 1887)

Clarias melas Boulenger, 1887a: 148. Type locality: Lower Congo. Holotype: BMNH 1887.1.13.6. Illustrated in Boulenger (1902e: pl. 22, fig. 2), possibly based on the holotype.

Clariallabes melas lembesseensis Fowler, 1949: 259, figs. 48-52. Type locality: Oka, Congo system. Holotype: ANSP 71906.

Distribution: Congo River system (Teugels, 1986b).

Clariallabes mutsindoziensis Taverne \& De Vos, 1998

Clariallabes mutsindoziensis Taverne \& De Vos, 1998: 212, figs. 1-3. Type locality: Rivière Mutsindozi près de son

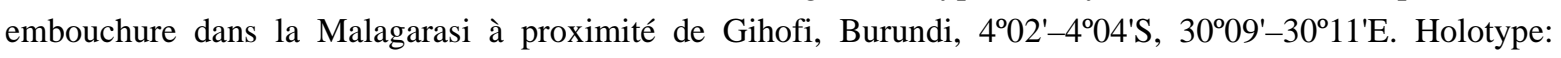


MRAC 96-31-P-53.

Distribution: Mutsindozi River, Lake Tanganyika basin, Burundi (Taverne \& De Vos, 1998).

Clariallabes petricola Greenwood, 1956

Clariallabes petricola Greenwood, 1956: 556, fig. 1. Type locality: Nile, about half a mile below the Owen-falls dam, Jinja, Uganda. Holotype: BMNH 1956.10.9.204.

Distribution: Lake Victoria and the Victoria Nile, near the Owen-falls dam (Teugels, 1986b).

Clariallabes pietschmanni (Güntert, 1938)

Allabenchelys pietschmanni Güntert, 1938: 299. Type locality: Aus dem Njong [Cameroon]. Syntypes (26): NMW 46063, ? NMW 47933 (1). Also appeared in Güntert (1939: 25).

Distribution: Nyong River; known only from type locality (Teugels, 1986b).

Clariallabes platyprosopos Jubb, 1965

Clariallabes platyprosopos Jubb, 1965: 393, fig. 1. Type locality: Upper Zambezi River, about 15 miles above the Victoria Falls, Rhodesia. Holotype: AMG P1050.

Distribution: Upper Zambezi River (Teugels, 1986b).

Clariallabes simeonsi Poll, 1941

Clariallabes simeonsi Poll, 1941a: 4, figs. 2, 5. Type locality: Uélé, rivières Mosongolia, Mekokpa, et Matanga [Precise locality of holotype not stated], Congo belge. Holotype: IRSNB 61.

Distribution: Uélé River, Congo River basin (Teugels, 1986b).

\section{Clariallabes teugelsi nomen novum}

Clarias (Allabenchelys) dumerili longibarbis David \& Poll, 1937: 232, fig. 21. Type locality: Léopoldville, Congo Belge. Syntypes: MRAC 44011-013 (3). Preoccupied by Clarias longibarbis Worthington, 1933.

Distribution: central Congo River basin (Teugels, 1986b).

Remarks: The name Clarias (Allabenchelys) dumerili longibarbis David \& Poll is a junior primary homonym of Clarias longibarbis Worthington and, as such, was permanently invalid upon publication. The species was treated as valid, as Clariallabes dumerili David \& Poll, 1937, by Teugels (1986b: 67). However, Clariallabes dumerili was not proposed as an available name in David \& Poll and it also was not made available by action of Teugels. Teugels (1986b) lists no available synonyms for this name, so the name Clariallabes teugelsi is proposed here as a replacement for Clarias (Allabenchelys) dumerili longibarbis to provide a valid name for this species.

Clariallabes uelensis (Poll, 1941)

Clarias (Allabenchelys) uelensis Poll, 1941a: 14, fig. 6. Type locality: rivières Mosongolia, Mekokpa, et Matanga, région Ibembo, Uélé [precise locality of holotype not stated.]. Holotype: IRSNB 63.

Distribution: Mosongolia, Mekokpa or Matanga Rivers, known only from types (Teugels, 1986b).

Clariallabes variabilis Pellegrin, 1926

Clariallabes variabilis Pellegrin, 1926: 201. Type locality: Dumbi ..., Kabambaie ...., Belenge ...., Tshimbu ..., Kamaiembi; Congo Belge. Syntypes (13): MNHN 1926-0151(1), MNHN 1926-0152 (1), MNHN 1926-0153 (1), MNHN 1926-0154 (1), MRAC 19689 (1), MRAC 19690 (1), MRAC 19692 (1), MRAC 19694 (1), MRAC 19696 (1), MRAC 19702 (1), MRAC 19712 (1), MRAC 19713 (1), MRAC 19714 (1).

Distribution: Congo River system (Teugels, 1986b).

\section{CLARIAS Scopoli, 1777}

Chlarias Scopoli, 1777: 455. Type species: Silurus anguillaris Linnaeus 1758. Type by subsequent designation by Teugels \& Roberts (1987). Gender: Masculine. First published without any included species; names apparently first added by Cuvier, 1816, but spelled Clarias therein. Scopoli based his name on Clarias Gronovius, a name published in a work that was rejected as non binomial (ICZN, opinion 261), but with an unexplained change in spelling. Spelling in Scopoli not followed in recent literature and not used here.

Macropteronotus La Cepède, 1803: 84. Type species: Macropteronotus charmuth La Cepède, 1803. Type by subsequent designation, apparently by Jordan (1917). Gender: Masculine.

Clarias Cuvier, 1816: 206. Type species not clearly established and may not have been proposed inasmuch as the name has been generally attributed to Scopoli or Gronovius. Proposed originally with two species. Gender: 
Masculine.

Cossyphus M'Clelland, 1844a: 403. Type species: Cossyphus ater M'Clelland, 1844. Type by monotypy. Gender: Masculine. Preoccupied several times, first by Cossyphus Olivier, 1791, in Insecta; replaced by Phagorus M'Clelland, 1844.

Phagorus M'Clelland, 1844b: 225 [errata]. Type species: Cossyphus ater M'Clelland, 1844. Type by being a replacement name. Gender: Masculine. Replacement for Cossyphus M'Clelland, 1844; preoccupied by Cossyphus Olivier, 1791.

Dinotopteroides Fowler, 1930: 41. Type species: Dinotopteroides prentissgrayi Fowler, 1930. Type by original designation. Gender: Masculine.

Prophagorus Smith, 1939: 236. Type species: Clarias nieuhofii Valenciennes, 1840. Type by original designation. Gender: Masculine.

Anguilloclarias Teugels, 1982a: 13. Type species: Clarias theodorae Weber, 1897. Type by original designation. Gender: Masculine. Originally proposed as a subgenus of Clarias.

Brevicephaloides Teugels, 1982a: 14. Type species: Clarias camerunensis Lönnberg, 1895. Type by original designation. Gender: Masculine. Also appeared in Teugels (1982b: 739). Originally proposed as a subgenus of Clarias.

Clarioides Teugels, 1982a: 12. Type species: Clarias angolensis Steindachner, 1866. Type by original designation. Gender: Masculine.

Platycephaloides Teugels, 1982a: 11. Type species: Clarias platycephalus Boulenger, 1902. Type by original designation. Gender: Masculine. Originally as a subgenus of Clarias. Also appeared in Teugels (1982b: 736).

Remarks: Clarioides was first proposed as subgenus of Clarias in David \& Poll (1937: 231) but without a type species and, therefore, cannot be treated as available from that publication [ICZN Art. 13.3]. Heterobranchoides was first proposed as a subgenus of Clarias by David (1935: 82, 99), but was not assigned a type species and the name is, therefore, not available from that publication. The name appears again in David \& Poll (1937: 230), but as a synonym of Clarias and not as a valid name. It is, therefore, also not available from that reference. See Teugels (1983) for comments on the validity of Dinotopteroides as a natural subgroup of Clarias.

Review: Debouche et al. (1979), Teugels (1982c, 1986a, African species).

Clarias agboyiensis Sydenham, 1980

Clarias (Clarioides) agboyiensis Sydenham, 1980: 660, figs. 1-2. Type locality: Agboyi Creek, a ditributary of the Ogun, Lagos State, south-west Nigeria, 6³6'N, 5²4'E. Holotype: BMNH 1959.8.18.66.

Clarias (Clarioides) isheriensis Sydenham, 1980: 663, figs. 3-4. Type locality: Energo Project site, Lagos-Badagry expressway, Lagos State, Nigeria, 6²9'N, 3¹5'E. Holotype: BMNH 1976.3.17.3.

Distribution: West Africa, from Ghana to Nigeria (Teugels, 1986b).

Clarias albopunctatus Nichols \& La Monte, 1953

Clarias albopunctatus Nichols \& La Monte, 1953: 1, fig. 1. Type locality: Kotto River, about 225 miles north of Bangassou, eastern French Equatorial Africa. Holotype: AMNH 19840; holotype illustrated by Teugels (1986a: 94, fig. 49).

Distribution: Lake Chad, Benue River and central Congo River (Teugels, 1986b).

Clarias alluaudi Boulenger, 1906

Clarias Alluaudi Boulenger, 1906b: 437. Type locality: Entebbe and Bunuako; [and] Kavirondo Bay, Lake Victoria [restricted to Bunuako by lectotype designation]. Lectotype: BMNH 1906.5.30.159, designated by Teugels (1986a: 120); lectotype illustrated by Seegers (1996: fig. 144).

Clarias hilgendorfi Boulenger, 1911c: 54. Type locality: Lake Rukwa. Holotype: BMNH 1911.3.27.41; illustrated in Seegers (1996: fig. 146).

Distribution: Lakes Victoria, Kyoga, Edward, Rukwa and Tanganyika (Teugels, 1986b).

Clarias anfractus $\mathrm{Ng}, 1999$

Clarias anfractus Ng, 1999d: 18, figs. 1a, 2a, 3a, 4a. Type locality: Borneo: Sabah, Danum, forest stream $600 \mathrm{~m}$ into conservation area, tributary of Sungai Segama. Holotype: ZRC 42598.

Distribution: Segama and Kalabakan River drainages, northeastern Borneo (Ng, 1999d).

Clarias angolensis Steindachner, 1866 
Clarias angolensis Steindachner, 1866: 766, pl. 13 (figs. 4, 7). Type locality: Angola. Holotype: NMW 46891; holotype illustrated in Teugels (1986a: 68, fig. 35).

Clarias brevinuchalis Lönnberg \& Rendahl, 1922: 123, fig. 2. Type locality: Upper Congo. Holotype: NRM 9640.

Clarias confluentus Nichols \& La Monte, 1933b: 3, fig. 3. Type locality: Luluabourg, Kasai District, Belgian Congo. Holotype: AMNH 12340.

Clarias (Clarioides) curtus Matthes, 1964: 102, pl. 4a. Type locality: Rivière Kululu (mare), Zaïre. Holotype: MRAC 138667.

Distribution: Lower and Central Congo River system (Teugels, 1986b).

Clarias anguillaris (Linnaeus, 1758)

Silurus anguillaris Linnaeus, 1758: 305. Type locality: Nile. Syntype: NRM LP 71.

Clarias Hasselquistii Valenciennes, in Cuvier \& Valenciennes, 1840b: 362 (280 in Strasbourg deluxe edition), pl. 446. Type locality: Nil. Syntypes: MNHN b-0287 (1), MNHN b-0288 (2).

Clarias Senegalensis Valenciennes, in Cuvier \& Valenciennes, 1840b: 376 (279 in the Strasbourg deluxe edition). Type locality: Sénégal. Syntypes: MNHN a-8667 (1, dry), MNHN a-9429 (1, dry).

Clarias parvimanus Günther, 1864: 15. Type locality: Nile. Holotype: BMNH 1859.7.29.21.

Clarias budgetti Boulenger, 1900e: 513. Type locality: River Gambia. Syntypes (3): BMNH 1901.7.17.4-6 (3).

Clarias anguillaris nigeriensis Pellegrin, 1923a: 213. Type locality: Branche nontante de la boucle du Niger entre Mopti et Niafunké. Holotype: MNHN 1922-0161 (skull).

Distribution: Niger, Benoue, Gambia, lower Senegal and lower and middle Nile Rivers; Chad system; coastal rivers of Benin, Togo, Ghana and Côte d'Ivoire; and relict populations in Mauritania and southern Algeria (Teugels, 1986b).

Remarks: Teugels \& Roberts (1987: 95) list NRM LP 71 as the holotype of Silurus anguillaris, however, the original description of that species lists a range of meristic values that implies that more than one specimen contributed to the diagnosis. Thus, the specimen mentioned by Teugel \& Roberts must be considered a syntype. See Benech et al. (1993) for characters that distinguish this species from Clarias gariepinus.

Clarias batrachus (Linnaeus, 1758)

Silurus batrachus Linnaeus, 1758: 305. Type locality: Africa, Asia. Syntypes: Whereabouts unknown [not NRM LP 71 as reported by Fernholm \& Wheeler (1983: 219-220); see discussion in Teugels \& Roberts (1987: 95)].

Macropteronotus jagur Hamilton, 1822: 145, 374. Type locality: in ponds and ditches [Ganges River]. No types known.

Macropteronotus magur Hamilton, 1822: 146, 374, pl. 26 (fig. 45). Type locality: in ponds and ditches [Ganges River]. No types known.

Plotosus (Clarias) hamiltonii Swainson, 1839: 307. Type locality: in ponds and ditches [Ganges River]. No types known. On "Ham. 146, pl. 26, f. 45" [= Hamilton (1822: 146, pl. 26, fig. 45)]. Unneeded new name for Macropteronotus magur Hamilton, 1822.

Clarias punctatus Valenciennes, in Cuvier \& Valenciennes, 1840b: 384 (285 in the Strasbourg deluxe edition). Type locality: Java. Holotype (10 pouces): at MNHN, whereabouts unknown.

Distribution: Widely distributed in South and Southeast Asia.

Remarks: More than one species is included within what is currently called Clarias batrachus. A clarification of the situation requires both demarcation of species and replacement of the missing primary types of Macropteronotus jagur and M. magur.

Clarias batu Lim \& Ng, 1999

Clarias batu Lim \& Ng, 1999: 157, figs. 1, 2, 4a, 5a, 7. Type locality: Sungai Baharu, on right side of Tekek-Juara trail, towards Juara, Pulau Tioman, Pahang, Malaysia. Holotype: ZRC 40087.

Distribution: Peninsular Malaysia.

? Clarias brachysoma Günther, 1864

Clarias brachysoma Günther, 1864: 20. Type locality: Ceylon. Syntypes: BMNH 1859.5.31.6-8 (3).

Distribution: Sri Lanka.

Clarias buettikoferi Steindachner, 1894

Clarias bulumae Steindachner, 1894: 55. Type locality: Buluma, Liberia. Holotype: RMNH 3340. Originally as: 
Clarias bulumae n. sp. ?

Clarias Büttikoferi Steindachner, 1894: 53. Type locality: Buluma, Liberia. Holotype: registered at RMNH, but apparently not returned by Steindachner (Teugels, 1986a: 88).

Clarias liberiensis Steindachner, 1894: 54, pl. 3 (fig. 2). Type locality: Buluma und aus dem Junk-flusse, Liberia [restricted to Junk-Flusse by lectotype designation]. Lectotype: RMNH 3342, designated by Sydenham (1978: 355); lectotype illustrated by Teugels (1986a: 47, fig. 47).

Clarias guineensis Osório, 1909: 104, pl. (fig. 3). Type locality: Guinée, Bolama, Postugo. Holotype: MB (destroyed).

Clarias dorsimarmoratus Ahl, 1937a: 239. Type locality: 40-50 km landeinwärts Freetown, Sierra Leone. Holotype: ZMB 20939.

Distribution: Comoe River, Côte d'Ivoire, to Guinea Bissau (Teugels, 1986b).

Remarks: Teugels (1986a: 88) appears to be the first to treat all three Steindachner names as synonyms and thus acted as first reviser in selecting Clarias buettikoferi as the valid name for the species.

Clarias buthupogon Sauvage, 1879

Clarias buthupogon Sauvage, 1879: 96. Type locality: Doumé [Fleuve Ogôoué]. Holotype: MNHN a-0896; holotype illustrated by Teugels (1986a: 80, fig. 44).

Clarias megapogon Sauvage, 1880c: 39. Type locality: Doumé [Fleuve Ogôoué]. Holotype: MNHN a-0896. Either an emendation or lapsus calami for Clarias buthupogon Sauvage.

Clarias zygouron Nichols \& Griscom, 1917: 704, fig. 18. Type locality: Malela, Congo. Holotype: AMNH 6698.

Clarias lindicus Boulenger, 1920a: 26, fig. 13. Type locality: Bafwasende sur la Lindi, Congo Belge. Holotype: MRAC 7112.

Clarias obscurus Poll, 1948: 22, figs. 8-9. Type locality: Pweto, Lac Moero. Holotype: IRSNB 86.

Chlarias angolensis macronema Fowler, 1949: 257, figs. 43-47. Type locality: Oka, 18 mi. north of Eovo, Congo system. Holotype: ANSP 71903.

Distribution: Coastal rivers, from Nigeria to the Congo River system (Teugels, 1986b).

Clarias camerunensis Lönnberg, 1895

Clarias camerunensis Lönnberg, 1895: 182. Type locality: Cameroons. Lectotype: ZMUU 283 (88 mm TL, largest specimen); lectotype illustrated in, and designated by, Teugels (1986a: 154, fig. 85).

Clarias walkeri Günther, 1896a: 274, pl. 14 (fig. b). Type locality: Ogowe River. Holotype: BMNH 1896.5.5.54.

Clarias breviceps Boulenger, 1900a: 135, pl. 48 (fig. 6). Type locality: Marais Scala N'toto, Mayumbe. Syntypes: BMNH 1899.11.27.93 (1), MRAC 271 (1).

Clarias poensis Boulenger, 1908b: 1078, fig. 252. Type locality: Fernando Po. Holotype: BMNH 1904.7.23.49.

Clarias monkei Keilhack, 1910: 119. Type locality: in einem Kleinen Bache im Urwald 2 km südlich von Logobaba (4²'N, 945'Ö), Kamerun. Syntypes (3): ZMB 17769 (3).

Clarias duchaillui Fowler, 1915a: 226, fig. 7. Type locality: Gaboon. Holotype: ANSP 8567 [but published as ANSP 8568].

Clarias nigeriae Popta, 1919: 4. Type locality: Wari, mouth of the Niger, West Africa. Holotype: RMNH 10332.

Clarias (Allabenchelys) submarginatus thysvillensis David \& Poll, 1937: 231. Type locality: Grottes de Thysville, Congo Belge. Holotype: MRAC 37550.

Distribution: Coastal rivers of Togo to the lower and central Congo River system (Teugels, 1986b).

Clarias cataractus (Fowler, 1939)

Phagorus cataractus Fowler, 1939: 54, figs. 1-3. Type locality: Waterfall at Trang, Siam. Holotype: ANSP 68462.

Distribution: Peninsular Thailand and, possibly, Cambodia (Rainboth, 1996).

Clarias cavernicola Trewavas, 1936

Clarias cavernicola Trewavas, 1936, 70, pl. 2 (figs. 7-9). Type locality: Aigamas Cave, north of Otavi, South-West Africa. Lectotype: BMNH 1935.3.20.174, designated by Teugels (1986a: 134).

Distribution: Caves of south west Africa (Teugels, 1986b).

Clarias dayi Hora, 1936

Clarias dayi Hora, 1936b: 350, figs. 3, 4c. Type locality: Wynaad [India]. Holotype: at ZSI [on “Day's Wynaad specimen"]. 
Distribution: Tamil Nadu, India (Manimekalan \& Arunachalam (2002).

Remarks: Treated in current literature as valid or as a synonym of Clarias dussumieri Valenciennes, 1840.

Clarias dhonti (Boulenger, 1920)

Allabenchelys dhonti Boulenger, 1920c: 402, fig. 8. Type locality: Kabeke, a village 30 miles south of Tumbwe, on the Niemba River, near the west coast of Lake Tanganyika. Syntypes (10): BMNH 1919.7.24.20-27 (8), MNHN 1919-0467 (1), MRAC 6991 (1). One syntype (MRAC 6991) illustrated in Teugels (1986a: 176, fig. 91) but incorrectly listed therein as the holotype.

Distribution: Niemba River, Democratic Republic of the Congo (Teugels, 1986b).

Clarias dialonensis Daget, 1962

Clarias dialonensis Daget, 1962: 120, fig. 39; pl. 11 (fig. 26). Type locality: Bafing près Mamou (Fouta Dialon). Lectotype: MNHN 1959-0107; lectotype illustrated in, and designated by, Teugels (1986a: 145, fig. 81) as Clarias laeviceps dialonensis.

Distribution: Fouta Dialon, Guinea, and coastal rivers of Sierra Leone (Teugels, 1986b).

Remarks: Treated by Teugels (1986a: 145; 1986b: 88) as a subspecies of Clarias laeviceps.

Clarias dumerilii Steindachner, 1866

Clarias Dumerilii Steindachner, 1866: 766, pl. 14 (fig. 5). Type locality: Angola. Neotype: BMNH 1864.7.13.42, designated by Teugels (1986a: 148).

Clarias ngola Lönnberg \& Rendahl, 1920: 170, fig. 1. Type locality: Kingoyi, Lower Congo. Holotype: NRM 9908. Clarias lualae Lönnberg \& Rendahl, 1922: 122, fig. 1. Type locality: Luala River, tributary of the Congo, near Kinkenge, Congo basin. Syntypes: NRM 39931 (2).

Distribution: central and upper Congo River system and the Luapula system (Teugels, 1986b).

Clarias dussumieri Valenciennes, 1840

Clarias Dussumieri Valenciennes, in Cuvier \& Valenciennes, 1840b: 382 (283 in the Strasbourg deluxe edition). Type locality: Malabar, ... Mahé, ... Pondicherry. Syntypes: MNHN b-0686 (3), MNHN b-0687 (1).

Distribution: South Asia.

Clarias ebriensis Pellegrin, 1920

Clarias ebriensis Pellegrin, 1920: 118. Type locality: Eau stagnante près Bingerville, Côte d'Ivoire. Lectotype: MNHN 1920-0037, designated by Teugels \& Thys van den Audenaerde (1981: 18); lectotype illustrated by Teugels (1986a: 131, fig. 72).

Clarias dahomeyensis Güntert, 1938: 296. Type locality: Porto Novo, Dahomey. Syntypes: NMW 46922 (18), NMW 469223 (9).

Distribution: Coastal rivers and lagoons from Nigeria to Côte d'Ivoire (Teugels, 1986b).

Clarias engelseni (Johnsen, 1926)

Allabenchelys engelseni Johnsen, 1926: 8, figs. 2-3. Type locality: Aluma, Ramadalla and Yei, Sudan. Syntypes (15): BMNH 1925.12.30.11 (1), ZMUB 4131 a-b (2), ZMUB 4132 a-e (3 + 2 skeletons).

Distribution: Sudan (Teugels, 1986b).

$\dagger$ Clarias falconeri Lydekker, 1886

$\dagger$ Clarias falconeri Lydekker, 1886: 247, pl. 37, fig. 1. Type locality: Siwalik Hills, India. Lower Pliocene. Holotype: BMNH 16402b (middle part of skull).

Distribution: Siwalik Hills, India; Lower Pliocene.

Clarias fuscus (La Cepède, 1803)

Macropteronotus fuscus La Cepède, 1803: 84, 88, pl. 2 (fig. 2). Type locality: China. No types known; based on an unpublished painting.

Clarias pulicaris Richardson, 1845: 135, pl. 62 (figs. 5-6). Type locality: Canton, China. Holotype: BMNH 1968.3.11.21 (larger of two specimens).

Distribution: Ishigakijama, Japan; Taiwan, and south China (Masuda et al., 1984); northeastern Laos, and northern Vietnam (Kottelat, 2001a).

Clarias gabonensis Günther, 1867

Clarias Gabonensis Günther, 1867b: 111. Type locality: River Ogowe, Gaboon. Holotype: BMNH 1867.5.22.3.

Clarias dolloi Boulenger, 1896a: 311. Type locality: Monsembe, upper Congo. Syntypes: BMNH 1896.3.9.18-20 
(3).

Clarias congicus Poll, 1942a: 6, figs. 2-3. Type locality: Lac Tumba, Congo belge. Holotype: MRAC 57759.

Distribution: Lower and middle Congo River system, including Lake Tumba and the Pool Malebo; Chiloango system and Ogowe River (Teugels, 1986b).

Remarks: Redescription and comments on status (Teugels, 1982b).

Clarias gariepinus (Burchell, 1822)

Silurus (Heterobranchus) Gariepinus Burchell, 1822: 425 (footnote), figure on p. 445. Type locality: Vaal River at Smidtsdrift, above confluence with Riet River, Cape Province, South Africa (28 $\left.42^{\prime} 10^{\prime \prime} S, 24^{\circ} 04^{\prime} 29^{\prime \prime} E\right)$, by neotype designation. Neotype: SAIAB 520, illustrated in Skelton \& Teugels (1992: fig. 2). Neotype designated by Teugels (1986a: 35), or Skelton \& Teugels (1992: 1) [see Remarks, below].

Clarias capensis Valenciennes, in Cuvier \& Valenciennes, 1840b: 377 (279 in the Strasbourg deluxe edition). Type locality: Cap de Bonne-Espérience (in error). Holotype: MNHN a-9431 (dry).

Clarias lazera Valenciennes, in Cuvier \& Valenciennes, 1840b: 372 (275 in the Strasbourg deluxe edition). Type locality: Nil. Syntypes (3, plus one skeleton): MNHN 0000-1578 (1).

Clarias Syriacus Valenciennes, in Cuvier \& Valenciennes, 1840b: 375 (275 in the Strasbourg deluxe edition). Type locality: Syrie. Holotype: MNHN a-9426.

Clarias mossambicus Peters, 1852: 682. Type locality: Tette, Mozambique. Syntypes: possibly FMNH 54274 (1), ZMB 3068 (1), ZMB 6591 (1), ZMB 6592 (1), ZMB 6593 (1).

Clarias macracanthus Günther, 1864: 16. Type locality: Upper Nile. Syntypes (5, one a skeleton): BMNH 1855.12.26.453 (1), BMNH 1862.6.17.51 (1), BMNH 1866.9.9.67-68 (2).

Clarias orontis Günther, 1864: 15. Type locality: Orontes. Syntype: BMNH 1855.6.25.3. On Silurus anguillaris of Russell (1794: 217, pl. 8) and Clarias of Gronovius (1763: 100).

Clarias xenodon Günther, 1864: 16. Type locality: Senegal, West Africa. Holotype: BMNH 1863.7. 30.3.

Clarias robecchii Vinciguerra, 1893: 450. Type locality: Uebi Scebeli [Somalia]. Syntypes: MSNG 8138 (2).

Clarias smithii Günther, 1896b: 219, fig. 1. Type locality: middle course of the Shebeli Somaliland. Holotype: BMNH 1895.12.31.65.

Clarias guentheri Pfeffer, 1896: 28. Type locality: Sansibar. Syntypes: Based on specimens identified as Clarius gariepinus in Playfair \& Günther (1867: 113), including BMNH 1867.3.9.388 (1, stuffed) and BMNH 1867.3.9.581 (stuffed, not found) and literature sources.

Clarias microphthalmus Pfeffer, 1896: 28. Type locality: Mbake, Ugogo, Bubu, Deutsch Ost-Africa. Holotype: ZMH H477.

Clarias longiceps Boulenger, 1899b: 103, pl. 41 (fig. 1). Type locality: Boma swamps, [Congo basin]. Syntypes: BMNH 1899.11.27.15-17 (3), MRAC 902-905 (4), MRAC 906-910 (5), MRAC 911-915 (5), MRAC 916-920 (5), MRAC 921-922 (2).

Clarias moorii Boulenger, 1901d: 13. Type locality: Lake Albert. Holotype: BMNH 1905.9.7.47.

Clarias tsanensis Boulenger, 1902c: 438. Type locality: Lake Tsana, Abyssinia. Syntypes (5): BMNH 1902.12.13.441-443 (3).

Clarias vinciguerrae Boulenger, 1902c: 438. Type locality: Awhorra, Kassam River, Abyssinia. Holotype: BMNH 1902.12.13.444.

Clarias malaris Nichols \& Griscom, 1917: 705, fig. 19. Type locality: Stanleyville, Congo. Holotype: AMNH 6441.

Clarias notozygurus Lönnberg \& Rendahl, 1922: 125, fig. 3. Type locality: Lukosi, a tributary to Luala, Lower Congo. Holotype: NRM 9933.

Clarias depressus Myers, 1925a: 12. Type locality: water hole of a hippopotamus, on the Albano River about $6 \mathrm{~km}$. west of Addagalla, Abyssinia. Syntypes: AMNH 8204 (2).

Clarias mülleri Pietschmann, 1939: 184. Type locality: Eritrea. Holotype: at Museo di Trieste.

Distribution: Nearly Pan African, as well as Asia Minor (Teugels, 1986b); introduced into Laos (Kottelat, 2001b).

Remarks: Teugels (1986a: 35) cites "Bruton \& Teugels (1986)" as source of neotype designation of Silurus (Heterobranchus) gariepinus Burchell. That publication has not been found and may not have preceded Teugels (1986a). Tentatively, Teugels (1986a) is considered the actual source of neotype designation. Seegers (1996: 206) was similarly unable to locate the Bruton \& Teugels source and, instead, cited Skelton \& Teugels (1992) as 
source of the actual neotype designation. See Benech et al. (1993) for characters that distinguish this species from Clarias anguillaris.

Clarias hilli Fowler, 1936

Clarias hilli Fowler, 1936b: 299, figs. 59-62. Type locality: Saidi's Village, Belgian Congo. Holotype: ANSP 65879.

Clarias gracilis Fowler, 1936b: 303, figs. 75-78. Type locality: Saidi's Village, Belgian Congo. Holotype: ANSP 66098.

Distribution: middle Congo River system and Lake Albert (Teugels, 1986b).

Remarks: Teugels (1986b: 181) gave precedence to Clarias hilli and placed C. gracilis as a synonym, thereby acting as first reviser.

Clarias insolitus $\mathrm{Ng}, 2003$

Clarias insolitus Ng, 2003h: 2, figs. 1, 2a. Type locality: Borneo: Kalimantan Tengah, Barito River drainage, small stream flowing into Sungai Rekut (tributary of Sungai Busang) about $1.5 \mathrm{~km}$ upstream from the Project Barito Ulu base camp on Sungai Busang. Holotype: MZB 6112.

Distribution: Barito River basin, southern Borneo (Ng, 2003h).

Clarias intermedius Teugels, Sudarto \& Pouyaud, 2001

Clarias intermedius Teugels, Sudarto \& Pouyaud, 2001: 86, fig. 5. Type locality: Palangkaraya market, Central Kalimantan, Indonesia. Holotype: MZB 10574.

Distribution: Central Kalimantan, between the Sampit and Barito river basins, Borneo (Teugels et al., 2001).

Clarias jaensis Boulenger, 1909

Clarias jaensis Boulenger, 1909: 188. Type locality: Ja River at Bitye, South Cameroon. Lectotype: BMNH 1909.4.29.77, designated by Teugels (1986a: 55). Illustrated in Boulenger (1911a: 242, fig. 201).

Clarias cameronensis Holly, 1927c: 422, unnumbered figure. Type locality: Nachtigalschnellen des Sanagaflussses. Holotype: NMW 13374. Considered by Pellegrin (1929b) to be preoccupied by Clarias camerunensis Lönnberg, 1895; replaced by Clarias Hollyi Pellegrin, 1929.

Clarias Hollyi Pellegrin, 1929b: 359 (footnote). Type locality: Nachtigalschnellen des Sanagaflussses. Holotype: NMW 13374. Replacement for Clarias cameronensis Holly, 1927; considered preoccupied by Clarias camerunensis Lönnberg, 1895.

Clarias (Clarioides) aboinensis Sydenham \& Olawoye, 1981: 234, fig. 1. Type locality: Ugbenu market, near Ologbo, Bendel State, Nigeria. Holotype: BMNH 1980.5.12.1.

Distribution: southeast Nigeria, south Cameroon and Gabon (Teugels, 1986b).

Clarias kapuasensis Sudarto, Teugels \& Pouyaud, 2003

Clarias kapuasensis Sudarto, Teugels \& Pouyaud, 2003: 156, figs. 2a-b. Type locality: Nanga Pinoh in peat swamp bordering the river Melawi in the upper part of the Kapuas River, West Kalimantan, Indonesia. Holotype: MZB 10965.

Distribution: Kapuas River basin, Borneo (Sudarto, Teugels \& Pouyaud, 2003).

\section{Clarias laeviceps Gill, 1862}

Clarias laeviceps Gill, 1862: 139, footnote. Type locality: [probably from Liberia]. Holotype: USNM 4099; holotype illustrated by Teugels (1986a: 143, fig. 79).

Clarias kingsleyae Günther, 1903: 334. Type locality: Odumasi [and] Infoan on the R. Offim, Gold Coast. Syntypes (2): BMNH 1903.4.24.65 (1), BMNH 1903.4.24.66 (1).

Clarias vandenhoutei Poll, 1941b: 137, fig. 3. Type locality: confluence de la rivière Masei et la rivière Bon de Boan, Côte d'Ivoire. Holotype: MRAC 66360.

Distribution: from Saint Pauls River, Liberia, to Volta River system, Ghana (Teugels, 1986b).

Remarks: Redescribed in Teugels (1981) with comments on the status of the species.

Clarias lamottei Daget \& Planquette, 1967

Clarias lamottei Daget \& Planquette, 1967: 279, fig. 1. Type locality: Aoué Bla, marigot affluent du Nzi, bassin du Bandama près Lamto, Côte d'Ivoire. Holotype: MNHN 1966-1104; holotype illustrated by Teugels (1986a: 20, fig. 15).

Distribution: Nzi River, tributary of the Bandama, Côte d'Ivoire (Teugels, 1986b). 
Remarks: Considered by Teugels (2003b) as likely to be an intergeneric hybrid between a species of Clarias and Heterobranchus.

Clarias leiacanthus Bleeker, 1851

Clarias leiacanthus Bleeker, 1851e: 430. Type locality: Sambas, in fluviis. Holotype (160 mm TL): possibly BMNH 1863.12.4.85 (124 mm SL).

Clarias liacanthus Günther, 1864: 20. Type locality: Sambas, in fluviis. Holotype: possibly BMNH 1863.12.4.85 (124 mm SL). Unjustified emendation of Clarias leiacanthus Bleeker, 1851.

Distribution: Kapuas River (Roberts, 1989a).

Clarias liocephalus Boulenger, 1898

Clarias liocephalus Boulenger, 1898d: 496. Type locality: Lake Tanganyika. Lectotype or Holotype: BMNH 1898.9.9.70. Described in more detail and illustrated in Boulenger (1898f: 24, pl. 7, figs. 2, 2a) with locality as Kinyamkolo. BMNH 1898.9.9.70 illustrated in Seegers (1996: 209, fig. 149) as holotype. The original description did not indicate how many specimens were included in the concept of this species. Recent authors (e. g., Teugels, 1986; Eschmeyer et al., 1998) have interpreted BMNH 1898.9.9.70 as a holotype, but BMNH 1898.9.9.71 was registered as the same species from the same locality. Therefore, the two specimens should probably have been treated as syntypes. Since it was unclear whether more than one specimen was involved in the original description, Teugels (1986) interpretation of a holotype made that specimen the lectotype.

Clarias carsonii Boulenger, 1903b: 362. Type locality: Fwambo, British Central Africa. Holotype: BMNH 1894.12.20.20.

Clarias neumanni Hilgendorf, 1905: 409. Type locality: Utim, im Guasso Nyiro in den Nguruman-Salzee fliessend, unter 36º̈ L., 150'S Br. Holotype: at Nairobi Museum, Kenya, but Seegers (1996: 214, fig. 152, bottom) illustrated BMNH 1905.7.25.49 as the holotype.

Clarias phillipsi Norman, 1925a: 189, pl. 11. Type locality: Lake Chahafi, S.O. Uganda. Holotype: MCZ 31519.

Clarias ornatus Poll, 1943b: 306. Type locality: Bururi, Haute Malagarazi, Lac Tanganika. Holotype: MRAC 56002; illustrated in Poll (1946: 230, fig. 25).

Clarias youngicus Ricardo-Bertram, 1943: 208. Type locality: Lake Young, Shiwa Ngandu, (Bangweulu region). Syntypes: BMNH 1943.7.27.377-382 (6).

Distribution: Lakes Victoria, Edward, George, Kivu, Tanganyika, Malawi and small lakes of Uganda and Rwanda; Kagera system, Malagarazi System, Ruzizi River, Tana River, Bangweulu-Moero system (Teugels, 1986b); Lake Rukwa basin (Seegers, 1996).

Clarias longior Boulenger, 1907

Clarias longior Boulenger, 1907b: 51. Type locality: Kribi and Lobi Rivers, South Cameroon [restricted to Kribi River, by lectotype designation]. Lectotype: BMNH 1904.10.26.63, designated by Sydenham (1978: 369).

Clarias melanopogon Fowler, 1936b: 301, figs. 71-74. Type locality: Thirty kilometers east of Kribi, Cameroons. Holotype: ANSP 66075.

Distribution: Kribi, Lobi and Ntem Rivers of South Cameroon (Teugels, 1986b).

Clarias maclareni Trewavas, 1962

Clarias maclareni Trewavas, 1962: 157, fig. 3. Type locality: L. Barombi-ma-Mbu, northwestern Cameroons. Holotype: BMNH 1959.8.18.174.

Distribution: Lake Barombi-ma-Mbu; known only from the type locality (Teugels, 1986b).

Clarias macrocephalus Günther, 1864

Clarias macrocephalus Günther, 1864: 18. Type locality: Siam. Lectotype: BMNH 1862.11.1.216; lectotype designated by Teugels et al. (1999: 294).

Distribution: Mekong and Chao Phraya River basins, and possibly Philippines (Kottelat, 2001b).

Remarks: Redescribed in Teugels et al. (1999: 294).

Clarias macromystax Günther, 1864

Clarias macromystax Günther, 1864: 17. Type locality: Gambia [in error]. Holotype: BMNH 1862.5.20.4.

Distribution: Oueme River, Benin, to the Niger delta and the Niger and Benue Rivers (Teugels, 1986b).

Remarks: Teugels (1986a: 76) indicated that the stated type locality appears to be in error.

Clarias meladerma Bleeker, 1846 
Clarias meladerma Bleeker, 1846a: 178. Type locality: Batavia. Syntypes (size and number not stated): RMNH 13709 (1), RMNH 16413 (1). Illustrated in Bleeker (1862-63: pl. 97 [ Silur. pl. 49], fig. 2) as Clarias melanoderma.

Clarias melasoma Bleeker, 1852b: 427. Type locality: Prabukarta, Borneo austro-orientalis, in flumne Kusan; Palembang, Sumatrae austro-orientalis, in flumine Mussi. Syntypes (2, 170-300 mm TL): RMNH 6802 (1).

Clarias melanoderma Bleeker, 1858b: 341. Type locality: Batavia. Syntypes: RMNH 13709 (1), RMNH 16413 (1), RMNH 6802 (1). Unneeded new name for Clarias meladerma Bleeker, and Clarias melasoma Bleeker.

Distribution: Mekong River basin, and Sumatra, Borneo and Java (Kottelat, 2001b); also the Philippines (Rainboth, 1996).

Clarias microstomus $\mathrm{Ng}, 2001$

Clarias microstomus Ng, 2001c: 158, fig. 1. Type locality: Borneo: Kalimantan Timur, Kayan River drainage, Ladang near Sungai Pingai, 20'9.6"N, 1159'13.8"E. Holotype: MZB 9336.

Distribution: Mahakam and Kayan River basins, eastern Borneo (Ng, 2001c).

Clarias ngamensis Castelnau, 1861

Ciarias N'gamensis Castelnau, 1861: 63. Type locality: lac N'gami, [now: Lake Ngami, S. W. Africa (1750'W, $21^{\circ} 00$ 'E), by neotype designation]. Neotype: BMNH 1910.5.31.27, designated by Teugels (1983: 17). Published name clearly intended as Clarias $N^{\prime}$ gamensis.

Clarias Mellandi Boulenger, 1905b: 644. Type locality: Lake Bangwelo. Holotype: BMNH 1905.11.10.7.

Dinotopterus jallae Gilchrist \& Thompson, 1917: 556, fig. 165. Type locality: Sheskeke, southern Rhodesia. Holotype: SAM 14297 [now at AMG].

Dinotopteroides prentissgrayi Fowler, 1930: 41, fig. 10. Type locality: Chouzo, Quanza River, Angola. Holotype: ANSP 51798.

Distribution: Quanza, Cunene, Okavango, Chobe, Zambezi above Victoria Falls, upper Lualaba, Luapula, Pungwe, Buzi, Save, Limpopo, Incomati, lower Pongolo, and lower Sabi River; Lake Ngami, Moero, Bangweulu, and Malawi (Teugels, 1986b).

Clarias nieuhofii Valenciennes, 1840

Clarias Nieuhofii Valenciennes, in Cuvier \& Valenciennes, 1840b: 386 (287 in the Strasbourg deluxe edition). Type locality: Indies. Holotype: MNHN b-0300.

Clarias pentapterus Bleeker, 1851d: 206. Type locality: Bandjarmassing, in fluviis. Holotype (350 mm TL): Whereabouts unknown.

Clarias gilli Smith \& Seale, 1906: 74, figs. a-b. Type locality: Rio Grande, Mindanao, Philippines. Holotype: USNM 55620.

Distribution: Sumatra, Kalimantan, India, Thailand and the Philippines (Sudarto et al., 2004); Coastal and, probably, Mekong side of Cardamom Range, Cambodia (Rainboth, 1996).

Clarias nigricans $\mathrm{Ng}, 2003$

Clarias nigricans Ng, 2003g: 393, figs. 1, 2, 3a. Type locality: Borneo: Kalimantan Timur, market in Samarinda. Holotype: MZB 10705.

Distribution: Mahakam River basin, Borneo (Ng, 2003e).

Clarias nigromarmoratus Poll, 1967

Clarias nigromarmoratus Poll, 1967: 229, fig. 106. Type locality: Riv. Luíta, Angola. Holotype: MD 6484.

Distribution: central Congo River system (Teugels, 1986b).

Clarias olivaceus Fowler, 1904

Chlarias olivaceus Fowler, 1904: 499, pl. 28 (lower figure). Type locality: Padang, Sumatra. Holotype: ANSP 27280.

Distribution: Highland rivers of western Sumatra (Tan \& Ng, 2000).

Clarias pachynema Boulenger, 1903

Clarias pachynema Boulenger, 1903c: 438. Type locality: Ja River, southern Cameroon. Lectotype: BMNH 1903.7.28.191, illustrated in, and designated by, Teugels (1980: 686, fig. 61; 214.5 mm TL).

Clarias esamesae Boulenger, 1911e: 372. Type locality: Esamesa, south Cameroon. Holotype: BMNH 1911.5.30.28. Clarias oxycephalus Boulenger, 1913: 67. Type locality: Nyong River, S. Cameroon. Holotype: BMNH 
1913.10.29.7.

Distribution: Coastal rivers of south Cameroon, Oowe system and central Congo River system (Teugels, 1986b).

Clarias planiceps $\mathrm{Ng}, 1999$

Clarias planiceps Ng, 1999d: 22, figs. 4b, 5, 6. Type locality: Borneo: Sarawak, Belakin area, Ulu Sungai Anap.

Holotype: SMK uncataloged.

Distribution: Upper reaches of the Rajang and Kapuas Rivers, western Borneo, and Kayan River, eastern Borneo $(\mathrm{Ng}, 1999 \mathrm{~d})$.

Clarias platycephalus Boulenger, 1902

Clarias platycephalus Boulenger, 1902d: 35, pl. 10 (fig. 1). Type locality: Monsembé, Haut-Congo. Holotype: BMNH 1901.12.21.43.

Clarias ekibondoi Fowler, 1936b: 300, figs. 63-66. Type locality: Ekibondo's Village, Belgain Congo. Holotype: ANSP 65886.

Clarias varispinis Fowler, 1936b: 301, figs. 67-70. Type locality: Nola, Middle Congo. Holotype: ANSP 65887.

Distribution: lower and central Congo River basin (Teugels, 1986b).

Clarias pseudoleiacanthus Sudarto, Teugels \& Pouyaud, 2003

Clarias pseudoleiacanthus Sudarto, Teugels \& Pouyaud, 2003: 156, figs. 2a-b. Type locality: Satong, peat swamp at ca. $30 \mathrm{~km}$ after Ketapang on the road to Sudadana, Borneo, West Kalimantan, Indonesia. Holotype: MZB 10964.

Distribution: Borneo.

Clarias pseudonieuhofii Sudarto, Teugels \& Pouyaud, 2004

Clarias pseudonieuhofii Sudarto, Teugels \& Pouyaud, 2004: 15, fig. 3. Type locality: Semitau, near the Danau Sentarum Reserve, upstream Kapuas R. on the road between Sintang and Putussibau, West Kalimantna, Borneo, Indonesia. Holotype: MZB 10966.

Distribution: Upper Kapuas River, West Kalimantan, Borneo (Sudarto et al., 2004: 16).

Clarias salae Hubrecht, 1881

Clarias salae Hubrecht, 1881: 68. Type locality: St. Paul's River, Liberia. Lectotype: RMNH 5373 (smallest specimen); lectotype illustrated in, and designated by, Teugels (1986a: 103, fig. 54).

Distribution: Konkoure system, Guinea, to the Cavally River, Côte d'Ivoire (Teugels, 1986b).

Clarias stappersii Boulenger, 1915

Clarias Stappersii Boulenger, 1915: 168. Type locality: Ruisseau affluent de la Lukinda, Tanganika-Moero. Holotype: MRAC 14139.

Distribution: Luapula-Moero system, Kafue River, upper Zambezi River and Cunene River (Teugels, 1986b).

Clarias submarginatus Peters, 1882

Clarias submarginatus Peters, 1882: 74. Type locality: Westafrica (Tooxlong River). Lectotype: ZMB 11895 (largest specimen), designated by Sydenham (1978: 358); lectotype illustrated by Teugels (1986a: 107, fig. 56).

Clarias hollyi Fowler, 1936b: 304, figs. 79-82. Type locality: Thirty kilometers east of Kribi, Cameroons. Holotype: ANSP 66118. Preoccupied by Clarias hollyi Pellegrin, 1929; apparently not replaced.

Distribution: Kribi and Lobi Rivers, south Cameroon (Teugels, 1986b).

Clarias sulcatus $\mathrm{Ng}, 2004$

Clarias sulcatus Ng, 2004d: 290, fig. 1. Type locality: Malaysia: Terengganu: Pulau Redang, stream on east slope of west ridge. Holotype: ZRC 22666.

Distribution: Rendang Island, off northeastern Malay Peninsula (Ng, 2004d).

Clarias teijsmanni Bleeker, 1857

Clarias Teijsmanni Bleeker, 1857a: 344. Type locality: Tjikoppo, provinciae Buitenzorg, 900 metr. p.m. supra mare [Java]. Holotype (110 mm TL): possibly AMS B.8031 (1), or BMNH 1863.12.4.62 (1).

Clarias pulcher Popta, 1904: 179. Type locality: le Howong, Bornéo central. Holotype RMNH 7542; described in more detail, with illustration of holotype, in Popta (1906: pl. 1, fig. 2).

Distribution: Kapuas River, Borneo (Roberts, 1989a), and Java.

Clarias theodorae Weber, 1897

Clarias theodorae Weber, 1897: 150. Type locality: Natal: Umhloti-Fluss, Süd-Afrika. Holotype: ZMA 100647; 
holotype illustrated by Teugels (1986a: 111, fig. 58).

Clarias amplexicauda Boulenger, 1902d: 36, pl. 10 (fig. 2). Type locality: Banzyville, [Haut Ubangi]. Holotype: MRAC 1158.

Clarias Fouloni Boulenger, 1905b: 644. Type locality: Lake Bangwelo. Holotype: BMNH 1905.11.10.8.

Clarias macrurus Boulenger, 1915: 168. Type locality: Ruisseau affluent de la Lukinda, Tanganika-Moero. Holotype: MRAC 14256.

Distribution: Zambezi, Kafue, Shire, upper Congo, Chobe, Okavango, Cunene, Pungwe, Sabi, Lundi, Limpopo, Incomati, Pongola, Umgeni rivers; Lakes Tanganyika, Bangweulu Kobo, Niumbe, Mweru, Malawi, and Sibaya (Teugels, 1986b).

Clarias werneri Boulenger, 1906

Clarias Werneri Boulenger, 1906c: 569. Type locality: Gondokoro, and Lake Victoria (at Bunjako, Buganga, and Munyonyo), Uganda [restricted to Buganga, by lectotype designation]. Lectotype: BMNH 1906.5.30.165; designated by Teugels (1986a: 126).

Distribution: Lakes Victoria, Kioga, Edward, and Tanganyika; upper Nile River (Teugels, 1986b).

\section{Species inquirendae, Clarias}

Clarias abbreviatus Valenciennes, in Cuvier \& Valenciennes, 1840b: 386 (286 in the Strasbourg deluxe edition). Type locality: Macao. Syntypes: MNHN a-9592 (2).

Clarias Assamensis Day, 1877 (in Day, 1875-78): 485. Type locality: Upper and lower Assam. Syntypes: Possibly AMS B.7485 (1), ZSI F1268 (1, lost).

Cossyphus ater M'Clelland, 1844a: 403, pl. 22 (fig. 3). Type locality: Chusan, China. Holotype: Whereabouts unknown.

Macropteronotus charmuth La Cepède, 1803: 84, 85. Type locality: Nile R. Types: based on numerous literature sources. Possibly a synonym of Clarias anguillaris (Linnaeus, 1758) or Clarias gariepinus (Burchell, 1822) (Teugels, 1986a: 35; Teugels, 1986b: 71, 79; Seegers, 1996: 205), depending on the selection of a single primary type specimen.

Macropteronotus hexacicinnus La Cepède, 1803: 84, 88, pl. 2 (fig. 3). Type locality: China. No types known; based on an unpublished painting.

Clarias marpus Valenciennes, in Cuvier \& Valenciennes, 1840b: 378 (280 in the Strasbourg deluxe edition). Type locality: Calcutta,.... Pondichéry ...[Vizagapatam], .... l'Irouaddi.... . Syntypes: MNHN b-0685 (2).

Clarias nebulosus Deraniyagala, 1958: 133, pl. 2 (figs. 3-5, 7). Type locality: Kaluganga at Ratnapura, Ceylon. Holotype: NMSL FF721 (lost).

Clarias sauteri Regan, 1908b: 151. Type locality: Kagi, Formosa. Syntypes: BMNH 1908.5.27.16-22 (7).

Clarias tenuis Güntert, 1938: 302. Type locality: China. Syntypes (2): NMW 47074 (2).

Clarias thienemanni Ahl, 1934: 235. Type locality: Porsea, Toba-See, Sumatra. Syntypes (4): ZMB 20931, ZMB 20934, ZMB 20944.

\section{DINOTOPTERUS Boulenger, 1906}

Dinotopterus Boulenger, 1906f: 550. Type species: Dinotopterus cunningtoni Boulenger, 1906. Type by monotypy. Gender: Masculine.

Revision: Greenwood (1961); sensu lato, including species now in Bathyclarias.

Dinotopterus atribranchus Greenwood, 1961

Dinotopterus atribranchus Greenwood, 1961: 228. Type locality: Lake Nyasa. Holotype: BMNH 1960.2.29.17.

Distribution: Lake Malawi (Teugels, 1986b).

Dinotopterus cunningtoni Boulenger, 1906

Dinotopterus cunningtoni Boulenger, 1906f: 550, pl. 31. Type locality: Mpala, Lake Tanganyika. Syntypes: BMNH 1906.9.8.62 (1), BMNH 1906.9.8.63 (1).

Distribution: Lake Tanganyika (Teugels, 1986b).

Dinotopterus filicibarbis (Jackson, 1959)

Bathyclarias filicibarbis Jackson, 1959: 120, fig. 5. Type locality: Nkata Bay, Lake Nyasa. Holotype: BMNH 
1955.6.14.1.

Distribution: Lake Malawi (Teugels, 1986b).

Dinotopterus foveolatus (Jackson, 1955)

Clarias foveolatus Jackson, 1955: 681, unnumbered figure. Type locality: Nkata Bay, Lake Nyasa. Holotype: BMNH 1955.6.15.1.

Distribution: Lake Malawi (Teugels, 1986b).

Dinotopterus gigas (Jackson, 1959)

Bathyclarias gigas Jackson, 1959: 125, fig. 7. Type locality: [Nkata Bay] Lake Nyasa. Holotype: BMNH 1960.2.29.35 (head and skin).

Distribution: Lake Malawi (Teugels, 1986b).

Dinotopterus jacksoni Greenwood, 1961

Dinotopterus jacksoni Greenwood, 1961: 222. Type locality: Nkata Bay, Lake Nyasa. Holotype: BMNH 1960.2.29.26.

Distribution: Lake Malawi (Teugels, 1986b).

Dinotopterus loweae (Jackson, 1959)

Bathyclarias loweae Jackson, 1959: 114, fig. 1. Type locality: Nkata Bay, Lake Nyasa. Holotype: BMNH 1960.2.29.3.

Distribution: Lake Malawi (Teugels, 1986b).

Dinotopterus nyasensis (Worthington, 1933)

Clarias nyasensis Worthington, 1933b: 308, fig. 9. Type locality: Lake Nyasa. Lectotype: BMNH 1932.11.15.584, designated by Greenwood (1961: 221).

Distribution: Lake Malawi (Teugels, 1986b).

DOLICHALLABES Poll, 1942

Dolichallabes Poll, 1942c: 95. Type species: Dolichallabes microphthalmus Poll, 1942. Type by monotypy. Gender: Masculine.

Dolichallabes microphthalmus Poll, 1942

Dolichallabes microphthalmus Poll, 1942c: 97, figs. a, d. Type locality: Kunungu, Congo belge. Holotype: MRAC 44655.

Distribution: Congo River system (Teugels, 1986b).

Remarks: Redescribed in Devaere et al. (2004).

ENCHELOCLARIAS Herre \& Myers, 1937

Encheloclarias Herre \& Myers, 1937: 66. Type species: Heterobranchus tapeinopterus Bleeker, 1852. Type by original designation. Gender: Masculine.

Revision: Ng \& Lim (1993).

Encheloclarias baculum $\mathrm{Ng} \& \mathrm{Lim}, 1993$

Encheloclarias baculum Ng \& Lim, 1993: 27, fig. 5. Type locality: Probably Borneo: Sambas, Kalimantan Barat. Holotype: BMNH 1863.12.4.162.

Distribution: Probably Sambas, Borneo; known only from holotype (Ng \& Lim, 1993).

Remarks: Register number of holotype of Encheloclarias baculum incorrectly reported as BMNH 1863.12.11.162 in $\mathrm{Ng} \&$ Lim (1993) and Eschmeyer et al. (1998).

Encheloclarias curtisoma $\mathrm{Ng} \& \mathrm{Lim}, 1993$

Encheloclarias curtisoma Ng \& Lim, 1993: 32, figs. 9-10. Type locality: Peninsular Malaysia: Selangor, north Selangor peat swamp forest, blackwater stream at $39 \mathrm{~km}$ milestone on road to Tanjong Malim from Sungei Basar. Holotype: ZRC 14886.

Distribution: streams north of Selangor peat forest, Selangor, Malaysia (Ng \& Lim, 1993).

Encheloclarias kelioides $\mathrm{Ng} \& \mathrm{Lim}, 1993$

Encheloclarias kelioides Ng \& Lim, 1993: 30, figs. 7-8. Type locality: Peninsular Malaysia: Pahang, south of Pekan, blackwater stream across road, at $69 \mathrm{~km}$ stone on road from Mersing to Pekan. Holotype: ZRC 29401. 
Distribution: Pahang, and Mawai, Johor, southeastern peninsular Malaysia (Ng \& Lim, 1993).

Encheloclarias prolatus $\mathrm{Ng} \& \mathrm{Lim}, 1993$

Encheloclarias prolatus Ng \& Lim, 1993: 29, fig. 6. Type locality: Borneo: Sarawak, Kuching. Holotype: SMK 5686.

Distribution: Western Sarawak, Borneo (Ng \& Lim, 1993).

Encheloclarias tapeinopterus (Bleeker, 1852)

Heterobranchus tapeinopterus Bleeker, 1852e: 732. Type locality: Provincia Toboali, in fluviis [Sumatra, Indonesia]. Holotype (124 mm TL): RMNH 6806; holotype illustrated in Ng \& Lim (1993: fig. 3).

Distribution: Banka, Sumatra (Ng \& Lim, 1993).

Encheloclarias velatus $\mathrm{Ng} \& \mathrm{Tan}, 2000$

Encheloclarias velatus Ng \& Tan, 2000: 536, fig. 1. Type locality: Sumatra: Jambi, Angso Duo market. Holotype: MZB 9335.

Distribution: Batang Hari drainage, central Sumatra, and Binton (Ng \& Tan, 2000).

GYMNALLABES Günther, 1867

Gymnallabes Günther, 1867b: 111. Type species: Gymnallabes typus Günther, 1867. Type by original designation. Gender: Feminine.

Gymnallabes alvarezi Roman, 1970

Gymnallabes alvarezi Roman, 1970: 5, fig. 1. Type locality: Rio Kie, circa de Ebebiyin, Rio Muni, Guinea Ecuatorial. Holotype: at Inst. Biol. Aplicada, Barcelona.

Distribution: Kie and Muni Rivers, Equatorial Guinea (Teugels, 1986b).

Gymnallabes nops Roberts \& Stewart, 1976

Gymnallabes nops Roberts \& Stewart, 1976: 277, pl. 7 (fig. e), pl. 8 (fig. a). Type locality: Near Tadi, about 50 km downstream from Luozi. Lat. 5 14'S, long. 1356'E; lower Zaïre or Congo River. Holotype: MCZ 50298; skeleton of holotype illustrated in Devaere et al. (2005).

Distribution: Lower Congo River (Teugels, 1986b).

Remarks: Redescribed in Devaere et al. (2005).

Gymnallabes typus Günther, 1867

Gymnallabes typus Günther, 1867b: 111, pl. 2 (fig. a). Type locality: West Africa, probably Old Calabar. Syntypes: BMNH 1866.12.4.1-2 (2).

Gymnallabes typus heterocercalis Lönnberg, 1895: 184. Type locality: Cameroons. Syntypes (2): ZMUU 566. Originally as Gymnallabes typus forma heterocercalis.

Clariallabes longicaudatus Pappenheim, 1911: 519, fig. 3. Type locality: in der Mabelle, Süd-Kamerun. Holotype: ZMB 18401.

Distribution: Niger River to Cameroon (Teugels, 1986b).

HETEROBRANCHUS Geoffroy Saint-Hilaire, 1809

Heterobranchus Geoffroy Saint-Hilaire, 1809: pls. 16-17. Type species: Heterobranchus bidorsalis Geoffroy SaintHilaire, 1809. Type by monotypy. Gender: Masculine.

Revision: Teugels, Denayer \& Legendre (1990).

Monophyly: Agnèse \& Teugels (2001b).

Heterobranchus bidorsalis Geoffroy Saint-Hilaire, 1809

Heterobranchus bidorsalis Geoffroy Saint-Hilaire, 1809: pls. 16-17. Type locality: Nil. Holotype: at MNHN (skeleton).

Heterobranchus Geoffroyi Valenciennes, in Cuvier \& Valenciennes, 1840b: 392 (291 in the Strasbourg deluxe edition). Type locality: Nil. Holotype: at MNHN (skeleton). New name for Heterobranchus bidorsalis Geoffroy Saint-Hilaire, 1809.

Heterobranchus Senegalensis Valenciennes, in Cuvier \& Valenciennes, 1840b: 397 (295 in the Strasbourg deluxe edition). Type locality: Sénégal. Holotype: at MNHN (skull).

Heterobranchus intermedius Günther, 1864: 22. Type locality: Khartoum, Upper Nile. Syntypes (3, one a skeleton): 
BMNH 1862.1.17.54 (1), BMNH 1863.10.6.1 (1).

Distribution: Nile, Niger, Upper Volta, Guinea and Senegal Rivers, Chad basin (Teugels, 1986b).

Heterobranchus boulengeri Pellegrin, 1922

Heterobranchus Boulengeri Pellegrin, 1922d: 274. Type locality: Rivière Lukonzolwa. Syntypes (2): MRAC 14763 (1), MRAC 14764 (1).

Distribution: Lake Moero, Lukonzolwa River and upper Congo River, Zimbabwe (Teugels, 1986b).

Heterobranchus isopterus Bleeker, 1863

Heterobranchus isopterus Bleeker, 1863a: 108, pl. 22 (fig. 1). Type locality: Guinea [Rio Boutry]. Holotype: RMNH 2977, or ZMB 6590.

Heterobranchus macronema Bleeker, 1863a: 109, pls. 21 (fig. 1), 22 (fig. 2). Type locality: Guinea [Dabo-Crom]. Syntypes (4): RMNH 2978 (3).

Distribution: West Africa, from Senegal to Ghana (Teugels, 1986b).

Heterobranchus longifilis Valenciennes, 1840

Heterobranchus longifilis Valenciennes, in Cuvier \& Valenciennes, 1840b: 394 (293 in the Strasbourg deluxe edition), pl. 447. Type locality: Nil (Egypte). Holotype: MNHN b-0273.

Heterobranchus laticeps Peters, 1852: 682. Type locality: Tette, Boror (Mossambique). Syntypes: ZMB 3071 or 3072 (2), possibly ZMB 4741 (1), ZMB 6590 (1). Illustrated in Peters (1868c: 37, pl. 7, fig. 1).

Heterobranchus platycephalus Nichols \& La Monte, 1934: 2, fig. 2. Type locality: Luluabourg, Kasai District, Belgian Congo. Holotype: AMNH 12356.

Clarias loangwensis Worthington, 1933a: 46, unnumbered figure. Type locality: Loangwa River, Zambezi system. Holotype: BMNH 1932.12.16.501.

Distribution: Nile, Niger, Senegal, Congo and upper and middle Zambezi Rivers (Teugels, 1986b).

$\dagger$ Heterobranchus palaeindicus Lydekker, 1886

$\dagger$ Heterobranchus palceindicus Lydekker, 1886: 248, pl. 36, fig. 4. Type locality: Siwalik Hills, India. Lower Pliocene. Holotype: BMNH (paleo) 16402c (middle part of skull).

Distribution: Siwalik Hills, India; Lower Pliocene.

HETEROPNEUSTES Müller, 1840

Heteropneustes Müller, 1840: 243. Type species: Silurus fossilis Bloch, 1794. Type by monotypy. Gender: Masculine.

Saccobranchus Valenciennes, in Cuvier \& Valenciennes, 1840b: 399 (296 in Strasbourg deluxe edition). Type species: Silurus singio Hamilton, 1822. Type by monotypy. Gender: Masculine.

Clarisilurus Fowler, 1937: 133. Type species: Clarisilurus kemratensis Fowler, 1937. Type by original designation. Gender: Masculine.

Phylogenetic position: Diogo et al. (2003a).

Review: Hora (1936a).

Remarks: The relative dates of publication of the names Heteropneustes and Saccobranchus have not been clearly established and, therefore, priority of one name over the other is unknown. Herein, Heteropneustes is treated as valid because of its widespread usage in recent times.

Heteropneustes fossilis (Bloch, 1794)

Silurus fossilis Bloch, 1794: 46, pl. 370 (fig. 2). Type locality: Tranquebar. Lectotype: ZMB 3074, designated by, and illustrated in, Paepke (1999: 87, pl. 25, fig. 3).

Silurus singio Hamilton, 1822: 147, 374, pl. 37 (fig. 46). Type locality: Bengal. No types known.

Silurus laticeps Swainson, 1838: 345, fig. 84. Type locality: India. No types known. See also, Swainson (1839: 393).

Silurus biserratus Swainson, 1839: 306. Type locality: Bengal. No types known. Based on "Ham. pl. 37 . f 46 " [=

Hamilton (1822: pl. 37, fig. 46)]. Unneeded new name for Silurus singio Hamilton, 1822.

Saccobranchus microcephalus Günther, 1864: 31. Type locality: Ceylon. Holotype: BMNH 1864.3.18.1.

Saccobranchus microps Günther, 1864: 31. Type locality: Ceylon. Syntypes: BMNH 1859.5.31.9-11 (3).

Distribution: Widely distributed in South Asia.

Remarks: Synonymy of Saccobranchus microps based on Pethiyagoda \& Bahir (1998). 
Heteropneustes kemratensis (Fowler, 1937)

Clarisilurus kemratensis Fowler, 1937: 133, figs. 5-7. Type locality: Kemrat, Siam. Holotype: ANSP 67880.

Distribution: Mekong, Chao Phraya and Tapi River basins (Kottelat, 2001b).

\section{Species inquirenda, Heteropneustes}

Heteropneustes longipectoralis Rema Devi \& Raghunathan, 1999: 109, pl. 2 (figs. 1-5). Type locality: Thirumurthi

Dam, Anamalai Hills, Western Ghats, Tamil Nadu, India. Holotype: ZSI/SRS F.4935.

HORAGLANIS Menon, 1950

Horaglanis Menon, 1950: 60. Type species: Horaglanis krishnai Menon, 1950. Type by original designation. Gender: Masculine.

Horaglanis alikunhii Subhash Babu \& Nayer, 2004

Horaglanis alikunhii Subhash Babu \& Nayer, 2004: 296, fig. 1. Type locality: Parappukara $\left(10^{\circ} 23^{\prime} \mathrm{N}, 7^{\circ} 15^{\prime} \mathrm{E}\right)$, Trichur district, Kerala [India]; from a well. Holotype: ZSI Calicut. Regn. V/F 13137.

Distribution: Trichur District, Kerala, India (Subhash Babu \& Nayer, 2004).

Horaglanis krishnai Menon, 1950

Horaglanis krishnai Menon, 1950: 64, pl. 1 (figs. 1-3). Type locality: Well at Kottayam, Kerala [India]. Holotype: ZSI F313/2.

Distribution: Two separate locations in Kottayam, Kerala State, India (Subhash Babu \& Nayer, 2004).

PLATYALLABES Poll, 1977

Platyallabes Poll, 1977: 129. Type species: Gymnallabes tihoni Poll, 1944. Type by original designation. Gender: Feminine.

Platyallabes tihoni (Poll, 1944)

Gymnallabes tihoni Poll, 1944b: 79, fig. on p. 81. Type locality: Poste de peche de Kingabwa, Stanley-Pool. Neotype: MRAC 103307, designated by Derijst (1991: 43).

Distribution: Malebo Pool (Teugels, 1986b).

Remarks: Described initially on an illustration. Redescribed by Poll (1957: 240) with a new "holotype" which Derijst (1991: 43) regarded as, and effectively designated as, the neotype. Species redescribed in Devaere et al. (2005).

PLATYCLARIAS Poll, 1977

Platyclarias Poll, 1977: 136. Type species: Platyclarias machadoi Poll, 1977. Type by original designation. Gender: Masculine.

Platyclarias machadoi Poll, 1977

Platyclarias machadoi Poll, 1977: 141, figs. 7-8. Type locality: Haut Cuango, Cafunfo, rivière Borio, Angola. Holotype: MRAC 78-6-P-1345.

Distribution: Upper Cuango River (Teugels, 1986b).

TANGANIKALLABES Poll, 1943

Tanganikallabes Poll, 1943a: 127. Type species: Tanganikallabes mortiauxi Poll, 1943. Type by monotypy. Gender: Feminine.

Tanganikallabes mortiauxi Poll, 1943

Tanganikallabes mortiauxi Poll, 1943a: 131, figs. 1-4. Type locality: lac Tanganyika, Albertville. Holotype: MRAC 63731; also illustrated in Poll (1946: 234, figs. 27, 28).

Distribution: Lake Tanganyika (Teugels, 1986b).

UEGITGLANIS Gianferrari, 1923

Uegitglanis Gianferrari, 1923: 1. Type species: Uegitglanis zammaranoi Gianferrari, 1923. Type by original desig- 
nation. Gender: Masculine.

Phylogeny: David (1936a).

Uegitglanis zammaranoi Gianferrari, 1923

Uegitglanis Zammaranoi Gianferrari, 1923: 1, pl. 1. Type locality: Pozzi di Uegit (El Uegit) Somalia italiana. Holotype: MSNM 37.

Distribution: Caves near the Uegit and the Uebi Scebeli rivers, Somalia (Teugels, 1986b).

Remarks: Systematic position discussed in David (1936a: 369).

XENOCLARIAS Greenwood, 1958

Xenoclarias Greenwood, 1958: 321. Type species: Clarias eupogon Norman, 1928. Type by original designation. Gender: Masculine.

Xenoclarias eupogon (Norman, 1928)

Clarias eupogon Norman, 1928a: 105. Type locality: Lake Victoria $\left(0^{\circ} 22^{\prime} \mathrm{S}, 34^{\circ} 141{ }^{\prime}{ }^{\prime} \mathrm{E}\right)$. Holotype: BMNH 1928.5.24.57.

Distribution: Lake Victoria, in deep waters (Teugels, 1986b).

Xenoclarias holobranchus Greenwood, 1958

Xenoclarias holobranchus Greenwood, 1958: 322. Type locality: off the south-western tip of Ukerewe Island, Lake Victoria. Holotype: BMNH 1958.7.8.1.

Distribution: Lake Victoria (Teugels, 1986b).

\section{CLAROTEIDAE Bleeker, 1862}

Claroteini Bleeker, 1862 (in Bleeker, 1862-63): 4. Type genus: Clarotes Kner, 1855.

Chrysichthyinae Regan, 1911: 561. Type genus: Chrysichthys Bleeker, 1858.

Auchenoglanidinae Jayaram, 1966b: 1119. Type genus: Auchenoglanis Günther, 1865.

Gephyroglanidini Jayaram, 1966b: 1071, 1099. Type genus: Gephyroglanis Boulenger, 1899.

Chrysichthyini Jayaram, 1966b: 1071.Type genus: Chrysichthys Bleeker, 1858; preoccupied by Regan (1911).

Taxonomic summaries: Risch (1986, as Bagridae, in part).

Reviews: Jayaram (1955a, 1966b, African genera); Risch (1992b, West Africa); Bailey \& Stewart (1984, Lake Tanganyika).

Phylogeny: Jayaram (1976); Jayaram \& Singh (1982); Mo (1991); Ng (2003j).

Key to genera: Poll \& Gosse (1994).

17 genera, 83 species; including 1 fossil genus and 3 fossil species.

AMARGINOPS Nichols \& Griscom, 1917

Amarginops Nichols \& Griscom, 1917: 713. Type species: Amarginops platus Nichols \& Griscom, 1917. Type by monotypy. Gender: Masculine.

Amarginops hildae (Bell-Cross, 1973)

Chrysichthys hildae Bell-Cross, 1973: 7, fig. 1. Type locality: Buzi River, Mozambique, at approximately $20^{\circ} 22^{\prime}$ S, 30²0'E. Holotype: QVMS 1827.

Distribution: Lower reaches of Buzi River, Mozambique (Skelton, 1993).

Remarks: Also treated in recent literature as Chrysichthys hildae.

Amarginops platus Nichols \& Griscom, 1917

Amarginops platus Nichols \& Griscom, 1917: 713, fig. 22-23. Type locality: Stanleyville, Congo. Holotype: AMNH 6528.

Distribution: Kisangani, Congo; known only from types (Risch, 1986).

ANASPIDOGLANIS Teugels, Risch, de Vos \& Thys van den Audenaerde, 1991

Anaspidoglanis Teugels, Risch, de Vos \& Thys van den Audenaerde, 1991: 513. Type species: Auchenoglanis mac- 
rostoma Pellegrin, 1909. Type by original designation. Gender: Masculine.

Revision: Mbega \& Teugels (1998: 233).

Key: Geerinckx et al. (2003).

Anaspidoglanis akiri (Risch, 1987)

Auchenoglanis akiri Risch, 1987: 33, fig. 4. Type locality: Umayara Mba, Etche, Rivers State, Nigeria $\left(5^{\circ} 12^{\prime} \mathrm{N}\right.$,

706'E). Holotype: MRAC 84-15-P-9; holotype illustrated in Geerinckx et al. (2003: 24, fig. 8c).

Distribution: Niger River delta, New Calabar and Bony Rivers, Nigeria (Geerinckx et al. 2003).

Remarks: Redescribed and assigned to Anaspidoglanis in Geerinckx et al. (2003).

Anaspidoglanis boutchangai (Thys van den Audenaerde, 1965)

Parauchenoglanis boutchangai Thys van den Audenaerde, 1965b: 347, fig. 1. Type locality: Rapides de la Ngounié ou de la Louetsi à Lelamba, 2²12'S, 11³0'E [Gabon]. Holotype: MRAC 153160.

Distribution: Ngounié or Louetsi River, Gabon; known only from the type locality (Risch, 1986).

Anaspidoglanis macrostomus (Pellegrin, 1909)

Auchenoglanis macrostoma Pellegrin, 1909a: 67. Type locality: Ngomo (Ogôoué) [Gabon]. Holotype: MNHN 1909-0017. Also described as new in Pellegrin (1909d: 48, figs. 1a, 1b). Name spelled Auchenauglanis macrostom on p. 66.

Parauchenoglanis ansorgii Boulenger, 1912b: 20, pl. 22 (fig. 3). Type locality: N'Kutu sur les chutes du Loango. Holotype: BMNH 1912.4.1.447.

Distribution: S. Cameroon, Gabon, and Congo River system (Risch, 1986).

AUCHENOGLANIS Günther, 1865

Auchenaspis Bleeker, 1858b: 198, 205, 208. Type species: Pimelodus biscutatus Geoffroy St. Hilaire, 1809. Type by subsequent designation by Bleeker, 1862 (in Bleeker, 1862-63: 12). Gender: Feminine. Preoccupied by $\dagger$ Auchenaspis Egerton, 1857, in fossil fishes; replaced by Auchenoglanis Günther, 1865.

Auchenoglanis Günther, 1865b: 165. Type species: Pimelodus biscutatus Geoffroy St. Hilaire, 1809. Type by being a replacement name. Gender: Masculine. Replacement for Auchenaspis Bleeker, 1858, preoccupied by $\dagger$ Auchenaspis Egerton, 1857, in fossil fishes.

Oxyglanis Vinciguerra, 1898: 249. Type species: Oxyglanis sacchii Vinciguerra, 1898. Type by monotypy. Gender: Masculine.

Remarks: Included species and synonymy follows Teugels et al. (1991).

Auchenoglanis biscutatus (Geoffroy Saint-Hilaire, 1809)

Pimelodus biscutatus Geoffroy Saint-Hilaire, 1809: pl. 14 (fig. 1). Type locality: Nil. Holotype: MNHN a-8969.

Distribution: Nile, Niger, and Senegal rivers, and Lake Chad basin (Risch, 1986).

Auchenoglanis occidentalis (Valenciennes, 1840)

Pimelodus occidentalis Valenciennes, in Cuvier \& Valenciennes, 1840b: 203 (150 in Strasbourg deluxe edition). Type locality: Sénégal. Holotype: MNHN a-8971.

Oxyglanis Sacchii Vinciguerra, 1898: 250. Type locality: fiume Omo a Murzù. Holotype: MSNG 14506.

Auchenoglanis occidentalis tanganicanus Boulenger, 1906f: 553, pl. 33. Type locality: Kalambo,... the middle of the lake [Lake Tanganyika] and .... Mtomdwe Bay. Syntypes (4): BMNH 1906.9.6.37-38 (2), BMNH 1906.9.6.39 (1), BMNH 1906.9.8.69 (1). Originally as Auchenoglanis occidentalis var. tanganicanus.

Auchenoglanis occidentalis tchadiensis Pellegrin, 1909b: 244. Type locality: Lac Chad à Bol. Syntypes: MNHN 1909-0425 (3), MNHN 1909-0426 (4). Originally as Auchenoglanis occidentalis var. tchadiensis.

Auchenoglanis acuticeps Pappenheim, in Pappenheim \& Boulenger, 1914: 249, pl. 5 (fig. 1). Type locality: Albertsee. Syntypes (7): ZMB 19094 (1), ZMB 21317 (3).

Auchenoglanis Wittei Giltay, 1930a: 92, figs. 1-2. Type locality: Stanleyville. Holotype: MRAC 19802.

Distribution: East African lakes; Lake Chad; Nile, Congo, Omo and Giuba rivers (Risch, 1986).

BATHYBAGRUS Bailey \& Stewart, 1984

Bathybagrus Bailey \& Stewart, 1984: 8. Type species: Bathybagrus tetranema Bailey \& Stewart, 1984. Type by original designation. Gender: Masculine. 
Bathybagrus tetranema Bailey \& Stewart, 1984

Bathybagrus tetranema Bailey \& Stewart, 1984: 11, figs. 1-4. Type locality: Zambian waters of Lake Tanganyika. Holotype: UMMZ 196110.

Distribution: Lake Tanganyika, Zambian portion (Risch, 1986).

\section{CHRYSICHTHYS Bleeker, 1858}

Chrysichthys Bleeker, 1858b: 60, 65. Type species: Pimelodus auratus Geoffroy St. Hilaire, 1809. Type by subsequent designation by Bleeker (1862-63: 9). Gender: Masculine.

Melanodactylus Bleeker, 1858b: 60, 65. Type species: Arius acutivelis Valenciennes, 1840. Type by subsequent designation by Bleeker (1862-63: 9). Gender: Masculine.

Chrysobagrus Boulenger, 1899a: 40. Type species: Chrysobagrus brevibarbis Boulenger, 1899. Type by subsequent designation, apparently by Jordan (1920: 485); see Eschmeyer et al. (1998) for details. Gender: Masculine.

Key to species: Jayaram (1966b).

Chrysichthys aluuensis Risch, 1985

Chrysichthys aluuensis Risch, 1985b: 185, figs. 2-3. Type locality: Omuehuechi-Aluu, New Calabar River, Rivers State, Nigeria. Holotype: MRAC 84-20-P-1.

Distribution: New Calabar River; known only from type locality (Risch, 1986).

Chrysichthys ansorgii Boulenger, 1910

Chrysichthys ansorgii Boulenger, 1910: 558. Type locality: Quanza River at Dondo and from the Bengo River. Syntypes (numerous): ANSP 37906 (1), BMNH 1911.6.1.116-124 (9), BMNH 1911.6.1.126 (1), USNM 86636 (1), ZMB 18228 (1).

Distribution: Cuanza and Bengo Rivers, Angola (Risch, 1986).

Chrysichthys auratus (Geoffroy Saint-Hilaire, 1809)

Pimelodus auratus Geoffroy Saint-Hilaire, 1809: pl. 14 (figs. 3-4). Type locality: Nil. Holotype: at MNHN. Name available from caption to plate, described as Porcus auratus in Geoffroy Saint-Hilaire (1827: 302).

Bagrus capito Valenciennes, in Cuvier \& Valenciennes, 1840a: 430 (319 of Strasbourg deluxe edition). Type locality: Nil. Syntypes: MNHN b-0605 (1), MNHN b-0606 (1).

Chrysichthys macrops Günther, 1864: 71. Type locality: Nile, Chartoum. Syntypes (7): BMNH 1860.11.9.125 (1), BMNH 1862.6.17.60-64 (5).

Gephyroglanis tilhoi Pellegrin, 1909b: 243. Type locality: Bol, Lac Chad. Holotype: MNHN 1909-0424.

Chrysichthys filamentosus Boulenger, 1912b: 19, pl. 18 (fig. 2). Type locality: Chiloango ville, Loango à N'Kutu, Luali à Lundo et Buco Zau, Lebuzi à Kuka Muno. Syntypes: BMNH 1912.4.1.440 (1), BMNH 1912.4.1.441 (1), BMNH 1912.4.1.442-443 (2), BMNH 1912.4.1.444 (1), BMNH 1912.4.1.445-446 (2), MRAC 1668-69 (2), MRAC 1670 (1), ZMB 8820 (1).

Chrysichthys longifilis Pfaff, 1933: 289, figs. 5-6. Type locality: Kabara. Holotype (29.2 cm): ZMUC P 28668.

Distribution: Nile, Niger, Volta, Senegal rivers, Lake Chad basin, and coastal rivers of western Africa, from Liberia to Angola (Risch, 1986).

Remarks: The name Bagrus auratus in Hyrtl (1859: 17) is sometimes treated as an available name but is more likely intended as a reference to this species.

Chrysichthys bocagii Boulenger, 1910

Chrysichthys bocagii Boulenger, 1910: 557. Type locality: Bengo River at Dondo, Angola. Syntypes (2): BMNH 1911.6.1.114-115 (2).

Distribution: Bengo River, Angola (Risch, 1986).

Chrysichthys brachynema Boulenger, 1900

Chrysichthys brachynema Boulenger, 1900c: 480. Type locality: Kalambo [Lake Tanganyika]. Syntype or lectotype: BMNH 1906.9.6.33 [see Remarks].

Chrysichthys myriodon Boulenger, 1900a: 139, pl. 51. Type locality: Albertville, Tembwi [Lake Tanganyika]. Syntypes: BMNH 1906.9.6.27 (1), BMNH 1906.9.6.28-31 (4), BMNH 1906.9.6.32 (1, skeleton), MRAC 325 (1).

Distribution: Lake Tanganyika (Risch, 1986).

Remarks: Risch (1986: 15) and Eschmeyer et al. (1998) stated that a lectotype was designated for Chrysichthys 
brachynema Boulenger, but neither indicated the source of that designation and I have not found an explicit lectotype designation; it may have been inadvertently designated by Risch (1986). The publication date for Boulenger (1900c) was reported as 1 Nov. 1900, while that of Boulenger (1900a) was December, 1900, which gives Chrysichthys brachynema priority.

Chrysichthys brevibarbis (Boulenger, 1899)

Chrysobagrus brevibarbis Boulenger, 1899a: 40, pl. 21 (fig. 1). Type locality: Boma. Holotype: MRAC 16.

Distribution: Congo River basin, below Stanley Falls (Risch, 1986).

Chrysichthys cranchii (Leach, 1818)

Pimelodus Cranchii Leach, in Tuckey, 1818: 409. Type locality: Lower Zaire. Holotype: BMNH 2005.5.17.4.

Chrysichthys börressoni Lönnberg, 1924: 620. Type locality: Lubosi river, a tributary to Luala river, Lower Congo. Holotype: NRM 9942.

Distribution: Lualaba River system, Congo basin (Risch, 1986).

Chrysichthys dageti Risch, 1992

Chrysichthys dageti Risch, 1992a: 152, fig. 1. Type locality: Riv. Nanga entre Mboukoumassi et le lac Nanga, Congo (04 $\left.15^{\circ} \mathrm{S}, 11^{\circ} 48^{\prime} \mathrm{E}\right)$. Holotype: MRAC 90-57-P-1273.

Distribution: Nanga River and tributaries, Congo (Risch, 1992a).

Chrysichthys delhezi Boulenger, 1899

Chrysichthys delhezi Boulenger, 1899b: 108, pl. 42 (fig. 2). Type locality: Boma. Lectotype: MRAC 952 (1)or MRAC 953 (1). Boulenger (1911: 225, fig. 262) illustrated a specimen and referred to it as the type, from the MRAC collection. If identifiable, that specimen is the lectotype of the species. If the illustrated specimen cannot be identified, the two speciemens listed above, plus BMNH 1899.6.27.49 (1), are syntypes.

? Chrysichthys delhezi thomasi Chabanaud, 1934: 209. Type locality: Congo. Syntypes: MNHN 1937-0074 (1), MNHN 1937-0075 (1).

Distribution: Lower Congo River system (Risch, 1986).

Chrysichthys duttoni Boulenger, 1905

Chrysichthys Duttoni Boulenger, 1905a: 641. Type locality: Lusambo, on the Kasai River. Holotype: MRAC 1342.

Distribution: Lualaba River system, Congo basin (Risch, 1986).

Chrysichthys furcatus Günther, 1864

Chrysichthys furcatus Günther, 1864: 430. Type locality: West Africa. Holotype: BMNH 1864.7.22.1.

Distribution: Coastal rivers of west Africa, from Liberia to Cabinda, Angola (Risch, 1986).

Chrysichthys grandis Boulenger, 1917

Chrysichthys grandis Boulenger, 1917b: 367. Type locality: Kilewa Bay [Lake Tanganyika]. Holotype: MRAC 14347.

Distribution: Lake Tanganyika (Risch, 1986).

Chrysichthys graueri Steindachner, 1911

Chrysichthys graueri Steindachner, 1911c: 529. Type locality: Tanganyika, am Ende des nordwestlichen Ufers. Syntypes: NMW 47106 (1), NMW 47107 (1). Species illustrated and described in more detail in Steindachner (1911e: 1178, pl. 3).

Distribution: Lake Tanganyika (Risch, 1986).

Chrysichthys habereri Steindachner, 1912

Chrysichthys habereri Steindachner, 1912: 445. Type locality: Dscha, Nebenfluss des Kongo, im Bezirke Molundu. Holotype: NMW 47108. Species also described as new and illustrated in Steindachner (1913: 32, fig. 8 and pl. 4).

Distribution: Congo River system, below Stanley Falls (Risch, 1986).

Chrysichthys helicophagus Roberts \& Stewart, 1976

Chrysichthys helicophagus Roberts \& Stewart, 1976: 276, pl. 6 (fig. e). Type locality: Near Tadi, about 50 km downstream from Luozi. Lat. 5²14'S, Long. 1356'E; lower Zaïre or Congo River. Holotype: MCZ 50292.

Distribution: Lower Congo River, below Malebo Pool (Risch, 1986).

Chrysichthys johnelsi Daget, 1959

Chrysichthys johnelsi Daget, 1959: 691, fig. 2. Type locality: Gambie à Bafoulabé (parc national du Niokolo-Koba). 
Syntypes (3): MNHN 1959-0146 (2) plus specimen described in Johnels (1954: 386), now NRM 13899.

Distribution: Rivers of the upper Guinea region, including the Geba, Taja, Moa, Mano, Loffa, St. Pauls, St. Johns, Nipoué and Tano rivers (Risch, 1986).

Remarks: Redescribed, with comments on relationships and zoogeography, by Risch \& Thys van den Audenaerde (1985a).

Chrysichthys laticeps Pellegrin, 1932

Chrysichthys laticeps Pellegrin, 1932: 166. Type locality: Congo, près de Brazzaville. Holotype: MNHN 1931-0159 (but listed as 0158 in the publication).

Distribution: Congo River system (Risch, 1986).

Chrysichthys levequei Risch, 1988

Chrysichthys levequei Risch, 1988: 3, fig. 1. Type locality: du Samou, (bassin du Konkouré) en Guinée. Holotype: MNHN 1986-0563.

Distribution: Konkouré River basin, Guinea (Risch, 1988).

Chrysichthys longibarbis (Boulenger, 1899)

Chrysobagrus longibarbis Boulenger, 1899a: 41, pl. 21 (fig. 2). Type locality: Léopoldville. Syntypes: BMNH 1899.2.20.18 (1), MRAC 108 (1).

Distribution: Congo River basin, below Stanley Falls (Risch, 1986).

Chrysichthys longidorsalis Risch \& Thys van den Audenaerde, 1981

Gephyroglanis velifer Thys van den Audenaerde, 1965a: 265, fig. 1. Type locality: Ekongolo, Cameroon. Holotype: MRAC 153158. Preoccupied in Chrysichthys by Chrysichthys velifer Norman, 1923; replaced by Chrysichthys longidorsalis Risch \& Thys van den Audenaerde, 1981.

Chrysichthys longidorsalis Risch \& Thys van den Audenaerde, 1981: 247. Type locality: Ekongolo. Holotype: MRAC 153158. Replacement for Gephyroglanis velifer Thys van den Audenaerde, 1965, preoccupied in Chrysichthys by Chrysichthys velifer Norman, 1923 (a junior synonym of Chrysichthys maurus).

Distribution: Nyong River (Risch \& Thys van den Audenaerde, 1985b), Middle Sanaga River, Cameroon (Risch, 1986).

Chrysichthys longipinnis (Boulenger, 1899)

Gephyroglanis longipinnis Boulenger, 1899b: 109, pl. 41 (fig. 4). Type locality: Dolo, Stanley Pool [Congo River]. Lectotype: MRAC 958, designated by Risch (1981: 510).

Gephyroglanis gigas Pellegrin, 1922d: 278. Type locality: Kwamouth. Holotype: MRAC 14771.

Chrysichthys magnus Pellegrin, 1922d: 276. Type locality: Kwamouth. Syntypes (4): MRAC 14766 (1), MRAC 14767 (1), MRAC 14768-69 (2).

Distribution: Congo-Lualaba River system (Risch, 1986).

Chrysichthys mabusi Boulenger, 1905

Chrysichthys mabusi Boulenger, 1905b: 645. Type locality: Lake Bangwelo. Holotype: BMNH 1905.11.10.9.

Distribution: Congo-Lualaba River system, above Stanley Falls (Risch, 1986).

Chrysichthys macropterus Boulenger, 1920

Chrysichthys macropterus Boulenger, 1920a: 27, pl. 24. Type locality: Stanley Falls, ... d'Avakubi sur Ituri, et ... Bafwasende sur la Lindi; Congo Belge. Syntypes (6): BMNH 1919.9.10.252 (1), BMNH 1919.9.10.253 (1), MRAC 7118 (1), MRAC 7577 (1), MRAC 7578 (1).

Distribution: Congo River basin (Risch, 1986).

$\dagger$ Chrysichthys macrotis Van Neer, 1994

$\dagger$ Chrysichthys macrotis Van Neer, 1994: 100, text figs. 3-5, pl. 3 (fig. 1). Type locality: Nkondo-Kaiso area, Knondo Formation, Albertine Rift Valley. Holotype: [apparently at MRAC] NK 1365'89 (neurocranium).

Distribution: Nkondo-Kaiso area, Lake Albert Rift, Uganda; Pliocene, Nkondo Formation (Van Neer, 1994; Murray \& Budney, 2003).

Remarks: Considered to be a member of the Claroteidae by Gayet \& Meunier (2003).

$\dagger$ Chrysichthys mahengeensis Murray \& Budney, 2003

$\dagger$ Chrysichthys mahengeensis Murray \& Budney, 2003: 985, fig. 1. Type locality: Mahenge, Singida Plateau, Tanzania, $4^{\circ} 47^{\prime} 38^{\prime \prime} \mathrm{S}, 34^{\circ} 15^{\prime} 28^{\prime \prime}$, about $53 \mathrm{~km}$ west of the town of Singida; Eocene. Holotype: National Museum of 
Tanzania WM 386/96.

Distribution: Known only from type locality.

Chrysichthys maurus (Valenciennes, 1840)

Bagrus maurus Valenciennes, in Cuvier \& Valenciennes, 1840a: 431 (319 of Strasbourg deluxe edition). Type locality: Sénégal. Holotype: MNHN 0000-1198.

Gephyroglanis lowei Norman, 1923b: 582. Type locality: Bandama River. Holotype: BMNH 1923.3.2.40.

Chrysichthys velifer Norman, 1923b: 582. Type locality: Bandama River. Holotype: BMNH 1923.3.2.41.

Distribution: West Africa (Risch, 1986).

Chrysichthys nigrodigitatus (La Cepède, 1803)

Pimelodus nigrodigitatus La Cepède, 1803: 95, 103. Type locality: [No locality stated; Senegal, according to Risch, 1986]. Holotype: MNHN 0000-0108.

Arius acutivelis Valenciennes, in Cuvier \& Valenciennes, 1840b: 85 (64 of Strasbourg deluxe edition). Type locality: Gorée, ... haut Sénégal. Syntypes: MNHN a-8968 (2) and possibly MNHN 0000-0108.

Chrysichthys acutirostris Günther, 1864: 431. Type locality: Golungo Alto, Angola. Holotype: BMNH 1864.7.13.41.

Chrysichthys Büttikoferi Steindachner, 1894: 60, pl. 3 (fig. 1). Type locality: Fischermann-See [Liberia]. Holotype: RMNH 5341; figured by Boeseman (1963: pl. 1, fig. 2).

Chrysichthys camaronensis Günther, 1899: 729, pl. 44. Type locality: Camaroons. Holotype: BMNH 1871.11.20.21. As Chrysichthys camaronensis in text (p. 729, 732) and Chrysichthys cameronensis on plate; first reviser may be Eschmeyer et al. (1998).

Chrysichthys ogowensis Günther, 1899: 723, fig. 5. Type locality: Kondo-Kondo, on the Ogowe River [Gabon]. Holotype: BMNH 1896.5.5.66.

Distribution: West Africa from Senegal to Cabinda, Angola (Risch, 1986).

Remarks: See Adépo-Gourène et al. (1997) for population differentiation.

Chrysichthys nyongensis Risch \& Thys van den Audenaerde, 1985

Chrysichthys longidorsalis nyongensis Risch \& Thys van den Audenaerde, 1985: 91. Type locality: Ebongo, Akonolinga et en amont des chutes, 12 km SE d'Esaka. Holotype: MRAC 73-29-P-1404 [or 1407].

Distribution: Nyong River, Cameroon (Risch, 1986).

Remarks: Risch (1986) reported catalog number of the holotype as MRAC 73-29-P-1404, but Eschmeyer et al. (1998) listed MRAC 73-29-P-1407 as holotype, with -1404 as a paratype.

Chrysichthys ogooensis (Pellegrin, 1900)

Gephyroglanis ogooensis Pellegrin, 1900: 180. Type locality: Adouma (Ogôoué). Holotype: MNHN 1886-0422.

Gephyroglanis ogoensis Boulenger, 1901a: 227. Type locality: Adouma (Ogôoué). Holotype: MNHN 1886-0422. Unjustified emendation or misspelling of Gephyroglanis ogooensis Pellegrin, 1900.

Distribution: Ogowe-Nyanga River basin, Gabon (Risch, 1986).

Remarks: Redescribed as Gephyroglanis ogooensis in Risch (1985a).

Chrysichthys okae Fowler, 1949

Chrysichthys okae Fowler, 1949: 261, figs. 53-58. Type locality: Oka, Congo system. Holotype: ANSP 71909.

Distribution: Central Congo basin, in tributary streams (Risch, 1986).

Chrysichthys ornatus Boulenger, 1902

Chrysichthys ornatus Boulenger, 1902d: 39, pl. 11 (fig. 2). Type locality: l'Ubangi à Banzyville [et] Monsembé, Haut Congo. Syntypes (4): BMNH 1901.12.21.48-50 (3), MRAC 1174 (1).

Distribution: Congo River, below Stanley Falls (Risch, 1986).

Chrysichthys persimilis Günther, 1899

Chrysichthys persimilis Günther, 1899: 727, pl. 43; fig. 7. Type locality: Gaboon. Holotype: BMNH 1867.5.22.1.

Distribution: Gabon, Congo (Risch, 1986).

Remarks: Treated in Paugy \& Bénech (1989: 302) as a synonym of Chrysichthys auratus (Geoffroy Saint-Hilaire, 1809).

Chrysichthys platycephalus Worthington \& Ricardo, 1937

Chrysichthys platycephalus Worthington \& Ricardo, 1937: 1092, fig. 6. Type locality: Mpulungu-Niamkolo and the 
estuary of the Ngere River, Lake Tanganyika basin. Syntypes (38): BMNH 1936.6.15.849-852 (4), BMNH 1936.6.15.853-856 (4), BMNH 1936.6.15.857-878 (22).

Distribution: Lake Tanganyika (Risch, 1986).

Chrysichthys polli Risch, 1987

Chrysichthys polli Risch, 1987: 25, fig. 2. Type locality: Yelelola (falls). Lower Zaire (543'S, 13³4'E). Holotype: MRAC 47814.

Distribution: Lower Congo River basin (Risch, 1987).

Chrysichthys punctatus Boulenger, 1899

Chrysichthys punctatus Boulenger, 1899b: 107, pl. 42 (fig. 1). Type locality: Stanley Pool and Kutu. Syntypes (9): BMNH 1899.11.27.25-26 (2), MRAC 950 (1), MRAC 951 (1).

Distribution: Congo River system below Stanley Falls (Risch, 1986).

Chrysichthys rueppelli Boulenger, 1907

Chrysichthys rueppelli Boulenger, 1907a: 337, pl. 60 (fig. 2). Type locality: Lower Nile and freshwater pools near Ghet-el-Nassara, Lake Menzaleh. Syntypes (4): BMNH 1850.7.29.17 (1), BMNH 1907.12.2.2131-2132 (2).

Distribution: Nile River (Risch, 1986).

Chrysichthys sharpii Boulenger, 1901

Chrysichthys Sharpii Boulenger, 1901b: 81. Type locality: Lake Mwero. Holotype: BMNH 1900.12.31.14 (dry skin).

Distribution: Lake Mweru, Congo Basin; known only from holotype (Risch, 1986).

Chrysichthys sianenna Boulenger, 1906

Chrysichthys sianenna Boulenger, 1906f: 551, pl. 32 (fig. 2). Type locality: Niamkolo and .... Mbete [Lake Tanganyika]. Syntypes (3): BMNH 1906.9.8.65-66 (2), BMNH 1906.9.8.67 (1).

Distribution: Lake Tanganyika (Risch, 1986).

Chrysichthys stappersii Boulenger, 1917

Chrysichthys stappersii Boulenger, 1917b: 366. Type locality: Kilewa Bay [Lake Tanganyika]. Holotype: MRAC 14236; illustrated in Poll (1946: 211, figs. 18, 19).

Distribution: Lake Tanganyika (Risch, 1986).

Chrysichthys teugelsi Risch, 1987

Chrysichthys teugelsi Risch, 1987: 22, fig. 1. Type locality: Cavally River at Tai (552'N, $\left.7^{\circ} 27^{\prime} \mathrm{W}\right)$, Ivory Coast. Holotype: MRAC 80-19-P-189.

Distribution: Cavally River, Côte d'Ivoire (Risch, 1987).

Chrysichthys thonneri Steindachner, 1912

Chrysichthys thonneri Steindachner, 1912: 446. Type locality: Dschaflusse und oberen Kongo. Syntypes (3): NMW 10643 (1), NMW 10645 (1). Illustrated and described in more detail in Steindachner (1913: 33, fig. 9 and pl. 5).

Distribution: Congo-Lualaba River system (Risch, 1986).

Chrysichthys thysi Risch, 1985

Chrysichthys thysi Risch, 1985b: 189, figs. 5-6. Type locality: Makokou, riv. Ivindo. Holotype: MRAC 73-2-P1791.

Distribution: Ogowe-Nyanga River basin, Gabon (Risch, 1986).

Chrysichthys uniformis Pellegrin, 1922

Chrysichthys uniformis Pellegrin, 1922d: 277. Type locality: Poko (Uelé). Holotype: MRAC 7477.

Distribution: Congo, known only from holotype (Risch, 1986).

Chrysichthys wagenaari Boulenger, 1899

Chrysichthys wagenaari Boulenger, 1899a: 39, pl. 20 (fig. 1). Type locality: Upoto [Congo basin]. Holotype: MRAC 147.

Distribution: Ubangi River and Middle Congo River system below Stanley Falls (Risch, 1986).

Chrysichthys walkeri Günther, 1899

Chrysichthys walkeri Günther, 1899: 720, fig. 3. Type locality: River Prah, Gold Coast. Syntypes (3): BMNH 1899.12.22.20-22 (3).

Distribution: Western Africa, from Ghana to Cabinda, Angola (Risch, 1986). 


\section{Species inquirendae, Chrysichthys}

Chrysichthys coriscanus Günther, 1899: 724. Type locality: Corisco Isld. [Equatorial Guinea]. Syntypes (2): BMNH 1896.5.5.64-65 (2). Treated in recent literature as a synonym of either Chrysichthys auratus or Chrysichthys nigrodigitatus.

Chrysichthys kingsleyae Günther, 1899: 728, pl. 45 (fig. a); fig. 8. Type locality: River Ogowe. Holotype: BMNH 1899.12.23.1. Treated in recent literature either as a synonym of Chrysichthys walkeri Günther, 1899 (Risch, 1986 : 27) or Chrysichthys auratus (Geoffroy Saint-Hilaire, 1809) (Paugy \& Bénech 1989: 302).

Chrysichthys lagoensis Günther, 1899: 725, fig. 6. Type locality: Lagos. Holotype: BMNH 1866.3.8.16. Treated as synonym of Chrysichthys nigrodigitatus or Chrysichthys maurus.

\section{CLAROTES Kner, 1855}

Clarotes Kner, 1855b: 313. Type species: Clarotes heuglini Kner, 1855. Type by monotypy. Gender: Masculine.

Gonocephalus Kner, 1855b: 313. Type species: Clarotes heuglini Kner, 1855. Type by monotypy. Gender: Masculine. Alternate new name for Clarotes. Preoccupied by Gonocephalus Kaup, 1825, in Reptilia.

Octonematichthys Bleeker, 1858b: 60, 65. Type species: Bagrus nigrita Valenciennes, 1840. Type by monotypy. Gender: Masculine.

Clarotes bidorsalis Pellegrin, 1938

Clarotes bidorsalis Pellegrin, 1938b: 220, fig. on p. 221. Type locality: Giuba [Somalia]. Holotype: MNHN 19380139.

Distribution: Juba River, Somalia (Risch, 1986).

Clarotes laticeps (Rüppell, 1829)

Pimelodus laticeps Rüppell, 1829: 7, pl. 1 (fig. 2). Type locality: Cairo. Holotype: SMF 226.

Bagrus nigrita Valenciennes, in Cuvier \& Valenciennes, 1840a: 426 (316 of Strasbourg deluxe edition), pl. 416. Type locality: Sénégal. Syntypes: MNHN 0000-1204 (1), MNHN a-1113 (2).

Clarotes heuglini Kner, 1855b: 313, pls. 1-2. Type locality: bei Girf in der Nahe von Chartoum [Sudan]. Holotype: at NMW.

Chrysichthys macropogon Norman, 1928b: 385. Type locality: Kiyawa River (near Katagum), Northern Provinces, Nigeria. Holotype: BMNH 1928.7.3.70.

Chrysichthys pitmani Fowler, 1936b: 312, figs. 97-100. Type locality: Athi River Crossing 16 miles north-northeast of Kibwezi, Kenya Colony. Holotype: ANSP 65888.

Distribution: East Africa, Nile, Niger and Senegal Rivers; Lake Chad (Risch, 1986).

Clarotes macrocephalus Daget, 1954

Clarotes macrocephalus Daget, 1954: 257, fig. 95. Type locality: Diafarabé [Niger River]. Syntypes (10): MNHN 1954-0003 (1).

Distribution: Niger, Volta and Senegal Rivers (Risch, 1986).

Remarks: MNHN 1954-0003 is listed in MNHN catalog as a holotype.

$\dagger$ EAGLESOMIA White, 1934

$\dagger$ Eaglesomia White, 1934: 70. Type species: † Chrysichthys eaglesomei White, 1926, by original designation. Gender: Feminine.

Remarks: Included in the Claroteidae in Gayet \& Meunier (2003).

$\dagger$ Eaglesomia eaglesomei (White, 1926)

$\dagger$ Chrysichthys eaglesomei White, 1926: 59, pls. 14-15. Type locality: Ameki, Nigeria, Eocene. Holotype: BMNH P.11944-11946 (posterior half of skull with anterior vertebral complex).

Remarks: Redescribed in White (1934: 70, pl. 8, 9, pl. 10, fig. 28).

GEPHYROGLANIS Boulenger, 1899

Gephyroglanis Boulenger, 1899a:42. Type species: Gephyroglanis congicus Boulenger, 1899. Type by monotypy. 
Gender: Masculine.

Gephyroglanis congicus Boulenger, 1899

Gephyroglanis congicus Boulenger, 1899a: 42, pl. 20 (fig. 2). Type locality: Upoto [Congo basin]. Holotype: MRAC 146.

Distribution: Congo-Lualaba River system (Risch, 1986).

Gephyroglanis gymnorhynchus Pappenheim, 1914

Gephyroglanis gymnorhynchus Pappenheim, in Pappenheim \& Boulenger, 1914: 248, pl. 4 (fig. 6). Type locality: Aruwimi bei Basoko. Holotype: ZMB 19092.

Distribution: Aruwimi River and Port Congo (Risch, 1986).

Gephyroglanis habereri Steindachner, 1912

Gephyroglanis habereri Steindachner, 1912: 449. Type locality: Dscha, Nebenflusse des Kongo, im Bezirke Molundu. Holotype: NMW 46570. Species illustrated and described in more detail in Steindachner (1913: 36, pl. 6).

Distribution: Congo River; known only from holotype (Risch, 1986).

GNATHOBAGRUS Nichols \& Griscom, 1917

Gnathobagrus Nichols \& Griscom, 1917: 711. Type species: Gnathobagrus depressus Nichols \& Griscom, 1917. Type by monotypy. Gender: Masculine.

Gnathobagrus depressus Nichols \& Griscom, 1917

Gnathobagrus depressus Nichols \& Griscom, 1917: 712, fig. 21. Type locality: Boma, Congo. Holotype: AMNH 6647.

Distribution: Congo River at Boma; known only from type locality (Risch, 1986).

LIAUCHENOGLANIS Boulenger, 1916

Liauchenoglanis Boulenger, 1916: 314. Type species: Liauchenoglanis maculatus Boulenger, 1916. Type by monotypy. Gender: Masculine.

Remarks: Treated in recent literature as valid or as a synonym of Notoglanidium.

Liauchenoglanis maculatus Boulenger, 1916

Liauchenoglanis maculatus Boulenger, 1916: 314, fig. 186. Type locality: N. Sherbo dist., Sierra Leone. Syntypes: BMNH 1915.5.19.9-12 (4).

Distribution: Sierra Leone (Risch, 1986).

Remarks: Also treated in recent literature as Notoglandium maculatus [sic, N. maculatum].

LOPHIOBAGRUS Poll, 1942

Lophiobagrus Poll, 1942b: 318. Type species: Lophiobagrus lestradei Poll, 1942. Type by monotypy. Gender: Masculine.

Lophiobagrus aquilus Bailey \& Stewart, 1984

Lophiobagrus aquilus Bailey \& Stewart, 1984: 24, figs. 5b, 6b, 8. Type locality: Lake Tanganyika. Holotype: UMMZ 199928.

Distribution: Lake Tanganyika; known only from type series (Risch, 1986).

Lophiobagrus asperispinis Bailey \& Stewart, 1984

Lophiobagrus asperispinis Bailey \& Stewart, 1984: 33, figs. 6d, 9. Type locality: Lake Tanganyika. Holotype: MRAC 14359A.

Distribution: Lake Tanganyika (Risch, 1986).

Lophiobagrus brevispinis Bailey \& Stewart, 1984

Lophiobagrus brevispinis Bailey \& Stewart, 1984: 35, figs. 5c, 6c, 10. Type locality: Lake Tanganyika. Holotype: UMMZ 199930.

Distribution: Lake Tanganyika (Risch, 1986).

Lophiobagrus cyclurus (Worthington \& Ricardo, 1937)

Chrysichthys cyclurus Worthington \& Ricardo, 1937: 1093, fig. 7. Type locality: Lake Tanganyika. Lectotype: BMNH 1936.6.15.879 (49 mm SL), designated by Bailey \& Stewart (1984: 20). 
Lophiobagrus lestradei Poll, 1942b: 320, fig. on p. 321. Type locality: Nyanza, Lake Tanganyika. Holotype: MRAC 54924.

Distribution: Lake Tanganyika (Risch, 1986).

NOTOGLANIDIUM Günther, 1903

Notoglanidium Günther, 1903: 336. Type species: Notoglanidium walkeri Günther, 1903. Type by monotypy. Gender: Neuter.

Notoglanidium pallidum Roberts \& Stewart, 1976

Notoglanidium pallidum Roberts \& Stewart, 1976: 276, pl. 7 (figs. a-c). Type locality: Near Bulu, about 15 km downstream from Luozi. Lat. 501'S, Long. 1401'E; lower Zaïre or Congo River. Holotype: MCZ 50236.

Distribution: Lower Zaire (Risch, 1986).

Notoglanidium thomasi Boulenger, 1916

Notoglanidium thomasi Boulenger, 1916: 313, fig. 185. Type locality: Victoria and Pujehun [Sierra Leone]. Syntypes: BMNH 1915.5.21.10 (1), BMNH 1915.5.27.3-4 (2).

Distribution: Sierra Leone (Risch, 1986).

Notoglanidium walkeri Günther, 1903

Notoglanidium walkeri Günther, 1903: 337, pl. 32. Type locality: River Ibbi [Ghana]. Syntypes (2): BMNH 1903.4.24.78-79 (2).

Distribution: Ghana and Côte d'Ivoire (Risch, 1986).

PARAUCHENOGLANIS Boulenger, 1911

Parauchenoglanis Boulenger, 1911a: 364. Type species: Pimelodus guttatus Lönnberg, 1895. Type by subsequent designation by Jordan (1920: 538). Gender: Masculine.

Remarks: Included species follows Teugels et al. (1991); species synonymies follow Risch (1986).

Revision: Geerinckx et al. (2004), with key to species.

Parauchenoglanis ahli (Holly, 1930)

Auchenoglanis ahli Holly, 1930: 201, pl. 1 (fig. 9). Type locality: Gebirchsbache von Bakoko, Kamerun. Syntypes: NMW 13895-99 (5); syntype illustrated in Geerinckx et al. (2004: 787, fig. 8i).

Distribution: Cross River basin, western Cameroon (Geerinckx et al., 2004; known only from types).

Parauchenoglanis altipinnis (Boulenger, 1911)

Auchenoglanis altipinnis Boulenger, 1911e: 372, fig. 288. Type locality: Ja River at Esamesa, Cameroon. Holotype: BMNH 1911.5.30.30; illustrated in Geerinckx et al. (2004: 787, fig. 8d).

Distribution: Congo, Nyong, Ogowe and Chiloango River basins (Geerinckx et al., 2004: 798).

Parauchenoglanis balayi (Sauvage, 1879)

Pimelodus Balayi Sauvage, 1879: 98. Type locality: Lopé [Ogôoué River]. Holotype: MNHN a-0898. Described in more detail in Sauvage (1880c: 44).

Pimelodus Guirali Thominot, 1886: 162. Type locality: [Not stated; as Rivière San Benito in MNHN catalog]. Holotype: MNHN 1885-0427; illustrated in Geerinckx et al. (2004: 787, fig, 8a).

Auchenoglanis ballayi Boulenger, 1901: 295. Type locality: Lopé [Ogôoué River]. Holotype: MNHN a-0898. Incorrect subsequent spelling or unjustified emendation of Pimelodus balayi Sauvage, 1879.

Auchenoglanis pulcher Boulenger, 1902g: 267, pl. 29 (figs. 2-2a). Type locality: Lindi River, which flows into the Congo at the Stanley Falls. Syntypes (several): BMNH 1902.4.14.19-20 (2), IRSNB 10 (2), MSNM 35 (1).

Auchenoglanis ubangensis Boulenger, 1902d: 41, pl. 11 (fig. 3). Type locality: l'Ubangi à Banzyville. Holotype: MRAC 1215.

Auchenoglanis ballayi gravoti Pellegrin, 1907b: 321. Type locality: N'Tem, Cameroun. Syntypes: MNHN 19070158 (1), MNHN 1907-0159 (1), MNHN 1907-0160 (1). Originally proposed as Auchenoglanis ballayi var. gravoti.

Auchenoglanis pietschmanni Holly, 1926: 158. Type locality: Bamfluss, Flußgebiet des Sanaga in Kamerun. Syntypes (5): NMW 7802 (1), NMW 7803 (1), NMW 7804 (1), NMW 78025 (1), NMW 7806 (1). Illustrated and described in more detail in Holly (1927b: 208, fig. 3). 
Distribution: Rivers of South Cameroon, Gabon and Congo (Geerinckx et al., 2004: 791).

Parauchenoglanis buettikoferi (Popta, 1913)

Auchenoglanis büttikoferi Popta, 1913: 237, pl. 10. Type locality: Warri, in Upper Nigeria. Holotype: RMNH 8860; illustrated in Geerinckx et al. (2004: 787, fig. 8g).

Distribution: Upper Nigeria; known only from the holotype (Risch, 1986; Geerinckx et al., 2004).

Parauchenoglanis longiceps (Boulenger, 1913)

Auchenoglanis longiceps Boulenger, 1913: 68. Type locality: Nyong River, S. Cameroon. Holotype: BMNH 1913.10.29.12.

Auchenoglanis maculosus Holly, 1927b: 211, fig. 4. Type locality: Kamerun. Syntypes: NMW 7800 (1), NMW 7801 (1).

Distribution: Nyong River, central and southern Cameroon (Geerinckx et al., 2004: 800).

Parauchenoglanis monkei (Keilback, 1910)

Pimelodus guttatus Lönnberg, 1895: 184. Type locality: river N'Dian [Cameroon]. Syntypes: NRM (2, apparently lost), ZMUU 567 (2). One syntype illustrated in Teugels et al. (1991: 502, fig. 1). Preoccupied by Pimelodus guttatus La Cepède, 1803; replaced by Parauchenoglanis loennbergi Fowler, 1958.

Auchenoglanis monkei Keilhack, 1910: 120. Type locality: in einem Kleinen Bache im Urwald 2 km südlich von Logobaba (4²'N, 945'Ö), Kamerun. Syntypes (3): ZMB 17771 (1), ZMB 21318 (2).

Parauchenoglanis loennbergi Fowler, 1958: 14. Type locality: river N'Dian [Cameroon]. Syntypes: NRM (2, lost?), ZMUU 567 (2). Replacement name for Pimelodus guttatus Lönnberg, 1895, preoccupied by Pimelodus guttatus La Cepède, 1803.

Auchenoglanis fasciatus Gras, 1961: 404, fig. 2. Type locality: Bokoutou, Bas-Dahomey. Syntypes (2): MNHN 1960-0392 (2); one syntype illustrated in Geerinckx et al. (2004: 787, fig. 8b).

Distribution: Ouémé River, Benin, to the Sanaga River, Cameroon (Geerinckx et al., 2004 [as Parauchenoglanis guttatus]).

Parauchenoglanis ngamensis (Boulenger, 1911)

Auchenoglanis ngamensis Boulenger, 1911a: 371, fig. 287. Type locality: Okovango River. Holotype: BMNH 1910.5.31.35; illustrated in Geerinckx et al. (2004: 787, fig. 8e).

Distribution: Okavango and upper Zambezi basins, Kasai River, Congo River basin (Skelton, 1993; Geerinckx et al., 2004).

Remarks: First transferred into Parauchenoglanis by Skelton (1993).

Parauchenoglanis pantherinus (Pellegrin, 1929)

Auchenoglanis pantherinus Pellegrin, 1929b: 363. Type locality: Nyabessan (Ntem), Cameroun. Syntypes (4): MNHN 1929-0029 (1), MNHN 1929-0030 (1), MNHN 1929-0031 (2).

Distribution: Ntem River, Cameroon (Risch, 1986; Geerinckx et al., 2004: 801).

Parauchenoglanis punctatus (Boulenger, 1902)

Auchenoglanis punctatus Boulenger, 1902g: 267, pl. 29 (figs. 1-1a). Type locality: Lindi River, which flows into the Congo at the Stanley Falls. Holotype: IRSNB 9.

Auchenoglanis iturii Steindachner, 1911b: 530. Type locality: Aus dem Ituriflüss bei Mawambi. Syntypes: NMW 47474 (1), NMW 47475 (1), NMW 47476 (1); NMW 47475 illustrated in Geerinckx et al. (2004: 787, fig. 8c). Species described in more detail in Steindachner (1911e: 1185).

Auchenoglanis grandis Fowler, 1936b: 312, fig. 96. Type locality: Nola, Middle Congo. Holotype: ANSP 66125. Distribution: lower and central Congo River system; San Benito and Ogowe River basins (Geerinckx et al., 2004: 796).

PARDIGLANIS Poll, Lanza \& Romoli Sassi, 1972

Pardiglanis Poll, Lanza \& Romoli Sassi, 1972: 330. Type species: Pardiglanis tarabinii Poll, Lanza \& Romoli Sassi, 1972. Type by original designation. Gender: Masculine.

Pardiglanis tarabinii Poll, Lanza \& Romoli Sassi, 1972

Pardiglanis tarabinii Poll, Lanza \& Romoli Sassi, 1972: 338, figs. 1-7. Type locality: Gelib, fleuve Juba, Somalie méridionale. Holotype: MZUF 1579. 
Distribution: Juba River, Somalia (Risch, 1986).

Remarks: See De Vos (2001b) for comments on this species.

PHYLLONEMUS Boulenger, 1906

Phyllonemus Boulenger, 1906f: 552. Type species: Phyllonemus typus Boulenger, 1906. Type by original designation. Gender: Masculine.

Phyllonemus brichardi Risch, 1987

Phyllonemus brichardi Risch, 1987: 29, fig. 3. Type locality: Ubwari, face est, lac Tanganyika (Zaire). Holotype: MRAC 83-19-P-14.

Distribution: Lake Tanganyika (Risch, 1987).

Phyllonemus filinemus Worthington \& Ricardo, 1937

Phyllonemus filinemus Worthington \& Ricardo, 1937: 1096, fig. 9. Type locality: Kibwesi [and] Kigoma, Lake Tanganyika. Syntypes (8): BMNH 1936.6.15.925 (8).

Distribution: Lake Tanganyika (Risch, 1986).

Phyllonemus typus Boulenger, 1906

Phyllonemus typus Boulenger, 1906f: 552, pl. 32 (fig. 3). Type locality: Niamkolo [Lake Tanganyika]. Holotype: BMNH 1906.9.8.68.

Distribution: Lake Tanganyika (Risch, 1986).

PLATYGLANIS Daget, 1979

Platyglanis Daget, 1979: 821. Type species: Platyglanis depierrei Daget, 1979. Type by original designation. Gender: Masculine.

Platyglanis depierrei Daget, 1979

Platyglanis depierrei Daget, 1979: 821, figs. 1-2. Type locality: Djim (affluent du Mbam lequel se jette dans la Sanaga en aval des chutes Nachtingal) aux environs du pont franchissant la rivière sur la piste qui mène de Ngila a Ngorro [Cameroon]. Holotype: MNHN 1978-0759.

Distribution: Sanaga Basin, Cameroon; known only from the type locality (Risch, 1986).

RHEOGLANIS Poll, 1966

Rheoglanis Poll, 1966: 425. Type species: Rheoglanis dendrophorus Poll, 1966. Type by original designation. Gender: Masculine.

Rheoglanis dendrophorus Poll, 1966

Rheoglanis dendrophorus Poll, 1966: 425, unnumbered figure. Type locality: Kinsuka, rapides du fleuve. Holotype: MRAC 153744.

Distribution: Lower Congo River, in rapids (Risch, 1986).

\section{Species inquirendae, Claroteidae or Bagridae}

Mystus fluviatilis Klein 1775 [Not seen].

Leptoglanis bouilloni Poll, 1959: 97, pl. 25 (fig. 1). Type locality: Stanley Pool. Holotype: MRAC 118507. Although originally considered an amphiliid, Roberts (2003) suggested that the holotype was a claroteid or bagrid.

\section{CRANOGLANIDIDAE Myers, 1931}

Cranoglanidae Myers, 1931: 261. Type genus: Cranoglanis Peters, 1881.

Taxonomic overview: Jayaram (1956b); Ng \& Kottelat (2000d).

1 genus, 3 species; no named fossil taxa.

CRANOGLANIS Peters, 1881

Cranoglanis Peters, 1881b: 1030. Type species: Cranoglanis sinensis Peters, 1881. Type by monotypy. Gender: 
Masculine.

Anopleutropius Vaillant, 1893b: 198. Type species: Anopleutropius henrici Vaillant, 1893. Type by monotypy. Gender: Masculine.

Pseudeutropichthys Koller, 1926: 74. Type species: Pseudeutropichthys multiradiatus Koller, 1926. Type by original designation. Gender: Masculine. Also appeared in Koller (1927: 28).

Remarks: Considered to be a monotypic genus by Liu et al. (2005), with only Cranoglanis bouderius as valid.

Cranoglanis bouderius (Richardson, 1846)

Bagrus bouderius Richardson, 1846: 283. Type locality: Lian Jiang, Lianzhou, Guangdong Prov., China. Neotype: ZMB 11325, designated by Ng \& Kottelat (2000d: 851). Originally as ? Bagrus (an Pimelodus ?) bouderius. Based solely on an illustration by Reeves; which was reproduced in Jayaram (1956b: pl. 18) and Whitehead (1970: pl. 19b).

Macrones sinensis Bleeker, 1872: 153. Type locality: Lian Jiang, Guangdon Prov., Lianzhou, China. Neotype: ZMB 11325 designated by $\mathrm{Ng} \&$ Kottelat (2000d: 851). Based originally on a painting, which was reproduced in Jayaram \& Boeseman (1976: pl. 1).

Cranoglanis sinensis Peters, 1881b: 1030, [pl. 1] (fig. 1). Type locality: Lian Jiang, Lianzhou, Guangdong Prov., China. Holotype: ZMB 11325. Preoccupied in Cranoglanis by Macrones sinensis Bleeker, 1872.

Distribution: Southern China.

Cranoglanis henrici (Vaillant, 1893)

Anopleutropius henrici Vaillant, 1893b: 199. Type locality: Rivière Noir, Tonkin [Vietnam]. Syntypes: MNHN 1892-0259 (1), MNHN 1892-0260 (1).

Distribution: Red River basin, northern Vietnam and southern China (Ng \& Kottelat, 2000d).

Cranoglanis multiradiatus (Koller, 1926)

Pseudeutropichthys multiradiatus Koller, 1926: 74. Type locality: Kan-Kongfluß auf Hainan [China]. Syntypes: NMW 5059-5061 (3). Also appeared as new in Koller (1927: 29, pl. 1, fig. 2).

Distribution: Hainan Island, China.

\section{DIPLOMYSTIDAE Eigenmann, 1890}

Diplomystidae Eigenmann, 1890: 14. Type genus: Diplomystes Bleeker, 1858.

Reviews: Azpelicueta (1994a, 1994b), Argentina; Arratia (1987) Chile.

Phylogeny: Arratia (1987).

Taxonomic summary: Ferraris (2003a).

2 genera, 6 species; no named fossil taxa.

\section{DIPLOMYSTES Bleeker, 1858}

Diplomyste Duméril, 1856: 487. Type species: Arius papillosus Valenciennes, 1840. Type by monotypy. Gender: Masculine. Predates Diplomystes Bleeker, 1858, but the latter in wide use.

Diplomystes Bleeker, 1858b: 63. Type species: Arius papillosus Valenciennes, 1840. Type by monotypy. Gender: Masculine.

Diplomystax Günther, 1864: 180. Type species: Arius papillosus Valenciennes, 1840. Type by original designation. Gender: Masculine. Unneeded replacement for Diplomyste Duméril and Diplomystes Bleeker.

Diplomystes camposensis Arratia, 1987

Diplomystes camposensis Arratia, 1987: 44, fig. 19. Type locality: Lago Riñihue, Chile. Holotype: IZUA 3302.

Distribution: Valdivia region, southern Chile (Arratia, 1987).

Diplomystes chilensis (Molina, 1782)

Silurus chilensis Molina, 1782: 225. Type locality: Chile. Type(s): Whereabouts unknown.

Arius papillosus Valenciennes in Cuvier \& Valenciennes, 1840b: 118 (88 in the Strasbourg deluxe edition), pl. 431.

Type locality: Rivières de Valparaíso et de San-Jago du Chili. Syntypes: MNHN 0000-1212 (1), MNHN b-0584

(1), MNHN b-0585 (4), MNHN a-9028 (1), MNHN uncataloged (skeleton, missing). 
Arius cacharioides Leybold, 1859: 1083, pls. 1-2. Type locality: Ad thermos Colina, dictas in flumine, Río Seco (Chile). Syntypes (100): Whereabouts unknown.

? Arius micropterus Philippi, 1866: 713. Type locality: (Chile). Type(s): Whereabouts unknown.

Arius squalus Philippi, 1866: 713. Type locality: Peine, Santiago, Chile. Type(s): Whereabouts unknown.

? Arius synodon Philippi, 1866: 714. Type locality: (Chile). Type(s): Whereabouts unknown.

? Arius villosus Philippi, 1866: 712. Type locality: (Chile). Type(s): Whereabouts unknown.

Distribution: Rivers near Valparaiso and Santiago, central Chile (Arratia 1987).

Remarks: Arratia (1987) reports the species as either extinct or endangered. The imprecise type localities of many of the names listed in the synonymy make it impossible to know whether the names belong here or with another species of Diplomystes.

Diplomystes nahuelbutaensis Arratia, 1987

Diplomystes nahuelbutaensis Arratia, 1987: 33, fig. 11. Type locality: Río Cautín, Lautaro, Chile. Holotype: CAS 55423.

Distribution: Bío-Bío River basin and Loncomilla River, Chile (Arratia, 1987; Ruiz \& Berra, 1994, with biological notes).

OLIVAICHTHYS Arratia, 1987

Olivaichthys Arratia, 1987: 66. Type species: Diplomystes viedmensis MacDonagh, 1931. Type by original designation. Gender: Masculine.

Key: Azpelicueta (1994a, as Diplomystes).

Review: Azpelicueta (1994b, as Diplomystes).

Olivaichthys cuyanus (Ringuelet, 1965)

Diplomystes viedmensis cuyanus Ringuelet, 1965: 91. Type locality: Arroyo Yaucha (Vilucó, Mendoza) [Argentina]. Holotype: MLP 286.

Distribution: Colorado River and tributaries; Desaguadero-Salado River basin, including the rivers San Juan, Mendoza, Tunuyán and temporary environments connected to those rivers, Argentina (Azelpelicueta, 1994b).

Olivaichthys mesembrinus (Ringuelet, 1982)

Diplomystes viedmensis mesembrinus Ringuelet, 1982: 349, figured on p. 350. Type locality: Desembocadura del Río Senguer en el lado Musters (4560'S, 49¹0'W) [Chubut, Argentina]. Holotype: ILPLA 8452.

Distribution: Chubut and Senguer River basins, Argentina (Azpelicueta, 1994b).

Remarks: Redescribed in Azpelicueta \& Gosztonyi (1998) as Diplomystes mesembrinus.

Olivaichthys viedmensis (MacDonagh, 1931)

Diplomystes viedmensis MacDonagh, 1931: 65. Type locality: río Negro, Argentina, frente a Viedma. Holotype: MLP 214.

Distribution: South America: Negro River and tributaries in Argentina (Azpelicueta, 1994b).

\section{DORADIDAE Bleeker, 1858}

Doradini Bleeker, 1858b: 48, 52. Type genus: Doras La Cepède, 1803.

Remarks: The family-group names Centrochirinae, Hemidoradinae, and Lithodoradinae were proposed by Fowler (1951b: 3) in a manner that made the names available when published, but the names were rendered unavailable in 1961 by Art. 13 of the Second Edition of the Code (ICZN, 1961). The names apparently remain unavailable, despite Art. 13.2.1 of the current version of the Code (ICZN, 1999), because they were treated as unavailable in Ferraris \& de Pinna (1999: 8).

Revision: Eigenmann (1925).

Review: Fernández-Yépez (1968, Venezuela).

Phylogeny: Higuchi (1992), Moyer et al. (2004).

Taxonomic summary: Sabaj (2002); Sabaj \& Ferraris (2003).

30 genera, 77 species; no named fossil taxa. 


\section{Incertae sedis}

Doras fimbriatus Kner, 1855

Doras loricatus Kner, 1853c: 146, fig. 2 on unnumbered plate. Type locality: [Not stated]. Holotype: NMW 4540709 ( 1 of 4). Name made available from caption to illustration of a swimbladder; associated specimen is the holotype, if recognizable. Illustrated and described in more detail, as Doras fimbriatus, in Kner (1855: 134, pl. 3, fig. 5) with locality given as Rio Guaporé. Considered a nomen oblitum in Sabaj \& Ferraris (2003).

Doras fimbriatus Kner, 1855a: 134, pl. 3 (fig. 5). Type locality: Rio Guaporé. Syntypes: NMW 45407-09 (4).

Distribution: Guaporé River basin, Bolivia and Brazil (Sabaj \& Ferraris, 2003).

Remarks: Generic placement of this species unresolved (Sabaj \& Ferraris, 2003).

Doras punctatus Kner, 1853

Doras (Corydoras) punctatus Kner, 1853c: 146, fig. 5 on unnumbered plate. Type locality: [Not stated]. Holotype: NMW 45434-41 (1 of 14) or RMNH 2966 (1). Name made available from caption to illustration of a swimbladder; associated specimen is the holotype, if recognizable. Illustrated and described in more detail in Kner (1855: 136, pl. 6, fig. 10), with locality given as Mato-grosso und Rio Guaporé.

Distribution: Amazon River basin (Sabaj \& Ferraris, 2003).

Remarks: Generic placement of this species unresolved (Sabaj \& Ferraris, 2003).

Oxydoras eigenmanni Boulenger, 1895

Oxydoras eigenmanni Boulenger, 1895b: 524. Type locality: Descalvados, Matto Grosso. Syntypes: BMNH 1895.5.17.50-52 (3), BMNH 1895.5.17.53 (1); one syntype illustrated in Boulenger (1896c: pl. 4, fig. 3).

Distribution: Amazon and upper Paraguay River basins (Sabaj \& Ferraris, 2003).

Remarks: Generic placement of this species unresolved (Sabaj \& Ferraris, 2003).

ACANTHODORAS Bleeker, 1862

Cataphractus Edwards, in Catesby, 1771: appendix. Type species: Silurus cataphractus Linnaeus, 1758. Type by absolute tautonymy. Gender: Masculine. Considered a nomen oblitum (Sabaj \& Ferraris, 2003).

Acanthodoras Bleeker, 1862 (in Bleeker, 1862-63): 5. Type species: Silurus cataphractus Linnaeus, 1758. Type by original designation. Gender: Masculine.

Acanthodoras cataphractus (Linnaeus, 1758)

Silurus cataphractus Linnaeus, 1758: 307. Type locality: America. Syntype: BMNH 1853.11.12.193 (1). BMNH syntype illustrated in Gronovius (1754: pl. 3, figs. 3 \& 4); another syntype illustrated in Catesby (1731-1743: 19, pl. 9).

Cataphractus Americanus Bloch \& Schneider, 1801: 107, pl. 28. Type locality: America, pedalis. Syntype: BMNH 1853.11.12.193 (1). BMNH syntype figured in Gronovius (1754: pl. 3, figs. 3 \& 4). Unneeded new name for Silurus cataphractus Linnaeus.

Cataphractus americanus La Cepède, 1803: 124, 126. Type locality: dans la Caroline. Syntypes: On several literature accounts, including Catesby (1731-1743), Gronovius (1754), Bonnaterre (1788), and Gmelin (1789); only extant syntype: BMNH 1853.11.12.193 (1) illustrated in Gronovius (1754: pl. 3, figs. 3 \& 4); another syntype illustrated in Catesby (1731-1743: 19, pl. 9). Name apparently proposed independently of Cataphractus americanus Bloch \& Schneider. Type locality reported by La Cepède was based on account in Catesby.

Doras Blochii Valenciennes, in Cuvier \& Valenciennes, 1840b: 277 (207 of Strasbourg deluxe edition). Type locality: [Not stated]. Syntype: BMNH 1853.11.12.193 (1) and literature sources. BMNH syntype figured in Gronovius (1754: pl. 3, figs. 3 \& 4).

Callichthys asper Gronow, in Gray, 1854: 157. Type locality: Americes Meridionalis rivulis. Syntype: BMNH 1853.11.12.193 (1). BMNH syntype figured in Gronovius (1754: pl. 3, figs. 3 \& 4).

Distribution: Amazon River basin and coastal drainages of French Guiana, Guyana and Suriname (Sabaj \& Ferraris, 2003).

Acanthodoras depressus (Steindachner, 1881)

Doras (Rhinodoras) depressus Steindachner, 1881c: 103, pl. 1 (fig. 3). Type locality: Ausstände (Lago Alexo) am 
mittleren Laufe des Amazonen-Stromes. Holotype: NMW 46870.

Distribution: Amazon and Negro River basins, Brazil (Sabaj \& Ferraris, 2003).

Acanthodoras spinosissimus (Eigenmann \& Eigenmann, 1888)

Doras brunnescens Jardine, in Schomburgk, 1841: 163. Type locality: Upper Essequibo [Guyana]. No types known.

Doras spinosissimus Eigenmann \& Eigenmann, 1888b: 161. Type locality: Coary [Brazil]. Holotype: MCZ 7222.

Distribution: Amazon and Essequibo River basins (Sabaj \& Ferraris, 2003).

Remarks: Acanthodoras spinosissimus treated as valid, following Sabaj \& Ferraris (2003), in which Doras brunnescens is treated as a nomen oblitum.

\section{Species inquirendae, Acanthodoras}

Doras castaneo-ventris Jardine, in Schomburgk, 1841: 161, pl. 3. Type locality: river Pasawiri. No types known.

Doras polygramma Kner, 1853c: 145, fig. 1 on unnumbered plate. Type locality: [Not stated]. Holotype: NMW 46846

(99 mm SL, 1 of 2). Name made available from caption to illustration of a swimbladder; associated specimen is the holotype, if recognizable. Treated in Kner (1855: 126-127) as Doras cataphractus, based on specimens from "Rio Guaporé and Barra do Rio negro."

Remarks: Catalog number for type of Doras polygramma incorrectly reported as NMW 46844 by Sabaj and Ferraris (2003).

\section{AGAMYXIS Cope, 1878}

Agamyxis Cope, 1878: 679. Type species: Doras pectinifrons Cope, 1870. Type by monotypy. Gender: Feminine.

Agamyxis albomaculatus (Peters, 1877)

Doras albomaculatus Peters, 1877: 470. Type locality: Calabozo [Venezuela]. Syntypes (2): ZMB 10043 (1).

Distribution: Orinoco River basin, Venezuela (Sabaj \& Ferraris, 2003).

Agamyxis pectinifrons (Cope, 1870)

Doras pectinifrons Cope, 1870b: 568. Type locality: Pebas, Ecuador [now Peru]. Holotype: ANSP 8346.

Doras (Agamyxis) flavopictus Steindachner, 1908c: 84. Type locality: Iquitos [Amazonasgebiet]. Syntypes: NMW 46598-99 (2).

Distribution: Amazon River basin (Sabaj \& Ferraris, 2003).

\section{AMBLYDORAS Bleeker, 1862}

Amblydoras Bleeker, 1862 (in Bleeker, 1862-63): 5. Type species: Doras affinis Kner, 1855. Type by original designation. Gender: Masculine.

Zathorax Cope, 1871b: 112. Type species: Zathorax monitor Cope, 1872. Type by subsequent monotypy. Gender: Masculine. Appeared first with no included species, subsequently described with one species (Cope, 1872a: 271).

Amblydoras affinis (Kner, 1855)

Doras affinis Kner, 1855a: 121, pl. 2 (fig. 1). Type locality: Rio branco und Guaporé. Syntypes (11): NMW 4682223 (4), NMW 46826 (3), RMNH 2973 (1).

Amblydoras truncatus Bleeker, 1863b: 18. Type locality: flumine Guapore. Holotype: RMNH 2973. Holotype is also a syntype of Doras affinis Kner.

Distribution: Guaporé, Branco and Essequibo River basins (Sabaj \& Ferraris, 2003).

Amblydoras bolivarensis (Fernández-Yépez, 1968)

Hildadoras bolivarensis Fernández-Yépez, 1968: 43, fig. 18. Type locality: Qda. El Pilón, al este del Río Parguaza, afluente (margen derecho) del Río Orinoco, frente a Pararuma [Estado Bolívar, Venezuela]. Holotype: FMNH 84067.

Distribution: Orinoco River basin, Venezuela (Sabaj \& Ferraris, 2003).

Amblydoras gonzalezi (Fernández-Yépez, 1968)

Zathorax gonzalezi Fernández-Yépez, 1968: 70, fig. 36. Type locality: Laguna "Punta Vista," a orillas del Río Caroní (margen izquierda) en el Parque Cachamay [Venezuela]. Holotype: MBUCV V-3940. 
Distribution: Orinoco River basin and Casiquiare Canal (Sabaj \& Ferraris, 2003).

Amblydoras monitor (Cope, 1872)

Zathorax monitor Cope, 1872a: 272, pl. 4 (figs. 1a-1c). Type locality: small streams tributary to the Ambyiacu, as well as ... the river itself ... which empties into the Amazon near Pebas, in Eastern Ecuador, some distance east of the Napo. Lectotype: ANSP 8296, designated by and illustrated in Eigenmann (1925: 363, pl. 16, fig. 3).

Distribution: Upper Amazon River basin (Sabaj \& Ferraris, 2003).

Amblydoras nauticus (Cope, 1874)

Zathorax nauticus Cope, 1874b: 133. Type locality: Nauta [Upper Amazon]. Syntypes: ANSP 21390-95 (6).

Distribution: Upper Amazon River basin, Peru (Sabaj \& Ferraris, 2003).

Remarks: Syntype series contains two species; with four specimens of Amblydoras monitor Cope and two representing the species indicated here (Sabaj \& Ferraris, 2003). A lectotype designation is necessary to formalize the validity of this name.

ANADORAS Eigenmann, 1925

Anadoras Eigenmann, 1925: 327. Type species: Doras grypus Cope, 1872. Type by original designation. Gender: Masculine.

Anadoras grypus (Cope, 1872)

Doras grypus Cope, 1872a: 270, pl. 15 (figs. 1, 1a). Type locality: small streams tributary to the Ambyiacu, as well as ... the river itself ... which empties into the Amazon near Pebas, in Eastern Ecuador, some distance east of the Napo. Syntypes: ANSP 8345 (1), ANSP 16460 (1).

Distribution: Upper Amazon River basin (Sabaj \& Ferraris, 2003).

Anadoras regani (Steindachner, 1908)

Doras regani Steindachner, 1908f: 163. Type locality: Fischmerke von Pará [Amazonasgebiete innerhalb Brasiliens]. Syntypes: NMW 46889 (4).

Distribution: Amazon and Oyapock River basins (Sabaj \& Ferraris, 2003).

Anadoras weddellii (Castelnau, 1855)

Doras weddellii Castelnau, 1855: 48, pl. 17 (fig. 1). Type locality: petites flaques d'eau de la province des Chiquitos. Holotype: MNHN 0000-4155.

Distribution: Upper Mamoré, Paraguay and Pilcomayo River basins (Sabaj \& Ferraris, 2003).

ANDUZEDORAS Fernández-Yépez, 1968

Anduzedoras Fernández-Yépez, 1968: 28. Type species: Anduzedoras arleoi Fernández-Yépez, 1968. Type by original designation. Gender: Masculine.

Anduzedoras oxyrhynchus (Valenciennes, 1821)

Doras Oxyrhynchus Valenciennes, in Humboldt \& Valenciennes, 1821: 184. Type locality: Probablement ... originaires d'Amérique. Holotype: MNHN 0000-4190.

Doras (Corydoras) ophthalmus Kner, 1853c: 146, fig. 3 on unnumbered plate. Type locality: [Not stated]. Holotype: NMW 45418-20 (1 of 4). Name made available from caption to illustration of a swimbladder; associated specimen is the holotype, if recognizable. Illustrated and described in more detail, as Doras (Oxydoras) lipophthalmus, in Kner (1855: 147, pl. 5, fig. 8), with locality given as: Rio negro.

Doras (Oxydoras) lipophthalmus Kner, 1855a: 147, pl. 5 (fig. 8). Type locality: Rio negro [Brazil]. Syntypes: NMW 45418-20 (4).

Opsodoras steindachneri Eigenmann, 1925: 354. Type locality: Mouth of Rio Negro [Brazil]. Holotype: NMW 46379.

Anduzedoras arleoi Fernández-Yépez, 1968: 29, fig. 9. Type locality: Río Autana, al este de Puerto Ayacucho, Terr. Fed. Amazonas [Venezuela]. Holotype: FMNH 84068.

Distribution: Negro and upper Orinoco River basins, Brazil and Venezuela (Sabaj \& Ferraris, 2003).

ASTRODORAS Bleeker, 1862

Astrodoras Bleeker, 1862 (in Bleeker, 1862-63): 5. Type species: Doras asterifrons Kner, 1853. Type by original 
designation. Gender: Masculine.

Astrodoras asterifrons (Kner, 1853)

Doras asterifrons Kner, 1853c: 146, fig. 4 on unnumbered plate. Type locality: not stated. Holotype: NMW 46412 (1 of 2), NMW 46611 (1 of 6), NMW 58211 (1 of 3) or RMNH 2972 (1). Name made available from caption to illustration of a swimbladder; associated specimen is the holotype, if recognizable. Species later illustrated described in more detail in Kner (1855: 123, pl. 2, fig. 2), with the locality given as: Barra do Rio negro und R. Guaporé.

Distribution: Amazon River basin (Sabaj \& Ferraris, 2003).

\section{CENTROCHIR Spix \& Agassiz, 1829}

Centrochir Spix \& Agassiz, 1829: 14. Type species: Doras crocodili Humboldt, 1821. Type by monotypy. Gender: Feminine.

Centrochir crocodili (Humboldt, 1821)

Doras Crocodili Humboldt, in Humboldt \& Valenciennes, 1821: 181, pl. 48 (fig. 2). Type locality: Rio Magdalena...Nouvelle-Grenade...entre Pinto et Mompox, par les $9^{\circ}$ et $9^{\circ} 1 / 2$ de latitude...[\&] près du confluent du Rio Cauca [Colombia]. No types known.

Doras longispinis Steindachner, 1878a: 89. Type locality: Magdalenen-Stromes [Colombia]. Syntypes: NMW 46624

(4). Species illustrated and described in more detail in Steindachner (1879d: 39, pl. 4, fig. 2, and pl. 5, fig. 1).

Distribution: Magdalena River basin, Colombia (Sabaj \& Ferraris, 2003).

Remarks: Redescribed in Schmidt (1928).

\section{CENTRODORAS Eigenmann, 1925}

Centrodoras Eigenmann, 1925: 309. Type species: Doras brachiatus Cope, 1872. Type by original designation. Gender: Masculine.

Centrodoras brachiatus (Cope, 1872)

Doras brachiatus Cope, 1872a: 270. Type locality: Between the mouth of the Rio Negro and the Huallaga, in the Marañon. Holotype: ANSP 8342. Appeared first as name only in Cope (1871a: 55).

Rhinodoras amazonum Steindachner, 1875: 141, pl. 2. Type locality: Amazonenstrom bei Teffé [brasilianische]. Holotype: at NMW, but not found in 2006 (M. Sabaj, pers. commun.).

Distribution: Amazon River basin, except Negro River (Sabaj \& Ferraris, 2003).

Centrodoras hasemani (Steindachner, 1915)

Oxydoras (Rhinodoras) amazonum hasemani Steindachner, 1915e: 68. Type locality: Mündung des Rio Negro. Holotype: at NMW, but not found in 2006 (M. Sabaj, pers. comm.). Originally as Oxydoras (Rhinodoras) amazonum var. hasemani.

Distribution: Negro River basin, Brazil (Sabaj \& Ferraris, 2003).

DORAOPS Schultz, 1944

Doraops Schultz, 1944c: 270. Type species: Doraops zuloagai Schultz, 1944. Type by original designation. Gender: Masculine.

Doraops zuloagai Schultz, 1944

Doraops zuloagai Schultz, 1944c: 271, pl. 7 (fig. a). Type locality: Río de Los Pajaros, 3 km above Lago Maracaibo. Holotype: USNM 121015.

Distribution: Lake Maracaibo basin including lake itself and Apon, Santa Ana, Catatumbo, Escalante and de Los Pajaros River basins, Colombia (Sabaj \& Ferraris, 2003).

DORAS La Cepède, 1803

Doras La Cepède, 1803: 116. Type species: Silurus carinatus Linnaeus, 1766. Type by subsequent designation by Bleeker (1862-63: 5). Gender: Masculine.

Mormyrostoma Miranda Ribeiro, 1911: 192. Type species: Silurus carinatus Linnaeus, 1766. Type by original designation. Gender: Neuter. 
Doras carinatus (Linnaeus, 1766)

Silurus carinatus Linnaeus, 1766: 504. Type locality: Surinami. Type(s): Whereabouts unknown.

Distribution: Essequibo River basin and other coastal drainages east to mouth of Amazon River, possibly in lower Amazon and lower Orinoco River basins (Sabaj \& Ferraris, 2003).

Doras micropoeus (Eigenmann, 1912)

Hemidoras micropoeus Eigenmann, 1912b: 195. Type locality: Wismar, British Guiana. Holotype: CM 1636 (whereabouts unknown).

Distribution: Essequibo, Demerara and Corantijn River basins, possibly other coastal drainages of northern South America above mouth of Amazon River (Sabaj \& Ferraris, 2003).

FRANCISCODORAS Eigenmann, 1925

Franciscodoras Eigenmann, 1925: 317. Type species: Doras marmoratus Reinhardt, 1874. Type by original designation. Gender: Masculine.

Franciscodoras marmoratus (Reinhardt, 1874)

Doras marmoratus Reinhardt, in Lütken, 1874c: 30. Type locality: in flumine Rio das Velhas [in provincia Minasgeraës circa oppidulum Lagoa Santa, Brasiliae centralis]. Holotype: ZMUC P 29669. Illustrated and described in Lütken (1875: 146 (and p. III of summary), pl. 1, fig. 1).

Distribution: São Francisco River basin, Brazil (Sabaj \& Ferraris, 2003).

HASSAR Eigenmann \& Eigenmann, 1888

Hassar Eigenmann \& Eigenmann, 1888b: 158. Type species: Oxydoras orestes [= Oxydoras orestis] Steindachner, 1875. Type by subsequent designation by Eigenmann (1910: 394). Gender: Masculine. Originally proposed as a subgenus of Hemidoras.

Hassar affinis (Steindachner, 1881)

Oxydoras affinis Steindachner, 1881c: 107, pl. 1 (fig. 1). Type locality: Rio Puty [Brazil]. Holotype: NMW 45394. Originally as: Oxydoras affinis ? (an Oxyd. Orestes var.?).

Hassar woodi Fowler, 1941a: 139, figs. 35-37. Type locality: Rio Parnahyba, Therezina, Piauhy [Brazil]. Holotype: ANSP 69392.

Hassar iheringi Fowler, 1941a: 140, figs. 38-40. Type locality: Rio Parnahyba, Therezina, Piauhy [Brazil]. Holotype: ANSP 69393.

Distribution: Parnaíba River basin, Brazil (Sabaj \& Ferraris, 2003).

Hassar orestis (Steindachner, 1875)

Oxydoras Orestis Steindachner, 1875: 138, pl. 1. Type locality: Rio Xingu (bei den Wasserfällen) und Rio Iça [brasilianische]. Syntypes: NMW 45427-30 (7), NMW 78651 (1). Steindachner consistently used Oxydoras orestis in 1875 , but later changed the spelling to Oxydoras orestes, which had been widely adopted.

Hemidoras notospilus Eigenmann, 1912b: 196, pl. 19 (fig. 2). Type locality: Crab Falls, British Guiana. Holotype: FMNH 53184 (missing).

? Hassar ucayalensis Fowler, 1940a: 228, figs. 15-17. Type locality: Ucayali River basin, Contamana, Peru. Holotype: ANSP 68647.

Distribution: Amazon, Orinoco and Essequibo River basins (Sabaj \& Ferraris, 2003).

Remarks: Inclusion of Hassar ucayalensis Fowler and, therefore, the distribution of the species into Peru must be considered tentative (Sabaj \& Ferraris, 2003).

Hassar wilderi Kindle, 1895

Hassar wilderi Kindle, 1895: 251. Type locality: Trocera, on Tocantins, Brazil. Lectotype: CAS 60711; designated by, and illustrated in, Eigenmann (1925: 364, pl. 22, fig. 2); subsequent lectotype designation in Myers \& Weitzman (1956: 4) not valid.

Distribution: Tocantins River basin, Brazil (Sabaj \& Ferraris, 2003).

HEMIDORAS Bleeker, 1858

Hemidoras Bleeker, 1858b: 53, 54. Type species: Doras (Oxydoras) stenopeltis Kner, 1855. Type by monotypy. 
Gender: Masculine.

Hemidoras morrisi Eigenmann, 1925

Hemidoras morrisi Eigenmann, 1925: 347, pl. 27 (fig. 3). Type locality: Iquitos. Holotype: CAS 52130.

Distribution: Upper Amazon River basin (Sabaj \& Ferraris, 2003).

Remarks: Status uncertain, possibly a junior synonym of Opsodoras boulengeri (Steindachner) (Sabaj \& Ferraris, 2003).

Hemidoras stenopeltis (Kner, 1855)

Doras (Oxydoras) stenopeltis Kner, 1855a: 142, pl. 4 (fig. 7). Type locality: Rio negro. Syntypes: NMW 45443 (2).

Distribution: Amazon River basin (Sabaj \& Ferraris, 2003).

HYPODORAS Eigenmann, 1925

Hypodoras Eigenmann, 1925: 329. Type species: Hypodoras forficulatus Eigenmann, 1925. Type by original designation. Gender: Masculine.

Hypodoras forficulatus Eigenmann, 1925

Hypodoras forficulatus Eigenmann, 1925: 330, pl. 25 (fig. 3). Type locality: Iquitos. Holotype: CAS 37246.

Distribution: Upper Amazon River basin, Peru (Sabaj \& Ferraris, 2003).

KALYPTODORAS Higuchi, Britski \& Garavello, 1990

Kalyptodoras Higuchi, Britski \& Garavello, 1990: 220. Type species: Kalyptodoras bahiensis Higuchi, Britski \& Garavello, 1990. Type by original designation. Gender: Masculine.

Kalyptodoras bahiensis Higuchi, Britski \& Garavello, 1990

Kalyptodoras bahiensis Higuchi, Britski \& Garavello, 1990: 222, fig. 1. Type locality: Brazil, Bahia State, Rio Paraguaçu, lower course, downstream from Bananeiras dam, portion just before construction site of Pedra do Cavalo dam (12²'05"S, 38²9'30"W to $12^{\circ} 35^{\prime} 50^{\prime}$ 'S, 3905'15"W). Holotype: MZUSP 38565.

Distribution: Paraguaçu River in Bahia State, Brazil (Sabaj \& Ferraris, 2003).

\section{LEPTODORAS Boulenger, 1898}

Leptodoras Boulenger, 1898b: 477. Type species: Oxydoras acipenserinus Günther, 1868. Type by subsequent designation by Eigenmann (1910: 395). Gender: Masculine.

Taxonomy: Böhlke (1970).

Revision, with key to species: Sabaj (2005).

Leptodoras acipenserinus (Günther, 1868)

Oxydoras acipenserinus Günther, 1868a: 475. Type locality: Xeberos [Upper Amazons]. Holotype: BMNH 1867.6.13.32. Species illustrated and described in more detail in Günther (1868b: 230, pl. 20).

Distribution: Upper Amazon River basin, Brazil, Ecuador, and Peru (Sabaj, 2005).

Leptodoras cataniai Sabaj, 2005

Leptodoras cataniai Sabaj, 2005: 667, fig. 22. Type locality: Venezuela, Amazonas, río Casiquiare from mouth of río Pamoni to $4 \mathrm{~km}$ below mouth, west of Pamoni ( $\left.2^{\circ} 48^{\prime} \mathrm{N}, 65^{\circ} 57^{\prime} \mathrm{W}\right)$. Holotype: ANSP 180918.

Distribution: Rio Negro and Casiquiare Canal in Brazil and Venezuela (Sabaj, 2005).

Leptodoras copei (Fernández-Yépez, 1968)

Anduzedoras copei Fernández-Yépez, 1968: 31, fig. 10. Type locality: una laguna al lado del Río Capanaparo [Venezuela]. Holotype: FMNH 84069.

Distribution: upper Orinoco, Negro, and Amazon River basins, Brazil, Peru and Venezuela (Sabaj, 2005).

Leptodoras hasemani (Steindachner, 1915)

Hemidoras hasemani Steindachner, 1915b: 218. Type locality: Amazonas, Südamerikas. Lectotype: NMW 46381 (82 mm SL, largest of 4 specimens); lectotype designated by Sabaj (2005: 654). Species illustrated and described in more detail in Steindachner (1915e: 75, pl. 10, figs. 4-7), with locality as: des Rio branco bei Boa Vista und Serra grande und an der Mündung des Rio negro.

Distribution: Branco, Orinoco and Essequibo River basins (Sabaj, 2005).

Leptodoras juruensis Boulenger, 1898 
Leptodoras juruensis Boulenger, 1898b: 478. Type locality: Jurua River, Brazil. Holotype: BMNH 1898.10.11.25.

Distribution: Main channel of Amazon River basin (Sabaj, 2005).

Leptodoras linnelli Eigenmann, 1912

Leptodoras linnelli Eigenmann, 1912b: 191, pl. 17, fig. 1; pl. 18, fig. 1. Type locality: Tumatumari [lower Potaro River], British Guiana. Holotype: FMNH 53561.

Distribution: Upper Orinoco (Mavaca River), Essequibo and Demerara River basins and northern (left bank) tributaries to lower Amazon (Sabaj, 2005).

Leptodoras myersi Böhlke, 1970

Leptodoras myersi Böhlke, 1970: 54, fig. 1. Type locality: Peru, vicinity of Iquitos, Rio Amazonas (Marañon) between Isla Iquitos and Isla Lapuna, near Isla Lapuna shore. Holotype: ANSP 112318.

Distribution: Upper Amazon River basin, near Iquitos, Peru (Sabaj, 2005).

Leptodoras nelsoni Sabaj, 2005

Leptodoras nelsoni Sabaj, 2005: 663, fig. 20. Type locality: Colombia, Meta, río Metica (Meta-Orinoco dr.), ca. 1.5 km east of Rajote, Plancha 267 (356' N, 73³'W). Holotype: ANSP 180917.

Distribution: middle Orinoco River basin, Colombia and Venezuela (Sabaj, 2005).

Leptodoras praelongus (Myers \& Weitzman, 1956)

Hassar praelongus Myers \& Weitzman, 1956: 2, fig. 1. Type locality: São Gabriel Rapids of the Rio Negro, Amazonas, Brazil. Holotype: SU 48679.

Distribution: Negro and Orinoco River basins, Brazil and Venezuela (Sabaj, 2005).

Leptodoras rogersae Sabaj, 2005

Leptodoras rogersae Sabaj, 2005: 665, fig. 21. Type locality: Venezuela, Bolivar, río Orinoco (Atlantic Dr.), river channel at Palua just below mouth of río Caroni, 181 nautical miles upstream from sea buoy $\left(8^{\circ} 21^{\prime} \mathrm{N}, 62^{\circ} 43^{\prime} \mathrm{W}\right)$. Holotype: USNM 226307.

Distribution: Lower to middle Orinoco River, primarily in main channel (Sabaj, 2005).

\section{LITHODORAS Bleeker, 1862}

Lithodoras Bleeker, 1862 (in Bleeker, 1862-63): 5. Type species: Doras lithogaster Kner, 1855. Type by original designation. Gender: Masculine.

Lithodoras dorsalis (Valenciennes, 1840)

Doras dorsalis Valenciennes, in Cuvier \& Valenciennes, 1840b: 284 (211 in Strasbourg deluxe edition). Type locality: [Not stated]. Holotype: MNHN 0000-4156. On Doras carinatus of Valenciennes (in Humboldt \& Valenciennes, 1821: p. 184).

Doras papilionatus De Filippi, 1853: 167. Type locality: in flumine Amazonum. Holotype: MZUT 229.

Doras lithogaster Kner, 1855a: 132. Type locality: Forte do Rio branco. Syntypes (2): at NMW, but not found in 2006 (M. Sabaj, pers. commun.).

Megalodoras paucisquamatus Van der Stigchel, 1946: 71, fig. 1. Type locality: Brazil. Holotype: RMNH 15480.

Distribution: Amazon River basin and estuary near Cayenne, French Guiana (Keith \& Merona, 2000; Sabaj \& Ferraris, 2003).

MEGALODORAS Eigenmann, 1925

Megalodoras Eigenmann, 1925: 306. Type species: Megalodoras irwini Eigenmann, 1925. Type by original designation. Gender: Masculine.

Hoplodoras Eigenmann, 1925: 310. Type species: Doras uranoscopus Eigenmann \& Eigenmann, 1888. Type by original designation. Gender: Masculine. Spelled Haplodoras on p. 311, Hoplodaros on fig. 12c, and Hoplodoras in key and main account. First reviser apparently Eschmeyer \& Bailey, in Eschmeyer (1990).

Deltadoras Fernández-Yépez, 1968: 36. Type species: Deltadoras guayoensis Fernández-Yépez, 1968. Type by original designation. Gender: Masculine.

Remarks: Sabaj \& Ferraris (2003: 462) served as first reviser in selecting Megalodoras as valid over Hoplodoras.

Megalodoras guayoensis (Fernández-Yépez, 1968)

Deltadoras guayoensis Fernández-Yépez, 1968: 37, fig. 14. Type locality: Caño de Guayo, Territorio Delta Ama- 
curo, Venezuela. Holotype: MBUCV V-15211.

Hoplodoras ramirezi Fernández-Yépez, 1968: 47, fig. 19. Type locality: bajo Orinoco [Venezuela]. Holotype: AFY 66117 (missing).

Distribution: Orinoco River basin, Venezuela (Sabaj \& Ferraris, 2003).

Remarks: Sabaj \& Ferraris (2003: 462) served as first reviser in selecting Deltadoras guayoensis as the valid name for this species.

Megalodoras uranoscopus (Eigenmann \& Eigenmann, 1888)

Doras uranoscopus Eigenmann \& Eigenmann, 1888b: 159. Type locality: Lake Hyanuary [Brazil]. Holotype: MCZ 7235.

? Oxydoras (Rhinodoras) huberi Steindachner, 1911a: 324. Type locality: Rio Tocantins, bei Cametá [Amazonasgebiete]. Holotype: NMW 12615.

Doras libertatis Miranda Ribeiro, 1912: 20. Type locality: Manáos [Brazil]. Holotype (112 mm): MNRJ 718 (missing). Holotype illustrated in Eigenmann (1925: pl. 7).

Megalodoras irwini Eigenmann, 1925: 308, pl. 25 (fig. 2). Type locality: Iquitos. Holotype: CAS 80243. Species account appears twice in paper; the first (p. 307) based on a single specimen (CAS 20735) that was apparently intended as the holotype of another new species description, which was changed before publication, except for word "holotype"in the species account, which was first treated as lapsus calami by Sabaj \& Ferraris (2003).

Distribution: Amazon, Tocantins and Essequibo River basins (Sabaj \& Ferraris, 2003).

NEMADORAS Eigenmann, 1925

Nemadoras Eigenmann, 1925: 359. Type species: Oxydoras elongatus Boulenger, 1898. Type by original designation. Gender: Masculine.

Nemadoras elongatus (Boulenger, 1898)

Oxydoras elongatus Boulenger, 1898e: 424, pl. 40 (fig. 4). Type locality: Rio Jurua, an affluent of the Amazons, Brazil. Holotype: BMNH 1897.12.1.47.

Opsodoras parallelus Eigenmann, 1925: 350, pl. 19 (fig. 3). Type locality: Iquitos. Holotype: CAS 60229.

Distribution: Amazon River basin (Sabaj \& Ferraris, 2003).

Remarks: Holotype of Oxydoras elongatus Boulenger was apparently registered twice, with the second registration number, BMNH 1971.4.13.1, appearing in several recent publications.

Nemadoras hemipeltis (Eigenmann, 1925)

Opsodoras hemipeltis Eigenmann, 1925: 349, pl. 19 (fig. 2). Type locality: Rio Ucayali at Contamana. Holotype: CAS 60236.

Distribution: Amazon River basin (Sabaj \& Ferraris, 2003).

Nemadoras humeralis (Kner, 1855)

Doras humeralis Kner, 1855a: 140, pl. 4 (fig. 6). Type locality: Barra do Rio negro. Syntypes: NMW 45410 (2).

Oxydoras bachi Boulenger, 1898e: 423, pl. 40 (fig. 3). Type locality: Rio Jurua, an affluent of the Amazons, Brazil. Holotype: BMNH 1897.12.1.46.

Distribution: Amazon River basin (Sabaj \& Ferraris, 2003).

Nemadoras leporhinus (Eigenmann, 1912)

Hemidoras leporhinus Eigenmann, 1912b: 195, pl. 19 (fig. 1). Type locality: Tumatumari [lower Potaro River], British Guiana. Holotype: FMNH 53185.

Distribution: Orinoco, Branco and Essequibo River basins (Sabaj \& Ferraris, 2003).

Nemadoras trimaculatus (Boulenger, 1898)

Oxydoras trimaculatus Boulenger, 1898e: 422, pl. 40 (fig. 1). Type locality: Rio Jurua, an affluent of the Amazons, Brazil. Syntypes: BMNH 1897.12.1.41-43 (3).

Leptodoras trimaculatus Fowler, 1914: 264, fig. 14. Type locality: Rupununi River, British Guiana ... in the highlands of British Guiana, approximately secured in North Latitude $2^{\circ}$ to $3^{\circ}$, and West Longitude $50^{\circ} 20^{\prime}$. Holotype: ANSP 39342. Originally considered by Fowler (1958) to have been preoccupied in Opsodoras by Opsodoras trimaculatus (Boulenger, 1898); and replaced by Opsodoras ogilviei Fowler, 1958. More recently, considered to be preoccupied in Nemadoras (Sabaj \& Ferraris, 2003). 
Opsodoras ogilviei Fowler, 1958: 13. Type locality: Rupununi River, British Guiana. Holotype: ANSP 39342. Replacement for Leptodoras trimaculatus Fowler, 1914; then preoccupied in Opsodoras by Opsodoras trimaculatus (Boulenger, 1898).

Distribution: Amazon, Orinoco and Essequibo River basins (Sabaj \& Ferraris, 2003).

\section{OPSODORAS Eigenmann, 1925}

Opsodoras Eigenmann, 1925: 348. Type species: Opsodoras orthacanthus Eigenmann, 1925. Type by original designation. Gender: Masculine.

Opsodoras boulengeri (Steindachner, 1915)

Hemidoras boulengeri Steindachner, 1915b: 218. Type locality: Mündung des Rio negro. Holotype: at NMW, but not found in 2006 (M. Sabaj, pers. comm.). Species illustrated and described in more detail, as Hemidoras (Leptodoras) boulengeri, in Steindachner (1915e: 63, pl. 8, figs. 1-3).

Distribution: Amazon River basin, Bolivia and Brazil (Sabaj \& Ferraris, 2003).

Opsodoras morei (Steindachner, 1881)

Oxydoras Morei Steindachner, 1881c: 106, pl. 1 (fig. 2). Type locality: Rio Negro. Holotype: NMW 45433.

Distribution: Negro River basin, Brazil (Sabaj \& Ferraris, 2003).

Opsodoras stuebelii (Steindachner, 1882)

Oxydoras Stübelii Steindachner, 1882b: 175. Type locality: Aus dem Huallaga. Syntypes (3): at NMW, but not found in 2006 (M. Sabaj, pers. commum.). Species illustrated and described in more detail in Steindachner (1882c: 5, pl. 3, figs. 1-1b).

Opsodoras orthacanthus Eigenmann, 1925: 351, pl. 22 (fig. 3). Type locality: Iquitos. Holotype: CAS 60231.

Distribution: Amazon River basin (Sabaj \& Ferraris, 2003).

Opsodoras ternetzi Eigenmann, 1925

Opsodoras ternetzi Eigenmann, 1925: 353. Type locality: Tapajos at Santarem. Holotype: CAS 60230.

Distribution: Amazon River basin (Sabaj \& Ferraris, 2003).

\section{ORINOCODORAS Myers, 1927}

Orinocodoras Myers, 1927: 124. Type species: Orinocodoras eigenmanni Myers, 1927. Type by original designation. Gender: Masculine.

Orinocodoras eigenmanni Myers, 1927

Orinocodoras eigenmanni Myers, 1927: 124. Type locality: Venezuela: Caño de Quiribana near Caicara. Holotype: SU 58747.

Distribution: Orinoco River basin, Venezuela (Sabaj \& Ferraris, 2003).

\section{OXYDORAS Kner, 1855}

Oxydoras Kner, 1855a: 115. Type species: Doras niger Valenciennes, 1821. Type by subsequent designation by Eigenmann \& Eigenmann (1890: 246). Gender: Masculine. Designation of Oxydoras kneri Bleeker, 1862 [=Doras (Oxydoras) niger of Kner, 1855a] as type species in Bleeker (1862-63: 5) is invalid because O. kneri was not an available name in Kner (1855).

Pseudodoras Bleeker, 1858b: 53, 54. Type species: Doras niger Valenciennes, 1821. Type by subsequent designation by Jordan (1919a: 278). Gender: Masculine.

Hildadoras Fernández-Yépez, 1968: 41. Type species: Hildadoras orinocensis Fernández-Yépez, 1968. Type by original designation. Gender: Masculine.

Oxydoras kneri Bleeker, 1862

Oxydoras kneri Bleeker, 1862 (in Bleeker, 1862-63): 5. Type locality: Cujaba. Holotype: at NMW. Based on Doras (Oxydoras) niger of Kner (1855), from Cujaba.

Distribution: Paraná River basin (Sabaj \& Ferraris, 2003).

Oxydoras niger (Valenciennes, 1821)

Doras niger Valenciennes, in Humboldt \& Valenciennes, 1821: 184. Type locality: Probablement ... Originaires d'Amérique. Holotype: MNHN 0000-4189. 
? Corydoras edentatus Spix, in Spix \& Agassiz, 1829: pl. 5. Type locality: [Not stated]. Holoype: Whereabouts unknown (Kottelat, 1988). Name available from plate, with illustrated specimen the holotype. Corresponds to Doras humboldti Spix \& Agassiz, 1829.

? Doras humboldti Spix \& Agassiz, 1829: 14, pl. 5. Type locality: fluvio S. Francisci mediae Brasiliae. Syntypes: Whereabouts unknown (Kottelat, 1988).

Rhinodoras prianomus Cope, 1874b: 134. Type locality: Nauta [upper Amazon]. Holotype: ANSP 21203.

Rhinodoras teffeanus Steindachner, 1875: 145, pl. 3. Type locality: Teffé am Amazonenstrom [brasilianische]. Syntypes: NMW 44568-69 (2).

? Oxydoras holdeni Fernández-Yépez, 1968: 54, fig. 24. Type locality: Río Apure, frente a Mango Verde [Venezuela]. Holotype: AFY 51265 (missing).

Distribution: Amazon, São Francisco and Essequibo River basins, possibly Orinoco River basin, (Sabaj \& Ferraris, 2003).

Remarks: Synonymy based on Sabaj \& Ferraris (2003). Inclusion of Doras humboldti and Corydoras edentatus in synonymy tentative, in the absence of types. Agassiz (1831: conspectus) serves as first reviser in treating Doras humboldti as valid over Corydoras edentatus.

Oxydoras sifontesi Fernández-Yépez, 1968

Oxydoras sifontesi Fernández-Yépez, 1968: 58, fig. 28. Type locality: Laguna rebalsera "El Medio," Ciudad Bolívar [Venezuela]. Holotype: MBUCV V-1584.

Hildadoras orinocensis Fernández-Yépez, 1968: 41, fig. 17. Type locality: Boca del caño San Juan, Terr. Delta Amacuro [Venezuela]. Holotype: MBUCV V-3939.

Distribution: Orinoco River basin (Sabaj \& Ferraris, 2003).

Remarks: Sabaj \& Ferraris (2003) acted as first reviser in giving precedence to $O$. sifontesi.

\section{PHYSOPYXIS Cope, 1871}

Physopyxis Cope, 1871b: 112. Type species: Physopyxis lyra Cope, 1871. Type by monotypy. Gender: Feminine. More complete description in Cope (1872a: 273).

Revision, with key to species: de Sousa \& Rapp Py-Daniel (2005).

Physopyxis ananas de Sousa \& Rapp Py-Daniel, 2005

Physopyxis ananas de Sousa \& Rapp Py-Daniel, 2005: 630, fig. 5. Type locality: Jutaí River, Solimões River basin, Amazonas State, Brazil (0245'40"S; 66²4'59"W). Holotype: INPA 24324.

Distribution: Amazon (including Negro River) and Essequibo River basins (de Sousa \& Rapp Py-Daniel, 2005).

Physopyxis cristata de Sousa \& Rapp Py-Daniel, 2005

Physopyxis cristata de Sousa \& Rapp Py-Daniel, 2005: 632, fig. 7. Type locality: mouth of igarapé Zamula, Negro River, Amazonas State, Brazil (0051'48"S; 6246'44"W). Holotype: INPA 24325.

Distribution: Negro River basin (Amazon River drainage), Amazonas State, Brazil (de Sousa \& Rapp Py-Daniel, 2005).

Physopyxis lyra Cope, 1871

Physopyxis lyra Cope, 1871b: 112. Type locality: Amazon. Holotype: ANSP 8282. Illustrated and described in more detail in Cope (1872a: 273, pl. 5, figs. 1a-1c), with locality as Ambyiacu River, Ecuador [now Peru].

Distribution: Amazon River basin, Peru and Brazil (de Sousa \& Rapp Py-Daniel, 2005).

\section{PLATYDORAS Bleeker, 1862}

Platydoras Bleeker, 1862 (in Bleeker, 1862-63): 5. Type species: Silurus costatus Linnaeus, 1758. Type by original designation. Gender: Masculine.

Platydoras armatulus (Valenciennes, 1840)

Doras armatulus Valenciennes, in Cuvier \& Valenciennes, 1840b: 273 (204 in Strasbourg deluxe edition). Type locality: Brésil, ... le Parana, mais non au-dessous du 27³0' de latitude sud. Holotype: MNHN 0000-4152.

Distribution: Paraná River basin (Sabaj \& Ferraris, 2003).

Platydoras costatus (Linnaeus, 1758)

Silurus costatus Linnaeus, 1758: 306. Type locality: Indiis. Type(s): Whereabouts unknown. Based on account in 
Gronovius (1756: 24-25, pl. 5, figs. 1 \& 2).

Mystus ascita Gronow, in Gray, 1854: 156. Type locality: Not stated. Type(s): Whereabouts unknown. Based on account in Gronovius (1756: 24-25, pl. 5, figs. 1 \& 2). Preoccupied by Mystus ascita Walbaum (1792); apparently not replaced.

? Doras dentatus Kner, 1855a: 118, pl. 3 (fig. 3). Type locality: Surinam. Holotype: NMW 46869. Originally proposed as Doras dentatus?

? Doras helicophilus Günther, 1868a: 475. Type locality: Surinam [Maroni River]. Syntypes: BMNH 1866.8.10.1-3 (3).

Distribution: Amazon, Tocantins, Parnaíba, Orinoco, Essequibo River basins and coastal drainages in French Guiana and Suriname (Sabaj \& Ferraris, 2003).

\section{Species inquirenda, Platydoras}

Doras Hancockii Valenciennes, in Cuvier \& Valenciennes, 1840b: 279 (207 in Strasbourg deluxe edition). Type locality: not stated. Holotype: BMNH 1857.6.13.163. Based on Doras costata of Hancock (1828), from Demerara [Guyana].

\section{PTERODORAS Bleeker, 1862}

Pterodoras Bleeker, 1862 (in Bleeker, 1862-63): 5. Type species: Doras granulosus Valenciennes, 1821. Type by original designation. Gender: Masculine.

Apuredoras Fernández-Yépez, 1950c: 195. Type species: Apuredoras rivasi Fernández-Yépez, 1950. Type by original designation. Gender: Masculine.

Parapterodoras Risso \& Morra, 1964: 1. Type species: Parapterodoras paranensis Risso \& Morra, 1964. Type by original designation. Gender: Masculine.

Sachsdoras Fernández-Yépez, 1968: 66. Type species: Sachsdoras apurensis Fernández-Yépez, 1968. Type by original designation. Gender: Masculine.

Pterodoras granulosus (Valenciennes, 1821)

Doras granulosus Valenciennes, in Humboldt \& Valenciennes, 1821: 184. Type locality: Probablement .... Originaires d'Amérique. Holotype: MNHN 0000-4187.

? Doras maculatus Valenciennes, 1836, (in Valenciennes, 1835-47): pl. 5 (fig. 3). Type locality: not stated. Holotype: MNHN b-0201 (1 of 2). Name available from plate that, according to Valenciennes (in Cuvier \& Valenciennes) 1840b: 281 (210 in Strasbourg deluxe edition), was based on single specimen, but description in Cuvier \& Valenciennes (1840b: 281 (209-211 in Strasbourg deluxe edition) based on holotype (MNHN 0000-4187) of Doras granulosus Valenciennes, 1821, and two specimens (MNHN b-0201) with locality as Buénos-Ayres.

? Doras murica Kner, 1855a: 129. Type locality: Cujaba. Holotype: at NMW. Name credited to Natterer; however, availability of name appears attributable solely to Kner.

? Doras laevigatulus Berg, 1901: 298. Type locality: Dock Sud, que comunica con el Río de la Plata y el Riachuelo de la Boca. Holotype: MACN 2118.

? Doras lentiginosus Eigenmann, 1917a: 401, pl. 40. Type locality: Santarem [Brazil]. Holotype: FMNH 58056.

? Silurus 12-radiatus Larrañaga, 1923: 386. Type locality: [Uruguay]. No types known.

? Silurus armatus Larrañaga, 1923: 377. Type locality: [Uruguay]. No types known. Originally as Silurus (armado) armatus.

? Parapterodoras paranensis Risso \& Morra, 1964: 2, pl. 1. Type locality: Río Paraná, frente a Corrientes. Holotype: Mus. Cien. Nat. del Chaco X-64-1.

Distribution: Amazon and Paraná River basins and coastal drainages in Guyana and Suriname (Sabaj \& Ferraris, 2003).

Pterodoras rivasi (Fernández-Yépez, 1950)

Apuredoras rivasi Fernández-Yépez, 1950c: 195, fig. on p. 196. Type locality: Río Apure, 2 km al este de San Fernando. Holotype: AFY 51062 (whereabouts unknown).

Pterodoras angeli Fernández-Yépez, 1968: 65, fig. 32. Type locality: Río Arauca, Edo. Apure [Venezuela]. Holo- 
type: AFY 66803 (whereabouts unknown). Name also spelled Ptedoras angelis in original description. Pterodoras angeli selected as the valid name by Sabaj \& Ferraris (2003: 465).

Sachsdoras apurensis Fernández-Yépez, 1968: 66, fig. 33. Type locality: Río Orinoco, cerca de la boca del Río Apure [Venezuela]. Holotype: AFY 66802 (whereabouts unknown).

Distribution: Orinoco River basin, Colombia and Venezuela (Sabaj \& Ferraris, 2003).

\section{RHINODORAS Bleeker, 1862}

Rhinodoras Bleeker, 1862 (in Bleeker, 1862-63): 5. Type species: Doras (Oxydoras) dorbignyi Kner, 1855. Type by original designation. Gender: Masculine.

Rhinodoras boehlkei Glodek, Whitmire \& Orcés, 1976

Rhinodoras boehlkei Glodek, Whitmire \& Orcés, 1976: 3, fig. 1. Type locality: Eastern Ecuador, Río Bobonaza between Montalvo and Chicherato. Holotype: FMNH 79203.

Distribution: Upper Amazon River basin (Sabaj \& Ferraris, 2003).

Rhinodoras dorbignyi (Kner, 1855)

Doras (Oxydoras) d'Orbignyi Kner, 1855a: 149, pl. 5 (fig. 9). Type locality: Rio de la Plata. Holotype: NMW 44555. Name also spelled Doras (Oxydoras) d'Orbigny in original description. Rhinodoras dorbignyi selected as the valid name over Rhinodoras dorbigny by Sabaj \& Ferraris (2003: 465). Name credited to Krøyer in Heckel manuscript; however, availability of name appears attributable solely to Kner.

Doras nebulosus Eigenmann \& Kennedy, 1903: 500. Type locality: either in Mato Grosso [at Descalvados, Brazil] or [in the Paraguay at] Asuncion. Holotype: CAS 31186.

Distribution: Paraná River basin (Sabaj \& Ferraris, 2003).

Remarks: Catalog number of holotype of Doras (Oxydoras) d'Orbignyi incorrectly reported as NMW 44551 in Eschmeyer et al. (1998) and Sabaj \& Ferraris (2003).

Rhinodoras thomersoni Taphorn \& Lilyestrom, 1984

Rhinodoras thomersoni Taphorn \& Lilyestrom, 1984a: 87, fig. 1. Type locality: Venezuela noroccidental. Cuenca del Lago Maracaibo, Estado Zulia, desembocadura del Río Catatumbo, cerca de El Congo. Holotype: MCNG 368.

Distribution: Santa Ana, Catatumbo and Escalante river basins, along southwest shore of Lake Maracaibo (Sabaj \& Ferraris, 2003).

RHYNCHODORAS Klausewitz \& Rössel, 1961

Rhynchodoras Klausewitz \& Rössel, 1961: 45. Type species: Rhynchodoras xingui Klausewitz \& Rössel, 1961. Type by original designation. Gender: Masculine.

Rhynchodoras woodsi Glodek, 1976

Rhynchodoras woodsi Glodek, 1976: 44, fig. 1. Type locality: Eastern Ecuador, Moreta Bobonaza, between Sarayacu and Montalvo. Río Bobonaza, a tributary of Río Pastaza, itself a tributary of Río Marañon in Peru. Holotype: FMNH 77008.

Distribution: Pastaza River basin in Marañon River drainage, Ecuador (Sabaj \& Ferraris, 2003).

Rhynchodoras xingui Klausewitz \& Rössel, 1961

Rhynchodoras xingui Klausewitz \& Rössel, 1961: 46, fig. 1. Type locality: Südamerika, Brasilien, Oberlauf des Rio Xingu. Holotype: SMF 5281.

Distribution: Upper Xingu River basin, Brazil (Sabaj \& Ferraris, 2003).

\section{SCORPIODORAS Eigenmann, 1925}

Scorpiodoras Eigenmann, 1925: 324. Type species: Doras heckelii Kner, 1855. Type by original designation. Gender: Masculine.

Autanadoras Fernández-Yépez, 1950a: 8. Type species: Autanadoras milesi Fernández-Yépez, 1950. Type by original designation. Gender: Masculine.

Scorpiodoras heckelii (Kner, 1855)

Doras Heckelii Kner, 1855a: 125, pl. 3 (fig. 4). Type locality: R. negro. Holotype: NMW 46613. 
Doras Calderonensis Vaillant, 1880b: 154. Type locality: Caldéron (Haute-Amazone). Holotype: MNHN a-1980.

Autanadoras milesi Fernández-Yépez, 1950a: 8, pl. 1 (fig. 4). Type locality: Río Autana [Venezuela]. Holotype: MBUCV V-15210.

Distribution: Negro, Orinoco, and upper Amazon River basins, Brazil and Venezuela (Sabaj \& Ferraris, 2003).

\section{TRACHYDORAS Eigenmann, 1925}

Trachydoras Eigenmann, 1925: 337. Type species: Trachydoras atripes Eigenmann, 1925. Type by original designation. Gender: Masculine.

\section{Trachydoras brevis (Kner, 1853)}

Doras (Corydoras) brevis Kner, 1853c: 146, fig. 8 on unnumbered plate. Type locality: [Not stated]. Holotype: NMW 45397-99 (1 of 5), NMW 46375 (1 of 2) or RMNH 2965 (1). Name made available from caption to illustration of a swimbladder; associated specimen is the holotype, if recognizable. Species later described and illustrated in Kner (1855a: 138-140, pl. 6, fig. 11), on 8 specimens with locality given as Barra do Rio negro; and swimbladder illustration in Kner (1853c) corrected from fig. 6 to fig. 8.

Distribution: Negro and Essequibo River basins (Sabaj \& Ferraris, 2003).

Trachydoras microstomus (Eigenmann, 1912)

Hemidoras microstomus Eigenmann, 1912b: 193, pl. 18 (fig. 2). Type locality: Rockstone, British Guiana. Holotype: FMNH 53206.

Distribution: Amazon, Orinoco and Essequibo River basins (Sabaj \& Ferraris, 2003).

Trachydoras nattereri (Steindachner, 1881)

Oxydoras Nattereri Steindachner, 1881c: 104, pl. 2 (fig. 1). Type locality: Amazonen-Strome bei Teffe [Brazil]. Holotype: at NMW, but not found in 2006 (M. Sabaj, pers. commun.).

Distribution: Amazon River basin (Sabaj \& Ferraris, 2003).

Trachydoras paraguayensis (Eigenmann \& Ward, 1907)

Hemidoras paraguayensis Eigenmann \& Ward in Eigenmann, McAtee \& Ward, 1907: 116, pl. 34 (fig. 1). Type locality: Paraguay River at ... Corumba [Brazil]. Holotype: CAS 37216.

Distribution: Paraná River basin (Sabaj \& Ferraris, 2003).

Trachydoras steindachneri (Perugia, 1897)

Oxydoras Steindachneri Perugia, 1897: 20. Type locality: Rio Beni, Bolivia. Syntypes: MSNG 8834 (17).

Oxydoras trachyparia Boulenger, 1898e: 423, pl. 40 (fig. 2). Type locality: Rio Jurua, an affluent of the Amazons, Brazil. Syntypes: BMNH 1897.12.1.44-45 (2).

Trachydoras atripes Eigenmann, 1925: 339, pl. 26 (fig. 4). Type locality: Brook near River Itaya, above Iquitos [Peru]. Holotype: CAS 60234.

Distribution: Amazon River basin (Sabaj \& Ferraris, 2003).

WERTHEIMERIA Steindachner, 1877

Wertheimeria Steindachner, 1877b: 659. Type species: Wertheimeria maculata Steindachner, 1877. Type by monotypy. Gender: Feminine.

Relationships: Higuchi et al. (1990).

Wertheimeria maculata Steindachner, 1877

Wertheimeria maculata Steindachner, 1877b: 660, pl. 10. Type locality: Rio Jequitinhonha [Brazil]. Syntypes: NMW 43460 (1, lost), NMW 43461 (1).

Distribution: Jequitinhonha River basin, Brazil (Sabaj \& Ferraris, 2003).

\section{Species inquirenda, Doradidae}

Doras insculptus Miranda Ribeiro, 1912: 22. Type locality: Manáos [Brazil]. Lectotype: MNRJ 656a, designated by Miranda Ribeiro (1953: 398). Validity and generic placement considered uncertain in Sabaj \& Ferraris (2003); likely as Anadoras or Amblydoras. 


\section{HEPTAPTERIDAE Gill, 1861}

Pteronotidae Adams, in Adams et al., 1854: 107. Type genus: Pteronotus Swainson, 1839. Permanently invalid, because Pteronotus Swainson is a junior homonym of Pteronotus Gray, 1837 (Mammalia).

Heptapterinae Gill, 1861c: 54. Type genus: Heptapterus Bleeker, 1858.

Rhamdiae Bleeker, 1862 (in Bleeker, 1862-63): 11, 60. Type genus: Rhamdia Bleeker, 1858.

Taxonomic summary: Gosline (1941), Bockmann \& Guazzelli (2003).

Phylogeny: Lundberg \& Mc Dade (1986), Ferraris (1988a, Nemuroglanis group); Bockmann (1994, 1998).

24 genera, 189 species; no named fossil taxa.

\section{Incertae sedis}

Imparales panamensis Bussing, 1970

Imparales panamensis Bussing, 1970: 7, fig. 2. Type locality: Panamá: Veraguas Prov., creek crossing road on s side of Santa Fe (elev. 305 m). Holotype: USNM 204692.

Distribution: River basin(s) draining Veraguas Province, Tuira River basin, and Frijoles River, Panama (Bockmann \& Guazzelli, 2003).

Remarks: Not related to Imparales, or its senior synonym Nemuroglanis (Bockmann \& Ferraris, 2005), but not placed elsewhere.

Pimelodus (Rhamdia) parvus Boulenger, 1898

Pimelodus (Rhamdia) parvus Boulenger, 1898c: 7. Type locality: du Rio Santiago, du Rio Zamora et du Rio Bomboiza. Syntypes: BMNH 1898.11.4.5-6, BMNH 1898.11.4.7, ZMUT 1543 (2), ZMUT 1544 (2), ZMUT 1691 (4).

Distribution: Marañon River basin, Ecuador.

Remarks: Silfvergrip (1996: 29) referred this species to Nannorhamdia, but Bockmann \& Guazzelli (2003) disagreed with that placement and, instead, left the species unassigned.

ACENTRONICHTHYS Eigenmann \& Eigenmann, 1889

Acentronichthys Eigenmann \& Eigenmann, 1889b: 28. Type species: Acentronichthys leptos Eigenmann \& Eigenmann, 1889. Type by original designation. Gender: Masculine.

Remarks: Treated as a synonym of Heptapterus by Miranda Ribeiro (1962b).

Acentronichthys leptos Eigenmann \& Eigenmann, 1889

Acentronichthys leptos Eigenmann \& Eigenmann, 1889b: 29. Type locality: Sao Matheos [=São Mateus, State of Espírito Santo, Brazil]. Holotype: MCZ 7532.

Distribution: Coastal streams from Rio de Janeiro to Santa Catarina State, and in São Mateus, in Espírito Santo State, Brazil (Bockmann \& Guazzelli, 2003).

BRACHYGLANIS Eigenmann, 1912

Brachyglanis Eigenmann, 1912b: 156. Type species: Brachyglanis frenata Eigenmann, 1912. Type by original designation. Gender: Masculine.

Remarks: First published as Breviglanis in Eigenmann (1910: 384), but without diagnosis or available species; name was apparently never made available by its use with an available species name.

Brachyglanis frenatus Eigenmann, 1912

Brachyglanis frenata Eigenmann, 1912b: 156. Type locality: Amatuk [Essequibo River basin], British Guiana. Holotype: CM 1670, not found at FMNH.

Distribution: Essequibo, Negro, Orinoco, and Urubu River basins of Brazil, Guyana, and Venezuela (Bockmann \& Guazzelli, 2003).

Brachyglanis magoi Fernández-Yépez, 1967

Brachyglanis magoi Fernández-Yépez, 1967: 162, pl. 1. Type locality: río Uruyén medio. Holotype: AFY 56611. 
Distribution: Caroní River basin in Orinoco River drainage, Venezuela (Bockmann \& Guazzelli, 2003).

Brachyglanis melas Eigenmann, 1912

Brachyglanis melas Eigenmann, 1912b: 157, pl. 11 (fig. 2). Type locality: Crab Falls, British Guiana. Holotype: FMNH 53217.

Distribution: Essequibo River basin, Guyana (Bockmann \& Guazzelli, 2003).

Brachyglanis microphthalmus Bizerril, 1991

Brachyglanis microphthalmus Bizerril, 1991: 2, fig. 1. Type locality: Brasil, Pará, Município de Oriximiná, Bacia do rio Trombetas, drenagem do Lago Sapucuá, Igarapé Saracá. Holotype: MNRJ 11788.

Distribution: Trombetas River basin, Brazil (Bockmann \& Guazzelli, 2003).

Brachyglanis nocturnus Myers, 1928

Brachyglanis nocturnus Myers, 1928: 84. Type locality: Rock-pools at São Gabriel Rapids, Rio Negro, Brazil. Holotype: CAS 63393.

Distribution: Negro River basin, Brazil (Bockmann \& Guazzelli, 2003).

Brachyglanis phalacra Eigenmann, 1912

Brachyglanis phalacra Eigenmann, 1912b: 157, pl. 12 (fig. 1), pl. 13 (fig. 1). Type locality: Amatuk [Essequibo River basin], British Guiana. Holotype: FMNH 53216.

Distribution: Essequibo River basin, Guyana (Bockmann \& Guazzelli, 2003).

BRACHYRHAMDIA Myers, 1927

Brachyrhamdia Myers, 1927: 123. Type species: Brachyrhamdia imitator Myers, 1927. Type by original designation. Gender: Feminine.

Brachyrhamdia heteropleura (Eigenmann, 1912)

Pimelodus heteropleurus Eigenmann, 1912b: 176, pl. 16 (fig. 2). Type locality: Rupununi Pan [Essequibo River basin], British Guiana. Holotype: FMNH 53336.

Distribution: Corantijn, Essequibo and Negro River basins (Bockmann \& Guazzelli, 2003).

Brachyrhamdia imitator Myers, 1927

Brachyrhamdia imitator Myers, 1927: 123. Type locality: Caño de Quiribana, near Caicara [Venezuela]. Holotype: CAS 54359.

Distribution: Caura River basin in Orinoco River drainage, Venezuela (Bockmann \& Guazzelli, 2003).

Brachyrhamdia marthae Sands \& Black, 1985

Brachyrhamdia marthae Sands \& Black, 1985: 58 (4), illustrated on p. 58 (7). Type locality: Peru, aquarium import. Holotype: RMNH 29424.

Distribution: Juruá and Mamoré/Madeira River basins (Bockmann \& Guazzelli, 2003).

Remarks: Redescribed, as Rhamdia marthae, in Zarske (2003).

Brachyrhamdia meesi Sands \& Black, 1985

Brachyrhamdia meesi Sands \& Black, 1985: 58 (5), illustrated on p. 58 (7). Type locality: Near Belem, Brazil. Holotype: RMNH 29425.

Distribution: Amazon River basin, Brazil (Bockmann \& Guazzelli, 2003).

Brachyrhamdia rambarrani (Axelrod \& Burgess, 1987)

Pimelodella rambarrani Axelrod \& Burgess, in Axelrod, 1987: 24, figures on pp. 24- 25. Type locality: reportedly from the Rio Unini, a tributary of the Rio Negro, Amazonas, Brazil. Holotype: MZUSP 37693.

Distribution: Unini River basin in upper Negro River drainage, Brazil (Bockmann \& Guazzelli, 2003).

CETOPSORHAMDIA Eigenmann \& Fisher, 1916

Cetopsorhamdia Eigenmann \& Fisher, in Eigenmann, 1916: 83. Type species: Cetopsorhamdia nasus Eigenmann \& Fisher, 1916. Type by original designation. Gender: Feminine.

Cetopsorhamdia boquillae Eigenmann \& Fisher, 1922

Cetopsorhamdia boquillae Eigenmann \& Fisher, in Eigenmann, 1922b: 37, pl. 1 (fig. 3). Type locality: Boquilla [Colombia]. Holotype: FMNH 55212.

Distribution: Cauca River basin, Colombia (Maldonado-Ocampo et al., 2005). 
Cetopsorhamdia filamentosa Fowler, 1945

Cetopsorhamdia filamentosa Fowler, 1945c: 4, figs. 5-6. Type locality: San Ramón, Vallé de Chanchamayo, at 800 meters elevation, Peru. Holotype: ANSP 71637.

Distribution: Tulumayo River basin in upper Ucayali River drainage, Peru (Bockmann \& Guazzelli, 2003).

Cetopsorhamdia iheringi Schubart \& Gomes, 1959

Cetopsorhamdia iheringi Schubart \& Gomes, 1959: 1, fig. 1. Type locality: Ribeirão Areia Branca, próximo à estrada carroçável para Piraçununga, bacia do Rio Mogi Guaçu, Município de Descalvado [Brazil]. Holotype: EEBP 368.

Distribution: Upper reaches of the Paraná and São Francisco River basins, Brazil (Bockmann \& Guazzelli, 2003).

Cetopsorhamdia insidiosa (Steindachner, 1915)

Imparfinis insidiosus Steindachner, 1915e: 61, pl. 12 (fig. 7). Type locality: Rio branco bei Bem Querer und im Rio Surumú, einem Nebenflusse des Rio Miang [Brazil]. Syntypes: NMW 46095.

Distribution: Branco River basin, Brazil (Bockmann \& Guazzelli, 2003).

Cetopsorhamdia molinae Miles, 1943

Cetopsorhamdia molinae Miles, 1943a: 26. Type locality: Bugalagrande [Colombia]. Holotype: at ICNMHN probably lost (Cala, 1981: 1).

Cetopsorhamdia shermani Schultz, 1944c: 220, pl. 2 (fig. c). Type locality: Río Guárico and tributaries between San Sebastián and San Casimiro (Orinoco system), Estado de Aragua, Venezuela. Holotype: USNM 121216.

Distribution: Magdalena, Orinoco and Tocantins River basins (Bockmann \& Guazzelli, 2003); also Cauca River, Colombia (Maldonado-Ocampo et al., 2005).

Cetopsorhamdia nasus Eigenmann \& Fisher, 1916

Cetopsorhamdia nasus Eigenmann \& Fisher, in Eigenmann, 1916: 83. Type locality: Honda, Colombia. Holotype: FMNH 58126; holotype illustrated in Eigenmann (1922b: 36, fig. 10; pl. 4, fig. 1).

Distribution: Magdalena and Cauca River basins, Colombia (Maldonado-Ocampo et al., 2005).

Cetopsorhamdia orinoco Schultz, 1944

Cetopsorhamdia orinoco Schultz, 1944c: 225, pl. 3 (fig. a). Type locality: Río Torbes, 1 km. above Táriba, Orinoco system, Venezuela. Holotype: USNM 121214.

Distribution: Napo and Orinoco River basins (Bockmann \& Guazzelli, 2003).

Cetopsorhamdia phantasia Stewart, 1985

Cetopsorhamdia phantasia Stewart, 1985a: 340, fig. 1. Type locality: Ecuador, Río Jivino, west of Limoncocha, approx. 0²4.6'S, 76³9.0'W. Holotype: FMNH 94601.

Distribution: Napo River basin, Ecuador (Bockmann \& Guazzelli, 2003).

Cetopsorhamdia picklei Schultz, 1944

Cetopsorhamdia picklei Schultz, 1944c: 222, pl. 2 (fig. d). Type locality: Río Motatán, 4 km. above Motatán, Maracaibo basin [Venezuela]. Holotype: USNM 121217.

Distribution: Maracaibo Lake basin, Venezuela (Bockmann \& Guazzelli, 2003).

\section{CHASMOCRANUS Eigenmann, 1912}

Chasmocranus Eigenmann, 1912b: 160. Type species: Chasmocranus longior Eigenmann, 1912. Type by original designation. Gender: Masculine.

Remarks: Named Chasmocephalus in Eigenmann (1910: 384) but without diagnosis or included available species; name was apparently never made available by its use with an available species name.

Chasmocranus brachynemus Gomes \& Schubart, 1958

Chasmocranus brachynema Gomes \& Schubart, 1958: 413, fig. 1. Type locality: Rio Mogi Guaçu, Cachoeira de Emas, Município de Piraçununga, Estado de São Paulo [Brazil]. Holotype: EEBP 617.

Distribution: Mogi Guaçu River basin in upper Paraná River drainage, Brazil (Bockmann \& Guazzelli, 2003).

Chasmocranus brevior Eigenmann, 1912

Chasmocranus brevior Eigenmann, 1912b: 162, pl. 15 (fig. 1). Type locality: Waratuk, British Guiana. Holotype: FMNH 53213.

Distribution: Mana and Maroni River basins, and Potaro River, Guianas (Bockmann \& Guazzelli, 2003). 
Chasmocranus chimantanus Inger, 1956

Chasmocranus chimantanus Inger, 1956: 425, fig. 92. Type locality: Rio Abácapa on west side of Chimantá-tepui [Venezuela]. Holotype: FMNH 45704.

Distribution: Caroní and Carapo River basins, Orinoco River drainage, Venezuela (Bockmann \& Guazzelli, 2003).

Chasmocranus longior Eigenmann, 1912

Chasmocranus longior Eigenmann, 1912b: 160, pl. 14 (fig. 2). Type locality: Amatuk, British Guiana. Holotype: FMNH 53208.

Distribution: Caribbean coastal drainages from Guyana to Surinam; Negro, Orinoco and Xingu River basins (Bockmann \& Guazzelli, 2003).

Chasmocranus lopezi Miranda Ribeiro, 1968

Chasmocranus Lopezi Miranda Ribeiro, 1968: 4, fig. 2. Type locality: Corrego Quente — Cubatão ? - Estado de São Paulo - Brasil. Holotype: MNRJ 9705.

Distribution: São Paulo State, Brazil (Bockmann \& Guazzelli, 2003).

Chasmocranus peruanus Eigenmann \& Pearson, 1942

Chasmocranus peruanus Eigenmann \& Pearson, in Eigenmann \& Allen, 1942: 91, pl. 3 (figs. 3-4). Type locality: Puerto Melendez below Pongo de Manseriche, Rio Marañon. Syntypes: CAS 63627 (2).

Distribution: Upper Amazon River basin, Peru (Bockmann \& Guazzelli, 2003).

Chasmocranus quadrizonatus Pearson, 1937

Chasmocranus quadrizonatus Pearson, 1937: 93. Type locality: Tingo de Pauca, Rio Marañon, Peru. Holotype: CAS 63628.

Distribution: Marañon River basin, Peru (Bockmann \& Guazzelli, 2003).

Chasmocranus rosae Eigenmann, 1919

Chasmocranes Rosae Eigenmann, 1919a: 127. Type locality: Río Negro, Villavicencio [Colombia]. Holotype: FMNH 55140; holotype illustrated in Eigenmann (1922b: pl. 1, fig. 4).

Distribution: Meta River basin, Colombia (Bockmann \& Guazzelli, 2003).

Chasmocranus surinamensis (Bleeker, 1862)

Heptapterus surinamensis Bleeker, 1862a: 387. Type locality: Surinama. Holotype: RMNH 2984; holotype illustrated in Bleeker (1864: 91, pl. 15, fig. 1).

Distribution: Suriname River basin (Bockmann \& Guazzelli, 2003).

Remarks: Mees (1967: 226) interpreted the type locality as the Suriname River, presumably not far from Paramaribo (Bockmann \& Guazzelli, 2003).

Chasmocranus truncatorostris Borodin, 1927

Chasmocranus truncatorostris Borodin, 1927b: 5, fig. 4. Type locality: Col. Hansa Joinville, Sta. Catharina Prov., Brazil. Holotype: AMNH 8640.

Distribution: Sta. Catharina Province, Brazil; known only from the type locality (Bockmann \& Guazzelli, 2003).

GLADIOGLANIS Ferraris \& Mago-Leccia, 1989

Gladioglanis Ferraris \& Mago-Leccia, 1989: 166. Type species: Gladioglanis machadoi Ferraris \& Mago-Leccia, 1989. Type by original designation. Gender: Masculine.

Gladioglanis conquistador Lundberg, Bornbusch \& Mago-Leccia, 1991

Gladioglanis conquistador Lundberg, Bornbusch \& Mago-Leccia, 1991: 192, fig. 7A. Type locality: Ecuador, Napo Province, Río Napo basin, Río Aguarico sub-basin, north tributary of Río Cuyabeno, ca 3 km above Laguna Grande de Cuyabeno, 0¹.5'S, 76²13.2'W. Holotype: FMNH 99288.

Distribution: Capim, Napo, Purus and Solimões Rivers, Amazon basin (Bockmann \& Guazzelli, 2003).

Gladioglanis machadoi Ferraris \& Mago-Leccia, 1989

Gladioglanis machadoi Ferraris \& Mago-Leccia, 1989: 167, fig. 1. Type locality: Venezuela, Territorio Federal Amazonas, Departamento Río Negro, Caño Manu of Casiquiare canal, approx. $250 \mathrm{~m}$ upstream of Solano, $2^{\circ} 00^{\prime} \mathrm{N}, 6^{\circ} 57^{\prime} \mathrm{W}$. Holotype: MBUCV V-16000.

Distribution: Negro and Orinoco River basins, Brazil and Venezuela (Bockmann \& Guazzelli, 2003). 
GOELDIELLA Eigenmann \& Norris, 1900

Goeldiella Eigenmann \& Norris, 1900: 353. Type species: Pimelodus eques Müller \& Troschel, 1848. Type by original designation. Gender: Feminine.

Goeldiella eques (Müller \& Troschel, 1848)

Pimelodus eques Müller \& Troschel, in Schomburgk, 1848: 628. Type locality: in allen Flüssen Guiana's. Holotype: ZMB 3049. Also described as new in Müller \& Troschel (1849: 5).

Distribution: Coastal Rivers of the Guianas and Amazon River basin (Bockmann \& Guazzelli, 2003).

HEPTAPTERUS Bleeker, 1858

Heptapterus Bleeker, 1858b: 197, 208. Type species: Pimelodus mustelinus Valenciennes, 1835. Type by monotypy. Gender: Masculine.

Review: Buckup (1988, southern Brazil and Uruguay); Mees (1967, Suriname).

Heptapterus bleekeri Boeseman, 1953

Heptapterus bleekeri Boeseman, 1953: 3, fig. 1a. Type locality: Marowijne basin, Nassau Mountains in brooklet [Suriname]. Holotype: RMNH 19421 (larger of 2 specimens).

Distribution: Maroni River basin, and State of Amapá, Brazil (Bockmann \& Guazzelli, 2003).

Heptapterus fissipinnis Miranda Ribeiro, 1911

Heptapterus fissipinnis Miranda Ribeiro, 1911: 240. Type locality: corrego affluente do Rio Estrella [probably in the State of Rio de Janeiro, Brazil]. Holotype: at MNRJ (current whereabouts unknown). Misspelled Heptapterus ñssipinnis in heading for account.

Distribution: Stream tributary to the Estrela River, Brazil (Bockmann \& Guazzelli, 2003).

Heptapterus multiradiatus Ihering, 1907

Heptapterus multiradiatus Ihering, 1907: 20. Type locality: ribeirão do Alto da Serra, Estado de S. Paulo [Brazil]. Holotype: MZUSP 294.

Distribution: Upper Tietê River basin, Brazil (Bockmann \& Guazzelli, 2003).

Heptapterus mustelinus (Valenciennes, 1835)

Pimelodus mustelinus Valenciennes, 1835, in Valenciennes, 1835-47: pl. 2 (fig. 1). Type locality: Rio de la Plata. Holotype: at MNHN. Name available from caption to plate, with illustrated specimen the holotype, if recognizable; species later described in Cuvier \& Valenciennes (1840b: 165; 122 of Strasbourg deluxe edition).

Heptapterus eigenmanni Steindachner, 1907e: 487. Type locality: Maldonado. Lectotype: MCZ 7597, designated by Buckup (1988: 650), but listed in the publication as MCZ 7579.

Distribution: La Plata and Uruguay River basins, and coastal drainages of southern Brazil (Bockmann \& Guazzelli, 2003).

Heptapterus stewarti Haseman, 1911

Heptapterus stewarti Haseman, 1911b: 376, pl. 74. Type locality: Serrinha Paraná, in a creek about one-half mile from the Rio Iguassú. Holotype: FMNH 54234.

Distribution: Iguaçu River basin, Brazil (Bockmann \& Guazzelli, 2003).

Heptapterus sympterygium Buckup, 1988

Heptapterus sympterygium Buckup, 1988: 644, fig. 1. Type locality: Canal at east side of highway BR-471 near pumping house of the Departamento Nacional de Obras de Saneamento (DNOS), 52³ $31^{\prime} \mathrm{W}, 32^{\circ} 32^{\prime} \mathrm{S}$, Ecological Station of Taim (EET), Rio Grande, State of Rio Grande do Sul (RS), Brazil. Holotype: MZUSP 19179.

Distribution: Coastal lowlands of eastern Rio Grande do Sul State, Brazil (Bockmann \& Guazzelli, 2003).

Heptapterus tapanahoniensis Mees, 1967

Heptapterus tapanahoniensis Mees, 1967: 223, fig. 2. Type locality: Tapanahoni, about two kilometres downstream of its confluence with the Paloemeu [Marowijne River basin, Suriname]. Holotype: RMNH 25546.

Distribution: Marowijne/Maroni and Sinnamary River basins (Bockmann \& Guazzelli, 2003).

Heptapterus tenuis Mees, 1986

Heptapterus tenuis Mees, 1986: 322, fig. 3. Type locality: Crique Cascade Moyen, Maroni [French Guiana]. Holotype: RMNH 29422.

Distribution: Maroni River basin, French Guiana (Bockmann \& Guazzelli, 2003). 


\section{Species inquirenda, Heptapterus}

Heptapterus ornaticeps Ahl, 1936: 110. Type locality: Rio de Janeiro [probably incorrect]. Holotype: ZMB 20933. Known only from holotype, which Bockmann \& Guazzelli (2003) indicated was likely to have been incorrectly reported to have originated from the vicinity of Rio de Janeiro.

HORIOMYZON Stewart, 1986

Horiomyzon Stewart, 1986b: 47. Type species: Horiomyzon retropinnatus Stewart, 1986. Type by original designation. Gender: Masculine.

Horiomyzon retropinnatus Stewart, 1986

Horiomyzon retropinnatus Stewart, 1986b: 48, fig. 1. Type locality: Ecuador, Napo Province, Río Napo at Añangu, just downstream from confluence with Río Añangu Cocha, lat. $0^{\circ} 31.6$ 'S, long. $76^{\circ} 22.9^{\prime} \mathrm{W}$. Holotype: FMNH 96553.

Distribution: Amazon and Napo River basins (Bockmann \& Guazzelli, 2003).

IMPARFINIS Eigenmann \& Norris, 1900

Imparfinis Eigenmann \& Norris, 1900: 351. Type species: Imparfinis piperatus Eigenmann \& Norris, 1900. Type by monotypy. Gender: Masculine.

Nannorhamdia Regan, 1913d: 467. Type species: Nannorhamdia spurrellii Regan, 1913. Type by monotypy. Gender: Feminine.

Imparfinis borodini Mees \& Cala, 1989

Imparfinis longicauda Borodin, 1927b: 4, fig. 3. Type locality: Franca, Rio Grande, Prov. of S. Paulo, Brazil. Holotype: AMNH 8639. Preoccupied in Imparfinis by Pimelodus (Rhamdia) longicauda Boulenger, 1887; replaced by Imparfinis borodini Mees \& Cala, 1989.

Imparfinis borodini Mees \& Cala, 1989: 387. Type locality: Franca, Rio Grande, Prov. of S. Paulo, Brazil. Holotype: AMNH 8639. Replacement for Imparfinis longicauda Borodin, 1927.

Distribution: Upper Paraná, São Francisco and Tocantins River basins, Brazil (Bockmann \& Guazzelli, 2003).

Imparfinis cochabambae (Fowler, 1940)

Pimelodella cochabambae Fowler, 1940c: 80, figs. 35-37. Type locality: Boca Chapare, Cochabamba, Bolivia. Holotype: ANSP 69066.

Distribution: Beni River basin, Bolivia (Bockmann \& Guazzelli, 2003).

Imparfinis guttatus (Pearson, 1924)

Nannorhamdia guttatus Pearson, 1924: 11, pl. 2 (fig. 2). Type locality: Popoi River, Upper Beni [Bolivia]. Holotype: CAS 63689.

Distribution: Mamoré and Pilcomayo River basins, Bolivia and Peru (Bockmann \& Guazzelli, 2003).

Imparfinis hasemani Steindachner, 1915

Imparfinis hasemani Steindachner, 1915e: 45, figs. 1-3. Type locality: Rio Surumú bei Serra do Mello, dem Rio branco bei Bem Querer und dem Rio Tapajos bei Santarem [Brazil]. Syntype: NMW 46093:1 (1).

Distribution: Branco and Tapajós River basins, Brazil (Bockmann \& Guazzelli, 2003).

Imparfinis hollandi Haseman, 1911

Imparfinis hollandi Haseman, 1911b: 383, pl. 48 (fig. 2). Type locality: Porto União da Victoria, Rio Iguassú. Holotype: FMNH 54244.

Distribution: Iguaçu River basin (Bockmann \& Guazzelli, 2003).

Imparfinis lineatus (Bussing, 1970)

Nannorhamdia lineata Bussing, 1970: 1, fig. 1. Type locality: Costa Rica: Puntarenas Prov., Quebrada 36 (elev. 80 m) $12 \mathrm{~km} \mathrm{~W}$ of Pueblo Río Claro at Interamerican Highway. Holotype: LACM 30688-1.

Distribution: Streams in Puntarenas Province, Costa Rica (Bockmann \& Guazzelli, 2003).

Imparfinis longicauda (Boulenger, 1887)

Pimelodus (Rhamdia) longicauda Boulenger, 1887c: 275, pl. 20 (fig. 2). Type locality: Canelos [Ecuador]. Syn- 
types: BMNH 1880.12.8.100-104 (5).

Distribution: Bobonaza River basin in upper Pastaza River drainage, Ecuador (Bockmann \& Guazzelli, 2003).

Imparfinis microps Eigenmann \& Fisher, 1916

Imparfinis microps Eigenmann \& Fisher, in Eigenmann, 1916: 82. Type locality: Rio Negro at Villavicencio, Colombia. Holotype: FMNH 57793, illustrated in Eigenmann (1922b: 285, pl. 4, figs. 2-3). Also described as new in Eigenmann (1919a: 128).

Distribution: Meta River basin, Colombia (Bockmann \& Guazzelli, 2003).

Imparfinis minutus (Lütken, 1874)

Rhamdia minuta Lütken, 1874c: 35. Type locality: in provincia Minas-geraës circa oppidulum Lagoa Santa, praecipue in flumine Rio das Velhas et affluentibus. Syntypes: ZMUC P 29661 (1), ZMUC P 29662 (1), ZMUC P 29663 (1). Illustrated and described in more detail in Lütken (1875: 179-180, pl. 3, fig. 6).

Distribution: Upper São Francisco River basin, Brazil (Bockmann \& Guazzelli, 2003).

Imparfinis mirini Haseman, 1911

Imparfinis mirini Haseman, 1911a: 318, pl. 47. Type locality: Near Piracicaba above the falls of Rio Piracicabamirini [Brazil]. Holotype: FMNH 54335.

Distribution: Upper Araguaia and Paraná River basins, Brazil (Bockmann \& Guazzelli, 2003).

Imparfinis nemacheir (Eigenmann \& Fisher, 1916)

Nannorhamdia nemacheir Eigenmann \& Fisher, in Eigenmann, 1916: 83. Type locality: Girardot, Colombia. Holotype: FMNH 58127, illustrated in Eigenmann (1922b: 41, fig. 13; pl. 4, fig. 4).

Distribution: Upper Amazon and Orinoco River basins (Bockmann \& Guazzelli, 2003); Magdalena, Cauca and Patia River basins, Colombia (Maldonado-Ocampo et al., 2005).

Imparfinis pijpersi (Hoedeman, 1961)

Cetopsorhamdia pijpersi Hoedeman, 1961: 132, fig. 2. Type locality: Surinam: ... Sipaliwini river, sta. 43, 20 km from frontier with Brazil. Holotype: ZMA 102230.

Distribution: Corantijn River basin, Suriname (Bockmann \& Guazzelli, 2003).

Imparfinis piperatus Eigenmann \& Norris, 1900

Imparfinis piperatus Eigenmann \& Norris, 1900: 352. Type locality: S. Paulo, Brazil. Holotype: CAS 63636.

Distribution: In tributaries of upper Paraná River and/or coastal streams of São Paulo State, Brazil (Bockmann \& Guazzelli, 2003).

Imparfinis pristos Mees \& Cala, 1989

Imparfinis pristos Mees \& Cala, 1989: 391, fig. 3. Type locality: Colombia: ... second tributary, left side, from the mouth of the Rio Tomo, Vichada. Holotype: ICNMHN 1401.

Distribution: Negro and Orinoco River basins (Bockmann \& Guazzelli, 2003).

Imparfinis pseudonemacheir Mees \& Cala, 1989

Imparfinis pseudonemacheir Mees \& Cala, 1989: 387, fig. 2. Type locality: Venezuela: ... Rio Tucuragua, near Caicara, Bolívar. Holotype: RMNH 30489 (37.5 mm SL specimen).

Distribution: Orinoco and Ucayali River basins (Bockmann \& Guazzelli, 2003).

Imparfinis schubarti (Gomes, 1956)

Nannorhamdia schubarti Gomes, 1956: 404, fig. 1. Type locality: da Cachoeira de Emas, Rio Mogi Guaçu, e do Córrego do Tijuco Prêto, um seu afluente, Município de Pirassununga, São Paulo [Brazil]. Holotype: EEBP 379.

Distribution: Upper Paraná River basin, Brazil (Bockmann \& Guazzelli, 2003).

Imparfinis spurrellii (Regan, 1913)

Nannorhamdia spurrellii Regan, 1913d: 467. Type locality: R. Condoto [Colombia]. Holotype: BMNH 1913.10.1.41.

Distribution: San Juan River basin, Colombia (Bockmann \& Guazzelli, 2003).

Imparfinis stictonotus (Fowler, 1940)

Nannorhamdia stictonotus Fowler, 1940c: 66, figs. 21-22. Type locality: Todos Santos, Rio Chapare, Bolivia. Holotype: ANSP 68892.

Distribution: Mamoré/Madeira, Paraguay, and Ucayali River basins (Bockmann \& Guazzelli, 2003). 


\section{LEPTORHAMDIA Eigenmann, 1918}

Leptoglanis Eigenmann, 1912b: 158. Type species: Leptoglanis essequibensis Eigenmann, 1912. Type by original designation. Gender: Masculine. Preoccupied by Leptoglanis Boulenger, 1902, in fishes; replaced by Leptorhamdia Eigenmann, 1918.

Leptorhamdia Eigenmann, 1918a: 260. Type species: Leptoglanis essequibensis Eigenmann, 1912. Type by being a replacement name. Gender: Feminine. Replacement for Leptoglanis Eigenmann, 1912; preoccupied by Leptoglanis Boulenger, 1902.

Leptorhamdia essequibensis (Eigenmann, 1912)

Leptoglanis essequibensis Eigenmann, 1912b: 158, pl. 13 (fig. 2). Type locality: Crab Falls, British Guiana. Holotype: FMNH 53331.

Distribution: Essequibo and lower Tocantins River basins (Bockmann \& Guazzelli, 2003).

Leptorhamdia marmorata Myers, 1928

Leptorhamdia marmorata Myers, 1928: 83. Type locality: Rock-pools at São Gabriel Rapids, Rio Negro, Brazil. Holotype: CAS 63671.

Distribution: Negro River basin, Brazil and Venezuela (Bockmann \& Guazzelli, 2003).

Leptorhamdia schultzi (Miranda Ribeiro, 1964)

Rhamdella schultzi Miranda Ribeiro, 1964: 1, fig. 1. Type locality: Alto Rio Xingú [Brazil]. Holotype: MNRJ 9549.

Distribution: Upper Xingu River basin, Brazil (Bockmann \& Guazzelli, 2003).

Remarks: Placement in Leptorhamdia follows Bizerril \& Borba (1994).

MASTIGLANIS Bockmann, 1994

Mastiglanis Bockmann, 1994: 762. Type species: Mastiglanis asopos Bockmann, 1994. Type by original designation. Gender: Masculine.

Mastiglanis asopos Bockmann, 1994

Mastiglanis asopos Bockmann, 1994: 762, fig. 1. Type locality: Brazil, Pará, Igarapé Saracazinho, tributary of rio Trombetas, near Porto Trombetas. Holotype: MNRJ 12227.

Distribution: Amazon, Capim and Orinoco River basins (Bockmann \& Guazzelli, 2003).

MYOGLANIS Eigenmann, 1912

Myoglanis Eigenmann, 1912b: 159. Type species: Myoglanis potaroensis Eigenmann, 1912. Type by original designation. Gender: Masculine.

Myoglanis aspredinoides Do Nascimiento \& Lundberg, 2005

Myoglanis aspredinoides Do Nascimiento \& Lundberg, 2005: 39, fig. 1. Type locality: Venezuela, Estado Amazonas, Río Ventuari, Laguna Pavón, in pool behind beach of Río Ventuari, on south side of river, ca. $12 \mathrm{~km}$ from its confluence with Río Orinoco; 44'N, 6656'W. Holotype: MBUCV V-25388.

Distribution: Ventuari River, Orinoco River basin, Venezuela (Do Nascimiento \& Lundberg, 2005).

Myoglanis koepckei Chang, 1999

Myoglanis koepckei Chang, 1999: 434, fig. 1. Type locality: Peru, Departamento Loreto, Provincia Maynas, Quebrada of the Mishana camp, small tributary of the Río Nanay, 0352'S, 73²9'W, 107 m elevation. Holotype: MUSM 10045.

Distribution: Upper Amazon River basin, Peru (Bockmann \& Guazzelli, 2003).

Myoglanis potaroensis Eigenmann, 1912

Myoglanis potaroënsis Eigenmann, 1912b: 159, pl. 14 (fig. 1). Type locality: Creek at Tukeit, British Guiana. Holotype: FMNH 53333.

Distribution: Essequibo River basin, Guyana (Bockmann \& Guazzelli, 2003).

NANNOGLANIS Boulenger, 1887

Nannoglanis Boulenger, 1887c: 278. Type species: Nannoglanis fasciatus Boulenger, 1887. Type by monotypy. Gender: Masculine. 
Nannoglanis fasciatus Boulenger, 1887

Nannoglanis fasciatus Boulenger, 1887c: 278, pl. 21 (fig. 3). Type locality: eastern Ecuador. Syntypes: BMNH 1880.12.8.149-150 (2).

Distribution: Napo River basin, Ecuador (Bockmann \& Guazzelli, 2003).

NEMUROGLANIS Eigenmann \& Eigenmann, 1889

Nemuroglanis Eigenmann \& Eigenmann, 1889b: 29. Type species: Nemuroglanis lanceolatus Eigenmann \& Eigenmann, 1889. Type by original designation. Gender: Masculine.

Imparales Schultz, 1944b: 93. Type species: Imparales mariai Schultz, 1944. Type by original designation. Gender: Masculine.

Medemichthys Dahl, 1961: 490. Type species: Medemichthys guayaberensis Dahl, 1961. Type by original designation. Gender: Masculine.

Revision: Bockmann \& Ferraris (2005).

Nemuroglanis lanceolatus Eigenmann \& Eigenmann, 1889

Nemuroglanis lanceolatus Eigenmann \& Eigenmann, 1889b: 29. Type locality: Jutahy [Brazil]. Holotype: MCZ 8169.

Distribution: Napo and Solimões River basins, Brazil and Ecuador (Bockmann \& Guazzelli, 2003).

Nemuroglanis mariai (Schultz, 1944)

Imparales mariai Schultz, 1944b: 94, fig. 1. Type locality: Río Meta system at Villavicencio, Colombia (Orinoco drainage). Holotype: USNM 121251, holotype illustrated in Bockmann \& Ferraris (2005: fig. 1c).

Medemichthys guayaberensis Dahl, 1961: 491, figure on p. 493. Type locality: small brook near the Expedition's camp I, on the left side of the Guayabero River [Colombia]. Holotype: probably lost (Cala, 1981).

Distribution: Meta and Guaviare River basins, Colombia (Bockmann \& Guazzelli, 2003, Bockmann \& Ferraris, 2005).

Nemuroglanis pauciradiatus Ferraris, 1988

Nemuroglanis pauciradiatus Ferraris, 1988a: 510, fig. 1. Type locality: Venezuela: Territorio Federal Amazonas; morichal $26.9 \mathrm{~km}$ from Puerto Ayacucho along Puerto Ayacucho to Caicara highway. Holotype: MBUCV V16450.

Distribution: Negro, Orinoco and Sanabani River basins, Brazil and Venezuela (Bockmann \& Guazzelli, 2003).

PARIOLIUS Cope, 1872

Pariolius Cope, 1872a: 289. Type species: Pariolius armillatus Cope, 1872. Type by monotypy. Gender: Masculine.

Pariolius armillatus Cope, 1872

Pariolius armillatus Cope, 1872a: 289. Type locality: the Ambyiacu [Peru]. Holotype: at ANSP; not ANSP 22004.

Distribution: Upper Amazon River basin, Brazil and Peru (Bockmann \& Guazzelli, 2003).

Remarks: Redescribed in Gosline (1940a). ANSP 22004 is a trichomycterid, thus cannot be the holotype.

PHENACORHAMDIA Dahl, 1961

Phenacorhamdia Dahl, 1961: 504. Type species: Phenacorhamdia macarenensis Dahl, 1961. Type by original designation. Gender: Feminine.

Phenacorhamdia anisura (Mees, 1987)

Heptapterus anisurus Mees, 1987a: 451, fig. 1. Type locality: Río Guarapiche near Maturin, Monagas, Venezuela. Holotype: RMNH 30499.

Distribution: Orinoco River basin, Venezuela (Bockmann \& Guazzelli, 2003).

Phenacorhamdia boliviana (Pearson, 1924)

Imparfinis bolivianus Pearson, 1924: 12, pl. 2 (fig. 3). Type locality: Huachi [Bolivia]. Syntypes: CAS 63632 (2).

Distribution: Upper Mamoré/Madeira River basin, Bolivia (Bockmann \& Guazzelli, 2003).

Phenacorhamdia hoehnei (Miranda Ribeiro, 1914)

Nannoglanis hoehnei Miranda Ribeiro, 1914: 3, pl. 1 (fig. 1). Type locality: Rio Taquary, Matto Grosso [Brazil]. Lectotype: MNRJ 787A, designated by Miranda Ribeiro (1953: 403). 
Distribution: Taquari River basin, Paraguay River drainage, Brazil (Bockmann \& Guazzelli, 2003).

Phenacorhamdia macarenensis Dahl, 1961

Phenacorhamdia macarenensis Dahl, 1961: 504, figure on p. 507. Type locality: Caño Lozada [Colombia]. Holotype: probably lost (Cala, 1981: 1).

Distribution: Guaviare River basin, Colombia (Bockmann \& Guazzelli, 2003).

Phenacorhamdia nigrolineata Zarske, 1998

Phenacorhamdia nigrolineata Zarske, 1998: 27, fig. 1. Type locality: Tierra Roja na der Strabe Campoverde Tournavista, nahe Pucallpa, Departamento Ucayali, Peru. Holotype: MTD F 20728.

Distribution: Ucayali River basin, Peru (Bockmann \& Guazzelli, 2003).

Phenacorhamdia somnians (Mees, 1974)

Heptapterus somnians Mees, 1974: 184, fig. 36. Type locality: Sangadina, a tributary of the Rio das Mortes, $2 \mathrm{~km}$ from Xaventina, Mato Grosso, Brazil. Holotype: BMNH 1971.7.29.4.

Distribution: Das Mortes River basin, Brazil (Bockmann \& Guazzelli, 2003).

Phenacorhamdia tenebrosa (Schubart, 1964)

Imparfinis tenebrosus Schubart, 1964a: 9, fig. 3. Type locality: Rio Mogi Guaçu, entre o pesqueiro da EE [=Estação Experimental de Piscicultura de Pirassununga] e o pesqueiro do Sr. Firmino (Faz. Graciosa) ca. 1 km abaixo da Cachoeira [Brazil]. Syntypes: EEBP 109 (1), EEBP 197 (5), EEBP 630 (4).

Distribution: Upper Paraná and São Francisco River basins, Brazil (Bockmann \& Guazzelli, 2003).

Phenacorhamdia unifasciata Britski, 1993

Phenacorhamdia unifasciata Britski, 1993: 42, fig. 1. Type locality: Ribeirão Santana, afluente do Rio São Bartolomeu (1550'N, 4740'W), Distrito Federal [Brazil]. Holotype: MZUSP 36691.

Distribution: Upper Paraná River basin, Brazil (Bockmann \& Guazzelli, 2003).

PIMELODELLA Eigenmann \& Eigenmann, 1888

Pimelodella Eigenmann \& Eigenmann, 1888b: 131. Type species: Pimelodus cristatus Müller \& Troschel, 1848. Type by original designation. Gender: Feminine.

Typhlobagrus Miranda Ribeiro, 1907b: [1]. Type species: Typhlobagrus kronei Miranda Ribeiro, 1907. Type by monotypy. Gender: Masculine.

Caecorhamdella Borodin, 1927a: 1. Type species: Caecorhamdella brasiliensis Borodin, 1927. Type by original designation. Gender: Feminine.

Revision: Eigenmann (1917b).

Review: Guazzelli (1997, southeastern Brazil).

Pimelodella altipinnis (Steindachner, 1864)

Pimelodus altipinnis Steindachner, 1864a: 37. Type locality: Britisch-Guiana. Holotype: NMW 45601. Illustrated and described in more detail in Steindachner (1864b: 213, pl. 2, figs. 3-4).

Distribution: Essequibo River basin, Guyana (Bockmann \& Guazzelli, 2003).

Pimelodella australis Eigenmann, 1917

Pimelodella laticeps australis Eigenmann, 1917b: 243. Type locality: Uruguayana [State of Rio Grande do Sul, Brazil]. Holotype: FMNH 57962.

Pimelodela garbei Miranda Ribeiro, 1918d: 639. Type locality: Itaqui —Rio Grande do Sul [Brazil]. Lectotype: MNRJ 923A, designated by Miranda Ribeiro (1953: 403). Name clearly is a lapsus for Pimelodella garbei.

Distribution: Uruguay River basin, and coastal river drainages of southeastern Brazil (Bockmann \& Guazzelli, 2003).

Pimelodella avanhandavae Eigenmann, 1917

Pimelodella avanhandavae Eigenmann, 1917b: 240, pl. 29 (fig. 3). Type locality: Rio Tietê at Salto Avanhandava, above the fall [Brazil]. Holotype: FMNH 57981.

Distribution: Tietê River basin, Brazil (Bockmann \& Guazzelli, 2003).

Pimelodella boliviana Eigenmann, 1917

Pimelodella boliviana Eigenmann, 1917b: 245, pl. 31 (fig. 2). Type locality: Santa Cruz de la Sierra, Bolivia. Holotype: FMNH 57976. 
Distribution: Mamoré River basin, Bolivia (Bockmann \& Guazzelli, 2003).

Pimelodella boschmai Van der Stigchel, 1964

Pimelodella boschmai Van der Stigchel, 1964: 327, fig. 1. Type locality: Mogi-Guassu (river), below the Emas falls, ... Pirassununga, Brazil. Holotype: RMNH 23248.

Pimelodella insignis Schubart, 1964a: 6, fig. 1. Type locality: Rio Mogi Guaçu, abaixo do pesqueiro da EEBP; Cochoeira [sic] de Emas, na topava; Rio Mogi Guaçu, abaixo do pesqueiro; Rio Mogi Guaçu, abaixo da cachoeira; Rio Mogi Guaçu; Rio Mogi Guaçu, pesqueiro da EEBP [Brazil]. Syntypes: EEBP 497, EEBP 497 [bis], EEBP 498, EEBP 499, EEBP 620, EEBP 620a, EEBP 647.

Distribution: Mogi Guaçu River basin, Brazil (Bockmann \& Guazzelli, 2003).

Pimelodella brasiliensis (Steindachner, 1877)

Pimelodus (Pseudorhamdia) brasiliensis Steindachner, 1877b: 608, pl. 7. Type locality: Rio Parahyba [Brazil]. Holotype: NMW 45612.

Distribution: Paraíba do Sul River basin, Brazil (Bockmann \& Guazzelli, 2003).

Pimelodella breviceps (Kner, 1857)

Pimelodus breviceps Kner, 1857: 418. Type locality: Marabitanos [Brazil]. Holotype: NMW 45615.

Distribution: Upper Negro River basin, Brazil (Bockmann \& Guazzelli, 2003).

Pimelodella buckleyi (Boulenger, 1887)

Pimelodus buckleyi Boulenger, 1887c: 275, pl. 20 (fig. 1). Type locality: Canelos [Ecuador]. Syntypes: BMNH 1880.12.8.98-99 (2).

Pimelodella copei Fowler, 1915a: 216, fig. 5. Type locality: Ambyiacu River, near Pebas, Ecuador. Holotype: ANSP 8362.

Distribution: Ambyiacu and Bobonaza River basins, upper Amazon basin (Bockmann \& Guazzelli, 2003).

Pimelodella chagresi (Steindachner, 1876)

Pimelodus (Pseudorhamdia) Chagresi Steindachner, 1876: 584. Type locality: Rio Chagres und dessen Nebenflusse bei Obispo [Panama]. Syntypes: at NMW.

Distribution: Chagres River basin, Panama (Bockmann \& Guazzelli, 2003); Magdalena, Cauca, Patia, and Catatumbo River basins, Colombia (Maldonado-Ocampo et al., 2005).

Pimelodella chaparae Fowler, 1940

Pimelodella chaparae Fowler, 1940c: 75, figs. 29-30. Type locality: Boca Chapare, Cochabamba, Bolivia. Holotype: ANSP 69021.

Distribution: Beni River basin, Bolivia (Bockmann \& Guazzelli, 2003).

Pimelodella conquetaensis Ahl, 1925

Pimelodella conquetaensis Ahl, 1925: 106. Type locality: Rio Coquetá [sic Caquetá], S.O. Columbien. Holotype: ZMB 32030.

Distribution: Caquetá River basin in upper Japurá River drainage, Colombia (Bockmann \& Guazzelli, 2003).

Pimelodella cristata (Müller \& Troschel, 1848)

Pimelodus cristatus Müller \& Troschel, in Schomburgk, 1848: 628. Type locality: Takutu und Mahu [Branco River basin, Guyana]. Syntypes: ZMB 3052 (1), ZMB 3053 (1).

Distribution: Guyana and French Guiana (Bockmann \& Guazzelli, 2003).

Remarks: Also cited as new in Müller \& Troschel (1849: 4).

Pimelodella cruxenti Fernández-Yépez, 1950

Pimelodella cruxenti Fernández-Yépez, 1950a: 5, pl. 1 (fig. 2). Type locality: Río Autana, Venezuela. Holotype: AFY 48161; whereabouts unknown.

Distribution: Autana River, Orinoco River drainage, Venezuela (Bockmann \& Guazzelli, 2003).

Pimelodella cyanostigma (Cope, 1870)

Rhamdia cyanostigma Cope, 1870b: 569. Type locality: Pebas, Ecuador. Syntypes: ANSP 8381-8383 (3).

Distribution: Ampyiacu River, upper Amazon basin, Peru (Bockmann \& Guazzelli, 2003).

Pimelodella dorseyi Fowler, 1941

Pimelodella dorseyi Fowler, 1941a: 127, figs. 4-7. Type locality: Rio Salgade, Icó, Ceará [Brazil]. Holotype: ANSP 69375. 
Distribution: Jaguaribe River basin, Brazil (Bockmann \& Guazzelli, 2003).

Pimelodella eigenmanni (Boulenger, 1891)

Pimelodus (Pimelodella) eigenmanni Boulenger, 1891: 232, footnote. Type locality: Macacos [Brazil]. Holotype: BMNH 1889.11.14.6.

Distribution: Paraíba do Sul River basin, Brazil (Bockmann \& Guazzelli, 2003).

Pimelodella eigenmanniorum (Miranda Ribeiro, 1911)

Rhamdia eigenmanniorum Miranda Ribeiro, 1911: 273. Type locality: from the Rio Parahyba and from Macacos [Brazil]. Syntypes (5): possibly MCZ 7438 (5), MCZ 7510 (1).

Distribution: Paraíba do Sul River basin and coastal rivers of Rio de Janeiro State, Brazil (Bockmann \& Guazzelli, 2003).

Pimelodella elongata (Günther, 1860)

Pimelodus elongatus Günther, 1860: 238, pl. 10 (fig. b). Type locality: Fresh waters of Esmeraldas, Ecuador. Syntypes: BMNH 1860.6.16.182-185, BMNH 1860.6.16.186-189, BMNH 1860.6.16.192, BMNH 1860.6.16.194.

Distribution: Esmeraldas River basin, Ecuador (Bockmann \& Guazzelli, 2003).

Pimelodella enochi Fowler, 1941

Pimelodella enochi Fowler, 1941a: 130, figs. 12-15. Type locality: Açude Piloes, Parahyba, Brazil. Holotype: ANSP 69378.

Distribution: Paraíba River basin in Paraíba State, Brazil (Bockmann \& Guazzelli, 2003).

Pimelodella eutaenia Regan, 1913

Pimelodella eutaenia Regan, 1913d: 466. Type locality: Rio Condoto and the Rio Sipi [Colombia]. Syntypes (5, 75145 mm TL): BMNH 1913.10.1.37-40.

Distribution: San Juan River basin, Colombia (Bockmann \& Guazzelli, 2003).

Pimelodella figueroai Dahl, 1961

Pimelodella figueroai Dahl, 1961: 498, figure on p. 500. Type locality: Caño Lozada, approx. 17 kilometers above its junction with Guayabero River [Colombia]. Holotype: probably lost (Cala, 1981).

Distribution: Guaviare River basin, Colombia (Bockmann \& Guazzelli, 2003).

Pimelodella geryi Hoedeman, 1961

Pimelodella geryi Hoedeman, 1961: 134. Type locality: French Guiana: ... mainland, Litany River, village Aloiké, sta. 29S. Holotype: ZMA 100168.

Distribution: Maroni River basin, Suriname (Bockmann \& Guazzelli, 2003).

Pimelodella gracilis (Valenciennes, 1835)

Pimelodus gracilis Valenciennes, 1835 (in Valenciennes, 1835-47): pl. 2 (fig. 5). Type locality: [not stated]. Holotype: Possibly MNHN a-9284 or MNHN 1-9285. Name available from plate; with illustrated specimen the holotype, if identifiable. Described in Cuvier \& Valenciennes (1840b: 181 (134 in Strasbourg deluxe edition)), with the locality as: Corrientes dans le Parana et les autres rivières au-dessus de $28^{\circ}$ de latitud sud [Argentina].

Distribution: Orinoco, Amazon and La Plata River basins (Bockmann \& Guazzelli, 2003).

Remarks: Bockmann \& Guazzelli (2003) list MNHN a-9284 as the holotype of Pimelodus gracilis, but without justifying this statement. If the illustrated specimen cannot be identified, the statement of Bockmann \& Guazzelli (2003) cannot be taken to be a de facto lectotype designation.

Pimelodella griffini Eigenmann, 1917

Pimelodella griffini Eigenmann, 1917b: 250, pl. 32 (fig. 3). Type locality: mountain rills near Sapucay, Paraguay. Holotype: FMNH 57974.

Distribution: Lower Paraguay River basin, Paraguay (Bockmann \& Guazzelli, 2003).

Pimelodella grisea (Regan, 1903)

Pimelodus (Pimelodella) griseus Regan, 1903b: 625. Type locality: Durango, Sapayo and Vaqueria Rivers, NW Ecuador. Syntypes: BMNH 1902.5.27.36 (1), BMNH 1902.7.29.47 (1), BMNH 1902.7.29.58 (1).

Distribution: Durango, Sapayo and Vaqueria Rivers, NW Ecuador (Bockmann \& Guazzelli, 2003).

Pimelodella hartii (Steindachner, 1877)

Pimelodus (Pseudorhamdia) Hartii Steindachner, 1877b: 611. Type locality: Rio Parahyba [Brazil]. Holotype: NMW 45784. 
Distribution: Paraíba do Sul River basin, Brazil (Bockmann \& Guazzelli, 2003).

Pimelodella hartwelli Fowler, 1940

Pimelodella hartwelli Fowler, 1940a: 222, figs.4-7. Type locality: Ucayali River basin, Contamana, Peru. Holotype: ANSP 68644.

Distribution: Ucayali River basin, Peru (Bockmann \& Guazzelli, 2003).

Pimelodella hasemani Eigenmann, 1917

Pimelodella hasemani Eigenmann, 1917b: 241, pl. 30 (fig. 1). Type locality: San Antonio de Rio Madeira. Holotype: FMNH 57980.

Distribution: Madeira River basin (Bockmann \& Guazzelli, 2003).

Pimelodella howesi Fowler, 1940

Pimelodella howesi Fowler, 1940c: 77, figs. 32-34. Type locality: Boca Chapare, Chimore River, Bolivia. Holotype: ANSP 69036.

Distribution: Beni River basin, Bolivia (Bockmann \& Guazzelli, 2003).

Pimelodella itapicuruensis Eigenmann, 1917

Pimelodella itapicuruensis Eigenmann, 1917b: 247, pl. 31 (fig. 3). Type locality: Queimadas, Rio Itapicurú [Brazil]. Holotype: FMNH 57986.

Distribution: Itapicurú River basin, Bahia, Brazil (Bockmann \& Guazzelli, 2003).

Pimelodella kronei (Miranda Ribeiro, 1907)

Typhlobagrus kronei Miranda Ribeiro, 1907b: [1], fig. on p. [1]. Type locality: Iguape, em aguas das cavernas do Iporanga, também no estado de S. Paulo [Brazil]. Holotype: MNRJ 836.

Caecorhamdella brasiliensis Borodin, 1927a: 1, fig. 1c. Type locality: Estado de São Paulo, Brazil. Holotype: AMNH 8604.

Distribution: Caves in the Ribeira do Iguape River basin, in São Paulo State, Brazil (Bockmann \& Guazzelli, 2003).

Remarks: Synonymy based on Trajano \& Britski (1992); If Caecorhamdella brasiliensis is later determined to be a distinct species of Pimelodella, it would require a new name, inasmuch as it would be preoccupied in Pimelodella by Pimelodella brasiliensis (Steindachner, 1877).

Pimelodella lateristriga (Lichtenstein, 1823)

Pimelodes lateristrigus Lichtenstein, 1823: 112. Type locality: Brasilien. Holotype: ZMB 3038.

Pimelodus lateristrigus Müller \& Troschel, 1849: 3. Type locality: Brasilien. Holotype: Probably ZMB 3038.

Distribution: Paraíba do Sul River basin, Brazil (Bockmann \& Guazzelli, 2003).

Pimelodella laticeps Eigenmann, 1917

Pimelodella laticeps Eigenmann, 1917b: 243, pl. 30 (fig. 2). Type locality: Sapucay, Paraguay. Holotype: FMNH 57969.

Distribution: Lower Paraguay River basin, Paraguay (Bockmann \& Guazzelli, 2003).

Pimelodella laurenti Fowler, 1941

Pimelodella laurenti Fowler, 1941a: 132, figs. 16-19. Type locality: Jatobá, Rio São Francisco, Pernambuco [Brazil]. Holotype: ANSP 69380 [incorrectly published as 19380].

Distribution: Lower São Francisco River basin, Brazil (Bockmann \& Guazzelli, 2003).

Pimelodella linami Schultz, 1944

Pimelodella linami Schultz, 1944c: 210, pl. 1 (fig. d). Type locality: Río Torbes, 1 km. above Táriba, Orinoco system [Venezuela]. Holotype: USNM 121132.

Distribution: Orinoco River basin, Venezuela (Bockmann \& Guazzelli, 2003).

Pimelodella macrocephala (Miles, 1943)

Nannorhamdia macrocephala Miles, 1943a: 25. Type locality: Valle del Cauca [Colombia]. Holotype: ICNMHN, probably lost (Cala, 1981).

Distribution: Cauca River basin, Colombia (Bockmann \& Guazzelli, 2003; Maldonado-Ocampo et al., 2005).

Pimelodella macturki Eigenmann, 1912

Pimelodella macturki Eigenmann, 1912b: 170, pl. 16 (fig. 1). Type locality: Creek in Mora Passage, British Guiana. Holotype: FMNH 53234.

Distribution: Approuague, Corantijn, and Nickerie River basins, and eastern coastal drainages of Suriname (Bock- 
mann \& Guazzelli, 2003).

Pimelodella martinezi Fernández-Yépez, 1970

Pimelodella martinezi Fernández-Yépez, 1970: pl. 35. Type locality: bajo Unare [Venezuela]. Holotype: AFY 70403; whereabouts unknown.

Distribution: Unare River basin, Venezuela (Bockmann \& Guazzelli, 2003).

Pimelodella meeki Eigenmann, 1910

Pimelodella eigenmanni Meek, 1905: 241. Type locality: Sao Paulo, Brazil. Holotype: FMNH 3400; holotype illustrated in Eigenmann (1917b: pl. 32, fig. 1). Preoccupied in Pimelodella by Pimelodus (Pimelodella) eigenmanni Boulenger, 1891; replaced by Pimelodella meeki Eigenmann, 1910.

Pimelodella meeki Eigenmann, 1910: 389. Type locality: São Paulo. Holotype: FMNH 3400; holotype illustrated in Eigenmann (1917b: pl. 32, fig. 1). Replacement for Pimelodella eigenmanni Meek, 1905.

Distribution: Tietê River basin, Brazil (Bockmann \& Guazzelli, 2003).

Pimelodella megalops Eigenmann, 1912

Pimelodella megalops Eigenmann, 1912b: 169, pl. 15 (fig. 2). Type locality: Tumatumari, British Guiana. Holotype: FMNH 53231.

Distribution: Approuague River, French Guiana, and Essequibo River, Guyana (Bockmann \& Guazzelli, 2003).

Pimelodella megalura Miranda Ribeiro, 1918

Pimelodella megalura Miranda Ribeiro, 1918d: 638. Type locality: S. Luiz de Caceres: Matto-Grosso [Brazil]. Lectotype: MNRJ 865A, designated by Miranda Ribeiro (1953: 403).

Distribution: Upper Paraguay River basin, Brazil (Bockmann \& Guazzelli, 2003).

Pimelodella metae Eigenmann, 1917

Pimelodella metae Eigenmann, 1917b: 244, pl. 31 (fig. 1). Type locality: Rio Negro, Villavicencio [Colombia]. Holotype: FMNH 58441.

Distribution: Meta River basin, Colombia (Bockmann \& Guazzelli, 2003).

Pimelodella modesta (Günther, 1860)

Pimelodus modestus Günther, 1860: 239, pl. 10 (fig. c). Type locality: Fresh waters of Esmeraldas, Ecuador. Syntypes: BMNH 1860.6.16.190-191 (2).

Distribution: Esmeraldas River basin, Ecuador (Bockmann \& Guazzelli, 2003); Patia River, Colombia (MaldonadoOcampo et al., 2005).

Pimelodella montana Allen, 1942

Pimelodella montana Allen, in Eigenmann \& Allen, 1942: 100, pl. 13 (fig. 2). Type locality: Rio Huallaga, Huánuco [Peru]. Syntypes: CAS 63719 (5).

Distribution: Upper Marañon River basin, Peru (Bockmann \& Guazzelli, 2003).

Pimelodella mucosa Eigenmann \& Ward, 1907

Pimelodella mucosa Eigenmann \& Ward, in Eigenmann, McAtee \& Ward, 1907: 114, pl. 32 (fig. 1). Type locality: Bahia Negra [Paraguay]. Holotype: CAS 63720.

Distribution: Paraguay River basin, Paraguay (Bockmann \& Guazzelli, 2003).

Pimelodella nigrofasciata (Perugia, 1897)

Pimelodus nigrofasciatus Perugia, 1897: 18. Type locality: Rio Beni, Missioni Mosetenes, Bolivia. Holotype: MSNG 8039 (reported to be missing in Eschmeyer et al., 1998).

Distribution: Beni River basin, Bolivia (Bockmann \& Guazzelli, 2003).

Pimelodella notomelas Eigenmann, 1917

Pimelodella notomelas Eigenmann, 1917b: 244, pl. 30 (fig. 3). Type locality: San Luiz de Caceres [Brazil]. Holotype: FMNH 57967.

Distribution: Upper Paraguay River basin, Brazil (Bockmann \& Guazzelli, 2003).

Pimelodella odynea Schultz, 1944

Pimelodella chagresi odynea Schultz, 1944c: 213, pl. 2 (fig. a). Type locality: Río San Juan at the bridge south of Mene Grande, Motatán system, Maracaibo basin [Venezuela]. Holotype: USNM 121133.

Distribution: Lake Maracaibo basin, Venezuela (Bockmann \& Guazzelli, 2003).

Pimelodella ophthalmica (Cope, 1878) 
Pimelodus ophthalmicus Cope, 1878: 675. Type locality: Peruvian Amazon. Holotype: ANSP 21102.

Distribution: Upper Amazon River basin, Peru (Bockmann \& Guazzelli, 2003).

Pimelodella pallida Dahl, 1961

Pimelodella pallida Dahl, 1961: 501, figure on p. 503. Type locality: Caño Lozada, about 500 meters above its junction with Guayabero River [Colombia]. Holotype: probably lost (Cala, 1981).

Distribution: Guaviare River basin, Colombia (Bockmann \& Guazzelli, 2003).

Pimelodella pappenheimi Ahl, 1925

Pimelodella pappenheimi Ahl, 1925: 107. Type locality: Rio Pedro bei Humboldt, Sta Catharina, Brasilien. Syntypes: ZMB 31951 (2).

Distribution: Itajaí-Açú, Itapocú and Cubatão River basins in Santa Catarina State, and Paranaguá River basin in Paraná State, Brazil (Bockmann \& Guazzelli, 2003).

Pimelodella parnahybae Fowler, 1941

Pimelodella parnahybae Fowler, 1941a: 129, figs. 8-11. Type locality: Rio Parnahyba, Therezina, Piauhy [Brazil]. Holotype: ANSP 69377.

Distribution: Parnaíba River basin, Brazil (Bockmann \& Guazzelli, 2003).

Pimelodella parva Güntert, 1942

Pimelodella parva Güntert, 1942: 34, fig. 4. Type locality: Río Paraguay, Paraguay. Holotype: NMBA 5302.

Distribution: Paraguay River basin, Paraguay (Bockmann \& Guazzelli, 2003).

Pimelodella pectinifer Eigenmann \& Eigenmann, 1888

Pimelodella pectinifer Eigenmann \& Eigenmann, 1888b: 132. Type locality: Campos [Brazil]. Holotype: MCZ 7508.

Distribution: Muriaé River basin, Brazil (Bockmann \& Guazzelli, 2003).

Pimelodella peruana Eigenmann \& Myers, 1942

Pimelodella peruana Eigenmann \& Myers, in Eigenmann \& Allen, 1942: 101, pl. 3 (fig. 5). Type locality: Inahuaya, Rio Ucayali [Peru]. Holotype: CAS 63721.

Distribution: Ucayali River basin, Peru (Bockmann \& Guazzelli, 2003).

Pimelodella peruensis Fowler, 1915

Pimelodella peruense Fowler, 1915a: 214, fig. 4. Type locality: Peruvian Amazon. Holotype: ANSP 21932.

Distribution: Upper Amazon River basin, Peru (Bockmann \& Guazzelli, 2003).

Pimelodella procera Mees, 1983

Pimelodella procera Mees, 1983: 49, fig. 3. Type locality: French Guiana: Crique Balaté. Holotype: RMNH 28588.

Distribution: Maroni River basin, French Guiana (Bockmann \& Guazzelli, 2003).

Pimelodella reyesi Dahl, 1964

Pimelodella reyesi Dahl, in Dahl \& Medem, 1964: 37, figure on p. 503. Type locality: Río Manso del Sinú, Colombia. Holotype: Whereabouts unknown.

Distribution: Sinú River basin, Colombia (Bockmann \& Guazzelli, 2003).

Pimelodella roccae Eigenmann, 1917

Pimelodella roccae Eigenmann, 1917b: 240, pl. 35 (fig. 7). Type locality: Lower Urubamba Valley. Holotype: MCZ 30975. Illustrated and described more fully in Eigenmann \& Allen (1942: 98, pl. 10, fig. 4).

Distribution: Urubamba and Beni River basins of Bolivia and Peru (Bockmann \& Guazzelli, 2003).

Remarks: Eigenmann \& Allen (1942: 98) reported that the holotype came from: Rio Comberciato, 1800 feet elevation.

Pimelodella rudolphi Miranda Ribeiro, 1918

Pimelodella rudolphi Miranda Ribeiro, 1918d: 637. Type locality: Mercado de São Paulo [Brazil]. Lectotype: MNRJ 857A, designated by Miranda Ribeiro (1953: 404).

Distribution: São Paulo State, Brazil (Bockmann \& Guazzelli, 2003).

Pimelodella serrata Eigenmann, 1917

Pimelodella serrata Eigenmann, 1917b: 235, pl. 29 (fig. 1). Type locality: San Joaquin, Bolivia. Holotype: FMNH 57979.

Distribution: Possibly from the Machupo River basin in upper Guaporé River drainage, Bolivia (Bockmann \& Guaz- 
zelli, 2003).

Pimelodella spelaea Trajano, Reis \& Bichuette, 2004

Pimelodella spelaea Trajano, Reis \& Bichuette, 2004: 317, fig. 1. Type locality: Brazil, State of Goiás, São Domingos, upper Tocantins River basin, subterranean stream tributary to São Bernardo River inside São Bernardo Cave, $13^{\circ} 49^{\prime} \mathrm{S}, 4^{\circ} 21^{\prime} \mathrm{W}$. Holotype: MZUSP 81726.

Distribution: subterranean stream tributary to São Bernardo River inside São Bernardo Cave, Tocantins River basin, Brazil (Trajano et al., 2004: 319).

Pimelodella steindachneri Eigenmann, 1917

Pimelodella steindachneri Eigenmann, 1917b: 237. Type locality: Maues, Rio Madeira... Para... Cudajas... Santarem... Rio Puty... Manacapurú [Brazil]. Syntypes: MCZ 7542a, MCZ 7566a-b, MCZ 7567a, MCZ 7547a, MCZ 7587a, MCZ 7588a.

Distribution: Amazon River basin, Brazil (Bockmann \& Guazzelli, 2003).

Pimelodella taeniophora (Regan, 1903)

Pimelodus (Pimelodella) taeniophorus Regan, 1903b: 625. Type locality: Descalvados, Matto Grosso [Brazil]. Syntypes: BMNH 1895.5.17.27-28 (2).

Distribution: Paraguay River basin, Brazil (Bockmann \& Guazzelli, 2003).

Pimelodella taenioptera Miranda Ribeiro, 1914

Pimelodella taenioptera Miranda Ribeiro, 1914: 5, figure on p. 5. Type locality: Tapyrapoan, Rio Sepotuba [Mato Grosso, Brazil]. Lectotype: MNRJ 691A, designated by Miranda Ribeiro (1953: 404).

Distribution: Upper Paraguay River basin, Brazil (Bockmann \& Guazzelli, 2003).

Pimelodella tapatapae Eigenmann, 1920

Pimelodella tapatapae Eigenmann, 1920a: 5. Type locality: Mouth of Rio Tapa Tapa, Venezuela. Holotype: CAS 57469.

Distribution: Tapa Tapa River basin in Lake Valencia drainage, Venezuela (Bockmann \& Guazzelli, 2003).

Pimelodella transitoria Miranda Ribeiro, 1907

Pimelodella transitoria Miranda Ribeiro, 1907a: 186. Type locality: Ribeirão do Alambary —Iporanga [Brazil]. Holotype: at MNRJ.

Distribution: Ribeira de Iguape River basin, Brazil (Bockmann \& Guazzelli, 2003).

Pimelodella vittata (Lütken, 1874)

Pseudorhamdia vittatus Lütken, 1874c: 34. Type locality: in flumine Rio das Velhas, in rivulis affluentibus, lacusculisque vicinis [Brazil]. Syntypes: ZMB 9175, ZMUC P 29652-ZMUC P 29657 (1 each).. Illustrated and described in more detail in Lütken (1875: 173-174, figure on p. 173).

Distribution: Upper São Francisco River basin, Brazil (Bockmann \& Guazzelli, 2003).

Pimelodella wesselii (Steindachner, 1877)

Pimelodus (Pseudorhamdia) Wesselii Steindachner, 1877b: 614, footnote. Type locality: Essequibo [Guyana]. Holotype: NMW 79188.

Distribution: Essequibo River basin, Guyana (Bockmann \& Guazzelli, 2003).

Pimelodella witmeri Fowler, 1941

Pimelodella witmeri Fowler, 1941a: 133, figs. 20-22. Type locality: Rio Jaguaribe, Orós, Ceará [Brazil]. Holotype: ANSP 69383.

Distribution: Jaguaribe River basin, Brazil (Bockmann \& Guazzelli, 2003).

Pimelodella yuncensis Steindachner, 1902

Pimelodella yuncensis Steindachner, 1902: 135. Type locality: Pacasmayo, Nord Peru. Holotype: at NMW.

Distribution: Jequetepeque River basin, Peru (Bockmann \& Guazzelli, 2003).

\section{Species inquirenda, Pimelodella}

Pimelodus bahianus Castelanu, 1855: 35, pl. 16, fig. 2. Type locality: eaux douces des environs de Bahia [Brazil]. Syntypes: MNHN b-0612 (2). Tentatively assigned to Pimelodella in Silfvergrip (1996: 16). Not treated in Bockmann \& Guazzelli, 2003). 
Pimelodella rendahli Ahl, 1925: 106. Type locality: [No locality stated]. Holotype: ZMB 32031; known only from holotype.

RHAMDELLA Eigenmann \& Eigenmann, 1888

Rhamdella Eigenmann \& Eigenmann, 1888b: 129. Type species: Rhamdia eriarcha Eigenmann \& Eigenmann, 1888. Type by original designation. Gender: Feminine. Originally proposed as a subgenus of Rhamdia.

Rhamdella aymarae Miquelarena \& Menni, 1999

Rhamdella aymarae Miquelarena \& Menni, 1999: 203, fig. 1. Type locality: Argentina, Salta Province, Río Itiyuro, $500 \mathrm{~m}$ below the Itiyuro Dam, route 34 between Estación Pocitos and Araguay, 22 $07^{\prime} \mathrm{S}, 6^{\circ} 11^{\prime} \mathrm{W}$. Holotype: ILPLA 611.

Distribution: Itiyuro River basin, Argentina (Bockmann \& Guazzelli, 2003).

Rhamdella eriarcha (Eigenmann \& Eigenmann, 1888)

Rhamdia eriarcha Eigenmann \& Eigenmann, 1888b: 129. Type locality: Rio Grande do Sul [Brazil]. Holotype: MCZ 27272.

Rhamdella straminea Cope, 1894: 93, pl. 8 (fig. 10). Type locality: Rio Grande do Sul [Brazil]. Syntypes (5): Possibly ANSP 21604 (1), ANSP 39835-36 (2), ANSP 21581-84 (4), ANSP 23216 (1).

Rhamdella lemai Bertoletti, 1967: 78, fig. 2. Type locality: Pôrto Alegre, Guaíba River (Ponta Grossa) [Brazil]. Holotype: MRCN 1897.

Distribution: Jacuí River basin, Brazil (Bockmann \& Guazzelli, 2003).

Rhamdella exsudans (Jenyns, 1842)

Pimelodus exsudans Jenyns, 1842 in Jenyns, 1840-42: 111. Type locality: ? Rio de Janeiro [Brazil]. Syntypes: Department of Zoology, University of Cambridge V.132 (2).

Distribution: Rio de Janeiro State, Brazil (Bockmann \& Guazzelli, 2003).

Rhamdella gilli (Starks, 1906)

Rhamdia gilli Starks, 1906: 769, pl. 65 (fig. 1). Type locality: Eten, Peru, in the Rio Eten. Holotype: USNM 53472.

Distribution: Eten River basin, Peru (Bockmann \& Guazzelli, 2003).

Rhamdella ignobilis Steindachner, 1907

Rhamdella ignobilis Steindachner, 1907e: 484. Type locality: Flusse Cubataõ um Staate Santa Catharina bei Theresopolis (Brasilien). Syntypes: at NMW.

Distribution: Cubatão River basin in Santa Catarina State, Brazil (Bockmann \& Guazzelli, 2003).

Rhamdella jenynsii (Günther, 1864)

Pimelodus jenynsii Günther, 1864: 128. Type locality: Rio de Janeiro [Brazil]. Type(s): at Department of Zoology, University of Cambridge. Name based on one or more specimens identified in Jenyns (1840-42: 110) as Pimelodus gracilis, which were deposited at the University of Cambridge.

Distribution: Rio de Janeiro State, Brazil (Bockmann \& Guazzelli, 2003).

Rhamdella leptosoma Fowler, 1914

Rhamdella leptosoma Fowler, 1914: 260, fig. 12. Type locality: Rupununi River, British Guiana ... in the highlands of British Guiana, approximately secured in North Latitude $2^{\circ}$ to $3^{\circ}$, and West Longitude $50^{\circ} 20^{\prime}$. Holotype: ANSP 39340.

Distribution: Rupununi River basin, Guyana (Bockmann \& Guazzelli, 2003).

Rhamdella longipinnis Borodin, 1927

Rhamdella longipinnis Borodin, 1927b: 6. Type locality: Prov. St. Paulo, Brazil. Holotype: AMNH 8642.

Distribution: São Paulo State, Brazil (Bockmann \& Guazzelli, 2003).

Rhamdella longiuscula Lucena \& da Silva, 1991

Rhamdella longiuscula Lucena \& da Silva, 1991: 32, figs. 2a, 3a. Type locality: Brasil: Rio Grande do Sul: arroio

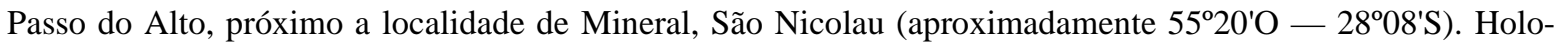
type: MCP 14441.

Distribution: Middle Uruguay River basin, Brazil (Bockmann \& Guazzelli, 2003).

Rhamdella montana Eigenmann, 1913

Rhamdella montana Eigenmann, 1913: 421, pl. 32 (fig. 1). Type locality: Queta. Small brook in highlands southeast 
of Tarma [Peru]. Holotype: FMNH 56067.

Distribution: Ucayali River basin, Peru (Bockmann \& Guazzelli, 2003).

Rhamdella papariae Fowler, 1941

Rhamdella papariae Fowler, 1941a: 135, figs. 27-30. Type locality: Lago Papary, Rio Grande do Norte, Brazil. Holotype: ANSP 69387.

Distribution: Lake Parary basin (?), Brazil (Bockmann \& Guazzelli, 2003).

Rhamdella robinsoni Fowler, 1941

Rhamdella robinsoni Fowler, 1941a: 135, figs. 23-26. Type locality: São José do Egito, Pernambuco [Brazil]. Holotype: ANSP 69386.

Distribution: Lower São Francisco River basin, Brazil (Bockmann \& Guazzelli, 2003).

Rhamdella rusbyi Pearson, 1924

Rhamdella rusbyi Pearson, 1924: 12, pl. 2 (fig. 4). Type locality: Rio Colorado, Lower Bopi, Bolivia. Holotype: CAS 63728.

Distribution: Beni River basin, Bolivia (Bockmann \& Guazzelli, 2003).

Rhamdella wolfi Fowler, 1941

Rhamdella wolfi Fowler, 1941a: 136, figs. 31-34. Type locality: Rio Choró, Ceará [Brazil]. Holotype: ANSP 69388. Distribution: Choró River basin, Brazil (Bockmann \& Guazzelli, 2003).

RHAMDIA Bleeker, 1858

Pteronotus Swainson, 1839: 190, 309. Type species: Heterobranchus 5-tentaculatus Sp. Ag. [sic Heterobranchus 6tentaculatus Spix \& Agassiz, 1829; = Heterobranchus sextentaculatus ]. Type by original designation. Gender: Masculine. Preoccupied by Pteronotus Gray, 1837 in Mammalia, not replaced. See Miller (1905) for comments.

Rhamdia Bleeker, 1858b: 197, 207. Type species: Pimelodus sebae Cuvier, 1829. Type by subsequent designation by Gill (1861c: 48). Gender: Feminine.

Pimelenotus Gill, 1858: 387. Type species: Pimelenotus vilsoni Gill, 1858. Type by subsequent designation by Eigenmann \& Eigenmann (1890: 116). Gender: Masculine. Listed a Pimelotus on p. 387, but corrected in printed corrigenda to Pimelenotus.

Caecorhamdia Norman, 1926c: 325. Type species: Caecorhamdia urichi Norman, 1926. Type by monotypy. Gender: Feminine.

Revision: Silfvergrip (1996).

Phylogeny: Perdices et al. (2002, Central American species).

Remarks: The name Rhamdia micayi Eigenmann (in Pearson, 1924: 11) has been treated in some literature as available, and as a valid species. However, the name was originally published without distinguishing features (Bockmann \& Guazzelli, 2003) and has apparently not yet been made available.

Rhamdia enfurnada Bichuette \& Trajano, 2005

Rhamdia enfurnada Bichuette \& Trajano, 2005: 589, fig. 1. Type locality: Brazil, southwestern State of Bahia, Serra

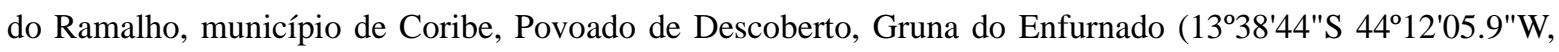
elevation 628 m), middle São Francisco River basin. Holotype: MZUSP 87776.

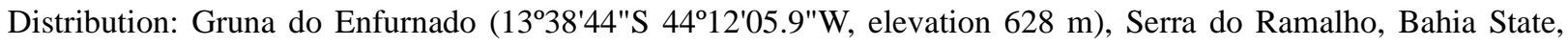
middle São Francisco River basin, northeastern Brazil (Bichuette \& Trajano, 2005).

Rhamdia foina (Müller \& Troschel, 1848)

Pimelodus foina Müller \& Troschel, in Schomburgk, 1848: 628. Type locality: an den felsenreichen Stellen des Takutu [Guyana]. Holotype: ZMB 3039. Also cited as new in Müller \& Troschel (1849: 5).

Rhamdia holomelas rupununi Fowler, 1914: 258, fig. 11. Type locality: Rupununi River, British Guiana ... in the highlands of British Guiana, approximately secured in North Latitude $2^{\circ}$ to $3^{\circ}$, and West Longitude $50^{\circ} 20^{\prime}$. Holotype: ANSP 39339.

Distribution: Essequibo River, Guyana; Branco, Negro, Tocantins, and Trombetas River basins, Brazil (Bockmann \& Guazzelli, 2003).

Rhamdia guasarensis Do Nascimiento, Provenzano \& Lundberg, 2004

Rhamdia guasarensis Do Nascimiento, Provenzano \& Lundberg, 2004: 565, figs. 1-4. Type locality: Surgencia del 
Tigre at $2.5 \mathrm{~km}$ W of Cerro Yolanda, Río Guasare basin, Sierra de Perijá, Estado Zulia, Venezuela (1052'53"N, 72³0'03"W. Holotype: MBUCV V-29604.

Distribution: Guasare River basin, northwestern Venezuela (Do Nascimiento et al., 2004).

Rhamdia humilis (Günther, 1864)

Pimelodus humilis Günther, 1864: 129. Type locality: Venezuela. Lectotype: BMNH 1965.2.19.1, designated by Silfvergrip (1996: 25).

Rhamdia guairensis Eigenmann, 1920a: 6. Type locality: Rio Guaire near Caracas [Venezuela]. Lectotype: CAS 76655, illustrated in, and designated by, Silfvergrip (1996: 34, pl. 1, figs. 3, 4).

Nannorhamdia benedettii Fernández-Yépez \& Martín Salazar, 1952: 35, figure on p. 37. Type locality: Quebrada Ojo de Agua, Baruta [Venezuela]. Holotype: MHNLS 82.

Distribution: Rivers around Caracas, along the Caribbean coast of Venezuela (Bockmann \& Guazzelli, 2003).

Rhamdia itacaiunas Silfvergrip, 1996

Rhamdia itacaiunas Silfvergrip, 1996: 83, pl. 2 (fig. 1). Type locality: Brazil, Est. Amazonas, Rio Tocantins basin, 'Igarapé Repartimento'. Holotype: INPA 7985.

Distribution: Middle Tocantins River basin, Brazil (Bockmann \& Guazzelli, 2003).

Rhamdia jequitinhonha Silfvergrip, 1996

Rhamdia jequitinhonha Silfvergrip, 1996: 84, pl. 3 (fig. 2). Type locality: Brasil, Est. Minas Gerais, Rio Araçuaí, Santa Rita. Holotype: MZUSP 38630.

Distribution: Jequitinhonha River basin, Brazil (Bockmann \& Guazzelli, 2003).

Rhamdia laluchensis Weber, Allegrucci \& Sbordoni, 2003

Rhamdia laluchensis Weber, Allegrucci \& Sbordoni, 2003: 275, fig. 1. Type locality: Mexico: Chiapas: Municipio Ocozocoautla, subterranean watercourse in Sistema de La Lucha (17 $\left.03^{\prime} 40^{\prime \prime} \mathrm{N}, 93^{\circ} 53^{\prime} 23^{\prime \prime} \mathrm{W}\right)$, entrance approximately $5 \mathrm{~km}$ southwest of village of Aguablanca situated at extreme eastern shore of Malpaso Reservoir (Presa Nezahualcoyotl). Holotype: ZMH 9373.

Distribution: Chiapas, Mexico; known only from type locality (Weber et al., 2003).

Rhamdia laticauda (Kner, 1857)

Pimelodus laticaudus Kner, 1857: 420. Type locality: Mexico. Syntypes: at NMW (not found; Silfvergrip, 1996: 16).

Pimelodus hypselurus Günther, 1864: 126. Type locality: Mexico. Holotype: BMNH 1858.11.22.32.

Pimelodus motaguensis Günther, 1864: 127. Type locality: Rio Motagua. Holotype: BMNH 1865.4.29.39.

Pimelodus petenensis Günther, 1864: 126. Type locality: Lake Peten. Holotype: BMNH 1864.1.26.371.

Pimelodus polycaulus Günther, 1864: 131. Type locality: Rio San Geronimo, Guatemala. Holotype: BMNH 1864.1.26.93.

Pimelodus salvini Günther, 1864: 130. Type locality: Rio de San Geronimo, Guatemala. Holotype: BMNH 1861.8.2.16.

Pimelodus (Rhamdia) brachypterus Cope, 1867: 404. Type locality: Orizava, Mexico. Holotype: ANSP 16471.

Rhamdia sacrificii Barbour \& Cole, 1906: 156, pl. 2. Type locality: Sacrificial Cenote, near Chichen Itza, Yucatan [Mexico]. Lectotype: MCZ 29073, designated by Silfvergrip (1996: 30).

Rhamdia cabrerae Meek, 1906: 93. Type locality: Amatitlan, Guatemala. Holotype: FMNH 5501.

Pimelodus brachycephalus Regan, 1907b: 258. Type locality: Guatemala, Rio Nacasil. Lectotype: BMNH 1875.6.9.5, designated by Silfvergrip (1996: 31).

Pimelodus Rogersi Regan, 1907b: 259. Type locality: Costa Rica, Irazu. Lectotype: BMNH 1907.2.11.1, designated by Silfvergrip (1996: 31).

Rhamdia regani Meek, 1907b: 144. Type locality: Turrialba, Costa Rica. Holotype: FMNH 6019.

Rhamdia underwoodi Regan, 1907, in Regan, 1906-08: 135, pl. 23 (fig. 4). Type locality: Costa Rica, Juan Viñas. Lectotype: BMNH 1907.6.28.33, designated by Silfvergrip (1996: 32).

Rhamdia amatitlanensis Fowler, 1936a: 518, fig. 10. Type locality: Stream issuing from Lake Amatitlan [Guatemala]. Holotype: ANSP 64136.

Rhamdia laticauda typhla Greenfield, Greenfield \& Woods, 1982: 564, fig. 1. Type locality: A cave in Belize, in an area known as Las Cuevas, near Millionario in the Mountain Pine Ridge (16 $\left.45^{\prime} \mathrm{N} 89^{\circ} 00^{\prime} \mathrm{W}\right)$. Holotype: FMNH 
71605.

Rhamdia zongolicensis Wilkens, 1993: 375, fig. 1. Type locality: Cueva del Ostoc, Sierra de Zongolica (Veracruz, Mexico), road from Comalapa to Tezonapa. Holotype: ZMH 9249.

Distribution: Atlantic slope river basins from central Mexico to northern Panama, in (Miller et al., 2005).

Rhamdia laukidi Bleeker, 1858

Rhamdia laukidi Bleeker, 1858b: 208. Type locality: Venezuela, Est. Amazonas, first Río Casiquiare caño, ca. 5 min. from confluence of Río Casiquiare and Río Orinoco left side, Caño Caripo, ca. 3 km from mouth; 03 06'N, 65 50'W. Neotype: ANSP 174652, illustrated in, and designated by, Silfvergrip (1996: 17, pl. 3, figs. 3, 4).

Pimelodus holomelas Günther, 1863: 442. Type locality: Essequibo [Guyana]. Lectotype: BMNH 1864.1.21.8, designated by Silfvergrip (1996: 23).

Rhamdia tenella Eigenmann \& Eigenmann, 1888b: 127. Type locality: Cudajas [Brazil]. Holotype: MCZ 7547.

Distribution: Amazon, Essequibo, and Orinoco River basins (Bockmann \& Guazzelli, 2003).

Rhamdia macuspanensis Weber \& Wilkens, 1998

Rhamdia macuspanensis Weber \& Wilkens, 1998: 1000, fig. 3. Type locality: Watercourse in the Grutas de Agua Blanca, Balneario de Agua Blanca at km 64.9 of the road 186 (carretera federal) from Villahermosa to Chetumal, ca. $20 \mathrm{~km}$ se. of Macuspana, Tabasco, Mexico (17³7'15.5"N, 92²8'22.6"W). Holotype: ZMH 8874.

Distribution: Grutas de Agua Blanca, at the northern edge of the Sierra del Norte de Chiapas, Tabasco, Mexico (Weber \& Wilkens, 1998).

Rhamdia muelleri (Günther, 1864)

Pimelodus mülleri Günther, 1864: 119. Type locality: Para, Brazil; River Capin, Para, Brazil; Surinam [restricted to River Capin by lectotype designation]. Lectotype: BMNH 1849.11.8.101 (133.6 mm SL), designated by Silfvergrip (1996: 23).

Pimelodus (Rhamdia) Knerii Steindachner, 1877b: 631, footnote. Type locality: Marabitanos [Brazil]. Lectotype: NMW 45790, designated by Silfvergrip (1996: 27).

Rhamdia obesa Eigenmann \& Eigenmann, 1888b: 124. Type locality: Teffé [Brazil]. Holotype: MCZ 7518.

Distribution: Amazon, Ampyiacu, Aripuanã, Capim, Essequibo, Madeira, Negro, Orinoco, Solimões, Tocantins and Trombetas River basins (Bockmann \& Guazzelli, 2003).

Remarks: Lectotype of Pimelodus mülleri Günther, 1864, incorrectly listed by Silfvergrip (1996) and elsewhere as: BMNH 1849.11.8.

Rhamdia nicaraguensis (Günther, 1864)

Pimelodus nicaraguensis Günther, 1864: 125. Type locality: Lake of Nicaragua. Holotype: BMNH 1864.1.26.212.

Pimelodus managuensis Günther, 1867c: 603. Type locality: Lake Managua [Nicaragua]. Holotype: BMNH 1865.7.20.37.

Rhamdia alfaroi Fowler, 1932b: 382, figure on p. 381. Type locality: Escobal, 400 meters elevation, Costa Rica. Holotype: ANSP 53934.

Rhamdia luigiana Villa, 1977: 133, fig. 1. Type locality: Nicaragua: Departamento de Granada; Lake Nicaragua, between Ometepe and Zapatera Islands. Holotype: USNM 217535.

Distribution: Rivers of Costa Rica and Nicaragua (Bockmann \& Guazzelli, 2003).

Rhamdia parryi Eigenmann \& Eigenmann, 1888

Rhamdia Parryi Eigenmann \& Eigenmann, 1888b: 130. Type locality: Rio Zanaleneo, near Tonala, Chiapas, Mexico. Lectotype: MCZ 27273, designated by Silfvergrip (1996: 29).

Distribution: Pacific draining rivers of Central America, from Tapanatepec River, Mexico, to southern Guatemala (Miller et al., 2005).

Remarks: Treated by Silfvergrip (1996) and Bockmann \& Guazzelli (2003) as a synonym of Rhamdia laticauda (Kner), but valid in Miller et al. (2005).

Rhamdia poeyi Eigenmann \& Eigenmann, 1888

Rhamdia poeyi Eigenmann \& Eigenmann, 1888b: 127. Type locality: Goyaz [Brazil]. Holotype: MCZ 8196.

Distribution: Mamoré, and Tocantins River basins, Brazil, and upper Napo River, Ecuador (Bockmann \& Guazzelli, 2003).

Rhamdia quelen (Quoy \& Gaimard, 1824) 
? Silurus quadrimaculatus Bloch, 1794: 37, pl. 368 (fig. 2). Type locality: Amerika. Holotype: ZMB 2944 (probably lost; Silfvergrip, 1996: 9).

? Silurus erythropterus Bloch, 1794: 42, pl. 369, fig. 2. Type locality: Amerika. Syntypes: ZMB 3048 (1), ZMB 8786 (1).

Pimelodus quelen Quoy \& Gaimard, 1824, in Freycinet, 1824-1825: 228, pl. 49 (fig. 3). Type locality: Peru, Depto Loreto, right bank quebradita tributary to R. Samiria between Caño Pastos and Hamburgo, by neotype designation. Neotype: NRM 16091, illustrated in, and designated by, Silfvergrip (1996: 12, 97, 139: pl. 7, figs. 2, 3).

Pimelodus sebae Cuvier, 1829: 294. Type locality: Peru, Depto Loreto, right bank quebradita tributary to R. Samiria between Caño Pastos and Hamburgo, by neotype designation. Neotype: NRM 16091, designated by Silfvergrip (1996: 13). Name made available by reference to Seba (1734-1765: pl. 29, fig. 5), for which the underlying specimen is apparently lost.

Pimelodus namdia Cuvier, 1829: 294. Type locality: Peru, Depto Loreto, right bank quebradita tributary to R. Samiria between Caño Pastos and Hamburgo, by neotype designation. Neotype: NRM 16091, designated by Silfvergrip (1996: 12).

? Heterobranchus sextentaculatus Spix \& Agassiz, 1829: 28, pl. 11. Type locality: Peru, Depto Loreto, right bank quebradita tributary to Río Samiria between Caño Pastos and Hamburgo, by neotype designation. Neotype: NRM 16091, designated by Silfvergrip (1996: 13). As Heterobranchus 6-tentaculatus on caption to plate.

Pimelodus sapo Valenciennes, 1835 (in Valenciennes, 1835-47): pl. 2 (fig. 6). Type locality: [not stated]. Holotype: MNHN 0000-1576. Name available from plate; described in Cuvier \& Valenciennes (1840b: 179; 133 of Strasbourg deluxe edition), with type locality given as: Buénos-Ayres.

Pimelodus Hilarii Valenciennes, in Cuvier \& Valenciennes, 1840b: 180 (134 in Strasbourg deluxe edition). Type locality: les riviéres qui se jettent dans celle de Saint-François au Brésil ... Monté-Vidéo [restricted to: SaintFrançois, by lectotype designation]. Lectotype: MNHN a-9415, designated by Silfvergrip (1996: 14, 147).

Pimelodus Pentlandii Valenciennes, in Cuvier \& Valenciennes, 1840b: 183 (135 in the Strasbourg deluxe edition), pl. 435. Type locality: de l'Apurimac. Lectotype: BMNH 1862.11.15.11, designated by Silfvergrip (1996: 14, 139).

Pimelodus Stegelichii Müller \& Troschel, in Schomburgk, 1848: 628. Type locality: Britisch-Guiana. Lectotype: ZMB 3043 (182.2 mm SL), designated by Silfvergrip (1996: 15). Also reported as new, with types listed as coming from Surinam in Müller \& Troschel (1849: 3).

Pimelodus Sellonis Müller \& Troschel, 1849: 2. Type locality: Brasilien. Holotype: ZMB 3041.

Pimelodus Deppei Müller \& Troschel, 1849: 3. Type locality: Sandwich-Inseln [in error; (Silfvergrip, 1996: 15) indicated that the specimens were probably from southern Mexico]. Lectotype: ZMB 3046, designated by Silfvergrip (1996: 15, 133).

Pimelodus musculus Müller \& Troschel, 1849: 4. Type locality: America. Holotype: ZMB 3048. Holotype is also a syntype of Silurus erythropterus Bloch, 1794.

Pimelenotus Vilsoni Gill, 1858: 391. Type locality: Trinidad. Holotype: possibly USNM 5927 (Silfvergrip, 1996 : 22).

Pimelodus cinerascens Günther, 1860: 237, pl. 10 (fig. a). Type locality: Fresh waters of Guayaquil and Esmeraldas, Ecuador. Lectotype: BMNH 1860.6.16.193, designated by Silfvergrip (1996: 22, 134).

Pimelodus guatemalensis Günther, 1864: 122. Type locality: Guatemala, Huamuchal. Lakes with brackish water. Lectotype: BMNH 1864.1.26.210, designated by Silfvergrip (1996: 24).

Pimelodus wuchereri Günther, 1864: 123. Type locality: Bahia. Lectotype: BMNH 1864.1.19.18, designated by Silfvergrip (1996: 24).

Pimelodus godmanni Günther, 1864: 124. Type locality: Guatemala, Mexico ... Lower Vera Paz ... Rio Motagua ... Mexico. Syntypes: BMNH 1856.3.17.39-40 (2), BMNH 1856.3.17.38 (1), BMNH 1864.1.26.99-100 (2). Lectotype designation of BMNH 1864.1.26.94 by Silfvergrip (1996: 24, 133), is invalid, inasmuch as the stated locality of that specimen: Rio San Geronimo is not one of the localities reported in Günther and, therefore, the specimen was not one of the original syntypes.

? Pimelodus micropterus Günther, 1864: 124. Type locality: Guatemala ... River of San Geronimo. Holotype: BMNH 1864.1.26.92. 
Pimelodus (Rhamdia) Baronis Mülleri Troschel, in Müller, 1865: 636. Type locality: Still. Oc. Type (s): Probably lost (Silfvergrip, 1996: 25). Silfvergrip (1996: 25) interpreted the locality of the original types as western Mexico.

Pimelodus wagneri Günther, 1868c: 474. Type locality: Pacific and Atlantic rivers of Panama. Type(s): at NMW. Type series may include NMW 45618, 45619 (Silfvergrip, 1996: 26). Species based on specimens from "Rio Chagres, Guajaquil und Neu-Granada" in Kner \& Steindachner (1864: 52) identified as Pimelodella cinerascens.

Rhamdia dorsalis Gill, 1870: 94. Type locality: the Maranon, or Upper Amazon, and Napo Rivers. Holotype: USNM 35334.

Pimelodus (Rhamdia) Parahybae Steindachner, 1877b: 615. Type locality: Rio Parahyba, Brasilien. Syntypes: NMW 45852.

Pimelodus (Rhamdia) Queleni cuprea Steindachner, 1877b: 623. Type locality: aus dem Parahyba bei Juiz de Fora (im mittleren lauf des Stromes). Lectotype: NMW 45877: 1, designated by Silfvergrip (1996: 146). Originally as Pimelodus (Rhamdia) Queleni (var. cuprea).

Pimelodus (Rhamdia) Cuyabae Steindachner, 1877b: 633, footnote. Type locality: Cuyaba. Lectotype: NMW 45919: 1, designated by Silfvergrip (1996: 28).

Rhamdia bransfordii Gill, 1877: 337. Type locality: Panama ... Camp Marie Caretta. Holotype: USNM 16674.

Pimelodus bathyurus Cope, 1878: 674. Type locality: Peruvian Amazon. Lectotype: ANSP 21437, designated by Silfvergrip (1996: 28).

Rhamdia oaxacae Meek, 1902: 74, pl. 14. Type locality: Rio Quiotepec, Cuicatlan, Oaxaca, Mexico. Holotype: FMNH 3716 (Originally published as 3717, see FMNH online catalog).

Rhamdia depressa Barbour \& Cole, 1906: 155, pl. 1. Type locality: Ikil Cenote, near Chichen-Itza, Yucatan [Mexico]. Lectotype: MCZ 29072, designated by Silfvergrip (1996: 30, 133).

Rhamdia barbata Meek, 1907a: 106. Type locality: San Francisco, Guatemala. Holotype: FMNH 5906.

Pimelodus Boucardi Regan, 1907b: 258. Type locality: Yucatan. Holotype: BMNH 1880.7.13.33.

Rhamdia heteracantha Regan, 1907, in Regan, 1906-08: 134. Type locality: Costa Rica, Juan Viñas. Holotype: BMNH 1907.6.28.32.

Rhamdia nasuta Meek, 1909: 207. Type locality: Buenos Aires de Terraba, Costa Rica. Holotype: FMNH 6480.

Rhamdia branneri Haseman, 1911b: 377, pl. 75. Type locality: Creek of the Rio Iguassú near Serrinha, Paraná, Brazil. Holotype: FMNH 54235.

Rhamdia branneri voulezi Haseman, 1911b: 378, pl. 76. Type locality: Porto União da Victoria, Rio Iguassú. Holotype: FMNH 54238.

Rhamdia mounseyi Regan, 1913c: 282. Type locality: River Ucayali, Peru. Lectotype: BMNH 1913.7.30.13, designated by Silfvergrip (1996: 33, 139).

Rhamdia riojae Fowler, 1915a: 209, fig. 2. Type locality: Rioja, near Moyabamba and Baka Puerto, on or near the lower course of the Huallagua River, Peru. Holotype: ANSP 21101.

Rhamdia ortoni Fowler, 1915a: 211, fig. 3. Type locality: Peruvian Amazon. Holotype: ANSP 21928.

Rhamdia microps Eigenmann, in Eigenmann \& Fisher, 1917: 394, pl. 38. Type locality: Uruguayana. Holotype: FMNH 58285.

Rhamdia pubescens Miranda Ribeiro, 1920: 11, sixth unnumbered plate. Type locality: Urucum (proximo de Corumbá), Matto-Grosso [Brazil]. Lectotype: MNRJ 925A, designated by Miranda Ribeiro (1953: 404), but perhaps specimen not isolated. Lectotype also selected by Silfvergrip (1996: 34), and listed as MNRJ 925.

Silurus ribularis Larrañaga, 1923: 376. Type locality: Peru, Depto Loreto, right bank quebradita tributary to R. Samiria between Caño Pastos and Hamburgo, by neotype designation. Neotype: NRM 16091, designated by Silfvergrip (1996: 35). Name spelled Silurus rivularis in Silfvergrip (1996) and Bockmann \& Guazzelli (2003), the latter of which includes a comment that ribularis is a misspelling, but no internal evidence supporting that claim was found.

Silurus 9-radiatus Larrañaga, 1923: 385. Type locality: Peru, Depto Loreto, right bank quebradita tributary to R. Samiria between Caño Pastos and Hamburgo. Neotype: NRM 16091, designated by Silfvergrip (1996: 35). Apparently an alternate new name for Silurus ribularis Larrañaga, 1923. 
Caecorhamdia urichi Norman, 1926c: 325, fig. 1. Type locality: Pool in interior of the Guacharo Cave, Trinidad. Lectotype: BMNH 1926.7.28.1 (99.7 mm SL, smaller of 2 specimens), designated by Silfvergrip (1996: 35).

Rhamdia guatemalensis muriei Hubbs, 1935: 7, pl. 4 (fig. 1). Type locality: a stagnant aguada ... at edge of swamp, in the high tropical "bush" region at Uaxactun, Petén, Guatemala. Holotype: UMMZ 97881.

Rhamdia guatemalensis decolor Hubbs, 1936: 201, pl. 1 (fig. 3). Type locality: Mexico ... San Bulha Cave, Motul, Yucatan. Holotype: UMMZ 102217.

Rhamdia guatemalensis stygaea Hubbs, 1936: 203, pl. 1 (fig. 2). Type locality: Mexico ... San Isidro Cave in Salar Colony, near Merida. Holotype: UMMZ 102218.

Rhamdia saijaensis Rendahl, 1941: 2. Type locality: Rio Saija, südl. von Buenaventura, zwischen B. und Guapi, Kolumbiens. Holotype: NRM 10675.

Rhamdia duquei Eigenmann \& Pearson, in Eigenmann \& Allen, 1942: 93. Type locality: Rio Urubamba, Santa Ana. Syntypes: SU 57895 (36). Although the original description indicates the type series consisted of a "type" and paratypes, there were no characteristics presented to indicate which of the specimens was considered the holotype. As such, the type series must be treated as syntypes.

Rhamdia sebae Martyi Güntert, 1942: 33. Type locality: Riactis Canâwé, Dept. Ita, Paraguay. Holotype: NMBA 5279.

Rhamdia lehmanni Dahl, 1961: 487, figure on p. 489. Type locality: a small pool in a brook tributary to the Guayabero River, approx. 1500 meters from Expedition's camp No. 1, and about 1000 meters from the mouth of the brook, on the left side of the river. Holotype: probably lost (Cala, 1981; Silfvergrip, 1996: 37).

Distribution: Rivers from Mexico to central Argentina (Bockmann \& Guazzelli, 2003).

Rhamdia reddelli Miller, 1984

Rhamdia reddelli Miller, 1984: 136, fig. 1. Type locality: Cueva del Nacimiento del Río San Antonio, ca. 9 km SW of Acatlán, Oaxaca, on Atlantic slope of eastern Mexico. Holotype: UMMZ 211164; illustrated in Miller et al. (2005: 175, fig. 6-185).

Distribution: Caves of Papaloapan River basin, Atlantic slope, Mexico (Miller et al., 2005).

Remarks: Treated as a synonym of Rhamdia laticauda (Kner) by Silfvergrip (1996) and Bockmann \& Guazzelli, 2003).

Rhamdia xetequepeque Silfvergrip, 1996

Rhamdia xetequepeque Silfvergrip, 1996: 98, pl. 2 (fig. 3). Type locality: Peru, Jequetepeque River, just W of Chilete (06'19'90"S, 78²5'90"W). Holotype: ROM 70112.

Distribution: Jequetepeque River basin, Peru (Bockmann \& Guazzelli, 2003).

RHAMDIOGLANIS Ihering, 1907

Rhamdioglanis Ihering, 1907: 16. Type species: Rhamdioglanis frenatus Ihering, 1907. Type by original designation. Gender: Masculine.

Rhamdioglanis frenatus Ihering, 1907

Rhamdioglanis frenatus Ihering, 1907: 16. Type locality: riverlets of the Island of S. Sebastião, State of S. Paulo. Lectotype: MZUSP 154, designated by Britski (1969: 205).

Distribution: Streams in the Island of São Sebastião, São Paulo State, Brazil (Bockmann \& Guazzelli, 2003).

Rhamdioglanis transfasciatus Miranda Ribeiro, 1908

Rhamdioglanis transfasciatus Miranda Ribeiro, 1908b: [3]. Type locality: Rio Bethary [Brazil]. Syntypes: MNRJ 965 (2).

Distribution: Coastal streams of Brazil from Rio de Janeiro to Santa Catarina State (Bockmann \& Guazzelli, 2003).

RHAMDIOPSIS Haseman, 1911

Rhamdiopsis Haseman, 1911b: 375. Type species: Rhamdiopsis moreirai Haseman, 1911. Type by monotypy. Gender: Feminine.

Rhamdiopsis microcephala (Reinhardt, 1874)

Rhamdia microcephala Reinhardt, in Lütken, 1874c: 35. Type locality: in flumine Rio das Velhas. Syntypes: ZMUC P 29658 (1), ZMUC P 29659 (1), ZMUC P 29660 (1). Illustrated and described in more detail in in Lütken 
(1875: 177-179, pl. 3, fig. 7).

Distribution: Upper Paraná and São Francisco River basins, Brazil (Bockmann \& Guazzelli, 2003).

Rhamdiopsis moreirai Haseman, 1911

Rhamdiopsis moreirai Haseman, 1911b: 375, pl. 73. Type locality: Serrinha Paraná, Rio Iguassú. Holotype: FMNH 54372.

Distribution: Iguaçu and Ribeira de Iguape River basins, Brazil (Bockmann \& Guazzelli, 2003).

TAUNAYIA Miranda Ribeiro, 1918

Taunayia Miranda Ribeiro, 1918d: 642. Type species: Taunayia marginata Miranda Ribeiro, 1918. Type by monotypy. Gender: Feminine.

Taunayia bifasciata (Eigenmann \& Norris, 1900)

Nannoglanis bifasciatus Eigenmann \& Norris, 1900: 350. Type locality: S. Paulo, Brazil. Holotype: CAS 75771.

Taunayia marginata Miranda Ribeiro, 1918d: 642. Type locality: Piquete [Brazil]. Holotype: MZUSP 2273; holotype illustrated in Oliveira \& Britski (2000: 129, fig. 1).

Distribution: Upper Paraíba do Sul and Tietê River basins, Brazil (Bockmann \& Guazzelli, 2003).

Remarks: Synonymy based on Oliveira \& Britski (2000), in which the species is redescribed.

\section{Species inquirenda, Heptapteridae}

Pimelodus velifer Humboldt, in Humboldt \& Valenciennes, 1821: 171. Type locality: Río Magdalena, Colombia. No types known.

\section{$\dagger$ HYPSIDORIDAE Grande, 1987}

$\dagger$ Hypsidoridae Grande, 1987: 28. Type genus: †Hypsidoris Lundberg \& Case, 1970.

1 genus, 2 species, all taxa based only on fossils.

$\dagger$ HYPSIDORIS Lundberg \& Case, 1970

$\dagger$ Hypsidoris Lundberg \& Case, 1970: 452. Type species: $†$ Hypsidoris farsonensis Lundberg \& Case, 1970. Type by original designation. Gender: Feminine.

$\dagger$ Hypsidoris farsonensis Lundberg \& Case, 1970.

$\dagger$ Hypsidoris farsonensis Lundberg \& Case, 1970: 452, pls. 1- 2; text figs. 1- 2. Type locality: Laney Shale member, Green River Formation, Sweetwater County, near Farson, Wyoming. Holotype: PU 20570 (impressions of complete skeleton, in dorsal and ventral view); holotype illustrated in Grande (1987: figs. 1-2).

Distribution: Early Middle Eocene Lake Gosiute deposits of the Laney Shale Member (Grande, 1987).

$\dagger$ Hypsidoris oregonensis Grande \& de Pinna, 1998

$\dagger$ Hypsidoris oregonensis Grande \& de Pinna, 1998: 454, figs. 3-10. Type locality: Clarno Formation, Jefferson County, north central Oregon. Holotype: FMNH PF10719 (impression of nearly complete skeleton).

\section{ICTALURIDAE Gill, 1861}

Ictaluri Gill, 1861c: 49. Type genus: Ictalurus Rafinesque, 1820.

Ameiurina Günther, 1864: 98. Type genus: Ameiurus Rafinesque, 1820.

Ichthaelurinae Meek, 1904: 10. Type genus: Ichthaelurus Cope, 1868.

$\dagger$ Astephinae Grande \& Lundberg, 1988: 146. Type genus: † Astephus Cope, 1873.

Reviews: Lundberg (1975, fossil forms).

Identification guide: Page \& Burr (1991, Canada and U.S.).

Checklist: Espinosa Pérez, et al. (1993, Mexico).

Phylogeny: Le Grande (1981, chromosomal evolution); Hardman (2002); Lundberg (1982, 1992). 
7 genera, 64 species; 1 fossil genus and 14 fossil species.

AMEIURUS Rafinesque, 1820

Ameiurus Rafinesque, 1820c: 359. Type species: Silurus lividus Rafinesque, 1820. Type designated by plenary powers (ICZN, Opinion 1584; see Bailey \& Robins, 1988, for justification). Gender: Masculine. Also described as new in Rafinesque (1820a: 65). Originally proposed as subgenus of Ictalurus.

Amiurus Agassiz, 1846: 17. Type species: Silurus lividus Rafinesque, 1820. Unjustified emendation of Ameiurus Rafinesque, 1820. Gender: Masculine.

Gronias Cope, 1864: 231. Type species: Gronias nigrilabris Cope, 1864. Type by monotypy. Gender: Masculine.

Remarks: See Bailey \& Robins (1988) for information on type species.

Relationships: Hardman \& Page (2003).

Ameiurus brunneus Jordan, 1877

Amiurus brunneus Jordan, 1877a: 366. Type locality: South Fork of Ocmulgee R., at Flat Shoals, Georgia. Lectotype: USNM 20148, designated by Jordan \& Evermann (1896: 142).

Distribution: Atlantic coast basins from southern Virginia to Georgia; Gulf Coast basins of Georgia, Alabama and Florida (Page \& Burr, 1991).

Ameiurus catus (Linnaeus, 1758)

Silurus catus Linnaeus, 1758: 305. Type locality: America; Asia [the latter in error]. No types known; based on illustrations in Catesby (1731-1743: pl. 23), and Marcgravius (1648: 173).

Pimelodus albidus Lesueur, 1819: 148. Type locality: la Delaware [River]. Type(s): Whereabouts unknown (Gilbert, 1998).

Pimelodus lynx Girard, 1859b: 160. Type locality: Potomac River, in a deep run, a tributary of Patapsco River, and at Carlisle, Pa [U. S. A.]. Syntypes: at USNM, but not found.

Ichthaelurus Kevinskii Stauffer, 1869: 578. Type locality: Susquehanna River drainage, Lancaster Co., Pennsylvania. Holotype: Whereabouts unknown.

Ichthaelurus McCaskei Stauffer 1869: 578. Type locality: Shenk's Ferry, Susquehanna River (or tributary thereof), Lancaster Co., Pennsylvania. Holotype: Whereabouts unknown.

Amiurus lophius Cope, 1870a: 487. Type locality: Washington, D. C. market, which came from the lower course of the Potomac River. Syntypes (3): ANSP 8461-62 (2).

Amiurus niveiventris Cope, 1870a: 488. Type locality: Neuse River, N. Ca. [North Carolina, U.S.A.]. Syntypes: ANSP 8466-67 (2).

Distribution: Atlantic and Gulf coast drainages from Hudson River to Pascagoula River, Mississippi; introduced elsewhere (Page \& Burr, 1991).

$\dagger$ Ameiurus hazenensis (Baumgartner, 1982)

$\dagger$ Ictalurus (Amiurus) hazenensis Baumgartner, 1982: 38. Type locality: Laminated diatomaceous beds of Miocene Middle Truckee Formation (ca. 39 31'36"N, 119 06'27"W), Two Tips Quadrangle, T19N, R26E, Sec. 7 (center), near Nazen, Churchill County. Nevada. Holotype: UMMP V74320 (anterior portion of fish).

Distribution: Churchill County, Nevada, Middle Truckee Formation; Miocene.

$\dagger$ Ameiurus lavetti (Lundberg, 1975)

$\dagger$ Ictalurus lavetti Lundberg, 1975: 31, fig. 4 a-e; pl. 11, fig. a. Type locality: Sec. 7, T. 11 S, R. 37 W, Old Vincent Ranch, 22 mi. NE of Wallace, Logan County, Kansas; middle Pliocene.

Distribution: Olallala Group, Logan County Kansas, and Basal Ash Hollow formation, South Dakota; lower to middle Pliocene (Lundberg, 1975).

$\dagger$ Ameiurus leidyi (Lundberg, 1975)

$\dagger$ Ictalurus leidyi Lundberg, 1975: 35, fig. 4 f; pl. 5, fig. a, pl. 8, fig. k. Type locality: Observation Quarry, NW 1/4, SE 1/4, Sec. 4, T. 31 N, R. 47 W. Dawes County, Nebraska; early Miocene. Holotype: AMNH 10509 (nearly complete skull).

Distribution: Observation Quarry, Nebraska; Valentine Formation, Nebraska; Ash Hollow Formation, Nebraska; lower Pliocene (Lundberg, 1975).

$\dagger$ Ameiurus macgrewi (Lundberg, 1975) 
$\dagger$ Ictalurus macgrewi Lundberg, 1975: 29, fig. 3; pl. 10, fig. a. Type locality: Banks of Horse Creek, Sec. 3, T. 17 N,

R. 66 W, Laramie County, Wyoming; middle Miocene. Holotype: University of Wyoming 3298.

Distribution: Banks of Horse Creek, Laramie County, Wyoming; middle Miocene (Lundberg, 1975).

Ameiurus melas (Rafinesque, 1820)

Silurus melas Rafinesque, 1820b: 51. Type locality: Ohio River. No types known. Also described as new as Pimelodus melas in Rafinesque (1820a: 66 and 1820c: 360).

Pimelodus catulus Girard, 1858b: 208, pl. 41 (figs. 4-6). Type locality: Fort Smith, Arkansas. Syntypes: USNM 926 (1), USNM 927 (5, missing).

Pimelodus confinis Girard, 1859b: 159. Type locality: Root River, near Racine, Wis. Syntypes: MCZ 35993 (1), USNM 1514 (7), USNM 1515 (3).

Pimelodus cupreoides Girard, 1859b: 159. Type locality: Aux Plaines, Ill. Syntypes: USNM 1497 (4).

Amiurus obesus Gill 1861b: 45. Type locality: Nebraska. Syntypes: USNM 8968 (3).

Amiurus brachyacanthus Cope, 1880: 35. Type locality: Wallace Creek, headwaters of Medina River, Bandera Co., Texas. Syntypes: ANSP 20527-28 (2).

Amiurus cragini Gilbert, 1884: 10. Type locality: "The Lake" (a blind arm of the Arkansas River), at Garden City, Finney Co., Kansas. Holotype: USNM 36814. Also appeared as new (and synonymized in a footnote) in Gilbert (1885: 512).

Distribution: North American Great Lakes, Hudson Bay, Mississippi River basin and Gulf Coast basins; introduced elsewhere (Page \& Burr, 1991).

Ameiurus natalis (Lesueur, 1819)

Pimelodus natalis Lesueur, 1819: 154. Type locality: Haut-Canada. No types known. Placed on Official List (ICZN, Opinion 1584).

Silurus lividus Rafinesque, 1820b: 50. Type locality: Ohio River. No types known. As Pimelodus lividus in Rafinesque (1820a: 65; and 1820c: 359).

Silurus lividus Fuscatus Rafinesque, 1820b: 51. Type locality: Ohio River. No types known. Originally as Silurus lividus var. Fuscatus.

Silurus (Pimelodus) coenosus Richardson, 1836: 132. Type locality: Penetanguisheue, Lake Huron. Holotype: Whereabouts unknown.

Pimelodus ailurus Girard, 1858b: 210. Type locality: Lake Amelia, near Fort Snelling, Minnesota. Syntypes: USNM 904 (2, originally 1), USNM 905 (1, missing).

Pimelodus antoniensis Girard, 1858b: 209. Type locality: Near San Antonio, Tex. Holotype: USNM 923.

Pimelodus felinus Girard, 1858b: 209. Type locality: Trib. of Gypsum creek, Canadian. Lectotype: USNM 924, designated by Jordan \& Evermann (1896: 140).

Pimelodus puma Girard, 1859b: 160. Type locality: Charleston, S. C. [South Carolina]. Holotype: USNM 1536.

Amiurus natalis analis Jordan, 1877c: 87, pl. 18 (fig. 31). Type locality: Little Red River, Judsonia, White Co., Arkansas. Holotype: USNM 31088 (missing).

Amiurus erebennus Jordan, 1877c: 85, pl. 13 (figs. 19-20). Type locality: St. John's River, Florida. Holotype: USNM 19093.

Amiurus bolli Cope, 1880: 35. Type locality: Little Wichita River, in northern Texas. Syntypes: ANSP 20512-13 (2). Amiurus prosthistius Cope, 1884a: 132. Type locality: Batsto River, Mullica System, New Jersey. Syntypes: ANSP 20546-49 (4).

Distribution: Atlantic and Gulf coast drainages, from New York to northern Mexico, Great Lakes and Mississippi River basin (Page \& Burr, 1991).

Remarks: Amiurus erebennus Jordan, 1877, was treated as a valid subspecies in Gilbert (1998).

Ameiurus nebulosus (Lesueur, 1819)

Pimelodus nebulosus Lesueur, 1819: 149. Type locality: Philadelphie .... la Delaware. No types known.

Pimelodus atrarius De Kay, 1842: 185, pl. 36 (fig. 116). Type locality: Wappinger's Creek, trib. to Hudson R., Dutchess Co., New York. Syntypes: New York State coll. (whereabouts unknown; Gilbert, 1998).

Pimelodus pullus DeKay, 1842: 184, pl. 37 (fig. 117). Type locality: Lake Pleasant, Lake Janet and other lakes in n. districts, New York. Syntypes: New York State coll. (whereabouts unknown; Gilbert, 1998). 
Pimelodus vulgaris Thompson, 1842: 138. Type locality: Lake Champlain, Vermont. Holotype: Whereabouts unknown.

? Pimelodus felis Agassiz, 1850: 281. Type locality: Lake Superior. Type(s): Whereabouts unknown.

Pimelodus marmoratus Holbrook, 1855: 54, pl. 6 (fig. 4). Type locality: Altamaha River, Georgia. Holotype: USNM 9031. As Pimelodus marginatus on table of plates (p. 58).

Pimelodus marginatus Holbrook, 1855: 58, pl. 6 (fig. 4). Type locality: Altamaha River, Georgia. Holotype: USNM 9031. Name made available from table of plates for illustration corresponding to species account of Pimelodus marmoratus Holbrook.

Pimelodus dekayi Girard, 1859b: 160. Type locality: Oswego, Lake Ontario, and in four mile creek, near Oswego [New York]. Syntypes: USNM 1512 (missing), USNM 1513 (4).

Pimelodus hoyi Girard, 1859b: 159. Type locality: In the neighborhood of Racine, Wis. Syntypes: MCZ 35945 (1), USNM 1539 (3).

Pimelodus vulpeculus Girard, 1859b: 160. Type locality: Charleston, S.C. [South Carolina]. Holotype: USNM 1530.

Gronias nigrilabris Cope, 1864: 231. Type locality: Conestoga Creek, trib. to Susquehanna River, Lancaster Co., Pennsylvania. Syntypes: ANSP 22082-83 (2).

Amiurus mispilliensis Cope, 1870a: 486. Type locality: Mispillion Creek, a sluggish stream in the southern part of the State of Delaware. Holotype: ANSP 8536.

Ictalurus nebulosus pannonicus Harka \& Pinter, 1990: 69, figs. 3-4. Type locality: Tisza R. at Poroszló, Hungary. Holotype: MNSB 87.1.1. [Name based on an introduced population.]

Distribution: Atlantic and Gulf coast drainages, from Canada to Alabama; Great Lakes, Hudson Bay, and Mississippi River basin; introduced elsewhere in North America (Page \& Burr, 1991); also in Europe and New Zealand.

Remarks: Pimelodus marmoratus Holbrook, 1855, was treated as a valid subspecies, with no synonyms, in Gilbert (1998).

$\dagger$ Ameiurus pectinatus (Cope, 1874)

$\dagger$ Ictalurus pectinatus Cope, 1874a: 49. Type locality: Florissant lake beds, Oligocene, Florissant, Colorado. Holotype: USNM 4086.

Distribution: Florissant lake beds, Colorado; Oligocene (Lundberg, 1975).

Ameiurus platycephalus (Girard, 1859)

Pimelodus platycephalus Girard, 1859b: 161. Type locality: In the neighborhood of Anderson, S.C. [South Carolina]. Lectotype: ANSP 8473, designated by Yerger \& Relyea (1968: 364).

Distribution: Atlantic drainages of Virginia, the Carolinas, and Georgia (Page \& Burr, 1991).

$\dagger$ Ameiurus reticulatus Smith, Morgan \& Gustafson, 2000

$\dagger$ Ameiurus reticulatus Smith, Morgan and Gustafson, 2000: 16, figs. 9 b, c. Type locality: Blufftop local fauna, Ringold Formation, Franklin Co., Washington; middle Pliocene. Holotype: UMMP 104009, complete mandible.

Distribution: Ringold Formation, Washington; Miocene (Smith, Morgan and Gustafson, 2000).

$\dagger$ Ameiurus sawrockensis (Smith, 1962)

$\dagger$ Ictalurus sawrockensis Smith, 1962: 527, fig. 8. Type locality: Beaver locality, Sawrock Canyon, west side of Sec. 36, T. 34 S, 312 W. Seward County, Kansas; upper Pliocene. Holotype: UMMP V37066 (pectoral-fin spine).

$\dagger$ Ictalurus benderensis Smith, 1962: 527, fig. 9. Type locality: Bender local fauna, Rexroad formation above Rexroad local fauna, Cottrell pasture NW 1/4, SE 1/4, Sec. 16., T. 33 S, R. 29 W. Mead County, Kansas, upper Pliocene. Holotype: UMMP V37664 (pectoral-fin spine).

Distribution: Sawrock Canyon local fauna, Rexroad Formation, Kansas; upper Pliocene (Lundberg, 1975).

Ameiurus serracanthus (Yerger \& Relyea, 1968)

Ictalurus serracanthus Yerger \& Relyea, 1968: 373, fig. 4. Type locality: N. shore of Lake Talquin, 6.8 mi. westsouthwest of Ochlockonee R., Gadsden Co., Florida. Holotype: USNM 231711.

Distribution: Gulf Coast drainages of northern Florida, western Georgia, and eastern Alabama (Page \& Burr, 1991).

$\dagger$ Ameiurus vespertinus (Miller \& Smith, 1967)

$\dagger$ Ictalurus vespertinus Miller \& Smith, 1967: 15. Type locality: roadcuts on state highway 45 about $1.5 \mathrm{mi}$. SE of Fossil Butte, sec. 16, T. 4 S, R 1 W. Glenns Ferry Formation, lacustrine facies, late Pliocene. Holotype: UMMP 
V55561 (right lower jaw).

$\dagger$ Ictalurus peregrinus Lundberg, 1975: 39, fig. 5 a-e; pl. 7, fig. c, d; pl. 8, fig. a. Type locality: Sec. 14, T. 21 S, R.

37 E, Malheur County, Oregon. Upper member of the Juntura Formation, Black Butte local fauna. Holotype: UO 28035 (pectoral spine).

Distribution: Glenns Ferry Formation; Chalk Hills Formation; Chalk Butte Formation; Rome Beds, Oregon; Juntura Formation, Black Butte local fauna, Pliocene-Pleistocene (Lundberg, 1975).

Remarks: Synonymy based on Lundberg (1992).

\section{Species inquirenda, Ameiurus}

Silurus Xanthocephalus Rafinesque, 1820b: 51. Type locality: Ohio, Kentucky and Licking rivers. No types known. See also Rafinesque (1820a: 66; and 1820c: 360) as Pimelodus Xanthocephalus.

\section{$\dagger$ ASTEPHUS Cope, 1873}

$\dagger$ Astephus Cope, 1873: 638. Type species: Pimelodus antiquus Leidy, 1873. Type by subsequent designation by Lundberg (1975: 5). Gender: Masculine. Originally proposed as a subgenus of Rhineastes Cope.

Remarks: Placement within the Ictaluridae follows Grande \& Lundberg (1988).

Revision: Grande \& Lundberg (1988).

Phylogenetic position: Grande \& Lundberg (1988).

$\dagger$ Astephus antiquus (Leidy, 1873)

$\dagger$ Pimelodus antiquus Leidy, 1873a: 99. Type locality: Bridger Formation, junction of Big Sandy and Green River, Wyoming. Holotype: USNM 2179 (pectoral spine, dentary). Illustrated and described in more detail in Leidy (1873b: 193, pl. 322, figs. 44-45). Holotype also illustrated in Lundberg (1975: fig. 1d; pl. 7, fig. f) and Grande \& Lundberg (1988: fig. 1a).

$\dagger$ Rhineastes arcuatus Cope, 1873: 641. Type locality: Bridger Formation, Wyoming. Holotype: USNM 3985 (pectoral spine); holotype illustrated in Grande \& Lundberg (1988: fig. 1b).

$\dagger$ Rhineastes calvus Cope, 1873: 640. Type locality: Bridger Formation, Mammoth Buttes, South Bitter Creek, Wyoming. Holotype: USNM 3980 (partial neurocranium); holotype illustrated in Cope (1884b: 65, pl. 5, figs. 3-4), Lundberg (1975: pl. 4, fig. c), and Grande \& Lundberg (1988: fig. 1c).

$\dagger$ Ameiurus primaevus Eastman, 1917: 293. Type locality: not data [almost certainly Green River Shales, Wyoming]. Holotype: USNM 8122 (nearly complete fish); holotype illustrated in Grande \& Lundberg (1988: fig. 1e).

Distribution: Bridger Formation and Green River Formation (Lundberg, 1975).

Remarks: Synonymy follows Grande \& Lundberg (1988), which treats the brief comments in Leidy (1873a: 99) as sufficient to make the name Pimelodus antiquus available. However Woodward (1901: 329) treated Rhineastes arcuatus as valid, with Pimelodus antiquus as a synonym, based on the observation that the account of $P$. antiquus in Leidy (1873a: 99) did not make the name available (nomen nudum) and the first description by Leidy (1873b: 193) that made the name available was published after that of $R$. arcuatus Cope.

\section{$\dagger$ Astephus resimus Lundberg, 1975}

$\dagger$ Astephus resimus Lundberg, 1975: 9, pl. 4, figs. a, b. Type locality: Bridger B Formation, middle Eocene; Pinnacle Rock between Little America and Twin Buttes, Bridger Basin, Sweetwater County, Wyoming. Holotype: USNM 18104 (nearly complete neurocranium); holotype illustrated in Grande \& Lundberg (1988: fig. 2).

Distribution: Bridger B formation, Wyoming; middle Eocene; known only from type specimen (Lundberg, 1975).

\section{ICTALURUS Rafinesque, 1820}

Ictalurus Rafinesque, 1820c: 355. Type species: Silurus cerulescens Rafinesque, 1820. Type by subsequent designation by Gill (1861a: 49). Gender: Masculine. Originally proposed as a subgenus of Pimelodus.

Elliops Rafinesque, 1820c: 356. Type species: Silurus maculatus Rafinesque, 1820. Type by subsequent designation by Jordan \& Gilbert (1877: 87). Gender: Masculine. Proposed as a section of the subgenus Ictalurus. Also proposed as new in Rafinesque (1820a: 62).

Synechoglanis Gill, 1859a: 39. Type species: Synechoglanis beadlei Gill, 1859. Type by monotypy. Gender: Mascu- 
line.

Ichthaelurus Cope, 1868: 237. Type species: Silurus cerulescens Rafinesque, 1820. Type by being a replacement name. Unjustified emendation of Ictalurus Rafinesque, 1820. Gender: Masculine.

Villarius Rutter, 1896: 256. Type species: Villarius pricei Rutter, 1896. Type by subsequent designation by Jordan \& Evermann (1898: 2789). Gender: Masculine.

Haustor Jordan \& Evermann, 1896: 135. Type species: “Gadus lacustris Walbaum, 1792", now Pimelodus nigricans Le Sueur, 1819 [see Remarks]. Type fixed by provision of Art. 70.3 (ICZN, 1999). Gender: Masculine. Originally proposed as a subgenus of Ameiurus.

Istlarius Jordan \& Snyder, 1899: 118. Type species: Istlarius balsanus Jordan \& Snyder, 1899. Type by original designation. Gender: Masculine.

Ichthyaelurus Meek, 1904: 10. Type species: Silurus cerulescens Rafinesque, 1820. Type by being a replacement name. Unjustified emendation of Ictalurus Rafinesque, 1820. Gender: Masculine.

Systematics: Smith (1987, fossil forms).

Remarks: The generic name Haustor was originally proposed as a subgenus of Ameiurus, with the type species Gadus lacustris Walbaum, a species that, at that time, was considered to be an ictalurid catfish [but see Speirs (1952) for the current placement of that name as a species of Lota]. Jordan (1920: 473) clarified his earlier usage of Gadus lacustris as type species by equating that name with Pimelodus nigricans Le Sueur, 1819, which is treated here as a junior synonym of Ictalurus punctatus. I interpret Jordan's action as consistent with the provisions of the Code that cover the discovery of a misidentified type species (Art. 70.3) and selection of a new type species that reflects the "taxonomic species actually involved in the misidentification (Art. 70.3.2)." As such, the name Haustor must be retained as a genus of ictalurid catfish with Pimelodus nigricans Le Sueur, 1819 as its type.

Ictalurus australis (Meek, 1904)

Amiurus australis Meek, 1904: 13. Type locality: Rio Forlón, Forlón, Tamaulipas, México. Holotype: FMNH 4474.

Distribution: Blanco to Panuco Rivers, Vera Cruz, Mexico (Espinosa Pérez, et al., 1993).

Ictalurus balsanus (Jordan \& Snyder, 1899)

Istlarius balsanus Jordan \& Snyder, 1899: 118, fig. 2. Type locality: Río Ixtla at Puente de Ixtla, Morelos, Mexico. Holotype: SU 6149.

Istlarius balsanus occidentalis de Buen, 1946b: 119. Type locality: Río Huámito at La Huacana, Río Balsas drainage, Michoacan, Mexico. Syntypes: Whereabouts unknown.

Distribution: Balsas River basin, Mexico (Espinosa Pérez, et al., 1993).

Ictalurus dugesii (Bean, 1880)

Amiurus dugèsii Bean, 1880b: 304. Type locality: Río Turbio, Guanajuato, Mexico. Lectotype: USNM 23123, illustrated in, and designated by (in plate caption), Jordan \& Evermann (1900: 3236, pl. 26).

Distribution: Lerma-Santiago, Ameca, Armeria basins and Chapala Lake, Mexico (Espinosa Pérez, et al., 1993).

$\dagger$ Ictalurus echinatus Lundberg, 1975

$\dagger$ Ictalurus echinatus Lundberg, 1975: 25, pl. 7, figs. a, i. Type locality: Verdigre Quarry on the E bank of an unnamed tributary of Verdigre Creek, in the NW 1/4, SE 1/4, Sec. 16, T. 29 N, R. 7 W, approximately 7.5 mi S and $5.5 \mathrm{mi} \mathrm{W}$ of the town of Verdigre, Knox County, Nebraska. Valentine Formation, late Miocene or early Pliocene. Holotype: UW 3290.

Distribution: Valentine Formation, Nebraska; late Miocene or early Pliocene (Lundberg, 1975).

Ictalurus furcatus (Valenciennes, 1840)

Pimelodus furcatus Valenciennes, in Cuvier \& Valenciennes, 1840b: 136 (102 in Strasbourg deluxe edition). Type locality: 1'Ohio ... le Wabash, ... le Mississipi. Syntypes: MNHN a-8834 (1), MNHN a-9458 (1), MNHN a-8402 (1).

Pimelodus affinis Baird \& Girard, 1854: 26. Type locality: Rio Grande del Norte. Lectotype: USNM 838, designated by Gilbert (1998: 206). Specimen listed as a type illustrated in Evermann \& Kendall (1894: 126, pl. 13, top), possibly qualifies as an earlier lectotype designation if illustrated specimen is identifiable.

Amiurus meridionalis Günther, 1864: 102. Type locality: Rio Usumacinta, Guatemala. Syntypes: BMNH 1865.4.29.38 (1), BMNH 1865.4.29.41-42 (2); one syntype illustrated in Günther (1868c: pl. 81, fig. 1). 
Amiurus pondersosus Bean, 1880a: 286. Type locality: Mississippi River near St. Louis. Holotype: USNM 287439 (skeleton) and USNM 23388 (cast).

Distribution: Mississippi River basin; Gulf Coast basins from Alabama to Mexico (Page \& Burr, 1991), and southward to the Usumacinta River basin, Guatemala (Miller et al., 2005).

Remarks: Although the name Pimelodus furcatus has long been treated as valid in Ictalurus, it is actually an unneeded new name for Pimelodus cauda-furcatus Lesueur, which makes the name a junior synonym of Ictalurus punctatus. The name is retained here as valid, pending action to correct this problem.

$\dagger$ Ictalurus lambda Hubbs \& Hibbard, 1951

$\dagger$ Ictalurus lambda Hubbs \& Hibbard, 1951: 9. Type locality: Ogallala Formation, Trego County, Kansas. Holotype: KU 6887 (partial left pectoral-fin spine).

Distribution: Ogallala Formation; Box Butte Co. and Verdigre Quarry, Knox Co., Nebraska; Lapara Creek Locality, Bee Co. Texas (Lower Pliocene or upper Miocene); McGehee Fauna Locality, Alachua Co. Florida (middle Pliocene)(Lundberg, 1975).

Ictalurus lupus (Girard, 1858)

Pimelodus lupus Girard, 1858b: 211. Type locality: Headwaters of Rio Pecos, Texas. Syntypes: USNM 915 (1), USNM 916 (3).

Distribution: Rio Grande River basin (Page \& Burr, 1991).

Ictalurus mexicanus (Meek, 1904)

Amiurus mexicanus Meek, 1904: 15. Type locality: Tributary to Rio Valles, Rascon, San Luis Potosi, Rio Panuco basin, Mexico. Holotype: FMNH 4507.

Distribution: Panuco River basin, Mexico (Miller et al., 2005).

Ictalurus ochoterenai (de Buen, 1946)

Haustor ochoterenai de Buen, 1946a: 271, fig. 1. Type locality: Isla Patos, Mexico. Holotype: at Instituto de Biologia, Mexico.

Distribution: Isla Patos, Mexico (de Buen, 1946a).

Remarks: Treated as valid in Lundberg (1992), but as a synonym of Ictalurus dugesii in Alverez (1966) and Miller et al. (2005).

Ictalurus pricei (Rutter, 1896)

Villarius pricei Rutter, 1896: 257. Type locality: San Bernardino Creek, Rio Yaqui, Arizona, or n. border of Sonora, Mexico. Syntypes (7): SU 4826 (5), BMNH 1900.9.29.179 (1).

Amiurus meeki Regan, 1907, in Regan, 1906-08: 140, pl. 23 (fig. 5). Type locality: Río Paphigochic, Miñaca, Chihuahua, Mexico. Holotype: BMNH 1905.12.6.348.

Distribution: Yaqui and Casas Grandes River basins, Mexico and southern U. S. A. (Page \& Burr, 1991).

Ictalurus punctatus (Rafinesque, 1818)

Silurus punctatus Rafinesque, 1818a: 355. Type locality: Ohio River. No types known.

Pimelodus cauda-furcatus Lesueur, 1819: 152. Type locality: l'Ohio ... Pittsbourg [Gilbert (1998: 210) stated that the types were collected from Wabash and Mississippi Rivers]. Syntypes: MNHN a-8402 (1), MNHN a-8834 (1).

Pimelodus nigricans Lesueur, 1819: 153, pl. 11 (lowest fig.). Type locality: Lac Erié, l'Ontario, etc. No types known.

Silurus argentinus Rafinesque, 1820b: 50. Type locality: Lower parts of Ohio River. No types known.

Pimelodus argyrus Rafinesque, 1820c: 358. Type locality: [Ohio River]. No types known. Also in Rafinesque (1820a: 64).

Silurus cerulescens melanurus Rafinesque, 1820b: 49. Type locality: Ohio River. No types known. Originally as Silurus cerulescens var. melanurus.

Silurus maculatus Rafinesque, 1820b: 48. Type locality: Pittsburg and in Kentucky River, Ohio River. No types known. Also in Rafinesque (1820a: 62 and 1820c: 356) as Pimelodus maculatus. Preoccupied by Silurus maculatus Thunberg 1792.

Silurus maculatus Erythroptera Rafinesque, 1820a: 49. Type locality: Ohio River. No types known. Originally as Silurus maculatus var. Erythroptera. 
Silurus pallidus Rafinesque, 1820b: 49. Type locality: Ohio River. No types known.

Silurus pallidus Lateralis Rafinesque, 1820b: 49. Type locality: Ohio River. No types known. Originally as Silurus pallidus var. Lateralis. Described as new as Pimelodus pallidus var. Lateralis in Rafinesque (1820a: 63; and 1820c: 358).

Silurus pallidus Leucoptera Rafinesque, 1820b: 49. Type locality: Ohio River. No types known. Originally as Silurus pallidus var. Leucoptera. Described as new as Pimelodus pallidus var. Leucoptera in Rafinesque (1820a: 63; and 1820c: 358).

Silurus pallidus Marginatus Rafinesque, 1820b: 49. Type locality: Ohio River. No types known. Originally as Silurus pallidus var. Marginatus. Described as Pimelodus pallidus var. Marginata in Rafinesque (1820a: 63 and 1820c: 358).

Silurus (Pimelodus) borealis Richardson, 1836: 135. Type locality: Pine Island Lake, lat. 54N. Holotype: Whereabouts unknown (Gilbert, 1998).

Silurus (Pimelodus) nigrescens Richardson, 1836: 134. Apparently an unintentional new name for Pimelodus nigricans Lesueur.

Pimelodus furcifer Valenciennes, in Cuvier \& Valenciennes, 1840b: 139 (103 of Strasbourg deluxe edition). Type locality: Surinam [in error, actually North America]. Syntype: MNHN 0000-1192 (1).

Pimelodus gracilis Hough, 1852: 26. Type locality: Oswegatchie River and Indian rivers, and other streams tributary to St. Lawrence River [New York]. Syntype: USNM 1532 (1). Preoccupied by Pimelodus gracilis Valenciennes, 1835; replaced by Pimelodus houghi Girard, 1859.

Pimelodus olivaceus Girard, 1858b: 211, pl. 41 (figs. 1-3). Type locality: Ft. Pierre, and Yellowstone Nebraska; Milk R., ?Montana; near mouth of Poteau R., and Arkansas R., Arkansas. Syntypes: MCZ 35911; USNM 906 (3), USNM 8449 (1), USNM 908 (1), USNM 909 (5), USNM 910 (1), USNM 911 (1, missing). Illustration of one of the types appeared in Suckley (1860: pl. 42).

Synechoglanis Beadlei Gill, 1859a: 40. Type locality: St. Catherines, between Hamilton and Niagara Falls, Ontario, Canada. Holotype: USNM 5115.

Pimelodus graciosus Girard, 1859b: 161. Type locality: Prairie mer Rouge, La. [Louisiana]. Syntypes: USNM 1511 (2).

Pimelodus houghi Girard, 1859b: 159. Type locality: Oswegatchie River and Indian rivers, and other streams tributary to St. Lawrence River [New York]. Syntype: USNM 1532 (1). Replacement for Pimelodus gracilis Hough, 1852; preoccupied by Pimelodus gracilis Valenciennes, 1835.

Pimelodus megalops Girard, 1859b: 161. Type locality: Black Warrior and Bigsby rivers, Ala. Syntype: USNM 1535 (1).

Pimelodus Hammondii Abbott, 1861: 568. Type locality: Fort Reily. Syntypes: ANSP 22065-66 (2).

Ictalurus simpsonii Gill, 1861b: 43. Type locality: Big Sandy R., Kansas. Syntypes (2): Whereabouts unknown (Gilbert, 1998).

Pimelodus notatus Abbott, 1861: 569. Type locality: Fort Reily, Kansas [probably in error, Gilbert (1998: 222)]. Holotype: ANSP 8449. Preoccupied by Pimelodus notatus Jardine, 1841.

Ichthaelurus robustus Jordan, 1877c: 76, pls. (fig. 3), 2 (fig. 4 \& 4b). Type locality: Exact locality unknown [Ohio or Mississippi rivers]. Holotype: USNM 20056.

Ictalurus okeechobeensis Heilprin, 1887: 129, pl. 18. Type locality: Kissimmee River, tributary to Lake Okeechobee, Florida. Holotype: ANSP 8443.

Ictalurus anguilla Evermann \& Kendall, 1898: 125, pl. 6 (fig. 1). Type locality: Atchafalaya River, above Morgan City, Pointe Coupee Parish, Louisiana. Holotype: USNM 48788.

$\dagger$ Amiurus decorus Hay, 1924: 1, pl. 1, fig. 1. Type locality: Garvin Gulley locality, 2 1/4 miles north of Navasota, Texas, Upper Miocene. Holotype: partial pectoral-fin spine; Whereabouts unknown.

Distribution: Great Lakes/Saint Lawrence basin, Hudson Bay, and Mississippi River basin; possibly native to Atlantic and Gulf Coast basins; widely introduced (Page \& Burr, 1991).

Remarks: Inclusion of Amiurus decorus in the synonymy is based on Lundberg (1975: 25).

$\dagger$ Ictalurus rhaeas (Cope, 1891)

$\dagger$ Rhineastes rhaeas Cope, 1891: 3. Type locality: Cypress Hills Formation, middle lower Oligocene, Cypress Hills, 
Saskatchewan, Canada. Holotype: NMC 6209 (vertebral centrum).

$\dagger$ Ameiurus cancellatus Cope, 1891: 4. Type locality: Cypress Hills Formation, middle lower Oligocene, Cypress Hills, Saskatchewan, Canada. Holotype: at Canadian Geol. Surv. Museum, Ottawa (vertebrae).

$\dagger$ Ameiurus maconnellii Cope, 1891: 5. Type locality: middle lower Oligocene, Cypress Hills, Saskatchewan, Canada. Holotype: at Canadian Geol. Surv. Museum, Ottawa (vertebrae).

Distribution: Cypress Hills Formation, middle lower Oligocene, Cypress Hills, Saskatchewan, Canada (Lundberg, 1975).

Remarks: $\dagger$ Rhineastes rhaeas selected as valid name by first reviser action of Lundberg (1975: 17).

$\dagger$ Ictalurus spodius Smith, 1987

$\dagger$ Ictalurus spodius Smith, 1987: 381, fig. 1. Type locality: Gravel pit near Jocotepec, Jalisco, Mexico. Holotype: UMMP V62528 (nearly complete neurocranium).

Distribution: Jocotepec, Jalisco, Mexico (Smith, 1987).

\section{Species inquirendae, Ictalurus}

Silurus Cerulescens Rafinesque, 1820b: 49. Type locality: Ohio River. No types known. Described as Pimelodus cerulescens in Rafinesque (1820a: 63 and 1820c: 357). Possibly a senior synonym of Ictalurus punctatus or Ictalurus furcatus.

Pimelodus vulpes Girard, 1858a: 170. Type locality: Live Oak Creek, Camanche Spring, Rio Leona Piedra Painte, Devil's R., Rio Salado, Texas. Syntypes: MCZ 35937 (1), USNM 843 (2), USNM 844 (1, missing), USNM 845 (4), USNM 846 (4), USNM 847 (1, missing), USNM 848 (1, missing), USNM 849 (2, missing); syntypes illustrated in Girard (1859a: 33, pl. 18). Thought by Gilbert (1998: 227) to have been based on specimens of two species (Ictalurus punctatus and Ictalurus lupus). In the absence of a lectotype, the status of this name is unresolved.

NOTURUS Rafinesque, 1818

Noturus Rafinesque, 1818b: 41. Type species: Noturus flavus Rafinesque, 1818. Type by monotypy. Gender: Masculine.

Schilbeodes Bleeker, 1858b: 258. Type species: Silurus gyrinus Mitchill, 1817. Type by monotypy. Gender: Masculine.

Pimelodon Lesueur, in Vaillant, 1896a: 28. Type species: Pimelodon insignarius Vaillant, 1896. Type by monotypy. Gender: Masculine.

Rabida Jordan \& Evermann, 1896: 144. Type species: Noturus furiosus Jordan \& Meek, 1889. Type by original designation. Originally proposed as a subgenus of Schilbeodes. Gender: Feminine.

Revision: Taylor (1969).

Phylogeny: LeGrande (1981), Grady (1987), Grady \& LeGrande (1992), Hardman (2004).

Remarks: The name Noturus latifrons appears in Jordan (1885) but was not made available therein and must be treated as a nomen nudum; see Gilbert (1998: 217) for discussion.

\section{Noturus albater Taylor, 1969}

Noturus albater Taylor, 1969: 144, frontispiece, pls. 4 (fig. 4), 11 (fig. 2). Type locality: White River, at Forsyth, on Hwy. M80, Taney Co., Missouri. Holotype: UMMZ 151171.

Distribution: White and Saint Francis River systems, Arkansas and Missouri (Taylor, 1969).

Noturus baileyi Taylor, 1969

Noturus baileyi Taylor, 1969: 141, pl.4 (fig. 3), pl. 11 (fig. 1). Type locality: The lower portion of Abrams Creek, Great Smoky Mountains National Park. Holotype: USNM 201602.

Distribution: Citico Creek, Little Tennessee River basin, Tennessee; Abrams Creek population extirpated (Page \& Burr, 1991).

Noturus crypticus Burr, Eisenhour \& Grady, 2005

Noturus crypticus Burr, Eisenhour \& Grady, 2005: 794, fig. 2c. Type locality: Tennessee, Greene County, Little Chucky Creek at mouth of Jackson Branch, 3607'11"N, 8302'04"W. Holotype: SIUC 52379.

Distribution: French Broad River basin, eastern Tennessee (Burr et al., 2005). 
Noturus elegans Taylor, 1969

Noturus elegans Taylor, 1969: 150, pl. 4 (figs. 5-6), pl. 12. Type locality: Fallen Timber Creek, at Hwy. 90, 8 mi. SE of Glasgow, Barren Co., Kentucky. Holotype: UMMZ 167597.

Distribution: Green River system, Kentucky and Tennessee River systems, Tennessee (Page \& Burr, 1991; Burr et al., 2005).

Noturus eleutherus Jordan, 1877

Noturus eleutherus Jordan, 1877a: 371. Type locality: French Broad River, Tennessee. Holotype: USNM 29678; holotype illustrated in Jordan (1877c: 73, pl. 40, figs. 62-63a).

Schilbeodes gallowayi Fowler, 1945d: 122, figs. 155-157. Type locality: South Fork of Holston River above Bluff City, Sullivan Co., Tennessee. Holotype: ANSP 54723.

Distribution: Ohio, Mississippi, Tennessee, and Ouachita and Red River systems (Page \& Burr, 1991).

Noturus exilis Nelson, 1876

Noturus exilis Nelson, 1876: 51. Type locality: Mackinaw Cr., McLean Co., Illinois. Lectotype: INHS 37651, designated by Sabaj et al. (1997: 270).

Noturus elassochir Swain \& Kalb, 1883: 639. Type locality: Illinois River, Napierville, Illinois. Holotype: USNM 29677.

Distribution: Mississippi River basin, in upland creeks (Taylor, 1969).

Phylgeography: Hardy et al. (2002).

Noturus fasciatus Burr, Eisenhour \& Grady, 2005

Noturus fasciatus Burr, Eisenhour \& Grady, 2005: 785, fig. 2a. Type locality: Tennessee, Hickman County, Brushy Fork Creek, off Brushy Road, $1.6 \mathrm{~km} \mathrm{~W}$ of junction with Tennessee Highway 48, N of Aetna, 35 40'25"N, 87³1'03"W. Holotype: SIUC 52378.

Distribution: Duck River basin and nearby tributaries of the Tennessee River, Tennessee (Burr et al., 2005).

Noturus flavater Taylor, 1969

Noturus flavater Taylor, 1969: 204, pl. 4 (fig. 15), pl. 20 (fig. 2). Type locality: Flat Creek, at Hwy. M39, 12 mi. NE. of Cassville, Barry Co., Missouri. Holotype: UMMZ 151322.

Distribution: Upper White River system, Arkansas and Missouri (Page \& Burr, 1991).

Noturus flavipinnis Taylor, 1969

Noturus flavipinnis Taylor, 1969: 201, pl. 4 (fig. 14), pl. 20 (fig. 1). Type locality: Hines Creek, Clinton, Tennessee. Holotype: USNM 163801

Distribution: Upper Tennessee River basin (Taylor, 1969).

Noturus flavus Rafinesque, 1818

Noturus flavus Rafinesque, 1818b: 41. Type locality: Ohio River [now: Eagle Creek (a tributary to the lower Kentucky River which is a tributary to the Ohio River), from a pool below bridge at state highway 36, 3.5 miles east of Jonesville in Grant Co., Kentucky, by neotype designation]. Neotype: USNM 202494, designated by Taylor (1969: 118).

Noturus luteus Rafinesque, 1819: 421. Type locality: [No locality stated]. No types known.

Noturus occidentalis Gill, 1861b: 45. Type locality: Platte River. Holotype: Whereabouts unknown (Gilbert, 1998).

Noturus platycephalus Günther, 1864: 104. Type locality: North America. Syntypes (2): BMNH 2005.9.21.1.

Distribution: Mississippi River system, Mohawk and Hudson Rivers, Great Lakes-Saint Lawrence River basin (Taylor, 1969).

Noturus funebris Gilbert \& Swain, 1891

Noturus funebris Gilbert \& Swain, in Gilbert, 1891: 153. Type locality: in a Spring Run tributary to North River near Tuscaloosa, Alabama. Lectotype: USNM 36696, designated by Taylor (1969: 98).

Distribution: Gulf Coast basins east of Mississippi River (Taylor, 1969).

Noturus furiosus Jordan \& Meek, 1889

Noturus furiosus Jordan \& Meek, in Jordan, 1889: 351, pl. 43 (figs. 1-1b). Type locality: Neuse River at Millburnie, near Raleigh, North Carolina. Lectotype: USNM 39932, illustrated in, as designated by (in plate caption), Jordan \& Evermann (1900: 3237, pl. 29, figs. 69, 69a, 69b); same specimen designated as lectotype by Taylor (1969: 186). 
Distribution: Neuse and Tar River systems, North Carolina (Taylor, 1969; Page \& Burr, 1991).

Noturus gilberti Jordan \& Evermann, 1889

Noturus gilberti Jordan \& Evermann, in Jordan, 1889: 352, pl. 43 (figs. 2-2b). Type locality: Roanoke River, Salem, Virginia. Lectotype: USNM 3993, designated by (in caption to plate) Jordan \& Evermann (1900: 3236, pl. 28); same specimen designated as lectotype by Taylor (1969).

Distribution: Roanoke River basin, Virginia; possibly introduced elsewhere (Page \& Burr, 1991).

Noturus gladiator Thomas \& Burr, 2004

Noturus gladiator Thomas \& Burr, 2004: 353, figs. 2-3. Type locality: Tennessee: Hardeman County: Mississippi River basin, Pleasant Run (Hatchie River) at Summerville-Bolivar Rd., just upstream of Potters Creek, ca. 2.5 km W of Bolivar; 35¹5'14"N, 08902'05"W. Holotype: SIUC 47997.

Distribution: Mississippi River tributaries of western Tennessee and Mississippi (Thomas \& Burr, 2004).

Noturus gyrinus (Mitchill, 1817)

Silurus gyrinus Mitchill, 1817: 289. Type locality: Wallkill [New York]. No types known.

Noturus sialis Jordan, 1877c: 102, pl. 42 (fig. 68), pl. 43 (fig. 69). Type locality: White River, Indianapolis, Indiana. Holotype: Whereabouts unknown.

Distribution: Atlantic draining Rivers, below Fall line; Mississippi River basin; rivers draining Gulf of Mexico (north of Rio Grande); and Great Lakes; introduced into many additional rivers (Taylor, 1969).

Noturus hildebrandi (Bailey \& Taylor, 1950)

Schilbeodes hildebrandi Bailey \& Taylor, 1950: 32, pl. 1; pl. 2 (figs. e-f). Type locality: Brushy Creek, about 1 mile above its mouth in Homochitto River, T.4N, R2E., Sect. 14, at the tiny hamlet of Homochitto, Amite County, Mississippi. Holotype: UMMZ 157620.

Noturus hildebrandi lautus Taylor, 1969: 138, pl. 4 (fig. 2), pl. 10 (fig. 2). Type locality: North Fork of Obion River, at Tennessee Highway 69, Henry County, Tennessee. Holotype: USNM 201665.

Distribution: Left bank tributaries of Mississippi River, in Mississippi, Kentucky, and Tennessee (Taylor, 1969).

Noturus insignis (Richardson, 1836)

Pimelodus insigne Richardson, 1836: 132. Type locality: Philadelphia. Holotype: MNHN 0000-3053. Available from reference to "Pimelodus ... insigne (livrée, Le Sueur)."

Pimelodus lemniscatus Valenciennes, in Cuvier \& Valenciennes, 1840b: 144 (107 in Strasbourg deluxe edition). Type locality: Philadelphia. Holotype: MNHN 0000-3053. On Pimélode livrée of Lesueur (1819).

Noturus marginatus Baird, in Cope, 1868: 237. Type locality: Sinking Creek of Kanawha River; head of James River, Virginia; and Susquehanna River at Carlisle, Pennsylvania. Syntypes (more than 20): ANSP 8431-32 (2), possibly ANSP 8435 (1), MCZ 8371 (1), MCZ 35955 (1), MNHN 0000-0340 (1), MNHN 0000-0341 (1), UMMZ 138221 (1), USNM 1571 (9).

Pimelodon insignarius Vaillant, 1896a: 28, pl. 24. Type locality: Philadelphia. Holotype: MNHN 0000-3053.

Schilbeodes marginatus atrorus Hubbs \& Raney, 1944: 7, 10, pl. 1. Type locality: Wolf Creek, between Bastian and Novis, Bland Co., Virginia. Holotype: UMMZ 139452.

Distribution: Atlantic coastal rivers between New York and Georgia, generally at or above Fall Line and New and Kanawha River basins; introduced elsewhere (Talyor, 1969),

Remarks: The name Pimelode livrée in Lesueur (1819: 155) was considered by Taylor (1969: 83) to be an informal name and therefore not available. The name was accompanied by a brief description which, when assigned a name by Richardson (1836: 132), was sufficient to make Richardson's name available, with the specimen examined by Lesueur as the holotype.

Noturus lachneri Taylor, 1969

Noturus lachneri Taylor, 1969: 54, pl. 3 (fig. 2), pl. 5 (fig. 2). Type locality: Middle Fork of the Saline River at Arkansas Highway 7 crossing, 11.2 miles north of Mountain Valley, Garland Co., Arkansas. Holotype: USNM 201592.

Distribution: Saline and Ouachita River systems, Arkansas (Page \& Burr, 1991).

Noturus leptacanthus Jordan, 1877

Noturus leptacanthus Jordan, 1877a: 352. Type locality: Silver Creek, 1 mi. above its junction with the Etowah R., near Rome, Georgia. Holotype: apparently destroyed (Taylor, 1969). 
Distribution: Southeastern United States, from Atlantic and Gulf of Mexico draining rivers (Taylor, 1969).

Noturus miurus Jordan, 1877

Noturus miurus Jordan, 1877a: 371. Type locality: Maumee River basin, lower Wabash River basin and White River near Indianapolis, Indiana [restricted to White River near Indianapolis, by lectotype designation]. Lectotype: MNHN a-1308, designated by Taylor (1969: 197).

Distribution: Great Lakes basin, Mississippi River basin, Pearl River system, and smaller rivers between the Pearl and Mississippi (Taylor, 1969).

Noturus munitus Suttkus \& Taylor, 1965

Noturus munitus Suttkus \& Taylor, 1965: 171, figs. 1, 2. Type locality: Pearl River, 2.6 mi. east of Sandy Hook, Marion Co., Mississippi. Holotype: TU 26250.

Distribution: Gulf Coast basins from Georgia to Louisiana, in several disjunct populations (Page \& Burr, 1991).

Noturus nocturnus Jordan \& Gilbert, 1886

Noturus nocturnus Jordan \& Gilbert, 1886: 6. Type locality: Poteau River, ... Washita and Saline Rivers, [Arkansas River tributaries]; [restricted to Saline River near Benton railroad bridge by lectotype designation]. Lectotype: USNM 36461, designated by use of term "type" in figure caption in Evermann \& Kendall (1894: pl. 11, top), and approximate size of illustrated specimen which were used by Taylor (1969: 79) to identify the individual.

Distribution: Lower and central Mississippi River basin and other rivers draining into Gulf of Mexico (Taylor, 1969).

Noturus phaeus Taylor, 1969

Noturus phaeus Taylor, 1969: 100, pl. 3 (fig. 8), pl. 7 (fig. 2). Type locality: North Fork of the Obion River at state highway 69, Henry County, Tennessee. Holotype: USNM 202143.

Distribution: Lower Mississippi River valley and headwaters of Bayou Teche, Louisiana (Taylor, 1969).

Noturus placidus Taylor, 1969

Noturus placidus Taylor, 1969: 168, pls. 4 (fig. 9), 15 (fig. 2), 17 (fig. 2). Type locality: Neosho River, just S. of U.S. Hwy. 50, near Emporia, sec. 7, T. 19 S., R. 12 E., Lyon Co., Kansas. Holotype: UMMZ 167653.

Distribution: Neosho River and tributaries, Cottonwood River and Illinois River, Mississippi River basin (Taylor, 1969).

Noturus stanauli Etnier \& Jenkins, 1980

Noturus stanauli Etnier \& Jenkins, 1980: 19, figs. 1-2. Type locality: Clinch R. at Frost Ford, Hancock Co., Tennessee. Holotype: TU 110929.

Distribution: Clinch and Duck Rivers, Tennessee River basin, Tennessee (Page \& Burr, 1991).

Noturus stigmosus Taylor, 1969

Noturus stigmosus Taylor, 1969: 173, pls. 2 (fig. 4), 4 (fig. 11), 16 (fig. 1), 18 (fig. 1). Type locality: Huron River, just south of pool, below North Territorial Road, north of Dexter, sec. 13, T. 1 S., R. 4 E., Washtenaw Co., Michigan. Holotype: UMMZ 165843.

Distribution: Lake Erie and Ohio River basin (Thomas \& Burr, 2004).

Noturus taylori Douglas, 1972

Noturus taylori Douglas, 1972: 785, fig. 1. Type locality: South Fork of Caddo R., $1.6 \mathrm{~km}$ southeast of Hopper and $0.8 \mathrm{~km}$ south of state hwy. 240, Montgomery Co., Arkansas. Holotype: USNM 206181.

Distribution: Caddo, Ouachita and Little Missouri River basins, Arkansas (Page \& Burr, 1991).

Noturus trautmani Taylor, 1969

Noturus trautmani Taylor, 1969: 156, pls. 4 (fig. 7), 13 (fig. 1). Type locality: Big Darby Creek, 1 mile south of Fox, southeastern Jackson Township, Pickaway County, Ohio. Holotype: UMMZ 187098.

Distribution: Big Darby Creek, Scioto River basin, Ohio (Taylor, 1969).

\section{Species inquirenda, Noturus}

Silurus mollis Hermann, 1804: 309. Type locality: America. No types known. Treated by Hubbs \& Raney (1944) and, subsequently, Gilbert (1998: 220) as a senior synonym of Silurus gyrinus Mitchell, but Taylor (1969: 52) rejected that conclusion and, instead, questioned whether the name could be unambiguously associated with any species. In 
the absence of a primary type specimen, the name is listed here as species inquirenda.

PRIETELLA Carranza, 1954

Prietella Carranza, 1954: 130. Type species: Prietella phreatophila Carranza, 1954. Type by original designation. Gender: Feminine.

Prietella lundbergi Walsh \& Gilbert, 1995

Prietella lundbergi Walsh \& Gilbert, 1995: 851, figs. 1 (top), 2 c-d. Type locality: a subsurface thermal spring (Nacimiento de San Rafael de Los Castros) approximately 15 km WNW of Ciudad Mantae Tamaulipas state, México (Río Tamesí drainage). Holotype: UNAM P-7705.

Distribution: Two springs of Tamesí River drainage, Tamaulipas state, Mexico (Miller et al., 2005).

Prietella phreatophila Carranza, 1954

Prietella phreatophila Carranza, 1954: 132, pl. 1. Type locality: Well at base of Sierra de Santa Rosa, ca. 27 $55^{\prime} \mathrm{N}$, $101^{\circ} 07^{\prime} \mathrm{W}$, Múzquiz, Coahuila state, Mexico. Holotype: Author's personal coll.; illustrated in Miller et al. (2005: 165, fig. 6.171).

Distribution: Cases in Bravo River basin, Coahuila, from near Múzquiz northward to about $50 \mathrm{~km} \mathrm{SW}$ of Ciudad Acuña and to near Allende, Mexico (Miller et al., 2005).

PYLODICTIS Rafinesque, 1819

Pylodictis Rafinesque, 1819: 422. Type species: Pylodictis limosus Rafinesque, 1819. Type by monotypy. Gender: Masculine. Placed on the Official List (ICZN, Opinion 1584). Proposed as new, but spelled Pilodictis in Rafinesque (1820c: 361, 1820a: 67).

Ilictis Rafinesque, 1820c: 360. Type species: Silurus limosus Rafinesque, 1820 [=Pylodictis limosus Rafinesque, 1819]. Type by monotypy. Gender: Masculine. Originally proposed as a section of the subgenus Ictalurus. Also in Rafinesque (1820: 66).

Leptops Rafinesque, 1820c: 358. Type species: Silurus viscosus Rafinesque, 1820. Type by subsequent designation by Jordan \& Gilbert (1877: 87). Gender: Masculine. Originally proposed as a section of the subgenus Ictalurus. Also proposed as new in Rafinesque (1820a: 64).

Opladelus Rafinesque, 1820c: 359. Type species: Silurus nebulosus Rafinesque, 1820. Type by monotypy. Gender: Masculine. Originally proposed as a subgenus of Ictalurus. Also in Rafinesque (1820a: 64-65).

Hopladelus Gill 1862: 51. Type species: Silurus nebulosus Rafinesque, 1820. Type by monotypy. Gender: Masculine. Unjustified emendation or misspelling of Opladelus Rafinesque, 1820.

Pelodichthys Jordan, 1877b: 10. Type species: Pylodictis limosus Rafinesque, 1819. Type by monotypy. Gender: Masculine. Unjustified emendation of Pylodictis Rafinesque, 1819.

Remarks: The name Pylodictis is treated as valid, with the type species Pylodictis limosus (with Silurus olivaris as a senior subjective synonym) following Opinion 1584 of the Code. However, see Markle (1997: 446) in which it shown that Pylodictis limosus was a mythical fish, based on a drawing made byAudubon.

Pylodictis olivaris (Rafinesque, 1818)

Silurus olivaris Rafinesque, 1818a: 355. Type locality: Ohio River. No types known. Placed on the Official List (ICZN, Opinion 1584).

Pylodictis limosus Rafinesque, 1819: 422. Type locality: Ohio River mouth. No types known. Described as new as Pimelodus limosus in Rafinesque (1820a: 67; and 1820c: 361); and as Silurus limosus in Rafinesque (1820b: 51).

Pimelodus aeneus Lesueur, 1819: 150. Type locality: l'Ohio. Type(s): Whereabouts unknown (Gilbert, 1998).

Silurus cupreus Rafinesque, 1820b: 51. Type locality: Ohio River. No types known. Described as Pimelodus cupreus in Rafinesque (1820a: 65 and 1820c: 359).

Silurus nebulosus Rafinesque, 1820b: 50. Type locality: Ohio River. No types known. Described as Pimelodus nebulosus in Rafinesque (1820a: 64 and 1820c: 358).

Silurus viscosus Rafinesque, 1820b: 50. Type locality: Near Louisville, Kentucky, at falls, Ohio River. No types known. Described as Pimelodus viscosus in Rafinesque (1820a: 64; and 1820c: 358).

Pimelodus lutescens Rafinesque, 1832: 20. Type locality: Tennessee River. No types known. 
Pimelodus punctulatus Valenciennes, in Cuvier \& Valenciennes, 1840b: 134 (100 of Strasbourg deluxe edition). Type locality: Newharmony. Lectotype: MNHN a-9459. Lectotype designated by Eschmeyer (in Eschmeyer $e t$ al. 1998: 1406). Although the description of the species was based entirely on one specimen, a second specimen, MNHN a-8965, from New Orleans, was listed, making both specimens syntypes. Bertin \& Esteve (1950: 24) listed MNHN a-9459 as the holotype and Gilbert (1998: 225) incorrectly treated that statement as a defacto lectotype designation.

Distribution: Great Lakes and Mississippi River basins, Gulf Coast drainages from Georgia to Mexico; introduced elsewhere (Page \& Burr, 1991); reported from the Pleistocene of Florida by Young \& Laerm (1993).

SATAN Hubbs \& Bailey, 1947

Satan Hubbs \& Bailey, 1947: 4. Type species: Satan eurystomus Hubbs \& Bailey, 1947. Type by original designation. Gender: Masculine.

Satan eurystomus Hubbs \& Bailey, 1947

Satan eurystomus Hubbs \& Bailey, 1947: 8, pl. 1 (figs. 1-3). Type locality: An artesian well (depth 381 m), near San Antonio, ca. 29³0'N, 98³0'W, Bexar Co., Texas. Holotype: UMMZ 190922.

Distribution: Artesian wells near San Antonio, Texas (Page \& Burr, 1991).

TROGLOGLANIS Eigenmann, 1919

Trogloglanis Eigenmann, 1919b: 397. Type species: Trogloglanis pattersoni Eigenmann, 1919. Type by monotypy. Gender: Masculine.

Trogloglanis pattersoni Eigenmann, 1919

Trogloglanis pattersoni Eigenmann, 1919b: 397, figs. 1-2. Type locality: Artesian well in San Antonio, Edwards Aquifer, ca. 29³0'N, 98³0'W, Bexar Co., Texas. Holotype: CAS 58675.

Distribution: Artesian wells near San Antonio, Texas (Page \& Burr, 1991).

Remarks: Anatomical description in Lundberg (1982).

\section{LACANTUNIIDAE Rodiles-Hernández, Hendrickson \& Lundberg, 2005}

Lacantuniidae Rodiles-Hernández, Hendrickson \& Lundberg, in Rodiles-Hernández et al., 2005: 6. Type genus: Lacantunia Rodiles-Hernández, Hendrickson \& Lundberg, 2005.

1 Genus, 1 species; no fossil species.

LACANTUNIA Rodiles-Hernández, Hendrickson \& Lundberg, 2005

Lacantunia Rodiles-Hernández, Hendrickson \& Lundberg, in Rodiles-Hernández et al., 2005: 6. Type species: Lacantunia enigmatica Rodiles-Hernández, Hendrickson \& Lundberg. Type by original designation. Gender: Feminine.

Remarks: Treated as the type of the family Lacantuniidae, in Rodiles-Hernandez et al. (2005: 5), or as incertae sedis within the Siluriformes (2005: 6).

Lacantunia enigmatica Rodiles-Hernández, Hendrickson \& Lundberg, 2005

Lacantunia enigmatica Rodiles-Hernández, Hendrickson \& Lundberg, in Rodiles-Hernández et al., 2005: 10, fig. 1. Type locality: México, Chiapas, Río Usumacinta basin, Río Lacantún, Selva Lacandona, Reserva de la Biosfera Montes Azules, 1608.083'N, 9055.317'W. Holotype: ECO-SC 3859.

Distribution: Usumacinta River basin, Chiapas, Mexico (Rodiles-Hernández et al., 2005).

\section{LORICARIIDAE Rafinesque, 1815}

Loricaria Rafinesque, 1815: 89. Type genus: Loricaria Linnaeus, 1758.

Hypostomiden Kner, 1853b: 279. Type genus: Hypostomus La Cepède, 1803.

Ancistri Kner, 1854: 6. Type genus: Ancistrus Kner, 1854. 
Acanthicini Bleeker, 1862 (in Bleeker, 1862-63): 2. Type genus: Acanthicus Spix \& Agassiz, 1829.

Acestrini Bleeker, 1862 (in Bleeker, 1862-63): 4. Type genus: Acestra Kner, 1853. Permanently invalid, following Art. 39 of the Code, because the underlying generic name is preoccupied.

Hypoptopominae [sic, Hypoptopomatinae] Eigenmann \& Eigenmann, 1890: 8, 12, 353, 388. Type genus: Hypoptopoma Günther, 1868.

Neoplecostominae Regan, 1904b: 306. Type genus: Neoplecostomus Eigenmann \& Eigenmann, 1888.

Lithogeneinae [sic, for Lithogeninae] Gosline, 1947: 80. Type genus: Lithogenes Eigenmann, 1909.

Chaetostomidi Fowler, 1958: 14. Type genus: Chaetostomus Kner, 1846.

Farlowellidi Fowler, 1958: 14. Type genus: Farlowella Eigenmann \& Eigenmann, 1889.

Harttiinae Boeseman, 1971: 4, 10. Type genus: Harttia Steindachner, 1877.

Acestridiinae Isbrücker \& Nijssen, 1974a: 67, 68. Type genus: Acestridium Haseman, 1911.

Loricariichthyina Isbrücker, 1979a: 87, 89. Type genus: Loricariichthys Bleeker, 1862.

Metaloricariina Isbrücker, 1979a: 88, 90. Type genus: Metaloricaria, Isbrücker, 1975.

Hemiodontichthyina Isbrücker, 1979a: 88, 89. Type genus: Hemiodontichthys Bleeker, 1862.

Otocinclini Isbrücker, 1979b: 114. Type genus: Otocinclus Cope, 1871.

Planiloricariina Isbrücker, 1979a: 87, 89. Type genus: Planiloricaria Isbrücker, 1971.

Reganellina Isbrücker, 1979a: 87, 89. Type genus: Reganella Eigenmann, 1905.

Rineloricariina Isbrücker, 1979a: 87, 89. Type genus: Rineloricaria Bleeker, 1862.

Lithoxina Isbrücker, 1980: 77. Type genus: Lithoxus Eigenmann, 1910.

Pseudacanthicini Isbrücker, 1980: 76. Type genus: Pseudacanthicus Bleeker, 1862.

Ricolina Isbrücker, 1981: 54. Type genus: Ricola Isbrücker \& Nijssen, 1978.

Hopliancistrini Isbrücker \& Nijssen, 1989: 543. Type genus: Hopliancistrus Isbrücker \& Nijssen, 1989.

Spectracanthicina Isbrücker \& Nijssen, 1989: 544. Type genus: Spectracanthicus Nijssen \& Isbrücker, 1987.

Otothyrini Schaefer, 1991: 31. Type genus: Otothyris Myers, 1927.

Lampiellini Isbrücker, in Isbrücker et al., 2001: 20. Type genus Lampiella Isbrücker, 2001.

Corymbophanini Armbruster, 2004a: 59. Type genus: Corymbophanes Eigenmann, 1909.

Pterygoplichthini [sic, for Pterygoplichthyini] Armbruster, 2004a: 61. Type genus: Pterygoplichthys Gill, 1858.

Rhinelepini Armbruster, 2004a: 61. Type genus: Rhinelepis Spix \& Agassiz, 1829 [not Valenciennes, as stated in Armbruster].

Taxonomic summary: Gosline (1947); Isbrücker (1979b, 1980, 1992a, 2001); Reis et al. (2003); Ferraris (2003b, Neoplecostominae; 2003c, Loricariinae); Fisch-Muller (2003, Ancistrinae); Schaefer (2003c, Hypoptopomatinae); Weber (2003, Hypostominae).

Phylogeny: Boeseman (1971); Schaefer (1987, 1998); Montoya-Burgos et al. (1997, 1998, 2002, molecular phylogeny); Armbruster (1998a, Rhinelepis group); Schaefer (1991, Hypoptopomatinae); Armbruster (2004a, Ancistrinae and Hypostominae); Gauger \& Buckup (2005, Otothyrini).

Review: Aquino (1997, Hypoptopomatinae of Argentina), Weber et al. (1992, Paraguay).

Remarks: The taxonomy of this some portions of this family is very unsettled at this time. Monophyly of the family appears to be strongly supported, except, possibly, for the inclusion of Lithogenes (see Schaefer, 2003d, and Armbruster, 2004a for comments). Two subfamilies, Loricariinae and Hypoptopomatinae, as used in Reis et al. (2003), appear to be generally regarded as monophyletic, but the monophyly and composition of the other subfamilies in Reis et al. (2003) are currently being examined and likely to be altered substantially in the future (see, for example, Armbruster, 2004a). Isbrücker \& Nijssen (1992b) summarized the extent of sexual dimorphism in the family; Muller $\&$ Weber (1992) examined the possible taxonomic value of dental characteristics.

96 genera, 716 species; no named fossil taxa.

\section{Incertae sedis}

Hypostomus itacua Valenciennes, 1836

Hypostomus itacua Valenciennes, 1836, in Valenciennes, 1835-47: pl. 7 (fig. 1). Type locality: not stated [considered to be restricted to tributaries of La Plata]. Holotype not found in MNHN. Name available from caption to 
plate, with illustrated specimen the holotype.

Distribution: Middle Paraná River basin.

Remarks: Type locality from description of species in Cuvier \& Valenciennes (1840: 506), but Weber (2003) indicated that redescription in Cuvier \& Valenciennes (1840: 505) does not agree with the illustration in Valenciennes (1836). Redescription is apparently the basis of decisions by Günther (1864: 243) and Isbrücker (1980: 50) to place the species in the ancistrin genus Hemiancistrus.

Plecostomus obtusirostris (Steindachner, 1907)

Plecostomus obtusirostris Steindachner, 1907e: 490. Type locality: Rio Cubatao, Santa Catherina by Theresopolis, Brazil. Holotype: at NMW (not found; Weber, 2003).

Distribution: Coastal drainages of southeastern Brazil.

Remarks: Weber (2003) disagreed with the placement of this species in Hypostomus by Isbrücker (1980: 29) inasmuch he indicated that the description included mention of hypertrophied odontodes on the sides of the head of the examined specimen.

ACANTHICUS Spix \& Agassiz, 1829

Acanthicus Spix \& Agassiz, 1829: 2. Type species: Acanthicus hystrix Spix \& Agassiz, 1829. Type by monotypy. Gender: Masculine.

Review: Isbrücker \& Nijssen (1988).

Acanthicus adonis Isbrücker \& Njissen, 1988

Acanthicus adonis Isbrücker \& Nijssen, 1988: 166, fig. 5. Type locality: Brasilien, Est. Pará, Rio Tocantins bei Cametá $\left(0^{\circ} 14^{\prime} \mathrm{S}, 49^{\circ} 30.5^{\prime} \mathrm{W}\right)$. Holotype: MZUSP 38580.

Distribution: Lower Tocantins River basin, Brazil (Fisch-Muller, 2003).

Acanthicus hystrix Spix \& Agassiz, 1829

Acanthicus hystrix Spix \& Agassiz, 1829: 3, pl. 1 (fig. 1). Type locality: flumine Amazonum. Holotype: Whereabouts unknown (Kottelat, 1988) and possibly destroyed (Terofal, 1983).

Rinelepis acanthicus Valenciennes, in Cuvier \& Valenciennes, 1840b: 487 (360 in Strasbourg deluxe edition). Type locality: flumine Amazonum. Holotype: destroyed (Terofal, 1983). Replacement name for Acanthicus hystrix Spix \& Agassiz, 1829, then considered congeneric with, and preoccupied by, Rinelepis histrix Valenciennes (1840), which was based on the older, but unpublished, Vandelli manuscript name Loricaria histrix.

Distribution: Amazon River basin (Fisch-Muller, 2003).

ACESTRIDIUM Haseman, 1911

Acestridium Haseman, 1911a: 319. Type species: Acestridium discus Haseman, 1911. Type by monotypy. Gender: Neuter.

Revision: Retzer et al. (1999).

Acestridium colombiense Retzer, 2005

Acestridium colombiensis Retzer, 2005: 2, fig. 1. Type locality: Colombia: Guainia State: Orinoco Basin: Río Inirida, lagoon at $1 \mathrm{~km}$ up river from Puerto Inirida. Holotype: FMNH 115255.

Distribution: Orinoco River basin, Colombia (Retzer, 2005).

Acestridium dichromum Retzer, Nico \& Provenzano, 1999

Acestridium dichromum Retzer, Nico \& Provenzano, 1999: 316, fig. 3(a-c). Type locality: Pozo Lucas, tributary of Río Orinoco, $7 \mathrm{~km}$ south of San Fernando de Atabapo, $4^{\circ} 02^{\prime} \mathrm{N}, 67^{\circ} 42^{\prime} \mathrm{W}$, Amazonas State, Venezuela. Holotype: MBUCV V-26780.

Distribution: Upper Orinoco and Negro River basins, Venezuela (Schaefer, 2003c).

Acestridium discus Haseman, 1911

Acestridium discus Haseman, 1911a: 319, pls. 50 (fig. 1), 51. Type locality: Igarapé de Cachoeira Grande, near Manaos, Brazil. Holotype: FMNH 54339.

Distribution: Lower Negro River basin, Brazil (Schaefer, 2003c).

Acestridium martini Retzer, Nico \& Provenzano, 1999

Acestridium martini Retzer, Nico \& Provenzano, 1999: 318, fig. 3 (d-f). Type locality: Caño Pozo Azul, tributary of 
Río Orinoco, near Balneario Pozo Azul, 545'49"N, 67²9'21"W, Amazonas State, Venezuela, elev. 75 m. Holotype: MCNG 36484.

Distribution: Upper Orinoco and Negro River basins, Venezuela (Schaefer, 2003c).

\section{ANCISTRUS Kner, 1854}

Ancistrus Kner, 1854: 272. Type species: Hypostomus cirrhosus Valenciennes, 1836. Type by subsequent designation by Bleeker (1862-63: 2). Gender: Masculine.

Xenocara Regan, 1904b: 251. Type species: Chaetostomus latifrons Günther, 1869. Type by subsequent designation by Eigenmann (1910: 405, 410). Gender: Neuter.

Thysanocara Regan, 1906a: 96. Type species: Hypostomus cirrhosus Valenciennes, 1836. Type by monotypy. Gender: Neuter. Proposed as a subgenus of Xenocara.

Pristiancistrus Fowler, 1945a: 121. Type species: Pristiancistrus eustictus Fowler, 1945. Type by original designation. Gender: Masculine.

Monophyly and systematics: Schaefer (1986); Fisch-Muller (1999).

Key: Fisch-Muller et al. (2005, species lacking adipose fin).

Ancistrus aguaboensis Fisch-Muller, Mazzoni \& Weber, 2001

Ancistrus aguaboensis Fisch-Muller, Mazzoni \& Weber, 2001: 293, fig. 4 (middle). Type locality: Brazil: Goiás: Minaçú: córrego Água Boa: small tributary of rio Bonito, a left bank tributary of the upper rio Tocantins, $10 \mathrm{~km}$ from Minaçú on road Minaçú-Campinaçu (13³5'S 48¹4'W). Holotype: MNRJ 20850.

Distribution: Upper Tocantins River basin, Brazil (Fisch-Muller, 2003).

Ancistrus bodenhameri Schultz, 1944

Ancistrus brevifilis bodenhameri Schultz, 1944c: 305, pl. 10 (fig. A). Type locality: Río San Pedro at the bridge south of Mene Grande, Motatán system; Venezuela. Holotype: USNM 121066.

Distribution: Eastern and western Maracaibo lake tributaries, Venezuela (Fisch-Muller, 2003).

Ancistrus bolivianus (Steindachner, 1915)

Xenocara boliviana Steindachner, 1915e: 95, pl. 9 (figs. 5-6). Type locality: Rio Songo [= Zongo], Nord Yungas, Bolivia. Syntypes: NMW 43475 (2), NMW 43476 (27). Species described conditionally "Xenocara bufonia (C.V.) Regan, juv.? (an Xenocara boliviana n. sp.).”

Distribution: Beni, Mamoré and Madre de Dios River basins (Fisch-Muller, 2003).

Ancistrus brevifilis Eigenmann, 1920

Ancistrus brevifilis Eigenmann, 1920a: 7, pl. 1. Type locality: El Concejo, Rio Tiquirito, Venezuela. Holotype: CAS 64609.

Distribution: Tuy River basin, Venezuela (Fisch-Muller, 2003).

Ancistrus brevipinnis (Regan, 1904)

Xenocara brevipinnis Regan, 1904b: 257. Type locality: Rio Grande do Sul [Sistema da Laguna dos Patos, Brazil]. Holotype: BMNH 1891.3.16.76, illustrated in Muller (1989: 894, fig. 3, tab. 3).

Distribution: Laguna dos Patos basin, Brazil (Fisch-Muller, 2003).

Ancistrus bufonius (Valenciennes, 1840)

Hypostomus bufonius Valenciennes, in Cuvier \& Valenciennes, 1840b: 511 (377 in Strasbourg deluxe edition). Type locality: Rio Apurimac, qui descend des montagnes du haut Pérou, à environ 2000 mètres de hauteur. Syntypes: MNHN 0000-2228 (2).

Hypostomus calamita Valenciennes, in Cuvier \& Valenciennes, $1840 \mathrm{~b}$ : 515 (380 in Strasbourg deluxe edition). Type locality: dans les montagnes du haut Pérou, dans le rio Apurimac. Syntypes: MNHN 0000-2227 (2).

Distribution: Apurimac River basin, Peru (Fisch-Muller, 2003).

Ancistrus caucanus Fowler, 1943

Ancistrus caucanus Fowler, 1943a: 253, figs. 41-43. Type locality: Sonsón, Cauca River basin, Colombia. Holotype: ANSP 70516.

Distribution: Cauca River basin, Colombia; known only from the holotype (Fisch-Muller, 2003).

Ancistrus centrolepis Regan, 1913

Ancistrus centrolepis Regan, 1913d: 470. Type locality: River Choco, San Juan, Colombia. Syntypes: BMNH 
1910.7.11.122 (1), BMNH 1913.10.1.58 (1).

Ancistrus melas Eigenmann, 1916: 83. Type locality: Condoto [San Juan basin, Colombia]. Holotype: FMNH 58339, illustrated in Eigenmann (1922b: 87, fig. 20; pl. 12, fig. 2).

Ancistrus baudensis Fowler, 1945a: 122, fig. 33-36. Type locality: Alto Rio Baudó, Pacific slope at 3000 feet elevation, Colombia. Holotype: ANSP 71709.

Distribution: Coastal drainages of northern Andes, rivers of northern Colombia (Fisch-Muller, 2003).

Ancistrus chagresi Eigenmann \& Eigenmann, 1889

Ancistrus chagresi Eigenmann \& Eigenmann, 1889b: 47. Type locality: Rio Chagres [Panama]. Syntypes (2, 14-19 cm): MCZ 8026 (2). Name made available by diagnostic features in key on p. 47.

Distribution: Panama Canal Zone, Chagres, Gatun and Chorrera River basins, Panama (Fisch-Muller, 2003).

Ancistrus cirrhosus (Valenciennes, 1836)

Hypostomus cirrhosus Valenciennes, 1836, in Valenciennes, 1835-47: pl. 7 (fig. 3). Type locality: Not stated. Holotype: at MNHN; not found (Fisch-Muller, 2003). Name available from caption to plate, with the illustrated specimen the holotype.

Distribution: Paraná River basin, Argentina (Fisch-Muller, 2003).

Remarks: Species subsequently described in Cuvier \& Valenciennes (1840b: 511, 378 in Strasbourg deluxe edition), with the statement that the species was found in Missiones and Buénos-Ayres. However, Fisch-Muller (2003) presented evidence that the collection was likely to have come from Corrientes. Isbrücker's (1980: 68) designation of MNHN b-0603 as lectotype is invalid, since the illustrated specimen must be considered the holotype. Fisch-Muller (2003) suggested, instead, that the holotype was not preserved.

Ancistrus claro Knaack, 1999

Ancistrus claro Knaack, 1999a: 71, fig. 1. Type locality: Brazil, Mato Grosso, Rio Claro, above the bridge, in the direction of Chappada dos Guimaraes. Holotype: MCP 28667.

Distribution: Cuiabá River basin in upper Paraguay River drainage, Brazil (Fisch-Muller, 2003).

Ancistrus clementinae Rendahl, 1937

Ancistrus clementinae Rendahl, 1937: 4, fig. 2 (upper). Type locality: Ecuador, Syst. Rio de Clementina [=Río Pozuelos], nw. von Babahoyo. Holotype: NRM 10369.

Distribution: Pozuelos River basin in the Guayas River drainage, Ecuador (Fisch-Muller, 2003).

Ancistrus cryptophthalmus Reis, 1987

Ancistrus cryptophthalmus Reis, 1987: 82, fig. 1b. Type locality: Caverna Passa Três (about $300 \mathrm{~m}$ into the cave), Rio São Vicente system, São Domingos, Goias, Brazil. Holotype: MZUSP 28809.

Distribution: São Vicente and Angélica-Bezerra Cave system in upper Paranã River, Tocantins River drainage, Brazil (Fisch-Muller, 2003).

Remarks: See Reis (1992) for additional information.

Ancistrus cuiabae Knaack, 1999

Ancistrus cuiabae Knaack, 1999d: 151, figure on p. 150. Type locality: Brazil, Mato Grosso, Pantanal, 36 km SE Pocone, in a permanent remnant water of Rio Bento Gome [=Gomes]. Holotype: MCP 28671.

Distribution: Cuiabá River basin in upper Paraguay River drainage, Brazil (Fisch-Muller, 2003).

Ancistrus damasceni (Steindachner, 1907)

Xenocara damasceni Steindachner, 1907d: 292. Type locality: dem Parnahyba bei Victoria und Sa. Filomena [Brazil]. Syntypes: NMW 43489 (4), NMW 43490 (7), ZSM 7579, and additional specimens.

Distribution: Upper Parnaíba River basin, Brazil (Fisch-Muller, 2003).

Ancistrus dolichopterus Kner, 1854

Hypostoma punctatum Jardine, in Schomburgk, 1841: 145, fig. on p. 145 (Schomb. Drawings, No 68). Type locality: Rio Branco [Brazil]. Holotype: not preserved (Isbrücker, 1980: 72). Preoccupied by Hypostomus punctatus Valenciennes, 1840.

Ancistrus dolichopterus Kner, 1854: 274, pl. 3 (fig. 1). Type locality: Barra do Rio negro [Brazil]. Syntypes: NMW 46276: 1-2 (2), NMW 47164: 1-2 (2). Originally as Anc. dolichopterus.

Distribution: Upper and middle Brazilian Amazon River basin; Negro, lower Trombetas, Tefé, Madeira and Tapajós River basins, (Fisch-Muller, 2003). 
Ancistrus dubius Eigenmann \& Eigenmann, 1889

Ancistrus cirrhosus dubius Eigenmann \& Eigenmann, 1889b: 48. Type locality: Gurupa [Brazil]. Holotype: MCZ 7993 (but published as 7983). Name made available by diagnostic features in key on p. 47.

Distribution: Amazon River basin; middle Paraná and Paraguay River basins (Fisch-Muller, 2003).

Remarks: Eschmeyer et al. (1998: 498), Fisch-Muller (2003: 375), and the MCZ fish collection catalog, list MCZ 7993 as a syntype of Ancistrus cirrhosus dubius. However, the notation in Eigenmann \& Eigenmann (1889b: 48) includes the word "type" only in front of the number 7983 [sic for 7993] for a single specimen from Gurupa, which appropriately makes that specimen the holotype.

Ancistrus erinaceus (Valenciennes, 1840)

Hypostomus erinaceus Valenciennes, in Cuvier \& Valenciennes, 1840b: 510 (376 in Strasbourg deluxe edition). Type locality: Chili [in error]. Holotype: MNHN a-9568.

Distribution: Unknown; known only from holotype (Fisch-Muller, 2003).

Remarks: Type locality, if intended to refer to Chile, must be in error inasmuch as no specimens of Ancistrus have subsequently been found in any of the river basins of that country.

Ancistrus eustictus (Fowler, 1945)

Pristiancistrus eustictus Fowler, 1945a: 121, figs. 29-32. Type locality: Alto Rio Baudó, Pacific slope at 3000 feet elevation, Colombia. Holotype: ANSP 71710.

Distribution: Upper Baudó River basin, Colombia; known only from the holotype (Fisch-Muller, 2003).

Ancistrus formoso Sabino \& Trajano, 1997

Ancistrus formoso Sabino \& Trajano, 1997: 74, fig. 4. Type locality: Buraco do Ducho cave, Serra da Bodoquena, Município de Bonito $\left(21^{\circ} 08^{\prime} \mathrm{S}, 56^{\circ} 28^{\prime} \mathrm{W}\right)$, State of Mato Grosso do Sul, Brazil. Holotype: MZUSP 51386.

Distribution: Buraco do Ducho Cave in Formoso phreatic system, Brazil (Fisch-Muller, 2003).

Ancistrus fulvus (Holly, 1929)

Xenocara fulva Holly, 1929: 119. Type locality: Alto Rio Acara (Brasilien). Holotype: NMW 57203.

Distribution: Acará River basin, lower Amazon drainage, Brazil; known only from the holotype (Fisch-Muller, 2003).

Ancistrus galani Pérez \& Viloria, 1994

Ancistrus galani Pérez \& Viloria, 1994: 105, fig. 3. Type locality: Cueva de Los Laureles (Zu. 31), Río Socuy, Sierra de Perijá, Zulia State; Venezuela, 72²7'42"W, 1045'04"N; elevation 650 m. Holotype: MBLUZ 04351.

Distribution: Los Laureles Cave in Socuy River basin, Lake Maracaibo drainage Venezuela (Fisch-Muller, 2003).

Ancistrus gymnorhynchus Kner, 1854

Ancistrus gymnorhynchus Kner, 1854: 275. Type locality: Puerto Cabello [Venezuela]. Holotype: NMW 43495. Originally as Anc. gymnorhynchus; redescribed and illustrated by Steindachner (1917: 93, pl. 9, figs. 7-8).

Xenocara rothschildi Regan, 1905b: 242. Type locality: San Esteban, near Porto Cabello, Venezuela. Syntypes: BMNH 1904.11.9.27-31 (5).

Distribution: Coastal rivers of Carabobo and Yaracuy States; upper Pao River basin in Orinoco River drainage, Venezuela (Fisch-Muller, 2003).

Ancistrus heterorhynchus (Regan, 1912)

Xenocara heterorhynchus Regan, 1912b: 668, pl. 76 (fig. 2). Type locality: Uruhuasi [Inambari basin] Peru (4000 ft). Syntypes: BMNH 1911.12.20.35-36 (2).

Distribution: Inambari River basin in Madre de Dios River drainage, Peru; known only from type series (FischMuller, 2003).

Ancistrus hoplogenys (Günther, 1864)

Chatostomus hoplogenys Günther, 1864: 247. Type locality: River Capin, Para [Brazil]. Syntypes: BMNH 1849.11.8.89-91 (3).

Chaetostomus alga Cope, 1872a: 287, pl. 15 (fig. 3). Type locality: The Ambyiacu river, which empties into the Amazon near to Pebas, in Eastern Equador, some distance east of the Napo [Peru]. Syntypes: ANSP 16461 (1), ANSP 16462 (1).

Chaetostomus tectirostris Cope, 1872a: 288, pl. 15 (fig. 2). Type locality: The Ambyiacu river, which empties into the Amazon near to Pebas, in Eastern Equador, some distance east of the Napo [Peru]. Syntypes: ANSP 8298 
(1), ANSP 8300 (1).

Chaetostomus (Ancistrus) cirrhosus punctata Steindachner, 1881c: 123. Type locality: Rio Branco und Rio Guapore in Mato Grosso, Coary und Tabatinga [Amazonas, Brazil]. Syntypes: NMW 47163 (3), NMW 47279: 1-3 (3), NMW 47280: 1-2 (2), NMW 47281: 1-2 (2), NMW 47288 (1). Originally as Chaetostomus (Ancistrus) cirrhosus var. punctata.

Distribution: Amazon, Essequibo, and Paraguay River basins (Fisch-Muller, 2003).

Ancistrus jataiensis Fisch-Muller, Cardoso, da Silva \& Bertaco, 2005

Ancistrus jataiensis Fisch-Muller, Cardoso, da Silva \& Bertaco, 2005b: 567, fig. 4. Type locality: Brazil: Goias:

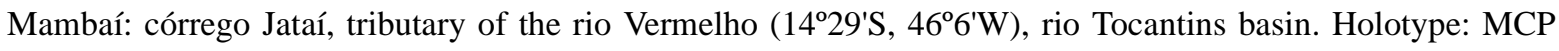
35244.

Distribution: upper Tocantins River basin, Brazil (Fisch-Muller et al., 2005b).

Ancistrus jelskii (Steindachner, 1876)

Chaetostomus Jelskii Steindachner, 1876: 603. Type locality: Amable Maria, Monterico, den Gebirgsbächen der hohen Anden in Peru [Tulumayo River basin, tributary of Péréné River]. Syntypes: at NMW.

Distribution: Tulumayo River basin in upper Ucayali River drainage, Peru (Fisch-Muller, 2003).

Remarks: Validity of species questioned by Fisch-Muller (2003).

Ancistrus latifrons (Günther, 1869)

Chaetostomus latifrons Günther, 1869: 426. Type locality: Upper Amazons, Peruvian Amazons. Holotype: BMNH 1869.5.21.4; holotype illustrated in Regan (1904b: 253, pl. 15, fig. 1).

Distribution: Upper Amazon and Solimões River basins, Brazil, Peru (Fisch-Muller, 2003).

Ancistrus leucostictus (Günther, 1864)

Chaetostomus leucostictus Günther, 1864: 248. Type locality: Essequibo [Guyana]. Possible holotype: BMNH 1864.1.21.85.

Distribution: Essequibo River basin, possibly all main rivers of Guianas (Fisch-Muller, 2003).

Remarks: Type status of specimen registered as holotype questioned by Muller (1989: 886; also Fisch-Muller, 2003).

Ancistrus lineolatus Fowler, 1943

Ancistrus lineolatus Fowler, 1943a: 255, figs. 44-47. Type locality: Florencia, Rio Orteguasa, Colombia. Holotype: ANSP 70517.

Distribution: Orteguaza River basin in upper Japurá River drainage, Colombia; known only from holotype (FischMuller, 2003).

Ancistrus lithurgicus Eigenmann, 1912

Ancistrus lithurgicus Eigenmann, 1912b: 241, pl. 25 (fig. 3). Type locality: Crab Falls; Lower Essequibo, British Guiana. Holotype: FMNH 53091

Distribution: Essequibo River basin, Guyana (Fisch-Muller, 2003).

Ancistrus macrophthalmus (Pellegrin, 1912)

Xenocara macrophthalma Pellegrin, 1912: 271, fig. 1. Type locality: 1'Orénoque [Venezuela]. Holotype: MNHN 1887-0650.

Distribution: Orinoco River and lower parts of some of its tributaries, Venezuela (Fisch-Muller, 2003).

Ancistrus maculatus (Steindachner, 1881)

Chaetostomus (Ancistrus) cirrhosus maculatus Steindachner, 1881c: 123. Type locality: Cudajas, Obidos, dem Rio Tajapouru [Brazil], und dem Rio Chagres [Panama]. Syntypes: NMW 47282 (1), NMW 47289: 1-2 (2), NMW 47290 (1), NMW 55035 (1). Originally as Chaetostomus (Ancistrus) cirrhosus var. maculatus.

Distribution: Amazon River basin, Brazil (Fisch-Muller, 2003).

Remarks: Type series mixed; specimens from Chagres River belong to Ancistrus chagresi (Fisch-Muller, 2003). A lectotype designation of a specimen from Brazil is necessary to stabilize the validity of this name.

Ancistrus malacops (Cope, 1872)

Chaetostomus malacops Cope, 1872a: 287, pl. 5 (figs. 2a-b). Type locality: The Ambyiacu river, which empties into the Amazon near to Pebas, in Eastern Equador, some distance east of the Napo [Peru]. Syntypes: ANSP 8299 (2). 
Distribution: Ampiyacu River basin, Peru (Fisch-Muller, 2003).

Remarks: Validity of species uncertain (Fisch-Muller, 2003).

Ancistrus maracasae Fowler, 1946

Ancistrus maracasae Fowler, 1946: 2, figs. 1-4. Type locality: Maracas River, Trinidad. Holotype: ANSP 71723.

Distribution: Maracas River basin, Trinidad and Tobago; known only from the holotype (Fisch-Muller, 2003).

Ancistrus martini Schultz, 1944

Ancistrus triradiatus martini Schultz, 1944c: 302, pl. 9 (fig. C). Type locality: Río Táchira, 7 km. north of San Antonio, Catatumbo system; Venezuela. Holotype: USNM 121064.

Distribution: Zulia River basin in the Catatumbo River drainage, Lake Maracaibo drainage, Venezuela (FischMuller, 2003).

Ancistrus megalostomus Pearson, 1924

Ancistrus megalostomus Pearson, 1924: 23, pl. 7 (fig. 1). Type locality: Huachi; at the junction of the Bopi and Cochabamba rivers, altitude 2,235 feet, Bolivia. Syntypes: CAS 64614 (2).

Distribution: Beni River basin in Madeira River drainage, Bolivia; known only from the syntypes (Fisch-Muller, 2003).

Ancistrus minutus Fisch-Muller, Mazzoni \& Weber, 2001

Ancistrus minutus Fisch-Muller, Mazzoni \& Weber, 2001: 298, fig. 5 (middle). Type locality: Brazil: Goiás: Minaçú: córrego Batéias, a left bank tributary of the upper rio Tocantins (1349'S 48²0'W). Holotype: MNRJ 20851.

Distribution: Upper Tocantins tributaries in Goiás State upstream of the dam of Serra da Mesa, Brazil (Fisch-Muller, 2003).

Ancistrus montanus (Regan, 1904)

Xenocara montana Regan, 1904b: 258, pl. 14 (fig. 6). Type locality: Tumupara [=Tumupasa], Andes of Bolivia, $1500 \mathrm{ft}$. Holotype: BMNH 1902.12.18.4.

Distribution: Beni River basin in upper Madeira River drainage, Bolivia (Fisch-Muller, 2003).

Ancistrus multispinis (Regan, 1912)

Xenocara multispinis Regan, 1912b: 668, pl. 76 (fig. 1). Type locality: Humboldt and Novo Rivers, Sta. Catherina, S. E. Brazil [restricted to Humboldt River, Rio Itapocú basin by lectotype designation]. Lectotype: BMNH 1910.7.26.32, illustrated in, and designated by, Muller (1989: 894, fig. 4, tab. 3).

Hemiancistrus albocinctus Ahl, 1936: 111. Type locality: Umbegung von Rio de Janeiro [Brazil]. Holotype: ZMB 20955.

Distribution: Atlantic coastal drainages of eastern Brazil, from Macacú River to Maquiné River (Fisch-Muller, 2003).

Ancistrus nationi (Fernández-Yépez, 1972)

Lasiancistrus nationi Fernández-Yépez, 1972a: 23, pl. 23. Type locality: Venezuela, la estación No 140 del Complejo Hidrográfico (4), Río Yaracuy. Holotype: personal collection of Fernández-Yépez, current whereabouts unknown.

Distribution: Yaracuy River basin, Venezuela (Fisch-Muller, 2003).

Remarks: Placement in Ancistrus follows Armbruster (2005: 567).

Ancistrus nudiceps (Müller \& Troschel, 1848)

Hypostomus nudiceps Müller \& Troschel, 1848: 631. Type locality: dem Takutu, Britisch-Guiana. Holotype: ZMB 3180.

Distribution: Takutu River basin in upper Branco River drainage, Guyana, known only from the holotype (FischMuller, 2003).

Remarks: Redescribed in Eigenmann (1912b: 236, footnote 24).

Ancistrus occidentalis (Regan, 1904)

Xenocara occidentalis Regan, 1904b: 257, pl. 14 (fig. 5). Type locality: Canelos [=Chambria], E. Ecuador. Syntypes: BMNH 1880.12.8.69-74 (6).

Distribution: Bobonaza River basin, Ecuador (Fisch-Muller, 2003).

Ancistrus occloi Eigenmann, 1928

Ancistrus occloi Eigenmann, in Myers, 1928: 86. Type locality: Ollantaytambo, Rio Urubamba, Peru, 9000 feet. 
Holotype: CAS 66847.

Distribution: Upper Urubamba River basin, Peru (Fisch-Muller, 2003).

Remarks: Redescribed in Eigenmann \& Allen (1942: 197, pl. 10 figs. 1-2).

Ancistrus parecis Fisch-Muller, Cardoso, da Silva \& Bertaco, 2005

Ancistrus parecis Fisch-Muller, Cardoso, da Silva \& Bertaco, 2005a: 529, fig. 3. Type locality: Brazil: Mato Grosso:

Campos de Júlio: rio Formiga (tributary of rio Juruena, upper rio Tapajós basin), on the road BR-364 (MT-235),

between Campos de Júlio and Sapezal, 13²41'01"S 59¹2'11"W. Holotype: MCP 35570.

Distribution: Upper Tapajós River basin, Mato Grosso, Brazil (Fisch-Muller, Cardoso, da Silva \& Bertaco, 2005a).

Ancistrus pirareta Muller, 1989

Ancistrus pirareta Muller, 1989: 891, fig. 2 (middle). Type locality: Salto Pirareta, sud de Piribebuy, affluent du Rio

Tebicuary-mi (Cordillera, Paraguay), 2530'S/56 $55^{\circ} \mathrm{W}$. Holotype: MHNG 2450.10.

Distribution: Upper Tebicuary-mi River basin in lower Paraguay River drainage, Paraguay (Fisch-Muller, 2003).

Remarks: Redescribed in Muller (1990).

Ancistrus piriformis Muller, 1989

Ancistrus piriformis Muller, 1989: 887, fig. 1 (middle). Type locality: Rio Acaray, en dessous du lac de barrage (Alto Paraná, Paraguay), $25^{\circ} 23^{\prime} \mathrm{S} / 54^{\circ} 42^{\prime} \mathrm{W}$. Holotype: MHNG 2450.11.

Distribution: Acaray River basin in middle Paraná River drainage, Paraguay (Fisch-Muller, 2003).

Remarks: Redescribed in Muller (1990).

Ancistrus ranunculus Muller, Rapp Py-Daniel \& Zuanon, 1994

Ancistrus ranunculus Muller, Rapp Py-Daniel \& Zuanon, 1994: 290, fig. 1 (middle). Type locality: Brazil, Pará, Rio

Xingú, furo do Tucum Seco, near Arroz Cru. Holotype: INPA 9509.

Distribution: Xingu and Tocantins River basins, Brazil (Fisch-Muller, 2003).

Ancistrus reisi Fisch-Muller, Cardoso, da Silva \& Bertaco, 2005

Ancistrus reisi Fisch-Muller, Cardoso, da Silva \& Bertaco, 2005b: 564, fig. 3. Type locality: Brazil: Goias: Mambaí: córrego das Dores, tributary of the rio Vermelho $\left(14^{\circ} 29^{\prime} \mathrm{S}, 46^{\circ} 6^{\prime} \mathrm{W}\right)$, rio Tocantins basin. Holotype: MCP 34818 .

Distribution: upper Tocantins River basin, Brazil (Fisch-Muller et al., 2005b).

Ancistrus spinosus Meek \& Hildebrand, 1916

Ancistrus spinosus Meek \& Hildebrand, 1916: 252. Type locality: Rio Calobre, tributary of the Rio Bayano, Panama. Holotype: FMNH 8942.

Distribution: Pacific and Caribbean river drainages of eastern Panama (Fisch-Muller, 2003).

Remarks: Fisch-Muller (2003) questioned the identity of the specimen registered as holotype.

Ancistrus stigmaticus Eigenmann \& Eigenmann, 1889

Ancistrus stigmaticus Eigenmann \& Eigenmann, 1889b: 48. Type locality: Sao Matheos; Goyaz [restricted to Goyaz, = Rio Araguaia drainage, upper course of Rio Vermelho at Goias, $15^{\circ} 47^{\prime} \mathrm{S}, 50^{\circ} 07^{\prime} \mathrm{W}$, by lectotype designation]. Lectotype: MCZ 77659, illustrated in, and designated by, Muller (1989: 895, fig. 5). Name made available by diagnostic features in key on p. 47.

Distribution: Upper Araguaia River basin, Brazil (Fisch-Muller, 2003).

Ancistrus tamboensis Fowler, 1945

Ancistrus tamboensis Fowler, 1945c: 9, figs. 13-15. Type locality: Satipo, at 600 meters elevation in upper Tambo basin, Rio Ucayali drainage, Peru. Holotype: ANSP 71643.

Distribution: Tambo River basin in upper Ucayali drainage, Peru, known only from holotype (Fisch-Muller, 2003).

Ancistrus taunayi Miranda Ribeiro, 1918

Ancistrus taunayi Miranda Ribeiro, 1918d: 631. Type locality: Itaqui, Rio Lageado, Rio Grande do Sul [Brazil]. Lectotype: MZUSP 975, designated by Britski (1969: 208) and illustrated in Muller (1989: 896, fig. 6, tab. 3).

Distribution: Middle Uruguay River basin (Fisch-Muller, 2003).

Ancistrus temminckii (Valenciennes, 1840)

Hypostomus Temminckii Valenciennes, in Cuvier \& Valenciennes, 1840b: 514 (380 in Strasbourg deluxe edition). Type locality: Cayenne [in error, interpreted as Suriname, region near Paramaribo, by Boeseman (1972: 305)]. Holotype: RMNH 3123.

Plecostomus aculeatus Gronow, in Gray, 1854: 158. Type locality: America Meridionali. Based on descriptions and 
illustrations in Gronovius (1763: no. 393) and Seba (1734-65: pl. 29, fig. 12). Syntypes: Whereabouts unknown.

Distribution: Saramacca, Suriname, and Maroni River basins, Suriname (Fisch-Muller, 2003).

Ancistrus tombador Fisch-Muller, Cardoso, da Silva \& Bertaco, 2005

Ancistrus tombador Fisch-Muller, Cardoso, da Silva \& Bertaco, 2005b: 560, fig. 1. Type locality: Brazil: Mato Grosso: Porto dos Gaúchos, irarapé Ribeirão Preto, on the road MT-338, about 26 km SE from Porto dos

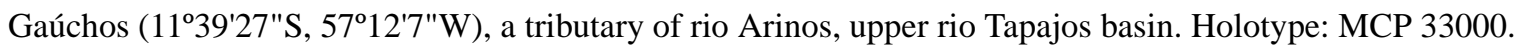

Distribution: Arinos River tributaries, upper Tapajós River basin, Brazil (Fisch-Muller, et al., 2005b).

Ancistrus trinitatis (Günther, 1864)

Chaetostomus trinitatis Günther, 1864: 246. Type locality: Trinidad. Syntypes: based on specimens reported on by Gill (1858: 409) as Ancistrus guacharote, possibly deposited at USNM, but whereabouts unknown.

Distribution: Trinidad Island, Trinidad and Tobago (Fisch-Muller, 2003).

Remarks: See comments on types and question about validity of species in Regan (1904b: footnote on p. 238; 1906 : 95). Generic allocation based on Armbruster (2004a: 13), although Armbruster (2005: 567) suggests that even that is uncertain.

Ancistrus triradiatus Eigenmann, 1918

Ancistrus triradiatus Eigenmann, 1918b: 680. Type locality: Quebrada Cramalote, Villavicencio; Andes east of Bogotá [upper Meta basin, Colombia]. Holotype: CAS 60164; holotype illustrated in Eigenmann (1922b: 224, pl. 13, figs. 3-4).

Distribution: Middle and lower Orinoco River tributaries; Valencia Lake and Los Guayos River basin; southern tributaries of Lake Maracaibo basin (Fisch-Muller, 2003).

Ancistrus variolus (Cope, 1872)

Chaetostomus variolus Cope, 1872a: 288. Type locality: The Ambyiacu river, which empties into the Amazon near to Pebas, in Eastern Equador, some distance east of the Napo [Peru]. Syntypes: ANSP 21284 (1), ANSP 21285 (1).

Distribution: Ampiyacu River basin, Peru (Fisch-Muller, 2003).

Ancistrus verecundus Fisch-Muller, Cardoso, da Silva \& Bertaco, 2005

Ancistrus verecundus Fisch-Muller, Cardoso, da Silva \& Bertaco, 2005a: 527, fig. 2. Type locality: Brazil: Rondônia: Vilhena: igarapé Piracolina, about $6 \mathrm{~km} \mathrm{~W}$ of Vilhena, on the road BR-364, upper rio Madeira basin, 1243'33"S 60¹1'34"W. Holotype: MCP 35572.

Distribution: Upper Madeira River basin, Rondônia, Brazil (Fisch-Muller et al., 2005a).

APHANOTORULUS Isbrücker \& Nijssen, 1983

Aphanotorulus Isbrücker \& Nijssen, 1983a: 105. Type species: Aphanotorulus frankei Isbrücker \& Nijssen, 1983. Type by original designation. Gender: Masculine.

Revision: Armbruster \& Page (1996) and Armbruster (1998b).

Aphanotorulus ammophilus Armbruster \& Page, 1996

Aphanotorulus ammophilus Armbruster \& Page, 1996: 385, fig. 2 (middle). Type locality: Venezuela, Estado Cojedes, Río San Carlos, R. Portuguesa drainage at Caño Hondo, $2 \mathrm{~km}$ west of Las Vegas on the road from Las Vegas to Campo Allegre - 9³1'51"N, 68³9'39"W. Holotype: INHS 32035.

Distribution: Portuguesa River basin, Venezuela (Weber, 2003).

Aphanotorulus unicolor (Steindachner, 1908)

Plecostomus unicolor Steindachner, 1908f: 164. Type locality: Rio Purús. Lectotype: NMW 44271: 2, designated by Armbruster (1998b: 254).

Plecostomus madeira Fowler, 1913: 571, fig. 23. Type locality: Madeira River, about 200 miles east of W. long. 635' $\mathrm{W}$, Brazil. Holotype: ANSP 39312.

Plecostomus popoi Pearson, 1924: 20, pl. 3 (fig. 2). Type locality: Popoi River, Upper Río Beni, Bolivia. Holotype: CAS 77346.

Plecostomus micropunctatus La Monte, 1935: 1, fig. 1. Type locality: Rio Purus: [...] in the vicinity of the mouth of Rio Macauhan, a tributary of Rio Iaco which, in turn, is a tributary of Rio Purus $\left(70^{\circ} 15^{\prime} \mathrm{W}, 7^{\circ} 30^{\prime} \mathrm{S}\right)$. Holotype: 
AMNH 12598.

Plecostomus chaparae Fowler, 1940c: 81, figs. 38-40. Type locality: Boca Chapare, Río Chimore, Cochabamba, Bolivia. Holotype: ANSP 69067.

Aphanotorulus frankei Isbrücker \& Nijssen, 1983a: 108, figs. 1-2. Type locality: [...] du Pérou, Dept. Ucayali, Prov. Coronel Portillo, système du Rio Aguaytia. Rio Neshua (environ $08^{\circ} 36^{\prime} \mathrm{S}, 74^{\circ} 50^{\prime} \mathrm{W}$ ), $60 \mathrm{~km} \mathrm{S.O}$. de Pucallpa, le long de la route de Tingo Maria. Holotype: ZMA 116.640.

Distribution: Upper Amazon River basin (Weber, 2003).

Remarks: Synonymy based on Armbruster (1998b: 254).

APISTOLORICARIA Isbrücker \& Nijssen, 1986

Apistoloricaria Isbrücker \& Nijssen, 1986b: 103. Type species: Apistoloricaria condei Isbrücker \& Nijssen, 1986. Type by original designation. Gender: Feminine.

Revision and key to species: Nijssen \& Isbrücker (1988).

Apistoloricaria condei Isbrücker \& Nijssen, 1986

Apistoloricaria condei Isbrücker \& Nijssen, 1986b: 104, fig. 1. Type locality: L’Equateur, Prov. Napo, bassin du

Napo (haut Amazone) embouchure du Rio Tiputini dans le Rio Napo au confluent de l'affluent principal, en eau profunde $\left(00^{\circ} 48.9^{\prime} \mathrm{S}, 75^{\circ} 32.5^{\prime} \mathrm{O}\right)$. Holotype: FMNH 94683.

Distribution: Upper Amazon drainage, Napo River basin, Ecuador (Nijssen \& Isbrücker, 1988).

Apistoloricaria laani Nijssen \& Isbrücker, 1988

Apistoloricaria laani Nijssen \& Isbrücker, 1988: 34, figs. 2-4. Type locality: Colombie, Dept. Meta, Río Metica, à El Aviso, environ $3^{\circ} 59^{\prime} \mathrm{N}, 72^{\circ} 59^{\prime} \mathrm{O}$. Holotype: ANSP 131483.

Distribution: Orinoco River drainage, Meta River basin, Colombia (Nijssen \& Isbrücker, 1988).

Apistoloricaria listrorhinos Nijssen \& Isbrücker, 1988

Apistoloricaria listrorhinos Nijssen \& Isbrücker, 1988: 36, figs. 5-7. Type locality: Colombie, Dept. Meta, Río Metica, à environ. 1.5 km E. de Rajote, $3^{\circ} 56^{\prime} \mathrm{N}, 7^{\circ} 03^{\prime} \mathrm{O}$. Holotype: ANSP 131482.

Distribution: Orinoco River drainage, Metica River basin, Colombia (Nijssen \& Isbrücker, 1988).

Apistoloricaria ommation Nijssen \& Isbrücker, 1988

Apistoloricaria ommation Nijssen \& Isbrücker, 1988: 37, figs. 8-10. Type locality: Pérou, Dept. Loreto, environs d'Iquitos, Rio Amazonas (Maranon) entre l'île Iquitos et l'île Lapuna, près du rivage de l'île Lapuna. Holotype: ANSP 138932.

Distribution: Lower Marañon River, Upper Amazon River drainage, Peru (Nijssen \& Isbrücker, 1988).

APOSTURISOMA Isbrücker, Britski, Nijssen \& Ortega, 1983

Aposturisoma Isbrücker, Britski, Nijssen \& Ortega, 1983: 34. Type species: Aposturisoma myriodon Isbrücker, Britski, Nijssen \& Ortega, 1983. Type by original designation. Gender: Neuter.

Aposturisoma myriodon Isbrücker, Britski, Nijssen \& Ortega, 1983

Aposturisoma myriodon Isbrücker, Britski, Nijssen \& Ortega, 1983: 35, figs. 3-6. Type locality: Pérou, Dept. Ucayali, Prov. Coronel Portillo, bassin du Aguaytia, Rio Huacamayó (environ $09^{\circ} 00^{\prime} \mathrm{S}, 75^{\circ} 29.0^{\prime} \mathrm{O}$ ) près de la roule de Pucallpa à Tingo Mario, environ 8 km N.E. du village d'Aguaytia. Holotype: MZUSP 15328.

Distribution: Upper Amazon River drainage, Aguaytia River basin, Peru (Isbrücker et al., 1983).

BARYANCISTRUS Rapp Py-Daniel, 1989

Baryancistrus Rapp Py-Daniel, 1989: 245. Type species: Hypostomus niveatus Castelnau, 1855. Type by original designation. Gender: Masculine.

Baryancistrus demantoides Werneke, Sabaj, Lujan \& Armbruster, 2005

Baryancistrus demantoides Werneke, Sabaj, Lujan \& Armbruster, 2005: 535, fig. 2. Type locality: Venezuela, Amazonas, río Orinoco drainage, río Ventuari near ornamental fish market in river, $04.07565^{\circ},-066.89285^{\circ}$. MCNG 54029.

Distribution: Orinoco River at confluence with Ventuari River and lower Ventuari River, Venezuela (Werneke, Sabaj, Lujan \& Armbruster, 2005). 
Baryancistrus longipinnis (Kindle, 1895)

Hemiancistrus longipinnis Kindle, 1895: 255. Type locality: Trocera on Tocantins, Brazil. Holotype: MCZ 29051.

Distribution: Tocantins River basin, Brazil (Fisch-Muller, 2003).

Baryancistrus niveatus (Castelnau, 1855)

Hypostomus niveatus Castelnau, 1855: 43, pl. 21 (fig. 3). Type locality: du Rio Araguay, de la province de Goyaz

[Brazil]. Possible syntypes: MNHN a-9453 and MNHN a-9454 (dry specimens).

Distribution: Tocantins, Xingu, Tapajós, and Trombetas River basins, Brazil (Fisch-Muller, 2003).

Remarks: Literature mentions 2 syntypes (e.g., Regan, 1904b, Bertin \& Estève, 1950), but the description appears to be based on a single alcohol-preserved specimen (Fisch-Muller, 2003) which was not found at MNHN.

BROCHILORICARIA Isbrücker \& Nijssen, 1979

Brochiloricaria Isbrücker \& Nijssen, in Isbrücker, 1979a: 90. Type species: Brochiloricaria chauliodon Isbrücker, 1979. Type by original designation. Gender: Feminine.

Brochiloricaria chauliodon Isbrücker, 1979

Brochiloricaria chauliodon Isbrücker, 1979a: 102, fig. 15. Type locality: Argentine, Prov. Entre Rios, Isla El Dorado, Paraná Guaza (localité non précisée). Holotype: ZSM 23342.

Distribution: La Plata River basin, Argentina (Isbrücker, 1979).

Brochiloricaria macrodon (Kner, 1853)

Loricaria macrodon Kner, 1853a: 12, 15, pl. 2. Type locality: Aus dem Cujabaflusse [Brazil]. Holotype: NMW 45087. Originally as Lor. macrodon.

Distribution: Paraguay River basin, Brazil.

\section{CHAETOSTOMA Tschudi, 1846}

Chaetostoma Tschudi, 1846: 25. Type species: Chaetostoma loborhynchos Tschudi, 1846. Type by monotypy. Gender: Neuter.

Chaetostomus Kner, 1854: 271, 286. Type species: Chaetostoma loborhynchos Tschudi, 1846. Type by being a replacement name. Unjustified emendation of Chaetostoma Tschudi, 1846. Gender: Masculine.

Hypocolpterus Fowler, 1943a: 259. Type species: Hypocolpterus analis Fowler, 1943. Type by original designation. Gender: Masculine.

Keys: Schultz (1944c: 287, Venezuela) and Fernández-Yépez (1945: 29, Venezuela).

Monophyly: Schaefer (1986).

Biogeography: Lasso \& Provenzano (1998: 62).

Chaetostoma aburrense (Posada, 1909)

Hypostomus aburrensis Posada, 1909: 297. Type locality: Medellín (?). Holotype: Whereabouts unknown.

Distribution: Cauca River basin, Colombia (Fisch-Muller, 2003).

Chaetostoma aequinoctiale Pellegrin, 1909

Chaetostomus aequinoctialis Pellegrin, 1909c: 518. Type locality: Rio Pove, Santo Domingo de los Colorados (560 mètres), Équateur. Holotype: MNHN 1904-0017.

Distribution: Guayas River basin, Ecuador (Fisch-Muller, 2003).

Chaetostoma alternifasciatum Fowler, 1945

Chaetostoma alternifasciatum Fowler, 1945a: 116, fig. 21-24. Type locality: Morelia, Rio Caquetá basin, Colombia. Holotype: ANSP 71711.

Distribution: Caqueta River basin in upper Japurá River drainage, Colombia (Fisch-Muller, 2003).

Chaetostoma anale (Fowler, 1943)

Hypocolpterus analis Fowler, 1943a: 261, figs. 56-59. Type locality: Florencia, Rio Orteguasa, Colombia. Holotype: ANSP 70525.

Distribution: Orteguasa River basin, upper Japurá River drainage, Colombia (Fisch-Muller, 2003).

Chaetostoma anomalum Regan, 1903

Chaetostomus anomalus Regan, 1903a: 599. Type locality: Merida, Venezuela, altitude 1500 meters and from the Albireggas and Milla rivers, above Merida, altitude 3500 meters. Syntypes: BMNH 1903.6.30.91-101, USNM 
133135.

Distribution: Lake Maracaibo basin, upper Chama, Capazon and Escalante River basins, Venezuela (Fisch-Muller, 2003).

Chaetostoma branickii Steindachner, 1881

Chaetostomus branickii Steindachner, 1881c: 118, pl. 6 (fig. 1). Type locality: Callacate [Peru]. Syntypes: NMW 47270 (2), NMW 47271 (3).

Distribution: Upper Chamaya River basin in upper Marañon River drainage, Peru (Fisch-Muller, 2003).

Remarks: Fisch-Muller (2003) clarified the type locality, as follows: Cajamarca: Rio Chota (= rio Chotano), alt. ca. 1465-1585 m., ca. $12 \mathrm{~km}$ from Cutervo N of Chota; $06^{\circ} 25^{\prime} \mathrm{S} / 78^{\circ} 56^{\prime} \mathrm{W}$.

Chaetostoma breve Regan, 1904

Chaetostomus brevis Regan, 1904b: 247, pl. 13 (fig. 3). Type locality: Bomboiza and Zamora Rivers [Santiago River basin], Eastern Ecuador. Syntypes (6): BMNH 1898.11.4.33-36 (4).

Distribution: Zamora River basin in upper Marañon River drainage, Ecuador (Fisch-Muller, 2003).

Chaetostoma brevilabiatum Dahl, 1942

Chaetostomus brevilabiatus Dahl, 1942: 80, fig. 1. Type locality: Rio Volcán near its junction to Rio San Bartolomé (tributary to Rio Magdalena on the left side, between Rios Nare and Ité) municipio of Remedios, department of Antióquia, Republic of Colombia. Altitude approximately 600 m. Magdalena system. Holotype: at ZMUL.

Distribution: Magdalena River basin, Colombia; known only from the holotype (Fisch-Muller, 2003).

Chaetostoma dermorhynchum Boulenger, 1887

Chaetostomus dermorhynchus Boulenger, 1887c: 277, pl. 22 (upper fig.). Type locality: Canelos [Bobonaza River]; eastern Ecuador. Syntypes: BMNH 1880.12.8.64-66 (3).

Distribution: Pastaza, upper Bobonaza, Napo, and upper Curaray River basins, Ecuador (Fisch-Muller, 2003).

Chaetostoma dorsale Eigenmann, 1922

Chaetostomus dorsalis Eigenmann, 1922b: 226. Type locality: Quebrada Cramalote, Villavicencio [Meta River basin, Colombia]. Holotype: CAS 77093.

Distribution: Upper Meta and upper Apuré River basins, Colombia and Venezuela (Fisch-Muller, 2003).

Chaetostoma dupouii Fernández-Yépez, 1945

Chaetostoma dupouii Fernández-Yépez, 1945: 27. Type locality: el rio Encantado, en el sitio indicado en los planos adjuntos y más o menos a un kilómetro más arriba de la confluencia do los ríos Encantado y Río Grande, tributario éste del Tuy; Venezuela. Holotype: collection of AFY; current whereabouts unknown.

Distribution: Tuy River basin, Venezuela (Fisch-Muller, 2003).

Chaetostoma fischeri Steindachner, 1879

Chaetostomus Fischeri Steindachner, 1879b: 150. Type locality: Mamoni-Fluss, bei Chepo im Isthmus von Panama. Syntypes: NMW 47170 (1), NMW 47171 (1), NMW 47172 (2), NMW 47173 (1), NMW 54991 (2). Illustrated and described in more detail in Steindachner (1879f: 162, pl. 4).

Distribution: Pacific versant rivers: Bayano, Tuira, San Juan and Guyas River basins; Caribbean versant Rivers: Chagres, Atrato, Sinú and Magdalena River basins (Fisch-Muller, 2003).

Chaetostoma greeni Isbrücker, 2001

Chaetostomus maculatus Regan, 1904b: 246, pl. 14 (fig. 4). Type locality: Rozmaiu, Upper Peru. Syntypes: BMNH 1903.10.12.3-4 (2). Preoccupied by Chaetostomus cirrhosus var. maculata Steindachner, 1881; replaced by Chaetostoma greeni Isbrücker (2001).

Chaetostoma greeni Isbrücker in Isbrücker et al., 2001: 24. Type locality: Rozmaiu, Upper Peru. Syntypes: BMNH 1903.10.12.3-4 (2). Replacement for Chaetostomus maculatus Regan, 1904, preoccupied by Chaetostomus (Ancistrus) cirrhosus var. maculata Steindachner, 1881.

Distribution: Roz River basin, Peru (Fisch-Muller, 2003).

Chaetostoma guairense Steindachner, 1881

Chaetostomus guairensis Steindachner, 1881c: 121, pl. 3 (fig. 1). Type locality: dem Guaire bei Carácas [Venezuela]. Syntypes: NMW 47183 (2), NMW 47184 (2), NMW 47185 (3).

Distribution: Tuy and Guaire River and Lake Valencia basins, Venezuela (Fisch-Muller, 2003).

Chaetostoma jegui Rapp Py-Daniel, 1991 
Chaetostoma jegui Rapp Py-Daniel, 1991: 240, fig. 2 (middle). Type locality: Brazil: Federal Territory of Roraima: Rio Uraricoera, Maracá Island, rocks above Furo Maracá, 321'N 61²6'W. Holotype: INPA 2822.

Distribution: Uraricoera and Tacutu River basins, Branco River drainage, Brazil (Fisch-Muller, 2003).

Chaetostoma lepturum Regan, 1912

Chaetostomus lepturus Regan, 1912b: 667, pl. 75 (fig. 1). Type locality: Rio Tamana, Rio San Juan, Choco, S. W. Colombia. Syntypes: BMNH 1910.7.11.116-118 (3).

Distribution: Upper San Juan River basin, Colombia (Fisch-Muller, 2003).

Chaetostoma leucomelas Eigenmann, 1918

Chaetostomus leucomelas Eigenmann, 1918b: 681. Type locality: Rio Patia, halfway between the Rios Magui and Telembi [Colombia]. Holotype: CAS 60167; holotype illustrated in Eigenmann (1922b: 84, pl. 12, figs. 5-6).

Distribution: Patia and upper Cauca River basins, Colombia (Fisch-Muller, 2003, Maldonado-Ocampo et al., 2005).

Chaetostoma lineopunctatum Eigenmann \& Allen, 1942

Chaetostoma lineopunctata Eigenmann \& Allen, 1942: 192, pl. 6 (fig. 1). Type locality: Rio Azupizú [Rio Pachitea drainage, Peru]. Holotype: CAS 64650.

Distribution: Aguaytia, Pachitea and Pisqui River basins, Ucayali River drainage, Upper Amazon system, Peru (Fisch-Muller, 2003).

Chaetostoma loborhynchos Tschudi, 1846

Chaetostoma loborhynchos Tschudi, 1846: 26, pl. 4. Type locality: dem Rio Tullumayo in der Montaña de Vitoc, am Ostabhang der Anden; Peru. Holotype: NMW 47190.

Distribution: Tambo River basin, Ucayali River drainage, Peru (Fisch-Muller, 2003).

Chaetostoma machiquense Fernández-Yépez \& Martín Salazar, 1953

Chaetostoma machiquensis Fernández-Yépez \& Martín Salazar, 1953: 238, fig. on p. 239. Type locality: Río Negro, a 16 kms. Oeste de Machiques; Perijá; Hoya del Lago de Maracaibo, Edo. Zulia, Venezuela. Holotype: MHNLS 1558.

Distribution: Negro River basin in the Lake Maracaibo drainage, Venezuela (Fisch-Muller, 2003).

Chaetostoma marcapatae Regan, 1904

Chaetostomus marcapatae Regan, 1904b: 246, pl. 14 (fig. 1). Type locality: Marcapata Valley, E. Peru. Holotype: BMNH 1902.5.29.211.

Distribution: Inambari River basin in the Madeira River drainage, Peru; known only from the holotype (FischMuller, 2003).

Chaetostoma marginatum Regan, 1904

Chaetostomus marginatus Regan, 1904b: 249, pl. 13 (fig. 1). Type locality: Salidero, N.W. Ecuador. Syntypes (3): BMNH 1901.8.3.27-28 (2), possibly BMNH 1902.5.27.44 (1), but recorded as coming from Rio Durango in register.

Distribution: Bogota, Chimbo and San Juan River basins, Colombia, Ecuador (Fisch-Muller, 2003).

Remarks: Fisch-Muller (2003) clarified type locality as follows: Ecuador: Esmeraldas: rio Bogota, near Bulún (= Pulún), alt. 50-110 m, ca. $01^{\circ} 05^{\prime} \mathrm{N} 78^{\circ} 40^{\prime} \mathrm{W}$.

Chaetostoma marmorescens Eigenmann \& Allen, 1942

Chaetostoma marmorescens Eigenmann \& Allen, 1942: 193. Type locality: Huanacachupa Creek, near Huánuco, elevation 6000 feet. ... A small tumultuous stream from the eastern slope of the Central Cordillera to the Huallaga, a league above Huánuco [Peru]. Holotype: IU 15403 (135 mm specimen); whereabouts unknown, apparently not at CAS.

Distribution: Upper Huallaga River basin, Marañon River drainage, Peru (Fisch-Muller, 2003).

Chaetostoma microps Günther, 1864

Chaetostomus microps Günther, 1864: 250. Type locality: Andes of Western Ecuador. Syntypes: BMNH 1860.6.16.137-143 (7). Syntypes redescribed and illustrated in Regan (1904b: 251, pl. 14, fig. 3)

Distribution: Andes of western Ecuador (Fisch-Muller, 2003).

Chaetostoma milesi Fowler, 1941

Chaetostomus milesi Fowler, 1941c: 2, figs. 1-5. Type locality: Honda, Colombia. Holotype: ANSP 69330.

Distribution: Magdalena River, Colombia, and Apuré River basin, Venezuela (Fisch-Muller, 2003). 
Chaetostoma mollinasum Pearson, 1937

Chaetostomus mollinasus Pearson, 1937: 96, pl. 13 (fig. 1). Type locality: Balsas, 3500 feet, and Cajamarca, 9843 feet, Peru. Syntypes: CAS 64653 (8), CAS 64654 (5).

Distribution: Marañon and Cajamarca River basins, Peru (Fisch-Muller, 2003).

Chaetostoma niveum Fowler, 1944

Chaetostoma niveum Fowler, 1944b: 238, figs. 14-16. Type locality: clear waters of Rio Jurubidá, Nuquí [northwestern Colombia]. Holotype: ANSP 71432.

Distribution: Jurubidá River basin, Colombia (Fisch-Muller, 2003).

Chaetostoma nudirostre Lütken, 1874

Chaetostomus nudirostris Lütken, 1874a: 207. Type locality: Valencia [Venezuela]. Holotype: ZMUC P 30168, illustrated by Steindachner (1881c: 120, pl. 5 figs. 2, 2a).

Distribution: Lake Valencia basin, Venezuela (Fisch-Muller, 2003).

Chaetostoma palmeri Regan, 1912

Chaetostomus palmeri Regan, 1912b: 667, pl. 75 (fig. 3). Type locality: Rio Tamana, Rio San Juan, Choco, S. W. Colombia. Syntypes: BMNH 1910.7.11.120-121 (2).

Distribution: Tamana River basin in the San Juan River drainage, Colombia (Fisch-Muller, 2003).

Chaetostoma patiae Fowler, 1945

Chaetostoma patiae Fowler, 1945a: 118, figs. 25-28. Type locality: Rio Patia, at 3000 feet elevation, Pacific slope of southwestern Colombia. Holotype: ANSP 71716.

Distribution: Patia River basin, Colombia (Fisch-Muller, 2003, Maldonado-Ocampo et al., 2005).

Chaetostoma paucispinis Regan, 1912

Chaetostomus paucispinis Regan, 1912b: 667, pl. 75 (fig. 2). Type locality: Tado, Rio San Juan, Choco, Colombia. Holotype: BMNH 1910.7.11.119.

Distribution: San Juan River basin, Colombia (Fisch-Muller, 2003).

Chaetostoma pearsei Eigenmann, 1920

Chaetostomus pearsei Eigenmann, 1920a: 8, pl. 2. Type locality: Rio Castaño [Lake Valencia basin] at Maracay, under rocks, Venezuela. Holotype: CAS 64655

Distribution: Lake Valencia and Tuy River basins, Venezuela (Fisch-Muller, 2003).

Chaetostoma sericeum Cope, 1872

Chaetostomus sericeus Cope, 1872a: 288. Type locality: The Ambyiacu river, which empties into the Amazon near to Pebas, in Eastern Equador, some distance east of the Napo [Peru]. Lectotype: ANSP 22005, designated by Fowler (1915a: 234)

Distribution: Ampiyacu River basin, Peru (Fisch-Muller, 2003).

Chaetostoma sovichthys Schultz, 1944

Chaetostoma anomala sovichthys Schultz, 1944c: 292, pl. 8 (fig. b). Type locality: near the bridge over the Río San Pedro, a tributary of the Río Motatán, southeast of Mene Grande, in the Maracaibo basin; Venezuela. Holotype: USNM 121053.

Distribution: Lake Maracaibo, Motatán River and tributaries, Venezuela (Fisch-Muller, 2003); Catatumbo River basin, Colombia (Maldonado-Ocampo et al., 2005).

Chaetostoma stannii, 1874

Chaetostomus Stannii Lütken, 1874a: 206. Type locality: Puerto Cabello [Venezuela]. Holotype: ZMUC P 30169, illustrated in Steindachner (1881c: 120, pl. 5 fig. 4, 4a).

Distribution: Caribbean coastal drainages of Venezuela: Aroa, Tocuyo, Urama and Yaracuy River basins (FischMuller, 2003).

Chaetostoma tachiraense Schultz, 1944

Chaetostoma tachiraensis Schultz, 1944c: 288, pl. 7 (fig.c). Type locality: Río Táchira, 7 km. north of San Antonio, Estado de Táchira, Venezuela; the Río Táchira is a tributary of the Río Zulia, Catatumbo system, Maracaibo basin. Holotype: USNM 121052.

Distribution: Catatumbo River basin in the Lake Maracaibo drainage (up to altitudes between 1,000 and 2,000 m above sea level), Colombia and Venezuela (Fisch-Muller, 2003). 
Chaetostoma taczanowskii Steindachner, 1882

Chaetostomus Taczanowskii Steindachner, 1882b: 177. Type locality: Rio de Tortora [Peru]. Species illustrated and described in more detail in Steindachner (1882c: 23, pl. 5, figs. 2, 2a). Lectotype: NMW 47219 (9 cm); designated by Isbrücker (1980: 64).

Distribution: Huallaga and Urubamba River basins, Peru (Fisch-Muller, 2003).

Remarks: Isbrücker (1980: 64) interpreted original description to be based on a single specimen, which he concluded must be the holotype. The later description (Steindachner, 1882c: 23, pl. 5, figs. 2, 2a) indicated that more than two specimens were examined, which requires that the examined specimens be considered as syntypes. However, since there was no clear indication of multiple specimens in the original description, Isbrücker's (1980: 64) interpretation of the $9 \mathrm{~cm}$ specimen as the holotype became a de facto lectotype designation. Type locality interpreted by Fisch-Muller (2003) as: Amazon: Upper rio Huambo basin, Río de la Tortora, 06² $26^{\prime} \mathrm{S}^{7} 7^{\circ} 18^{\prime} \mathrm{W}$. Redescribed in Eigenmann \& Allen (1942: 192).

Chaetostoma thomsoni Regan, 1904

Chaetostomus thomsoni Regan, 1904b: 250, pl. 14 (fig. 2). Type locality: Villeta [Magdalena basin], Colombia. Syntypes: BMNH 1902.5.15.28-30 (3).

Distribution: Magdalena River basin, Colombia (Fisch-Muller, 2003, Maldonado-Ocampo et al., 2005).

Chaetostoma vagum Fowler, 1943

Chaetostomus vagus Fowler, 1943a: 258, figs. 52-55. Type locality: Florencia, Rio Orteguasa, Colombia. Holotype: ANSP 70521.

Distribution: Orteguaza River basin, upper Japurá River drainage, Colombia (Fisch-Muller, 2003).

Chaetostoma vasquezi Lasso \& Provenzano, 1998

Chaetostoma vasquezi Lasso \& Provenzano, 1998: 55, fig. 1b. Type locality: Raudales del Caño El Cambur, afluente del Río Caura, $20 \mathrm{~km}$ al suroeste del puente del mismo río, Estado Bolívar, Venezuela. Holotype: MHNLS 8791.

Distribution: Orinoco, Caura and Caroni River basins, Venezuela (Fisch-Muller, 2003).

Chaetostoma venezuelae (Schultz, 1944)

Corymbophanes venezuelae Schultz, 1944d: 41, pl. 1 (fig. 1). Type locality: Río Caripe, Caripito, Venezuela. Holotype: USNM 120752.

Distribution: San Juan River basin, Venezuela (Fisch-Muller, 2003).

Chaetostoma yurubiense Ceas \& Page, 1996

Chaetostoma yurubiense Ceas \& Page, 1996: 672, fig. 2 (top). Type locality: Río Mayorica (Río Yaracuy system) at Hwy 3, 9 km N Albarico near the town of Mayorica, State of Lara, Venezuela (10²5'43"N, 68 $\left.40^{\prime} 47^{\prime \prime} \mathrm{W}\right)$. Holotype: INHS 34942.

Distribution: Yaracuy, Urama and Aroa River basins, Venezuela (Fisch-Muller, 2003).

CORDYLANCISTRUS Isbrücker, 1980

Cordylancistrus Isbrücker, 1980: 48. Type species: Pseudancistrus torbesensis Schultz, 1944. Type by original designation. Gender: Masculine.

Monophyly: Schaefer (1986: 103).

Cordylancistrus daguae (Eigenmann, 1912)

Hemiancistrus daguae Eigenmann, 1912a: 11. Type locality: Caldas, Colombia. Holotype: FMNH 56052, illustrated in Eigenmann (1922b: 78, pl. 12, figs. 1-2).

Distribution: Dagua River basin, Colombia (Fisch-Muller, 2003).

Cordylancistrus perijae Pérez \& Provenzano, 1996

Cordylancistrus perijae Pérez \& Provenzano, 1996: 29, fig. 2 (top). Type locality: Venezuela, Zulia State, Maracaibo basin, Sierra de Perijá, Caño Colorado, tributary to the Palmar River, 100 meters from dam site (under construction), approx. 10³7'12"N 72²5'34"W; elevation 150 m. Holotype: MBLUZ 4413.

Distribution: Palmar and Socuy River basins, Lake Maracaibo drainage, Venezuela (Fisch-Muller, 2003).

Cordylancistrus platycephalus (Boulenger, 1898)

Chaetostomus platycephalus Boulenger, 1898c: 12. Type locality: Rio Bomboiza [Zamora basin], Equateur oriental. 
Syntypes: BMNH 1898.11.4.42 (1), MZUT 1551 (1).

Distribution: Zamora River basin in upper Marañon River drainage, Ecuador (Fisch-Muller, 2003).

Cordylancistrus platyrhynchus (Fowler, 1943)

Hemiancistrus platyrhynchus Fowler, 1943a: 252, figs. 36-39. Type locality: Florencia, Colombia. Holotype: ANSP 70512.

Distribution: Upper Caquetá River basin, upper Japurá River drainage, Colombia (Fisch-Muller, 2003).

Cordylancistrus torbesensis (Schultz, 1944)

Pseudancistrus torbesensis Schultz, 1944c: 296, pl. 8 (fig. c). Type locality: 1 km. above Táriba in the Río Torbes

[Apuré River basin], Orinoco system, Venezuela. Holotype: USNM 121001.

Distribution: Torbes River basin in Apuré River drainage, Venezuela (Fisch-Muller, 2003).

CORUMBATAIA Britski, 1997

Corumbataia Britski, 1997: 231. Type species: Corumbataia cuestae Britski, 1997. Type by original designation. Gender: Feminine.

Corumbataia cuestae Britski, 1997

Corumbataia cuestae Britski, 1997: 234, fig. 3. Type locality: Cachoeira de Analândia, rio Corumbataí, Analândia, SP [Brazil]. Holotype: MZUSP 51222.

Distribution: Tietê River basin, Brazil (Schaefer, 2003c).

Corumbataia tocantinensis Britski, 1997

Corumbataia tocantinensis Britski, 1997: 237, fig. 4. Type locality: rio Vermelho, Goiás, GO [Brazil]. Holotype: MZUSP 51223.

Distribution: Araguaia and Tocantins River basins, Brazil (Schaefer, 2003c).

\section{CORYMBOPHANES Eigenmann, 1909}

Corymbophanes Eigenmann, 1909a: 5. Type species: Corymbophanes andersoni Eigenmann, 1909. Type by monotypy. Gender: Masculine.

Revision: Armbruster et al. (2000).

Corymbophanes andersoni Eigenmann, 1909

Corymbophanes andersoni Eigenmann, 1909a: 5. Type locality: Aruataima Falls, Upper Potaro River, British Guiana. Holotype: FMNH 52675, illustrated in Eigenmann (1912b: pl. 27, fig.1).

Distribution: Essequibo River drainage, upper Potaro River, upstream Kaieteur Falls, Guyana (Weber, 2003).

Corymbophanes kaiei Armbruster \& Sabaj, 2000

Corymbophanes kaiei Armbruster \& Sabaj, in Armbruster et al., 2000: 1002, fig. 3 (middle). Type locality: Guyana, Potaro-Siparuni Region, Oung Creek (Chenapou River-Potaro River Drainage) about one hour hike southwest of coordinates $04^{\circ} 58^{\prime 2} 26^{\prime \prime N}, 59^{\circ} 34^{\prime} 41^{\prime \prime}$ W (mouth of Chenapou River). Holotype: UG/CSBD F644.

Distribution: Essequibo river drainage, upper Potaro River basin, Guyana (Weber, 2003).

CROSSOLORICARIA Isbrücker, 1979

Crossoloricaria Isbrücker, 1979a: 90. Type species: Loricaria variegata Steindachner, 1879. Type by original designation. Gender: Feminine.

Crossoloricaria bahuaja Chang \& Castro, 1999

Crossoloricaria bahuaja Chang \& Castro, 1999: 83, fig. 1. Type locality: Peru, Departamento Madre de Dios, Provincia Tambopata, Río Tambopata, 1247'05"S, 69¹8'55"W. Holotype: MUSM 9916.

Distribution: Madre de Dios River basin in Peru, Grande River in Bolivia and Manuripe River in Pando (Chang \& Castro, 1999).

Crossoloricaria cephalaspis Isbrücker, 1979

Crossoloricaria cephalaspis Isbrücker, 1979a: 102, fig. 18. Type locality: Colombie, Río Magdalena. Holotype: BMNH 1947.7.1.228.

Distribution: Magdalena River basin, Colombia (Isbrücker, 1979).

Crossoloricaria rhami Isbrücker \& Nijssen, 1983 
Crossoloricaria rhami Isbrücker \& Nijssen, 1983b: 9, fig. 1. Type locality: Pérou, Dept. Ucayali, Prov. Coronel Portillo, Bassin du Rio Aguaytia, Rio Huacamayo (ca. $9^{\circ} \mathrm{S}, 7^{\circ} 29^{\prime} \mathrm{O}$ ) près de la route de Pucallpa à Tingo Maria (Carretera Federico Badadre), à environ 8 km au N. E. du village Aguaytia. Holotype: ZMA 116391.

Distribution: Upper Amazon River basin (Isbrücker \& Nijssen, 1983).

Crossoloricaria variegata (Steindachner, 1879)

Loricaria variegata Steindachner, 1879b: 151. Type locality: Mamoni (Mamoni-Flusses, bei Chepo in Isthmus von Panama). Holotype: NMW 45138. Species illustrated and described in more detail in Steindachner (1879f: 165, pl. 3).

Distribution: Mamoni, Tuira and Yape River basins in Panama; San Juan and Sinu River basins in Colombia (Ferraris, 2003c).

Crossoloricaria venezuelae (Schultz, 1944)

Loricaria variegata venezuelae Schultz, 1944c: 329, pl. 12 (fig. C). Type locality: Río Palmar at the bridge about 70 km southwest of Maracaibo [Venezuela]. Holotype: USNM 121108.

Distribution: Lake Maracaibo drainage (Schultz, 1944); also Magdalena River basin, Colombia (MaldonadoOcampo et al., 2005).

CTENILORICARIA Isbrücker \& Nijssen, 1979

Cteniloricaria Isbrücker \& Nijssen, in Isbrücker, 1979a: 91. Type species: Loricaria platystoma Günther, 1868. Type by original designation. Gender: Feminine.

Cteniloricaria fowleri (Pellegrin, 1908)

Oxyloricaria Fowleri Pellegrin, 1908: 126. Type locality: Rivière Camopi (Guyane française). Holotype: MNHN 1901-0372.

Distribution: Oyapock River basin, Brazil and French Guiana (le Bail et al., 2000).

Remarks: Species redescribed in le Bail et al. (2000).

Cteniloricaria maculata (Boeseman, 1971)

Parasturisoma maculata Boeseman, 1971: 33, pl. 5. Type locality: Sipaliwini, near airstrip, upper Corantijn River basin, Surinam. Holotype: RMNH 26381.

Distribution: Maroni and Corantijn River basins (le Bail et al., 2000).

Remarks: Species redescribed in le Bail et al. (2000).

Cteniloricaria platystoma (Günther, 1868)

Loricaria platystoma Günther, 1868a: 478. Type locality: Surinam. Lectotype: BMNH 1866.8.14.124, designated by Isbrücker (1979a: 113). Illustrated and described in more detail in Günther (1868b: 236, fig. 4).

Distribution: Suriname.

DASYLORICARIA Isbrücker \& Nijssen, 1979

Dasyloricaria Isbrücker \& Nijssen, in Isbrücker, 1979a: 90. Type species: Loricaria filamentosa Steindachner, 1878. Type by original designation. Gender: Feminine.

Dasyloricaria capetensis (Meek \& Hildebrand, 1913)

Loricaria capetensis Meek \& Hildebrand, 1913: 80. Type locality: Río Capetí, Río Tuyra basin, Panama. Holotype: FMNH 7582.

Distribution: Tuira River basin, Panama (Ferraris, 2003c).

Dasyloricaria filamentosa (Steindachner, 1878)

Loricaria filamentosa Steindachner, 1878a: 90. Type locality: Magdalenen-Stromes [Colombia]. Lectotype: NMW 44874, designated by Isbrücker (1979a: 113). Illustrated and described in more detail in Steindachner (1879d: 45, pl. 9).

Distribution: Magdalena River basin and Catatumbo River, Colombia (Maldonado-Ocampo et al., 2005).

Dasyloricaria latiura (Eigenmann \& Vance, 1912)

Loricaria filamentosa latiura Eigenmann \& Vance, in Eigenmann, 1912a: 13. Type locality: Boca de Certegai, Colombia. Syntypes (12): originally CM 3806 and IU 12695, now FMNH 55115 (7), USNM 79219 (1), plus 4 additional specimens (whereabouts unknown). 
Distribution: Tuira River basin, Panama, and Atrato River basin, Colombia (Ferraris, 2003c).

Dasyloricaria seminuda (Eigenmann \& Vance, 1912)

Loricaria filamentosa seminuda Eigenmann \& Vance, in Eigenmann, 1912a: 13. Type locality: Colombia, Girardot. Holotype: FMNH 55117; holotype illustrated in Eigenmann (1922b: 91, pl. 14, figs. 1, 2).

Distribution: Magdalena River basin, Colombia (Maldonado-Ocampo et al., 2005).

Dasyloricaria tuyrensis (Meek \& Hildebrand, 1913)

Loricaria tuyrensis Meek \& Hildebrand, 1913: 81. Type locality: Rio Tuyra, Boca de Cupe, Panama. Holotype: FMNH 7583.

Distribution: Tuira River basin, Panama (Ferraris, 2003c).

DEKEYSERIA Rapp Py-Daniel, 1985

Dekeyseria Rapp Py-Daniel, 1985: 178. Type species: Dekeyseria amazonica Rapp Py-Daniel, 1985. Type by original designation. Gender: Feminine.

Zonancistrus Isbrücker in Isbrücker et al., 2001: 23. Type species: Ancistrus brachyurus Kner, 1854. Type by original designation. Gender: Masculine.

Remarks: The recently proposed genus Zonancistrus Isbrücker was considered by Fisch-Muller (2003) to be invalid.

Dekeyseria amazonica Rapp Py-Daniel, 1985

Dekeyseria amazonica Rapp Py-Daniel, 1985: 180, fig. 2. Type locality: Complexo do Janauacá, Rio Solimões, Amazonas, Brasil. Holotype: INPA 104.4.

Distribution: Amazon River basin, primarily above Manaus, Brazil (Fisch-Muller, 2003).

Dekeyseria brachyura (Kner, 1854)

Ancistrus brachyurus Kner, 1854: 279, pl. 4 (fig. 1). Type locality: Barra do Rio negro [=Manaus, Brazil]. Holotype: NMW 47268. Originally as Anc. brachyurus.

Distribution: Lower Negro River basin, Brazil (Fisch-Muller, 2003).

Dekeyseria niveata (La Monte, 1929)

Plecostomus niveatus La Monte, 1929: 3, fig. 2. Type locality: Caño Pescado; about five miles north of Esmeralda, at an elevation of 325 feet, Upper Orinoco drainage, Venezuela. Holotype: AMNH 9601.

Distribution: Upper Orinoco River basin, Venezuela (Fisch-Muller, 2003).

Dekeyseria picta (Kner, 1854)

Ancistrus pictus Kner, 1854: 277, pl. 4 (fig. 2). Type locality: Barra do Rio negro [=Manaus, Brazil]. Syntypes: NMW 47200 (2), NMW 47201 (1), NMW 47202 (1). Originally as Anc. pictus.

Distribution: Lower Negro River basin, Brazil (Fisch-Muller, 2003).

Dekeyseria pulchra (Steindachner, 1915)

Aneistrus (Hemiancistrus) pulcher Steindachner, 1915b: 219. Type locality: Rio Negro bei Moura [Brazil]. Holotype: at NMW. Described in more detail and illustrated in Steindachner (1915e: 89, pl. 11, figs. 1, 2). Generic name clearly a misspelling of Ancistrus.

Distribution: Upper Negro and Orinoco rivers and Canal Casiquiare basin, Brazil, Colombia, Venezuela (FischMuller, 2003).

Dekeyseria scaphirhyncha (Kner, 1854)

Ancistrus scaphirhynchus Kner, 1854: 280, pl. 3 (fig. 2). Type locality: Barra do Rio negro [Manaus, Brazil]. Syntypes (4): NMW 47207-10. Originally as Anc. scaphirhynchus.

Distribution: Negro River basin, Brazil (Fisch-Muller, 2003).

Remarks: Redescribed by Rapp Py-Daniel (1985: 181, figs.).

DELTURUS Eigenmann \& Eigenmann, 1889

Delturus Eigenmann \& Eigenmann, 1889b: 45. Type species: Delturus parahybae Eigenmann \& Eigenmann, 1889. Type by original designation. Gender: Masculine.

Carinotus La Monte, 1933a: 1. Type species: Plecostomus (Carinotus) carinotus La Monte, 1933. Type by original designation. Gender: Masculine.

Delturus angulicauda (Steindachner, 1877) 
Plecostomus angulicauda Steindachner, 1877b: 672, pl. 12. Type locality: Rio Mucuri bei Santa Clara; Rio Parahyba. Syntypes: NMW 44069-70 (only 2 found).

Distribution: Mucuri River basin, Brazil (Weber, 2003).

Delturus carinotus (La Monte, 1933)

Plecostomus (Carinotus) carinotus La Monte, 1933a: 2, fig. 1(a). Type locality: Rio Doce, Estado do Espirito Santo, eastern Brazil. Holotype: AMNH 11911.

Distribution: Doce River basin, Brazil.

Delturus parahybae Eigenmann \& Eigenmann, 1889

Delturus parahyba Eigenmann \& Eigenmann, 1889b: 45. Type locality: Parahyba [Brazil]. Syntypes (2, $28 \mathrm{~cm})$ : MCZ 7726 (2).

Distribution: Paraíba do Sul River basin, Brazil (Weber, 2003).

DENTECTUS Martín Salazar, Isbrücker \& Nijssen, 1982

Dentectus Martín Salazar, Isbrücker \& Nijssen, 1982: 127. Type species: Dentectus barbarmatus Martín Salazar, Isbrücker \& Nijssen, 1982. Type by original designation. Gender: Masculine.

Dentectus barbarmatus Mártin Salazar, Isbrücker \& Nijssen, 1982

Dentectus barbarmatus Martín Salazar, Isbrücker \& Nijssen, 1982: 130, fig. 3. Type locality: Venezuela, Estado Cojedes, Río Salinas, a tributary of the Río Pao Viejo, N.E. of El Baul (9¹3'N, $\left.68^{\circ} 07^{\prime} \mathrm{W}\right)$. Holotype: MBUCV V-12780.

Distribution: Western Orinoco River basin, Venezuela (Mártin Salazar, Isbrücker \& Nijssen, 1982).

DOLICHANCISTRUS Isbrücker, 1980

Dolichancistrus Isbrücker, 1980: 47. Type species: Pseudancistrus pediculatus Eigenmann, 1918. Type by original designation. Gender: Masculine.

Monophyly: Schaefer (1986: 103).

Dolichancistrus atratoensis (Dahl, 1960)

Pseudancistrus atratoênsis Dahl, 1960a: 455, fig. on p. 456. Type locality: Quebrada La Noche, tributary to the upper Atrato, approximate height above sea level $550 \mathrm{~m}$; western Colombia. Holotype: ICNMHN 51.

Distribution: Upper Atrato River basin, Colombia (Fisch-Muller, 2003).

Dolichancistrus carnegiei (Eigenmann, 1916)

Pseudancistrus carnegiei Eigenmann, 1916: 85. Type locality: Rio San Gil, Santander, Colombia. Holotype: FMNH 58350, illustrated in Eigenmann (1922b: 79, pl. 10, fig. 3).

Distribution: Magdalena River basin, Colombia (Fisch-Muller, 2003).

Dolichancistrus cobrensis (Schultz, 1944)

Pseudancistrus pediculatus cobrensis Schultz, 1944c: 299, pl. 9 (fig. B). Type locality: Río Cobre, tributary to Río Quinta, latter tributary to Río La Grita, below La Grita, Catatumbo system, Maracaibo basin, Venezuela. Holotype: USNM 121036.

Distribution: Catatumbo River basin, Lake Maracaibo drainage, Venezuela (Fisch-Muller, 2003).

Dolichancistrus fuesslii (Steindachner, 1911)

Ancistrus füsslii Steindachner, 1911b: 373. Type locality: Sosomoco, Ostkolumbien, in 800 m Höhe. Holotype: NMW 48026.

Distribution: Western Colombia; known only from the holotype (Fisch-Muller, 2003).

Dolichancistrus pediculatus (Eigenmann, 1918)

Pseudancistrus pediculatus Eigenmann, 1918b: 679. Type locality: Río Negro at Villavicencio, Colombia. Holotype: FMNH 58352; holotype illustrated in Eigenmann (1922b: 78, 224, fig. 17; pl. 10, fig. 4)

Distribution: Upper Meta River basin, Colombia (Fisch-Muller, 2003); Catatumbo River basin (Maldonado-Ocampo et al., 2005).

Dolichancistrus setosus (Boulenger, 1887)

Chaetostomus setosus Boulenger, 1887b: 349. Type locality: Andes of Columbia. Syntypes: BMNH 1880.2.26.9-10 (2). Redescription of syntypes in Regan (1904b: 239, pl. 12, fig. 2). 
Distribution: Andes of Colombia (Fisch-Muller, 2003).

EPACTIONOTUS Reis \& Schaefer, 1998

Epactionotus Reis \& Schaefer, 1998: 4. Type species: Epactionotus bilineatus Reis \& Schaefer, 1998. Type by original designation. Gender: Masculine.

Epactionotus aky Azpelicueta, Casciotta, Almirón \& Körber, 2004

Epactionotus aky Azpelicueta, Casciotta, Almirón \& Körber, 2004: 82, figs. 1-2. Type locality: Argentina, Misiones, Río Uruguay basin, Arroyo Garibaldi (26³8'46.1"S, 5359'55"W). Holotype: AI 124.

Distribution: Yabotí-Guazú basin, Uruguay River system, Misiones, Argentina (Azpelicueta et al., 2004).

Epactionotus bilineatus Reis \& Schaefer, 1998

Epactionotus bilineatus Reis \& Schaefer, 1998: 5, fig. 3. Type locality: Mouth of arroio do Ouro, which flows into rio Maquiné, between Barra do Ouro and Maquiné, 29³5'S, 50¹7'W, Rio Grande do Sul State, Brazil. Holotype: MCP 20279.

Distribution: Maquiné and Três Forquilhas River basins, Brazil (Schaefer, 2003c).

Epactionotus gracilis Reis \& Schaefer, 1998

Epactionotus gracilis Reis \& Schaefer, 1998: 12, fig. 6. Type locality: Rio Jordão at Jordão Alto, 28³6'S, 49²9'W, Nova Veneza, Santa Catarina State, Brazil. Holotype: MCP 20282.

Distribution: Araranguá River basin, Brazil (Schaefer, 2003c).

Epactionotus itaimbezinho Reis \& Schaefer, 1998

Epactionotus itaimbezinho Reis \& Schaefer, 1998: 9, fig. 5. Type locality: Rio Canoas, tributary of rio Mampituba, ca. $8 \mathrm{~km}$ from Praia Grande in the direction of Mãe dos Homens, 29¹4'S, 5001'W, Rio Grande do Sul, Brazil. Holotype: MCP 20281.

Distribution: Mampituba River basin, Brazil (Schaefer, 2003c).

Epactionotus yasi Almirón, Azpelicueta \& Casciotta, 2004

Epactionotus yasi Almirón, Azpelicueta \& Casciotta, 2004: 138, figs. 1-3. Type locality: Argentina, province of

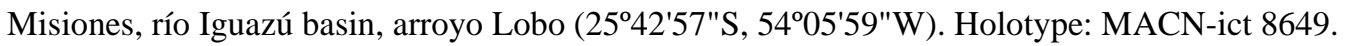

Distribution: Iguaçu River basin (Almirón, Azpelicueta \& Casciotta, 2004).

EURYCHEILICHTHYS Reis \& Schaefer, 1993

Eurycheilus Reis \& Schaefer, 1992: 216. Type species: Eurycheilus pantherinus Reis \& Schaefer, 1992. Type by monotypy. Gender: Masculine. Preoccupied by Eurycheilus Septfontaine, 1970, in fossil cephalopods; replaced by Eurycheilichthys Reis \& Schaefer, 1993.

Eurycheilichthys Reis \& Schaefer, 1993: 894. Type species: Eurycheilus pantherinus Reis \& Schaefer, 1992. Type by being a replacement name. Gender: Masculine. Replacement for Eurycheilus Reis \& Schaefer, 1992; preoccupied by Eurycheilus Septfontaine, 1970, in fossil cephalopods.

Eurycheilichthys limulus Reis \& Schaefer, 1998

Eurycheilichthys limulus Reis \& Schaefer, 1998: 15, fig. 7. Type locality: Rio Jacuí on road RS-010, between Marau and Passo Fundo, $28^{\circ} 18^{\prime} \mathrm{S}, 52^{\circ} 18^{\prime} \mathrm{W}$, Rio Grande do Sul State, Brazil. Holotype: MCP 20283.

Distribution: Upper Jacuí River basin, Brazil (Schaefer, 2003c).

Eurycheilichthys pantherinus (Reis \& Schaefer, 1992)

Eurycheilus pantherinus Reis \& Schaefer, 1992: 217, figs. 1-5. Type locality: Brazil: Rio Grande do Sul, creek tributary of rio dos Touros, at road from Silveira to Rondinha, Bom Jesus, approx. $28^{\circ} 42^{\prime} \mathrm{S}, 50^{\circ} 10^{\prime} \mathrm{W}$. Holotype: MCP 13077.

Distribution: Upper and middle Uruguay River basin, Brazil (Schaefer, 2003c).

EXASTILITHOXUS Isbrücker \& Nijssen, 1979

Exastilithoxus Isbrücker \& Nijssen, in Isbrücker, 1979a: 91. Type species: Pseudacanthicus fimbriatus Steindachner, 1915. Type by original designation. Gender: Masculine.

Monophyly: Schaefer (1986: 103).

Review: Isbrücker \& Nijssen (1985). 
Exastilithoxus fimbriatus (Steindachner, 1915)

Pseudocanthicus (Lithoxus) fimbriatus Steindachner, 1915a: 201. Type locality: Coquenanfluss [=Kukenan River, also Cuquenan, Caroni basin, Venezuela]. Holotype: NMW 44418. Species illustrated and described in more detail in Steindachner (1915e: 92, pl. 10, figs. 1-3) with name as Pseudacanthicus (Lithoxus) fimbriatus. Holotype illustrated in Isbrücker \& Nijssen (1985).

Distribution: Caroni and Matacuni River basins, Orinoco drainage, Venezuela (Fisch-Muller, 2003).

Exastilithoxus hoedemani Isbrücker \& Nijssen, 1985

Exastilithoxus hoedemani Isbrücker \& Nijssen, 1985: 227, fig. 3 (middle). Type locality: Brazil, Est. Amazonas, Rio Marauiá, foot of the Sierra Neblina, Cachoeira "Pora Comeschie", Rio Negro basin. Holotype: INPA 506.

Distribution: Maurauiá River in the upper Negro River drainage, Brazil (Fisch-Muller, 2003).

FARLOWELLA Eigenmann \& Eigenmann, 1889

Acestra Kner, 1853a: 29. Type species: Acestra acus Kner, 1853. Type by subsequent designation by Bleeker (186263: 4). Gender: Feminine. Preoccupied by Acestra Bonaparte, 1846 in fishes and by Dallas, 1852 in Hemiptera; replaced by Farlowella Eigenmann \& Eigenmann, 1889.

Farlowella Eigenmann \& Eigenmann, 1889b: 32. Type species: Acestra acus Kner, 1853. Type by being a replacement name. Gender: Feminine. Replacement for Acestra Kner, 1853.

Revision: Retzer \& Page (1997).

Farlowella acus (Kner, 1853)

Acestra acus Kner, 1853a: 13, 29, pl. 8 (fig. 1). Type locality: Caracas [Venezuela]. Holotype: NMW 47795.

Loricaria scolopacina De Filippi, 1853: 166. Type locality: in rivis prov. Venezuelae, Caracas. Holotype: MZUT 22.

Distribution: Lake Valencia and Torito River basins, Venezuela (Retzer \& Page, 1997).

Farlowella amazona (Günther, 1864)

Acestra amazonum Günther, 1864: 261. Type locality: Santarem, River Amazons [Brazil]. Holotype: BMNH 1856.3.25.22.

Acestra gladiolus Günther, 1864: 261. Type locality: Rio Cupai [Brazil]. Lectotype: BMNH 1853.3.19.66, designated by Isbrücker (1979a: 114).

Farlowella carinata Garman in Eigenmann \& Eigenmann, 1889b: 32. Type locality: Santarem, Teffé, Gurupa, Obidos, Juthay, Tabatinga [restricted to Gurupá [Brazil] by lectotype designation]. Lectotype: MCZ 9830, designated by Isbrücker (1979a: 114).

Farlowella pseudogladiolus Steindachner, 1910c: 405, figured on p. 405. Type locality: Amazonenstromes ohne nähere Angabe des Fundortes, wahrscheinlich aus dem Rio negro. Holotype: NMW 46498.

Farlowella paranaënse Meinken, 1937: 78. Type locality: mittleren Paranà -gebiete [Argentina]. Holotype: lost (Eschmeyer et al., 1998).

Farlowella pleurotaenia Miranda Ribeiro, A., 1939: 360. Type locality: Pará [Brazil]. Holotype: MNRJ 758.

Farlowella oliveirae Miranda Ribeiro, A., 1939: 359. Type locality: Pará [Brazil]. Holotype: MNRJ 757.

Distribution: Amazon, Tocantins, and La Plata River basins (Retzer \& Page, 1997).

Remarks: Van der Stigchel (1946: 185) tentatively placed Farlowella gladiolus as a synonym of Farlowella amazonum, thereby acting as first reviser and making $F$. amazona the valid name for this species.

Farlowella colombiensis Retzer \& Page, 1997

Farlowella colombiensis Retzer \& Page, 1997: 62, fig. 33. Type locality: Colombia, Meta Department, area of Guaicaramo, Río Upia drainage. Holotype: SU 23733.

Distribution: Orinoco River drainage, Meta River basin, Colombia (Retzer \& Page, 1997).

Farlowella curtirostra Myers, 1942

Farlowella curtirostra Myers, 1942: 102, fig. 11. Type locality: Quebrada Tabor, tributary of the Río Motatan system, $30 \mathrm{~km}$ north of Trujillo, Maracaibo basin, Venezuela. Holotype: SU 36508.

Distribution: Lake Maracaibo drainage, Venezuela (Retzer \& Page, 1997).

Farlowella gracilis Regan, 1904

Farlowella gracilis Regan, 1904b: 303, pl. 20 (fig. 3). Type locality: Río Caqueta, Cauca Valley, s. Colombia. Holotype: BMNH 1902.5.29.180. 
Farlowella boliviana Steindachner, 1910c: 404. Type locality: Kolombien. Holotype: at NMW, but apparently lost, Isbrücker (1979a: 114).

Distribution: Caqueta River basin, Upper Amazon River basin, Colombia (Retzer \& Page, 1997).

Farlowella hahni Meinken, 1937

Farlowella hahni Meinken, 1937: 77, fig. 2. Type locality: Río Paranà, ca. 2 km east of Ayolas, 27²4'S, 56 $46^{\prime} \mathrm{W}$, Misiones Dept., Paraguay. Neotype: UMMZ 228132, designated by Retzer \& Page (1997: 76).

Distribution: Middle Paraná River basin, Argentina and Paraguay (Retzer \& Page, 1997).

Farlowella hasemani Eigenmann \& Vance, 1917

Farlowella hasemani Eigenmann \& Vance, 1917: 301, pl. 30 (figs. 1-2). Type locality: Para [Brazil]. Holotype: FMNH 55089.

Distribution: Pará State, Brazil (Retzer \& Page, 1997).

Farlowella henriquei Miranda Ribeiro, 1918

Farlowella henriquei Miranda Ribeiro, 1918d: 636. Type locality: Rio Vermelho, aff. do Araguaya (Sta. Rita das Antas) [Brazil]. Holotype: MZUSP 2159 [reported as MNRJ 2159 in Retzer \& Page (1997: 71)].

Distribution: Araguaia River basin, Brazil (Retzer \& Page, 1997).

Farlowella isbrueckeri Retzer \& Page, 1997

Farlowella isbruckeri Retzer \& Page, 1997: 55, fig. 24. Type locality: Brazil, Mato Grosso State, small river on highway from Cuibá to Porto Velho, approximately $32 \mathrm{~km}$ from Lacerda. Holotype: MZUSP 37641.

Distribution: Upper Paraguay River basin, Brazil (Retzer \& Page, 1997).

Farlowella jauruensis Eigenmann \& Vance, 1917

Farlowella jauruënsis Eigenmann \& Vance, 1917: 300, pl. 30 (fig. 3). Type locality: Jaura [Brazil]. Holotype: FMNH 55088.

Distribution: Upper Paraguay River basin, Brazil (Retzer \& Page, 1997).

Farlowella knerii (Steindachner, 1882)

Acestra Knerii Steindachner, 1882b: 177. Type locality: Canelos [Ecuador]. Lectotype: NMW 47796, designated by Isbrücker (1979a: 114). Illustrated and described in more detail in Steindachner (1882c: 26, pl. 7, figs. 1, 1a).

Distribution: Upper Amazon River drainage, Napo and Pastaza River basins, Ecuador (Retzer \& Page, 1997).

Farlowella mariaelenae Martín Salazar, 1964

Farlowella mariaelenae Martín Salazar, 1964: 246, fig. 1. Type locality: río Salinas, brazo del río Pao Viejo, al noreste de El Baúl, brazo del río Portuguesa, Edo. Cojedes [Venezuela]. Holotype: MBUCV V-11938.

Distribution: Western Orinoco River basin and, possibly, Caribbean coastal rivers, Venezuela (Retzer \& Page, 1997).

Farlowella martini Fernández-Yépez, 1972

Farlowella martini Fernández-Yépez, 1972a: 22, pl. 15. Type locality: la Estación 114 del Complejo Hidrográfico (04) "Rio Yaracuy" [Coastal Caribbean drainage, Venezuela]. Syntypes (2; as male and female "holotypes"): originally in personal collection of Fernández-Yépez, current whereabouts unknown.

Distribution: Caribbean coastal rivers in Venezuela (Retzer \& Page, 1997).

Farlowella nattereri Steindachner, 1910

Farlowella nattereri Steindachner, 1910c: 403, figured on p. 404. Type locality: Aus dem mittleren Amazonas-Gebiete ohne nähere Angabe des Fundortes, wahrsheinlich aus dem Rio negro. Holotype: NMW 46497.

Farlowella hargreavesi Eigenmann, 1912b: 252, pl. 32 (fig. 3). Type locality: British Guiana. Holotype: Museum of Georgetown (apparently lost); holotype illustrated in Eigenmann (1912b: pl. 32, fig. 3).

Farlowella azygia Eigenmann \& Vance, 1917: 299, pl. 29 (figs. 1-2). Type locality: Santarem. Holotype: FMNH 55091.

Farlowella acestrichthys Pearson, 1924: 25, pl. 8 (fig. 2). Type locality: Rurrenabaque, Bolivia. Syntypes: CAS 77322 (2), UMMZ 66480 (1).

Distribution: Upper Amazon and Essequibo River basins (Retzer \& Page, 1997).

Farlowella odontotumulus Retzer \& Page, 1997

Farlowella odontotumulus Retzer \& Page, 1997: 57, fig. 28. Type locality: Ecuador, Sucumbios Province, Río Aguarico, $4.7 \mathrm{~km}$ north of Lago Agrio at bridge on road to El Conejo, 007'42"S, 76 52'42"W. Holotype: FMNH 99135. 
Distribution: Aguarico River basin in Ecuador and Mavaca River basin in Venezuela (Retzer \& Page, 1997).

Farlowella oxyrryncha (Kner, 1853)

Acestra oxyrryncha Kner, 1853a: 13, 31, pl. 8 (fig. 2). Type locality: Rio Mamoré - Cachoeira da Bananeira [Brazil]. Holotype: NMW 47797. Name spelled Acestra oxyrrhyncha in figure captions, Acestra oxyrryncha in text; Acestra oxyrryncha treated as valid following first reviser action of Isbrücker (1980: 100).

Acestra gladius Boulenger, 1898e: 425, pl. 41 (fig. 2). Type locality: Rio Jurua, an affluent of the Amazons, Brazil. Lectotype: BMNH 1897.12.1.80, designated by Isbrücker (1979a: 114).

Distribution: Amazon and Orinoco River basins and coastal rivers of NE Brazil (Retzer \& Page, 1997).

Farlowella paraguayensis Retzer \& Page, 1997

Farlowella paraguayensis Retzer \& Page, 1997: 78, fig. 54. Type locality: Paraguay, Canendiyu Department, Arroyo Carimbatay, 15.6 km WSW Curuguaty, 24³1'06"S, 56²43'30"W. Holotype: UMMZ 228129.

Distribution: Paraguay River basin (Retzer \& Page, 1997).

Farlowella platorynchus Retzer \& Page, 1997

Farlowella platorynchus Retzer \& Page, 1997: 71, fig. 44. Type locality: Peru, Ucayali Department, Pucallpa, Yarinachocha, Río Ucayali drainage, $8^{\circ} 23^{\prime}$ S, 74³2'W. Holotype: MHNG 2389.57.

Distribution: Upper and lower Amazon River basin (Retzer \& Page, 1997).

Farlowella reticulata Boeseman, 1971

Farlowella reticulata Boeseman, 1971: 37, pl. 7 (right). Type locality: Maka Creek, left tributary of the Lawa River $10 \mathrm{~km} \mathrm{~S}$ of Stoelmanseiland, Marowijne River basin, Surinam. Lectotype: ZMA 106174, designated by Isbrücker (1979a: 114).

Distribution: Essequibo, Marowijne, and Oyapock River basins (Retzer \& Page, 1997).

Farlowella rugosa Boeseman, 1971

Farlowella rugosa Boeseman, 1971: 40, pl. 7 (middle). Type locality: Kamaloea (or Saloea) Creek, right tributary of the Marowijne River, $9 \mathrm{~km}$ SE of the outlet of Gran Creek, French Guyane. Lectotype: ZMA 106208, designated by Isbrücker (1979a: 114).

Farlowella parvicarinata Boeseman, 1971: 42, pl. 7 (left). Type locality: Right tributary of the Nickerie River, 12 km WSW of Stondansie Falls, Surinam. Lectotype: ZMA 105823, designated by Isbrücker (1979a: 114).

Distribution: Essequibo, Marowijne, and Corantijn River (Retzer \& Page, 1997).

Remarks: Farlowella rugosa treated as the valid name following first reviser action of Retzer \& Page (1997: 72).

Farlowella schreitmuelleri Ahl, 1937

Farlowella schreitmülleri Ahl, 1937b: 446. Type locality: Santarem [Brazil]: Lectotype ZMB 20956, designated by Isbrücker (1979a: 114).

Farlowella latisoma Miranda Ribeiro, A., 1939: 361. Type locality: Oriximiná, Amazonas [Brazil]. Holotype: MNRJ 761.

Distribution: Lower Amazon River basin, Brazil (Retzer \& Page, 1997).

Farlowella smithi Fowler, 1913

Farlowella smithi Fowler, 1913: 574, fig. 24. Type locality: Rio Madeira, about 200 miles east of W. Long. 62²0', Brazil. Holotype: ANSP 39313.

Distribution: Middle Madeira River basin, Brazil (Retzer \& Page, 1997).

Farlowella taphorni Retzer \& Page, 1997

Farlowella taphorni Retzer \& Page, 1997: 51, fig. 19. Type locality: Venezuela, Merida State, Río Muyapas (Río Torondoy-Lago Maracaibo drainage), $4.5 \mathrm{~km} \mathrm{~S}$ of Nueva Bolivai on highway 1. Holotype: INHS 60352.

Distribution: Torondoy River basin, Maracaibo Lake drainage, Venezuela (Retzer \& Page, 1997).

Farlowella venezuelensis Martín Salazar, 1964

Farlowella acus venezuelensis Martín Salazar, 1964: 250. Type locality: río Colorado, de la Hoya del río Guarapiche, en San Antonio de Maturín, Monagas [Venezuela]. Lectotype: MBUCV V-26150, designated by Retzer \& Page (1997: 64).

Distribution: Guaripiche River basin, lower Orinoco River basin, Venezuela (Retzer \& Page, 1997).

Farlowella vittata Myers, 1942

Farlowella vittata Myers, 1942: 103, fig. 12. Type locality: tributaries of the Rio Uribanto, from San Cristobal to 
Ilanos, Venezuela. Holotype: SU 36512.

Farlowella angosturae Martín Salazar, 1964: 251, fig. 3. Type locality: Caño Largo al oeste de Cuidad Bolívar, Edo. Bolívar [Venezuela]. Holotype: MBUCV V-11939.

Farlowella agustini Martín Salazar, 1964: 254, fig. 5. Type locality: Una quebrada límite entre los Estados Cojedes y Carabobo, carretera Campo Carabobo Taguanes, afluente del río Chirigua que a su vez lo es del río Pao [Venezuela]. Holotype: MBUCV V-11940.

Farlowella guaricensis Martín Salazar, 1964: 253, fig. 4. Type locality: Río Guárico, en el puente de Uverito, carreteria San Juan de Los Morros a Ortiz, Guárico [Venezuela]. Holotype: MBUCV V-11941.

Farlowella roncallii Martín Salazar, 1964: 258, fig. 6. Type locality: la quebrada El Ahorcado Aguirre, afluente del Río Tirgua Carabobo [Venezuela]. Holotype: MBUCV V-11949.

Distribution: Orinoco River basin (Retzer \& Page, 1997).

FONCHIIICHTHYS Isbrücker \& Michels, 2001

Fonchiiichthys Isbrücker \& Michels, in Isbrücker et al., 2001: 18. Type species: Loricaria uracantha Kner, 1863. Type by original designation. Gender: Masculine.

Fonchiiichthys rupestris (Schultz, 1944)

Loricaria uracantha rupestre Schultz, 1944c: 325, pl. 12 (figs. a-b). Type locality: Río San Pedro at bridge south of Mene Grande, Río Motatán system, Maracaibo basin [Venezuela]. Holotype: USNM 121102.

Distribution: Rivers of the Lake Maracaibo basin, in lower and middle reaches of rivers, Venezuela (Ferraris, 2003c).

Fonchiiichthys uracanthus (Kner, 1863)

Loricaria uracantha Kner, 1863: 228, fig. 18. Type locality: Rio Chagres, Nordseite von Panama. Syntypes (apparently 2): at NMW, probably lost (Isbrücker, 1979a: 112). Originally as Lor. uracantha. Also appeared in Kner \& Steindachner (1864: 56, pl. 6, fig. 3).

Loricaria bransfordi Gill, 1877: 338. Type locality: Isthmus of Panama, Empire Station. Holotype: USNM 16673.

Distribution: Atlantic and Pacific versant rivers of Costa Rica and Panama (Bussing, 1998).

Remarks: Diagnosis and ecological notes in Bussing (1998: 163), as Rineloricaria uracantha.

FURCODONTICHTHYS Rapp Py-Daniel, 1981

Furcodontichthys Rapp Py-Daniel, 1981: 2. Type species: Furcodontichthys novaesi Rapp Py-Daniel, 1981. Type by original designation. Gender: Masculine.

Furcodontichthys novaesi Rapp Py-Daniel, 1981

Furcodontichthys novaesi Rapp Py-Daniel, 1981: 4, figs. 1-6. Type locality: rio Solimões, lago Tefé, Caititu, Amazonas, Brasil. Holotype: INPA T.79-014.

Distribution: Solimões River basin, Brazil (Rapp Py-Daniel, 1981).

HARTTIA Steindachner, 1877

Harttia Steindachner, 1877b: 668. Type species: Harttia loricariformis Steindachner, 1877. Type by monotypy. Gender: Feminine.

Quiritixys Isbrücker, in Isbrücker et al., 2001: 21. Type species: Harttia leiopleura Oyakawa, 1993. Type by original designation. Gender: Feminine.

Harttia carvalhoi Miranda Ribeiro, 1939

Harttia carvalhoi Miranda Ribeiro, P., 1939a: 11, pl. 2 (upper). Type locality: Rio Paquequer, Estado do Rio de Janeiro [Brazil]. Syntypes: MNRJ 1038 (11).

Distribution: Paquequer River basin in Paraíba do Sul River drainage, Brazil.

Harttia depressa Rapp Py-Daniel \& Oliveira, 2001

Harttia depressa Rapp Py-Daniel \& Oliveira, 2001: 87, fig. 5. Type locality: Brazil: Amazonas, rio Pitinga, rio Uatumã basin, below the Paranapanema Enterprise dam, Cachoeira 40 Ilhas (59³4'24"W, 053'20"S). Holotype: INPA 3901.

Distribution: Uatumã River basin, Brazil (Rapp Py-Daniel \& Oliveira, 2001). 
Harttia dissidens Rapp Py-Daniel \& Oliveira, 2001

Harttia dissidens Rapp Py-Daniel \& Oliveira, 2001: 91, fig. 8. Type locality: Brazil: Pará: rio Tapajós, Pimental (56²15'16"W, 4³5'22"S). Holotype: INPA 7739.

Distribution: Tapajós River basin, Brazil (Rapp Py-Daniel \& Oliveira, 2001).

Harttia duriventris Rapp Py-Daniel \& Oliveira, 2001

Harttia duriventris Rapp Py-Daniel \& Oliveira, 2001: 84, fig. 3. Type locality: Brazil: Pará: rio Tocantins, Igarapé Bacuri (49³0'32"W, 4²9'44"S). Holotype: INPA 2833.

Distribution: Tocantins River basin, above Tucurui dam, Brazil (Rapp Py-Daniel \& Oliveira, 2001).

Harttia garavelloi Oyakawa, 1993

Harttia garavelloi Oyakawa, 1993: 13, fig. 4. Type locality: Rio Fanado, ponte de cidade de Minas Novas, $17^{\circ} 15^{\prime} \mathrm{S}$, 42³5'W, MG [Brazil]. Holotype: MZUSP 43266.

Distribution: Fanado and Araçuaí River basins, Brazil (Oyakawa, 1993).

Harttia gracilis Oyakawa, 1993

Harttia gracilis Oyakawa, 1993: 16, fig. 5. Type locality: Riacho afluente dos rio São João municipio de Fortaleza de Minas, próximo à estrada de ferro Minas-Perobas, 2058'S, 46²47'W, MG [Brazil]. Holotype: MZUSP 43267.

Distribution: São João and upper Paraná River basin, Brazil (Oyakawa, 1993).

Harttia guianensis Rapp Py-Daniel \& Oliveira, 2001

Harttia guianensis Rapp Py-Daniel \& Oliveira, 2001: 88, fig. 6. Type locality: French Guiana: Approuague River, Saut Athanase (52¹'W, 4¹1'N). Holotype: MNHN 1998-0395.

Distribution: Sinnamary and Approuague River basins (Rapp Py-Daniel \& Oliveira, 2001).

Harttia kronei Miranda Ribeiro, 1908

Harttia kronei Miranda Ribeiro, 1908b: [2], figs. 3-3a. Type locality: Rio Bethary [Brazil]. Lectotype: MNRJ 713A, designated by Miranda Ribeiro (1953: 400).

Distribution: Ribeira de Iguape River basin, Brazil.

Harttia leiopleura Oyakawa, 1993

Harttia leiopleura Oyakawa, 1993: 5, fig. 1. Type locality: Riacho afluente do Córrego Mutuca, à direita da estrada Belo Horizonte Nova Lima, km 20, 2060'S, 435' W, bacia do rio das Velhas MG [Brazil]. Holotype: MZUSP 43264.

Distribution: Das Velhas River basin, Brazil (Oyakawa, 1993).

Harttia longipinna Langeani, Oyakawa \& Montoya-Burgos, 2001

Harttia longipinna Langeani, Oyakawa \& Montoya-Burgos, 2001: 137, fig. 1. Type locality: Brazil: Minas Gerais State: rio Pará, bridge on road BR 262 between Nova Serrana and Pará de Minas, approximately 1953'S, 4452'W. Holotype: MZUSP 54579.

Distribution: Upper São Francisco River basin, Brazil (Langeani, Oyakawa \& Montoya-Burgos, 2001).

Harttia loricariformis Steindachner, 1877

Harttia loricariformis Steindachner, 1877b: 669, pl. 6 (figs. 2, 2a-b). Type locality: Oberer Lafu des Rio Parahyba und dessen Nebenflüsse. Lectotype: NMW 46346, designated by Isbrücker (1979a: 113).

Distribution: Paraíba do Sul River basin, Brazil.

Harttia merevari Provenzano, Machado-Allison, Chernoff, Willink \& Petry, 2005

Harttia merevari Provenzano, Machado-Allison, Chernoff, Willink \& Petry, 2005: 520, figs. 1-2. Type locality: Venezuela, Bolívar State, Caura River at the top of Salto Pará waterfalls, among rocks on the eastern side of the river. Holotype: MBUCV V-26578.

Distribution: Upper Caura River, Orinoco River basin, Venezuela (Provenzano, et al., 2005).

Harttia novalimensis Oyakawa, 1993

Harttia novalimensis Oyakawa, 1993: 8, fig. 2. Type locality: Riacho afluente do Córrego Mutuca, à direita da estrada Belo Horizonte Nova Lima, km 20, 2060'S, 43⒌' W, bacia do rio das Velhas, MG [Brazil]. Holotype: MZUSP 43262.

Distribution: Das Velhas River basin, Brazil (Oyakawa, 1993).

Harttia punctata Rapp Py-Daniel \& Oliveira, 2001

Harttia punctata Rapp Py-Daniel \& Oliveira, 2001: 82, fig. 2. Type locality: Brazil: Pará: rio Tocantins, on rocks 
just below the Tucurui dam (49³8'10"W, 3º9'43"S). Holotype: INPA 4714.

Distribution: Upper and middle Tocantins River basin, Brazil (Rapp Py-Daniel \& Oliveira, 2001).

Harttia rhombocephala Miranda Ribeiro, 1939

Harttia rhombocephala Miranda Ribeiro, P., 1939a: 11, pl. 2 (lower). Type locality: Rio Farias [Brazil]. Holotype: MNRJ 712.

Distribution: Farias River basin, Brazil (Miranda Ribeiro, P., 1939a).

Harttia surinamensis Boeseman, 1971

Harttia surinamensis Boeseman, 1971: 28, pl. 3. Type locality: Grandam, Gran Rio, upper Suriname River, Surinam. Holotype: RMNH 26388 (188 mm specimen, holotype may not have been separated from paratypes).

Distribution: Coastal rivers from Suriname River east to Approuague River, French Guiana (le Bail et al., 2000).

Remarks: Species redescribed by le Bail et al. (2000).

Harttia torrenticola Oyakawa, 1993

Harttia torrenticola Oyakawa, 1993: 10, fig. 3. Type locality: Riacho afluente do rio Paraopebá, Água Limpa, município de Noeda, $3 \mathrm{~km}$ da BR 040, acima das cachoeiras, $44^{\circ} 02^{\prime} \mathrm{W}, 20^{\circ} 20^{\prime} \mathrm{S}$, bacia do rio São Francisco, MG [Brazil]. Holotype: MZUSP 43283.

Distribution: Upper São Francisco River basin, Brazil (Oyakawa, 1993).

Harttia trombetensis Rapp Py-Daniel \& Oliveira, 2001

Harttia trombetensis Rapp Py-Daniel \& Oliveira, 2001: 89, fig. 7. Type locality: Brazil: Pará: rio Trombetas, Cach-

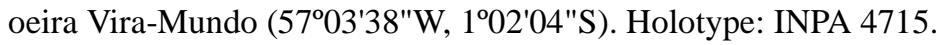

Distribution: Trombetas River basin, above Porteira Falls, Brazil (Rapp Py-Daniel \& Oliveira, 2001).

Harttia uatumensis Rapp Py-Daniel \& Oliveira, 2001

Harttia uatumensis Rapp Py-Daniel \& Oliveira, 2001: 85, fig. 4. Type locality: Brazil: Amazonas: rio Uatumã, at Santa Luzia (59²8'36"W, 150'13"S). Holotype: INPA 2974.

Distribution: Uatumã River basin, Brazil (Rapp Py-Daniel \& Oliveira, 2001).

HARTTIELLA Boeseman, 1971

Harttiella Boeseman, 1971: 25. Type species: Harttia crassicauda Boeseman, 1953. Type by original designation. Gender: Feminine.

Harttiella crassicauda (Boeseman, 1953)

Harttia crassicauda Boeseman, 1953: 10, fig. 2. Type locality: Nassau Mountains, in creek [Suriname]. Holotype: RMNH 19418 (largest of 15 specimens). Holotype may not have been separated from paratypes.

Distribution: Marowijne River basin, Suriname (le Bail et al., 2000).

Remarks: Species redescribed in le Bail et al. (2000).

HEMIANCISTRUS Bleeker, 1862

Hemiancistrus Bleeker, 1862 (in Bleeker, 1862-63): 2. Type species: Ancistrus medians Kner, 1854. Type by original designation. Gender: Masculine.

Remarks: Monophyly of genus is not supported by anatomical characters (Schaefer, 1986: 105) or mitochondrial DNA (Montoya-Burgos et al., 2002: 379), but alternate taxonomy has not yet been proposed for the placement of all of the species listed below. However, Armbruster (2004) indicated that some species were clearly distinct and he listed them in the "Hemiancistrus annectens group."

Hemiancistrus annectens (Regan, 1904)

Ancistrus annectens Regan, 1904b: 225, pl. 11 (fig. 2). Type locality: St. Javier [San Javier de Cachaví] and the Rio Durango [tributary of Rio Bogotá], N. W. Ecuador. Syntype (2): BMNH 2005.9.20.1 (1), BMNH 1902.5.27.49 (1).

Distribution: Pacific coastal rivers of NW Ecuador (Cayapas basin) and of SW Colombia (Patia basin), Colombia, Ecuador (Fisch-Muller, 2003).

Hemiancistrus aspidolepis (Günther, 1867)

Chaetostomus aspidolepis Günther, 1867c: 603. Type locality: Veragua [probably Veraguas province], Panama. Holotype: BMNH 1866.11.5.1 (skin). 
Plecostomus plecostomus panamensis Eigenmann, 1922b: 69. Type locality: the Rio Gatun [...] at Monte Liria and at Gatun, Panama; and New Granada. Syntypes: FMNH 26265 (14) and others [see Remarks].

Distribution: Gatun River basin, Caribbean coastal drainage, Panama (Fisch-Muller, 2003).

Remarks: Specimens from several literature sources cited by Eigenmann (1922b: 69) from Panama, including about 150 specimens of Plecostomus plecostomus mentioned by Meek \& Hildebrand (1916: 247) should be considered as part of the syntype series of Plecostomus plecostomus panamensis, but only those listed above appear to have been examined by Eigenmann.

Hemiancistrus chlorostictus Cardoso \& Malabarba, 1999

Hemiancistrus chlorostictus Cardoso \& Malabarba, 1999: 144, fig. 3. Type locality: Passo Fundo dam, Gerasul UHPF, Ronda Alta, Rio Grande do Sul, Brazil (approximately 2740'09"S, 52 45'25"W). Holotype: MCP 21153.

Distribution: Upper Uruguay River basin, Passo Fundo dam, Brazil (Fisch-Muller, 2003).

Hemiancistrus fugleri Ovchynnyk, 1971

Hemiancistrus fugleri Ovchynnyk, 1971: 108, figs. 12-15. Type locality: Ecuador, Province Esmeraldas, Rio Bogota, Parroquia Cadondelet (78 $45^{\prime}$ west longitude. $1^{\circ} 6^{\prime}$ north latitude). Holotype: MSUM 5133.

Distribution: Cayapas River basin, Ecuador, known only from holotype (Fisch-Muller, 2003).

Hemiancistrus fuliginosus Cardoso \& Malabarba, 1999

Hemiancistrus fuliginosus Cardoso \& Malabarba, 1999: 148, fig. 6. Type locality: rio Jacutinga, road Seara-Concórdia (BR283), Concórdia, Santa Catarina, Brazil (approx. 27010'S, 5209'W). Holotype: MCP 21155.

Distribution: Upper and middle Uruguay River basin, Brazil (Fisch-Muller, 2003).

Hemiancistrus guahiborum Werneke, Armbruster, Lujan \& Taphorn, 2005

Hemiancistrus guahiborum Werneke, Armbruster, Lujan \& Taphorn, 2005: 544, fig. 2. Venezuela, Amazonas, río Ventuari at raudales Tencua $56 \mathrm{~km}$ ESE of San Juan de Manapiare, 05.04968 ${ }^{\circ},-065.62722^{\circ}$ Holotype: MCNG 51994.

Distribution: middle and upper Orinoco River basin (Werneke, Armbruster, Lujan \& Taphorn, 2005).

Hemiancistrus hammarlundi Rendahl, 1937

Hemiancistrus hammarlundi Rendahl, 1937: 2, fig. 1 (up). Type locality: Ecuador, Syst. Rio de Clementina [= Rio Pozuelos] nw. von Babahoyo. Holotype: NRM 10370.

Distribution: Guayas River basin, Ecuador (Fisch-Muller, 2003).

Hemiancistrus holostictus Regan, 1913

Hemiancistrus holostictus Regan, 1913d: 469. Type locality: Not stated [San Juan River basin, Colombia]. Holotype: BMNH 1913.10.1.57.

Distribution: San Juan River basin, Colombia (Fisch-Muller, 2003).

Remarks: Redescribed by Eigenmann (1922b: 73, pl. 9 fig. 4).

Hemiancistrus landoni Eigenmann, 1916

Hemiancistrus landoni Eigenmann, 1916: 84. Type locality: Naranjito [Chimbo tributary, Babahoyo basin], Ecuador. Holotype: CAS 59939; holotype illustrated in Eigenmann (1922b: 72, text fig. 16; pl. 9 fig. 3, pl. 10 fig. 2).

Distribution: Guayas River basin, Ecuador (Fisch-Muller, 2003).

Hemiancistrus macrops (Lütken, 1874)

Chaetostomus macrops Lütken, 1874a: 209. Type locality: Surinam. Holotype: ZMUC P 30142, illustrated in Steindachner (1881c: 124, pl. 5, figs. 3-3a).

Distribution: Suriname (Fisch-Muller, 2003).

Hemiancistrus maracaiboensis Schultz, 1944

Hemiancistrus maracaiboensis Schultz, 1944c: 317, pl. 10 (fig. c). Type locality: near the mouth of the Río Concha in Lago Maracaibo; Venezuela. Holotype: USNM 121012.

Distribution: Lake Maracaibo basin, Venezuela (Fisch-Muller, 2003).

Hemiancistrus medians (Kner, 1854)

Ancistrus medians Kner, 1854: 256. Type locality: [No locality stated]. Holotype: at NMW. Originally as Anc. medians.

Distribution: Maroni River basin, French Guiana and Suriname (Fisch-Muller, 2003). 
Remarks: Type locality interpreted by Isbrücker (1992b) as Rivière Marouini, Maroni system, French Guiana.

Hemiancistrus megacephalus (Günther, 1868)

Chaetostomus megacephalus Günther, 1868a: 476. Type locality: Surinam. Holotype: BMNH 1978.9.12.3. Described in more detail in Günther (1868b: 232).

Distribution: imprecise locality in Suriname, and Essequibo basin, Guyana (Fisch-Muller, 2003).

Hemiancistrus megalopteryx Cardoso, 2004

Hemiancistrus megalopteryx Cardoso, 2004: 174, fig. 1. Type locality: Brazil: Santa Catarina: Braço do Norte: rio Braço do Norte, downstream dam (tributary of rio Tubarão), approx. 28¹6'38"S 49¹1'1"W. Holotype: MCP 32229.

Distribution: Tubarão River basin, Santa Catarina State, Brazil (Cardoso, 2004).

Hemiancistrus meizospilos Cardoso \& Pezzi da Silva, 2004

Hemiancistrus meizospilos Cardoso \& Pezzi da Silva, 2004: 4, fig. 6. Type locality: Brazil: Santa Catarina: Coronel Freitas: rio Chapecó (tributary of the rio Uruguai basin). Holotype: MCP 34091.

Distribution: Chapecó River system, Uruguay River basin, Brazil (Cardoso \& Pezzi da Silva, 2004).

Hemiancistrus micrommatos Cardoso \& Lucinda, 2003

Hemiancistrus micrommatos Cardoso \& Lucinda, 2003: 77, fig. 3 (lower). Type locality: Brazil: Goiás; rio Tocantins, at site of future hydroelectric dam of Serra da Mesa, between Minaçu \& Cavalcante, Minaçu, approximatively 1350'S 48 $19^{\prime}$ W. Holotype: MNRJ 20873.

Distribution: Lower Amazon Basin, Upper Rio Tocantins and tributaries, Brazil (Fisch-Muller, 2003).

Hemiancistrus punctulatus Cardoso \& Malabarba, 1999

Hemiancistrus punctulatus Cardoso \& Malabarba, 1999: 152, fig. 8. Type locality: rio dos Sinos, João Fernandes beach, Caraá, Rio Grande do Sul, Brazil (approx. 2945'87"S, 50²5'68"W). Holotype: MCP 21154.

Distribution: Laguna dos Patos basin, Brazil (Fisch-Muller, 2003).

Hemiancistrus spilomma Cardoso \& Lucinda, 2003

Hemiancistrus spilomma Cardoso \& Lucinda, 2003: 75, fig. 2 (lower). Type locality: Brazil: Tocantins; Porto Franco, rio Tocantins near mouth of rio Lajeado. Holotype: MCP 28425.

Distribution: Lower Amazon Basin, lower and upper Tocantins drainage, Brazil (Cardoso \& Lucinda, 2003).

Hemiancistrus spinosissimus Cardoso \& Lucinda, 2003

Hemiancistrus spinosissimus Cardoso \& Lucinda, 2003: 79, fig. 4 (lower). Type locality: Brazil: Goiás; rio Maranhão, near mouth of the rio Tocantinzinho, border of Niquelândia and Campinaçu, 1356'25"S 48º'19'08"W. Holotype: MCP 26239.

Distribution: Lower Amazon Basin, Middle and Upper Rio Tocantins and tributaries, Brazil (Cardoso \& Lucinda, 2003).

Hemiancistrus subviridis Werneke, Sabaj, Lujan \& Armbruster, 2005

Hemiancistrus subviridis Werneke, Sabaj, Lujan \& Armbruster, 2005: 538, fig. 4. Type locality: Venezuela, Amazonas, río Orinoco $117 \mathrm{~km} \mathrm{~W}$ of La Esmeralda, 03.28998 , -66.60004. Holotype: MCNG 54032.

Distribution: Upper Orinoco River, Venezuela (Werneke, Sabaj, Lujan \& Armbruster, 2005).

Hemiancistrus votouro Cardoso \& Pezzi da Silva, 2004

Hemiancistrus votouro Cardoso \& Pezzi da Silva, 2004: 2, fig. 1. Type locality: Brazil: Rio Grande do Sul: Benjamin Constant: arroio Lageado Grande (tributary of the rio Passo Fundo, Rio Uruguai basin), ca. $2.5 \mathrm{~km} \mathrm{NE}$ from Votouro Indian Reserve (27²6'50"S, 52³7'5"W). Holotype: MCP 33594.

Distribution: Passo Fundo system, Uruguay River basin, Brazil (Cardoso \& Pezzi da Silva, 2004).

Hemiancistrus wilsoni Eigenmann, 1918

Hemiancistrus wilsoni Eigenmann, 1918b: 678. Type locality: Truando [tributary of Río Atrato, Colombia]. Holotype: FMNH 58550; holotype illustrated in Eigenmann (1922b: 74, pl. 10, fig. 1).

Distribution: Truando River basin, Atrato River drainage, Colombia (Fisch-Muller, 2003).

HEMILORICARIA Bleeker, 1862

Hemiloricaria Bleeker, 1862 (in Bleeker, 1862-63): 3. Type species: Hemiloricaria caracasensis Bleeker, 1862. Type by original designation. Gender: Feminine. 
Remarks: Removed from the synonymy of Rineloricaria in Isbrücker (2001: 23).

Hemiloricaria altipinnis (Breder, 1925)

Loricaria altipinnis Breder, 1925: 1, fig. 1. Type locality: Río Chico, Darien, Panama. Holotype: AMNH 8404.

Distribution: Chico River basin, Panama (Ferraris, 2003c).

Hemiloricaria aurata Knaack, 2002

Hemiloricaria aurata Knaack, 2002b: 58, unnumbered figures. Type locality: Mündang eines lkeinen Zuflusses in den Rio Aquidaban, Paraguay, Dpto. de Concepcion, Strecke Loreto-San Carlos. Holotype: ZMA 123591.

Distribution: Paraguay (Knaack, 2002b).

Hemiloricaria beni (Pearson, 1924)

Loricaria beni Pearson, 1924: 25, pl. 5 (fig. 4). Type locality: Lagoons, Lake Rogoagua [Beni River basin, Bolivia]. Holotype: CAS 28772.

Distribution: Beni River basin, Bolivia (Ferraris, 2003c).

Hemiloricaria cacerensis (Miranda Ribeiro, 1912)

Loricaria cacerensis Miranda Ribeiro, 1912: 11. Type locality: Caceres, M. Grosso (Aguas do Paraguay) [Brazil]. Lectotype: MNRJ 645A, designated by Miranda Ribeiro (1953: 400).

Distribution: Mato Grosso, Brazil (Ferraris, 2003c).

Hemiloricaria caracasensis Bleeker, 1862

Loricaria rostrata van der Hoeven, 1849: 90. Type locality: Caracas [Venezuela]. Holotype: RMNH 3120. Preoccupied by Loricaria rostrata Spix \& Agassiz, 1829.

Hemiloricaria caracasensis Bleeker, 1862 (in Bleeker, 1862-63): 3. Type locality: Caracas [Venezuela]. Holotype: RMNH 3120; holotype illustrated by Boeseman (1972: pl. 1), as Hemiloricaria caracassensis.

Distribution: Caribbean coastal drainages, Venezuela (Ferraris, 2003c).

Remarks: Hemiloricaria caracasensis was incorrectly reported to be a replacement name for Loricaria rostrata in Ferraris (2003c). The two names were actually independently based on the same specimen.

Hemiloricaria castroi (Isbrücker \& Nijssen, 1984)

Rineloricaria castroi Isbrücker \& Nijssen, 1984a: 93, fig. 1. Type locality: Brazil, Est. Pará, Trapiche da Reserva Biológica de Trombetas, Rio Trombetas. Holotype: MZUSP 15731.

Distribution: Trombetas River basin, Brazil (Ferraris, 2003c).

Hemiloricaria eigenmanni (Pellegrin, 1908)

Loricaria Eigenmanni Pellegrin, 1908: 125. Type locality: Sarare (Venezuela). Lectotype: MNHN 1899-0118, designated by Isbrücker (1979a: 113).

Distribution: Venezuela (?).

Hemiloricaria fallax (Steindachner, 1915)

Loricaria (Loricariichthys) fallax Steindachner, 1915e: 101, pl. 13 (figs. 1-4). Type locality: Rio Rupununi, dem Rio Parimé dei Serra do Banco, aus einem Igarapé bei Capivari, dem Rio Branco bei Bem Querer und Conçeicão, einem Flusse bei Magauarý an der Braganzabahn bei Pará. Lectotype: NMW 44864, designated by Isbrücker \& Nijssen (1979: 198).

Distribution: Upper Rupununi and Branco River basins (Ferraris, 2003c).

Hemiloricaria formosa (Isbrücker \& Njissen, 1979)

Rineloricaria formosa Isbrücker \& Nijssen, 1979: 192, fig. 1. Type locality: Colombia, Lagoon about 1 km upriver from Puerto Inírida, Río Orinoco basin. Holotype: FMNH 83713.

Distribution: Western Orinoco and upper Amazon River basins (Ferraris, 2003c).

Hemiloricaria hasemani (Isbrücker \& Nijssen, 1979)

Rineloricaria hasemani Isbrücker \& Nijssen, 1979: 200, fig. 4. Type locality: Brazil, Est. Pará, Maguarý near Belém, along the Bragança railroad (Bragança, $1^{\circ} 02^{\prime} \mathrm{S}, 46^{\circ} 46^{\prime} \mathrm{W}$ ), in forest streams. Holotype: NMW 44865.

Distribution: Lower Amazon or Tocantins River basin, Brazil (Ferraris, 2003c).

Hemiloricaria hoehnei (Miranda Ribeiro, 1912)

Loricaria hoehnei Miranda Ribeiro, 1912: 11. Type locality: Coxim, no Rio Paraguay [Brazil]. Holotype: MNRJ 650.

Distribution: Paraguay River basin, Brazil (Ferraris, 2003c). 
Hemiloricaria jubata (Boulenger, 1902)

Loricaria jubata Boulenger, 1902a: 70. Type locality: Northwestern Ecuador, St. Javier (60 feet), and the Rio Durango, elev. (350 feet) [restricted to the Durango River by lectotype designation]. Lectotype: BMNH 1902.5.27.45, designated by Isbrücker (1979a: 112).

Distribution: Mira and Esmeraldas River basins, Pacific drainage, Ecuador (Ferraris, 2003c); and Patia River basin, Colombia (Maldonado-Ocampo et al., 2005).

Hemiloricaria konopickyi (Steindachner, 1879)

Loricaria konopickyi Steindachner, 1879a: 34. Type locality: dem mittleren Laufe des Amazonenstromes. Holotype: NMW 44934. Illustrated and described in more detail in Steindachner (1879e: 45 pl. 6, fig. 3).

Distribution: Amazon River basin, Brazil (Ferraris, 2003c).

Hemiloricaria lanceolata (Günther, 1868)

Loricaria lanceolata Günther, 1868a: 477. Type locality: Xeberos. Holotype: BMNH 1867.6.13.79. Described in more detail in Günther (1868b: 235, fig. 3). Holotype redescribed and illustrated in Isbrücker (1973: 78, fig. 1) as Rineloricaria lanceolata.

Distribution: Upper Amazon River basin, Peru (Ferraris, 2003c).

Hemiloricaria magdalenae (Steindachner, 1879)

Loricaria Magdalenae Steindachner, 1879d: 74. Type locality: Magdalenen-Stromes [Colombia]. Lectotype: NMW 45080, designated by Isbrücker (1979a: 112); syntype illustrated in Boeseman (1972: pl. 2).

Distribution: Sinú, Magdalena and, possibly, Catatumbo River basins, Colombia (Ferraris, 2003c).

Hemiloricaria melini (Schindler, 1959)

Loricariichthys melini Schindler, 1959: 387, fig. 1. Type locality: Rio Solimoes beim Ort Manacapuru, nahe der Mündung des Rio Negro [Brazil]. Holotype: NRM 10830.

Distribution: Amazon River basin, Brazil (Ferraris, 2003c).

Hemiloricaria morrowi (Fowler, 1940)

Rhineloricaria morrowi Fowler, 1940a: 244, figs. 39- 41. Type locality: Ucayali River basin, Contamana, Peru. Holotype: ANSP 68663.

Distribution: Ucayali River basin, Peru (Ferraris, 2003c).

Hemiloricaria nigricauda (Regan, 1904)

Loricaria nigricauda Regan, 1904b: 275, pl. 16 (fig. 2). Type locality: Porto Real, Province Rio Janeiro [Brazil]. Lectotype: BMNH 1891.6.16.32, designated by Isbrücker (1979a: 113).

Distribution: Coastal streams in Rio de Janeiro State, Brazil (Ferraris, 2003c).

Hemiloricaria parva (Boulenger, 1895)

Loricaria parva Boulenger, 1895b: 527. Type locality: Descalvados, Matto Grosso [Brazil]. Lectotype: BMNH 1895.5.17.91, designated by Isbrücker (1979a: 112); one syntype illustrated in Boulenger (1896: pl. 7, fig. 1).

Distribution: Paraguay River basin, Argentina and Brazil (Ferraris, 2003c).

Hemiloricaria phoxocephala (Eigenmann \& Eigenmann, 1889)

Loricaria phoxocephala Eigenmann \& Eigenmann, 1889b: 37. Type locality: Coary [Brazil]. Lectotype: MCZ 8030, designated by Isbrücker (1979a: 112).

Distribution: Amazon River basin, Brazil (Ferraris, 2003c).

Hemiloricaria platyura (Müller \& Troschel, 1848)

Loricaria platyura Müller \& Troschel, 1848: 631. Type locality: dem Rupununi [Guyana]. Holotype: ZMB 3166.

Loricaria submarginatus Eigenmann, 1909a: 10. Type locality: Creek below Potaro Landing [Guyana]. Holotype: FMNH 53080.

Distribution: Various coastal rivers from near Amazon mouth to Essequibo River, Guyana (le Bail et al., 2000).

Remarks: Species redescribed in le Bail et al. (2000).

Hemiloricaria sneiderni (Fowler, 1944)

Loricaria sneiderni Fowler, 1944b: 240, figs. 17-19. Type locality: Río Jurubidá, Nuquí, in brook [Colombia]. Holotype: ANSP 71433.

Distribution: Jurubidá River basin, Pacific Drainage, Colombia (Ferraris, 2003c).

Hemiloricaria stewarti (Eigenmann, 1909) 
Loricaria stewarti Eigenmann, 1909a: 9. Type locality: Chipoo Creek, a tributary of the Ireng [Guyana]. Holotype: FMNH 53330; holotype illustrated in Eigenmann (1912b: pl. 30, fig. 5).

Distribution: Coastal rivers of the Guianas, and upper Branco River Basin (Ferraris, 2003c).

Remarks: Species redescribed by le Bail et al. (2000).

Hemiloricaria teffeana (Steindachner, 1879)

Loricaria teffeana Steindachner, 1879a: 34. Type locality: Teffé, Amazonenstrom [Brazil]. Lectotype: NMW 45134:

1, designated by Isbrücker (1979a: 112). Illustrated and described in more detail in Steindachner (1879e: 44, pl.

6, fig. 2).

Loricaria Valenciennesii Vaillant, 1880b: 157. Type locality: Calderon [Brazil]. Holotype: MNHN a-1985.

Distribution: Amazon River basin, Brazil (Ferraris, 2003c).

Hemiloricaria wolfei (Fowler, 1940)

Rhineloricaria wolfei Fowler, 1940a: 241, figs.33-35. Type locality: Ucayali River basin, Contamana, Peru. Holotype: ANSP 68660.

Distribution: Ucayali River basin, Peru (Ferraris, 2003c).

\section{HEMIODONTICHTHYS Bleeker, 1862}

Hemiodontichthys Bleeker, 1862 (in Bleeker, 1862-63): 4. Type species: Hemiodon acipenserinus Kner, 1853. Type by original designation. Gender: Masculine.

Hemiodontichthys acipenserinus (Kner, 1853)

Hemiodon acipenserinus Kner, 1853a: 12, 28, pl. 7 (fig. 2a-c). Type locality: Rio Guaporé, Matogrosso (Juquia) [Brazil]. Lectotype: NMW 46139, designated by Isbrücker \& Nijssen (1974b: 204). Originally as Hem. acipenserinus.

Distribution: Amazon, Essequibo, Oyapock, and Paraguay River basins (Ferraris, 2003c).

Remarks: Species redescribed by le Bail et al. (2000).

HEMIPSILICHTHYS Eigenmann \& Eigenmann, 1889

Xenomystus Lütken, 1874a: 217. Type species: Xenomystus gobio Lütken, 1874. Type by original designation. Gender: Masculine. Preoccupied by Xenomystus Günther, 1868, in fishes; replaced by Hemipsilichthys Eigenmann \& Eigenmann, 1889.

Hemipsilichthys Eigenmann \& Eigenmann, 1889b: 46. Type species: Xenomystus gobio Lütken, 1874. Type by being a replacement name. Gender: Masculine. Replacement name for Xenomystus Lütken, 1874, preoccupied by Xenomystus Günther, 1868, in fishes.

Upsilodus Miranda Ribeiro, 1924: 365. Type species: Upsilodus victori Miranda Ribeiro, 1924. Type by monotypy. Gender: Masculine.

Remarks: The name Psilichthys is found in Steindachner (1907a: 82) and treated in subsequent literature as the proposal of a new generic name, with Psilichthys cameroni Steindachner, 1907, as type. However the name is apparently a lapsus calami for Hemipsilichthys, which is used extensively in Steindachner (1907e), including mention of its being a replacement for Xenomystus Lütken.

Revision and key to species: Pereira \& Reis (2002), but with most species subsequently transferred into Pareiorhaphis by Pereira (2005).

Hemipsilichthys gobio (Lütken, 1874)

Xenomystus gobio Lütken, 1874a: 217, pl. 4 (fig. 1). Type locality: not stated. Holotype: ZMUC 30173.

Upsilodus victori Miranda Ribeiro, 1924: 366. Type locality: Paquequer ad Theresopolis, Rio de Janeiro [Brazil]. Lectotype: MNRJ 639, designated by Gosline (1947: 100).

Distribution: Paraíba do Sul River basin, Brazil (Weber, 2003).

Remarks: Type locality of Xenomystus gobio given by Pereira et al. (2000: 382) as Paraíba do Sul River basin, Brazil.

Hemipsilichthys nimius Pereira, Reis, Souza \& Lazzarotto, 2003

Hemipsilichthys nimius Pereira, Reis, Souza \& Lazzarotto, 2003: 5, fig. 1. Type locality: Brazil: Rio de Janeiro, Parati, rio Carrasquinho below the Cachoeira do Tobogã, upper Perequê-Açu Basin, Penha, ca. 7.5 km west of 
highway BR 101, on road from Parati to Cunha (2312'51"S, 44²47'28"W. Holotype: MCP 33049.

Distribution: Perequê-Açu and Taquari Rivers, Rio de Janeiro State, Brazil (Pereira et al., 2003).

Hemipsilichthys papillatus Pereira, Oliveira \& Oyakawa, 2000

Hemipsilichthys papillatus Pereira, Oliveira \& Oyakawa, 2000: 378, fig. 1. Type locality: Brazil: Minas Gerais: rio Paraíba do Sul drainage, County of Rio Preto, ribeirão Santana, tributary of rio Preto (approx. $22^{\circ} 02^{\prime} S$ 434' W). Holotype: MZUSP 53085.

Distribution: Preto River basin of Paraíba do Sul River drainage, Brazil (Weber, 2003).

HISONOTUS Eigenmann \& Eigenmann, 1889

Hisonotus Eigenmann \& Eigenmann, 1889b: 40. Type species: Hisonotus notatus Eigenmann \& Eigenmann, 1889. Type by original designation. Gender: Masculine.

Hisonotus depressicauda (Miranda Ribeiro, 1918)

Otocinclus depressicauda Miranda Ribeiro, 1918d: 635. Type locality: Sorocaba [Paraná River basin, Brazil]. Lectotype: MZUSP 1004, designated by Britski (1969: 208).

Distribution: Tietê River basin, Brazil (Schaefer, 2003c).

Hisonotus depressinotus (Miranda Ribeiro, 1918)

Microlepidogaster depressinotus Miranda Ribeiro, 1918d: 633. Type locality: Piracicaba [São Paulo State, Brazil]. Holotype: MZUSP 2156.

Distribution: Tietê River basin, Brazil (Schaefer, 2003c).

Hisonotus francirochai (Ihering, 1928)

Otocinclus francirochai Ihering, 1928a: 2, fig. 1. Type locality: Creeks by Pirangy, headwaters of the rio Turvo, Brazil. Lectotype: MZUSP 3258, designated by Britski (1969: 209).

Distribution: Grande River basin, Brazil (Schaefer, 2003c).

Hisonotus insperatus Britski \& Garavello, 2003

Hisonotus insperatus Britski \& Garavello, 2003: 588, fig. 1. Type locality: Brazil, São Paulo State, Botucatu, Rio Capivara, Rio Tietê drainage. Holotype: MZUSP 78957.

Distribution: Upper Paraná River basin, São Paulo and Mato Grosso states, Brazil (Britski \& Garavello, 2003).

Hisonotus laevior Cope, 1894

Hisonotus laevior Cope, 1894: 95, pl. 8 [sic, 7] (figs. 12, 12a-b). Type locality: Rio Jacuhy, Rio Grande do Sul, Brazil. Holotype: ANSP 21563.

Distribution: Jacuí River basin, Brazil (Schaefer, 2003c).

Hisonotus leptochilus Cope, 1894

Hisonotus leptochilus Cope, 1894: 96, pl. 8 [sic, 7] (figs. 11 a-b). Type locality: Rio Grande do Sul, Brazil. Holotype: ANSP 21564.

Distribution: Jacuí River basin, Brazil (Schaefer, 2003c).

Hisonotus leucofrenatus (Miranda Ribeiro, 1908)

Otocinclus leucofrenatus Miranda Ribeiro, 1908b: [2]. Type locality: Rio das Pedras [Brazil]. Type(s): at MNRJ, current whereabouts unknown.

Distribution: Ribeira de Iguape River basin, Brazil (Schaefer, 2003c).

Hisonotus maculipinnis (Regan, 1912)

Otocinclus maculipinnis Regan, 1912b: 668, pl. 77 (fig. 3). Type locality: Rio de la Plata, South America. Syntypes: BMNH 1909.4.2.19-22 (4).

Distribution: Paraná/Paraguay and La Plata River basins, Argentina (Schaefer, 2003c).

Hisonotus nigricauda (Boulenger, 1891)

Otocinclus nigricauda Boulenger, 1891: 234, pl. 25 (fig. 3). Type locality: Rio Grande do Sul, Brazil. Syntypes: BMNH 1891.3.16.53-62.

Distribution: Paraná/Paraguay River basin, Brazil (Schaefer, 2003c).

Hisonotus notatus Eigenmann \& Eigenmann, 1889

Hisonotus notatus Eigenmann \& Eigenmann, 1889b: 41. Type locality: Santa Cruz and Juiz de Fora [Brazil]. Syntypes (96): ANSP 166924 (1), BMNH 1904.1.28.13-16 (4), MCZ 7764 (91), MCZ 8177 (1). 
Distribution: Southeastern Brazil (Schaefer, 2003c).

Remarks: Eigenmann \& Eigenmann (1890: 391) included the name minutus [interpreted in context as Hisonotus minutus] in the discussion of H. notatus. This has been interpreted as a lapsus for H. notatus by Isbrücker (1980: 84) and Schaefer (1996b: 1034) although not so noted in the printed corrigenda associated with the volume. The name is not associated with an indication and not treated as valid elsewhere in the volume, so there is no reason to consider this as an available name.

Hisonotus paulinus (Regan, 1908)

Otocinclus paulinus Regan, 1908f: 799, fig. 208. Type locality: Rio Piracicaba, San Paulo, Brazil. Holotype: BMNH 1907.7.6.9.

Distribution: Tietê River basin, Brazil (Schaefer, 2003c).

Hisonotus ringueleti Aquino, Schaefer \& Miquelarena, 2001

Hisonotus ringueleti Aquino, Schaefer \& Miquelarena, 2001: 3, fig. 1. Type locality: Uruguay, Rivera State, upper Uruguay River drainage, Quaraí River drainage, creek at Km 18 of route joining Santa do Livramento, Brazil, and Rivera, Uruguay; close to border (ca. $31^{\circ} 00^{\prime} \mathrm{S}, 55^{\circ} 30^{\prime} \mathrm{W}$ ). Holotype: ILPLA 886.

Distribution: Upper Uruguay River basin, Uruguay (Schaefer, 2003c).

Hisonotus taimensis (Buckup, 1981)

Microlepidogaster taimensis Buckup, 1981: 22, figs. 1-7. Type locality: Novo canal do Arroio Taim, Estação Ecológica do Taim, Rio Grande do Sul, Brazil. Holotype: MZUSP 14133.

Distribution: Mirim Lagoon drainage, Brazil (Schaefer, 2003c).

HOPLIANCISTRUS Isbrücker \& Nijssen, 1989

Hopliancistrus Isbrücker \& Nijssen, 1989: 543. Type species: Hopliancistrus tricornis Isbrücker \& Nijssen, 1989. Type by original designation. Gender: Masculine.

Hopliancistrus tricornis Isbrücker \& Nijssen, 1989

Hopliancistrus tricornis Isbrücker \& Nijssen, 1989: 543, fig. 6. Type locality: Brasilien, Est. Pará, Poça de Pedra no Rio Tapajós, São Luis. Holotype: MZUSP 22007.

Distribution: Tapajós and Xingu River basins, Brazil (Fisch-Muller, 2003).

HYPANCISTRUS Isbrücker \& Nijssen, 1991

Hypancistrus Isbrücker \& Nijssen, 1991: 347. Type species: Hypancistrus zebra Isbrücker \& Nijssen, 1991. Type by original designation. Gender: Masculine.

Monophyly: Armbruster (2002).

Hypancistrus inspector Armbruster, 2002

Hypancistrus inspector Armbruster, 2002: 87, fig. 2 (middle). Type locality: Venezuela, Amazonas, Dpto. Casiquiare, río Casiquiare, río Negro-río Amazonas drainage, approximatively 10 river km above the río Negro (below Solano), $1^{\circ} 58^{\prime} \mathrm{N}, 67^{\circ} 05^{\prime} \mathrm{W}$. Holotype: MCNG 12133.

Distribution: Upper Negro, Casiquiare, and upper Orinoco River basin, Venezuela (Fisch-Muller, 2003).

Hypancistrus zebra Isbrücker \& Nijssen, 1991

Hypancistrus zebra Isbrücker \& Nijssen, 1991: 348, fig. 1 (middle). Type locality: Brazil, Est. Pará, anastomoses of Rio Xingú, about one hour upstream of Altamira by speedboat (Altamira: $03^{\circ} 13^{\prime} \mathrm{S} 53^{\circ} 15^{\prime} \mathrm{W}$ ). Holotype: MZUSP 41668.

Distribution: Xingu River basin, Brazil (Fisch-Muller, 2003).

НYРОРТОРОМА Günther, 1868

Hypoptopoma Günther, 1868a: 477. Type species: Hypoptopoma thoracatum Günther, 1868. Type by monotypy. Gender: Neuter.

Aristommata Holmberg, 1893a: 96. Type species: Aristommata inexpectata Holmberg, 1893. Type by monotypy. Gender: Feminine.

Diapeltoplites Fowler, 1915a: 237. Type species: Hypoptopoma gulare Cope, 1878. Type by original designation. Gender: Masculine. Originally proposed as a subgenus of Hypoptopoma. 
Hypoptopoma bilobatum Cope, 1870

Hypoptopoma bilobatum Cope, 1870b: 566. Type locality: Pebas, Ecuador [now Peru]. Syntypes: ANSP 8280 (2).

Distribution: Amazon River basin, Peru (Schaefer, 2003c).

Hypoptopoma guianense Boeseman, 1974

Hypoptopoma guianense Boeseman, 1974: 259, fig. 1; pl. 1. Type locality: Left tributary of the Nickerie River, a few

km upstream from the Stondansi Falls, Surinam. Holotype: RMNH 26919.

Distribution: Nickerie River basin, Suriname (Schaefer, 2003c).

Hypoptopoma gulare Cope, 1878

Hypoptopoma gulare Cope, 1878: 679. Type locality: Peruvian Amazon. Holotype: ANSP 21477.

Distribution: Amazon River basin (Schaefer, 2003c).

Hypoptopoma inexspectatum (Holmberg, 1893)

Aristommata inexpectata Holmberg, 1893a: 96. Type locality: Río Paraguay, Formosa Prov., Argentina. Holotype: MACN 5164. Also in Holmberg (1893b: 354).

Hypoptopoma guentheri Boulenger, 1895b: 526. Type locality: Descalvados, Mato Grosso [Brazil]. Syntypes: BMNH 1895.5.17.77-82 (6).

Distribution: Paraná/Paraguay River basin (Schaefer, 2003c).

Remarks: Redescribed in Aquino \& Miquelarena (2001), as Hypoptopoma inexpectata.

Hypoptopoma joberti (Vaillant, 1880)

Otocinclus joberti Vaillant, 1880a: 147. Type locality: Calderon, Brésil. Holotype: MNHN a-1966.

Distribution: Amazon River basin, Brazil (Schaefer, 2003c).

Hypoptopoma psilogaster Fowler, 1915

Hypoptopoma psilogaster Fowler, 1915a: 235, fig. 9. Type locality: Peruvian Amazon. Holotype: ANSP 21922.

Distribution: Amazon River basin, Colombia and Peru (Schaefer, 2003c).

Hypoptopoma steindachneri Boulenger, 1895

Hypoptopoma steindachneri Boulenger, 1895b: 526. Type locality: Amazonenstrome zunächst der mündung des Rio

Negro. Holotype: NMW 46272; holotype illustrated in Schaefer (1996b:1033, fig. 1). Based on specimen described and illustrated in Steindachner (1879e: 47, pl. 7, fig. 1) as Hypoptopoma thoracatum.

Distribution: Amazon River basin (Schaefer, 2003c).

Hypoptopoma thoracatum Günther, 1868

Hypoptopoma thoracatum Günther, 1868a: 477. Type locality: Xeberos [upper Amazon River, Peru]. Holotype:

BMNH 1867.6.13.38. Illustrated and described in more detail in Günther (1868b: 234, fig. 2).

Distribution: Amazon River basin (Schaefer, 2003c).

HYPOSTOMUS La Cepède, 1803

Hypostomus La Cepède, 1803: 145. Type species: Hypostomus guacari La Cepède, 1803. Type by monotypy. Gender: Masculine.

Cochliodon Heckel, in Kner, 1854: 265. Type species: Hypostomus cochliodon Kner, 1854. Type by monotypy. Gender: Masculine.

Cheiridodus Eigenmann, 1922b: 70. Type species: Plecostomus hondae Regan, 1912. Type by original designation. Gender: Masculine.

Watawata Isbrücker \& Michels, in Isbrücker et al., 2001: 21. Type species: Hypostomus microstomus Weber, 1987. Type by original designation. Gender: Feminine.

Remarks: Plecostomus Gronovius (1763: 127) was published in a work rejected by the ICZN (Opinion 261) for nomenclatural purposes; the name appears on the Official Index of Rejected and Invalid Generic Names In Zoology (Melville \& Smith, 1987). Subsequent uses of Plecostomus were summarized in Isbrïcker (1980). The recently proposed genus Watawata Isbrücker \& Michels was considered by Weber (2003) to be invalid.

Revision: Armbruster (2003a, Hypostomus cochliodon group).

Reviews: Boeseman (1968, Suriname), Weber (1986, Paraguay), Reis et al. (1990, Rio Grande do Sul), López \& Miquelarena (1991a, b, Argentina); Mazzoli et al. (1994, Paraiba do Sul, Brazil), and Le Bail et al. (2000, French Guiana). 
Molecular phylogeny: Montoya-Burgos et al. (2002, mitochondrial D-loop sequences).

Historical biogeography: Montoya-Burgos (2003).

Hypostomus affinis (Steindachner, 1877)

Plecostomus affinis Steindachner, 1877b: 685. Type locality: aus dem Rio Mucury bei Santa Clara [...] im Rio Parahyba und dessen Nebenflusse Muriahé, im Rio Mucury und S. Antonio bei S. Antonio de Ferros [restricted to Rio Parahyba (as Campos, in the rio Paraíba do Sul basin), Brazil, by lectotype designation ]. Lectotype: NMW 44041:1; designated by Mazzoni et al. (1994: 5 and 7).

Distribution: Paraíba do Sul River basin, Brazil (Weber, 2003).

Hypostomus agna (Miranda Ribeiro, 1907)

Plecostomus ãgnã Miranda Ribeiro, 1907a: 188. Type locality: Rio da Ribeira: da Iporanga —S. Paulo [Brazil]. Holotype: at MNRJ.

Distribution: Ribeira de Iguapé River basin, Brazil (Weber, 2003; Oyakawa et al., 2005).

Hypostomus alatus Castelanau, 1855

Hypostomus alatus Castelnau, 1855: 41, pl. 20 (fig. 1). Type locality: rio Sabara, dans la province de Minas Geraës (Brésil). Holotype: MNHN a-9441.

Distribution: Upper São Francisco River basin, Brazil (Weber, 2003).

Hypostomus albopunctatus (Regan, 1908)

Plecostomus albopunctatus Regan, 1908f: 797, pl. 49 (fig. 1). Type locality: Rio Piracicaba, Sao Paulo, Brazil. Lectotype: BMNH 1907.7.6.15; designated by Weber (1987: 279).

Distribution: Upper Paraná River basin, (Weber, 2003).

Hypostomus ancistroides (Ihering, 1911)

Plecostomus ancistroides Ihering, 1911: 396. Type locality: Rio Tatuhy, affluente do lado esquerdo do Rio Sorocaba, Rio Piracicaba, Est. de S. Paulo [Brazil]. Syntypes: Whereabouts unknown, not found at MZUSP (Britski, 1969: 200).

Distribution: Tietê River basin, Brazil (Weber, 2003); Ribeira de Iguape River basin (Oyakawa et al., 2005).

Hypostomus angipinnatus (Leege, 1922)

Plecostomus angipinnatus Leege, 1922: 152, pl. 9 (fig. 1). Type locality: Mato Grosso (?) [Brazil]. Holotype: at Phyletisch Museum, Jena (not found, Weber, 2003).

Distribution: La Plata drainage: possibly Paraguay basin (Weber, 2003).

Hypostomus argus (Fowler, 1943)

Plecostomus argus Fowler, 1943a: 249, figs. 28-31. Type locality: Villavicencio, Río Meta basin, Colombia. Holotype: ANSP 70510

Distribution: Upper Meta River basin, Colombia (Weber, 2003).

Hypostomus asperatus Castelnau, 1855

Hypostomus asperatus Castelnau, 1855: 41, pl. 20 (fig. 2). Type locality: de l'Araguay [Brazil]. Holotype: MNHN a9442.

Distribution: Tocantins River basin, Brazil (Weber, 2003).

Hypostomus aspilogaster (Cope, 1894)

Plecostomus aspilogaster Cope, 1894: 100, pl. 7 [pl. 7 reversed with pl. 8] (fig. 14). Type locality: from the Jacuhy, Rio Grande do Sul. Lectotype: ANSP 21781 designated by Reis et al. (1990: 733).

Distribution: Uruguay River basin and Laguna dos Patos drainage, Brazil (Weber, 2003).

Remarks: Redescribed by Reis et al. (1990: 733).

Hypostomus atropinnis (Eigenmann \& Eigenmann, 1890)

Plecostomus lima atropinnis Eigenmann \& Eigenmann, 1890: 399, 410. Type locality: Goiás, Brazil. Holotype: MCZ 27265.

Distribution: Tocantins River basin, Brazil (Weber, 2003).

Hypostomus auroguttatus Kner, 1854

Hypostomus auroguttatus Kner, 1854: 269, pl. 2 (fig. 3). Type locality: [Not explicitly given, apparently Rio Parahyba, Brazil (Weber, 2003)]. Syntypes: at NMW. Originally proposed as Hyp. auroguttatus.

Plecostomus Lütkeni Steindachner, 1877c: 217. Type locality: Im Rio Parahyba, R. Mucuri, R. San Antonio, R. 
Quenda, R. de Pedral [restricted to Rio Parahyba (= rio Paraíba do Sul), by lectotype designation]. Lectotype: NMW 44196: 1; designated by Mazzoni et al. (1994: 10).

Distribution: Paraíba do Sul River basin, Brazil (Weber, 2003).

Hypostomus bolivianus (Pearson, 1924)

Plecostomus bolivianus Pearson, 1924: 21, pl. 4 (fig. 1). Type locality: Popoi River, Upper Beni, Bolivia. Holotype: CAS 77347.

Distribution: Beni River basin, Bolivia (Weber, 2003).

Hypostomus borellii (Boulenger, 1897)

Plecostomus borellii Boulenger, 1897c: 2. Type locality: Mission de San Francisco [Pilcomayo River, Bolivia]. Syntypes: BMNH 1897.1.27.19 (1), MZUT 1393 (1).

Distribution: Upper and middle Pilcomayo River basin, (Weber, 2003).

Hypostomus boulengeri (Eigenmann \& Kennedy, 1903)

Plecostomus boulengeri Eigenmann \& Kennedy, 1903: 502. Type locality: Matto Grosso or Asuncion. Holotype: CAS 58554.

Distribution: Paraguay River basin (Weber, 2003).

Remarks: Redescription in Weber (1986: 982).

Hypostomus brevicauda (Günther, 1864)

Plecostomus brevicauda Günther, 1864: 235. Type locality: Bahia [Brazil]. Syntypes: BMNH 1864.1.19.16-17 (2).

Distribution: coastal drainages of Eastern Brazilian (Weber, 2003).

Hypostomus brevis (Nichols, 1919)

Plecostomus brevis Nichols, 1919a: 413, 423. Type locality: no Estado de S. Paulo [...] in the state of São Paulo [Brazil]. Holotype: AMNH 7150.

Distribution: Upper Paraná River basin or eastern Brazilian coastal drainage; known only from holotype (Weber, 2003).

Hypostomus carinatus (Steindachner, 1881)

Plecostomus carinatus Steindachner, 1881c: 108, pl. 4 (fig. 2). Type locality: aus dem Amazonen-Strome, ohne nähere Angabe des Fundortes; [...], von Jatuarana [=probably in the Parintins, near Barreirinha], Ueranduba [=lago Iranduba], und aus dem See Saraca [=lago de Sarac]. Syntypes: ?MCZ 27266 (1), NMW 44094 (1), NMW 44096 (1).

Distribution: Middle Amazon River basin, Brazil (Weber, 2003).

Remarks: Some diagnostic characters in Rapp Py-Daniel (1988: 17 and tab. 3).

Hypostomus carvalhoi (Miranda Ribeiro, 1937)

Plecostomus carvalhoi Miranda Ribeiro, 1937: 54. Type locality: Rio Granjeiro- Crato- Ceará. Syntypes: MNRJ 924 (4, of which only 2 were reportedly found by Weber, 2003).

Distribution: Jaguaribe River basin, Brazil (Weber, 2003).

Remarks: Lectotype designation of specimen as MNRJ 924A by Miranda Ribeiro (1953: 401) considered not to clearly refer to a single specimen and therefore not valid by Weber (2003: 357).

Hypostomus cochliodon Kner, 1854

Hypostomus cochliodon Kner, 1854: 265, pl. 2 (fig. 1). Type locality: Rio Cuyaba. Syntypes: NMW 44101 (1), NMW 46277 (1), NMW 59395 (1), RMNH D1897 (1).

Distribution: Paraguay and middle Paraná River basins (Weber, 2003).

Remarks: The names Loricaria melanoptera and Cochliodon hypostomus were published by Kner (1854: 265) as synonyms of $H$. cochliodon and, therefore, not available. Species redescribed in Armbruster (2003a), but see Hollanda Carvalho \& Weber (2004: 972) for comments on the identity of Armbruster's material.

Hypostomus commersoni Valenciennes, 1836

Hypostomus Commersoni Valenciennes, 1836, in Valenciennes, 1835-47: pl. 7 (fig. 2). Type locality: not stated [considered to have come from La Plata River, Uruguay]. Holotype or lectotype: MNHN a-9444. Name made available from caption to plate, with illustrated specimen the holotype (if recognizable; see Remarks). Described in Cuvier \& Valenciennes (1840b: 495, 366 of Strasbourg deluxe edition).

Plecostomus spiniger Hensel, 1870: 73. Type locality: Rio Cadea [=Cadeia River, Rio Grande do Sul, Brazil]. Holo- 
type: ZMB 7444.

Plecostomus limosus Eigenmann \& Eigenmann, 1888b: 167. Type locality: Rio Grande do Sul [Brazil]. Lectotype: MCZ 7869, designated by Reis et al. (1990: 737). Name made available by diagnostic features in key on p. 168. Distribution: Middle and lower Paraná and Uruguay River basins; Laguna dos Patos basin (Weber, 2003).

Remarks: Weber (1986) selected MNHN a-9444 as a lectotype for Hypostomus commersoni. If this specimen was the specimen illustrated in Valenciennes (1836), it is actually the holotype, and no lectotype would have been necessary. However, if the illustrated specimen could not be identified, Weber's lectotype designation would stand. Type locality of Hypostomus commersoni discussed in Weber (1986: 994).

Hypostomus coppenamensis Boeseman, 1969

Hypostomus coppenamensis Boeseman, 1969: 120, fig. 3. Type locality: tributary of Left Coppename River, 354'N, $56^{\circ} 46^{\prime} \mathrm{W}$, Surinam. Holotype: ZMA 105856.

Distribution: Upper Coppename River basin, Suriname (Weber, 2003).

Hypostomus corantijni Boeseman, 1968

Hypostomus corantijni Boeseman, 1968: 40, pl. 4 (fig. 1). Type locality: Sipaliwini River, Surinam. Holotype: RMNH 25471 (largest specimen, if not separated).

Distribution: Corantijn River basin, upstream first cataracts, Suriname (Weber, 2003).

Hypostomus crassicauda Boeseman, 1968

Hypostomus crassicauda Boeseman, 1968: 42, pl. 17 (fig. 1). Type locality: Sipaliwini River, Surinam. Holotype: RMNH 25489.

Distribution: Upper Sipaliwini River basin, Suriname (Weber, 2003).

Hypostomus derbyi (Haseman, 1911)

Plecostomus derbyi Haseman, 1911b: 384, pl. 79. Type locality: Porto União da Victoria [Iguaçu River basin, Brazil]. Holotype: FMNH 54246.

Distribution: Iguaçu River and Uruga-í Creek basins, Paraguayan tributaries of Paraná River, (Weber, 2003).

Remarks: Redescribed in Gomez et al. (1990) from Argentina.

Hypostomus dlouhyi Weber, 1985

Hypostomus dlouhyi Weber, 1985: 956, fig. 1 (middle). Type locality: système du Rio Paraná, sur un bras du lac de retenue du Rio Yguazú [non Iguaçu, Brésil] à la hauteur de la localité Juan E. O'Leary, 55²0' Ouest, 2525'30" Sud, Paraguay, dèp. Alto Parana. Holotype: MHNG 2229.43.

Distribution: Yguazú River basin in middle Paraná River drainage, Paraguay (Weber, 2003).

Hypostomus eptingi (Fowler, 1941)

Chaetostomus eptingi Fowler, 1941a: 158, figs. 66-68. Type locality: Forteleza, Ceará [Brazil]. Holotype: ANSP 69447.

Distribution: Coastal drainages of northern Brazil (Weber, 2003).

Hypostomus ericae Hollanda Carvalho \& Weber, 2004

Hypostomus ericae Hollanda Carvalho \& Weber, 2004: 955, fig. 5. Type locality: Brazil: Goiás: Tocantins River basin: Rio Tocantins, Porto Alfredinho, 1402' 22.1"S, 48³1'28.6"W. Holotype: MNRJ 27861.

Distribution: Upper Tocantins River basin, Brazil (Hollanda Carvalho \& Weber, 2004).

Remarks: Included in Hypostomus cochliodon group by Hollanda Carvalho \& Weber (2004).

Hypostomus ericius Armbruster, 2003

Hypostomus ericius Armbruster, 2003a: 25, fig. 9. Type locality: Peru. Amazonas: Río Marañon dr. 1 km upstream from Caterpiza. Holotype: MUSM 17441.

Distribution: Maranon, Itaya, and Nanay Rivers, Upper Amazon River basin, Peru (Armbruster, 2003a).

Remarks: Included in the Hypostomus cochliodon species group by Armbruster (2003a).

Hypostomus fluviatilis (Schubart, 1964)

Plecostomus fluviatilis Schubart, 1964b: 7, figs. 1-4. Type locality: Corrego da Lazica, perto de Ouro Fino (MG); Cachoeìra do Espraiado, no alto Mogi Guaçu, acima do Soledade, Ouro Fino (MG); Alto Mogi Guaçu, 5 km jusante de Inconfidentes (mun. Ouro Fino, MG). Syntypes: Whereabouts unknown, not found (Weber, 2003).

Distribution: Grande River basin, Brazil (Weber, 2003).

Hypostomus fonchii Weber \& Montoya-Burgos, 2002 
Hypostomus fonchii Weber \& Montoya-Burgos, 2002: 357, fig. 3 (b). Type locality: Peru: Ucayali: Quebrada John, lower part, near mouth in the Rio Pauya, in shallow water with emerging rocks, $06^{\circ} 36^{\prime} 7.0^{\prime \prime} \mathrm{S} / 75^{\circ} 56^{\prime} 26.3^{\prime \prime} \mathrm{W}$ (GPS), alt. 360 m., Rio Cushabatay drainage, Rio Ucayali basin (Station 1). Holotype: MHNG 2613.66.

Distribution: Cushabatay River basin, Peru (Weber, 2003).

Hypostomus francisci (Lütken, 1874)

Plecostomus francisci Lütken, 1874c: 30. Type locality: in flumine Sti. Francisci [=São Francisco River, Brazil]. Holotype: ZMUC P 30171. Originally proposed as Pl. francisci.

Distribution: Upper São Francisco River basin, Brazil (Weber, 2003).

Hypostomus garmani (Regan, 1904)

Plecostomus garmani Regan, 1904b: 214, pl. 10 (fig. 1). Type locality: Rio das Velhas, Eastern Brazil. Holotype: BMNH 1904.1.28.3.

Distribution: São Francisco River basin, Brazil (Weber, 2003).

Hypostomus goyazensis (Regan, 1908)

Plecostomus goyazensis Regan, 1908f: 798, fig. 207(a). Type locality: Goyaz [Brazil]. Holotype: BMNH 1889.11.14.49.

Distribution: Upper Araguaia River basin, Brazil (Weber, 2003).

Hypostomus gymnorhynchus (Norman, 1926)

Plecostomus gymnorhynchus Norman, 1926b: 95. Type locality: Iponcin Creek, into Approuague River, French Guiana. Holotype: BMNH 1926.3.2.745.

Distribution: Guianan coastal drainages, from Oyapock westward to Maroni basin, French Guiana (Weber, 2003).

Remarks: Redescribed in Le Bail et al. (2000: 254).

Hypostomus hemicochliodon Armbruster, 2003

Hypostomus hemicochliodon Armbruster, 2003a: 29, fig. 12. Type locality: Peru. Amazonas: Río Nanay, Río Amazonas dr. at Pampa Chica, $4.54 \mathrm{~km}$ from Iquitos center, $269^{\circ}$ bearing, 0345'07"S, 73¹6'59"W. Holotype: MUSM 17442.

Distribution: Marañon, Itaya, and Nanay, Orosa and Napo Rivers, Upper Amazon River basin, Negro, Tapajos and Xingu Rivers of Brazil, and Orinoco River basin, (Armbruster, 2003a).

Remarks: Included in the Hypostomus cochliodon species group by Armbruster (2003a).

Hypostomus hemiurus (Eigenmann, 1912)

Plecostomus hemiurus Eigenmann, 1912b: 224, fig. 34, pl. 25 (fig. 1). Type locality: Amatuk [Potaro River], British Guiana. Holotype: FMNH 53110.

Distribution: Coastal drainages of Guyana (Weber, 2003).

Remarks: Appeared first as name only in Eigenmann (1910: 407).

Hypostomus hermanni (Ihering, 1905)

Plecostomus Hermanni Ihering, 1905: 560. Type locality: Rio Piracicaba, São Paulo, Brazil. Holotype: BMNH 1905.6.9.5.

Distribution: Tietê River basin, Brazil (Weber, 2003).

Hypostomus hondae (Regan, 1912)

Plecostomus hondae Regan, 1912b: 666, pl. 76 (fig. 3). Type locality: Honda, Colombia (300-400 ft.). Syntypes: BMNH 1909.7.23.43-44 (2).

Cochliodon pospisili Schultz, 1944c: 312, pl. 11 (figs. c-d). Type locality: Rio Palmar near Totuma, about 100 km. southwest of Maracaibo, Venezuela. Holotype: USNM 121003.

Distribution: Magdalena River and Lake Maracaibo basins (Weber, 2003); also Sinú and Atrato basins (Armbruster, 2003a).

Remarks: Redescribed in Lilyestrom (1984) and Armbruster (2003a); included by Armbruster in the Hypostomus cochliodon species group.

Hypostomus hoplonites Rapp Py-Daniel, 1988

Hypostomus hoplonites Rapp Py-Daniel, 1988: 14, fig. 2(6). Type locality: Brasil: Amazon (Rio Solimões, complexo de lagos do Janauacá). Holotype: INPA 109.2.

Distribution: Middle Amazon basin, Brazil (Weber, 2003). 
Hypostomus iheringii (Regan, 1908)

Plecostomus iheringii Regan, 1908f: 795, pl. 47 (fig. 1). Type locality: Rio Piracicaba, San Paulo, Brazil. Holotype: BMNH 1907.7.6.13.

Distribution: Tietê River basin, Brazil (Weber, 2003).

Hypostomus interruptus (Miranda Ribeiro, 1918)

Plecostomus interruptus Miranda Ribeiro, 1918d: 632. Type locality: Rio Juquiá [Brazil]. Holotype: MZUSP 2110. Distribution: Ribeira de Iguape River basin, Brazil (Weber, 2003; Oyakawa et al., 2005).

Remarks: Type locality interpreted by Britski (1969: 209) as rio Juquiá, Poço Grande, Estado de São Paulo, Brazil.

Hypostomus isbrueckeri Reis, Weber \& Malabarba, 1990

Hypostomus isbrueckeri Reis, Weber \& Malabarba, 1990: 752, fig. 22. Type locality: Rio Conceição, Ijui, Rio Grande do Sul, Brazil. Holotype: MCP 10488.

Distribution: Middle and upper Uruguay River basin, Brazil (Weber, 2003).

Hypostomus jaguribensis (Fowler, 1915)

Plecostomus jaguribensis Fowler, 1915b: 264, fig. 3. Type locality: Rio Jaguaribé at Barro Alto, Brazil. Holotype: ANSP 39930.

Distribution: Jaguaribe River basin, Brazil (Weber, 2003).

Hypostomus johnii (Steindachner, 1877)

Plecostomus Johnii Steindachner, 1877b: 691. Type locality: Rio Puty und Rio Preto [Brazil]. Syntypes: MCZ 7831 (1), MCZ 7863 (4), MCZ 7864 (2), NMW 44191 (2), NMW 44192 (2), NMW 44193 (2).

Distribution: Parnaíba and São Francisco River basins (Weber, 2003).

Hypostomus laplatae (Eigenmann, 1907)

Plecostomus laplatae Eigenmann, 1907: 450, pl. 21 (fig. 1). Type locality: Buenos Aires [Argentina]. Holotype: at Princeton Univ.

Plecostomus taeniatus Regan, 1908d: 358. Type locality: Río La Plata, Argentina. Syntypes: BMNH 1908.8.29.17 (1), ZMB 16817 (1).

Plecostomus rachovii Regan, 1913e: 555. Type locality: Near Rio Janeiro [Probably incorrect (Weber, 2003)]. Holotype: BMNH 1913.10.30.15.

Plecostomus commersonoides Marini, Nichols \& La Monte, 1933: 3. Type locality: Dársena Norte, Buenos Aires, Argentina. Holotype: AMNH 12243.

Distribution: La Plata River basin (Weber, 2003).

Remarks: Redescribed in López \& Miquelarena (1991a: 28).

Hypostomus latifrons Weber, 1986

Hypostomus latifrons Weber, 1986: 991, fig. 6 (middle). Type locality: Río Aguaray-guazú, bassin du río Paraguay système du rio Paraná, Transchaco 117 km 58 00'00" Ouest, 2422'50" Sud, dép. Presidente Hayes, Paraguay. Holotype: MHNG 2256.67.

Distribution: Paraguay River basin, Paraguay (Weber, 2003).

Hypostomus latirostris (Regan, 1904)

Plecostomus latirostris Regan, 1904b: 213, pl. 11 (fig. 1). Type locality: River Jungada, Matto Grosso ... and from Goyaz [Brazil]. Syntypes (3): BMNH 1892.4.20.26-27 (2).

Distribution: Paraguay River basin (Weber, 2003).

Hypostomus levis (Pearson, 1924)

Rhinelepis levis Pearson, 1924: 22, pl. 5 (fig. 1). Type locality: Huachi, altitude 2,235 feet. Huachi overlooks the junction of the Bopi and Cochabamba rivers which here form the Beni, Bolivia. Holotype: CAS 77349.

Distribution: Cochabamba and Popoi Rivers, upper Beni River basin, Madeira River drainage of Bolivia (Armbruster, 2003a).

Remarks: Redescribed and placed in the Hypostomus cochliodon species group by Armbruster (2003a).

Hypostomus lexi (Ihering, 1911)

Plecostomus lexi Ihering, 1911: 387. Type locality: Rio Pardo, perto de Barretos (Est. S. Paulo) quasi na foz do rio que é affluente do Rio Grande, por sua vez tributario do lado esquerdo do Rio Paraná [Brazil]. Holotype: MZUSP 2126. 
Distribution: Grande River basin, Brazil (Weber, 2003).

Hypostomus lima (Reinhardt, 1874)

Plecostomus lima Reinhardt, in Lütken, 1874c: 29. Type locality: in rivulis flumini Rio da Velhas affluentibus [as "Ribeirão do Mato" og andre smaa Bifloder til Rio d. Velhas, in Lütken, 1875]. Syntypes: BMNH 1876.1.10.12 (2), MNHN 0000-9573 (1), NMW 44194 (1), NMW 44195 (1), SMNS 2050 (1), ZMUC P 30143-ZMUC P 30148 (1 each).

Distribution: São Francisco River basin, Brazil (Weber, 2003).

Hypostomus longiradiatus (Holly, 1929)

Plecostomus longiradiatus Holly, 1929: 118. Type locality: aus dem Rio Guaná [sic, Guamá (Weber, 2003)], vermutlich einem Nebenflusse des Madeira, der selbst ein Nebenfluß des Amazonenstromes ist [...] (Brasilien). Holotype: Whereabouts unknown.

Distribution: Guamá River basin, Brazil (Weber, 2003).

Hypostomus luteomaculatus (Devincenzi 1942)

Plecostomus luteomaculatus Devincenzi, in Devincenzi \& Teague, 1942: 20, pl. 3 (fig. 3). Type locality: Río Uruguay [at Paysandú, Uruguay]. Holotype: MHNM CI 359.

Distribution: Uruguay River basin, (Weber, 2003).

Hypostomus luteus (Godoy, 1980)

Plecostomus luteus Godoy, 1980: 29, fig. 15. Type locality: Rio Pelotas, Volta Grande 2, Marcelino Ramos, RS [Brazil]. Holotype: personal collection of M. Godoy.

Distribution: Upper Uruguay River basin, Brazil (Weber, 2003).

Remarks: Redescribed by Reis et al. (1990: 741).

Hypostomus macrophthalmus Boeseman, 1968

Hypostomus pseudohemiurus macrophthalmus Boeseman, 1968: 56, pl. 10 (fig. 1). Type locality: Sipaliwini River, near air strip, Surinam. Holotype: RMNH 25514.

Distribution: Sipaliwini River basin, Suriname (Weber, 2003).

Hypostomus macrops (Eigenmann \& Eigenmann, 1888)

Plecostomus macrops Eigenmann \& Eigenmann, 1888b: 170. Type locality: Rio das Velhas [Brazil]. Holotype: MCZ 7888. Name made available by diagnostic features in key on p. 170.

Distribution: Das Velhas River basin, Brazil (Weber, 2003).

Hypostomus macushi Armbruster \& de Souza, 2005

Hypostomus macushi Armbruster \& de Souza, 2005: 3, fig. 1. Type locality: Guyana, Rupunini (Region 9). Ireng River, 6.9 km WW Karasabai, Takutu River - Negro River drainage, 04.01957º, $059.60170^{\circ}$. Holotype: UG/ CSBD 11047.

Distribution: Lower and middle Rupununi River, Essequibo River, Takutu River and Ireng River, Guyana (Armbruster \& de Souza, 2005).

Remarks: Placed into the Hypostomus cochliodon species group by Armbruster \& de Souza (2005) .

Hypostomus margaritifer (Regan, 1908)

Plecostomus margaritifer Regan, 1908f: 796, pl. 47 (fig. 2). Type locality: Rio Piracicaba, San Paulo, Brazil. Holotype: BMNH 1907.7.6.14.

Plecostomus margaritifer butantanis Ihering, 1911: 394. Type locality: no Rio Pinheiros, perto de São Paulo (Capital) [Brazil]. Holotype: possibly at MZUSP, but not found.

Distribution: Upper and middle Paraná River basin, (Weber, 2003).

Hypostomus meleagris (Marini, Nichols \& La Monte, 1933)

Plecostomus meleagris Marini, Nichols \& La Monte, 1933: 4. Type locality: Southeastern Brazil [apparently in upper Paraná drainage]. Holotype: AMNH 12246.

Distribution: Upper and Middle Paraná River basin (Weber, 2003).

Hypostomus micromaculatus Boeseman, 1968

Hypostomus micromaculatus Boeseman, 1968: 49, 77 [addendum], pl. 7 (figs. 1-4). Type locality: Mamadam (falls), Surinam River, Surinam. Holotype: RMNH 25487 (largest specimen).

Distribution: Upper and middle Suriname River basin, Suriname (Weber, 2003). 


\section{Hypostomus microstomus Weber, 1987}

Hypostomus microstomus Weber, 1987: 275, fig. 2 (middle). Type locality: système du río Paraná, río Alto-Paraná, Paraguay, dpt Itapua, $15 \mathrm{~km}$ SE Encarnacion, Campichuelo, 55 $45^{\prime}$ Ouest, $27^{\circ} 26^{\prime}$ Sud. Holotype: MHNG 2367.90

Distribution: Middle Paraná River basin (Weber, 2003).

Hypostomus mutucae Knaack, 1999

Hypostomus mutucae Knaack, 1999b: 102, fig. p. 102, Type locality: Brazil, Mato Grosso, rio Mutuca. Holotype: MCP 28669.

Distribution: Cuiabá River basin, Brazil (Weber, 2003).

Hypostomus myersi (Gosline, 1947)

Plecostomus myersi Gosline, 1947: 116, pl. 5 (fig. 9). Type locality: Rio Iguaçu at Porto União, State of Paraná [Brazil]. Holotype: MNRJ 4251.

Distribution: Iguaçu River and Uruga-í Creek basins, Brazil (Weber, 2003); Argentina (Gomez et al., 1990).

Remarks: Redescribed in Gomez et al. (1990) from Argentina.

Hypostomus nematopterus Isbrücker \& Nijssen, 1984

Hypostomus nematopterus Isbrücker \& Nijssen, 1984c: 9, fig. 1 (middle). Type locality: French Guyana, Alikene Creek, left bank tributary to Camopi River, Oyapock river system. Holotype: IRSNB 689.

Distribution: Oyapock River basin, French Guiana; known only from the types (Weber, 2003).

Hypostomus niceforoi (Fowler, 1943)

Hemiancistrus niceforoi Fowler, 1943a: 250, figs. 32-35. Type locality: Florencia, Río Orteguasa, Colombia. Holotype: ANSP 70511.

Distribution: Upper Japurá River basin, Colombia (Weber, 2003).

Hypostomus nickeriensis Boeseman, 1969

Hypostomus nickeriensis Boeseman, 1969: 125, fig. 4a. Type locality: Stondansie Falls, Nickerie River, Surinam. Holotype: ZMA 105.765.

Distribution: Upper Nickerie River basin, Suriname (Weber, 2003).

Hypostomus niger (Marini, Nichols \& La Monte, 1933)

Plecostomus niger Marini, Nichols \& La Monte, 1933: 4. Type locality: Southeastern Brazil. Holotype: AMNH 12245.

Distribution: Rivers of southeastern Brazil; known only from holotype (Weber, 2003).

Hypostomus nigromaculatus (Schubart, 1964)

Plecostomus nigromaculatus Schubart, 1964b: 2, fig. 2. Type locality: Rio Mogi Guaçu, Cachoeira de Emas, na região da corredeira (topava) (Mun. Pirassununga, Est. São Paulo) [Brazil]. Holotype: EEBP 304 (whereabouts unknown).

Distribution: Upper and middle Paraná River basin, Brazil (Weber, 2003).

Hypostomus nudiventris (Fowler, 1941)

Plecostomus nudiventris Fowler, 1941a: 147, figs. 44-46. Type locality: Rio Choró, Ceará, near Forteleza [Brazil]. Holotype: ANSP 69402

Distribution: Coastal drainages of northern Brazil (Weber, 2003).

Hypostomus occidentalis Boeseman, 1968

Hypostomus gymnorhynchus occidentalis Boeseman, 1968: 47, pl. 6 (figs. 1-4). Type locality: Surinam River near Brokopondo, Surinam. Holotype: RMNH 25520.

Distribution: Suriname River basin, Suriname (Weber, 2003).

Hypostomus oculeus (Fowler, 1943)

Panaque oculeus Fowler, 1943a: 256, figs.48-51. Type locality: Florencia, Rio Orteguasa, Colombia. Holotype: ANSP 70518.

Distribution: Marañon, Napo, and Orteguasa River basins, upper Amazon drainage (Armbruster, 2003a); upper Japurá River basin, Colombia (Weber, 2003).

Remarks: Redescribed in Armbruster (2003a) and placed in the Hypostomus cochliodon species group.

Hypostomus pagei Armbruster, 2003 
Hypostomus pagei Armbruster, 2003a: 41, figs. 14 b, 18. Type locality: Venezuela, Yaracuy, Rio Tupe, Rio Aroa dr. ca. 12 km N Aroa on Highway 3; 10³0'31"N, 6852'55"W. Holotype: MCNG 24696.

Distribution: Aroa, Tocuyo and Yaracuy River basins, Caribbean drainage, Venezuela (Armbruster, 2003a).

Remarks: Included in the Hypostomus cochliodon species group by Armbruster (2003a) .

Hypostomus pantherinus Kner, 1854

Hypostomus pantherinus Kner, 1854: 267. Type locality: Rio Guaporé, ohne nähere Angaben [Brazil]. Holotype: NMW 44206. Originally proposed as Hyp. pantherinus.

Distribution: Madeira River basin, Brazil; known only from holotype (Weber, 2003).

Hypostomus papariae (Fowler, 1941)

Plecostomus plecostomus papariae Fowler, 1941a: 145, figs. 41-43. Type locality: Lago Papary, Rio Grande do Norte [Brazil]. Holotype: ANSP 69398.

Distribution: Grande do Norte River basin, Brazil (Weber, 2003).

Hypostomus paranensis Weyenbergh, 1877

Hypostomus paranensis Weyenbergh, 1877: 9. Type locality: las lagunas al rededor de Sante Fe [Argentina]. Syntypes: Whereabouts unknown.

Plecostomus cordovae Günther, 1880a: 11. Type locality: Cordova [Argentina]. Holotype: BMNH 1878.4.4.1.

Distribution: Bermejo, Dulce, Uno, Secundo and middle Paraná River basins (Weber, 2003).

Remarks: Hypostomus paranensis Weyenbergh treated as nomen oblitum by Weber (2003), without explanation.

Hypostomus paucimaculatus Boeseman, 1968

Hypostomus paucimaculatus Boeseman, 1968: 52, pl. 8 (fig. 1). Type locality: Surinam River, near Brokopondo, Surinam. Holotype: RMNH 25468.

Distribution: Upper and middle Suriname River basin, Suriname (Weber, 2003).

Hypostomus paucipunctatus Hollanda Carvalho \& Weber, 2004

Hypostomus paucipunctatus Hollanda Carvalho \& Weber, 2004: 962, fig. 7. Type locality: Brazil: Pará: municipality of Caldeirão, Itacaiúnas River, Igarapé Pojuca, Serra dos Carajás $\left(5^{\circ} 52^{\prime} \mathrm{S}, 50^{\circ} 32^{\prime} \mathrm{W}\right.$, coordinates of Itacaiúnas River, near Castanhal). Holotype: MZUSP 82271.

Distribution: Itacaiúnas River basin, Pará, Brazil (Hollanda Carvalho \& Weber, 2004).

Remarks: Included in Hypostomus cochliodon species group by Hollanda Carvalho \& Weber (2004).

Hypostomus paulinus (Ihering, 1905)

Plecostomus paulinus Ihering, 1905: 560. Type locality: Rio Piracicaba, São Paulo, Brazil. Holotype: BMNH 1905.6.9.4.

Distribution: Tietê River basin, Brazil, Paraná River basin, Paraguay (Weber, 2003).

Hypostomus piratatu Weber, 1986

Hypostomus piratatu Weber, 1986: 987, fig. 3 (middle). Type locality: Bras intermittant du Río Tebicuary, bassin du rio Paraguay, systeme du Río Paraná; Sapucái, dép. Paraguari, Paraguay. Holotype: MHNG 2265.03.

Distribution: Eastern Paraguay River tributaries, Paraguay (Weber, 2003).

Hypostomus plecostomoides (Eigenmann, 1919)

Cochliodon plecostomoides Eigenmann, 1919a: 133. Type locality: Quebrada Cramalote, Villavicencio [Colombia]. Holotype: CAS 82501 [lost in mail (Eschmeyer et al., 1998)]. Also described as new in Eigenmann (1922b: 225 , pl. 11, figs. 1-3).

Distribution: Orinoco River basin and Tuy River and Valencia Lake basins of Venezuela (Armbruster, 2003a).

Remarks: Redescribed in Lilyestrom (1984) and Armbruster (2003a); placed by Armbruster in Hypostomus cochliodon species group.

Hypostomus plecostomus (Linnaeus, 1758)

Acipenser plecostomus Linnaeus, 1758: 238. Type locality: India [probably Suriname]. Syntypes: NRM 32 (3, of which one is illustrated in Linnaeus (1754: pl. 28, fig. 4)), and specimen also illustrated in Gronovius (1754: 24, pl. 3, figs. 1-2).

Hypostomus guacari La Cepède, 1803: 144, 145, pl. 4 (fig. 2). Type locality: Les rivieres de l'Amerique. Syntypes: Based on numerous literature sources, including account of Acipenser plecostomus Linnaeus, 1758; therefore syntypes of that species are also syntypes of this one. 
Loricaria flava Shaw, 1804: 38, pl. 101. Type locality: Indian Seas. Based on numerous literature sources, including account of Acipenser plecostomus Linnaeus, 1758; therefore syntypes of that species are also syntypes of this one.

Plecostomus bicirrosus Gronow, in Gray, 1854: 158. Type locality: Americes Meridionali fluminibus. Holotype or syntype: Specimen illustrated in Gronovius (1754: 24, pl. 3, figs. 1-2).

Plecostomus brasiliensis Bleeker, 1862 (in Bleeker, 1862-63): 2. Type locality: [not stated]. Based on description of Hypostomus plecostomus in Cuvier \& Valenciennes (1840). Described in more detail in Bleeker (1864: 7), with localities listed as follows: Surinama, Mejico, Cujaba, Chili. Boeseman (1968: 38) designated a lectotype, RMNH 3102, but its validity needs to be clarified, as it was apparently based on the description in Bleeker (1864) and not the earlier paper.

Distribution: Coastal drainages of the Guianas (Weber, 2003).

Remarks: Proposed neotype designations for Acipenser plecostomus and Hypostomus guacari (RMNH 18240 for both names) in Boeseman (1968: 11) are invalid inasmuch as the NRM syntypes are still extant.

Hypostomus pseudohemiurus Boeseman, 1968

Hypostomus pseudohemiurus Boeseman, 1968: 54, pl. 9 (fig. 1). Type locality: Kabalebo River, Corantijn River basin, Surinam. Holotype: RMNH 25516 (largest specimen).

Distribution: Corantijn River basin, Suriname (Weber, 2003).

Hypostomus punctatus Valenciennes, 1840

Hypostomus punctatus Valenciennes, in Cuvier \& Valenciennes, 1840b: 493 (364 in Strasbourg deluxe edition). Type locality: Rio-Janéiro. Holotype: at MNHN, but not found by Weber (2003).

Distribution: coastal drainages of southeastern Brazil (Weber, 2003).

Hypostomus pusarum (Starks, 1913)

Plecostomus pusarum Starks, 1913: 36, pl. 6. Type locality: at Ceará Mirim [Brazil]. Holotype: SU 22225.

Distribution: coastal drainages of northern Brazil (Weber, 2003).

Hypostomus pyrineusi (Miranda Ribeiro, 1920)

Cochliodon pyrineusi Miranda Ribeiro, 1920: 9, pls. 3\& 4. Type locality: provavelmente Jamary [Brazil]. Holotype: MNRJ 863.

Distribution: Madeira, Napo, Ucayali, Marañon and upper Amazon River basins (Armbruster, 2003a).

Remarks: Redescribed and included in Hypostomus cochliodon species group in Armbruster (2003a).

Hypostomus regani (Ihering, 1905)

Plecostomus regani Ihering, 1905: 558. Type locality: Rio Piracicaba, São Paulo, Brazil. Lectotype: BMNH 1905.6.7.2, designated by Reis et al. (1990: 745).

Distribution: Paraná, Paraguay and Uruguay River basins (Weber, 2003).

Remarks: Redescribed by Reis et al. (1990: 745).

Hypostomus robinii Valenciennes, 1840

Hypostomus Robinii Valenciennes, in Cuvier \& Valenciennes, 1840b: 501 (370 in Strasbourg deluxe edition). Type locality: de la Trinité [...] des affluens da la Plata [restricted to La Trinité by lectotype designation]. Lectotype: MNHN a-9569, designated by Boeseman (1968: 36).

Distribution: Trinidad Island, Trinidad and Tobago (Weber, 2003).

Hypostomus rondoni (Miranda Ribeiro, 1912)

Plecostomus rondoni Miranda Ribeiro, 1912: 6. Type locality: S. Manoel— Rio Tapajós [Brazil]. Holotype: MNRJ 741. Specific name spelled Plecostomus rondini in heading of account, but corrected in attached printed corrigendum.

Distribution: Tapajós River basin, Brazil (Weber, 2003).

Hypostomus roseopunctatus Reis, Weber \& Malabarba, 1990

Hypostomus roseopunctatus Reis, Weber \& Malabarba, 1990: 756, figs. 2, 26. Type locality: Rio Pelotas at road from Esmeralda to Anita Garibaldi, Rio Grande do Sul, Brazil. Holotype: MCP 12239.

Distribution: Uruguay River basin, (Weber, 2003).

Hypostomus saramaccensis Boeseman, 1968

Hypostomus saramaccensis Boeseman, 1968: 58, pl. 2 (fig. 1). Type locality: Feddiprati (rapids), middle Saramacca 
River, Surinam. Holotype: RMNH 25488 (largest).

Distribution: Coastal drainages of Suriname (Weber, 2003).

Hypostomus scabriceps (Eigenmann \& Eigenmann, 1888)

Plecostomus commersonii scabriceps Eigenmann \& Eigenmann, 1888b: 168. Type locality: Sao Matheos [Brazil].

Syntypes: MCZ 7894 (2), BMNH 1904.1.28.2 (1). Name made available from characters in key on p. 168.

Distribution: São Mateus River basin, Brazil (Weber, 2003).

Hypostomus scaphyceps (Nichols, 1919)

Plecostomus scaphyceps Nichols, 1919a: 425. Type locality: Cerqueira Cezar, Estado de São Paulo [Brazil]. Holotype: AMNH 7152. Name spelled Plecostomus scaplyceps on p. 416 [in Portuguese portion of original description].

Distribution: Paranapanema River basin, Brazil (Weber, 2003).

Remarks: Fowler (1954: 194) acted as first reviser and selected Plecostomus scaphyceps as the valid name for this species.

Hypostomus sculpodon Armbruster, 2003

Hypostomus sculpodon Armbruster, 2003a: 49, fig. 21. Type locality: Venezuela, Amazonas, Río Casiquiare, Río Negro dr., Departmento Río Negro, at Piedra Carguana (at second camp, left descending bank of river in portion of river between mouths of Río Siapa and Río Pasimoni), 0158.611'N, 66³2.359'W. Holotype: MCNG 37042.

Distribution: Upper Negro and upper Orinoco River basins, Venezuela (Armbruster, 2003a).

Remarks: Included in the Hypostomus cochliodon species group by Armbruster (2003a).

Hypostomus seminudus (Eigenmann \& Eigenmann, 1888)

Plecostomus seminudus Eigenmann \& Eigenmann, 1888b: 169. Type locality: Brazil (?). Holotype: MCZ 28981.

Name available from description in key on p. 169.

Distribution: Probably Brazil; known only from holotype (Weber, 2003).

Hypostomus simios Hollanda Carvalho \& Weber, 2004

Hypostomus simios Hollanda Carvalho \& Weber, 2004: 967, fig. 9. Type locality: Brazil: Amapá: bridge on the road to Serra do Navio, Cupixi river. Holotype: MZUSP 82268.

Distribution: Cupixi River basin, Amapá, Brazil (Hollanda Carvalho \& Weber, 2004).

Remarks: Included in Hypostomus cochliodon species group by Hollanda Carvalho \& Weber (2004).

Hypostomus sipaliwinii Boeseman, 1968

Hypostomus sipaliwinii Boeseman, 1968: 60, pl. 12 (fig. 1). Type locality: Sipaliwini River, upper Corantijn River basin, Surinam. Holotype: RMNH 25481 (larger specimen).

Distribution: Upper Corantijn River basin, Suriname (Weber, 2003).

Hypostomus soniae Hollanda Carvalho \& Weber, 2004

Hypostomus soniae Hollanda Carvalho \& Weber, 2004: 965, fig. 8. Type locality: Brazil: Pará: temporary pools on the river sides of the Rio Tapajós, between Vila Nova and Urua. Holotype: MZUSP 82272.

Distribution: Tapajós River basin, Pará, Brazil (Hollanda Carvalho \& Weber, 2004).

Remarks: Included in Hypostomus cochliodon species group by Hollanda Carvalho \& Weber (2004).

Hypostomus strigaticeps (Regan, 1908)

Plecostomus strigaticeps Regan, 1908f: 796, pl. 48 (fig. 1). Type locality: Rio Piracicaba, San Paulo, Brazil. Syntypes: BMNH 1907.7.6.10-12 (2 or 3).

Distribution: Tietê River basin, Brazil (Weber, 2003).

Hypostomus subcarinatus Castelnau, 1855

Hypostomus subcarinatus Castelnau, 1855: 42, p. 21 (fig. 1). Type locality: des rivières de la province des Mines [Brazil]. Holotype: MNHN a-9575.

Distribution: Coastal drainages of eastern Brazil, possibly including São Francisco River basin (Weber, 2003).

Hypostomus surinamensis Boeseman, 1968

Hypostomus surinamensis Boeseman, 1968: 61, pl. 13 (fig. 1). Type locality: Surinam River near Brokopondo, Surinam. Holotype: RMNH 25497 (larger specimen).

Distribution: Suriname River basin, Suriname (Weber, 2003).

Hypostomus tapanahoniensis Boeseman, 1969 
Hypostomus gymnorhynchus tapanahoniensis Boeseman, 1969: 129, figs. 5 (b, d). Type locality: Upper Tapanahoni River, Surinam. Holotype: RMNH 25476. Originally proposed as H. gymnorhynchus tapanahoniensis.

Distribution: Maroni River basin (Weber, 2003).

Hypostomus taphorni (Lilyestrom, 1984)

Cochliodon taphorni Lilyestrom, 1984: 43, fig. 8. Type locality: Río Botanamo, cerca del puente en la vía a Bochinche, Edo, Bolívar, Venezuela. Holotype: MCNG 8084.

Distribution: Cuyuni River basin, Essequibo drainage, Venezuela (Armbruster, 2003a), Rupununi River and Takutu River basins, Guyana (Armbruster \& de Souza, 2005).

Remarks: Redescribed and included in Hypostomus cochliodon species group in Armbruster (2003a).

Hypostomus tapijara Oyakawa, Akama \& Zanata, 2005

Hypostomus tapijara Oyakawa, Akama \& Zanata, 2005: 19, fig. 7. Type locality: Brazil: São Paulo, rio Catas Altas, Ribeira. Holotype: MZUSP 55204.

Distribution: Ribeira de Iguape River basin, Brazil (Oyakawa et al., 2005).

Hypostomus ternetzi (Boulenger, 1895)

Plecostomus ternetzi Boulenger, 1895b: 525. Type locality: Paraguay. Holotype: BMNH 1895.5.17.64; holotype illustrated in Boulenger (1896c: pl. 5).

Distribution: Middle Paraná, Paraguay, and Uruguay River basins (Weber, 2003).

Hypostomus tietensis (Ihering, 1905)

Plecostomus tietensis Ihering, 1905: 559. Type locality: Rio Tietè, São Paulo, Brazil. Holotype: BMNH 1905.6.9.1.

Distribution: Tietê River basin, Brazil (Weber, 2003).

Hypostomus topavae (Godoy, 1969)

Plecostomus topavae Godoy, 1969: 176, fig. 1. Type locality: na região da topava [...] Cachoeira de Emas, Rio Mogi Guassu, Estado de São Paulo, Brasil. Holotype: EEBP 315a.

Distribution: Grande River basin, Brazil; known only from holotype (Weber, 2003).

Hypostomus unae (Steindachner, 1878)

Plecostomus Unae Steindachner, 1878b: 383. Type locality: Rio Una (südlich von Bahia) [Brazil]. Syntypes: BMNH 1862.11.23.12 (1), NMW 44259 (4).

Distribution: Una River basin in Bahia State, Brazil (Weber, 2003).

Remarks: This name was based on descriptions of Plecostomus Robinii (not Valenciennes) in Günther (1864: 236) and Steindachner (1877b: 676).

Hypostomus uruguayensis Reis, Weber \& Malabarba, 1990

Hypostomus uruguayensis Reis, Weber \& Malabarba, 1990: 760, fig. 30. Type locality: Rio Uruguay at "Rancho da Amizade”, São Borja, Rio Grande do Sul, Brazil. Holotype: MCP 11874.

Distribution: Uruguay River basin (Weber, 2003).

Hypostomus vaillanti (Steindachner, 1877)

Plecostomus Vaillanti Steindachner, 1877c: 225. Type locality: Rio Preto [at Villa de Santa Rita, Bahia, Brazil]. Syntypes: MCZ 7842 (2), NMW 44273 (1), NMW 44276 (3), NMW 44277 (1).

Distribution: Preto River basin of São Francisco River drainage, Brazil (Weber, 2003).

Hypostomus variipictus (Ihering, 1911)

Plecostomus variipictus Ihering, 1911: 390. Type locality: Rio Pardo, perto de Barretos, Estado de São Paulo, quasi em sua foz no Rio Grande, tributario do lado esquerdo do Rio Paraná, Brazil. Holotype: MZUSP 2114.

Distribution: Grande River basin, Brazil (Weber, 2003).

Hypostomus varimaculosus (Fowler, 1945)

Plecostomus varimaculosus Fowler, 1945a: 113, fig. 14-16. Type locality: Morelia, Río Caquetá drainage, Colombia. Holotype: ANSP 71707.

Distribution: Upper Japurá River basin, Upper Amazon drainage of Brazil and Colombia (Weber, 2003).

Hypostomus variostictus (Miranda Ribeiro, 1912)

Plecostomus variostictus Miranda Ribeiro, 1912: 6. Type locality: Coxim, M[ato] Grosso [Brazil]. Holotype: MNRJ 1072.

Distribution: Upper Paraguay River basin, Brazil; only known from holotype (Weber, 2003). 
Hypostomus ventromaculatus Boeseman, 1968

Hypostomus ventromaculatus Boeseman, 1968: 65, pl. 15 (fig. 1). Type locality: Surinam River between Afobaka and Brokopondo, Surinam. Holotype: RMNH 25507.

Distribution: Coastal drainages of northern South America from Oyapock River to Suriname River (Weber, 2003).

Remarks: Redescribed in Le Bail et al. (2000: 256).

Hypostomus vermicularis (Eigenmann \& Eigenmann, 1888)

Plecostomus vermicularis Eigenmann \& Eigenmann, 1888b: 171. Type locality: Rio Parahyba; Rio Janeiro; Mendez; Macacos, Goiás [Brazil]. Syntypes (37, 8-30 cm): BMNH 1904.1.28.4-5 (2), MCZ 7814 (1), MCZ 7848 (12), MCZ 7849 (1), MCZ 7850 (4), MCZ 7851 (1), MCZ 7857 (1), MCZ 91352 (12), NMW 44279 (1), USNM 123010 (3).

Distribution: Coastal drainages of eastern Brazil (Weber, 2003).

Remarks: Type series apparently mixed (Weber, 2003), and in need of a lectotype designation to stabilize current usage.

Hypostomus waiampi Hollanda Carvalho \& Weber, 2004

Hypostomus waiampi Hollanda Carvalho \& Weber, 2004: 955, fig. 2. Type locality: Brazil: Amapá, bridge on the road to Serra do Navioo, Cupixi river. Holotype: MZUSP 82269.

Distribution: Cupixi River, Amapá, Brazil (Hollanda Carvalho \& Weber, 2004).

Remarks: Included in Hypostomus cochliodon species group by Hollanda Carvalho \& Weber (2004).

Hypostomus watwata Hancock, 1828

Hypostomus watwata Hancock, 1828: 246. Type locality: Demerara [...] on the sea-shores [now: Berbice River, Guyana, by neotype designation]. Neotype: BMNH 1932.11.10.31; designated by, and illustrated in, Boeseman (1868: 71, pl. 16, fig. 1).

Hypostomus verres Valenciennes in Cuvier \& Valenciennes, 1840b: 494 (365 in Strasbourg deluxe edition). Type locality: envoyés de Cayenne [French Guiana]. Lectotype: MNHN a-9450, designated by Boeseman (1968: 70).

Distribution: coastal drainages of northern South America from Oyapock River to Demerara River, and Venezuela (Weber, 2003).

Remarks: Redescribed in Le Bail et al. (2000: 260).

Hypostomus winzi (Fowler, 1945)

Plecostomus winzi Fowler, 1945b: 9, figs. 4-7. Type locality: Honda, Colombia. Holotype: ANSP 71623.

Distribution: Magdalena River basin, Colombia, known only from holotype (Weber, 2003).

Hypostomus wuchereri (Günther, 1864)

Plecostomus wuchereri Günther, 1864: 235. Type locality: Bahia, Brazil. Syntypes (4): BMNH 1863.3.27.15 (1).

Distribution: Paraguaçu River basin (?), Brazil (Weber, 2003).

\section{ISBRUECKERICHTHYS Derijst, 1996}

Isbrueckerichthys Derijst, 1996: 64. Type species: Hemipsilichthys duseni Miranda Ribeiro, 1907. Type by original designation. Gender: Masculine.

Revision and key to species: Pereira \& Reis (2002).

Isbrueckerichthys alipionis (Gosline, 1947)

Pareiorhaphis alipionis Gosline, 1947: 104. Type locality: Rio Bethary, São Paulo [Brazil]. Holotype: MNRJ 4241.

Distribution: Betari River basin, Ribeira de Iguape River drainage, Brazil (Weber, 2003).

Isbrueckerichthys duseni (Miranda Ribeiro, 1907)

Hemipsilichthys duseni Miranda Ribeiro, 1907a: 187, figure on page 187. Type locality: do Paraná ..[\&]... do Ribeirão do Monjolinho, á 300 metros sobre o mar [Iporanga River, São Paulo, Brazil]. Lectotype: MNRJ 772, designated by Gosline (1947: 102).

Distribution: Upper Ribeira de Iguape River in Paraná State, Brazil (Weber, 2003).

Isbrueckerichthys epakmos Pereira \& Oyakawa, 2003

Isbrueckerichthys epakmos Pereira \& Oyakawa, 2003: 6, fig. 2. Type localilty: Brazil. São Paulo: Tapiraí: rio Ribeira de Laguna drainage, rio Verde at Piúva, on road to Rio Verde, 2358'22"S, 47³4'23"W. Holotype: MZUSP 79804. 
Distribution: Ribeira de Iguape River basin, Brazil (Pereira \& Oyakawa, 2003).

ISORINELORICARIA Isbrücker, 1980

Isorineloricaria Isbrücker, 1980: 15. Type species: Plecostomus spinosissimus Steindachner, 1880. Type by original designation. Gender: Feminine.

Isorineloricaria spinosissima (Steindachner, 1880)

Plecostomus spinosissimus Steindachner, 1880b: 98, pl. 5 (figs. 1, 1a). Type locality: Guayaquil [Ecuador]. Holotype: NMW 55027.

Plecostomus festae Boulenger, 1898c: 11. Type locality: Rio Vinces and Rio Peripa, Equateur. Syntypes: BMNH 1898.11.4.32 (1), ZMUT 1518 (1).

Distribution: Pacific versant rivers of Ecuador (Weber, 2003).

IXINANDRIA Isbrücker \& Nijssen, 1979

Ixinandria Isbrücker \& Nijssen, in Isbrücker, 1979a: 91. Type species: Loricaria steinbachi Regan, 1906. Type by original designation. Gender: Feminine.

Ixinandria montebelloi (Fowler, 1940)

Canthopomus montebelloi Fowler, 1940c: 55, figs. 9-11. Type locality: Monte Bello, Tarija, Bolivia. Holotype: ANSP 68832.

Distribution: Southern Bolivia.

Ixinandria steinbachi (Regan, 1906)

Loricaria Steinbachi Regan, 1906a: 97. Type locality: Salta, Argentina. Lectotype: BMNH 1906.5.31.37, designated by Isbrücker (1979a: 113), and illustrated in Isbrücker (1979a: 91, figs. 1 a-c, 2, 3).

Distribution: Salado River basin, Argentina.

Remarks: Species reviewed by Ringuelet et al. (1967).

KRONICHTHYS Miranda Ribeiro, 1908

Kronichthys Miranda Ribeiro, 1908b: [1]. Type species: Kronichthys subteres Miranda Ribeiro, 1908. Type by monotypy. Gender: Masculine.

Remarks: Also described as new in Miranda Ribeiro (1908a).

Kronichthys heylandi (Boulenger, 1900)

Plecostomus heylandi Boulenger, 1900b: 165. Type locality: Mountain stream in the Province São Paulo [...] 400 feet above sea-level near Santos [Brazil]. Holotype: BMNH 1899.12.18.1.

Distribution: coastal streams of eastern Brazil, between Santos and Rio de Janeiro (Weber, 2003).

Remarks: Redescribed in Gosline (1947: 106).

Kronichthys lacerta (Nichols, 1919)

Plecostomus lacerta Nichols, 1919a: 414. Type locality: Poço Grande (Estado de São Paulo) Rio Juquia, Brazil. Holotype: AMNH 7151.

Distribution: Baia de Paranaguá basin and Ribeira de Iguape River basin, Brazil (Weber, 2003).

Remarks: Redescribed in Armbruster \& Page (1997: 228).

Kronichthys subteres Miranda Ribeiro, 1908

Kronichthys subteres Miranda Ribeiro, 1908b: [2], fig. 1. Type locality: Rios Bethary, Pardo e Iporanga [Brazil]. Syntypes: MNRJ 655 (4).

Distribution: Ribeira de Iguape River basin, Brazil (Weber, 2003).

Remarks: Also described as new in Miranda Ribeiro (1908a).

LAMONTICHTHYS Miranda Ribeiro, 1939

Lamontichthys Miranda Ribeiro, P., 1939a: 12. Type species: Harttia filamentosa La Monte, 1935. Type by original designation. Gender: Masculine.

Revision: Isbrücker \& Nijssen (1978b).

Lamontichthys filamentosus (La Monte, 1935) 
Harttia filamentosa La Monte, 1935: 5, fig. 4. Type locality: Brazilian Amazonas, Rio Jurua, in the vicinity of the mouth of the Rio Embira, a tributary of Rio Tarauaca, which, in turn, is a tributary of Rio Jurua, $70^{\circ} 15^{\prime} \mathrm{W}$, 7³0'S. Holotype: AMNH 12616.

Harttia filamentissima Eigenmann \& Allen, 1942: 211, pl. 8 (figs. 1-2). Type locality: Rio Huallaga [Peru]. Holotype: CAS 28541.

Distribution: Western Amazon River basin (Ferraris, 2003c).

Lamontichthys llanero Taphorn \& Lilyestrom, 1984

Lamontichthys llanero Taphorn \& Lilyestrom, 1984: 96, fig. 2. Type locality: Venezuela, Estado Portuguesa, Río Guanare Viejo cerca de Hoyada. Holotype: MCNG 10000.

Distribution: Guanare Viejo River basin, Orinoco River drainage, Venezuela (Taphorn \& Lilyestrom, 1984).

Lamontichthys maracaibero Taphorn \& Lilyestrom, 1984

Lamontichthys maracaibero Taphorn \& Lilyestrom, 1984b: 94, fig. 1. Type locality: Venezuela, Estado Zulia, Distrito Miranda, Río Motatán, en el puente en Agua Viva. Holotype: MCNG 3592.

Distribution: Lake Maracaibo basin, Venezuela (Taphorn \& Lilyestrom, 1984).

Lamontichthys stibaros Isbrücker \& Nijssen, 1978

Lamontichthys stibaros Isbrücker \& Nijssen, 1978b: 66, fig. 8. Type locality: Ecuador, Prov. Pastaza, Río Amazonas system, Río Bobonaza at Chicherota, 2²5'S, 76³8'W, altitude approximately 260-280 m., upper Río Pastaza. Holotype: USNM 167914.

Distribution: Bobonaza River basin, Ecuador (Isbrücker \& Nijssen, 1978b).

LAMPIELLA Isbrücker, 2001

Lampiella Isbrücker, in Isbrücker et al., 2001: 19. Type species: Otocinclus gibbosus Miranda Ribeiro, 1908. Type by original designation. Gender: Feminine.

Remarks: Genus created to accommodate Otocinclus gibbosus Miranda Ribeiro, which Schaefer (1997) removed from Otocinclus, but did not place elsewhere.

Lampiella gibbosa (Miranda Ribeiro, 1908)

Otocinclus gibbosus Miranda Ribeiro, 1908b: [2], figs. 2-2a. Type locality: Rio Bethary [Brazil]. Holotype: MNRJ 1048.

Distribution: Ribeira de Iguape River basin, Brazil (Schaefer, 2003c).

\section{LASIANCISTRUS Regan, 1904}

Lasiancistrus Regan, 1904b: 224. Type species: Chaetostomus heteracanthus Günther, 1869. Type by subsequent designation by Eigenmann (1910: 409). Gender: Masculine. Proposed originally as a subgenus of Ancistrus Kner.

Phylogeny: Armbruster \& Provenzano (2000).

Revision: Heitmans, et al. (1983), Armbruster (2005).

Key to species: Armbruster (2005: 552).

Lasiancistrus caucanus Eigenmann, 1912

Lasiancistrus caucanus Eigenmann, 1912a: 11. Type locality: Cartago; the Cauca, Colombia. Holotype: FMNH 56034; holotype illustrated in Eigenmann (1922: 76, pl. 11, fig. 4).

Hemiancistrus mayoloi Eigenmann, 1912a: 10. Type locality: Istmina, San Juan River, Colombia. Holotype: FMNH 56036.

Ancistrus planiceps Meek \& Hildebrand, 1913: 79. Type locality: Rio Tuyra, Boca de Cupe, Panama. Holotype: FMNH 7580.

Lasiancistrus volcanensis Dahl, 1942: 83, fig. 3. Type locality: Rio Volcán near its junction to Rio San Bartolomé ...tributary of Rio Magdalena, on the left side, between Rios Narés and Ité, municipio of Remedios, department of Antióquia, Republic of Colombia). Altitude approx. 600 m. Holotype: at ZMUL.

Distribution: Magdalena, Atrato, San Juan, Cauca, Tuyra, and Bayano River basin, Colombia and Panama (Armbruster, 2005).

Remarks: Armbruster (2005: 555) acted as first reviser in selecting Lasiancistrus caucanus as the valid name for the 
species; Maldonado-Ocampo et al. (2005), however, continue to treat Lasiancistrus mayoloi and L. volcanensis as distinct species.

Lasiancistrus guacharote (Valenciennes, 1840)

Hypostomus guacharote Valenciennes, in Cuvier \& Valenciennes, 1840b: 508 (375 in Strasbourg deluxe edition). Type locality: Les eaux douces de Porto-Ricco [=Puerto Rico, in error]. Syntype: MNHN a-9567 (1).

Ancistrus mystacinus Kner, 1854: 276. Type locality: Carracas [Venezuela]. Holotype: NMW 44200. Originally as Anc. mystacinus.

Lasiancistrus maracaiboensis Schultz, 1944c: 314, pl. 11 (fig. a). Type locality: Río Socuy, 3 km. above its mouth. Venezuela. Holotype: USNM 121038.

Distribution: Lake Maracaibo basin, Venezuela (Armbruster, 2005).

Lasiancistrus heteracanthus (Günther, 1869)

Chaetostomus heteracanthus Günther, 1869: 425, fig. 3. Type locality: Upper Amazons, Peruvian Amazons. Holotype: BMNH 1869.5.21.3.

Distribution: Napo River, Ecuador and Peru (Armbruster, 2005).

Remarks: Type locality interpreted by Eigenmann \& Eigenmann (1891: 41; see also Eigenmann \& Allen 1942: 18) as Marañon River, but see different interpertation in Armbruster (2005).

Lasiancistrus saetiger Armbruster, 2005

Lasiancistrus saetiger Armbruster, 2005: 559, fig. 7. Type locality: Brazil, Pará, rio Guamá near Ourém, 1³4'07"S, 470108"W. Holotype: MCP 37942.

Distribution: Guama River, Capim River basin, Brazil (Armbruster, 2005).

Lasiancistrus schomburgkii (Günther, 1864)

Hypostomus pictus Castelnau, 1855: 44, pl. 22 (fig. 2). Type locality: 1'Ucayale [Peru]. Holotype: MNHN a-9573. Preoccupied in Hemiancistrus by Ancistrus pictus Kner, 1854; replaced by Hemiancistrus castelnaui Miranda Ribeiro, 1911.

Chaetostomus schomburgkii Günther, 1864: 245. Type locality: British Guiana. Syntypes: BMNH 1845.3.5.26-27 (2).

Hemiancistrus castelnaui Miranda Ribeiro, 1911: 58. Type locality: 1'Ucayale [Peru]. Holotype: MNHN a-9573. Replacement for Hypostomus pictus Castelnau, 1855, which was then preoccupied in Hemiancistrus by Ancistrus pictus Kner, 1854.

Ancistrus multispinis Holly, 1929: 119. Type locality: Mercado Blèin (Brasilien). Holotype: at NMW.

Hemiancistrus caquetae Fowler, 1945a: 115, figs. 17-20. Type locality: Morelia, Río Caquetá drainage, Colombia. Holotype: ANSP 71708.

Lasiancistrus scolymus Nijssen \& Isbrücker, 1985: 242, fig. 1. Type locality: Brazil, Est. Mato Grosso do Sul, Rio Aripuanã, Humboldt (10¹0'S 59²7'W), Rio Madeira system. Holotype: MZUSP 26809.

Lasiancistrus guapore Knaack, 2000a: 57, figure on p. 58. Type locality: Brasilien, Mato Grosso, im Hauptstrom des Rio Guaporé im Stadtgebiet von Pontes E Lacerda; S 1507.627', W 5857.786'. Holotype: MCP 28678.

Distribution: Amazon, Upper Orinoco and Upper Essequibo River basins (Armbruster, 2005).

Lasiancistrus tentaculatus Armbruster, 2005

Lasiancistrus tentaculatus Armbruster, 2005: 565, fig. 9. Type locality: río Las Marias, near El Portrero, below the metal bridge. $09^{\circ} 05^{\prime} \mathrm{N}, 69^{\circ} 39^{\prime} \mathrm{W}$ [Venezuela]. Holotype: MCNG 19744.

Distribution: Orinoco River basin and Valencia Lake basin, Venezuela and Colombia (Armbruster, 2005).

\section{LEPORACANTHICUS Isbrücker \& Nijssen, 1989}

Leporacanthicus Isbrücker \& Nijssen, 1989: 544. Type species: Leporacanthicus galaxias Isbrücker \& Nijssen, 1989. Type by original designation. Gender: Masculine.

Leporacanthicus galaxias Isbrücker \& Nijssen, 1989

Leporacanthicus galaxias Isbrücker \& Nijssen, 1989: 546, fig. 10. Type locality: Brasilien, Est. Pará, Rio Tocantins, Lagoa em frenta a Jacobal. Holotype: MZUSP 24136.

Distribution: Southern tributaries of middle and lower Amazon, including Madeira, Tocantins and Guamá rivers; Ventuari River basin, upper Orinoco drainage (Provenzano, 1995; Fisch-Muller, 2003). 
Leporacanthicus heterodon Isbrücker \& Nijssen, 1989

Leporacanthicus heterodon Isbrücker \& Nijssen, 1989: 547, fig. 12. Type locality: Brasilien, Est. Mato Grosso, Rio Xingú, Cachoeira von Martius, oberer Xingú. Holotype: IRSNB 693.

Distribution: Xingu River basin, Brazil (Fisch-Muller, 2003).

Leporacanthicus joselimai Isbrücker \& Nijssen, 1989

Leporacanthicus joselimai Isbrücker \& Nijssen, 1989: 546, fig. 11. Type locality: Brasilien, Est. Pará, Maloquinha, perta de Itaituba, Rio Tapajós. Holotype: MZUSP 21921.

Distribution: Tapajós River basin, Brazil (Fisch-Muller, 2003).

Leporacanthicus triactis Isbrücker, Nijssen \& Nico, 1992

Leporacanthicus triactis Isbrücker, Nijssen \& Nico, 1992: 3, fig. 3. Type locality: Venezuela, Territorio Federal Amazonas, oberer Orinoco, Caño Mavaquita etwa einen Kilometer flussaufwärts von der Mündung in den Río Mavaca, ungefähr 2¹2'30"N, 6505'30"W. Holotype: MCNG 25357.

Distribution: Upper Orinoco River basin (Fisch-Muller, 2003).

LEPTOANCISTRUS Meek \& Hildebrand, 1916

Leptoancistrus Meek \& Hildebrand, 1916: 254. Type species: Acanthicus canensis Meek \& Hildebrand, 1913. Type by original designation. Gender: Masculine.

Monophyly: Schaefer (1986: 108).

Leptoancistrus canensis (Meek \& Hildebrand, 1913)

Acanthicus canensis Meek \& Hildebrand, 1913: 80. Type locality: Rio Cana, Cana, Darien, Panama. Holotype: FMNH 7581. Species illustrated and described in more detail by Meek \& Hildebrand (1916: 254, pl. 11).

Distribution: Pacific drainages of eastern Panama; Armila River basin on Caribbean slope, Panama (Fisch-Muller, 2003).

Leptoancistrus cordobensis Dahl, 1964

Leptoancistrus cordobensis Dahl, in Dahl et al., 1964: 32, unnum. plate. Type locality: río Batatal, Alto río Uré [upper San Jorge tributary, Magdalena basin, Colombia]. Holotype: Whereabouts unknown (possibly at ICNMHN).

Distribution: Upper Sinú River basin, and upper San Jorge River basin, Magdalena River drainage, Colombia (Fisch-Muller, 2003).

LIMATULICHTHYS Isbrücker \& Nijssen, 1979

Limatulichthys Isbrücker \& Nijssen, in Isbrücker, 1979a: 91. Type species: Loricaria punctata Regan, 1904. Type by original designation. Gender: Masculine.

Limatulichthys griseus (Eigenmann, 1909)

Loricaria griseus Eigenmann, 1909a: 8. Type locality: Conawaruk [=Konawaruk, Guyana]. Holotype: FMNH 53077; holotype illustrated in Eigenmann (1912b: pl. 30, fig. 2; pl. 32, fig. 2).

Distribution: Essequibo and western Orinoco River basins (Ferraris, 2003c).

Limatulichthys petleyi (Fowler, 1940)

Loricaria punctata Regan, 1904b: 285, pl. 17 (fig. 1). Type locality: Manaos, Rio Negro, middle Amazon, and Porto do Moz, lower Amazon. Syntypes: BMNH 1893.4.24.18, and at MCZ. Preoccupied by Loricaria punctata Kner, 1854 [now Pterygoplichthys punctatus], apparently not replaced.

Rhineloricaria petleyi Fowler, 1940a: 243, figs. 36-38. Type locality: Ucayali River basin, Contamana, Peru. Holotype: ANSP 68661.

Loricariichthys parnahybae Fowler, 1941a: 161, figs. 69-71. Type locality: Rio Parnahyba, Therizina, Piauhy [Brazil]. Holotype: ANSP 69451.

Distribution: Amazon, Tocantins and Parnaíba River basins (Ferraris, 2003c).

Remarks: Recently removed from the synonymy of Limatulichthys griseus (Eigenmann, 1909) by Isbrücker (2001).

LIPOPTERICHTHYS Norman, 1935

Lipopterichthys Norman, 1935b: 627. Type species: Lipopterichthys carrioni Norman, 1935. Type by original desig- 
nation. Gender: Masculine.

Monophyly: Schaefer (1986: 109).

Lipopterichthys carrioni Norman, 1935

Lipopterichthys carrioni Norman, 1935b: 628, fig. on p. 628 (top). Type locality: the Zamora River, near Loja, Ecuador. Holotype: BMNH 1933.5.29.1.

Distribution: Marañon River basin, Ecuador (Fisch-Muller, 2003).

\section{LITHOGENES Eigenmann, 1909}

Lithogenes Eigenmann, 1909a: 6. Type species: Lithogenes villosus Eigenmann, 1909. Type by original designation. Gender: Masculine.

Phylogenetic position: Schaefer (2003d).

Lithogenes valencia Provenzano, Schaefer, Baskin \& Royero, 2003

Lithogenes valencia Provenzano, Schaefer, Baskin \& Royero, 2003: 564, fig. 1. Type locality: Venezuela, Est. Carabobo, Lago de Valencia basin. Holotype: MBUCV V-30740.

Distribution: Lake Valencia basin, northern Venezuela (Provenzano et al., 2003).

Lithogenes villosus Eigenmann, 1909

Lithogenes villosus Eigenmann, 1909a: 6. Type locality: Aruataima Falls, Upper Potaro. [Guyana]. Holotype: FMNH 52960; holotype illustrated in Eigenmann (1912b: pl. 26, figs. 2-4) and Schaefer (2003d: fig. 2).

Distribution: Essequibo River basin, Potaro River, Guyana.

Remarks: Redescribed in Schaefer (2003d: 8).

\section{LITHOXUS Eigenmann, 1910}

Lithoxus Eigenmann, 1910: 405. Type species: Lithoxus lithoides Eigenmann, 1910. Type by original designation (p. 412). Gender: Masculine. Diagnostic characters given in key in footnote.

Paralithoxus Boeseman, 1982: 46. Type species: Ancistrus bovallii Regan, 1906. Type by original designation. Gender: Masculine. Proposed as a subgenus of Lithoxus. Paralithoides appears in the abstract of Boeseman (1982: 41) and is treated here as an error.

Revision: Boeseman (1982), Nijssen \& Isbrücker (1990)

Monophyly: Schaefer (1986: 110).

Lithoxus boujardi Muller \& Isbrücker, 1993

Lithoxus boujardi Muller \& Isbrücker, 1993: 72, fig. 1. Type locality: Guyane française, bassin de l'Approuague, Arataye, entre le Saut Japigny et le Saut Pararé. Holotype: MNHN 1992-1321.

Distribution: Approuague and Oyapock River basins, French Guiana (Fisch-Muller, 2003).

Remarks: Redescribed in Le Bail et al. (2000: 242, fig.).

Lithoxus bovallii (Regan, 1906)

Ancistrus Bovallii Regan, 1906a: 96. Type locality: the Kaat River, tributary to the Treng River, Upper Potaro, British Guiana. Lectotype: BMNH 1905.11.1.43, designated by Nijssen \& Isbrücker (1990: 332).

Distribution: Ireng River basin in upper Negro River drainage, Guyana (Fisch-Muller, 2003).

Remarks: Redescribed in Schindler (1996).

Lithoxus lithoides Eigenmann, 1910

Lithoxus lithoides Eigenmann, 1910: 412. Type locality: Cataracts of British Guiana. Holotype: FMNH 53557; holotype illustrated in Eigenmann (1912b: 242, pl. 29, figs. 1, 2, 4).

Distribution: Essequibo River, Guyana and upper Corantijn River basin, Suriname (Fisch-Muller, 2003).

Lithoxus pallidimaculatus Boeseman, 1982

Lithoxus (Paralithoxus) pallidimaculatus Boeseman, 1982: 50, pl. 3 (top). Type locality: Kwambaolo Creek, right tributary of Sara Creek above Dam, Suriname River system, Surinam. Holotype: RMNH 28368.

Distribution: Suriname River basin, Suriname (Fisch-Muller, 2003).

Lithoxus planquettei Boeseman, 1982

Lithoxus (Paralithoxus) planquettei Boeseman, 1982: 53, pl. 4 (top). Type locality: Crique Boulenger, Comté system, French Guyana. Holotype: RMNH 28304 (1 of 3). 
Distribution: Atlantic coastal drainages from Maroni to Kaw River basins, French Guiana (Fisch-Muller, 2003).

Remarks: Redescribed in Le Bail et al. (2000: 244).

Lithoxus stocki Nijssen \& Isbrücker, 1990

Lithoxus stocki Nijssen \& Isbrücker, 1990: 329, fig. 2. Type locality: Marouini River, downstream of village Epoia, French Guiana. Holotype: IRSNB 639.

Distribution: Maroni and Mana River basins, French Guiana (Fisch-Muller, 2003).

Remarks: Redescribed in Le Bail et al. (2000: 246).

Lithoxus surinamensis Boeseman, 1982

Lithoxus (Paralithoxus) surinamensis Boeseman, 1982: 54, pl. 5 (top). Type locality: near Awaradam, Gran Rio, upper Suriname River system, Surinam. Holotype: RMNH 28361 (largest specimen).

Distribution: Gran Rio River basin in upper Suriname River drainage, Suriname (Fisch-Muller, 2003).

LORICARIA Linnaeus, 1758

Loricaria Linnaeus, 1758: 307. Type species: Loricaria cataphracta Linnaeus, 1758. Type by monotypy. Gender: Feminine.

Fusiloricaria Fowler, 1940a: 247. Type species: Loricaria clavipinna Fowler, 1940. Type by original designation. Gender: Feminine. Originally proposed as a subgenus of Loricaria.

Revision: Isbrücker (1981).

Loricaria apeltogaster Boulenger, 1895

Loricaria apeltogaster Boulenger, 1895b: 528. Type locality: Paraguay. Lectotype: BMNH 1895.5.17.105, designated by Isbrücker (1979a: 110) and illustrated in Isbrücker (1979a: 107, figs. 20a, 21a); one syntype illustrated in Boulenger (1896c: pl. 7, fig. 2).

Distribution: Uruguay, Paraná and Paraguay River basins (Isbrücker, 1981).

Loricaria cataphracta Linnaeus, 1758

Loricaria cataphracta Linnaeus, 1758: 307. Type locality: In America meridionali. Lectotype: NRM 33, designated by Isbrücker (1979a: 110). Earlier neotype designation by Isbrücker (1972: 173) invalidated by rediscovery of syntype series.

Loricaria cirrhosa Bloch \& Schneider, 1801: 125, pl. 34. Type locality: America meridionali. Lectotype: ZMB 3160, designated by, and illustrated in, Isbrücker (1972: 170, figs. 4-5).

Loricaria setifera La Cepède, 1803: 140, 141. Type locality: In America meridionali. Syntype: NRM 33. On Gronovius (1763: n. 69), Bloch (1794: pl. 375, fig. 3), Loricaria cataphracta Linnaeus, Seba (1734-65: pl. 29, fig. 14), and other literature sources.

Loricaria Dentata Shaw, 1804: 37. Type locality: Indian Seas, in error [South America]. Types: On Gronovius (1763: 127, pl. 2, figs. 1-2), Bloch (1794: pl. 375, figs. 3-4), and Loricaria cataphracta Linnaeus.

Plecostomus flagellaris Gronow in Gray, 1854: 158. Type locality: Surinam. Holotype: BMNH 1863.11.12.195 (dorsal surface of body) and 196 (ventral surface); holotype redescribed and illustrated in Isbrücker (1972: fig. 7).

Loricaria carinata Castelnau, 1855: 46, pl. 23 (fig. 3). Type locality: De la rivières Amazones. Holotype: MNHN a9562; holotype redescribed and illustrated in Isbrücker (1972: 178, fig. 8).

Loricaria dura Bleeker, 1862 (in Bleeker, 1862-63): 3. Type locality: Surinama. Lectotype: NRM 33. Unneeded new name for Loricaria cataphracta Linnaeus.

Distribution: Amazon River basin, and coastal rivers of the Guianas, Brazil, French Guiana, Guyana, Suriname (Isbrücker, 1981).

Remarks: Species redescribed by le Bail et al. (2000); see Isbrücker (1972) for comments on the identity of the species.

Loricaria clavipinna Fowler, 1940

Loricaria clavipinna Fowler, 1940a: 247, figs. 44-47. Type locality: Ucayali River basin, Contamana, Peru. Holotype: ANSP 68665; holotype illustrated in Isbrücker (1981: figs. 9-10).

Distribution: Amazon River basin, Brazil, Peru (Isbrücker, 1981).

Loricaria lata Eigenmann \& Eigenmann, 1889 
Loricaria lata Eigenmann \& Eigenmann, 1889b: 36. Type locality: Goyaz [Brazil]. Lectotype: MCZ 46721, designated by, and illustrated in, Isbrücker (1972: 179, figs. 9-10).

Distribution: Araguaia River basin, Brazil (Isbrücker, 1981).

Loricaria nickeriensis Isbrücker, 1979

Loricaria nickeriensis Isbrücker, 1979a: 97, figs. 11-13. Type locality: Surinam, district Nickerie, rapide dans la rivière Fallawatra, 5 km SSW des chutes Stondansie. Holotype: ZMA 107561.

Distribution: Nickerie and Marowijne River basins (Isbrücker, 1981).

Loricaria parnahybae Steindachner, 1907

Loricaria parnahybae Steindachner, 1907b: 153. Type locality: dem Rio Parnahyba an der Mündung eines Baches bei Victoria [Brazil]. Lectotype: NMW 44854, designated by Isbrücker (1979a: 110).

Loricaria piauhiae Fowler, 1941a: 163, figs. 72-74. Type locality: Rio Parnahyba, Therezina, Piauhy [Brazil]. Holotype: ANSP 69452; holotype illustrated in Isbrücker (1981: fig. 7).

Distribution: Coastal rivers of NE Brazil and French Guiana (Isbrücker, 1981).

Loricaria piracicabae Ihering, 1907

Loricaria piracicabae Ihering, 1907: 28. Type locality: Piracicaba, Piracicaba River, State of S. Paulo [Brazil]. Holotype: MZUSP 2182.

Distribution: Piracicaba River basin, Brazil (Isbrücker, 1981).

Loricaria simillima Regan, 1904

Loricaria simillima Regan, 1904b: 292, pl. 17 (fig. 2). Type locality: Canelos, e. Ecuador. Lectotype: BMNH 1880.12.8.77, designated by Isbrücker (1979a: 110).

Distribution: Amazon, Orinoco and La Plata River basins (Isbrücker, 1981).

Loricaria tucumanensis Isbrücker, 1979

Loricaria tucumanensis Isbrücker, 1979a: 98, fig. 14. Type locality: Argentine, Prov. Tucuman, San Miguel de Tucuman, 26 $46^{\circ} \mathrm{S}, 6^{\circ} 15^{\prime} \mathrm{W}$ (Rio Sali ?). Holotype: USNM 88669.

Distribution: Dulce River basin, Argentina (Isbrücker, 1981).

\section{LORICARIICHTHYS Bleeker, 1862}

Plecostomus Swainson, 1839: 304. Type species: Loricaria maculata Bloch, 1794. Type by monotypy. Gender: Masculine. Originally as a subgenus of Loricaria. Predates Loricariichthys Bleeker, but not treated as valid since before 1899 and, therefore, permanently suppressed.

Loricariichthys Bleeker, 1862 (in Bleeker, 1862-63): 3. Type species: Loricaria maculata Bloch, 1794. Type by original designation. Gender: Masculine.

Parahemiodon Bleeker, 1862: 373. Type species: Parahemiodon typus Bleeker, 1862. Type by original designation. Gender: Masculine.

Loricariichthys acutus (Valenciennes, 1840)

Loricaria acuta Valenciennes, in Cuvier \& Valenciennes, 1840b: 472 (349 of Strasbourg deluxe edition), pl. 452. Type locality: On peut croire qu'it venait du Brésil. Holotype: at MNHN (but apparently lost).

Distribution: Brazil (?).

Loricariichthys anus (Valenciennes, 1836)

Loricaria anus Valenciennes, 1836, in Valenciennes, 1835-47: pl. 6 (fig. 1). Type locality: [Not stated]. Holotype: MNHN a-9456 or MNHN a-9455. Name made available from illustration of single specimen, which must be considered the holotype if it can be recognized. Described in Cuvier \& Valenciennes (1840b: 470 [347 of Strasbourg deluxe edition]).

Distribution: Coastal rivers of southern Brazil, Uruguay and lower Paraná River basins (Ferraris, 2003c).

Remarks: Redescribed in Cuvier \& Valenciennes (1840: 470 (347 in Strasbourg deluxe edition) from two specimens, with locality stated as: sur les bordes de la rivière de La Plata près de Buénos-Ayres. Lectotype designation by Isbrücker (1979a: 113) of MNHN a-9456 (396 mm) would be valid, if illustrated specimen can not be identified.

Loricariichthys brunneus (Hancock, 1828)

Loricaria brunnea Hancock, 1828: 247. Type locality: Venezuela, the branches and lakes of the Orinoko [sic]. 
Type(s): Whereabouts unknown (Isbrücker, 1979a: 113).

Distribution: Orinoco River basin (Ferraris, 2003c).

Loricariichthys cashibo (Eigenmann \& Allen, 1942)

Loricaria cashibo Eigenmann \& Allen, 1942: 205. Type locality: Lago Cashiboya [Peru]. Holotype: IU 15416 (131 mm FL; whereabouts unknown, not found by Isbrücker \& Nijssen, 1976b: 110).

Distribution: Upper Amazon River basin, Peru (Ferraris, 2003c).

Loricariichthys castaneus (Castelnau, 1855)

Loricaria castanea Castelnau, 1855: 46, pl. 23 (fig. 4). Type locality: donné a Rio de Janeiro, comme venant des rivières du voisinage [Brazil]. Holotype: MNHN a-9561.

Loricaria Spixii Steindachner, 1881b: 97. Type locality: Rio Parahyba, Santa Cruz, Rio Quenda, Rio Muriahe, Sao Matheos, Sambaia, Rio Grande do Sul [restricted to Rio Quenda by lectotype designation] . Lectotype: NMW 45113, designated by Isbrücker (1979a: 113). Illustrated and described in more detail in Steindachner (1881d: 4, pl. 2).

Distribution: Coastal streams of SE Brazil between southern São Paulo and northern Espírito Santo, including the Paraíba do Sul River basin, Brazil (Ferraris, 2003c).

Loricariichthys chanjoo (Fowler, 1940)

Parahemiodon chanjoo Fowler, 1940a: 240, figs. 30-32. Type locality: Ucayali River basin, Contamana, Peru. Holotype: ANSP 68656.

Distribution: Ucayali River basin, Peru (Ferraris, 2003c).

Loricariichthys derbyi Fowler, 1915

Loricariichthys derbyi Fowler, 1915b: 267, fig. 4. Type locality: Rio Jaguaribe at Barro Alto, Brazil. Holotype: ANSP 39932.

Distribution: Jaguaribe River basin, Brazil (Ferraris, 2003c).

Loricariichthys edentatus Reis \& Pereira, 2000

Loricariichthys edentatus Reis \& Pereira, 2000: 1041, fig. 8. Type locality: rio Uruguay at brazo Itape, south of Isla Canario, Concepción del Uruguay, Argentina (32²9'S, 58¹4'W). Holotype: MACN 4041.

Distribution: Lower Uruguay River basin, Argentina (Reis \& Pereira, 2000).

Loricariichthys hauxwelli Fowler, 1915

Loricariichthys hauxwelli Fowler, 1915a: 238, fig. 10. Type locality: Ambyiacu River, Ecuador. Holotype: ANSP 8301.

Distribution: Ampyiacu River basin, Peru (Ferraris, 2003c).

Loricariichthys labialis (Boulenger, 1895)

Loricaria labialis Boulenger, 1895b: 527. Type locality: Paraguay. Lectotype: BMNH 1895.5.17.98, designated by Isbrücker (1979a: 113); one syntype illustrated in Boulenger (1896c: pl. 7, fig. 1).

Distribution: Paraguay and middle Paraná River basin (Ferraris, 2003c).

Remarks: Reviewed in Ringuelet et al. (1967), diagnosed in Britski et al. (1999).

Loricariichthys maculatus (Bloch, 1794)

Loricaria maculata Bloch, 1794: 73, pl. 375 (figs. 1-2). Type locality: [No locality stated]. Lectotype: ZMB 3163, designated by, and illustrated in, Isbrücker (1971a: 12, figs. 3-4), with the locality stated as Surinam .... District Suriname, ditches at Cultuurtuin at Paramaribo West.

Loricaria accipenser Shaw, 1804: 36. Type locality: Indian seas, in error [South America]. Types: On Bloch (1794: pl. 375, fig. 1), Gronovius (1763: 127, no. 391), and Loricaria cataphracta Linnaeus.

Plecostomus cataphractus Gronow in Gray, 1854: 158. Type locality: [No locality stated]. Neotype: ZMB 3163, designated by, and illustrated in, Isbrücker (1971a: 15, figs. 3-4).

Loricaria amazonica Castelnau, 1855: 46, pl. 23 (fig. 2). Type locality: [Not stated]. Holotype: MNHN a-9560.

? Parahemiodon typus Bleeker, 1862a: 373. Type locality: Surinama. Holotype: RMNH 3121; holotype illustrated in Bleeker (1864a: 20, pl. 6, fig. 1; pl. 13, fig. 1).

? Loricaria parahemiodon Günther, 1864: 258. Type locality: Surinama. Holotype: RMNH 3121. Unneeded new name for Parahemiodon typus Bleeker.

Distribution: Rivers of Suriname (Ferraris, 2003c). 
Remarks: Redescribed in Isbrücker (1971a: 12).

Loricariichthys melanocheilus Reis \& Pereira, 2000

Loricariichthys melanocheilus Reis \& Pereira, 2000: 1042, fig. 9. Type locality: mouth of rio Ijuí-Mirim, tributary of rio Ijuí, Pirapó, Rio Grande do Sul, Brazil (rio Uruguay drainage, 2803'S, 55²11'W). Holotype: MCP 16961.

Distribution: Lower Paraná and Uruguay River basins, Brazil (Reis \& Pereira, 2000).

Loricariichthys microdon (Eigenmann, 1909)

Loricaria microdon Eigenmann, 1909a: 7. Type locality: Rupununi, British Guiana. Holotype: FMNH 53555; holotype illustrated in Eigenmann (1912b: pl. 30, fig. 1; pl. 32, fig. 1).

Distribution: Rupununi River basin, Guyana (Ferraris, 2003c).

Loricariichthys nudirostris (Kner, 1853)

Loricaria nudirostris Kner, 1853a: 12, 22, pl. 4. Type locality: Barra do Rio negro [Manaus, Brazil]. Lectotype: NMW 45092: 1, designated by Isbrücker (1979a: 113). Originally as Lor. nudirostris.

Distribution: Amazon River basin, Brazil (Ferraris, 2003c).

Loricariichthys platymetopon Isbrücker \& Nijssen, 1979

Loricariichthys platymetopon Isbrücker \& Nijssen, 1979: 203, fig. 7. Type locality: Paraguay, Lago Ypacarai, San Bernardino, 25ำ'S, 57²16'W, Río Paraguay system. Holotype: USNM 181580.

Distribution: La Plata River basin (Ferraris, 2003c).

Loricariichthys rostratus Reis \& Pereira, 2000

Loricariichthys rostratus Reis \& Pereira, 2000: 1044, fig. 10. Type locality: rio São Francisco Falso, limit between Diamante do Oeste and Santa Helena, Paraná, Brazil (upper rio Paraná drainage, $24^{\circ} 54^{\prime} \mathrm{S}, 5^{\circ} 12^{\prime} \mathrm{W}$ ). Holotype: MCP 16962.

Distribution: Paraná River basin, above Paraguay River mouth (Reis \& Pereira, 2000).

Loricariichthys stuebelii (Steindachner, 1882)

Loricaria Stübelii Steindachner, 1882b: 175. Type locality: Aus dem Huallaga [Peru]. Lectotype: NMW 45110, designated by Isbrücker (1979a: 113). Illustrated and described in more detail in Steindachner (1882c: 7, pl. 3, figs. $2,2 \mathrm{a}, 2 \mathrm{~b})$.

Distribution: Huallaga River basin, Upper Amazon drainage, Peru (Ferraris, 2003c).

Loricariichthys ucayalensis Regan, 1913

Loricariichthys ucayalensis Regan, 1913c: 282. Type locality: River Ucayali, Peru. Lectotype: BMNH 1913.7.30.36, designated by Isbrücker \& Nijssen (1979: 208).

Distribution: Ucayali River basin, Upper Amazon drainage, Peru (Ferraris, 2003c).

MACROTOCINCLUS Isbrücker \& Seidel, 2001

Macrotocinclus Isbrücker \& Seidel, in Isbrücker et al., 2001: 20. Type species: Otocinclus affinis Steindachner, 1877. Type by original designation. Gender: Masculine.

Remarks: This genus corresponds to the "affinis" clade of Otocinclus as diagnosed in Schaefer (1997) and is the sister group to Otocinclus sensu stricto.

Macrotocinclus affinis (Steindachner, 1877)

Otocinclus affinis Steindachner, 1877c: 221, pl. 1 (figs. 1, 1a, 1b). Type locality: Flüssen bei S. Crux in der umgebung von Rio de Janeiro, Brazil. Lectotype: NMW 45368:1, designated by, and illustrated in [but labeled as NMW 45368:2], Schaefer (1997: 49).

Distribution: Vicinity of Rio de Janeiro, Brazil (Schaefer, 2003c).

Macrotocinclus flexilis (Cope, 1894)

Otocinclus fimbriatus Cope, 1894: 98, pl. 9 (figs. 16, 16a-b). Type locality: Rio Jacuhy [Jacuí], Rio Grande do Sul, Brazil. Syntypes: ANSP 21752-55 (4), ANSP 21585-97 (14); syntype ANSP 21754 illustrated in Schaefer (1997: fig. 34a).

Otocinclus flexilis Cope, 1894: 97, pl. 8 (figs. 13, 13a-b). Type locality: Rio Jacuhy [Jacuí], Rio Grande do Sul, Brazil. Lectotype: ANSP 21756, designated by, and illustrated in, Schaefer (1997: 53, fig. 33).

Otocinclus arnoldi Regan, 1909: 234. Type locality: Rio de la Plata, South America. Holotype: BMNH 1908.12.5.13; holotype ilustrated in Schaefer (1997: fig. 34b). 
Distribution: Middle and lower Paraná/Paraguay, Uruguay and La Plata basins and Atlantic coastal streams of Southeastern Brazil (Schaefer, 2003c).

Remarks: Otocinclus flexilis treated as valid by first reviser action of Eigenmann (1910: 412). Redescribed in Aquino (1996).

MEGALANCISTRUS Isbrücker, 1980

Megalancistrus Isbrücker, 1980: 52. Type species: Chaetostomus gigas Boulenger, 1895. Type by original designation. Gender: Masculine.

Megalancistrus barrae (Steindachner, 1910)

Ancistrus barrae Steindachner, 1910a: 58. Type locality: Rio San Francisco bei Barra [Brazil]. Syntypes: NMW 48019 (1), NMW 48020 (1).

Distribution: São Francisco River basin, Brazil (Fisch-Muller, 2003).

Megalancistrus parananus (Peters, 1881)

Pterygoplichthys (Ancistrus) parananus Peters, 1881a: 17. Type locality: Parana fluss bei La Paz in Entre-Rios [Argentina]. Holotype: ZMB 11328.

Chaetostomus aculeatus Perugia, 1891: 637. Type locality: Asuncion (Rio Paraguay) [Paraguay]. Holotype: MSNG 8937.

Chaetostomus gigas Boulenger, 1895b: 526. Type locality: Paraguay. Holotype: BMNH 1895.5.17.72; holotype illustrated in Boulenger (1896c: pl. 6).

Distribution: Paraguay, Paraná, and Uruguay River basins (Fisch-Muller, 2003).

Remarks: Redescribed as Pterygoplichthys aculeatus in Pignalberi et al. (1970: 379).

METALORICARIA Isbrücker, 1975

Metaloricaria Isbrücker, 1975b: 2. Type species: Metaloricaria paucidens Isbrücker, 1975. Type by original designation. Gender: Feminine.

Review: Isbrücker \& Nijssen (1982).

Metaloricaria nijsseni (Boeseman, 1976)

Harttia nijsseni Boeseman, 1976: 170, pl. 8. Type locality: Sipaliwini River, southwestern Surinam. Holotype: RMNH 27498.

Distribution: Suriname, Saramacca, Nickerie, and Corantijn River basins, Suriname (Isbrücker \& Nijssen, 1982).

Metaloricaria paucidens Isbrücker, 1975

Metaloricaria paucidens Isbrücker, 1975b: 2, pls 1-3. Type locality: French Guiana, creek at right bank of Ouaqui River, upstream of Saut Bali, Maroni River system. Holotype: IRSNB 549.

Distribution: Oyapock, Sinnamary and Marowijne River basins (le Bail et al., 2000).

Remarks: Species redescribed by le Bail et al. (2000).

MICROLEPIDOGASTER Eigenmann \& Eigenmann, 1889

Microlepidogaster Eigenmann \& Eigenmann, 1889a: 8. Type species: Microlepidogaster perforatus Eigenmann \& Eigenmann, 1889. Type by original designation. Gender: Feminine.

Remarks: Type species written as Microlepidogaster perforated in generic account, but as M. perforatus in species account.

Microlepidogaster perforatus Eigenmann \& Eigenmann, 1889

Microlepidogaster perforatus Eigenmann \& Eigenmann, 1889a: 9. Type locality: Rio Carandahy, Brazil. Holotype: MCZ 8181.

Distribution: Carandaí River in upper Paraná River basin, Brazil (Schaefer, 2003c).

NANNOPTOPOMA Schaefer, 1996

Nannoptopoma Schaefer, 1996a: 915. Type species: Otocinclus spectabilis Eigenmann, 1914. Type by original designation. Gender: Neuter.

Nannoptopoma spectabile (Eigenmann, 1914) 
Otocinclus spectabilis Eigenmann, 1914b: 229. Type locality: Quebrada Cramalote, 407'N, 73³6'W, Dpto. Meta, Villavicencio, Colombia. Holotype: CAS 33806.

Distribution: Amazon and Orinoco River basins, Colombia (Schaefer, 2003c).

Nannoptopoma sternoptychum Schaefer, 1996

Nannoptopoma sternoptychum Schaefer, 1996a: 920, fig. 4. Type locality: Quebrada Mariposa at Cusco-Amazonico Lodge, 12³6'S, 69¹1'W, Puerto Maldonado, Tambopata, Madre de Dios, Peru. Holotype: MUSM 4097.

Distribution: Lower Amazon River basin and tributaries below 200 m elevation (Schaefer, 2003c).

NEBLINICHTHYS Ferraris, Isbrücker \& Nijssen, 1986

Neblinichthys Ferraris, Isbrücker \& Nijssen, 1986: 70. Type species: Neblinichthys pilosus Ferraris, Isbrücker \& Nijssen, 1986. Type by original designation. Gender: Masculine.

Neblinichthys pilosus Ferraris, Isbrücker \& Nijssen, 1986

Neblinichthys pilosus Ferraris, Isbrücker \& Nijssen, 1986: 70, fig. 1 (top). Type locality: Venezuela, Territorio Federal Amazonas; Dept. Rio Negro, Rio Baria basin. Rio Mawarinuma tributary at Neblina base camp, on right bank in riffle, $0^{\circ} 55^{\prime} \mathrm{N}, 66^{\circ} 10^{\prime} \mathrm{W}$, elevation $120 \mathrm{~m}$. Holotype: AMNH 56137.

Distribution: Upper Baria River basin, Venezuela (Ferraris et al., 1986).

Neblinichthys yaravi (Steindachner, 1915)

Ancistrus (Hemiancistrus) yaravi Steindachner, 1915e: 87. Type locality: Rio Coquenan [=Kukenan, Caroni basin], Venezuela. Holotype: at NMW.

Neblinichthys roraima Provenzano, Lasso \& Ponte, 1995: 245, fig. 1. Type locality: Venezuela: Estado Bolivar: río Caroni system, first creek (quebrada) NW of base camp Roraima tepui, tributary of río Kukenan $\left(5^{\circ} 15^{\prime} \mathrm{N}\right.$, $\left.60^{\circ} 40^{\prime} \mathrm{W}\right)$, at an elevation of 1200-1400 m. Holotype: MHNLS 8753.

Distribution: Upper Caroni drainage, Venezuela (Fisch-Muller, 2003).

Remarks: Synonymy follows Armbruster (pers. commun., 2005).

NEOPLECOSTOMUS Eigenmann \& Eigenmann, 1888

Neoplecostomus Eigenmann \& Eigenmann, 1888b: 171. Type species: Plecostomus microps Steindachner, 1877. Type by original designation. Gender: Masculine. Proposed originally as a subgenus of Plecostomus.

Neoplecostomus espiritosantensis Langeani, 1990

Neoplecostomus espiritosantensis Langeani, 1990: 16, fig. 3. Type locality: rio Jacu (braço sul), Vitor Hugo, Mun. de Domingos Martins, ES [Brazil]. Holotype: MZUSP 38573.

Distribution: South America: Jacu and São Lourenço River basins, eastern slope of Serra do Mar, Brazil (Langeani, 1990).

Neoplecostomus franciscoensis Langeani, 1990

Neoplecostomus franciscoensis Langeani, 1990: 22, fig. 5. Type locality: riacho afluente do córrego da Mutuca, à direita da estrada Belo Horizonte-Nova Lima, Km 20, MG, 2006'S,435' $\mathrm{W}$ [Brazil]. Holotype: MZUSP 38577 .

Distribution: Headwaters of Das Velhas and Paraopeba River basins, São Francisco River drainage, Brazil (Langeani, 1990).

Neoplecostomus granosus (Valenciennes, 1840)

Hypostomus granosus Valenciennes in Cuvier \& Valenciennes, 1840b: 502 (371 in Strasbourg deluxe edition). Type locality: Cayenne, ... Rio-Janéiro [both localities apparently in error (Langeani, 1990)]. Syntypes: MNHN a9566 (3), MNHN b-0597 (6), MNHN b-0598 (6).

Distribution: Southeastern Brazil (?).

Neoplecostomus microps (Steindachner, 1877)

Plecostomus microps Steindachner, 1877b: 688, pl. 13. Type locality: der Umgebung von Rio Janeiro [Brazil]. Lectotype: NMW 45337.1, designated by Langeani (1990: 8).

Distribution: Paraíba do Sul River basin, Brazil (Langeani, 1990).

Neoplecostomus paranensis Langeani, 1990

Neoplecostomus paranensis Langeani, 1990: 12, figs. 2, 6. Type locality: Rio Cubatão, Faz. Santa Carlota, Cajurú, 
SP [Brazil]. Holotype: MZUSP 38572.

Distribution: Upper Paraná River basin, Brazil (Langeani, 1990).

Neoplecostomus ribeirensis Langeani, 1990

Neoplecostomus ribeirensis Langeani, 1990: 19, fig. 4. Type locality: riacho afluente do rio Bananal, km 7 da rodovia Biguá-Iguape (SP-222), SP [Brazil]. Holotype: MZUSP 38576.

Distribution: Ribeira de Iguape River basin, Brazil (Langeani, 1990).

Neoplecostomus variipictus Bizerril, 1995

Neoplecostomus variipictus Bizerril, 1995b: 695, figs. 1-2. Type locality: rio Santo Antônio, tributário do rio Bengala, bacia do rio Paraíba do Sul, Firburgo, RJ [Brazil]. Holotype: MNRJ 12806.

Distribution: Paraíba do Sul River basin, Brazil (Bizerril, 1995b).

NIOBICHTHYS Schaefer \& Provenzano, 1998

Niobichthys Schaefer \& Provenzano, 1998: 222. Type species: Niobichthys ferrarisi Schaefer \& Provenzano, 1998. Type by original designation. Gender: Masculine.

Niobichthys ferrarisi Schaefer \& Provenzano, 1998

Niobichthys ferrarisi Schaefer \& Provenzano, 1998: 226, fig. 4. Type locality: Municipio Autónomo Río Negro, Río Baria, approximately $200 \mathrm{~m}$ above Neblina base camp, $0^{\circ} 55^{\prime} \mathrm{N}, 66^{\circ} 10^{\prime} \mathrm{W}$, Amazonas State, Venezuela. Holotype: MBUCV V-20225.

Distribution: Upper Baria River, Negro River basin, Venezuela (Schaefer, 2003c).

OLIGANCISTRUS Rapp Py-Daniel, 1989

Oligancistrus Rapp Py-Daniel, 1989: 246. Type species: Chaetostomus punctatissimus Steindachner, 1881. Type by original designation. Gender: Masculine.

Oligancistrus punctatissimus (Steindachner, 1881)

Chaetostomus punctatissimus Steindachner, 1881c: 119, pl. 3 (fig. 3). Type locality: aus dem Amazonen-Strome ohne nähere Angabe des Fundortes [Brazil]. Holotype: NMW 47206.

Distribution: Xingu River basin, Brazil (Fisch-Muller, 2003).

\section{OTOCINCLUS Cope, 1871}

Otocinclus Cope, 1871b: 112. Type species: Otocinclus vestitus Cope, 1872. Type by subsequent monotypy. Gender: Masculine. Genus originally proposed without species; species name first added in Cope (1872a).

Revision, phylogeny and biogeography: Schaefer (1997).

Otocinclus bororo Schaefer, 1997

Otocinclus bororo Schaefer, 1997: 69, fig. 43. Type locality: Barra do Bugres, stream crossing road between Barra do Bugres and Cáceres, $51 \mathrm{~km} \mathrm{~S}$ of Barra Do Bugres, ca. $15^{\circ} 20^{\prime} \mathrm{S}, 5^{\circ} 12^{\prime} \mathrm{W}$, Mato Grosso, Brazil. Holotype: MCP 19285.

Distribution: Upper Paraguay River basin, Brazil (Schaefer, 2003c).

Otocinclus caxarari Schaefer, 1997

Otocinclus caxarari Schaefer, 1997: 71, fig. 44. Type locality: Region between Guajara-Mirim and Mato Grosso, ca. $10^{\circ} 48^{\prime} \mathrm{S}, 65^{\circ} 23^{\prime} \mathrm{W}$, Rio Solimoes/Madeira/Guaporé drainage, Brazil/Bolivia border. Holotype: MZUSP 51102.

Distribution: Upper Madeira River basin, Brazil (Schaefer, 2003c).

Otocinclus cocama Reis, 2004

Otocinclus cocama Reis, 2004: 110, figs. $1-4$. Type locality: quebrada Yanayacu (approx. $04^{\circ} 55^{\prime} \mathrm{S}, 073^{\circ} 43^{\prime} \mathrm{W}$ ), tributary to the caño of the cocha Supay in Jenaro Herrera, Provincia Requena, Departamento Loreto, Peru. Holotype: MUSM 20686.

Distribution: Upper Amazon River basin, Peru (Reis, 2004).

Otocinclus hasemani Steindachner, 1915

Otocinclus hasemani Steindachner, 1915c: 348. Type locality: Engenhão de Agua, im flußgebiete des Paranahyba [Maranhão State, Brazil]. Lectotype: NMW 45369: 1, designated by, and illustrated in, Schaefer (1997: 62, fig. 38a). 
Distribution: Tocantins and Parnaíba River basins, Brazil (Schaefer, 2003c).

Otocinclus hoppei Miranda Ribeiro, 1939

Otocinclus hoppei Miranda Ribeiro, A., 1939: 359. Type locality: Belém, Pará, Brasil-Norte. Lectotype: MNRJ 1030, designated by Miranda Ribeiro (1953: 401); lectotype illustrated in Schaefer (1997: fig. 39a).

Distribution: Amazon River basin, Brazil (Schaefer, 2003c).

Otocinclus huaorani Schaefer, 1997

Otocinclus huaorani Schaefer, 1997: 74, fig. 46. Type locality: Trib. to Río San Miguel (km 50, 4.5 km S of Tipischca), ca. $0^{\circ} 10^{\prime} \mathrm{N}, 76^{\circ} 13^{\prime} \mathrm{W}$, Río Amazonas/Napo drainage, Napo, Ecuador. Holotype: FMNH 101555.

Distribution: Western upper Amazon and Orinoco River basins (Schaefer, 2003c).

Otocinclus macrospilus Eigenmann \& Allen, 1942

Otocinclus macrospilus Eigenmann \& Allen, 1942: 201, pl. 13 (fig. 1). Type locality: Rio Morona [Upper Amazon basin, Peru]. Holotype: CAS 77335; holotype illustrated in Schaefer (1997: fig. 42a).

Distribution: Amazon River basin, Peru (Schaefer, 2003c).

Otocinclus mariae Fowler, 1940

Otocinclus mariae Fowler, 1940c: 83, figs. 41-43. Type locality: Boca Chapare, Río Chimore [Río Mamoré system] Bolivia. Holotype: ANSP 69068; holotype illustrated in Schaefer (1997: fig. 41a).

Distribution: Upper Madeira and lower Amazon River basins, Bolivia (Schaefer, 2003c).

Otocinclus mimulus Axenrot \& Kullander, 2003

Otocinclus mimulus Axenrot \& Kullander, 2003: 251, fig. 1. Type locality: Paraguay: Departamento Caaguazú: río Paraná drainage, small stream (arroyo) at Estancia María Belén, $8 \mathrm{~km}$ from Colone Patricio Colman, 2540'13"S, 555'52"W. Holotype: MNHNP uncat (currently as NRM 43480).

Distribution: Monday River, Paraná River basin, Paraguay (Axenrot \& Kullander, 2003).

Otocinclus mura Schaefer, 1997

Otocinclus mura Schaefer, 1997: 77, fig. 47. Type locality: Rio Sanabani, munícìio de Silves (Lago Canaçari drainage into Rio Solimões mainstem), ca. $2^{\circ} 51^{\prime} 40^{\prime \prime S}, 58^{\circ} 15^{\prime} 40^{\prime \prime} \mathrm{W}$, rio Solimões/Sanabani/Lago Canaçari drainage, Brazil. Holotype: MZUSP 51101.

Distribution: Upper Amazon River basin, Brazil (Schaefer, 2003c).

Otocinclus tapirape Britto \& Moreira, 2002

Otocinclus tapirape Britto \& Moreira, 2002: 1064, fig. 1. Type locality: Brazil: Goiás, córrego Água Parada, tributary of Rio Pintado, $11 \mathrm{~km}$ west from Novo Planalto, rio Araguaia system, $13^{\circ} 7^{\prime} \mathrm{S}, 49^{\circ} 62^{\prime} \mathrm{W}$. Holotype: MZUSP 73975.

Distribution: Upper and middle Araguaia River basin, Brazil (Schaefer, 2003c).

Otocinclus vestitus Cope, 1872

Otocinclus vestitus Cope, 1872a: 283, pl. 4 (figs. 2a-c). Type locality: Tributaries of the Ambyiacu [which empties into the Amazon near to Pebas, in Eastern Equador, some distance east of the Napo]. Lectotype: ANSP 8283, designated by, and illustrated in, Schaefer (1997: 46, fig. 29).

Distribution: Amazon and lower [sic, upper] Paraná River basins (Schaefer, 2003c).

Otocinclus vittatus Regan, 1904

Otocinclus vittatus Regan, 1904b: 267, pl. 15 (fig. 3). Type locality: Descalvados, Mato Grosso, Brazil. Lectotype: BMNH 1895.5.17.83, designated by, and illustrated in, Schaefer (1997: 57, fig. 36a).

Rhinolepis paraguensis Borodin, 1927b: 7. Type locality: Paraguay. Holotype: AMNH 8641.

Distribution: Amazon, Orinoco, Paraná/Paraguay, Xingu and Tocantins River basins (Schaefer, 2003c).

Otocinclus xakriaba Schaefer, 1997

Otocinclus xakriaba Schaefer, 1997: 79, fig. 48. Type locality: Rio Pau de Colher, on road between Guanambi and Mutas, 14¹2'48"S, 4249'29"W, rio São Francisco drainage, Bahia, Brazil. Holotype: MZUSP 51103.

Distribution: São Francisco River basin, Brazil (Schaefer, 2003c).

OTOTHYRIS Myers, 1927

Otothyris Myers, 1927: 128. Type species: Otothyris canaliferus Myers, 1927. Type by original designation. Gender: Feminine. 
Revision: Garavello et al. (1998).

Otothyris juquiae Garavello, Britski \& Schaefer, 1998

Otothyris juquiae Garavello, Britski \& Schaefer, 1998: 8, fig. 4. Type locality: Juquiá creek at Cantagalo farm, São Paulo State, Brazil. Holotype: MZUSP 10309.

Distribution: Ribeira de Iguape River basin, Brazil (Schaefer, 2003c).

Otothyris lophophanes (Eigenmann \& Eigenmann, 1889)

Rhinelepis lophophanes Eigenmann \& Eigenmann, 1889a: 9. Type locality: Santa Cruz, Brazil. Holotype: MCZ 8164.

Otocinclus cephalacanthus Miranda Ribeiro, 1911: 93. Type locality: Brazil. Holotype: MNRJ 1050.

Otothyris canaliferus Myers, 1927: 128. Type locality: Hills in the vicinity of Rio de Janeiro, Brazil. Lectotype: MCZ 31577, designated in Garavello et al. (1998: 7).

Distribution: Atlantic coastal streams of Rio de Janeiro State, Brazil (Schaefer, 2003c).

Otothyris rostrata Garavello, Britski \& Schaefer, 1998

Otothyris rostrata Garavello, Britski \& Schaefer, 1998: 10, fig. 5. Type locality: Osório, Canal between lagoons Emboaba and Emboabinha, on the highway between Osório and Tramandai, Rio Grande do Sul State, Brazil. Holotype: MCP 14812.

Distribution: Atlantic coast of Santa Catarina and Rio Grande do Sul States, Brazil (Schaefer, 2003c).

Otothyris travassosi Garavello, Britski \& Schaefer, 1998

Otothyris travassosi Garavello, Britski \& Schaefer, 1998: 12, fig. 6. Type locality: Barra Sêca River at Linhares to São Mateus Road, Espírito Santo State, Brazil. Holotype: MNRJ 10177.

Distribution: Atlantic coast of southeastern Brazil (Schaefer, 2003c).

OTOTHYROPSIS Ribeiro, Carvalho \& Melo, 2005

Otothyropsis Ribeiro, Carvalho \& Melo, 2005: 491. Type species: Otothyropsis marapoama Ribeiro, Carvalho \& Melo, 2005, by original designation. Gender: Feminine.

Otothyropsis marapoama Ribeiro, Carvalho \& Melo, 2005

Otothyropsis marapoama Ribeiro, Carvalho \& Melo, 2005: 491, fig. 1. Type locality: Brazil, São Paulo, rio Tietê

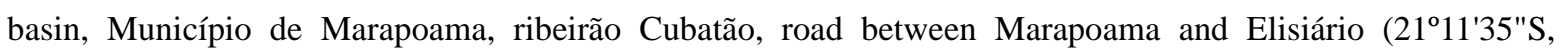
4907'22"W). Holotype: LIRP 5640.

Distribution: Tietê River, Paraná River basin, Brazil (Ribeiro et al., 2005).

OXYROPSIS Eigenmann \& Eigenmann, 1889

Oxyropsis Eigenmann \& Eigenmann, 1889b: 39. Type species: Oxyropsis wrightiana Eigenmann \& Eigenmann, 1889. Type by original designation. Gender: Feminine.

Revision: Aquino \& Schaefer (2002).

Oxyropsis acutirostris Miranda Ribeiro, 1951

Oxyropsis acutirostris Miranda Ribeiro, 1951a: 2, pl. 1 (fig. 2), pl. 2 (fig. 2). pl. 3 (fig. 2). Type locality: Paricachoeira, rio Taquié, afl. do Vaupes que é afl. do Negro, Amazonas [Brazil]. Holotype: MNRJ 5745.

Distribution: Orinoco and Negro River basins (Schaefer, 2003c).

Oxyropsis carinata (Steindachner, 1879)

Hypoptopoma carinatum Steindachner, 1879e: 48, pl. 4 (fig. 3). Type locality: Einem nebenflusse des Amazonenstromes an der Peruanische Grenze. Holotype: NMW 46267; holotype illustrated in Aquino \& Schaefer (2002: fig. 6).

Distribution: Middle and upper Amazon River basin (Schaefer, 2003c).

Oxyropsis wrightiana Eigenmann \& Eigenmann, 1889

Oxyropsis wrightiana Eigenmann \& Eigenmann, 1889b: 39. Type locality: Lago Hyanuary [Brazil]. Holotype: MCZ 8055; holotype illustrated in Aquino \& Schaefer (2002: fig. 4).

Oxyropsis wrightii Eigenmann \& Eigenmann, 1891: 40. Type locality: Lago Hyanuary. Holotype: MCZ 8055. Unjustified emendation of Oxyropsis wrightiana.

Distribution: Middle and upper Amazon River basin (Schaefer, 2003c). 
PANAQOLUS Isbrücker \& Schraml, 2001

Panaqolus Isbrücker \& Schraml, in Isbrücker et al., 2001: 20. Type species: Panaque gnomus Schaefer \& Stewart, 1993. Type by original designation. Gender: Masculine.

Revision: Schaefer \& Stewart (1993) as Panaque dentex species group.

Species key: Chockley \& Armbruster (2002), as Panaque dentex species group.

Remarks: This genus corresponds to the monophyletic assembledge called the Panaque dentex species group by Schaefer \& Stewart (1993). The recognition of this group as a separate genus was rejected by Chockley \& Armbruster (2002) and Weber (2003).

Panaqolus albomaculatus (Kanazawa, 1958)

Panaque albomaculatus Kanazawa, 1958: 327, fig. 1 (top). Type locality: Ecuador, tributary of the Rio Suno, upper Napo River, $0^{\circ} 47^{\prime}$ south latitude, $77^{\circ} 16^{\prime}$ west longitude, Rio Pucuno. Holotype: USNM 167909.

Distribution: Headwaters of Napo, Marañon and Ucayali rivers, Ecuador, Peru (Fisch-Muller, 2003).

Remarks: Redescribed by Schaefer \& Stewart (1993: 327, fig. 13).

Panaqolus changae (Chockley \& Armbruster, 2002)

Panaque changae Chockley \& Armbruster, 2002: 83, fig. 1 (middle). Type locality: Peru: Loreto: río Itaya, río Ama-

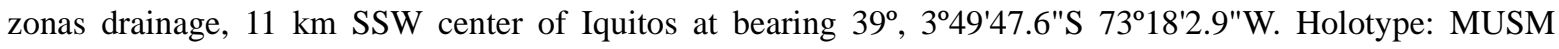
17107.

Distribution: Itaya and Momon River basins, Peru (Fisch-Muller, 2003).

Panaqolus dentex (Günther, 1868)

Chaetostomus dentex Günther, 1868a: 477. Type locality: Xeberos. Holotype: BMNH 1867.6.13.37. Described in more detail in Günther (1868b: 233).

Distribution: Napo, Pastaza and Marañon River basins (Fisch-Muller, 2003).

Remarks: Redescribed by Schaefer \& Stewart (1993: 321, fig. 9), with type locality given as: Peru, Departamento Loreto, Xeberos (or Jeberos), upper Río Aipena system, tributary to Río Huallaga near its confluence with Río Marañon, approx. $5^{\circ} 18^{\prime} \mathrm{S} 76^{\circ} 17^{\prime} \mathrm{W}$.

Panaqolus gnomus (Schaefer \& Stewart, 1993)

Panaque gnomus Schaefer \& Stewart, 1993: 333, fig. 17 (top). Type locality: Ecuador, Morona-Santiago Province,

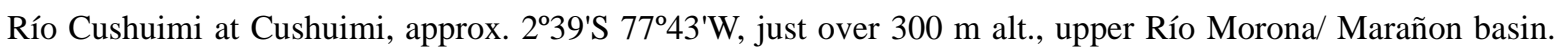
Holotype: FMNH 70860.

Distribution: Pastaza and upper Marañon basins (Fisch-Muller, 2003).

Panaqolus maccus (Schaefer \& Stewart, 1993)

Panaque maccus Schaefer \& Stewart, 1993: 335, figs. 18, 19. Type locality: Venezuela, Estado Portuguesa, Río Las Marinas, upstream from bridge on Route 5 east of Guanare, tributary of Río Portuguesa, Río Orinoco basin, approx. $9^{\circ} 05^{\prime} \mathrm{N} 69^{\circ} 40^{\prime} \mathrm{W}$. Holotype: MBUCV V-24010.

Distribution: Apuré and Caroni River basins, Venezuela (Fisch-Muller, 2003).

Panaqolus nocturnus (Schaefer \& Stewart, 1993)

Panaque nocturnus Schaefer \& Stewart, 1993: 330, fig. 15. Type locality: Peru, Departamento Amazonas, Río San-

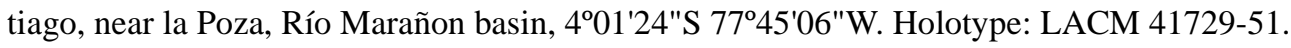

Distribution: Santiago and Pastaza River basins, upper Napo drainage (Fisch-Muller, 2003).

Panaqolus purusiensis (La Monte, 1935)

Panaque purusiensis La Monte, 1935: 4, fig. 3. Type locality: Rio Purus, Brazil; in the vicinity of the mouth of Rio Macauhan [=Macauá], a tributary of Rio Yaco [=Iaco], which, in turn, is a tributary of Rio Purus $\left(69^{\circ} \mathrm{W}, 9^{\circ} 20^{\prime} \mathrm{S}\right)$. Holotype: AMNH 12600; holotype illustrated in Schaefer \& Stewart (1991: fig. 12).

Distribution: Macauá River basin, upper Purus drainage, Brazil (Fisch-Muller, 2003).

PANAQUE Eigenmann \& Eigenmann, 1889

Panaque Eigenmann \& Eigenmann, 1889b: 44. Type species: Chaetostomus nigrolineatus Peters, 1877. Type by original designation. Gender: Masculine.

Panaque cochliodon (Steindachner, 1879) 
Chaetostomus cochliodon Steindachner, 1879c: 194. Type locality: Aus dem Cauca, dem grössten Nebenflusse des Magdalenen-Stromes [Colombia]. Syntypes: NMW 47297 (1), NMW 47298 (1). Also described as new in Steindachner (1879g: 187); described in more detail and illustrated in Steindachner (1880b: 63, pl. 4).

Chaetostomus gibbosus Steindachner, 1880b: 63, pl. 4. Type locality: Aus dem Cauca, dem grössten Nebenflusse des Magdalenen-Stromes [Colombia]. Syntypes: NMW 47297 (1), NMW 47298 (1). Alternate name for Chaetostomus cochliodon proposed as Chaetostomus cochliodon (sive gibbosus).

Distribution: Cauca and Magdalena River basins, Colombia (Fisch-Muller, 2003).

Panaque nigrolineatus (Peters, 1877)

Chaetostomus nigrolineatus Peters, 1877: 471, fig. on an unnumb. pl. ff. p. 556. Type locality: Calabozo [Guaricó River], Venezuela. Syntypes: ZMB 10046 (1), ZMB 10047 (1).

Distribution: Orinoco River and middle and lower Amazon River tributaries (Fisch-Muller, 2003).

Panaque suttonorum Schultz, 1944

Panaque suttoni Schultz, 1944c: 308, pl. 10 (fig. b). Type locality: Río Negro below the mouth of the Río Yasa, Maracaibo basin, Venezuela. Holotype: USNM 121033.

Distribution: Western and eastern tributaries of Maracaibo Lake, Venezuela (Fisch-Muller, 2003).

Remarks: Schultz (1944c: 311) indicated that the species was to be named in honor of "Dr. and Mrs. Fredrick A. Sutton," which prompted Isbrucker (2001: 30) to correct the name to suttonorum.

PARALORICARIA Isbrücker, 1979

Paraloricaria Isbrücker, 1979a: 87, 90. Type species: Loricaria vetula Valenciennes, 1836. Type by original designation. Gender: Feminine.

Paraloricaria agastor Isbrücker, 1979

Paraloricaria agastor Isbrücker, 1979a: 103, figs. 20 (right) -21 (right). Type locality: Paraguay (rio ?) Paraguay. Holotype: BMNH 1895.5.17.108.

Distribution: Paraguay River basin, Paraguay (Ferraris, 2003c).

Paraloricaria commersonoides (Devincenzi, 1943)

Loricaria commersonoides Devincenzi, 1943: 3, fig. 2. Type locality: río Uruguay, frente a la ciudad de Paysandú [Uruguay]. Holotype: at MHNM (apparently lost, Olazarri et al., 1970).

Distribution: Uruguay River basin (Ferraris, 2003c).

Remarks: Redescribed in Ringuelet et al. (1967).

Paraloricaria vetula (Valenciennes, 1836)

Loricaria vetula Valenciennes, 1836, in Valenciennes, 1835-47: pl. 6 (fig. 2). Type locality: [Not stated]. Holotype: MNHN a-8996. Described in Cuvier \& Valenciennes (1840b: 466, 344 in Strasbourg deluxe edition) with locality stated as: des environs de Buénos-Ayres.

Distribution: La Plata River basin (Ferraris, 2003c).

Remarks: Reviewed in Ringuelet et al. (1967).

\section{PARANCISTRUS Bleeker, 1862}

Parancistrus Bleeker, 1862 (in Bleeker, 1862-63): 2. Type species: Hypostomus aurantiacus Castelnau, 1855. Type by original designation. Gender: Masculine.

Acanthodemus Marschall, 1873: 63. Type species: Hypostomus aurantiacus Castelnau, 1855. Type by subsequent designation by Jordan (1919a: 264). Gender: Masculine.

Monophyly: Rapp Py-Daniel (1989).

Parancistrus aurantiacus (Castelnau, 1855)

Hypostomus aurantiacus Castelnau, 1855: 43, pl. 21 (fig. 2). Type locality: l'Ucayale. Holotype: MNHN a-9452 (dry). Name spelled Hypostomus aurentiacus on figure caption. Eschmeyer, et al. (1998: 165) acted as first reviser in selecting the spelling $H$. aurantiacus over $H$. aurentiacus.

Hypostomus nigricans Castelnau, 1855: 44, pl. 22 (fig. 1). Type locality: l'Amazone. Holotype: MNHN a-9576. Hypostomus vicinus Castelnau, 1855: 45, pl. 23 (fig. 1). Type locality: l'Ucayale. Holotype: MNHN a-9572. Distribution: Ucayali, Tocantins and Xingu Rivers (Fisch-Muller, 2003). 
Remarks: Redescribed by Rapp Py-Daniel (1989: 239), in which the three names are placed together in synonymy for the first time. Therefore, Rapp Py-Daniel acted as first reviser in selecting H. aurantiacus as valid.

Parancistrus nudiventris Rapp Py-Daniel \& Zuanon, 2005

Parancistrus nudiventris Rapp Py-Daniel \& Zuanon, 2005: 573, fig. 1. Type locality: Brazil; Pará State; rio Xingu, bedrock at ilha do Bacabal, 323'19" S, 51²3'24" W. Holotype: INPA 15037.

Distribution: Xingu River, Brazil (Rapp Py-Daniel \& Zuanon, 2005).

PAREIORHAPHIS Miranda Ribeiro, 1918

Pareiorhaphis Miranda Ribeiro, 1918a: 106. Type species: Hemipsilichthys calmoni [= Psilichthys cameroni Steindachner, 1907]. Type by subsequent designation by Regan (1920b: 14). Gender: Feminine. Type designation made with the unjustified emended name of Hemipsilichthys calmoni, and, erroneously, with Ribeiro as author. Subsequent type designation by Gosline (1947: 102) of Hemipsilichthys duseni is invalid.

Revision and key to species: Pereira \& Reis (2002) as Hemipsilichthys, in part.

Pareiorhaphis azygolechis (Pereira \& Reis, 2002)

Hemipsilichthys azygolechis Pereira \& Reis, 2002: 129, fig. 20 (middle). Type locality: Brazil: Paraná: Guaratuba; rio Araraquara ca. $8 \mathrm{~km}$ N of Guaruva (255'58"S 4849'43"W). Holotype: MCP 19670.

Distribution: São João River basin, Brazil (Weber, 2003).

Pareiorhaphis bahianus (Gosline, 1947)

Corymbophanes bahianus Gosline, 1947: 107, pl. 3 (fig. 6). Type locality: Fazenda Almada, Ilheus, Bahia [Brazil]. Holotype: MNRJ 4243.

Distribution: Small drainages near Ilhéus, between Pado and Contas River basins, Brazil (Weber, 2003).

Pareiorhaphis cameroni (Steindachner, 1907)

Psilichthys cameroni Steindachner, 1907a: 82. Type locality: aus dem Fluss Cubataõ im Staate Santa Catharina, Brazilien. Lectotype: ZSM 4837, designated by Pereira \& Reis (2002: 104, 106). Illustrated and described in more detail in Steindachner (1907e: 475, pls. 1, 2) as Hemipsilichthys cameroni.

Hemipsilichthys calmoni Steindachner, 1908a: 31. Emendation of Psilichthys cameroni [see Remarks].

Distribution: Cubatão (southern), Tubarão and Araranguá River basins, Brazil (Weber, 2003).

Remarks: Steindachner (1908a: 31) proposed to correct the name cameroni to calmoni in order to reflect the correct spelling of the patronym which was stated in Steindachner (1907c: 418) to honor "Dr. Miguel Calmon." However, the spelling cameroni appears in the minutes of the meeting, which constitutes the original proposal of the name (Steindachner, 1907a) as well as the invariant spelling in the text and plate captions of the subsequent description (Steindachner, 1907e). Despite this, Pereira \& Reis (2002: 104, 106), and Weber (2003: 353) treat cameroni as an incorrect spelling. Species redescribed, as Hemipsilichthys calmoni, in Steindachner (1910d: 430).

Pareiorhaphis cerosus (Miranda Ribeiro, 1951)

Hemipsilichthys cerosus A. Miranda Ribeiro, in P. Miranda Ribeiro, 1951c: xxxix. Type locality: Not explicitly given. Lectotype: MNRJ 648. Lectotype illustrated in, and designated, by Pereira \& Reis (2002: 115, fig. 11). Earlier lectotype proposal in Miranda Ribeiro (1953: 400) considered to be invalid by Pereira \& Reis (2002: 117) and Weber (2003).

Distribution: Eastern Brazilian coastal basin; known only from two type specimens (Weber, 2003).

Pareiorhaphis eurycephalus (Pereira \& Reis, 2002)

Hemipsilichthys eurycephalus Pereira \& Reis, 2002: 131, fig. 21 (middle). Type locality: Brazil: Santa Catarina: Urubici: creek tributary of Rio Canoas East of vila São José, on road to serra do Corvo Branco (2803'33"S 49²5'42"W). Holotype: MCP 19778.

Distribution: Upper Canoas River basin, Brazil (Weber, 2003).

Pareiorhaphis garbei (Ihering, 1911)

Hemipsilichthys garbei Ihering, 1911: 399. Type locality: no curso superior, montanha acima, do Rio Macahé, no Est. do Rio de Janeiro [...] im Staat Rio de Janiero, im Gebirgeflusse Macahé (ca. 10-15m. Breite, flach und Steinig) im Oberlaufe, d.h. am Gebirgsrande [Brazil]. Lectotype: MZUSP 1163, designated by, and illustrated in, Oliveira (1997: 117, fig. 1). 
Distribution: Macaé River basin, Brazil (Weber, 2003).

Remarks: Redescribed in Oliveira (1997).

Pareiorhaphis hypselurus (Pereira \& Reis, 2002)

Hemipsilichthys hypselurus Pereira \& Reis, 2002: 136, fig. 23 (middle). Type locality: Brazil: Rio Grande do Sul: Maquiné; rio Forqueta tributary to rio Maquiné, 29³2'42"S 50¹4'21"W. Holotype MCP 19665.

Distribution: Maquiné, Três Forquillas, Mampituba and Araranguá River basins, Brazil (Weber, 2003).

Pareiorhaphis hystrix (Pereira \& Reis, 2002)

Hemipsilichthys hystrix Pereira \& Reis, 2002: 134, fig. 22 (middle). Type locality: Brazil: Rio Grande do Sul: Bom Jesus; creek tributary of rio dos Touros, on road from Silveira to Rondinha, 28³9'18"S 50¹8'25"W. Holotype MCP 19779.

Distribution: Headwaters of Pelotas, Ijui and Piratinim River basins; upper Taquari River basin, Brazil (Weber, 2003).

Pareiorhaphis mutuca (Oliveira \& Oyakawa, 1999)

Hemipsilichthys mutuca Oliveira \& Oyakawa, 1999: 75, fig. 1 (top). Type locality: Brazil: Minas Gerais: Rio das Velhas drainage, County of Nova Lima, Córrego Mutuca, at right side of the road from Belo Horizonte to Nova Lima, km 20 (approx. 2060'S, 4355'W). Holotype: MZUSP 37148.

Distribution: Headwaters of Das Velhas River basin, Brazil (Weber, 2003).

Pareiorhaphis nudulus (Reis \& Pereira, 1999)

Hemipsilichthys nudulus Reis \& Pereira, 1999: 46, fig. 2 (top). Type locality: Rio Jordão, at Jordão Alto (approx. $28^{\circ} 35^{\prime}$ S, 49²7'W), Nova Veneza, Santa Catarina, Brazil. Holotype: MCP 20278.

Distribution: Araranguá, Mapituba, Três Forquilhas, and Maquiné River basins, Brazil (Weber, 2003).

Pareiorhaphis parmula Pereira, 2005

Pareiorhaphis parmula Pereira, 2005: 272, fig. 1. Type locality: Brazil: Paraná: Lapa: rio Iguaçu basin, rio dos Patos, tributary to rio da Várzea, on road PR-427 from Lapa to Campo do Tenente, 2550'36.8"S 04943'39.2"W. Holotype: MCP 35826.

Distribution: Dos Patos River, tributary of Iguaçu River, Parana River basin, Brazil (Pereira, 2005).

Pareiorhaphis regani (Giltay, 1936)

Hemipsilichthys regani Giltay, 1936: 6, fig. 2. Type locality: Rio Curi Curiay, au S. du Rio Negro (Amazone, Brésil). Holotype: IRSNB 47.

Distribution: Curicuriari and possibly Jaú River basins in the Negro River drainage, Brazil (Weber, 2003).

Pareiorhaphis splendens (Bizerril, 1995)

Hemipsilichthys splendens Bizerril, 1995a: 118, fig. 2. Type locality: Brazil: State of Santa Catarina, river Cubatão: near to the city of Joinville. Holotype: MNRJ 13325.

Distribution: Cubatão, Itajaí-Açu, Cubatão, Nhundiaquara and São João River basins, Brazil (Weber, 2003).

Pareiorhaphis steindachneri (Miranda Ribeiro, 1918)

Hemipsilichthys steindachneri Miranda Ribeiro, 1918a: 107. Type locality: Flum. Rio Itapucú (Santa Catharina) [Brazil]. Lectotype: NMW 92790, designated by Pereira \& Reis (2002: 109). Originally proposed as H. steindachneri, but included in a new genus Pareiorhaphis.

Pseudancistrus luderwaldti Miranda Ribeiro 1918d: 632. Type locality: Hansa, Santa Catharina, Brasil. Holotype: MZUSP 2109.

Distribution: Cubatão, Itapocu, and Itajaí-Açu River basins, Brazil (Weber, 2003).

Remarks: Priority of Hemipsilichthys steindachneri over Pseudancistrus luderwaldti has not been clearly established and may not be resolved by dates of publication; precedence in the absence of a clear indiction of priority may be by first reviser action of Pereira \& Reis (2002), which is tentatively followed here.

Pareiorhaphis stephanus (Oliveira \& Oyakawa, 1999)

Hemipsilichthys stephanus Oliveira \& Oyakawa, 1999: 77, fig. 4 (top). Type locality: Brazil: Minas Gerais: Rio Jequitinhonha drainage, County of Diamantina, ribeirão das Pedras, ca. 1300 masl, $3 \mathrm{~km}$ north of Diamantina

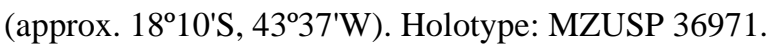

Distribution: Jequitinhonha River basin, Brazil (Weber, 2003).

Pareiorhaphis stomias (Pereira \& Reis, 2002) 
Hemipsilichthys stomias Pereira \& Reis, 2002: 139, fig. 24 (middle). Type locality: Brazil: Santa Catarina: Treviso: rio Mãe Luzia at Forquilha, 28²7'66"S 49³0'06"W. Holotype MCP 19666.

Distribution: Ararangua and Tubarão River basins, Brazil (Weber, 2003).

Pareiorhaphis vestigipinnis (Pereira \& Reis, 1992)

Hemipsilichthys vestigipinnis Pereira \& Reis, 1992: 113, fig. 2(b). Type locality: Creek tributary of the Rio Caveiras at Painel, on the road from São Joaquim to Lares (approximately $27^{\circ} 55^{\prime} \mathrm{S}, 50^{\circ} 05^{\prime} \mathrm{W}$ ) Lajes, Santa Catarina, Brazil. Holotype: MCP 14344.

Distribution: Headwaters of Canoas River basin, Brazil (Weber, 2003).

PAREIORHINA Gosline, 1947

Pareiorhina Gosline, 1947: 104. Type species: Rhinelepis rudolphi Miranda Ribeiro, 1911. Type by original designation. Gender: Feminine.

Key to species: Chamon et al. (2005: 555).

Pareiorhina brachyrhyncha Chamon, Aranda \& Buckup, 2005

Pareiorhina brachyrhyncha Chamon, Aranda \& Buckup, 2005: 551, fig. 1. Type locality: Brazil, State of São Paulo, Município Pindamonhangaba, Bairro Ribeirão Grande (or Teteqüera) and adjacent tributary ditch (Rio Paraíba do Sul drainage), Vera Cruz farm, 2246'14.1"S, 45²6'42.5"W. Holotype: MNRJ 25033.

Distribution: Paraíba do Sul River basin, São Paulo State, Brazil (Chamon et al., 2005).

Pareiorhina carrancas Bockmann \& Ribeiro, 2003

Pareiorhina carrancas Bockmann \& Ribeiro, 2003: 232, fig. 1. Type locality: Brazil, Minas Gerais: minicípio of Carrancas, córrego Debaixo da Serra (an upper course tributary of córrego do Beijinho, a left margin affluent of

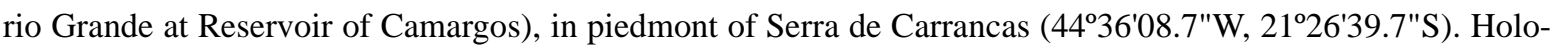
type: LIRP 2280.

Distribution: Upper Rio Grande basin, of Upper Parana River system, Brazil (Bockmann \& Ribeiro, 2003).

Pareiorhina rudolphi (Miranda Ribeiro, 1911)

Plecostomus (Rhinelepis) microps Ihering, 1907: 24. Type locality: Piquete (Lorena), Estado de São Paulo [Brazil]. Holotype: MZUSP 2154. Previously unpublished illustration of holotype reproduced in Bockmann \& Ribeiro (2003: fig. 6). Preoccupied by Plecostomus microps Steindachner, 1876; replaced by Rhinelepis rudolphi Miranda Ribeiro, 1911.

Rhinelepis rudolphi Miranda Ribeiro, 1911: 84. Type locality: Piquete (Lorena), Estado de São Paulo [Brazil]. Holotype: MZUSP 2154. Replacement name for Plecostomus microps Ihering, 1907.

Distribution: Paraíba do Sul River near Lorena, São Paulo State, Brazil (Weber, 2003).

PAROTOCINCLUS Eigenmann \& Eigenmann, 1889

Parotocinclus Eigenmann \& Eigenmann, 1889b: 41. Type species: Otocinclus maculicauda Steindachner, 1877.

Type by monotypy. Gender: Masculine. Proposed originally as a subgenus of Hisonotus.

Revision: Garavello (1976).

Reviews: Schaefer \& Provenzano (1993, Guiana Shield); Lacerda \& Evers (1996, Brazil).

Key: Garavello \& Britski (2003, eastern Brazilian coastal rivers).

Parotocinclus amazonensis Garavello, 1976

Parotocinclus amazonensis Garavello, 1976: 7, figs. 21-22. Type locality: Ilha Sorubim, Rio Solimões, Amazonas, Brazil. Holotype: MZUSP 10145.

Distribution: Amazon River basin, Brazil (Schaefer, 2003c).

Parotocinclus aripuanensis Garavello, 1988

Parotocinclus aripuanensis Garavello, 1988: 122, fig. 3. Type locality: Ingazeiro, $20 \mathrm{~km}$ upstream of Boca do Rio Canumã, Aripuanã, MT, Brazil. Holotype: MZUSP 36899.

Distribution: Aripuanã River basin, Brazil (Schaefer, 2003c).

Parotocinclus bahiensis (Miranda Ribeiro, 1918)

Microlepidogaster bahiensis Miranda Ribeiro, 1918d: 635. Type locality: Villa Nova, E. da Bahia [Brazil]. Syntypes: MZUSP 1071, lost (Britski, 1969). 
Distribution: Bahia State, Brazil (Schaefer, 2003c).

Parotocinclus bidentatus Gauger \& Buckup, 2005

Parotocinclus bidentatus Gauger \& Buckup, 2005: 512, fig. 5. Type locality: rio Pirapetinga (left bank tributary of rio Paraíba do Sul), Resende (22²8'08"S 44²6'48"W), Rio de Janeiro State, Brazil. Holotype: MNRJ 27962.

Distribution: Pirapetinga and Calçado Rivers, Paraíba do Sul River basin, Rio de Janeiro, Brazil (Gauger \& Buckup, 2005).

Parotocinclus britskii Boeseman, 1974

Parotocinclus britskii Boeseman, 1974: 267, fig. 2; pl. 2. Type locality: Left tributary of Coppename River, Surinam, 3ํ5' N, 5655'W. Holotype: ZMA 106593.

Distribution: Orinoco River basin and Atlantic coastal drainages of the Guianas (Schaefer, 2003c).

Parotocinclus cearensis Garavello, 1976

Parotocinclus cearensis Garavello, 1976: 14, figs. 19-20. Type locality: Cachoeira do Gusmão, Ipu, Ceará, Brazil. Holotype: MNRJ 10176.

Distribution: Ceará State, Brazil (Schaefer, 2003c).

Parotocinclus cesarpintoi Miranda Ribeiro, 1939

Paraotocinclus cesarpintoi Miranda Ribeiro, P., 1939b: 364, fig. Type locality: Vila de Quebrângulo, no Estado de Alagôas [Brazil]. Syntypes (2, 45-55 mm TL): possibly MNRJ 1022 (2 of 7), or MNRJ 1024 (2).

Distribution: Paraíba do Sul River basin, Brazil (Schaefer, 2003c).

Parotocinclus collinsae Schmidt \& Ferraris, 1985

Parotocinclus collinsae Schmidt \& Ferraris, 1985: 341, figs. 1-3. Type locality: Tributary to Takutu River, ca. 2 mi. from Mazarhally Takutu Lumber Camp in Takutu mountains, 6 ${ }^{\circ} 15^{\prime} \mathrm{N}, 59^{\circ} 5^{\prime} \mathrm{W}$, Essequibo, Guyana. Holotype: AMNH 55433.

Distribution: Essequibo River basin, Guyana (Schaefer, 2003c).

Parotocinclus cristatus Garavello, 1976

Parotocinclus cristatus Garavello, 1976: 6, figs. 9-10. Type locality: Fazenda Almada, Ilhéus, Bahia, Brazil. Holotype: MNRJ 10132.

Distribution: Coastal rivers near Ilhéus, Bahia State, Brazil (Schaefer, 2003c).

Parotocinclus doceanus (Miranda Ribeiro, 1918)

Microlepidogaster doceanus Miranda Ribeiro, 1918d: 634. Type locality: Rio Doce, E. Santo [Brazil]. Holotype: MZUSP 1016.

Distribution: Doce River basin, Brazil (Schaefer, 2003c).

Parotocinclus eppleyi Schaefer \& Provenzano, 1993

Parotocinclus eppleyi Schaefer \& Provenzano, 1993: 46, figs. 5-6. Type locality: Caño Curicurito, ca. $1 \mathrm{~km}$ above its mouth into the Río Autana, Amazonas, Venezuela, 4²47'N, 67²5'W. Holotype: MBUCV V-22530.

Distribution: Upper and middle Orinoco River basin, Venezuela (Schaefer, 2003c).

Parotocinclus haroldoi Garavello, 1988

Parotocinclus haroldoi Garavello, 1988: 118, fig. 1. Type locality: Córrego do Otaviano, Poço do Sanharó, Riacho Sanharó, Piauí, Brazil. Holotype: MNRJ 10531.

Distribution: Piauí State, Brazil (Schaefer, 2003c).

Parotocinclus jimi Garavello, 1976

Parotocinclus jimi Garavello, 1976: 9, figs. 25-26. Type locality: Rio do Peixe, small tributary of Rio de Contas, Fazenda Pedra Branca, Itagibá, Bahia, Brazil. Holotype: MZUSP 12133.

Distribution: Bahia State, Brazil (Schaefer, 2003c).

Parotocinclus jumbo Britski \& Garavello, 2002

Parotocinclus jumbo Britski \& Garavello, 2002: 280, fig. 1. Type locality: Brazil: Paraíba: rio Paraíba do Norte, at bridge on road PB 408, NW of Umbuzeiro (7 38'27"S 35 42'30"W). Holotype: MZUSP 69513.

Distribution: Coastal rivers in Ceará, Paraíba, Pernambuco and Alagoas States, Brazil (Schaefer, 2003c).

Parotocinclus longirostris Garavello, 1988

Parotocinclus longirostris Garavello, 1988: 120, fig. 2. Type locality: Rio Preto da Eva, Manaus-Itacoatiara highway, km 80, Manaus, Amazonas, Brazil. Holotype: MZUSP 36891. 
Distribution: Amazon River basin, Brazil (Schaefer, 2003c).

Parotocinclus maculicauda (Steindachner, 1877)

Otocinclus maculicauda Steindachner, 1877c: 222, pl. 1 (figs. 2-2b). Type locality: Santa Cruz, Rio Quenda in vicinity of Rio de Janeiro, Brazil. Lectotype: NMW 45381: 1, designated by, and illustrated in, Schaefer (1996b: 1034, fig. 2).

Parotocinclus steindachneri Di Caporiacco, 1948: 201, fig. 2. Type locality: Rio Novo, Santa Catarina, Brazil. Syntypes: MZUF 5565 (1).

Distribution: Coastal rivers between Santa Catarina and Rio de Janeiro States, Brazil (Schaefer, 2003c).

Parotocinclus minutus Garavello, 1976

Parotocinclus minutus Garavello, 1976: 15, figs. 13-14. Type locality: Rio Vasa-Barris, Canudos, Bahia, Brazil. Holotype: MNRJ 10135.

Distribution: Vasa-Barris River basin, Brazil (Schaefer, 2003c).

Parotocinclus muriaensis Gauger \& Buckup, 2005

Parotocinclus muriaensis Gauger \& Buckup, 2005: 514, fig. 7. Type locality: rio Muriaé river (left bank tributary of rio Paraíba do Sul), approx. $21^{\circ} 15^{\prime} \mathrm{S} 41^{\circ} 45^{\prime} \mathrm{W}$, Município de Itaperuna, Rio de Janeiro State, Brazil. Holotype: MNRJ 28528.

Distribution: Itaperuna River, Paraiba do Sul River basin, Rio de Janeiro, Brazil (Gauger \& Buckup, 2005).

Parotocinclus planicauda Garavello \& Britski, 2003

Parotocinclus planicauda Garavello \& Britski, 2003: 254, fig. 1. Type locality: Brazil. Minas Gerais, Coroaci, Suaçui Pequeno River, barro do Rochedo, at Antonio Pereira de Oliveira farm, $20 \mathrm{~km}$ from Coroaci. Holotype: MZUSP 75071.

Distribution: Doce river basin, Minas Gerais, Brazil (Garavello \& Britski, 2003).

Parotocinclus polyochrus Schaefer, 1988

Parotocinclus polyochrus Schaefer, 1988: 184, figs. 1-2. Type locality: Río Mawarinuma tributary at Neblina base camp, Terr. Federal Amazonas, Venezuela, 055'N, 66¹0'W, elev. 120 m. Holotype: AMNH 74482.

Distribution: Baria River, Upper Negro River basin, Venezuela (Schaefer, 2003c).

Parotocinclus prata Ribeiro, Melo \& Pereira, 2002

Parotocinclus prata Ribeiro, Melo \& Pereira, 2002: 218, fig. 1. Type locality: Brazil: Minas Gerais: rio São Francisco basin, município de Presidente Olegário, headwaters of rio da Prata, stream tributary of ribeirão Quiricó, fazenda São Zeferino, near Galena; approximately 18²2'S 46²14.3'W. Holotype: MZUSP 68359.

Distribution: Upper São Francisco River basin, Brazil (Schaefer, 2003c).

Parotocinclus spilosoma (Fowler, 1941)

Plecostomus spilosoma Fowler, 1941a: 152, figs. 53-55. Type locality: Campina Grande, Parahyba, Brazil. Holotype: ANSP 69410.

Distribution: Paraíba State, Brazil (Schaefer, 2003c).

Parotocinclus spilurus (Fowler, 1941)

Plecostomus spilurus Fowler, 1941a: 148, figs. 47-49. Type locality: Rio Salgade, Icó, Ceará, Brazil. Holotype: ANSP 69403.

Distribution: Ceará State, Brazil (Schaefer, 2003c).

PECKOLTIA Miranda Ribeiro, 1912

Peckoltia Miranda Ribeiro, 1912: 7. Type species: Chaetostomus vittatus Steindachner, 1881. Type by monotypy. Gender: Feminine.

Peckoltichthys Miranda Ribeiro, 1917: 49. Type species: Peckoltichthys filicaudatus Miranda Ribeiro, 1917. Type by monotypy. Gender: Masculine.

Ancistomus Isbrücker \& Seidel, in Isbrücker et al., 2001: 17. Type species: Ancistrus snethlageae Steindachner, 1911. Type by original designation. Gender: Masculine.

Sophiancistrus Isbrücker \& Seidel, in Isbrücker et al., 2001: 21. Type species: Hemiancistrus ucayalensis Fowler, 1940. Type by original designation. Gender: Masculine.

Remarks: Miranda Ribeiro submitted a manuscript for publication in which Peckoltichthys was proposed as a 
replacement for Peckoltia, which he considered to be preoccupied by an identical generic name in Botany. The publication of that manuscript was delayed until1920 (Miranda Ribeiro, 1920) and was preceded by Miranda Ribeiro (1917), in which Peckoltichthys was published with only one included species, P. filicaudatus, and no indication that the name was intended as a replacement. Therefore, Peckoltichthys must be considered to have been proposed with P. filicaudatus as type, by monotypy and not, as indicated in Miranda Ribeiro (1920) as a replacement name with Chaetostomus vittatus as type. The newly proposed generic names Sophiancistrus Isbrücker \& Seidel and Ancistomus Isbrücker \& Seidel were considered by Fisch-Muller (2003) to be unwarranted.

Peckoltia arenaria (Eigenmann \& Allen, 1942)

Hemiancistrus arenarius Eigenmann \& Allen, 1942: 185, pl. 6 (fig. 2). Type locality: Yurimaguas. Holotype: CAS 77323.

Distribution: Upper Amazon River basin, Peru (Fisch-Muller, 2003).

Peckoltia bachi (Boulenger, 1898)

Chaetostomus bachi Boulenger, 1898e: 425, pl. 41 (fig. 1). Type locality: Rio Jurua, an affluent of the Amazons, Brazil. Holotype: BMNH 1897.12.1.61.

Distribution: Juruá River basin, Brazil (Fisch-Muller, 2003).

Peckoltia braueri (Eigenmann, 1912)

Hemiancistrus braueri Eigenmann, 1912b: 232, pl. 28 (fig. 1). Type locality: Takutu, British Guiana. Holotype: ZMB 3174 (larger specimen).

Distribution: Upper Negro and Branco River basins; Brazil and Guyana (Armbruster \& Werneke, 2005).

Remarks: Redescribed in Armbruster \& Werneke (2005:2).

Peckoltia brevis (La Monte, 1935)

Hemiancistrus brevis La Monte, 1935: 3, fig. 2. Type locality: Rio Purus, Brazil; in the vicinity of the mouth of Rio Macauhan, a tributary of Rio Yaco, which, in turn, is a tributary of Rio Purus $\left(69^{\circ} \mathrm{W} .9^{\circ} 20^{\prime}\right.$ S. $)$. Holotype: AMNH 12602.

Distribution: Purus River basin, Brazil (Fisch-Muller, 2003).

Peckoltia cavatica Armbruster \& Werneke, 2005

Peckoltia cavatica Armbruster \& Werneke, 2005: 8, figs. 2 (bottom), 4. Type locality: Guyana, Rupununi (Region 9), 3.7 km SSE Massara, 03.86228,$-059.28439^{\circ}$. Holotype: UG/CSBD 11043.

Distribution: Upper Rupununi River, Guyana (Armbruster \& Werneke, 2005).

Peckoltia filicaudata (Miranda Ribeiro, 1917)

Peckoltichthys filicaudatus Miranda Ribeiro, 1917: 49. Type locality: Fluvio Solimões [Brazil]. Holotype: MNRJ 969.

Distribution: Upper Amazon River basin, Brazil; known only from holotype (Fisch-Muller, 2003).

Peckoltia furcata (Fowler, 1940)

Chaetostomus furcatus Fowler, 1940a: 238, figs. 28-29. Type locality: Ucayali River basin, Contamana, Peru. Holotype: ANSP 68655.

Distribution: Ucayali River basin, Peru; known only from holotype (Fisch-Muller, 2003).

Peckoltia kuhlmanni (Miranda Ribeiro, 1920)

Peckoltichthys kuhlmanni Miranda Ribeiro, 1920: 10, pl. 5 (middle). Type locality: Tapajóz [Brazil]. Lectotype: MNRJ 2044A, designated by Miranda Ribeiro (1953: 401), but specimen not isolated.

Distribution: Tapajós River basin, Brazil (Fisch-Muller, 2003).

Peckoltia oligospila (Günther, 1864)

Chaetostomus oligospilus Günther, 1864: 244. Type locality: River Capin [Brazil]. Holotype: BMNH 1849.11.8, illustrated in Regan (1904b: 232, pl. 12 fig. 1).

Distribution: Lower Amazon River basin, Brazil (Fisch-Muller, 2003).

Peckoltia sabaji Armbruster, 2003

Peckoltia sabaji Armbruster, 2003b: 6, figs. 2-4. Type locality: Guyana: Rupununi (Region 9), Essequibo River Dr., Rupununi R. 5.9 km WSW village of Sand Creek, 02.96656 ${ }^{\circ},-059.56943^{\circ}$. Holotype: UG/CSBD 11041.

Distribution: Rupununi, Essequibo and Takutu River basins, Guyana, and Casiquiare and Orinoco basins, Venezuela 
(Armbruster, 2003b).

Peckoltia snethlageae (Steindachner, 1911)

Ancistrus snethlageae Steindachner, 1911a: 328. Type locality: Rio Tapajoz bei Villa Braga und Goyana [Brazil].

Syntypes: NMW 48049 (1), NMW 48050 (1).

Distribution: Tapajós River basin, Brazil (Fisch-Muller, 2003).

Remarks: Recently placed by Isbrücker \& Seidel (in Isbrücker, 2001a: 17) in the monotypic genus Ancistomus.

Peckoltia ucayalensis (Fowler, 1940)

Hemiancistrus ucayalensis Fowler, 1940a: 235, figs. 24-25. Type locality: Ucayali River, Contamana, Peru. Holotype: ANSP 68651.

Distribution: Ucayali River basin, Peru (Fisch-Muller, 2003).

Peckoltia vermiculata (Steindachner, 1908)

Ancistrus vittatus vermiculata Steindachner, 1908f: 166. Type locality: dem mittleren Laufe des Amazonestrommes, den Gewässern um Pará, Brasiliens. Syntypes: NMW 48056 (1), NMW 48059 (1), NMW 48064 (1). Originally as Ancistrus vittatus var. vermiculata.

Distribution: Middle and lower Amazon River basin, Brazil (Fisch-Muller, 2003).

Peckoltia vittata (Steindachner, 1881)

Chaetostomus vittatus Steindachner, 1881c: 115, pl. 2 (fig. 5). Type locality: Amazonen-Strom, Tajapouru, Xingu bei Porto de Moz, Rio Madeira [Brazil]. Syntypes: MCZ 7999 (1), MCZ 8017 (1), NMW 47225 (1), NMW 47226 (1), NMW 47227 (1), NMW 47228 (2).

Distribution: Middle and lower Amazon River basin, Brazil (Fisch-Muller, 2003).

PLANILORICARIA Isbrücker, 1971

Planiloricaria Isbrücker, 1971b: 276. Type species: Pseudohemiodon (Planiloricaria) cryptodon Isbrücker, 1971.

Type by original designation. Gender: Feminine. Originally proposed as a subgenus of Pseudohemiodon.

Remarks: Diagnosed and distinguished in key from related genera in Isbrücker \& Nijssen (1986a).

Planiloricaria cryptodon (Isbrücker, 1971)

Pseudohemiodon (Planiloricaria) cryptodon Isbrücker, 1971b: 278, fig. 3. Type locality: Peru: Río Ucayali near

Pucallpa. Holotype: ZFMK 1865. Holotype illustrated in Isbrücker (1979b: fig. 4).

Distribution: Ucayali, Purus, and Mamoré River basins, Upper Amazon drainage (Isbrücker \& Nijssen, 1986a).

Remarks: Redescribed in Isbrücker \& Nijssen (1986a).

POGONOPOMA Regan, 1904

Pogonopoma Regan, 1904b: 205. Type species: Plecostomus wertheimeri Steindachner, 1867. Type by subsequent designation by Eigenmann (1910: 407). Gender: Neuter. Originally proposed as subgenus of Plecostomus.

Pogonopomoides Gosline, 1947: 109. Type species: Rhinelepis parahybae Steindachner, 1877. Type by original designation. Gender: Neuter.

Revision: Quevedo \& Reis (2002).

Pogonopoma obscurum Quevedo \& Reis, 2002

Pogonopoma obscurum Quevedo \& Reis, 2002: 405, fig. 3 (middle). Type locality: Rio Canoas at road from Anita Garibaldi to Abdon Batista (approximatively $27^{\circ} 39^{\prime} \mathrm{S}, 5^{\circ}{ }^{\circ} 5^{\prime} \mathrm{W}$ ) Santa Catarina, Brazil. Holotype MCP 25036.

Distribution: Upper Uruguay River basin, Brazil (Weber, 2003).

Pogonopoma parahybae (Steindachner, 1877)

Rhinelepis Parahybae Steindachner, 1877c: 218, pl. 2. Type locality: aus dem Parahyba [Brazil]. Syntypes: MCZ

7756 (4), NMW 44556 (1), NMW 44557 (1), NMW 44558 (1).

Distribution: Paraíba do Sul River basin, Brazil (Weber, 2003).

Remarks: Redescribed (in Pogonopomoides) in Armbruster (1998a: 629).

Pogonopoma wertheimeri (Steindachner, 1867)

Plecostomus Wertheimeri Steindachner, 1867c: 119. Type locality: dem Fluss Mucuri im gleichnamige Districte bei

Santa Clara, in Brasilien. Holotype: NMW 44288; illustrated in Steindachner (1867f: 701, pl. 1).

Distribution: Mucuri and São Mateus River basins, Brazil (Weber, 2003).

Remarks: Redescription in Armbruster (1998a: 629). 
PROLORICARIA Isbrücker, 2001

Proloricaria Isbrücker, in Isbrücker et al., 2001: 21. Type species: Loricaria prolixa Isbrücker \& Nijssen, 1978. Type by original designation. Gender: Feminine.

Proloricaria lentiginosa (Isbrücker, 1979)

Loricaria prolixa lentiginosa Isbrücker, 1979a: 97, fig. 9. Type locality: Brésil, Est. Sao Paulo, haut bassin du Rio Parana, Represa de Volta Grande, Rio Grande. Holotype: MZUSP 13188.

Distribution: Upper Paraná River basin, Brazil (Isbrücker, 1979).

Proloricaria prolixa (Isbrücker \& Nijssen, 1978)

Loricaria prolixa Isbrücker \& Nijssen, 1978a: 188, fig. 5. Type locality: Brazil, Est. São Paulo, Rio Piracicaba through River Tietê, Rio Paraná system. Holotype: NMW 45091.

Distribution: Upper Paraná River drainage, La Plata basin, Brazil (Isbrücker \& Nijssen, 1978).

PSEUDACANTHICUS Bleeker, 1862

Pseudacanthicus Bleeker, 1862 (in Bleeker, 1862-63): 2. Type species: Hypostomus serratus Valenciennes, 1840. Type by original designation. Gender: Masculine.

Stoneiella Fowler, 1914: 271. Type species: Stoneiella leopardus Fowler, 1914. Type by original designation. Gender: Feminine.

Monophyly: Schaefer (1986: 113).

Pseudacanthicus fordii (Günther, 1868)

Chaetostomus Fordii Günther, 1868a: 476. Type locality: Surinam [Probably Suriname (Günther, 1868b)]. Syntypes (4): BMNH 1866.8.14.148 and BMNH 1866.8.14.152 (4), BMNH 1866.8.14.150 (1), BMNH 1866.8.14.151

(1). Species illustrated and described in more detail, with additional comments on type locality, in Günther (1868b: 231, pl. 21).

Distribution: Coastal drainages of Suriname (Fisch-Muller, 2003).

Pseudacanthicus histrix (Valenciennes, 1840)

Rinelepis histrix Valenciennes, in Cuvier \& Valenciennes, 1840b: 486 (359 in Strasbourg deluxe edition). Type locality: [No locality stated]. No types known; description based on unpublished manuscript and illustrations by Vandelli. Illustration of type reproduced by Capello (1870: pl. 7), and França (1922: fig. 9).

Loricaria spinosae Ferreira, in França, 1922: 84, fig. 9. Type locality: in Fluminis Amazonici. No types known.

Distribution: Negro and lower Amazon River basins, Brazil (Fisch-Muller, 2003).

Pseudacanthicus leopardus (Fowler, 1914)

Stoneiella leopardus Fowler, 1914: 271, fig. 17. Type locality: Rupununi River, British Guiana ... in the highlands of British Guiana, approximately secured in North Latitude $2^{\circ}$ to $3^{\circ}$, and West Longitude $50^{\circ} 20^{\prime}$. Holotype: ANSP 39345.

Distribution: Rupununi River basin, Guyana (Fisch-Muller, 2003).

Pseudacanthicus serratus (Valenciennes, 1840)

Hypostomus serratus Valenciennes, in Cuvier \& Valenciennes, 1840b: 503 (372 in Strasbourg deluxe edition). Type locality: rapportée de Surinam. Holotype: RMNH 3125.

Distribution: Coastal drainages of the Guianas (Fisch-Muller, 2003).

Pseudacanthicus spinosus (Castelnau, 1855)

Hypostomus spinosus Castelnau, 1855: 45, pl. 22 (fig. 3). Type locality: la rivière des Amazones. Holotype: MNHN a-9577.

Distribution: Amazon River basin, Brazil (Fisch-Muller, 2003).

PSEUDANCISTRUS Bleeker, 1862

Pseudancistrus Bleeker, 1862 (in Bleeker, 1862-63): 2. Type species: Hypostomus barbatus Valenciennes, 1840. Type by original designation. Gender: Masculine.

Lithoxancistrus Isbrücker, Nijssen \& Cala, 1988: 14. Type species: Lithoxancistrus orinoco Isbrücker, Nijssen \& Cala, 1988. Type by original designation. Gender: Masculine. 
Guyanancistrus Isbrücker, in Isbrücker et al., 2001: 19. Type species: Lasiancistrus brevispinis Heitmans, Nijssen \& Isbrücker, 1983. Type by original designation. Gender: Masculine.

Monophyly: Synonymy and species composition follows Armbruster (2004a, b).

Pseudancistrus barbatus (Valenciennes, 1840)

Hypostomus barbatus Valenciennes, in Cuvier \& Valenciennes, 1840b: 506 (374 in Strasbourg deluxe edition). Type locality: La Mana [French Guiana]. Holotype: MNHN a-9564.

Hypostomus guttatus Valenciennes, in Cuvier \& Valenciennes, 1840b: 508 (375 in Strasbourg deluxe edition). Type locality: Surinam. Holotype: RMNH 3126, illustrated in Bleeker (1864: 10, pl. 2 fig. 2, pl. 3 fig. 3 ).

Distribution: Major river basins of Guianas: Oyapock, Mana, Maroni, Suriname, Corantijn, and Essequibo (FischMuller, 2003).

Pseudancistrus brevispinis (Heitmans, Nijssen \& Isbrücker, 1983)

Lasiancistrus brevispinis Heitmans, Nijssen \& Isbrücker, 1983: 38, fig. 4 (middle). Type locality: Surinam, Nickerie River system, district Nickerie, Fallawatra River, rapid 5 km S. W. of Stondansie Fall. Holotype: ZMA 107.740.

Distribution: Atlantic coastal drainages of the Guianas, from Nickerie to Oyapock River basins, (Fisch-Muller, 2003).

Remarks: Redescribed in Le Bail et al. (2000: 236, fig.).

Pseudancistrus coquenani (Steindachner, 1915)

Ancistrus (Pseudancistrus) coquenani Steindachner, 1915e: 90, pl. 11 (fig. 3). Type locality: dem Coquenanflusse, einem Nebenflusse des Caroni in Venezuela. Syntypes (3): NMW 48023 (2). Name proposed conditionally as “coquenani n. sp.? an A. güntheri Regan female."

Distribution: Kukenan River basin in upper Caroni River drainage, Venezuela (Fisch-Muller, 2003).

Pseudancistrus depressus (Günther, 1868)

Chaetostomus depressus Günther, 1868a: 476. Type locality: Surinam. Holotype: BMNH 1866.8.14.139. Described in more detail in Günther (1868b: 232).

Distribution: Unknown locality in Suriname (Fisch-Muller, 2003).

Pseudancistrus genisetiger Fowler, 1941

Pseudancistrus genisetiger Fowler, 1941a: 155, figs. 59-62. Type locality: Rio Jaguaribe, Orós, Ceará [Brazil]. Holotype: ANSP 69441.

Distribution: Jaguaribe River basin, Brazil (Fisch-Muller, 2003).

Pseudancistrus guentheri (Regan, 1904)

Ancistrus guentheri Regan, 1904b: 241, pl. 12 (fig. 3). Type locality: British Guiana. Holotype: BMNH 1978.3.2.1.

Distribution: Unknown locality in Guyana (Fisch-Muller, 2003).

Pseudancistrus longispinis (Heitmans, Nijssen \& Isbrücker, 1983)

Lasiancistrus longispinis Heitmans, Nijssen \& Isbrücker, 1983: 45, fig. 8 (middle). Type locality: French Guiana, Oyapock River system: Camopi River at Pauwé Jean-Jean, upstream of Saut Mauvais $\left(03^{\circ} 11^{\prime} \mathrm{N} 52^{\circ} 22^{\prime} \mathrm{W}\right)$. Holotype: IRSNB 612.

Distribution: Oyapock River basin, French Guiana (Fisch-Muller, 2003).

Pseudancistrus niger (Norman, 1926)

Hemiancistrus niger Norman, 1926b: 96. Type locality: Oyapock River at Sant [sic] Cafesoca, French Guiana. Lectotype: BMNH 1926.3.2.756, designated by Heitmans et al. (1983: 35).

Distribution: Oyapock River basin, French Guiana (Fisch-Muller, 2003).

Pseudancistrus nigrescens Eigenmann, 1912

Pseudancistrus nigrescens Eigenmann, 1912b: 234, pl. 25 (fig. 2). Type locality: Amatuk, British Guiana. Holotype: FMNH 53105.

Distribution: Upper Potaro River basin, Guyana (Fisch-Muller, 2003).

Pseudancistrus orinoco (Isbrücker, Nijssen \& Cala, 1988)

Lithoxancistrus orinoco Isbrücker, Nijssen \& Cala, 1988: 14, fig. 1 (top). Type locality: Colombie, Province de Vichada, Rio Orinoco, à la cascade (=raudal) près de l'embouchure du Rio Tuparro. Holotype: ICNMHN 1200.

Distribution: Orinoco River basin (Fisch-Muller, 2003).

Pseudancistrus papariae Fowler, 1941 
Pseudancistrus papariae Fowler, 1941a: 157, figs. 63-65. Type locality: Lago Papary, Rio Grande do Norte [Brazil]. Holotype: ANSP 69442.

Distribution: Lake Papari and Jaguaribe River basin, Brazil (Fisch-Muller, 2003).

Pseudancistrus sidereus Armbruster, 2004

Pseudancistrus sidereus Armbruster, 2004b: 8, fig. 3. Type locality: Venezuela, Amazonas, Rio Orinoco dr.: 175.6, Río Siapa from 10 to $15 \mathrm{~km}$ downstream, Río Casiquiare - Río Negro dr., $01.50000^{\circ},-065.71667^{\circ}$. Holotype: MCNG 26125.

Distribution: Upper Orinoco River and Casiquaire, Amazonas, Venezuela (Armbruster, 2004b).

PSEUDOHEMIODON Bleeker, 1862

Pseudohemiodon Bleeker, 1862 (in Bleeker, 1862-63): 3. Type species: Hemiodon platycephalus Kner, 1853. Type by original designation. Gender: Masculine.

Pseudohemiodon amazonus (Delsman, 1941)

Loricaria apeltogaster amazonum Delsman, 1941: 80. Type locality: Trombetas-river, near Obidos [Brazil]. Holotype: IRSNB 546. Originally as Loricaria apeltogaster var. amazonum.

Distribution: Lower Amazon River basin, Brazil.

Pseudohemiodon apithanos Isbrücker \& Nijssen, 1978

Pseudohemiodon apithanos Isbrücker \& Nijssen, 1978a: 195, fig. 6. Type locality: Ecuador, Prov. Napo, Río Conejo, a tributary of Río San Miguel, vicinity of Santa Cecilia, $0^{\circ} 06^{\prime} \mathrm{N}, 76^{\circ} 51^{\prime} \mathrm{W}$, upper Río Amazonas system. Holotype: ANSP 134370.

Distribution: San Miquel River basin, Western Amazon River drainage, Ecuador.

Pseudohemiodon devincenzii (Soriano-Señorans, 1950)

Loricaria devincenzii Soriano-Señorans, 1950: 265. Type locality: sobre fondo de arena fangosa con red de playa, a 200 metros aqua abajo d la desembocadura del arroyo Espinillar, en el Río Uruguay, Rep. Oriental del Uruguay, departamento de Salto. Holotype: MHNM (apparently lost, Olazarri et al., 1970).

Distribution: Uruguay River basin, Uruguay.

Pseudohemiodon laminus (Günther, 1868)

Loricaria lamina Günther, 1868a: 478. Type locality: Xeberos (Upper Amazon). Lectotype: BMNH 1867.6.13.33, designated by Isbrücker (1973: 186). Illustrated and described in more detail in Günther (1868b: 239, figs. 6, 7).

Distribution: Upper Amazon River basin, Peru.

Pseudohemiodon laticeps (Regan, 1904)

Loricaria laticeps Regan, 1904b: 295, pl. 20 (fig. 1). Type locality: Paraguay. Lectotype: BMNH 1895.5.17.113, designated by Isbrücker (1973: 187).

Distribution: Uruguay, Paraná and Paraguay River basins.

Pseudohemiodon platycephalus (Kner, 1853)

Hemiodon platycephalus Kner, 1853a: 12, 25, pl. 1 (fig. 6), pl. 6 (fig. 2a-b). Type locality: Rio Cuiaba [Brazil]. Holotype: lost (Isbrücker, 1980: 122). Originally as Hem. ? platycephalus.

Distribution: Paraguay River basin, Brazil.

Pseudohemiodon thorectes Isbrücker, 1975

Pseudohemiodon thorectes Isbrücker, 1975a: 87, fig. 1. Type locality: Bolivia, Est. Santa Cruz, Buena Vista, $17^{\circ} 28^{\prime} \mathrm{S}, 63^{\circ} 37^{\prime} \mathrm{W}$, west of Río Palacios, tributary of Río Mamoré, which is a tributary of Río Madeira, Rio Amazonas system. Holotype: BMNH 1927.10.4.43.

Distribution: Mamoré River basin, Bolivia.

PSEUDOLITHOXUS Isbrücker \& Werner, 2001

Pseudolithoxus Isbrücker \& Werner, in Isbrücker et al., 2001: 21. Type species: Lasiancistrus tigris Armbruster \& Provenzano, 2000. Type by original designation. Gender: Masculine.

Remarks: This genus corresponds to the Lasiancistrus anthrax species group of Armbruster \& Provenzano (2000).

Pseudolithoxus anthrax (Armbruster \& Provenzano, 2000)

Lasiancistrus anthrax Armbruster \& Provenzano, 2000: 243, fig. 1B. Type locality: Venezuela, Estado Amazonas, 
Río Orinoco backwater behind sand beach ca. $0.5 \mathrm{hr}$. upstream from Isla Temblador, $03^{\circ} 04^{\prime} \mathrm{N} 66^{\circ} 28^{\prime} \mathrm{W}$. Holotype: MBUCV V-18384.

Distribution: Upper and lower Orinoco tributaries, Caura and Aro rivers, Venezuela (Fisch-Muller, 2003).

Pseudolithoxus dumus (Armbruster \& Provenzano, 2000)

Lasiancistrus dumus Armbruster \& Provenzano, 2000: 246, fig. 3B. Type locality: Venezuela, Estado Amazonas, Río Orinoco, backwater behind sand playa circa $0.5 \mathrm{hr}$. upstream from Isla Temblador, $03^{\circ} 04^{\prime} \mathrm{N} 66^{\circ} 28^{\prime} \mathrm{W}$. Holotype: MBUCV V-17544.

Distribution: Upper Orinoco, upper Negro and Casiquiare River basins, Venezuela (Fisch-Muller, 2003).

Pseudolithoxus nicoi (Armbruster \& Provenzano, 2000)

Lasiancistrus nicoi Armbruster \& Provenzano, 2000: 249, fig. 4B. Type locality: Venezuela, Estado Amazonas, Departamento Río Negro, Río Manipitare (Río Siapa drainage), from about 5 to $8 \mathrm{~km}$ upstream from confluence with Río Siapa. Holotype: MCNG 37033.

Distribution: Upper Negro River basin, including Canal Casiquiare basin, Venezuela (Fisch-Muller, 2003).

Pseudolithoxus tigris (Armbruster \& Provenzano, 2000)

Lasiancistrus tigris Armbruster \& Provenzano, 2000: 251, fig. 5B. Type locality: Venezuela, Estado Amazonas, backwater of Río Orinoco behind sand playa circa $0.5 \mathrm{hr}$. upstream from Isla Temblador, $03^{\circ} 04^{\prime} \mathrm{N} 66^{\circ} 28^{\prime} \mathrm{W}$. Holotype: MBUCV V-17546.

Distribution: Upper Orinoco and Ventuari River basins, Venezuela (Fisch-Muller, 2003).

\section{PSEUDOLORICARIA Bleeker, 1862}

Pseudoloricaria Bleeker, 1862 (in Bleeker, 1862-63): 3. Type species: Loricaria laeviuscula Valenciennes, 1840. Type by original designation. Gender: Feminine.

Revision: Isbrücker \& Nijssen (1976a).

Pseudoloricaria laeviuscula (Valenciennes, 1840)

Loricaria laviuscula Valenciennes, in Cuvier \& Valenciennes, 1840b: 476 (p. 352 of Strasbourg deluxe edition). Type locality: [Locality unknown]. Holotype: MNHN b-0365.

Distribution: Lower and middle Amazon basin, including Negro and Branco rivers, Brazil (Isbrücker \& Nijssen, 1976a).

\section{PSEUDORINELEPIS Bleeker, 1862}

Pseudorinelepis Bleeker, 1862 (in Bleeker, 1862-63): 3. Type species: Rinelepis genibarbis Valenciennes, 1840. Type by original designation. Gender: Feminine.

Canthopomus Eigenmann, 1910: 407. Type species: Rhinelepis genibarbis Valenciennes, 1840. Type by original designation. Gender: Feminine. Designation by Eigenmann \& Allen (1942: 183) of Rhinelepis agassizii Steindachner as type is invalid.

Monistiancistrus Fowler, 1940a: 236. Type species: Monistiancistrus carachama Fowler, 1940. Type by original designation. Gender: Masculine.

Relationships: Armbruster (1998a: 631).

Pseudorinelepis genibarbis (Valenciennes, 1840)

Rinelepis genibarbis Valenciennes, in Cuvier \& Valenciennes, 1840b: 484 (357 in Strasbourg deluxe edition), pl. 453. Type locality: probablement du Brésil. Holotype (15 pouces): at MNHN.

Rhinelepis Agassizii Steindachner, 1877c: 228. Type locality: am See Manacapouru [Brazil]. Syntypes: MCZ 8007 (1), NMW 44559 (3), NMW 445560 (1), NMW 44561 (1).

Plecostomus pellegrini Regan, 1904b: 218. Type locality: from Upper Amazon. Holotype: MNHN a-3956.

Monistiancistrus carachama Fowler, 1940a: 236, figs. 26-27. Type locality: Ucayali River basin, Contamana, Peru. Holotype: ANSP 68654.

Distribution: Upper Amazon River basin (Weber, 2003).

Remarks: Redescribed in Armbruster \& Hardman (1999).

PSEUDOTOCINCLUS Nichols, 1919 
Pseudotocinclus Nichols, 1919b: 534. Type species: Pseudotocinclus intermedius Nichols, 1919. Type by monotypy. Gender: Masculine.

Revision, with key to species (Takako et al., 2005: 501).

Pseudotocinclus juquiae Takako, Oliveira \& Oyakawa, 2005

Pseudotocinclus juquiae Takako, Oliveira \& Oyakawa, 2005: 504, fig. 4. Type locality: Brazil, São Paulo, first tributary of the rio Juquiá, near ribeirão das Antas on the road from São Lourenço da Serra to Juquitiba, 2359'49.1"S, 4656'01.0"W, Juquitiba. Holotype: MZUSP 49333.

Distribution: Juquiá River basin, Brazil (Takako et al., 2005).

Pseudotocinclus parahybae Takako, Oliveira \& Oyakawa, 2005

Pseudotocinclus parahybae Takako, Oliveira \& Oyakawa, 2005: 503, fig. 3. Type locality: Brazil, São Paulo, a tributary of the ribeirão Grande, Fazenda São Sebastião do Ribeirão Grande, rio Paraíba do Sul basin, approximately $22^{\circ} 46^{\prime} S, 45^{\circ} 27^{\prime} \mathrm{W}$, Pindamonhangaba. Holotype: MZUSP 49122.

Distribution: Ribeirão Grande tributary, Paraíba do Sul River basin, Brazil (Takako et al., 2005).

Pseudotocinclus tietensis (Ihering, 1907)

Otocinclus (Microlepidogaster) tietensis Ihering, 1907: 26. Type locality: Rio Tieté, São Paulo, Brazil. Holotype: at DZSASP [now MZUSP] (not found).

Pseudotocinclus intermedius Nichols, 1919b: 534. Type locality: Campo Grande, Mato Grosso, Brazil. Holotype: AMNH 7177.

Distribution: Upper Tietê River at Paranapiacaba, Brazil (Schaefer, 2003c).

PSEUDOTOTHYRIS Britski \& Garavello, 1984

Pseudotothyris Britski \& Garavello, 1984: 232. Type species: Otocinclus obtusus Miranda Ribeiro, 1911. Type by original designation. Gender: Feminine.

Pseudotothyris janeirensis Britski \& Garavello, 1984

Pseudotothyris janeirensis Britski \& Garavello, 1984: 234, figs. 9-10. Type locality: Rio dos Macacos, Represa Engenho da Serra, Paulo de Frontin, Rio de Janeiro, Brazil. Holotype: MNRJ 10278.

Distribution: Rio de Janeiro State, Brazil (Schaefer, 2003c).

Pseudotothyris obtusa (Miranda Ribeiro, 1911)

Otocinclus obtusus Miranda Ribeiro, 1911: 95, pl. 29 (fig. 3). Type locality: Brazil. Lectotype: MNRJ 1026A, designated by Miranda Ribeiro (1953: 401). As Otocinclus obtusos at heading of account and index (p. 498), and Otocinclus obtusus in etymology, index (p. 497), figure caption, and elsewhere in the text. Britski \& Garavello (1984: 226) treated $O$. obtusa as the valid name, with obutsos as a misspelling, thereby acting as first reviser.

Distribution: Southeastern Brazil (Schaefer, 2003c).

PTEROSTURISOMA Isbrücker \& Nijssen, 1978

Pterosturisoma Isbrücker \& Nijssen, 1978b: 69. Type species: Harttia microps Eigenmann \& Allen, 1942. Type by original designation. Gender: Neuter.

Pterosturisoma microps (Eigenmann \& Allen, 1942)

Harttia microps Eigenmann \& Allen, 1942: 211, pl. 8 (figs. 3-4); pl. 9 (fig. 1). Type locality: Iquitos [Peru]. Lectotype: CAS 28543, designated by Isbrücker \& Nijssen (1978b: 71).

Distribution: Upper Amazon River basin, Peru (Eigenmann \& Allen, 1942).

\section{PTERYGOPLICHTHYS Gill, 1858}

Pterygoplichthys Gill, 1858: 408. Type species: Hypostomus duodecimalis Valenciennes, 1840. Type by subsequent designation in Bleeker (1862-63: 2) with genus spelled Pterygophlichtys. Gender: Masculine.

Liposarcus Günther, 1864: 238. Type species: Hypostomus multiradiatus Hancock, 1828. Type by subsequent designation by Jordan (1919b: 332). Gender: Masculine

Glyptoperichthys Weber, 1991: 639. Type species: Ancistrus lituratus Kner, 1854. Type by original designation. Gender: Masculine.

Remarks: Revised by Weber (1992), with Liposarcus and Glyptoperichthys also treated as valid. Generic synonymy 
herein based on Armbruster (2004a).

Pterygoplichthys ambrosettii (Holmberg, 1893)

Liposarcus ambrosettii Holmberg, 1893b: 354. Type locality: Río Paraguay, in front of Formosa. No types known.

Pterygoplichthys anisitsi Eigenmann \& Kennedy, 1903: 503. Type locality: Laguna of the Rio Paraguay at Asuncion. Holotype: IU 9873; whereabouts unknown.

Pterygoplichthys juvens Eigenmann \& Kennedy, 1903: 504. Type locality: Asuncion, Río Paraguay, Paraguay. Holotype: CAS 59784.

Ancistrus multiradiatus alternans Regan, 1904b: 229. Type locality: Paraguay and Southern Bolivia [restricted by Weber (1992: 28) to: Paraguyan Chaco [...] région Vila Concepción (=Concepción) - Caraya Vuelta -2/3 Piste Pozo Colorado, probablement Waikthlatingmayalwa (=? Mission Inglesia)]. Lectotype: BMNH 1898.7.4.5, designated by Weber (1992: 28). Originally proposed as A. multiradiatus var. alternans.

Distribution: Paraguay, middle Paraná, Bermejo, and Uruguay River basins (Weber, 2003).

Remarks: Weber $(1992,2003)$ treated Liposarcus ambrosettii Holmberg, 1893, as a nomen oblitum, but its use in several publications negate that status. Redescribed as Liposarcus anisitsi in Weber (1992: 13).

Pterygoplichthys disjunctivus (Weber, 1991)

Liposarcus disjunctivus Weber, 1991: 638. Type locality: Rio Madeira, système de l'Amazonas, Restauracão, Amazonas, Brésil. Holotype: MZUSP 28360; illustrated in Weber (1992: pl. 9).

Distribution: Madeira River basin, Bolivia and Brazil (Weber, 2003).

Pterygoplichthys etentaculatus (Spix \& Agassiz, 1829)

Hypostoma etentaculatum Spix \& Agassiz, 1829: 7, pl. 4 (fig. 1). Type locality: in Brasilae septentrioralis fluviis [now: São Francisco River at Januaria, Minas Gerais State, Brazil, by neotype designation]. Neotype: MZUSP 35821, designated by Weber (1992: 25).

Hypostomus duodecimalis Valenciennes in Cuvier \& Valenciennes, 1840b: 498 (367 in Strasbourg deluxe edition), pl. 454. Type locality: dans la rivière Saint-François au Brésil. Holotype: MNHN a-9446.

Hypostomus brevitentaculatus Ranzani, 1841: 63. Type locality: nelle aqua dolci della Provincia di S. Paulo nel Brasile. Holotype: at MZUB. Described in more detail in Ranzani (1842: 329).

Ancistrus longimanus Kner, 1854: 283, pl. 5 (fig. 1). Type locality: [Not stated]. Syntypes (2): at NMW. Originally proposed as Anc. longimanus.

Distribution: São Francisco River basin, Brazil (Weber, 2003).

Pterygoplichthys gibbiceps (Kner, 1854)

Ancistrus gibbiceps Kner, 1854: 284, pl. 5 (fig. 2). Type locality: aus dem Rio Negro bei Marabitanos [Brazil]. Holotype: at NMW. Originally proposed as Anc. gibbiceps.

Liposarcus altipinnis Günther, 1864: 239. Type locality: Rio Cupai [Brazil]. Holotype: BMNH 1853.3.19.34.

Distribution: Middle and upper Amazon and Orinoco basins (Weber, 2003).

Remarks: Redescribed in Weber (1992: 17), with type locality of Liposarcus altipinnis as: Cupari River, Tapajós River basin, Amazon drainage, Amazonas State, Brazil.

Pterygoplichthys joselimaianus (Weber, 1991)

Glyptoperichthys joselimaianus Weber, 1991: 640, illustrated in Weber (1992: 20, pl. 15b). Type locality: Rio Araguaya, affl. du Tocantins, système de l’Amazone, Aruanã, Goiàs, Brésil. Holotype: MZUSP 4873.

Distribution: Tocantins River basin, Brazil (Weber, 2003).

Pterygoplichthys lituratus (Kner, 1854)

Ancistrus lituratus Kner, 1854: 285, pl. 5 (fig. 3). Type locality: aus dem Rio Guaporé bei cidade do Matogrosso [Amazon system, Brazil]. Lectotype: NMW 16416; designated by Weber (1992: 28). Originally as Anc. lituratus.

Distribution: Madeira River basin, Bolivia and Brazil (Weber, 2003).

Remarks: Redescribed in Weber (1992: 28).

Pterygoplichthys multiradiatus (Hancock, 1828)

Hypostomus multiradiatus Hancock, 1828: 246. Type locality: Demerara [in title] [...] Lakes [...] on the slime. Holotype: BMNH 1857.6.13.162.

Distribution: Orinoco River basin, possibly also in Guyana (Weber, 2003). 
Remarks: Redescription and type locality interpretation in Weber (1992: 9, 27).

Pterygoplichthys pardalis (Castelnau, 1855)

Hypostomus pardalis Castelnau, 1855: 42, pl. 20 (fig. 3). Type locality: l'Amazone. Holotype: MNHN a-9574.

Liposarcus varius Cope, 1872a: 284. Type locality: The Ambyiacu river, which empties into the Amazon near to Pebas, in Eastern Equador, some distance east of the Napo [Peru]. Lectotype: ANSP 21931, designated by Fowler (1915: 233).

Liposarcus jeanesianus Cope, 1874b: 135. Type locality: Nauta, Peru [= Marañon River, Amazon basin]. Lectotype: ANSP 8241, designated by Weber (1992: 27).

Distribution: Lower, middle and upper Amazon River basin (Weber, 2003).

Remarks: Redescribed in Weber (1992: 10).

Pterygoplichthys parnaibae (Weber, 1991)

Glyptoperichthys parnaibae Weber, 1991: 641. Type locality: Lac de Parnaguá, Rio Paraim, bassin de Parnaíba, sys. côtier brésilien; Maranhão, Brésil. Holotype: NMW 48034, illustrated in Weber (1992: 21, pl. 16 b).

Distribution: Parnaíba River basin, Brazil (Weber, 2003).

Pterygoplichthys punctatus (Kner, 1854)

Loricaria punctata Kner, 1854: 281. Type locality: S. Vincente [...] aus einer Lache [Brazil]. Holotype: NMW 76587.

Distribution: Madeira, Purus, Jurua and Marañon River basins (Weber, 2003).

Remarks: Redescribed Weber (1992: 22).

Pterygoplichthys scrophus (Cope, 1874)

Liposarcus scrophus Cope, 1874b: 136. Type locality: Nauta [Peru]. Syntypes: USNM 132587 (2).

Distribution: Marañon and Ucayali River basins, Peru (Weber, 2003).

Remarks: Redescribed in Page et al. (1996: 186).

Pterygoplichthys undecimalis (Steindachner, 1878)

Chaetostomus undecimalis Steindachner, 1878a: 90. Type locality: Cienaga Grande de Santa Marta, Magdalena basin, 40 km east of Barranquilla, Colombia. Lectotype: NMW 47224, designated in Weber (1992: 26); illustrated in Steindachner (1879d: 43, pl. 8).

Distribution: Magdalena River basin, Colombia (Weber, 2003); also Cauca and Catatumbo River basins (Maldonado-Ocampo et al., 2005).

Pterygoplichthys xinguensis (Weber, 1991)

Glyptoperichthys xinguensis Weber, 1991: 640. Type locality: Rio Fresco, affl. du Rio Xingu, système de l'Amazone; Aldeio Gorotire, municipe de São Felix do Xingu, Pará, Brésil. Holotype: MZUSP 35961, illustrated in Weber (1992: pl. 14, fig. b).

Distribution: Xingu River basin, Brazil (Weber, 2003).

Pterygoplichthys zuliaensis Weber, 1991

Pterygoplichthys zuliaensis Weber, 1991: 638. Type locality: Río Santa Ana, bassin du Maracaibo, Hacienda Río Grande, $9^{\circ} 36^{\prime} 20^{\prime \prime N}, 72^{\circ} 07^{\prime} 00^{\prime} \mathrm{W}$, état de Zulia, Venezuela. Holotype: MBUCV V-14653; holotype illustrated in Weber (1992: pl. 4b).

Distribution: Lake Maracaibo basin, Venezuela (Weber, 2003).

PYXILORICARIA Isbrücker \& Nijssen, 1984

Pyxiloricaria Isbrücker \& Nijssen, 1984b: 163. Type species: Pyxiloricaria menezesi Isbrücker \& Nijssen, 1984. Type by original designation. Gender: Feminine.

Pyxiloricaria menezesi Isbrücker \& Nijssen, 1984

Pyxiloricaria menezesi Isbrücker \& Nijssen, 1984b: 164, fig. 1. Type locality: Brazil, Mato Grosso do Sul, Lagoas marginais da rodovia Transpantaneira, município de Miranda $\left(20^{\circ} 10^{\prime} \mathrm{S}, 56^{\circ} 19^{\prime} \mathrm{W}\right)$.

Distribution: Paraguay River basin, Brazil (Isbrücker \& Nijssen, 1984).

REGANELLA Eigenmann, 1905

Hemiodon Kner, 1853a: 24. Type species: Hemiodon depressus Kner, 1853. Type by subsequent designation by 
Bleeker (1862-63: 4). Gender: Masculine. Preoccupied by Hemiodon Swainson, 1840, in Mollusca; replaced by Reganella Eigenmann, 1905.

Reganella Eigenmann, 1905: 793. Type species: Hemiodon depressus Kner, 1853. Type by being a replacement name. Gender: Feminine. Replacement for Hemiodon Kner, 1853.

Reganella depressa (Kner, 1853)

Hemiodon depressus Kner, 1853a: 12, 27, pl. 7 (fig. 1a-c). Type locality: Rio negro und Marabitanos [restricted to Marabitanos by lectotype designation]. Lectotype: NMW 9438, designated by Isbrücker \& Nijssen (1974b: 219). Originally as Hem. depressus.

Distribution: Negro, Branco, and Tapajós River basins, Brazil (Ferraris, 2003c).

RHADINOLORICARIA Isbrücker \& Nijssen, 1974

Rhadinoloricaria Isbrücker \& Nijssen, 1974a: 73. Type species: Loricaria macromystax Günther, 1869. Type by original designation. Gender: Feminine.

Rhadinoloricaria macromystax (Günther, 1869)

Loricaria macromystax Günther, 1869: 426, figs. 5-6. Type locality: Peruvian Amazonas. Holotype: BMNH 1869.5.21.8.

Distribution: Upper Amazon River basin (Ferraris, 2003c).

RHINELEPIS Spix \& Agassiz, 1829

Rhinelepis Spix \& Agassiz, 1829: 2. Type species: Rhinelepis aspera Spix \& Agassiz, 1829. Type by monotypy. Gender: Feminine.

Phylogenetic position: Armbruster (1998b).

Rhinelepis aspera Spix \& Agassiz, 1829

Rhinelepis aspera Spix \& Agassiz, 1829: 4, pl. 2 (figs. 1-2). Type locality: in flumine S. Francisci [Brazil]. Holotype: Whereabouts unknown (Kottelat, 1988).

Distribution: São Francisco and upper Paraná River basins, Brazil (Weber, 2003).

Rhinelepis strigosa Valenciennes, 1840

Rhinelepis strigosa Valenciennes, in Cuvier \& Valenciennes, 1840: 480 [354 in Strasbourg deluxe edition]. Type locality: dans le Parana et d'autres rìvières de la province de Corrientes [Argentina]. Holotype: MNHN a-9571.

Distribution: Paraná and Uruguay River basins (Weber, 2003).

RICOLA Isbrücker \& Nijssen, 1978

Ricola Isbrücker \& Nijssen, 1978a: 182. Type species: Loricaria macrops Regan, 1904. Type by original designation. Gender: Feminine.

Ricola macrops (Regan, 1904)

Loricaria macrops Regan, 1904b: 290, pl. 17 (fig. 3). Type locality: the R. de la Plata. Lectotype: BMNH 1868.9.16.1, designated by Isbrücker \& Nijssen (1978a: 184).

Distribution: Lower Paraná River basin (Isbrücker \& Nijssen, 1978).

Remarks: Reviewed in Ringuelet et al. (1967).

RINELORICARIA Bleeker, 1862

Rineloricaria Bleeker, 1862 (in Bleeker, 1862-63): 3. Type species: Loricaria lima Kner, 1853. Type by original designation. Gender: Feminine.

Leliella Isbrücker, in Isbrücker et al., 2001: 20. Type species: Rineloricaria heteroptera Isbrucker \& Nijssen, 1975. Type by original designation. Gender: Feminine.

Rineloricaria aequalicuspis Reis \& Cardoso, 2001

Rineloricaria aequalicuspis Reis \& Cardoso, 2001: 324, fig. 3. Type locality: Brazil: Rio Grande do Sul: rio Três Forquilhas, opposie to church of Vila Itati (29³0'43"S 5005'31"W). Holotype: MCP 25852.

Distribution: Três Forquilhas and Maquiné River basins, Brazil (Reis \& Cardoso, 2001).

Rineloricaria cadeae (Hensel, 1868) 
Loricaria cadeae Hensel, 1868: 369. Type locality: Rio Cadéa [Rio Grande do Sul, Brazil]. Lectotype: ZMB 7430, designated by Isbrücker (1979a: 112).

Distribution: Laguna dos Patos basin, Brazil (Ferraris, 2003c).

Rineloricaria catamarcensis (Berg, 1895)

Loricaria catamarcensis Berg, 1895: 137. Type locality: Provincia Catamarca, Argentina. Holotype: at MACN (not found, Isbrücker, 1979a: 113).

Distribution: Tala and Salí River basins in Catamarca Province, Argentina (Ringuelet et al., 1967).

Remarks: Reviewed in Ringuelet et al. (1967).

Rineloricaria cubataonis (Steindachner, 1907)

Loricaria cubataonis Steindachner, 1907e: 489. Type locality: aus dem Flusse Cubatão, im Staate Santa Catharina bei Theoresopolis (Brasilien). Holotype: NMW (not found, Isbrücker, 1979a: 113).

Distribution: Coastal Atlantic rivers of southeastern Brazil (Ferraris, 2003c).

Rineloricaria felipponei (Fowler, 1943)

Loricaria felipponei Fowler, 1943b: 319, figs. 7-9. Type locality: Uruguay. Holotype: ANSP 70324.

Distribution: Lower La Plata River basin, Uruguay (Ferraris, 2003c).

Rineloricaria henselii (Steindachner, 1907)

Loricaria henselii Steindachner, 1907e: 488. Type locality: Aus dem Flusse Cubatão im Staate Santa Catherina bei Theresopolis (Brasilien). Holotype: at NMW (not found, Isbrücker, 1979a: 113).

Distribution: Cubatão River in southern Santa Catarina State, Brazil (Ferraris, 2003c).

Rineloricaria heteroptera Isbrücker \& Nijssen, 1976

Rineloricaria heteroptera Isbrücker \& Nijssen, 1976b: 112, fig. 1. Type locality: Brazil, Est. Amazonas. Creek in reserve Ducke, near Manaus (0306'S, 6000'W). Holotype: IRSNB 555.

Distribution: Amazon River basin, near Manaus, Brazil (Ferraris, 2003c).

Remarks: Recently designated as type of a monotypic genus, Leliella, by Isbrücker (2001: 20).

Rineloricaria jaraguensis (Steindachner, 1909)

Loricaria jaraguensis Steindachner, 1909a: 196. Type locality: dem Jaraguá und dessen Nebenflussen in brasilianischen Staate Sa. Catharina. Lectotype: NMW 44886, designated by Isbrücker (1979a: 113).

Distribution: Jaraguá River basin in Santa Catarina State, Brazil (Ferraris, 2003c).

Rineloricaria kronei (Miranda Ribeiro, 1911)

Loricaria kronei Miranda Ribeiro, 1911: 132, pl. 34 (fig. 2). Type locality: Rio Iporanga, S. Paulo [Brazil]. Holotype: MNRJ (not found).

Distribution: Ribeira de Iguape River basin, Brazil (Ferraris, 2003c).

Rineloricaria latirostris (Boulenger, 1900)

Loricaria latirostris Boulenger, 1900b: 165. Type locality: Southern Brazil, Province São Paulo, Mogy-guassu River about 250 miles inland of Santos. Lectotype: BMNH 1899.12.18.6, designated by Isbrücker (1979a: 112).

Loricaria paulina Boulenger, 1900b: 166. Type locality: Southern Brazil, Province São Paulo, Mogy-guassu River. Lectotype: BMNH 1899.12.18.8, designated by Isbrücker (1979a: 112).

Distribution: Upper Paraná River basin, Brazil (Ferraris, 2003c).

Remarks: Loricaria latirostris treated as valid following first reviser action of Regan (1904b: 283).

Rineloricaria lima (Kner, 1853)

Loricaria lima Kner, 1853a: 12, 25, pl. 6 (fig. 1a-b). Type locality: aus Brasilien durch Natterer, ober ohne nähere Angabe des Fundortes. Holotype: NMW (not found, Isbrücker, 1980: 107). Originally as Lor.? lima.

Distribution: Brazil (Ferraris, 2003c).

Rineloricaria longicauda Reis, 1983

Rineloricaria longicauda Reis, 1983: 64, figs. 5-7. Type locality: Banhado do Taim, município de Rio Grande, RS [Brazil]. Holotype: MZUSP 16078.

Distribution: Coastal drainages of Rio Grande do Sul State, between Chuí and Tramandaí, Brazil (Reis, 1983).

Rineloricaria maquinensis Reis \& Cardoso, 2001

Rineloricaria maquinensis Reis \& Cardoso, 2001: 321, fig. 1. Type locality: Brazil: Rio Grande do Sul: arroio Água Parada, tributary to rio Maquiné (approximately $29^{\circ} 40^{\prime} \mathrm{S} 50^{\circ} 12^{\prime} \mathrm{W}$ ), Maquiné. Holotype: MCP 25817. 
Distribution: Maquiné and Araranguá River basins in southern Brazil (Reis \& Cardoso, 2001).

Rineloricaria microlepidogaster (Regan, 1904)

Loricaria microlepidogaster Regan, 1904b: 283, pl. 15 (fig. 4). Type locality: Rio Grande do Sul [Brazil]. Holotype: BMNH 1884.2.5.41.

Distribution: Laguna dos Patos basin, Brazil (Ferraris, 2003c).

Rineloricaria microlepidota (Steindachner, 1907)

Loricaria lima microlepidota Steindachner, 1907b: 154. Type locality: dem Juruá [Brazil]. Syntypes: at NMW (not found: Isbrücker, 1979a: 113). Originally as Loricaria lima var. microlepidota.

Distribution: Juruá River basin, Brazil (Ferraris, 2003c).

Rineloricaria misionera Rodríguez \& Miquelarena, 2005

Rineloricaria misionera Rodríguez \& Miquelarena, 2005: 5, fig. 1. Type locality: arroyo Cuña-Pirú (2708'S, 5454'W) Canguás Department, Misiones Province, Argentina. Holotype: ILPLA 1698.

Distribution: Paraná and Uruguay River basins, Misiones, Argentina (Rodríguez \& Miquelarena, 2005).

Rineloricaria pareiacantha (Fowler, 1943)

Loricaria pareiacantha Fowler, 1943b: 321, figs. 10-12. Type locality: Rio Santa Lucia, Canelones [Uruguay]. Holotype: ANSP 67815.

Distribution: Santa Lucia River basin, Uruguay (Ferraris, 2003c).

Rineloricaria pentamaculata Langeani \& Araujo, 1994

Rineloricaria pentamaculata Langeani \& Araujo, 1994: 153, fig. 1. Type locality: rio Turvo, Ourinhos, São Paulo, Brasil. Holotype: MZUSP 43051.

Distribution: Turvo River basin in São Paulo State, Brazil (Langeani \& Araujo, 1994).

Rineloricaria quadrensis Reis, 1983

Rineloricaria quadrensis Reis, 1983: 66, figs. 15-17. Type locality: Lagoa dos Quadros, município de Osório, RS [Brazil]. Holotype: MZUSP 14363.

Distribution: Coastal streams and lagoons in Rio Grande do Sul, between Tramandaí and Torres, Brazil (Reis, 1983).

Rineloricaria steindachneri (Regan, 1904)

Loricaria steindachneri Regan, 1904b: 281. Type locality: Eastern Brazil, from the R. Preto to the R. Parahyba. Lectotype: NMW 45016: 1, designated by Isbrücker (1979a: 113).

Distribution: Coastal rivers of northeastern Brazil (Ferraris, 2003c).

Rineloricaria strigilata (Hensel, 1868)

Loricaria strigilata Hensel, 1868: 368. Type locality: Südbrasilien, in eimen steinigen Bache bei Santa Cruz. Holotype: ZMB 7429.

Distribution: Laguna dos Patos basin, Brazil (Ferraris, 2003c).

Rineloricaria thrissoceps (Fowler, 1943)

Loricaria thrissoceps Fowler, 1943b: 322, figs. 13-15. Type locality: Río Santa Lucia, Canelones [Uruguay]. Holotype: ANSP 67796.

Distribution: Santa Lucia River basin, Uruguay (Ferraris, 2003c).

SCHIZOLECIS Britski \& Garavello, 1984

Schizolecis Britski \& Garavello, 1984: 228. Type species: Microlepidogaster guntheri Miranda Ribeiro, 1918. Type by original designation. Gender: Feminine.

Schizolecis guntheri (Miranda Ribeiro, 1918)

Microlepidogaster guntheri Miranda Ribeiro, 1918d: 634. Type locality: Praia do Piraíque, Ilha de São Sebastião, Estado de São Paulo [Brazil]. Lectotype: MZUSP 1015, designated by Britski (1969: 208).

Pseudotocinclus ribeiroi Gomes, 1955: 222, pl. 1. Type locality: Rio Branco, Bacia do rio Itanhaem, São Paulo, Brazil. Holotype: EEBP 110.

Distribution: Southeastern Brazil (Schaefer, 2003c).

Remarks:There is some controversy regarding the correct spelling of the valid name for this species. The name was listed as Microlepidogaster guntheri Miranda Ribeiro (1918d: 634) with a statement in a footnote on that page that the species name was a patronym for a "Sr. F. Gunther." However, Isbrücker (1980: 85-86) reported that the 
species was listed as Microlepidogaster güntheri in a later article (Miranda Ribeiro, 1918e: 717) in the same volume of the journal. If the two papers were published simultaneously, the two spellings would need to be treated as alternate spellings that require further action. Isbrücker (1980: 85-86) noted both spellings and treated $M$. guentheri as valid, and would thereby act as first reviser, if necessary.

SCOBINANCISTRUS Isbrücker \& Nijssen, 1989

Scobinancistrus Isbrücker \& Nijssen, 1989: 542. Type species: Scobinancistrus pariolispos Isbrücker \& Nijssen, 1989. Type by original designation. Gender: Masculine.

Scobinancistrus aureatus Burgess, 1994

Scobinancistrus aureatus Burgess, 1994: 237, figure on p. 236. Type locality: Ilha da Fazenda, Rio Xingu, Estado Para, Brazil. Holotype: MZUSP 47690.

Distribution: Xingu River basin, Brazil (Fisch-Muller, 2003).

Scobinancistrus pariolispos Isbrücker \& Nijssen, 1989

Scobinancistrus pariolispos Isbrücker \& Nijssen, 1989: 542, fig. 1. Type locality: Brasilien, Est. Pará, Rio Tocantins, Jatobal. Holotype: INPA 1076.

Distribution: Tocantins and Tapajós River basins, Brazil (Fisch-Muller, 2003).

SPATULORICARIA Schultz, 1944

Spatuloricaria Schultz, 1944c: 287, 334. Type species: Spatuloricaria phelpsi Schultz, 1944. Type by original designation. Gender: Feminine.

Euacanthagenys Fowler, 1945a: 123. Type species: Euacanthagenys caquetae Fowler, 1945. Type by original designation. Gender: Feminine.

Spatuloricaria atratoensis Schultz, 1944

Spatuloricaria atratoensis Schultz, 1944a: 155. Type locality: Río Truando, tributary of Río Atrato, Colombia. Holotype: USNM 93810.

Distribution: Atrato River basin, Colombia (Ferraris, 2003c).

Spatuloricaria caquetae (Fowler, 1943)

Loricaria caquetae Fowler, 1943a: 261, figs. 60-62. Type locality: Florencia, Rio Orteguasa, Colombia. Holotype: ANSP 70526.

Distribution: Orteguasa River basin, Colombia (Ferraris, 2003c).

Spatuloricaria curvispina (Dahl, 1942)

Loricaria curvispina Dahl, 1942: 85, figs. 5-7. Type locality: Río Batatal, tributary to Río San Jorge, which is next to Rio Cauca the largest tributary to Río Magdalena. Department of Bolivar, Republic of Colombia. Holotype: possibly at ZMUL.

Distribution: Magdalena River basin, Colombia (Ferraris, 2003c).

Spatuloricaria euacanthagenys Isbrücker, 1979

Euacanthagenys caquetae Fowler, 1945a: 125, figs. 37-40. Type locality: Morelia, Rio Caquetá drainage, Colombia. Holotype: ANSP 71718; holotype illustrated in Isbrücker (1979a: 111, figs. 24, 25, 26, 27b). Preoccupied in Spatuloricaria by Loricaria caquetae Fowler, 1943; replaced by Spatuloricaria euacanthagenys Isbrücker, 1979.

Spatuloricaria euacanthagenys Isbrücker, 1979a: 113, figs. 24-26. Type locality: Morelia, Río Caquetá drainage, Colombia. Holotype: ANSP 71718; holotype illustrated in Isbrücker (1979a: 113, figs 24, 25, 26, 27b). Replacement for Euacanthagenys caquetae Fowler, 1945.

Distribution: Caquetá River basin, Colombia (Ferraris, 2003c).

Spatuloricaria evansii (Boulenger, 1892)

Loricaria Evansii Boulenger, 1892b: 10, pl. 1. Type locality: Jangada [Matto Grosso, Brazil]. Holotype: BMNH 1892.4.20.29; holotype illustrated in Isbrücker (1973: 174, fig. 1).

? Loricaria cirrhosa Perugia, 1897: 22. Type locality: Rio Beni, Missioni Mosetenes, Bolivia. Lectotype: MSNG 8850 (1); lectotype designated by, and illustrated in, Isbrücker (1973: 174, fig. 2). Preoccupied by Loricaria cirrhosa Bloch \& Schneider, 1801; apparently not replaced. 
Distribution: Paraguay and Beni River basins (Ferraris, 2003c).

Spatuloricaria fimbriata (Eigenmann \& Vance, 1912)

Loricaria fimbriata Eigenmann \& Vance, in Eigenmann, 1912a: 12. Type locality: Boca de Certegai [Colombia]. Holotype: FMNH 55117; holotype illustrated in Eigenmann (1922b: 93, pl. 15, figs.1, 2).

Distribution: Tuira River, Panama and Magdalena River and Boca de Certegai, Colombia (Ferraris, 2003c).

Spatuloricaria gymnogaster (Eigenmann \& Vance, 1912)

Loricaria gymnogaster Eigenmann \& Vance, in Eigenmann, 1912a: 12. Type locality: Apulo [Colombia]. Holotype: CAS 28773; holotype illustrated in Eigenmann (1922b: 92, pl. 14, figs. 3-4).

Distribution: Upper Magdalena River basin, Colombia (Maldonado-Ocampo et al., 2005).

Spatuloricaria lagoichthys (Schultz, 1944)

Loricaria gymnogaster lagoichthys Schultz, 1944c: 331, pl. 13. Type locality: Río Palmar near Totuma, about 100 km southwest of Maracaibo [Venezuela]. Holotype: USNM 121092. Teeth of holotype illustrated in Isbrücker (1979a: 111, fig. 27a).

Distribution: Lake Maracaibo basin, Venezuela (Ferraris, 2003c).

Spatuloricaria nudiventris (Valenciennes, 1840)

Loricaria nudiventris Valenciennes, in Cuvier \& Valenciennes, 1840b: 469 (346 of Strasbourg deluxe edition). Type locality: la rivière de Saint-François au Brésil. Holotype: MNHN a-9558.

Distribution: São Francisco River basin, Brazil (Ferraris, 2003c).

Spatuloricaria phelpsi Schultz, 1944

Spatuloricaria phelpsi Schultz, 1944c: 335, pl. 14. Type locality: Río Socuy, $3 \mathrm{~km}$ above its mouth, Maracaibo basin, Venezuela. Holotype: USNM 121121. Teeth of holotype illustrated in Isbrücker (1979a: 112, fig. 27c).

Distribution: Lake Maracaibo basin, Venezuela (Ferraris, 2003c).

Spatuloricaria puganensis (Pearson, 1937)

Loricaria puganensis Pearson, 1937: 96. Type locality: Pusoc, Rio Marañon, Peru. Lectotype: CAS 28776, designated by Isbrücker (1979a: 113).

Distribution: Marañon River basin, Peru (Ferraris, 2003c).

SPECTRACANTHICUS Nijssen \& Isbrücker, 1987

Spectracanthicus Nijssen \& Isbrücker, 1987: 93. Type species: Spectracanthicus murinus Nijssen \& Isbrücker, 1987. Type by original designation. Gender: Masculine.

Spectracanthicus murinus Nijssen \& Isbrücker, 1987

Spectracanthicus murinus Nijssen \& Isbrücker, 1987: 94, fig. 1 (middle). Type locality: Brésil, Est. Pará, système du Rio Tapajós: Poça de Pedra no Rio Tapajós, São Luis. Holotype: MZUSP 22011.

Distribution: Tapajós River basin, Brazil (Fisch-Muller, 2003).

SQUALIFORMA Isbrücker \& Michels, 2001

Squaliforma Isbrücker \& Michels, in Isbrücker et al., 2001: 22. Type species: Hypostomus horridus Kner, 1854. Type by original designation. Gender: Feminine.

Squaliforma annae (Steindachner, 1881)

Plecostomus annae Steindachner, 1881c: 112, pl. 3 (figs. 2-2a). Type locality: Pará [= Belem, Brazil]. Holotype: NMW 44073. Listed as Chaetostomus annae in figure caption (p. 146).

Distribution: Guamá River basin, Brazil (Weber, 2003).

Squaliforma biseriata (Cope, 1872)

Plecostomus biseriatus Cope, 1872a: 285. Type locality: the Amazon, between the mouth of the Rio Negro and the Peruvian Amazon or Ucayale River. Holotype: ANSP 8279.

Distribution: Upper Amazon River basin, Brazil (Weber, 2003).

Squaliforma emarginata (Valenciennes, 1840)

Hypostomus emarginatus Valenciennes, in Cuvier \& Valenciennes, 1840b: 500 (369 in Strasbourg deluxe edition).

Type locality: Probablement orìginaire du Brésil. Holotype: MNHN a-9447 (dry).

Distribution: Lower, middle and upper Amazon River basin (Weber, 2003). 
Squaliforma gomesi (Fowler, 1941)

Plecostomus iheringi Fowler, 1941a: 150, figs. 50-52. Type locality: Ceará [Brazil]. Holotype: ANSP 69409. Preoccupied by Plecostomus iheringii Regan, 1908; replaced by Plecostomus gomesi Fowler, 1942.

Plecostomus gomesi Fowler, 1942: [1]. Type locality: Ceará [Brazil]. Holotype: ANSP 69409. Replacement name for Plecostomus iheringi Fowler, 1941; preoccupied by Plecostomus iheringii Regan, 1908.

Distribution: Jaguaribe River basin, Brazil (Weber, 2003).

Squaliforma horrida (Kner, 1854)

Hypostomus horridus Kner, 1854: 259, pl. 1 (fig. 1). Type locality: Forte do Principe am Rio Guaporé, Brazil. Syntypes (3): NMW 16325 (1), NMW 86604 (1). Originally proposed as Hyp. horridus.

Distribution: Madeira River basin, Brazil (Weber, 2003).

Squaliforma phrixosoma (Fowler, 1940)

Plecostomus phrixosoma Fowler, 1940a: 233, figs. 21-23. Type locality: Ucayali River basin, Contamana, Peru. Holotype: ANSP 68650.

Distribution: Upper Amazon River basin, Peru (Weber, 2003).

Squaliforma scopularia (Cope, 1871)

Plecostomus scopularius Cope, 1871a: 55. Type locality: the Amazon above the mouth of the Rio Negro. Lectotype: ANSP 8081, designated by Fowler (1915: 233); illustrated in Cope (1872a: pl. 16, no. 1).

Distribution: Middle Amazon River basin, Brazil (Weber, 2003).

Squaliforma squalina (Jardine, 1841)

Hypostoma squalinum Jardine, in Schomburgk, 1841: 142, pl. 2. Type locality: Rios Branco, Negro, and Essequibo. No types known.

Distribution: Negro and Branco River basins; Essequibo River basin (Weber, 2003).

Squaliforma tenuicauda (Steindachner, 1878)

Plecostomus tenuicauda Steindachner, 1878a: 90. Type locality: Magdalenen-Stromes [Colombia]. Syntypes: MSNG 8856 (1), NMW 42596 (1), NMW 44263 (1), NMW 44264 (1), NMW 44265 (1), NMW 44266 (3), NMW 44268 (1), NMW 44294 (1), ZMUC P 30172 (1). One syntype illustrated in Steindachner (1879d: pl. 6). Distribution: Magdalena River basin, Colombia (Weber, 2003).

Squaliforma tenuis (Boeseman, 1968)

Hypostomus tenuis Boeseman, 1968: 64, pl. 14 (fig. 3). Type locality: Near Paramaribo, Surinam. Holotype: RMNH 16198.

Distribution: Possibly Suriname River basin; known only from holotype (Weber, 2003).

Remarks: Type locality considered to be in error by Weber (2003).

Squaliforma villarsi (Lütken, 1874)

Plecostomus villarsi Lütken, 1874a: 211. Type locality: Caracas. Syntypes: ZMUC P 30149 (1), ZMUC P 30150 (1).

Distribution: Venezuela (Weber, 2003).

Squaliforma virescens (Cope, 1874)

Plecostomus virescens Cope, 1874b: 137. Type locality: not explicitly given [Upper Amazon]. Syntypes: ANSP $21280(4)$

Distribution: Upper Amazon River basin, Peru (?)(Weber, 2003).

STURISOMA Swainson, 1838

Sturisoma Swainson, 1838: 333. Type species: Loricaria rostrata Spix \& Agassiz, 1829. Type by monotypy. Gender: Neuter. See also Swainson (1839: 304).

Oxyloricaria Bleeker, 1862 (in Bleeker, 1862-63): 3. Type species: Loricaria barbata Kner, 1853. Type by original designation. Gender: Feminine.

Parasturisoma Miranda Ribeiro, 1911: 109. Type species: Loricaria (Rineloricaria) brevirostris Eigenmann \& Eigenmann, 1889. Type by monotypy. Gender: Neuter.

Sturisoma aureum (Steindachner, 1900)

Loricaria aurea Steindachner, 1900: 206. Type locality: Bodega central, Rio Magdalena [Colombia]. Holotype: at NMW. 
Distribution: Magdalena, San Jorge and Cesar River basins, Colombia (Ferraris, 2003c).

Sturisoma barbatum (Kner, 1853)

Loricaria barbata Kner, 1853a: 12, 23, pl. 5. Type locality: Rio Cuiabá, Cujaba-Fluss [Brazil]. Holotype: NMW 46155. Originally Lor. barbata.

Distribution: Paraguay River basin, Brazil (Ferraris, 2003c).

Sturisoma brevirostre (Eigenmann \& Eigenmann, 1889)

Loricaria brevirostris Eigenmann \& Eigenmann, 1889b: 35. Type locality: Iça [Brazil]. Holotype: MCZ 8095.

Distribution: Içá River basin, Brazil (Ferraris, 2003c).

Sturisoma dariense (Meek \& Hildebrand, 1913)

Oxyloricaria dariensis Meek \& Hildebrand, 1913: 81. Type locality: Rio Tuyra, Boca de Cupe, Panama. Holotype: FMNH 7584.

Distribution: Tuira River basin, Panama (Ferraris, 2003c).

Sturisoma festivum Myers, 1942

Sturisoma festivum Myers, 1942: 100, fig. 8. Type locality: Río Monay, $35 \mathrm{~km}$ north of Trujillo, Motatan system, Maracaibo basin, Venezuela. Holotype: SU 36505.

Distribution: Lake Maracaibo basin (Ferraris, 2003c).

Sturisoma frenatum (Boulenger, 1902)

Loricaria frenata Boulenger, 1902a: 69. Type locality: Northwestern Ecuador, St. Javier (60 feet), Saildero (350 feet), and Rio Durango (350 feet) [restricted to Salidero, Ecuador by lectotype designation]. Lectotype: BMNH 1901.8.3.29, designated by Isbrücker (1979a: 113).

Distribution: Pacific versant rivers, Ecuador (Ferraris, 2003c).

Sturisoma guentheri (Regan, 1904)

Oxyloricaria guentheri Regan, 1904b: 299, pl. 18 (fig. 1). Type locality: Xeberos, Upper Amazon [Peru]. Holotype: BMNH 1867.6.13.36.

Distribution: Upper Amazon River basin, Peru (Ferraris, 2003c).

Sturisoma kneri Ghazzi, 2005

Sturisoma kneri Ghazzi, 2005: 559, fig. 1. Type locality: Venezuela, Lago Maracaibo. Holotype: MZUT 764.

Distribution: Maracaibo Lake basin, Venezuela (Ghazzi, 2005).

Remarks: Previous citations of this name (as well as Loricaria kneri) were shown to be unavailable for purposes of zoological nomenclature (Ghazzi, 2005).

Sturisoma lyra (Regan, 1904)

Oxyloricaria lyra Regan, 1904b: 300, pl. 19 (fig. 2). Type locality: Rio Jurua. Lectotype: BMNH 1897.12.1.64, designated by Isbrücker (1979a: 113).

Distribution: Juruá River basin, middle Amazon River drainage (Ferraris, 2003c).

Sturisoma monopelte Fowler, 1914

Sturisoma monopelte Fowler, 1914: 274, fig. 18. Type locality: Rupununi River, British Guiana ... in the highlands of British Guiana, approximately secured in North Latitude $2^{\circ}$ to $3^{\circ}$, and West Longitude $50^{\circ} 20^{\prime}$. Holotype: ANSP 39346.

Distribution: Rupununi River basin, Guyana (Ferraris, 2003c).

Sturisoma nigrirostrum Fowler, 1940

Sturiosoma nigrirostrum Fowler, 1940a: 249, figs. 48-50. Type locality: Ucayali River basin, Contamana, Peru. Holotype: ANSP 68666.

Distribution: Ucayali River basin, Peru (Ferraris, 2003c).

Sturisoma panamense (Eigenmann \& Eigenmann, 1889)

Loricaria panamensis Eigenmann \& Eigenmann, 1889b: 34. Type locality: Panama. Holotype: MCZ 8126.

Distribution: Pacific slope rivers of Panama, Colombia, and Ecuador (Ferraris, 2003c); Cauca River basin, Colombia (Maldonado-Ocampo et al., 2005).

Sturisoma robustum (Regan, 1904)

Oxyloricaria robusta Regan, 1904b: 300, pl. 19 (fig. 1). Type locality: Rio Paraguay. Lectotype: BMNH 1895.5.17.89, designated by Isbrücker (1979a: 113). 
Distribution: La Plata River basin (Ringuelet et al., 1967).

Remarks: Reviewed in Ringuelet et al. (1967).

Sturisoma rostratum (Spix \& Agassiz, 1829)

Loricaria rostrata Spix \& Agassiz, 1829: 5, pl. 3. Type locality: Brasiliae fluviis. Holotype: lost (Isbrücker, 1979a: 113).

Distribution: Brazil (Ferraris, 2003c).

Sturisoma tenuirostre (Steindachner, 1910)

Oxyloricaria tenuirostris Steindachner, 1910b: 410. Type locality: dem Rio Meta in Venezuela. Holotype: NMW 45453.

Distribution: Meta River basin, Venezuela (Ferraris, 2003c).

STURISOMATICHTHYS Isbrücker \& Nijssen, 1979

Sturisomatichthys Isbrücker \& Nijssen, in Isbrücker, 1979a: 91. Type species: Oxyloricaria leightoni Regan, 1912. Type by original designation. Gender: Masculine.

Sturisomatichthys caquetae (Fowler, 1945)

Harttia caquetae Fowler, 1945a: 126, figs. 41-43. Type locality: Morelia, Rio Caquetá drainage, Colombia. Holotype: ANSP 71719.

Distribution: Caquetá River basin, Colombia (Ferraris, 2003c).

Sturisomatichthys citurensis (Meek \& Hildebrand, 1913)

Oxyloricaria citurensis Meek \& Hildebrand, 1913: 82. Type locality: Río Cupe at Cituro, Panama, Tuyra River basin. Holotype: FMNH 7585.

Distribution: Tuira and Bayano River basins, Panama (Ferraris, 2003c).

Sturisomatichthys leightoni (Regan, 1912)

Oxyloricaria leightoni Regan, 1912b: 669, pl. 77 (fig. 2). Type locality: Honda, Colombia, 300-400 ft. Lectotype: BMNH 1909.7.23.45, designated by Isbrücker (1979a: 113).

Distribution: Upper Magdalena and Cauca River basins, Colombia (Maldonado-Ocampo et al., 2005).

Sturisomatichthys tamanae (Regan, 1912)

Oxyloricaria tamanae Regan, 1912b: 669, pl. 77 (figs.1, 1a \& b). Type locality: the Rio Tamana, Río San Juan, Choco, S.W. Colombia. Lectotype: BMNH 1910.7.11.133, designated by Isbrücker (1979a: 113), and illustrated in Isbrücker (1979a: 96, fig. 7).

Distribution: San Juan River basin, Colombia (Ferraris, 2003c).

\section{Species inquirendae, Loricariidae}

Microlepidogaster bourguyi Miranda Ribeiro, 1911: 90. Type locality: Brasil. Syntypes (12): possibly NMRJ 1036.

Ancistrus salgadae Fowler, 1941: 154. Fig. 57. Type locality: Rio Salgado, Icá, Ceará; eastern Brazil. Holotype: ANSP 69440. A juvenile, identification uncertain (S. Fisch-Muller, 2003).

Ancistrus mattogrossenssis Miranda Ribeiro, 1912: 5. Type locality: Not stated. Syntypes (2): Whereabouts unknown, not found at MNRJ or MZUSP (Fisch-Muller, 2003). Original spelling of name considered to be incorrect by FischMuller (2003) and corrected the spelling to Ancistrus mattogrossensis, following Miranda Ribeiro (1918c: 114).

\section{MALAPTERURIDAE Bleeker, 1858}

Malapterurini Bleeker, 1858b: ix. Type genus: Malapterurus La Cepède, 1803.

Revision: Poll \& Gosse (1969); Norris (2002).

Taxonomic summary: Gosse (1986a).

Phylogeny: Howes (1985).

Reviews: Teugels (1992b), west Africa.

2 genera, 19 species; no named fossil taxa. 
MALAPTERURUS La Cepède, 1803

Malapterurus La Cepède, 1803: 90. Type species: Silurus electricus Gmelin, 1789. Type by designation under plenary powers; placed on Official List (ICZN Opinion 93, Direction 56). Gender: Masculine.

Anacanthus Minding 1832: 117. Type species: Silurus electricus Gmelin, 1789. Type by monotypy. Preoccupied by Anacanthus Gray, 1830, in fishes. Gender: Masculine.

Malopterurus Agassiz, 1846: 223. Type species: Silurus electricus Gmelin, 1789. Type by being a replacement name. Unjustified emendation of Malapterurus La Cepède, 1803. Gender: Masculine.

Remarks: Malapterus Jarocki, 1822, Malapturus Swainson, 1838, and Malacopterurus Gill, 1890, are considered to be incorrect subsequent spellings, and therefore unavailable names, in ICZN Direction 56. Forsskål (1775: 16) applied the generic name of the electric ray, Torpedo, to his account of the electric catfish of the Nile.

Malapterurus barbatus Norris, 2002

Malapterurus barbatus Norris, 2002: 88, fig. 49. Type locality: Sierra Leone, Sewa River system, Bagbe River at Yfin, $8^{\circ} 42^{\prime} \mathrm{N}, 11^{\circ} 05^{\prime} \mathrm{W}$. Holotype: MNHN 1990-100.

Distribution: Western Guinean rivers from the Kolente River, Sierra Leone, to the Borlor River, Liberia (Norris, 2002).

Malapterurus beninensis Murray, 1855

Malapterurus Beninensis Murray, 1855: 20, pl. Type locality: Old Calabar. Syntypes: BMNH 1856.1.16.1 (2), possibly NMSZ 1855.27 (2).

? Malapterurus affinis Günther, 1864: 220. Type locality: Old Calabar. Syntypes: BMNH 1859.11.20.1 (1), BMNH 1864.7.9.1 (1).

Distribution: Coastal plains of west central Africa, from the Volta River, Ghana, to the Shiloango River, Angola (Norris, 2002).

Remarks: Synonymy of Malapterurus affinis with M. beninensis considered tentative by Norris (2002).

Malapterurus cavalliensis Roberts, 2000

Malapterurus cavalliensis Roberts, 2000: 8. Type locality: Ivory Coast: rivière Cavally directly W of Tiéouléoula about $18 \mathrm{~km}$ S of Tai. Holotype: BMNH 2000.3.3.7.

? Malapterurus thysi Norris, 2002: 81, fig. 45. Type locality: Ivory Coast, Cess (Nipoué) River at Toyebli, $6^{\circ} 38^{\prime} \mathrm{N}$, $8^{\circ} 29^{\prime}$ W. Holotype: MRAC 73-10-P-4144.

Distribution: Cess and Cavally Rivers, western Côte d'Ivoire (Norris, 2002).

Malapterurus electricus (Gmelin, 1789)

Silurus electricus Bonnaterre, 1788: 156, pl. 62 (fig. 245). Type locality: Nile and rivers of Africa. No types known.

Silurus electricus Gmelin, 1789: 1354. Type locality: Rosetta, branch of the Nile River, Egypt. Neotype: BMNH 1907.12.2.2547, designated in Poll \& Gosse (1969: 5). Placed on Official list as type of Malapterurus (ICZN Direction 57).

Distribution: Nile River basin; Western African rivers south to the Niger River basin (Norris, 2002).

Malapterurus leonensis Roberts, 2000

Malapterurus leonensis Roberts, 2000: 9. Type locality: Sierra Leone, Moyamba. Holotype: BMNH 1976.11.12.122.

? Malapterurus stiassnyae Norris, 2002: 48, fig. 23. Type locality: Sierra Leone, River Moa drainage, Tiwai Island, $7^{\circ} 33^{\prime} \mathrm{N}, 11^{\circ} 21^{\prime} \mathrm{W}$. Holotype: AMNH 59488.

Distribution: Upper Guinean basins of western Africa, between the Bofon River, Guinea, and St. Paul River, Liberia (Norris, 2002).

Malapterurus melanochir Norris, 2002

Malapterurus melanochir Norris, 2002: 109, fig. 65. Type locality: Congo, Congo R. basin, Ruki River at Eala, $00^{\circ} 04^{\prime} \mathrm{N}, 18^{\circ} 20^{\prime} \mathrm{E}$. Holotype: MRAC 46166.

Distribution: Central Congo River basin and upper Lualaba River system (Norris, 2002).

Malapterurus microstomus Poll \& Gosse, 1969

Malapterurus microstoma Poll \& Gosse, 1969: 8, pls. 1, 2 (fig. b), 3 (fig. b). Type locality: Yangambi, Congo R., Zaire. Holotype: MRAC 164701.

Distribution: Congo River basin (Norris, 2002). 
Malapterurus minjiriya Sagua, 1987

Malapterurus minjiriya Sagua, 1987: 78, fig. 1. Type locality: Lake Kainji, lower Niger basin, Nigeria. Holotype: BMNH 1979.3.5.405.

Distribution: Niger and Volta River basins; possibly also White Nile and Omo River (Norris, 2002).

Malapterurus monsembeensis Roberts, 2000

Malapterurus monsembeensis Roberts, 2000: 9. Type locality: Upper Congo at Monsembe. Holotype: BMNH 1899.8.22.6.

? Malapterurus gossei Norris, 2002: 102, fig. 60. Type locality: Congo, Congo R. basin, Léopoldville (Ouest) (= Kinshasa), 4º' $20^{\prime} \mathrm{N}, 15^{\circ} 15^{\prime} \mathrm{E}$. Holotype: MRAC 88017.

Distribution: Congo River system (Norris, 2002).

Malapterurus occidentalis Norris, 2002

Malapterurus occidentalis Norris, 2002: 73, fig. 40. Type locality: Gambia, Mc Carthy Id. 13³2' [N], 1446' E [sic, W]. Holotype: BMNH 1901.12.28.77.

Distribution: Middle Gambia River, Gambia, and Geba River, Guinea-Bissau (Norris, 2002).

Malapterurus oguensis Sauvage, 1879

Malapterurus electricus oguensis Sauvage, 1879: 99. Type locality: Lopé, pays des Okanda, fleuve Ogôoué. Holotype: MNHN a-0889. Originally proposed as Malapterurus electricus var. oguensis. Described in more detail and illustrated in Sauvage (1880c: 45, pl. 1, fig. 3) as Malapterurus electricus, var. ogooensis.

Distribution: Ivindo River, Ogowe River basin; and Nyanga River and Kouilou-Niari Systems of western Africa.

Malapterurus punctatus Norris, 2002

Malapterurus punctatus Norris, 2002: 91, fig. 51. Type locality: Liberia, Cavally River drainage, unspecified tributary waters of Duobe River at Duo Town, ca. 541'N, $8^{\circ} 06^{\prime}$ W. Holotype: MRAC 80-36-P-2154.

Distribution: Eastern Guinean rivers, from the St. Paul River, Sierra Leone, to the Cavally River, Liberia (Norris, 2002).

Malapterurus shirensis Roberts, 2000

Malapterurus shirensis Roberts, 2000: 10. Type locality: Chiromo, lower Shire. Holotype: BMNH 1921.9.6.53.

? Malapterurus zambeziensis Norris, 2002: 44, fig. 20. Type locality: Zambia/Zimbabwe, Zambezi River drainage, Lake Kariba at Andora Harbor. Holotype: MRAC 187094.

Distribution: Zambezi River basin (Norris, 2002).

Malapterurus tanganyikaensis Roberts, 2000

Malapterurus tanganyikaensis Roberts, 2000: 11. Type locality: Lake Tanganyika. Holotype: BMNH 1936.6.15.1234.

Malapterurus polli Norris, 2002: 111, fig. 67. Type locality: Tanzania: au large de Karema, à 660-700 m de la côte, $\pm 6^{\circ} 49^{\prime} \mathrm{N}, 30^{\circ} 26^{\prime} \mathrm{E}$. Holotype: MRAC 90328 .

Distribution: Lake Tanganyika (Norris, 2002).

Malapterurus tanoensis Roberts, 2000

Malapterurus tanoensis Roberts, 2000: 11. Type locality: Unvaiye lagoon, Tano basin, sw. Ghana. Holotype: USNM 316021.

? Malapterurus murrayi Norris, 2002: 94, fig. 54. Type locality: Ghana, Tano River near Wiawso, slow pool, $6^{\circ} 12^{\prime} \mathrm{N}$ $2^{\circ} 29^{\prime}$ W. Holotype: BMNH 1969.4.28.29.

Distribution: Ofin and Tano Rivers, Ghana (Norris, 2002).

Malapterurus teugelsi Norris, 2002

Malapterurus teugelsi Norris, 2002: 52, fig. 26. Type locality: Guinea, Ndyarendi, Kogon River, ca. $11^{\circ} 21^{\prime} \mathrm{N}$, 14³0'W. Holotype: MRAC 92-59-P-1680.

Distribution: Kogon River basin, Guinea (Norris, 2002).

PARADOXOGLANIS Norris, 2002

Paradoxoglanis Norris, 2002: 117. Type species: Paradoxoglanis caudivittatus Norris, 2002. Type by original designation. Gender: Masculine.

Paradoxoglanis caudivittatus Norris, 2002 
Paradoxoglanis caudivittatus Norris, 2002: 120, fig. 72. Type locality: Congo, Lomela, N. Sankuru, petits ruisseaux affl. riv. Lomela, $2^{\circ} 00^{\prime}-2^{\circ} 19^{\prime} \mathrm{S}, 22^{\circ} 58^{\prime}-23^{\circ} 15^{\prime} \mathrm{E}$. Holotype: MRAC 98452.

Distribution: Central portions of the Congo Basin (Norris, 2002).

Paradoxoglanis cryptus Norris, 2002

Paradoxoglanis cryptus Norris, 2002: 128, fig. 78. Type locality: Congo, Aketi, riv. Kagala, affl. riv. Itimbiri, rive droite, $2^{\circ} 44^{\prime} \mathrm{N}, 2^{\circ} 46^{\prime} \mathrm{E}$. Holotype: MRAC 88-25-P-2231.

Distribution: Kagal River, Itimbiri basin, Congo System (Norris, 2002).

Paradoxoglanis parvus Norris, 2002

Paradoxoglanis parvus Norris, 2002: 126, fig. 76. Type locality: Congo, Congo R. drainage, Mawuya, riv. Mawuya, terr. de Libenge, $3^{\circ} 14^{\prime} \mathrm{N} 18^{\circ} 41^{\prime} \mathrm{E}$. Holotype: MRAC 167535.

Distribution: middle Congo River basin (Norris, 2002).

\section{MOCHOKIDAE Jordan, 1923}

Synodontini Bleeker, 1862 (in Bleeker, 1862-63): 6. Type genus: Synodontis Cuvier, 1816.

Rhinoglanina Günther, 1864: 4, 10, 216. Type genus: Rhinoglanis Günther, 1864.

Mochokidae Jordan, 1923: 150. Type genus: Mochokus Joannis, 1835.

Chiloglanidinae Riehl \& Baensch, 1990: 396. Type genus: Chiloglanis Peters, 1868.

Remarks: The name Simuldentinae, published in Taverne \& Aloulou-Triki (1974) is not available as a family group name because it is not based on an available generic name.

Taxonomic summary: Gosse (1986b).

Reviews: Paugy \& Roberts (1992, West Africa); Skelton (1993, 2001, Southern Africa); Seegers (1996, Lake Rukwa basin); Skelton, et al. (1985, Okavango basin).

Key to genera: Poll \& Gosse (1994).

10 genera, 188 species; no named fossil taxa.

ACANTHOCLEITHRON Nichols \& Griscom, 1917

Acanthocleithron Nichols \& Griscom, 1917: 720. Type species: Acanthocleithron chapini Nichols \& Griscom, 1917. Type by monotypy. Gender: Neuter.

Acanthocleithron chapini Nichols \& Griscom, 1917

Acanthocleithron chapini Nichols \& Griscom, 1917: 721, fig. 26. Type locality: Avakubi [Ituri River], Congo. Holotype: AMNH 6548.

Distribution: Ituri River and Congo River from Kisangani to Stanley Pool (Gosse, 1986b).

ATOPOCHILUS Sauvage, 1879

Atopochilus Sauvage, 1879: 97. Type species: Atopochilus savorgnani Sauvage, 1879. Type by monotypy. Gender: Masculine.

Atopochilus chabanaudi Pellegrin, 1938

Atopochilus Chabanaudi Pellegrin, 1938a: 375, fig. 1. Type locality: Brazzaville (Congo). Holotype: MNHN 19370111.

Distribution: Congo River at Stanley Pool (Gosse, 1986b).

Remarks: Catalog number for holotype of Atopochilus Chabanaudi mistakenly reported as MNHN 37-311 by Bertin \& Estève (1950).

Atopochilus christyi Boulenger, 1920

Atopochilus Christyi Boulenger, 1920a: 33, fig. 17. Type locality: Stanley-Falls, ... d'Avakubi sur l'Ituri. Syntypes (2): Possibly BMNH 1919.9.10.282 (1), MRAC 7239 (1), MRAC 7350 (1).

Distribution: Congo River basin (Gosse, 1986b).

Atopochilus macrocephalus Boulenger, 1906

Atopochilus macrocephalus Boulenger, 1906a: 112. Type locality: Fort Don Carlos, in the province of Loanda, at the 
junction of the Cambo and Kwango (or Cuango) Rivers, Angola. Holotype: BMNH 1904.5.2.182.

Distribution: Congo River basin (Gosse, 1986b).

Atopochilus mandevillei Poll, 1959

Atopochilus mandevillei Poll, 1959: 104, pl. 18 (figs. 2a-c). Type locality: Stanley Pool. Holotype: MRAC 100503.

Distribution: Congo River at Stanley Pool (Gosse, 1986b).

Atopochilus pachychilus Pellegrin, 1924

Atopochilus pachychilus Pellegrin, 1924c: 487. Type locality: Kanda kanda, dans la Lubilonji, tributaire du Kasaï, affluent du Congo. Syntypes (2): MNHN 1924-0213 (1), MRAC 14920 (1).

Distribution: Kasai River, Congo basin; known only from type locality (Gosse, 1986b).

Atopochilus savorgnani Sauvage, 1879

Atopochilus Savorgnani Sauvage, 1879: 98. Type locality: Doumé [Fl. Ogôoué, aux chutes de Doumé (Congo français)]. Holotype: MNHN a-0899. Described in more detail and illustrated in Sauvage (1880c: 43. pl. 3, fig. 3).

Distribution: Upper Ogowe River, Muni River Basin, Equatorial Guinea, and Ntem River, southern Cameroon (Gosse, 1986b).

Atopochilus vogti Pellegrin, 1922

Atopochilus Vogti Pellegrin, 1922c: 350. Type locality: Riv. Wamé, Afrique orientale. Holotype: MNHN $1922-0021$.

Distribution: Wami River, Tanzania (Gosse, 1986b; Seegers, 2003).

\section{BRACHYSYNODONTIS Bleeker, 1862}

Brachysynodontis Bleeker, 1862 (in Bleeker, 1862-63): 6. Type species: Synodontis batensoda Rüppell, 1832. Type by original designation. Gender: Feminine.

Remarks: Treated in Willoughby (1994) as a synonym of Synodontis, without comment.

Brachysynodontis batensoda (Rüppell, 1832)

Synodontis batensoda Rüppell, 1832: 6, pl. 3 (fig. 2). Type locality: Nil, bei Kaïro. Holotype: SMF 2636 (dry).

Synodontes Ruppelli Swainson, 1838: 339, fig. 82. Type locality: [No locality stated]. No types known.

Distribution: Nile, Chad, Niger, Senegal and Gambia river basins (Gosse, 1986b).

Remarks: Synonymy follows Grant (2004).

CHILOGLANIS Peters, 1868

Chiloglanis Peters, 1868b: 599. Type species: Chiloglanis deckenii Peters, 1868. Type by monotypy. Gender: Masculine.

Reviews: Jubb \& Le Roux (1969, southern Africa); Roberts (1989b, Cameroon), Seegers (1996, Lake Rukwa).

Remarks: The name Chiloglanis athiensis appears in Copley (1941: 15) but as name only and therefore not an available name.

Chiloglanis angolensis Poll, 1967

Chiloglanis angolensis Poll, 1967: 255, fig. 120. ruisseau à forêt-galerie, traversant la route Chibia-Jau, à la bifurcation Jau-Onguéria, quelques km au sud de la Chibia, environs de Sá de Bandeira, alt. 1.500 m., Angola. Holotype: MD 1875.

Distribution: known only from type locality (Gosse, 1986b).

Chiloglanis anoterus Crass, 1960

Chiloglanis anoterus Crass, 1960: 446, fig. 2 (a-d). Type locality: Upper Pivaan River (Pongola River system), altitude $\pm 4600 \mathrm{ft}$. $\left(30^{\circ} 28^{\prime} \mathrm{E}, 27^{\circ} 30\right.$ 'S), Natal. Holotype: AMG P1157.

Distribution: Phongolo and Incomati River system tributaries (Skelton, 1993).

Chiloglanis asymetricaudalis De Vos, 1993

Chiloglanis asymetricaudalis De Vos, 1993: 154, figs. 2-4. Type locality: Nyakabuye, rivière Rubyiro, (bassin de la Ruzizi), près de la confluence avec la rivière Ntandwe, Rwanda ( $\left.2^{\circ} 34^{\prime} \mathrm{S}, 2^{\circ} 02^{\prime} \mathrm{E}\right)$. Holotype: MRAC 87-05-P263.

Distribution: Ruzizi River basin, Lake Tanganyika drainage (De Vos, 1993).

Chiloglanis batesii Boulenger, 1904 
Chiloglanis Batesii Boulenger, 1904a: 19. Type locality: South Cameroon; Efulen and stream tributaries of the Lobi River, 15 or 20 miles S. W. of Efulen. Syntypes (5): BMNH 1904.7.1.97-100 (4), BMNH 1904.7.1.127 (1).

Chiloglanis micropogon Poll, 1952: 228, figs. 3-4. Type locality: riv. Nzokwe, affluent del la riv. Ulindi (Territ. Kabare). Holotype: MRAC 91479.

Distribution: Lualaba and Kasai River, Congo Basin; Upper Niger; Meme, Mungo, Lobi Rivers and coastal rivers of Cameroon; Kaduna River, Nigeria (Gosse, 1986b).

Remarks: Chiloglanis micropogon has been generally treated as valid, but recently treated by Roberts (1989b: 169) as a synonym of $C$. batesii.

Chiloglanis benuensis Daget \& Stauch, 1963

Chiloglanis benuensis Daget \& Stauch, 1963: 98, fig. 3. Type locality: Bénoué à Lakdo. Holotype: MNHN 19621273.

Distribution: Niger River basin; known only from type locality (Gosse, 1986b).

Chiloglanis bifurcus Jubb \& Le Roux, 1969

Chiloglanis bifurcus Jubb \& Le Roux, 1969: 17, figs. 9-9a. Type locality: Crocodile River, Incomati River system, Montrose Farm, Nelspruit District, Southern Africa. Holotype: AMG P996.

Distribution: Crocodile-Incomati basin (Skelton, 1993).

Remarks: See Kelynhans \& James (1995) for comments on species.

Chiloglanis brevibarbis Boulenger, 1902

Chiloglanis brevibarbis Boulenger, 1902f: 224, pl. 17 (figs. 2, 2a, 2b). Type locality: Mathoiya River, in the Kenya district, East Africa. Holotype: BMNH 1902.5.26.19.

Distribution: Tana System and Athi River (Gosse, 1986b).

Chiloglanis cameronensis Boulenger, 1904

Chiloglanis cameronensis Boulenger, 1904a: 18. Type locality: Efulen, South Cameroon. Syntypes (7): BMNH 1904.7.1.90-96 (7).

Distribution: coastal rivers of Cameroon, Equatorial Guinea, and Gabon, Congo River basin (Gosse, 1986b).

Chiloglanis carnosus Roberts \& Stewart, 1976

Chiloglanis carnosus Roberts \& Stewart, 1976: 282, pl. 8 (figs. b-d). Type locality: Near village of Inga, a few kilometers upstream and on the opposite side of the river from Inga hydroelectric dam. Lat. 5 27.5'S, Long. $13^{\circ} 36.0^{\prime} \mathrm{E}$; lower Zaïre or Congo River. Holotype: MCZ 50464.

Distribution: Lower Congo River basin; known only from type locality (Gosse, 1986b).

Chiloglanis congicus Boulenger, 1920

Chiloglanis congicus Boulenger, 1920a: 32, fig. 16. Type locality: Stanley-Falls, Congo Belge. Holotype: MRAC 7159.

Distribution: Stanley Falls, Stanley Pool, Congo River basin (Gosse, 1986b).

Chiloglanis deckenii Peters, 1868

Chiloglanis Deckenii Peters, 1868b: 599, pl. 2. Type locality: Africa orientalis. Syntypes: ZMB 6844 (3), ZMB 16387.

Synodontis eurystomus Pfeffer, 1889: 14. Type locality: Rufu bei Korogwe. Lectotype: ZMH H387, designated by Ladiges et al. (1958: 159).

Distribution: Kenya and Tanzania (Gosse, 1986b).

Chiloglanis disneyi Trewavas, 1974

Chiloglanis disneyi Trewavas, 1974: 365, figs. 6 (left), 8. Type locality: R. Wowe, Mungo system, Cameroon. Holotype: BMNH 1973.5.14.342.

Distribution: Wowe River, Mungo basin; Known only from type locality (Gosse, 1986b).

Chiloglanis elisabethianus Boulenger, 1915

Chiloglanis elisabethianus Boulenger, 1915: 171. Type locality: Rivière Lubumbashi, à Elisabethville. Holotype: MRAC 12126.

Distribution: Luapula-Moero River, Congo River basin (Gosse, 1986b).

Chiloglanis emarginatus Jubb \& Le Roux, 1969

Chiloglanis emarginatus Jubb \& Le Roux, 1969: 21, figs. 12-12a. Type locality: Lekkerloop River, tributary of the 
Komati River of the Incomati River system, on the farm Vergelegen, Carolina District, Southern Africa. Holotype: AMG P953.

Distribution: Phongolo and Incomati Rivers, South Africa and Swaziland, and Pungwe and Zambezi Rivers, Zimbabwe (Skelton, 1993).

Chiloglanis fasciatus Pellegrin, 1936

Chiloglanis fasciatus Pellegrin, 1936a: 58. Type locality: Cubango, Angola. Holotype: MNHN 1936-0158.

Distribution: Zambezi River system, Angola and Botswana (Gosse, 1986b).

Chiloglanis harbinger Roberts, 1989

Chiloglanis harbinger Roberts, 1989b: 175, fig. 13. Type locality: Cameroun, R. Lokunje near Bipindi. Holotype: CAS 60811.

Distribution: Lokunje River basin, southeastern Cameroon (Roberts, 1989b).

Chiloglanis kalambo Seegers, 1996

Chiloglanis kalambo Seegers, 1996: 377, figs. 279-281. Type locality: Kanyele River, a southeastern affluent of the upper Kalambo River, on road from Sumbawanga to Mbala, $34 \mathrm{~km}$ N of the Tanzania-Zambia border, Lake Tanganyika drainage, Rukwa region, western Tanzania (8²5'S, 31³0'E). Holotype: MRAC 94-34-P-1079.

Distribution: Upper Kalambo River basin, Tanzania (Seegers, 1996).

Chiloglanis lamottei Daget, 1948

Chiloglanis lamottei Daget, 1948: 38, fig. 12. Type locality: Guineé Fse: Mt. Nimba, Zié, alt. 475-500 m. Holotype: MNHN 1949-0056.

Distribution: Known only from the type locality (Gosse, 1986b).

Chiloglanis lufirae Poll, 1976

Chiloglanis lufirae Poll, 1976: 97, fig. 52. Type locality: Muye (affluent droit de la Lufira), alt. $800-900$ m. [Upemba National Park]. Holotype: MRAC 79-01-P-4728.

Distribution: Lufira River tributary; known only from type locality (Gosse, 1986b).

Chiloglanis lukugae Poll, 1944

Chiloglanis lukugae Poll, 1944a: 7, figs. 3-5. Type locality: Sange, région d'Albertville, Congo belge. Holotype: IRSNB 72; illustrated in Poll (1946: 224, fig. 23).

Distribution: Luapula River basin; tributaries of Lake Tanganyika, Angola, affl. of Cuango River (Gosse, 1986b).

Remarks: Gosse (1986b) stated that the type locality is a small tributary of the Luvua River on road between Albertville and Kiambi but not near Albertville as has been stated elsewhere.

Chiloglanis macropterus Poll \& Stewart, 1975

Chiloglanis macropterus Poll \& Stewart, 1975: 152, fig. 1. Type locality: Luongo River, at ferry crossing, $53 \mathrm{~km}$. S. of Kawambwa, Zambia. Holotype: ROM 28035.

Distribution: Luongo River, Zambia (Gosse, 1986b).

Chiloglanis marlieri Poll, 1952

Chiloglanis marlieri Poll, 1952: 226, figs. 1-2. Type locality: riv. Ndakirwa à Meshe, affluent de la Luhoho. Holotype: MRAC 91478.

Distribution: Lualaba basin (Gosse, 1986b).

Chiloglanis mbozi Seegers, 1996

Chiloglanis mbozi Seegers, 1996: 224, figs. 161-162. Type locality: Hanseketwa River, a brook which is an affluent of the Momba River, draining the Mbozi block via the Msangano trough, $25 \mathrm{~km}$ east of Tunduma on the road to Mbeya, Mbeya Region, western Tanzania (908'S, 3252'E). Holotype: MRAC 94-34-P-927.

Distribution: Hanseketwa River, Momba River basin, Tanzania (Seegers, 1996).

Chiloglanis microps Matthes, 1965

Chiloglanis microps Matthes, 1965: 188, figs. 3-4. Type locality: riv. Lukima à Kiamakoto (Lufira). Holotype: MRAC 140908.

Distribution: Lufira River basin (Gosse, 1986b).

Chiloglanis modjensis Boulenger, 1904

Chiloglanis modjensis Boulenger, 1904b: 332, pl. 31 (figs. 3, 3a, 3b). Type locality: Modjo River [Webi Shebeli basin]. Syntypes (2): BMNH 1905.7.25.103 (1). 
Distribution: Webi Shebeli basin, southern Ethiopia (Gosse, 1986b).

Chiloglanis neumanni Boulenger, 1911

Chiloglanis neumanni Boulenger, 1911a: 481, fig. 359. Type locality: Upper Bubu River, Masailand. Lectotype: BMNH 1905.7.25.49; designated by, and illustrated in, Seegers (1996: 214, fig. 152).

Distribution: Limpopo River system, Cunene, Kafue, Zambezi, Kiavango, and upper Congo Rivers, Lakes Malawi and Kariba (Gosse, 1986b; Skelton, 1993).

Chiloglanis niger Roberts, 1989

Chiloglanis niger Roberts, 1989b: 173, figs. 1f, 2f, 4e-f, 6f, 12. Type locality: Cameroun, Niger basin, R. Menchum below high waterfall near Befang, Bamenda highlands. Holotype: CAS 60809.

Distribution: Menchum River, Niger River basin, Cameroon (Roberts, 1989b).

Chiloglanis niloticus Boulenger, 1900

Chiloglanis niloticus Boulenger, 1900d: 522. Type locality: island of Arko, Soudan. Syntypes (several): BMNH 1907.12.2.2466-73 (8), MNHN 1907-0230 (1), MSNG 14420 (1).

Distribution: Nile and Niger River basins (Gosse, 1986b).

Chiloglanis normani Pellegrin, 1933

Chiloglanis normani Pellegrin, 1933a: 113, fig. on p. 114. Type locality: Danané (Côte d'Ivoire). Syntypes (2): MNHN 1932-0301 (1), NMBA 4253 (1).

Distribution: Cavally River system; known only from type locality (Gosse, 1986b).

Chiloglanis occidentalis Pellegrin, 1933

Chiloglanis occidentalis Pellegrin, 1933a: 112, fig. on p. 112. Type locality: Douékoué, ... Man (Côte d'Ivoire). Syntypes (2): MNHN 1932-0300 (1), NMBA 4252 (1).

Chiloglanis niloticus waterloti Daget, 1954: 304, fig. 115. Type locality: Banamanan, cercle de Kissidougou [Guinea]. Syntypes: MNHN 1935-0224 (1), MNHN 1960-0472 (5), MNHN 1960-0473 (29 or 32), MNHN 1960-0474 (18 or 15).

Distribution: Bafing and Baoule rivers, upper Senegal, upper Niger, Sassandra, upper St. Paul, Kolente and Konkoure Rivers (Gosse, 1986b).

Chiloglanis paratus Crass, 1960

Chiloglanis paratus Crass, 1960: 452, fig. 4 (a-d). Type locality: Concrete wall of Pongola River barrage, altitude \pm 1000 ft. (31 $30^{\circ} \mathrm{E}, 27^{\circ} 23$ 'S), Natal. Holotype: AMG P1154.

Distribution: Phongolo, Incomati and Limpopo river systems, southern Africa (Skelton, 1993).

Chiloglanis pojeri Poll, 1944

Chiloglanis pojeri Poll, 1944a: 8, figs. 6-8. Type locality: Mambwe, région d'Albertville. Holotype: IRSNB 74; illustrated in Poll (1946: 225, fig. 24).

Distribution: Luvua and Lualaba River, upper Congo Basin; Koki River of Lake Tanganyika (Gosse, 1986b).

Chiloglanis polyodon Norman, 1932

Chiloglanis polyodon Norman, 1932: 184, fig. 3. Type locality: Headwaters of Bagbwe River, Sierra Leone. Holotype: BMNH 1932.5.18.64.

Distribution: Bagbwe River basin, Sierra Leone; known only from type locality (Gosse, 1986b).

Chiloglanis polypogon Roberts, 1989

Chiloglanis polypogon Roberts, 1989b: 168, figs. 1b, 2b, 3c-d, 6a-b, 8. Type locality: Cameroun, Cross basin, high gradient stream along road from Mamfe to Bamenda, 88-94 km SW of Bamenda. Holotype: CAS 60790.

Distribution: Cross River basin, Cameroon (Roberts, 1989b).

Chiloglanis pretoriae van der Horst, 1931

Chiloglanis pretoriae van der Horst, 1931: 248, fig. 2. Type locality: Crocodile River, Pretoria District, Transvaal. Holotype: at TM, current whereabouts unknown.

Chiloglanis pumilus van der Horst, 1931: 250, fig. 4. Type locality: Aapies River and Crocodile River, Pretoria Dist., Transvaal. Syntypes: SAIAB 30011 (1), SAIAB 30012 (1).

Distribution: Incomati, Limpopo, middle and lower Zambezi, Pungwe and Busi basins, southern Africa (Skelton, 1993).

Chiloglanis reticulatus Roberts, 1989 
Chiloglanis reticulatus Roberts, 1989b: 165, figs. 1a, 2a, 3a-b, 5, 7. Type locality: Cameroun, Congo basin, R. Mwamedjwel, a very small stream 2-3 km W of Yokadouma. Holotype: CAS 60786.

Distribution: Northwestern portion of the Congo River basin, Cameroon and Congo (Roberts, 1989b).

Chiloglanis rukwaensis Seegers, 1996

Chiloglanis rukwaensis Seegers, 1996: 227, figs. 163-164. Type locality: Chiwanda River (also spelled Chuwanda or Chwanda River), a small river, Momba drainage, $32 \mathrm{~km}$ northwest of Tunduma near Chiwanda (or Chuwanda), on the road to Sumbawanga, western Lake Rukwa drainage, Mbeya Region, western Tanzania, (09¹0'S, 32³3'E). Holotype: MRAC 94-34-P-932.

Distribution: Chiwanda River, Momba River basin, Tanzania (Seegers, 1996).

Chiloglanis ruziziensis De Vos, 1993

Chiloglanis ruziziensis De Vos, 1993: 162, figs. 6-7. Type locality: Nyakabuye, rivière Rubyiro (bassin de la Ruzizi), pres de la confluence avec la rivière Ntandwe, Rwanda (2³4'S, 2902'E). Holotype: MRAC 87-05-P-264.

Distribution: Ruzizi River basin, Lake Tanganyika drainage (De Vos, 1993).

Chiloglanis sanagaensis Roberts, 1989

Chiloglanis sanagaensis Roberts, 1989b: 169, figs. 1c, 2c, 3 (e-f), 6 (c-d), 9. Type locality: Cameroun, Sanaga basin, gravel bars and riffles in lower 1-2 km of R. Nchit where it flows into R. Mbam. Holotype: CAS 60794.

Distribution: Sanaga River basin (Roberts, 1989b).

Chiloglanis sardinhai Ladiges \& Voelker, 1961

Chiloglanis sardinhai Ladiges \& Voelker, 1961: 139, pl. 7 (figs. 12 and 13). Type locality: Mujije (der Mujije ist ein Zufluss des Longa, Cuanza-Sul, Angolas). Holotype: ZMH H1317.

Distribution: Longa River basin; known only from type locality (Gosse, 1986b).

Chiloglanis somereni Whitehead, 1958

Chiloglanis somereni Whitehead, 1958: 199, figs. 2-3. Type locality: Waroya River, Nyanza Province, Kenya; at $34^{\circ} 30^{\prime} \mathrm{E}, 0^{\circ} 10^{\prime} \mathrm{N}$. Holotype: BMNH 1958.7.18.1.

Distribution: Westward flowing rivers of Kenya into Lake Victoria (Gosse, 1986b).

Chiloglanis swierstrai van der Horst, 1931

Chiloglanis swierstrai van der Horst, 1931: 249, fig. 3. Type locality: Crocodile River, Pretoria District, Transvaal. Holotype: SAIAB 30013.

Chiloglanis engiops Crass, 1960: 451, fig. 3 (a-d). Type locality: Lower Pivaan Rier (Pongola River system), altitude $\pm 2000 \mathrm{ft}$. $\left(31^{\circ} 11^{\prime} \mathrm{E}, 27^{\circ} 25^{\prime} \mathrm{S}\right)$, Natal. Holotype: AMG P1156.

Distribution: Lowveld and warmer portions of Phongolo, Incomati and Limpopo river basins, southern Africa (Skelton, 1993).

Chiloglanis trilobatus Seegers, 1996

Chiloglanis trilobatus Seegers, 1996: 230, figs. 165-167. Type locality: Piti River, an affluent of the Rungwa River, $63 \mathrm{~km} \mathrm{~S}$ of Rungwa Village on the road from Itigi to Makambako or Mbeya, eastern Lake Rukwa drainage, western Tanzania $\left(07^{\circ} 27^{\prime} \mathrm{S}, 33^{\circ} 25^{\prime} \mathrm{E}\right)$. Holotype: MRAC 94-34-P-938.

Distribution: Rungwizi River basin, Lake Rukwa basin, Tanzania (Seegers, 1996).

Chiloglanis voltae Daget \& Stauch, 1963

Chiloglanis voltae Daget \& Stauch, 1963: 99, fig. 4. Type locality: Bougouri Ba au pont de Nabéré. Holotype: MNHN 1962-1280.

Distribution: Volta and upper Bénoué River systems (Gosse, 1986b).

EUCHILICHTHYS Boulenger, 1900

Euchilichthys Boulenger, 1900d: 522. Type species: Atopochilus guentheri Schilthuis, 1891. Type by subsequent designation by Jordan (1920: 488). Gender: Masculine.

Euchilichthys astatodon (Pellegrin, 1928)

Atopochilus astatodon Pellegrin, 1928b: 107, fig. 1. Type locality: Luluaburg Saint-Joseph, Congo belge. Syntypes (6): MNHN 1928-0017 (1), MNHN 1928-0018 (1), MRAC 19942 (1), NMBA 3721 (1), NMBA 3723-24 (2), NMBA 3726 (1).

Distribution: Kasai River (Gosse, 1986b). 
Euchilichthys boulengeri Nichols \& La Monte, 1934

Euchilichthys boulengeri Nichols \& La Monte, 1934: 3, fig. 3. Type locality: Luluabourg, Kasai District, Belgian Congo. Holotype: AMNH 12357.

Distribution: Kasai River (Gosse, 1986b).

Euchilichthys dybowskii (Vaillant, 1892)

Chiloglanis Dybowskii Vaillant, 1892b: 2. Type locality: l'Oubanghi. Syntypes: MNHN 1892-0081 (2).

Euchilichthys habereri Steindachner, 1912: 447. Type locality: Dscha, Süd. Kamerun. Holotype: NMW 10700.

Illustrated and described in more detail in Steindachner (1913: 45, fig. 9 and pl. 1 (figs. 2-2a).

Distribution: Ja River, Congo basin, Cameroon (Gosse, 1986b).

Euchilichthys guentheri (Schilthuis, 1891)

Atopochilus güntheri Schilthuis, 1891: 86, pl. 6 (fig. 2). Type locality: Stanley Pool. Holotype: BMNH 1899.9.6.6.

Distribution: Congo River basin, up to Bangweulu River system (Gosse, 1986b).

Euchilichthys royauxi Boulenger, 1902

Euchilichthys royauxi Boulenger, 1902d: 46, pl. 13. Type locality: l'Ubangi à Banzyville. Syntypes (3): BMNH 1901.12.26.46-47 (2), MRAC 1186 (1).

Distribution: Congo River basin, up to Moero Lake system (Gosse, 1986b).

\section{HEMISYNODONTIS Bleeker, 1862}

Hemisynodontis Bleeker, 1862 (in Bleeker, 1862-63): 6. Type species: Pimelodus membranaceus Geoffroy St. Hilaire, 1809. Type by original designation. Gender: Feminine.

Remarks: Treated in Willoughby (1994) as a synonym of Synodontis, without comment.

Hemisynodontis membranacea (Geoffroy Saint-Hilaire, 1809)

Pimelodus membranaceus Geoffroy Saint-Hilaire, 1809: pl. 13 (figs. 1-2). Type locality: Fl. Nil (Egypte). Holotype: MNHN 0000-4197. Name available from caption on plate, described as Synodontis membranaceus in Geoffroy Saint-Hilaire (1827: 297).

Synodontis Guentheri Vaillant, 1893a: 16. Type locality: Karthoum. Syntypes: BMNH 1862.6.17.160 (1), BMNH 1862.6.17.161-162 (2), BMNH 1865.11.15.13 (1).

Distribution: Nile, Niger, Senegal, Gambia, and Volta River basins, Chad system (Gosse, 1986b).

MICROSYNODONTIS Boulenger, 1903

Microsynodontis Boulenger, 1903e: 26. Type species: Microsynodontis batesii Boulenger, 1903. Type by monotypy. Gender: Feminine.

Microsynodontis armatus $\mathrm{Ng}, 2004$

Microsynodontis armatus Ng, 2004h: 10, fig. 6. Type locality: Gabon: Ogooué-Ivindo province, Ivindo River drainage, Balé Creek, 0³1'19"N, 1247'58"E. Holotype: CU 89392.

Distribution: Ivindo River basin, Gabon (Ng, 2004h: 15).

Microsynodontis batesii Boulenger, 1903

Microsynodontis batesii Boulenger, 1903e: 26, pl. 4. Type locality: Mvile River, southern Cameroon. Syntypes (several; apparently more than 6, from notes found in BMNH register): BMNH 1903.7.28.105-110 (6).

Microsynodontis Christyi Boulenger, 1920a: 32, fig. 15. Type locality: Poko, Congo Belge. Syntypes (4): BMNH 1919.9.10.281 (1), MRAC 7168-70 (3).

Distribution: Ntem River basin, southern Cameroon and northern Gabon, and Campo. Ivindo, Lobé, Nyong and Sanaga River basins, southern and central Cameroon (Ng, 2004h: 9).

Remarks: Ng (2004h: 2) stated that Microsynodontis christyi should be treated as valid, but provided no further comments. The BMNH syntype of $M$. christyi has had an incorrect jar label, with the registration number listed as BMNH 1919.9.10.381.

Microsynodontis emarginata $\mathrm{Ng}, 2004$

Microsynodontis emarginatus Ng, 2004h: 15, fig. 8. Type locality: Gabon: Haut-Ogooué Province, Motobo I village, Kiéne creek, 1³2'14.1"S, 13³2'43.5"E. Holotype: CU 89393.

Distribution: Ogooué River basin, Gabon (Ng, 2004h: 19). 
Microsynodontis hirsuta $\mathrm{Ng}, 2004$

Microsynodontis hirsutus Ng, 2004h: 20, fig. 10. Type locality: Gabon: Woleu-Ntem province, Ngomo creek, where it crosses Oyem-Minvoul road, 141'30.0"N, 11³9'18.9"E. Holotype: CU 87040.

Distribution: Nye River of Ntem River basin, Gabon (Ng, 2004h: 23).

Microsynodontis laevigata $\mathrm{Ng}, 2004$

Microsynodontis laevigatus Ng, 2004h: 24, fig. 11. Type locality: Gabon: Ogooué-Ivindo province, Ivindo River drainage, small creek flowing into Ivindo River, Makokou, 0³5'8"N, 1251'22"E. Holotype: CU 88265.

Distribution: Ivindo River basin, Gabon (Ng, 2004h: 27).

Microsynodontis lamberti Poll \& Gosse, 1963

Microsynodontis lamberti Poll \& Gosse, 1963: 61, pl. 4 (fig. 1). Type locality: Rivière Lilanda, Yangole, Congo centrale. Holotype: MRAC 137837.

Distribution: Lilanda River, Congo River basin; known only from type locality (Gosse, 1986b).

Microsynodontis nannoculus $\mathrm{Ng}, 2004$

Microsynodontis nannoculus Ng, 2004h: 27, fig. 12. Type locality: Equatorial Guinea: Mami River, a tributary of Kyé River. Holotype: MRAC 173145.

Distribution: Kyé River basin, Ntem River drainage, Equatorial Guinea (Ng, 2004h: 31).

Microsynodontis nasutus $\mathrm{Ng}, 2004$

Microsynodontis nasutus Ng, 2004h: 32, fig. 14. Type locality: Gabon: Woleu-Ntem province, Okano River on rapids $0.5 \mathrm{~km} \mathrm{~S}$ of village of $\mathrm{Na}, 0^{\circ} 48^{\prime} 35^{\prime \prime} \mathrm{N}, 1^{\circ} 38^{\prime} 47^{\prime E}$. Holotype: CU 89394.

Distribution: Okano River, Ogooué River basin, Gabon (Ng, 2004h: 35).

Microsynodontis notata $\mathrm{Ng}, 2004$

Microsynodontis notatus Ng, 2004h: 35, fig. 15. Type locality: Gabon: Ezanga River, about midway between Lake Ezanga and Ogooué mainstream. Holotype: MRAC 80-51-P-839.

Distribution: Lower Ogooué River, Gabon (Ng, 2004h: 39).

Microsynodontis polli Lambert, 1958

Microsynodontis polli Lambert, 1958: 42, fig. 1. Type locality: Rivière Gbin, Guinée Forestière, Guinée Française. Holotype: MRAC 119457.

Distribution: Gbin River, Upper Niger; known only from type locality (Gosse, 1986b).

Microsynodontis vigilis $\mathrm{Ng}, 2004$

Microsynodontis vigilis Ng, 2004h: 39, fig. 16. Type locality: Gabon: Moyen-Ogooué province, $12 \mathrm{~km} \mathrm{~N}$ of Lambaréné, branch of Nzorbang creek near village of same name, 0³4'6.2"N, 10¹2'46.4"E. Holotype: CU 87039.

Distribution: Lower Ogooué River basin, Gabon (Ng, 2004h: 43).

MOCHOKIELLA Howes, 1980

Mochokiella Howes, 1980: 165. Type species: Mochokiella paynei Howes, 1980. Type by original designation. Gender: Feminine.

Mochokiella paynei Howes, 1980

Mochokiella paynei Howes, 1980: 165, figs. 1, 2b. Type locality: Kassawe Forest Reserve, Sierra Leone. Holotype: BMNH 1979.8.22.1.

Distribution: Kassawe Forest Reserve, Sierra Leone; known only from type locality (Gosse, 1986b).

MOCHOKUS Joannis, 1835

Mochokus Joannis, 1835: [Cl. IV, pl. 8]. Type species: Mochokus niloticus Joannis, 1835. Type by monotypy. Gender: Masculine.

Mochocus Günther, 1864: 217. Type species: Mochokus niloticus Joannis, 1835. Gender: Masculine. Unjustified emendation of Mochokus Joannis, 1835.

Rhinoglanis Günther, 1864: 216. Type species: Rhinoglanis typus Günther, 1864. Type by original designation. Gender: Masculine.

Mochokus brevis Boulenger, 1906

Mochocus brevis Boulenger, 1906d: 148. Type locality: Fashoda and Lake No, White Nile. Syntypes (43): BMNH 
1907.12.2.2529-2542 (14), BMNH 1907.12.2.2544 (1), MNHN 1907-0233 (2).

Distribution: Nile and Chad basins (Gosse, 1986b).

Mochokus niloticus Joannis, 1835

Mochokus Niloticus Joannis, 1835: [Cl. IV, pl. 8]. Type locality: Nil. Holotype: Whereabouts unknown; not found in MNHN by Boulenger (1900d: 526) or subsequently.

Rhinoglanis typus Günther, 1864: 216, unnumbered figure. Type locality: Gondokoro. Holotype: BMNH 1981.4.13.1.

Rhinoglanis Vannutellii Vinciguerra, 1898: 254. Type locality: lago Rodolfo. Syntypes: MSNG 14419 (4).

Distribution: Nile and Niger basins (Gosse, 1986b).

SYNODONTIS Cuvier, 1816

Synodontis Cuvier, 1816: 203. Type species: Silurus clarias Linnaeus, 1758. Type by subsequent designation by Bleeker, 1862 (in Bleeker, 1862-63): 6. Gender: Feminine.

Leiosynodontis Bleeker, 1862 (in Bleeker, 1862-63): 6. Type species: Synodontis maculosus Rüppell, 1829. Type by original designation. Gender: Feminine.

Pseudosynodontis Bleeker, 1862 (in Bleeker, 1862-63): 6. Type species: Synodontis serratus Rüppell, 1829. Type by original designation. Gender: Feminine.

Revision: Vaillant (1895b, 1896b); Poll (1971, with keys to species).

Reviews: Willoughby (1994, Lake Kainji, Nigeria); Bruwer \& van der Bank (2003, southern Africa).

Keys: Skelton \& White (1990, southern Africa); Bruwer \& van der Bank (2003).

Remarks: The name Synodontes published in Minding (1832: 116) is considered an incorrect subsequent spelling and not an available name.

Synodontis acanthomias Boulenger, 1899

Synodontis acanthomias Boulenger, 1899a: 46, pl. 23. Type locality: Boma et Léopoldville [restricted to Boma by lectotype designation]. Lectotype: MRAC 18, designated by Poll (1971: 282).

Synodontis pfefferi Steindachner, 1912: 447. Type locality: Dscha, Nebenfluss des Congo im Bezirke Molundu des südlichen Kamerun. Holotype: NMW 9023. Species illustrated and described in more detail in Steindachner (1913: 43, pl. 8).

Distribution: Congo River basin, except central forest (Gosse, 1986b).

Synodontis afrofischeri Hilgendorf, 1888

Synodontis Afro-Fischeri Hilgendorf, 1888: 77. Type locality: Victoria-Nyanza (Ukerewe-See). Holotype: ZMB 12745. Holotype illustrated in Poll (1971: fig. 28) and Seegers (1996: fig. 171).

Distribution: Lakes Victoria, Nabugabo, Kioga, Ihema; Nile, Kagera, Kingani Malagarasi Rivers (Gosse, 1986b); Piti River, Lake Rukwa basin (Seegers, 1996).

Synodontis alberti Schilthuis, 1891

Synodontis alberti Schilthuis, 1891: 88. Type locality: Kinshasa, Stanley Pool. Lectotype: BMNH 1899.9.6.9, designated by, and illustrated in, Poll (1971: 252, fig. 114).

Distribution: Congo River basin (Gosse, 1986b).

Synodontis albolineata Pellegrin, 1924

Synodontis albolineatus Pellegrin, 1924b: 320, fig. 1. Type locality: Madjingo, rivière Djoua (Gabon). Holotype: MNHN 1924-0147, illustrated in Poll (1971: fig. 68).

Distribution: Ogowe River basin; known only from type locality (Gosse, 1986b).

Synodontis angelica Schilthuis, 1891

Synodontis angelica Schilthuis, 1891: 87. Type locality: Léopoldville, Stanley Pool. Holotype: BMNH 1899.9.6.8; holotype illustrated in Poll (1971: fig. 110, top).

Synodontis tholloni Boulenger, 1901a: 304. Type locality: bassin de l'Ogooué (?). Holotype: MNHN 1890-0030.

Synodontis angelicus zonatus Poll, 1933: 134, fig. 11. Type locality: Lukulu (riv. Lukulu). Holotype: MRAC 37904; holotype illustrated in Poll (1971: fig. 110, bottom). Originally as Synodontis angelicus var. zonatus.

Synodontis werneri Boeseman, 1957: 146, pl. 5. Type locality: Stanley Pool, Belgian Congo. Holotype: RMNH 20913. 
Distribution: Congo River basin, except in central forest (Gosse, 1986b).

Synodontis annectens Boulenger, 1911

Synodontis annectens Boulenger, 1911d: 56. Type locality: Nianimaru, Gambia; Culufi and Geba Rivers, Portuguese Guinea [restricted to Nianimaru by lectotype designation]. Lectotype: BMNH 1901.12.28.60, designated by Poll (1971: 131).

Distribution: Gambia; Geba basin, Guinea Bissau; and Taja basin, Sierra Leone (Gosse, 1986b).

Synodontis ansorgii Boulenger, 1911

Synodontis ansorgii Boulenger, 1911b: 375. Type locality: Geba and Culufi Rivers, Portuguese Guinea [restricted to Geba River, by lectotype designation]. Lectotype: BMNH 1912.4.1.128, designated by,and illustrated in, Poll (1971: 135, fig. 56).

Distribution: Geba River basin, Guinnea-Bissau; and Taja River basin, Sierra Leone (Gosse, 1986b).

Synodontis arnoulti Roman, 1966

Synodontis arnoulti Roman, 1966: 141, pl. 7 (fig. 14). Type locality: le Kou, affluent de la Volta Noire, à 30 km au Nord de Bobo-Dioulasso. Holotype: MRAC 141742.

Distribution: Volta River basin (Gosse, 1986b).

Synodontis aterrima Poll \& Roberts, 1968

Synodontis aterrimus Poll \& Roberts, 1968: 297, figure on p. 298. Type locality: Bokuma, Bassin du Congo. Holotype: MRAC 101466.

Distribution: Central Congo River basin (Gosse, 1986b).

Synodontis bastiani Daget, 1948

Synodontis bastiani Daget, 1948: 35, fig. 10. Type locality: Côte d’Ivoire: Bouaflé. Holotype: MNHN 1949-0055.

Synodontis dageti Poll \& Roman, 1967: 185, fig. 3. Type locality: [68 km south of Bolo-Dioulasso], Haut Comoé. Holotype: MRAC 141982.

Synodontis eburneensis Daget, 1965: 473. Type locality: le Bandama au lieu dit Lamto, à mi-chemin entre Toumodi et Tiassalé (Côte d'Ivoire). Holotype: MNHN 1964-0266; holotype illustrated in Poll (1971: fig. 92).

Distribution: Sassandra, Bandama, Bia, and Comoré basins, Côte d'Ivoire (Gosse, 1986b).

Remarks: Synonymy of these three names first proposed by Paugy \& Roberts (1992).

Synodontis batesii Boulenger, 1907

Synodontis Batesii Boulenger, 1907b: 50. Type locality: Ja River, South Cameroon. Lectotype: BMNH 1907.5.22.179; designated by, and illustrated in, Poll (1971: 331, fig. 150).

Distribution: Central Congo River basin; Ja River and Nyong River, Cameroon; Muni River, Equatorial Guinea (Gosse, 1986b).

Synodontis brichardi Poll, 1959

Synodontis brichardi Poll, 1959: 100, pl. 17 (figs. 2 a-c). Type locality: Léopoldville, début des rapides à Kinsuka. Holotype: MRAC 100494.

Distribution: Lower Congo River rapids (Gosse, 1986b).

Synodontis budgetti Boulenger, 1911

Synodontis budgetti Boulenger, 1911a: 403, fig. 305. Type locality: Lokoja, Upper Niger [Nigeria]. Holotype: BMNH 1904.1.20.42.

Distribution: Niger and Oueme Rivers, Nokuoue Lake (Gosse, 1986b).

Synodontis camelopardalis Poll, 1971

Synodontis camelopardalis Poll, 1971: 430, fig. 202; pls. 7 (fig. 4), 12 (fig. 14). Type locality: Eala, riv. Ruki. Holotype: MRAC 47654.

Distribution: Central Congo River basin; known only from type locality (Gosse, 1986b).

Synodontis caudalis Boulenger, 1899

Synodontis caudalis Boulenger, 1899a: 44, pl. 22 (fig. 1). Type locality: Boma, Matadi, Léopoldville [restricted to Matadi by lectotype designation]. Lectotype: MRAC 57, designated by, and illustrated in, Poll (1971: 274, fig. 124).

Distribution: (Gosse, 1986b).

Synodontis caudovittata Boulenger, 1901 
Synodontis caudovittatus Boulenger, 1901c: 10. Type locality: Mouth of Lake No, White Nile. Lectotype: BMNH 1907.12.2.2360, designated by, and illustrated in, Poll (1971: 104).

Distribution: Nile River basin (Gosse, 1986b).

Synodontis centralis Poll, 1971

Synodontis centralis Poll, 1971: 435, fig. 204; pls. 7(fig. 7), 12 (fig. 12). Type locality: Ndwa village, Kunungu, Zaire. Holotype: MRAC 57198.

Distribution: Central Congo River basin (Gosse, 1986b).

Synodontis clarias (Linnaeus, 1758)

Silurus clarias Linnaeus, 1758: 306. Type locality: habitat in Americae, Africae fluviis. Types: See Remarks, below.

Silurus callarias Bloch \& Schneider, 1801: 379. Type locality: in fluviis Americae australis et Nilo. Syntypes: on Silurus clarias Linnaeus, and Bloch (1782: pl. 35, figs. 1-2). An unneeded new name for Silurus clarias Linnaeus, 1758.

Pimelodus synodontis Geoffroy Saint-Hilaire, 1809: pl. 12 (figs. 5-6). Type locality: Nil. Holotype: MNHN 00004194. Name available on caption to plate. Species described as Synodontis macrodon by Geoffroy Saint-Hilaire (1827: 295).

Synodontis macrodon Geoffroy Saint-Hilaire, 1827: 295. Type locality: Fl. Nil (Egypte). Holotype: MNHN 00004194. New name for Pimelodus synodontis Geoffroy Saint-Hilaire, 1809.

Distribution: Nile, Niger, Senegal, Gambia, and Volta River basins, Chad system (Gosse, 1986b).

Remarks: Silfvergrip (1996: 8-9) discussed the specimens that formed the type series for Silurus clarias. Two of the four type specimens are extant, neither of which is a species of Synodontis. Silfvergrip (1996: 9) selected "Hasselquist's specimen" (NRM 7044), which was subsequently identified as a specimen of Bagrus docmak (Silfvergrip, pers. commun., 2003), as the lectotype of Silurus clarias. The nomenclatural impact of this selection would result in Bagrus docmak (Forsskål, 1775) becoming a junior synonym of Silurus clarias, and the genera Synodontis Cuvier, 1816, and Bagrus Bosc, 1816, becoming synonyms. Because of the nomenclatural impact of Silfvergrip's lectotype designation on two relatively well known African catfishes, his designation is not followed here but it left for a more thorough review in the future.

Synodontis comoensis Daget \& Lévêque, 1981

Synodontis comoensis Daget \& Lévêque, 1981: 45, figs. 1-5. Type locality: rivière Comoé au niveau du bac de Gansé, Parc National de la Comoé (Côte d'Ivoire), 8³9'N, 356'W. Holotype: MNHN 1980-1636.

Distribution: Comoé River, Côte d'Ivoire; known only from type locality (Gosse, 1986b).

Synodontis congica Poll, 1971

Synodontis congicus Poll, 1971: 440, fig. 206; pls. 7 (fig. 6), 12 (fig. 16). Type locality: Gangala na Bodio, riv. Dungu. Holotype: MRAC 165695.

Distribution: Congo River basin, except lower Congo, central basin, Luapula-Moero, and high Katanga (Gosse, 1986b).

Synodontis contracta Vinciguerra, 1928

Synodontis contractus Vinciguerra, 1928: 24, pl. 1. Type locality: fiume Rubi a Buta [upper Congo basin]. Lectotype: MSNG 26493, designated by Poll (1971: 383).

Synodontis davidi Axelrod, 1970: 33, figs. Type locality: Stanley Pool near Kinshasha, Congo. Type(s): Whereabouts unknown.

Distribution: Congo River basin (Gosse, 1986b).

Synodontis courteti Pellegrin, 1906

Synodontis Courteti Pellegrin, 1906b: 473. Type locality: Fort-Archambault (Chari) [Soudan]. Holotype: MNHN 1904-0083; illustrated in Poll (1971: 129, fig. 54).

Synodontis thomasi Chabanaud, 1934: 219. Type locality: Afrique équatorial française. Type(s) (510 mm): Whereabouts unknown.

Distribution: Chad and Niger basins (Gosse, 1986b).

Synodontis cuangoana Poll, 1971

Synodontis cuangoanus Poll, 1971: 446, fig. 208; pl. 7 (fig. 10), pl. 12 (fig. 17). Type locality: Cuango, Cafunfo ( $\left.8^{\circ} 47^{\prime} \mathrm{S}, 18^{\circ} 01^{\prime} \mathrm{E}\right)$. Holotype: MRAC 172253. 
Distribution: Congo River basin, south of Kasai, Zaire and Angola (Gosse, 1986b).

Synodontis decora Boulenger, 1899

Synodontis decorus Boulenger, 1899a: 49, pl. 25. Type locality: Léopoldville et Nouvelle-Anvers; [restricted to Nouvelle-Anvers by lectotype designation]. Lectotype: MRAC 154, designated by Poll (1971: 291).

Synodontis vittatus Boulenger, 1920a: 31, pl. 25. Type locality: Stanleyville. Lectotype: MRAC 7119, designated by Poll (1971: 295).

Distribution: Congo River basin, except Luapula River system (Gosse, 1986b).

Synodontis dekimpei Paugy, 1987

Synodontis dekimpei Paugy, 1987: 357, fig. 1. Type locality: rivière Konkouré à Soukya (aval du pont routier Kindia-Telimélé en amont de Tondo) (Guinée). Holotype: MRAC 81-20-P-11.

Distribution: Konkouré River, Guinea (Paugy, 1987).

Synodontis depauwi Boulenger, 1899

Synodontis depauwi Boulenger, 1899a: 45, pl. 22 (fig. 2). Type locality: Léopoldville. Lectotype: MRAC 111, designated by Poll (1971: 278).

Distribution: Congo River at Stanley Pool (Gosse, 1986b).

Synodontis dhonti Boulenger, 1917

Synodontis dhonti Boulenger, 1917b: 367. Type locality: Kilewa Bay [Lake Tanganyika]. Holotype: MRAC 14344; holotype illustrated in Poll (1946: 211, figs. 21-22, and 1971: fig. 168).

Synodontis irsacae Matthes, 1959b: 78. Type locality: Kalundu, nord du Lac Tanganyika. Holotype: MRAC 130315. Distribution: Lake Tanganyika (Gosse, 1986b).

Synodontis dorsomaculata Poll, 1971

Synodontis dorsomaculatus Poll, 1971: 449, fig. 210; pl. 7 (fig. 8), pl. 12 (fig. 18). Type locality: Kadia, Kisale. Holotype: MRAC 71395.

Distribution: Upper Lualaba (Katanga), Congo River basin (Gosse, 1986b).

Synodontis euptera Boulenger, 1901

Synodontis eupterus Boulenger, 1901c: 11. Type locality: Mouth of Lake No, White Nile. Holotype (147 mm SL): BMNH 1907.12.2.2376; holotype redescribed and illustrated in Boulenger (1907a: 370, pl. 68), with locality as Goz-abu-Gumah, White Nile; holotype also illustrated in Poll (1971: fig. 44).

Synodontis macrepipterus Pellegrin, 1922b: 222. Type locality: Fort Crampel (Gribingui). Holotype: MNHN 19220031.

Distribution: White Nile, Chad Basin (Gribinqui), Niger and Volta Rivers (Gosse, 1986b).

Synodontis fascipinna Nichols \& La Monte, 1953

Synodontis fascipinna Nichols \& La Monte, 1953: 2, fig. 2. Type locality: Birao, eastern French Equatorial Africa. Holotype: AMNH 19841.

Distribution: Headwaters of the Chari River basin; known only from type locality (Gosse, 1986b).

Synodontis filamentosa Boulenger, 1901

Synodontis filamentosus Boulenger, 1901c: 10. Type locality: Mouth of Lake No, White Nile. Holotype: BMNH 1907.12.2.2377; holotype illustrated in Boulenger (1907: pl. 69) and Poll (1971: fig. 42).

Synodontis augierasi Pellegrin, 1929a: 136, fig.1. Type locality: [Niger R. at Koulikoro, Mali, w. Africa.] Syntypes: MNHN 1928-0243 (1), MNHN 1928-0244 (1).

Distribution: Nile, Chad, Niger, and Volta basins (Gosse, 1986b).

Synodontis flavitaeniata Boulenger, 1919

Synodontis flavitaeniatus Boulenger, 1919: 161. Type locality: Eala [Congo River basin]. Holotype: MRAC 1369; holotype illustrated in Poll (1971: fig. 170).

Distribution: Central Congo River basin (Gosse, 1986b).

Synodontis frontosa Vaillant, 1895

Synodontis frontosus Vaillant, 1895a: 48. Type locality: Nil [White Nile, Sudan]. Holotype: MNHN 0000-4208. Illustrated in Vaillant (1895b: pl. 10, fig. 8) and described in more detail in Vaillant (1896b: 146).

Synodontis Citernii Vinciguerra, 1898: 247. Type locality: Lago Rodolfo. Holotype: MSNG 14423 (dry).

Distribution: Nile basin; Lakes Rudolf, Albert, Eduard and Kioga; Chad basin; Uebi Giupa (Uebi Shebeli) (Gosse, 
1986b).

Synodontis fuelleborni Hilgendorf \& Pappenheim, 1903

Synodontis fuelleborni Hilgendorf \& Pappenheim, 1903: 265. Type locality: Rukwa-See. Lectotype: ZMB 16309, designated by, and illustrated in, Poll (1971: 320, fig. 146); lectotype also illustrated in Seegers (1996: fig. 177).

Distribution: Rukwa Lake and Rufigi basin (Gosse, 1986b).

Synodontis gambiensis Günther, 1864

Synodontis gambiensis Günther, 1864: 214. Type locality: Gambia. Holotype: BMNH 2005.9.9.1; holotype illustrated in Poll (1971: fig. 20).

Synodontis gambiensis latifrons Blache, 1964: 201, fig. 112. Type locality: Lake Tchad basin. Syntypes: Possibly MNHN 1959-0495 (2), MNHN 1959-0500 (5), MNHN 1959-0524 (8).

Distribution: Niger, Chad, Gambia, Geba, Taja, Waange, Volta, and Oueme basins (Gosse, 1986b).

Remarks: Considered to be a synonym of Synodontis schall by Lévêque et al. (1989: 120) and Paugy (in Lévêque $e t$ al., 1992: 561). Register number for holotype of Synodontis gambiensis Günther, given in Poll (1971) and Eschmeyer et al. (1998) as BMNH 1863.16.4.40, is incorrect; it not a possible register number in the BMNH system.

Synodontis geledensis Günther, 1896

Synodontis geledensis Günther, 1896b: 220, fig. 2. Type locality: Geledi on the Shebeli. Holotype: BMNH 1895.12.31.69.

Distribution: Uebi Shebeli, Somalia; Lorian Swamps and Uasso Nyiro (Uebi Giuba basin), Kenya (Gosse, 1986b).

Synodontis gobroni Daget, 1954

Synodontis gobroni Daget, 1954: 301, fig. 113. Type locality: Mopti, Markala. Syntypes (4): MNHN 1954-0005 (1), MNHN1960-0471 (1).

Distribution: Niger River basin (Gosse, 1986b).

Remarks: Treated in Willoughby (1994: 28) as a synonym of Synodontis guttatus Günther, 1865.

Synodontis granulosa Boulenger, 1900

Synodontis granulosus Boulenger, 1900c: 480. Type locality: North end of Lake Tanganyika. Lectotype: BMNH 1906.9.6.40, designated by, and illustrated in, Poll (1971: 303, fig. 138).

Distribution: Lake Tanganyika (Gosse, 1986b).

Synodontis greshoffi Schilthuis, 1891

Synodontis greshoffi Schilthuis, 1891: 87. Type locality: Kinshasa, Stanley Pool. Holotype: BMNH 1899.9.6.7; holotype illustrated in Poll (1971: fig. 112).

Synodontis holopercnus Boulenger, 1920a: 28. Type locality: Bosabangi, Avakubi,\& Poko, Congo Belge. Syntypes (5): BMNH 1919.9.10.264-265 (2), MRAC 7616 (1), MRAC 7775 (1), MRAC 7829 (1).

Synodontis pantherinus Boulenger, 1920b: 149. Type locality: Ituri à Avakubi. Holotype: MRAC 14513.

Distribution: Congo River basin, except Luapula River system and lower Congo (Gosse, 1986b).

Synodontis guttata Günther, 1865

Synodontis guttatus Günther, 1865a: 452. Type locality: Niger. Holotype: BMNH 1865.4.6.4 (29 inches, dry); holotype illustrated in Boulenger (1911a: fig. 335) and Poll (1971: fig. 24)

Distribution: Lower Niger basin (Gosse, 1986b).

Synodontis haugi Pellegrin, 1906

Synodontis Haugi Pellegrin, 1906a: 470. Type locality: Ngomo (Ogôoué). Holotype: MNHN 1906-0209; holotype illustrated in Poll (1971: fig. 52).

Distribution: Ogowe River basin (Gosse, 1986b).

Synodontis iturii Steindachner, 1911

Synodontis melanostictus iturii Steindachner, 1911c: 534. Type locality: Ituriflüsse. Holotype: NMW 46049; holotype illustrated in Poll (1971: fig. 160). Originally as Synodontis melanostictus var. iturii.

Synodontis ituriensis Boulenger, 1920a: 28. Type locality: Mawambi sur l'Ituri, Congo Belge. Holotype: MRAC 7576.

Distribution: Ituri River (Gosse, 1986b).

Synodontis katangae Poll, 1971 
Synodontis katangae Poll, 1971: 454, fig. 212; pls. 7 (fig. 11), 12 (fig. 19). Type locality: Lukonzolwa, lac Moëro. Holotype: MRAC 14231.

Distribution: Congo River basin, high Katanga and Luapula-Moero (Gosse, 1986b).

Synodontis khartoumensis Abu Gideiri, 1967

Synodontis khartoumensis Abu Gideiri, 1967: 133, fig. 1. Type locality: Blue Nile, two miles from place where the Blue Nile joins the White Nile to form the main Nile at Khartoum, Sudan. Holotype: at Sudan Natural History Museum.

Distribution: Nile basin and Lake Albert (Gosse, 1986b).

Synodontis koensis Pellegrin, 1933

Synodontis koensis Pellegrin, 1933a: 110, fig. on p. 111. Type locality: Man (rivière Ko). Holotype: NMBA 4500; holotype illustrated in Poll (1971: fig. 72).

Distribution: Nzo River of Sassandra River basin, Côte d'Ivoire (Gosse, 1986b).

Synodontis laessoei Norman, 1923

Synodontis laessoei Norman, 1923c: 696. Type locality: Kokema River, a tributary of the Quanza River, Angola; altitude 4000 ft. Holotype: BMNH 1923.8.15.3; holotype illustrated in Poll (1971: fig. 176).

Distribution: Kolema River of Quanza River basin, Angola; known only from type locality (Gosse, 1986b).

Synodontis leopardina Pellegrin, 1914

Synodontis leopardinus Pellegrin, 1914: 26. Type locality: Pays des Barotsés (Ht.-Zambèze). Holotype: MNHN 1913-0321; holotype illustrated in Poll (1971: fig. 162).

Synodontis jallae Gilchrist \& Thompson, 1917: 561. Type locality: Shesheke, S. Rhodesia. Holotype: SAM 14290 [now at AMG].

Distribution: Cunene, Upper Zambezi and Okovango river basins (Skelton, 1993).

Synodontis leoparda Pfeffer, 1896

Synodontis leopardus Pfeffer, 1896: 35. Type locality: Rufu bei Korogwe. Holotype: at ZMH (Gosse, 1986: 132).

Distribution: Coastal rivers of Tanzania and Somalia (Gosse, 1986b).

Remarks: Considered by De Vos (2001a: 49) as a possible synonym of Synodontis zanzibaricus Peters, 1868.

Synodontis levequei Paugy, 1987

Synodontis levequei Paugy, 1987: 361, fig. 3. Type locality: rivière Kakrima (basin du Konkouré), près de Kasséri (Guinée). Holotype: MNHN 1987-0931.

Distribution: Kakrima River (Paugy, 1987).

Synodontis longirostris Boulenger, 1902

Synodontis longirostris Boulenger, 1902d: 44, pl. 12 (fig. 2). Type locality: Rivière Yembe à Banzyville. Holotype: MRAC 1243.

Synodontis ovidius Lönnberg \& Rendahl, 1920: 173, figs 4-5. Type locality: Mukimbungu, Lower Congo. Holotype: NRM 9913.

Distribution: Congo River basin (Gosse, 1986b).

Synodontis longispinis Pellegrin, 1930

Synodontis Batesi longispinis Pellegrin, 1930: 207. Type locality: la Sangha. Holotype: MNHN 1929-0245; holotype illustrated in Poll (1971: fig. 182). Originally as Synodontis Batesi var. longispinis nov. var.

Distribution: Sangha River, Congo River basin (Gosse, 1986b).

Synodontis lufirae Poll, 1971

Synodontis lufirae Poll, 1971: 459, fig. 214; pl. 7 (fig. 12). Type locality: Lac de Koni, Lufira. Holotype: MRAC 168400.

Distribution: Lufira River, Congo River basin (Gosse, 1986b).

Synodontis macrophthalma Poll, 1971

Synodontis macrophthalmus Poll, 1971: 207, fig. 98; pls. 3 (fig. 15), 10 (fig. 5). Type locality: Ampem, Volta Lake (Ghana). Holotype: MRAC 168500.

Distribution: Volta Lake, Ghana (Gosse, 1986b).

Synodontis macrops Greenwood, 1963

Synodontis macrops Greenwood, 1963: 66. Type locality: tributary stream of the Aswa River, Teso district, Uganda. 
Holotype: BMNH 1961.12.1.319; holotype illustrated in Poll (1971: fig. 88).

Distribution: Aswa River basin; Nile River system, Uganda (Gosse, 1986b).

Synodontis macrostigma Boulenger, 1911

Synodontis macrostigma Boulenger, 1911a: 432, fig. 325. Type locality: Okovango River. Lectotype: BMNH 1910.5.31.37, designated by Poll (1971: 345). A previous lectotype designation may have been made by Gilchrist \& Thompson (1913: 462) in the caption to fig. 109, in which the illustrated specimen is labeled as the type, if the illustrated specimen can be identified.

Distribution: Kafue and Mashi Rivers, Zambezi River basin; Okovango and Cunene Rivers (Gosse, 1986b; Skelton, 1993).

Synodontis macrostoma Skelton \& White, 1990

Synodontis macrostoma Skelton \& White, 1990: 281, fig. 3. Type locality: Upper Zambezi River at Katima Mulilo, Caprivi, Namibia. Holotype: AMG P11687.

Distribution: Cunene, Okavango, upper Zambezi and Kafur river basins (Skelton, 1993).

Synodontis manni De Vos, 2001

Synodontis manni De Vos, 2001a: 42, figs. 2-3. Type locality: Kenya: Tana River at Baomo area near Baomo village, ${ }^{\circ} 55^{\prime} \mathrm{S}, 40^{\circ} 08^{\prime} \mathrm{E}$. Holotype: NMK FW-600/1.

Distribution: Tana River, Kenya (De Vos, 2001a).

Synodontis marmorata Lönnberg, 1895

Synodontis marmoratus Lönnberg, 1895: 186. Type locality: Muddy rivulet at Bonge [Cameroon]. Lectotype: ZMUU, 38.5 mm SL specimen, designated by, and illustrated in, Poll (1971: 93, fig. 34).

Synodontis marmoratus truncatus Holly, 1927a: 8. Type locality: Njongfluß, Kamerun. Treated by Gosse (1986) as possible synonym of $S$. marmoratus.

Distribution: Sanaga and Nyong Rivers (Gosse, 1986b).

Synodontis matthesi Poll, 1971

Synodontis matthesi Poll, 1971: 462, fig. 216; pls. 7 (fig. 14), 12 (fig. 20). Type locality: Mtera, riv. Ruaha (Tanzania). Holotype: ZMA 109743.

Distribution: Rufigi River basin, Tanzania (Gosse, 1986b).

Synodontis melanoptera Boulenger, 1903

Synodontis melanopterus Boulenger, 1903d: 327, pl. 29 (fig. 1). Type locality: Oguta, Niger Delta. Lectotype: BMNH 1902.10.25.3, designated and illustrated in Poll (1971: 114, fig. 46).

Distribution: Porto Novo, Benin; Niger, Ouémé and Ogun River basins (Gosse, 1986b).

Synodontis multimaculata Boulenger, 1902

Synodontis multimaculatus Boulenger, 1902d: 43, pl. 12 (fig. 1). Type locality: l'Ubangi à Banzyville. Holotype: MRAC 1314; illustrated in Poll (1971: 306, fig. 140).

Distribution: Ubangi River, Congo River basin (Gosse, 1986b).

Synodontis multipunctata Boulenger, 1898

Synodontis multipunctatus Boulenger, 1898d: 497. Type locality: Lake Tanganyika. Holotype: BMNH 1898.9.9.76; illustrated and described in more detail in Boulenger (1898f: 24, pl. 8), with locality as Sumba.

Distribution: Lake Tanganyika (Gosse, 1986b).

Synodontis nebulosa Peters, 1852

Synodontis nebulosus Peters, 1852: 682. Type locality: Tette [Zambezi River, Mozambique]. Holotype: ZMB 3120; illustrated in Peters (1868c: 28, pl. 5, fig. 1).

Distribution: Lower and middle Zambezi River basin (Skelton, 1993).

Synodontis nigrita Valenciennes, 1840

Synodontis nigrita Valenciennes, in Cuvier \& Valenciennes, 1840b: 265 (197 in Strasbourg deluxe edition), pl. 441. Type locality: Sénégal. Holotype: MNHN 0000-3051; illustrated in Poll (1971: 59, fig. 14).

Synodontis ornatus Pappenheim, in Pappenheim \& Boulenger, 1914: 251, pl. 5 (fig. 2). Type locality: Albert See. Syntypes: ZMB 19097 (8).

Distribution: Nile, Chad, Niger, Senegal, Gambia, Casamance, Geba, Kolente and Volta River basins; coastal rivers from Ghana to Nigeria (Gosse, 1986b). 
Synodontis nigriventris David, 1936

Synodontis nigriventris David, 1936b: 417. Type locality: Mongende; Ikengo; Basongo; Koteli; Buta; Budjala; Léopoldville; Flandria [restricted to Buta by lectotype designation]. Lectotype: MRAC 30312, designated by, and illustrated in, Poll (1971: 397, fig. 186).

Distribution: Central Congo River basin (Gosse, 1986b).

Synodontis nigromaculata Boulenger, 1905

Synodontis nigromaculatus Boulenger, 1905b: 645. Type locality: Lake Bangwelo. Holotype: BMNH 1905.11.10.10; holotype illustrated in Poll (1971: fig. 148).

Synodontis melanostictus Boulenger, 1906f: 553, pl. 34. Type locality: Lofu, Lake Tanganyika. Holotype: BMNH 1906.9.8.72.

Synodontis colyeri Boulenger, 1923: 438. Type locality: Mansa River, N. Rhodesia. Holotype: SAM 14529 [now at AMG].

Distribution: Okavango and upper Zambezi River basins; Upper Congo River basin, Zambia; Lake Tanganyika and Kasai River (Skelton, 1993).

Synodontis njassae Keilhack, 1908

Synodontis njassae Keilhack, 1908: 168.Type locality: Njassa-See. Lectotype: ZMB 18191, designated by, and illustrated in, Poll (1971: 337, fig. 154).

Distribution: Lake Malawi (Gosse, 1986b).

Synodontis notata Vaillant, 1893

Synodontis Maculatus Vaillant, 1892b: 2. Type locality: Riv. Oubangui à Bangui. Holotype: apparently MNHN 1892-0080. Nomen oblitum, see Remarks.

Synodontis notatus Vaillant, 1893a: 17. Type locality: Riv. Oubangui à Bangui. Holotype: apparently MNHN 18920080. Proposed as a replacement for Synodontis maculatus, Vaillant, 1892, considered by Vaillant to be effectively preoccupied by Synodontis maculosus Rüppell, 1829. Poll (1971: fig. 116) illustrated MNHN 1886-0436 and referred to it as the lectotype. Nomen protectum.

Synodontis notatus binotata Pellegrin, 1926: 205. Type locality: Bolobo, Congo belge. Syntypes (2): MRAC 49686, MNHN 1926-0195. Originally as Synodontis notatus var. binotata.

Synodontis notatus ocellatus Poll, 1938: 404, fig. 9. Type locality: environs de Maka, riv. Londo [Katanga, bassin du Congo]. Holotype: MRAC 49686. Originally as Synodontis notatus var. ocellatus.

Distribution: Congo River basin (Gosse, 1986b).

Remarks: Synodontis notata Vaillant, 1893, has been in wide use since its proposal (see Gosse, 1986b, for list of publications) and considered here to be a nomen protectum. The holotype for Synodontis maculatus Vaillant, 1892, and S. notatus Vaillant, 1893, is listed by Bertin \& Estève (1950) and other sources as MNHN 1886-0436, but Gosse (1986: 135) indicated that the holotype was actually MNHN 1892-0080, which was collected by Dybowski from the Oubangui, as stated in Vaillant (1892).

Synodontis nummifer Boulenger, 1899

Synodontis nummifer Boulenger, 1899a: 47, pl. 24. Type locality: Léopoldville. Holotype: MRAC 107.

Distribution: Congo River basin (Gosse, 1986b).

Synodontis obesus Boulenger, 1898

Synodontis obesus Boulenger, 1898a: 415. Type locality: Gaboon and Opobo River, Old Calabar [restricted to Opobo River by lectotype designation]. Lectotype: BMNH 1896.5.5.67, designated by and illustrated in Poll (1971: 96, fig. 36).

Synodontis loppei Pellegrin, 1927: 365. Type locality: Edea [Cameroon]. Syntypes: MHNLR P.197 (1), MNHN 1927-0278 (1). Illustrated in Pellegrin (1928a: 5, unnumbered figure).

Distribution: Coastal rivers from Ghana to Gabon; basins of the Comoë, Pra, Volta, Momo, Cross, Wouri, Sanaga Nyong, Kribi and Lobé Rivers (Gosse, 1986b).

Synodontis ocellifer Boulenger, 1900

Synodontis ocellifer Boulenger, 1900e: 514. Type locality: Kunchow Creek [River Gambia]. Lectotype: BMNH 1901.7.17.7; designated by, and illustrated in, Poll (1971: 101, fig. 38).

Distribution: Senegal, Gambia, Volta, Chad, and Niger basins (Gosse, 1986b). 
Synodontis omias Günther, 1864

Synodontis omias Günther, 1864: 213. Type locality: West Africa [Upper Niger River]. Holotype: BMNH 1863.12.9.3 (skeleton).

Distribution: Niger River basin (Gosse, 1986b).

Synodontis ornatipinnis Boulenger, 1899

Synodontis ornatipinnis Boulenger, 1899b: 111, pl. 43 (fig. 2). Type locality: Coquilhatville et Bikoro (Lac Tumba) [restricted to Coquilhatville by lectotype designation]. Lectotype: MRAC 968, designated by Poll (1971: 299).

Distribution: Congo River system (Gosse, 1986b).

Synodontis ornatissima Gosse, 1982

Synodontis ornatus Boulenger, 1920a: 29, fig. 14. Type locality: Poko, Congo Belge. Holotype: MRAC 7144. Preoccupied by Synodontis ornatus Pappenheim, 1914; replaced by Synodontis ornatissimus Gosse, 1982.

Synodontis ornatissimus Gosse, 1982: 48. Type locality: Poko, Congo Belge. Holotype: MRAC 7144. Replacement for Synodontis ornatus Boulenger, 1920; preoccupied by Synodontis ornatus Pappenheim, 1914.

Distribution: Ubangi River and its tributaries, Congo River basin (Gosse, 1986b).

Synodontis pardalis Boulenger, 1908

Synodontis pardalis Boulenger, 1908a: 30. Type locality: a waterfall of the Libi River, near the Ja River (Congo System), South Cameroon. Lectotype: BMNH 1909.4.29.91, designated by, and illustrated in, Poll (1971: 334, fig. 152).

Distribution: Ja River, Congo River basin, Cameroon (Gosse, 1986b).

Synodontis petricola Matthes, 1959

Synodontis petricola Matthes, 1959b: 78. Type locality: lac Tanganyika. Holotype: MRAC 130357; holotype illustrated in Poll (1971: fig. 192).

Distribution: Lake Tanganyika (Gosse, 1986b).

Synodontis pleurops Boulenger, 1897

Synodontis pleurops Boulenger, 1897b: 423. Type locality: Stanley Falls, upper Congo. Holotype: BMNH 1897.9.30.13; holotype illustrated in Poll (1971: fig. 120).

Distribution: Congo River basin, except the Luapula system and the lower Congo River (Gosse, 1986b).

Synodontis polli Gosse, 1982

Synodontis eurystomus Matthes, 1959b: 77. Type locality: lacTanganyika, confiné au littoral rocheux du lac. Holotype: MRAC 130440. Holotype illustrated in Matthes (1962: pl. 1, fig. c) and Poll (1971: fig. 190). Preoccupied by Synodontis eurystomus Pfeffer, 1889; replaced by Synodontis polli Gosse, 1982.

Synodontis polli Gosse, 1982: 48. Type locality: Luhanga, Lake Tanganyika. Holotype: MRAC 130440. Replacement for Synodontis eurystomus Matthes, 1959.

Distribution: Lake Tanganyika (Gosse, 1986b).

Synodontis polyodon Vaillant, 1895

Synodontis polyodon Vaillant, 1895a: 48. Type locality: Ogôoué. Holotype: MNHN 1886-0433; holotype illustrated in Vaillant (1895b: pl. 9, fig. 1), and described in more detail in Vaillant (1896b: 127).

Distribution: Ogowe River basin (Gosse, 1986b).

Synodontis polystigma Boulenger, 1915

Synodontis polystigma Boulenger, 1915: 170. Type locality: Rivière Luapula, à Kasenga; lac Moero, à Lukonzolwa [restricted to lac Moëro by lectotype designation]. Lectotype: MRAC 14174, designated by, and illustrated in, Poll (1971: 356, fig. 164).

Distribution: Luapula-Moëro Rivers, Congo River basin (Gosse, 1986b).

Synodontis pulcher Poll, 1971

Synodontis pulcher Poll, 1971: 465, fig. 218; pls. 7 (fig. 13), 12 (fig. 21). Type locality: Stanley Pool. Holotype: MRAC 168399.

Distribution: Stanley Pool, Congo River basin (Gosse, 1986b).

Synodontis punctifer Daget, 1965

Synodontis punctifer Daget, 1965: 472. Type locality: le Nzo à Guiglo, Côte d'Ivoire. Holotype: MNHN 1964-0265; holotype illustrated in Poll (1971: fig. 90). 
Distribution: Nzo River, Sassandra River basin (Gosse, 1986b).

Synodontis punctulata Günther, 1889

Synodontis punctulatus Günther, 1889: 71, pl. 8 (fig. a). Type locality: River Ruva, in the Arusha country. Lectotype: BMNH 1887.11.3.47; designated by, and illustrated in, Poll (1971: 233, fig. 108).

Distribution: Ruwa River, Tanzania; Uebi Shebeli, Somalia (Gosse, 1986b).

Remarks: Considered to be a possible synonym of Synodontis zanzibaricus Peters, 1868 (De Vos 2001a: 49).

Synodontis rebeli Holly, 1926

Synodontis rebeli Holly, 1926: 157. Illustrated in Holly (1927b: 215, figs. 5-6). Type locality: Bamfluß, Flußgebiet des Sanaga in Kamerun. Lectotype: NMW 7796: 1; designated by, and illustrated in, Poll (1971: 155, fig. 70).

Distribution: Sanaga River basin, Cameroon (Gosse, 1986b).

Synodontis resupinata Boulenger, 1904

Synodontis resupinatus Boulenger, 1904c: 199, pl. 8. Type locality: Lokoja, Northern Nigeria. Holotype: BMNH 1904.1.20.52.

Distribution: Niger River basin (Gosse, 1986b).

Synodontis ricardoae Seegers, 1996

Synodontis ricardoae Seegers, 1996: 245, figs. 178-180. Type locality: Kazizi, some kilometers northeast of Muze, at the northwestern shore of Lake Rukwa, Rukwa region, western Tanzania $\left(07^{\circ} 37^{\prime} \mathrm{N}, 31^{\circ} 36^{\prime} \mathrm{E}\right)$. Holotype: MRAC 94-34-P-1093.

Distribution: Lake Rukwa basin (Seegers, 1996).

Synodontis robbianus Smith, 1875

Synodontis robbianus Smith, 1875: 92, fig. 1. Type locality: Ikorofiong, Old Calabar River. Syntype: BMNH 1874.5.23.3 (1); illustrated in Poll (1971: fig. 26) as holotype.

Distribution: Lower Niger River and Cross River (Gosse, 1986b).

Synodontis robertsi Poll, 1974

Synodontis robertsi Poll, 1974: 442, fig. 1. Type locality: Riv. Lukenie à Elombe, ferry landing, lat. $2^{\circ} 49^{\prime}$ S, long. 18²1'E. Holotype: MRAC 189535.

Distribution: Lukenie River, Congo River basin; known only from type locality (Gosse, 1986b).

Synodontis ruandae Matthes, 1959

Synodontis ruandae Matthes, 1959a: 62. Type locality: Riv. Kagera à Rusumu, Ruanda. Holotype: MRAC 130345; holotype illustrated in Poll (1971: 178, fig. 82).

Distribution: Kagera River basin, Nile-Victoria system (Gosse, 1986b).

Synodontis rufigiensis Bailey, 1968

Synodontis rufigiensis Bailey, 1968: 346, fig. 1. Type locality: Lake Lugongwe, at Utete on the lower Rufiji, Tanzania. Holotype: BMNH 1968.6.12.1; holotype illustrated in Poll (1971: 428, fig. 200).

Distribution: Rufigi River basin, Tanzania (Gosse, 1986b).

Synodontis rukwaensis Hilgendorf \& Pappenheim, 1903

Synodontis zambezensis rukwaensis Hilgendorf \& Pappenheim, 1903: 267. Type locality: Rukwa Sees. Lectotype: ZMB 16311; designated by, and illustrated in, Seegers (1996: 249, fig. 182).

Synodontis maculipinna Norman, 1922: 687. Type locality: Mpanganye, Rufigi River, Tanganyika Territory. Lectotype: BMNH 1922.4.18.27, designated by, and illustrated in, Poll (1971: 376, fig. 174); lectotype also illustrated in Seegers (1996: fig. 184).

Synodontis wamiensis Lohberger, 1930: 91, fig. 1. Type locality: aus dem Wamiflusse und Nebenflüssen [30 km. north of Bahamojo, Tanzania]. Holotype: NMW 18866; illustrated in Seegers (1996: fig. 185).

Distribution: Lake Rukwa basin, Ugalla basin Malagarasi system, and coastal drainages of Tangazni south of the Pangani River (Seegers, 1996).

Remarks: Synonymy based on Seegers (1996).

Synodontis schall (Bloch \& Schneider, 1801)

Silurus schall Bloch \& Schneider, 1801: 385. Type locality: Habitat Nilum. Holotype: ZMB 3110 (lost; Paepke, 1999: 99).

Pimelodus clarias Geoffroy Saint-Hilaire, 1809: pl. 13 (figs. 3-4). Type locality: Nil, Egypt. Holotype: Possibly 
MNHN 0000-4203 (1) or MNHN 0000-4205 (1 of 2). Name available from plate, with illustrated specimen the holotype, if identifiable. Described as Synodontis clarias in Geoffroy Saint-Hilaire (1827: 299). Independence of this name and Silurus clarias Linnaeus not clearly established, but treated as independent in literature (e. g., Poll, 1971).

Synodontis maculosus Rüppell, 1829: 10, pl. 3 (fig. 1). Type locality: die Märkte von Cairo. Lectotype: SMF 653, designated by Poll (1971: 50).

Synodontis arabi Valenciennes, in Cuvier \& Valenciennes, 1840b: 261 (194 in Strasbourg deluxe edition). Type locality: Nil, Egypte. Syntypes: MNHN 0000-4203 (1), MNHN 0000-4205 (2). Unneeded new name for Silurus schall Bloch \& Schneider, 1801.

Synodontis smithii Günther, 1896b: 222, pl. 9. Type locality: [Not stated; introduction indicates specimen obtained "en route to Lake Rudolph"; BMNH register lists Lake Stephanie [sic, Lac Stefanie, Kenya] and Poll (1971) listed Lake Rudolph, but these are in contradiction to statements in introduction]. Holotype: BMNH 1895.12.31.68.

Distribution: Nile basin, Abaia, Stephanie and Rudolf Lakes, Chad, Niger, Senegal, Uebi Guiba (Uebi Shebeli) basins and possibly Tana and Volta rivers (Gosse, 1986b).

Synodontis schoutedeni David, 1936

Synodontis schoutedeni David, 1936b: 416. Type locality: Kungungu; Koteli; Basongo; Mongende; Budjala; Bumba; Flandria [restricted to Kungungu by lectotype designation]. Lectotype: MRAC 38093; designated by, and illustrated in, Poll (1971: 393, fig. 184).

Distribution: Central Congo River basin (Gosse, 1986b).

Synodontis serpentis Whitehead, 1962

Synodontis serpentis Whitehead, 1962b: 100, figs. 1a, 1b. Type locality: Athi River at Jilore, thirty miles from river mouth, Kenya. Holotype: BMNH 1962.1.19.1.

Distribution: Athi and Tana River basins, Kenya (Gosse, 1986b).

Synodontis serrata Rüppell, 1829

Synodontis serratus Rüppell, 1829: 8, pl. 2 (fig. 1). Type locality: Cairo. Holotype: SMF 2649 (dry).

Synodontis humeratus Valenciennes, in Cuvier \& Valenciennes, 1840b: 264 (196 in Strasbourg deluxe edition). Type locality: Nil. No types known; based on a drawing.

Distribution: Nile basin (Gosse, 1986b).

Synodontis smiti Boulenger, 1902

Synodontis smiti Boulenger, 1902d: 45, pl. 12 (fig. 3). Type locality: l'Ubangi à Banzyville. Lectotype: MRAC 1266, designated in Poll (1971: 316).

Synodontis tenuis Nichols \& Griscom, 1917: 717, fig. 25. Type locality: Stanleyville, Congo. Holotype: AMNH 6535.

Distribution: Ubangi and Lualaba Rivers, Congo River basin (Gosse, 1986b).

Synodontis soloni Boulenger, 1899

Synodontis soloni Boulenger, 1899b: 110, pl. 43 (fig. 1). Type locality: Congo. Lectotype: MRAC 155385, designated by Poll (1971: 296).

Distribution: Stanley Pool rapids and Libenge, Congo River basin (Gosse, 1986b).

Synodontis sorex Günther, 1864

Synodontis sorex Günther, 1864: 211. Type locality: Chartoum, upper Nile. Lectotype: BMNH 1862.6.17.69, designated by Poll (1971: 65).

Distribution: Nile, Chad, Niger and Volta basins (Gosse, 1986b).

Remarks: Lectotype register number incorrectly listed as BMNH 1862.1.17.69 in Poll (1971) and Eschmeyer et al. (1998).

Synodontis steindachneri Boulenger, 1913

Synodontis steindachneri Boulenger, 1913: 69. Type locality: Nyong River, S. Cameroon. Holotype: BMNH 1913.10.29.16; holotype illustrated in Boulenger (1916: fig. 188) and Poll (1971: fig. 162).

Distribution: Nyong River, Cameroon (Gosse, 1986b).

Synodontis tanganaicae Borodin, 1936 
Synodontis serratus tanganaicae Borodin, 1936: 9. Type locality: Kasanga, Lake Tanganyika. Lectotype: MCZ 32538; designated by De Vos \& Thys van den Audenaerde (1998: 148).

Synodontis lacustricolus Poll, 1953: 157, fig. 18a; pl. 6 (fig. 3). Type locality: Stat. 123, au large de Karéma [Lac Tanganyika]. Holotype: IRSNB 197.

Distribution: Lake Tanganyika (De Vos \& Thys van den Audenaerde, 1998: 153).

Remarks: Synonymy first proposed in De Vos \& Thys van den Audenaerde (1998: 153), with valid name as Synodontis tanganyicae.

Synodontis tessmanni Pappenheim, 1911

Synodontis tessmanni Pappenheim, 1911: 523, fig. 5. Type locality: Akonangi, im Kje [Ntem basin]. Holotype (12.8 cm SL): ZMB 18413; illustrated in Poll (1971: fig. 62), as lectotype, but a holotype was clearly stated (as "Type der Art") in description.

Synodontis hollyi ntemensis Pellegrin, 1929b: 367. Type locality: Nyabessan (Ntem), Cameroun. Holotype: MNHN 1929-0051. Originally as Synodontis Hollyi var. ntemensis nov. var.

Distribution: Ntem River, Cameroon (Gosse, 1986b).

Synodontis thamalakanensis Fowler, 1935

Synodontis thamalakanensis Fowler, 1935a: 274, fig. 12. Type locality: Thamalakane River, Maun, Bechuanaland Protectorate. Holotype: ANSP 53245.

Distribution: Okavango and Upper Zambezi River basins (Skelton, 1993).

Synodontis thysi Poll, 1971

Synodontis thysi Poll, 1971: 210, fig. 100; pls. 3 (fig. 16), 10 (fig. 6). Type locality: Mange-Ferry, riv. Little Scarcies ou Kaba (Sierra Leone). Holotype: MRAC 168314.

Distribution: Little Scarcies River, Sierra Leone; known only from type locality (Gosse, 1986b).

Synodontis tourei Daget, 1962

Synodontis tourei Daget, 1962: 113, fig. 38; pl. 11 (figs. 24-25). Type locality: Bafing à Ballay, Guinée. Lectotype: MNHN 1959-0109; designated by Poll (1971: 185).

Distribution: Bafing River, Senegal River basin, Guinea (Gosse, 1986b).

Synodontis unicolor Boulenger, 1915

Synodontis unicolor Boulenger, 1915: 170. Type locality: Lac Moero et rivière Luapula, à Kasenga [restricted to Kasenga by lectotype designation]. Lectotype: MRAC 14223; designated by, and illustrated in, Poll (1971: 359 , fig. 166).

Distribution: Luapula-Moero system, Congo River basin (Gosse, 1986b).

Synodontis vanderwaali Skelton \& White, 1990

Synodontis vanderwaali Skelton \& White, 1990: 284, fig. 5. Type locality: Okavango River, Namibia. Holotype: AMG P5796.6.

Distribution: Cunene, Okavango and upper Zambezi basins (Skelton, 1993).

Synodontis velifer Norman, 1935

Synodontis velifer Norman, 1935a: 219, fig. 3. Type locality: Ejura, Ashanti Forest, Gold Coast. Holotype: BMNH 1934.8.31.146; holotype illustrated in Poll (1971: 164, fig. 74).

Distribution: Sassandra, Bandama and Volta River basins (Gosse, 1986b).

Synodontis vermiculata Daget, 1954

Synodontis vermiculatus Daget, 1954: 292, fig. 109. Type locality: Mopti, riv. Niger (Mali). Neotype: MNHN 19611129; designated by Poll (1971: 170).

Distribution: Niger River basin (Gosse, 1986b).

Synodontis victoriae Boulenger, 1906

Synodontis victoriae Boulenger, 1906b: 438. Type locality: Entebbe and Buganga, Lake Victoria [restricted to Buganga by lectotype designation]. Lectotype: BMNH 1906.5.30.190; designated by, and illustrated in, Poll (1971: 120, fig. 50).

Distribution: Victoria Lake basin, Kioga Lake and Victoria Nile, Malagarasi River (Gosse, 1986b).

Synodontis violacea Pellegrin, 1919

Synodontis violaceus Pellegrin, 1919b: 212. Type locality: Gribingui. Lectotype: MNHN 1919-0246, designated by, 
and illustrated in, Poll (1971: 149, fig. 66).

Distribution: Chad, Niger and Volta basins (Gosse, 1986b).

Synodontis voltae Roman, 1975

Synodontis voltae Roman, 1975: 45, unnumbered figure. Type locality: dans la Bougouriba, affluent de la Volta Noire. Holotype: MHV V5641.

Distribution: Upper Volta River basin; known only from type locality (Gosse, 1986b).

Synodontis waterloti Daget, 1962

Synodontis waterloti Daget, 1962: 111, fig. 37; pl. 10 (fig. 23). Type locality: Friguiagbé, près Kindia, Guinée. Holotype: MNHN 1935-0223.

Distribution: Waanje and Taja Rivers, Sierra Leone; St. John River basin, Liberia; Cess River basin, Côte d'Ivoire; Pra River basin, Ghana (Gosse, 1986b).

Synodontis woosnami Boulenger, 1911

Synodontis woosnami Boulenger, 1911a: 424, fig. 319. Type locality: Okovango [Botswana]. Holotype: BMNH 1910.5.31.36.

Distribution: Cunene, Okavango and upper Zambezi River basins (Skelton, 1993).

Synodontis xiphias Günther, 1864

Synodontis xiphias Günther, 1864: 215. Type locality: West Africa. Holotype: BMNH 1863.11.9.1 (dry); holotype illustrated in Boulenger (1911a: fig. 339) and Poll (1971: fig. 22).

Synodontis labeo Günther, 1865a: 453. Type locality: West Africa, probably Niger R. Holotype: BMNH 1865.5.9.8 (stuffed); illustrated in Boulenger (1911a: fig. 337).

Distribution: Niger River basin (Gosse, 1986b).

Remarks: Synodontis labeo Günther, 1865, is treated by Willoughby (1994: 28) as probably valid.

Synodontis zambezensis Peters, 1852

Synodontis zambezensis Peters, 1852: 682. Type locality: riv. Zambèze, Mozambique. Lectotype: ZMB 3119; designated by, and illustrated in, Poll (1971: 225, fig. 104). Illustrated in Peters (1868c: 31, pl. 5, figs. 2-3).

Synodontis zambesensis Günther, 1864: 214. Type locality: Zambezi. Lectotype: ZMB 3119, designated by, and illustrated in, Poll (1971: 225, fig. 104). Unjustified emendation of Synodontis zambezensis Peters, 1852.

Distribution: Pongolo, Lompopo, Pungwe and Zambezi River basins, Rukwa Lake, Luangwe River of Moero basin. (Gosse, 1986b).

Synodontis zanzibarica Peters, 1868

Synodontis zanzibaricus Peters, 1868b: 600. Type locality: Wahrscheinlich von Mombas [Kenya]. Lectotype: ZMB 6846; designated by, and illustrated in, Poll (1971: 229, fig. 106).

Distribution: Mombassa, Kenya (Gosse, 1986b).

\section{Species inquirendae, Synodontis}

Synodontis vaillanti Boulenger, 1897b: 424. Type locality: Congo français (Bangui). Holotype: MNHN 1892-0275. Treated by Gosse (1986:148) as doubtfully valid.

Synodontis Hollyi Pellegrin, 1929b: 365. Type locality: Ndjibula ..., Nanga Eboko (Sanaga), Cameroun. Syntypes: MNHN 1929-0047 (1), MNHN 1929-0048 (1), MNHN 1929-0049 (1), MNHN 1929-0050 (1). Type series apparently mixed; with specimens representing both Synodontis obesus Boulenger, 1898, and Synodontis rebeli Holly, 1926.

Synodontis galinae Kochetov, 1998: 4, 2 figs. Type locality: White Nile. Type(s): Whereabouts unknown.

\section{NEMATOGENYIIDAE Bleeker, 1862}

Nematogenyini Bleeker, 1862 (in Bleeker, 1862-63): 16. Type genus: Nematogenys Girard, 1855.

Taxonomic summary: de Pinna (2003).

1 genus, 2 species; including 1 named fossil species. 
NEMATOGENYS Girard, 1855

Nematogenys Girard, 1855: 198. Type species: Trichomycterus inermis Guichenot, 1848. Type by monotypy. Gender: Feminine.

Phylogeny: Arratia \& Chang (1975).

$\dagger$ Nematogenys cuivi Azpelicueta \& Rubilar, 1998

$\dagger$ Nematogenys cuivi Azpelicueta \& Rubilar, 1998: 476, figs. 2, 3. Type locality: Cura-Mallín Formation, Miocene, Northern slope of the Cerro Rucañanco ( $\left.38^{\circ} 41^{\prime} \mathrm{S}, 71^{\circ} 11^{\prime} \mathrm{W}\right)$, about 1,250 m a.s.1., Lonquimay, Chile. Holotype: CPUC Lonq (R)/7 (neurocranium with Weberian complex, branchial and hyoid arches, pectoral girdle and spine, some vertebrae, and ribs).

Distribution: Cura-Mallin Formation, south-central Chile (Azpelicueta \& Rubilar, 1998).

Nematogenys inermis (Guichenot, 1848)

Trichomycterus inermis Guichenot, 1848: 312, pl. 9 (fig. 2). Type locality: Chile. No types known.

Nematogenys nigricans Philippi, 1866: 716. Type locality: Chile. No types known.

Nematogenys pallidus Philippi, 1866: 716. Type locality: Chile. No types known.

Distribution: Isolated localities in Concepción, Rancagua and Angol, Chile. Formerly widespread throughout much of Central Chile (de Pinna, 2003).

\section{PANGASIIDAE Bleeker, 1858}

Pangasini Bleeker, 1858b: 49, 63. Type genus: Pangasius Valenciennes, 1840.

Pangasianodonidi Fowler, 1958: 14. Type genus: Pangasianodon Chevey, 1931.

Revision: Roberts \& Vidthayanon (1991).

Review: Vidthayanon \& Roongthongbaisuree (1993, Thailand).

Identification guide: Jayaram (1977d, South Asia).

Phylogeny: Pouyaud et al. (2000); Pouyaud et al. (2004).

Remarks: Several phylogenetic studies indicate that the species included in this family may constitute a natural group, but the group is likely nested within the Schilbidae. As such, the Pangasiidae may not deserve continued recognition as a family but is so recognized herein due to current usage. See Fumihito (1989) for morphological comparisons among some species.

5 genera, 30 species; including 1 named fossil genus and 2 fossil species.

$\dagger$ CETOPANGASIUS Roberts \& Jumnongthai, 1999

$\dagger$ Cetopangasius Roberts \& Jumnongthai, 1999: 177. Type species: †Cetopangasius chaetobranchus Roberts \& Jumnongthai, 1999. Type by original designation. Gender: Masculine. Also spelled Cetopagansius in text. Cetopangasius treated here as the valid spelling.

$\dagger$ Cetopangasius chaetobranchus Roberts \& Jumnongthai, 1999

$\dagger$ Cetopangasius chaetobranchus Roberts \& Jumnongthai, 1999: 177, figs.16-21. Type locality: Thailand, Northcentral Phetchabun Province, Ban Nong Pla; Miocene. Holotype: Department of Natural Resources, Thailand (DNR) TF 5013 (entire skeleton).

Distribution: Thailand; Miocene (Roberts \& Jumnongthai, 1999).

HELICOPHAGUS Bleeker, 1858

Helicophagus Bleeker, 1858b: 28, 45. Type species: Helicophagus typus Bleeker, 1858. Type by original designation. Gender: Masculine.

Helicophagus leptorhynchus $\mathrm{Ng} \&$ Kottelat, 2000

Helicophagus leptorhynchus $\mathrm{Ng} \&$ Kottelat, 2000c: 55, figs. 1, 2a. Type locality: Thailand Ubon Ratchathani Provinve, Mun River at Bung Wai, about $7 \mathrm{~km}$ W of Ubon Ratchathani (15'12'30"N, 104'47'30"E). Holotype: USNM 288676.

Distribution: Mekong and Chao Phraya River basins (Kottelat, 2001b). 
Helicophagus typus Bleeker, 1858

Helicophagus typus Bleeker, 1858a: 46. Type locality: Palembang, in fluvimine Mussi. Holotype: BMNH 1863.12.4.118; illustrated in Bleeker (1862-63: pl. 79 [= Silur. pl. 31], fig. 2).

Distribution: Sumatra and southeastern Borneo (Roberts \& Vidthayanon, 1991; Musikasinthorn et al., 1998).

Helicophagus waandersii Bleeker, 1858

Helicophagus Waandersii Bleeker, 1858b: 175. Type locality: Palembang, in fluviis [Sumatra]. Holotype (340 mm

TL): BMNH 1863.12.4.89; illustrated in Bleeker (1862-63: pl. 80 [= Silur. pl. 32]).

Distribution: Mekong and Chao Phraya Rivers, and Sumatra (Roberts \& Vidthayanon, 1991).

PANGASIANODON Chevey, 1931

Pangasianodon Chevey, 1931: 538. Type species: Pangasianodon gigas Chevey, 1931. Type by monotypy. Gender: Masculine.

Remarks: Treatment of this genus as valid follows Rainboth (1996).

Pangasianodon gigas Chevey, 1931

Pangasianodon gigas Chevey, 1931: 538, figs. 1-2, pl. 1. Type locality: Cambodge. No types known (cast of type in Musée Economique de Phnom-Pehn).

Pangasius paucidens Fang \& Chaux, in Chaux \& Fang, 1949b: 344, fig. 6. Type locality: Cambodge. Holotype: MNHN 1966-0730.

Distribution: Mekong River basin; stocked in various reservoirs in Thailand (Kottelat, 2001b).

Pangasianodon hypophthalmus (Sauvage, 1878)

Helicophagus hypophthalmus Sauvage, 1878: 235. Type locality: Laos. Lectotype: MNHN a-0745, designated by Kottelat (1984b: 812).

Pangasius sutchi Fowler, 1937: 141, figs. 27-29. Type locality: Bangkok, Siam. Holotype: ANSP 67902.

Distribution: Mekong and Chao Phraya River basin (Kottelat, 2001b); in cultivation elsewhere in Asia, including Viet Nam and Myanmar.

PANGASIUS Valenciennes, 1840

Pangasius Valenciennes, in Cuvier \& Valenciennes, 1840b: 45 (34 of Strasbourg deluxe edition). Type species: Pangasius buchanani Valenciennes, 1840 (= Pimelodus pangasius Hamilton, 1822). Type by monotypy. Gender: Masculine.

Pseudopangasius Bleeker, 1862c: 399. Type species: Pangasius polyuranodon Bleeker, 1852. Type by original designation. Gender: Masculine. Also in Bleeker (1862-63: 14, 75).

Neopangasius Popta, 1904: 180. Type species: Neopangasius nieuwenhuisii Popta, 1904. Type by monotypy. Gender: Masculine.

Sinopangasius Chang \& Wu, 1965: 11, 13. Type species: Sinopangasius semicultratus Chang \& Wu, 1965. Type by original designation. Gender: Masculine.

Pangasius bocourti Sauvage, 1880

Pangasius (Pseudopangasius) Bocourti Sauvage, 1880b: 229. Type locality: Phnom-Penh [Cambodia]. Holotype: MNHN 0000-9528.

Pangasius altifrons Durand, 1940: 23, pl. 5. Type locality: Tonlé-Sap [Cambodia]. Holotype: at Institut Océanographique Nhatrang.

Distribution: Mekong and Chao Phraya River basins (Roberts \& Vidthayanon, 1991; Kottelat, 2001b).

Pangasius conchophilus Roberts \& Vidthayanon, 1991

Pangasius conchophilus Roberts \& Vidthayanon, 1991: 114, figs. 1b, 2j, 5. Type locality: Thabo, Nongkhai prov. [Thailand]. Holotype: NIFI 2227.

Distribution: Mekong, Bangpakong, and Chao Phraya River basins (Roberts \& Vidthayanon, 1991; Kottelat, 2001b).

Pangasius djambal Bleeker, 1846

Pangasius djambal Bleeker, 1846b: 290. Type locality: Batavia, in fluviis. Possible syntypes: BMNH 1863.12.4.81 (1, 400+ mm TL), RMNH 6854 (1), RMNH 31192 (6), RMNH 8069 (1).

Pangasius bedado Roberts, 1999: 110, figs. 1-3. Type locality: Palembang, Sumatra. Holotype: MZB 2598. 
Distribution: Java and Borneo (Roberts \& Vidthayanon, 1991), Sumatra (Roberts, 1999).

Remarks: Roberts \& Vidthayanon (1991:116) designated a neotype (RMNH 6854) for Pangasius djambal although several specimens from Bleeker's collections, some of which may be syntypes, were reported on therein. The "neotype" was incorrectly listed as a lectotype by Eschmeyer et al. (1998). Synonymy follows Gustiano et al. (2004). Register number of syntype of Pangasius djambal incorrectly reported as BMNH 1863.12.11.81 in Eschmeyer et al. (1998).

Pangasius elongatus Pouyaud, Gustiano \& Teugels, 2002

Pangasius elongatus Pouyaud, Gustiano \& Teugels, 2002: 248, fig. 5. Type locality: Vietnam: Lower Mekong River Delta. Holotype: MZB 10890.

Distribution: Lower reaches of the Mekong, Chao Phraya, and Bangpakong Rivers (Pouyaud et al., 2002).

Pangasius humeralis Roberts, 1989

Pangasius humeralis Roberts, 1989a: 131, figs. 100, 101c. Type locality: Kapuas basin, fish market at Sintang, Western Borneo (Kalimantan Barat, Indonesia). Holotype: MZB 3680.

Distribution: Kapuas River basin, western Borneo (Roberts, 1989a; Roberts \& Vidthayanon, 1991).

$\dagger$ Pangasius indicus (Marck, 1876)

$\dagger$ Brachyspondylus indicus Marck, 1876a: 412, pl. 24 (fig. 2). Type locality: Padang, Sumatra; Tertiary. Holotype: at Royal Geol. Mus. Dresden (imperfect fish). Also in Marck (1876b), priority of publication not established.

$\dagger$ Pseudeutropius verbeekii Günther, 1876: 435, pl. 15 (fig. 2). Type locality: Highlands of Padang, Sumatra; Tertiary. Syntypes (2): BMNH 47527 (postcranial fish).

Distribution: Padang, Sumatra; Tertiary.

Remarks: Gunther (1876: 436) noted that his specimens were likely conspecific with that of Marck, but considered Marck's name inaccurately reflected the geographic distribution and was, therefore, inappropriate. Woodward (1901: 326) lists $\dagger$ Brachyspondylus indicus, as a junior synonym of $\dagger$ Pseudeutropius verbeekii, which was followed in the literature. Also see Sanders (1934) for summary of the taxonomic history of these names. Roberts \& Jumnongthai (1999) treated this as a species of Pangasius, apparently following Sanders (1934: 22). Patterson (1993) expressed skepticism of the reported Eocene age of these fossils.

Pangasius kinabatanganensis Roberts \& Vidthayanon, 1991

Pangasius kinabatanganensis Roberts \& Vidthayanon, 1991: 123, figs. 2P, 10. Type locality: Kinabatangan River at Deramakot [Borneo, Malaysia]. Holotype: FMNH 68042.

Distribution: Kinabatangan River basin, northeastern Borneo (Roberts \& Vidthayanon, 1991).

Pangasius krempfi Fang \& Chaux, 1949

Pangasius krempfi Fang \& Chaux, in Chaux \& Fang, 1949b: 343, fig. 5. Type locality: en mer .... à Bong-Lao [Vietnam]. Holotype: MNHN 1966-0729.

Sinopangasius semicultratus Chang \& Wu, 1965: 11, 13, figs. 1-4. Type locality: Off Paihai, Kwangtung, China. Holotype: ASIZB 56-1174.

Distribution: Mekong and Hue River basins, and along coast of South China Sea of Vietnam and Guandong, China (Kottelat, 2001b).

Pangasius kunyit Pouyaud, Teugels \& Legendre, 1999

Pangasius kunyit Pouyaud, Teugels \& Legendre, 1999: 251, fig. 2. Type locality: Sangasanga Village, 30 km southeast of the town of Samarinda, delta of Mahakam River, East Kalimantan, Indonesia. Holotype: MZB 10009.

Distribution: Mahakam River delta, Borneo, Indonesia (Pouyaud et al., 1999).

Pangasius larnaudii Bocourt, 1866

Pangasius Larnaudii Bocourt, 1866: 15, pl. 1 (figs. 2, 2a). Type locality: Ajuthia. Syntypes: MNHN 0000-1549 (1), MNHN a-9423 (1).

Pangasius taeniura Fowler, 1935b: 98, fig. 19. Type locality: Bangkok, Siam. Holotype: ANSP 61753.

Pangasius burgini Fowler, 1937: 141, figs. 24-26. Type locality: Bangkok, Siam. Holotype: ANSP 67901.

Distribution: Mekong, Meklong and Chao Phraya River basins (Roberts \& Vidthayanon, 1991; Kottelat, 2001 b).

Pangasius lithostoma Roberts, 1989

Pangasius lithostoma Roberts, 1989a: 132, fig. 102. Type locality: Market at Sintang, Western Borneo (Kalimantan Barat, Indonesia). Holotype: MZB 3678. 
Distribution: Kapuas River basin, western Borneo (Roberts, 1989a; Roberts \& Vidthayanon, 1991).

Pangasius macronema Bleeker, 1851

Pangasius macronema Bleeker, 1851a: 11. Type locality: Banjermassing, in fluviis. Holotype (116 mm): possibly NMV 45892 (1), RMNH 6855 (10), BMNH 1863.12.4.66 (1).

Pangasius siamensis Steindachner, 1878c: 393. Type locality: Menam-Fluss bei Bangkok. Syntypes: NMW 45469 (1), NMW 76998 (1).

? Pangasius aequilabialis Fowler, 1937: 140, figs. 20-23. Type locality: Bangkok, Siam. Holotype: ANSP 67897.

Distribution: Mekong and Chao Phraya River basins, Java, and Borneo (Kottelat, 2001b).

Remarks: BMNH lists BMNH 1863.12.4.66 (1, $144 \mathrm{~mm} \mathrm{SL)} \mathrm{as} \mathrm{a} \mathrm{type} \mathrm{of} \mathrm{Pangasius} \mathrm{macronema} \mathrm{but} \mathrm{is} \mathrm{too} \mathrm{large} \mathrm{to}$ be the holotype.

Pangasius mahakamensis Pouyaud, Gustiano \& Teugels, 2002

Pangasius mahakamensis Pouyaud, Gustiano \& Teugels, 2002: 246, fig. 4. Type locality: Indonesia, East Kalimantan Province, Mahakam River at Samarinda. Holotype: MZB 10886.

Distribution: Asia: Mahakam River of eastern Kalimantan, Indonesia (Pouyaud et al., 2002).

Pangasius mekongensis Gustiano, Teugels \& Pouyaud, 2003

Pangasius mekongensis Gustiano, Teugels \& Pouyaud, 2003: 370, fig. 7. Type locality: [Lower Mekong River, Vietnam]. Holotype: MZB 10847.

Distribution: Mekong River, Vietnam (Gustiano, Teugels \& Pouyaud, 2003).

Pangasius myanmar Roberts \& Vidthayanon, 1991

Pangasius myanmar Roberts \& Vidthayanon, 1991: 131, fig. 17. Type locality: Rangoon, [Myanmar]. Holotype: SU 33787.

Distribution: Myanmar, known only from types (Roberts \& Vidthayanon, 1991).

Pangasius nasutus (Bleeker, 1863)

Pseudopangasius nasutus Bleeker, 1863f: 72. Type locality: Bandjermassin, in fluviis. Holotype (270 mm TL): possibly BMNH 1863.12.4.113 (207 mm SL, $255 \mathrm{~mm} \mathrm{TL).} \mathrm{Also} \mathrm{described} \mathrm{as} \mathrm{new} \mathrm{in} \mathrm{Bleeker} \mathrm{(1863e).}$

Pangasius ponderosus Myers, in Herre \& Myers, 1937: 67, pl. 6. Type locality: Chandra Dam, Perak, Malay Peninsula. Holotype: SU 14162.

Distribution: Sumatra, Borneo and Malay Peninsula (Roberts \& Vidthayanon, 1991).

Pangasius nieuwenhuisii (Popta, 1904)

Neopangasius Nieuwenhuisii Popta, 1904: 180. Type locality: le Bo, Bornéo central. Holotype: RMNH 7546; described in more detail, with illustration of holotype, in Popta (1906: 30, pl. 1, figs. 3a, 3b).

Distribution: Mahakam River basin, eastern Borneo (Roberts \& Vidthayanon, 1991).

Pangasius pangasius (Hamilton, 1822)

Pimelodus pangasius Hamilton, 1822: 163, 376, pl. 33 (fig. 52). Type locality: Estuaries of Bengal. No types known.

Pachypterus luridus Swainson 1839: 306. No types known. Made available by reference to "Ham. p. 163, f. 62 [sic, 52]" [= Hamilton (1822, 163, pl. 33, fig. 52)]. Unneeded new name for Pimelodus pangasius Hamilton, 1822.

Pangasius Buchanani Valenciennes, in Cuvier \& Valenciennes, 1840b: 45 (34 of Strasbourg deluxe edition), pl. 425. Type locality: Estuaries of Bengal. No types known. Unneeded new name for Pimelodus pangasius Hamilton, 1822, apparently to avoid tautonymy.

Pangasius pangasius godavarii David, 1962: 151, fig. 3g. Type locality: Godavary River, at Rajahmundry [India]. Syntypes: at Central Inland Fisheries Research Institute's Tank Fisheries Unit, Bangalore, India.

Pangasius pangasius upiensis Srivastava, 1968: 97, fig. 60. Type locality: Bale-ka-Maidan, R. Rohini, Gorakhpur, Uttar Pradesh, India. Holotype: at Zool. Mus. Gorakhpur Univ., India.

Distribution: Rivers and estuaries of Indian subcontinent (Roberts \& Vidthayanon, 1991).

Remarks: Redescribed in Hora (1938b).

Pangasius polyuranodon Bleeker, 1852

Pangasius polyuranodon Bleeker, 1852b: 425. Type locality: Bandjermassing, in fluviis. Holotype (160 mm TL): possibly RMNH 6855.

Pangasius juaro Bleeker, 1852d: 589. Type locality: Palembang, in fluviis. Holotype (336 mm TL): possibly BMNH 1863.12.4.79 (1) or RMNH 6855 (1 of larger specimens). 
Distribution: Rivers of Sumatra and northern, western and southern Borneo (Pouyaud et al., 2002).

Pangasius rheophilus Pouyaud \& Teugels, 2000

Pangasius rheophilus Pouyaud \& Teugels, 2000: 194, figs. 1-2. Type locality: Bahau River, tributary of upper Kayan at Longpujungan, Bulungan Regency, Kalimantan Timur, Indonesia. Holotype: MZB 10010.

Distribution: Bahau River, tributary of upper Kayan at Longpujungan, Kalimantan Timur, Indonesia (Pouyaud \& Teugels, 2000).

Pangasius sabahensis Gustiano,Teugels \& Pouyaud, 2003

Pangasius sabahensis Gustiano,Teugels \& Pouyaud, 2003: 372, fig. 8. Type locality: [Kinabatangan River basin, North Borneo, Sabah State, Malaysia]. Holotype: MZB 10847.

Distribution: Kinabatangan River basin, North Borneo, Sabah State, Malaysia (Gustiano et al., 2003).

Pangasius sanitwongsei Smith, 1931

Pangasius sanitwongsei Smith, 1931: 29, figs. 13-14. Type locality: Menam Chao Phya at Koh Yai, Central Siam. Holotype: Siam Department of Fisheries (apparently lost).

Pangasius beani Smith, 1931: 26. Type locality: Klong Ban Poh, off Lopburi, near Ayuthia, Central Siam. Holotype: USNM 90308.

Distribution: Mekong and Chao Phraya River basins (Roberts \& Vidthayanon, 1991; Kottelat, $2001 b$ ).

Remarks: Roberts \& Vidthayanon (1991: 138) acting as first reviser, selected Pangasius sanitwongsei as valid.

\section{Species inquirendae, Pangasius}

Pangasius delicatissimus Bleeker, 1863 (in Bleeker, 1862-63): 73, footnote. Type locality: Krawang, Java. No types known, based on a drawing.

Pangasius hoeksi Hardenberg, 1948: 412. Type locality: Kapuas River (W. Borneo). Holotype (275 mm): Whereabouts unknown.

PSEUDOLAIS Vaillant, 1902

Pseudolais Vaillant, 1902: 51. Type species: Pseudolais tetranema Vaillant, 1902. Type by monotypy. Gender: Masculine.

Pteropangasius Fowler, 1937:142. Type species: Pangasius cultratus Smith, 1931. Type by original designation. Gender: Masculine.

Pseudolais micronemus (Bleeker, 1847)

Pangasius micronemus Bleeker, 1847b: 8. Type locality: Java. Syntypes: Possibly BMNH 1863.12.4.82 (1, $250 \mathrm{~mm}$ SL), RMNH 6856 (3). Also in Bleeker (1847: 166).

Pangasius rios Bleeker, 1851d: 205. Type locality: Bandjermassing, in fluviis. Holotype (115 mm TL): possibly BMNH 1863.12.4.92 ( $104 \mathrm{~mm}$ SL).

Pseudolais tetranema Vaillant, 1902: 52, fig. 3. Type locality: Tepoe, bords du Mahakam, Bornéo central. Holotype: Possibly RMNH 7821.

Pangasius de Zwaani Weber \& de Beaufort, 1912: 535, pl. 12 (fig. 3). Type locality: Taluk, Sumatra. Holotype: ZMA 113011; illustration of holotype reproduced in Weber \& de Beaufort (1913: fig. 103).

Pangasius tubbi Inger \& Chin, 1959: 287, fig. 47. Type locality: Confluence of the Deramakot River with the Kinabatangan River, Kinabatangan District, North Borneo. Holotype: FMNH 68047.

Distribution: Mekong and Hue Rivers, Malay Peninsula, Sumatra, Java, and Borneo (Roberts \& Vidthayanon, 1991).

Pseudolais pleurotaenia (Sauvage, 1878)

Pangasius pleurotaenia Sauvage, 1878: 235. Type locality: Laos. Lectotype: MNHN 0000-9529 designated by, and illustrated in, Kottelat (1984b: 813, fig. 8a). One of two syntypes illustrated in Sauvage (1881:169, pl. 8, fig. 6).

Pangasius cultratus Smith, 1931: 25. Type locality: Tapi River near Bandon, peninsular Siam. Holotype: USNM 90306.

Pangasius fowleri Smith, 1931: 28. Type locality: Lopburi River at Lopburi, Central Siam. Holotype: USNM 90309. Distribution: Mekong, Meklong, Tapi and Chao Phraya River basins (Roberts \& Vidthayanon, 1991; Kottelat, 
2001b).

\section{PIMELODIDAE}

Pimelodini Bonaparte, 1838: 131. Type genus: Pimelodus La Cepède, 1803.

Pimelodinae Swainson, 1838: 331, 338. Type genus: Pimelodus La Cepède, 1803.

Sorubinae Swainson, 1838: 356. Type genus: Sorubium Swainson, 1838.

Hypophthalmini Bleeker, 1862 (in Bleeker, 1862-63): 15. Type genus: Hypophthalmus Spix \& Agassiz, 1829.

Calophysinae Eigenmann, 1890: 12. Type genus: Calophysus Müller \& Troschel, 1842.

Luciopimelodinae Driver, 1919: 451. Type genus: Luciopimelodus Eigenmann \& Eigenmann, 1888.

Pinirampidae Fernández-Yépez, 1965: 12. Type genus: Pinirampus Bleeker, 1858.

Brachyplatystomatini Lundberg \& Akama, 2005: 496. Type genus: Brachyplatystoma Bleeker, 1862.

Remarks: Priority of the publications by Bonaparte and Swainson has not yet been established. Thus, the authorship of the valid family name is not fixed. Callophysidae, based on the unjustified emendation Callophysus, has been used on occasion.

Taxonomic summary: Lundberg \& Littmann (2003).

Phylogeny: Nass (1991).

Review: Mago-Leccia et al. (1986, Venezuela); Castro (1986a, Colombian Amazon and Orinoco basins).

29 genera, 93 species; 4 named fossil species.

AGUARUNICHTHYS Stewart, 1986

Aguarunichthys Stewart, 1986a: 662. Type species: Aguarunichthys torosus Stewart, 1986. Type by original designation. Gender: Masculine.

Aguarunichthys inpai Zuanon, Rapp Py-Daniel \& Jégu, 1993

Aguarunichthys inpai Zuanon, Rapp Py-Daniel \& Jégu, 1993: 258, figs. 2b, 6. Type locality: Brazil: Amazonas State: Solimões River drainage, north of the Marchantaria Island, at about $15 \mathrm{~km}$ above confluence with Rio Negro drainage. Holotype: INPA 5398.

Distribution: Middle Amazon River basin, Brazil (Lundberg \& Littmann, 2003).

Aguarunichthys tocantinsensis Zuanon, Rapp Py-Daniel \& Jégu, 1993

Aguarunichthys tocantinsensis Zuanon, Rapp Py-Daniel \& Jégu, 1993: 252, figs. 2a, 4. Type locality: Brazil: Pará State: Tocantins River drainage, rapids above Marabá. Holotype: INPA 5400.

Distribution: Tocantins River basin, Brazil (Lundberg \& Littmann, 2003).

Aguarunichthys torosus Stewart, 1986

Aguarunichthys torosus Stewart, 1986a: 663, figs. 7-8. Type locality: Peru, Departamento Amazonas, Río Cenepa, $1.6 \mathrm{~km}$ west of Huampami and near Peruvian military camp Chavez Valdivia, about $210 \mathrm{~m}$ elev., Approx. $4^{\circ} 28^{\prime} \mathrm{S}, 78^{\circ} 10^{\prime} \mathrm{W}$. Holotype: LACM 39651-1.

Distribution: Cenepa River basin, Amazon River drainage, Peru (Lundberg \& Littmann, 2003).

BAGROPSIS Lütken, 1874

Bagropsis Lütken, 1874c: 32. Type species: Bagropsis reinhardti Lütken, 1874. Type by monotypy. Gender: Feminine.

Bagropsis reinhardti Lütken, 1874

Bagropsis Reinhardti Lütken, 1874a: 32. Type locality: in flumine Rio das Velhas. Syntypes: BMNH 1876.1.10.9 (1), NMW 45905 (1), ZMUC P 29630 (1), ZMUC P 29631 (1), ZMUC P 29632 (1). Species illustrated and described in Lütken (1875: 160 (and p. V of summary), pl. 1, fig. 2).

Distribution: Das Velhas River basin, São Francisco River drainage, Brazil (Lundberg \& Littmann, 2003).

BERGIARIA Eigenmann \& Norris, 1901

Bergiella Eigenmann \& Norris, 1900: 355. Type species: Pimelodus westermanni Reinhardt, 1874. Type by original 
designation. Gender: Feminine. Preoccupied by Bergiella Baker, 1897 (Hymenoptera), replaced by Bergiaria Eigenmann \& Norris, 1901.

Bergiaria Eigenmann \& Norris, 1901: 272. Type species: Pimelodus westermanni Reinhardt, 1874. Type by being a replacement name. Gender: Feminine. Replacement for Bergiella Eigenmann \& Norris, 1900.

Bergiaria platana (Steindachner, 1908)

Bergiella platana Steindachner, 1908d: 111. Type locality: La Plata. Holotype: at NMW.

Distribution: Paraná River basin, Argentina (Lundberg \& Littmann, 2003).

Bergiaria westermanni (Reinhardt, 1874)

Pimelodus Westermanni Reinhardt, in Lütken, 1874a: 33. Type locality: in flumine Rio das Velhas. Syntypes: ZMUC P 29638 (1), ZMUC P 29639 (1). Species illustrated and described in more detail in Lütken (1875: 167 (and p. VI of summary), pls. 2 (fig. 4), pl. 3 (fig. 4a-b)).

Distribution: Das Velhas River basin in São Francisco River drainage, Brazil (Lundberg \& Littmann, 2003).

BRACHYPLATYSTOMA Bleeker, 1862

Piramutana Bleeker, 1858b: 356. Type species: Bagrus piramuta Kner, 1857. Type by monotypy. Gender: Feminine.

Piratinga Bleeker, 1858b: 355. Type species: Bagrus reticulatus Kner, 1857. Type by subsequent designation by Bleeker (1862-63): 11. Gender: Feminine.

Brachyplatystoma Bleeker, 1862 (in Bleeker, 1862-63): 10. Type species: Platystoma vaillanti Valenciennes, 1840. Type by original designation. Gender: Neuter.

Malacobagrus Bleeker, 1862 (in Bleeker, 1862-63): 11. Type species: Pimelodes filamentosus Lichtenstein, 1819. Type by original designation. Gender: Masculine.

Taenionema Eigenmann \& Bean, 1907: 662. Type species: Taenionema steerei Eigenmann \& Bean, 1907. Type by original designation. Gender: Neuter. Preoccupied by Taenionema Banks, 1905 (Plecoptera), and by Taenionema Bolivar, 1906 (Orthoptera); replaced by Goslinia Myers, 1941.

Goslinia Myers, 1941: 88. Type species: Taenionema steerei Eigenmann \& Bean, 1907. Type by being a replacement name. Gender: Feminine. Replacement for Taenionema Eigenmann \& Bean, 1907, preoccupied by Taenionema Banks, 1905.

Ginesia Fernández-Yépez, 1951: [1]. Type species: Ginesia cunaguaro Fernández-Yépez, 1951. Type by original designation. Gender: Feminine.

Merodontotus Britski, 1981: 109. Type species: Merodontotus tigrinus Britski, 1981. Type by original designation. Gender: Masculine.

Review: Miranda Ribeiro (1918f, Brazil).

Remarks: Treatment of Brachyplatystoma as valid over older names follows Lundberg \& Littmann (2003) and Lundberg \& Akama (2005). Synonymy of Merodontodus and Goslinia follows Lundberg \& Akama (2005).

Brachyplatystoma capapretum Lundberg \& Akama, 2005

Brachyplatystoma capapretum Lundberg \& Akama, 2005: 501, fig. 8. Type locality: Brazil, Amazonas State, Rio Tefé sand beach, Lago Mucura, Supiã-Pucu, Tefé, 3²2'S, 64²43'W. Holotype: MZUSP 78481.

Distribution: Amazon River basin of Brazil and Peru (Lundberg \& Akama, 2005).

Brachyplatystoma filamentosum (Lichtenstein, 1819)

Pimelodes filamentosus Lichtenstein, 1819: 60. Type locality: Brasilien. Holotype: ZMB 2973.

Platystoma affine Valenciennes, in Cuvier \& Valenciennes, 1840b: 24 (18 in the Strasbourg deluxe edition). Type locality: [not stated; apparently from Rio de Janeiro, Brazil]. Holotype: MNHN a-9360 (mounted).

Platystoma gigas Günther, 1872: 450. Type locality: River Huallaga, Upper Amazon [Peru]. Holotype: BMNH 1872.12.3.1 (stuffed).

Piratinga pirá-ä̈ba Goeldi, 1898: 464, 477, pl. (fig. 4). Type locality: [Pará, Brazil]. Holotype: Whereabouts unknown. Name available from caption on plate.

Brachyplatystoma goeldii Eigenmann \& Bean, 1907: 661, fig. 1. Type locality: Brazil: Amazon River, between Para and Manaos. Holotype: USNM 52561.

Distribution: Amazon and Orinoco River basins and major rivers of the Guianas and NE Brazil (Lundberg \& Litt- 
mann, 2003).

Brachyplatystoma juruense (Boulenger, 1898)

Platystoma juruense Boulenger, 1898e: 421, pl. 39. Type locality: Rio Jurua, an affluent of the Amazons, Brazil. Holotype: BMNH 1897.11.26.11.

Ginesia cunaguaro Fernández-Yépez, 1951: [2], unnumbered figure. Type locality: Río Apure, 3 km east of San Fernando de Apure, Venezuela. Holotype: AFY 51189; current whereabouts unknown.

Distribution: Amazon and Orinoco River basins (Lundberg \& Littmann, 2003).

Brachyplatystoma platynemum Boulenger, 1898

Brachyplatystoma platynema Boulenger, 1898b: 477. Type locality: Brazil, Pará. Holotype: BMNH 1898.10.11.20.

Taenionema steerei Eigenmann \& Bean, 1907: 662, fig. 2. Type locality: Brazil, Amazon River, between Para and Manaos. Holotype: USNM 52571.

Distribution: Amazon and Orinoco River basins (Lundberg \& Littmann, 2003).

$\dagger$ Brachyplatystoma promagdalena Lundberg, 2005

$\dagger$ Brachyplatystoma promagdalena Lundberg, 2005: 599, figs. 1a, 2a, 3, 4a, 5a, 6a. Type locality: Miocene Villavieja Formation of the Honda Group in the southern end of the río Magdalena basin near the town of Villavieja,

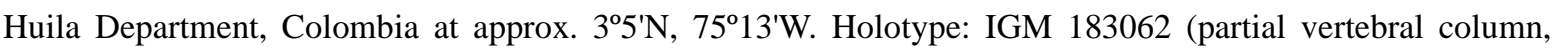
Weberian complex).

Distribution: La Venta formation, central Colombia; middle Miocene (Lundberg, 2005).

Brachyplatystoma rousseauxii (Castelnau, 1855)

Bagrus rousseauxii Castelnau, 1855: 32, pl. 14 (fig. 1). Type locality: rivière des Amazones. Holotype: MNHN a9457 (dry).

Bagrus Goliath Kner, 1857: 379. Type locality: Salto Theotonio [Brazil]. Holotype: at NMW.

Brachyplatystoma paraense Steindachner, 1909a: 195. Type locality: Fischmarkt von Pará [Brazil]. Holotype: NMW 47590. Illustrated and described in more detail in Steindachner (1915e: 65, fig. 4, pl. 13, fig. 5).

Distribution: Amazon and Orinoco River basins and other major rivers of South America east of the Andes mountains (Lundberg \& Littmann, 2003).

Brachyplatystoma tigrinum (Britski, 1981)

Merodontotus tigrinus Britski, 1981: 110, figs. 1-2. Type locality: Brazil, Cachoeira do Teotônio, rio Madeira, Território de Rondônia. Holotype: MZUSP 14004.

Distribution: Amazon River basin (Lundberg \& Littmann, 2003).

Brachyplatystoma vaillantii (Valenciennes, 1840)

? Silurus Vaillantii Cuvier, 1816: 204. Type locality: Brazil. Based on "bagre primus” Marcgravius (1648: 173). Originally as Sil. Nob. Vaillantii.

Platystoma Vaillantii Valenciennes, in Cuvier \& Valenciennes, 1840b: 21 (16 of Strasbourg deluxe edition), pl. 423. Type locality: de Cayenne et de Suriname. Syntypes: MNHN b-0158 (1), MNHN b-0159 (1). Based in part on "bagre primus" Marcgravius (1648: 173).

Bagrus reticulatus Kner, 1857: 376, pl. 1 (fig. 1). Type locality: Salto Theotonio, am Flusse Araguay, Forte do Rio branco und Rio Madeira [Brazil]. Holotype: at NMW.

Bagrus piramuta Kner, 1857: 382. Type locality: Barra do Rio negro und Borba Rio Madeira [Brazil]. Syntypes: at NMW.

Brachyplatystoma parnahybae Steindachner, 1908e: 126. Type locality: Rio Parnahyba. Holotype: at NMW.

Distribution: Amazon and Orinoco River basins and major rivers of the Guianas and NE Brazil (Lundberg \& Littmann, 2003).

Remarks: Inclusion of Silurus Vaillanti in the synonymy is based on the citation of the same species from Marcgravius (1648) in the text of that species as well as Platystoma Vaillantii Valenciennes.

\section{Species inquirenda, Brachyplatystoma}

Bagrus punctulatus Castelnau, 1855: 33, pl. 14 (fig. 2). Type locality: de la rivière des Amazones [Brazil]. Holotype: at MNHN, but current whereabouts unknown. 
CALOPHYSUS Müller \& Troschel, 1842

Calophysus Müller \& Troschel, in Müller, 1842b: 310. Type species: Pimelodes macropterus Lichtenstein, 1819. Type by subsequent designation by Bleeker (1862-63: 12). Gender: Masculine. Also as new in Müller (1843: 318).

Callophysus Müller \& Troschel, 1848: 629. Type species: Pimelodes macropterus Lichtenstein, 1819. Type by subsequent designation by Bleeker (1862-63: 12). Gender: Masculine. Unjustified emendation of Calophysus, and a spelling that was in wide use.

Pimeletropis Gill, 1859b: 196. Type species: Pimeletropis lateralis Gill, 1859. Type by monotypy. Gender: Feminine.

Pseudocallophysus Bleeker, 1862 (in Bleeker, 1862-63): 12. Type species: Pimelodus ctenodus Spix \& Agassiz, 1829. Type by original designation. Gender: Masculine.

Calophysus macropterus (Lichtenstein, 1819)

Pimelodes macropterus Lichtenstein, 1819: 59. Type locality: Brasilien. Holotype: ZMB 3055.

Pimelodus ctenodus Spix \& Agassiz, 1829: 21, pl. 8a. Type locality: aequitorialis fluviis [Brazil]. Holotype: Whereabouts unknown (Kottelat, 1988).

Pimeletropis lateralis Gill, 1859b: 196. Type locality: Amazon River. Holotype: at Lyceum Natural History, NY (whereabouts unknown).

Distribution: Amazon and Orinoco River basins (Lundberg \& Littmann, 2003).

\section{CHEIROCERUS Eigenmann, 1917}

Cheirocerus Eigenmann, 1917a: 398. Type species: Cheirocerus eques Eigenmann, 1917. Type by original designation. Gender: Masculine.

Sovichthys Schultz, 1944c: 190. Type species: Sovichthys abuelo Schultz, 1944. Type by original designation. Gender: Masculine.

Revision: Stewart \& Pavlik (1985).

Cheirocerus abuelo (Schultz, 1944)

Sovichthys abuelo Schultz, 1944c: 191, pl. 1 (fig. a). Type locality: Venezuela, Río de Los Pájaros, 3 km above Lago Maracaibo, at depth of $15 \mathrm{ft}$. Holotype: USNM 121183.

Distribution: Lake Maracaibo basin (Lundberg \& Littmann, 2003).

Cheirocerus eques Eigenmann, 1917

Cheirocerus eques Eigenmann, 1917a: 398, pl. 39. Type locality: Villa Bella [Bolivia]. Holotype: FMNH 58255.

Distribution: Amazon River basin (Lundberg \& Littmann, 2003).

Cheirocerus goeldii (Steindachner, 1908)

Pimelodina goeldii Steindachner, 1908c: 83. Type locality: Rio Purus [Brazil]. Holotype: NMW 45503.

Pimelodus leptus Eigenmann \& Pearson, in Eigenmann \& Allen, 1942: 104, pl. 4 (fig. 3). Type locality: Río Pachitea [Peru]. Holotype: CAS 47288.

Distribution: Purus River basin (Lundberg \& Littmann, 2003).

DUOPALATINUS Eigenmann \& Eigenmann, 1888

Duopalatinus Eigenmann \& Eigenmann, 1888b: 136. Type species: Platystoma emarginatum Valenciennes, 1840. Type by monotypy. Gender: Masculine.

Duopalatinus emarginatus (Valenciennes, 1840)

Platystoma emarginatum Valenciennes, in Cuvier \& Valenciennes, 1840b: 25 (19 of Strasbourg deluxe edition). Type locality: rivière de Saint-François. Holotype: MNHN a-9353 (mounted).

Distribution: São Francisco River basin, Brazil (Lundberg \& Littmann, 2003).

Duopalatinus peruanus Eigenmann \& Allen, 1942

Duopalatinus peruanus Eigenmann \& Allen, 1942: 107, pl. 4 (fig. 4). Type locality: Rio Puinagua, mouth of Rio Pacaya [Río Ucayali system, Peru]. Holotype: CAS 63630.

Distribution: Amazon and Orinoco River (Lundberg \& Littmann, 2003). 
EXALLODONTUS Lundberg, Mago-Leccia \& Nass, 1991

Exallodontus Lundberg, Mago-Leccia \& Nass, 1991: 843. Type species: Exallodontus aguanai Lundberg, MagoLeccia \& Nass, 1991. Type by original designation. Gender: Masculine.

Exallodontus aguanai Lundberg, Mago-Leccia \& Nass, 1991

Exallodontus aguanai Lundberg, Mago-Leccia \& Nass, 1991: 847, figs. 2-10, 13. Type locality: Venezuela, Territorio Federal Delta Amacuro, Río Orinoco near Los Castillos, $159 \mathrm{n}$ mi from sea buoy at the terminus of the ship navigation channel in Boca Grande, $8^{\circ} 32^{\prime} \mathrm{N}, 62^{\circ} 23^{\prime} \mathrm{W}, 20-30 \mathrm{~m}$. Holotype: MBUCV V-18930.

Distribution: Amazon and Orinoco River basins (Lundberg \& Littmann, 2003).

HEMISORUBIM Bleeker, 1862

Hemisorubim Bleeker, 1862 (in Bleeker, 1862-63): 10. Type species: Platystoma platyrhynchos Valenciennes, 1840. Type by original designation. Gender: Masculine.

Hemisorubim platyrhynchos (Valenciennes, 1840)

Platystoma platyrhynchos Valenciennes in Cuvier \& Valenciennes, 1840b: 27 (20 of Strasbourg deluxe edition). Type locality: [Not stated]. Holotype: MNHN 0000-1203.

Distribution: Amazon, Maroni, Orinoco, and Paraná River basins (Lundberg \& Littmann, 2003).

\section{HYPOPHTHALMUS Cuvier, 1829}

Hypophthalmus Cuvier, 1829: 293. Type species: Hypophthalmus edentatus Spix \& Agassiz, 1829. Type by subsequent designation by Bleeker (1862-63: 15). Gender: Masculine.

Hypophthalmus Spix \& Agassiz, 1829: 16. Type species: Hypophthalmus edentatus Spix \& Agassiz, 1829. Type by subsequent designation Bleeker (1862-63: 15). Gender: Masculine.

Notophthalmus Hyrtl, 1859: 17. Type species: Hypophthalmus marginatus Valenciennes, 1840. Type by monotypy. Gender: Masculine. Preoccupied by Notophthalmus Rafinesque, 1820 (Amphibia); apparently not replaced.

Pseudohypophthalmus Bleeker, 1862 (in Bleeker, 1862-63): 15. Type species: Hypophthalmus fimbriatus Kner, 1857. Type by original designation. Gender: Masculine.

Review: Lopez-Fernandez \& Winemiller (2000, Venezuela).

Phylogeny: Howes (1983a), Lundberg et al. (1991).

Hypophthalmus edentatus Spix \& Agassiz, 1829

Hypophthalmus edentatus Spix \& Agassiz, 1829: 16, pl. 9. Type locality: influviis Brasiliae equitorialis. Syntypes (2): Possibly MHNN 706 (2); see Kottelat (1988: 24) for discussion.

Hypophthalmus Spixii Valenciennes in Cuvier \& Valenciennes, 1840b: 231 (172 of Strasbourg deluxe edition). Type locality: la partie la plus septentrionale du Brésil. Possible syntypes: MHNN 706 (2). Unneeded new name for Hypophthalmus edentatus Spix \& Agassiz, 1829.

Distribution: Amazon and Orinoco River basins and Atlantic coastal rivers of Guyana and Suriname (Lundberg \& Littmann, 2003).

Hypophthalmus fimbriatus Kner, 1857

Hypophthalmus fimbriatus Kner, 1857: 444, pl. 9 (fig. 30). Type locality: Rio negro [Brazil]. Syntype: NMW 50519 (1).

Distribution: Amazon River at Santarém and Negro River basin in Brazil and Venezuela (Lundberg \& Littmann, 2003).

Hypophthalmus marginatus Valenciennes, 1840

Hypophthalmus marginatus Valenciennes, in Cuvier \& Valenciennes, 1840b: 225 (168 of Strasbourg deluxe edition), pl. 439. Type locality: Cayenne, ... Surinam. Syntypes: MNHN a-8961 (1), MNHN a-8963 (1), RMNH 1932 (1), RMNH 1933 (1).

Distribution: Amazon and Orinoco River basins and major rivers of French Guiana and Suriname (Lundberg \& Littmann, 2003).

Hypophthalmus oremaculatus Nani \& Fuster, 1947

Hypophthalmus oremaculatus Nani \& Fuster, 1947: 3, figs. 2-3. Type locality: Puerto Gaboto, Rio Paraná, curso 
inferior [Argentina]. Holotype: MACN 3496.

Distribution: Paraná River basin, Brazil and Argentina (Lundberg \& Littmann, 2003).

\section{Species inquirendae, Hypophthalmus}

Hypophthalmus longifilis Valenciennes in Cuvier \& Valenciennes, 1840b: 230 (171 of Strasbourg deluxe edition). Type locality: Surinam. Syntypes: RMNH 1931 (1), RMNH 2974 (1), RMNH 2988 (1).

Hypophthalmus perporosus Cope, 1878: 673. Type locality: Peru, probably Nauta. Holotype: USNM 132589.

Hypophthalmus devall Röhl, 1942: 383. Type locality: Venezuela, rios Aupre y Orinoco. Type(s): Whereabouts unknown.

IHERINGICHTHYS Eigenmann \& Norris, 1900

Iheringichthys Eigenmann \& Norris, 1900: 354. Type species: Pimelodus labrosus Lütken, 1874. Type by original designation. Gender: Masculine.

Iheringichthys labrosus (Lütken, 1874)

Pimelodus labrosus Lütken, 1874b: 200. Type locality: La Plata. Syntypes: ZMUC P 29633 (1), ZMUC P 29634 (1), ZMUC P 29635 (1), ZMUC P 29636 (1)..

Distribution: Paraná River basin (Lundberg \& Littmann, 2003).

Iheringichthys megalops Eigenmann \& Ward, 1907

Iheringichthys megalops Eigenmann \& Ward, in Eigenmann, McAtee \& Ward, 1907: 115, pl. 32 (figs. 3-4). Type locality: Paraguay, Bahia Negra, Río Paraguay. Holotype: CAS 63631.

Distribution: Paraná River basin, Paraguay (Lundberg \& Littmann, 2003).

\section{LEIARIUS Bleeker, 1862}

Leiarius Bleeker, 1862 (in Bleeker, 1862-63): 10. Type species: Arius longibarbis Castelnau, 1855. Type by original designation. Gender: Masculine.

Sciadeoides Eigenmann \& Eigenmann, 1888b: 136. Type species: Sciades marmoratus Gill, 1870. Type by monotypy. Gender: Masculine. Originally proposed as a subgenus of Sciades.

\section{Leiarius marmoratus (Gill, 1870)}

Sciades marmoratus Gill, 1870: 95. Type locality: Peru or Ecuador, Amazon system, Río Marañon or Río Napo. Holotype: USNM 8447.

Distribution: Amazon, Essequibo, and Orinoco River basins (Lundberg \& Littmann, 2003).

Leiarius pictus (Müller \& Troschel, 1849)

Bagrus (Sciades) pictus Müller \& Troschel, 1849: 8, pl. 1 (fig. 1). Type locality: unbekannt. Holotype: ZMB 2991.

Distribution: Amazon and Orinoco River basins (Lundberg \& Littmann, 2003).

\section{Species inquirendae, Leiarius}

Pimelodus arekaima Jardine, in Schomburgk, 1841: 178, pl. 5. Type locality: Upper Essequibo, Rio Branco. No types known.

Arius longibarbis Castelnau, 1855: 36, pl. 15 (fig. 2). Type locality: rivière des Amazones. Holotype: MNHN 0000-1189. Originally as Arius? longibarbis.

Pimelodus multiradiatus Kner, 1857: 414. Type locality: Borba am Rio Madeira und Forte do Rio branco am Rio Facutu [Brazil]. Syntypes: NMW 16515 (1), NMW 16518 (1).

LUCIOPIMELODUS Eigenmann \& Eigenmann, 1888

Luciopimelodus Eigenmann \& Eigenmann, 1888b: 122. Type species: Pimelodus pati Valenciennes, 1835. Type by original designation. Gender: Masculine.

Luciopimelodus pati (Valenciennes, 1835)

Pimelodus Pati Valenciennes, 1835, in Valenciennes, 1835-47: pl. 1 (figs. 7-9). Type locality: Not stated [Corri- 
entes, Argentina]. Probable holotype: MNHN a-9419 (1, dry). Name available from plate; mentioned in Valenciennes (1847: 7) from Corrientes. Described in Cuvier \& Valenciennes (1840b: 176 (131 of Strasbourg deluxe edition)).

Silurus 11-radiatus Larrañaga, 1923: 386. Type locality: Uruguay. No types known. Corresponds to Silurus pati on p. 376.

Distribution: La Plata and Blanco River basins (Lundberg \& Littmann, 2003).

MEGALONEMA Eigenmann, 1912

Megalonema Eigenmann, 1912b: 150. Type species: Megalonema platycephalum Eigenmann, 1912. Type by original designation. Gender: Neuter.

Megalonema argentina (MacDonagh, 1938)

Perugia argentina MacDonagh, 1938: 157. Type locality: Argentina, rio Paraná, Posadas, Territorio de Misiones. Holotype: MLP 5.V.I.35.

Distribution: Paraná River basin, Argentina (Lundberg \& Littmann, 2003).

Megalonema pauciradiatum Eigenmann, 1919

Megalonema pauciradiatum Eigenmann, in Driver, 1919: 455. Type locality: Paraguay, Villa Rica. Holotype: CAS 63672.

Distribution: Paraná River basin, Paraguay (Lundberg \& Littmann, 2003).

Megalonema platanum (Günther, 1880)

Pimelodus platanus Günther, 1880a: 10. Type locality: Parana. Holotype: BMNH 1872.6.8.18.

Distribution: Paraná River basin (Lundberg \& Littmann, 2003).

Megalonema platycephalum Eigenmann, 1912

Megalonema platycephalum Eigenmann, 1912b: 150, fig. 31, pl. 10 (fig. 2). Type locality: Tumatumari, British Guiana. Holotype: FMNH 53224.

Distribution: Amazon, Essequibo, and Orinoco River basins (Lundberg \& Littmann, 2003).

Megalonema psammium Schultz, 1944

Megalonema platycephalum psammium Schultz, 1944c: 216, pl. 2 (fig. b). Type locality: Venezuela, Rio Palmar at the bridge $70 \mathrm{~km}$ southwest of Maracaibo. Holotype: USNM 121175.

Distribution: Lake Maracaibo basin, Colombia, Venezuela (Lundberg \& Littmann, 2003).

Megalonema xanthum Eigenmann, 1912

Megalonema xanthum Eigenmann, 1912a: 16. Type locality: Girardot [Colombia]. Holotype: FMNH 56032; holotype illustrated in Eigenmann (1922b: 35, pl. 3, fig. 3) as Perugia xanthus.

Distribution: Magdalena River basin, Colombia (Lundberg \& Littmann, 2003; Maldonado-Ocampo et al., 2005).

PARAPIMELODUS La Monte, 1933

Parapimelodus La Monte, 1933b: 226. Type species: Pimelodus valenciennis Lütken, 1874. Type by original designation. Gender: Masculine.

Revision: Lucena et al. (1992).

Parapimelodus nigribarbis (Boulenger, 1889)

Pimelodus (Pseudorhamdia) nigribarbis Boulenger, 1889: 266. Type locality: Brazil, Rio Grande do Sul, Camaquam River. Lectotype: BMNH 1889.8.24.6, designated by Lucena et al. (1992: 145).

Distribution: Laguna dos Patos basin, Brazil (Lundberg \& Littmann, 2003).

Parapimelodus valenciennis (Lütken, 1874)

Pimelodus valenciennis Lütken, 1874b: 200. Type locality: La Plata. Holotype: ZMUC P 29637.

Pimelodus Spegazzinii Perugia, 1891: 632. Type locality: Rio Durazno. Syntypes: MSNG 8026 (2).

Distribution: Paraná River basin (Lundberg \& Littmann, 2003).

PERRUNICHTHYS Schultz, 1944

Perrunichthys Schultz, 1944c: 229. Type species: Perrunichthys perruno Schultz, 1944. Type by original designation. Gender: Masculine. 


\section{Perrunichthys perruno Schultz, 1944}

Perrunichthys perruno Schultz, 1944c: 230, pl. 3 (fig. b); fig. 3. Type locality: Venezuela, Río Negro, below the mouth of the Río Yasa, about $75 \mathrm{~km}$. south of Rosario, west side of Lago Maracaibo. Holotype: USNM 121189.

Distribution: Lake Maracaibo basin (Lundberg \& Littmann, 2003).

PHRACTOCEPHALUS Spix \& Agassiz, 1829

Phractocephalus Spix \& Agassiz, 1829: 10. Type species: Phractocephalus bicolor Spix \& Agassiz, 1829. Type by monotypy. Gender: Masculine.

Pirarara Spix \& Agassiz, 1829: 23. Type species: Silurus hemioliopterus Bloch \& Schneider, 1801. Type by monotypy. Gender: Feminine. Appeared first as name in synonymy under Phractocephalus and in legend for pl. 6; made available by Bleeker (1862-63: 11) but with authorship to Spix \& Agassiz.

Remarks: Phractocephalus selected as valid by first reviser action of Agassiz (in Spix \& Agassiz, 1831: Conspectus); see Kottelat (1989b: 321) for details.

Phractocephalus hemioliopterus (Bloch \& Schneider, 1801)

Silurus hemioliopterus Bloch \& Schneider, 1801: 385. Type locality: [in flumine Maranham Brasiliae].

Pimelodus grunniens Humboldt, in Humboldt \& Valenciennes, 1821: 172. Type locality: le Bas-Orénoque. No types known.

Phractocephalus bicolor Spix \& Agassiz, 1829: 23, pl. 6. Type locality: in fluvio Amazonum [Brazil]. Whereabouts unknown (Kottelat, 1988). As Pirarara bicolor on plate.

Distribution: Amazon and Orinoco River basins (Lundberg \& Littmann, 2003).

Remarks: See Boeseman (1983b) for comments on taxonomy and nomenclature.

$\dagger$ Phractocephalus nassi Lundberg \& Aguilera, 2003

$\dagger$ Phractocephalus nassi Lundberg \& Aguilera, 2003: 101, figs. 3, 5a, 5b, 5e, 5h, 5k, 5l. Type locality: El Mamón oil field, $350 \mathrm{~m}$ north of oil well number 1 , locality USB $56-\mathrm{FU}, 11^{\circ} 15^{\prime} \mathrm{N}, 70^{\circ} 13^{\prime} \mathrm{W}$, Urumaco Formation, middle member, Falcón State, northwestern Venezuela. Holotype: MCN.USB OL-2142.

Distribution: Urumaco Formation, Falcón State, Venezuela, upper Miocene (Lundberg \& Aguilera, 2003).

Remarks: Described earlier by Lundberg et al. (1988) as Phractocephalus hemioliopterus.

PIMELODINA Steindachner, 1877

Pimelodina Steindachner, 1877a: 149. Type species: Pimelodina flavipinnis Steindachner, 1877. Type by monotypy. Gender: Feminine.

Revision: Stewart (1986a).

Pimelodina flavipinnis Steindachner, 1877

Pimelodina flavipinnis Steindachner, 1877a: 150, pl. 13 (fig. 2) [as Pimelodus (Pimelodina) flavipinnis]. Type locality: Amazonenstrom bei Para [Brazil]. Holotype: NMW 45498.

Pimelodina nasus Eigenmann \& Eigenmann, 1888b: 120. Type locality: Para [Brazil]. Holotype: MCZ 7490.

Distribution: Amazon and Orinoco River basins (Lundberg \& Littmann, 2003).

\section{PIMELODUS La Cepède, 1803}

Pimelodus La Cepède, 1803: 93. Type species: Pimelodus maculatus La Cepède, 1803. Type by subsequent designation, by Gill (1861b). Gender: Masculine.

Pseudariodes Bleeker, 1862 (in Bleeker, 1862-63): 11. Type species: "Pseudariodes clarias = Silurus clarias B1." [= Pimelodus Blochii Valenciennes, 1840, nec. Silurus clarias Linnaeus, 1758]. Type by original designation. Gender: Masculine.

Pseudorhamdia Bleeker, 1862 (in Bleeker, 1862-63): 11. Type species: Pimelodus maculatus La Cepède, 1803. Type by original designation. Gender: Feminine.

Pimelodus absconditus Azpelicueta, 1995

Pimelodus absconditus Azpelicueta, 1995: 72, figs. 1-2. Type locality: Argentina, Misiones, San Javier, Uruguay River. Holotype: MLP 8781.

Distribution: Paraná River basin (Lundberg \& Littmann, 2003). 
Pimelodus albicans (Valenciennes, 1840)

Arius albidus Valenciennes, 1835, in Valenciennes, 1835-47: pl. 3, fig. 2. Type locality: Not stated. Holotype: MNHN a-9400. Name made available by caption on plate.

Arius albicans Valenciennes, in Cuvier \& Valenciennes, 1840b: 80 (60 of Strasbourg deluxe edition). Type locality: Buénos-Ayres. Syntype: MNHN a-9400 (dry). Unneeded new name or lapsus calami for Arius albidus Valenciennes.

Silurus muticus Larrañaga, 1923: 386. Type locality: Uruguay. No types known.

Distribution: Paraná River basin, Argentina (Lundberg \& Littmann, 2003).

Remarks: Treatment of Pimelodus albicans as valid follows Lundberg \& Littmann (2003), in which Arius albidus Valenciennes, 1835, was treated as a nomen oblitum.

Pimelodus albofasciatus Mees, 1974

Pimelodus albofasciatus Mees, 1974: 137, pl. 4. Type locality: Suriname, Sipaliwini. Holotype: RMNH 26156.

Distribution: Amazon, Orinoco, upper Corantijn and Sipaliwini River basins (Lundberg \& Littmann, 2003).

Pimelodus altissimus Eigenmann \& Pearson, 1942

Pimelodus altissimus Eigenmann \& Pearson, in Eigenmann \& Allen, 1942: 106, pl. 5 (fig. 5). Type locality: Rio Ucayali, near Orellana [Peru]. Holotype: CAS 55369.

Distribution: Amazon River basin (Lundberg \& Littmann, 2003).

Pimelodus atrobrunneus Vidal \& Lucena, 1999

Pimelodus atrobrunneus Vidal \& Lucena, 1999: 123, figs. 1-3. Type locality: Brasil: rio Ligeiro na estrada entre Marcelino Ramos e Maximiliano de Almeida, Marcelino Ramos, 27³8'S-51 $52^{\prime} \mathrm{O}$, Rio Grande do Sul. Holotype: MCP 19678.

Distribution: Upper Uruguay River basin, Brazil (Lundberg \& Littmann, 2003).

Pimelodus blochii Valenciennes, 1840

Pimelodus Blochii Valenciennes, in Cuvier \& Valenciennes, 1840b: 188 (139 of Strasbourg deluxe edition). Type locality: Surinam. Syntypes: MNHN b-0072 (1), ZMB 2995 (1). Based in part on Silurus clarias of Bloch (1782: pl. 35, figs. 1-2), not Silurus clarias Linnaeus, 1758.

Pseudorhamdia macronema Bleeker, 1864a: 79, pl. 13 (fig. 7), pl. 14. Type locality: Surinama. Lectotype: RMNH 3069, designated by Boeseman (1972: 317).

Pseudorhamdia piscatrix Cope, 1870b: 569. Type locality: Pebas [Peru]. Syntypes: ANSP $8386-87$ (2).

Distribution: Gulf of Paria, Amazon, Corantijn, Essequibo and Orinoco River basins (Lundberg \& Littmann, 2003); Magdalena River basin (Maldonado-Ocampo et al., 2005).

Remarks: Eschmeyer et al. (1998) and Lundberg \& Littmann (2003) list MNHN b-0072 as lectotype of Pimelodus blochii, without citing a published source of the lectotype designation and, instead, apparently relying on a notation in the MNHN catalog to that effect. No published designation has been found, so the two specimens are tentatively retained as syntypes here.

Pimelodus brevis Marini, Nichols \& La Monte, 1933

Pimelodus brevis Marini, Nichols \& La Monte, 1933: 1, fig. 1. Type locality: Argentina, Rio de la Plata, San Fernando. Holotype: AMNH 12240 (missing).

Distribution: Paraná River basin; Durazno River, Argentina (Lundberg \& Littmann, 2003).

Pimelodus coprophagus Schultz, 1944

Pimelodus clarias coprophagus Schultz, 1944c: 203, fig. 2. Type locality: Venezuela, Río Agua Caliente, 2 to 3 km above the southwestern corner of Lago Maracaibo. Holotype: USNM 121150.

Distribution: Lake Maracaibo basin (Lundberg \& Littmann, 2003).

Pimelodus fur (Reinhardt, 1874)

Pseudorhamdia fur Reinhardt, in Lütken, 1874c: 33. Type locality: in flumine Rio das Velhas. Syntypes: NMW 44443 (3), NMW 44763 (2), SMNS 2026 (1), ZMB 9181 (2), ZMUC P 29643-ZMUC P 29651 (1 each). Illustrated and described in more detail in Lütken (1875: 169 (and p. VI of summary), pl. 2, fig. 3; pl. 3, fig. 3a).

Distribution: Das Velhas River basin, São Francisco River drainage, Brazil (Lundberg \& Littmann, 2003).

Pimelodus garciabarrigai Dahl, 1961

Pimelodus garcia-barrigai Dahl, 1961: 494. Type locality: Colombia, Deep pool in Caño Lozada, about $11 \mathrm{~km}$ 
above its junction with the Guayabero River. Holotype: ICNMHN 744 (Cala, 1981).

Distribution: Guayabero River basin, Orinoco River drainage, Colombia (Lundberg \& Littmann, 2003).

Pimelodus grosskopfii Steindachner, 1879

Pimelodus (Pimelodus) Grosskopfii Steindachner, 1879c: 194. Type locality: Río Cauca [Colombia]. Syntypes: NMW 45781 (4), NMW 45782 (1). Also described as new in Steindachner (1879g: 186); described in more detail and illustrated in Steindachner (1880b: 57, pl. 1 figs. 1, 1a).

Pimelodus longifilis Posada, 1909: 294. Type locality: aus dem Cauca [Colombia]. No types known.

Distribution: Magdalena River basin and Lake Maracaibo basins (Lundberg \& Littmann, 2003).

Pimelodus heraldoi Azpelicueta, 2001

Pimelodus heraldoi Azpelicueta 2001: 194, figs. 1-2 Type locality: Brazil, Estado de São Paulo: Município de Pirassununga, rio Mogi Guaçu in Emas. Holotype: MZUSP 22713.

Distribution: Upper Paraná River basin, Brazil (Lundberg \& Littmann, 2003).

Pimelodus jivaro Eigenmann \& Pearson, 1942

Pimelodus jivaro Eigenmann \& Pearson, in Eigenmann \& Allen, 1942: 105, pl. 4 (fig. 2). Type locality: Rio Morona [Peru]. Syntypes (5; 85-105 mm): CAS 55891 (3).

Distribution: Upper Amazon River basin, Ecuador, Peru (Lundberg \& Littmann, 2003).

Pimelodus maculatus La Cepède, 1803

Pimelodus maculatus La Cepède, 1803: 94. Type locality: Le grand fleuve de la Plata, ... Buénos-Ayres, ainsi qu’à la Encenada. No types known.

Distribution: Paraná and São Francisco River basins (Lundberg \& Littmann, 2003).

Pimelodus microstoma Steindachner, 1877

Pimelodus microstoma Steindachner, 1877b: 604, footnote. Type locality: Brazil, von Irisanga, Rio branco und Barra do Rio negro. Syntypes: NMW 45823 (1), NMW 45824 (2).

Distribution: Amazon River basin, Brazil (Lundberg \& Littmann, 2003).

Pimelodus mysteriosus Azpelicueta, 1998

Pimelodus mysteriosus Azpelicueta, 1988: 88, figs. 1-3, 5-6, 8, 10, 12, 14. Type locality: Argentina, Misiones, Candelaria, arroyo Anselmo. Holotype: MLP 9191.

Distribution: Paraná River basin (Lundberg \& Littmann, 2003).

Pimelodus navarroi Schultz, 1944

Pimelodus grosskopfii navarroi Schultz, 1944c: 207, pl. 1 (fig. c). Type locality: Venezuela, Río Palmar at the bridge $70 \mathrm{~km}$ southwest of Maracaibo. Holotype: USNM 121174.

Distribution: Lake Maracaibo basin (Lundberg \& Littmann, 2003).

Pimelodus ornatus Kner, 1857

Pimelodus ornatus Kner, 1857: 411, pl. 6 (fig. 18). Type locality: Surinam, dem Rio negro und Cujaba [Brazil]. Syntypes: NMW 45832 (2), NMW 45843 (1).

Megalonema rhabdostigma Fowler, 1914: 256, fig. 10. Type locality: Rupununi River, British Guiana ... in the highlands of British Guiana, approximately secured in North Latitude $2^{\circ}$ to $3^{\circ}$, and West Longitude $50^{\circ} 20^{\prime}$. Holotype: ANSP 39338.

Distribution: Amazon, Corantijn, Essequibo, Orinoco and Paraná River basins and major rivers of the Guianas (Lundberg \& Littmann, 2003).

Pimelodus ortmanni Haseman, 1911

Pimelodus ortmanni Haseman, 1911b: 379, pl. 50 (fig. 2). Type locality: Brazil, Porto União da Victoria, Rio Iguassú. Holotype: FMNH 54240.

Distribution: Paraná River basin, Brazil (Lundberg \& Littmann, 2003).

Pimelodus paranaensis Britski \& Langeani, 1988

Pimelodus paranaensis Britski \& Langeani, 1988: 410, figs. 1-2. Type locality: Ilha Solteira, Rio Paraná, SP [Brazil]. Holotype: MZUSP 23089.

Distribution: Upper Paraná River basin, Brazil (Lundberg \& Littmann, 2003).

Pimelodus pictus Steindachner, 1877

Pimelodus pictus Steindachner, 1877a: 144. Type locality: aus dem Amazonenstrome auf peruanishen Gebiete, und 
aus dem Hyavary. Syntypes: NMW 45859 (3).

Distribution: Amazon and Orinoco River basins (Lundberg \& Littmann, 2003).

Pimelodus platicirris Borodin, 1927

Pimelodus platicirris Borodin, 1927c: 2. Type locality: Salto de Pirassunungo, Rio Mogy Guassu, São Paulo Prov., Brazil. Holotype: AMNH 8628.

Distribution: Paraná River basin, Brazil; known only from the holotype (Lundberg \& Littmann, 2003).

Pimelodus punctatus (Meek \& Hildebrand, 1913)

Megalonema punctatum Meek \& Hildebrand, 1913: 77. Type locality: Río Tuyra, Boca de Cupe, Panama. Holotype: FMNH 7577.

Megalonema robustum Meek \& Hildebrand, 1913: 78. Type locality: Río Tuyra, Marriganti, Panama. Holotype: FMNH 7578.

Distribution: Tuira River basin, Panama (Lundberg \& Littmann, 2003).

\section{Species inquirendae, Pimelodus}

Pimelodus rigidus Spix \& Agassiz, 1829: 19, pl. 7 (fig. 2). Type locality: Brazil aequatoriali. Holotype: Whereabouts unknown (Kottelat, 1988).

Pseudariodes pantherinus Lütken, 1874b: 192. Type locality: Caracas. Syntypes: BMNH 1876.1.10.10 (1), NMW 45851 (1), USNM 44970 (1), ZMUC P 29640 (1), ZMUC P 29641 (1), ZMUC P 29642 (1).

Piramutana macrospila Günther, 1880a: 10, pl. 2. Type locality: Rio de la Plata. Holotype: BMNH 1878.9.10.14.

PINIRAMPUS Bleeker, 1858

Pinirampus Bleeker, 1858b: 198. Type species: Pimelodus pinirampus Agassiz [= Pimelodus pirinampu Spix \& Agassiz, 1829]. Type by monotypy. Gender: Masculine.

Pirinampus Günther, 1864: 135. Type species: Pimelodus pirinampu Spix \& Agassiz, 1829. Type by being a replacement name. Gender: Masculine. Unjustified emendation of Pinirampus Bleeker, 1858.

Perugia Eigenmann \& Norris, 1900: 355. Type species: Pirinampus [sic] agassizii Steindachner, 1876. Type by original designation. Gender: Feminine.

Pinirampus pirinampu (Spix \& Agassiz, 1829)

Pimelodus barbancho Humboldt, in Humboldt \& Valenciennes, 1821: 172. Type locality: Guarico, l'Apure et d'autres rivières des steppes de Venezuela. Holotype: at MNHN.

Pimelodus pirinampu Spix \& Agassiz, 1829: 20, pl. 8. Type locality: Brasiliae fluviis. Type(s): Whereabouts unknown (Kottelat, 1988).

Pimelodus insignis Jardine, in Schomburgk, 1841: 180, pl. 6. Type locality: Rio Branco [Brazil]. No types known.

Galeichthys araguayensis Castelnau, 1855: 37, pl. 17 (fig. 3). Type locality: Rio Araguay [Brazil]. Holotype: MNHN a-9366 (mounted).

Pinirampus typus Bleeker, 1862 (in Bleeker, 1862-63): 11. Type locality: Brasiliae fluviis. Type(s): Whereabouts unknown. Unneeded replacement for Pimelodus pinirampu Spix \& Agassiz, 1829, apparently to avoid Stricklandian tautonymy.

Pirinampus Agassizii Steindachner, 1876: 607, pl. 12. Type locality: Pará [Brazil]. Holotype: NMW 45955.

Distribution: Amazon, Essequibo, Orinoco, and Paraná River basins (Lundberg \& Littmann, 2003).

Remarks: Pimelodus barbancho treated here as a nomen oblitum, following Lundberg \& Littmann (2003).

PLATYNEMATICHTHYS Bleeker, 1858

Platynematichthys Bleeker, 1858b: 356. Type species: Bagrus punctulatus Kner, 1857. Type by monotypy. Gender: Masculine.

Platypogon Starks, 1913: 28. Type species: Platypogon caerulorostris Starks, 1913. Type by monotypy. Gender: Masculine.

Platynematichthys notatus (Jardine, 1841)

Pimelodus notatus Jardine, in Schomburgk, 1841: 181, pl. 7. Type locality: Fort St. Joaquim on the Rio Branco [Bra- 
zil]. No types known.

Bagrus punctulatus Kner, 1857: 380. Type locality: Forte do Principe am Rio Guaporé und Rio branco [Brazil]. Holotype: at NMW. Preoccupied by Bagrus punctulatus Castelnau, 1855; replaced by Bagrus nigropunctatus Kner, 1858.

Bagrus nigropunctatus Kner, 1858: 345. Type locality: Forte do Principe am Rio Guapore und Rio branco [Brazil]. Holotype: at NMW. Replacement for Bagrus punctulatus Kner, 1857; preoccupied by Bagrus punctulatus Castelnau, 1855.

Platypogon caerulorostris Starks, 1913: 29, pl. 5. Type locality: Brazil, Pará. Holotype: SU 2222.

Distribution: Amazon and Orinoco River basins (Lundberg \& Littmann, 2003).

PLATYSILURUS Haseman, 1911

Platysilurus Haseman, 1911a: 320. Type species: Platysilurus barbatus Haseman, 1911. Type by monotypy. Gender: Masculine.

Platysilurus malarmo Schultz, 1944

Platysilurus malarmo Schultz, 1944c: 234, fig. 3g, pl. 3 (fig. c). Type locality: Venezuela, Lago Maracaibo near the mouth of the Río Concho. Holotype: USNM 121179.

Distribution: Lake Maracaibo basin (Lundberg \& Littmann, 2003).

Platysilurus mucosus (Vaillant, 1880)

Platystoma mucosa Vaillant, 1880b: 151. Type locality: à Caldéron (Haute-Amazone). Holotype: MNHN a-1955.

Duoplatinus goeldii Steindachner, 1908b: 65. Type locality: Rio Purus [Brazil]. Holotype: at NMW.

Platysilurus barbatus Haseman, 1911: 320, pl. 52. Type locality: São Antonio de R. Madeira [Brazil]. Holotype: FMNH 60306.

Distribution: Amazon and Orinoco River basins (Lundberg \& Littmann, 2003).

Platysilurus olallae (Orcés, 1977)

Duopalatinus olallae Orcés, 1977: 78, fig. 4. Type locality: el bajo Bobonaza [Ecuador]. Holotype: at MEPN (132 $\mathrm{mm}$, catalog number not stated).

Distribution: Ecuador.

Remarks: Not treated in Lundberg \& Littmann (2003).

PLATYSTOMATICHTHYS Bleeker, 1862

Platystomatichthys Bleeker, 1862 (in Bleeker, 1862-63): 10. Type species: Platystoma sturio Kner, 1857. Type by original designation. Gender: Masculine.

Platystomatichthys sturio (Kner, 1857)

Platystoma sturio Kner, 1857: 395, pl. 3 (figs. 9-9a). Type locality: Rio branco [Brazil]. Holotype: at NMW.

Distribution: Amazon River basin (Lundberg \& Littmann, 2003).

PROPIMELODUS Lundberg \& Parisi, 2002

Propimelodus Lundberg \& Parisi, 2002: 77. Type species: Pimelodus eigenmanni van der Stigchel, 1946. Type by original designation.

Propimelodus eigenmanni (van der Stigchel, 1946)

Pimelodus eigenmanni van der Stigchel, 1946: 64. Type locality: Brazil. Holotype: RMNH 15465. Preoccupied by Pimelodus eigenmanni Boulenger, 1891 [now Pimelodella eigenmanni, in the Heptapteridae]; apparently not replaced.

Distribution: Amazon, Approuague, Kourou, and Oyapock River basins (Lundberg \& Littmann, 2003).

Remarks: Pimelodus eigenmanni is tentatively treated as valid, despite being a junior primary homonym, following Art. 23.9.5 of the Code of Zoological Nomenclature. Redescribed in Lundberg \& Parisi (2002).

PSEUDOPLATYSTOMA Bleeker, 1862

Pseudoplatystoma Bleeker, 1862 (in Bleeker, 1862-63): 10. Type species: Silurus fasciatus Linnaeus, 1766. Type by original designation. Gender: Neuter. 
Hemiplatystoma Bleeker, 1862 (in Bleeker, 1862-63): 10. Type species: Platystoma tigrinum Valenciennes, 1840. Type by original designation. Gender: Neuter.

Remarks: Eigenmann \& Eigenmann (1888b) serve as first revisers in giving precedence to Pseudoplatystoma over Hemiplatystoma.

Pseudoplatystoma corruscans (Spix \& Agassiz, 1829)

Platystoma corruscans Spix \& Agassiz, 1829: 26 [pl. 13]. Type locality: in flumine S. Francisci [Brazil]. Holotype: Whereabouts unknown (Kottelat, 1988). As Sorubim caparary on plate.

Sorubim caparary Spix in Spix \& Agassiz, 1829: pl. 13. Type locality: in flumine S. Francisci [Brazil]. Holotype: Whereabouts unknown (Kottelat, 1988). Name available from caption on plate.

Platystoma coruscans Valenciennes, in Cuvier \& Valenciennes, 1840b: 17 (13 in the Strasbourg deluxe edition). Misspelling or emendation of Platystoma corruscans Spix \& Agassiz.

Silurus macrocephalus Larrañaga, 1923: 386. Type locality: Uruguay. No types known. Appears to correspond to Silurus mangrullo on p. 377, which is a nomen nudum.

Distribution: São Francisco and Paraná River basins (Lundberg \& Littmann, 2003)

Remarks: Agassiz (in Spix \& Agassiz, 1831: conspectus) serves as first reviser in giving precedence to Platystoma corruscans over Sorubim caparary.

Pseudoplatystoma fasciatum (Linnaeus, 1766)

Silurus fasciatus Linnaeus, 1766: 505. Type locality: Brasilia, Surinami. Holotype: Whereabouts unknown.

Platystoma artedii Günther, 1864: 106. Type locality: South America. Syntypes: On Mystus no. 6 in Seba (1734-65: 84, pl. 29, fig. 6) and Mystus no. 386 of Gronovius (1784: pl. 125).

Pseudoplatystoma fasciatum intermedium Eigenmann \& Eigenmann, 1888b: 138. Type locality: Obidos. Holotype: MCZ 7321.

Pseudoplatystoma fasciatum brevefile Eigenmann \& Eigenmann, 1889b: 31. Type locality: Goyaz [Brazil]. Holotype: MCZ 7317.

Pseudoplatystoma fasciatum reticulatum Eigenmann \& Eigenmann, 1889b: 30. Type locality: Rio Negro [Brazil]. Holotype: MCZ 23813.

Pseudoplatystoma fasciatum nigricans Eigenmann \& Eigenmann, 1889b: 31. Type locality: Xingu. Syntypes (2): MCZ 7301 (1), MCZ 7311 (1).

Distribution: Amazon, Corantijn, Essequibo, Orinoco, and Paraná River basins (Lundberg \& Littmann, 2003); Magdalena River basin (Maldonado-Ocampo et al., 2005).

Pseudoplatystoma tigrinum (Valenciennes, 1840)

Platystoma truncatum Spix \& Agassiz, 1829: 27, pl. 13a. Type locality: Brazil, Japurá and Solimoen. Type (s): Whereabouts unknown (Kottelat, 1988).

Platystoma tigrinum Valenciennes, in Cuvier \& Valenciennes, 1840b: 10 (8 of Strasbourg deluxe edition), pl. 422. Type locality: probablement originaire du Brésil. Holotype: MNHN a-9354 (mounted).

Distribution: Amazon and Orinoco River basins (Lundberg \& Littmann, 2003).

Remarks: Synonymy follows Lundberg \& Littmann (2003) which differs from most previous accounts, in which Platystoma truncatum was treated as a synonym of Pseudoplatystoma fasciatum. Because of this, Platystoma truncatum became the senior synonym for a widely used Pseudoplatystoma tigrinum. $P$. truncatum was treated as a nomen oblitum in Lundberg \& Littmann (2003), but without supporting documentation. Use of $P$. truncatum as a valid name has not been found in surveyed compilations of late $19^{\text {th }}$ or $20^{\text {th }}$ century and, therefore, the use of $P$. tigrinum as valid is continued here.

\section{Species inquirendae, Pseudoplatystoma}

Platystoma pardalis Valenciennes, 1836, in Valenciennes, 1835-47: pl. 4 (fig. 2). Type locality: Buenos Aires, Argentina. Holotype: MNHN a-8833. Name available from plate, mentioned in Valenciennes (1847: 6), as Platystoma panthale. Described in Cuvier \& Valenciennes (1840b: 15 (12 of Strasbourg deluxe edition)), as Platystoma pardale.

Platystoma orbignianus Valenciennes, 1836, in Valenciennes, 1835-47: pl. 4 (fig. 3). Type locality: Brazil. Holotype: MNHN b-0160. Name available from plate, description in Valenciennes (1847: 6), as Platystoma orbignyanum. 
Description in Cuvier \& Valenciennes (1840b: 12, [9 of Strasbourg deluxe edition]).

Platystoma panthale Valenciennes, 1847: 6. Type locality: [Not stated]. Holotype: MNHN a-8833. New name proposed for Platystoma pardalis Valenciennes, 1836, without explanation.

Platystoma punctifer Castelnau, 1855: 40, pl. 19 (fig. 2). Type locality: de l' Amazone. Holotype: MNHN 0000-1582.

\section{SORUBIM Cuvier, 1829}

Sorubim Cuvier, 1829: 293. Type species: Silurus lima Bloch \& Schneider, 1801. Type by subsequent designation, apparently by Kottelat (1988). Gender: Masculine.

Platystoma Spix \& Agassiz, 1829: 23. Type species: Silurus lima Bloch \& Schneider, 1801. Type by subsequent designation by Jordan (1917: 131). Gender: Neuter. Preoccupied by Platystoma Meigen, 1803 (Diptera).

Sorubim Spix \& Agassiz, 1829: 24. Type species: Silurus lima Bloch \& Schneider, 1801. Type by subsequent designation by Bleeker (1862-63: 10). Gender: Masculine. First appeared in synonymy of Platystoma Spix \& Agassiz and therefore not available, but made available because of use as a valid name by Bleeker (1862-63: 10) [ICZN art. 11.6.1]. Preoccupied by Sorubim Cuvier, 1829.

Sorubium Swainson, 1838: 356. Type species: Silurus lima Bloch \& Schneider, 1801. Type by being a replacement name. Unneeded replacement for Sorubim Spix \& Agassiz, 1829.

Abron Gistel, 1848: X. Type species: Silurus lima Bloch \& Schneider, 1801. Type by being a replacement name. Gender: Neuter. Replacement for Platystoma Valenciennes [= Spix \& Agassiz, 1829].

Remarks: Sorubim was apparently adopted by Cuvier (1829) from the Spix \& Agassiz (1829) manuscript, but the name was published first in the former book.

Revision: Littmann (1998).

Sorubim cuspicaudus Littmann, Burr \& Nass, 2000

Sorubim cuspicaudus Littmann, Burr \& Nass, 2000: 903, fig. 2b, 3, 4c. Type locality: Colombia, Departamento de Tolima, Río Magdalena drainage, at Puerto Soplaviento. Holotype: FMNH 56223.

Distribution: Magdalena and Sinu River basins, Colombia, and Lake Maracaibo (Lundberg \& Littmann, 2003).

Sorubim elongatus Littmann, Burr, Schmidt \& Isern, 2001

Sorubim elongatus Littmann, Burr, Schmidt \& Isern, 2001: 4, fig. 2. Type locality: Peru: Departamento Loreto, Maynas Province, Ullpa Caño, 50 m upstream of confluence with Moena Caño, Río Itaya drainage, Río Amazonas basin (approx. 346'20"S 73¹4'17"W). Holotype: SIUC 30303.

Distribution: Amazon, Essequibo, and Orinoco River basins (Lundberg \& Littmann, 2003).

Sorubim lima (Bloch \& Schneider, 1801)

Silurus lima Bloch \& Schneider, 1801: 384. Type locality: in flumine Maranham Brasiliae. Holotype: ZMB 3185 (stuffed).

Sorubim infraoculare Spix, in Spix \& Agassiz, 1829: pl. 15. Type locality: Brasiliae aequatorialis fluviis. Holotype: Whereabouts unknown (Kottelat, 1988). Name available from caption on plate.

Platystoma Luceri Weyenbergh, 1877: 10, pl. 3 (figs. 1-3). Type locality: Santa-Fe [Argentina]. Holotype: Whereabouts unknown.

Sorubim latirostris Miranda Ribeiro, 1920: 14, pl. 12. Type locality: Amazonas [Brazil]. Holotype: MNRJ 631.

Distribution: Amazon, Orinoco, Paraná and Parnaíba River basin (Lundberg \& Littmann, 2003).

Sorubim maniradii Littmann, Burr \& Buitrago-Suárez, 2001

Sorubim maniradii Littmann, Burr \& Buitrago-Suárez, 2001: 88, fig. 1. Type locality: Ecuador, Napo State, Río Yasuni, Río Napo drainage, 059'06"S 75²5'36"W. Holotype: FMNH 108814.

Distribution: Amazon River basin (Lundberg \& Littmann, 2003).

Sorubim trigonocephalus Miranda Ribeiro, 1920

Sorubim trigonocephalus Miranda Ribeiro, 1920: 12, pls. 10, 11. Type locality: Porto Velho [Brazil]. Holotype: MNRJ 882.

Distribution: Madeira and Tapajós River basins, Brazil (Lundberg \& Littmann, 2003).

\section{SORUBIMICHTHYS Bleeker, 1862}

Sorubimichthys Bleeker, 1862, in Bleeker, 1862-63: 10. Type species: Sorubim jandia Spix \& Agassiz, 1829. Type 
by original designation. Gender: Masculine.

Pteroglanis Eigenmann \& Pearson, in Pearson, 1924: 9. Type species: Pteroglanis manni Eigenmann \& Pearson, 1924. Type by monotypy. Gender: Masculine.

Sorubimichthys planiceps (Spix \& Agassiz, 1829)

Platystoma planiceps Spix \& Agassiz, 1829: 25, pl. 12. Type locality: Brazil: in fluminae Amazonum, Solimoëns, Rio Negro. Possible syntype or lectotype: MHNN 811(Kottelat, 1988).

Sorubim Pirauáca Spix, in Spix \& Agassiz, 1829: pl. 12. Type locality: Brazil: in fluminae Amazonum, Solimoëns, Rio Negro. Possible holotype: MHNN 811(Kottelat, 1988). Name available from caption on plate; as Platystoma planiceps in text.

Sorubim jandia Spix \& Agassiz, 1829: pl. 14. Type locality: equatorialis fluviis [Brazil]. Holotype: Whereabouts unknown (Kottelat, 1988). Name available from caption on plate; as Platystoma spatula in text.

Platystoma spatula Spix \& Agassiz, 1829: 26, pl. 14. Type locality: equatorialis fluviis [Brazil]. Types: Whereabouts unknown (Kottelat, 1988).

Sorubimichthys ortoni Gill, 1870: 94. Type locality: Maranon, or Upper Amazon, and Napo Rivers. Holotype: USNM 8238.

Pteroglanis manni Eigenmann \& Pearson, in Pearson, 1924: 9, pl. 1 (fig. 1). Type locality: Bolivia, Little Rio Negro, tributary to Rio Beni. Holotype: CAS 59623.

Distribution: Amazon and Orinoco River basins (Lundberg \& Littmann, 2003).

Remarks: Lundberg et al. (1989) reviewed the nomenclature of this species. Agassiz (in Spix \& Agassiz, 1831: conspectus) serves as first reviser in treating Platystoma planiceps as valid over Sorubim Pirauáca, and Platystoma spatula over Sorubim jandia. Precedence of Platystoma planiceps over Platystoma spatula may be by action of Lundberg et al. (1989).

STEINDACHNERIDION Eigenmann \& Eigenmann, 1919

Steindachneria Eigenmann \& Eigenmann, 1888b: 137. Type species: Steindachneria amblyurus Eigenmann \& Eigenmann, 1888. Type by original designation. Gender: Feminine. Preoccupied by Steindachneria Goode \& Bean, 1888 (in fishes), replaced by Steindachneridion Eigenmann \& Eigenmann, 1919.

Steindachneridion Eigenmann \& Eigenmann, 1919: 525. Type species: Steindachneria amblyurus Eigenmann \& Eigenmann, 1888. Type by being a replacement name. Gender: Neuter. Replacement for Steindachneria Eigenmann \& Eigenmann, 1888.

Revision, with key to species: Garavello (2005).

Steindachneridion amblyurum (Eigenmann \& Eigenmann, 1888)

Steindachneria amblyurus Eigenmann \& Eigenmann, 1888b: 137. Type locality: Jequitinhonha [Brazil]. Syntypes (2, 35-38 cm): MCZ 7324 (2).

Distribution: Jequitinhonha River basin, Brazil (Garavello, 2005).

Steindachneridion doceana (Eigenmann \& Eigenmann, 1889)

Steindachneria doceana Eigenmann \& Eigenmann, 1889b: 30. Type locality: Rio Doce [Brazil]. Syntypes (9): MCZ 23792 (1), MCZ 23793 (1), MCZ 23794 (7); one syntype illustrated in Garavello (2005: 614, fig. 6).

Distribution: Doce River basin, Brazil (Garavello, 2005).

$\dagger$ Steindachneridion iheringi (Woodward, 1899)

$\dagger$ Arius iheringi Woodward, 1899: 64, pl. 2 (fig. 1), pl. 3 (fig. 2). Type locality: Taubaté, Estado do Sao Paulo, Brazil; Tertiary Lignite. Syntypes: BMNH P.9220 (skull), Museu Paulista (nearly complete specimen), Senckenberg Museum (partial specimen).

Distribution: Paraíba River basin, Estado do São Paulo, Brazil; Tertiary (Santos, 1973).

Remarks: Species assigned to the Pimelodidae and the genus Steindachneridion by Santos (1973).

Steindachneridion melanodermatum Garavello, 2005

Steindachneridion melanodermatum Garavello, 2005: 618, fig. 9. Type locality: Brazil, Paraná State, municipality of Quedas do Iguaçu, rio Iguaçu, down river of the Salto Osório dam (approx. $25^{\circ} 35^{\prime} \mathrm{S} 5^{\circ} 05^{\prime} \mathrm{W}$ ). Holotype: MZUSP 87983.

Distribution: Iguaçu River, upper Paraná River Basin, Brazil (Garavello, 2005). 
Steindachneridion parahybae (Steindachner, 1877)

Platystoma Parahybae Steindachner, 1877b: 640, pl. 9. Type locality: Rio Parahyba bei Juiz de Fora; Rio Jequitinhonha [Brazil]. Syntypes: MCZ 25521 (1, skeleton), MCZ 7295 (1), MCZ 7323 (2), NMW (?).

Distribution: Paraíba do Sul River basin, Brazil (Garavello, 2005).

Remarks: Garavello (2005: 610) incorrectly reported the catalog number of one lot of the types as MCZ 73231. Redescribed in Oliveira \& de Moraes (1997).

Steindachneridion punctatum (Miranda Ribeiro, 1918)

Steindachneria scripta punctata Miranda Ribeiro, 1918d: 642. Type locality: Itaqui, Rio Grande do Sul [Brazil]. Holotype: MNRJ 1167. Originally Steindachneria scripta var. punctata.

Distribution: Upper Uruguay and Paraná River basin, Brazil (Garavello, 2005).

Steindachneridion scriptum (Miranda Ribeiro, 1918)

Steindachneria scripta Miranda Ribeiro, 1918d: 640. Type locality: Itaqui [Rio Grande do Sul, Brazil]. Lectotype: MZUSP 2286, designated by Britski (1969).

Distribution: Upper Paraná and Uruguay River basins, Brazil (Garavello, 2005).

$\dagger$ Steindachneridion silvasantosi Figueiredo \& Costa-Carvlho, 1999

† Steindachneridion silvasantosi Figueiredo \& Costa-Carvlho, 1999: 685, figs. 1-4. Type locality: Tremembé Formation, Lacustrine sedimentary deposit that outcrops in the mine Nossa Mina Senhora da Guia, Tremembé county, city of Taubaté, São Paulo State [Brazil]; Tertiary (? Oligocene). Holotype: DGM 1291-P; partial skull, including neurocranium and suspensorium.

Distribution: Paraíba River basin, São Paulo State, Brazil; Tertiary (Figueiredo \& Costa-Carvlho, 1999).

ZUNGARO Bleeker, 1858

Zungaro Bleeker, 1858b: 196. Type species: Zungaro humboldtii Bleeker [=Pimelodus zungaro Humboldt, 1821]. Type by absolute tautonymy. Gender: Masculine.

Paulicea Ihering, 1898: 108. Type species: Paulicea jahu Ihering, 1898. Type by subsequent designation by Eigenmann (1910). Gender: Feminine.

Remarks: Generic synonymy based on Silfvergrip (1992: 306).

Zungaro jahu (Ihering, 1898)

Paulicea jahu Ihering, 1898: 108. Type locality: Sao Paulo [Brazil]. Types: Whereabouts unknown.

Distribution: Paraná-Paraguay River basin (Lundberg \& Littmann, 2003).

Zungaro zungaro (Humboldt, 1821)

Pimelodus zungaro Humboldt, in Humboldt \& Valenciennes, 1821: 170, pl. 46 (fig. 1). Type locality: Tomependa, Río Marañon [Amazon River basin, Peru]. Holotype: Whereabouts unknown.

Bagrus flavicans Castelnau, 1855: 31, pl. 13 (fig. 2). Type locality: Brazil, l'Amazone. Holotype: MNHN a-8823 (mounted).

Zungaro humboldtii Bleeker, 1858b: 207. Type locality: Tomedenda, Río Marañon [Amazon River basin, Peru]. Holotype: Whereabouts unknown. Unneeded replacement name for Pimelodus zungaro, presumably to avoid tautomymy.

Platystoma Lütkeni Steindachner, 1876: 609, pl. 13. Type locality: Laufe des Amazonenstromes gefangen. Syntypes: at NMW.

Distribution: Amazon and Orinoco River basins (Lundberg \& Littmann, 2003).

ZUNGAROPSIS Steindachner, 1908

Zungaropsis Steindachner, 1908b: 67. Type species: Zungaropsis multimaculatus Steindachner, 1908. Type by monotypy. Gender: Feminine.

Zungaropsis multimaculatus Steindachner, 1908

Zungaropsis multimaculatus Steindachner, 1908b: 67. Type locality: Rio Xingu. Holotype: NMW 43537.

Remarks: Considered to be a valid species, in a genus near to Zungaro by A. Silfvergrip (pers. commun., 2003). 


\section{Species inquirenda, Pimelodidae}

Pimelodus (Bagrus) maculatus Jardine, in Schomburgk, 1841: 175, fig. Type locality: Most rivers of Guyana, also Rio Negro and Amazon River. No types known. Preoccupied by Pimelodus maculatus La Cepède, 1803.

\section{PLOTOSIDAE Bleeker, 1858}

Plotosichthyoidei Bleeker, 1858b: 49, 310. Type genus: Plotosus La Cepède, 1803.

Taxonomic summaries: Taylor \& Gomon (1986, Africa); Tilak (1970a, South Asia); Hoese \& Hanley, in Paxton et al. (1989, Australia).

Reviews: Allen (1989, Australia); Allen (1991, New Guinea); Ferraris (1999a, western central Pacific).

Identification guide: Jayaram (1982, South Asia).

Remarks: Whitley (1940b: 407) noted that the names Plotosus laticeps, Copidoglanis labiosus, Copidoglanis labrosus, Copidoglanis levis and Copidoglanis curtus, all found in Saville-Kent (1889), are nomina nuda and therefore unavailable. The name Plotosus flavolineatus found in Whitley (1941a: 311) is also a nomen nudum and not available.

10 genera, 35 species; no named fossil taxa.

\section{Incertae sedis}

Plotosus argenteus Zietz, 1896

Plotosus argenteus Zietz, 1896: 410, pl. 16 (fig. 7). Type locality: Barcoo River, Finke River at Hermannsburg and Idracowra, and in Plam Creek and Ilpilla Creek, [Central Australia]; [ restricted to Cooper Creek, near Innamincka, (which is apparently the locality listed by Zeitz (1896:411) as Barcoo River) by lectotype designation]. Lectotype: SAMA F1090, designated by Unmack (2001: 57).

Distribution: Cooper Creek, Bulloo and Finke Rivers, internal drainage system, Australia (Allen, 1989).

Remarks: Treated in recent literature as valid either as Neosilurus argenteus or Porochilus argenteus.

Copidoglanis rendahli Whitley, 1928

Copidoglanis obscurus Rendahl, 1922: 173, fig. 5. Type locality: Glencoe, Hermit Hill, nw. Australia. Lectotype: ZMUO J6341, designated by Pethon (1969: 5). Preoccupied by Copidoglanis obscurus Günther, 1864, replaced by Copidoglanis rendahli Whitley, 1928.

Copidoglanis rendahli Whitley, 1928: 214. Type locality: Glencoe, Hermit Hill, N.W. Australia. Lectotype: ZMUO J6341 designated by Pethon (1969: 5) for Copidoglanis obscurus Rendahl. Replacement for Copidoglanis obscurus Rendahl 1922; preoccupied by Copidoglanis obscurus Günther, 1864.

Distribution: Widely distributed in northern Australia in isolated localities, including Fitzroy, Ord, Jardin Rivers, and streams of Arnhem Land (Allen, 1989).

Remarks: Treated in recent literature as valid either as Neosilurus rendahli or Porochilus rendahli.

ANODONTIGLANIS Rendahl, 1922

Anodontiglanis Rendahl, 1922: 168. Type species: Anodontiglanis dahli Rendahl, 1922. Type by original designation. Gender: Masculine.

Anodontiglanis dahli Rendahl, 1922

Anodontiglanis dahli Rendahl, 1922: 169, figs. 2-3. Type locality: Glencoe, Northern Territory, Australia. Holotype: ZMUO J361.

Distribution: Northern Australia, including Fitzroy, Daly, East Alligator, Roper, Mitchell and Archer Rivers (Allen, 1989), in lentic and lotic freshwater habitats (Paxton et al., 1989).

CNIDOGLANIS Günther, 1864

Cnidoglanis Günther, 1864: 27. Type species: Plotosus megastomus Richardson, 1845. Type by subsequent designation by Jordan (1919b: 332). Gender: Masculine. 
Choeroplotosus Kner, 1866: 545. Choeroplotosus decemfilis Kner, 1867. Type by subsequent monotypy. Name appeared first without an available species name. Also described as new in Kner (1867: 300). Gender: Masculine.

Neoplotosus Castelnau, 1875: 45. Type species: Neoplotosus waterhousii Castelnau, 1875. Type by monotypy. Gender: Masculine.

Ostophycephalus Ogilby, 1899: 155. Type species: Ostophycephalus duriceps Ogilby, 1899. Type by original designation. Gender: Masculine.

Remarks: Eschmeyer \& Bailey (1990: 92) interpreted the availability of Choeroplotosus as follows: "In 1866: 545, Kner listed one species as, "Choeroplotosus limbatus (Plotos. limbatus ? C. V.)", but in 1867 (p. 300) he described the species as Choeroplotosus decemfilis n. sp., with "Syn.? an Plotosus limbatus C. V. Apparently the genus can date to Kner (1866: 545) with no definitely-included named species, with species added in 1867."

Cnidoglanis macrocephalus (Valenciennes, 1840)

Plotosus macrocephalus Valenciennes, in Cuvier \& Valenciennes, 1840b: 428 (317 in Strasbourg deluxe edition), pl. 449. Type locality: prise à Timor [in error, possibly for Tasmania, Australia]. Holotype: MNHN a-8921.

Plotosus megastomus Richardson, 1845, in Richardson, 1844-48: 31, pl. 21 (figs. 1-3). Type locality: Sydney Cove; Australia. Holotype: BMNH 1974.5.22.1.

Choeroplotosus decemfilis Kner, 1867: 300, pl. 12 (fig. 1 [as Choeroplotosus limbatus]). Type locality: Von Sidney. Syntypes (2): NMW 47098 (1), NMW 47100 (1).

Cnidoglanis bostockii Castelnau, 1873: 140. Type locality: Fremantle, Western Australia. Syntypes (2): Whereabouts unknown.

Neoplotosus waterhousii Castelnau, 1875: 45. Type locality: Adelaide, South Australia. Holotype: MNHN a-9545.

Ostophycephalus duriceps Ogilby, 1899: 156. Type locality: St. Vincent's Gulf, South Australia. Holotype: SAMA F1093.

Distribution: Kirra, Queensland to Jervis Bay, New South Wales, and Kingston, South Australia to Houtman Abrolhos Islands, Western Australia and Duck River, Tasmania, in near-shore and reef habitats (Paxton et al., 1989).

Remarks: Kowarsky (1976) discussed the valid name for, and distribution of, this species.

EURISTHMUS Ogilby, 1899

Euristhmus Ogilby, 1899: 154. Type species: Plotosus elongatus Castelnau, 1878. Type by original designation. Gender: Masculine.

Exilichthys Whitley, 1933: 65. Type species: Cnidoglanis nudiceps Günther, 1880. Type by original designation. Gender: Masculine.

Euristhmus lepturus (Günther, 1864)

Cnidoglanis lepturus Günther, 1864: 28. Type locality: Sydney, New South Wales [Australia]. Syntypes (2, one stuffed): BMNH 1864.1.17.33 (1).

Plotosus elongatus Castelnau, 1878a: 237. Type locality: Brisbane River [Queensland, Australia]. Holotype: Whereabouts unknown [possibly MNHN a-2783].

Distribution: Exmouth Gulf, Western Australia to Sydney, New South Wales, and New Guinea, in freshwater, estuarine and near-shore marine habitats (Paxton et al., 1989; Allen, 1997: 60).

Euristhmus microceps (Richardson, 1845)

Plotosus microceps Richardson, 1845, in Richardson, 1844-48: 31, pl. 21 (figs. 4-7). Type locality: North-west coast of Australia. Holotype: BMNH 1846.3.3.2.

Cnidoglanis microcephalus Günther, 1864: 28. Type locality: North-west coast of Australia. Holotype: BMNH 1846.3.3.2. Unjustified emendation of Plotosus microceps Richardson, 1845.

Distribution: Shark Bay to Broome, Western Australia, in near-shore habitats over soft bottoms (Paxton et al., 1989).

Euristhmus nudiceps (Günther, 1880)

Cnidoglanis nudiceps Günther, 1880b: 49. Type locality: Arafura Sea. Holotype or lectotype: BMNH 1879.5.14.590; illustrated in Weber \& de Beaufort (1913: 232).

Distribution: Shark Bay, Western Australia to Brisbane Queensland, Australia, in near-shore habitats over soft bottoms (Paxton et al., 1989); also New Guinea (Allen, 1997: 60). 
Remarks: Paxton et al. (1989: 223) list a holotype for Cnidoglanis nudiceps, but the Natural History Museum in London lists a second specimen (BMNH 1890.2.26.165) from the Challenger Expedition taken in the Arafura Sea that may also have been examined by Günther. There is no evidence in the original description whether more than one specimen was examined. Therefore, even if the second specimen was originally a syntype, it would have been rendered a paralectotype by the de facto lectotype designation in Paxton et al. (1989).

NEOSILUROIDES Allen \& Feinberg, 1998

Neosiluroides Allen \& Feinberg, 1998: 11. Neosiluroides cooperensis Allen \& Feinberg, 1998. Type by original designation. Gender: Masculine.

Neosiluroides cooperensis Allen \& Feinberg, 1998

Neosiluroides cooperensis Allen \& Feinberg, 1998: 12, fig. 2. Type locality: Callamurra Waterhole, Coopers Creek, $11.3 \mathrm{~km}$ east of Innamincka, South Australia, ca. 2741'S, 14051'E. Holotype: AMS I.18699-001.

Distribution: Coopers Creek system, Lake Eyre drainage (Allen \& Feinberg, 1998).

NEOSILURUS Steindachner, 1867

Neosilurus Steindachner, 1867a: 7. Type species: Neosilurus hyrtlii Steindachner, 1867. Type by monotypy. Gender: Masculine. Also appeared as new in Steindachner (1867d: 11).

Neosilurus Castelnau, 1878a: 238. Type species: Neosilurus australis Castelnau, 1878. Type by monotypy. Gender: Masculine. Preoccupied by Neosilurus Steindachner, 1867, in fishes; replaced by Cainosilurus Macleay, 1881.

Eumeda Castelnau, 1878b: 143. Type species: Eumeda elongata Castelnau, 1878. Type by monotypy. Gender: Feminine.

Cainosilurus Macleay, 1881: 211. Type species: Neosilurus australis Castelnau, 1878. Type by being a replacement name. Gender: Masculine. Replacement for Neosilurus Castelnau, 1878; preoccupied by Neosilurus Steindachner, 1867.

Lambertia Perugia, 1894: 550. Type species: Lambertia atra Perugia, 1894. Type by monotypy. Gender: Feminine. Preoccupied by Lambertia Robineau-Desvoidy, 1863, in Diptera; replaced by Lambertichthys Whitley, 1938.

Anyperistius Ogilby, 1908: 3, 11. Type species: Anyperistius perugiae Ogilby, 1908. Type by original designation. Gender: Masculine. Spelled two ways originally: Anyperistius and Anyperisteus. First reviser apparently Eschmeyer \& Bailey (in Eschmeyer, 1990) who selected Anyperistius.

Lambertichthys Whitley, 1938: 223. Type species: Lambertia atra Perugia, 1894. Type by being a replacement name. Gender: Masculine. Replacement for Lambertia Perugia, 1894; preoccupied by Lambertia RobineauDesvoidy, 1863, in Diptera.

Neosilurus ater (Perugia, 1894)

Lambertia atra Perugia, 1894: 551. Type locality: Inawi, Papuasia orientale. Syntypes: MSNG 1663 (1), MSNG 8140 (1, missing), ZMA 113360 (1).

Neosilurus mediobarbis Ogilby, 1908: 12. Type locality: ? Queensland, Australia. Holotype: QM (lost: Paxton et al., 1989).

Lambertichthys ater sepikensis Whitley, 1956b: 68. Type locality: Upper Sepik River, New Guinea. Holotype: AMS IA.7278; illustrated in Whitley (1956a: 44, fig. 4).

Distribution: Northern Australia and central-southern New Guinea; in freshwater (Allen, 1989).

Neosilurus brevidorsalis (Günther, 1867)

Copidoglanis brevidorsalis Günther, 1867a: 66. Type locality: Nicol Bay, Cape York, Queensland, Australia. Holotype: BMNH 1867.5.13.11.

Anyperistius perugiae Ogilby, 1908: 11. Type locality: Inawe, St. Joseph River, New Guinea. Type(s): at MSNG and/or BMNH 1965.1.26.1 (1). Based on specimen(s) identified by Perugia (1894: 552) as Eumeda elongata (not of Castelnau).

Neosilurus bartoni Regan, 1908c: 153. Type locality: Sogeri, New Guinea. Syntypes: BMNH 1905.8.15.9-10 (2).

Distribution: Cape York Peninsula, northern Australia, and central-southern New Guinea; in streams, turbid backwaters, and lagoons (Allen, 1989).

Remarks: BMNH 1965.1.26.1 was sent to BMNH on exchange from MSNG and is apparently a type of Anyperistius 
perugiae Ogilby, 1908.

Neosilurus coatesi (Allen, 1985)

Tandanus coatesi Allen, 1985: 252, fig. 3. Type locality: Ninar River, ca. 6 km west of Maprik, Papua New Guinea, ca. $3^{\circ} 37^{\prime} \mathrm{S}, 143^{\circ} 00^{\prime} \mathrm{E}$. Holotype: WAM P.27839-007.

Distribution: Sepik River basin, in streams of Torracelli Mountains (Allen et al., 1992).

Neosilurus equinus (Weber, 1913)

Copidoglanis equinus Weber, 1913: 527, fig. 11. Type locality: Lorentz-Fluss [New Guinea]. Syntypes (13): ZMA 111105 (7), ZMA 111106 (1), ZMA 111107 (4), ZMA 111108 (2).

Distribution: Southern Papua (formerly Irian Jaya), New Guinea (Allen, 1996).

Neosilurus gjellerupi (Weber, 1913)

Copidoglanis gjellerupi Weber, 1913: 528. Type locality: Kaiserin Augusta-fluss [New Guinea]. Holotype: ZMA 111091.

Distribution: Ramu River basin, Papua New Guinea (Allen et al., 1992) and northern Papua (formerly Irian Jaya), New Guinea (Allen, 1996).

Remarks: The name Copidoglanis papuensis that is found in the caption of figures 11 and 12 in Hase (1914: 540) appears to have been published accidentally inasmuch as the species account indicates that the specimens were regarded as having been identified as Copidoglanis gjellerupi (Weber, 1913). This was treated by Eschmeyer et al. (1998: 1283) as unavailable, because it was not treated as the valid name of a species when proposed.

Neosilurus gloveri Allen \& Feinberg, 1998

Neosilurus gloveri Allen \& Feinberg, 1998: 13, fig. 3. Type locality: Main Spring, Dalhousie Springs, South Australia, ca. $26^{\circ} 25^{\prime} \mathrm{S}, 135^{\circ} 30^{\prime}$ E. Holotype: SAMA F.4159.

Distribution: Dalhousie artesian springs, Finke River basin, southern Australia (Allen \& Feinberg, 1998).

Neosilurus hyrtlii Steindachner, 1867

Neosilurus Hyrtlii Steindachner, 1867d: 14, pl. 1 (figs. 3-3a). Type locality: Fitzroy-Flusse bei Rockhampton, OstAustralien. Syntypes: NMW 45340 (5), NMW 45341 (2).

Silurichthys australis Castelnau, 1875: 45. Type locality: Cape York [Queensland, Australia]. Holotype: Whereabouts unknown.

Neosilurus australis Castelnau, 1878a: 239. Type locality: Freshwater lagoons of Rockhampton, Queensland, Australia. Holotype: Whereabouts unknown.

Eumeda elongata Castelnau, 1878b: 144. Type locality: Brisbane River, Rockhampton [Queensland, Australia]. Holotype: MNHN a-2173.

Neosilurus robustus Ogilby, 1908: 13. Type locality: Keppel Bay, Queensland, Australia. Holotype: Whereabouts unknown, not at QM.

Copidoglanis glencoensis Rendahl, 1922: 170, fig. 4. Type locality: Glencoe R., nw. Australia. Lectotype: ZMUO J5254; Lectotype designated by Pethon (1969: 4).

Neosilurus mortoni Whitley, 1941b: 7, fig. 7. Type locality: Yam Creek (nine miles from Brock's Creek, railway line south from Darwin) Northern Territory of Australia. Holotype: AMS IA.4824.

Distribution: Widely distributed in northern Australia; in freshwater (Allen, 1989).

Neosilurus idenburgi (Nichols, 1940)

Copidoglanis idenburgi Nichols, 1940: 1. Type locality: Bernhard Camp (altitude 75 meters), Idenburg River, Netherland New Guinea. Holotype: AMNH 15034.

Distribution: Ramu River basin, Papua New Guinea (Allen, 1992) and northern Papua (formerly Irian Jaya), New Guinea (Allen, 1996).

Neosilurus mollespiculum Allen \& Feinberg, 1998

Neosilurus mollespiculum Allen \& Feinberg, 1998: 16, fig. 6. Type locality: Running River, Burdekin River system, Queensland, ca. 1907'S, 14550'E, Australia. Holotype: QM I.30685.

Distribution: Isdell, Carson, Drysdale, Katherine, Fergusson, and Burdekin Rivers, northern Australia (Allen \& Feinberg, 1998).

Neosilurus novaeguineae (Weber, 1907)

Copidoglanis novae-guineae Weber, 1907: 226. Type locality: Sentani-See, Nord-Neu-Guinea. Syntypes (13): 
FMNH 52388 (1), NMW 46696 (1), RMNH 7980 (1), ZMA 112670 (8).

Copidoglanis novae-guineae niger Nichols, 1940: 1. Type locality: Bernhard Camp (altitude 75 meters), Idenburg River, Netherland New Guinea. Holotype: AMNH 15035.

Distribution: Ramu River basin, Papua New Guinea (Allen et al., 1992) and northern Papua (formerly Irian Jaya), New Guinea (Allen, 1996).

Neosilurus pseudospinosus Allen \& Feinberg, 1998

Neosilurus pseudospinosus Allen \& Feinberg, 1998: 15, figs. 4-5. Type locality: Rocky pool of Ord R. on Old Lissadel Station, Kimberley Dist., Western Australia, ca. 16²40'S, 12883'E. Holotype: WAM P.28505-007.

Distribution: Ord River, Western Australia (Allen \& Feinberg, 1998).

OLOPLOTOSUS Weber, 1913

Oloplotosus Weber, 1913: 521 Type species: Oloplotosus mariae Weber, 1913. Type by monotypy. Gender: Masculine.

Key: Allen (1985; 1991).

Oloplotosus luteus Gomon \& Roberts, 1978

Oloplotosus luteus Gomon \& Roberts, in Roberts, 1978: 47, fig. 25. Type locality: Shallow, turbid backwater of Ok Tedi and lowermost half km of small tributary about $16 \mathrm{~km}$ NE of Nigerum, $915 \mathrm{~km}$ upriver from Toro Pass, 5³4.1'S, 141¹5.0'E; Fly River, Papua New Guinea. Holotype: AMS I.27093-001.

Distribution: Upper Fly River, New Guinea (Roberts, 1978).

Oloplotosus mariae Weber, 1913

Oloplotosus Mariae Weber, 1913: 522, fig. 3. Type locality: Lorentz-Fluss, Sabang; Lorentz-Fluss, Alkmaar [New Guinea]. Syntypes (5): AMNH 9500 (1), ZMA 111103 (1), ZMA 111104 (2).

Distribution: Southern Papua (formerly Irian Jaya), New Guinea (Allen, 1996).

Oloplotosus torobo Allen, 1985

Oloplotosus torobo Allen, 1985: 248, fig. 1. Type locality: Soro River at n. end of Lake Kutubu, ca. 4 km east of Moro landing strip, Papua New Guinea, ca. 6²3'S, 143ํㄷ'ㄹ, 0-3 m. Holotype: WAM P.28158-003.

Distribution: Soro River, Papua New Guinea (Allen, 1985).

PARAPLOTOSUS Bleeker, 1863

Paraplotosus Bleeker, 1863 (in Bleeker, 1862-63): 100. Type species: Plotosus albilabris Valenciennes, 1840. Type by monotypy. Gender: Masculine.

Endorrhis Ogilby, 1898b: 283. Type species: Copidoglanis longifilis Macleay, 1881. Type by original designation. Gender: Feminine.

Revision: Allen (1998).

Paraplotosus albilabris (Valenciennes, 1840)

Plotosus albilabris Valenciennes, in Cuvier \& Valenciennes, 1840b: 427 (316 in Strasbourg deluxe edition). Type locality: Batavia. Holotype: MNHN a-9544.

Plotosus macrophthalmus Bleeker, 1846a: 179. Type locality: Batavia. Syntypes (size and number not stated): Whereabouts unknown.

Copidoglanis longifilis Macleay, 1881: 207. Type locality: Long Island, Torres Strait, Queensland, Australia. Syntypes: AMS I.16269-001 (2).

Distribution: Indo-Australian Archipelago, New Guinea and Australia; often in coral reef habitats (Allen, 1998).

\section{Paraplotosus butleri Allen, 1998}

Paraplotosus butleri Allen, 1998: 129, fig. 3. Type locality: Near Broome, ca. 17º58'S, 122²14'E, Western Australia. Holotype: WAM P.27368-021.

Distribution: Northern Australia, in coastal reefs (Allen, 1998).

Paraplotosus muelleri (Klunzinger, 1880)

Cnidoglanis mülleri Klunzinger, 1880: 411. Type locality: Port Darwin [Australia]. Holotype: SMNS 2519.

Distribution: Dampier Archipelago to the eastern Gulf of Carpentaria, northern Australia; in turbid coastal reefs (Allen, 1998). 
PLOTOSUS La Cepède, 1803

Plotosus La Cepède, 1803: 129. Type species: Platystacus anguillaris Bloch, 1794. Type by monotypy. Gender: Masculine.

Deportator Gistel, 1848: X. Type species: Platystacus anguillaris Bloch, 1794. Type by being a replacement name. Gender: Masculine. Unneeded replacement for Plotosus La Cepède, 1803, which was considered by Gistel to be preoccupied by Plotus Linnaeus, 1766, in Aves.

Remarks: Plotosis (in Duméril, 1856: 340), Plotoseus (in Lesson, 1826, in Lesson 1826-31: pl. 31, 1831: 435) and Plotosius (in Siebold, 1846: 228) are incorrect subsequent spellings of Plotosus and not available names.

Key: Gomon \& Taylor (1982).

Plotosus canius Hamilton, 1822

Plotosus canius Hamilton, 1822: 142, 374, pl. 15 (fig. 44). Type locality: rivers of the southern parts of Bengal. No types known.

Plotosus unicolor Valenciennes, in Cuvier \& Valenciennes, 1840b: 426 (316 in Strasbourg deluxe edition). Type locality: Java. Holotype: MNHN a-8924.

Plotosus viviparus Bleeker, 1846a: 182. Type locality: Batavia. Syntypes (size and number not stated): possibly RMNH 8066 (1), RMNH 15875 (6), SMNS 10624 (1).

Plotosus horridus Bleeker, 1846a: 183. Type locality: Batavia. Type(s) (size and number not stated): Whereabouts unknown.

Plotosus multiradiatus Bleeker, 1846a: 183. Type locality: Batavia. Type (s) (size and number not stated): Whereabouts unknown.

Distribution: Coastal regions of Thailand, Sundaland, Sulawesi, Moluccas, and India, and into lower Mekong River (Kottelat, 2001b).

Remarks: Synonymy based on Bleeker (1862-63: 99). Plotosus caesius in Cuvier (1829, 1836, etc.) and Hyrtl (1859: 5, 6, 17) are considered to be incorrect spellings for $P$. canius and not the proposal of an available name. Silurus unitius of Hora (1933: 133) is not available, as it was not treated as a valid species.

Plotosus fisadoha $\mathrm{Ng} \&$ Sparks, 2002

Plotosus fisadoha Ng \& Sparks, 2002: 565, fig. 1. Type locality: Madagascar: southeastern coast of Fanarantsoa Province; Farafangana market. Holotype: UMMZ 235269.

Distribution: Southeastern Madagascar (Ng \& Sparks, 2002).

Plotosus limbatus Valenciennes, 1840

Plotosus limbatus Valenciennes in Cuvier \& Valenciennes, 1840b: 422 (313 in Strasbourg deluxe edition). Type locality: Côte de Malabar ... Pondichéry; [restricted to Malabar by lectotype designation]. Lectotype: MNHN a9546, designated by Gomon \& Taylor (1982: 9).

Copidoglanis obscurus Günther, 1864: 26. Type locality: Probably from Australia. Holotype: BMNH 1852.9.13.164.

Distribution: Western Indian Ocean and Arabian Sea, in marine and brackish waters (Taylor \& Gomon, 1986).

Plotosus lineatus (Thunberg, 1787)

Silurus lineatus Thunberg, 1787: 31, footnote 13. Type locality: [East Indian Seas]. Type(s): ZMUC P 28555. Described in more detail and illustated in Thunberg (1791: 191, pl. 6).

Platystacus anguillaris Bloch, 1794: 61, pl. 373 (figs. 1, 2). Type locality: Not stated [given as Tranquebar in Bloch \& Schneider, 1801]. Lectotype: ZMB 3078, designated by Taylor \& Gomon (1986: 160).

Plotosus thunbergianus La Cepède, 1803: 693, 694. Type locality: La mer des grandes Indes. Holotype: ZMUC P 28555. Unneeded new name for Silurus lineatus Thunberg, 1787.

Plotoseus ikapor Lesson, 1826, in Lesson 1826-31: pl. 31, fig. 3. Type locality: baie d'Offeach de l'ile de Waigiou. Holotype: Whereabouts unknown. Name made available by figure caption, with illustrated specimen the holotype; described in Lesson (1831: 132).

Plotosus marginatus Bennett, 1830: 691. Type locality: Sumatra. Syntype: BMNH 1855.12.26.452 (1).

Plotosus vittatus Swainson, 1839: 307. Type locality: Not stated [given as Tranquebar in Bloch \& Schneider, 1801]. Unneeded new name for Platystacus anguillaris Bloch. Based on "Bl. 373, fig. 1 " [= Bloch (1794: pl. 373 (fig. $1)]$. 
Plotosus castaneus Valenciennes, in Cuvier \& Valenciennes, 1840b: 421 (312 in Strasbourg deluxe edition). Type locality: Mahé sur la côte malabare. Holotype: MNHN a-8929.

Plotosus lineatus Valenciennes in Cuvier \& Valenciennes, 1840b: 412 (306 in the Strasbourg deluxe edition). Type locality: la mer des Indes ... la mer Rouge ... la Séchelles ... la côte de Malabar, Trincomalé, Pondichéry, Amboine ... Célèbes, îles des Amis, îles de la Société, Macao ... Philippines. Syntypes:MNHN a-8930 (1), MNHN a-8931 (2), MNHN a-8932 (10 or 11), MNHN a-8936 (1). Preoccupied in Plotosus by Silurus lineatus Thunberg, 1787.

Plotosus castaneö̈des Bleeker, 1851f: 490. Type locality: Rio [=Riouw], in mari. Syntypes (2, 160-170 mm TL): at RMNH.

Plotosus arab Bleeker, 1862a: 111. Type locality: Not stated [given as Tranquebar in Bloch \& Schneider, 1801]. Syntypes: at MNHN, ZMB 3078 (1), ZMB 3079 (1). Name made available by reference to Plotosus anguillaris of La Cepède (1803: 130, pl. 3 fig. 2), which is based on specimens and Platystacus anguillaris Bloch, 1794.

Plotosus brevibarbus Bessednov, 1967: 446, fig. 3. Type locality: 21¹0' с. ш, 108³0'в. д, 29 m [Gulf of Tonkin, South China Sea]. Holotype: Mus. TINRO 3667.

Distribution: Indian Ocean and Western Pacific, in marine waters, but sometimes entering freshwaters in East Africa and Madagascar (Taylor \& Gomon, 1986); eastern Mediterranean (Golani, 2002).

Remarks: The name Silurus Arab in Forsskål (1775: xvi) is not available (M. Kottelat, pers. commun.). The name Plotosus malignus appears in the account of Plotosus lineatus Valenciennes, but it not treated as valid and is therefore not available from that publication. The name does not appear to have been treated as valid subsequently. See Smith (1941) for nomenclatural comments regarding this species.

Plotosus nkunga Gomon \& Taylor, 1982

Plotosus nkunga Gomon \& Taylor, 1982: 3, pl. 1. Type locality: South Africa, Transkei, Bashee River (32¹5'S, 285'). Holotype: SAM 18457.

Distribution: Southern Africa from Boknes to Boteler Point, and possibly to Zanzibar, in marine waters but also entering freshwater (Taylor \& Gomon, 1986).

Plotosus papuensis Weber, 1910

Plotosus papuensis Weber, 1910: 228. Type locality: Lorentz-Fluss [New Guinea]. Syntypes: FMNH 52494 (1), ZMA 111093-97 (5), ZMA 111098-100 (5), ZMA 111513 (1).

Distribution: Southern Papua (formerly Irian Jaya), New Guinea (Allen, 1996).

\section{Species inquirenda, Plotosus}

Plotosus abbreviatus Boulenger, 1895a: 247. Type locality: Mouth of Baram River, Sarawak. Holotype: BMNH 1894.8.3.35.

POROCHILUS Weber, 1913

Porochilus Weber, 1913: 523. Type species: Porochilus obbesi Weber, 1913. Type by monotypy. Gender: Masculine.

Porochilus meraukensis (Weber, 1913)

Copidoglanis meraukensis Weber, 1913: 529. Type locality: Merauke, Süd-Neu Guinea. Syntypes: RMNH 10865 (1), ZMA 111092 (1).

Distribution: Southern Papua (formerly Irian Jaya), New Guinea (Allen, 1996).

Porochilus obbesi Weber, 1913

Porochilus Obbesi Weber, 1913: 523, figs. 4-5. Type locality: Lorentz-Fluss, ... bei Sabang, New Guinea. Syntypes (6): ZMA 111101 (3), ZMA 111102 (1), RMNH 10864 (1).

Distribution: Southern Papua (formerly Irian Jaya), New Guinea (Allen, 1996).

TANDANUS Mitchell, 1838

Tandanus Mitchell, 1838: 95. Type species: Plotosus (Tandanus) tandanus Mitchell, 1838. Type by monotypy. Gender: Masculine. Originally proposed as a subgenus of Plotosus.

Copidoglanis Günther, 1864: 25. Type species: Plotosus (Tandanus) tandanus Mitchell, 1838. Type by subsequent 
designation by Jordan (1919b: 332) with name was misprinted Copiodoglanis. Gender: Masculine.

Tandanus bostocki Whitley, 1944

Plotosus unicolor Castelnau, 1873: 141. Type locality: Interior of Western Australia. Syntype: NMV 51851 (1). Preoccupied by Plotosus unicolor Valenciennes, 1840, replaced by Tandanus bostocki Whitley, 1944.

Tandanus bostocki Whitley, 1944: 260. Type locality: Interior of Western Australia. Syntype: NMV 51851 (1). Replacement for Plotosus unicolor Castelnau, 1873, preoccupied by Plotosus unicolor Valenciennes, 1840.

Remarks: The statement in Whitley (1944: 260) that designates a holotype for the species is in error, since he clearly states elsewhere that Tandanus bostocki is intended to replace the preoccupied name of Castelnau and, thereby, takes the same specimen as holotype.

Distribution: Coastal drainages of southwestern Australia, from the Frankland River to Moore River (Allen, 1989).

Tandanus tandanus (Mitchell, 1838)

Plotosus (Tandanus) tandanus Mitchell, 1838: 44, 95, pl. 5 (fig. 2). Type locality: Lagoon near Tangulda, Namoi River, New South Wales; river between Gwydir River and MacIntyre River, New South Wales. No types known.

Distribution: Murray-Darling River system and coastal drainages of eastern Australia (Allen, 1989).

Remarks: See Musyl \& Keenan (1996) for comments on populational variation that may represent unrecognized additional species.

\section{Species inquirenda, Plotosidae}

Plotosus nigricans Valenciennes, in Cuvier \& Valenciennes, 1840b: 412, 417 (310 in Strasbourg deluxe edition). Type locality: Not stated. Holotype: at MNHN (skeleton). Species name mentioned in generic account as well as account of Plotosus lineatus. Reference made to published comments about the skeleton by Cuvier in Regne Animal, which are adequate to act as an indication, even though Valenciennes never provides a more complete treatment of the species. Gomon \& Taylor (1982: 10), disagree and treated the name as a nomen nudum, but later argue that a specimen previously treated as the holotype of the species, MNHN a-9433, must not be considered as one of the "original type-specimens."

\section{PSEUDOPIMELODIDAE Lundberg, Bornbusch \& Mago-Leccia, 1991}

Pseudopimelodinae Lundberg, Bornbusch \& Mago-Leccia, 1991: 204. Type genus: Pseudopimelodus Bleeker, 1858.

Remarks: The name Pseudopimelodidae was proposed first by Fernández-Yépez \& Martín Salazar (1953) in a manner that made the name available when published, but was rendered unavailable in Art. 13 of the Second Edition of the Code (ICZN, 1961). The name apparently remains unavailable, despite Art. 13.2.1 of the current version of the Code (ICZN, 1999), because the name was treated as unavailable in Ferraris \& de Pinna (1999: 8).

Taxonomic summary: Shibatta (2003a).

Phylogeny: Shibatta (1998, 2003b).

5 genera, 29 species; no named fossil taxa.

\section{BATROCHOGLANIS Gill, 1858}

Batrochoglanis Gill, 1858: 389. Type species: Pimelodus raninus Valenciennes, 1840. Type by original designation. Gender: Masculine.

Remarks: Batrachoglanis, first used in Gill (1861c), is either a misspelling or unjustified emendation of Batrochoglanis Gill, 1858.

Batrochoglanis acanthochiroides (Güntert, 1942)

Pseudopimelodus acanthochiroides Güntert, 1942: 29. Type locality: Santander, Colombia. Syntypes: NMBA 527778 (2).

Pseudopimelodus villosus butcheri Schultz, 1944c: 199, pl. 1 (fig. b). Type locality: Río San Juan near bridge south of Mene Grande, tributary to Rio Motatán, Maracaibo basin, Venezuela. Holotype: USNM 121270.

Distribution: Catatumbo River basin in Lake Maracaibo drainage (Shibatta, 2003a). 
Batrochoglanis melanurus Shibatta \& Pavanelli, 2005

Batrochoglanis melanurus Shibatta \& Pavanelli, 2005: 23, figs. 1-2. Type locality: Córrego Cancela, affluent of rio Cuiabá, rio Paraguai basin; $1^{\circ} 42.501 S / 56^{\circ} 15.850 W$; Nobres; State of Mato Grosso; Brazil. Holotype: MZUSP 87240.

Distribution: Cuiabá River, Paraguay River basin, Mato Grosso. Brazil (Shibatta \& Pavanelli, 2005).

Batrochoglanis raninus (Valenciennes, 1840)

Pimelodus raninus Valenciennes, in Cuvier \& Valenciennes, $1840 \mathrm{~b}: 157$ (117 in the Strasbourg deluxe edition), pl. 434. Type locality: La Mana ... Rio-Janéiro. Syntypes: MNHN a-9942 (3).

Pseudopimelodus acanthochirus Eigenmann \& Eigenmann, 1888b: 122. Type locality: Brazil. Syntypes (5): MCZ 8133 (1), MCZ 8157 (1), MCZ 7732 (2), MCZ 7332 (1).

Distribution: Amazon River basin (Shibatta, 2003a).

Batrochoglanis transmontanus (Regan, 1913)

Pseudopimelodus transmontanus Regan, 1913d: 467. Type locality: Condoto ..., San Juan and the Tamana ...., and the Durango, W. Ecuador. Syntypes (9): BMNH 1910.7.11.104, BMNH 1910.7.11.105, BMNH 1913.10.1.36, BMNH 1902.5.27.37-40.

Distribution: Baudó, San Juan, Patia and Durango River basins, Pacific slope drainages (Shibatta, 2003a).

Batrochoglanis villosus (Eigenmann, 1912)

Pseudopimelodus villosus Eigenmann, 1912b: 152, fig. 32, pl. 10 (fig. 1). Type locality: Potaro Landing, British Guiana. Holotype: FMNH 53219.

Distribution: Demerara, Essequibo, Orinoco and Amazon River basins (Shibatta, 2003a).

CEPHALOSILURUS Haseman, 1911

Cephalosilurus Haseman, 1911a: 317. Type species: Cephalosilurus fowleri Haseman, 1911. Type by monotypy. Gender: Masculine.

Cephalosilurus albomarginatus (Eigenmann, 1912)

Pseudopimelodus albomarginatus Eigenmann, 1912b: 153, pl. 11 (fig. 1). Type locality: Tukeit, British Guiana. Holotype: FMNH 53221.

Distribution: Tukeit, Guyana (Shibatta, 2003a).

Cephalosilurus apurensis (Mees, 1978)

Pseudopimelodus apurensis Mees, 1978b: 253, pl. 1. Type locality: Río Arichuna, near San Pedro, Rincón hondo, Apure, Venezuela. Holotype: RMNH 27644.

Distribution: Arichuna River basin, Apure State, Venezuela (Shibatta, 2003a).

Cephalosilurus fowleri Haseman, 1911

Cephalosilurus fowleri Haseman, 1911a: 317, pl. 46. Type locality: Rio São Francisco, Cidade de Barra, Bahia, Brazil. Holotype: FMNH 54254.

Distribution: São Francisco River basin, Brazil (Shibatta, 2003a).

Cephalosilurus nigricaudus (Mees, 1974)

Pseudopimelodus nigricauda Mees, 1974: 218, figs. 37d, 38d; pls. 11-12. Type locality: Sipaliwini, Suriname. Holotype: RMNH 26739.

Distribution: Sipaliwini River basin, Suriname (Shibatta, 2003a).

\section{LOPHIOSILURUS Steindachner, 1877}

Lophiosilurus Steindachner, 1877a: 154. Type species: Lophiosilurus alexandri Steindachner, 1877. Type by monotypy. Gender: Masculine.

Lophiosilurus alexandri Steindachner, 1877

Lophiosilurus Alexandri Steindachner, 1877a: 154, pl. 15. Type locality: Aus Brasilien, wahrscheinlich aus dem Amazonenstrome. Holotype: NMW 46123.

Pseudopimelodus Agassizi Steindachner, 1880b: 61. Type locality: Aus Brasilien, wahrscheinlich aus dem Amazonenstrome. Holotype: NMW 46123. Unneeded new name, or lapsus calami for Lophiosilurus alexandri Steindachner, 1877, originally written as Pseudop. Agassizi. 
Distribution: São Francisco River basin, Brazil (Shibatta, 2003a).

Remarks: See Pinto \& Marzulo (1975) for comments on morphology.

MICROGLANIS Eigenmann, 1912

Microglanis Eigenmann, 1912b: 130, 155. Type species: Microglanis poecilus Eigenmann, 1912. Type by original designation. Gender: Masculine.

Key: Bertaco \& Cardoso (2005), and Shibatta \& Benine (2005), southern Brazil.

Microglanis ater Ahl, 1936

Microglanis ater Ahl, 1936: 109. Type locality: Brazil. Holotype: ZMB 20932.

Distribution: Brazil (Shibatta, 2003a).

Microglanis cibelae Malabarba \& Mahler, 1998

Microglanis cibelae Malabarba \& Mahler, 1998: 249, figs. 1, 4-6. Type locality: arroio do Ouro, Rio Maquiné, between Maquiné and Barra do Ouro, Rio Grande do Sul, Brasil. Holotype: MZUSP 48653.

Distribution: Coastal drainages in northern Rio Grande do Sul and Santa Catarina States, Brazil (Shibatta, 2003a; Bertaco \& Cardoso, 2005).

Microglanis cottoides (Boulenger, 1891)

Pimelodus (Pseudopimelodus) cottoides Boulenger, 1891: 233, pl. 25 (fig. 2). Type locality: Rio Camaquã, Rio Grande do Sul, Brazil. Syntypes: BMNH 1891.3.16.36-45 and BMNH 1891.3.16.106 (lots combined, 12 specimens) and BMNH 1889.8.24.5 (1).

Distribution: Laguna dos Patos and Uruguay River basins, Brazil (Shibatta, 2003a; Bertaco \& Cardoso, 2005).

Microglanis eurystoma Malabarba \& Mahler, 1998

Microglanis eurystoma Malabarba \& Mahler, 1998: 248, fig. 3. Type locality: Rio Uruguay, Itá, Santa Catarina, Brazil. Holotype: MCP 13405.

Distribution: Upper Uruguay River basin, Brazil (Shibatta, 2003a; Bertaco \& Cardoso, 2005).

Microglanis garavelloi Shibatta \& Benine, 2005

Microglanis garavelloi Shibatta \& Benine, 2005: 580, figs. 1-2. Type locality: Brazil, Paraná: Taquari stream, 23ํ12'24"S 5056'50"W, Município de Jataizinho. Holotype: MZUSP 88006.

Distribution: Paranapanema and Tietê River basins, Brazil (Shibatta \& Benine, 2005).

Microglanis iheringi Gomes, 1946

Microglanis iheringi Gomes, 1946: 9, pl. 1. Type locality: Río Turmero, Aragua, Venezuela. Holotype: FMNH 35350.

Distribution: Turmero River basin, Venezuela (Shibatta, 2003a).

Microglanis malabarbai Bertaco \& Cardoso, 2005

Microglanis malabarbai Bertaco \& Cardoso, 2005: 62, fig. 2. Type locality: arroio Albino, tributary of rio Ijuí (rio Uruguay drainage), 2808'10"S, 5455'28"W, São Pedro do Butiá, Rio Grande do Sul, Brazil. Holotype: MCP 35941.

Distribution: Middle Uruguay River basin, Brazil (Bertaco \& Cardoso, 2005).

Microglanis nigripinnis Bizerril \& Perez-Neto, 1992

Microglanis nigripinnis Bizerril \& Perez-Neto, 1992: 97, figs. 1-2. Type locality: Brazil, Estado do Rio de Janeiro, rio Macacu, Município de Cachoeira do Macacu, Cidade de Papucaia. Holotype: MZUSP 42308.

Distribution: Macacu River basin in Rio de Janeiro State, Brazil (Shibatta, 2003a).

Microglanis parahybae (Steindachner, 1880)

Pseudopimelodus Parahybae Steindachner, 1880b: 60 footnote, pl. 1 (figs. 2-2b). Type locality: Rio Parahyba und von Santa Cruz [Brazil]. Syntypes: MCZ 8161 (25), MCZ 8162 (6), NMW 44433 (9), NMW44436 (1).

Distribution: Paraíba do Sul River basin, Brazil (Shibatta, 2003a).

Microglanis pellopterygius Mees, 1978

Microglanis pellopterygius Mees, 1978b: 256, pl. 2. Type locality: Tributary stream of Río Aguarico at Santa Cecilia, $0^{\circ} 06^{\prime} \mathrm{N}, 7^{\circ} 51^{\prime} \mathrm{W}$, Napo, Ecuador. Holotype: ANSP 130437.

Distribution: Aguarico River basin, Ecuador (Shibatta, 2003a).

Microglanis poecilus Eigenmann, 1912 
Microglanis poecilus Eigenmann, 1912b: 155, pl. 12 (fig. 2). Type locality: Below Packeoo Falls, British Guiana. Holotype: FMNH 46365.

Distribution: Essequibo River basin and rivers of French Guiana (Shibatta, 2003a).

Microglanis secundus Mees, 1974

Microglanis secundus Mees, 1974: 235, fig. 40 e; pl. 14. Type locality: Sipaliwini, Suriname. Holotype: RMNH 26525.

Distribution: Catatumbo River basin; Venezuela and Colombia; Sipaliwini River basin, Suriname (Shibatta, 2003a).

Microglanis variegatus Eigenmann \& Henn, 1914

Microglanis variegatus Eigenmann \& Henn, in Eigenmann, Henn \& Wilson, 1914: 14. Type locality: Near Vinces, Ecuador. Holotype: CAS 17971; holotype illustrated in Eigenmann (1922b: 33, pl. 2, figs. 3-4).

Distribution: Forest pool near Vinces, Ecuador (Shibatta, 2003a).

Microglanis zonatus Eigenmann \& Allen, 1942

Microglanis zonatus Eigenmann \& Allen, 1942: 89, pl. 3 (figs. 1-2). Type locality: Rio Morona (?). Holotype: CAS 17970.

Distribution: Upper Amazon River basin, Peru (Shibatta, 2003a).

\section{PSEUDOPIMELODUS Bleeker, 1858}

Pseudopimelodus Bleeker, 1858b: 196, 204, 207. Type species: Pimelodus bufonius Valenciennes, 1840. Type by subsequent designation by Gill (1861c). Gender: Masculine.

Pseudopimelodus bufonius (Valenciennes, 1840)

Pimelodus Bufonius Valenciennes, in Cuvier \& Valenciennes, 1840b: 154 (115 in the Strasbourg deluxe edition). Type locality: Cayenne. Holotype: RMNH (lost).

Distribution: Rivers of northeastern South America from Lake Maracaibo basin to eastern Brazil (Shibatta, 2003a) and Magdalena and Cauca River basins, Colombia (Maldonado-Ocampo et al., 2005).

Pseudopimelodus charus (Valenciennes, 1840)

Pimelodus charus Valenciennes, in Cuvier \& Valenciennes, 1840b: 159 (118 in the Strasbourg deluxe edition). Type locality: Rio Sabara [Brazil]. No types known; description based on an unpublished illustration, reproduced in Mees (1974, frontispiece).

Distribution: São Francisco River basin, Brazil (Shibatta, 2003a).

Pseudopimelodus mangurus (Valenciennes, 1835)

Pimelodus mangurus Valenciennes, 1835, in Valenciennes, 1835-47: pl. 1 (figs. 4-6). Type locality: Río de la Plata. Possible holotype: MNHN a-8401(1), MNHN a-9417 (1). Name available from caption on plate, so illustrated specimen is the holotype, if identifiable. Described in Cuvier \& Valenciennes (1840b: 156 (116 of Strasbourg deluxe edition)), with locality given as Rio-Janéiro.

Pseudopimelodus roosevelti Borodin, 1927b: 1, fig. 1. Type locality: Parassununga, Estado São Paulo, Brazil. Holotype: AMNH 8638.

Distribution: Uruguay, Paraná, Paraguay and La Plata River basins (Shibatta, 2003a).

Pseudopimelodus pulcher (Boulenger, 1887)

Pimelodus (Pseudopimelodus) pulcher Boulenger, 1887c: 276, pl. 21 (fig. 1). Type locality: Canelos, e. Ecuador. Syntypes: BMNH 1880.12.8.105-107 (3).

Pseudopimelodus variolosus Miranda Ribeiro, 1914: 4, pl. 1 (fig. 2), pl. 2 (figs. 1-2). Type locality: Coxim, Rio Taquary, M. Grosso [Brazil]. Lectotype: MNRJ 818A, designated by Miranda Ribeiro (1953: 404).

Distribution: Upper Amazon River basin (Shibatta, 2003a).

Pseudopimelodus schultzi (Dahl, 1955)

Zungaro zungaro schultzi Dahl, 1955: 13. Type locality: Cereté, Colombia. Holotype: Whereabouts unknown (possibly at ICNMHN).

Distribution: Magdalena River basin, Colombia (Shibatta, 2003a).

Species inquirenda, Pseudopimelodidae 
Zungaro mathisoni Fernández-Yépez, 1972a: 22, pl. 41. Type locality: Río Yaracuy drainage, Venezuela. Holotype: author's personal collection; whereabouts unknown.

\section{SCHILBIDAE Bleeker, 1858}

Ailichthyoidei Bleeker, 1858b: ix, 49, 248. Type genus: Ailia Gray, 1830.

Schilbeini Bleeker, 1858b: 49, 250, 256. Type genus: Schilbe Oken, 1817.

Siluranodontinae Regan, 1911: 568. Type genus: Siluranodon Bleeker, 1858.

Taxonomic summaries: De Vos (1984b, 1986, Africa).

Reviews: Reizer et al. (1980, Senegal), De Vos (1995, Africa); De Vos (1992, West Africa).

Identification guide: Jayaram (1977, South Asia).

Key to genera: Trewavas (1943, Africa); Poll \& Gosse (1994, Africa).

Remarks: Although the family group name is often spelled Schilbeidae, the spelling used here, Schilbidae, was considered to be correct by Steyskal (1980: 174) and is followed herein. The monophyly of this family, as currently populated, is unlikely. Species of the Pangasiidae and possibly Horabagrus may be nested within the family, and the species of Neotropius and Pseudeutropius may be more closely related to some species of the Bagridae. See Mo (1991), Pouyaud et al. (2000, 2004), Diogo et al. (2004) for details. Hardmann (2005) further suggested that African species of the Schilbidae may be more closely related to other African catfishes than to any Asian member of the Schilbidae, in which case the name Ailiidae would be the valid name for the Asian group.

14 genera, 62 species; no named fossil taxa.

AILIA Gray, 1830

Acanthonotus Gray, 1830: pl. 85 (fig. 1). Type species: Silurus (Acanthonotus) cuvieri Gray, 1830. Type by monotypy. Gender: Masculine. Name made available by figure caption. Originally proposed as a subgenus of Silurus. Subsequently published in Gray (1831: 8), but with Acanthonotus Hardwickii as the only included species. Preoccupied by Acanthonotus Goldfuss, 1809 (and possibly earlier), in Mammalia.

Ailia Gray, 1830: pl. 85 (fig. 2). Type species: Malapterus (Ailia) bengalensis Gray, 1830. Type by monotypy. Gender: Feminine. Originally proposed as a subgenus of Malapterus. Name made available by caption of plate; text published later in Gray (1831: 8).

Ailiichthys Day, 1872: 712. Type species: Ailiichthys punctata Day, 1872. Type by monotypy. Gender: Masculine.

Review: Hora (1941); Jayaram (1963).

Ailia coila (Hamilton, 1822)

Malapterurus coila Hamilton, 1822: 158, 375. Type locality: Fresh water rivers of Bengal. No types known. Unpublished Hamilton illustration reproduced in Gray (1830: pl. 85, fig. 2), as Malapterus (Ailia) bengalensis.

Malapterus (Ailia) bengalensis Gray, 1830: pl. 85 (fig. 2). Type locality: India. No types known; name based on previously-unpublished illustration by Hamilton. Name made available by figure caption; brief diagnosis published in Gray (1831: 8), with locality stated as "Rivers Bengal."

Silurus (Acanthonotus) Cuvieri Gray, 1830: pl. 85 (fig. 1). Type locality: India. No types known. Name made available by plate legend; text published later in Gray (1831: 8), as Acanthonotus Hardwickii.

Acanthonotus Hardwickii Gray, 1831: 8 [pl. 85 (fig. 1), of Gray, 1830]. Type locality: India. No types known. Unneeded new name for Silurus (Acanthonotus) Cuvieri Gray, 1830.

Ailia affinis Günther, 1864: 56. Type locality: Kulu and Assam, India. Syntypes (3, in two lots): BMNH 1860.3.19.867-868 (2), BMNH 2005.5.17.3 (1).

Distribution: Rivers of northern India and Deccan, including Indus, Ganges, Yamuna, Brahmaputra, Mahanadi and Krishna Rivers (Jayaram, 1963).

Ailia punctata (Day, 1872)

Ailiichthys punctata Day, 1872: 713. Type locality: Jumna at and below Delhi, also in the lower Punjaub rivers. Syntypes: AMS B.7570 (1), BMNH 1889.2.1.2585-87 (3), BMNH 1889.2.1.2588-90 (3), BMNH 1889.2.1.2591 (1, skeleton), BMNH 1889.2.1.2592-93 (2), NMW 47849 (1), NMW 47863 (1), RMNH 2763 (1), ZMB 11213 (1), ZSI 420 (1, lost). 
Distribution: Indus, Yamuna and Ganges Rivers, northern India (Jayaram, 1963).

\section{CLUPISOMA Swainson, 1838}

Clupisoma Swainson, 1838: 347, 351, 354. Type species: Silurus (Clupisoma) argentata Swainson, 1839. Type by subsequent monotypy. Gender: Neuter. Originally proposed as a subgenus of Silurus, without any included species; first species added in Swainson (1839: 306).

Schilbeichthys Bleeker, 1858b: 255, 256. Type species: Silurus garua Hamilton, 1822. Type by monotypy. Gender: Masculine.

Key to species: Datta \& Karmakar (1980).

Clupisoma bastari Datta \& Karmakar, 1980

Clupisoma bastari Datta \& Karmakar, 1980: 193, figs. 1-2. Type locality: Indravati River (a tributary of Godavari River) at Lohandigura, $33 \mathrm{kms}$ west of Jagdalpur, Bastar District, Madhya Pradesh, India. Holotype: ZSI FF1342.

Distribution: Godavari River basin, Madhya Pradesh, India (Jayaram, 1999).

Clupisoma garua (Hamilton, 1822)

Silurus garua Hamilton, 1822: 156, 375, pl. 21 (fig. 50). Type locality: Fresh water rivers of the Gangetic provinces. No types known.

Silurus (Clupisoma) argentata Swainson, 1839: 306. Type locality: Fresh water rivers of the Gangetic provinces. No types known. Made available by reference to "Ham. pl. 21, f. 50" [= Hamilton, 1822: pl. 21 (fig. 50)]. Unneeded new name for Silurus garua Hamilton, 1822.

Distribution: Throughout northern India, but not south of the Mahanadi River, Pakistan, Bangladesh, and Nepal (Jayaram, 1999).

Clupisoma longianalis (Huang, 1981)

Platytropius longianalis Huang, 1981: 438, fig. 5. Type locality: Puer county, Xiaoganlanba, Yunnan, China. Holotype: KIZ 735118.

Distribution: Lancangjiang [= Mekong River] (Chu et al., 1999).

Clupisoma montana Hora, 1937

Clupisoma montana Hora, 1937b: 673, figs. 7-8. Type locality: Teesta River, below Darjeeling, India. Type(s): ZSI F 12472/1.

? Pseudeutropius murius batarensis Shrestha, 1980: 212, fig. 97. Type locality: Batar, Trisuli river, Nuwakot Dist., Bagmati Zone, Nepal. Holotype $(220 \mathrm{~mm})$ : at Museum of Zoology Department, Institute of Science, Tribuhaven University, Nepal.

Distribution: Teesta, Jamuna and Sone Rivers, India, Nepal (Jayaram, 1999).

Clupisoma naziri Mirza \& Awan, 1973

Clupisoma naziri Mirza \& Awan, 1973: 152, fig. 2. Type locality: Indus River at Jinnah Barrage, Pakistan. Holotype: GCM 10.

Distribution: NWFP, Punjab, Azad Kashmir, Indus River basin, Pakistan (Mirza, 2003).

Clupisoma nujiangense Chen, Ferraris \& Yang, 2005

Clupisoma nujiangense Chen, Ferraris \& Yang, 2005: 566, fig. 1. Type locality: China, Yunnan Province, Baoshan City, Longling County, Mungnuo Township, Sanjiangkou, Nu Jiang (=Salween River), 24²5'18.6"N, 9858'21'2"E. Holotype: KIZ 200310118.

Distribution: Upper Salween River, Yunnan, China (Chen et al., 2005).

Clupisoma prateri Hora, 1937

Clupisoma prateri Hora, 1937b: 671, fig. 6. Type locality: Burma. Holotype: Asiatic Soc. Bengal 213 or 219 [now at ZSI].

Distribution: Lower and middle reaches of Irrawaddy River, Sittang, Bago and Salween river basins, Myanmar (Ferraris, 2004).

Remarks: Redescribed in Ferraris (2004: 6, fig. 2).

Clupisoma roosae Ferraris, 2004

Clupisoma roosae Ferraris, 2004: 2, fig. 1. Type locality: Myanmar, Kachin State, Myitkyina market. Holotype: 
NRM 40030.

Distribution: Upper Irrawaddy River basin, Myanmar (Ferraris, 2004).

Clupisoma sinense (Huang, 1981)

Platytropius sinensis Huang, 1981: 437, figs. 1-4. Type locality: Puer county, Xiaoganlanba, Yunnan, China. Holotype: KIZ 735124.

Distribution: Mekong River basin (Rainboth, 1996), including Lancangjiang (= upper Mekong River of Yunnan)

(Chu et al., 1999), and Malay Peninsula (Zakaria-Ismail, 1992; Kottelat, 2001b).

Remarks: Placement of this species in Clupisoma follows Ng (1999b) and Chen et al. (2005).

EUTROPIICHTHYS Bleeker, 1862

Eutropiichthys Bleeker, 1862b: 398. Type species: Pimelodus vacha Hamilton, 1822. Type by original designation. Gender: Masculine. Also proposed as new in Bleeker (1862-63: 14).

Revision: Hora (1937e).

Eutropiichthys burmannicus Day, 1877

Eutropiichthys burmannicus Day, 1877 (in Day, 1875-78): 490. Type locality: Burma. Possible syntypes: ZSI B.3839 (2), ZSI B.219 (1, lost), ZSI B.306 (1). Originally as: "Variety Eutropiichthys burmannicus," in the account of Eutropiichthys vacha.

Distribution: Irrawaddy, lower Salween and smaller intervening river basins.

Eutropiichthys murius (Hamilton, 1822)

Pimelodus murius Hamilton, 1822: 195, 378. Type locality: Mahananda river [Bengal]. No types known. Unpublished Hamilton illustration reproduced in Hora (1929: pl. 22, fig. 2).

Pachypterus melanurus Swainson, 1839: 306. Type locality: Mahananda river [Bengal]. No types known. Unneeded new name for Pimelodus murius Hamilton, 1822.

Distribution: Northern India, Bangladesh and Pakistan (Jayaram, 1999).

Eutropiichthys vacha (Hamilton, 1822)

Pimelodus vacha Hamilton, 1822: 196, 378, pl. 19 (fig. 64). Type locality: Larger fresh water rivers of the Gangetic provinces. No types known.

Pachypterus punctatus Swainson, 1839: 306. Type locality: Larger fresh water rivers of the Gangetic provinces. No types known. Made available by reference to "Ham. p. 196, f. 64 " [= Hamilton, 1822: 196, p. 19, fig. 64)]. Unneeded new name for Pimelodus vacha Hamilton, 1822.

Distribution: Northern India, south to the Mahanadi River, Bangladesh, Nepal and Pakistan (Jayaram, 1999).

IRVINEIA Trewavas, 1943

Irvineia Trewavas, 1943: 165. Type species: Irvineia voltae Trewavas, 1943. Type by monotypy. Gender: Feminine.

Irvineia orientalis Trewavas, 1964

Irvineia orientalis Trewavas, 1964: 390, figs. 1, 2, 3 b-c. Type locality: Villaggio Duca delgi Abruzzi, Uebi Scebeli

(Shebeli) system. Holotype: MSNG 39551; holotype illustrated in De Vos (1995: 358, fig. 226).

Distribution: Juba-Uebi Shebeli system (De Vos, 1995).

Irvineia voltae Trewavas, 1943

Irvineia voltae Trewavas, 1943: 165, fig. 1. Type locality: Above the Senchi Rapids, River Volta, Gold Coast. Syntypes (3): BMNH 1944.2.9.19-21 (3); one syntype illustrated in De Vos (1995: 362, fig. 229).

Distribution: lower Volta River basin (De Vos, 1995).

LAIDES Jordan, 1919

Laïs Bleeker, 1857b: 473. Type species: Pangasius hexanema Bleeker, 1852. Type by monotypy. Gender: Masculine. Preoccupied by Lais Gistel, 1848, in Tunicata, replaced by Laides Jordan, 1919.

Laides Jordan, 1919b: 293. Type species: Pangasius hexanema Bleeker, 1852. Type by being a replacement name. Gender: Masculine. Replacement for Lais Bleeker, 1857; preoccupied by Lais Gistel, 1848, in Tunicata.

Laides hexanema (Bleeker, 1852)

Pangasius hexanema Bleeker, 1852d: 588. Type locality: Palembang, Batavia. Syntypes (2, 102-152 mm TL): pos- 
sibly BMNH 1863.12.4.1007 (1, 157 mm TL), NMV A928 (1).

Distribution: Thailand to Indonesia, in large rivers, including the Mekong (Rainboth, 1996).

Laides longibarbis (Fowler, 1934)

Pangasius longibarbis Fowler, 1934a: 87, fig. 27. Type locality: Me Kong at Chieng Sen, North Siam. Holotype: ANSP 59441.

Distribution: Mekong, Mehklong, and Chao Phraya River basins (Ng, 1999b).

Remarks: Redescribed by Ng (1999b).

\section{NEOTROPIUS Kulkarni, 1952}

Pachypterus Swainson, 1838: 346 et seq. Type species: Silurus atherinoides Bloch, 1794. Type by subsequent designation by Swain (1882: 281). Gender: Masculine. Originally without any included species. Species first added in Swainson (1839: 306).

Neotropius Kulkarni, 1952: 231. Type species: Neotropius khavalchor Kulkarni, 1952. Type by monotypy. Gender: Masculine.

Review: Hora (1941).

Remarks: Swain (1882: 281) and (following Swain) Jordan (1919a: 205) treated Pachypterus Swainson as preoccupied in Coleoptera, presumably basing their decision on the following notation in Agassiz (1846: 271): "Pachypterus Sol. Col., 1833." However, it appears that Agassiz was referring to usage of the name in Dejean, 1834, in which a manuscript by Solier was cited as the source of Pachypterus. According to Neave (1940: 515) Dejean's use of the name was as a nomen nudum and Pachypterus was not used in a way that would have made it available in Coleoptera until Lucas, 1846, which postdates the first proposal of Pachypterus. However, the writings of Swain and Jordan may have stifled use of Pachypterus Swainson as valid, and the younger name Neotropius, which has been in widespread usage for the past one-half century, is used here. The species listed below are similar overall to those of Pseudeutropius and the two genera may not both represent natural groups.

Neotropius acutirostris (Day, 1870)

Pseudeutropius acutirostris Day, 1870c: 618. Type locality: Throughout Burma. Syntypes: AMS B.7967 (1), BMNH 1889.2.1.2413 (1), BMNH 1889.2.1.2462 (1), NMW 48327 (1), ZSI F484 (1), ZSI A.505 (1 lost).

Distribution: Irrawaddy, Sittang, and Bago Rivers, Myanmar.

Neotropius atherinoides (Bloch, 1794)

Silurus Atherinoides Bloch, 1794: 48, pl. 371 (fig. 1). Type locality: Tranquebar. Holotype: ZMB 3013.

Pimelodus angius Hamilton, 1822: 180, 377, pl. 29 (fig. 59). Type locality: Rivers of Bengal. No types known.

Pimelodus urua Hamilton, 1822: 177, 377. Type locality: Rivers and ponds of the northern parts of Bengal. No types known. Unpublished Hamilton illustration reproduced in Hora (1929: pl. 21, fig. 4).

Pachypterus trifasciatus Swainson, 1839: 306. Type locality: Rivers of Bengal. No types known. Name made available by reference to "Ham. P. 180, f. 59 " [= Hamilton (1822: 180, pl. 29, fig. 59)]. Unneeded new name for Pimelodus angius Hamilton, 1822.

Pseudeutropius atherinoides walkeri Chaudhuri, 1912: 444, pl. 41 (fig. 3). Type locality: Siripur, Saran, Bihar [North India]. Syntypes (2): at ZSI. Originally as Pseudeutropius atherinoides var. walkeri.

Distribution: Widely distributed in India, except for Assam and Kerala; also in Pakistan, Nepal, and Bangladesh (Jayaram, 1999).

Neotropius khavalchor Kulkarni, 1952

Neotropius khavalchor Kulkarni, 1952: 232, fig. 1. Type locality: Panchaganga River, near Kolhapur, Bombay State, India. Holotype: ZSI F647/2.

Distribution: Krishna River basin, Maharashtra and Andhra Pradesh, India (Jayaram, 1999).

\section{Species inquirenda, Neotropius}

Bagrus Exodon Cuvier \& Valenciennes, in Valenciennes, 1832b: 385, pl. 4 (fig. 1). Type locality: Bengal. Syntypes: MNHN a-8960 (2), MNHN b-0680 (1). Also in Cuvier \& Valenciennes (1840a: 395). 
PARAILIA Boulenger, 1899

Parailia Boulenger, 1899b: 105. Type species: Parailia congica Boulenger, 1899. Type by monotypy. Gender: Feminine.

Physailia Boulenger, 1901e: 445. Type species: Physailia pellucida Boulenger, 1901. Type by monotypy. Gender: Feminine.

Parailia congica Boulenger, 1899

Parailia congica Boulenger, 1899b: 106, pl. 41 (fig. 2). Type locality: Ebinga (rivière du lac Léopold II). Lectotype: MRAC 942, designated by De Vos (1995: 297).

Parailia longifilis Boulenger, 1902d: 37, pl. 10 (fig. 3). Type locality: l'Ubangi à Banzyville. Lectotype: MRAC 1309 (1), designated by De Vos (1995: 295).

Distribution: Congo River basin (De Vos, 1995).

Parailia occidentalis (Pellegrin, 1901)

Ailia occidentalis Pellegrin, 1901: 331. Type locality: Cap Lopez, Congo français. Holotype: MNHN 1885-0404.

Physailia ansorgii Boulenger, 1910: 557. Type locality: Quanza River at Cunga, Angola. Syntypes (2): BMNH 1911.6.1.107 (1), NMW 45490 (1); NMW syntype illustrated in De Vos (1995: 305, fig. 208).

Physailia villiersi Boulenger, 1912b: 17, pl. 17 (fig. 6). Type locality: de la Lucala près de Cabinda, du Chiloango à Mayili, de la Luali à Lundo, et de la Luculla. Syntypes: ANSP 38756-58 (3), BMNH 1912.4.1.419-420 (2), BMNH 1912.4.1.421 (1), BMNH 1912.4.1.422-424 (3), BMNH 1912.4.1.425-429 (5), FMNH 56151 (2), MRAC 1656-1659 (4), MRAC 1660-1661 (2), MRAC 1662 (1), NMW 45489 (16), ZMB 18818 (9).

Distribution: Ogowe, Congo, Quanza, Luculla, Chiloango Rivers (De Vos, 1995).

Parailia pellucida (Boulenger, 1901)

Physailia pellucida Boulenger, 1901e: 445. Type locality: Omdurman, Nile River. Syntypes (numerous): BMNH 1907.12.2.1942-1956 (14 or 15), BMNH 1907.12.2.1940-1941 (2), ? MCZ 32074 (1), MNHN 1907-0215 (3), MNHN 1907-0216 (3).

Distribution: Nile River, Chad Basin, several west African river basins (De Vos, 1995).

Parailia somalensis (Vinciguerra, 1897)

Ailia somalensis Vinciguerra, 1897: 346. Type locality: Lugh, Fl. Ganana, Somaliland. Syntypes: BMNH 1961.5.3.12 (1), MSNG 14612 (4); one MSNG syntype illustrated in de Vos (1995: 321, fig. 212).

Physailia somalensis tanensis Whitehead, 1962a: 101, fig. 2. Type locality: Tana River at Hola, Kenya. Holotype: BMNH 1961.5.3.7.

Distribution: Ganana and lower Tana Rivers (De Vos, 1995).

Parailia spiniserrata Svensson, 1933

Parailia spiniserrata Svensson, 1933: 73, pl. 4 (fig. 2); fig. 24. Type locality: MacCarthy Island Area, Gambia River, British West Africa. Syntypes (about 25): NRM 11144 (4), NRM 14453 (1), NRM 14454 (8), NRM 21144 (2), NRM 31144 (1), NRM 31145 (1), NRM 31146 (4), NRM 31147 (1); one syntype illustrated in De Vos (1995: 326, fig. 215).

Distribution: Gambia, Geba, and Jong River basins (De Vos, 1995).

\section{PAREUTROPIUS Regan, 1920}

Ansorgia Boulenger, 1912b: 17. Type species: Ansorgia vittata Boulenger, 1912. Type by monotypy. Gender: Feminine. Preoccupied by Ansorgia Warren, 1899, in Lepidoptera; replaced by Ansorgiichthys Whitley, 1935.

Pareutropius Regan, 1920a: 105. Type species: Pareutropius micristius Regan, 1920. Type by monotypy. Gender: Masculine.

Eutropiellus Nichols \& La Monte, 1933b: 5. Type species: Eutropiellus kasai Nichols \& La Monte, 1933. Type by original designation. Gender: Masculine.

Ansorgiichthys Whitley, 1935: 249. Type species: Ansorgia vittata Boulenger, 1912. Type by being a replacement name. Gender: Masculine. Replacement for Ansorgia Boulenger, 1912; preoccupied by Ansorgia Warren, 1899 , in Lepidoptera.

Revision: Thys van den Audenaerde (1964, as Eutropiellus).

Pareutropius buffei (Gras, 1961) 
Eutropiellus buffei Gras, 1961: 406, fig. 3. Type locality: Bas-Ouémé, Bas-Dahomey. Syntypes (5): Lab. d'Hydrobiol. Cotonou, Benin (1), MNHN 1958-0001 (4).

Eutropiellus vandeweyeri Thys van den Audenaerde, 1964: 225, fig. 3. Type locality: Environment de Aba, Lower Nigeria Delta, Nigeria. Holotype: MRAC 140281.

Distribution: Lower Ouémé River, Cross and Niger Rivers (De Vos, 1995).

Pareutropius debauwi (Boulenger, 1900)

Eutropius debauwi Boulenger, 1900a: 138, pl. 50 (fig. 2). Type locality: Uerre. Syntypes: BMNH 1900.12.13.8 (1), MRAC 347 (1).

Ansorgia vittata Boulenger, 1912b: 17, pl. 19 (fig. 2). Type locality: Lower Congo. Syntypes: ANSP 38734-35 (2), BMNH 1912.4.1.414 (1), BMNH 1912.4.1.415-417 (3), BMNH 1912.4.1.418 (1, skeleton), MRAC 1653 (1), MRAC 1654 (1), MRAC 1655 (1), ZMB 18817 (1). Name as Ansorgia vitata on p. 17, A. vittata on plate legend (p. 28) and on plate; De Vos (1986: 37) cited both spellings and used Ansorgia vittata, thereby acting as first reviser.

Ansorgia vittata bistriata Giltay, 1930b: 393, fig. 1. Type locality: Buta. Holotype: MRAC 20584.

Eutropiellus kasai Nichols \& La Monte, 1933b: 5, fig. 4. Type locality: Luluabourg, Kasai, Belgian Congo. Holotype: AMNH 12338.

Distribution: Congo River system, Chiloango River, the Kouilou and Ogowe Rivers (De Vos, 1995).

Pareutropius longifilis (Steindachner, 1914)

Eutropius longifilis Steindachner, 1914: 537. Type locality: Deutsch-Ostafrika [Kiperege]. Syntypes: NMW 46507 (2). Species illustrated and described in more detail in Steindachner (1915d: 75, pl. 3, fig. 2), with locality as: Wasserlauf bei dem Dorfe Keperege; one syntype illustrated in De Vos (1995: 347, fig. 222).

Pareutropius micristius Regan, 1920a: 105. Type locality: Tanganyika Territory, Morogoro. Syntypes (7): BMNH 1920.3.8.10-12 (3).

Distribution: eastward flowing rivers north of the Ruvuma, Lake Chiuta, Ruvuma system, Lake Chilwa (De Vos, 1995).

Pareutropius mandevillei Poll, 1959

Pareutropius mandevillei Poll, 1959: 92, pl. 16 (fig. 4a). Type locality: Stanley Pool. Holotype: MRAC 98387.

Distribution: Congo River basin (De Vos, 1995).

\section{PLATYTROPIUS Hora, 1937}

Platytropius Hora, 1937a: 352. Type species: Pseudeutropius siamensis Sauvage, 1883. Type by original designation. Gender: Masculine.

Nemasiluroides Fowler, 1937: 137. Type species: Nemasiluroides furcatus Fowler, 1937. Type by original designation. Gender: Masculine.

Remarks: Platytropius Hora was published in January, 1937, and therefore has priority over Nemasiluroides Fowler, 1937, which was published on 19 May, 1937. A second paper by Hora (1937c: 39) which has been cited as the original proposal of Platytropius (e. g., Eschmeyer, 1990) was not published until November, 1937.

Platytropius siamensis (Sauvage, 1883)

Pseudeutropius siamensis Sauvage, 1883b: 154. Type locality: Mé-Nam, Siam. Holotype: MNHN a-5002.

Nemasiluroides furcatus Fowler, 1937: 138, figs. 16-19. Type locality: Bangkok, Siam. Holotype: ANSP 67893.

Distribution: Chao Phraya River basin, Thailand.

\section{PROEUTROPIICHTHYS Hora, 1937}

Proeutropiichthys Hora, 1937a: 353. Type species: Eutropius macropthalmos Blyth, 1860. Type by original designation. Gender: Masculine.

Review: Hora (1941).

? Proeutropiichthys buchanani (Valenciennes, 1840)

Bagrus Buchanani Valenciennes, 1840, in Jacquemont, 1835-44: pl. 16 (fig. 3). Type locality: Hindustan, India. Holotype: MNHN a-5721(1 of 3) or MNHN a-5722 (1 of 3). Name made available by figure caption.

Distribution: Hindustan, India. 
Remarks: Illustrated specimen should be treated as the holotype, if identifiable from among the specimens listed herein. Inclusion into Proeutropiichthys based on personal observation of types.

? Proeutropiichthys goongwaree (Sykes, 1839)

Hypophthalmus Goongwaree Sykes, 1839: 163. Type locality: the Mota Mola near Poona, India. No types known. Also described as new in Sykes (1840: 60); illustrated and described in more detail in Sykes (1841: 369, pl. 64, fig. 3).

Distribution: Southern India (Jayaram, 1999).

Remarks: Placed in Pseudeutropius by Günther (1864) and Day (1875-78), then into Eutropiichthys by Hora (1937e), where it has remained until now. However, the described species and illustrations are clearly not of a species of either of these genera and more likely that of a species of Proeutropiichthys.

Proeutropiichthys macropthalmos (Blyth, 1860)

Eutropius macropthalmos Blyth, 1860: 156. Type locality: Tenasserim [Myanmar]. Type(s): Whereabouts unknown. Proeutropiichthys taakree burmanicus Tilak, 1982: 35, figs. 1, 3, 5. Type locality: Burma. Holotype: ZSI FF771.

Distribution: Irrawaddy, Sittang, and Bago River basins, Myanmar.

Proeutropiichthys taakree (Sykes, 1839)

Hypophthalmus taakree Sykes, 1839: 163. Type locality: Deccan, India. No types known. Also described as new in Sykes (1840: 60); illustrated and described more fully in Sykes (1841: 369, pl. 64, fig. 4).

Schillee Sykesii Jerdon, 1849: 335. Type locality: Cavery River, southern India. No types known.

Pseudeutropius longimanus Günther, 1864: 60. Type locality: India. Holotype: BMNH 1857.6.13.88 (skin).

Pseudeutropius megalops Günther, 1864: 60. Type locality: Godaveri at Mahadespur, Orissa, Central India. Holotype: BMNH 1860.3.19.784.

Distribution: Western Ghats, Kerala, Maharashtra, India (Jayaram, 1999).

\section{PSEUDEUTROPIUS Bleeker, 1862}

Pseudeutropius Bleeker, 1862b: 398. Type species: Eutropius brachypopterus Bleeker, 1858. Type by original designation. Gender: Masculine. Also in Bleeker (1862-63: 14, 74).

\section{Pseudeutropius brachypopterus (Bleeker, 1858)}

Eutropius brachypopterus Bleeker, 1858b: 169. Type locality: Palembang [Sumatra]. Holotype (115 mm TL): Whereabouts unknown; holotype illustrated in Bleeker (1862-63: 71, pl. 75 [= Silur. pl. 27], fig. 1).

Distribution: Kapuas River (Roberts, 1989a); Sumatra.

Pseudeutropius mitchelli Günther, 1864

Pseudeutropius mitchelli Günther, 1864: 59. Type locality: Madras Presidency [India]. Syntypes: BMNH 1863.12.18.134-135 (2).

Distribution: Kerala, India (Jayaram, 1999).

Pseudeutropius moolenburghae Weber \& de Beaufort, 1913

Pseudeutropius moolenburghae Weber \& de Beaufort, 1913: 249, fig. 100. Type locality: Sumatra (Batang Hari river) [Indonesia]. Syntypes: AMNH 9283 (1), ZMA 112681 (6).

Distribution: Batang Hari River, Sumatra (Weber \& de Beaufort, 1913); Kapuas River (Roberts, 1989a).

SCHILBE Oken, 1817

Schilbe Oken, 1817: 1183. Type species: Silurus mystus Linnaeus, 1758. Type by monotypy. Gender: Masculine.

Pusichthys Swainson, 1838: 348, et seq. Type species: Schilbe uranoscopus Rüppell, 1832. Type by subsequent monotypy. Gender: Masculine. Originally proposed as a subgenus of Silurus, without any included species. Species first added in Swainson (1839: 307).

Eutropius Müller \& Troschel, 1849: 6. Type species: "Bagrus schilboides Val. (Hypophthalmus niloticus Rüppell)" [= Bagrus schilbeides Valenciennes, 1840]. Type by monotypy. Gender: Masculine. Originally proposed as a subgenus of Bagrus.

Proeutropius Fowler, 1936b: 307. Type species: Silurus congensis Leach, 1818. Type by original designation. Gender: Masculine. Originally proposed as a subgenus of Eutropius.

Review: De Vos \& Lévêque (1983), west Africa (as Eutropius). 
Key: De Vos (1984a) Quanza and Bengo rivers, Angola (as Eutropius).

Schilbe angolensis (De Vos, 1984)

Eutropius angolensis De Vos, 1984a: 13, figs. 4, 5d. Type locality: Quanza river at Quimbango, Angola, 1051'S, 17²30'E. Holotype: MRAC 78-6-P-899.

Distribution: Upper Quanza River, Angola (De Vos, 1995).

Schilbe banguelensis (Boulenger, 1911)

Eutropius banguelensis Boulenger, 1911a: 282, fig. 231. Lake Bangwelu. Holotype: BMNH 1907.9.30.8.

Eutropius nasalis Boulenger, 1915: 169. Type locality: Lac Moero. Lectotype: MRAC 14163; lectotype designated by, and illustrated in, De Vos (1995: 124, fig. 158, bottom) as lectoholotype.

Distribution: Chambezi River, Lake Bangweulu, Luapula River and Lake Mweru (De Vos, 1995).

Schilbe bocagii (Guimarães, 1884)

Eutropius Bocagii Guimarães, 1884a: 85, unnumbered pl. Type locality: Dondo (Quanza fl.), Angola. Holotype: MB (destroyed, De Vos, 1995: 128). Illustration of holotype reproduced in De Vos (1984a: 5, fig. 1a).

Eutropius ansorgii Boulenger, 1910: 555. Type locality: Quanza River at Cunga, Angola. Syntypes (3): BMNH 1911.6.1.100-101 (2), NMW 46502 (1).

Eutropius seraoi Boulenger, 1910: 556. Type locality: Bengo River and from the Lucalla River at Lucalla, Angola. Syntypes (10): ANSP 37956 (1), BMNH 1911.6.1.102-105 (4), BMNH 1911.6.1.106 (1), NMW 46524 (2), ZMB 18226 (1); one NMW syntype illustrated in De Vos (1984a: fig. 3, and 1995: 129, fig. 160f).

Eutropius eclipsis Fowler, 1919b: 270, fig. 12. Type locality: Quanza River at Cunga, Angola. Holotype: USNM 42342. Illustration of holotype reproduced in De Vos (1984a: 5, fig. 1b).

Distribution: Lower Quanza River and Bengo Rivers, Angola (De Vos, 1995).

Schilbe brevianalis (Pellegrin, 1929)

Eutropius brevianalis Pellegrin, 1929b: 362. Type locality: Dehane (Nyong), Cameroun. Syntypes: MNHN 19280354 (1), MNHN 1928-0355 (1).

Distribution: coastal rivers in Nigeria and Cameroon (De Vos, 1995).

Schilbe congensis (Leach, 1818)

Silurus Congensis Leach, in Tuckey, 1818: 409. Type locality: Lower Congo. Holotype: BMNH 2005.5.17.6.

Eutropius congoensis Boulenger, 1899b: 105. Type locality: Lower Congo. Holotype: BMNH 2005.5.17.6. Unjustified emendation of Silurus congensis (De Vos, 1995).

Eutropius congolensis Boulenger, 1901a: 268. Type locality: Lower Congo. Holotype: BMNH 2005.5.17.6. Unjustified emendation of Silurus congensis Leach, 1818.

Distribution: Congo River system (De Vos, 1995).

Schilbe djemeri (Thys van den Audenaerde \& De Vos, 1982)

Eutropius djemeri Thys van den Audenaerde \& De Vos, 1982: 179, figs. 1-2. Type locality: River Djerem, below falls at the rapids, 6-7 km south of Mbakaou, Cameroon. Holotype: MRAC 73-18-P-2310.

Distribution: Upper Sanaga River basin, Cameroon (De Vos, 1995).

Schilbe durinii (Gianferrari, 1932)

Eutropius Durinii Gianferrari, 1932: 141. Type locality: Lago Tanganyika. Holotype: MSNM 36; illustrated in De Vos (1995: 146, fig. 169).

Distribution: Unknown (De Vos, 1995).

Remarks: Validity of species questioned by De Vos $(1986,1995)$, who indicated that the type locality is almost certainly wrong.

Schilbe grenfelli (Boulenger, 1900)

Eutropius grenfelli Boulenger, 1900a: 137, pl. 50 (fig. 1). Type locality: Bolobo. Holotype: BMNH 1900.6.23.6.

Eutropius gastratus Nichols \& Griscom, 1917: 708, fig. 20. Type locality: Rungu, Congo. Holotype: AMNH 6404.

Eutropius bomae Lönnberg \& Rendahl, 1922: 126. Type locality: Boma, lower Congo. Holotype: NRM 6024.

Distribution: Congo River basin, Ogowe, Nyanga, and Ntem-Campo River basins, Nyong and Lokondje basins (De Vos, 1995).

Schilbe intermedius Rüppell, 1832

Schilbe intermedius Rüppell, 1832: 6. Type locality: Nil. Syntypes: BMNH 1850.7.29.14 (1), SMF 202 (1), SMF 
2625 (1, dry), SMF 6403 (1, skeleton). BMNH specimen illustrated by Seegers (1996: 184, fig. 129) as holotype.

Schilbe auratus Joannis, 1835: [Cl. IV, pl. 5]. Type locality: Nil. Possible holotype: MNHN b-0346.

Schilbe Senegallus Valenciennes, in Cuvier \& Valenciennes, 1840a: 378 (281 of Strasbourg deluxe edition). Type locality: Sénégal. Syntypes (huit pouces): at MNHN.

Bagrus depressirostris Peters, 1852: 682. Type locality: Mozambique. Syntypes: BMNH 1863.4.30.15 (1), ZMB 3015 (2), ZMB 3016 (1). Illustrated and described in more detail in Peters (1868c: 25, pl. 4, fig. 5) as Eutropius depressirostris.

Schilbe dispila Günther, 1864: 51. Type locality: River Niger, West Africa; Upper Nile, 500 miles south of Chartoum. Syntypes: BMNH 1852.2.22.17 (1), BMNH unregistered (2).

Schilbe senegalensis Günther, 1864: 51. Type locality: Senegal. Syntypes:at MNHN (not found). Unjustified emendation of Schilbe senegallus Valenciennes, 1840.

Schilbe senegalensis fasciata Steindachner, 1870: 983, pl. 6 (figs. 1-2). Type locality: Senegal bei St. Louis, Dagana, Podor, und Bakel. Syntypes: NMW 44579-81 (61). Originally as Schilbe senegalensis var. fasciata.

Schilbe steindachneri Guimaraes, 1884b: 1, pl. 1 (figs. 1-2). Type locality: Cunene. Holotype: MB (destroyed; De Vos, 1995: 162).

Schilbe bouvieri Rochebrune, 1885: 95. Type locality: [Casamance R., Senegal, w. Africa]. Type(s): at Museo Bouvieri.

Schilbe Emini Pfeffer, 1896: 32. Type locality: Muhale-Bach, Uniamwesi. Holotype: ZMB 12851.

Eutropius lemairii Boulenger, 1900a: 138, pl. 50 (fig. 3). Type locality: Lofoi, Katanga. Syntypes: BMNH 1900.12.13.24 (1), MRAC 293 (1).

Schilbe palmeri Svensson, 1933: 71, pl. 3 (fig. 4). Type locality: Gambia River, Mc Carthy Island area. Syntypes: NRM 11145 (3), NRM 14452 (1), NRM 21145 (1).

Distribution: Widely distributed in Sub-Saharan Africa (De Vos, 1995).

Remarks: See De Vos \& Skelton (1990) for discussion of validity and correct name of the species.

Schilbe laticeps (Boulenger, 1899)

Eutropius laticeps Boulenger, 1899b: 105, pl. 41 (fig. 3) [not fig. 2 as stated in text]. Type locality: Kutu, Lac Léopold II. Holotype: MRAC 941.

Distribution: Congo River basin (De Vos, 1995).

Schilbe mandibularis (Günther, 1867)

Eutropius mandibularis Günther, 1867b: 112. Type locality: Bossumprah River, Gold Coast. Holotype: deposited at Liverpool Museum. Holotype considered to be lost by Boulenger (1911: 290) and Trewavas (1943: 168).

Eutropius liberiensis Hubrecht, 1881: 69. Type locality: St. Paul's River, Liberia. Holotype: RMNH 5328.

Eutropius mentalis Boulenger, 1901a: 269. Type locality: River Prah, Gold Coast. Syntypes: BMNH 1899.12.22.810 (3).

Distribution: West Africa, from the St. Paul to the Prah River (De Vos, 1995).

Remarks: See De Vos (1983) for comments on taxonomy.

Schilbe marmoratus Boulenger, 1911

Schilbe marmoratus Boulenger, 1911f: 222. Type locality: la rivière Sankuru (Kasaï). Holotype (70 mm): at MHNL. Schilbe congolensis Steindachner, 1912: 445. Type locality: Moloundou, Dscha, Kamerun. Syntypes: NMW 1069899 (2). Species described in more detail in Steindachner (1913: 30). Preoccupied in Schilbe by Eutropius congolensis Boulenger, 1901.

Distribution: Congo River basin and Shiloango system (De Vos, 1995).

Schilbe micropogon (Trewavas, 1943)

Eutropius micropogon Trewavas, 1943: 168. Type locality: River Volta, Gold Coast. Syntypes (3): BMNH 1944.2.9.16-17 (2), BMNH 1944.2.9.18 (1).

Distribution: lower course of west African rivers, from Senegal to Cameroon (De Vos, 1995).

Schilbe moebiusii (Pfeffer, 1896)

Eutropius möbiusii Pfeffer, 1896: 30. Type locality: Kingani-Fluss. Syntypes: ZMB 13680 (8, ? now 3); one syntype illustrated in De Vos (1995: 235, fig. 185). 
Eutropius moebii Boulenger, 1911a: 293. Type locality: Kingani -Fluss. Syntypes: ZMB 13680 (8, ? now 3). Unjustified emendation of Eutropius möbiusii Pfeffer, 1896.

Distribution: Coastal rivers of Tanzania, including the Kingani, Rufiji, and Wani basins (De Vos, 1995).

Schilbe multitaeniatus (Pellegrin, 1913)

Eutropius multitaeniatus Pellegrin, 1913b: 273. Type locality: l'Ogôoué (Ngomo). Syntypes: MNHN 1908-0230 (1), MNHN 1908-0231 (1), MNHN 1913-0266 (1).

Eutropius multilineatus Boulenger, 1916: 293. Unjustified emendation of Eutropius multitaeniatus Pellegrin, 1913 (de Vos, 1995).

Eutropius cameronensis Pellegrin, 1929b: 361. Type locality: Akonolinga (Nyong), ... Mfida (Nyong), .... Nyabessan (Ntem); Cameroun. Syntypes: MNHN 1928-0351 (1), MNHN 1928-0352 (1), MNHN 1928-0353 (1).

Distribution: Dja, Nyong, Igowe, Nyang, and N'dogo Rivers (De Vos, 1995).

Remarks: See De Vos (1983) for comments on taxonomy.

Schilbe mystus (Linnaeus, 1758)

Silurus mystus Linnaeus, 1758: 305. Type locality: In Nilo. Holotype: NRM 70; illustrated in De Vos (1995: 247, fig. 191).

Hypophthalmus niloticus Rüppell, 1829: 6, pl. 1 (fig. 1). Type locality: Nil. Syntypes: BMNH 1850.7.29.16 (1), possibly RMNH 2985 (1), SMF 752 [or 725] (1), SMF 2637 (1, dry), SMF 6050 (1, skeleton), SMF 6051 (1, skeleton), SMF 6053 (1).

Bagrus Adansonii Valenciennes, in Cuvier \& Valenciennes, 1840a: 391 (290 of Strasbourg deluxe edition), pl. 414. Type locality: Sénégal. Holotype: MNHN a-8670 (dry).

Schilbe Hasselquistii Valenciennes, in Cuvier \& Valenciennes, 1840a: 377 (280 of Strasbourg deluxe edition). Type locality: Nil. Holotype: MNHN b-0599.

Bagrus schilbeides Valenciennes, in Cuvier \& Valenciennes, 1840a: 389 (289 of Strasbourg deluxe edition). Type locality: Nil. Syntypes: MNHN b-0600 (1), MNHN b-0601 (1). Unneeded new name for Hypophthalmus niloticus Rüppell, 1829.

Eutropius obtusirostris Günther, 1864: 53. Type locality: India [in error]. Syntypes: BMNH 1842.3.26.28-29 (2).

Eutropius altipinnis Steindachner, 1894: 57, pl. 1 (fig. 1). Type locality: St. Paul -Flusse, bei Soforch Place. Syntypes: NMW 46501 (1), RMNH 5397 (2).

Distribution: Nile River basin and West Africa (De Vos, 1995).

Remarks: De Vos \& Skelton (1990: 324, fig. 1) illustrated the holotype of Silurus mystus Linnaeus, and discussed nomenclatural history of the specimen but, unfortunately, mislabeled the photographed specimen as NRM 63 (rather than the correct number: NRM 70). De Vos (1995) concluded that Eutropius obtusirostris types probably originated in West Africa.

Schilbe nyongensis (De Vos, 1981)

Eutropius nyongensis De Vos, 1981: 968, fig. 4. Type locality: Nyong river, above falls at 12 km. S.-E. of Eseka, Cameroon. Holotype: MRAC 75-4-P-43.

Distribution: Nyong River, Cameroon, known only from type locality (De Vos, 1995).

Schilbe tumbanus (Pellegrin, 1926)

Eutropius tumbanus Pellegrin, 1926: 203. Type locality: Tondu (Lac Tumba); Congo belge. Syntypes (7): MNHN 1926-0161(1), MNHN 1926-0162 (1), MNHN 1926-0163 (1), MRAC 19685-86 (1), MRAC 19686 (1), MRAC 19687 (1), MRAC 19688 (1).

Distribution: Middle Congo River basin, abundant in Lake Tumba (De Vos, 1995).

Schilbe uranoscopus Rüppell, 1832

Schilbe uranoscopus Rüppell, 1832: 4, pl. 1 (fig. 1). Type locality: Ägypten, Nil bei Cairo. Lectotype: BMNH 1850.7.29.22, designated by De Vos (1986: 45).

Schilbe Isidori Valenciennes, in Cuvier \& Valenciennes, 1840a: 375 (278 of Strasbourg deluxe edition), pl. 412. Type locality: Egypte. Syntypes (no larger than 10 pouces): at MNHN.

Distribution: Nile River basin, Turkana system, Chad basin, Niger system, and Cross River (De Vos, 1995).

Schilbe yangambianus (Poll, 1954)

Eutropius yangambianus Poll, 1954: 61, fig. 4. Type locality: riv. Lubulu Iles du fleuve. Holotype: MRAC 123625. 
Distribution: Congo River basin and one location in upper Zambezi River (Skelton, 1993; De Vos, 1995).

Schilbe zairensis De Vos, 1995

Schilbe zairensis De Vos, 1995: 285, fig. 201. Type locality: Lemfu, Inkisi River, Lower Zaire Basin, Zaire, ca. $5^{\circ} 18$ 'S, 15²13'E. Holotype: MRAC 86-21-P-98.

Distribution: Lower Congo River basin (De Vos, 1995).

SILONIA Swainson, 1838

Silonia Swainson, 1838: 345 et seq. Type species: Ageniosus (Silonia) lurida Swainson, 1838. Type by monotypy. Gender: Feminine. Originally proposed as a subgenus of Ageniosus.

Silundia Valenciennes, in Cuvier \& Valenciennes, 1840b: 48 (36 of Strasbourg deluxe edition). Type species: Silundia gangetica Valenciennes, 1840. Type by subsequent designation by Bleeker (1862b: 399). Gender: Feminine.

Silondia Günther, 1864: 65. Type species: Silundia gangetica Valenciennes, 1840. Unjustified emendation of Silundia Valenciennes, 1840. Gender Feminine.

Silonopangasius Hora, 1937a: 352. Type species: Ageneiosus childreni Sykes, 1839. Type by original designation. Gender: Masculine.

Silonia childreni (Sykes, 1839)

Ageneiosus childreni Sykes, 1839: 165. Type locality: Deccan, India. No types known. Also proposed as new in Sykes (1840: 62). Illustrated and described in more detail in Sykes (1841: 375, pl. 66, fig. 3).

Silundia Sykesii Day, 1876: 569. Type locality: Deccan and Kurnool, India. Syntypes: AMS B.8084 (1), NMW 44624 (1), ZSI F1230 (1). Originally as "Silundia Sykesii, n.s.? = ? Ageneiosus Childreni, Sykes, Bleeker, \& Jerdon."

Distribution: Cauvery, Godavari and Krishna River basins, India (Jayaram, 1999).

Silonia silondia (Hamilton, 1822)

Pimelodus silondia Hamilton, 1822: 160, 375, pl. 7 (fig. 50). Type locality: Gangetic estuaries. No types known.

Ageniosus (Silonia) lurida Swainson, 1838: 345, fig. 85. Type locality: Gangetic estuaries. No types known. Name made available to reference to Ham. pl. 7 fig. 50 [= Hamilton, 1822, pl. 7, fig. 50]. Unneeded new name for Pimelodus silondia Hamilton.

Silundia Gangetica Valenciennes, in Cuvier \& Valenciennes, 1840b: 49 (36 of Strasbourg deluxe edition), pl. 426. Unneeded new name for Pimelodus silondia Hamilton, 1822, apparently to avoid tautonymy.

Distribution: Northern India, Bangladesh, Myanmar and Nepal (Jayaram, 1999).

Remarks: Redescribed in Hora (1938a).

SILURANODON Bleeker, 1858

Siluranodon Bleeker, 1858b: 253, 255, 256. Type species: Silurus auritus Geoffroy St. Hilaire, 1809. Type by monotypy. Gender: Masculine.

Siluranodon auritus (Geoffroy Saint-Hilaire, 1809)

Silurus auritus Geoffroy Saint-Hilaire, 1809: pl. 11 (figs. 1-2). Type locality: fl. Nil, Egypt. Possible holotype or syntypes: MNHN a-8954 (4), MNHN a-8956 (1). Name available from caption on plate, with illustrated specimen the holotype, if identifiable. Description of species in Geoffroy St. Hilaire (1827: 291-299).

Distribution: Nile, Chad, Niger, Volta, Comoe Rivers (De Vos, 1995).

\section{Genus inquirendum, Schilbidae}

Schillee Jerdon, 1849: 335. Type species: apparently not designated; name based on two species. Name credited to Cuvier, which may refer to Schilbe, but included species belong to two other genera.

\section{SCOLOPLACIDAE Bailey \& Baskin, 1976}

Scoloplacinae Bailey \& Baskin, 1976: 5. Type genus: Scoloplax Bailey \& Baskin, 1976.

Revision: Schaefer et al. (1989). 
Phylogeny: Schaefer (1990).

Taxonomic summary: Schaefer (2003a).

1 genus, 4 species; no named fossil taxa.

SCOLOPLAX Bailey \& Baskin, 1976

Scoloplax Bailey \& Baskin, 1976: 5. Type species: Scoloplax dicra Bailey \& Baskin, 1976. Type by original designation. Gender: Feminine.

Scoloplax dicra Bailey \& Baskin, 1976

Scoloplax dicra Bailey \& Baskin, 1976: 7, figs. 1-3. Type locality: Isolated Ox-Bow lagoon off the Río Iténez, ca. $400 \mathrm{~m}$ southwest of the river at a point opposite Costa Marques (Brazil), Dept. of Beni, Bolivia, 12 28.38'S, 64¹6.59'W. Holotype: AMNH 32482.

Distribution: Amazon and Paraguay River basins (Schaefer, 2003a).

Scoloplax distolothrix Schaefer, Weitzman \& Britski, 1989

Scoloplax distolothrix Schaefer, Weitzman \& Britski, 1989: 191, figs. 5, 6. Type locality: Small tributary of Rio Batovi, upper Rio Xingu, Mato Grosso, Brazil, approx. 1258'S, 53³7'W. Holotype: MZUSP 39065.

Distribution: Tocantins/Araguaia, Xingu and Paraguay River basins (Schaefer, 2003a).

Scoloplax dolicholophia Schaefer, Weitzman \& Britski, 1989

Scoloplax dolicholophia Schaefer, Weitzman \& Britski, 1989: 196, figs. 9, 10. Type locality: In igarapé, trib. of Rio Tarumãzinho, approximately $45 \mathrm{~km}$ north of Manaus, Amazonas, Brazil, 242'S, 6003'W. Holotype: MZUSP 6788.

Distribution: Negro River basin, Brazil (Schaefer, 2003a).

Scoloplax empousa Schaefer, Weitzman \& Britski, 1989

Scoloplax empousa Schaefer, Weitzman \& Britski, 1989: 194, figs. 7, 8. Type locality: Rio Ivinheima $70 \mathrm{~km}$ upstream from its confluence with Rio Paraná and Rio dos Bandeirantes, Mato Grosso, Brazil, approx. 22³5'S, 53³0'W. Holotype: MZUSP 39075.

Distribution: Amazon and Paraguay/Paraná River basins (Schaefer, 2003a).

\section{SILURIDAE Cuvier, 1816}

Siluroïdes Cuvier, 1816: 199. Type genus: Silurus Linnaeus, 1758.

Kryptopterini Bleeker, 1862 (in Bleeker, 1862-63): 18, 85. Type genus: Kryptopterus Bleeker, 1857.

Phalacronotini Bleeker, 1862 (in Bleeker, 1862-63):18, 90. Type genus: Phalacronotus Bleeker, 1857.

Monophyly: Bornbusch (1991b).

Phylogeny: Bornbusch (1991b; 1995).

Revision: Kobayakawa (1989, Silurus sensu lato, including Pterocryptis).

Review: Chen (1977, China).

Checklist: Haig (1952, with key to genera).

Identification guide: Jayaram (1977d, South Asia).

Remarks: Although the monophyly of the family appears to be well supported (see Bornbusch, 1991b), the limits of many of the included genera are not clear. As such, the composition of the genera as listed herein are likely to change with additional study.

12 genera, 94 species; 3 named fossil species of uncertain status.

\section{Incertae sedis}

Kryptopterus indicus Datta, Barman \& Jayaram, 1987

Kryptopterus indicus Datta, Barman \& Jayaram, 1987: 29, fig. 1. Type locality: Hornbill Point, Namdapha River, Namdapha Wildlife Sanctuary, Arunachal Pradesh, India. Holotype: ZSI FF1699.

Distribution: Known only from type locality (Jayaram, 1999). 
Remarks: Generic placement undetermined. Illustration of type clearly not that of a species of Kryptopterus.

BELODONTICHTHYS Bleeker, 1857

Belodontichthys Bleeker, 1857b: 472. Type species: Belodontichthys macrochir Bleeker, 1857. Type by monotypy. Gender: Masculine.

Phylogeny: Howes \& Fumihito (1991).

Belodontichthys dinema (Bleeker, 1851)

Wallago dinema Bleeker, 1851d: 202. Type locality: Banjermasin. Syntypes (2, 190, 195 mm TL): possibly RMNH 6834 (1 or 2 of 5. Syntype illustrated in Bleeker (1862-63: pl.86 [= Silur. 38], fig. 2) as Belodontichthys macrochir.

Belodontichthys macrochir Bleeker, 1857b: 472. Type locality: Banjermasin. Syntypes (2, 190, 195 mm TL): possibly RMNH 6834 (1 or 2 of 5). Unneeded new name for Wallago dinema Bleeker, 1851.

Distribution: Laos, Vietnam, Thailand, Malay Peninsula, Sumatra and Borneo (Roberts, 1989a).

Remarks: BMNH 1863.12.4.54 (1, $272 \mathrm{~mm} \mathrm{SL)}$ is incorrectly registered as a type of Belodontichthys macrochir [= Wallago dinema], but is too long to be either of the syntypes.

Belodontichthys truncatus Kottelat \& Ng, 1999

Belodontichthys truncatus Kottelat \& Ng, 1999: 388, fig. 1. Type locality: Chao Phraya River, flood-waters 17.5 km north of Ayuthya, Maharaj Prov., Thailand. Holotype: UMMZ 235105.

Distribution: Mekong and Chao Phraya River basins (Kottelat, 2001b).

\section{CERATOGLANIS Myers, 1938}

Ceratoglanis Myers, 1938: 98. Type species: Hemisilurus scleronema Bleeker, 1863. Type by original designation. Gender: Masculine.

Ceratoglanis pachynemus $\mathrm{Ng}, 1999$

Ceratoglanis pachynema Ng, 1999c: 390, figs. 3, 5, 6. Type locality: Prachinburi Market, Thailand. Holotype: CAS 96577.

Distribution: Chao Phraya and Mekong River basins (Ng, 1999c).

Ceratoglanis scleronemus (Bleeker, 1863)

Hemisilurus scleronema Bleeker, 1863 (in Bleeker, 1862-63): 93, pl. 101 [= Silur. pl. 53]. Type locality: Java (Krawang). Holotype (399 mm TL): possibly RMNH 2918 (1). Also described as new in Bleeker (1863e and 1863f).

Distribution: Pahang River basin, peninsular Malaysia, Baram, Barito, Kapuas and Rejang river basins, Borneo, Citarum River basin, Java, and the Batang Hari and Siak River basins, Sumatra (Ng, 1999c).

\section{HEMISILURUS Bleeker, 1857}

Hemisilurus Bleeker, 1857b: 472. Type species: Wallago heterorhynchus Bleeker, 1853. Type by monotypy. Gender: Masculine.

Diastatomycter Vaillant, 1891: 182. Type species: Diastatomycter chaperi Vaillant, 1891. Type by monotypy. Gender: Masculine. Also appeared in Vaillant (1893c: 61).

Phylogeny and biogeography: Bornbusch \& Lundberg (1989).

Hemisilurus heterorhynchus (Bleeker, 1853)

Wallago heterorhynchus Bleeker, 1853b: 514. Type locality: Moara kompeh, Sumatrae orientalis, in fluviis. Holotype (326 mm TL): RMNH 6849.

Diastatomycter Chaperi Vaillant, 1891: 182. Type locality: Knapei River, Borneo. Holotype: MNHN 1891-0458. Also described as new in Vaillant (1893c: 61); redescribed and illustrated in Vaillant (1894b: 70, pl. 2, fig. 2).

Distribution: Sumatra, and Borneo (Roberts, 1989a).

Remarks: BMNH 1863.12.4.58 ( 205 mm SL) is incorrectly listed as a type of Wallago heterorhynchus as the species was named on a single individual and this specimen is too small.

Hemisilurus mekongensis Bornbusch \& Lundberg, 1989

Hemisilurus mekongensis Bornbusch \& Lundberg, 1989: 435, figs. 1-4. Type locality: Thailand, Ubon Ratchathani 
Province, Khong Chiam District, Mun River at Ban Dan, upstream of confluence with Mekong River, $15^{\circ} 18^{\prime} \mathrm{N}$, 105³1'E. Holotype: UMMZ 214565.

Distribution: Mekong River basin (Kottelat, 2001b).

Hemisilurus moolenburghi Weber \& de Beaufort, 1913

Hemisilurus moolenburghi Weber \& de Beaufort, 1913: 212, figs. 84-85. Type locality: Batang Hari, Sumatra. Syntypes: ZMA 113564 (2).

Distribution: Batang Hari, Sumatra, and western Borneo (Roberts, 1989a).

\section{KRYPTOPTERUS Bleeker, 1857}

Kryptopterus Bleeker, 1857b: 472. Type species: Kryptopterus micropus Bleeker, 1857. Type by subsequent designation by Bleeker (1862: 395, or 1862-63: 18). Gender: Masculine.

Kryptopterichthys Bleeker, 1857b: 472. Type species: Silurus palembangensis Bleeker, 1852. Type by subsequent designation by Bleeker (1862: 395, or 1862-63: 18). Gender: Masculine.

Cryptopterus Günther, 1864: 6, 38. Type species: Kryptopterus micropus Bleeker, 1857. Type by being a replacement name. Gender: Masculine. Unjustified emendation of Kryptopterus. Preoccupied by Cryptopterus Kaup, 1860 , in fishes.

Cryptopterella Fowler, 1944a: 1. Type species: Cryptopterella beldti Fowler, 1944. Type by original designation. Gender: Feminine.

Remarks: First reviser action in making Kryptopterus the valid name for this taxon appears to have been accomplished in Haig (1952: 106), in which Kryptopterichthys is treated as a junior synonym of Kryptopterus, although the names were attributed to Bleeker (1858) which was, until recently, thought to be the source of both names.

\section{Kryptopterus baramensis $\mathrm{Ng}, 2002$}

Kryptopterus baramensis Ng, 2002c: 68, figs. 1-2. Type locality: Borneo: Sarawak, Sungai Akah, above Long Tebangan, $300 \mathrm{~m}$ below confluence with Sungai Pahang, below falls, 3²2'12"N, 11456'06"E. Holotype: ROM 72477.

Distribution: Baram River basin, northern Borneo (Ng, 2002c).

Kryptopterus bicirrhis (Valenciennes, 1840)

Silurus bicirrhis Valenciennes, in Cuvier \& Valenciennes, 1840a: 367 (272 of Strasbourg deluxe edition), pl. 411. Type locality: Java. Holotype: MNHN a-9932; radiograph of holotype in Roberts (1989a: fig. 113, lower).

Cryptopterus amboinensis Günther, 1864: 40, 429. Type locality: Amboyna [apparently in error]. Syntypes (2): BMNH 1855.3.24.14 (1).

Distribution: Mekong and Chao Phraya River basins, and Sundaland (Kottelat, 2001b).

Kryptopterus cryptopterus (Bleeker, 1851)

Silurus cryptopterus Bleeker, 1851b: 270. Type locality: Bandjarmassing. Holotype (110 mm TL): RMNH 6840 (1 of 16).

Kryptopterus micropus Bleeker, 1857b: 472. Type locality: Bandjarmassing. Holotype (110 mm TL): RMNH 6840 (1 of 16). Unneeded replacement name for Silurus cryptopterus Bleeker, 1851, apparently to avoid tautonymy.

Distribution: Borneo and Sumatra, and possibly Tapi River (Ng, 2003i: 8).

Kryptopterus dissitus $\mathrm{Ng}, 2001$

Kryptopterus dissitus Ng, 2001a: 198, fig. 1. Type locality: Laos: Champasak Province, Mekong River at Ban Hang Khone, just downstream from Khone falls. Holotype: UMMZ 238017.

Distribution: Chao Phraya and Mekong River basins (Ng, 2001a).

Kryptopterus eugeneiatus (Vaillant, 1893)

Callichrous eugeneiatus Vaillant, 1893c: 61. Type locality: riv. Knapei et Sebroeang, près Smitow, Bornéo. Syntypes: MNHN 1891-0459 (1), MNHN 1891-0460 (1).

Distribution: Kapuas River basin (Roberts, 1989a).

Kryptopterus geminus $\mathrm{Ng}, 2003$

Kryptopterus geminus Ng, 2003i: 2, figs 1, 2a. Type locality: Cambodia: Stung Treng, Mekong River 2 km downstream from mouth of Tonle San on sandbars, Mekong River drainage, $13^{\circ} 31^{\prime} \mathrm{N}, 105^{\circ} 56^{\prime} \mathrm{E}$. Holotype: UMMZ 
234664.

Distribution: Mekong, Mae Khlong, Bang Pakong and Chao Phraya River basins (Ng, 2003i).

Kryptopterus hesperius $\mathrm{Ng}, 2002$

Kryptopterus hesperius Ng, 2002c: 70, fig. 3. Type locality: Thailand: Kanchanaburi Province, Kwae Noi River at Sai Yok. Holotype: ZMUC P28551.

Distribution: Mae Khlong River basin, western Thailand (Ng, 2002c).

Kryptopterus lais (Bleeker, 1851)

Silurus laïs Bleeker, 1851e: 428. Type locality: Sambas, in fluviis. Holotype (125 mm TL): RMNH 6839 (larger of 2).

Distribution: Sambas, Kapuas, Kahajan and Barito rivers, Borneo (Roberts, 1989a).

Kryptopterus limpok (Bleeker, 1852)

Silurus limpok Bleeker, 1852d: 583. Type locality: Palembang, Sumatra. Holotype (175 mm TL): possibly BMNH 1863.12.4.100 (150 mm SL).

Distribution: Mekong and Chao Phraya River basins, Malay Peninsula, Sumatra and Borneo (Kottelat, 2001b).

Kryptopterus lumholtzi Rendahl, 1922

Cryptopterus lumholtzi Rendahl, 1922: 200. Type locality: Bulungan, northeastern Borneo. Holotype: ZMUO J5310.

Distribution: Bulungan, northeastern Borneo (Rendahl, 1922).

Kryptopterus macrocephalus (Bleeker, 1858)

Kryptopterichthys macrocephalus Bleeker, 1858b: 293. Type locality: Sumatra ?; Padang ?, in fluviis. Holotype (113 $\mathrm{mm}$ TL): possibly BMNH 1863.12.4.99 (96 mm SL) or BMNH 1863.12.4.101. Illustrated in Bleeker (1862-63: pl. 89 [= Silur. pl. 41], fig. 1).

Distribution: Peninsular Thailand, Malay Peninsula, Sumatra and Borneo (Roberts, 1989a).

Kryptopterus minor Roberts, 1989

Kryptopterus minor Roberts, 1989a: 149, fig. 115. Type locality: Mainstream of Sungai Pinoh at Nanga Saian, 45 $\mathrm{km} \mathrm{S}$ of Nangapinoh, Western Borneo (Kalimantan Barat, Indonesia). Holotype: MZB 3638. Name spelled Kryptopterus minimus on p. 146. As there has not been a first reviser action to fix the name, it is done here, with Kryptopterus minor as the valid name.

Distribution: Kapuas River, western Borneo (Roberts, 1989a).

Kryptopterus mononema (Bleeker, 1847)

Silurus mononema Bleeker, 1847b: 8. Type locality: Surakarta, Java. Syntypes: possibly BMNH 1863.12.4.93 (1, 145 mm SL), RMNH 6835 (2). One syntype illustrated in Bleeker (1862-63: 87, pl. 91 [= Silur pl. 43], fig. 1). Also described as new in Bleeker (1847: 166).

Distribution: Java, Indonesia.

Kryptopterus palembangensis (Bleeker, 1852)

Silurus palembangensis Bleeker, 1852d: 584. Type locality: Palembang, in fluviis. Holotype (169 mm TL): Whereabouts unknown.

Distribution: Sumatra.

Remarks: BMNH 1863.12.4.101 (120 mm SL) is incorrectly registered as a type of Silurus palembangensis but appears to be too small to be the holotype.

Kryptopterus paraschilbeides $\mathrm{Ng}, 2003$

Kryptopterus paraschilbeides Ng, 2003d: 3, fig. 1. Type locality: Cambodia: Kompong Chhnang, Tonle Sap River, $17 \mathrm{~km}$ upstream from Kompong Chhnang. Holotype: UMMZ 238788.

Distribution: Mekong River basin (Ng, 2003d).

Kryptopterus piperatus $\mathrm{Ng}$, Wirjoatmodjo \& Hadiaty, 2004

Kryptopterus piperatus Ng, Wirjoatmodjo \& Hadiaty, 2004: 92, fig. 1. Type locality: Indonesia, Sumatra: Aceh Selatan: Sungai Lembang in front of camp, Suag Balimbing Research Station, Gunung Leuser National Park. Holotype: MZB 8717.

Distribution: Lembang River, Alas River basin, Sumatra, Indonesia (Ng et al., 2004).

Kryptopterus sabanus (Inger \& Chin, 1959) 
Ompok sabanus Inger \& Chin, 1959: 282. Type locality: Segama River at the Segama Estate near Lahad Datu, Lahad Datu District, East Coast Residency, North Borneo. Holotype: FMNH 44828.

Distribution: Kapuas and Kinabatangan Rivers, northern Borneo (Roberts, 1989a).

Kryptopterus schilbeides (Bleeker, 1858)

Hemisilurus schilbeides Bleeker, 1858b: 297. Type locality: Palembang, Sumatra; Bandjermassin, Borneo. Syntypes (2: 96-99 mm TL): possibly BMNH 1863.12.4.157 (1, 79 mm SL, 91 mm TL), BMNH 1864.5.15.6 (1). Illustrated in Bleeker (1862-63: pl. 90 [=Silur. pl. 42], fig. 4).

Distribution: Lower Mekong River to Indonesia, in rivers, canals, ditches and swamps (Rainboth, 1996).

Remarks: Register number of syntype of Hemisilurus schilbeides incorrectly reported as BMNH 1863.12.11.157 in Eschmeyer et al. (1998).

\section{Species inquirendae, Kryptopterus}

Cryptopterella beldti Fowler, 1944a: 2, unnumbered figure. Type locality: Borneo (aquarium fish). Holotype: ANSP 71571.

MICRONEMA Bleeker, 1857

Micronema Bleeker, 1857b: 472. Type species: Silurus hexapterus Bleeker, 1851. Type by monotypy. Gender: Neuter.

Micronema cheveyi (Durand, 1940)

Cryptopterus Cheveyi Durand, 1940: 19, pl. 4. Type locality: Rivière de Kaskos [ Cambodia]. Holotype: possibly at Institut Océanographique Nhatrang.

Distribution: Mekong River basin (Chu et al., 1999), in rivers and canals (Rainboth, 1996).

Micronema hexapterus (Bleeker, 1851)

Silurus hexapterus Bleeker, 1851d: 203. Type locality: Bandjermassing. Holotype (145 mm TL): Whereabouts unknown.

Distribution: Thailand to Indonesia, in rivers, streams, and canals (Rainboth, 1996).

Remarks: BMNH 1863.12.4.73 is incorrectly registered as the holotype of Silurus hexapterus Bleeker, but it is much larger (159 mm SL) than the listed length of the holotype.

Micronema moorei (Smith, 1945)

Kryptopterus moorei Smith, 1945: 342, fig. 78. Type locality: Menam Chao Phya, near Paknampo, central Thailand. Holotype: USNM 109787.

Distribution: Chao Phraya and lower Mekong River basins, in streams and canals of the floodplain (Rainboth, 1996). Remarks: Treated as a probable synonym of Kryptopterus cheveyi in Kottelat (2001b).

Micronema platypogon $(\mathrm{Ng}, 2004)$

Kryptopterus platypogon Ng, 2004f: 2, figs. 1, 2a. Type locality: Borneo: Sarawak, Rajang River drainage, market at Sibu, 2¹7'18.6"N 11149'49.2"E. Holotype: ZRC 45838.

Distribution: Rajang River basin, northern Borneo (Ng, 2004f).

OMPOK La Cepède, 1803

Ompok La Cepède, 1803: 49. Type species: Ompok siluroides La Cepède, 1803. Type by monotypy. Gender: Masculine.

Callichrous Hamilton, 1822: 149. Type species: Silurus pabda Hamilton, 1822. Type by subsequent designation by Bleeker (1862: 395 or 1862-63: 17). Gender: Masculine. Originally proposed as a subgenus of Silurus.

Pseudosilurus Bleeker, 1857b: 472. Type species: Wallago leiacanthus Bleeker, 1853. Type by monotypy. Gender: Masculine.

Silurodes Bleeker, 1857b: 472. Type species: Silurus macronema Bleeker, 1851. Type by monotypy. Gender: Masculine. Also in Bleeker (1858b: 255, 256, 271).

Revision: Ng (2003b, Ompok hypophthalmus group); Tan \& Ng (1996, Ompok leiacanthus species group).

Review: Parameswaran et al. (1967, Indian species). 
Ompok bimaculatus (Bloch, 1794)

Silurus bimaculatus Bloch, 1794: 24, pl. 364. Type locality: Tranquebar. Lectotype: ZMB 2916, designated by, and illustrated in, Paepke (1999: 139, pl. 25, fig. 2).

Callichrous ceylonensis Günther, 1864: 46. Type locality: Ceylon. Syntypes: BMNH 1852.2.19.107-108 (2), BMNH 1853.3.30.60 (1).

Distribution: Widely distributed in south and Southeast Asia, including Mekong River (Kottelat, 2001b), but probably more restricted to just south Asia and Sri Lanka.

Remarks: See Jayaram (1977a) for comments on type locality of Silurus bimaculatus, and Rema Devi \& Emilyamma (1997) for comments on its identity.

Ompok binotatus $\mathrm{Ng}, 2002$

Ompok binotatus Ng, 2002b: 26, fig. 1. Type locality: Indonesia: Borneo, Kalimantan Barat, Sungai Mandai Kechil near its confluence with Kapuas mainstream, $18 \mathrm{~km}$ west southwest of Putussibau, $0^{\circ} 48^{\prime} \mathrm{N}, 112^{\circ} 47^{\prime} \mathrm{E}$. Holotype: FMNH 94243.

Distribution: Kapuas River basin, Borneo (Ng, 2002b).

Ompok borneensis (Steindachner, 1901)

Callichrous (Silurodes) borneensis Steindachner, 1901: 445, pl. 18 (fig. 3). Type locality: Baram-Flusse, Borneo. Holotype $(8.4 \mathrm{~cm})$ : at NMW or SMF.

Distribution: Baram River basin, Borneo (Steindachner, 1901).

Ompok fumidus Tan \& Ng 1996

Ompok fumidus Tan \& Ng 1996: 537, figs. 3, 4c. Type locality: North Selengor peat swamp forest, $43 \mathrm{~km}$ on road from Tanjong Malim to Sungai Besar, Selangor, Malaysia. Holotype: ZRC 15049.

Distribution: peninsular Malaysia (Tan \& Ng, 1996).

Ompok hypophthalmus (Bleeker, 1846)

Silurus hypophthalmus Bleeker, 1846a: 149. Type locality: Batavia. Type(s) (size and number not stated): Whereabouts unknown.

Silurus macronema Bleeker, 1851: 203. Type locality: Bandjermassing. Holotype (140 mm TL): possibly BMNH 1863.12.4.155 (121 mm SL); illustrated in Bleeker (1862-63: 83, pl. 88 [= Silur. pl. 40], fig. 2), as Silurodes macronema.

Distribution: Ciliwung and Brantas River basins, Java, and Barito River basin, southern Borneo (Ng, 2003b).

Ompok jaynei Fowler, 1905

Ompok jaynei Fowler, 1905: 466, fig. 3. Type locality: Baram basin, Baram region of Sarawak, Borneo. Holotype: ANSP 114890.

Distribution: Baram River basin, Borneo (Fowler, 1905).

Ompok leiacanthus (Bleeker, 1853)

Wallago leiacanthus Bleeker, 1853a: 189. Type locality: Marwang, in fluviis [now: East end of Danau Arang Arang, Jambi Prov., Sumatra, Indonesia, by neotype designation]. Neotype: ZRC 38538, designated by Tan \& Ng (1996: 532).

Distribution: Sumatra.

Ompok malabaricus (Valenciennes, 1840)

Silurus Malabaricus Valenciennes, in Cuvier \& Valenciennes, 1840a: 353 (262 of Strasbourg deluxe edition). Type locality: Malabar. Syntypes: MNHN b-0607 (2).

Distribution: Goa, Kerala, India (Jayaram, 1999).

Ompok miostomus Vaillant, 1902

Wallago miostoma Vaillant, 1902: 44. Type locality: Tepoe, bords du Mahakam, Bornéo central. Syntypes: RMNH 7811 (2); syntype illustrated in Roberts (1982b: fig. 3b).

Distribution: Mahamam River basin, central Borneo (Vaillant, 1902).

Ompok pabda (Hamilton, 1822)

Silurus pabda Hamilton, 1822: 150, 374, pl. 25 (fig. 47). Type locality: ponds and rivers of Bengal. No types known.

Silurus (Callichrus) vittatus Swainson 1839: 306. Type locality: ponds and rivers of Bengal. No types known. Name made available by reference to "Ham. pl. 25, f. 47 " [= Hamilton (1822: p. 25, fig. 47)]. Unneeded new name for 
Silurus pabda Hamilton, 1822.

Distribution: Brahmaputra and Ganges River basins, India and Bangladesh; Pakistan; Myanmar (Jayaram, 1999).

Ompok pabo (Hamilton, 1822)

Silurus pabo Hamilton, 1822: 153, 375, pl. 22 (fig. 48). Type locality: Brahmaputra River, towards Assam. No types known.

Silurus (Callichrus) erythrogaster Swainson, 1839: 306. Type locality: Brahmaputra River, towards Assam. No types known. Name made available by reference to "Ham. pl. 17 f. 48 " [sic pl. 22, fig. 48] (= Hamilton, 1822: pl. 22, fig. 48). Unneeded new name for Silurus pabo Hamilton, 1822.

Distribution: Brahmaputra, Ganges, and Yamuna River basins, India and Bangladesh; Pakistan; Myanmar (Jayaram, 1999).

Ompok pinnatus $\mathrm{Ng}, 2003$

Ompok pinnatus Ng, 2003f: 48, fig. 1. Type locality: Cambodia, Tonle Sap at Koompong Chhnang, fishing lot 9 in second channel E of town. Holotype: UMMZ 232679.

Distribution: Chao Phraya River, Thailand and Mekong River basin, Cambodia (Ng, 2003f).

Ompok platyrhynchus Ng \& Tan, 2004

Ompok platyrhynchus Ng \& Tan, 2004: 2, figs. 1, 2a. Type locality: Borneo: Brunei Darussalam, Temburong district: Belalong sub-basin Sungai Esu, about 15 minutes upstream of Kuala Belalong Field Studies Centre (04³2'17.9"N 11509'35.2"E). Holotype: ZRC 48678.

Distribution: Temburong River basin, northern Borneo (Ng \& Tan, 2004).

Ompok pluriradiatus $\mathrm{Ng}, 2002$

Ompok pluriradiatus Ng, 2002b: 28, fig. 3. Type locality: Indonesia: Borneo: Kalimantan Timur: Mahakam River drainage, a swift blackwater stream entering Mahakam River from the left side downriver of Nuarapahu $\left(0^{\circ} 14^{\prime} \mathrm{S}, 116^{\circ} 07^{\prime} \mathrm{E}\right)$. Holotype: MZB 5951.

Distribution: Mahakam River basin, eastern Borneo (Ng, 2002b).

Ompok rhadinurus $\mathrm{Ng}, 2003$

Ompok rhadinurus Ng, 2003b: 1299, figs. 3, 5b. Type locality: Peninsular Malaysia, Selangor, North Selangor Peat Swamp Forest, irrigation canal on western boundary. Holotype: ZRC 14897.

Distribution: Bernam, Endau, Pahang, Pattani and Perak River basins, peninsular Malaysia, Kapuas River basin, Borneo, and Batang Hari, Deli, Indragiri and Musi River basins, Sumatra (Ng, 2003b).

Ompok sindensis (Day, 1877)

Callichrous Sindensis Day, 1877 (in Day, 1875-78): 476, pl. 110 (fig. 1). Type locality: Sind, from the Indus [Pakistan]. Holotype: ZSI 505.

Distribution: Sindh, Pakistan (Mirza, 2003).

Ompok urbaini (Fang \& Chaux, 1949)

Cryptopterus urbaini Fang \& Chaux, in Chaux \& Fang, 1949a: 197, fig. 2. Type locality: Cambodge. Holotype: MNHN 1966-0706.

Distribution: Mekong, Chao Phraya and Pasak River basins, Southeast Asia (Ng, 2003b).

Remarks: Ng (2003b) lists three syntypes, but a holotype was clearly indicated in the description.

Ompok weberi (Hardenberg, 1936)

Callichrous weberi Hardenberg, 1936a: 232. Type locality: Padang Tikarbay, Kapuas River basin, western Borneo.

Holotype (79 mm): Whereabouts unknown (see Ng \& Siebert, 2002, for comments).

Distribution: Kapuas River, Borneo (Ng \& Siebert, 2002).

Remarks: Redescribed in Ng \& Siebert (2002).

\section{Species inquirendae, Ompok}

Ompok silurö̈des La Cepède, 1803: 49, 50, pl. 1 (fig. 2). Type locality: not stated [Asia]. Holotype: MNHN a-8669. Silurus lamghur Heckel, 1838: 82, pl. 12 (figs. 5-6). Type locality: Kashmir. Holotype: NMW 44689.

Silurus canio Hamilton, 1822: 151, 374. Type locality: Ponds in the north-east parts of Bengal. No types known. Unpublished Hamilton illustration reproduced in Hora (1929: pl. 20, fig. 5). 
Silurus chechra Hamilton, 1822: 152, 375. Type locality: Kusi river [India]. No types known.

Silurus duda Hamilton, 1822: 152, 375. Type locality: river Kusi [India]. No types known.

Silurus (Callichrus) affinis Swainson, 1839: 306. Type locality: Kusi River [India]. No types known. Name made available by reference to "Ham. 152 (duda)" [= Hamilton, 1822: 152]. Unneeded new name for Silurus duda Hamilton, 1822.

Silurus (Callichrus) immaculatus Swainson, 1839: 306. Type locality: Bengal. No types known. Name made available by reference to "Ham. 151 (canio)" [= Hamilton, 1822: 151]. Unneeded new name for Silurus canio Hamilton, 1822.

Silurus (Callichrus) nebulosus Swainson, 1839: 306. Type locality: Kusi River [India]. No types known. Name made available by reference to "Ham. 152 (nebulosus)" [=Hamilton, 1822: 152)]. Unneeded new name for Silurus chechra Hamilton, 1822.

Silurus anostomus Valenciennes, in Cuvier \& Valenciennes, 1840a: 363 (269 of Strasbourg deluxe edition), pl. 410. Type locality: Bengale. Syntypes: MNHN a-8949 (2), MNHN a-8950 (2).

Silurus microcephalus Valenciennes, in Cuvier \& Valenciennes, 1840a: 365 (271 of Strasbourg deluxe edition). Type locality: Bengale. Holotype (6 pouces): at MNHN.

Silurus Mysoricus Valenciennes, in Cuvier \& Valenciennes, 1840a: 364 (270 of Strasbourg deluxe edition). Type locality: Mysore. Syntypes: MNHN 0000-3107 (2).

Silurus indicus M'Clelland \& Griffith, in M'Clelland, 1842: 583. Type locality: Loodianah, the Punjab, and Cabool River at Jullalabad. Holotype: Whereabouts unknown, possibly at BMNH.

Cryptopterus latovittatus Playfair, 1867: 16. Type locality: Cachar, eastern provinces of British India, within the watershed of the Burhampooter. Syntypes: BMNH 1867.2.14.72-73.

Pseudosilurus macropthalmos Blyth, 1860: 156. Type locality: Tenasserim. Holotype: Whereabouts unknown.

Callichrous nigrescens Day, 1870c: 616. Type locality: Throughout the branches of the Irrawaddi, in the Pegu and Sittoung rivers, Burma. Syntypes: AMS B.7636 (1), ZSI A.500 (2, lost).

Callichrous notatus Day, 1870c: 616. Type locality: Rivers of Burma. Possible syntypes: AMS B.7982 (2), ZSI 1275 (1), ZSI A.499 (2, lost).

Callichrous egertonii Day, 1872: 710. Type locality: Subhimalayan range in the Punjaub. Syntype: AMS B.8065 (1).

Wallago krattensis Fowler, 1934b: 335, fig. 1. Type locality: Kratt, southeast Siam. Holotype: ANSP 60177.

Silurus goae Haig, 1952: 77, fig. on p. 78. Type locality: Goa, India. Holotype: SU 41889. Referred to Ompok rather than Silurus by Kobayakawa (1989: 176).

\section{PHALACRONOTUS Bleeker, 1857}

Phalacronotus Bleeker, 1857b: 472. Type species: Silurus phalacronotus Bleeker, 1851. Type by absolute tautonymy. Gender: Masculine. Type designations of Silurus leptonema in Bleeker (1862: 395 and 1862-63: 18) are invalid.

Phalacronotus apogon (Bleeker, 1851)

Silurus apogon Bleeker, 1851c: 67. Type locality: Banjermasin. Syntypes (4, 118-175 mm TL): RMNH 6843 (in part).

Silurus leptonema Bleeker, 1852: 584. Type locality: Palembang, Sumatra. Holotype (268 mm TL): possibly at RMNH.

Silurus micropogon Bleeker, 1855: 418, 419. Type locality: Pontianak; Bandjermasin, Borneo. Apparently an unneeded new name for Silurus apogon Bleeker, 1851.

Distribution: Mekong and Chao Phraya river basins, Malay Peninsula, Sumatra and Borneo (Kottelat, 2001b).

Phalacronotus bleekeri (Günther, 1864)

Cryptopterus bleekeri Günther, 1864: 44. Type locality: Siam. Syntypes (2): BMNH 1863.12.4.105, and possibly BMNH 1862.11.1.208.

Micronema bleekeri Bocourt, 1866: 17, pl. 1 (figs. 3, 3a-c). Type locality: [Menam R. at Bangkok]. Syntypes: MNHN 0000-1546 (3).

Distribution: Mekong and Chao Phraya River basins, and Sundaland (Kottelat, 2001b).

Remarks: Günther (1864) cited Micronema bleekeri Bocourt in his account of Cryptopterus bleekeri. However, he did not cite a Bocourt publication in his list of cited literature (pp. vii-x) and available evidence indicates that 
Bocourt's publication did not appear until 1866. Therefore, Günther's name has priority and Bocourt's must be treated as independently proposed and available.

Phalacronotus micronemus (Bleeker, 1846)

Silurus micronemus Bleeker, 1846b: 289. Type locality: Batavia. Holotype: RMNH 6841.

Silurus phalacronotus Bleeker, 1851e: 429. Type locality: Sambas. Holotype (220 mm TL): Whereabouts unknown.

Phalacronotus micruropterus Bleeker, 1857b: 473. Type locality: Sambas, in fluviis. Holotype (220 mm TL): Whereabouts unknown. Unneeded replacement name for Silurus phalacronotus Bleeker, 1851, to avoid Stricklandian tautonymy.

Micronema typus Bleeker, 1858b: 300. Type locality: Batavia. Holotype: RMNH 6841. Unneeded new name for Silurus micronemus Bleeker, 1846.

Kryptopterus deignani Fowler, 1937: 136, figs. 10-12. Type locality: Me Poon, Siam. Holotype: ANSP 67884.

Distribution: Mekong and Chao Phraya River basins, and Sundaland (Kottelat, 2001b).

Remarks: BMNH 1863.12.4.75 (209 mm SL), BMNH 1863.12.4.95 (222 mm SL, 249 mm TL), and BMNH 1863.12.4.96 (122 mm SL) are incorrectly registered as the types of Silurus micronemus.

Phalacronotus parvanalis (Inger \& Chin, 1959)

Kryptopterus parvanalis Inger \& Chin, 1959: 284, fig. 46. Type locality: Kinabatangan River at Deramakot, Kinabatangan District, North Borneo. Holotype: FMNH 68014.

Distribution: Kinabatangan River at Deramakot, North Borneo, Malaysia (Inger \& Chin, 1959).

PINNIWALLAGO Gupta, Jayaram \& Hajela, 1981

Pinniwallago Gupta, Jayaram \& Hajela, 1981: 291. Type species: Pinniwallago kanpurensis Gupta, Jayaram \& Hajela, 1981. Type by original designation. Gender: Masculine.

Pinniwallago kanpurensis Gupta, Jayaram \& Hajela, 1981

Pinniwallago kanpurensis Gupta, Jayaram \& Hajela, 1981: 290, fig. 1. Type locality: 'Bara Tal' near village of Bhitargaon, Tehsil, Ghatampur, Kanpur, Uttar Pradesh, India. Holotype: ZSI FF1443.

Distribution: Known only from the type locality in Uttar Pradesh, India (Jayaram, 1999).

\section{PTEROCRYPTIS Peters, 1861}

Pterocryptis Peters, 1861: 712. Type species: Pterocryptis gangelica Peters, 1861. Type by monotypy. Gender: Feminine.

Apodoglanis Fowler, 1905: 463. Type species: Apodoglanis furnessi Fowler, 1905. Type by original designation. Gender: Masculine.

Hito Herre, 1924a: 702. Type species: Hito taytayensis Herre, 1924. Type by original designation. Gender: Masculine.

Hitoichthys Herre 1924b: 1570. Type species: Hitoichthys taytayensis Herre, 1924. Type by original designation. Gender: Masculine.

Penesilurus Herre, 1924a: 703. Type species: Penesilurus palavanensis Herre, 1924. Type by original designation. Gender: Masculine. Also described as new in Herre (1924b: 1570); earliest publication not established.

Herklotsella Herre, 1933: 179. Type species: Herklotsella anomala, Herre, 1933. Type by original designation. Gender: Feminine. Publication date stated to be Dec. 1933, but perhaps did not appear until later (Eschmeyer, 1998); priority over Herklotsella Fowler, 1934, which was published in January, 1934, is not clearly established.

Review: Ng \& Freyhof (2001a), Vietnam.

Remarks: The revision by Kobayakawa (1989) included many of these species in an expanded Silurus sensu lato. Haig (1952: 82) gave precedence to Hito over Hitoichthys, after concluding that priority of publication could not be determined.

Pterocryptis anomala (Herre, 1933)

Herklotsella anomala Herre, 1933: 179. Type locality: Hong Kong market. Holotype: SU 26769.

Silurus sinensis Hora, 1937d: 343, figs. 8 (c-d). Type locality: Lunchow, China. Holotype: Originally ZMFMIB 13692, apparently now at ASIZB. Name proposed for specimen identified as Silurus wynaadensis in Tchang (1936: 35). Preoccupied by Silurus sinensis La Cepède, 1803; replaced by Silurus gilberti Hora, 1938. 
Silurus gilberti Hora, 1938c: 243. Type locality: Lunchow, China. Holotype: Originally ZMFMIB 13692, apparently now at ASIZB. Replacement name for Silurus sinensis Hora, 1937.

Distribution: Rivers draining southeastern China, from Minjiang and Pearl Rivers to vicinity of Hong Kong (Ng \& Chan, 2005).

Remarks: Synonymy follows Ng \& Chan (2005).

Pterocryptis berdmorei (Blyth, 1860)

Silurichthys Berdmorei Blyth, 1860: 156. Type locality: Tenasserim provinces. Holotype: ZSI 481 (whereabouts unknown).

Silurus morehensis Arunkumar \& Tombi Singh, 1997: 73, fig. 1. Type locality: Moreh Bazar, Moreh, Manipur, India. Holotype: MUMF 2211/1A.

Distribution: Southeastern Myanmar and possibly western Thailand (Ng \& Freyhof, 2001a), Manipur, India (Arunkumar \& Tombi Singh, 1997).

Pterocryptis bokorensis (Pellegrin \& Chevey, 1937)

Penesilurus bokorensis Pellegrin \& Chevey, 1937: 315. Type locality: [Bokor, Cambodia, elev. 800-1000 m]. Holotype: MNHN 1936-0167.

Distribution: Cambodia.

Remarks: Ng \& Kottelat (1998b) indicate that the species is known only from holotype, but Rainboth (1996) implies that it is a food fish, known from upland streams of the Mekong basin in Cambodia.

Pterocryptis buccata $\mathrm{Ng} \&$ Kottelat, 1998

Pterocryptis buccata Ng \& Kottelat, 1998b: 394, figs. 1, 2a, 3a-b. Type locality: Thailand: Kanchanaburi Province, Amphoe Sai Yok, Mae Khlong basin. Holotype: ZRC 41496.

Distribution: Meklong River basin, Kanchanaburi Province, Thailand; in caves and epigean habitats (Ng \& Kottelat, 1998b).

Pterocryptis burmanensis (Khin-Thant, 1966)

Silurus burmanensis Khin-Thant, 1966: 219, pls. 1-3. Type locality: Inlé Lake, s. Shan State, 2335'N, 96º57'E, Burma, elev. 2915 ft. Holotype: Zoological Museum, Art and Sciences University of Rangoon 332.

Distribution: Inle Lake, Myanmar.

Pterocryptis cochinchinensis (Valenciennes, 1840)

Silurus Cochinchinensis Valenciennes, in Cuvier \& Valenciennes, 1840a: 352 (262 of Strasbourg deluxe edition). Type locality: [Cochinchine]. Syntypes: MNHN 0000-0573 (1), MNHN b-0602 (1).

Distribution: Coastal Rivers of central Vietnam, between Vinh and An Lao (Ng \& Freyhof, 2001a); Xijiang [= West River], Jiulongjiang, Hainan Island (Chu et al., 1999); Nam Xam basin, Laos (Kottelat, 2001b).

Pterocryptis crenula Ng \& Freyhof, 2001

Pterocryptis crenula Ng \& Freyhof, 2001a: 630, fig. 6. Type locality: Vietnam: Quang Ninh province, Hai Ninh district, torrent at km 5 on road from Bac Phong Sinh to Mong Cai, 21³5'31"N, 107²43'52"E. Holotype: ZRC 46317.

Distribution: coastal rivers of northeastern Vietnam (Ng \& Freyhof, 2001a).

Pterocryptis cucphuongensis (Mai, 1978)

Silurus cucphuongensis Mai, 1978: 245, fig. 112. Type locality: Cuc Phurong, Vietnam. Holotype: DVZUT 345.

Distribution: Song Iuong drainage, northern Vietnam (Ng \& Freyhof, 2001a).

Pterocryptis furnessi (Fowler, 1905)

Apodoglanis furnessi Fowler, 1905: 463, fig. 2. Type locality: Baram River, Borneo. Holotype: ANSP 114894; holotype illustrated in Bornbusch (1991a: 1071, fig. 1).

Distribution: Niah and Baram River basins, Sarawak (Bornbusch, 1991a).

Remarks: Redescribed in Bornbusch (1991a).

Pterocryptis gangelica Peters, 1861

Pterocryptis gangelica Peters, 1861: 712. Type locality: Ganges River. Holotype: ZMB 4796; holotype illustrated in Bornbusch (1991a: 1077, fig. 4).

Silurus afghana Günther, 1864: 34. Type locality: Afghanistan [in error: Assam, India]. Holotype: BMNH 1860.3.19.755. 
Silurus dukai Day, 1873: 239. Type locality: Darjeeling, India. Syntypes: AMS B.7571 (1), ZSI 1118 (1, lost), ZSI $1232(1$, lost).

Distribution: Northern India (Ng \& Chan, 2005).

Remarks: Coad (1981b: 16) discussed the mistake in the reported type locality of Silurus afghana. Synonymy of $P$. gangelica and S. afghana follows $\mathrm{Ng} \&$ Chan (2005).

Pterocryptis inusitata $\mathrm{Ng}, 1999$

Pterocryptis inusitata Ng, 1999a: 372, figs. 1-2. Type locality: Laos: Mekong Basin, Nam Theun watershed, Nam Ong at Ban Don. Holotype: ZRC 41455.

Distribution: Nam Kading basin, Mekong River system, Laos (Ng, 1999a).

Pterocryptis taytayensis (Herre, 1924)

Hito taytayensis Herre, 1924a: 703. Type locality: Small fresh-water creek, near Taytay, Palawan, Philippines. Holotype: BSMP 9357 (presumed destroyed).

Hitoichthys taytayensis Herre 1924b: 1570. Type locality: Small fresh-water creek, near Taytay, Palawan, Philippines. Holotype: BSMP 9357 (presumed destroyed).

Penesilurus palavanensis Herre, 1924a: 704. Type locality: Lake Manguao, Palawan, Philippines. Holotype: BSMP (considered destroyed). Also described as new in Herre (1924b: 1570), earliest not established.

Distribution: Palawan, Philippines.

Pterocryptis torrentis (Kobayakawa, 1989)

Silurus torrentis Kobayakawa, 1989: 171, fig. 33. Type locality: Lampae stream, Khaoluk village, Trang, Thailand. Holotype: NSMT-P 50234.

Distribution: Trang and Chataburi, Thailand (Kobayakawa, 1989); Myanmar localities reported by Kobayakawa may refer to specimens of Pterocryptis berdmorei (Ng \& Freyhof, 2001a).

Pterocryptis verecunda $\mathrm{Ng} \&$ Freyhof, 2001

Pterocryptis verecunda Ng \& Freyhof, 2001a: 636, fig. 9. Type locality: Vietnam: Hai Phong province, Cat Ba Island, Stream near entrance of Trung Trang cave, (2047'17"N, 10700'04"E). Holotype: ZRC 46316.

Distribution: Cat Ba Island, northeastern Vietnam (Ng \& Freyhof, 2001a).

Pterocryptis wynaadensis (Day, 1873)

Silurus punctatus Day, 1868: 155. Type locality: Stream in Wynaad, India, elev. $3000 \mathrm{ft}$. Possible syntypes: AMS B.7990 (1), BMNH 1868.5.14.6-7 (2), BMNH 1889.2.1.2521-2522 (2), NMW 77251 (1), ZMB 11221 (1), ZSI 461 (1), ZSI 1233 (1), ZSI A.480 (1). Preoccupied by Silurus punctatus Rafinesque, 1818, and Silurus punctatus Cantor, 1842.

Silurus wynaadensis Day, 1873c: 237. Type locality: in the Wynaad, in a stream about $3000 \mathrm{ft}$. above the level of the sea [India]. Possible syntypes: AMS B.7990 (1), BMNH 1868.5.14.6-7 (2), BMNH 1889.2.1.2521-2522 (2), NMW 77251 (1), ZMB 11221 (1), ZSI 461 (1), ZSI 1233 (1), ZSI A.480 (1). Replacement for Silurus punctatus Day, 1868.

Distribution: Kerala, India (Ng \& Kottelat, 1998b; Jayaram, 1999).

SILURICHTHYS Bleeker, 1856

Silurichthys Bleeker, 1856: 417, 418. Type species: Silurus phaiosoma Bleeker, 1851. Type by monotypy. Gender: Masculine.

Revision: $\mathrm{Ng} \& \mathrm{Ng}$ (1998).

Silurichthys citatus Ng \& Kottelat, 1997

Silurichthys citatus Ng \& Kottelat, 1997: 204, fig. 1. Type locality: Kalimantan Barat, Sungai Sekumpai, a small forest stream flowing into Sungai Pinoh, 0³2'S, 111³9.5'E, Borneo. Holotype: MZB 3670.

Distribution: Kapuas River basin, western Borneo (Ng \& Ng, 1998).

Silurichthys gibbiceps $\mathrm{Ng} \& \mathrm{Ng}, 1998$

Silurichthys gibbiceps $\mathrm{Ng} \& \mathrm{Ng}$, 1998: 301, figs. 1b, 1j, 2b, 3b, 6. Type locality: Kalimantan Tengah, Sungai Barito basin, Sungai Paku-merah (0³5.171'S, 115¹1.398'E) [Indonesia]. Holotype: MZB 6101.

Distribution: Barito River basin, southern Borneo ( $\mathrm{Ng} \& \mathrm{Ng}, 1998)$.

Silurichthys hasseltii Bleeker, 1858 
Silurichthys Hasseltii Bleeker, 1858: 270. Type locality: Tjisekat, in fluviis. Neotype: RMNH 2992. Illustrated in Bleeker (1862-63: pl. 87 [= Silur. pl. 39], fig. 1).

Distribution: Western Java, Bangka, Bintan, Batam, Singapore, and southern peninsular Malaysia (Ng \& Ng, 1998).

Remarks: Species named on illustration that may have been based on RMNH 2992 or on another specimen that was not preserved. In the absence of conclusive evidence that the RMNH specimen was the basis of the illustration, $\mathrm{Ng} \& \mathrm{Ng}$ (1998: 305) selected RMNH 2992 as the neotype of the species.

Silurichthys indragiriensis Volz, 1904

Silurichthys indragiriensis Volz, 1904: 464. Type locality: Kwantang River, near Djapura (Indragari), Sumatra. Holotype: NMW 44622.

Distribution: Central Sumatra, Bintan, Bangka, Billiton and peninsular Malaysia ( $\mathrm{Ng} \& \mathrm{Ng}, 1998)$.

Silurichthys marmoratus $\mathrm{Ng} \& \mathrm{Ng}, 1998$

Silurichthys marmoratus $\mathrm{Ng} \& \mathrm{Ng}$, 1998: 310, figs. 1e, 1f, 2e, 3e, 11. Type locality: Sarawak: Sungai Sebiris, 13.8 $\mathrm{km}$ after Kampung Puteh turnoff, towards Lundu on Sematan-Lundu road (1 $\left.{ }^{\circ} 41^{\prime} 32^{\prime \prime N}, 109^{\circ} 47^{\prime} 0.8^{\prime \prime E}\right)$. Holotype: ZRC 40293.

Distribution: Northern and western Borneo (Sarawak, Brunei, and Kalimantan Barat) (Ng \& Ng, 1998).

Silurichthys phaiosoma (Bleeker, 1851)

Silurus phaiosoma Bleeker, 1851: 428. Type locality: Sambas, in fluviis. Holotype (82 mm TL): RMNH 6831.

Distribution: Northwestern Borneo (Ng \& Ng, 1998).

Remarks: BMNH 1863.12.4.109 (102 mm SL) and BMNH 1864.5.15.5 (79 mm SL, $100 \mathrm{~mm}$ TL) are registered as types, but are too large to be the holotype.

Silurichthys sanguineus Roberts, 1989

Silurichthys sanguineus Roberts, 1989a: 151, fig. 119. Type locality: Sungai Tekam, a small forest stream, where it enters Kapuas mainstream about 5-6 km upstream from Sanggau, Western Borneo (Kalimantan Barat, Indonesia). Holotype: MZB 3673; holotype illustrated in Ng \& Ng (1998: fig. 13).

Distribution: Kapuas River basin, western Borneo; known only from holotype (Ng \& Ng, 1998).

Silurichthys schneideri Volz, 1904

Silurichthys schneideri Volz, 1904: 463. Type locality: Upper Langkat, Danau near Sukaranda [Sumatra]. Holotype: NMW 44623.

Silurichthys leucopodus Fowler, 1939: 56, figs. 4-6. Type locality: Waterfall at Trang, Siam. Holotype: ANSP 68463.

Distribution: Northern Sumatra, northern peninsular Malaysia, southern and southeastern Thailand, and southern Cambodia ( $\mathrm{Ng} \& \mathrm{Ng}, 1998)$.

\section{SILURUS Linnaeus, 1758}

Silurus Linnaeus, 1758: 304. Type species: Silurus glanis Linnaeus, 1758. Type by Linnaean tautonymy. On Official List (ICZN Opinion 77 and Direction 56). Gender: Masculine.

Glanis Agassiz, 1857: 333. Type species: Glanis aristotelis Agassiz, 1857. Type by monotypy. Gender: Masculine. Preoccupied by Glanis Spix \& Agassiz, 1829, in fishes.

Parasilurus Bleeker, 1862b: 392, 394. Type species: Silurus japonicus Temminck \& Schlegel, 1846. Type by original designation. Gender: Masculine. Also in Bleeker (1862-63: 17).

Revision: Kobayakawa (1989), Silurus sensu lato, including Pterocryptis.

Remarks: The name Duanensis appears in the title of Hu et al. (2004) as a generic name for a species referred to in the text as a species of Silurus. Duanensis is considered a lapsus calami and, therefore, a nomen nudum.

Silurus aristotelis Agassiz, 1857

Glanis Aristotelis Agassiz, 1857: 333. Type locality: Greece. Syntypes: MCZ 7938 (6), USNM 55895 (1).

Silurus (Parasilurus) aristotelis Garman, 1890: 56. Type locality: Acheloüs River, Acarnania, Greece. Syntypes: MCZ 7938 (6), USNM 55895 (1).

Distribution: Balkan Peninsula, Greece (Kobayakawa, 1989).

Silurus asotus Linnaeus, 1758

Silurus Asotus Linnaeus, 1758: 304. Type locality: Asia. Type(s): Whereabouts unknown. Neotype designation by 
Chen (1977: 206, 216) apparently valid inasmuch as ten specimens were all designated as a collective neotype (M. Kottelat, pers. commun.).

Silurus inermis Houttuyn, 1782: 338. Type locality: Japanse. No types known. Preoccupied by Silurus inermis Linnaeus, 1766; effectively replaced by Silurus imberbis Gmelin, 1789.

Silurus dauuricus Pallas 1787: 359, pl. 11 (fig. 11). Type locality: Ingoda, Onone et Arguno Dauuriae fluuiis. Type(s) (20" 3'" TL): possibly at ZIN or ZMB.

Silurus imberbis Gmelin, 1789:1361. Type locality: Japonia. On Silurus inermis Houttuyn and effectively replacing that name. Apparently intended as a new name to avoid the homonymy with Silurus inermis Linnaeus, 1766.

Centranodon japonicus La Cepède, 1803: 138, 139. Type locality: Japan. On Silurus inermis Houttuyn (1782: 338, no. 27) and Silurus imberbis Gmelin.

Silurus punctatus Cantor, 1842: 485. Type locality: Chusan [China]. Syntypes: BMNH 1968.3.11.29 (3); possibly BMNH 1843.7.21.6, BMNH 1843.7.21.24, and BMNH 1843.7.21.25 (not found), also BMNH 1860.3.19.736737 (2), BMNH 1860.3.19.785-786 (2). Preoccupied by Silurus punctatus Rafinesque, 1818; apparently not replaced.

Silurus xanthosteus Richardson, 1845: 133, pl. 56 (figs. 12-14). Type locality: Chusan, Canton, China. Syntypes (3): BMNH 1968.3.11.29 (1) and specimen used for drawing by Reeves.

Silurus japonicus Temminck \& Schlegel, 1846, in Temminck \& Schlegel, 1843-50: 226, pl. 104 (fig. 1). Type locality: Nagasaki, Japan. Lectotype: RMNH D675, designated by Boeseman (1947: 169).

Silurus cinereus Dabry de Thiersant, 1872: 189, pl. 47 (fig. 1). Type locality: Yang-tsee-kiang [China]. Type(s): possibly at MNHN.

Silurus bedfordi Regan, 1908g: 61, pl. 2 (fig. 3). Type locality: Kimhoa [and] Chong-ju, Corea. Syntypes: BMNH 1907.12.10.66 (1), BMNH 1907.12.10.67 (1).

? Parasilurus asotus longus Wu, 1930a: 255, fig. 1. Type locality: Creek at Tian-Tai Mountain, Chekiang [Zhejiang], China. Syntypes (2): possibly at MNHN. Originally as Parasilurus asotus var. longus.

Distribution: Zhujiang [= Pearl River], Changjiang [=Yangtze River], Huanghe [= Yellow River], Heilongjiang [= Amur River], China (Chu et al., 1999); Southern Hokkaido to Kyushu, Japan, as well as Taiwan, and northern Vietnam (Matsuura et al., 2000); Common throughout Korea (Mori, 1952).

Remarks: Treated as valid as Parasilurus asotus in some recent publications.

Silurus biwaensis (Tomoda, 1961)

Parasilurus biwaensis Tomoda, 1961: 348, fig. 1. Type locality: Offshore of Onoé, Lake Biwa-ko, Japan. Holotype: MIKU 34407 [now at FAKU].

Distribution: Lake Biwa, Japan (Matsuura et al. 2000); reported from the Ueno Formation, Pliocene of ancient Lake Biwa, by Kobayakawa \& Okuyama (1994a, b).

Silurus duanensis Hu, Lan \& Zhang, 2004

Silurus duanensis Hu, Lan \& Zhang, 2004: 586, 589, figs. 1-2. Type locality: underground rivers of Disu Town $\left(23^{\circ} 34^{\prime} \mathrm{N}, 108^{\circ} 01^{\prime} \mathrm{E}\right)$, Du'an County, Guangxi [China]. Holotype: ASIZB 73176.

Distribution: Hongushui River basin, Pearl River drainage, China (Hu et al., 2004).

Silurus glanis Linnaeus, 1758

Silurus glanis Linnaeus, 1758: 304. Type locality: Oriente, minus frequens in Europae lacubus. Syntypes: BMNH 1853.11.12.168 (1, skin), NRM 59 (1). Placed on Official List as type of Silurus (ICZN Direction 57).

Silurus silurus Wulff, 1765: 33. Type locality: Germany. No types known.

Silurus glanis aralensis Kessler, 1872: 48. Type locality: Amu-Darya, Syr-Darya, and Zeravshan rivers, cent. Asia. Syntype (3): ZISP 2071 (1). Originally as Silurus glanis var. aralensis.

? Silurus chantrei Sauvage, 1882: 163. Type locality: Fleuve Koura à Tiflis. Syntypes: MNHN a-3932 (2); described in more detail and illustrated in Sauvage (1884b: 19, pl. 1, fig. 1).

Distribution: Danube basin, all Baltic basin drainages, Elbe River, Lake Constance, Murtensee, and Rivers draining the Caspian, Azov and Aral Seas (Lelek, 1987); Lake Urmia, Tedhzhen River and possibly Tigris River basin (Coad, 1995), widely introduced elsewhere in Europe, including Great Britain, France, Italy, the Iberian Peninsula, and rivers flowing into the Arctic Ocean.

Silurus grahami Regan, 1907 
Silurus grahami Regan, 1907a: 64. Type locality: Chien Kiung Lake, 30 mi. southeast of Yunnan Fu, China. Holotype: BMNH 1907.5.4.46.

Distribution: Fuxian Lake, Yunnan, China (Chu et al., 1999)

Silurus lanzhouensis Chen, 1977

Silurus lanzhouensis Chen, 1977: 210, pl. 2 (fig. 8). Type locality: [Yellow River system, Lanchow and Inner Mongolia, China]. Syntypes: Mus. Inst. Hydrobiol. Acad. Sinica, Hupei [LAN] 001-003 (3), [NEI] 004-005 (2), [NEI] 769739-42 (4).

Distribution: Changjiang [=Yangtze River], Zhujiang [= Pearl River], Minjiang (Chu et al., 1999).

Silurus lithophilus (Tomoda, 1961)

Parasilurus lithophilus Tomoda, 1961: 350, fig. 2. Type locality: Near Onoé, Lake Biwa-ko, Japan. Holotype: MIKU 34411 [now at FAKU].

Distribution: Lake Biwa and Lake Yogo, Japan (Masuda et al., 1984).

Silurus mento Regan, 1904

Silurus mento Regan, 1904a: 192. Type locality: Sea of Tien [Tien Chih], Yunnan Fu, China, elev. 6000 ft. Syntypes: BMNH 1904.1.26.40-41 (2).

Distribution: Lakes of Dianchi and Yilong, Yunnan, China (Chu et al., 1999)

Silurus meridionalis Chen, 1977

Silurus soldatovi meridionalis Chen, 1977: 209, pl. 2 (fig. 7). Type locality: [Yangtze R. system, China]. Syntypes: ? Mus. Inst. Hydrobiol. Acad. Sinica, Hupei 101-102 (2), 0719 (1), 66.5.194-195 (2), 731419 (1), 731494 (1), 746003 (1), 746008-09 (2).

Distribution: Yangtze River basin, China.

Silurus microdorsalis (Mori, 1936)

Parasilurus microdorsalis Mori, 1936: 671, pl. 24 (fig. 1). Type locality: River Rakuto at Ei-yo, South Chosen [Korea]. Holotype: At Preparatory Department of Keijo Imperial University.

Distribution: Yalujiang [= Amnok River], China (Chu et al., 1999); Upper reaches of the Yalu, Han, Kim, Naktong and Anpyeng Rivers, Korea (Mori, 1952).

Silurus soldatovi Nikolsky \& Soin, 1948

Silurus soldatovi Nikolsky \& Soin, 1948: 1359, fig. 1. Type locality: [Amur River, Lake Kabar at Elabuga, Khabarovskyi Krai, Russia]. Holotype: ZMMU P-6505.

Distribution: Heilongjiang [=Amur River] China and Russia; Liaohe, China (Chu et al., 1999)

Silurus triostegus Heckel, 1843

Silurus triostegus Heckel, 1843: 1090, pl. 13 (fig. 1). Type locality: dem Tigris bei Mossul. Syntypes (4): at NMW, SMF 2623 (1).

Distribution: Tigris River basin (Coad, 1995); Euphrates River, Turkey (Ünlü \& Bozkurt, 1996).

WALLAGO Bleeker, 1851

Wallago Bleeker, 1851b: 265. Type species: Silurus Mülleri Bleeker, 1846. Type by subsequent designation by Bleeker (1862: 17, 79). Gender: Masculine.

Silurodon Kner, 1866: 546. Type species: Silurodon hexanema Kner, 1866. Type by monotypy. Gender: Masculine. Also described as new in Kner (1867: 305).

Wallagonia Myers, 1938: 98. Type species: Wallago leerii Bleeker, 1851. Type by original designation. Gender: Feminine.

Remarks: See Myers (1938: 98, 1948: 19) and Kottelat (2000b: 87) concerning the history of problems surrounding designation of type species of Wallago.

Revision, with key to species: Roberts (1982b).

Wallago attu (Bloch \& Schneider, 1801)

Silurus attu Bloch \& Schneider, 1801: 378, pl. 75. Type locality: in lacubus Malabariae. Holotype: ZMB 8783 (dry, lost). As Silurus athu in text and index, Silurus attu on plate; first reviser apparently Bleeker (1862-63: 79) in which Wallago attu is treated as valid over Wallago atthu [sic.]).

Silurus boalis Hamilton, 1822: 154, 375, pl. 29 (fig. 49). Type locality: Rivers not only of the Gangetic provinces, 
but all over India, and is occasionally found in ponds. No types known.

Silurus (Callichrus) macrostomus Swainson, 1839: 306. Type locality: Rivers not only of the Gangetic provinces, but all over India, and is occasionally found in ponds. No types known. Name made available by reference to "Ham. 154, pl. 29, fig. 49 " [= Hamilton, 1822: 154, 375, pl. 29 (fig. 49)]. Unneeded new name for Silurus boalis Hamilton, 1822.

Silurus Wallagoo Valenciennes, in Cuvier \& Valenciennes, 1840a: 354. Type locality: Bengale, .... la côte de Coromandel, .... pays des Birmans. Syntypes: based on specimens at MNHN and literature accounts in Bloch \& Schneider (1801), Russell (1803), and Hamilton (1822).

Silurus Mülleri Bleeker, 1846b: 289. Type locality: Batavia. Type(s): Whereabouts unknown.

Wallago Russellii Bleeker, 1854c: 108. Type locality: Calcutta, in fluminae Hooghly ... Batavia, in fluviis. Syntypes (4, 285-485 mm TL): Whereabouts unknown, and numerous literature citations.

Wallago attu valeya Deraniyagala, 1953: 45. Type locality: Yakvala, Ceylon. Holotype: NMSL FF187; illustrated in Deraniyagala (1952: pl. 13, as Wallagonia attu).

Distribution: India, Malay Peninsula, Sumatra, Java, and Mekong and Chao Phraya River basins (Kottelat, 2001b).

Remarks: Redescribed in Hora (1939b) as Wallagonia attu.

Wallago leerii Bleeker, 1851

Wallago Leerii Bleeker, 1851e: 427. Type locality: Sambas et Palembang, in fluviis. Syntypes (2, 225, $230 \mathrm{~mm}$ TL): RMNH 6833 (2 of 4).

Wallago nebulosus Vaillant, 1902: 46. Type locality: Tepoe, bords du Mahakam, Bornéo central. Holotype: RMNH 7812; holotype illustrated by Roberts (1982b: fig. 3a).

Wallagonia tweediei Hora \& Misra, in Hora \& Gupta, 1941: 18, figs. 2-3. Type locality: Kuala Tahan, Pahang, Malaysia. Holotype: ZRC 350 (plaster cast with head and fins built in); ZSI F13365/1 (right anterior gill arch of holotype).

Distribution: Malay Peninsula, Sumatra and Borneo (Kottelat, 2001b; Ng, 2004a).

Wallago maculatus Inger \& Chin, 1959

Wallago maculatus Inger \& Chin, 1959: 279. Type locality: Kinabatangan River at Deramakot, Kinabatangan District, North Borneo. Holotype: FMNH 68038.

Distribution: Kinabatangan River, Borneo; known only from type series (Roberts (1982b).

Wallago micropogon $\mathrm{Ng}, 2004$

Wallago micropogon Ng, 2004a: 93, fig. 1. Type locality: Cambodia: Stung Treng morning market $\left(13^{\circ} 30.0^{\prime} \mathrm{N}\right.$, 10558.0'E). Holotype: UMMZ 232320.

Distribution: Mekong River basin of Cambodia, Laos and Vietnam, and middle Chao Phraya River basin (Ng, 2004a).

\section{Species inquirenda, Wallago}

Silurodon hexanema Kner, 1866: 546. Type locality: Von Schanghai. Holotype (6 1/3"): at NMW. Described in more detail and illustrated in Kner (1867: 305, pl. 12, fig. 2 [as Wallago attu ?]).

\section{Species inquirendae, Siluridae}

Silurus sinensis La Cepède, 1803: 58, 82, pl. 2 (fig. 1). Type locality: China. No types known: based on an unpublished drawing.

Silurus sinensis M'Clelland, 1844a: 402. Type locality: China. No types known. Preoccupied by Silurus sinensis La Cepède, 1803.

Silurichthys basilewskii Bleeker, 1858b: 256. Type locality: China borealis. Based on specimens identified as Silurus asotus in Basilewky (1855). Originally Silurichthys? basilewskii.

Belodontichthys javanensis Hardenberg, 1938: 311. Type locality: Fish market of Batavia. Holotype (215 mm): Whereabouts unknown.

$\dagger$ Silurus altus Sytchevskaya, 1989. [No other information; in Gayet \& Meunier, 2003]. 
Distribution: middle Miocene to lower Pliocene, Russia; Lower/Middle Miocene, China; Miocene or Pliocene of Tueirusia Formation, Russia (Gayet \& Meunier, 2003).

$\dagger$ Heterobranchus austriacus Thenius, 1952. [No other information; in Gayet \& Meunier, 2003].

Distribution: Bruun-Vösendorf, Austria, Pannonian (Gayet \& Meunier, 2003).

Remarks: Included in Siluridae and probably Silurus by Gayet \& Meunier (2003), following Gaudant (1994).

$\dagger$ Silurus glanis atavus Bogačew, 1924. [No additional information; from Weiler, 1956: 187.]

\section{SISORIDAE Bleeker, 1858}

Sisorichthyoidei Bleeker, 1858b: 48, 50. Type genus: Sisor Hamilton, 1822.

Glyptosterni Gill, 1861c: 53. Type genus: Glyptosternon M'Clelland, 1842.

Erethistides Bleeker, 1862 (in Bleeker, 1862-63): 13. Type genus: Erethistes Müller \& Troschel, 1849.

Bagarina Günther, 1864: 3, 9, 183. Type genus: Bagarius Bleeker, 1854.

Exostomatina Günther, 1864: 264. Type genus Exostoma Blyth, 1860.

Continae de Pinna, 1996: 64. Type genus: Conta Hora, 1950.

Glyptothoracini de Pinna, 1996: 64. Type genus: Glyptothorax Blyth, 1860.

Laguviini de Pinna, 1996: 65. Type genus: Laguvia Hora, 1921.

Pseudecheneidina de Pinna, 1996: 64. Type genus: Pseudecheneis Blyth, 1860.

Revision: Hora \& Silas (1952a, glyptosternoids).

Review: Chu (1986, western China); Chu \& Kuang (1990, Yunnan, China); Chu \& Mo (1999, China); Menon (1999, India); Mirza \& Hameed (1974, Pakistan).

Phylogeny: de Pinna (1996); Diogo et al. (2002, 2003b); Hora \& Silas (1952b, glyptosternoids), Chu (1979, glyptosternoids), He (1996b, glyptosternoids), Peng et al. (2004, glyptosternoids), Guo et al. (2005, glyptosternoids).

Identification guide: Jayaram (1979, South Asia).

Historical biogeography: Hora \& Silas (1952b), He (1995, glyptosternoids).

Remarks: In 1996, de Pinna removed several taxa from the Sisoridae and placed them into the family Erethistidae. Those taxa, placed herein within the genera Conta, Erethistes, Erethistoides, Hara and Pseudolaguvia, together with the subsequently named genera Ayarnangra and Caelatoglanis, appear instead to be part of a natural subgroup within the Sisoridae that includes the genus Glyptothorax and possibly others, but not the glyptosternoid genera (Ferraris \& Britz, 2005; pers. obs.; and pers. commun., H. H. Ng, 2005). Therefore, the taxa placed in the Erethistidae by de Pinna are returned to the Sisoridae.

22 genera, 167 species; 1 named fossil species.

\section{Incertae sedis}

Laguvia manipurensis Arunkumar, 2000

Laguvia manipurensis Arunkumar, 2000: 194, fig. 1. Type locality: Lairaok Maru stream near Moreh, 110 km from Imphal City, Manipur, India. Holotype: MUMF 3001/1A.

Distribution: Yu River basin, Chindwin River basin, Manipur, India (Arunkumar, 2000).

Remarks: Although described as a species of Laguvia, neither the description nor the illustration are sufficient to assign this species to genus or even family.

AYARNANGRA Roberts, 2001

Ayarnangra Roberts, 2001: 83. Type species: Ayarnangra estuarius Roberts, 2001. Type by original designation. Gender: Masculine.

Ayarnangra estuarius Roberts, 2001

Ayarnangra estuarius Roberts, 2001: 84, figs. 1-3. Type locality: Pathein Chaung (=Ngawan Chaung) near Pathein, lower Ayeyarwaddy basin, Myanmar. Holotype: KUMF 3190. 
Distribution: Coastal portions of Irrawaddy and Bago rivers, Myanmar.

BAGARIUS Bleeker, 1854

Bagarius Bleeker, 1854c: 121. Type species: Pimelodus bagarius Hamilton, 1822. Type by monotypy. Gender: Masculine.

Revision: Roberts (1983).

Bagarius bagarius (Hamilton, 1822)

Pimelodus bagarius Hamilton, 1822: 186, 378, pl. 7 (fig. 62). Type locality: Ganges River. No types known.

Pimelodus platespogon Valenciennes, 1840, in Jacquemont, 1835-44: pl. 18 (fig. 3). Type locality: l'Inde. Holotype: MNHN 0000-2904 (1 of 2). Name made available by plate caption, therefore the illustrated specimen is the holotype if distinguishable.

Bagarius Buchanani Bleeker, 1854c: 121. Type locality: Calcutta, in flumine Hooghly; Surakata, Javae centrals, in flumine Pepeh. Syntype (3): possibly NMV 46015 (1). Considered to be an unneeded new name for Pimelodus bagarius Hamilton by Roberts (1983), to avoid Stricklandian tautonymy.

Distribution: Mekong, Chao Phraya and Ganges River basins (Kottelat, 2001b).

Remarks: Redescribed in Hora (1939a).

$\dagger$ Bagarius gigas Gunther, 1876

$\dagger$ Bagarius gigas Gunther, 1876: 436, pl. 16, fig. 1. Type locality: Highlands of Padang, Sumatra; Tertiary. Holotype BMNH 47513 (Imperfect pectoral arch, etc.).

Distribution: Highlands of Padang, Sumatra; reportedly from Eocene, but age questioned by Gayet \& Meunier (2003).

Remarks: See Sanders (1934) for additional information.

Bagarius rutilus Ng \& Kottelat, 2000

Bagarius rutilus Ng \& Kottelat, 2000b: 10, fig. 3. Type locality: Market in Hanoi, Viet Nam. Holotype: ZRC 40440.

Distribution: Nam Xan and Nam Ma basins, Laos, and Red River basin, Viet Nam and China (Kottelat, 2001b).

Bagarius suchus Roberts, 1983

Bagarius suchus Roberts, 1983: 442, figs. 2c, 4. Type locality: Thailand, Kemrat. Holotype: ANSP 89521.

Distribution: Mekong and Chao Phraya River basins (Kottelat, 2001b).

Bagarius yarrelli (Sykes, 1839)

Bagrus Yarrelli Sykes, 1839: 163. Type locality: Mota Mola at Poona, Deccan, India. Type(s): Whereabouts unknown. Also described as new in Sykes (1840: 60); illustrated and described in more detail in Sykes (1841: 370, pl. 65, fig. 1).

Pimelodus Carnaticus Jerdon, 1849: 341. Type locality: Bowany River, southern India. No types known.

Bagarius lica Volz, 1903a: 557. Type locality: Moresi R. at Palembang, Sumatra. Holotype: at NMBE. Described in more detail in Volz (1903b: 391).

Bagarius Nieuwenhuisii Popta, 1904: 190. Type locality: le Bo, Bornéo central. Holotype: RMNH 7561; described in more detail, with illustration of holotype, in Popta (1906: 66, pl. 4, fig. 14).

Distribution: Widely distributed in southern and southeastern Asia (Kottelat, 2001b).

CAELATOGLANIS Ng \& Kottelat, 2005

Caelatoglanis Ng \& Kottelat, 2005: 14. Type species: Caelatoglanis zonatus Ng \& Kottelat, 2005. Type by original designation. Gender: Masculine.

Caelatoglanis zonatus $\mathrm{Ng} \&$ Kottelat, 2005

Caelatoglanis zonatus Ng \& Kottelat, 2005: 14, fig. 2. Type locality: Myanmar, Kayin State, stream "Chon Son" between Kyondaw and Phadaw, about $20 \mathrm{~km} \mathrm{NW}$ of Payathouzu, (at border with Thailand), 15² $25^{\prime} \mathrm{N}, 9^{\circ} 15^{\prime} \mathrm{E}$. Holotype: ZRC 49885.

Distribution: Ataran River basin, Myanmar (Ng \& Kottelat, 2005).

CONTA Hora, 1950

Conta Hora, 1950: 194. Type species: Pimelodus conta Hamilton, 1822. Type by original designation. Gender: Fem- 
inine.

Conta conta (Hamilton, 1822)

Pimelodus conta Hamilton, 1822: 191, 378. Type locality: River Mahananda [Bengal]. No types known. Previously unpublished Hamilton illustration reproduced in Hora (1929: pl. 21, fig. 8), and subsequently reproduced in Hora (1950: 297, fig. 4a) and $\mathrm{Ng}$ (2005a: 28, fig. 6).

Hara elongata Day, 1872: 704. Type locality: Stream near Garrow hills. Holotype: ZSI 436. Illustration of holotype first published in Day (1877: pl. 92, fig. 5) and reproduced in Ng (2005a: 28, fig. 7).

Distribution: Bhareli and Mahananda Rivers, northeast Bengal, Garo Hills, Meghalaya; and Bangladesh (Jayaram, 1979); also Sarda River, Uttar Pradesh (Tilak, 1987).

Conta pectinata $\mathrm{Ng}, 2005$

Conta pectinata Ng, 2005a: 24, fig. 1. Type locality: India: Assam: Dibrugarh. Holotype: ZRC 49672.

Distribution: Middle Brahmaputra River basin, Assam, India (Ng, 2005a).

ERETHISTES Müller \& Troschel, 1849

Erethistes Müller \& Troschel, 1849: 12. Type species: Erethistes pusillus Müller \& Troschel, 1849. Type by monotypy. Gender: Masculine.

Erethistes maesotensis Kottelat, 1983

Erethistes maesotensis Kottelat, 1983: 71, figs. 1-2. Type locality: Thailand: Tak province: Mae Nam Moei, $5 \mathrm{~km} \mathrm{~W}$ of Mae Sot (16²41'N, 98²31'E). Holotype: MHNG 2096.63.

Distribution: Mae Nam Moei, Salween River basin, Thailand (Kottelat, 1983).

Erethistes pusillus Müller \& Troschel, 1849

Erethistes pusillus Müller \& Troschel, 1849: 12, pl. 1 (fig. 3). Type locality: Assam. Holotype: ZMB 3102; holotype illustration reproduced by Hora (1950: 187, pl. 1, fig. 5, as Erethistes pussilus).

Distribution: Ganges River basin, Assam, India.

ERETHISTOIDES Hora, 1950

Erethistoides Hora, 1950: 190. Type species: Erethistoides montana Hora, 1950. Type by original designation. Gender: Masculine.

Erethistoides ascita $\mathrm{Ng}$ \& Edds, 2005

Erethistoides ascita Ng \& Edds, 2005a: 240, fig. 1. Type locality: Nepal: Jhapa, Bhadrapur, Mechi River at Bhadrapur, 2632'17.9"N, 886'6.1"E. Holotype: KU 35016.

Distribution: Rivers of lowland plains of southeastern Nepal (Ng \& Edds, 2005a).

Erethistoides cavatura $\mathrm{Ng} \&$ Edds, 2005

Erethistoides cavatura Ng \& Edds, 2005a: 243, fig. 5. Type locality: Nepal: Chitawan, Dhungre River at Sauraha. Holotype: OSUS 15572.

Distribution: Narayani River basin, Nepal (Ng \& Edds, 2005a).

Erethistoides montana Hora, 1950

Erethistoides montana Hora, 1950: 191, pl. 1 (figs. 10-12). Type locality: Streamlets round about Tangla, Darrang district, Assam [India]. Holotype: ZSI F314/2.

Distribution: Brahmaputra River basin, India (Ng, 2005f).

Erethistoides pipri Hora, 1950

Erethistoides montana pipri Hora, 1950: 193, pl. 1 (figs. 7-9). Type locality: Pipri, Rihand River, Mirzapur district, U. P. [India]. Holotype: ZSI F315/2.

Distribution: Rihand River, Uttar Pradesh, India (Hora, 1950); known only from holotype (Jayaram, 1979).

Erethistoides sicula $\mathrm{Ng}, 2005$

Erethistoides sicula Ng, 2005f: 2, fig. 1. Type locality: India: West Bengal, Schutunga River (tributary of the Mansai River) as Ansole, 26²2'24"N 89 ${ }^{\circ} 11^{\prime} 17 " E$. Holotype: UMMZ 243718.

Distribution: Mansai River drainage, Brahmaputra River basin, India (Ng, 2005f: 6).

EUCHILOGLANIS Regan, 1907 
Chimarrichthys Sauvage, 1874: 332. Type species: Chimarrichthys davidi Sauvage, 1874. Type by monotypy. Gender: Masculine.

Euchiloglanis Regan, 1907c: 158. Type species: Chimarrichthys davidi Sauvage, 1874. Type by being a replacement name. Gender: Masculine. Proposed as a replacement for Chimarrichthys Sauvage, 1874, then considered to be preoccupied by Cheimarrichthys Haast, 1874, in fishes.

Coraglanis Hora \& Silas, 1952a: 12. Type species: Euchiloglanis kishinouyei Kimura, 1934. Type by original designation. Gender: Masculine.

Remarks: Chimarrichthys Sauvage, 1874, is not actually preoccupied by Cheimarrichthys Haast, 1874, but the name has apparently not been used as valid since Regan (1907c) proposed Euchiloglanis as a replacement. The prevailing usage requirements of the Code require that Euchiloglanis be treated as valid here. Coraglanis Hora \& Silas is generally treated as valid as a monotypic genus for Coraglanis kishinouyei in Indian literature. He (1996b) indicated that Euchiloglanis kishinouyei shared derived characters uniquely with Euchiloglanis davidi and placed the two into Euchiloglanis.

Euchiloglanis davidi (Sauvage, 1874)

Chimarrichthys Davidi Sauvage, 1874: 333. Type locality: Yao-Tchy, Thibet oriental. Syntypes: BMNH 1923.3.13.1 (1), MNHN 0000-6273 (3), MNHN 0000-6274 (3).

Distribution: Chinijiang, Sichuan (Chu et al., 1999); eastern Tibet.

Euchiloglanis kishinouyei Kimura, 1934

Euchiloglanis Kishinouyei Kimura, 1934: 178, pl. 6. Type locality: Kwan-hsien, Szechwan Province, China. Holotype: at Laboratory of Biological Department of the Shanghai Science Institute, Shanghai.

Distribution: Jinshajiang [upper Yangtze River basin] (Chu et al., 1999).

EXOSTOMA Blyth, 1860

Exostoma Blyth, 1860: 155. Type species: Exostoma berdmorei Blyth, 1860. Type by subsequent designation, by Bleeker (1862-1863: 13). Gender: Neuter.

Remarks: See Regan (1923) for comments on this genus.

Exostoma berdmorei Blyth, 1860

Exostoma Berdmorei Blyth, 1860: 155. Type locality: Tenasserim [Burma]. Syntypes: ASB 597 (2), (now at ZSI). Possible syntype: ASB 600.

Distribution: Eastern Myanmar.

Exostoma labiatum (M'Clelland, 1842)

Glyptosternon labiatus M'Clelland, 1842: 588. Type locality: Mishmee Hills Upper Assam [India]. Holotype: BMNH 1860.3.19.97.

Distribution: Yaluzangbujiang [= upper Brahmaputra River], and Irrawaddy River (Chu et al., 1999).

\section{Species inquirendae, Exostoma}

Glyptosternum chaudhurii Hora, 1923b: 41, fig. 7. Type locality: Putao Plains, northern frontier of Burma. Holotype: ZSI F9741/1. Treated as a synonym of either E. labiatum or E. vinciguerrae.

Glyptosternum stuarti Hora, 1923b: 39, pl. 2 (figs. 4-6). Type locality: Nam-Yak River at Tanja, on the northern frontier of Burma. Holotype: ZSI F9742/1. Treated either as valid or as a synonym of E. labiatum.

Exostoma Vinciguerrae Regan, 1905a: 184. Type locality: Khakhyen Hills, upper Burma. Holotype: BMNH 1893.2.16.17. Treated either as valid or as a synonym of E. labiatum. See Steinitz (1961) for comments on taxonomy.

GAGATA Bleeker, 1858

Gagata Bleeker, 1858b: 204, 206. Type species: Pimelodus gagata Hamilton, 1822. Type by absolute tautonymy. Gender: Feminine.

Callomystax Günther, 1864: 218. Type species: Pimelodus gagata Hamilton, 1822. Type by being a replacement name. Gender: Masculine. Unneeded replacement for Gagata Bleeker, 1858. 
Revision: Hora \& Law (1941); Roberts \& Ferraris (1998).

Gagata cenia (Hamilton, 1822)

Pimelodus cenia Hamilton, 1822: 174, 376, pl. 31 (fig. 57). Type locality: Northern parts of Bengal, where it frequents rivers. No types known.

Distribution: Ganges, Indus and Mahanadi river basins (Roberts \& Ferraris, 1998).

Gagata dolichonema He, 1996

Gagata dolichonema He, 1996a: 380, fig. 1. Type locality: Daojieba of Baoshan County, $\left(24^{\circ} 41^{\prime} \mathrm{N}, 9^{\circ} 10^{\prime} \mathrm{E}\right)$, Yunnan Province, China. Holotype: IHASW 791.

Gagata gasawyuh Roberts \& Ferraris, 1998: 325, figs. 6-7. Type locality: Myanmar, Tenasserim River mainstream upstream from Kita (or Htee-tah). Holotype: CAS 95544.

Distribution: Upper Salween River basin, China and Irrawaddy, Salween and Tenasserim River basins, Myanmar (Roberts \& Ferraris, 1998).

Remarks: Synonymy based on recent examination of specimens from the vicinity of the type locality of Gagata dolichonema, and photograph of the holotype.

Gagata gagata (Hamilton, 1822)

Pimelodus gagata Hamilton, 1822: 197, 379, pl. 39 (fig. 65). Type locality: Fresh water rivers and estuaries of Bengal. No types known.

Gagata typus Bleeker, 1863c: 90. Type locality: Fresh water rivers and estuaries of Bengal. No types known. Unneeded new name for Pimelodus gagata Hamilton, 1822.

Distribution: Ganges River basin, India and Bangladesh (Roberts \& Ferraris, 1998).

Gagata itchkeea (Sykes, 1839)

Phractocephalus itchkeea Sykes, 1839: 164. Type locality: Deccan, India. Type(s): at BMNH. Also described as new in Sykes (1840: 61) Illustrated and described in more detail in Sykes (1841: 373, pl. 67, fig. 1).

Arius pumilus Valenciennes, 1840, in Jacquemont, 1835-44: pl. 18 (fig. 1). Type locality: Inde. Syntypes: MNHN 0000-1208 (8). Name made available from caption on plate.

Distribution: Narmada, Krishna and Cauvery River basins, India (Roberts \& Ferraris, 1998).

Remarks: Synonymy of Arius pumilus based on examination of types; illustrated specimen of A. pumilis is arguably a holotype, but it could not be readily distinguished from remaining specimens.

Gagata melanoptera Roberts \& Ferraris, 1998

Gagata melanopterus Roberts \& Ferraris, 1998: 330, fig. 10. Type locality: Myanmar: Yangon Division, Hlaing River, $16^{\circ} 53^{\prime} 41^{\prime \prime} \mathrm{N}, 96^{\circ} 05^{\prime} 28^{\prime \prime}$. Holotype: USNM 348852.

Distribution: Irrawaddy, Rangoon, Sittang and lower Salween River basins, Myanmar (Roberts \& Ferraris, 1998).

Gagata pakistanica Mirza, Parveen \& Javed, 1999

Gagata pakistanica Mirza, Parveen \& Javed, 1999: 1, fig. 1. Type locality: River Indus near Ghazi, Pakistan. Holotype: Disposition not stated in publication, but presumably deposited at GCM.

Distribution: Indus River, Pakistan (Mirza, 2003).

Gagata sexualis Tilak, 1970

Gagata sexualis Tilak, 1970b: 207, fig. 1. Type locality: North Koel River at Daltonganj (Chotanagpur) [South Bihar, India]. Holotype: ZSI F5592/2.

Gagata youssoufi Ataur Rahman, 1976: 5, fig. 1. Type locality: river Meghna near Chandpur, Bangladesh. Holotype: at Museum of Freshwater Fisheries Research Station, Chandpur, Bangladesh.

Distribution: Ganges and Brahmaputra River basins (Roberts \& Ferraris, 1998).

Remarks: Synonymy based on Heok Hee Ng (pers. comm., 2003).

GLARIDOGLANIS Norman, 1925

Glaridoglanis Norman, 1925b: 574. Type species: Exostoma andersonii Day, 1870. Type by monotypy. Gender: Masculine.

Glaridoglanis andersonii (Day, 1870)

Exostoma andersonii Day, 1870b: 524. Type locality: Hotham; and Ponsee, China. Possible syntypes: ZSI F917374/1 [? = A.595] (2), ZSI A.596 (2, lost), AMS B.8081 (1). 
Distribution: Yaluzangbujiang [= upper Brahmaputra River], and Irrawaddy River, China (Chu et al., 1999).

\section{GLYPTOSTERNON M'Clelland, 1842}

Glyptosternon M'Clelland, 1842: 584. Type species: Glyptosternon reticulatus M'Clelland, 1842. Type by subsequent designation by Bleeker (1862-63: 12). Gender: Neuter

Glyptosternum Agassiz, 1846: 164. Type species: Glyptosternon reticulatus M'Clelland, 1842. Unjustified emendation of Glyptosternon M'Clelland, 1842. Gender: Neuter.

Parexostoma Regan, 1905a: 182. Type species: Exostoma stoliczkae Day, 1877. Type by subsequent designation, apparently by Jordan (1920: 515). Gender: Neuter.

Remarks: Blyth (1860: 154) restricted Glyptosternon to a single species, but did not, by that action, designate that species, G. reticulatus, as type. See Regan (1923) for comments on this genus.

Glyptosternon akhtari Silas, 1952

Glyptosternum akhtari Silas, in Hora \& Silas, 1952a: 11, pl. 1 (figs. 4-6). Type locality: Bamian River, Oxus watershed, Afghanistan. Holotype: ZSI F643/2.

Distribution: Bamian River, Oxus watershed, Afghanistan (Hora \& Silas, 1952a).

Glyptosternon maculatum (Regan, 1905)

Parexostoma maculatum Regan, 1905a: 183. Type locality: Lhasa, Tibet. Syntypes: BMNH 1904.12.28.87-88 (2).

Distribution: Yaluzangbujiang [= upper Brahmaputra River] (Chu et al., 1999).

Glyptosternon malaisei Rendahl \& Vestergren, 1941

Glyptosternon malaisei Rendahl \& Vestergren, 1941: 213. Type locality: Kambaiti, Kachin State, Irrawaddy River drainage, Burma, 7000 ft. elev. Holotype: NRM 10721.

Distribution: Irrawaddy River drainage, northern Myanmar (Rendahl \& Vestergren, 1941).

Glyptosternon reticulatum M'Clelland, 1842

Glyptosternon reticulatus M'Clelland, 1842: 584. Type locality: Sir-i-Chusma, at the source of the Cabul River [Afghanistan]. No types preserved. Name spelled Glyptosternon retuculatus and Glyptosternon reticulatus in original description; Kullander et al. (1999: 146) apparently serve as first revisers in selecting reticulatus.

Exostoma stoliczkae Day, 1877b: 782. Type locality: Upper waters of the Indus River. Possible syntypes: AMS I.122 (1), ZSI F497 (1), ZSI 1196-98 (3, lost).

Exostoma gracile Gratzianov, 1907: 58. Type locality: Naryn R. drainage, upper Syr-Darya R. basin, Namanganskaya Obl., Uzbekistan. Holotype: ZMMU P-785.

Exostoma labrax Gratzianov, 1907: 59. Type locality: Garm dist., mountain spring Kartveng at kishlak Shulmak, Garmskaya Obl., Tadzhikistan. Syntypes: ZMMU P-2034 (2).

Distribution: Upper Jhelum basin and Gilgit River, Indus River drainage, Pakistan and Kashmir (Kullander, 1999).

Remarks: See Steinitz (1961) for comments on taxonomy. Redescribed in Hora (1932).

\section{GLYPTOTHORAX Blyth, 1860}

Glyptothorax Blyth, 1860: 154. Type species: Glyptosternon striatus M'Clelland, 1842. Type by subsequent designation by Bleeker (1862- 1863: 13). Gender: Masculine.

Aglyptosternon Bleeker, 1862 (in Bleeker, 1862-63): 12. Type species: Silurus cous Linnaeus, 1766. Type by original designation. Gender: Neuter.

Euclyptosternum Günther, 1864: 183. Type species: Silurus cous Linnaeus, 1766. Unjustified emendation of Aclyptosternon [sic, Aglyptosternon Bleeker, 1862]. Gender: Neuter.

Pteroglanis Fowler, 1934a: 92. Type species: Pteroglanis horai Fowler, 1934. Type by original designation. Preoccupied by Pteroglanis Eigenmann \& Pearson, 1924, in fishes, replaced by Pteropsoglanis Fowler, 1934. Gender: Masculine.

Pteropsoglanis Fowler, 1934b: 351. Type species: Pteroglanis horai Fowler, 1934. Type by being a replacement name. Replacement for Pteroglanis Fowler, 1934; preoccupied by Pteroglanis Eigenmann \& Pearson, 1924. Gender: Masculine.

Sundagagata Boeseman, 1966: 243. Type species: Sundagagata robusta Boeseman, 1966. Type by original designation. Gender: Feminine. 
Paraglyptothorax Li, 1986: 524. Type species: Glyptosternum pallozonum Lin, 1934. Type by original designation. Gender: Masculine. Originally proposed as a subgenus of Glyptothorax.

Superglyptothorax Li, 1986: 524. Type species: Glyptothorax coheni Ganguly, Datta \& Sen, 1972. Type by original designation. Gender: Masculine. Originally proposed as a subgenus of Glyptothorax.

Key: Mo \& Chu (1986, Chinese species).

Review: Menon, M. A. S. (1955, India and Burma, with key), Sufi (1963, Pakistan).

Remarks: Extension of the eastern limit of the distribution of this genus into the Black Sea of Turkey noted by Coad \& Delmastro (1985).

Glyptothorax alaknandi Tilak, 1969

Glyptothorax brevipinnis alaknandi Tilak, 1969: 42, figs. 8-11. Type locality: Alaknanda River, near Srinagar, dist. Pauri Garhwal, Uttar Pradesh, India. Holotype: ZSI F6154/2.

Distribution: Alaknanda River, Uttar Pradesh, India (Jayaram, 1999).

Glyptothorax anamalaiensis Silas, 1951

Glyptothorax anamalaiensis Silas, 1951b: 370. Type locality: Streams at base of Anamalai Hills, South India. Holotype: ZSI F629/2; illustrated in Silas (1951a: pl. 1, figs 1-3), as Glyptothorax prox. madraspatanus Day.

Distribution: Anamalai Hills, Kerala, India (Jayaram, 1999).

Glyptothorax annandalei Hora, 1923

Glyptothorax annandalei Hora, 1923b: 14, pl. 1 (fig. 3). Type locality: Nierolay stream, Bhavani River at the base of Nilgiri Hills [India]. Holotype: ZSI F10135/1.

Distribution: Yaluzanbujiang [= Brahmaputra River], China (Chu \& Mo, 1999).

Glyptothorax armeniacus (Berg, 1918)

Glyptosternum armeniacum Berg, 1918: 146. Type locality: River Mukhlassi-darasi, headwaters of Euphrates. Syntypes (5): possibly ZSI F11319/1 (1), ZIN 20806 (4); ZIN syntype illustrated in Berg (1931: pl. 1, figs. 3-5; fig. 6).

Distribution: Tigris River basin, Iran (Coad, 1995); Euphrates River basin, Turkey (Berg, 1918).

Glyptothorax botius (Hamilton, 1822)

Pimelodus botius Hamilton, 1822: 192, 378. Type locality: Northern rivers of Bengal [now India: west Bengal:

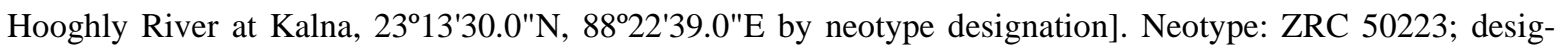
nated by, and illustrated in, $\mathrm{Ng}$ (2005e: 3 , fig. 1).

Distribution: Hooghly River, India (Ng, 2005e: 8).

Remarks: Redescribed in Ng (2005e).

Glyptothorax brevipinnis Hora, 1923

Glyptothorax brevipinnis Hora, 1923b: 16, pl. 1 (fig. 4). Type locality: Unknown locality [probably India]. Syntypes: ZSI F10134/1 (4).

Distribution: Not known (Jayaram, 1999).

Glyptothorax buchanani Smith, 1945

Glyptothorax buchanani Smith, 1945: 402, fig. 89. Type locality: The Metum, a small swift affluent of the Mechem, tributary of the Meping, in northern Thailand. Holotype: USNM 117754.

Distribution: Me Ping, Chao Phraya Basin, Thailand (Smith, 1945).

Glyptothorax burmanicus Prashad \& Mukerji, 1929

Glyptothorax burmanicus Prashad \& Mukerji, 1929: 184, pl. 7 (fig. 3); fig. 5. Type locality: Sankha, a large hillstream, midway between Kamaing and Mogaung, Myitkyina dist. [Burma]. Holotype: ZSI F10877/1.

Distribution: Irrawaddy and Salween Rivers (Chu \& Mo, 1999).

Glyptothorax callopterus Smith, 1945

Glyptothorax callopterus Smith, 1945: 400, fig. 87. Type locality: Waterfall stream on Kao Chong, near Trang, in Peninsular Thailand. Holotype: USNM 109820.

Distribution: Peninsular Thailand.

Glyptothorax cavia (Hamilton, 1822)

Pimelodus cavia Hamilton, 1822: 188, 378. Type locality: Northern rivers of Bengal. No types known.

Euglyptosternum lineatum Day, 1877 (in Day, 1875-78): 500, pl. 116 (fig. 7). Type locality: Jumna, near Suddya in 
upper Assam. Possible syntypes: AMS B.7509 (1), ZSI F1312 (1).

Distribution: Ganges River basin.

Glyptothorax coheni Ganguly, Datta \& Sen, 1972

Glyptothorax coheni Ganguly, Datta \& Sen, 1972: 342, figs. 3-4. Type locality: Subarnarekha River, Chotanagpur Plateau, Bihar, India. Holotype: USNM 205612.

Distribution: Subarnarekha River, Bihar, India (Jayaram, 1999).

Glyptothorax conirostris (Seindachner, 1867)

Glyptosternum conirostre Steindachner, 1867e: 532, pl. 6 (fig. 2). Type locality: Simla. Holotype: at NMW.

Glyptosternum modestum Day, 1872: 714. Type locality: upper portion of the Jumna. Possible syntypes: AMS B.7562 (1), AMS B.7564 (1), ZMB 2765 (1).

Distribution: Jamuna River, Mahananda River basin and Indus River basin (Jayaram, 1999).

Glyptothorax cous (Linnaeus, 1766)

Silurus cous Linnaeus, 1766: 504. Type locality: Syria. Possible syntype: BMNH 1955.6.25.2 (1).

Distribution: Tigris River basin, Iran (Coad, 1995).

Glyptothorax dakpathari Tilak \& Husain, 1976

Glyptothorax dakpathari Tilak \& Husain, 1976: 229, figs. 1-8. Type locality: Yamuna R., below Barrage, Dakpathar, District Dehra Dun, Uttar Pradesh, India. Holotype: ZSI NRS V-988.

Distribution: Yamuna River, Uttar Pradesh, India (Jayaram, 1999).

Remarks: Treated as a synonym of Glyptothorax gracilis by Menon (1999).

Glyptothorax davissinghi Manimekalan \& Das, 1998

Glyptothorax davissinghi Manimekalan \& Das, 1998: 87, unnumbered figure. Type locality: Karim Puzha, Maancheri, Nilambur Reserve Forest, Kerala, India. Holotype: ZSIC 6008.

Distribution: Kerala, India (Manimelakan \& Das, 1998).

Glyptothorax deqinensis Mo \& Chu, 1986

Glyptothorax deqinensis Mo \& Chu, 1986: 345, 350, fig. 6. Type locality: Deqin county (28³0'N, 9900'E) Yunnan [China]. Holotype: KIZ 748621.

Distribution: upper Lancangjiang [= Mekong River], China (Chu et al., 1999).

Glyptothorax dorsalis Vinciguerra, 1890

Glyptothorax dorsalis Vinciguerra, 1890: 246, pl. 7 (fig. 4). Type locality: Meetan, Birmania [Myanmar]. Holotype: MSNG 14417.

Distribution: Upper Irrawaddy River basin (pers. obs.); Nujiang [= Salween River], China (Chu et al., 1999).

Glyptothorax exodon $\mathrm{Ng} \&$ Rachmatika, 2005

Glyptothorax exodon Ng \& Rachmatika, 2005: 251, fig. 1. Type locality: Borneo: Kalimantan Barat, Sungai Tekelan. Holotype: MZB 9940.

Distribution: Kapuas River basin, western Borneo (Ng \& Rachmatika, 2005).

Glyptothorax fokiensis (Rendahl, 1925)

Glyptosternum fokiensis Rendahl, 1925: 307. Type locality: Lan-Hao, Lien-Cheng-Hsien, Fokien, s. China. Syntypes: NRM 10018 (2).

Glyptosternon punctatum Nichols, 1941: 1, figs. 1-2. Type locality: Kiating (Loshan), Szechwan Prov., China, elev. $1100 \mathrm{ft}$. Holotype: AMNH 15218.

Distribution: Changjiang [= Yangtze River], rivers east of Yuanjiang (Chu et al., 1999; as Glyptothorax fukiensis fukiensis).

Remarks: Redescribed as Glyptothorax fukiensis in Xie et al. (2001).

Glyptothorax fuscus Fowler, 1934

Glyptothorax fuscus Fowler, 1934a: 89, figs. 31-33. Type locality: Chantaboon, Southeast Siam. Holotype (59 mm): ANSP 59358.

Distribution: Mekong and Chao Phraya River basins (Kottelat, 2001b).

Glyptothorax garhwali Tilak, 1969

Glyptothorax garhwali Tilak, 1969: 37, figs. 1-4. Type locality: Alaknanda River, near Srinagar, dist. Pauri Garhwal, Uttar Pradesh, India. Holotype: ZSI F6152/2. 
Distribution: Alaknanda River, Uttar Pradesh, India (Jayaram, 1999).

Glyptothorax gracilis (Günther, 1864)

Glyptosternum gracile Günther, 1864: 186. Type locality: Nepal. Holotype: BMNH 1845.1.9.846.

Distribution: Sikkim, India and Nepal (Jayaram, 1999).

Glyptothorax hainanensis (Nichols \& Pope, 1927)

Glyptosternon hainanensis Nichols \& Pope, 1927: 333, fig. 7. Type locality: Nodoa, Hainan [China]. Holotype: AMNH 8362.

Distribution: Hainan Island, China (Chu et al., 1999, as Glyptothorax fukiensis hainanensis).

Glyptothorax honghensis Li, 1984

Glyptothorax fukiensis honghensis Li, 1984a: 66, fig. 1. Type locality: Hekou, Weishan, Nanjian, Hong River basin, Yunnan Province, China. Syntypes: KIZ 6440430 (1), KIZ 6440474 (1), KIZ 6507022 (1), KIZ 6507133 (1), KIZ 6507134 (1), KIZ 6507137 (1). Name spelled Glyptothorax fukiensis honghensis on p. 66 and Glyptothorax fukiensis honghenensis on p. 69; G. honghensis treated as the valid spelling here, based on presumed etymology of name.

Distribution: Northeastern Laos, and Red River basin of Vietnam and China (Kottelat, 2001b).

Glyptothorax horai (Fowler, 1934)

Pteroglanis horai Fowler, 1934a: 92, figs. 37-40. Type locality: Sop Lao, in Maun Luang, Southeast Shan States [Burma]. Holotype: ANSP 59462.

Distribution: Mekong River basin, Myanmar.

Glyptothorax housei Herre, 1942

Glyptothorax housei Herre, 1942: 117, fig. 1. Type locality: Rapids in a mountain stream in the Anamallai Hills, Puthototam Estate, four miles east of Valparai, Pollachi district, South India. Holotype: SU 36531.

Distribution: Kerala, India (Jayaram, 1999).

Glyptothorax indicus Talwar, 1991

Glyptothorax horai Shaw \& Shebbeare, 1936: 188, unnumbered pl. Type locality: Streams of Terai, northern Bengal. Holotype: ZSI F11376/1. Preoccupied in Glyptothorax by Pteroglanis horai Fowler, 1934; replaced by Glyptothorax indicus Talwar, 1991.

Glyptothorax indicus Talwar, in Talwar \& Jhingran, 1991: 654, fig. 210. Type locality: Streams of Terai, n. Bengal. Holotype: ZSI F11376/1. Replacement name for Glyptothorax horai Shaw \& Shebbeare, 1936, preoccupied in Glyptothorax by Pteroglanis horai Fowler, 1934.

Distribution: Kosi River, Rihand River and rivers of the Vindhya Mountain range, India, as well as the Punjab and Nepal (Jayaram, 1999).

Glyptothorax interspinalus (Mai, 1978)

Glyptosternon interspinalum Mai, 1978: 271, fig. Type locality: Creeks in n. Vietnam. Syntypes: probably at DVZUT.

? Glyptothorax merus Li, 1984b: 79, fig. 2. Type locality: Jingdong Co., Yunnan, China. Holotype: KIZ 737159.

Distribution: Nam Ma Basin, Laos, and Red River basin of China and Vietnam (Kottelat, 2001b).

Glyptothorax jalalensis Balon \& Hensel, 1970

Glyptothorax jalalensis Balon \& Hensel, 1970: 160, fig. 1. Type locality: Kabul River tributary, near Jalal-Abad, Afghanistan. Holotype: SNMB RY 2176.

Distribution: Kabul River basin, Afghanistan (Balon \& Hensel, 1970).

Glyptothorax kashmirensis Hora, 1923

Glyptothorax kashmirensis Hora, 1923b: 22, fig. 2. Type locality: Kashmir Valley. Syntypes: ZSI F10270/1 (2).

Distribution: Jhelum River, Kashmir (Kullander, 1999); NWFP, Azad Kashmir (Mirza, 2003).

Remarks: See Rashida et al. (1996) for comments on the taxonomic position of this species.

Glyptothorax kurdistanicus (Berg, 1931)

Glyptosternum kurdistanicum Berg, 1931: 1267, fig. 1; pl. 1 (fig. 2). Type locality: Kurdistan, at the village of Germau (or Germav), at the height of $1500 \mathrm{~m}, \ldots$ Germau is situated in latitude $36^{\circ}$ southeast of Serdesht on the Western slope of the Sur-kei Range, in the basin of the river Bané, tributary to the Little Zab, which is a tributary to the Tigris R. Holotype: ZIN 20780. 
Distribution: Tigris River basin, Iran (Coad, 1995).

Glyptothorax lampris Fowler, 1934

Glyptothorax lampris Fowler, 1934a: 91, figs. 34-36. Type locality: Chieng Mai, North Siam. Holotype: ANSP 59357.

Distribution: Mekong and Chao Phraya River basins, and rivers of southeastern Thailand (Kottelat, 2001b); in medium-sized upland rivers (Rainboth, 1996).

Glyptothorax laosensis Fowler, 1934

Glyptothorax laosensis Fowler, 1934a: 88, figs. 28-30. Type locality: Bua Yai, East Siam. Holotype: ANSP 59412.

Distribution: Mekong and Chao Phraya River basins (Kottelat, 2001b); in small to medium-sized streams (Rainboth, 1996).

Glyptothorax lonah (Sykes, 1839)

Bagrus lonah Sykes, 1839: 164. Type locality: Deccan, India. Holotype: BMNH 1860.3.19.756 or BMNH 1860.3.19.757. Also described as new in Sykes (1840: 61); illustrated and described in more detail in Sykes (1841: 371).

Glyptosternum dekkanense Günther, 1864: 187. Type locality: Dekkan. Holotype: BMNH 1860.3.19.757.

Distribution: Deccan, Godavari and Krishna River basins, India (Jayaram, 1999).

Remarks: Holotype of Bagrus lonah Sykes has not been clearly established; see Günther (1864: 187), and Day (1873d: 748) for comments.

Glyptothorax longicauda Li, 1984

Glyptothorax longicauda Li, 1984b: 82, fig. 3. Type locality: Tengchong, Yunnan, China. Holotype: KIZ 764126.

Distribution: Irrawaddy River basin, China (Chu et al., 1999).

Glyptothorax longjiangensis Mo \& Chu, 1986

Glyptothorax longjiangensis Mo \& Chu, 1986: 344, 349, fig. 5. Type locality: Longjiang river (upper tributary of

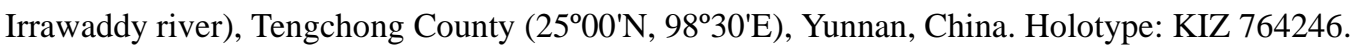

Distribution: Irrawaddy River basin, Yunnan, China (Chu et al., 1999).

Glyptothorax macromaculatus Li, 1984

Glyptothorax macromaculatus Li, 1984b: 82, fig. 7. Type locality: Yangbi Xian, Yunnan, China. Holotype: KIZ 748804.

Distribution: Mekong River basin (Chu et al., 1999; Kottelat, 2001b).

Glyptothorax madraspatanus (Day, 1873)

Glyptosternum madraspatanum Day, 1873b: 526. Type locality: Bowany River, Neilgherries, Madras State, India. Possible syntypes: AMS B.7759 (1), AMS B.8004 (1), NMW 46582-83 (2), RMNH 2739 (1), ZMB 10798 (1), ZMB 10829 (1), ZMB 11208 (1), ZSI F1235 (1), ZSI F1313 (1).

Distribution: Western Ghats, Anaimalai Hills, Nilgiri Hills, and Cauvery River (Jayaram, 1999).

Glyptothorax major (Boulenger, 1894)

Akysis major Boulenger, 1894a: 246. Type locality: Senah, Tagora and Baram rivers, Sarawak. Syntypes: BMNH 1892.9.2.59 (1), BMNH 1892.10.7.26 (1), BMNH 1893.3.6.173-177 (5), BMNH 1893.3.6.178 (1, skeleton).

Glyptosternum kükenthali Steindachner, 1901: 448, pl. 18 (figs. 5, 5a). Type locality: Baram-Flusse, Borneo. Holotype: SMF 752.

Distribution: Malay Peninsula and western Borneo (Roberts, 1989a).

Glyptothorax manipurensis Menon, 1955

Glyptothorax manipurensis Menon (A. G. K.), 1955: 23, fig. 1. Type locality: Barak River at Karong, Naga Hills, Manipur State, India. Holotype: ZSI F738/2.

Distribution: Barak River, Manipur, India (Jayaram, 1999).

Remarks: Redescribed in Kosygin \& Vishwanath (2005).

Glyptothorax minimaculatus Li, 1984

Glyptothorax minimaculatus Li, 1984b: 81, fig. 5. Type locality: Tengchong Xian, Yunnan, China. Holotype: KIZ 764336.

Distribution: Irrawaddy River basin, Yunnan, China (Chu et al., 1999).

Glyptothorax minutus Hora, 1921 
Glyptothorax minutus Hora, 1921a: 180, fig. 1. Type locality: Imphal stream near Karong [Kameng], Manipur Valley, India. Syntypes (4): lost (Hora, 1921a: 182).

Distribution: Manipur, India (Hora, 1921a).

Glyptothorax naziri Mirza \& Naik, 1969

Glyptothorax naziri Mirza \& Naik, 1969: 123, figs. 1-2. Type locality: Zhob River, Baluchistan, Pakistan. Holotype: GCM 6.

Distribution: NWFP, Punjab, Balochistan, Azad Kashmir, Pakistan (Mirza, 2003).

Glyptothorax nelsoni Ganguly, Datta \& Sen, 1972

Glyptothorax nelsoni Ganguly, Datta \& Sen, 1972: 341, fig. 1. Type locality: Subarnarekha River, Chotanagpur Plateau, Bihar, India. Holotype: USNM 205611.

Distribution: Subarnarekha River, Bihar, India (Jayaram, 1999).

Glyptothorax nieuwenhuisi (Vaillant, 1902)

Glyptosternon Nieuwenhuisi Vaillant, 1902: 72, 162, figs. 14-15. Type locality: Le Bloeoe, Bornéo central. Syntypes: MNHN 1891-0484 (1), MNHN 1891-0485 (1), MNHN 1891-0486 (1), MNHN 1891-0487 (1), MNHN 1903-0189 (1).

Distribution: Bloeoe River, Borneo.

Glyptothorax obscurus Li, 1984

Glyptothorax obscura Li, 1984b: 86, fig. 1. Type locality: Jingdon Xian, Yunnan, China. Holotype: KIZ 737197.

Distribution: Upper Red River basin, China (Li, 1984b).

Glyptothorax pallozonus (Lin, 1934)

Glyptosternum pallozonum Lin, 1934: 228, figs. 7-8. Type locality: Loh Fau Shan, Poh-lo District, Kwangtung, China. Holotype: Fisheries Experiment Station, Canton G10.

Distribution: Dongjiang, Guandong, China (Chu et al., 1999).

Glyptothorax panda Ferraris \& Britz, 2005

Glyptothorax panda Ferraris \& Britz, 2005: 376, fig. 1. Type locality: Myanmar, Kachin State: Upper Irrawaddy River drainage, hill stream 8 miles from Kamaing on road to Tanai, vicinity of Myitkyina. Holotype: USNM 384824.

Distribution: Upper Irrawaddy River basin, Myanmar (Ferraris \& Britz, 2005).

Glyptothorax pectinopterus (M'Clelland, 1842)

Glyptosternon pectinopterus M'Clelland, 1842: 587. Type locality: The mountains of Simla. No types known.

Distribution: Ganges, Sutlej and upper Indus River basins, south Asia (Kullander, 1999).

Glyptothorax platypogon (Valenciennes, 1840)

Pimelodus platypogon Valenciennes, in Cuvier \& Valenciennes, 1840b: 152 (113 of Strasbourg deluxe edition). Type locality: Java. Possible syntypes: MNHN b-0196 (2), MNHN 0000-2903 (2), MNHN 0000-2904 (2); RMNH (not found); SMF 649 (2).

Pimelodus cyanochloros Bleeker, 1847b: 11. Type locality: Java. Syntypes: SMNS 10569 (6). Also described as new in Bleeker (1847a: 168).

Sundagagata robusta Boeseman, 1966: 243, fig. 1. Type locality: River near Buitenzorg, Java. Holotype: RMNH 25264.

Distribution: Java; western Borneo (Roberts, 1989a).

Remarks: Sundagagata robusta Boeseman, 1966, was considered by Kottelat et al. (1993: 76) to be an abnormal specimen of Glyptothorax platypogon (Valenciennes, 1840).

Glyptothorax platypogonides (Bleeker, 1855)

Pimelodus platypogonides Bleeker, 1855a: 272. Type locality: Lahat, in fluviis. Syntypes (4, 70-85 mm TL): possibly BMNH 1863.12.4.154 (1), RMNH 6912 (4), RMNH 15289 (3).

Callomystax Schmidti Volz, 1904: 470. Type locality: Simbolon Gebirge, cent. Sumatra, Indonesia, elev. $1400 \mathrm{~m}$. Syntypes (5): MHNG 683.22 (1), NMBA 2827 (1).

Distribution: Sumatra (Ng \& Rachmatika, 2005).

Remarks: Register number of syntype of Pimelodus platypogonides incorrectly reported as BMNH 1863.12.11.154 in Eschmeyer et al. (1998). Name of species frequently cited as Glyptothorax platypogonoides. 
Glyptothorax prashadi Mukerji, 1932

Glyptothorax prashadi Mukerji, 1932: 281, fig. 1. Type locality: Kyenchaung, Mergui District, Lower Burma. Holotype: ZSI F11334/1.

Distribution: Southeastern Myanmar and Peninsular Thailand (Jayaram, 1999).

Glyptothorax punjabensis Mirza \& Kashmiri, 1971

Glyptothorax conirostris punjabensis Mirza \& Kashmiri, 1971: 88, fig. 1. Type locality: Rawal Dam, Rawalpindi, Pakistan. Holotype: GCM 7.

Distribution: Soan River, Jhelum canal, Kabul River, and Siran River, Pakistan, and Kotli, Azad Kashmir (Jayaram, 1999).

Remarks: Treated as a possible synonym of Glyptothorax kashmirensis Hora, 1923, by Kullander (1999).

Glyptothorax quadriocellatus (Mai, 1978)

Glyptosternon quadriocellatum Mai, 1978: 272, fig. 121. Type locality: Fast-running creeks, n. Vietnam. possibly at DVZUT.

Glyptosternon minutum Mai, 1978: 274, fig. 122. Type locality: Fast-running creeks, n. Vietnam. Type (s): at DVZUT. Preoccupied in Glyptothorax by Glyptothorax minutus Hora, 1921; replaced by Glyptothorax spectrum Kottelat, 2001.

Glyptothorax spectrum Kottelat, 2001a: 55. Type locality: Fast-running creeks, n. Vietnam. Type (s): at DVZUT. Replacement name for Glyptosternon minutum Mai, 1978, preoccupied in Glyptothorax by Glyptothorax minutus Hora, 1921.

Distribution: Yuanjiang, China (Chu et al., 1999); northern Viet Nam (Kottelat, 2001a).

Remarks: Kottelat (2001a: 54) synonymized G. quadriocellatum and G. minutum and selected G. quadriocellatum as the valid name.

Glyptothorax saisii (Jenkins, 1910)

Glyptosternum saisii Jenkins, 1910: 128, fig.; pl. 6 (fig. 6). Type locality: Sita Nullah stream, Paresnath Hills, Bihar, w. Bengal, elev. $2150 \mathrm{ft}$. Holotype: ZSI F2583/1.

Distribution: Bihar, Uttar Pradesh, and Maharashtra, India (Jayaram, 1999).

Remarks: Redescribed in Tilak \& Husain (1978).

Glyptothorax siamensis Hora, 1923

Glyptothorax siamensis Hora, 1923a: 168, pl. 12 (figs. 1-3). Type locality: Nakon Sritamarat Hills, Siam. Holotype: ZSI F10548/1.

Distribution: Thailand and Peninsular Malaysia (Ng \& Rachimatika, 2005).

Glyptothorax silviae Coad, 1981

Glyptothorax silviae Coad, 1981a: 291, figs. 1-3. Type locality: Iran, Khuzestan, stream 3 km south of Bagh-e Malek, tributary to Rud-e Zard or Ab-e Ala in the drainage of the Jarrahi River, $31^{\circ} 29^{\prime} \mathrm{N}, 4^{\circ} 54 ' 30^{\prime \prime E}$, elev. 660 m. Holotype: NMC 79-0390A.

Distribution: Tigris River and possibly Gulf basins (Coad, 1995).

Glyptothorax sinensis (Regan, 1908)

Glyptosternum sinense Regan, 1908a: 110, pl. 4 (fig. 3). Type locality: Tunting [=Tungting], China. Holotype: BMNH 1907.11.26.4.

Distribution: lower and middle Changjiang [= Yangtze River], China (Chu et al., 1999); also reported from Manipur, India (Kosygin \& Vishwanath, 2005) .

Remarks: Redescribed as Glyptothorax sinense in Xie et al. (2001).

Glyptothorax steindachneri (Pietschmann, 1913)

Glyptosternum steindachneri Pietschmann, 1913a: 93. Type locality: dem Tigris, Mosul. Syntypes (2): at NMW.

Distribution: Tigris River basin (Coad, 1995).

Glyptothorax stocki Mirza \& Nijssen, 1978

Glyptothorax stocki Mirza \& Nijssen, 1978: 79, fig. 1. Type locality: Bhed Nullah, small stream on G.T. road to Rawalpindi, 7 mi. from Lahore, Pakistan. Holotype: ZMA 114763.

Distribution: NWFP, Punjab, Azad Kashmir, Pakistan (Mirza, 2003).

Glyptothorax stolickae (Steindachner, 1867) 
Glyptosternum Stoličkae Steindachner, 1867e: 533, pl. 6 (fig. 1). Type locality: Simla. Syntypes: NMW 76606 (3).

Distribution: Western Himalaya, Shimla, Ganga, India (Jayaram, 1999).

Glyptothorax striatus (M'Clelland, 1842)

Glyptosternon Striatus M'Clelland, 1842: 587, pl. 6 (in part). Type locality: Kasyah mountains. Syntypes (3): BMNH 1860.3.19.95 (1, 78 mm SL).

Distribution: Kashi and Garro Hills, India (Jayaram, 1999).

Glyptothorax sufii Bashir \& Mirza, 1975

Glyptothorax telchitta sufii Bashir \& Mirza, 1975: 96, fig. 1. Type locality: River Sutlej, Lahore District, Pakistan. Holotype: GCM F-13.

Distribution: Indus River basin, Pakistan (Bashir \& Mirza, 1975).

Glyptothorax sykesi (Day, 1873)

Glyptosternum sykesi Day, 1873d: 748. Type locality: Deccan, India. Holotype: BMNH 1860.3.19.756. Name proposed conditionally for specimen in Günther (1864: 187) identified as Glyptosternum lonah, which Day contended was not the holotype and, in fact, not even conspecific with G. lonah.

Distribution: Peninsular India.

Glyptothorax telchitta (Hamilton, 1822)

Pimelodus telchitta Hamilton, 1822: 185, 378. Type locality: Fresh water rivers of Bengal and Behar [now: India: west Bengal: Hooghly River at Kalna, 23³'30.0"N, 88²2'39.0"E, by neotype designation]. Neotype: UMMZ 244946; designated by, and illustrated in, $\mathrm{Ng}$ (2005e:10, fig. 5). Unpublished Hamilton illustration reproduced in Hora (1929: pl. 21, fig. 3). Name spelled Pimelodus telchita on p. 378; first reviser may be Eschmeyer et al. (1998), but not yet clearly established to be earliest.

Distribution: Widely distributed in India (Jayaram, 1999).

Glyptothorax trewavasae Hora, 1938

Glyptothorax trewavasae Hora, 1938d: 373, pl. 7 (figs. 3-4). Type locality: Yenna Valley, Satara dist., Maharashtra, India. Holotype: ZSI F9723/1.

Distribution: Krishna River basin, Yenna, Kayana, and Tunga Rivers, India (Jayaram, 1999).

Glyptothorax trilineatus Blyth, 1860

Glyptothorax trilineatus Blyth, 1860: 154. Type locality: Tenasserim [Burma]. Syntypes: ZSI F10380/1 (2).

Glyptothorax trilineatoides Li, 1984b: 87, fig. 5. Type locality: Tengchong Xian, Yunnan, China. Holotype: KIZ 764336.

Distribution: Nujiang [=Salween River], Irrawaddy River (Chu et al., 1999).

Glyptothorax ventrolineatus Vishwanath \& Linthoingambi, 2005

Glyptothorax ventrolineatus Vishwanath \& Linthoingambi, 2005: 201, fig. 1. Type locality: Iril river, Ukhrul district, Manipur, India. Holotype: MUMFL022I.

Distribution: Iril River, Chindwin River basin, Manipur, India (Vishwanath \& Linthoingambi, 2005).

Glyptothorax zanaensis $\mathrm{Wu}, \mathrm{He} \& \mathrm{Chu}, 1981$

Glyptothorax zanaensis Wu, He \& Chu, 1981: 74, fig. 1 (1-b). Type locality: Qinghai-Xizang plateau region, China. Syntypes: IHASW 606164-66 (3), IHASW 606168 (1), IHASW 606170-74 (5), IHASW 606178 (1), IHASW 6006651-53 (3).

Glyptothorax longinema Li, 1984b: 81, fig. 6. Type locality: Lushui Xian, Yunnan, China. Holotype: KIZ 741097.

Glyptothorax rubermentus Li, 1984b: 83, 88, fig. 8. Type locality: Baoshan Xian, Yunnan, China. Holotype: KIZ 749356.

Distribution: Mekong and Salween River basins, China (Chu et al., 1999, as Glyptothorax zainaensis; Kottelat, 2001b).

Glyptothorax zhujiangensis Lin, 2003

Glyptothorax zhujiangensis Lin, 2003: 159, 161. Type locality: Baishuidai Streamin Yaxi Town $\left(22^{\circ} 15^{\prime} \mathrm{N}\right.$, 11259'25"E), Xinhui City, middle-southern Guandong, China. Holotype: ZX 970901 (housed in IHASW).

Distribution: Zhujiang [= Pearl River] China (Lin, 2003).

Species inquirendae, Glyptothorax 
Glyptosternum laak Popta, 1904: 190. Type locality: le Howong, Bornéo central. Syntypes: RMNH 7562 (3); described in more detail, with an illustration of one of the syntypes, in Popta (1906: 71, pl. 4, figs. 15a, 15b).

Glyptosternum tiong Popta, 1904: 191. Type locality: le Kajan, Bornéo central. Syntypes: RMNH 7564 (2); described in more detail, with an illustration of one of the syntypes in Popta (1906: 75, pl. 4, fig. 16).

Glyptothorax conirostre poonaensis Hora, 1938d: 368, pl. 7 (figs. 5-6). Type locality: Mula Mutha R. at Poona, Maharashtra, Bombay State, India. Holotype: ZSI F12126/1. Originally as Glyptothorax conirostre var. poonaensis. Treated as valid or as a synonym of Glyptothorax conirostris (Steindachner, 1867).

\section{GOGANGRA Roberts, 2001}

Gangra Roberts \& Ferraris, 1998: 333. Type species: Pimelodus viridescens Hamilton, 1822. Type by original designation. Gender: Feminine. Preoccupied by Gangra Walker, 1862; in Lepidoptera, replaced by Gogangra Roberts, 2001.

Gogangra Roberts, 2001: 83. Type species: Pimelodus viridescens Hamilton, 1822. Type by being a replacement name. Gender: Feminine. Replacement for Gangra Roberts \& Ferraris, 1998; preoccupied by Gangra Walker, 1862 , in Lepidoptera.

Gogangra laevis $\mathrm{Ng}, 2005$

Gogangra laevis Ng, 2005c: 280, fig. 1. Type locality: Bangladesh: Gowain River and Khal at Gowainghat. Holotype: UMMZ 244603.

Distribution: Yamuna and Meghna Rivers, lower Brahmaputra River basin, Bangladesh (Ng, 2005c).

Gogangra viridescens (Hamilton, 1822)

Pimelodus viridescens Hamilton, 1822: 173, 377, pl. 11 (fig. 56). Type locality: Rivers in the northern parts of Bengal. No types known.

Nangra punctata Day, 1877 (in Day, 1875-78): 494, pl. 115 (fig. 8). Type locality: Sone River at Bheer Bhoom in Bengal, India. Syntype: AMS B.7566 (1).

Distribution: Ganges and Brahmaputra River basins (Roberts \& Ferraris, 1998; Ng, 2005c).

HARA Blyth, 1860

Hara Blyth, 1860: 152. Type species: "Hara buchanani nobis; Pimelodus hara, B.H.” [= Pimelodus hara Hamilton, 1822]. Type by original designation. Gender: Feminine.

Laguvia Hora, 1921b: 739. Type species: Pimelodus asperus M'Clelland, 1844. Type by subsequent designation, by Jordan (1923: 148). Gender: Feminine.

Remarks: Jayaram (1973c, 1999) lists Laguvia shawi as the type species of Laguvia, by subsequent designation, but it is not stated where this designation was published. If published before 1923, it would have priority over the designation by Jordan (1923). Therefore, the type species as stated above must be considered tentative until the details of the designation of L. shawi are clarified. Synonymy of Laguvia in Hara follows Ng \& Kottelat (2005: 20).

Hara filamentosa Blyth, 1860

Hara filamentosa Blyth, 1860: 152. Type locality: Tenasserim [Burma]. Syntypes: ZSI 585 (6).

Distribution: Widely distributed in Myanmar.

Hara hara (Hamilton, 1822)

Pimelodus hara Hamilton, 1822: 190, 378. Type locality: River Kosi [now Hooghly River south of Ranaghat, by neotype designation]. Neotype: UMMZ 244697, designated by, and illustrated in, Ng \& Kottelat (2005: 20, fig. 5). Previously unpublished Hamilton illustration reproduced in Hora (1929: pl. 21, fig. 5) and reproduced in Ng \& Kottelat (2005: 20, fig. 6).

Pimelodus asperus M'Clelland, 1844a: 404, pl. 24 (fig. 2). Type locality: Chusan, China [now Hooghly River south of Ranaghat, by neotype designation]. Neotype: UMMZ 244697, designated by, and illustrated in, $\mathrm{Ng} \&$ Kottelat (2005: 19, fig. 5).

Hara buchanani Blyth, 1860: 152. Type locality: River Kosi [now Hooghly River south of Ranaghat, by neotype designation]. Neotype: UMMZ 244697, designated by, and illustrated in, Ng \& Kottelat (2005: 20, fig. 5) as 
neotype of Pimelodus hara. Unneeded new name for Pimelodus hara Hamilton, 1822.

Distribution: Bihar, Uttar Pradesh, Orissa, northern Bengal and Assam, India; Bangladesh and Myanmar [questionable] (Jayaram, 1979).

Remarks: Redescribed in Tilak (1978).

\section{Hara horai Misra, 1976}

Hara horai Misra, 1976: 245, pl. 9 (figs. 1-3). Type locality: Terai and Duars, n. Bengal. Syntype: ZSI FF955 (formerly ZSI F 11390/1), illustrated in Hora (1950: pl. 2, figs. 1-3) as Hara hara.

Distribution: Terai Duars, northern Bengal, India (Misra, 1976).

Remarks: Neotype designation by Tilak \& Talwar (1976: 246) [ZSI FF955] invalid inasmuch as that specimen is apparently one of the original syntypes (M. Kottelat, pers. commum.). Redescribed in Tilak (1978).

Hara jerdoni Day, 1870

Hara jerdoni Day, 1870a: 39, pl. 4 (figs. 2a-c). Type locality: Sylhet district. Syntypes (2): ZSI 431 (1).

Distribution: Sylhet district, Bangladesh (Jayaram, 1979).

Remarks: Redescribed in Husain \& Tilak (1978).

Hara saharsai Datta Munshi \& Srivastava, 1988

Hara saharsai Datta Munshi \& Srivastava, 1988: 265, fig. 5. Type locality: Kosi Belt, n. Bihar, India. Syntypes: Bhagalpur Univ., Dept. Zool. Mus. 67 (13). Also appeared as new in Srivastava (1989: 121, fig. 1).

Distribution: northern Bihar, India (Datta Munshi \& Srivastava, 1988).

Hara serrata Vishwanath \& Kosygin, 2000

Hara serratus Vishwanath \& Kosygin, 2000: 143, figs. 2-5. Type locality: Jiri river at Jiribam, Manipur, India. Holotype: MUMF 2508.

Distribution: Barak and Jiri Rivers, Manipur, India (Vishwanath \& Kosygin, 2000).

MYERSGLANIS Hora \& Silas, 1952

Myersglanis Hora \& Silas, 1952a: 19. Type species: Exostoma blythii Day, 1870. Type by original designation. Gender: Masculine.

Key: Vishwanath \& Kosygin (1999: 296).

Myersglanis blythii (Day, 1870)

Exostoma Blythii Day, 1870b: 525. Type locality: not stated. Syntypes (2): Asiatic Society of Bengal 599 (2) [now at ZSI] (2), possibly ZSI 2361 (1).

Distribution: Possibly Pharping, Nepal (Regan, 1907c; Hora \& Silas, 1952a).

Myersglanis jayarami Vishwanath \& Kosygin, 1999

Myersglanis jayarami Vishwanath \& Kosygin, 1999: 291, pl. 1. Type locality: Laniye river at Jessami, Manipur, India (94 $\left.32^{\prime} \mathrm{E}, 25^{\circ} 38^{\prime} \mathrm{N}\right)$. Holotype: MUMF 2138.

Distribution: Lainye River, Chindwin River basin, Manipur, India (Vishwanath \& Kosygin, 1999).

NANGRA Day, 1877

Nangra Day, 1877 (in Day, 1875-78): 493. Type species: Pimelodus nangra Hamilton, 1822. Type by absolute tautonymy. Gender: Feminine.

Revision: Hora \& Law (1941); Roberts \& Ferraris (1998).

Remarks: See Jayaram (1972a) for comments on the validity of the genus.

Nangra assamensis Sen \& Biswas, 1994

Nangra assamensis Sen \& Biswas, 1994: 441, fig. 1; pl. 1. Type locality: Brahmaputra River at Neematighat, 14 kms. from Jorhat, Assam [India]. Holotype: ZSI V/F/ERS/53.

Nangra carcharhinoides Roberts \& Ferraris, 1998: 338, fig. 16. Type locality: Ganges River at Patna, India. Holotype: CAS 95566.

Distribution: Ganges and Brahmaputra Rivers, India (Roberts \& Ferraris, 1998).

Nangra bucculenta Roberts \& Ferraris, 1998

Nangra bucculenta Roberts \& Ferraris, 1998: 336, fig. 14. Type locality: Ganges River delta, Tangail District, North Central Region, Bangladesh. Holotype: CAS 95564. 
Distribution: Ganges River delta, Bangladesh (Roberts \& Ferraris, 1998).

Nangra nangra (Hamilton, 1822)

Pimelodus nangra Hamilton, 1822: 193, 378, pl. 11 (fig. 63). Type locality: Kosi River [now: Ganges River at Patna, India, by neotype designation]. Neotype: CAS 96626, designated by Roberts \& Ferraris (1998: 341).

Nangra Buchanani Day, 1877 (in Day, 1875-78): 494, pl. 113 (fig. 3). Kosi River [now: Ganges River at Patna, India, by neotype designation]. Neotype: CAS 96626. Replacement for Pimelodus nangra Hamilton, 1822, to avoid tautomymy.

Distribution: Ganges and Indus River basins (Roberts \& Ferraris, 1998).

Nangra ornata Roberts \& Ferraris, 1998

Nangra ornata Roberts \& Ferraris, 1998: 341, fig. 19. Type locality: Bangladesh: Gowain River and Khal at Gowainghat, northern Sylhet Province (Surma or Meghna watershed). Holotype: UMMZ 233236.

Distribution: Surma or Meghna watershed, Bangladesh (Roberts \& Ferraris, 1998).

Nangra robusta Mirza \& Awan, 1973

Nangra robusta Mirza \& Awan, 1973: 145, fig. 1. Type locality: Indus River at Jinnah Barrage near Kalabagh, Pakistan. Holotype: GCM 9.

Distribution: Indus River, Pakistan (Mirza \& Awan, 1973).

OREOGLANIS Smith, 1933

Oreoglanis Smith, 1933: 70. Type species: Oreoglanis siamensis Smith, 1933. Type by original designation. Gender: Masculine.

Paroreoglanis Pellegrin, 1936: 244. Type species: Paroreoglanis delacouri Pellegrin, 1936. Type by monotypy. Gender: Masculine.

Oreoglanis delacouri (Pellegrin, 1936)

Paroreoglanis delacouri Pellegrin, 1936b: 244. Type locality: Xieng Khouang in upper Laos. Lectotype: MNHN 1936-0031 (103.4 mm SL), designated by Ng \& Kottelat (1999: 379).

Distribution: Nam Ngiap basin, Mekong drainage, Laos (Ng \& Rainboth, 2001).

Oreoglanis frenatus $\mathrm{Ng} \&$ Rainboth, 2001

Oreoglanis frenatus Ng \& Rainboth, 2001: 7, fig. 6. Type locality: Laos: Xieng Khouang Province, Nam Ka basin, Houay Kheua at Hwy 7 bridge, 19³8'N, 103²8'E. Holotype: UMMZ 236811.

Distribution: Nam Ka Basin, Mekong drainage, Laos (Ng \& Rainboth, 2001).

Oreoglanis hypsiurus $\mathrm{Ng} \&$ Kottelat, 1999

Oreoglanis hypsiurus Ng \& Kottelat, 1999: 376, fig. 1. Type locality: Upper Nam Theun, ca. 1 km upstream of confluence with Houay Nuok Lan, 1804'09"N, 105²9'44"E, Kham-mouan Province, Laos. Holotype: ZRC 40440. Distribution: Nam Kading basin, Mekong drainage, Laos (Kottelat, 2001b).

Oreoglanis infulatus $\mathrm{Ng} \&$ Freyhof, 2001

Oreoglanis infulatus Ng \& Freyhof, 2001b: 1165, figs. 1-3. Type locality: Vietnam: Ha Tinh Province, stream at Son Kim, a tributary of Song Lam (18²4'25"N, 105¹1'10"E). Holotype: ZFMK 35719.

Distribution: Lam River headwaters, central Vietnam (Ng \& Freyhof, 2001b).

Oreoglanis insignis $\mathrm{Ng} \&$ Rainboth, 2001

Oreoglanis insignis Ng \& Rainboth, 2001: 15, fig. 8. Type locality: China: Yunnan Province, Baoshan Prefecture, Longchuanjiang and Dajiang, near Qushi. Holotype: KIZ 9810191.

Distribution: Upper Irrawaddy River basin, China and Myanmar, possibly upper Salween basin (Ng \& Rainboth, 2001).

Oreoglanis lepturus $\mathrm{Ng} \&$ Rainboth, 2001

Oreoglanis lepturus Ng \& Rainboth, 2001: 17, fig. 10. Type locality: Laos: Bolikamsai Province, Nam Phao about 2 $\mathrm{km}$ from Vietnam border, 18²3'N, 105¹9'E. Holotype: UMMZ 236814.

Distribution: Nam Phao basin, Mekong drainage, northeastern Laos (Ng \& Rainboth, 2001).

Oreoglanis macronemus $\mathrm{Ng}, 2004$

Oreoglanis macronemus Ng, 2004e: 209, fig. 1. Type locality: Laos: Xieng Khouang. Holotype: BMNH 1933.8.19.51. 
Distribution: Nam Ngiap or Nam Sen River basins, Xieng Khouang region, Laos (Ng, 2004e).

Oreoglanis macropterus (Vinciguerra, 1890)

Exostoma macropterum Vinciguerra, 1890: 253, pl. 8 (fig. 5). Type locality: Paese dei Catcin, Birmania [Myanmar].

Syntypes: BMNH 1893.2.16.18 (1), MSNG 14410 (3), NMW 46488 (1), RMNH 10236 (1), USNM 44805 (1)

Distribution: Irrawaddy River basin; Salween River, China (Ng \& Rainboth, 2001).

Remarks: See Steinitz (1961) for comments on taxonomy.

Oreoglanis setiger $\mathrm{Ng} \&$ Rainboth, 2001

Oreoglanis setiger Ng \& Rainboth, 2001: 23, fig. 12. Type locality: Laos: Louang Namtha Province, Nam Ma Oun, $21^{\circ} 05^{\prime} \mathrm{N}, 101^{\circ} 04^{\prime} \mathrm{E}$. Holotype: UMMZ 236813.

Distribution: Nam Oun basin, Mekong River drainage, Laos, and upper Mekong River southwestern China (Ng \& Rainboth, 2001).

Oreoglanis siamensis Smith, 1933

Oreoglanis siamensis Smith, 1933: 72, pl. 3 (figs. 1-2); fig. 4. Type locality: Kang River near the base of Doi Angka, northern Siam. Holotype: KUMF 0172.

Distribution: Mae Nam Ping basin, Chao Phraya drainage, northern Thailand (Ng \& Rainboth, 2001).

Remarks: Statements in Menon \& Yazdani (1968) and Eschmeyer et al. (1998) which indicated that ZSI F12233/1 might be the holotype of Oreoglanis siamensis are in error. Smith (1933: 74) clearly stated that two specimens were examined, which he identified as the "type" and "topotype," and that the specimen deposited in the Indian Museum (now ZSI) was the topotype.

PARACHILOGLANIS Wu, He \& Chu, 1981

Parachiloglanis Wu, He \& Chu, 1981: 76, 79. Glyptosternum hodgarti Hora, 1923. Type by original designation. Gender: Masculine.

Parachiloglanis hodgarti (Hora, 1923)

Glyptosternum hodgarti Hora, 1923b: 38, pl. 2 (figs. 1-3). Type locality: Pharping, Nepal. Holotype: ZSI F1553/1.

Distribution: Yaluzangbujiang [= upper Brahmaputra River] (Chu et al., 1999); Abor Hills, Meghalaya, Darjeeling, Kali River, Teesta valley and Nepal (as Euchiloglanis hodgarti) (Jayaram, 1999).

PAREUCHILOGLANIS Pellegrin, 1936

Pareuchiloglanis Pellegrin, 1936b: 245. Type species: Pareuchiloglanis poilanei Pellegrin, 1936. Type by monotypy. Gender: Masculine.

Remarks: Included species follows He (1996b).

Review: Ding (2003, western China).

Pareuchiloglanis anteanalis Fang, Xu \& Cui, 1984

Pareuchiloglanis anteanalis Fang, Xu \& Cui, 1984: 209, fig.1. Type locality: Wudu County, Gansu Province, China. Holotype: Shaanxi Inst. Zool. 82VI9565.

Distribution: Wudu County, Gansu Province, China (Fang, et al., 1984).

Pareuchiloglanis feae (Vinciguerra, 1890)

Exostoma Feae Vinciguerra, 1890: 256, pl. 8 (fig. 6). Type locality: Taó, e Iadó, Birmania [Myanmar]. Syntypes: BMNH 1893.2.16.19-20 (2), BMNH 1893.2.16.21-22 (2), BMNH 1893.2.16.23 (1, skeleton), MSNG 14411 (4), MSNG 14412 (3), MSNM 38 (1), NMW 9064-65 (2), RMNH 10237 (3), USNM 44743 (3).

Distribution: Irrawaddy and Salween Rivers, China (Chu et al., 1999).

Pareuchiloglanis gongshanensis Chu, 1981

Pareuchiloglanis gongshanensis Chu, 1981: 28, 31, fig. 2. Type locality: Gongshan Xian, Yunnan, China. Holotype: KIZ 731202.

Distribution: Upper Nujiang [= Salween River], China (Chu et al., 1999).

Pareuchiloglanis gracilicaudatus (Wu \& Chen, 1979)

Euchiloglanis gracilicaudata Wu \& Chen, 1979: 294, fig. 4. Type locality: Za-Qu, upper Lancang Jiang, Nangqen, Qinghai, China. Syntypes: Qinghai Inst. Biol. (12).

Distribution: Lancangjiang [= Mekong River], China (Chu et al., 1999). 
Pareuchiloglanis kamengensis (Jayaram, 1966)

Euchiloglanis kamengensis Jayaram, 1966d: 85, fig. 1. Type locality: Norgum River at Kalaktang, $1370 \mathrm{~m}$ alt., Kameng Frontier Division, N.E.F.A. Holotype: ZSI F2105/2.

Distribution: Yaluzangbujiang [= upper Brahmaputra River], Irrawaddy, Nujiang [= Salween River], Lancangjiang [= Mekong River], China (Chu et al., 1999).

Pareuchiloglanis longicauda (Yue, 1981)

Euchiloglanis longicauda Yue, in Zheng, 1981: 183, fig. 151. Type locality: Panyang R. (Hong-Shui R. system), Bama Co., Guangxi Prov., China. Holotype: ASIZB and Fish. Res. Inst., Guangxi Zhuangzu Autonomous Region 750443.

Distribution: Nanpanjiang, Beipaniang, Hongshuihe, China (Chu et al., 1999).

Pareuchiloglanis macropterus $\mathrm{Ng}, 2004$

Pareuchiloglanis macropterus Ng, 2004g: 3, fig. 1. Type locality: China, Yunnan province, Salween River (Nujiang) drainage; Laowo River, a tributary of Salween River (Nujiang), ca. $5 \mathrm{~km}$ to Liuku on Yongping-Liuku Road, 2550'18.6"N 9853'46.8"E; 900 m asl. Holotype: ZRC 49124.

Distribution: Salween and Irrawaddy River basins, southern China (Ng, 2004g).

Pareuchiloglanis macrotremus (Norman, 1925)

Euchiloglanis macrotrema Norman, 1925b: 570. Type locality: Ngoi-Tio, Col des Nuages, Tonkin, Vietnam, elev. 4500-6500 ft. Syntypes: BMNH 1925.2.19.5-6 (2).

Distribution: Yuanjiang, China (Chu et al., 1999); Tonkin, Vietnam.

Pareuchiloglanis myzostomus (Norman, 1923)

Euchiloglanis myzostoma Norman, 1923a: 562. Type locality: Yunnan, China. Syntypes: BMNH 1923.2.21.40-49 (10).

Distribution: Lancangjiang [= Mekong River], China (Chu et al., 1999).

Pareuchiloglanis nebulifer $\mathrm{Ng} \&$ Kottelat, 2000

Pareuchiloglanis nebulifer $\mathrm{Ng} \&$ Kottelat, 2000b: 11, fig. 4. Type locality: Small creek, trib. of Houai Siam, upstream of Ban Kangpabong, Houaphan Prov., Laos, 20¹9'36"N, 104²5'01"E. Holotype: ZRC 45706.

Distribution: Nam Xam and Nam Ma basins, Ma River Drainage, Laos (Kottelat, 2001b).

Pareuchiloglanis poilanei Pellegrin, 1936

Pareuchiloglanis poilanei Pellegrin, 1936b: 246. Type locality: Annam [Vietnam]. Syntypes: MNHN 1936-0005 (1), MNHN 1936-0006 (1), MNHN 1936-0007 (1), MNHN 1936-0008 (1), MNHN 1936-0009 (1), MNHN 1936-0010 (1), MNHN 1936-0011 (1), MNHN 1936-0012 (1), MNHN 1936-0013 (1), MNHN 1936-0014 (1), MNHN 1936-0015 (1), MNHN 1935-0016 (1), MNHN 1936-0017 (1), MNHN 1936-0018 (1), MNHN 19360019 (1), MNHN 1936-0020 (4).

Distribution: Central Vietnam.

Pareuchiloglanis rhabdurus $\mathrm{Ng}, 2004$

Pareuchiloglanis rhabdurus Ng, 2004g: 7, fig. 3. Type locality: Vietnam: Ha Giang province, Red River (Song Hong) drainage, Vi Xuyen district, Cao Bo stream (Bac Trao river) near camp 1, 2245'18.0"N, 104'52'11'4"E. Holotype: AMNH 211153.

Distribution: Song Lo River, Red River basin, northern Vietnam (Ng, 2004g).

Pareuchiloglanis robustus Ding, Fu \& Ye, 1991

Pareuchiloglanis robusta Ding, Fu \& Ye, 1991: 369, fig. 1. Type locality: a stream (Qing Yijing), Sichuan, China. Holotype: SPNRI 8801.

Distribution: Yangtze River basin, China (Ding et al., 1991).

Pareuchiloglanis sichuanensis Ding, Fu \& Ye, 1991

Pareuchiloglanis sichuanensis Ding, Fu \& Ye, 1991: 371, fig. 2. Type locality: Baoxing, Sichuan Province, China. Holotype: Sichuan Agric. Univ. 6-1481.

Distribution: Yangtze River basin, China (Ding et al., 1991).

Pareuchiloglanis sinensis (Hora \& Silas, 1952)

Euchiloglanis sinensis Hora \& Silas, 1952a: 17, fig. 2. Type locality: presumably .... from some part of China, possibly Yunnan. Holotype: ZSI F12208/1. 
Distribution: Jinshajiang, China (Chu et al., 1999).

Pareuchiloglanis songdaensis Nguyen \& Nguyen, 2001

Pareuchiloglanis songdaensis Nguyen \& Nguyen, 2001: 67, fig. 1. Type locality:[Da River, Muong Te, Lai Chau Town and Phong Tho, Vietnam]. Holotype (115 mm SL): LM 2001.01 H.

Distribution: Da River, Vietnam (Nguyen \& Nguyen, 2001).

Pareuchiloglanis songmaensis Nguyen \& Nguyen, 2001

Pareuchiloglanis songmaensis Nguyen \& Nguyen, 2001: 68, fig. 2. Type locality: [Ma River, Song Ma district, Son La Province, Vietnam]. Holotype (82 mm SL): LM 2001.02H.

Distribution: Ma River, Vietnam (Nguyen \& Nguyen, 2001).

Pareuchiloglanis tianquanensis Ding \& Fang, 1997

Pareuchiloglanis tianquanensis Ding \& Fang, 1997: 17, fig. 1. Type locality: a stream (Upper Qing Yi River), altitude 2010 m., Tianquan County, Sichuan Province [China]. Holotype: SPNRI 920185.

Distribution: Upper Qing Yi River basin, Sichuan, China (Ding \& Fang, 1997).

PSEUDECHENEIS Blyth, 1860

Pseudecheneis Blyth, 1860: 154. Type species: Glyptosternon sulcatus M'Clelland, 1842. Type by monotypy. Gender: Feminine.

Parapseudecheneis Hora, in Hora \& Chabanaud, 1930: 216. Type species: Pseudecheneis paviei Vaillant, 1892. Type by monotypy. Gender: Feminine.

Propseudecheneis Hora, 1937d: 348.Type species: Propseudecheneis tchangi Hora, 1937. Type by original designation. Gender: Feminine.

Review: Hora (1952); Hora \& Chabanaud (1930); Chu (1982), China.

Phylogeny: Chu (1982), Zhou \& Zhou (2005).

Pseudecheneis crassicauda $\mathrm{Ng} \&$ Edds, 2005

Pseudecheneis crassicauda Ng \& Edds, 2005b: 2, fig. 1. Type locality: Nepal: Mewa Khola (River), Dhakuta District, $27^{\circ} 0^{\prime} \mathrm{N}, 8^{\circ} 20^{\prime} \mathrm{E}$. Holotype: BMNH 1958.9.1.8.

Distribution: Tamur River basin, Kosi drainage, Nepal (Ng \& Edds, 2005b).

Pseudecheneis immaculata Chu, 1982

Pseudecheneis immaculatus Chu, 1982: 428, 447, fig. 1. Type locality: Baijixun (upper Lancang River), Weixi County, Yunnan, China. Holotype: KIZ 748742.

Distribution: upper Langcanjiang [= Mekong River], China (Chu et al., 1999).

Pseudecheneis paviei Vaillant, 1892

Pseudecheneis paviei Vaillant, 1892a: 126. Type locality: Near Lai Chau, Tonkin, northern Vietnam. Holotype: MNHN 1892-0049; redescribed and illustrated in Vaillant (1904: 464, pl. 22, fig. 3). Holotype illustrated in Hora \& Chabanaud (1930: 217, fig. 2).

Pseudecheneis intermedius Chu, 1982: 430, 447, fig. 2. Type locality: Dongbao (a stream at upper reaches of Babian River, belonging to the Red River drainage), Jingdong County [Yunnan, China]. Holotype: KIZ 737173.

Distribution: Northern Vietnam and Yunnan, China (Kottelat, 2001a).

Remarks: Synonymy follows Ng \& Edds (2005b: 12).

Pseudecheneis serracula $\mathrm{Ng} \&$ Edds, 2005

Pseudecheneis serracula Ng \& Edds, 2005b: 6, fig. 3. Type locality: Nepal: Mugu/Bajura, Jhugala, Karnali River, purchased at Jhugala, 29³1'18.0"N, 81'46'48.0"E. Holotype: KU 29554.

Distribution: Karnali and Gandaki River basins, Nepal (Ng \& Edds, 2005).

Pseudecheneis sulcata (M'Clelland, 1842)

Glyptosternon sulcatus M'Clelland, 1842: 587, pl. 6 (in part). Type locality: Kasyah Hills. Holotype: BMNH 2005.5.17.5 (76 mm SL).

Distribution: Lancangjiang [ = Mekong River], Nujiang [= Salween River], Yaluzangbujiang [= upper Brahmaputra River], Irrawaddy River, China (Chu et al., 1999).

Pseudecheneis sulcatoides Zhou \& Chu, 1992

Pseudecheneis sulcatoides Zhou \& Chu, 1992: 111, 115, figs. 1-3. Type locality: Yangbi (2540'N, 9957'E), [Lan- 
cangjiang River (=upper Mekong)] Yunnan Province, China. Holotype: KIZ 839059.

Distribution: Upper Mekong River basin, Yunnan, China (Zhou \& Chu, 1992).

Pseudecheneis sympelvica Roberts, 1998

Pseudecheneis sympelvicus Roberts, 1998: 290, fig. 1. Type locality: Nam Veo, tributary of Nam Phao 25 km east of

Lak Sao, Nam Theun watershed, Mekong Basin, central Laos. Holotype: ZRC 40359.

Distribution: Nam Theun watershed, Mekong River basin, Laos (Roberts, 1998).

Pseudecheneis tchangi Hora, 1937

Propseudecheneis tchangi Hora, 1937d: 348, fig. 11b. Type locality: Yunnan, China. Holotype: Zoological Museum of Fan Memorial Institute, Beijing, 12016.

Distribution: Red River, Yunnan, China (Hora, 1937d).

PSEUDEXOSTOMA Chu, 1979

Pseudexostoma Chu, 1979: 78, 81. Type species: Glyptosternum yunnanensis Tchang, 1935. Type by original designation. Gender: Neuter.

Pseudexostoma brachysoma Chu, 1979

Pseudexostoma yunnanensis brachysoma Chu, 1979: 78, 81, fig. 3. Type locality: Hill stream running into the $\mathrm{Nu}$ River, near Laowo, Yunlong Xian [Yunnan, China]. Holotype: KIZ 742093.

Distribution: Nujiang [= Salween River], China (Chu et al., 1999).

Pseudexostoma yunnanense (Tchang, 1935)

Glyptosternum yunnanensis Tchang, 1935b: 174, fig. 1. Type locality: Yunnan, China. Holotype: ASIZB [= ZMFMIB] 12027.

Distribution: Irrawaddy River, China (Chu et al., 1999).

PSEUDOLAGUVIA Misra, 1976

Pseudolaguvia Misra, 1976: 253. Type species: Glyptothorax tuberculatus Prashad \& Mukerji, 1929. Type by original designation. Gender: Feminine.

Remarks: See Jayaram (1973c) for comments on this genus, but as Laguvia.

Pseudolaguvia foveolata $\mathrm{Ng}, 2005$

Pseudolaguvia foveolata Ng, 2005b: 174, fig. 1. Type locality: India: West Bengal: Tista River at Tista barrage, 2645'10"N, 88³4'11"E. Holotype: UMMZ 244867.

Distribution: Tista River, Brahamputra River basin, India (Ng, 2005b).

Pseudolaguvia inornata $\mathrm{Ng}, 2005$

Pseudolaguvia inornata Ng, 2005g: 36, fig. 1. Type locality: Bangladesh: Chittagong District, Koilla Khal (creek), $10 \mathrm{~km}$ E of Feni-Chittagong highway on road to Ramgarh, 2255'N, 91³6'E. Holotype: UMMZ 245580.

Distribution: Feni River basin, Bangladesh (Ng, 2005g).

Pseudolaguvia kapuri (Tilak \& Husain, 1975)

Laguvia ribeiroi kapuri Tilak \& Husain, 1975: 1, figs. 1-3. Type locality: Padhoi river, near Kalsia Ghat, Saharanpur, (U. P.) [India]. Holotype: ZSI/NRS 836.

Distribution: Padhoi River and Dhamola River, Saharanpur, Uttar Pradesh (Jayaram, 1999).

Remarks: Redescribed in Gupta (1982).

Pseudolaguvia muricata $\mathrm{Ng}, 2005$

Pseudolaguvia muricata Ng, 2005g: 41, fig. 3. Type locality: Bangladesh: Sylhet District, Rangapani Khal (creek), 6 km WNW of Sylhet-Shillong highway, $25^{\circ} 10^{\prime} \mathrm{N}, 92^{\circ} 6^{\prime} \mathrm{E}$. Holotype: UMMZ 245581.

Distribution: Brahmaputra River basin, Bangladesh (Ng, 2005g).

Pseudolaguvia ribeiroi (Hora, 1921)

Laguvia ribeiroi Hora, 1921b: 741, pl. 29 (fig. 3). Type locality: Khoila River, tributary of Teesta at Jalpaiguri [India]. Holotype: ZSI F 10086/1.

Distribution: Teesta River system, north Bengal; Kosi River system Behar; Rihand River system, Uttar Pradesh, and rivers of Nepal (Jayaram, 1979, 1999).

Pseudolaguvia shawi (Hora, 1921) 
Laguvia shawi Hora, 1921b: 740, pl. 29 (fig. 2). Type locality: Mahanadi River below Darjeeling, India. Holotype: ZSI F10085/1.

Distribution: North Bengal, India, and Bangladesh (Jayaram, 1999); Brahmaputra River basin, Assam, India (Arunkumar, 2000).

Pseudolaguvia tenebricosa Britz \& Ferraris, 2003

Pseudolaguvia tenebricosa Britz \& Ferraris, 2003: 2, fig. 1. Type locality: Myanmar, Kayin Division, Pathe Chaung, hill stream, 13 miles east of Taungoo, 1901'11"N, 96³5'33"E. Holotype: USNM 373293.

Distribution: Sittang and upper Irrawaddy River basins, Myanmar (Britz \& Ferraris, 2003).

Pseudolaguvia tuberculata (Prashad \& Mukerji, 1929)

Glyptothorax tuberculatus Prashad \& Mukerji, 1929: 182, pl. 7 (fig. 2); fig. 4. Type locality: Sankha, a large hillstream, midway between Kamaing and Mogaung, Myitkyina dist., Upper Burma. Holotype: ZSI F10876/1.

Distribution: Myitkyina District, Kachin State, Myanmar (Prashad \& Mukerji, 1929).

SISOR Hamilton, 1822

Sisor Hamilton, 1822: 208, 379. Type species: Sisor rabdophorus Hamilton, 1822. Type by monotypy. Gender: Masculine.

Revision: $\mathrm{Ng}$ (2003c).

Sisor barakensis Vishwanath \& Darshan, 2005

Sisor barakensis Vishwanath \& Darshan, 2005: 1952, fig. 1a. Type locality: River Barak, Jiri, Manipur, India. Holotype: MUMF 3131. Holotype illustrated as Image 1 on web version of paper.

Distribution: Barak River, Brahmaputra River basin, India (Vishwanath \& Darshan, 2005).

Sisor chennuah $\mathrm{Ng} \&$ Lahkar, 2003

Sisor chennuah Ng \& Lahkar, in Ng, 2003c: 2876, fig. 5. Type locality: India, Assam State, Brahmaputra River drainage, Dibrugarh. Holotype: NRM 40420.

Distribution: Brahmaputra River basin, India and Bangladesh (Ng, 2003c).

Sisor rabdophorus Hamilton, 1822

Sisor rabdophorus Hamilton, 1822: 208, 379. Type locality: Northern rivers of Bengal and Behar [now India: West Bengal State, Bhagirathi River at crossing point between Kalna (Barddhaman District) and Nisinghapur (Nadia District) (23 $\left.{ }^{\circ} 13^{\prime} 33^{\prime \prime} \mathrm{N}, 88^{\circ} 32^{\prime} 41.4^{\prime \prime} \mathrm{E}\right)$, by neotype designation]. Neotype: ZRC 45829, designated by Ng (2003c: 2873, 2881).

Distribution: Ganges River basin, West Bengal, India (Ng, 2003c).

Remarks: Name often misspelled Sisor rhabdophorus, following the incorrect subsequent spelling in Cuvier \& Valenciennes (1840).

Sisor rheophilus $\mathrm{Ng}, 2003$

Sisor rheophilus Ng, 2003c: 2877, fig. 6. Type locality: India: Uttar Pradesh State, Kali Nadi River, near Muzaffarnagar. Holotype: UMMZ 189651.

Distribution: Upper and middle Ganges River basin, Bihar State and Uttar Pradesh, India (Ng, 2003c).

Sisor tortosus $\mathrm{Ng}, 2003$

Sisor tortosus Ng, 2003c: 2878, fig. 7. Type locality: India: Bihar State, Ganges River at Patna. Holotype: CAS 96629.

Distribution: Middle Ganges River basin, Bihar and Deli States, India (Ng, 2003c).

\section{Species inquirenda, Sisoridae}

Exostoma Oschanini Herzenstein, 1889a: 70. Upper Amu-Darya R., upper Syr-Derya R. basin (Ugam R, Tashkent), Uzbekistan. Syntypes: BMNH 1888.11.24.1 (1), ZIN 8055 (1), ZIN 8056 (1), ZIN 8057 (2). Also in Herzenstein (1889b:120).

\section{TRICHOMYCTERIDAE Bleeker, 1858}


Eremophilini Bonaparte, 1846: 5. Type genus: Eremophilus Humboldt, 1805.

Trichomycterini Bleeker, 1858b: 49, 250, 257. Type genus: Trichomycterus Valenciennes, 1832.

Vandelliini Bleeker, 1862 (in Bleeker, 1862-63): 17. Type genus: Vandellia Valenciennes, 1846.

Stegophilina Günther, 1864: 5, 12, 276. Type genus: Stegophilus Reinhardt, 1859.

Pareiodontinae Eigenmann, 1918c: 261. Type genus: Pareiodon Kner, 1855.

Tridentinae Eigenmann, 1918c: 275. Type genus: Tridens Eigenmann \& Eigenmann, 1889.

Glanapteryginae Myers, 1944: 592. Type genus: Glanapteryx Myers, 1927.

Sarcoglanidinae Myers \& Weitzman, 1966: 278. Type genus: Sarcoglanis Myers \& Weitzman, 1966.

Trichogeninae Isbrücker, 1986: 276. Type genus: Trichogenes Britski \& Ortega, 1983.

Copionodontinae de Pinna, 1992b: 179. Type genus: Copionodon de Pinna, 1992.

Taxonomic summary: de Pinna \& Wosiacki (2003).

Biogeography: Arratia (1990).

Revision: Eigenmann (1918a); Tchernavin (1944, Trichomcyterinae).

Phylogeny: Baskin (1973), de Pinna (1989b, Glanapteryginae); de Pinna (1992b); Costa (1994); Bockmann et al. (2004).

Review: Miranda Ribeiro (1946, 1951b, Brazilian Stegophilinae); Miranda Ribeiro (1947, Brazilian Vandelliinae); Miranda Ribeiro (1949b, 1956, 1957, Brazilian Trichomycterinae).

41 genera, 207 species; no named fossil taxa (but see Propygidium in section below entitled "Names wrongly treated as Siluriformes").

ACANTHOPOMA Lütken, 1892

Acanthopoma Lütken, 1892: 57. Type species: Acanthopoma annectens Lütken, 1892. Type by monotypy. Gender:

Neuter.

Acanthopoma annectens Lütken, 1892

Acanthopoma annectens Lütken, 1892: 53, figures on p. 56. Type locality: Huallaga. Holotype: MTD F5245.

Distribution: Upper and middle Amazon River basin, Brazil and Peru (de Pinna \& Wosiacki, 2003).

AMMOGLANIS Costa, 1994

Ammoglanis Costa, 1994: 208. Type species: Ammoglanis diaphanus Costa, 1994. Type by original designation.

Gender: Masculine.

Ammoglanis diaphanus Costa, 1994

Ammoglanis diaphanus Costa, 1994: 208, fig. 1. Type locality: Stream trib. to Rio Javaés, Rio Araguaia basin, ca. 40

km north of Araguaçú, 12²4'S, 4958'W, Tocantin state, Brazil. Holotype: MNRJ 12442.

Distribution: Stream tributary to Javaés River, Araguaia River basin, Brazil (de Pinna \& Wosiacki, 2003).

Ammoglanis pulex de Pinna \& Winemiller, 2000

Ammoglanis pulex de Pinna \& Winemiller, 2000: 257, fig. 1. Type locality: Venezuela: Estado Amazonas: Río Paria

Grande at bridge on road between Pto. Ayacucho and Samariapo ( $\left.5^{\circ} 23^{\prime} \mathrm{N} 67^{\circ} 37^{\prime} \mathrm{W}\right)$. Holotype: MBUCV V29040.

Distribution: Paria Grande River, Pamoni River and Caño Garrapata, Venezuela (de Pinna \& Wosiacki, 2003).

APOMATOCEROS Eigenmann, 1922

Apomatoceros Eigenmann, 1922a: 113. Type species: Apomatoceros alleni Eigenmann, 1922. Type by monotypy. Gender: Masculine.

Apomatoceros alleni Eigenmann, 1922

Apomatoceros alleni Eigenmann, 1922a: 113, pls. 3 (figs. 1-4), 4 (fig. 9). Type locality: Río Morona, upper Amazon system, Peru. Holotype: CAS 56174.

Distribution: Amazon River basin, Peru (de Pinna \& Wosiacki, 2003).

BULLOCKIA Arratia, Chang, Menu-Marque \& Rojas, 1978

Bullockia Arratia, Chang, Menu-Marque \& Rojas, 1978: 162, 187. Type species: Hatcheria maldonadoi Eigenmann, 1920. Type by monotypy. Gender: Feminine. 
Bullockia maldonadoi (Eigenmann, 1920)

Hatcheria Maldonadoi Eigenmann, 1920c: 53. Type locality: lower course of the Rio Nonguen where it passes through the ground of the Agricultural School, at the outskirts of Concepción, Chile. Lectotype: CAS 63842. Illustrated and described in more detail in Eigenmann (1927: 39, pl. 8, figs. 2-2b); with the lectotype established in caption to pl. 8.

Hatcheria bullocki Fowler, 1940b: 180, figs. 14-15. Type locality: Angol, Chile. Holotype: ANSP 69145. Böhlke (1984: 163) noted that stated locality in the original description is Angol, but catalog and label say "El Vergal."

Distribution: Chile (de Pinna \& Wosiacki, 2003).

Remarks: The description of Hatcheria maldonadoi stated that the Type (= Holotype) was illustrated. However, there was no illustration associated with that publication, so all of the examined specimens need to be treated as syntypes. The illustrated specimen in Eigenmann (1927) was labeled as the type, which is sufficient to be treated as a lectotype designation.

COPIONODON de Pinna, 1992

Copionodon de Pinna, 1992b: 181. Type species: Copionodon pecten de Pinna, 1992. Type by original designation. Gender: Masculine.

Copionodon lianae Campanario \& de Pinna, 2000

Copionodon lianae Campanario \& de Pinna, 2000: 370, figs. 1-2. Type locality: Brazil: Bahia: Rio Grisante (Rio

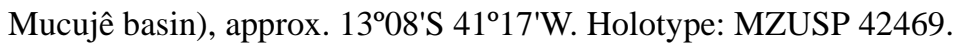

Distribution: Grisante River, tributary to Mucujê River, Paraguaçu River basin, Brazil (de Pinna \& Wosiacki, 2003).

Copionodon orthiocarinatus de Pinna, 1992

Copionodon orthiocarinatus de Pinna, 1992b: 188, figs. 3-4. Type locality: Rio Mucujê, trib. of Rio Paraguaçu, Bahia, Brazil, ca. 1300'S, 41²3'W, elev. 1200 m. Holotype: MZUSP 42463.

Distribution: Mucujê River, tributary of Paraguaçu River, Brazil (de Pinna \& Wosiacki, 2003).

Copionodon pecten de Pinna, 1992

Copionodon pecten de Pinna, 1992b: 182, figs. 1-2. Type locality: Rio Mucujê, trib. of R. Paraguaçu, Bahia, Brazil, ca. $13^{\circ} 00^{\prime} \mathrm{S}, 41^{\circ} 23^{\prime} \mathrm{W}$, elev. $1200 \mathrm{~m}$. Holotype: MZUSP 42461.

Distribution: Mucujê River, tributary of Paraguaçu River, Brazil (de Pinna \& Wosiacki, 2003).

\section{EREMOPHILUS Humboldt, 1805}

Eremophilus Humboldt, 1805a: 17. Type species: Eremophilus mutisii Humboldt, 1805. Type by monotypy. Gender: Masculine.

Thrichomycterus Humboldt, 1805a: 18. Type species: Eremophilus mutisii Humboldt, 1805. Type by monotypy. Gender: Masculine. Given as an alternative name, but never used subsequently, for Eremophilus Humboldt.

Trachypoma Giebel, 1871: 97. Type species: Trachypoma marmoratum Giebel, 1871. Type by original designation. Gender: Neuter. Preoccupied by Trachypoma Günther, 1859, in fishes; apparently not replaced.

Eremophilus mutisii Humboldt, 1805

Eremophilus mutisii Humboldt, 1805a: 18, pl. 6. Type locality: petite rivière de Bogota, qui forme la fameuse catarate de Tequendama, Colombia. No types known.

? Trachypoma marmoratum Giebel, 1871: 97. Type locality: obern Amazonenstrom. Type(s): Whereabouts unknown.

Distribution: Bogotá River basin; probably introduced to Ubaté, Chinquinquirá and Tundama valleys, Colombia (de Pinna \& Wosiacki, 2003).

GLANAPTERYX Myers, 1927

Glanapteryx Myers, 1927: 128. Type species: Glanapteryx anguilla Myers, 1927. Type by original designation. Gender: Feminine.

Glanapteryx anguilla Myers, 1927

Glanapteryx anguilla Myers, 1927: 129. Type locality: Rapids of São Gabriel, Rio Negro system, Brazil. Holotype: CAS 56048. 
Distribution: Negro and Orinoco River basins, Brazil and Venezuela (Nico \& de Pinna, 1996; de Pinna \& Wosiacki, 2003).

Remarks: Redescribed in de Pinna (1989b), with notes on distribution in Nico \& de Pinna (1996).

Glanapteryx niobium de Pinna, 1998

Glanapteryx niobium de Pinna, 1998b: 36, fig. 1. Type locality: Brazil, State of Amazonas, Pico da Neblina National Park, Morro dos Seis Lagos (approx. 017'N 66²41'W), Lago Esperança. Holotype: INPA 12421.

Distribution: Morro dos Seis Lagos, Negro River basin, Brazil (de Pinna \& Wosiacki, 2003).

GLAPHYROPOMA de Pinna, 1992

Glaphyropoma de Pinna, 1992b: 194. Type species: Glaphyropoma rodriguesi de Pinna, 1992. Type by original designation. Gender: Feminine.

Glaphyropoma rodriguesi de Pinna, 1992

Glaphyropoma rodriguesi, 1992b: 196, figs. 7-8. Type locality: Rio Mucujê, trib. of Rio Paraguaçu, Bahia, Brazil, ca. $13^{\circ} 00^{\prime} \mathrm{S}, 41^{\circ} 23^{\prime} \mathrm{W}$, elev. $1200 \mathrm{~m}$. Holotype: MZUSP 42465.

Distribution: Mucujê River, tributary of Paraguaçu River, Brazil (de Pinna \& Wosiacki, 2003).

HAEMOMASTER Myers, 1927

Haemomaster Myers, 1927: 131. Type species: Haemomaster venezuelae Myers, 1927. Type by original designation. Gender: Masculine.

Haemomaster venezuelae Myers, 1927

Haemomaster venezuelae Myers, 1927: 131. Type locality: Venezuela, Playa Matepalma, Orinoco. Holotype: CAS 55882.

Distribution: Amazon and Orinoco River basins, Brazil and Venezuela (de Pinna \& Wosiacki, 2003).

Remarks: Redescribed in Schmidt (1985).

HATCHERIA Eigenmann, 1909

Hatcheria Eigenmann, 1909b: 250. Type species: Hatcheria patagoniensis Eigenmann, 1909. Type by original designation. Gender: Feminine.

Revision: Arratia \& Menu-Marque (1981).

Hatcheria macraei (Girard, 1855)

Thrichomycterus macraei Girard, 1855: 245. Type locality: Near Uspullata, east side of the Cordilleras, Argentina, elev. 7000 ft. Syntypes: MCZ 8298 (1), USNM 1546 (orig. 3, now 1).

Pygidium Burmeisteri Berg, 1895: 128, pl. 2 (fig. 1). Type locality: Provincia Mendoza (Río Mendoza), Argentina. Holotype: at MACN.

Hatcheria patagoniensis Eigenmann, 1909b: 250, pl. 33 (fig. 2), pl. 34 (fig. 1). Type locality: Río Blanco at base of Andes, Patagonia, Argentina, $47^{\circ} 30^{\prime} \mathrm{S}, 72^{\circ} \mathrm{W}$. Holotype: Whereabouts unknown.

Hatcheria titcombi Eigenmann, 1918c: 692. Type locality: Rio Comajo; tributary of Lake Traful, tributary to Rio Limay, Argentina. Holotype: CAS 28557; holotype illustrated in Eigenmann (1918a: 284, pl. 44, fig. 2).

Hatcheria pique MacDonagh, 1938: 171, figs. 23-24. Type locality: Río Colorado cerca de su desembocadura, vecino a la estación Pedro Luro del F. C. S., Argentina. Holotype: MLP 15.X.36.

Distribution: Cis-Andean rivers of Argentina, between $29^{\circ}$ and 4530'S (de Pinna \& Wosiacki, 2003).

HENONEMUS Eigenmann \& Ward, 1907

Henonemus Eigenmann \& Ward, in Eigenmann, McAtee \& Ward, 1907: 118. Type species: Stegophilus intermedius Eigenmann \& Eigenmann, 1889. Type by original designation. Gender: Masculine.

Cobitiglanis Fowler, 1914: 268. Type species: Ochmacanthus taxistigma Fowler, 1914. Type by original designation. Gender: Masculine. Proposed originally as a subgenus of Ochmacanthus.

Henonemus intermedius (Eigenmann \& Eigenmann, 1889)

Stegophilus intermedius Eigenmann \& Eigenmann, 1889b: 54. Type locality: Goyaz [Brazil]. Holotype: MCZ 9842. Distribution: Araguaia River basin, Brazil (de Pinna \& Wosiacki, 2003). 
Henonemus macrops (Steindachner, 1882)

Stegophilus macrops Steindachner, 1882b: 178. Type locality: Amazonenstrom. Holotype: at NMW. Species illustrated and described in more detail in Steindachner (1882c: 28, pl. 6, figs. 2, 2a).

Distribution: Amazon River basin, Brazil (de Pinna \& Wosiacki, 2003).

Henonemus punctatus (Boulenger, 1887)

Stegophilus punctatus Boulenger, 1887c: 279, pl. 21 (fig. 4). Type locality: Canelos. Holotype: BMNH 1880.12.8.89.

Distribution: Amazon River basin of Brazil, Ecuador and Peru (de Pinna \& Wosiacki, 2003).

Henonemus taxistigma (Fowler, 1914)

Ochmacanthus taxistigma Fowler, 1914: 268, fig. 16. Type locality: Rupununi River, British Guiana ... in the highlands of British Guiana, approximately secured in North Latitude $2^{\circ}$ to $3^{\circ}$, and West Longitude $50^{\circ} 20^{\prime}$. Holotype: ANSP 39344.

Distribution: Rupununi River basin, Guyana (de Pinna \& Wosiacki, 2003).

HOMODIAETUS Eigenmann \& Ward, 1907

Homodiaetus Eigenmann \& Ward, in Eigenmann, McAtee \& Ward, 1907: 117. Type species: Homodiaetus anisitsi Eigenmann \& Ward, 1907. Type by original designation. Gender: Masculine.

Revision: Koch (2002).

Homodiaetus anisitsi Eigenmann \& Ward, 1907

Homodiaetus anisitsi Eigenmann \& Ward, in Eigenmann, McAtee \& Ward, 1907: 119, pl. 34 (figs. 2-3). Type locality: small creek at Villa Rica, Paraguay. Holotype: CAS 37276; holotype illustrated in Eigenmann (1918a: 351; pl. 56, fig. 3, 5).

Homodiaetus vazferreirai Devincenzi, in Devincenzi \& Vaz-Ferreira, 1939: 168, figured. Type locality: río Uruguay, en las inmediaciones de la ciudad de Paysandú, Uruguay. Holotype: MHNM CI 345.

Distribution: Paraná-Paraguay River basin (Koch, 2002).

Homodiaetus banguela Koch, 2002

Homodiaetus banguela Koch, 2002: 42, fig. 4. Type locality: Brasil, Rio de Janeiro: Silva Jardim (rio São João, no cruzamento com a BR-101). Holotype: MCP 19619.

Distribution: São João River, State of Rio de Janeiro, southeastern Brazil (Koch, 2002).

Homodiaetus graciosa Koch, 2002

Homodiaetus graciosa Koch, 2002: 44, fig. 5. Type locality: Brasil, Paraná, Morretes (rio Sagrado, posto florestal). Holotype: MCP 19618.

Distribution: Coastal basins of southeastern Brazil in states of Paraná and São Paulo (Koch, 2002).

Homodiaetus passarellii (Miranda Ribeiro, 1944)

Stegophilus passarellii Miranda Ribeiro, 1944c: 1, fig. on p. 2. Type locality: Córrego do Barro Branco (conhecido atualmente como Roncador) com nascentes na Serra do Barro Branco e desaguando no canal Saracuruna- um dos formadores do rio Estrêla (hoje também conhecido como Canal), e que se lança no fundo de baia de Guanabara no local denominado "Boqueirão" estrada de Magé, Estado do Rio de Janeiro [Brazil]. Holotype: MNRJ 3783.

Distribution: Coastal basins of Rio de Janeiro State, southeastern Brazil (Koch, 2002).

ITUGLANIS Costa \& Bockmann, 1993

Ituglanis Costa \& Bockmann, 1993: 44. Type species: Pygidium proops parahybae Eigenmann, 1918. Type by original designation. Gender: Masculine.

Ituglanis amazonicus (Steindachner, 1882)

Trichomycterus amazonicus Steindachner, 1882b: 178. Type locality: Cudajas [Brazil]. Holotype: NMW 43306. Species illustrated and described in more detail in Steindachner (1882c: 29, pl. 6, figs. 4, 4a).

Distribution: Amazon River basin, Brazil, and rivers of French Guiana (de Pinna \& Wosiacki, 2003).

Ituglanis bambui Bichuette \& Trajano, 2004

Ituglanis bambui Bichuette \& Trajano, 2004: 245, fig. 1. Type locality: Brazil: Goiás: São Domingos: Terra Ronca 
State Park: Angélica Cave: upper tributary of main subterranean stream, formed by infiltration of epikarstic water, $13^{\circ} 31^{\prime} \mathrm{W}, 46^{\circ} 23^{\prime} \mathrm{W}$. Holotype: MZUSP 79860.

Distribution: São Domingos karst area, Tocantins River basin, Brazil (Bichuette \& Trajano, 2004).

Ituglanis eichorniarum (Miranda Ribeiro, 1912)

Trichomycterus eichorniarum Miranda Ribeiro, 1912: 27. Type locality: Cáceres, nas margens do Paraguay (M. Grosso) [Brazil]. Lectotype: MNRJ 780A, designated by Miranda Ribeiro (1953: 405).

Distribution: Upper Paraguay River basin, Brazil (de Pinna \& Wosiacki, 2003).

Ituglanis epikarsticus Bichuette \& Trajano, 2004

Ituglanis epikarsticus Bichuette \& Trajano, 2004: 248, fig. 4. Type locality: Brazil: Goiás: São Domingos: Terra Ronca State Park: São Mateus Cave: rimstone dams (travertine basins) fed by infiltration of epikarstic water (1340'S, 46²2'W). Holotype: MZUSP 79869.

Distribution: São Domingos karst area, Tocantins River basin, Brazil (Bichuette \& Trajano, 2004).

Ituglanis gracilior (Eigenmann, 1912)

Pygidium gracilior Eigenmann, 1912b: 213. Type locality: Erukin, British Guiana. Holotype: FMNH 53264; holotype illustrated in Eigenmann (1918a: 326; pl. 50, fig. 3).

Distribution: Guyana (de Pinna \& Wosiacki, 2003).

Ituglanis guayaberensis (Dahl, 1960)

Pygidium metae guayaberensis Dahl, 1960b: 307, fig. 2. Type locality: Small pond in a brook trib. to Río Guayabero, ca. $1 \mathrm{~km}$ from mouth of the brook In a brook approx. $1500 \mathrm{~m}$. from Camp 1, same locality as the holotype of Leyvaichthys castaneus, Orinoco System, Colombia. Holotype: Whereabouts unknown (possibly at ICN$\mathrm{MHN})$.

Distribution: Guayabero River basin, Orinoco drainage, Colombia (de Pinna \& Wosiacki, 2003).

Ituglanis herberti (Miranda Ribeiro, 1940)

Trichomycterus Herberti Miranda Ribeiro, 1940: 60, fig. Type locality: Rio Bodoquena, Pantanal, sur de Mato Grosso [Brazil]. Syntypes (7): MNRJ 1428 (2), MNRJ 1429 (5).

Distribution: Bodoquena River, Paraguay River basin, Brazil (de Pinna \& Wosiacki, 2003).

Ituglanis laticeps (Kner, 1863)

Trichomycterus laticeps Kner, 1863: 228, fig. 17. Type locality: Fundort, wie die vorige Art [in reference to Trichomycterus taenia, from "Westabhange der Andes im Staate Ecuador"]. Also described in Kner \& Steindachner (1864: 54, pl. 6, fig. 2).

Distribution: Ecuador (de Pinna \& Wosiacki, 2003).

Ituglanis macunaima Datovo \& Landim, 2005

Ituglanis macunaima Datovo \& Landim, 2005: 456, fig. 1. Type locality: Brazil, Mato Grosso, Cocalinho; rio Araguaia basin, Corixo da Saudade (Corixinho); 14'17'20.6"S 519'12.1"W. Holotype: MZUSP 88452.

Distribution: Araguaia River basin, Brazil (Datovo \& Landim, 2005).

Ituglanis metae (Eigenmann, 1918)

Pygidium metae Eigenmann, 1918c: 694. Type locality: Barrigona, Colombia. Holotype: CAS 58138; holotype illustrated in Eigenmann (1918a: 312, pl. 47, fig. 5).

Distribution: Colombia (de Pinna \& Wosiacki, 2003).

Ituglanis nebulosus de Pinna \& Keith, 2003

Ituglanis nebulosus de Pinna \& Keith, 2003: 874, figs. 1, 2. Type locality: French Guiana, Arataye river (Tributary of Approuague river), near the natural preserve "Reserve naturelle des Nouragues" (04º0'22"N, 52³6'34"W). Holotype: MNHN 2001-1128.

Distribution: Approuague River basin, French Guiana (de Pinna \& Keith, 2003).

Ituglanis parahybae (Eigenmann, 1918)

Pygidium proops parahybae Eigenmann, 1918a: 332. Type locality: Rio Parahyba, Brazil. Holotype: FMNH 58576.

Distribution: Paraíba do Sul and São João River basins, Brazil (de Pinna \& Wosiacki, 2003).

Ituglanis parkoi (Miranda Ribeiro, 1944)

Pygidium parkoi Miranda Ribeiro, 1944b: 1, fig. Type locality: rio Iticoaí (entre os rios das Pedras e Ituí) que deságua no Javari, afluente do Amazonas, Benjamin Constant, Estados do Amazonas [Brazil]. Holotype: MNRJ 
3849.

Distribution: Amazon River basin, Brazil (de Pinna \& Wosiacki, 2003).

Ituglanis passensis Fernández \& Bichuette, 2002

Ituglanis passensis Fernández \& Bichuette, 2002: 274, fig. 1. Type locality: Brazil: Goiás: São Domingos: Passa

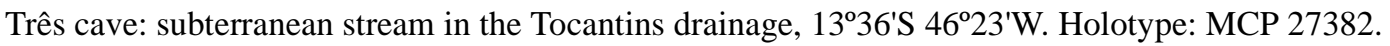

Distribution: Passa Três cave system in São Domingos, Goiás, Brazil (de Pinna \& Wosiacki, 2003).

Ituglanis proops (Miranda Ribeiro, 1908)

Trichomycterus proöps Miranda Ribeiro, 1908b: [3], fig. 4. Type locality: Rio Ribeira, Iguape, [Brazil]. Lectotype: MNRJ 781A, designated by Miranda Ribeiro (1953: 405).

Distribution: Ribeira de Iguape River basin, Brazil (de Pinna \& Wosiacki, 2003).

Ituglanis ramiroi Bichuette \& Trajano, 2004

Ituglanis ramiroi Bichuette \& Trajano, 2004: 250, fig. 7. Type locality: Brazil: Goiás: São Domingos: Terra Ronca

State Park: São Bernardo Cave: side pool fed by small water inlet $\left(13^{\circ} 49^{\prime} \mathrm{S}, 46^{\circ} 21^{\prime} \mathrm{W}\right)$, for about $700 \mathrm{~m}$ from cave entrance. Holotype: MZUSP 79865.

Distribution: São Domingos karst area, Tocantins River basin, Brazil (Bichuette \& Trajano, 2004).

LISTRURA de Pinna, 1988

Listrura de Pinna, 1988: 114. Type species: Listrura nematopteryx de Pinna, 1988. Type by original designation. Gender: Feminine.

Listrura boticario de Pinna \& Wosiacki, 2002

Listrura boticario de Pinna \& Wosiacki, 2002: 721, fig. 1. Type locality: Brazil, State of Paraná, Município de Guaraqueçaba, pool adjacent to Rio da Figueira (tributary of Rio Morato, itself tributary to Rio Guaraqueçaba, an isolated coastal basin that drains directly into the Baía das Laranjeiras), inside the nature preserve "Reserva Particular do Patrimônio Natural Salto Morato" (2516'S, 48¹2'W, UTM: 7.212.500-7.215.400). Holotype: MZUSP 69573.

Distribution: Da Figueira and Guaraqueçaba River basins, Paraná State, Brazil (de Pinna \& Wosiacki, 2003).

Listrura camposi (Miranda Ribeiro, 1957)

Eremophilus camposi Miranda Ribeiro, 1957: 72, fig. Type locality: Rio Poço Grande, [trib. of the Rio Juquiá,

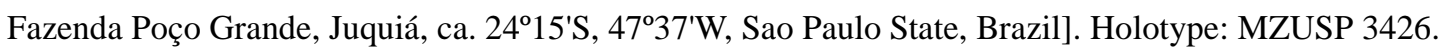

Distribution: Poço Grande River, tributary of the Juquiá River, São Paulo State, and Ribeirão da Ilha, Florianópolis, Santa Catarina State, Brazil (de Pinna \& Wosiacki, 2003).

Listrura nematopteryx de Pinna, 1988

Listrura nematopteryx de Pinna, 1988: 115, figs. 1-2. Type locality: Small marsh which is source of creek later joining Ribeirão Imbariê, trib. of Rio Estrela, near 58 km mark of old road leading to Petrópolis (Antiga Rio-Petrópolis, also called "Estrada Automóvel Club"), Município de Magé, Localidade de Piabetá, Rio de Janeiro, Brazil

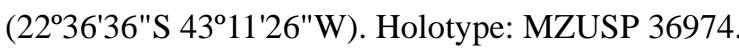

Distribution: Imbariê Creek, basin, Estrela River system, Piabetá, Rio de Janeiro State, and Picinguaba, São Paulo State, Brazil (de Pinna \& Wosiacki, 2003).

Listrura tetraradiata Landim \& Costa, 2002

Listrura tetraradiata Landim \& Costa, 2002: 152, fig. 1. Type locality: Brazil: Estado do Rio de Janeiro: Municipio de Saquarema, rio da Represa, a tributary of rio Bom Sucesso, rio Ibicuíba basin of the Lagoa de Araruama sys-

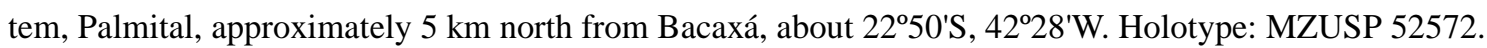

Distribution: Ibicuíba River, Araruama Lagoon system, Brazil (de Pinna \& Wosiacki, 2003).

MALACOGLANIS Myers \& Weitzman, 1966

Malacoglanis Myers \& Weitzman, 1966: 281. Type species: Malacoglanis gelatinosus Myers \& Weitzman, 1966. Type by original designation. Gender: Masculine.

Malacoglanis gelatinosus Myers \& Weitzman, 1966

Malacoglanis gelatinosus Myers \& Weitzman, 1966: 282, figs. 3-4. Type locality: Small caño of the Río Orteguaza, a short distance above its junction with the Rio Caquetá, Caquetá Province, Colombia. Holotype: SU 50754. 
Distribution: Caquetá River basin, Colombia (de Pinna \& Wosiacki, 2003).

MEGALOCENTOR de Pinna \& Britski, 1991

Megalocentor de Pinna \& Britski, 1991: 115. Type species: Megalocentor echthrus de Pinna \& Britski, 1991. Type by original designation. Gender: Masculine.

Megalocentor echthrus de Pinna \& Britski, 1991

Megalocentor echthrus de Pinna \& Britski, 1991: 116, figs. 3, 4 (a-b). Type locality: Rio Madeira, Calama, Praia do Caraparu, Estado do Amazonas, Brazil. Holotype: MZUSP 41879.

Distribution: Amazon and Orinoco River basins (de Pinna \& Wosiacki, 2003).

MICROCAMBEVA Costa \& Bockmann, 1994

Microcambeva Costa \& Bockmann, 1994a: 718. Type species: Microcambeva barbata Costa \& Bockmann, 1994. Type by original designation. Gender: Feminine.

Microcambeva barbata Costa \& Bockman, 1994

Microcambeva barbata Costa \& Bockmann, 1994a: 718, figs. 2-3. Type locality: Brazil: Estado do Rio de Janeiro: rio São João, near Gaviões, Município de Silva Jardim. Holotype: MZUSP 43678.

Distribution: Atlantic coastal drainages of Rio de Janeiro and Espírito Santo states, Brazil (de Pinna \& Wosiacki, 2003).

Microcambeva ribeirae Costa, Lima \& Bizerril, 2004

Microcambeva ribeirae Costa, Lima \& Bizerril, 2004: 3, figs. 1, 2. Type locality: Brazil: Estado de São Paulo: Município de Pedro de Toledo, Rio São Lourencinho, no Bairro São Lourenço. Holotype: MZUSP 84301.

Distribution: Ribeira do Iguape River basin, SE Brazil (Costa et al., 2004).

MIUROGLANIS Eigenmann \& Eigenmann, 1889

Miuroglanis Eigenmann \& Eigenmann, 1889b: 55. Type species: Miuroglanis platycephalus Eigenmann \& Eigenmann, 1889. Type by original designation. Gender: Masculine.

Miuroglanis platycephalus Eigenmann \& Eigenmann, 1889

Miuroglanis platycephalus Eigenmann \& Eigenmann, 1889b: 56. Type locality: Jutahy [Brazil]. Holotype: MCZ 8172.

Distribution: upper Amazon River basin, Brazil (de Pinna \& Wosiacki, 2003).

OCHMACANTHUS Eigenmann, 1912

Ochmacanthus Eigenmann, 1912: 213. Type species: Ochmacanthus flabelliferus Eigenmann, 1912. Type by original designation. Gender: Masculine.

Gyrinurus Miranda Ribeiro, 1912: 27. Type species: Gyrinurus batrachostoma Miranda Ribeiro, 1912. Type by monotypy. Gender: Masculine.

Ochmacanthus alternus Myers, 1927

Ochmacanthus alternus Myers, 1927: 129. Type locality: Venezuela: Caño de Quiribana, near Caicara. Lectotype: CAS 13522.

Distribution: Negro and Orinoco River basins, Brazil and Venezuela (de Pinna \& Wosiacki, 2003).

Ochmacanthus batrachostoma (Miranda Ribeiro, 1912)

Gyrinurus batrachostoma Miranda Ribeiro, 1912: 28, pl. Type locality: rio Paraguay em S. Luiz de Cáceres [ Mato Grosso, Brazil]. Holotype: MNRJ 786.

Distribution: Paraguay River basin, Brazil (de Pinna \& Wosiacki, 2003); la Plata basin, Argentina (Casciotta \& Almirón, 1996).

Ochmacanthus flabelliferus Eigenmann, 1912

Ochmacanthus flabelliferus Eigenmann, 1912b: 213. Type locality: Konawaruk, British Guiana. Holotype: FMNH 53263; holotype illustrated in Eigenmann (1918a: 357; pl. 55, fig. 5).

Distribution: River drainages in Guyana and Venezuela (de Pinna \& Wosiacki, 2003).

Ochmacanthus orinoco Myers, 1927 
Ochmacanthus orinoco Myers, 1927: 130. Type locality: Venezuela: Playa Matepalma, Orinoco. Holotype: CAS 76355.

Distribution: Negro and Orinoco River basins, Brazil and Venezuela (de Pinna \& Wosiacki, 2003).

Ochmacanthus reinhardtii (Steindachner, 1882)

Stegophilus Reinhardtii Steindachner, 1882b: 178. Type locality: Amazonenstrom und Rio Iça. Syntypes: NMW 44735 (2), NMW 50603 (3). Species illustrated and described in more detail in Steindachner (1882c: 28, pl. 6, fig. 1).

Distribution: Amazon River basin, Brazil, and drainages in French Guyana (de Pinna \& Wosiacki, 2003).

PARACANTHOPOMA Giltay, 1935

Paracanthopoma Giltay, 1935: 1. Type species: Paracanthopoma parva Giltay, 1935. Type by original designation. Gender: Neuter.

Phylogeny: Schmidt (1993).

Paracanthopoma parva Giltay, 1935

Paracanthopoma parva Giltay, 1935: 1, figs. 1-3. Type locality: Rio Catrymany supérieur [Brazil]. Holotype: IRSNB 43.

Distribution: Amazon basin, Brazil, and Essequibo River basin, Guyana (de Pinna \& Wosiacki, 2003).

Remarks: Redescribed, with notes on biology, in Schmidt (1993).

PARASTEGOPHILUS Miranda Ribeiro, 1946

Parastegophilus Miranda Ribeiro, 1946: 12. Type species: Stegophilus maculatus Steindachner, 1879. Type by original designation. Gender: Masculine.

Parastegophilus maculatus (Steindachner, 1879)

Stegophilus maculatus Steindachner, 1879a: 32. Type locality: La Plata, Prov. of Buenos Aires, Argentina. Holotype: NMW 57222. Species illustrated and described in more detail in Steindachner (1879e: 25, pl. 4, fig. 2).

Distribution: Lower Paraná and Uruguay River basins, Argentina (de Pinna \& Wosiacki, 2003).

Parastegophilus paulensis (Miranda Ribeiro, 1918)

Pseudostegophilus paulensis Miranda Ribeiro, 1918e: 727. Type locality: Avanhandava, Rio Tieté, Estado de São Paulo [Brazil]. Holotype: MZUSP 2272.

Pseudostegophilus scarificator Ihering, 1930: 100, pl. 13 (fig. 2). Type locality: São Paulo [Brazil]. Syntypes (2): Whereabouts unknown.

Distribution: Upper Paraná River basin, Brazil (de Pinna \& Wosiacki, 2003).

PARAVANDELLIA Miranda Ribeiro, 1912

Paravandellia Miranda Ribeiro, 1912: 28. Type species: Paravandellia oxyptera Miranda Ribeiro, 1912. Type by monotypy. Gender: Feminine.

Branchioica Eigenmann, 1918c: 702. Type species: Branchioica bertoni Eigenmann, 1918. Type by original designation. Gender: Feminine.

Pleurophysus Miranda Ribeiro, 1918d: 636. Type species: Pleurophysus hydrostaticus Miranda Ribeiro, 1918. Type by monotypy. Gender: Masculine.

Parabranchioica Devincenzi \& Vaz-Ferreira, 1939: 5. Type species: Parabranchioica teaguei Devincenzi \& VazFerreira, 1939. Type by monotypy. Gender: Feminine.

Paravandellia oxyptera Miranda Ribeiro, 1912

Paravandellia oxyptera Miranda Ribeiro, 1912: 29. Type locality: Caceres, margens do Rio Paraguay [Brazil]. Holotype: MNRJ 790.

Branchioica bertoni Eigenmann, 1918c: 703. Type locality: Asuncion, Paraguay. Holotype: CAS 63840; holotype illustrated in Eigenmann (1918a: 368, pl. 53, figs. 3-5) as Branchioica bertonii.

Pleurophysus hydrostaticus Miranda Ribeiro, 1918d: 636. Type locality: Rio Claro [Brazil]. Lectotype: MZUSP 2214, designated by Britski (1969: 206).

Vandellia hematophaga Guimarães, 1935: 300, figs. 1-4. Type locality: Río Tietê, Salto, São Paulo [Brazil]. Lecto- 
type: SU 16766, designated by Böhlke (1953: 45).

Parabranchioica teaguei Devincenzi \& Vaz-Ferreira, 1939: 5, unnumbered figure. Type locality: río Uruguay, en las inmediaciones de la ciudad de Paysandú [Uruguay]. Type(s): at MHNM.

Distribution: Paraná, Paraguay and Uruguay River basins, Brazil, Paraguay, and Uruguay (de Pinna \& Wosiacki, 2003).

Paravandellia phaneronema (Miles, 1943)

Branchioica phaneronema Miles, 1943a: 32, fig. 8. Type locality: various points in the Upper Cauca valley. Holotype: lost (Román-Valencia, 1998). Described again in Miles (1943b: 367, figs. 1-3).

Branchioica magdalenae Miles, 1943a: 33. Type locality: Río Magdalena, Colombia. Holotype: lost (Román-Valencia, 1998). Described again in Miles (1943b: 368).

Distribution: Magdalena and Cauca River basins, Colombia (de Pinna \& Wosiacki, 2003).

Remarks: Although both of these nominal species were described twice in 1943, the text of Miles (1943b) indicates that the names were first made available in Miles (1943a). Román-Valencia (1998) treated Branchioica magdalenae as a junior synonym of Paravandellia phaneronema, thereby acting as first reviser.

\section{PAREIODON Kner, 1855}

Pareiodon Kner, 1855a: 160. Type species: Pareiodon microps Kner, 1855. Type by monotypy. Gender: Masculine.

Centrophorus Kner, 1859: 167 (footnote). Type species: Pareiodon microps Kner, 1855. Type by being a replacement name. Gender: Masculine. Unneeded replacement for Pareiodon Kner, 1855, then considered preoccupied by Parodon. Preoccupied by Centrophorus Müller \& Henle, 1837, in Elasmobranchs.

Astemomycterus Guichenot, 1860: 525. Type species: Trichomycterus pusillus Castelnau, 1855. Type by original designation. Gender: Masculine.

\section{Pareiodon microps Kner, 1855}

Pareiodon microps Kner, 1855a: 160, fig. 2. Type locality: Borba (?) [Río Madeira]. Syntypes: NMW 45486 (2).

Trichomycterus pusillus Castelnau, 1855: 50, pl. 24 (fig. 4). Type locality: l'Araguay et [...] l'Amazone. Syntypes: MNHN 0000-1210 (4).

Distribution: Amazon River basin, Brazil (de Pinna \& Wosiacki, 2003).

Remarks: Priority of publication of the two included synonyms may not have yet been established, inasmuch as the precise dates of publication of the two works were not listed in Eschmeyer et al. (1998) or other relevant publications. If priority cannot be used to resolve this issue, precedence must be given to Pareiodon microps by first reviser action of Eigenmann \& Eigenmann (1889: 346).

PLECTROCHILUS Miranda Ribeiro, 1917

Plectrochilus Miranda Ribeiro, 1917: 50. Type species: Plectrochilus machadoi Miranda Ribeiro, 1917. Type by monotypy. Gender: Masculine.

Plectrochilus diabolicus (Myers, 1927)

Urinophilus diabolicus Myers, 1927: 132. Type locality: Iquitos, Peru. Holotype: CAS 59940.

Distribution: Amazon River basin, Brazil and Peru (de Pinna \& Wosiacki, 2003).

Plectrochilus machadoi Miranda Ribeiro, 1917

Plectrochilus machadoi Miranda Ribeiro, 1917: 50, three unnumbered figures. Type locality: Fluvio Solimões, [Brazil]. Holotype: MNRJ 978.

Vandellia hasemani Eigenmann, 1918a: 363, figs. 33-35, pl. 53 (fig. 3). Type locality: Río Mamoré, Alto Amazonas, Bolivia. Holotype: FMNH 58523.

Distribution: Amazon River basin, Brazil and Peru (de Pinna \& Wosiacki, 2003).

Plectrochilus wieneri (Pellegrin, 1909)

Vandellia wieneri Pellegrin, 1909e: 199, unnumbered figure. Type locality: [Río Napo, near mouth of Río Misahually, Ecuador]. Holotype: MNHN a-9934.

Distribution: Napo River basin, Ecuador (de Pinna \& Wosiacki, 2003).

PSEUDOSTEGOPHILUS Eigenmann \& Eigenmann, 1889 
Pseudostegophilus Eigenmann \& Eigenmann, 1889b: 54. Type species: Stegophilus nemurus Günther, 1869. Type by original designation. Gender: Masculine.

Pseudostegophilus haemomyzon (Myers, 1942)

Homodiaetus haemomyzon Myers, 1942: 98, fig. 4. Type locality: Río Guarico at Calabozo, Venezuela. Holotype: SU 36500.

Distribution: Orinoco River basin, Venezuela (de Pinna \& Wosiacki, 2003).

Pseudostegophilus nemurus (Günther, 1869)

Stegophilus nemurus Günther, 1869: 429. Type locality: Upper Amazon River, Peru. Holotype: BMNH 1869.5.21.9.

Distribution: Amazon River basin, Brazil and Peru (de Pinna \& Wosiacki, 2003).

PYGIDIANOPS Myers, 1944

Pygidianops Myers, 1944: 592. Type species: Pygidianops eigenmanni Myers, 1944. Type by original designation. Gender: Masculine.

Key: Schaefer et al. (2005: 5).

Pygidianops cuao Schaefer, Provenzano, de Pinna \& Baskin, 2005

Pygidianops cuao Schaefer, Provenzano, de Pinna \& Baskin, 2005: 5, figs. 1, 2. Type locality: Venezuela, Estado Amazonas, Río Cuao at Raudal Guacamaya, 8.1 miles upstream from Raudal El Danto, 0507.71'N, 67³1.53'W. Holotype: MBUCV V-30917.

Distribution: Cuao River, Orinoco basin, Venezuela (Schaefer et al., 2005).

Pygidianops eigenmanni Myers, 1944

Pygidianops eigenmanni Myers, 1944: 592, pl. 52 (fig. 1), pl. 53 (figs. 3-5). Type locality: Rock pools below São

Gabriel Rapids, Rio Negro, Brazil. Holotype: CAS 11120.

Distribution: Negro River basin, Brazil (de Pinna \& Wosiacki, 2003).

Pygidianops magoi Schaefer, Provenzano, de Pinna \& Baskin, 2005

Pygidianops magoi Schaefer, Provenzano, de Pinna \& Baskin, 2005: 10, figs. 5, 6. Type locality: Venezuela, Estado

Delta Amacuro, Río Orinoco at Puerto Cabrian, 8³4.8'N, 62²15.9'W. Holotype: MBUCV V-31035.

Distribution: Lower Orinoco River, between Ciudad Bolivar and Barrancas, Venezuela (Schaefer et al., 2005).

RHIZOSOMICHTHYS Miles, 1943

Bathophilus Miles, 1942: 57. Type species: Pygidium totae Miles, 1942. Type by monotypy. Gender: Masculine. Preoccupied by Bathophilus Giglioli, 1882, in fishes; replaced by Rhizosomichthys Miles, 1943, and Bathypygidium Whitley, 1947.

Rhizosomichthys Miles, 1943b: 369. Type species: Pygidium totae Miles, 1942. Type by being a replacement name. Gender: Masculine. Replacement for Bathophilus Miles, 1942; preoccupied by Bathophilus Giglioli, 1882.

Bathypygidium Whitley, 1947: 150. Type species: Pygidium totae Miles, 1942. Type by being a replacement name.

Gender: Neuter. Replacement for Bathophilus Miles, 1942; preoccupied by Bathophilus Giglioli, 1882.

Rhizosomichthys totae (Miles, 1942)

Pygidium totae Miles, 1942: 55, unnumbered figure. Type locality: Lago de Tota, Boyacá, Cordillera Oriental, Colombia, 3060 metros. Holotype: ICNMNH 20 [? now ICNMHN 353].

Distribution: Lake Tota basin, Colombia (de Pinna \& Wosiacki, 2003).

SARCOGLANIS Myers \& Weitzman, 1966

Sarcoglanis Myers \& Weitzman, 1966: 279. Type species: Sarcoglanis simplex Myers \& Weitzman, 1966. Type by original designation. Gender: Masculine.

Sarcoglanis simplex Myers \& Weitzman, 1966

Sarcoglanis simplex Myers \& Weitzman, 1966: 279, figs. 1-2. Type locality: Rock pools below São Gabriel Rapids, of the Rio Negro (below the town of Uaupés, formerly São Gabriel), Estado de Amazonas, Brazil. Holotype: SU 50189.

Distribution: Upper Negro River basin, Brazil (de Pinna \& Wosiacki, 2003). 
SCHULTZICHTHYS Myers \& Weitzman, 1966

Schultzichthys Dahl, 1960b: 312. Type species: Schultzichthys gracilis Dahl, 1960. Type by monotypy. Gender: Masculine.

Schultzichthys bondi (Myers, 1942)

Acanthopoma bondi Myers, 1942: 97, fig. 5. Type locality: Río Apuré at San Fernando de Apuré, Venezuela. Holotype: SU 36498.

Distribution: Amazon and Orinoco River basins (de Pinna \& Wosiacki, 2003).

Schultzichthys gracilis Dahl, 1960

Schultzichthys gracilis Dahl, 1960b: 312, figs. 4-5. Type locality: foot of a rapid in Caño Lozada, about $15 \mathrm{~km}$ above its junction with the Guayabero River [Río Orinoco system, Colombia]. Holotype: Whereabouts unknown (possibly at ICNMHN).

Distribution: Guayabero River, Orinoco River basin, Colombia (de Pinna \& Wosiacki, 2003).

SCLERONEMA Eigenmann, 1918

Scleronema Eigenmann, 1918c: 691. Type species: Scleronema operculatum Eigenmann, 1918. Type by original designation. Gender: Neuter.

Scleronema angustirostre (Devincenzi, 1942)

Pygidium angustirostris Devincenzi, in Devincenzi \& Teague, 1942: 30, pl. 4 (fig. 3). Type locality: La Cañeda de las Piedras, Uruguay. Holotype: at MHNM (not found).

Distribution: Uruguay (de Pinna \& Wosiacki, 2003).

Scleronema minutum (Boulenger, 1891)

Trichomycterus minutus Boulenger, 1891: 235, pl. 26 (fig. 3). Type locality: San Lorenzo district [= São Lourenço das Missões], Brazil. Syntypes: BMNH 1891.3.16.84-86 (3).

Distribution: Rio Grande do Sul State, Brazil (de Pinna \& Wosiacki, 2003); la Plata basin, Argentina (Casciotta \& Almirón, 1996).

Scleronema operculatum Eigenmann, 1918

Scleronema operculatum Eigenmann, 1918c: 691. Type locality: Cacequy, Brazil. Holotype: FMNH 58080; holotype illustrated in Eigenmann (1918a: 281, pl. 44, fig. 1).

Distribution: Rio Grande do Sul State, Brazil (de Pinna \& Wosiacki, 2003).

\section{SILVINICHTHYS Arratia, 1998}

Silvinichthys Arratia, 1998: 356. Type species: Trichomycterus mendozensis Arratia, Chang, Menu-Marque \& Rojas, 1978. Type by monotypy. Gender: Masculine.

Silvinichthys bortayro Fernández \& de Pinna, 2005

Silvinichthys bortayro Fernández \& de Pinna, 2005: 101, figs. 1-2. Type locality: Argentina, Provincia de Salta, Departamento Capital, artificial well near Río Arenales at San Luis, 1273 m elevation, 12 m depth, 2450'S, 65³0'W. Holotype: FML 2590.

Distribution: Artificial wells in Salta Province, Argentina (Fernández \& de Pinna, 2005).

Silvinichthys mendozensis (Arratia, Chang, Menu-Marque \& Rojas, 1978)

Trichomycterus mendozensis Arratia, Chang, Menu-Marque \& Rojas, 1978: 170, figs. 8-10. Type locality: freshwater of the preandean range of Mendoza, Argentina ..., where it occupies a restricted environment between 1,500 to $1,700 \mathrm{~m}$ above sea level. Holotype: FFSUC IC 118-27.

Distribution: Mendoza River basin, Argentina (de Pinna \& Wosiacki, 2003).

STAUROGLANIS de Pinna, 1989

Stauroglanis de Pinna, 1989a: 6. Type species: Stauroglanis gouldingi de Pinna, 1989. Type by original designation. Gender: Masculine.

Stauroglanis gouldingi de Pinna, 1989

Stauroglanis gouldingi de Pinna, 1989a: 7, figs. 1-3. Type locality: Cachoeira do Aracu, R. Daraá (R. Negro drainage system), Estado do Amazonas, Brazil. Holotype: MZUSP 31088. 
Distribution: Daraá River, Negro River basin, Brazil (de Pinna \& Wosiacki, 2003).

STEGOPHILUS Reinhardt, 1859

Stegophilus Reinhardt, 1859: 5 Type species: Stegophilus insidiosus Reinhardt, 1859. Type by monotypy. Gender: Masculine.

Stegophilus insidiosus Reinhardt, 1859

Stegophilus insidiosus Reinhardt, 1859: 5, pl. 2. Type locality: Rio das Velhas, Rio São Francisco system, Brazil. Syntypes: BMNH 1875.5.22.1 (1), ZMUC P 30152 (1), ZMUC P 30153 (1).

Distribution: São Francisco River basin, Brazil (de Pinna \& Wosiacki, 2003).

Stegophilus panzeri (Ahl, 1931)

Henonemus panzeri Ahl, 1931: 210, fig. 3. Type locality: Rio Capim [Brazil]. Holotype: at ZMB (orig. no. 656).

Distribution: Lower Amazon River basin, Brazil (de Pinna \& Wosiacki, 2003).

Stegophilus septentrionalis Myers, 1927

Stegophilus septentrionalis Myers, 1927: 130. Type locality: Venezuela: Santa Barbara, Orinoco. Holotype: CAS 64597.

Distribution: Orinoco River basin, Venezuela (de Pinna \& Wosiacki, 2003).

STENOLICMUS de Pinna \& Starnes, 1990

Stenolicmus de Pinna \& Starnes, 1990: 77. Type species: Stenolicmus sarmientoi de Pinna \& Starnes, 1990. Type by original designation. Gender: Masculine.

Stenolicmus sarmientoi de Pinna \& Starnes, 1990

Stenolicmus sarmientoi de Pinna \& Starnes, 1990: 77, fig. 1-2. Type locality: Río Matos, trib. of Río Apere, 48 km east of San Borja, Ballivia Prov., Depto. Beni, Bolivia, 1455'S, 66²17'W. Holotype: USNM 301664.

Distribution: Upper Apere River basin, Bolivia (de Pinna \& Wosiacki, 2003).

TRICHOGENES Britski \& Ortega, 1983

Trichogenes Britski \& Ortega, 1983: 211. Type species: Trichogenes longipinnis Britski \& Ortega, 1983. Type by original designation. Gender: Masculine.

Trichogenes longipinnis Britski \& Ortega, 1983

Trichogenes longipinnis Britski \& Ortega, 1983: 212, figs. 1-2. Type locality: Cachoeira do Amor, km 3 da estrada Parati-Ubatuba, SP [Brazil] Holotype: MZUSP 16099.

Distribution: Atlantic coastal drainages, northern São Paulo State, Brazil (de Pinna \& Wosiacki, 2003).

TRICHOMYCTERUS Valenciennes, 1832

Trichomycterus Valenciennes, 1832a: 348. Type species: Trichomycterus nigricans Valenciennes, 1832. Type by monotypy. Gender: Masculine.

Review: Fernández (1999, Argentina).

Trichomycterus albinotatus Costa, 1992

Trichomycterus albinotatus Costa, 1992: 102, figs. 1-2. Type locality: Estado do Rio de Janeiro: Visconde de Maua, $1 \mathrm{~km} O$ de la Ville de Maromba, rio Preto, bassin du rio Paraíba [22³0'S 44¹5'], Brazil. Holotype: MZUSP 42312.

Distribution: Preto River, Paraíba do Sul River basin, Brazil (de Pinna \& Wosiacki, 2003).

Trichomycterus alternatus (Eigenmann, 1918)

Pygidium alternatum Eigenmann, 1918c: 700. Type locality: Rio Doce, Brazil. Holotype: FMNH 58082; holotype illustrated in Eigenmann (1918a: 336, pl. 52, fig. 3).

Pygidium florense Miranda Ribeiro, 1943: 1, fig. Type locality: rio das Flores, próximo a Ipiabas (estação de Pandiá Calógeras), Estado do Rio de Janeiro [Brazil]. Holotype: MNRJ 3751.

Pygidium travassosi Miranda Ribeiro, 1949a: 145, fig. 2. Type locality: Rio das Pedras, Fazenda Penedo, Agulhas Negras, Estado do Rio, Rio de Janeiro, Brazil. Holotype: MNRJ 5424.

Distribution: Atlantic coastal river basins, Rio de Janeiro and Espírito Santo States, Brazil (de Pinna \& Wosiacki, 
2003), Jequitinhonha River basin, Minas Gerais (Triques \& Vono, 2004).

Trichomycterus alterus (Marini, Nichols \& La Monte, 1933)

Pygidium alterum Marini, Nichols \& La Monte, 1933: 2, fig. 2. Type locality: Rio de los Sauces, La Rioja, Argentina. Holotype: AMNH 12241.

Distribution: Humahuaca, Los Sauces River and Valle Guanchin (La Rioja), Argentina (de Pinna \& Wosiacki, 2003).

Trichomycterus areolatus Valenciennes, 1846

Trichomycterus areolatus Valenciennes, in Cuvier \& Valenciennes, 1846: 492. Type locality: la rivière de San Jago, Santiago. Syntypes: MNHN 0000-3167 (3), MNHN a-6310 (2).

Trichomycterus maculatus Valenciennes, in Cuvier \& Valenciennes, 1846: 493. Type locality: Santiago du Chili. Syntypes: MNHN 0000-3166 (1), MNHN 0000-4236 (10).

Trichomycterus marmoratus Philippi, 1866: 714. Type locality: Chile. No types known.

Trichomycterus palleus Philippi, 1866: 715. Type locality: Chile. No types known.

Trichomycterus tigrinus Philippi, 1866: 714. Type locality: Chile. No types known.

Distribution: Pacific draining rivers of Central Chile (de Pinna \& Wosiacki, 2003).

Trichomycterus arleoi (Fernández-Yépez, 1972)

Pygidium arleoi Fernández-Yépez, 1972a: 21, pl. 34. Type locality: Estación -140 — de la Cuenca del Rio Yaracuy, Venezuela. Holotype: collection of author, whereabouts unknown.

Distribution: Yaracuy River basin, Venezuela (de Pinna \& Wosiacki, 2003).

Trichomycterus auroguttatus Costa, 1992

Trichomycterus auroguttatus Costa, 1992: 105, fig. 6. Type locality: Rio de Janeiro Estado do Rio de Janeiro: 1 km à l'ouest de la ville de Visconde de Mauá, rio Marimbondo, affluent du rio Preto, bassin du rio Paraíba, Brazil. Holotype: MZUSP 43341.

Distribution: Marimbondo River, Preto River system, Paraíba do Sul River basin, Brazil (de Pinna \& Wosiacki, 2003).

Trichomycterus bahianus Costa, 1992

Trichomycterus bahianus Costa, 1992: 105, fig. 5. Type locality: Estado da Bahia: Una, 6,5 km au sud-est de la ville de Sao José, dans um ruisseau affluent du ribeirão Caveira, bassin du rio Una, Bahia, Brazil. Holotype: MZUSP 43340 .

Distribution: Ribeirão Caveira tributary, Una River basin, State of Bahia, Brazil (de Pinna \& Wosiacki, 2003).

Trichomycterus banneaui (Eigenmann, 1912)

Pygidium banneaui Eigenmann, 1912a: 19. Type locality: Bernal Creek near Honda, Colombia. Holotype: FMNH 56025; holotype illustrated in Eigenmann (1918a: 318, pl. 48, fig. 1).

Distribution: River drainages of Tolima and Cundinamarca, Colombia (Maldonado-Ocampo et al., 2005).

Remarks: Treated as a junior synonym of Trichomycterus bogotensis (Eigenmann, 1912), in de Pinna \& Wosiacki (2003), who also apparently acted as first reviser, but treated as a valid species in Maldonado-Ocampo et al. (2005).

Trichomycterus barbouri (Eigenmann, 1911)

Pygidium barbouri Eigenmann, 1911: 214, pl. 32. Type locality: Río Beni, e. Bolivia. Holotype: MCZ 29313.

Distribution: Amazon River basin, Bolivia, and la Plata River basin, Argentina (Fernández, 2000b); Beni River basin, Bolivia (de Pinna \& Wosiacki, 2003).

Remarks: Redescribed in Fernández (2000).

Trichomycterus belensis Fernández \& Vari, 2002

Trichomycterus belensis Fernández \& Vari, 2002: 739, fig. 1. Type locality: Argentina, Provincia de Catamarca, Departamento Belén, stream tributary to Laguna Blanca, $11 \mathrm{~km}$ northeast from Belén on provincial road 43 along route from Belén to Antogagasta de la Sierra, near Los Nacimientos de San Antonio (approximately

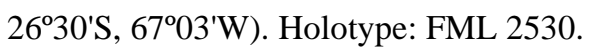

Distribution: Laguna Blanca basin, Argentina (de Pinna \& Wosiacki, 2003).

Trichomycterus bogotensis (Eigenmann, 1912)

Pygidium bogotense Eigenmann, 1912a: 18. Type locality: On the plains of Bogota, at an elevation of nearly nine thousand feet, ..., Chapinero, Colombia. Holotype: FMNH 56030; holotype illustrated in Eigenmann (1918a: 
315, pl. 49, fig. 3).

Distribution: Upper Cauca, Calima and San Juan rivers, Colombia (Maldonado-Ocampo et al., 2005).

Trichomycterus bomboizanus (Tortonese, 1942)

Pygidium bomboizanum Tortonese, 1942: 113, pl. 1. Type locality: Rio Bomboiza (Ecuador merid.) Ecuador. Holotype: MZUT 3553.

Distribution: Bomboiza River basin, Ecuador (de Pinna \& Wosiacki, 2003).

Trichomycterus borellii Boulenger, 1897

Trichomycterus borellii Boulenger, 1897c: 3. Type locality: Mission d'Aguairenda, Tala, and Lesser, Argentina. Syntypes: BMNH 1897.1.27.26 (1), ZMUT 1396 (6), ZMUT 1397 (2), ZMUT 13998 (1).

Pygidium Schmidti Berg, 1897: 266. Type locality: Río de Belén (Provincia de Catamarca), Argentina. Syntypes: BMNH 1898.9.23.3 (1), MACN 2361 (1), MACN 4595 (1), MACN 5164 (2), MACN 5176 (1).

Distribution: Salta, Catamarca and Mendoza, Argentina; Aguairenda, Bolivia (de Pinna \& Wosiacki, 2003).

Trichomycterus boylei Nichols, 1956

Pygidium boylei Nichols, 1956: 1, fig. 1. Type locality: dry country at Tilcara, Argentina, elevation 8000 feet, Argentina. Holotype: AMNH 20299.

Distribution: Grande River basin, Argentina (de Pinna \& Wosiacki, 2003).

Trichomycterus brasiliensis Reinhardt, 1874

Trichomycterus brasiliensis Reinhardt, in Lütken, 1874c: 29. Type locality: in Rio das Velhas. Syntypes: NMW 85270 (3), SMNS 2021 (1), ZMB 9171 (2), ZMUC P 30154-ZMUC P 30166 (1 each).

Trichomycterus brasiliensis tristis Lütken, 1875: 137. Type locality: Rio das Velhas, Minas Gerais, Brazil. Holotype: ZMUC P 30167.

Distribution: Upper São Francisco River, State of Minas Gerais and in smaller adjoining basins in Southeastern Brazil (de Pinna \& Wosiacki, 2003).

Trichomycterus caliensis (Eigenmann, 1912)

Pygidium caliense Eigenmann, 1912a: 18. Type locality: Cali, Colombia. Holotype: FMNH 56029.

Distribution: Calima River basin, Colombia (de Pinna \& Wosiacki, 2003).

Trichomycterus candidus (Miranda Ribeiro, 1949)

Eremophilus candidus Miranda Ribeiro, 1949b: 2, unnumbered plate. Type locality: pequeno córrego que cai no ribeirão Espírito Santo, afluente do Rio Claro e este do Sapucaí, que deságua no Rio Grande — Município de Conceição Aparecida, Estado de Minas Gerais, Brazil. Holotype: MNRJ 5209.

Distribution: Grande River basin, Minas Gerais State, Brazil (de Pinna \& Wosiacki, 2003).

Remarks: Redescribed as Eremophilus candidus in Barbosa \& Costa (2003a).

Trichomycterus castroi de Pinna, 1992

Trichomycterus castroi de Pinna, 1992a: 90, figs. 1-3. Type locality: Branch of the Rio Iguaçu, near the point crossed by Curitiba-Paranaguá Road, State of Paraná, Brazil, ca. 25²6’S, 4906’W. Holotype: MZUSP 36964.

Distribution: Iguaçu River basin, Brazil (de Pinna \& Wosiacki, 2003).

Trichomycterus catamarcensis Fernández \& Vari, 2000

Trichomycterus catamarcensis Fernández \& Vari, 2000: 990. Type locality: Provincia de Catamarca, Departamento Belén, stream tributary to Laguna Blanca, $11 \mathrm{~km}$ northeast from Belén on provincial road 43 along route from Belén to Antofagasta de la Sierra, near Los Nacimientos de San Antonio (approximately $26^{\circ} 30^{\prime} \mathrm{S}, 67^{\circ} 03^{\prime} \mathrm{W}$ ), elevation 3500m, Argentina. Holotype: FML 2507.

Distribution: Laguna Blanca basin in Catamarca Province, Argentina (de Pinna \& Wosiacki, 2003).

Trichomycterus caudofasciatus Alencar \& Costa, 2004

Trichomycterus caudofasciatus Alencar \& Costa, 2004: 5, fig. 2. Type locality: Brazil: Estado de Minas Gerais: Município de Alto Caparaó, Rio Caparaó, Rio Itabapoana basin, Alto Caparaó, 20²5'53.9"S, 4151'56.8"W. Holotype. UFRJ 6002.

Distribution: Upper Itabapoana River basin, southeastern Brazil (Alencar \& Costa, 2004).

Trichomycterus celsae Lasso \& Provenzano, 2003

Trichomycterus celsae Lasso \& Provenzano, 2003: 1140, fig. 1. Type locality: Venezuela, Estado Bolívar, río Kukenán, cabeceras, valle entre los tepuyes Roraima y Kukenán, Gran Sabana (0506'30"N, 6049'48"O). Holo- 
type: MHNLS 6453.

Distribution: Kukenán River, Caroní River basin, Venezuela; known only from type locality (Lasso \& Provenzano, 2003).

Trichomycterus chaberti Durand, 1968

Trichomycterus chaberti Durand, 1968: 344, fig. 3. Type locality: Umayalanta [Cave], Bolivia. Holotype: MNHN 1968-0217.

Distribution: Umayalanta Cave system, Bolivia (de Pinna \& Wosiacki, 2003).

Trichomycterus chapmani (Eigenmann, 1912)

Pygidium chapmani Eigenmann, 1912a: 18. Type locality: Boquia, Colombia. Holotype: FMNH 56027; holotype illustrated in Eigenmann (1918a: 309, fig. 13; pl. 47, fig. 2).

Distribution: Upper Cauca River basin, and Dagua, Calima and San Juan Rivers, Colombia (Maldonado-Ocampo et al., 2005).

Trichomycterus chiltoni (Eigenmann, 1920)

Pygidium chiltoni Eigenmann, 1920c: 54. Type locality: lower course of the Rio Nonguen where it passes through the ground of the Agricultural School, at the outskirts of Concepción, Chile. Lectotype: CAS 57596. Also described as new in Eigenmann (1927: 40, pl. 8, figs. 1, 1a, and pl. 13, figs. 5-6), with lectotype indicated in caption of pl. 8: “Type: 15059 I. U. M., 168 mm."

Distribution: Western drainages in central Chile (de Pinna \& Wosiacki, 2003).

Trichomycterus chungaraensis Arratia, 1983

Trichomycterus chungaraensis Arratia, 1983: 67, fig. 2. Type locality: Streams of Vertiente de Mal Paso, Chungará Lake, $4500 \mathrm{~m}$ above sea level, North Chile, South America, Chile. Holotype: FFSUC IC 290878A.

Distribution: Streams of Chungará Lake, Chile (de Pinna \& Wosiacki, 2003).

Trichomycterus concolor Costa, 1992

Trichomycterus concolor Costa, 1992: 107, fig. 9. Type locality: ruisseau $20 \mathrm{~km}$ au sud de la ville de Garapuava, bassin du rio São Francisco, Minas Gerais, Brazil. Holotype: MZUSP 43347.

Distribution: Upper São Francisco River basin, Brazil (de Pinna \& Wosiacki, 2003).

Trichomycterus conradi (Eigenmann, 1912)

Pygidium conradi Eigenmann, 1912b: 212. Type locality: Amatuk, British Guiana. Holotype: FMNH 53721; holotype illustrated in Eigenmann (1918a: 325; pl. 50, fig. 2).

Distribution: River drainages in Guyana and Venezuela (de Pinna \& Wosiacki, 2003).

Trichomycterus corduvensis Weyenbergh, 1877

Trichomycterus corduvensis Weyenbergh, 1877: 11, pl. 3 (figs. 1-2). Type locality: Rio Primero, y en las acequias de Córdoba, Argentina. Syntype: MSNG 9020 (1).

Distribution: Primeiro River basin, Argentina (de Pinna \& Wosiacki, 2003).

Trichomycterus davisi (Haseman, 1911)

Pygidium davisi Haseman, 1911b: 380, pl. 77; fig. 1. Type locality: Serrinha Paraná, Rio Iguaçu system, Brazil. Holotype: FMNH 60309; holotype illustrated in Eigenmann (1918a: 334; pl. 51, fig. 5).

Distribution: Iguaçu and Ribeira de Iguape River basins, Brazil (de Pinna \& Wosiacki, 2003).

Trichomycterus diabolus Bockmann, Casatti \& de Pinna, 2004

Trichomycterus diabolus Bockmann, Casatti \& de Pinna, 2004: 227, figs. 1-2. Type locality: Brazil: São Paulo: Rio Paranapanema basin, município de Teodoro Sampaio, Morro do Diabo State Park, Cóão Carlos (22³5'28.0"S, 52¹4'38.1"W). Holotype: MZUSP 78860.

Distribution: Paranapanema River basin, southeastern Brazil (Bockmann et al., 2004).

Trichomycterus dispar (Tschudi, 1846)

Pygidium dispar Tschudi, 1846: 22, pl. 3. Type locality: ... meisten Flüssen der Cordillera; ich habe ein Exemplar ahf einer Höhe von mehr als 14000 Fussü. M. gefangen; aber immer nur auf dem Hochlande zwischen den beiden Gebirgsketten und am Ostabhange der Anden wie an dem der Cordillera, Peru. Syntype: MHNN 767 (1). Distribution: Peruvian Andes (de Pinna \& Wosiacki, 2003).

Trichomycterus dorsotriatus (Eigenmann, 1918)

Pygidium dorsotriatum Eigenmann, 1918c: 695. Type locality: Villavicencio, Colombia. Holotype: FMNH 58096; 
holotype illustrated in Eigenmann (1918a: 320, pl. 48, fig. 3). Appeared only as Pygidium dorsotriatum in Eigenmann (1918c) but as Pygidium dorsostriatum in later Eigenmann publications (e. g., Eigenmann, 1918a: 320); dorsotriatum regarded as a typographical error in Eschmeyer et al. (1998) and de Pinna \& Wosiacki (2003), but no corrigendum by Eigenmann has been reported.

Distribution: River drainages in Villavicencio, Colombia (de Pinna \& Wosiacki, 2003).

Trichomycterus duellmani Arratia \& Menu-Marque, 1984

Trichomycterus duellmani Arratia \& Menu-Marque, 1984: 510, figs. 14-15. Type locality: Río Tupiza, 12.5 km SE Tupiza, Bolivia, ... 22³3'S, 6545'W. Holotype: KU 20191.

Distribution: Tupiza River basin, Bolivia (de Pinna \& Wosiacki, 2003).

Trichomycterus emanueli (Schultz, 1944)

Pygidium emanueli emanueli Schultz, 1944c: 259, pl. 5 (fig. c). Type locality: Río Chama at Estanques, Estado de Mérida, Venezuela. Holotype: USNM 121223.

Distribution: Chama River basin, Venezuela (de Pinna \& Wosiacki, 2003).

Trichomycterus fassli (Steindachner, 1915)

Pygidium fassli Steindachner, 1915a: 200. Type locality: Rio Songo im Distrikt Nord-Yungas, Bolivia. Syntypes (4): NMW 44470 (1). Illustrated and described in more detail as Pygidium fasslii in Steindachner (1915e: 97, pl. 13, figs. 1-2).

Distribution: Songo River basin, Bolivia (de Pinna \& Wosiacki, 2003).

Trichomycterus gabrieli (Myers, 1926)

Pygidium gabrieli Myers, 1926: 151. Type locality: São Gabriel rapids, Rio Negro, in rock-pools, Amazon system, Brazil. Syntypes: CAS 64583 (4), SU 36556 (1).

Distribution: Upper Negro River, Brazil (de Pinna \& Wosiacki, 2003).

Trichomycterus giganteus Lima \& Costa, 2004

Trichomycterus giganteus Lima \& Costa, 2004: 1, fig. 1. Type locality: Brazil: Estado do Rio de Janeiro: Município do Rio de Janeiro, Campo Grande, Rio Guandu-Mirim, Rio Guandu basin. Holotype: UFRJ 5999.

Distribution: Upper Guandu River basin, southeastern Brazil (Lima \& Costa, 2004).

Trichomycterus goeldii Boulenger, 1896

Trichomycterus goeldii Boulenger, 1896b: 154. Type locality: Colonia Alpina, Organ Mts in the Province Rio Janeiro, at an altitude of nearly 2600 feet, Brazil. Syntypes: BMNH 1896.4.4.7-8 (2).

Distribution: Mountain ranges of coastal basins of Rio de Janeiro State, Brazil (de Pinna \& Wosiacki, 2003).

Trichomycterus gorgona Fernández \& Schaefer, 2005

Trichomycterus gorgona Fernández \& Schaefer, 2005: 69, figs. 1-3. Type locality: Colombia, Departamento Cauca, Isla Gorgona, freshwater stream near northeast end of island, 0259'N, 78¹1'30"W. Holotype: ANSP 149946.

Distribution: Gorgona Island, Pacific coast of Colombia (Fernández \& Schaefer, 2005).

Trichomycterus guaraquessaba Wosiacki, 2005

Trichomycterus guaraquessaba Wosiacki, 2005: 51, fig. 2. Type locality: rio Bracinho, Fazenda Salto Dourado, Município de Guaraqueçaba, Paraná, Brazil. Holotype: MPEG 7916.

Distribution: Bracinho River, Atlantic coastal basin, southern Brazil (Wosiacki, 2005).

Trichomycterus guianensis (Eigenmann, 1909)

Pygidium guianensis Eigenmann, 1909a: 11. Type locality: Aruataima Falls, Upper Potaro, British Guiana. Holotype: FMNH 52676; holotype illustrated in Eigenmann (1918a: 325, pl. 50, fig. 1) as Pygidium guianense.

Distribution: Rivers of the Guianas and Venezuela (de Pinna \& Wosiacki, 2003).

Trichomycterus hasemani (Eigenmann, 1914)

Pygidium hasemani Eigenmann, 1914a: 48. Type locality: Santarem, Pará, Brazil. Holotype: FMNH 56424; holotype illustrated in Eigenmann (1918a: 326, pl. 50, fig. 4).

Distribution: Amazon River basin (de Pinna \& Wosiacki, 2003).

Trichomycterus heterodontus (Eigenmann, 1918)

Pygidium heterodontum Eigenmann, 1918c: 692. Type locality: Rio Mendoza, Palmira, Argentina, 900 m, Argentina. Holotype: CAS 58139; holotype illustrated in Eigenmann (1918a: 296, pl. 44, fig. 4).

Distribution: Mendoza River basin, Argentina (de Pinna \& Wosiacki, 2003). 
Trichomycterus iheringi (Eigenmann, 1918)

Pygidium iheringi Eigenmann, 1918c: 697. Type locality: São Paulo in coastal streams and Parana basin, Brazil. Holotype: CAS 64585; holotype illustrated in Eigenmann (1918a: 330, pl. 50, fig. 5) and Wosiacki (2005: 58, fig. 3).

Distribution: Ribeira do Iguape River basin, Brazil (de Pinna \& Wosiacki, 2003).

Remarks: Redescribed in Wosiacki (2005: 56).

Trichomycterus immaculatus (Eigenmann \& Eigenmann, 1889)

Pygidium immaculatum Eigenmann \& Eigenmann, 1889b: 52. Type locality: Juiz de Fora, Rio Parahybuna, Sao Matheos, Goyaz [Brazil]. Syntypes (14): MCZ 8266 (1), MCZ 8300 (10), MCZ 8302 (1), MCZ 8305 (1), MCZ 8307 (1). Species illustrated in Eigenmann (1918a: 334, pl. 52, fig.1).

Distribution: Paraíbuna River, Paraíba do Sul River basin, Brazil (de Pinna \& Wosiacki, 2003).

Trichomycterus itacambirussu Triques \& Vono, 2004

Trichomycterus itacambirussu Triques \& Vono, 2004: 165, fig. 2. Type locality: Brazil: Minas Gerais: County of

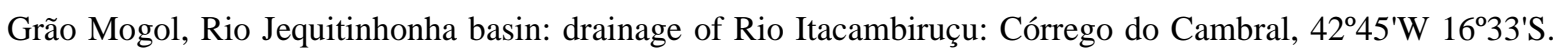
Holotype: MZUSP 58493.

Distribution: Jequitinhonha River basin, Minas Gerais, Brazil (Triques \& Vono, 2004).

Trichomycterus itacarambiensis Trajano \& de Pinna, 1996

Trichomycterus itacarambiensis Trajano \& de Pinna, 1996: 86, figs. 1-2. Type locality: Creek inside Olhos d'Agua cave, Município de Itacarambi, Minas Gerais, Brazil, 1506'06"S, 4409'30"W. Holotype: MZUSP 42469.

Distribution: Olhos d'Água Cave in State of Minas Gerais, Brazil (de Pinna \& Wosiacki, 2003).

Trichomycterus itatiayae Miranda Ribeiro, 1906

Trichomycterus brasiliensis itatiayae Miranda Ribeiro, 1906: 178, pl. 1 (figs. a-c). Type locality: ... do Itatiaya, ... em afluente do Parahyba, Rio de Janeiro State, Brazil. Lectotype: MNRJ 792, designated by Caramaschi \& Caramaschi (1991: 223).

Distribution: Rivers in Itatiaia Mountains, upper reaches of Paraíba do Sul River basin, Brazil (de Pinna \& Wosiacki, 2003).

Remarks: Type locality was interpreted by Caramaschi \& Caramaschi (1991: 223) as: Ribeirão da Tapera (tributary of the Ribeirão Bonito, Rio Paraíba do Sul drainage), Mont-Serrat, Itatiaia (ca. $22^{\circ} 27^{\prime} \mathrm{S}, 4^{\circ} 50^{\prime} \mathrm{W}$, ca. $800 \mathrm{~m}$ a.s.l.), Rio de Janeiro State, Brazil.

Trichomycterus jacupiranga Wosiacki \& Oyakawa, 2005

Trichomycterus jacupiranga Wosiacki \& Oyakawa, 2005: 469, figs. 5-6. Type locality: rio do Queimado, Parque Estadual de Jacupiranga, Cajati [São Paulo, Brazil]. Holotype: MZUSP 67818.

Distribution: Queimado River, São Paulo, Brazil (Wosiacki \& Oyakawa, 2005).

Trichomycterus jequitinhonhae Triques \& Vono, 2004

Trichomycterus jequitinhonhae Triques \& Vono, 2004: 167, fig. 3. Type locality: Brazil: Minas Gerais: County of Coronel Murta: Rio Jequitinhonha basin: Córrego Laranjeiras, 42¹8'W 16²45'S. Holotype: MZUSP 58497.

Distribution: Jequitinhonha River basin, Minas Gerais, Brazil (Triques \& Vono, 2004).

Trichomycterus johnsoni (Fowler, 1932)

Pygidium johnsoni Fowler, 1932a: 367, figure on p. 364. Type locality: Descalvados, Matto Grosso, Brazil. Holotype: ANSP 53873.

Distribution: Paraná River basin, Mato Grosso, Brazil, and Corrientes, Argentina (de Pinna \& Wosiacki, 2003).

Trichomycterus knerii Steindachner, 1882

Trichomycterus Knerii Steindachner, 1882a: 142. Type locality: Canelos [Ecuador]. Syntype: NMW 43328 (1). Species illustrated and described in more detail in Steindachner (1882d: 81, pl. 5, figs. 1, 1a).

Distribution: Canelos, Ecuador (de Pinna \& Wosiacki, 2003).

Trichomycterus landinga Triques \& Vono, 2004

Trichomycterus landinga Triques \& Vono, 2004: 162, fig. 1. Type locality: Brazil: Minas Gerais: County of Coronel Murta: Rio Jequitinhonha basin: Córrego Moquém, 42³5'W 16²45'S. Holotype: MZUSP 58496.

Distribution: Jequitinhonha River basin, Minas Gerais, Brazil (Triques \& Vono, 2004).

Trichomycterus latidens (Eigenmann, 1918) 
Pygidium latidens Eigenmann, 1918c: 693. Type locality: Small creek near mouth of Rio Calima, Colombia. Holotype: IU 13801 (whereabouts unknown); holotype illustrated in Eigenmann (1918a: 312, pl. 47, fig. 4).

Distribution: Calima River basin, Colombia (de Pinna \& Wosiacki, 2003).

Trichomycterus latistriatus (Eigenmann, 1918)

Pygidium latistriatum Eigenmann, 1918c: 696. Type locality: Quebrada de Pinchote, Santander, Colombia. Holotype: FMNH 58449; holotype illustrated in Eigenmann (1918a: 321, pl. 48, fig. 4).

Distribution: Gaira, Cesar and Ariguani rivers, Magdalena River basin, Colombia (Maldonado-Ocampo et al., 2005).

Trichomycterus laucaensis Arratia, 1983

Trichomycterus laucaensis Arratia, 1983: 74, fig. 8. Type locality: System of Lauca River, Parinacota, 4,390 m above sea level, northern Chile. Holotype: FFSUC IC 160878A.

Distribution: Lauca River basin, Chile (de Pinna \& Wosiacki, 2003).

Trichomycterus lewi Lasso \& Provenzano, 2003

Trichomycterus lewi Lasso \& Provenzano, 2003: 1144, fig. 7. Type locality: Venezuela, Estado Bolívar, río Kukenán, cabeceras, valle entre los tepuyes Roraima y Kukenán, Gran Sabana (0506'30"N, 6049'48"O). Holotype: MHNLS 6454.

Distribution: Kukenán River, Caroni River basin, Venezuela; known only from type locality (Lasso \& Provenzano, 2003).

Trichomycterus longibarbatus Costa, 1992

Trichomycterus longibarbatus Costa, 1992: 104, fig. 4. Type locality: près de la ville de Santa Tereza, Espirito Santo, Brazil. Holotype: MZUSP 43339.

Distribution: Near Santa Tereza, Espirito Santo State, Brazil (de Pinna \& Wosiacki, 2003).

Trichomycterus maracaiboensis (Schultz, 1944)

Pygidium banneaui maracaiboensis Schultz, 1944c: 262, pl. 6 (fig. b). Type locality: Río San Juan near bridge, south of Mene Grande, tributary to Río Motatán, Maracaibo basin, Venezuela. Holotype: USNM 121227.

Distribution: San Juan River, tributary to Motatán River, Lake Maracaibo basin, Venezuela (de Pinna \& Wosiacki, 2003).

Trichomycterus mboycy Wosiacki \& Garavello, 2004

Trichomycterus mboycy Wosiacki \& Garavello, 2004: 8, fig. 5. Type locality: Brazil: State of Paraná: município de Foz do Jordão, Jordão reservoir, rio Jordão near at its mouth, tributary of rio Iguaçu (rio Paraná Basin), $25^{\circ} 45^{\prime} \mathrm{S}$, $52^{\circ} 10^{\prime}$ W. Holotype: MPEG 6695.

Distribution: Jordão River, Iguaçu River basin, Brazil (Wosiacki \& Garavello, 2004).

Trichomycterus meridae Regan, 1903

Trichomycterus meridae Regan, 1903b: 624. Type locality: Merida, Venezuela, and from the Rio Albireggas above Merida, altitude 3500 metres, Venezuela. Syntypes: BMNH 1903.4.28.35-38 (4), BMNH 1906.6.30.77-79 (3), USNM 133136 (1).

Distribution: Albirregas River basin, Venezuela (de Pinna \& Wosiacki, 2003).

Trichomycterus migrans (Dahl, 1960)

Pygidium migrans Dahl, 1960b: 309, fig. 3. Type locality: in front of Camp 1, in the Guayabero River [Orinoco System, Colombia]. Holotype: ICNMHN 399.

Distribution: Guayabero River, Orinoco River basin, Colombia (de Pinna \& Wosiacki, 2003).

Trichomycterus mimonha Costa, 1992

Trichomycterus mimonha Costa, 1992: 106, fig. 7. Type locality: $2 \mathrm{~km}$ au nord de la ville de Piquete, rio Benfica, bassin du rio Paraíba, Sao Paulo, Brazil. Holotype: MZUSP 43343.

Distribution: Benfica River, Paraíba do Sul River basin, Brazil (de Pinna \& Wosiacki, 2003).

Trichomycterus mirissumba Costa, 1992

Trichomycterus mirissumba Costa, 1992: 107, fig. 8. Type locality: Visconde de Mauá, $1 \mathrm{~km}$ à l'est de la ville de Maromba, rio Preto, près du confluent avec le ruisseau Santa Clara, bassin du rio Paraíba, Rio de Janeiro, Brazil. Holotype: MZUSP 43345.

Distribution: Preto River, Paraíba do Sul River basin, Brazil (de Pinna \& Wosiacki, 2003). 
Trichomycterus mondolfi (Schultz, 1945)

Pygidium mondolfi Schultz, 1945: 29, fig. 1. Type locality: Quebrado Chacaito near Caracas but in Estado de Mirando, Río Tuy system, Venezuela. Holotype: USNM 120377.

Distribution: Tuy River basin, Caribbean Drainage, Venezuela (de Pinna \& Wosiacki, 2003).

Trichomycterus motatanensis (Schultz, 1944)

Pygidium emanueli motatanensis Schultz, 1944c: 260, pl. 6 (fig. a). Type locality: Río San Juan at the bridge south of Mene Grande, Motatán system, Maracaibo basin Venezuela. Holotype: USNM 121232.

Distribution: San Juan River, Motatán drainage, Lake Maracaibo basin, Venezuela (de Pinna \& Wosiacki, 2003).

Trichomycterus naipi Wosiacki \& Garavello, 2004

Trichomycterus naipi Wosiacki \& Garavello, 2004: 2, fig. 2. Type locality: Brazil: State of Paraná: município de Tijucas do Sul, rio São João, tributary of rio da Várzea (Iguaçu Basin), near Lagoinha, $25^{\circ} 55^{\prime} \mathrm{S}, 4^{\circ} 11^{\prime} \mathrm{W}$. Holotype: MPEG 6699.

Distribution: da Várzea River, Iguaçu River basin, Brazil (Wosiacki \& Garavello, 2004).

Trichomycterus nigricans Valenciennes, 1832

Trichomycterus nigricans Valenciennes, 1832a: 348. Type locality: ruisseaux de Sainte-Catherine du Brèsil, Santa Catarina, Brazil. Holotype: apparently MNHN b-0251.

Distribution: Coastal drainages in Santa Catarina State, Brazil (de Pinna \& Wosiacki, 2003).

Remarks: Species redescribed by Arratia (1998) with MNHN b-0251 listed as the holotype.

Trichomycterus nigromaculatus Boulenger, 1887

Trichomycterus nigromaculatus Boulenger, 1887b: 349. Type locality: Andes of Columbia. Syntypes: BMNH 1880.2.26.16-17 (2).

Distribution: Colombian Andes (de Pinna \& Wosiacki, 2003).

Trichomycterus pantherinus Alencar \& Costa, 2004

Trichomycterus pantherinus Alencar \& Costa, 2004: 2, fig. 1. Type locality: Brazil: Estado do Espírito Santo: Município de Sant Leopoldina, below waterfall near Fazenda Sete Quedas, Rio da Prata, Rio Santa Maria da Vitória basin, 2003'15.9"S, 40³2'20.5"W. Holotype: UFRJ 6001.

Distribution: Santa Maria da Vitória River basin, Southeastern Brazil (Alencar \& Costa, 2004).

Trichomycterus paolencis (Eigenmann, 1918)

Pygidium paolence Eigenmann, 1918c: 698. Type locality: Sâo Paulo in the Parana basin and (?) in coastal streams. ... Alto da Serra, Rio Tieté, Sâo Paulo. ... Mogy das Cruces, Rio Tieté, São Paulo, Brazil. Holotype: FMNH 58085; holotype illustrated in Eigenmann (1918a: 332, pl. 51, fig. 3).

Distribution: Paraná River basin, São Paulo State, Brazil (de Pinna \& Wosiacki, 2003).

Trichomycterus papilliferus Wosiacki \& Garavello, 2004

Trichomycterus papilliferus Wosiacki \& Garavello, 2004: 5, figs. 3, 4. Type locality: Brazil: State of Paraná: município de Foz do Jordão, Jordão reservoir, rio Jordão near at its mouth, tributary of rio Iguaçu (rio Paraná Basin), $25^{\circ} 45^{\prime} \mathrm{S}, 52^{\circ} 10^{\prime} \mathrm{W}$. Holotype: MPEG 6692.

Distribution: Jordão River, Iguaçu River basin, Brazil (Wosiacki \& Garavello, 2004).

Trichomycterus paquequerensis (Miranda Ribeiro, 1943)

Pygidium paquequerense Miranda Ribeiro, 1943: 2, fig. Type locality: rio Paquequer Grande, Estado do Rio de Janeiro [Brazil]. Holotype: MNRJ 1159.

Distribution: Paquequer River, Paraíba do Sul River basin, Brazil (de Pinna \& Wosiacki, 2003).

Trichomycterus piurae (Eigenmann, 1922)

Pygidium punctulatum piurae Eigenmann, 1922b: 63. Type locality: Piura, Peru. Holotype: CAS 58119.

Distribution: Piura River basin, Peru (de Pinna \& Wosiacki, 2003).

Trichomycterus plumbeus Wosiacki \& Garavello, 2004

Trichomycterus plumbeus Wosiacki \& Garavello, 2004: 13, fig. 8. Type locality: Brazil: State of Paraná: município de Foz do Jordão, Jordão reservoir, rio Jordão near at its mouth, tributary of rio Iguaçu (rio Paraná Basin), $25^{\circ} 45^{\prime} \mathrm{S}, 52^{\circ} 10^{\prime} \mathrm{W}$. Holotype: MPEG 6689.

Distribution: Jordão River, Iguaçu River basin, Brazil (Wosiacki \& Garavello, 2004).

Trichomycterus potschi Barbosa \& Costa, 2003 
Trichomycterus potschi Barbosa \& Costa, 2003b: 282, fig. 1. Type locality: Brazil: Estado do Rio de Janeiro: rio das Cachoeiras, serra do Matutu, Município de Mangaratiba. Holotype: MCP 29061.

Distribution: Coastal river basins between Mangaratiba and Itaguaí, Rio de Janeiro State, Brazil (Barbosa \& Costa, 2003b).

Trichomycterus pradensis Sarmento-Soares, Martins-Pinheiro, Aranda \& Chamon, 2005

Trichomycterus pradensis Sarmento-Soares, Martins-Pinheiro, Aranda \& Chamon, 2005: 291, fig. 1. Type locality: Brazil: Bahia: Jucuruçu, rio Jucuruçu, $2 \mathrm{~km}$ before the city of Jucuruçuon road Itamaraju-Jucuruçu, middle of rio Jucuruçu basin, 1650'10"S 4008'40"W. Holotype: MNRJ 28483.

Distribution: Jucuruçu River, a coastal river basin of SE Brazil (Sarmento-Soares et al., 2005).

Trichomycterus pseudosilvinichthys Fernández \& Vari, 2004

Trichomycterus pseudosilvinichthys Fernández \& Vari, 2004: 876, figs. 1-2. Type locality: Argentina, Provincia de La Rioja, Departamento Chilecito, Río Amarillo at Famatina, a small assemblage of houses near Fundición de Oro Santa Florentina (28 $\left.55^{\prime} \mathrm{S}, 6^{\circ} 31^{\prime} \mathrm{W}\right)$, on east slope of Sierra de Famatina. Holotype: FML 2588.

Distribution: La Rioja province, Argentina (Fernández \& Vari, 2004).

Trichomycterus punctatissimus Castelnau, 1855

Trichomycterus punctatissimus Castelnau, 1855: 49, pl. 24 (fig. 3). Type locality: De l'Araguay. Holotype: MNHN b-0610.

Distribution: Araguaia River basin, Brazil (de Pinna \& Wosiacki, 2003).

Trichomycterus punctulatus Valenciennes, 1846

Trichomycterus punctulatus Valenciennes, in Cuvier \& Valenciennes, 1846: 488, pl. 552. Type locality: la rivière de Lima, Peru. Syntypes: MNHN 0000-3168 (5).

Distribution: Western Peru (de Pinna \& Wosiacki, 2003).

Trichomycterus ramosus Fernández, 2000

Trichomycterus ramosus Fernández, 2000a: 350, fig. 1. Type locality: Catamarca: Departamento Belém: Laguna

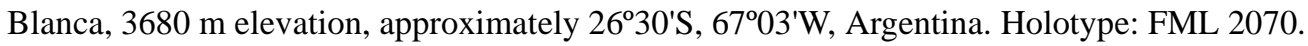

Distribution: Laguna Blanca basin, Catamarca Province, Argentina (de Pinna \& Wosiacki, 2003).

Trichomycterus regani (Eigenmann, 1918)

Pygidium regani Eigenmann, 1918c: 696. Type locality: Tado, Rio San Juan, Colombia. Holotype: CAS 64591; holotype illustrated in Eigenmann (1918a: 323, pl. 48, fig. 5).

Distribution: San Juan River basin, Colombia (de Pinna \& Wosiacki, 2003).

Trichomycterus reinhardti (Eigenmann, 1918)

Pygidium reinhardti Eigenmann, 1918c: 699. Type locality: Burmier on the Rio Itabira, a tributary of the Rio das Velhas, Brazil. Holotype: FMNH 58081; holotype illustrated in Eigenmann (1918a: 333, pl. 51, fig. 4).

Distribution: Upper São Francisco River basin, Brazil (de Pinna \& Wosiacki, 2003).

Trichomycterus retropinnis Regan, 1903

Trichomycterus retropinnis Regan, 1903b: 624. Type locality: St. Augustin, Andes of Colombia, elev. 5000 feet. Syntypes (2): BMNH 1899.8.21.12-13 (2).

Distribution: Upper Magdalena River basin, Colombia (Maldonado-Ocampo et al., 2005).

Trichomycterus riojanus (Berg, 1897)

Pygidium riojanum Berg, 1897: 269. Type locality: un arroyo de la Cordillera de La Rioja, Argentina. Holotype: MACN 5175 (destroyed, Fernández \& Schaefer, 2003: 357).

Distribution: La Rioja Range, Argentina (de Pinna \& Wosiacki, 2003).

Trichomycterus rivulatus Valenciennes, 1846

Trichomycterus rivulatus Valenciennes, in Cuvier \& Valenciennes, 1846: 495. Type locality: ruisseaux qui se jettent dans le lac de Titicaca, vaste mer alpine, peuplée par les Orestias sans ventrales, ou dans les afluens de l'Apurimac, l'une des sources de l'Amazone ... du Guasacona, Peru. Syntype: MNHN b-0586 (1).

Trichomycterus barbatula Valenciennes, in Cuvier \& Valenciennes, 1846: 498. Type locality: Guasacona et du Rio de Pontezualo près coroico, par une hauteur de terize à quatorze milles pieds et à una latitude de seize à dix-sept degrés nord. Syntypes: MNHN 0000-4077 (1), MNHN 0000-4078 (5) and MNHN b-0587 (3).

Trichomycterus gracilis Valenciennes, in Cuvier \& Valenciennes, 1846: 497. Type locality: Rio de Azangaro près de 
Guasacona, dans le Rio de Pontezualo près de Coroico, et enfin dans le lac de la Compucila dans les Andes, à l'ouest de Cuzco, par la hautes de quatorze mille pieds, Peru. Syntypes: MNHN 0000-3129 (2), MNHN 00004063 (2), MNHN a-9766 (6), MNHN b-0588 (2).

Trichomycterus incae Valenciennes, in Cuvier \& Valenciennes, 1846: 496. Type locality: Rio Guatanai à Cuzco, Peru. Syntype: MNHN a-8986 (1).

Trichomycterus pentlandi Castelnau, 1855: 49, pl. 24 (fig. 1). Type locality: lac situé prés de la mission de Sarayacu, qui communiqué avec la riviér d'Ucayale, Peru. Holotype: MNHN b-0608.

Trichomycterus pictus Castelnau, 1855: 50, pl. 24 (fig. 2). Type locality: grand lac de Titicaca. Syntypes: MNHN b0609 (3).

Trichomycterus pardus Cope, 1874b: 132. Type locality: Upper Amazon. Possible holotype: ANSP 22004.

Trichomycterus poeyanus Cope, 1877: 47. Type locality: Arequipa, Peru. Syntypes: ANSP 21382-83 (2).

Pygidium oroyae Eigenmann \& Eigenmann, 1889b: 51. Type locality: Pochachara, Oroya River [Brazil]. Syntypes (8): MCZ 3955 (8).

Trichomycterus eigenmanni Boulenger, 1898c: 8. Type locality: Cumbaca [Brazil]. Holotype: MCZ 8301.

Pygidium quechuorum Steindachner, 1900: 207. Type locality: Arequipa, Río Chile, Südperu. Syntypes (5): at NMW.

Pygidium tiraquae Fowler, 1940c: 92, fig. 52. Type locality: Tiraque, Cochabamba Department, Bolivia. Holotype: ANSP 69126.

Pygidium atochae Allen, in Eigenmann \& Allen, 1942: 156, pl. 13 (figs. 3-5). Type locality: Rio de Atocha, Atocha, Bolivia. Holotype: CAS 64576.

Distribution: High-altitude lakes and streams in the central Andean range (including Lakes Titicaca and Poopó), from Lake Junin in the north to Chilean region of Tarapacá in the south, spanning Western Bolivia, Peru and Northern Chile (de Pinna \& Wosiacki, 2003).

Trichomycterus roigi Arratia \& Menu-Marque, 1984

Trichomycterus roigi Arratia \& Menu-Marque, 1984: 494, figs. 1-10. Type locality: Río Pastos Chicos, Jujuy, north of Argentina; 23ํㄴ' $24^{\prime} \mathrm{S}-66^{\circ} 35^{\prime} \mathrm{W}$. Holotype: MLP 29-8-83-1.

Distribution: Pastos Chicos River basin, Argentina (de Pinna \& Wosiacki, 2003).

Trichomycterus romeroi (Fowler, 1941)

Pygidium romeroi Fowler, 1941c: 4, figs. 6-8. Type locality: Honda, Colombia. Holotype: ANSP 69331.

Distribution: Honda River basin, Colombia (de Pinna \& Wosiacki, 2003).

Trichomycterus santaeritae (Eigenmann, 1918)

Pygidium santae-ritae Eigenmann, 1918a: 341, pl. 52 (fig. 5). Type locality: Santa Rita, Rio Preto, Brazil. Holotype: FMNH 58577.

Distribution: Preto River, Paraíba do Sul River basin, Brazil (de Pinna \& Wosiacki, 2003).

Trichomycterus spegazzinii (Berg, 1897)

Pygidium Spegazzinii Berg, 1897: 267. Type locality: Río de Cachi (Provincia de Salta), ..., á una altura de 2500 á 2800 metros sobre el nivel del mar, Argentina. Syntypes: BMNH 1898.9.23.1-2 (2), MACN 4925 (19), MACN 5173 (2), SMF 831 (1).

Distribution: Provinces of Salta and Catamarca in Argentina (de Pinna \& Wosiacki, 2003).

Trichomycterus spelaeus Do Nascimiento, Villarreal \& Provenzano, 2001

Trichomycterus spelaeus Do Nascimiento, Villarreal \& Provenzano, 2001: 21, fig. 1. Type locality: Cueva Punto Fijo, en el caserío Punto Fijo a 7,5 km al N. del Cerro Yolanda. 590 m.s.n.m., cuenca del Río Guasare, Edo. Zulia, Venezuela (1057'10"N 72²8'06"O). Holotype: MBUCV V-29602.

Distribution: Punto Fijo Cave, upper Guasare River basin, Venezuela (de Pinna \& Wosiacki, 2003).

Trichomycterus spilosoma (Regan, 1913)

Pygidium spilosoma Regan, 1913d: 468. Type locality: Rio Sipi and Rio Tamana, Colombia. Syntypes (3, 130-250 mm TL): BMNH 1910.7.11.106-107 (2), BMNH 1910.7.11.108 (1, skeleton), BMNH 1910.7.11.15 (1).

Distribution: Sipi and Tamana River basins, Colombia (de Pinna \& Wosiacki, 2003).

Trichomycterus stawiarski (Miranda Ribeiro, 1968)

Pygidium stawiarski Miranda Ribeiro, 1968: 1. Type locality: Pequeno córrego pertencente á bacia do Rio Paraná — 
localidade de Bituruna —Est. do Paraná, Paraná, Brazil. Holotype: MNRJ 9739.

Distribution: Iguaçu River basin, Brazil (de Pinna \& Wosiacki, 2003).

Trichomycterus stellatus (Eigenmann, 1918)

Pygidium stellatum Eigenmann, 1918a: 308, pl. 47 (fig. 1). Type locality: Quebrada Sarjento, Colombia. Holotype: FMNH 58101.

Distribution: Rivers of Andean eastern cordillera, Colombia (de Pinna \& Wosiacki, 2003).

Trichomycterus straminius (Eigenmann, 1918)

Pygidium straminium Eigenmann, 1918c: 694. Type locality: Quebrada del Mango, Santander, Colombia. Holotype: FMNH 58105; holotype illustrated in Eigenmann (1918a: 313, pl. 49, fig. 1) as Pygidium stramineum.

Distribution: Andean streams of Cundinamarca and Santander, Colombia (Maldonado-Ocampo et al., 2005).

Trichomycterus striatus (Meek \& Hildebrand, 1913)

Pygidium striatum Meek \& Hildebrand, 1913: 78. Type locality: Río Cana at Cana, Panama. Holotype: FMNH 7579.

Pygidium septentrionale Behre, 1928: 309, pl. 18. Type locality: small streams tributary to Rio Chiriqui del Tire above Caldera, Pacific slope of Panama, altitude about 4,000 feet ... Quebrada Salão, Panama, ca. elev. $4000 \mathrm{ft}$. Holotype: FMNH 59522.

Distribution: Rivers of Panama and Costa Rica (de Pinna \& Wosiacki, 2003); Catatumbo, Magdalena, Cauca and Dagua rivers, Colombia (Maldonado-Ocampo et al., 2005).

Trichomycterus taczanowskii Steindachner, 1882

Trichomycterus Taczanowskii Steindachner, 1882b: 177. Type locality: Rio Huambo, Rio de Tortora [Peru]. Syntype: NMW 43387 (1). Species illustrated and described in more detail in Steindachner (1882c: 22, pl. 4, figs. 1, $1 \mathrm{a}, 1 \mathrm{~b})$.

Distribution: Peru (de Pinna \& Wosiacki, 2003).

Trichomycterus taenia Kner, 1863

Trichomycterus taenia Kner, 1863: 228, fig. 16. Type locality: Vom Westabhange der Andes im Staate Ecuador, Ecuador. Holotype: at NMW. Also appeared in Kner \& Steindachner (1864: 52, pl. 6, fig. 1).

Distribution: Ecuadorian Andes (de Pinna \& Wosiacki, 2003); Magdalena and Patia River basins, Colombia (Maldonado-Ocampo et al., 2005).

Trichomycterus taeniops Fowler, 1954

Pygidium tenue Fowler, 1945c: 6, figs. 7-9. Type locality: Acobamba, near Tarma at 2000 meters elevation, Rio Ucayali basin, Peru. Holotype: ANSP 71638. Preoccupied in Trichomycterus by Trichomycterus tenuis Weyenbergh, 1877; replaced by Trichomycterus taeniops Fowler, 1954.

Trichomycterus taeniops Fowler, 1954: 36, fig. 635. Type locality: Acobamba, near Tarma, at 2000 meters elevation, Rio Ucayali basin, Peru, elev. 2000 m, Peru. Replacement for Pygidium tenue Fowler, 1945; preoccupied in Trichomycterus by Trichomycterus tenuis Weyenburgh, 1877.

Distribution: Ucayali River basin (elevation 2000 m), Peru (de Pinna \& Wosiacki, 2003).

Trichomycterus taroba Wosiacki \& Garavello, 2004

Trichomycterus taroba Wosiacki \& Garavello, 2004: 10, fig. 6. Type locality: Brazil: State of Paraná: município de Foz do Jordão, Jordão reservoir, rio Jordão near at its mouth, tributary of rio Iguaçu (rio Paraná Basin), 25\%45'S, $52^{\circ} 10^{\prime}$ W. Holotype: MPEG 6689.

Distribution: Jordão River, Iguaçu River basin, Brazil (Wosiacki \& Garavello, 2004).

Trichomycterus tenuis Weyenbergh, 1877

Trichomycterus tenuis Weyenbergh, 1877: 12, pl. 3 (figs. a-c). Type locality: una pequeña laguna, en la Sierra de Córdoba, cerca de la Villa Cruz-del-eje, Argentina. Syntypes: MSNG 8852 (2).

Distribution: Córdoba Sierra, Argentina (de Pinna \& Wosiacki, 2003).

Trichomycterus transandianus (Steindachner, 1915)

Pygidium taenia transandianum Steindachner, 1915e: 100, pl. 12 (fig. 6). Type locality: Gebirgsbach im Cañon del Gallo, einem rechten Seitentale des Rio Combeima in der Zentral-Cordillere, Columbien, in einer Höhe von 1800 m. Syntypes (2): NMW 44475. Originally as Pygidium taenia Var. nova transandianum.

Distribution: Combeima River basin, central Andean cordillera, Colombia (de Pinna \& Wosiacki, 2003). 
Trichomycterus trefauti Wosiacki, 2004

Trichomycterus trefauti Wosiacki, 2004: 3, fig. 2. Type locality: riacho Andrequicé, tributary of rio Paraúna, itself a tributary of rio das Velhas (rio São Francisco basin), approximately $18^{\circ} 30^{\prime} \mathrm{S}, 43^{\circ} 30^{\prime} \mathrm{W}$, Município de Trinta Réis, Minas Gerais, Brazil. Holotype: MZUSP 79911.

Distribution: Upper São Francisco River basin, Brazil (Wosiacki, 2004).

Trichomycterus triguttatus (Eigenmann, 1918)

Pygidium triguttatum Eigenmann, 1918a: 339, pl. 52 (fig. 4). Type locality: Jacarehy, São Paulo, Brazil. Holotype: FMNH 58670.

Distribution: São Paulo State, Brazil (de Pinna \& Wosiacki, 2003).

Trichomycterus tupinamba Wosiacki \& Oyakawa, 2005

Trichomycterus tupinamba Wosiacki \& Oyakawa, 2005: 466, figs. 2-4. Type locality: Brazil, São Paulo: rio Betari, tributary of rio Ribeira de Iguape, in front of the camping area of Núcleo Santana, Parque Estadual Turístico do Alto Ribeira. Holotype: MZUSP 62382.

Distribution: Betari and Iporanga rivers, Ribeira de Iguape River basin, Brazil (Wosiacki \& Oyakawa, 2005).

Trichomycterus unicolor (Regan, 1913)

Pygidium unicolor Regan, 1913d: 468. Type locality: Condoto, Colombia. Syntypes: BMNH 1913.10.1.42-43 (2).

Distribution: San Juan River basin, Colombia (de Pinna \& Wosiacki, 2003).

Trichomycterus variegatus Costa, 1992

Trichomycterus variegatus Costa, 1992: 103, fig. 3. Type locality: Estado de Minas Gerais: ville de São Roque de Minas, rio do Peixe, affluent du haut rio Sao Francisco [Brazil]. Holotype: MZUSP 42316.

Distribution: Upper São Francisco River basin, Brazil (de Pinna \& Wosiacki, 2003).

Trichomycterus venulosus (Steindachner, 1915)

Pygidium venulosum Steindachner, 1915a: 199. Type locality: Paramo de Cruz verde, östliche Cordillere, Columbien, in 3000 m. Syntypes: NMW 44476 (2). Species illustrated and described in more detail in Steindachner (1915e: 85, pl. 121, figs. 3-4).

Distribution: Streams of eastern Andean cordillera, Colombia (Maldonado-Ocampo et al., 2005).

Trichomycterus vermiculatus (Eigenmann, 1918)

Pygidium vermiculatum Eigenmann, 1918c: 699. Type locality: Juiz de Fora, Rio Parahyba, Brazil. Holotype: FMNH 58077; holotype illustrated in Eigenmann (1918a: 335, pl. 52, fig. 2).

Distribution: Paraíba do Sul River, State of Minas Gerais, Brazil (de Pinna \& Wosiacki, 2003).

Trichomycterus vittatus Regan, 1903

Trichomycterus vittatus Regan, 1903b: 623. Type locality: Marcapata Valley, E. Peru. Holotype: BMNH 1902.5.29.210.

Distribution: Eastern Peru (de Pinna \& Wosiacki, 2003).

Trichomycterus weyrauchi (Fowler, 1945)

Pygidium weyrauchi Fowler, 1945c: 7, figs. 10-12. Type locality: Acobamba, near Tarma, at 2200 meters elevation, Rio Ucayali drainage, Peru. Holotype: ANSP 71639.

Distribution: Ucayali River basin (elev. 2900 m), Peru (de Pinna \& Wosiacki, 2003).

Trichomycterus yuska Fernández \& Schaefer, 2003

Trichomycterus yuska Fernández \& Schaefer, 2003: 355, figs. 1-2. Type locality: Argentina: Provincia de Catamarca, Departamento Tinogasta, Arroyo Aguas Calientes, 4050 m elevation. Holotype: FML 2535.

Distribution: Known only from the type locality, a high elevation stream in western Argentina (Fernández \& Schaefer, 2003).

Trichomycterus zonatus (Eigenmann, 1918)

Pygidium zonatum Eigenmann, 1918a: 330, pl. 51 (fig. 1). Type locality: Agua Quente, São Paulo, Brazil. Holotype: FMNH 58573.

Trichomycterus cubataonis Bizerril, 1994: 618, figs. 1-3. Type locality: Rio Cubatão, Joinville, Estado de Santa Catarina [Brazil]. Holotype: MNRJ 12490.

Distribution: Coastal rivers from Santa Catarina to São Paulo States, Brazil (de Pinna \& Wosiacki, 2003). 
TRIDENS Eigenmann \& Eigenmann, 1889

Tridens Eigenmann \& Eigenmann, 1889b: 53. Type species: Tridens melanops Eigenmann \& Eigenmann, 1889. Type by original designation. Gender: Masculine.

Tridens melanops Eigenmann \& Eigenmann, 1889

Tridens melanops Eigenmann \& Eigenmann, 1889b: 53. Type locality: Iça [Brazil]. Syntypes (27): CAS 64598 (1), BMNH 1889.11.14.73 (1), MCZ 8137 (1), MCZ 1566385 (6), MCZ 156639 (2, c\&s), MZUSP uncat [ex. MCZ 156638] (1), USNM 41522 (1), USNM 120296 (3).

Distribution: Amazon River basin, Brazil (de Pinna \& Wosiacki, 2003).

TRIDENSIMILIS Schultz, 1944

Tridensimilis Schultz, 1944c: 266. Type species: Tridensimilis venezuelae Schultz, 1944. Type by original designation. Gender: Masculine.

Tridensimilis brevis (Eigenmann \& Eigenmann, 1889)

Tridens brevis Eigenmann \& Eigenmann, 1889b: 54. Type locality: Tabatinga [Brazil]. Holotype: MCZ 8160.

Distribution: Amazon River basin, Brazil (de Pinna \& Wosiacki, 2003).

Tridensimilis venezuelae Schultz, 1944

Tridensimilis venezuelae Schultz, 1944c: 267, pl. 6 (fig. c). Type locality: Río Negro, below the mouth of the Río Yasa, Maracaibo basin, Venezuela. Holotype: USNM 121290.

Distribution: Orinoco River basin, Venezuela (de Pinna \& Wosiacki, 2003).

\section{TRIDENTOPSIS Myers, 1925}

Tridentopsis Myers, 1925b: 84. Type species: Tridentopsis pearsoni Myers, 1925. Type by original designation. Gender: Feminine.

Tridentopsis cahuali Azpelicueta, 1990

Tridentopsis cahuali Azpelicueta, 1990: 982, figs. 1-4. Type locality: Estancia El Bagual, Formosa Province, Argentina, 26¹0'53"S, 58 56'39"W. Holotype: MLP 5-IX-89-1.

Distribution: Paraguay River basin, Argentina (de Pinna \& Wosiacki, 2003).

Tridentopsis pearsoni Myers, 1925

Tridentopsis pearsoni Myers, 1925b: 84. Type locality: Lagoons at Lake Rogoagua, Bolivia. Holotype: CAS 28258.

Distribution: Upper Amazon River basin, Bolivia (de Pinna \& Wosiacki, 2003).

Tridentopsis tocantinsi La Monte, 1939

Tridentopsis tocantinsi La Monte, 1939: 1. Type locality: Rio Tocantins, northeastern Brazil. Holotype: AMNH 13967.

Distribution: Tocantins River basin, Brazil (de Pinna \& Wosiacki, 2003).

\section{TYPHLOBELUS Myers, 1944}

Typhlobelus Myers, 1944: 593. Type species: Typhlobelus ternetzi Myers, 1944. Type by original designation. Gender: Masculine.

Key: Schaefer et al. (2005).

Typhlobelus guacamaya Schaefer, Provenzano, de Pinna \& Baskin, 2005

Typhlobelus guacamaya Schaefer, Provenzano, de Pinna \& Baskin, 2005: 14, figs. 7, 8. Type locality: Venezuela, Estado Amazonas, Río Cuao at Raudal Guacamaya, 8.1 miles upstream from Raudal El Danto, $05^{\circ} 07.71$ 'N, 67³1.53'W. Holotype: MBUCV V-30936.

Distribution: Cuao River, Orinoco River basin, Venezuela (Schaefer et al., 2005).

Typhlobelus lundbergi Schaefer, Provenzano, de Pinna \& Baskin, 2005

Typhlobelus lundbergi Schaefer, Provenzano, de Pinna \& Baskin, 2005: 10, figs. 5, 6. Type locality: Venezuela, Estado Delta Amacuro, Río Orinoco at Los Castillos, upstream of east end of Caño Limon, $8^{\circ} 31.2^{\prime} \mathrm{N}, 62^{\circ} 35.1^{\prime} \mathrm{W}$. Holotype: MBUCV V-31040.

Distribution: Lower Orinoco River, between Ciudad Bolivar and Los Castillos, Venezuela (Schaefer et al., 2005).

Typhlobelus macromycterus Costa \& Bockmann, 1994 
Typhlobelus macromycterus Costa \& Bockmann, 1994b: 68, figs. 1-3. Type locality: Brazil: Estado do Pará, Rio Tocantins near Tucuruí. Holotype: MNRJ 12129.

Distribution: Tocantins River near Tucuruí, Pará State, Brazil (de Pinna \& Wosiacki, 2003).

Typhlobelus ternetzi Myers, 1944

Typhlobelus ternetzi Myers, 1944: 593, pl. 52 (fig. 2), pl. 53 (figs. 6-8). Type locality: Rock pools below São Gabriel Rapids, Rio Negro, Brazil. Holotype: CAS 11118.

Distribution: Upper Negro River basin, Brazil (de Pinna \& Wosiacki, 2003).

VANDELLIA Valenciennes, 1846

Vandellia Valenciennes, in Cuvier \& Valenciennes, 1846: 386. Type species: Vandellia cirrhosa Valenciennes, 1846. Type by monotypy. Gender: Feminine.

Urinophilus Eigenmann, 1918a: 358. Type species: Vandellia sanguinea Eigenmann, 1918. Type by subsequent designation by Eigenmann (1920d: 441). Gender: Masculine. Originally proposed without any included species; species first assigned, and a type species designated, in Eigenmann (1920d).

Vandellia beccarii Di Caporiacco, 1935

Vandellia beccarii Di Caporiacco, 1935: 59. Type locality: Rockstone, in flumine Essequibo dicto, in Guiana Britannica. Holotype: MZUF 5506.

Distribution: Orinoco River basin and rivers of Guyana (de Pinna \& Wosiacki, 2003).

Remarks: Redescribed in Schmidt (1987).

Vandellia cirrhosa Valenciennes, 1846

Vandellia cirrhosa Valenciennes, in Cuvier \& Valenciennes, 1846: 386, pl. 547. Type locality: [Probably from America]. Syntypes (2): MNHN a-6308.

Vandellia gigantea Cornalia, 1849: 15, figs. 4-5. Type locality: [Fl. Amazonum et Napo]. Type(s): lost (Cagnolaro \& Violani, 1988).

Vandellia plazaii Castelnau, 1855: 51, pl. 28 (fig. 1). Type locality: rio Ucayale (Pérou). Holotype: MNHN a-6309.

Vandellia Balzanii Perugia, 1897: 23. Type locality: Rio Beni, Missioni Mosetenes, Bolivia. Holotype: MSNG 8848.

Urinophilus erythrurus Eigenmann, 1922b: 114, pls. 3 (figs. 5-7), 4 (10-16). Type locality: Río Morona, Peru. Holotype: CAS 64599.

Distribution: Amazon River basin (de Pinna \& Wosiacki, 2003).

Remarks: Vandellia gigantea Cornalia, 1849, was treated as a nomen oblitum by Cagnolaro \& Violani (1988), but considered to be available, albeit a junior synonym, in de Pinna \& Wosiacki (2003).

Vandellia sanguinea Eigenmann, 1918

Vandellia sanguinea Eigenmann, 1918c: 701. Type locality: San Antonio de Rio Madeira. Holotype: FMNH 58086; holotype illustrated in Eigenmann (1918a: 365, pl. 53, fig. 2).

Distribution: Amazon, Orinoco and Essequibo River basins (de Pinna \& Wosiacki, 2003).

\section{Genus inquirendum, Trichomycteridae}

Pygidium Meyen, 1835, in Meyen, 1834-35: 475. Type species: Pygidium fuscum Meyen, 1835. Type by monotypy. Gender: Neuter. Familial assignment of this genus is uncertain (cf. Tchernavin, 1944).

Remarks: The family group name Pygidiidae was proposed in Eigenmann \& Eigenmann (1888a: 649) on Pygidium Meyen, 1835.

\section{Species inquirenda, Trichomycteridae}

Pygidium fuscum Meyen, 1835, in Meyen, 1834-35: 369. Type locality: Peru. Holotype: Whereabous unknown. Familial assignment of this species is uncertain (see Tchernavin, 1944).

\section{UNPLACED NAMES}

\section{SILURIFORMES Incertae sedis}


$\dagger$ BACHMANNIA Dolgopol de Saez, 1945

$\dagger$ Bachmannia Dolgopol de Saez, 1945: 453. Type species: † Bachmannia chabutensis Dolgopol de Saez, 1945. Type by Monotypy. Gender: Feminine.

$\dagger$ Bachmannia chabutensis Dolgopol de Saez, 1945

$\dagger$ Bachmannia chabutensis Dolgopol de Saez, 1945: 453, figure on p. 453. Type locality: de la laguna del Hunco y del Mirador de Chubut [Argentina]; Tertiary. Holotype: MLP 40-V-17-1a (impression).

$\dagger$ Arius argentinus Dolgopol de Saez, 1945: 455, figure on p. 455. Type locality: La Laguna del Huncho y del Niradorde, Chabut, Argentina; Tertiary. Holotype: MLP 40-V-17-3 (impression of cranium and partial vertebral column).

Distribution: Miocene of Argentina (Gayet \& Meunier, 2003).

Remarks: Synonymy from Gayet \& Meunier (2003), which appears to be based on Arratia \& Cione (1996).

\section{CONORHYNCHOS Bleeker, 1858}

Conostome Duméril, 1856: 484. Type species: Pimelodus conirostris Cuvier, 1836. Type by original designation. Gender: Feminine. Possibly preoccupied by Conostoma Hodgson, 1842, in birds and not treated as valid in recent times. Considered a nomen oblitum.

Conorhynchos Bleeker, 1858: 191, 205, 209. Type species: Pimelodus conirostris Cuvier, 1836. Type by monotypy. Gender: Neuter. New name for Conostome Duméril, which is apparently preoccupied by Conostoma Hodgson, 1842, in birds. Name often misspelled Conorhynchus following the spelling in Bleeker (1862-63).

Conorhynchus Bleeker, 1863 (in Bleeker, 1862-63): 12. Type species: Pimelodus conirostris Cuvier, 1836. Type by original designation. New spelling for Conorhynchos. Preoccupied by Conorhynchus Motschousky, 1860, in Coleoptera.

Conorhynchichthys Regan, 1908, in Regan, 1906-08: 192. Type species: Pimelodus conirostris Cuvier, 1836. Type by being a replacement name. Gender: Masculine. Replacement for Conorhynchus Bleeker, 1863 [=Conorhynchos Bleeker, 1858], preoccupied by Conorhynchus Motschousky, 1860.

Remarks: Initially included in the Pimelodidae, but that placement questioned in Reis et al. (2003).

Conorhynchos conirostris (Cuvier, 1836)

Pimelodus conirostris Cuvier, 1836: 541, footnote 12. Type locality: rivière de Saint-François. Holotype: MNHN a9413. Described and illustrated in Cuvier \& Valenciennes (1840b: 204 (156 in Strasbourg deluxe edition), pl. 436).

Conorhynchus glaber Steindachner, 1877b: 637, pl. 8. Type locality: einem flusse bei Porto Seguro [apparently in error]. Holotype: at NMW.

Distribution: São Francisco River basin, Brazil.

\section{$\dagger$ EOPEYERIA Whitley, 1947}

$\dagger$ Ariopsis Peyer, 1928: 43. Type species: † Ariopsis aegyptiacus Peyer, 1928. Type by monotypy. Gender: Feminine. Preoccupied by Ariopsis Gill, 1861, in Recent fishes. Replaced by $\dagger$ Peyeria Whitley, 1940.

$\dagger$ Peyeria Whitley, 1940a: 242. Type species: † Ariopsis aegyptiacus Peyer, 1928. Type by being a replacement name. Gender: Feminine. Replacement for $\dagger$ Ariopsis Peyer, 1928, preoccupied by Ariopsis Gill, 1861, in Recent fishes. But, $\uparrow$ Peyeria Whitley preoccupied by $\uparrow$ Peyeria Weiler, 1935, in fossil Chondrichthyes.

$\dagger$ Eopeyeria Whitley, 1947: 150. Type species: †Ariopsis aegyptiacus Peyer, 1928. Type by being a replacement name. Gender: Feminine. Replacement for $\dagger$ Peyeria Whitley, 1940, preoccupied by $\dagger$ Peyeria Weiler, 1935, in fossil Chondrichthyes.

$\dagger$ Eopeyeria aegyptiaca (Peyer, 1928)

$\dagger$ Ariopsis aegyptiacus Peyer, 1928: 43, pl. 5, pl. 6 (fig. 1). Type locality: Qasr-es-Sagha-Stufe, Norden des Fajum. Ägyptens; Eocene. Holotype: at Bayerische Staatssammlung, München.

Distribution: Fajum, Egypt; Eocene.

$\dagger$ FAJUMIA Stromer, 1904

$\dagger$ Fajumia Stromer, 1904: 3. Type species: $\dagger$ Fajumia schweinfurthi Stromer, 1904. Type by monotypy. Gender: 
Feminine.

Remarks: Placed by Jordan (1923) into the Bagridae, but placed in the Ariidae by Peyer (1928). Gayet \& Meunier (2003) place this genus as Siluriformes incertae sedis, possibly belonging within the Arioida.

$\dagger$ Fajumia menoni Sahni \& Mishra, 1975

$\dagger$ Fajumia menoni Sahni \& Mishra, 1975: 7, pl. 1 (figs. 3 a-c). Type locality: Bluish-grey stage (Middle Eocene) at Gabhatad, Western India; Middle-Eocene. Holotype: LUVP 11140 (cranium).

Distribution: Western India, middle Eocene (Sahni \& Mishra, 1975).

Remarks: Placed in the Bagridae by Sahni \& Mishra (1975), following placement of genus in that family by Jordan (1923).

$\dagger$ Fajumia misrai Sahni \& Mishra, 1975

$\dagger$ Fajumia misrai Sahni \& Mishra, 1975: 8, pl. 1 (figs. 4-5). Type locality: Bluish-grey shales of Babia Stage (Middle Eocene) at Nareda, Western India. Holotype: LUVP 11142 (cranium).

Distribution: Western India; middle Eocene (Sahni \& Mishra, 1975).

Remarks: Placed in the Bagridae by Sahni \& Mishra (1975), following placement of genus in that family by Jordan (1923).

$\dagger$ Fajumia schweinfurthi Stromer, 1904

$\dagger$ Fajumia Schweinfurthi Stromer, 1904: 3, pl. 1 (figs. 1-2). Type locality: Mitteleocän am Nordrande des Fajûm, Aegypten. Holotype: in Munich (? at Bayerische Staatssammlung, München) (nearly complete cranium).

Distribution: Fajum, Egypt; middle Eocene.

$\dagger$ Fajumia stromeri Peyer, 1928

$\dagger$ Fajumia Stromeri Peyer, 1928: 33, fig. 3, pl. 4 (fig. 1). Type locality: Qasr-es-Sagha-Stufe, Norden des Fajum. Ägyptens; Eocene. Holotype: at Bayerische Staatssammlung, München.

Distribution: Fajum, Egypt; middle Eocene.

HORABAGRUS Jayaram, 1955

Horabagrus Jayaram, 1955b: 261. Type species: Pseudobagrus brachysoma Günther, 1864. Type by original designation. Gender: Masculine.

Remarks: Horabagrus was initially placed in the Bagridae and has subsequently been suggested to be more closely related to species of the Schilbidae (K. C. Jayaram, pers. comm.) or as a separate family that also includes some species currently placed in the Schilbidae (de Pinna, 1998a, Hardmann, 2005).

Horabagrus brachysoma (Günther, 1864)

Pseudobagrus brachysoma Günther, 1864: 86. Type locality: Cochinchina. Holotype: BMNH 1862.9.18.5 (skin).

Pseudobagrus chryseus Day, 1865b: 290. Type locality: Kurriavanoor River, Kurriapudnam or Cochin, India. Holotype: Possibly AMS B.7922 (1), BMNH 1865.7.17.5-6 (2), MCZ 4280 (1), NMW 44177 (1), RMNH 6870 (1), ZMB 9575 (1), ZSI 475 (1, lost). Also described and illustrated in Day (1865a: 185, pl. 13, fig. 2).

Distribution: Vernbanad Lake and estuaries of Kerala and southwestern Karnataka, India (Pethiyagoda \& Kottelat, 1994).

Remarks: See Jayaram (1952) for taxonomic comments.

Horabagrus nigricollaris Pethiyagoda \& Kottelat, 1994

Horabagrus nigricollaris Pethiyagoda \& Kottelat, 1994: 110, fig. 13. Type locality: India: Kerala: Chalakudy River, $26 \mathrm{~km}$ upstream of Chalakudy town, near Vettilappara. Holotype: AMS I.34198-001.

Distribution: Chalakudy River, Kerala, India (Pethiyagoda \& Kottelat, 1994).

PHREATOBIUS Goeldi, 1905

Phreatobius Goeldi, 1905: 549. Type species: Phreatobius cisternarum Goeldi, 1905. Type by monotypy. Gender: Masculine.

Remarks: Originally treated as a genus of the Trichomycteridae, in recent years this genus has been placed within the Heptapteridae (e. g., Bockmann \& Guazzelli, 2003), following Buckup (1988) and the unpublished study by Bockmann (1998). However, Muriel-Cunha \& de Pinna (2005) suggests that the placement of this genus and its single named species is unsettled. The family-group name Phreatobinae was proposed for this genus by Reichel 
(1927: 383).

Phreatobius cisternarum Goeldi, 1905

Phreatobius cisternarum Goeldi, 1905: 549. Type locality: Wasser einer Binnenlandzisterne tief im Innern der Mündung des Amazonenstromes vorgelagertern Rieseninsel Marajó [Brazil]. Syntypes (2): MHNG 2623.30 (1), plus one of the following: FMNH 58580 (1), MHNG 1213.97 (1), or MHNG 1505.91 (2 entire specimens plus one sectioned and mounted on microscopic preparations).

Distribution: Marajó island, mouth of the Amazon River, Brazil, in cisterns (Bockmann \& Guazzelli, 2003).

Remarks: See Reichel (1927), Carvalho (1967) and Muriel-Cunha \& de Pinna (2005) for further information on this enigmatic species.

$\dagger$ PLIOSILURUS Weiler, 1956.

$\dagger$ Pliosilurus Weiler, 1956: 180. Type species: † Pliosilurus primus Weiler, 1956. Type by monotypy. Gender: Masculine.

Remarks: Listed as $\dagger$ Pliosilurus primus gen. \& sp. nov., which, if published before 1931, would have made the type designation by original designation, but not after 1930 .

$\dagger$ Pliosilurus primus Weiler, 1956

$\dagger$ Pliosilurus primus Weiler, 1956: 180, figs. 1-2; pl. 11. Type locality: Willershausen bek Göttingen [Pliocene, Germany]. Holotype: Geologischen Instituts der Universität Göttingen 4836 (head, pectoral girdle, portion of vertebral column and anal fin).

Distribution: Germany; Pliocene.

$\dagger$ RHINEASTES Cope, 1872

$\dagger$ Rhineastes Cope, 1872b: 486. Type species: $\dagger$ Rhineastes peltatus Cope, 1872 . Type by original designation. Gender: Masculine.

Remarks: $†$ Rhineastes has generally been placed in the Ariidae, but Lundberg (1992: 396) considered that placement in error and, instead, treated the genus as Incertae sedis in the Siluriformes, which is followed here.

$\dagger$ Rhineastes peltatus Cope, 1872

$\dagger$ Rhineastes peltatus Cope, 1872b: 486. Type locality: Bridger Formation, South Bitter Creek, Wyoming. Holotype USNM 3984 (supraoccipital bone and dorsal spine); holotype illustrated in Cope (1884b: 63, pl. 5, figs. 1-2).

Distribution: Bridger Formation, Wyoming; known only from type locality (Lundberg, 1975).

$\dagger$ Rhineastes smithii Cope, 1872

$\dagger$ Rhineastes smithii Cope, 1872b: 486. Type locality: Bridger Formation, South Bitter Creek, Wyoming. Holotype: USMN 3978 (pectoral spine); holotype illustrated in Cope (1884b: 64, pl. 5, figs. 5-11).

Distribution: Bridger Formation, Wyoming (Lundberg, 1975).

Remarks: Inclusion in $\dagger$ Rhineastes considered tentative by Lundberg (1975).

\section{Species inquirenda, $\dagger$ Rhineastes}

$\dagger$ Rhineastes radulus Cope, 1873: 639. Type locality: Bridger Formation at Cottonwood Creek, Wyoming; Eocene. Holotype: USNM 4099 (fragments); holotype illustrated in Cope (1884b: 67, pl. 5, figs. 14-17). Identity uncertain (Lundberg, 1975).

$\dagger$ SOCNOPAEA Stromer, 1904

$\dagger$ Socnopaea Stromer, 1904: 6. Type species: † Socnopaea grandis Stromer, 1904. Type by monotypy. Gender: Feminine.

Remarks: Jordan (1923) placed this genus and $\uparrow$ Fajumia into the Bagridae, but Peyer (1928) placed both genera in the Ariidae.

$\dagger$ Socnopaea grandis Stromer, 1904

$\dagger$ Socnopaea grandis Stromer, 1904: 6, pl. 1 (fig. 3). Type locality: Mitteleocän am Nordrande des Fajûm, Aegypten. Holotype: in Munich (partial cranium).

Distribution: Fajum, Egypt; middle Eocene. 
$†$ Socnopaea horai Sahni \& Mishra, 1975

$\dagger$ Socnopaea horai Sahni \& Mishra, 1975: 10, pl. 2 (fig. 1). Type locality: Ossiferus gypseous shales of Babia stage (Middle Eocene) at Harudi, Western India. Holotype: LUVP 11145 (cranium).

\section{Genera inquirendae, Siluriformes}

Tachysurus La Cepède, 1803: 150. Type species: Tachysurus sinensis La Cepède, 1803. Type by monotypy. Gender: Masculine. The type species is considered to be an unrecognizable siluriform by Wheeler \& Baddokwaya (1981) and Taylor (1986a).

Laimumena Sauvage, 1884a: 147. Type species: Laimumena barbonica Sauvage, 1884. Type by monotypy. Gender: Feminine. Description of type species is not recognizable to genus or even family.

Trachymochlus Hoedeman, 1961: 137. Type species: Trachymochlus cupido Hoedeman, 1961. Type by monotypy. Gender: Masculine.

\section{Species inquirendae, Siluriformes}

Silurus undecimalis Linnaeus, 1758: 305. Type locality: Not stated. Type(s): originally part of collection at Museum Adolphi Friderici but whereabouts unknown, apparently not at NRM. Type locality stated as "Surinami" in Linnaeus (1764: 97).

Silurus luvur Molina, 1782: 346. Type locality: Chile. No types known.

Silurus schilby Sonnini, 1799: 256, pl. 23 (fig. 1). Type locality: Nil. On p. 295 there is note about Silurus schilbe niloticus of Hasselquist that suggests that this may not be intended to be the proposal of a new name.

Silurus gurgu Bloch \& Schneider, 1801: 388. Type locality: in Nilo. No types known, based on literature account. Proposed conditionally.

Silurus minutus Schneider, in Bloch \& Schneider, 1801: 389. Type locality: India. No types known, based on unpublished literature source. Proposed conditionally.

Tachysurus sinensis La Cepède, 1803: 150, 151, pl. 5 (fig. 2). Type locality: China. No types known; based on a Chinese painting; illustration in La Cepède reproduced in Wheeler \& Baddokwaya (1981). Treated in many Chinese works as valid in Arius, but Wheeler \& Baddokwaya (1981) and Taylor (1986a) indicate that it is an unrecognizable siluriform from the description and/or illustration.

Silurus ichneumon Hermann, 1804: 309. Type locality: Cairo. No types known.

Pimelodes fossor Lichtenstein, 1823: 112. Type locality: Cape of Good Hope, South Africa. Type(s): at ZMB.

Pimelodus octocirrhus Cuvier, 1829: 294. Type locality: not stated. Available from footnote as, "Pim. octocirrhus, N., Seb., III, xxix, 1" [= Seba (1734-65: pl. 29, fig. 1)].

Pimelodus javus Valenciennes, in Cuvier \& Valenciennes, 1840b: 187 (139 in Strasbourg deluxe edition). Type locality: Java. Holotype (4 1/2 pouces): at MNHN. Status uncertain (Roberts, 1993: 47).

Pimelodus tachisurus Valenciennes, in Cuvier \& Valenciennes, 1840b: 163 (121 of Strasbourg deluxe edition). Type locality: China. No types known; based on a Chinese painting. Unneeded new name for Tachisurus chinensis [sic, for sinensis] La Cepède.

Pimelodus Cantonensis Valenciennes, in Cuvier \& Valenciennes, 1840b: 142 (106 of Strasbourg deluxe edition). Type locality: Canton [China]. No types known. Based solely on an illustration.

Bagrus chinta Valenciennes, in Cuvier \& Valenciennes, 1840a: 445 (330 of Strasbourg deluxe edition). Type locality:[Vizagapatam]. No types known, based on account and illustration in Russell (1803: pl. 167).

Pimelodus pusillus Ranzani, 1842: 332, pl. 27. Type locality: Unknown [apparently Brazil, based on other species in publication]. Holotype: MZUB 933. Name appeared first in Ranzani (1841: 64) as a nomen nudum.

$\dagger$ Pimelodus Sadleri Heckel, 1849: 19. Type locality: Bihar Comitat, Hungary; Miocene. Holotype ? (fin ray fragments). Illustrated and described in more detail in Heckel (1850: 213, pl. 16, fig. 3).

Silurus ferox Gronow, in Gray, 1854: 135. Type locality: not stated. No types known.

Rhamdia javanica Bleeker, 1858b: 139. Type locality: Java. Holotype: at MNHN. Unjustified emendation of Pimelodus javus Valenciennes, 1840. 
Laimumena barbonica Sauvage, 1884a: 147. Type locality: Réunion. No types known. Not found in catalogs of MNHN (pers. obs.) and not seen on shelves.

$\dagger$ Silurus Serdicensis Toula, 1889: 108, pl. 9. Type locality: im Westen von Sofia. Upper Tertiary. Syntypes: (fragmentary remains).

$\dagger$ Silurus gaudryi Leriche, 1900: 181, pl. 1, figs. 7-12. Type locality: Cuis, Monthelon [Marne, Lower Eocene]. Types: at laboratoire de Géologie de l'Université de Lille (dorsal fin spines, pectoral fin spine). Originally Silurus (?)gaudryi. Remarks: As Pimelodus gaudryi in Leriche (1901:165).

$\dagger$ Silurus pliocaenicus Leidenfrost, 1925, 121, text fig. 2 and pl. 6. Type locality: pannonisch-pontischen Tonen von Rákos bei Budapest [Hungary, Pliocene]. Holotype: at Samml. der Kön. ung. Geologischen Anstalt, Budapest, partial neurocranium.

Remarks: Possibly a bagrid (S. L.), or a silurid (Gayet \& Meunier, 2003: 503).

$\dagger$ Silurus stenocephalus Leidenfrost, 1925, 122, pl. 7. Type locality: Pannonisch-pontischen Tonen von Rákos bei Budapest [Hungary, Pliocene]. Holotype: at Samml. der Kön. ung. Geologischen Anstalt, Budapest, partial neurocranium.

$\dagger$ Rhineastes grangeri Hussakof, 1932: 17, fig. 26. Type locality: Tung Gur beds, 50 miles southeast of Iren Dabasu, Inner Mongolia; Pliocene. Holotype: at AMNH (right pectoral fin spine).

Distribution: Known only from the type locality, in central Asia.

Trachymochlus cupido Hoedeman, 1961: 137, fig. 4. Type locality: Nickerie, entrance of Cupido creek into Maratakka River, Suriname. Holotype: ZMA 102236.

$\dagger$ Schmidelia graciliformis Berry. [no other information; from Dolgopol de Saez (1945)].

$\dagger$ Clarias pliocaenicus Sauvage (in Depéret, 1885). [no other information; from Gayet \& Meunier (2003)]

$\dagger$ Ariopsis peyeria Eocene, North Africa [no additional information, From Romer. Probably a mistake for Ariopsis Peyer]

\section{Names wrongly treated as Siluriformes}

$\dagger$ Arius lemoinei of Eocene of France [No additional information]

Remarks: Considered an acipenserid by Gayet \& Meunier (2003).

$\dagger$ BRACHYSPONDYLUS Marck, in Marck \& Schlüter, 1868: 283. Type species: † Brachspondylus cretaceus Marck, 1868. Type by monotypy. Gender: Masculine.

Remarks: Listed by Jordan (1923: 643) as a cyprinid with $\dagger$ Brachyspondylus saropterix Marck, 1876, as orthotype. Brachyspondylus saropterix has been considered to be a siluriform taxon, most recently by Gayet \& Meunier (2003), but $\dagger$ Brachyspondylus is actually available from Marck \& Schlüter (1868: 283), with † Brachyspondylus cretaceus (283, pl. 43, fig. 2) as type by monotypy. This species is a scaly fish, probably a cyprinid, so that $\dagger$ Brachyspondylus is not a catfish name. See Sanders (1934: 30) for discussion of the taxonomic history of this name.

$\dagger$ BUCKLANDIUM König, 1825

$\dagger$ Bucklandium König, 1825: 4. Type species: $†$ Bucklandium diluvii König, 1825. Type by monotypy. Gender: Neuter.

$\dagger$ Glyptocephalus Agassiz, 1843 (in Agassiz, 1833-43): 264. Type species: $†$ Glyptocephalus radiatus Agassiz, 1843. Type by monotypy. Gender: Masculine. Preoccupied by Glyptocephalus Gottsche, 1834, in Recent fishes (Pleuronectidae).

$\dagger$ Glyptocara Gill, 1888: 926. Replacement for $†$ Glyptocephalus Agassiz, 1844, preoccupied by Glyptocephalus Gottsche, 1834.

Remarks: The name Ephippus Owenii appears in Agassiz (1843 (in Agassiz, 1833-43): 264) in the account of the newly proposed name Glyptocephalus radiatus. It is not treated as a valid name and is therefore not available from this usage. Gayet \& Meunier (2003) place $†$ Bucklandium as Siluriformes incertae sedis, possibly belonging within the Arioida, which appears to follow Woodward (1889b: 208), in which the name is treated as a siluriform fish near to Auchenoglanis. However, the illustration provided by Woodward is apparently not that of a 
siluriform fish and after close examination of the type specimen, Ralf Britz (pers. commun.) concluded that the bones are definitely not those of a catfish.

$\dagger$ Bucklandium diluvii König, 1825

$\dagger$ Bucklandium diluvii König, 1825: 4, pl. 8, no. 91. Type locality: Isle of Sheppey, Lower Eocene, London Clay. Holotype: BMNH P.9230 (cranium and pectoral arch). Redescribed and illustrated in Woodward (1889b: 208, pl. 22).

$\dagger$ Glyptocephalus radiatus Agassiz, 1843 (in Agassiz, 1833-43): 264. Type locality: [Argiles de Londres] Sheppy. Holotype: BMNH P.9230 (cranium and pectoral arch).

Distribution: London Clay, Lower Eocene.

Remarks: $\dagger$ Glyptocephalus radiatus Agassiz, 1843, is an unneeded new name for $\dagger$ Bucklandium diluvii König, 1825, which Agassiz apparently did not associate with the examined specimen.

$\dagger$ PROPYGIDIUM Bocchino, 1964: 186. Type species: † Propygidium primaevus Bocchino, 1964. Type by original designation. Gender: Neuter.

Remarks: Although originally described as a member of the siluriform family Trichomycteridae, the material was reexamined and interpreted as a member of the Perciformes by Cione \& Torno (1988).

$\dagger$ Propygidium primaevus Bocchino, 1964: 186, fig. on p. 189. Type locality: Serie Andesítica Lask Bayas, to the west of Rio Negro Province, Argentina; Eocene/Oligocene. [Corrected to: Cerro David, $0.5 \mathrm{~km}$ from the road from San Carlos de Bariloche to Chenqueniyeu, to the west of the Province of Rio Negro, from the middle levels of the Nirihuau Formation (Upper Oligocene-Lower Miocene), by Cione \& Torno (1988)]. Holotype: División de Paleontología Vertebrados del Museo de La Plata 21-871, partial skeletal impression.

$\dagger$ TREWAVASIA White \& Moy Thomas, 1941

$\dagger$ Xenopholis Davis, 1887: 548. Type species: † Xenopholis carinatus Davis, 1887, by monotypy. Gender: Feminine. Preoccupied by Xenopholis Peters, 1869, in Reptiles; replaced by $\dagger$ Trewavasia White \& Moy Thomas, 1941, and $†$ Xenopholoides Fowler, 1958.

$\dagger$ Trewavasia White \& Moy Thomas, 1941: 400. Type species: † Xenopholis carinatus Davis, 1887. Gender: Feminine. Type by being a replacement name. Replacement for $\dagger$ Xenopholis Davis, 1887; preoccupied by Xenopholis Peters, 1869, in Reptiles. Gender: Feminine.

$\dagger$ Xenopholoides Fowler, 1958: 13. Type species: $\dagger$ Xenopholis carinatus Davis, 1887. Gender: Feminine. Type by being a replacement name. Replacement for $\dagger$ Xenopholis Davis, 1887; preoccupied by Xenopholis Peters, 1869, in Reptiles.

Remarks: Described by Davis (1887) as probably showing a "closer connection with siluroids than with any other group" but clearly not a siluriform fish. Most likely an Acanthopterygian.

$\dagger$ Trewavasia carinata (Davis, 1887)

$\dagger$ Xenopholis carinatus Davis, 1887: 549, pl. 29, fig. 4. Type locality: Mt. Lebanon, Syria, Hard Chalk. 
Appendix 1. Siluriform genera and species named from otoliths. Unless otherwise noted, all names were found in Weiler's (1968) catalogue of fish otoliths as belonging to catfishes

\section{Generic names}

$\dagger$ Claibornichthys Frizzel \& Dante, 1965: 697. Type species: † Claibornichthys troelli Frizzel \& Dante, 1965. Type by original designation. Gender: Masculine.

Remarks: $\dagger$ Otolithus (Sciaenidarum) decipiens Koken, 1888, tentatively included as a second species of this genus by Frizzel \& Dante (1965).

$\dagger$ Vorhisia Frizzell, 1965: 179. Type species: $†$ Vorhisia vulpes Frizzell, 1965. Type by original designation. Gender: Feminine.

Remarks: The family group name $†$ Vorhisiidae was proposed in the same publication.

\section{Species names}

(Names listed by date of publication)

$\dagger$ Otolithus (incertae sedis) crassus Koken, 1884: 559, pl. 12, fig. 13. Type locality: Heaton Hill, Isle of Wight. Holotype: at Roy. Mus. Nat. Hist. Berlin (otolith), but apparently lost (Stinton, 1977: 71).

Remarks: Placed in Arius by Weiler (1968). Treated as valid as † Tachysurus crassus by Stinton (1977: 71).

$\dagger$ Otolithus (Sciaenidarum) decipiens Koken, 1888: 285, pl. 19 (figs. 5-6). Type locality: Calyborne-Schicten. Syntypes (2): Whereabouts unknown..

Remarks: Not listed among siluriform otolith based names in Weiler (1968), but treated a possible second species of $\dagger$ Claibornichthys (as † Otolithus (Arius) decipiens) in Frizzel \& Dante (1965).

$\dagger$ Arius baroni Newton, 1889: 207, pl. 21, fig. 7. Type locality: Ankoala, Madagascar; Eocene. Holotype: at BMNH, in the Baron collection ( 1 of 8 otoliths). Name available from figure caption, thus illustrated specimen is the holotype.

$\dagger$ Raja similis Woodward, 1889a: 86, pl. 4, figs. 4-5. Type locality: Hampshire, England; Eocene. (Otoliths).

Remarks: Name corrected to $\dagger$ Arius similis on plate caption. Treated as a synonym of $\dagger$ Tachysurus crassus (Koken) by Stinton (1977: 71).

$\dagger$ Otolithus (Arius) danicus Koken, 1891: 81, fig. 1. Type locality: Copenhagen; lower Eocene. Based on otolith identified as $\dagger$ Otolithus cf. crassus in Koken (1885: 116, pl. 5, fig. 29).

$\dagger$ Otolithus (Arius) germanicus Koken, 1891: 81, pl. 1, fig. 3, and pl. 6, fig. 8. Type locality: Lattorf, Westeregeln, and Osterwedingen, Lower Oligocene; Sollingern, Weinheim, Waldboeckelheim, Middle Oligocene. (otoliths).

$\dagger$ Otolithus (Arius) vanigonis Koken, 1891: 81, pl. 6, fig. 4. Type locality: Mitteloligöcan, Waldböeckelheim.

$\dagger$ Otolithus (Arius) Lerichei Priem, 1906: 277, figs. 46-47. Type locality: problement du Thanétien ou Yprésien ? des environs de Reims, éocènes du bassin Parisien. Holotype (partial Lapillus): Collection Bourdot.

† Otolithus (Siluridarum ?) incertus Priem, 1906: 277, figs. 48-49. Type locality: problement d'Hérouval (Yprésien supérieur), éocènes du bassin Parisien. Holotype: ? MNHN.

$\dagger$ Otolithus (Arius ?) moravicus Schubert, 1908: 106, abb. 3. Type locality: Marnes de Pausram (Lower Ologocene). Holotype: GBW 1908/01/4.

Remarks: Nolf (1981: 136) listed this name as a rejected species.

$\dagger$ Otolithus (Arius) angelicus Bassoli, 1909: 41, fig. 1. Type locality: Headon Member, Solent formation, Brockenhurst, Hampshire, Britain. Holotype: (Lapillus).

Remarks: Treated as valid as $\dagger$ Galeichthys angelicus by Stinton (1977: 73).

$\dagger$ Otolithus (Arius) crassus bartonensis Shepherd, 1916: 183, fig. 155 (5). Type locality: Ober- Eozän, England. Originally † Otolithus (Arius) crassus var. bartonensis.

Remarks: Treated as a synonym of $\dagger$ Tachysurus crassus (Koken) by Stinton (1977: 71).

$\dagger$ Otolithus (Arius) danicus bartonensis Shepherd, 1916: 180, fig. 157 (11). Type locality: Upper Eocene, England. Originally $\dagger$ Otolithus (Arius) danicus var. bartonensis.

Remarks: Treated as a synonym of $\dagger$ Tachysurus planus (Frost), as well $\dagger$ Tachysurus crassus (Koken) as by Stinton 
(1977: 70).

$\dagger$ Otolithus (Arius ?) parvus Schubert, 1916: 287, pl. 7 (fig. 24). Type locality: Barton Formation (Upper Eocene) at Barton Cliff, England. Syntypes: GBW 1916/01/25, 25a.

Remarks: Treated as a synonym of $\dagger$ Tachysurus crassus (Koken) by Stinton (1977: 71). Nolf (1981: 136) listed this name as a rejected species.

$\dagger$ Otolithus (Arius) newtoni Schubert, 1916: 286, pl. 7 (fig. 3). Type locality: Barton Formation (Upper Eocene) at Barton Cliff, England. Syntypes: GBW 1916/01/3 (or MGB 0427, see Stinton, 1977: 72).

Remarks: Treated as a synonym of $\dagger$ Tachysurus crassus (Koken) by Stinton (1977: 71). Nolf (1981: 136) treated this name as a synonym of $\dagger$ Arius crassus (Koken).

$\dagger$ Otolithus (Arius) tenuis Frost, 1925: 28, pl. 2 (fig. 37). Type locality: Hoofdsossiel-zone, Al. Geureugah (Nisam), Sumatra; upper Tertiary (Neogene). Holotype: (? Lapillus).

Remarks: Name spelled $\dagger$ Otolithus (Arius) tenius on plate, which is treated here as an invalid name.

$\dagger$ Otolithus (Arius) africanus Frost, 1926: 84, pl. 18, figs. 7-8. Type locality: Ameki, Nigeria; Eocene. Syntypes (5): (Lapillus).

$\dagger$ Otolithus (Arius) amekiensis Frost, 1926: 84, pl. 18, fig. 10. Type locality: Ameki, Nigeria; Eocene. Holotype: (Lapillus).

$\dagger$ Otolithus (Arius) angulatus Frost, 1926: 84, pl. 18, fig. 9. Type locality: Ameki, Nigeria; Eocene. Syntypes (3): (Lapillus).

$\dagger$ Otolithus (Arius ?) glaber Voigt, 1926: 177, pl. 2, figs. 23-24. Type locality: Bavaria, Cretaceous.

$\dagger$ Otolithus (Arius) jaekeli Richter 1928: 138, pl. 1, fig. 3. Type locality: Pomerania, Cretaceous.

$\dagger$ Otolithus (Arius) aequus Frost, 1934: 504, pl. 14 (fig. 15). Type locality: Barton Formation, Barton, Hampshire; Upper Eocene. Holotype: BMNH P 22732 (Lapillus).

Remarks: Treated as valid as $\dagger$ Galeichthys aequus by Stinton (1977: 71).

$\dagger$ Otolithus (Arius) planus Frost, 1934: 504, pl. 14 (fig. 14). Type locality: Barton Formation, Barton, Hampshire; Upper Eocene. Holotype: BMNH P 22730 (otolith).

Remarks: Treated as valid as † Tachysurus planus by Stinton (1977: 70).

$\dagger$ Arius rutschi Casier, 1958: 22, pl. 2 (figs. 12, 15). Type locality: l'Ile de la Barbade; formation de Scotland superieure. Holotype: TLLS 80. (Lapillus).

$\dagger$ Arius cavatus Stinton, 1962: 82, pl. 19 (fig. 6). Type locality: Borneo, Lower Pliocene. Holotype: (Lapillus).

$\dagger$ Tachysurus oblongus Stinton, 1962: 81, pl. 19 (fig. 3). Type locality: Borneo, Miocene-Pliocene. Type(s): Whereabouts unknown.

$\dagger$ Netuma radiata Stinton, 1962: 81, pl. 1 (fig. 17). Type locality: Borneo, Pliocene.

$\dagger$ Netuma regularis Stinton, 1962: 82, pl. 19 (fig. 5). Type locality: Borneo, Pliocene.

$\dagger$ Otolithus (Arius) rotundatus Roedel, 1930: 52, pl. 1, fig. 17. Type locality: Köthen und Frqankfurt [Germany], Eocene. Holotype: (Lapillus).

† Vorhisia vulpes Frizzell, 1965: 180, figs, 2, 3. Type locality: Upper Cretaceous (Maestrichtian): along left bank of Grand River; Sec. 19, T20N, R20W; Corson County, South Dakota. Holotype: Frizzell personal collection, no number (otolith: left lapillus).

Distribution: Fox Hill Formation: Irish Creek lithofacies, Bullhead lithofacies, and Colgate lithofacies, South Dakota, USA; Upper Cretaceous (Frizzell \& Koenig, 1973).

$\dagger$ Claibornichthys troelli Frizzel \& Dante, 1965: 697, pl. 86 (figs. 1, 2, 8, 17, 18, 19). Type locality: Stone City Beds: Brazos River, west of Bryan Texas. Holotype: USNM 23371 (otolith).

$\dagger$ Diplomystes rudis Stinton, 1966: 423, pl. 66, fig. 12. Type locality: London Clay, England.

Distribution: London Clay, England, Lower Eocene (Weiler, 1968). 
AFY Personal collection of Agustín Fernández-Yépez; portions now at SCN and MBUCV, Venezuela.

AI

AMG

AMNH

AMS

ANSP

ASIZB

BCUE

BKNU

BMNH

BSMP

CAS

CM

CPUC

CSIRO Commonwealth Science \& Industrial Research Organization, Division of Marine Research, Hobart, Tasmania, Australia.

CU Cornell University Museum of Vertebrates, Ithaca, New York, U.S.A.

DGM Paleontological collection of the Setor de Paleontologia, Departamento Nacional de Produço Mineral, Universisade do Estado do Rio de Janeiro, Brazil.

DVZUT Department of Vertebrate Zoology, University of Tông-Hop, Hanoi, Vietnam.

DZSASP Departamento de Zoologia, Secretaria da Agricultura, São Paulo, Brazil. Formerly Museu Paulista.

ECO-SC El Colegio de la Frontera Sur, San Cristóbal, Chiapas, Mexico.

EEBP Estaçao Experimental de Biologia e Piscicultura de Pirassununga, Brazil.

FAKU Kyoto University, Department of Fisheries, Faculty of Agriculture, Japan.

FFSUC Faculty of Forestry Sciences, Universidad de Chile, Ichthyological Collection, Santiago, Chile.

F/GUZ Department of Zoology, Gauhati University, Guwahati, Assam, India.

FML Instituto Fundacion Miguel Lillo, Tucuman, Argentina.

FMNH Field Museum of Natural History, Chicago, Illinois, U.S.A.

FRLM Faculty of Fisheries, Mie University, Fisheries Research Laboratory, Mie-ken, Japan.

GBW Collection du Gelogische Bundesanstald, Vienna, Austria.

GCM Government College, Department of Zoology, Lahore, Pakistan.

GSJ Geological Society of Japan.

ICNMHN Instituto de Ciencias Naturales, Museo de Historia Natural, Universidad Nacional de Colombia, Bogotá, Colombia. .

IHASW Institute Hydrobiology Academy Sinica, Wuhan, China.

ILPLA Museo de La Plata, Instituto de Limnologia, La Plata, Argentina.

IMCN Natural Sciences Museum Federico Carlos Lehmann V.- INCIVA Cali, Colombia

INHS Illinois Natural History Survey, Champaigne, Illinois, U.S.A.

INPA Instituto Nacional de Pesquisas da Amazonia, Manaus, Amazonas, Brazil.

INVEMAR Instituto de Investigaciones Marinas de Punta de Betin, Santa Marta, Colombia.

IRSNB Institut Royal des Sciences Natureles de Belgique, Brussels, Belgium.

IU Indiana University, Bloomington, Indiana, U.S.A. Now distributed among several institutions, primarily CAS, UMMZ and USNM; many IU types remain unaccounted for.

IUQ Laboratorio de Ictiología, Departamento de Biología, Universidad del Quindío, Armenia, Colombia.

IZUA Universidad Austral de Chile, Instituto de Zoologia, Valdivia, Chile.

JFBM Bell Museum Fish Collection at the University of Minnesota. 
JNU Department of Biology, Ji Nan University, China.

KIZ Kunming Institute of Zoology, Chinese Academy of Sciences, Kunming, Yunnan, China.

KU University of Kansas, Museum of Natural History, Lawrence, Kansas, U.S.A.

KUMF Kasetsart University Museum of Fisheries, Bangkok, Thailand.

LACM Los Angeles County Museum of Natural History, Los Angeles, California, U.S.A.

LIVCM World Museum Liverpool, U.K.

LUVP Vertebrate Palaeontology Laboratory, Geology Department, Luchnow Uiversity, Luchnow, India.

MACN Museo Argentino de Ciencias Naturales, Bernardino Rivadavia, Capital Federal, Argentina.

MB Universidade de Lisboa, Museu Bocage, Lisboa, Portugal.

MBLUZ Museo de Biología de la Universidad del Zulia, Venezuela.

MBUCV Universidad Central de Venezuela, Museo de Biologia, Caracas, Venezuela.

MCN.USB Museo de Ciencias Naturales, Universidad Simón Bolívar, Caracas, Venezuela.

MCNG Museo de Ciencias Naturales, Guanare, Venezuela.

MCP Pontifícia Universidade Católica do Rio Grande do Sul, Museu de Ciências, Rio Grande do Sul, Porto Alegre, Brazil.

MCZ Museum of Comparative Zoology, Harvard University, Cambridge, Massachusetts, U.S.A.

MD Museo do Dundo, Dundo, Angola.

MEPN Escuela Polytecnica Nacional, Quito, Ecuador.

MHNC Museo de Historia Naturale de Cochabamba, Bolivia.

MHNG Museum d'Histoire Naturelle, Geneve, Switzerland.

MHNL Musée d'Hisoire Naturelle du Luxembourg.

MHNLR Museum d'Histoire Naturelle, La Rochelle, France.

MHNLS Museo de Historia Natural de La Salle, Caracas, Venezuela.

MHNM Museo de Historia Natural de Montevideo, Montevideo, Uruguay.

MHNN Museum d'Histoire Naturelle, Neuchatel, Switzerland.

MHV Musée de Haute Volta, Ouagadougou, Upper Volta.

MIKU Marine Biological Institute, Kyoto University, Japan. Specimens now at FAKU.

MLP Museo de La Plata, La Plata, Argentina.

MNHN Muséum National d'Histoire Naturelle, Paris, France.

MNHNP Museo Nacional de Historia Natural de Paraguay.

MNKP Museo de Historia Natural Noel Kempff Mercado, Santa Cruz, Bolivia.

MNRJ Universidade Federal do Rio de Janeiro, Museu Nacional, Rio de Janeiro, Brazil.

MNSB Museum of Natural Sciences, Budapest, Hungary.

MPEG Museu Paraenese 'Emilio Goeldi', Para, Brazil.

MRAC Musée Royal de l'Afrique Centrale, Tervuren, Belgium.

MRCN Museu Rio-Grandense de Ciências Naturais, Brazil.

MSINR Museum Sichuan Institute of Natural Resources, China.

MSNG Museo Civico di Storia Naturale di Genova 'Giacomo Doria', Genova, Italy.

MSNM Museo Civico di Storia Naturale, Milano, Italy.

MSUM Michigan State University, University Museum, East Lansing, Michigan, U.S.A.

MTD Staatliches Museum für Tierkunde, Dresden, Germany.

MUMF Department of Life Sciences, Manipur University Museum of Fishes, Canchipur, Manipur, India.

MUSM Museo de Historia Natural de la Universidad Nacional Mayor de San Marcos, Lima, Peru.

MZB Museum Zoologicum Bogoriense, Bogor, Indonesia.

MZUF Universita di Firenze, Museo Zoologico de la Specola, Firenze, Italy.

MZS Universite de Strasbourg, Musée de Zoologie, Strasbourg, France.

MZUSP Universidade de São Paulo, Museu de Zoologia, São Paulo, Brazil.

MZUT Universita di Torino, Museo Zoologico, Torino, Italy.

NIFI National Inland Fisheries Institute, Fish Taxonomy Division, Bangkok, Thailand.

NMBA Naturhistorisches Museum Basel, Basel, Switzerland. 


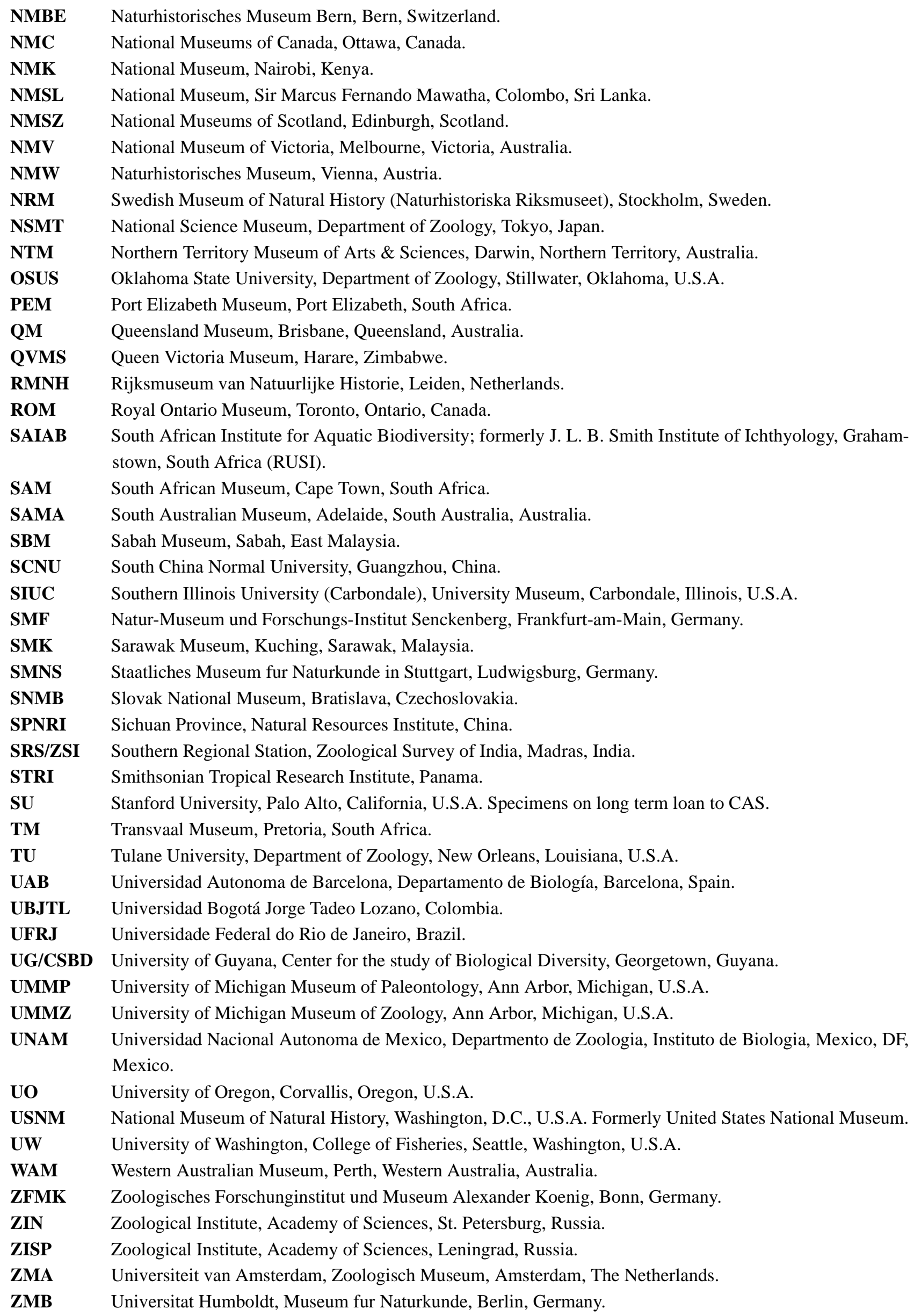


ZMFMIB Zoological Museum Fan Memorial Institute of Biology, Tsing Hua University, Peiping, China.

ZMH Universitat Hamburg, Zoologisches Institut und Museum, Hamburg, Germany.

ZMMU Zoological Museum, Moscow University, Russia.

ZMUB Universitetet i Bergen, Zoologisk Museum, Bergen, Norway.

ZMUC Kobenhavns Universitet Zoologisk Museum (Zoological Museum, University of Copenhagen), Copenhagen, Denmark.

ZMUL Universitetets Lund, Zoologiska Museet, Lund, Sweden.

ZMUO Universitetets I Oslo, Zoologisk Museum, Oslo, Norway.

ZMUT University of Tokyo, Department of Zoology, University Museum, Tokyo, Japan.

ZMUU Zoologiska Museet, Uppsala Universitet, Uppsala, Sweden.

ZRC Zoological Reference Collection, National University of Singapore, Singapore.

ZSI Zoological Survey of India, Kolkata [Calcutta], India.

ZSIC Zoological Survey of India, Chennai, India.

ZSI/NRS Northern Regional Station, Zoological Survey of India, Dehradun, India.

ZSI/SRS Southern Regional Station, Zoological Survey of India, Madras, India.

ZVC-P Facultad de Ciencias, Universidad de la República, Montevideo, República Oriental del Uruguay

ZSM Zoologische Museum Staatssammlung, München, Germany.

ZX Zhuhai City Fisheries Science Research Institute, Guandong Province, China. 


\section{Appendix 3. Taxonomic publications on Siluriformes issued in 2006}

The following publications were issued in 2006, after the cutoff date for this Checklist. The publications are listed in alphabetical order of authorship, with an abbreviated summary of the taxonomic and nomenclatural actions taken, to make the checklist as up to date as possible. New names and other names that were subject to nomenclatural action have been noted and are included in the index. However, new names and any other proposed taxonomic changes were not incorporated into the statistics provided in Table 1.

Alencar, A.R. \& Costa, W.J.E.M. (2006) Trichomycterus pauciradiatus, a new catfish species from the upper rio Paraná basin, southeastern Brazil (Siluriformes: Trichomycteridae). Zootaxa, 1269, 43-49.

Trichomycterus pauciradiatus Alencar \& Wilson, 2006: 44, fig. 1. Type locality: Brazil: Estado de Minas Gerais: Municipio de Carrancas, córrego Debaixo da Serra, stream tributary of córego Água Limba, rio Paraná basin, $21^{\circ} 26^{\prime} 40^{\prime \prime} \mathrm{S}$, 4436'09"W. Holotype: UFRJ 5831.

Distribution: Upper Paraná River basin, southeastern Brazil.

Almirón, A., Azpelicueta, M. de las M., Casciotta, J. \& Litz, T. (2006) A new species of Hisonotus (Siluriformes, Loricariidae, Otothyrini) from the Republica Oriental del Uruguay. Revue suisse de Zoologie, Annales de la Société zoologique suisse et du Muséum d'Histoire naturelle de Genève, 113, 87-94.

Hisonotus charrua Almirón Azpelicueta, Casciotta \& Litz, 2006: 88, figs. 1-3. Type locality: República Oriental del Uruguay, Departamento Tacuarembó, río Uruguay basin, Cañada de Los Peña (31 $\left.39.09^{\prime} \mathrm{S}, 56^{\circ} 12.32^{\prime} \mathrm{W}\right)$. Holotype: ZVC-P 5639.

Distribution: Uruguay River and La Plata River mouth, Uruguay.

Betancur-R., R. \& Acero P., A. (2006) A new species of Notarius (Siluriformes: Ariidae) from the Colombian Pacific. Zootaxa, 1249, 47-59.

Notarius armbrusteri Betancur-R. \& Acero P., 2006: 50, figs. 2-3. Type locality: fish market of Buenaventura, Valle del Cauca, Colombia. Holotype: INVEMAR-PEC 6677.

Distribution: Eastern Pacific coast, at Valle del Cauca, Colombia.

Key: Species of Notarius of Eastern Pacific on p. 58.

Casciotta, J., Azpelicueta, M. de las M., Almirón, A. \& Litz, T. (2006) Hisonotus candombe, a new species from the río Uruguay basin in the República Oriental del Uruguay (Siluriformes, Loricariidae, Otothyrini). Spixiana, 26 (2), 147-152.

Hisonotus candombe Casciotta, Azpelicueta, Almirón \& Litz, 2006: 147, figs. 1-3. Type locality: República Oriental del Uruguay, Departamento Salto, río Uruguay basin, arroyo Palomas (3104'43"S - 57³7'26"W. Holotype: ZVC-P 5595.

Distribution: lower Uruguay River basin, Uruguay.

Covain, R., Le Bail, P.Y., Sagnes, P. \& Fisch-Muller, S. (2006) Species of the genus Harttia (Siluriformes: Loricariidae) in French Guiana: Morphology, taxonomy and distribution. Cybium, 30, 3-18.

Day, J. J. \& Wilkinson, M. (2006) On the origin of the Synodontis catfish species flock from Lake Tanganyika. Biology Letters, doi:10.1098/rsbl.2006.0532, 1-5.

Diogo, R. \& Bills, R. (2006) Osteology and myology of the cephalic region and pectoral girdle of the South African catfish Austroglanis gilli, with comments on the autapomorphies and phylogenetic relationships of the Austroglanididae (Teleostei: Siluriformes). Animal Biology, 56, 39-62.

Diogo, R., Chardon, M. \& Vandewalle, P. (2006) Osteology and myology of the cephalic region and pectoral girdle of the Cetopsis coecutiens Spix \& Agassiz, 1829, comparison with other cetopsids, and comments on the synapomorphies and phylogenetic relationships of the Cetopsidae (Teleostei: Siluriformes). Belgian Journal of Zoology, $136,3-13$.

Diogo, R., Chardon, M. \& Vandewalle, P. (2006) Osteology and myology of the cephalic region and pectoral girdle of the Nematogenys inermis Guichenot, 1848, with comments on the autapomorphies and phylogenetic relationships of the Nematogenyidae (Teleostei: Siluriformes). Belgian Journal of Zoology, 136, 15-24.

Do Nascimiento, C. \& Provenzano, F. (2006) The genus Henonemus (Siluriformes: Trichomycteridae) with a description of a new species from Venezuela. Copeia, 2006, 198-205. 
Henonemus triacanthopomus Do Nascimiento \& Provenzano, 2006: 199, fig. 1. Type locality: Venezuela, Estado Delta Amacuro, beach at Caño Macareo, 849'16"N, 6200'00"W. Holotype: MBUCV-V 29526

Distribution: Apure and Arauca Rivers and in Caño Macareo in the Orinoco Delta, Venezuela.

Egge, J.J.D. \& Simons, A.M. (2006) The challenge of truly cryptic diversity: diagnosis and description of a new madtom catfish (Ictaluridae: Noturus). Zoologica Scripta, 35, 581-595.

Noturus maydeni Egge, 2006: 588, fig. 7. Type locality: Strawberry River at Simstown Public Access, 4 mi N of Evening Shade, 1.7 mi off U.S. Hwy 167 on Simstown Road (036 05'52"N, 09136'27"W), Sharp Co., AR [USA]. Holotype: JFBM 39465.

Distribution: Black and St. Francis river basins, Mississippi River drainage, Arkansas and Missouri, USA (Egge \& Simons, 2006).

Remarks: Not distinguishable morphologically from Noturus albater; diagnosis based on karyotypes, allozyme variation, and DNA sequences.

Fernández, L. \& Osinaga, K. (2006) A new Trichomycterus (Siluriformes: Trichomycteridae) from Aguarague National Park of the Bolivian preandean region, with comments on the relationships within the genus. Environmental Biology of Fishes, 75, 385-393.

Trichomycterus aguarague Fernández \& Osinaga, 2006: 386, fig. 1. Type locality: Bolivia, Tarija, Province Gran Chaco, National Park Aguarague, Quebrada Timboycito, a Río Caiguami tributary, elevation 700-800 m, 21 $30^{\prime} \mathrm{S}, 6^{\circ} 60^{\prime} \mathrm{W}$. Holotype: MNKP 4012.

Distribution: Caiguami River tributaries, Pilcomayo River basin, Paraná River system, Bolivia.

Friel, J.P. \& Vigliotta, T.R., (2006) Synodontis acanthoperca, a new species from the Ogôoué River system, Gabon with comments on spiny ornamentation and sexual dimorphism in mochokid catfishes (Siluriformes: Mochokidae). Zootaxa, 1125, 45-56.

Synodontis acanthoperca Friel \& Vigliotta, 2006: 47, figs. 1-3. Type locality: Gabon, Haut-Ogôoué Province, Ogôoué River at and below the Rapids of Massoukou (Masuku), 1³9'30"S 13³2'14"E. Holotype: CU 89005.

Distribution: Louétsi and Ogôoué Rivers, Ogôoué River basin, Gabon.

Jayaram, K.C. (2006) Catfishes of India, Narendra Publishing House, Delhi, xxii +323 p., 11 pl.

Pterocryptis barakensis Vishwanath \& Sharma, in Jayaram, 2006: 99, pl. 5, fig. 2. Type locality: India, Manipur, Tamenglong District, Barak River at Vanchengphai, village. Holotype: MUMF 4018.

Distribution: Barak River, Brahmaputra River basin, India.

Horabagrinae new subfamily [Schilbidae], Jayaram, 2006: 141. Type genus: Horabagrus Jayaram, 1955.

Horaglanidinae new subfamily [Clariidae], Jayaram, 2006: 309. Type genus: Horaglanis Menon, 1950.

Janssen, G., Devaere, S. Weekers, P. \& Adriaens, D. (2006) Phylogenetic and biogeographical analysis of African airbreathing catfish (Siluriformes: Clariidae): inferred from ribosomal genes and spacers sequences with emphasize on anguilliformity. Molecular Phylogeny and Evolution 38: 65-78.

Jerep, F.C., Shibatta, O.A., Pereira, E.H.L. \& Oyakawa, O.T. (2006) Two new species of Isbrueckerichthys Derijst, 1996 (Siluriformes: Loricariidae) from the rio Paranapanema basin, Brazil. Zootaxa, 1372, 53-68.

Isbrueckerichthys calvus Jerep, Shibatta, Pereira \& Oyakawa, 2006: 60, fig. 2. Type locality: Brazil; Paraná State; Apucarana; rio Tibagi basin; córrego Juruba; 23³4’44.6”S/51²2’12.6”W. Holotype: MZUEL 3714. Distribution: Tributary streams of the Taquara River, Tibagi River basin, Paraná State, Brazil.

Isbrueckerichthys saxicola Jerep, Shibatta, Pereira \& Oyakawa, 2006: 55, fig. 1. Type locality: Brazil; Paraná State;

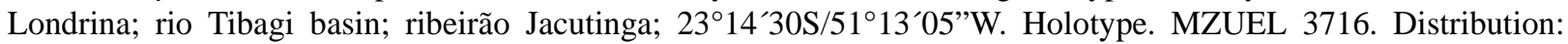
Ribeirão Jacutinga headwaters, Tibagi River basin, Paraná State, Brazil.

Koblmüller, S., Sturmbauer C., Verheyen, E., Meyer, A. \& Salzburger, W. (2006) Mitochondrial phylogeny and phylogeography of East African squeaker catfishes (Siluriformes: Synodontis). BMC Evolutionary Biology, 2006, 6 (49) (http://www.biomedcentral.com/1471-2148/6/49).

Mori, H. \& Shibatta, O.A. (2006) A new species of Microglanis Eigenmann, 1912 (Siluriformes, Pseudopimelodidae) from rio São Francisco basin, Brazil. Zootaxa, 1302, 31-42.

Microglanis leptostriatus Mori \& Shibatta, 2006: 33, fig. 1. Type locality: rio Verde Grande, $16^{\circ} 39^{\prime} \mathrm{S} / 46^{\circ} 11^{\prime} 57.8^{\prime \prime} \mathrm{W}$, Montes Claros, State of Minas Gerais, Brazil. Holotype: MZUSP 85985.

Distribution: São Francisco River basin, Brazil. 
Murdy, E.O. \& Ferraris, C.J., Jr. (2006) A revision of the marine catfish genus Euristhmus (Teleostei: Siluriformes: Plotosidae). The Beagle, Records of the Museums and Art Galleries of the Northern Territory, 22, 77-90.

Euristhmus microphthalmus Murdy \& Ferraris, 2006: 84, figs. 3, 6. Type locality: AUSTRALIA. Northern Territory. Woods Inlet, Darwin Harbour, $12^{\circ} 30^{\prime}$ S, $130^{\circ} 45^{\prime}$ E. Holotype: NTM S. 11242-001.

Distribution: Northern Territory, Australia and Irian Jaya, Indonesia, in nearshore habitats over soft bottoms.

Euristhmus sandrae Murdy \& Ferraris, 2006: 88, figs 3, 9. Type locality: AUSTRALIA. Western Australia. Exmouth Gulf, $21^{\circ} 42^{\prime} \mathrm{S}, 114^{\circ} 48^{\prime} \mathrm{E}, 9 \mathrm{~m}$. Holotype: WAM P.32730.001.

Distribution: Western Australia from south of Rowley Shoals and Exmouth Gulf, in waters as deep as 80m over soft bottoms.

Cnidoglanis lepturus Günther, 1864, Lectotype: BMNH 1864.1.17.33, designated on p. 82.

Key: species of Euristhmus on p. 80.

Near, T. J. \& Hardman, M. (2006) Phylogenetic relationships of Noturus stanauli and N. crypticus (Siluriformes: Ictaluridae), two imperiled freshwater fish species from the southeastern United States. Copeia, 2006, 378-383.

Ng, H.H. (2006) A Phylogenetic Analysis of the Asian Catfish Family Sisoridae (Teleostei: Siluriformes), and the Evolution of Epidermal Characters in the Group. Unpublished Ph.D. dissertation, University of Michigan, Ann Arbor.

Ng, H.H. (2006) Erethistoides infuscatus, a new species of catfish (Teleostei: Erethistidae) from South Asia. Ichthyological Explorations of Freshwaters, 17, 283-287.

Erethistoides infuscatus Ng, 2006: 284, fig. 1. Type locality: India: Meghalaya, East Khasi Hills, Umsing River. Holotype: UMMZ 245695.

Distribution: Brahmaputra and Meghna River drainages, northeast India and Bangladesh.

$\mathrm{Ng}$, H.H. (2006) The identity of Batasio tengana (Hamilton, 1822), with the description of two new species of Batasio from north-eastern India (Teleostei: Bagridae). Journal of Fish Biology, 68 (suppl. A), 101-118.

Batasio tengana Hamilton, 1822: Neotype designated by Ng, 2006: 103, fig. 1. Type locality: India: West Bengal: Tista River at Tista barrage; $26^{\circ} 45^{\prime} 10^{\prime \prime} \mathrm{N} ; 8^{\circ} 34^{\prime} 11^{\prime \prime E}$. Neotype: UMMZ 244796.

Distribution: Ganges and Brahmaputra River basins.

Batasio fasciolatus Ng, 2006: 107, fig. 4. Type locality: India: West Bengal, market at Malbazar: 26³2'30"N; 8844'17"E. Holotype: UMMZ 244798.

Distribution: Tista River basin, Brahmaputra River system.

Batasio spilurus Ng, 2006: 110, fig. 5. Type locality: India: Assam, Dibrugarh district, 27²9'N: 9454'E. Holotype: ZRC 49133.

Distribution: Brahmaputra River basin (Ng, 2006).

Ng, H.H. (2006) Pseudecheneis suppaetula, a new species of glyptosternine catfish (Teleostei: Sisoridae) from India. Zootaxa, 1267, 59-68.

Pseudecheneis suppaetula Ng, 2006: 60, fig. 1. Type locality: India: Himachal Pradesh, Ganges River drainage, upper reaches of Giri River, in Chhaila area (in the vicinity of Kotkhai), 31 ${ }^{\circ}$ ' $^{\prime \prime}$ "N $77^{\circ} 25^{\prime} 56^{\prime \prime E}$. Holotype: NRM 36977. Distribution: Ganges River basin, Himachal Pradesh, India.

Ng, H.H. (2006) Akysis longifilis, a new species of catfish (Teleostei: Akysidae) from Myanmar. Zootaxa, 1150, 19-30.

Akysis longifilis Ng, 2006: 20, fig. 1. Type locality: Myanmar: Bago division, Pyu township, Pyu stream (tributary of Sittang River) ca. 229 km from Yangon, 18²9'N, 96²6'E. Holotype: UMMZ 246172.

Distribution: Sittang River basin, Myanmar.

Ng, H.H. (2006) The identity of Pseudecheneis sulcata (M'Clelland, 1842), with descriptions of two new species of rheophilic catfish (Teleostei: Sisoridae) from Nepal and China. Zootaxa, 1254, 45-68.

Pseudecheneis eddsi Ng, 2006: 51, fig. 4. Type locality: Nepal: Tanahun, Khairenitar, Seti River (Ganges River drainage), $28^{\circ} 2^{\prime} 0.0^{\prime \prime} \mathrm{N} 84^{\circ} 4^{\prime} 0.0^{\prime \prime E}$. Holotype: KU 36872.

Distribution: Gandaki River basin, Nepal.

Pseudecheneis stenura Ng, 2006: 57, fig. 5. Type locality: China: Yunnan, Baoshan Prefecture, Longchuanjiang at Lianmengjie bridge (Irrawaddy River drainage). Holotype: KIZ 199811999.

Distribution: Irrawaddy River basin, Yunnan, China.

Ng, H.H. (2006) Pseudolaguvia ferula, a new species of sisoroid catfish (Teleostei: Erethistidae) from India. Zootaxa, 1229, 59-68.

Pseudolaguvia ferula Ng, 2006: 60, fig. 1. Type locality: India: West Bengal, Tista River at Tista Barrage, $26^{\circ} 45^{\prime} 10^{\prime \prime} \mathrm{N}$ 
88³4'11"E. Holotype: UMMZ 245985.

Distribution: Tista River, West Bengal, India.

Ng, H.H. \& Bailey, R.M. (2006) Chiloglanis productus, a new species of suckermouth catfish (Siluriformes: Mochokidae) from Zambia. Occasional Papers of the University of Michigan Museum of Zoology, 738, 1-13.

Chiloglanis productus Ng, \& Bailey, 2006: 2, figs. 1-3. Type locality: Zambia: Lunzua stream, $11.3 \mathrm{~km}$ SSE of Mpulungu, about $1 \mathrm{~km} \mathrm{~N}$ of bridge on Mbala-Mpulungu road, 849'S 31²10'E. Holotype: UMMZ 199816.

Distribution: Lunzua River basin, Lake Tanganyika drainage, Zambia.

Ng, H.H. \& Lim, K.K.P. (2006) Two new species of Leiocassis (Teleostei: Bagridae), riverine catfishes from northeast Borneo. Ichthyological Exploration of Freshwaters, 17, 165-172.

Leiocassis collinus Ng \& Lim, 2006: 166, fig. 1. Type locality: Borneo: Sabah: Danum Valley, Segama River drainage, Sungai Palum Tambun, tributary of Sungai Segama, upstream of Danum Valley Field Center. Holotype: ZRC 46154. Distribution: Kalabakan and Segama River basins, Sabah, Borneo.

Leiocassis tenebricus $\mathrm{Ng} \&$ Lim, 2006: 166, fig. 1. Type locality: Borneo: Kalimantan Timur: Kayan River drainage, Sungai Nah, tributary to Kayan River ca. 20 minutes upstream of confluence with Iwan River, $1^{\circ} 57^{\prime} 43.2^{\prime \prime} \mathrm{N}$ $115^{\circ} 6$ '35.4"E at $550 \mathrm{~m}$ asl;. Holotype: MZB 10718.

Distribution: Kayan River basin, northeastern Borneo.

Ortega-Lara, A. \& Lehmann A., P. (2006) Cruciglanis, a new genus of pseudopimelodid catfish (Ostariophysi: Siluriformes) with description of a new species from the Colombian Pacific coast. Neotropical Ichthyology, 4, $147-156$.

Cruciglanis Ortega-Lara \& Lehmann, 2006: 149. Type species: Cruciglanis pacifici Ortega-Lara \& Lehmann, 2006, by original designation. Gender: Masculine.

Cruciglanis pacifici Ortega-Lara \& Lehmann, 2006: 150, fig. 1. Type locality: Colombia, Valle del Cauca Department, near Buenaventura city, San Cipriano River where it crosses San Cipriano village, confluence of the La Sardina stream, approximately $500 \mathrm{~m}$ before the confluence with Dagua River, Dagua River basin, $03^{\circ} 50.543^{\prime} \mathrm{N}$ $76^{\circ} 54.068^{\prime} \mathrm{W}, 84 \mathrm{~m}$ of altitude. Holotype: IMCN 2359.

Distribution: Dagua River basin and Anchicayá River basin, Pacific versant, Colombia.

Parisi, B.M., Lundberg, J.G. \& DoNascimiento, C. (2006) Propimelodus caesius a new species of long-finned pimelodid catfish (Teleostei: Siluriformes) from the Amazon Basin, South America. Proceedings of the Academy Of Natural Sciences, Philadelphia, 155, 67-78.

Propimelodus caesius Parisi, Lundberg \& DoNascimiento, 2006: 68, figs. 1-2. Type locality: Brazil, Pará State, Rio Amazonas above Rio Trombetas, $20 \mathrm{~km}$ above Obidos, collected with $3 \mathrm{~m}$ bottom trawl in channel 4-7 m deep, $1^{\circ} 56^{\prime} 07.3 " \mathrm{~S}, 55^{\circ} 41^{\prime} 18.5^{\prime \prime W}$. Holotype: MZUSP 88582.

Distribution: Amazon River main stem and lower reaches of many tributary rivers, Brazil and Peru.

Peng, Z.-G., Ho, S.-W., Zhang, Y.-G. \& He, S.-P. (2006) Uplift of the Tibetan plateau: Evidence from divergence times of glyptosternoid catfishes. Molecular Phylogenetics and Evolution, 39, 568-572.

Provenzano, F. \& Milani, N. (2006) Cordylancistrus nephelion (Siluriformes, Loricariidae), a new and endangered species of suckermouth armored catfish from the Tuy River, north-central Venezuela. Zootaxa, 1116, 29-41.

Cordylancistrus nephelion Provenzano \& Milani, 2006: 31, figs. 1-2. Type locality: Venezuela, Caribbean Sea basin, Tuy River system, Mesia River, tributary of the Guare River, near Village Corocito, approximately $10^{\circ} 12^{\prime} \mathrm{N}$, $67^{\circ} 05^{\prime} \mathrm{W}$. Holotype: MBUCV-V-21800.

Distribution: Tuy River basin, Venezuela (Provenzano \& Milani, 2006).

Key: species of Cordylancistrus on p. 38.

Reis, R.E. \& Borges, T.A.K. (2006) The South American catfish genus Entomocorus (Ostariophysi: Siluriformes: Auchenipteridae), with the description of a new species from the Paraguay River basin. Copeia, 2006, 412-422.

Entomocorus radiosus Reis \& Borges, 2006: 416, figs. 6-7. Type locality: Brazil, Mato Grosso, Rio Paraguay at neighborhood of Cáceres, approx. $1^{\circ} 03^{\prime} \mathrm{S}, 057^{\circ} 42^{\prime} \mathrm{W}$. Holotype: MCP 35902.

Distribution: Paraguay River basin (Reis \& Borges, 2006).

Key: species of Entomocorus p. 419.

Reis, R.E., Pereira, E.H.L. \& Armbruster, J.A.W. (2006) Delturinae, a new loricariid catfish subfamily (Teleostei, Siluriformes), with revisions of Delturus and Hemipsilichthys. Zoological Journal of the Linnean Society, 147, $277-299$.

Delturus brevis Reis \& Pereira, 2006: 295, fig. 12. Type locality: Rio Araçuaí upstream of its mouth on Rio Jequitinhonha, Araçuaí, Minas Gerais, Brazil. Holotype: MZUSP 69858. 
Distribution: Jequitinhonha River basin, Minas Gerais, Brazil.

Delturinae new subfamily Armbruster, Reis \& Pereira, 2006: 279. Type genus: Delturus Eigenmann \& Eigenmann, 1889.

Key: subfamilies of Loricariidae, p. 279.

Key: Hemipsilichthys on p. 281.

Delturus parahybae Lectotype: MCZ 7726, designated on p. 293.

Delturus angulicauda Lectotype: NMW 44069, designated on p. 288.

Hemipsilichthys papillatus holotype illustrated on p. 285, fig. 6.

Hemipsilichthys nimius holotype illustrated on p. 287, fig. 8.

Retzer, M. E. (2006) A new species of Farlowella Eigenmann and Eigenmann (Siluriformes: Loricariidae), a stick catfish from Bolivia. Zootaxa, 1282, 59-68.

Farlowella altocorpus Retzer, 2006: 60, figs. 1, 2. Type locality: Bolivia: La Paz State: Río Beni Basin: Río Coroico, Caranavi. Holotype: INHS 99773.

Distribution: Coroico River, Beni River basin, Bolivia.

Salcedo, N.J. (2006) New species of Chaetostoma (Siluriformes: Loricariidae) from central Peru. Copeia, $2006,60-67$.

Chaetostoma changae, Salcedo, 2006: 61, fig. 1. Type locality: Peru, Departamento de Huánuco, vicinity of Tingo María, back-water near Puerto Nuevo, flowing into Río Tullamayo, 09¹8'S, 7559'W, 649 m. Holotype: ANSP 179125.

Distribution: Huallaga River basin at Tingo María, Peru.

Salcedo, N.J. (2006) Two new species of Chaetostoma (Siluriformes: Loricariidae) from the Huallaga River in central Peru. Ichthyological Exploration of Freshwaters, 17, 207-220 [not seen].

Chaetostoma daidalmatos Salcedo, 2006

Chaetostoma stroumpoulos Salcedo, 2006

Sarmento-Soares, L.M., Martins-Pinheiro, R.F., Aranda, A.T. \& Chamon, C.C. (2006) Microglanis pataxo, a new catfish from southern Bahia coastal rivers, northeastern Brazil (Siluriformes: Pseudopimelodidae). Neotropical Ichthyology, 4, 157-166.

Microglanis pataxo Sarmento-Soares, Martins-Pinheiro, Aranda \& Chamon, 2006: 158, Fig. 1. Type locality: Brazil, Bahia: Itamarajú, Jundiar creek on road BR-101 after joint with road to Jucuruçu, in the neighbourhoods of the city of Itamaraju (1701'35"S 39³5'57"W). Holotype: MNRJ 28397.

Distribution: Peruipe, Jucurucu and Cahy Rivers, northeastern Brazil.

Sarmento-Soares, L.M., Martins-Pinheiro, R.F., Aranda A.T. \& Chamon, C.C. (2006) Ituglanis cahyensis, a new catfish from Bahia, Brazil (Siluriformes: Trichomycteridae). Neotropical Ichthyology, 4, 309-318.

Ituglanis cahyensis Sarmento-Soares, Martins-Pinheiro, Aranda \& Chamon, 2006: 310, fig. 1. Type locality: Brazil, Bahia: Prado, rio Palmares on road Guarany- Corumbau, in direction to Corumbau after the joint with the road to the mouth of rio Cahy, (16²57'48, 39¹6'33"W). Holotype: MNRJ 28404.

Distribution: Palmares River, Cahy River basin, Southeastern Bahia State, Brazil.

Sullivan J.P., Lundberg, J.G. \& Hardman, M. (2006) A phylogenetic analysis of the major groups of catfishes (Teleostei: Siluriformes) using rag1 and rag2 nuclear gene sequences. Molecular Phylogenetics and Evolution, 41, 636-662.

Tamang, L., Chaudhury, S. \& Chauhury, D. (2006) On a new record of freshwater fish, Pseudolaguvia shawi (Hora) from Arunachal Pradesh, India (Teleostomi, Erethistidae). Zoos' Print Journal, 21 (11), 2443-2446.

Thomson, A.W. \& Page, L.M. (2006) Genera of the Asian catfish families Sisoridae and Erethistidae (Teleostei: Siluriformes). Zootaxa, 1345, 1-90.

Diagnoses and review of named taxa above species level.

Synonymy of Hara and Erethistes.

Vari, R. P. \& Ferraris, C.J., Jr. (2006) The Catfish genus Tetranematichthys (Auchenipteridae). Copeia, $2006,168-180$.

Tetranematichthys wallace $i$ Vari \& Ferraris, 2006: 169, figs. 1a, 2, 4. Type locality: Brazil, Amazonas, upper Rio Negro, São Pedro, mouth of Igarapé do Ibará, approximately 0¹5'S, 6646'W. Holotype: MZUSP 31096,

Distribution: Orinoco, Negro Amazon and Tocantins River basins.

Villa-Verde, L. \& Costa, W.J.E.M. (2006) A new glanapterygine catfish of the genus Listrura (Siluriformes: Trichomycteridae) from the southeastern Brazilian coastal plains. Zootaxa, 1142, 43-50. 
Listrura picinguabae Villa-Verde \& Costa, 2006: 44, figs. 1-3. Type locality: Brazil: Estado de São Paulo: Município de Ubatuba, serra do Mar, Picinguaba, small stream tributary to rio da Fazenda, on small road near Km 11 of the road BR-101, Parque Estadual da Serra do Mar, about 2320'S 44²5'W. Holotype: UFRJ 6111, 48.6 mm SL.

Distribution: da Fazenda River tributaries, São Paulo State, southeastern Brazil.

Vishwanath, W. \& Darshan, A. (2006) A new species of the genus Batasio Blyth (Teleostei: Bagridae) from Manipur, India. Zoos' Print Journal, 21, 2160-2163.

Batasio niger Vishwanath \& Darshan, 2006: 2160, fig. 1. Type locality: Khujailok stream, Chandel district, Manipur, India. Holotype: MUMF 9028.

Distribution: Chindwin River basin, Manipur, India.

Remarks: Publication includes illustration of tooth plates only; illustration of holotype available only on web version of paper.

Wright, J.J. \& Page, L.M. (2006) Taxonomic revision of Lake Tanganyikan Synodontis (Siluriformes: Mochokidae). Florida Museum of Natural History Bulletin, 46, 99-154.

Synodontis grandiops Wright \& Page, 2006: 109, figs. 3c, 8, 9, 10. Type locality: Mwakizega coastline, L. Tanganyika. Holotype: BMNH 1982.4.13.4785.

Distribution: Lake Tanganyika.

Synodontis ilebrevis Wright \& Page, 2006: 117, figs. 5d, 13, 14, 15. Type locality: Lake Tanganyika at Chaitika, Zambia. Holotype: UF 160942.

Distribution. Lake Tanganyika, only from the Cape Chaitika area, Zambia.

Synodontis lucipinnis Wright \& Page, 2006: 126, figs. 4a, 17, 18. Type locality: Zambia, Mpulungu, Musende Rocks, 0846'00"S, 031 ${ }^{\circ} 51^{\prime} 00^{\prime \prime E}$. Holotype: SAIAB 39577.

Distribution: Lake Tanganyika, only from the Musende Rocks area, Zambia.

Key: Synodontis species of Lake Tanganyika.

\section{References}

Abbott, C.C. (1861) Descriptions of two new species of Pimelodus from Kansas. Proceedings of the Academy of Natural Sciences, Philadelphia, 12, 568-569.

Abu Gideiri, Y.B. (1967) A new species of genus Synodontis (Siluroidea, Teleostei). Revue de Zoologie et de Botanique Africaines, 76, 132-136.

Acero-P., A. (2002) Ariidae. In: Carpenter, K.E. (Ed.), FAO Species Identification Guides for Fishery Purposes: The living Marine Resources of the Western Central Atlantic, FAO, Rome, pp. 831-852.

Acero-P., A. \& Betancur-R., R. (2002a) Arius cookei, a new species of ariid catfish from the tropical American Pacific. Aqua, Journal of Ichthyology and Aquatic Biology, 5, 133-138.

Acero-P., A. \& Betancur-R., R. (2002b) Description of Arius neogranatensis, a new species of sea catfish from Colombia, with an identification key for Caribbean ariid fishes. Aqua, Journal of Ichthyology and Aquatic Biology, 6, 133138.

Acero-P., A., Tavera, J.J. \& Reyes, J. (2005) Systematics of the genus Bagre (Siluriformes: Ariidae), a morphometric approach. Cybium (3e série), 29, 127-133.

Adams, A., Baikie, W.B. \& Barron, C. (1854) A Manual of Natural History for the Use of Travellers; Being a Description of the Families of the Animal and Vegetable Kingdoms: with Remarks on the Practical Study of Geology and Meteorology, John Van Voorst, Paternoster Row, London, viii + 749 p.

Adépo-Gourène, B., Teugels, G.G., Risch, L.M., Hanssens, M. \& Agnèse, J.-F. (1997) Morphological and genetic differentiation of 11 populations of the African catfish Chrysichthys nigrodigitatus (Siluroidei; Claroteidae), with consideration of their biogeography. Canadian Journal of Zoology, 75, 102-109.

Agassiz, L. (1844) Recherches sur les Poissons Fossiles, Part 4, Petitpierre, Neuchâtel and Soleure, xlix + 188 p.; atlas, 11 pls.

Agassiz, L. (1846) Nomenclatoris Zoologici. Index Universalis, Continens Nomina Systematica Classium, Ordinum, Familiarum et Generum Animalium Omnium, tam Viventium quam Fossilium, Jent et Gassmann, Soloduri, viii + 393 p.

Agassiz, L. (1850) Lake Superior: its Physical Character, Vegetation, and Animals, Compared with Those of Other Similar Regions, by Louis Agassiz; with a Narrative of the Tour, by J. Elliott Cabot; and Contributions by Other Scientific gentlemen, Gould, Kendall and Lincoln, Boston, 428 p., 8 pl. 
Agassiz, L. (1857) [The identity of the Glanis of Aristotle]. Proceedings of the American Academy of Arts and Sciences, $3,325-334$.

Agnèse, J.F. \& Teugels, G.G. (2001a) The Bathyclarias - Clarias species flock. A new model to understand rapid speciation in African Great lakes. Comptes Rendus de l'Academie des Sciences, Serie III: Sciences de la Vie, 324, 683688.

Agnèse, J.F. \& Teugels, G.G. (2001b) Genetic evidence for monophyly of the genus Heterobranchus and paraphyly of the genus Clarias (Siluriformes, Clariidae). Copeia, 2001, 548-552.

Agnèse, J.F. \& Teugels, G.G. (2005) Insights into the phylogeny of African Clariidae (Teleostei: Siluriformes): implications for their body shape evolution, biogeography, and taxonomy. Molecular Phylogenetics and Evolution, 36, 546553.

Ahl, E. (1925) Neue südamerikanische Fische aus dem Zool. Museum Berlin. Sitzungsberichte der Gesellschaft Naturforschender Freunde zu Berlin, 1925, 106-109.

Ahl, E. (1931) Neue Süsswasserfische aus dem Stromgebiet des Amazonenstromes. Sitzungsberichte der Gesellschaft Naturforschender Freunde zu Berlin, 1931, 206-211.

Ahl, E. (1934) Weitere Fische aus dem Toba-See in Sumatra. Sitzungsberichte der Gesellschaft Naturforschender Freunde zu Berlin, 1934, 235-238.

Ahl, E. (1936) Beschreibungen dreier neuer Welse aus Brasilien. Zoologischer Anzeiger, 116, 109-111.

Ahl, E. (1937a) Ueber eine kleine Fischsammlung aus Sierra Leone. Mitteilungen aus dem Zoologischen Museum in Berlin, 22, 239-243.

Ahl, E. (1937b) Zwei neue Süsswasserfische aus Südamerika. Sitzungsberichte der Gesellschaft Naturforschender Freunde zu Berlin, 1937, 445-447.

Akama, A. \& Ferraris, C.J., Jr. (2003) Entomocorus melaphareus, a new species of auchenipterid catfish (Osteichthyes: Siluriformes) from the lower and middle reaches of the Amazon River. Neotropical Ichthyology, 1, 77-82.

Alencar, A.R. \& Costa, W.J.E.M. (2004) Description of two new species of the catfish genus Trichomycterus from southeastern Brazil (Siluriformes: Trichomycteridae). Zootaxa, 744, 1-8.

Alexander, R.M. (1965) Structure and function in the catfish. Journal of Zoology (London), 148, 88-152.

Alfred, E.R. (1966) A new catfish of the genus Akysis from Malaya. Copeia, 1966, 467-470.

Allen, G.R. (1985) Descriptions of two new species of freshwater catfishes (Plotosidae) from Papua New Guinea. Records of the Western Australian Museum, 12, 247-256.

Allen, G.R. (1989) Die Süsswasserfische Neuguineas. Eine Kommentierte Checkliste. (Freshwater Fishes of New Guinea. An Annotated Checklist), 35 p. [not seen]

Allen, G.R. (1991) Field Guide to the Freshwater Fishes of New Guinea, Christensen Research Institute, Madang, Papua New Guinea, 268 p.

Allen, G.R. (1996) Freshwater fishes of Irian Jaya. In: Kitchener, D.J.\& Suyanto, A. (Eds.), Proceedings of the first international conference on eastern Indonesian-Australian vertebrate fauna, Manado, Indonesia, pp. 15-21. November 22-26, 1994

Allen, G.R. (1997) Marine Fishes of Tropical Australia and South-east Asia, Western Australian Museum, Perth, 292 p.

Allen, G.R. (1998) A review of the marine catfish genus Paraplotosus (Plotosidae) with the description of a new species from north-western Australia. The Raffles Bulletin of Zoology, 46, 123-124.

Allen, G.R. \& Coates, D. (1990) An ichthyological survey of the Sepik River, Papua New Guinea. Records of the Western Australian Museum, Supplement, 34, 31-116.

Allen, G.R. \& Feinberg, M.N. (1998) Descriptions of a new genus and four new species of freshwater catfishes (Plotosidae) from Australia. Aqua, Journal of Ichthyology and Aquatic Biology, 3, 9-18.

Allen, G.R., Parenti, L.R. \& Coates, D. (1992) Fishes of the Ramu River, Papua New Guinea. Ichthyological Exploration of Freshwaters, 3, 289-304.

Almirón, A.E., Azpelicueta, M. \& Casciotta, J.R. (2004) A new species of Epactionotus (Siluriformes: Loricariidae: Otothyrini) from the río Iguazú basin, Argentina. Zoologische Abhandlungen; Staatliches Museums für Tierkunde in Dresden, 54, 137-144.

Alonso de Arámburu, A. \& Arámburu, R.H. (1962) Una nueva especie de Xyliphius de la Argentina (Siluriformes, Bunocephalidae). Physis (Buenos Aires), 23, 219-222.

Anderson, J. (1879) Pisces. In: Anderson, J. (Ed.), Anatomical and Zoological Researches; Comprising an Account of the Zoological Results of the Two Expeditions to Western Yunnan in 1868 and 1875, and a Monograph of the Two Cetacean Genera, Platanista and Orcella, Bernard Quaritch, London, pp. 863-869, pl. 79.

Anseaume, L. \& Teugels, G.G. (1999) On the rehabilitation of the clariid catfish genus Bathyclarias endemic to the east 
African rift Lake Malawi. Journal of Fish Biology, 55, 405-419.

Anuradha, S. (1986) Contributions to the study of bagrid fishes, 19.- Systematic position of Macrones halepensis colvillii Hora \& Misra, 1943, with description of a new species (Siluriformes, Bagridae). Revue suisse de Zoologie, Annales de la Société zoologique suisse et du Muséum d'Histoire naturelle de Genève, 93, 291-296.

Anuradha, S. \& Jayaram, K.C. (1985) Contributions to the study of bagrid fishes. 18.- Redescription of Mystus pelusius Solander, the type species of the genus Mystus Scopoli. Bulletin of the Zoological Survey of India, 7, $107-111$.

Aquino, A.E. (1996) Redescripcíon de Otocinclus flexilis Cope, 1894 (Siluriformes, Loricariidae, Hypoptopomatinae) con un nuevo sinónimo. Iheringia, Série Zoologia, 81, 13-22.

Aquino, A.E. (1997) Las especies de Hypoptopomatinae (Pisces, Siluriformes, Loricariidae) en la Argentina. Revista de Ictiologia, 5, 5-21.

Aquino, A.E. \& Miquelarena, A.M. (2001) Redescription of Hypoptopoma inexspectata (Holmberg, 1883), with notes on its anatomy (Siluriformes: Loricariidae). Physis (Buenos Aires), Seccion B, 58, for 2000, 1-18.

Aquino, A.E. \& Schaefer, S.A. (2002) Revision of Oxyropsis Eigenmann and Eigenmann, 1889 (Siluriformes, Loricariidae). Copeia, 2002, 374-390.

Aquino, A.E., Schaefer, S.A. \& Miquelarena, A.M. (2001) A new species of Hisonotus (Siluriformes, Loricariidae) of the upper Río Uruguay basin. American Museum Novitates, 3333, 1-12.

Ardila Rodríguez, C.A. (2005) Callichthys oibaensis, una nueva especie de pez para el departamento de Santander Colombia (Siluriformes: Callichthyidae). Memoires del VIII Simposio Colombiano de Ictiologia, 31-43. [not seen]

Armbruster, J.W. (1998a) Phylogenetic relationships of the suckermouth armored catfishes of the Rhinelepis group (Loricariidae: Hypostominae). Copeia, 1998, 620-636.

Armbruster, J.W. (1998b) Review of the loricariid catfish genus Aphanotorulus and redescription of A. unicolor (Teleostei: Siluriformes). Ichthyological Exploration of Freshwaters, 8, 253-262.

Armbruster, J.W. (2002) Hypancistrus inspector: a new species of suckermouth armored catfish (Loricariidae: Ancistrinae). Copeia, 2002, 86-92.

Armbruster, J.W. (2003a) The species of the Hypostomus cochliodon group (Siluriformes: Loricariidae). Zootaxa, 249, $1-60$.

Armbruster, J.W. (2003b) Peckoltia sabaji, a new species from the Guyana Shield (Siluriformes: Loricariidae). Zootaxa, $344,1-12$.

Armbruster, J.W. (2004a) Phylogenetic relationships of the suckermouth armoured catfishes (Loricariidae) with emphasis on the Hypostominae and the Ancistrinae. Zoological Journal of the Linnean Society, 141, 1-80.

Armbruster, J.W. (2004b) Pseudancistrus sidereus, a new species from southern Venezuela (Siluriformes: Loricariidae) with a redescription of Pseudancistrus. Zootaxa, 628, 1-15.

Armbruster, J.W. (2005) The loricariid catfish genus Lasiancistrus with description of two new species. Neotropical Ichthyology, 3, 549-569.

Armbruster, J.W. \& Hardman, M. (1999) Redescription of Pseudorinelepis genibarbis (Loricariidae: Hypostominae) with comments on behavior as it relates to air-holding. Ichthyological Exploration of Freshwaters, 10, 53-61.

Armbruster, J.W. \& Page, L.M. (1996) Redescription of Aphanotorulus (Teleostei: Loricariidae) with description of one new species, A. ammophilus, from the Río Orinoco Basin. Copeia, 1996, 379-389.

Armbruster, J.W. \& Page, L.M. (1997) Generic reassignment of the loricariid species Monistiancistrus carachama Fowler 1940, Plecostomus lacerta Nichols, 1919, and Rhinelepis levis Pearson, 1924 (Teleostei: Siluriformes). Copeia, 1997, 227-232.

Armbruster, J.W. \& Provenzano, F. (2000) Four new species of the suckermouth armored catfish genus Lasiancistrus (Loricariidae: Ancistrinae). Ichthyological Exploration of Freshwaters, 11, 241-254.

Armbruster, J.W., Sabaj, M.H., Hardman, M., Page, L.M. \& Knouft, J.H. (2000) Catfish genus Corymbophanes (Loricariidae: Hypostominae) with description of one new species: Corymbophanes kaiei. Copeia, 2000, 997-1006.

Armbruster, J.W. \& de Souza, L.S. (2005) Hypostomus macushi, a new species of the Hypostomus cochliodon group (Siluriformes: Loricariidae) from Guyana. Zootaxa, 920, 1-12.

Armbruster, J.W. \& Werneke, D.C. (2005) Peckoltia cavatica, a new loricariid catfish from Guyana and a redescription of P. braueri (Siluriformes). Zootaxa, 882, 1-14.

Arratia, G. (1983a) Preferencias de habitat de peces Siluriformes de aguas continentales de Chile (Fam. Diplomystidae y Trichomycteridae). Studies on Neotropical Fauna and Environment, 18, 217-237.

Arratia, G. (1983b) Trichomycterus chungaraensis n. sp. and Trichomycterus laucaensis n. sp. (Pisces, Siluriformes, Trichomycteridae) from the high Andean range. Studies on Neotropical Fauna and Environment, 18, 65-87.

Arratia, G. (1987) Description of the primitive family Diplomystidae (Siluriformes, Teleostei, Pisces): Morphology, tax- 
onomy and phylogenetic implications. Bonner Zoologische Monographien, 24, 1-120.

Arratia, G. (1990) The South American Trichomycterinae (Teleostei: Siluriformes), a problematic group. In: Peters, G. \& Hutterer, R. (Eds.), Vertebrates in the Tropics: Proceedings of the International Symposium on Vertebrate Biogeography and Systematics in the Tropics, Bonn, June 5-8, 1989, Alexander Koenig Zoological Research Institute and Zoological Museum, Bonn, pp. 395-403.

Arratia, G. (1992) Development and variation of the suspensorium of primitive catfishes (Teleostei: Ostariophysi) and their phylogenetic relationships. Bonner Zoologische Monographien, 32, 1-149.

Arratia, G. (1998) Silvinichthys, a new genus of trichomycterid catfishes from the Argentinian Andes, with redescription of Trichomycterus nigricans. Ichthyological Exploration of Freshwaters, 9, 347-370.

Arratia, G. \& Chang, A. (1975) Osteocráneo de Nematogenys inermis Guichenot, 1848, y consideraciones acerca de la primitividad del género (Pisces, Siluriformes, Trichomycteridae). Museo Nacional de Historia Natural, Santiago de Chile, Publicación Ocasional, 19, 3-7.

Arratia, G., Chang, A., G., Menu-Marque, S. \& Rojas, G., M. (1978) About Bullockia gen. nov., Trichomycterus mendozensis n. sp. and revision of the family Trichomycteridae (Pisces, Siluriformes). Studies on Neotropical Fauna and Environment, 13, 157-194.

Arratia, G. \& Cione, A. (1996) The record of fossil fishes of southern South America. Münchner Geowissenschftliche Abhandlungen, Reihe A-Geologie und Paläontologie, 30, 9-72.

Arratia, G. \& Gayet, M. (1995) Sensory canals and related bones of Tertiary siluriform crania from Bolivia and North America and comparison with Recent forms. Journal of Vertebrate Paleontology, 15, 482-505.

Arratia, G. \& Huaquin, L. (1995) Morphology of the lateral line system and of the skin of diplomystid and certain primitive loricarioid catfishes and systematic and ecological considerations. Bonner Zoologische Monographien, 36, 1110 .

Arratia, G., Kapoor, B.G., Chardon, M. \& Diogo, R. (Eds.) (2003) Catfishes, Science Publishers, Enfield, NH, USA, 812 p.

Arratia, G. \& Menu-Marque, S. (1981) Revision of the freshwater catfishes of the genus Hatcheria (Siluriformes, Trichomycteridae) with commentaries on ecology and biogeography. Zoologischer Anzeiger, 207, 88-111.

Arratia, G. \& Menu-Marque, S. (1984) New catfishes of the genus Trichomycterus from the high Andes of South America (Pisces, Siluriformes) with remarks on distribution and ecology. Zoologische Jahrbücher. Abteilung für Systematik, Ökologie und Geographie der Tiere, 111, 493-520.

Arunkumar, L. (2000) Laguvia manipurensis, a new species of sisorid cat fish (Pisces: Sisoridae) from the Yu River system of Manipur. Indian Journal of Fisheries, 47, 193-200.

Arunkumar, L. \& Tombi Singh, H. (1997) Silurus morehensis, a new species of silurid cat fish (Order: Siluriformes, Family: Siluridae) from Manipur, India. Journal of Freshwater Biology, 9, 72-76.

Ataur Rahman, A.K. (1976) A new species of the genus Gagata Bleeker from river of Bangladesh. Bangladesh Journal of Biological Sciences, 5, 4-8.

Axelrod, H.R. (1970) Synodontis davidi, David's upside-down catfish. Tropical Fish Hobbyist, 18, 33-35.

Axelrod, H.R. (1987) Two new species of catfishes (Siluriformes, Callichthyidae and Pimelodidae) from the Rio Unini, Amazonas, Brazil. Tropical Fish Hobbyist, 35, 22-25.

Axenrot, T.E. \& Kullander, S.O. (2003) Corydoras diphyes (Siluriformes: Callichthyidae) and Otocinclus mimulus (Siluriformes: Loricariidae), two new species of catfishes from Paraguay, a case of mimetic association. Ichthyological Exploration of Freshwaters, 14, 249-272.

Azpelicueta, M.M. (1990) Tridentopsis cahuali n. sp. (Siluriformes, Trichomycteridae), a new miniature tridentine from Paraguay System, in Argentina. Revue suisse de Zoologie, Annales de la Société zoologique suisse et du Muséum d'Histoire naturelle de Genève, 97, 981-988.

Azpelicueta, M.M. (1994a) Los diplomistidos en Argentina (Siluriformes, Diplomystidae). Fauna de Agua Dulce de la República Argentina, 40, Pisces, 5-27.

Azpelicueta, M.M. (1994b) Three east-Andean species of Diplomystes (Siluriformes: Diplomystidae). Ichthyological Exploration of Freshwaters, 5, 223-240.

Azpelicueta, M.M. (1995) Pimelodus absconditus, a new species of pimelodid catfish from the la Plata basin (Siluriformes: Pimelodidae). Ichthyological Exploration of Freshwaters, 6, 71-76.

Azpelicueta, M.M. (1998) A new species of Pimelodus (Siluriformes: Pimelodidae) from the Paraguay and lower Paraná rivers. Neotropica, 44, 87-94.

Azpelicueta, M.M. (2001) A new species of Pimelodus (Siluriformes: Pimelodidae) from the upper Paraná basin, Brazil. Ichthyological Exploration of Freshwaters, 12, 193-200. 
Azpelicueta, M.M., Casciotta, J., Almirón, A. \& Körber, S. (2004) A new species of Otothyrini (Siluriformes: Loricariidae: Hypoptopomatinae) from the Río Uruguay basin, Argentina. — Eine neue Art des Stammes Otothyrini (Siluriformes: Loricariidae: Hypoptopomatinae) aus dem System des Rio Uruguay, Argentinien. Verhandlungen der Gesellschaft für Ichthyologie, 4, 81-90.

Azpelicueta, M.M. \& Gosztonyi, A.E. (1998) Redescription of Diplomystes mesembrinus (Siluriformes, Diplomystidae). Revue suisse de Zoologie, Annales de la Société zoologique suisse et du Muséum d'Histoire naturelle de Genève, 105, 901-910.

Azpelicueta, M.M. \& Rubilar, A. (1998) A Miocene Nematogenys (Teleostei: Siluriformes: Nematogenyidae) from South-Central Chile. Journal of Vertebrate Paleontology, 18, 475-483.

Bailey, R.G. (1968) A new species of Synodontis (Mochokidae) from Tanzania, East Africa. Revue de Zoologie et de Botanique Africaines, 77, 346-348.

Bailey, R.M. \& Baskin, J.N. (1976) Scoloplax dicra, a new armored catfish from the Bolivian Amazon. Occasional Papers of the Museum of Zoology, University of Michigan, 674, 1-14.

Bailey, R.M. \& Robins, C.R. (1988) Ameiurus Rafinesque, 1820 (Osteichthyes, Siluriformes): proposed designation of Silurus lividus Rafinesque, 1820 (= Pimelodus natalis Lesueur, 1819) as the type species. Bulletin of Zoological Nomenclature, 45, 135-137.

Bailey, R.M. \& Stewart, D.J. (1983) Bagrus Bosc, 1816 (Pisces, Siluriformes): Proposal to place on the Official List. Z.N.(S.) 2371. Bulletin of Zoological Nomenclature, 40, 167-172.

Bailey, R.M. \& Stewart, D.J. (1984) Bagrid catfishes from Lake Tanganyika, with a key and descriptions of new taxa. Miscellaneous Publications, Museum of Zoology, University of Michigan, 168, i-iv + 1-41.

Bailey, R.M. \& Taylor, W.R. (1950) Schilbeodes hildebrandi, a new ameiurid catfish from Mississippi. Copeia, 1950, 31-38, pls. 1-2.

Baird, S.F. \& Girard, C.F. (1854) Descriptions of new species of fishes collected in Texas, New Mexico and Sonora, by Mr. John H. Clark, on the U. S. and Mexican Boundary Survey, and in Texas by Capt. Stewart Van Vliet, U. S. A. Proceedings of the Academy of Natural Sciences, Philadelphia, 7, 24-29.

Balon, E.K. \& Hensel, K. (1970) Notes on a small collection of fishes from Afghanistan with a description of Glyptothorax jalalensis, sp. n. (Pisces, Sisoridae). Vestník Ceskoslovenské Spolecnosti Zoologické, 34, 159-163.

Barbosa, M.A. \& Costa, W.J.E.M. (2003a) Validade, relações filogenéticas e redescrição de Eremophilus candidus Ribeiro, 1949 (Teleostei, Siluriformes, Trichomycteridae). Arquivos do Museu Nacional do Rio de Janeiro, 61, 179188.

Barbosa, M.A. \& Costa, W.J.E.M. (2003b) Trichomycterus potschi (Siluriformes: Loricarioidei): a new trichomycterid catfish from coastal streams of southeastern Brazil. Ichthyological Exploration of Freshwaters, 14, 281-288.

Barbour, T. \& Cole, L.J. (1906) Vertebrata from Yucatan: Reptilia, Amphibia, and Pisces. Bulletin of the Museum of Comparative Zoology, 50, 146-159, pls. 1-2.

Bardack, J. (1961) New Tertiary teleosts from Argentina. American Museum Novitates, 2041, 1-27.

Barnard, K.H. (1943) Revision of the indigenous freshwater fishes of the S. W. Cape region. Annals of the South African Museum, 36, 101-262.

Bashir, K.A. \& Mirza, M.R. (1975) Fishes of the river Sutlej in Lahore District, Pakistan, with the description of a new subspecies. Bulletin of Hydrobiological Research, series 1, 9, 91-104.

Basilewsky, S. (1855) Ichthyographia Chinae Borealis. Nouveaux Mémoires de la Société Impériale des Naturalistes de Moscou, 10, 215-263, pls. 1-9.

Baskin, J.N. (1973) Structure and relationships of the Trichomycteridae. Unpublished Ph.D. dissertation, City University of New York, New York.

Baumgartner, J.V. (1982) A new fossil ictalurid catfish from the Miocene middle member of the Truckee Formation, Nevada. Copeia, 1982, 38-46.

Bean, T.H. (1880a) Description of a new species of Amiurus (A. ponderosus) from the Mississippi River. Proceedings of the United States National Museum, 2, 286-290.

Bean, T.H. (1880b) Descriptions of two species of fishes collected by Prof. A. Dugès in central Mexico. Proceedings of the United States National Museum, 2, 302-305.

Beaufort, L.F. de. (1931) Pisces, Reptilia and Aves. In: Onse palaeontoligische kennis van Nederlandsch Oost-Indiën 1930. Leidsche geologische Mededeelingen, 5, 461-470.

Beaufort, L.F. de. (1933) On some new or rare species of Ostariophysi from the Malay Peninsula and a new species of Betta from Borneo. Bulletin of the Raffles Museum, 8, 31-36.

Behre, E.H. (1928) A list of the fresh water fishes of western Panama between $81^{\circ} 45^{\prime}$ and $83^{\circ} 15^{\prime} \mathrm{W}$. Annals of the Carn- 
egie Museum, 18, 305-328, pl. 1.

Bell-Cross, G. (1973) The fish fauna of the Buzi River system in Rhodesia and Moçambique. Arnoldia (Rhodesia), 6, 114.

Bellotti, C. (1892) Un nuovo siluroide giapponese. Atti della Società Italiana di Scienze Naturali, Milano, 34, 99-101.

Benech, V., Teugels, G.G. \& Gourene, G. (1993) Critère pratique pour distinguer deux poissons-chats Africains, Clarias anguillaris et $C$. gariepinus (Siluriformes; Clariidae). Cybium (3e série), 17, 83-85.

Bennett, E.T. (1830) Class Pisces. In: Catalogue of Zoological Specimens, in: S. Raffles, Memoirs of the Life and Public Services of Sir Thomas Stamford Raffles [...], Particularly in the Government of Java, 1811-1816, and of Bencoolen and its Dependencies, 1817-1824; with Details of the Commerce and Resources of the Eastern Archipelago, and Selections from his Correspondence, by his Widow, Murray, London, pp. 686-694. [Authorship of Class Pisces indicated on p. [xi] and 629-630]

Berg, C. (1895) Sobre peces de agua dulce nuevos ó poco conocidos de la República Argentina. Anales del Museo Nacional de Buenos Aires, secunda Serie, 4, 121-165, pls. 2-3.

Berg, C. (1897) Contribuciones al conocimento de los peces Sudamericanos, especialmente de los de la República Argentina. Anales del Museo Nacional de Buenos Aires, secunda Serie, 5, 263-302.

Berg, C. (1901) Comunicaciones ictiológicas, IV. Comunicaciones del Museo Nacional de Buenos Aires, 1, $293-311$.

Berg, L.S. (1907) Beschreibungen einiger neuer Fische aus dem Stromgebiete des Amur. Ezhegodnik Zoologicheskii Muzei Akademiia Nauk, 12, 418-423.

Berg, L.S. (1918) [On a new siluroid fish, Glyplosternum armeniacum n. sp., from the Upper Euphrates]. Bulletin du Musée du Caucase, Tiflis, 11, 145-148. [In Russian, with English title and summary]

Berg, L.S. (1931) Description of a new siluroid fish, Glyptosternum kurdistanicum, from the basin of the Tigris River. Izvestiia Akademii Nauk SSSR. Otdelenie Matematicheskikh i Estestvennykh Nauk, 1931, 1267-1270, pl. 1.

Bertaco, V.A. \& Cardoso, A.R. (2005) A new species of Microglanis (Siluriformes: Pseudopimelodidae) from the rio Uruguay drainage, Brazil. Neotropical Ichthyology, 3, 61-67.

Bertin, L. \& Estève, R. (1950) Catalogue des Types de Poissons du Muséum National d'Histoire Naturelle. $5^{e}$ partie.Ostariophysaires (Siluriformes), Imp. Nationale, Paris, 85 p.

Bertoletti, J.J. (1967) Nova espécie de "Rhamdella" Eigenmann \& Eigenmann (Actinopterygii, Siluriformes, Pimelodidae). Revista Brasileira de Biologia, 27, 77-83.

Bertoletti, J.J., Pereira, E.H.L. \& da Silva, J.F.P. (1992) Nota sobre o gênero Trachelyopterus Valenciennes, 1840, no estado do Rio Grande do Sul, Brasil (Siluriformes, Auchenipteridae). Comunicações do Museu de Ciências da PUCRS, Série Zoologia, Porto Alegre, 5, 169-177.

Bertoletti, J.J., Pezzi da Silva, J.F. \& Pereira, E.H.L. (1995) A new species of the catfish genus Trachelyopterus (Siluriformes, Auchenipteridae) from southern Brazil. Revue Française d'Aquariologie et Herpetologie, 22, 71-74.

Bessednov, L.N. (1967) [Sea stinging catfishes of the genus Plotosus Lacépède from the Tonkin Bay]. Zoologicheskii Zhurnal, 46, 443-447. [In Russian, with English abstract.]

Betancur-R., R. (2003) Filogenia de los Bagres Marinos (Siluriformes: Ariidae) del Nuevo Mundo, M.Sc.Thesis, Universidad Nacional de Colombia, Bogotá.

Betancur-R., R. \& Acero P., A. (2004) Description of Notarius biffi n. sp. and redescription of N. insculptus (Jordan and Gilbert) (Siluriformes: Ariidae) from the eastern Pacific, with evidence of monophyly and limits of Notarius. Zootaxa, 703, 1-20.

Betancur-R., R. \& Acero P., A. (2005) Description of Cathorops mapale, a new species of sea catfish (Siluriformes: Ariidae) from the Colombian Caribbean, based on morphological and mitochondrial evidence. Zootaxa, 1045, 45-60.

Betancur-R., R., Acero-P., A. \& Mejia-Ladino, L.M. (2004) Analisis filogenético preliminar de algunos bagres marinos (Siluriformes: Ariidae) neotropicales. Preliminar [Preliminary] phylogenetic analysis of some neotropical marine catfishes (Siluriformes: Ariidae). Memoria de la Fundacion la Salle de Ciencias Naturales, 62, 61-85.

Bhatt, A. \& Jayaram, K.C. (2004) A new species of the genus Batasio Blyth (Siluriformes: Bagridae) from Sharavati River, Uttara Kannada, Karnataka. Zoos'Print Journal, 19, 1339-1342.

Bichuette, M.E. \& Trajano, E. (2004) Three new subterranean species of Ituglanis from Central Brazil (Siluriformes: Trichomycteridae). Ichthyological Exploration of Freshwaters, 25, 243-256.

Bichuette, M.E. \& Trajano, E. (2005) A new cave species of Rhamdia Bleeker, 1858 (Siluriformes: Heptapteridae) from Serra do Ramalho, northeastern Brazil, middle São Francisco River basin, with notes on ecology and behavior. Neotropical Ichthyology, 3, 587-595.

Bizerril, C.R.S.F. (1991) Descrição de uma nova espécie de Brachyglanis Eigenmann, 1912 da Bacia do rio Trombetas (Siluroidei, Pimelodidae). Boletim do Museu Nacional do Rio de Janeiro, Nova Série, zoologia, 347, 1-8. 
Bizerril, C.R.S.F. (1994) Descrição de uma nova especie de Trichomycterus (Siluroidei, Trichomycteridae) do Estado de Santa Catarina, com uma sinópse da composição da familia Trichomycteridae no leste Brasileiro. Arquivos de Biologia e Tecnologia (Curitiba), 37, 617-628.

Bizerril, C.R.S.F. (1995a) Description of new species of Hemipsilichthys (Loricariidae, Hypostominae) from the State of Santa Catarina, Brazil. Acta Biologica Leopoldensia, 17, 115-122.

Bizerril, C.R.S.F. (1995b) Descrição de uma nova espécie de Neoplecostomus (Loricariidae, Neoplecostominae) com uma sinópse da composição taxonômica dos Loricariidae no leste Brasileiro. Arquivos de Biologia e Tecnologia (Curitiba), 38, 693-704.

Bizerril, C.R.S.F. \& Borba, I.M.M. (1994) Status taxonômico de Rhamdella schultzi Ribeiro, 1964 (Siluriformes, Pimelodidae). Boletim do Museu Nacional do Rio de Janeiro, Nova Série, zoologia, 357, 1-6.

Bizerril, C.R.S.F. \& Perez-Neto, P.R. (1992) Description of a new species of Microglanis (Siluroidei, Pimelodidae) from eastern Brazil. Revue Française d'Aquariologie et Herpetologie, 18, for 1991, 97-100.

Blache, J. (1964) Les poissons du bassin du Tchad et du bassin adjacent du Mayo Kebbi. Étude systématique et biologique. Mémoires ORSTOM, 4, 1-483.

Black, B. (1987) A new species of Corydoras, Corydoras davidsandsi (Siluriformes, Callichthyidae) from the Rio Unini, Amazonas, Brazil. Practical Fishkeeping, 1987, 74-75.

Bleeker, P. (1846a) Overzigt der siluroïden, welke te Batavia voorkomen. Natuur-en Geneeskundig Archif voor Neerlandsch Indie, 3, 135-184.

Bleeker, P. (1846b) Siluroideorum bataviensium species nuperrime detectae. Natuur-en Geneeskundig Archif voor Neerlandsch Indië, 3, 284-293.

Bleeker, P. (1847a) Pharyngognathorum Siluroideorumque species novae Javanenses. Natuur-en Geneeskundig Archif voor Neerlandsch Indië, 4, 155-169.

Bleeker, P. (1847b) Nieuwe bijdrage tot de kennis der Siluroiden van Java. Verhandelingen van het Bataviaasch Genootschap van Kunsten en Wettenschappen, 21, 1-12.

Bleeker, P. (1847c) Siluroideorum bataviensium conspectus diagnosticus. Verhandelingen van het Bataviaasch Genootschap van Kunsten en Wettenschappen, 21, 1-60.

Bleeker, P. (1849) Bijdrage tot de kennis der ichthyologische fauna van het eiland Madura, met beschrijving van eenige neiuwe soorten. Verhandelingen van het Bataviaasch Genootschap van Kunsten en Wettenschappen, 22, 1-16.

Bleeker, P. (1851a) Bijdrage tot de kennis der ichthyologische fauna van Borneo, met beschrijving van 16 nieuwe soorten van zoetwatervisschen. Natuurkundig Tijdschrift voor Nederlandsch Indië, 1, 1-16.

Bleeker, P. (1851b) Nieuwe bijdrage tot de kennis der ichthyologische fauna van Borneo met beschrijving van eenige nieuwe soorten van zoetwatervisschen. Natuurkundig Tijdschrift voor Nederlandsch Indië, 1, 259-275.

Bleeker, P. (1851c) Derde bijdrage tot de kennis der ichthyologische fauna van Borneo, met beschrijving van eenige nieuwe soorten van zoetwatervisschen. Natuurkundig Tijdschrift voor Nederlandsch Indië, 2, 57-70.

Bleeker, P. (1851d) Vierde bijdrage tot de kennis der ichthyologische fauna van Borneo, met beschrijving van eenige nieuwe soorten van zoetwatervisschen. Natuurkundig Tijdschrift voor Nederlandsch Indië, 2, 193-208.

Bleeker, P. (1851e) Vijfde bijdrage tot de kennis der ichthyologische fauna van Borneo, met beschrijving van eenige nieuwe soorten van zoetwatervisschen. Natuurkundig Tijdschrift voor Nederlandsch Indië, 2, 415-442.

Bleeker, P. (1851f) Bijdrage tot de kennis der ichthyologische fauna van Riouw. Natuurkundig Tijdschrift voor Nederlandsch Indië, 2, 469-497.

Bleeker, P. (1852a) Bijdrage tot de kennis der ichthyologische fauna van Blitong (Billiton), met beschrijving van eenige nieuwe soorten van zoetwatervisschen. Natuurkundig Tijdschrift voor Nederlandsch Indië, 3, 87-100.

Bleeker, P. (1852b) Zesde bijdrage tot de kennis der ichthyologische fauna van Borneo. Visschen van Pamangkat, Bandjermassing, Praboekarta en Sampit. Natuurkundig Tijdschrift voor Nederlandsch Indië, 3, 407-442.

Bleeker, P. (1852c) Bijdrage tot de kennis der ichthyologische fauna van het eiland Banka. Natuurkundig Tijdschrift voor Nederlandsch Indië, 3, 443-460.

Bleeker, P. (1852d) Diagnostische beschrijvingen van nieuwe of weinig bekende vischsoorten van Sumatra. Tiental I IV. Natuurkundig Tijdschrift voor Nederlandsch Indië, 3, 569-608.

Bleeker, P. (1852e) Nieuwe bijdrage tot de kennis der ichthyologische fauna van het eiland Banka. Natuurkundig Tijdschrift voor Nederlandsch Indie, 3, 715-738.

Bleeker, P. (1853a) Nalezingen op de ichthyologische fauna van het eiland Banka. Natuurkundig Tijdschrift voor Nederlandsch Indië, 5, 175-193.

Bleeker, P. (1853b) Nieuwe tientallen diagnostische beschrijvingen van nieuwe of weinig bekende vischsoorten van Sumatra. Natuurkundig Tijdschrift voor Nederlandsch Indië, 5, 495-534. 
Bleeker, P. (1854a) Overzigt der ichthyologische fauna van Sumatra, met beschrijving van eenige nieuwe soorten. Natuurkundig Tijdschrift voor Nederlandsch Indië, 7, 49-108.

Bleeker, P. (1854b) Specierum piscium javanensium novarum vel minus cognitarum diagnoses adumbratae. Natuurkundig Tijdschrift voor Nederlandsch Indië, 7, 415-448.

Bleeker, P. (1854c) Nalezingen op de ichthyologische fauna van Bengalen en Hindostan. Verhandelingen van het Bataviaasch Genootschap van Kunsten en Wettenschappen, 25, 1-164, pls. 1-6.

Bleeker, P. (1855a) Nalezingen op de vischfauna van Sumatra. Visschen van Lahat en Sibogha. Natuurkundig Tijdschrift voor Nederlandsch Indië, 9, 257-280.

Bleeker, P. (1855b) Negende bijdrage tot de kennis der ichthyologische fauna van Borneo. Zoetwatervisschen van Pontianak en Bandjermasin. Natuurkundig Tijdschrift voor Nederlandsch Indië, 9, 415-430.

Bleeker, P. (1856) Verslag van eenige verzamelingen van zee- en zoetwatervisschen van het eiland Banka. Natuurkundig Tijdschrift voor Nederlandsch Indië, 11, 415-420.

Bleeker, P. (1857a) Descriptionum specierum piscium Javanensium novarum vel minus cognitarum diagnosticae. Natuurkundig Tijdschrift voor Nederlandsch Indië, 13, 323-368.

Bleeker, P. (1857b) Index: Descriptionum specierum piscium Bleekerianarum in voluminibus I ad XIV diarii Societatis Scientiarum Indo-Batavae. Natuurkundig Tijdschrift voor Nederlandsch Indië, 14, 447-486.

Bleeker, P. (1858a) Zesde bijdrage tot de kennis der vischfauna van Sumatra. Visschen van Padang, Troessan, Priaman, Sibogha en Palembang. Verhandelingen der Koninklijke Natuurkundige Vereeniging in Nederlandsch Indië [= Acta Societatis Regiae Scientiarum Indo-Neêrlandicae], 3, 1-50.

Bleeker, P. (1858b) De visschen van den Indischen Archipel. Beschreven en toegelicht. Siluri. Verhandelingen der Koninklijke Natuurkundige Vereeniging in Nederlandsch Indië.[= Acta Societatis Regiae Scientiarum Indo-Neêrlandicae], 4, i-xii + 1-370. [Also: Ichthyologiae Archipelagi Indici Prodromus, Vol 1. Siluri. Lange \& Co., Batavia. $\mathrm{xii}+370$.

Bleeker, P. (1858c) Twaalfde bijdrage tot de kennis der vischfauna van Borneo. Visschen van Sinkawang. Verhandelingen der Koninklijke Natuurkundige Vereeniging in Nederlandsch Indië. [ = Acta Societatis Regiae Scientiarum IndoNeêrlandicae], 5, 1-10.

Bleeker, P. (1858d) Vierde bijdrage tot de kennis der vischfauna van Biliton. Natuurkundig Tijdschrift voor Nederlandsch Indië, 15, 219-240.

Bleeker, P. (1858e) Enumeratio specierum piscium javanensium hucusque cognitarum. Natuurkundig Tijdschrift voor Nederlandsch Indië, 15, 359-456.

Bleeker, P. (1859) Enumeratio specierum piscium hucusque in Archipelago indico observatarum, adjectis habitationibus citationibusque, ubi descriptiones earum recentiores reperiuntur, nec non speciebus Musei Bleekeriani Bengalensibus, Japonicis, Capensibus Tasmanicisque. Verhandelingen der Koninklijke Natuurkundige Vereeniging in Nederlandsch Indië. [= Acta Societatis Regiae Scientiarum Indo-Neêrlandicae], 6, i-xxxvi, 1-276.

Bleeker, P. (1860) Negende bijdrage tot de kennis der vischfauna van Sumatra (Visschen uit de Lematang-Enim en van Benkoelen). Verhandelingen der Koninklijke Natuurkundige Vereeniging in Nederlandsch Indië.[= Acta Societatis Regiae Scientiarum Indo-Neêrlandicae], 8, 1-12.

Bleeker, P. (1861) Iets over de vischfauna van het eiland Pinang. Verslagen en Mededeelingen der Koninklijke Akademie van Wetenschappen (Afdeeling Natuurkunde), Amsterdam, 12, 64-80.

Bleeker, P. (1862-1863) Atlas Ichthyologique des Indes Orientales Néêrlandaises, Publié sous les Auspices du Gouvernement Colonial Néêrlandais. Tome II.-Siluroüdes, Chacoüdes et Hétérobranchoüdes, Amsterdam, 112 p., pls. 49-101. [Issued in installments. Text pages 1-32 and plates 49-72 date to 1862; pages 33-112 and plates 73-101 date to 1863. See Boeseman (1963: 4) for details.]

Bleeker, P. (1862a) Descriptions de quelques espèces nouvelles de Silures de Suriname. Verslagen en Mededeelingen der Koninklijke Akademie van Wetenschappen (Afdeeling Natuurkunde), Amsterdam, 14, 371-389.

Bleeker, P. (1862b) Notice sur les genres Parasilurus, Eutropiichthys, Pseudeutropius, et Pseudopangasius. Verslagen en Mededeelingen der Koninklijke Akademie van Wetenschappen (Afdeeling Natuurkunde), Amsterdam, 14, 390-399.

Bleeker, P. (1862c) Notice sur les genres Trachelyopterichthys, Hemicetopsis et Pseudocetopsis. Verslagen en Mededeelingen der Koninklijke Akademie van Wetenschappen (Afdeeling Natuurkunde), Amsterdam, 14, 400-403.

Bleeker, P. (1863a) Mémoire sur les poissons de la côte de Guinée. Natuurkundige Verhandelingen van de Hollandsche Maatschappij der Wetenschappen te Haarlem (Ser. 2), 18, 1-136, col. pls. 1-28.

Bleeker, P. (1863b) Sur quelques genres nouveaux du groupe des Doras. Nederlandsch Tijdschrift voor de Dierkunde, 1, 10-18.

Bleeker, P. (1863c) Systema Silurorum revisum. Nederlandsch Tijdschrift voor de Dierkunde, 1, 77-122. 
Bleeker, P. (1863d) Description de quelques espèces de poissons, nouvelles ou peu connues de Chine, envoyées au Musée de Leide par M.- G. Schlegel. Nederlandsch Tijdschrift voor de Dierkunde, 1, 135-150.

Bleeker, P. (1863e) Description de trois espèces nouvelles de Siluroïdes de l'Inde archipélagique. Nederlandsch Tijdschrift voor de Dierkunde, 1, 368-371.

Bleeker, P. (1863f) Description de trois espèces nouvelles de Siluroïdes de l'Inde archipélagique. Verslagen en Mededeelingen der Koninklijke Akademie van Wetenschappen (Afdeeling Natuurkunde), Amsterdam, 15, 70-76.

Bleeker, P. (1864a) Description des espèces de Silures de Suriname, conservées aux Musées de Leide et d'Amsterdam. Natuurkundige Verhandelingen van de Hollandsche Maatschappij der Wetenschappen te Haarlem (Ser. 2), 20, 1104, pls. 1-16.

Bleeker, P. (1864b) Notice sur la faune ichthyologique de Siam. Verslagen en Mededeelingen der Koninklijke Akademie van Wetenschappen (Afdeeling Natuurkunde), Amsterdam, 16, 352-358, 1 pl. [Also published in 1865 in Nederlandsch Tijdschrift voor de Dierkunde, 2, 96-99.]

Bleeker, P. (1864c) Rhinobagrus et Pelteobagrus deux genres nouveaux de Siluroïdes de Chine. Nederlandsch Tijdschrift voor de Dierkunde, 2, 7-10.

Bleeker, P. (1870) Description et figure d'une espèce inédite de Hemibagrus de Chine. Verslagen en Mededeelingen der Koninklijke Akademie van Wetenschappen (Afdeeling Natuurkunde), Amsterdam (Ser. 2), 4, 257-258, 1 pl.

Bleeker, P. (1872) Mémoire sur la faune ichthyologique de Chine. Nederlandsch Tijdschrift voor de Dierkunde, 4, 113154.

Bloch, M.E. (1794) Naturgeschichte der Ausländischen Fische, Vol. 8, Berlin, iv + 174 p., pls. 361-396.

Bloch, M.E. (1797) Icthyologie, ou Histoire Naturelle des Poissons, Vol. 2, Berlin.

Bloch, M.E. \& Schneider, J.G. (1801) M. E. Blochii, Systema Ichthyologiae Iconibus cx Illustratum. Post Obitum Auctoris Opus Inchoatum Absolvit, Correxit, Interpolavit Jo. Gottlob Schneider, Saxo, Sumtibus Austoris Impressum et Bibliopolio Sanderiano Commissum, Berolini, lx + 584 p., 110 pl.

Blyth, E. (1858) Report of Curator, Zoological Department, for May, 1858. Journal of the Asiatic Society of Bengal, 27, 267-290.

Blyth, E. (1860) Report on some fishes received chiefly from the Sitang River and its tributary streams, Tenasserim Provinces. Journal of the Asiatic Society of Bengal, 29, 138-174.

Bocchino, A. (1964) Sobre un Pygidiidae (Pisces, Siluriformes) del Eoceno de Río Negro. Ameghiniana, 3, $185-189$.

Bockmann, F.A. (1994) Description of Mastiglanis asopos, a new pimelodid catfish from northern Brazil, with comments on phylogenetic relationships inside the subfamily Rhamdiinae (Siluriformes: Pimelodidae). Proceedings of the Biological Society of Washington, 107, 760-777.

Bockmann, F.A. (1998) Análise Filogenética da Família Heptapteridae (Teleostei, Ostariophysi, Siluriformes) e Redefenição de seus Gêneros, Unpublished Ph.D. thesis, Universidade de São Paulo, São Paulo.

Bockmann, F.A., Casatti, L. \& Pinna, M.C.C., de. (2004) A new species of trichomycterid catfish from the Rio Paranapanema basin, southeastern Brazil (Teleostei: Siluriformes), with comments on the phylogeny of the family. Ichthyological Exploration of Freshwaters, 25, 225-242.

Bockmann, F.A. \& Ferraris, C.J., Jr. (2005) Systematics of the Neotropical catfish genera Nemuroglanis Eigenmann and Eigenmann 1889, Imparales Schultz 1944, and Medemichthys Dahl 1961 (Siluriformes: Heptapteridae). Copeia, 2005, 124-137.

Bockmann, F.A. \& Guazzelli, G.M. (2003) Heptapteridae. In: Reis, R.E., Kullander, S.O. \& Ferraris, C.J., Jr. (Eds.), Check list of the Freshwater Fishes of South and Central America. Edipucrs, Porto Alegre, Brazil, pp. 406-431.

Bockmann, F.A. \& Pinna, M.C.C., de. (2004) Heptapterus collettii Steindachner, 1881: a member of the Asian bagrid genus Olyra erroneously assigned to the Neotropical fauna (Siluriformes: Ostariophysi). Copeia, 2004, 665-675.

Bockmann, F.A. \& Ribeiro, A.C. (2003) Description of a new suckermouth armoured catfish of the genus Pareiorhina (Siluriformes: Loricariidae), from southeastern Brazil. Ichthyological Exploration of Freshwaters, 14, $231-242$.

Bocourt, F. (1866) Poissons. In: Notes sur les reptiles, les batraciens et les poissons recueillis pendant un voyage dans le royaume de Siam. Nouvelles Archives du Muséum d'Histoire Naturelle, Paris, 2, 11-20, pl. 1.

Boeseman, M. (1947) Revision of the fishes collected by Burger and Von Siebold in Japan. Zoologische Mededelingen (Leiden), 28, i-vii + 1-242, pls. 1-5. [Also issued as a book, with the same title, by E. J. Brill, Leiden.]

Boeseman, M. (1953) Scientific results of the Surinam Expedition 1948-1949. Part II._Zoology, no. 2: The Fishes (I). Zoologische Mededelingen (Leiden), 32, 1-24.

Boeseman, M. (1954) On Sciadeichthys (Selenaspis) walrechti, a new South American catfish. Zoologische Mededelingen (Leiden), 33, 59-62, pl. 3.

Boeseman, M. (1957) On a collection of fishes from Stanley Pool (Belgian Congo). Zoologische Mededelingen (Leiden), 
35, 139-151, pl. 5.

Boeseman, M. (1963) An annotated list of fishes from the Niger Delta. Zoologische Verhandelingen (Leiden), 61, 1-48, pls. $1-6$.

Boeseman, M. (1966) A new sisorid catfish from Java, Sundagagata robusta gen. et spec. nov. Proceedings of the Koninklijke Nederlandse Akademie van Wetenschappen (Series C), 69, 242-247 + table.

Boeseman, M. (1968) The genus Hypostomus Lacépède, 1803, and its Surinam representatives (Siluriformes, Loricariidae). Zoologische Verhandelingen (Leiden), 99, 1-89, pls. 1-18.

Boeseman, M. (1969) Additional new species of Hypostomus La Cepède, 1803, from Surinam; with remarks on the apparent "gymnorhynchus-complex" (Siluriformes, Loricariidae). Beaufortia, 16, 119-136.

Boeseman, M. (1971) The "comb-toothed" Loricariinae of Surinam, with reflections on the phylogenetic tendencies within the family Loricariidae (Siluriformes, Siluroidei). Zoologische Verhandelingen (Leiden), 116, 1-56, pls. 1-8.

Boeseman, M. (1972) Notes on South American catfishes, including remarks on Valenciennes and Bleeker types in the Leiden Museum. Zoologische Mededelingen (Leiden), 47, 293-320, pls. 1-2.

Boeseman, M. (1974) On two Surinam species of Hypoptopomatinae, both new to science (Loricariidae, Siluriformes, Ostariophysi). Proceedings of the Koninklijke Nederlandse Akademie van Wetenschappen (Series C), 77, $251-271$.

Boeseman, M. (1976) A short review of the Surinam Loricariinae; with additional information on Surinam Harttiinae, including the description of a new species (Loricariidae, Siluriformes). Zoologische Mededelingen (Leiden), 50, 153-177, pls. 1-8.

Boeseman, M. (1982) The South American mailed catfish genus Lithoxus Eigenmann, 1910, with the description of three new species from Surinam and French Guyana and records of related species (Siluriformes, Loricariidae). Proceedings of the Koninklijke Nederlandse Akademie van Wetenschappen (Series C), 85, 41-58, pls. 1-5.

Boeseman, M. (1983a) Introduction. In: P. Bleeker, Atlas Ichthyologique des Indes Orientales Néêrlandaises; Plates Originally Prepared for Planned Tomes XI-XIV, Smithsonian Institution Press, Washington, pp. 1-12.

Boeseman, M. (1983b) Some remarks on the South American pimelodid catfish usually known by the name of Phractocephalus hemiliopterus (Bloch \& Schneider) (Pimelodidae, Siluriformes). Zoologische Mededelingen (Leiden), 57, $105-114$.

Bogačew. (1924) Nowie materiali k istorii tretitschnich slonow w Jugo-Wostotschnej Rossii. Iswestij Aserbaidjanskogo Universiteta, Baku, 3, for 1923/24, [Not seen, from Weiler, 1956: 187.]

Böhlke, E.B. (1984) Catalog of type specimens in the ichthyological collection of the Academy of Natural Sciences of Philadelphia. Academy of Natural Sciences, Philadelphia, Special Publication, 14, i-viii + 1-246.

Böhlke, J.E. (1950) A new catfish of the genus Corydoras from the Peruvian Amazon. The Fish Culturist, 30, $26-27$.

Böhlke, J.E. (1951a) A new miniature catfish of the genus Corydoras from the Rio Orinoco in Venezuela. Annals and Magazine of Natural History (Ser. 12), 4, 824-827.

Böhlke, J.E. (1951b) Description of a new auchenipterid catfish of the genus Pseudepapterus from the Amazon Basin. Stanford Ichthyological Bulletin, 4, 38-40.

Böhlke, J.E. (1953) A catalogue of the type specimens of Recent fishes in the Natural History Museum of Stanford University. Stanford Ichthyological Bulletin, 5, 1-168.

Böhlke, J.E. (1959) Results of the Catherwood Foundation Peruvian Amazon Expedition. Petacara, a new genus for the bunocephalid catfish, Bunocephalus dolichurus Delsman. Notulae Naturae (Philadelphia), 318, 1-6.

Böhlke, J.E. (1970) A new species of the doradid catfish genus Leptodoras, with comments on related forms. Proceedings of the California Academy of Sciences, Series 2, 38, 53-61.

Böhlke, J.E. (1980) Gelanoglanis stroudi: a new catfish from the Rio Meta system in Colombia (Siluriformes, Doradidae, Auchenipterinae). Proceedings of the Academy of Natural Sciences, Philadelphia, 132, 150-155.

Bonaparte, C.L. (1838) Synopsis vertebratorum systematis. Nuovi Annali delle Scienze Naturali (Bologna), 2, $105-133$.

Bonaparte, C.L. (1846) Catalogo Metodico dei Pesci Europei. Stamperia e Cartieri del Fibreno, Napoli, 98 p.

Bonnaterre, J.P. (1788) Tableau Encyclopédique et Méthodique des Trois Règnes de la Nature.-Ichthyologie, Chez Panckoucke, Paris, lvi + 215 p., 102 pls.

Bornbusch, A.H. (1991a) Redescription and reclassification of the silurid catfish Apodoglanis furnessi Fowler (Siluriformes: Siluridae), with diagnoses of three intrafamilial silurid subgroups. Copeia, 1991, 1070-1084.

Bornbusch, A.H. (1991b) Monophyly of the catfish family Siluridae (Teleostei: Siluriformes), with a critique of previous hypotheses of the family's relationships. Zoological Journal of the Linnean Society, 101, 105-120.

Bornbusch, A.H. (1995) Phylogenetic relationships within the Eurasian catfish family Siluridae (Pisces: Siluriformes), with comments on generic validities and biogeography. Zoological Journal of the Linnean Society, 115, 1-46.

Bornbusch, A.H. \& Lundberg, J.G. (1989) A new species of Hemisilurus (Siluriformes, Siluridae) from the Mekong 
River, with comments on its relationships and historical biogeography. Copeia, 1989, 434-444.

Borodin, N.A. (1927a) A new blind catfish from Brazil. American Museum Novitates, 263, 1-5.

Borodin, N.A. (1927b) Some new catfishes from Brazil. American Museum Novitates, 266, 1-7.

Borodin, N.A. (1927c) Pimelodus platicirris, new species, and other notes on Brazilian catfishes. American Museum Novitates, 271, 1-4.

Borodin, N.A. (1934) Netuma hassleriana, a new catfish from Panama. Copeia, 1934, 33-34.

Borodin, N.A. (1936) On a collection of freshwater fishes from lakes Nyasa, Tanganyika and Viktoria in Central Afrika. Zoologische Jahrbücher. Abteilung für Systematik, Ökologie und Geographie der Tiere, 68, 1-34, pl. 1.

Bosc, L.A.G. (1816) [Pisces accounts] Vol. 3. Nouveau Dictionnaire d'Histoire Naturelle..., Nouv. éd., Chez Deterville, Paris.

Boulenger, G.A. (1887a) On new fishes from the lower Congo. Annals and Magazine of Natural History (Ser. 5), 19, 148-149.

Boulenger, G.A. (1887b) On new siluroid fishes from the Andes of Columbia. Annals and Magazine of Natural History (Ser. 5), 19, 348-350.

Boulenger, G.A. (1887c) An account of the fishes collected by Mr. C. Buckley in eastern Ecuador. Proceedings of the Zoological Society of London, 1887, 274-283, pls. 20-24.

Boulenger, G.A. (1889) Descriptions of a new snake and two new fishes obtained by Dr. H. von Ihering in Brazil. Annals and Magazine of Natural History (Ser. 6), 4, 265-267.

Boulenger, G.A. (1890a) List of the reptiles, batrachians and freshwater fishes collected by Professor Moesch and Mr. Iversen in the district of Deli, Sumatra. Proceedings of the Zoological Society of London, 1890, 31-40.

Boulenger, G.A. (1890b) Descriptions of two new species of the siluroid genus Arges. Proceedings of the Zoological Society of London, 1890, 450-452, pl. 41.

Boulenger, G.A. (1891) An account of the siluroid fishes obtained by Dr. H. von Ihering and Herr Sebastian Wolff in the Province Rio Grande do Sul, Brazil. Proceedings of the Zoological Society of London, 1891, 231-235, pls. 25-26.

Boulenger, G.A. (1892a) Description of a new siluroid fish from China. Annals and Magazine of Natural History (Ser. 6), 9, 247.

Boulenger, G.A. (1892b) On some new or little-known fishes obtained by Dr. J. W. Evans and Mr. Spencer Moore during their recent expedition to the Province of Matto Grosso, Brazil. Annals and Magazine of Natural History (Ser. 6), 10, 9-12, pls. 1-2.

Boulenger, G.A. (1894a) Descriptions of new freshwater fishes from Borneo. Annals and Magazine of Natural History (Ser. 6), 13, 245-251.

Boulenger, G.A. (1894b) Description of a new siluroid fish from Burma. Annals and Magazine of Natural History (Ser. 6), 14, 196.

Boulenger, G.A. (1895a) Descriptions of two new fishes obtained by Mr. C. Hose in Sarawak. Annals and Magazine of Natural History (Ser. 6), 15, 247.

Boulenger, G.A. (1895b) [Abstract of a report on a large collection of fishes formed by Dr. C. Ternetz in Matto Grosso and Paraguay, with descriptions of new species]. Proceedings of the Zoological Society of London, 1895, 523-529.

Boulenger, G.A. (1896a) Descriptions of new fishes from the upper Congo. Annals and Magazine of Natural History (Ser. 6), 17, 309-311.

Boulenger, G.A. (1896b) Description of a new siluroid fish from the Organ Mountains, Brazil. Annals and Magazine of Natural History (Ser. 6), 18, 154.

Boulenger, G.A. (1896c) On a collection of fishes from the Rio Paraguay. Transactions of the Zoological Society of London, 24, 25-39, pls.1-6.

Boulenger, G.A. (1897a) On a collection of fishes from the island of Marajo, Brazil. Annals and Magazine of Natural History (Ser. 6), 20, 294-299.

Boulenger, G.A. (1897b) Descriptions of new fishes from the upper Congo.- II. Annals and Magazine of Natural History (Ser. 6), 20, 422-427.

Boulenger, G.A. (1897c) Viaggio del Dott. Alfredo Borelli nel Chaco boliviano e nella Repubblica Argentina. III.Poissons. Bollettino dei Musei di Zoologia ed Anatomia Comparata della Università di Torino, 12, 1-4.

Boulenger, G.A. (1898a) Description of a new siluroid fish from West Africa. Annals and Magazine of Natural History (Ser. 7), 2, 415.

Boulenger, G.A. (1898b) Descriptions of two new siluroid fishes from Brazil. Annals and Magazine of Natural History (Ser. 7), 2, 477-478.

Boulenger, G.A. (1898c) Viaggio del Dr. Enrico Festa nell' Equateur e regioni vicine. Poissons de l'Équateur.— Part I. 
Bollettino dei Musei di Zoologia ed Anatomia Comparata della Università di Torino, 13, 1-13.

Boulenger, G.A. (1898d) Report on the fishes recently obtained by Mr. J. E. S. Moore in Lake Tanganyika. Proceedings of the Zoological Society of London, 1898, 494-497.

Boulenger, G.A. (1898e) On a collection of fishes from the Rio Jurua, Brazil. Transactions of the Zoological Society of London, 14, 421-428, pls. 39-42.

Boulenger, G.A. (1898f) Report on the collection of fishes made by Mr. J. E. S. Moore in Lake Tanganyika during his expedition, 1895-96. Transactions of the Zoological Society of London, 15, 1-30, pls. 1-8.

Boulenger, G.A. (1899a) Matériaux pour la faune du Congo. Poissons nouveaux du Congo. Troisième Partie. Silures, Acanthoptérygiens, Mastacembles, Plectognathes. Annales du Musée du Congo, Zoologie, 1, 39-58, pls. 20-29.

Boulenger, G.A. (1899b) Matériaux pour la faune du Congo. Poissons nouveaux du Congo. Cinquième Partie. Cyprins, Silures, Cyprinodontes, Acanthoptérygiens. Annales du Musée du Congo, Zoologie, 1, 97-128, pls. 40-47.

Boulenger, G.A. (1900a) Matériaux pour la faune du Congo. Poissons nouveaux du Congo. Sixième Partie. Mormyres, Characins, Cyprins, Silures, Acanthoptérygiens, Dipneustes. Annales du Musée du Congo, Zoologie, 1, 129-164, pls. 48-56.

Boulenger, G.A. (1900b) Descriptions of three new species of siluroid fishes from southern Brazil. Annals and Magazine of Natural History (Ser. 7), 5, 165-166.

Boulenger, G.A. (1900c) Diagnoses of new fishes discovered by Mr. J. E. S. Moore in Lake Tanganyika. I.-Cyprinidae, Siluridae. Annals and Magazine of Natural History (Ser. 7), 6, 478-481.

Boulenger, G.A. (1900d) On some little-known African silurid fishes of the subfamily Doradinae. Annals and Magazine of Natural History (Ser. 7), 6, 520-529.

Boulenger, G.A. (1900e) List of the fishes collected by Mr. J. S. Budgett in the river Gambia. Proceedings of the Zoological Society of London, 1900, 511-516.

Boulenger, G.A. (1901a) Les Poissons du Bassin du Congo. Publication de l'État Indépendant du Congo, Brussels, lxii + 532 p., 25 pls., 1 map.

Boulenger, G.A. (1901b) Descriptions of four new African freshwater fishes. Annals and Magazine of Natural History (Ser. 7), 7, 80-82.

Boulenger, G.A. (1901c) Descriptions of three new siluroid fishes of the genus Synodontis discovered by Mr. W. L. S. Loat in the White Nile. Annals and Magazine of Natural History (Ser. 7), 8, 10-12.

Boulenger, G.A. (1901d) Diagnoses of four new fishes discovered by Mr. J. E. S. Moore in Lakes Albert and Albert Edward. Annals and Magazine of Natural History (Ser. 7), 8, 12-14.

Boulenger, G.A. (1901e) Diagnoses of new fishes discovered by Mr. W. L. S. Loat in the Nile. Annals and Magazine of Natural History (Ser. 7), 8, 444-446.

Boulenger, G.A. (1901f) Description of a new silurid fish of the genus Anoplopterus, from Cameroon. Annals and Magazine of Natural History (Ser. 7), 8, 447-448.

Boulenger, G.A. (1901g) Description of a new silurid fish of the genus Gephyroglanis, from South Africa. Annals of the South African Museum, 2, 227-228, pl. 13.

Boulenger, G.A. (1902a) Descriptions of two new fishes of the genus Loricaria from north-western Ecuador. Annals and Magazine of Natural History (Ser. 7), 9, 69-71.

Boulenger, G.A. (1902b) Descriptions of new fishes and reptiles discovered by Dr. F. Silvestri in South America. Annals and Magazine of Natural History (Ser. 7), 9, 284-288.

Boulenger, G.A. (1902c) Descriptions of new fishes from the collection made by Mr. E. Degen in Abyssinia. Annals and Magazine of Natural History (Ser. 7), 10, 421-439.

Boulenger, G.A. (1902d) Additions à la faune ichthyologique de bassin du Congo. Matériaux pour la faune du Congo. Annales du Musée du Congo, Zoologie, 2, 19-57, pl. 7-16.

Boulenger, G.A. (1902e) Contributions to the ichthyology of the Congo. - I. On some new fishes from the French Congo. Proceedings of the Zoological Society of London, 1902, pt. 1, 234-237, pls. 22-24.

Boulenger, G.A. (1902f) On the fishes collected by Mr. S. L. Hinde in the Kenya District, East Africa, with descriptions of four new species. Proceedings of the Zoological Society of London, 1902, pt. 1, 221-224, pls. 16-17.

Boulenger, G.A. (1902g) Contributions to the ichthyology of the Congo- II. On a collection of fishes from the Lindi River. Proceedings of the Zoological Society of London, 1902, pt.1, 265-271, pl. 28-30.

Boulenger, G.A. (1902h) Descriptions of two new fishes discovered by Dr. W. J. Ansorge in southern Nigeria. Proceedings of the Zoological Society of London, 1902, pt. 2, 623-624, pl. 37.

Boulenger, G.A. (1903a) Description of a new fish of the genus Arges from Venezuela. Annals and Magazine of Natural History (Ser. 7), 11, 601-602. 
Boulenger, G.A. (1903b) Description of a new silurid fish of the genus Clarias from British Central Africa. Annals and Magazine of Natural History (Ser. 7), 12, 362-363.

Boulenger, G.A. (1903c) Descriptions of new freshwater fishes from southern Cameroon. Annals and Magazine of Natural History (Ser. 7), 12, 435-441.

Boulenger, G.A. (1903d) Second account of the fishes collected by Dr. W. J. Ansorge in the Niger Delta. Proceedings of the Zoological Society of London, 1902, pt. 2, 324-330, pls. 28-29.

Boulenger, G.A. (1903e) On the fishes collected by Mr. G. L. Bates in southern Cameroon. Proceedings of the Zoological Society of London, 1903, pt.1, 21-29, pls. 1-5.

Boulenger, G.A. (1904a) Descriptions of new West-African freshwater fishes. Annals and Magazine of Natural History (Ser. 7), 14, 16-20.

Boulenger, G.A. (1904b) Report on the fishes collected by Mr. Oscar Neumann and Baron Carlo von Erlanger in Gallaland and southern Ethiopia. Proceedings of the Zoological Society of London, 1903, pt. 2, 328-334, pls. 29-31.

Boulenger, G.A. (1904c) Descriptions of three new fishes discovered by the late Mr. J. S. Budgett in the Niger. Proceedings of the Zoological Society of London, 1904, pt. 1, 197-199, pls. 7-8.

Boulenger, G.A. (1905a) On a small collection of fishes from the Kasai river (Congo). Annals and Magazine of Natural History (Ser. 7), 16, 640-642.

Boulenger, G.A. (1905b) On a collection of fishes from Lake Bangwelo. Annals and Magazine of Natural History (Ser. 7), 16, 642-647.

Boulenger, G.A. (1905c) On a second collection of fishes made by Mr. S. L. Hinde in the Kenya District, East Africa. Proceedings of the Zoological Society of London, 1905, pt. 1, 62-64, pl. 7.

Boulenger, G.A. (1906a) On some fishes from the Kwango River (Congo System) in Angola, collected by Dr. W. J. Ansorge. Annals and Magazine of Natural History (Ser. 7), 17, 110-112.

Boulenger, G.A. (1906b) Descriptions of new fishes discovered by Mr. E. Degen in Lake Victoria. Annals and Magazine of Natural History (Ser. 7), 17, 433-452.

Boulenger, G.A. (1906c) Description of a new fish of the genus Clarias from Uganda. Annals and Magazine of Natural History (Ser. 7), 17, 569.

Boulenger, G.A. (1906d) On a second species of the silurid genus Mochocus. Annals and Magazine of Natural History (Ser. 7), 18, 147-148.

Boulenger, G.A. (1906e) Description of a new silurid fish of the genus Doumea, Sauvage, from Angola. Annals and Magazine of Natural History (Ser. 7), 18, 347-348.

Boulenger, G.A. (1906f) Fourth contribution to the ichthyology of Lake Tanganyika.- Report on the collection of fishes made by Dr. W. A. Cunnington during the Third Tanganyika Expedition, 1904-1905. Transactions of the Zoological Society of London, 17, 537-619, pls. 30-41.

Boulenger, G.A. (1907a) Zoology of Egypt: The Fishes of the Nile, Hugh Rees, Ltd., London, li + 578 p., 97 pls.

Boulenger, G.A. (1907b) Descriptions of three new freshwater fishes discovered by Mr. G. L. Bates in South Cameroon. Annals and Magazine of Natural History (Ser. 7), 20, 50-52.

Boulenger, G.A. (1907c) Descriptions of three new fishes from Central Africa. Annals and Magazine of Natural History (Ser. 7), 20, 487-489.

Boulenger, G.A. (1908a) Description of a new silurid fish of the genus Synodontis from South Cameroon. Annals and Magazine of Natural History (Ser. 8), 2, 30-31.

Boulenger, G.A. (1908b) A revision of the African silurid fishes of the subfamily Clariinae. Proceedings of the Zoological Society of London, 1907, 1062-1097.

Boulenger, G.A. (1909) Descriptions of new freshwater fishes discovered by Mr. G. L. Bates in South Cameroon. Annals and Magazine of Natural History (Ser. 8), 4, 186-188.

Boulenger, G.A. (1910) On a large collection of fishes made by Dr. W. J. Ansorge in the Quanza and Bengo rivers, Angola. Annals and Magazine of Natural History (Ser. 8), 6, 537-561.

Boulenger, G.A. (1911a) Catalogue of the Fresh-water Fishes of Africa in the British Museum (Natural History), Vol. 2, British Museum (Natural History) Trustees, London, xii +529 p.

Boulenger, G.A. (1911b) Descriptions of new freshwater fishes discovered by Dr. W. J. Ansorge in Portuguese Guinea. Annals and Magazine of Natural History (Ser. 8), 7, 373-376.

Boulenger, G.A. (1911c) Description of a new African fish of the genus Clarias from Lake Rukwa. Annals and Magazine of Natural History (Ser. 8), 8, 54.

Boulenger, G.A. (1911d) Further descriptions of new freshwater fishes discovered by Dr. W. J. Ansorge in Portuguese Guinea. Annals and Magazine of Natural History (Ser. 8), 8, 56-57. 
Boulenger, G.A. (1911e) Descriptions of three new freshwater fishes discovered by Mr. G. L. Bates in South Cameroon. Annals and Magazine of Natural History (Ser. 8), 8, 372-373.

Boulenger, G.A. (1911f) Liste des poissons représentés dans une nouvelle collection de la rivière Sankuru (Kasaï) reçue de M. E. Luja. Bulletins mensuels. Société des naturalistes luxembourgeois [= Monatsberichte / Gesellshaften Luxemburger Naturfreunde], n. s, 5, 218-225.

Boulenger, G.A. (1912a) Descriptions of two new fishes from the Nile system. Annals and Magazine of Natural History (Ser. 8), 10, 601-602.

Boulenger, G.A. (1912b) Poissons recueillis dans la Région du Bas-Congo par M. le Dr W. J. Ansorge. - Poissons, Batraciens et Reptiles. Matériaux pour la Faune du Congo. Annales du Musée du Congo Belge, Zoologie (Ser. 1), 2, 1-28, pls. 17-22.

Boulenger, G.A. (1912c) On a collection of fishes made by Mr. A. Blayney Percival in British East Africa to the East of Lake Baringo. Proceedings of the Zoological Society of London, 1912, 672-676, pls. 78-80.

Boulenger, G.A. (1913) Descriptions of four new fishes discovered by Mr. G. L. Bates in the Nyong River, S. Cameroon. Annals and Magazine of Natural History (Ser. 8), 12, 67-70.

Boulenger, G.A. (1915) Diagnoses de poissons nouveaux. II.- Mormyrides, Kneriides, Characinides, Cyprinides, Silurides. (Mission Stappers au Tanganika-Moero.). Revue de Zoologie Africaine, 4, 162-171.

Boulenger, G.A. (1916) Catalogue of the Fresh-water Fishes of Africa in the British Museum (Natural History), Vol. 4, Trustees, London, xxvii + 392 p.

Boulenger, G.A. (1917a) Description of a new silurid fish from Natal. Annals of the Durban Museum, 1, 432.

Boulenger, G.A. (1917b) Descriptions of new fishes from Lake Tanganyika forming part of the collection made by the late Dr. L. Stappers for the Belgian Government. Annals and Magazine of Natural History (Ser. 8), 20, 363-368.

Boulenger, G.A. (1918) On some fishes from the Shari River, with descriptions of two new species. Annals and Magazine of Natural History (Ser. 9), 2, 426-427.

Boulenger, G.A. (1919) Descriptions de poissons nouveaux du Congo. Revue de Zoologie Africaine, 7, 158-161.

Boulenger, G.A. (1920a) Poissons recueillis au Congo Belge par l'expédition du Dr. C. Christy. Zoologie. - Série I. Matériaux pour la Faune du Congo. Annales du Musée du Congo Belge, Zoologie (Ser. 1), 2, 1-38, pls. 23-25.

Boulenger, G.A. (1920b) Descriptions de deux poissons nouveaux de l'Ituri. Revue de Zoologie Africaine, 8, 148-150.

Boulenger, G.A. (1920c) On some new fishes from near the west coast of Lake Tanganyika. Proceedings of the Zoological Society of London, 1919, 399-404.

Boulenger, G.A. (1923) Descriptions of three new fresh-water fishes from northern Rhodesia. Annals of the South African Museum, 13, 437-438.

Bowdich, S.L. (1825) Fishes of Madeira. In: T. E. Bowdich, Excursions in Madeira and Porto Santo During the Autumn of 1823, While on His Third Voyage to Africa. G. B. Whittaker, London, pp. 121-125, 233-238.

Breder, C.M., Jr. (1925) New loricariate, characin and poeciliid fishes from the Rio Chucunaque, Panama. American Museum Novitates, 180, 1-9.

Breder, C.M. (1927) The fishes of the Rio Chucunaque drainage, Eastern Panama. Bulletin of the American Museum of Natural History, 57, 91-176, 6 pls.

Briggs, J.C. (1961) Emendated generic names in Berg's classification of fishes. Copeia, 1961, 161-166.

Britski, H.A. (1969) Lista dos tipos de peixes das coleções do Departamento de Zoologia da Secretaria da Agricultura de São Paulo. Papeis Avulsos de Zoologia, São Paulo, 22, 197-215.

Britski, H.A. (1972) Sistemática e Evolução dos Auchenipteridae e Ageneiosidae (Teleostei, Siluriformes), Doctoral Dissertation, Universidade de São Paulo, São Paulo, Brazil.

Britski, H.A. (1981) Sobre um novo gênero e espécie de Sorubiminae da Amazônia (Pisces, Siluriformes). Papeis Avulsos de Zoologia, São Paulo, 34, 109-114.

Britski, H.A. (1993) Uma nova espécie de Phenacorhamdia da bacia do alto Paraná (Pisces, Siluriformes). Comunicações do Museu de Ciências da PUCRS, Série Zoologia, Porto Alegre, 6, 41-50.

Britski, H.A. (1997) Descrição de um novo genero de Hypoptopomatinae, com duas especies novas (Siluriformes, Loricariidae). Papeis Avulsos de Zoologia, São Paulo, 40, 231-255.

Britski, H.A., de Silimon, K.Z. de S. \& Lopes, B.S. (1999) Peixes do Pantanal: Manual de Identificação, Embrapa, Brasília, 184 p.

Britski, H.A. \& Garavello, J.C. (1984) Two new southeastern Brazilian genera of Hypoptopomatinae and a redescription of Pseudotocinclus Nichols, 1919 (Ostariophysi, Loricariidae). Papeis Avulsos de Zoologia, São Paulo, 35, $225-41$.

Britski, H.A. \& Garavello, J.C. (2002) Parotocinclus jumbo, a new species of the subfamily Hypoptopomatinae from northeastern Brazil (Ostariophysi: Loricariidae). Ichthyological Exploration of Freshwaters, 13, $279-288$. 
Britski, H.A. \& Garavello, J.C. (2003) Hisonotus insperatus: new species, from the upper Rio Paraná basin (Pisces: Ostariophysi: Loricariidae). Copeia, 2003, 588-593.

Britski, H.A. \& Langeani, F. (1988) Pimelodus paranaensis sp. n., um novo Pimelodidae (Pisces, Siluriformes) do Alto Paraná, Brasil. Revista Brasileira de Zoologia, 5, 409-417.

Britski, H.A. \& Ortega, H. (1983) Trichogenes longipinnis, novo gênero e espéce de Trichomycterinae do sudeste do Brazil (Pisces, Siluriformes). Revista Brasileira de Zoologia, 1, 211-216.

Britski, H.A., Sato, Y. \& Rosa, A.B.S. (1986) Manual de Identificação de Peixes da Região de Três Marias (com Chaves de Identificação para os Peixes da Bacia do São Francisco). CODEVASF, Brasília, 115 p.

Britto, M.R. (1998) Two new species of the genus Aspidoras (Siluriformes: Callichthyidae) from central Brazil. Ichthyological Exploration of Freshwaters, 8, 359-368.

Britto, M.R. (2000) Aspidoras depinnai (Siluriformes: Callichthyidae): A new species from northeastern Brazil. Copeia, 2000, 1048-1055.

Britto, M.R. (2003) Phylogeny of the subfamily Cordoradinae Hoedeman, 1952 (Siluriformes: Callichthyidae), with a definition of its genera. Proceedings of the Academy of Natural Sciences, Philadelphia, 153, 119-154.

Britto, M.R. \& Castro, R.M.C. (2002) New corydorine catfish (Siluriformes: Callichthyidae) from the upper Paraná and São Francisco: the sister group of Brochis and most of Corydoras species. Copeia, 2002, 1006-1015.

Britto, M.R. \& Lima, F.C.T. (2003) Corydoras tukano, a new species of corydoradine catfish from the rio Tiquié, upper rio Negro basin, Brazil (Ostariophysi: Siluriformes: Callichthyidae). Neotropical Ichthyology, 1, 83-91.

Britto, M.R., Lima, F.C.T. \& Moreira, C.R. (2002) Aspidoras velites, a new catfish from the upper Rio Araguaia basin, Brazil (Teleostei: Siluriformes: Callichthyidae). Proceedings of the Biological Society of Washington, 115, 727-736.

Britto, M.R., Lima, F.C.T. \& Santos, A.C.A. (2005) A new Aspidoras (Siluriformes: Callichthyidae) from rio Paraguaçu basin, Chapada Diamantina, Bahia, Brazil. Neotropical Ichthyology, 3, 473-479.

Britto, M.R. \& Moreira, C.R. (2002) Otocinclus tapirape: a new hypoptopomatine catfish from central Brazil (Siluriformes: Loricariidae). Copeia, 2002, 1063-1069.

Britto, M.R. \& Reis, R.E. (2005) A new Scleromystax species (Siluriformes: Callichthyidae) from coastal rivers of Southern Brazil. Neotropical Ichthyology, 3, 481-488.

Britz, R. \& Ferraris, C.J., Jr. (2003) A new species of the Asian catfish genus Pseudolaguvia from Myanmar (Teleostei: Ostariophysi: Siluriformes: Erethistidae). Zootaxa, 388, 1-8.

Brown, B.A. \& Ferraris, C.J., Jr. (1988) Comparative osteology of the Asian catfish family Chacidae, with the description of a new species from Burma. American Museum Novitates, 2907, 1-16.

Brunet, M. \& Franco-Thacienne, M.P. (2000) Chad: discovery of a vertebrate fauna close to the Mio-Pliocene border. Journal of Vertebrate Paleontology, 20, 205-209.

Bruwer, E.E. \& van der Bank, F.H. (2003) A morphological key for the identification of southern African Synodontis species Cuvier, 1816 (Teleostei: Mochokidae). African Journal of Aquatic Science, 28, 183-186.

Buckup, P.A. (1981) Microlepidogaster taimensis sp. n., novo Hypoptopomatinae da Estação Ecológica do Taim, Rio Grande do Sul, Brasil (Ostriophysi, Loricariidae). Iheringia, Série Zoologia, 60, 19-31.

Buckup, P.A. (1988) The genus Heptapterus (Teleostei, Pimelodidae) in southern Brazil and Uruguay, with the description of a new species. Copeia, 1988, 641-653.

Buckup, P.A. \& Malabarba, L.R. (1990) Sobre as localidades-tipo de Characidium rachovii e Corydoras macropterus (Teleostei: Ostariophysi). Comunicações do Museu de Cièncias da PUCRS, Série Zoologia, Porto Alegre, 23, 103109.

Buen, F. de (1946a) Investigaciones sobre ictiologia Mexicana. III.- La ictiofauna del Lago de Chapala, con descripción de una nueva especie (Haustor ochoterenai de Buen). Anales del Instituto de Biología de la Universidad Nacional Autónoma de Mexico, 17, 261-281.

Buen, F. de (1946b) Ictiogeografia continental mexicana (I, II, y III). Revista de la Sociedad Mexicana de Historia Natural, 7, 87-138.

Burchell, W.J. (1822) Travels in the Interior of Southern Africa, 2 Vols, Longman, Hurst, Rees, Orme, and Brown, London.

Burgess, W.E. (1982) Corydoras adolfoi, a new species of catfish (Siluriformes, Callichthyidae) from the upper Rio Negro, Brazil, near São Gabriel da Cachoeira. Tropical Fish Hobbyist, 30, 15-16.

Burgess, W.E. (1983) Corydoras robineae, a new species of callichthyid catfish from Brazil. Tropical Fish Hobbyist, 31 , 42-43.

Burgess, W.E. (1989) An Atlas of Freshwater and Marine Catfishes. A Preliminary Survey of the Siluriformes, T.F.H. Publications, Neptune City, New Jersey, 784 p. 
Burgess, W.E. (1993) Three new species of catfishes of the genus Corydoras (Callichthyidae: Siluriformes). Tropical Fish Hobbyist, 41, 152-158.

Burgess, W.E. (1994) Scobinancistrus aureatus, a new species of loricariid catfish from the Rio Xingu (Loricariidae: Ancistrinae). Tropical Fish Hobbyist, 43, 236-242.

Burgess, W.E. (1997) Corydoras coriatae, a new species of callichthyid catfish related to Corydoras fowleri. Tropical Fish Hobbyist, 45, 138-147.

Burr, B.M., Eisenhour, D.J. \& Grady, J.M. (2005) Two new species of Noturus (Siluriformes: Ictaluridae) from the Tennessee River drainage: description, distribution, and conservation status. Copeia, 2005, 783-802.

Bussing, W.A. (1970) Two new species of catfishes of the genera Nannorhamdia and Imparales (family Pimelodidae) from Central America. Contributions in Science, Los Angeles County Museum, 196, 1-11.

Bussing, W.A. (1987) Peces de las Aguas Continentales de Costa Rica, Editorial de la Universidad de Costa Rica, San José, 271 p.

Bussing, W.A. (1998) Peces de las Aguas Continentales de Costa Rica.- Freshwater Fishes of Costa Rica, Second Edition, Editorial de la Universidad de Costa Rica, San José, 468 p. [Also, Revista de Biología Tropical, 46 (supplement 2): 1-468.]

Cagnolaro, L. \& Violani, C. (1988) Introduction to the anastatic reprint of "Vertebratorum Synopsis..." by E. Cornalia (1849). Atti della Società Italiana di Scienze Naturali, Milano, 129, 433-434 + 16 pp. and $1 \mathrm{pl}$.

Cala, P. (1981) Catalogo de los ejemplares tipo en la coleccion de peces del Instituto de Ciencias Naturales - Museo de Historia Natural de la Universidad Nacional de Colombia. Lozania (Acta Zoologica Colombiana), 34, 1-5.

Campanario, C.M. \& Pinna, M.C.C., de. (2000) A new species of the primitive trichomycterid subfamily Copionodontinae from northeastern Brazil (Teleostei: Trichomycteridae). Ichthyological Exploration of Freshwaters, 11, 369375.

Cantor, T.E. (1842) General features of Chusan, with remarks on the flora and fauna of that island. Annals and Magazine of Natural History (n. s.), 9, 265-278, 361-370, 481-493.

Cantor, T.E. (1849) Catalogue of Malayan fishes. Journal of the Asiatic Society of Bengal, 18, i-xii + 981-1443, pls. 114. [Also issued in 1850 , as a book with the same title, by J. Thomas, Calcutta.]

Capello, F.B. (1870) Noticia ácerca de um peixe pouco conhecido proveniente do Brasil. Jornal do Sciências Mathemáticas, Physicas e Naturaes, Lisboa, 2, 64-69, pl. 7.

Caramaschi, E.P. \& Caramaschi, U. (1991) Taxonomic status of the trichomycterid catfish Trichomycterus itatiayae. Copeia, 1991, 222-224.

Cardona, L. \& Guerao, G. (1994) Astroblepus riberae, una nueva especie de siluriforme cavernicola del Peru (Osteichthyes: Astroblepidae). Mémoires de Biospéologie, 21-24.

Cardoso, A.R. (2004) Hemiancistrus megalopteryx, a new species of loricariid catfish from the rio Tubarão drainage, Santa Catarina State, Brazil (Teleostei: Siluriformes: Trichomycteridae). Ichthyological Exploration of Freshwaters, $15,173-178$.

Cardoso, A.R. \& Lucinda, P.H.F. (2003) Three new species of Hemiancistrus (Teleostei: Siluriformes: Loricariidae) from the rio Tocantins basin with comments on the genus. Ichthyological Exploration of Freshwaters, 14, 73-84.

Cardoso, A.R. \& Malabarba, L.R. (1999) Description of three new species of Hemiancistrus Bleeker, 1862, from southern Brazil (Teleostei: Siluriformes: Loricariidae). Comunicações do Museu de Ciências da PUCRS, Série Zoologia, Porto Alegre, 12, 141-161.

Cardoso, A.R. \& Pezzi da Silva, J.F. (2004) Two new species of the genus Hemiancistrus Bleeker (Teleostei: Siluriformes: Loricariidae) from the upper rio Uruguai basin. Neotropical Ichthyology, 2, 1-8.

Carranza, J. (1954) Descripción del primer bagre anoftalmo y depigmentado encontrado en aguas mexicanas. Ciencia (Mexico City), 14, 129-136, pl. 1.

Carvalho, A.L. (1967) Novos dados para o conhecimento de Phreatobius cisternarum Goeldi (Pisces, Pygidiidae, Phreatobiinae). Atlas do Simpósio sôbre a Biota Amazonica 3 (Limnologia), 83-88.

Casciotta, J.R. \& Almirón, A.E. (1996) Scleronema minutum (Boulenger) y Ochmacanthus batrachostoma (M. Ribeiro) (Siluriformes: Trichomycteridae), dos citas nuevas para la cuenca del Plata en Argentina. Neotropica, 42, 51-54.

Casier, E. (1958) Contribution à l'étude des poissons fossiles des Antilles. Mémoires Suisses de Paléontologie, 74, 1-95, pls. $1-3$.

Casier, E. (1960) Note sure la collection des poissons Paléocènes et Eocènes de l'Enclave de Cabinda (Congo): recueillis par J. Bequaert au cours de sa mission in 1913. Annales du Musée Royal du Congo Belge, A-Minérologie, Géologie, Paléontologie, Sér. 3, 1, 1-47, 2 pl.

Castaño, M.L., Mojica C., J.I. \& Royero, R. (2005) Revisión taxonónomica del género Pimelodella, Eigenmann \& 
Eigenmann, 1888 (Pisces, Siluriformes: Heptapteridae) de la región transandina de Colombia. Acta Biologia Colombia, 19,79 .

Castelnau, F.L. (1855) Poissons. In: Animaux Nouveaux or Rares Recueillis Pendant l'Expédition dans les Parties Centrales de l'Amérique du Sud, de Rio de Janeiro à Lima, et de Lima au Para; Exécutée par Ordre du Gouvernement Français Pendant les Années 1843 a 1847, P. Bertrand, Paris, pp. i-xii + 1-112, 50 pl.

Castelnau, F.L. (1861) Mémoire sur les Poissons de l'Afrique Australe. J. Balliére et fils, Paris, vii + 78 p.

Castelnau, F.L. (1873) Contribution to the ichthyology of Australia.- No. VIII. Proceedings of the Zoological and Acclimatisation Society of Victoria, 2, 123-149.

Castelnau, F.L. (1875) Researches on the fishes of Australia. Intercolonial Exhibition Essays, 1875-6, 1-52.

Castelnau, F.L. (1878a) Australian fishes. New or little known species. Proceedings of the Linnean Society of New South Wales, 2, 225-248, pls. 2-3.

Castelnau, F.L. (1878b) On several new Australian (chiefly) fresh-water-fishes. Proceedings of the Linnean Society of New South Wales, 3, 140-144.

Castillo, G.O. \& Brull, G.O. (1989) Ageneiosus magoi una nueva especie de bagre ageneiosido (Teleostei, Siluriformes) para Venezuela y algunas notas sobre su historia natural. Acta Biologica Venezuelica, 12, 72-87.

Castro, D.M. (1986a) Los bagres de la subfamilia Sorubiminae de la Orinoquía y Amazonía Colombiana (Siluriformes Pimelodidae). Boletin Ecotrópica, 13, 1-40.

Castro, D.M. (1986b) Corydoras gomezi a new species from Colombia (Pisces, Siluriformes, Callichthyidae). Boletín Ecotrópica, 15, 33-38.

Castro, D.M. (1987) The fresh-water fishes of the genus Corydoras from Colombia, including two new species (Pisces, Siluriformes, Callichthyidae). Boletín Ecotrópica, 16, 23-57, pls. 1-11.

Catesby, M. (1731-1743) The Natural History of Carolina, Florida and the Bahama Islands; Containing the Figures of Birds, Beasts, Fishes, Serpents, Insects and Plants: Together with their Descriptions in English and French.etc., 2 vols., Privately printed, London.

Catesby, M. (1771) The Natural History of Carolina, Florida and the Bahama Islands; Containing the Figures of Birds, Beasts, Fishes, Serpents, Insects and Plants: ...Together with their Descriptions in English and French. ..., Revised by Mr. Edwards, $3^{\text {rd }}$ ed. 2 vols, Printed for B. White, London.

Ceas, P.A. \& Page, L.M. (1996) Chaetostoma yurubiense (Teleostei: Siluriformes), a new species of loricariid catfish from the Aroa, Urama, and Yaracuy River systems in Venezuela. Copeia, 1996, 671-677.

Chabanaud, P. (1934) Mission Jean Thomas en Afrique équatoriale française (septembre 1929 à mai 1930). Faune des Colonies Françaises, 5, 195-231.

Chakrabarty, P. \& Ng., H.H. (2005) The identity of catfishes identified as Mystus cavasius (Hamilton, 1822) (Teleostei: Bagridae), with a description of a new species from Myanmar. Zootaxa, 1093, 1-24.

Chamon, C.C., Aranda, A.T. \& Buckup, P.A. (2005) Pareiorhina brachyrhyncha (Loricariidae: Siluriformes): a new species of fish from the Paraíba do Sul slope of Serra da Mantiqueira, southeastern Brazil. Copeia, 2005 550-558.

Chang, F. (1999) New species of Myoglanis (Siluriformes, Pimelodidae) from the Río Amazonas, Peru. Copeia, 1999, 434-438.

Chang, F. \& Castro, E. (1999) Crossoloricaria bahuaja, a new loricariid fish from Madre de Dios, southeastern Peru. Ichthyological Exploration of Freshwaters, 10, 81-88.

Chang, M.-M. \& Zhou, J.-J. (1993) A brief survey of the Chinese Eocene ichthyofauna. Kaupia Darmstaedler Beiträge zur Naturgeschichte, 2, 157-162.

Chang, Y.-W. \& Wu, C.-T. (1965) [A new pangasid cat-fish, Sinopangasius semicultratus gen. et sp. nov., found in China]. Acta Zootaxonomica Sinica, 2, 11-14. [In Chinese and English.]

Chardon, M. (1968) Anatomie comparée de l'appareil de Weber et structures connexes chez les Siluriformes. Annales du Musée Royal de l'Afrique Centrale, Series in 8º Sciences Zoologiques, 169, 1-277.

Chaudhuri, B.L. (1911) Contributions to the fauna of Yunnan based on collections made by J. Coggin Brown, B.Sc., 1909-1910. Part II.- Fishes. Records of the Indian Museum, 6, 13-24, pl. 1.

Chaudhuri, B.L. (1912) Descriptions of some new species of freshwater fishes from north India. Records of the Indian Museum, 7, 437-444, pls. 38-41.

Chaudhuri, B.L. (1913) Zoological results of the Abor Expedition, 1911-12. XVIII.- Fish. Records of the Indian Museum, 8, 243-257, pls. 7-9.

Chaudhuri, B.L. (1916) Fauna of the Chilka Lake. Fish._- Part I. Memoirs of the Indian Museum, 5, 403-439.

Chaudhuri, B.L. (1919) Report on a small collection of fish from Putao (Hkamti Long) on the northern frontier of Burma. Records of the Indian Museum, 16, 271-287, pl. 22. 
Chaux, J. \& Fang, P.W. (1949a) Catalogue des Siluroidei d'Indochine de la collection du Laboratoire des Pêches Coloniales au Muséum, avec la description de six espèces nouvelles. Bulletin du Muséum National d'Histoire Naturelle (2 $2^{e}$ Série), 21, 194-201.

Chaux, J. \& Fang, P.W. (1949b) Catalogue des Siluroidei d'Indochine de la collection du Laboratoire des Pêches Coloniales au Muséum, avec la description de six espèces nouvelles. (Suite et fin). Bulletin du Muséum National d'Histoire Naturelle (2e Série), 21, 342-346.

Chen, H.-L. (1977) [A review of the Chinese Siluridae]. Acta Hydrobiologica Sinica, 6, 197-218, pls. 1-2. [In Chinese, with English abstract.]

Chen, X.-P. \& Lundberg, J.G. (1995) Xiurenbagrus, a new genus of amblycipitid catfishes (Teleostei: Siluriformes), and phylogenetic relationships among the genera of Amblycipitidae. Copeia, 1995, 780-800.

Chen, X.-Y., Ferraris, C.J., Jr. \& Yang, J.-X. (2005) A new species of catfish of the genus Clupisoma (Siluriformes: Schilbidae) from the Salween River, Yunnan, China. Copeia, 2005 566-570.

Chevey, P. (1931) Sur un nouveau silure géant du Bassin du Mékong Pangasianodon gigas nov. g., nov. sp. Bulletin de la Société Zoologique de France, 55, for 1930, 536-542, pl. 1.

Chevey, P. (1932) Poissons des Campagnes du "de Lanessan" (1925-1929). I Ie Partie. Travaux de l'Institut Océanographique de l'Indochine, $4^{\circ}$ Mémoire. Gouvernement General de l'Indochine, Saigon, 155 p., 50 pls.

Chockley, B.R. \& Armbruster, J.W. (2002) Panaque changae, a new species of catfish (Siluriformes: Loricariidae) from eastern Peru. Ichthyological Exploration of Freshwaters, 13, 81-90.

Choo, I.-Y. (1987) Systematic Studies on the Torrent Catfish, Family Amblycipitidae (Pisces: Siluriformes) from Korea. Department of Biology, Choong Ang University.

Chu, X.-L. (1979) [Systematics and evolutionary pedigree of the glyptosternoid fishes (family Sisoridae)]. Acta Zootaxonomica Sinica, 4, 72-82. [In Chinese.]

Chu, X.-L. (1981) [Taxonomic revision of the genera Pareuchiloglanis and Euchiloglanis]. Zoological Research, 2, 2531. [In Chinese.]

Chu, X.-L. (1982) [Phylogeny of the genus Pseudecheneis (Siluriformes: Sisoridae), with descriptions of two new species]. Acta Zootaxonomica Sinica, 7, 428-437. [In Chinese.]

Chu, X.-L. (1986) [Glyptosternoid fishes in the Himalaya-Hengduan Mountain region]. Freshwater Fishes, 12 , $39-44$. [In Japanese.]

Chu, X.-L. \& Chen, Y.-R. (Eds.) (1990) The Fishes of Yunnan, China. Part II - Cyprinidae [sic, non-Cyprinidae], Science Press, Beijing.

Chu, X.-L. \& Kuang, P.R. (1990) Sisoridae (Siluriformes). In: Chu, X.-L. \& Chen, Y.-R. (Eds.), The Fishes of Yunnan, China. Part II - Cyprinidae [sic, non-Cyprinidae], Science Press, Beijing, pp. 170-225. [In Chinese.]

Chu, X.-L. \& Mo, T.-P. (1999) Sisoridae. In: Chu, X.-L., Zheng, B.-S., Dai, D.-Y. \& al. (Eds.), Fauna Sinica: Osteichthyes: Siluriformes, Science Press, Beijing, pp. 114-181. [In Chinese.]

Chu, X.-L., Zheng, B.-S. \& Dai, D.-Y. (1999) Fauna Sinica. Osteichthyes: Siluriformes, Science Press, Beijing, vii + 230 p. [In Chinese.]

Cione, A.L. (1982) Peces del Pleistoceno tardío de la provincia de Buenos Aires. Consideraciones biogeográficas. Circular Informativa de la Asociación Paleontologica Argentina, 8, 12.

Cione, A.L. (1986) Los peces continentales del Cenozoico de Argentina. Su significación paleoambiental y paleobiogeográfica. Congreso Argentino de Paleontología y Bioestratigrafia, No. 4, Actas. Vol. 2, Mendoza, pp. 101-106.

Cione, A.L., Azpelicueta, M.M. \& Caille, G. (1996) An ariid catfish (Osteichthyes: Siluriformes) from marine middle Miocene beds of Patagonia; Recent ariid biogeography in southern South America. Cienc. Nat. Bernardino Rivadavia, Paleontologie [ ? Revista del Museo Argentino de Ciencias Naturales "Bernardino Rivadavia" e Instituto Nacional de Investigación de las Ciencias Naturales. Paleontología.], 3, 111-120.

Cione, A.L., Pereira, S.M., Alonso, R. \& Arias, J. (1985) Los bagres (Osteichthyes, Siluriformes) de la Formacion Yacoraite (Cretacico tardio) del Noroeste Argentino: Consideraciones biogeographificas y bioestratigraficas. Ameghiniana, 21, 294-304.

Cione, A.L. \& Prashad, G.V.R. (2002) The oldest known catfish (Teleostei: Siluriformes) from Asia (India, Late Cretaceous). Journal of Paleontology, 76, 190-193.

Cione, A.L. \& Torno, A.E. (1988) Assignment of the bony fish "Propygidium primaevus"(a supposed siluriform from the Tertiary of Patagonia) to the Order Perciformes. Journal of Palenotology, 62, 656-657.

Cloquet, H. (1816) [Pisces accounts. vol. 4, supplement]. Dictionnaire des Sciences Naturelles, Imprint Le Normant, Paris, pp. 52-53. [Not seen, citation from Bailey \& Stewart, 1983]

Coad, B.W. (1981a) Glyptothorax silviae, a new species of sisorid catfish from southwestern Iran. Japanese Journal of 
Ichthyology, 27, 291-295.

Coad, B.W. (1981b) Fishes of Afghanistan, an annotated checklist. Publications in Zoology, National Museum of Natural Sciences (Canada), 14, i-v + 1-26.

Coad, B.W. (1995) Freshwater fishes of Iran. Acta Scientiarum Naturalium Academiae Scientarium Bohemicae, Brno, 29, 1-64.

Coad, B.W. \& Delmastro, G.B. (1985) Notes on a sisorid catfish from the Black Sea drainage of Turkey. Cybium (3e série), 9, 221-224.

Coad, B.W. \& Holcík, J. (2000) On Silurus species from Iran (Actinopterygii: Siluridae). Folia Zoologica, 49, $139-148$.

Cockerell, T. (1925) A fossil fish of the family Callichthyidae. Science (n. s.), 62, 397-398.

Collette, B.B. (1962) Astroblepus pholeter, a new species of cave-dwelling catfish from eastern Ecuador. Proceedings of the Biological Society of Washington, 75, 311-314.

Cope, E.D. (1864) On a blind silurid, from Pennsylvania. Proceedings of the Academy of Natural Sciences, Philadelphia, 16, 231-233.

Cope, E.D. (1867) Supplement on some new species of American and African fishes. Transactions of the American Philosophical Society, 13, 400-407.

Cope, E.D. (1868) On the distribution of fresh-water fishes in the Allegheny region of southwestern Virginia. Journal of the Academy of Natural Sciences of Philadelphia (Ser. 2), 6, 207-247, pls. 26-28 [listed as 22-24].

Cope, E.D. (1870a) Partial synopsis of the fishes of the fresh waters of North Carolina. Proceedings of the American Philosophical Society, 11, 448-495.

Cope, E.D. (1870b) Contribution to the ichthyology of the Marañon. Proceedings of the American Philosophical Society, $11,559-570$.

Cope, E.D. (1871a) [Fishes from the Amazon above the mouth of the Rio Negro]. Proceedings of the Academy of Natural Sciences, Philadelphia, 23, 55.

Cope, E.D. (1871b) [Some anatomical points of importance in the classification of the siluroids of the Amazon]. Proceedings of the Academy of Natural Sciences, Philadelphia, 23, 112-113.

Cope, E.D. (1872a) On the fishes of the Ambyiacu River. Proceedings of the Academy of Natural Sciences, Philadelphia, 23 , for $1871,250-294$, pls 3-16.

Cope, E.D. (1872b) Notices of new Vertebrata from the upper waters of Bitter Creek, Wyoming Territory. Proceedings of the American Philosophical Society, 7, 483-486.

Cope, E.D. (1873) On the extinct Vertebrata of the Eocene of Wyoming observed by the expedition of 1872, with notes on the geology. Sixth Report. U. S. Geological and Geographic Survey of the Territories, 546-649.

Cope, E.D. (1874a) Supplementary notices of fishes from the freshwater Tertiaries of the Rocky Mountains. Bulletin of the U. S. Geological and Geographic Survey of the Territories, 1, 49-51.

Cope, E.D. (1874b) On some Batrachia and Nematognathi brought from the upper Amazon by Prof. Orton. Proceedings of the Academy of Natural Sciences, Philadelphia, 26, 120-137.

Cope, E.D. (1877) Synopsis of the cold blooded Vertebrata, procured by Prof. James Orton during his exploration of Peru in 1876-77. Proceedings of the American Philosophical Society, 17, 33-49.

Cope, E.D. (1878) Synopsis of the fishes of the Peruvian Amazon, obtained by Professor Orton during his expeditions of 1873 and 1877. Proceedings of the American Philosophical Society, 17, 673-701.

Cope, E.D. (1880) On the zoological position of Texas. Bulletin of the U. S. National Museum, 17, 1-51.

Cope, E.D. (1884a) The fishes of the Batsto River, N. J. Proceedings of the Academy of Natural Sciences, Philadelphia, $35,132-133$.

Cope, E.D. (1884b) The Vertebrata of the Tertiary formations of the West. Book 1. Report, U. S. Geological and Geographic Survey of the Territories, 3, 1-1009, $76 \mathrm{pl}$.

Cope, E.D. (1891) On Vertebrata from the Tertiary and Cretaceous rocks of the Northwest Territory. I.- The species from the Oligocene of lower Miocene beds of the Cypress Hills. Contributions to Canadian Palaeontology, 3, 1-25.

Cope, E.D. (1894) On the fishes obtained by the Naturalist Expedition in Rio Grande do Sul. Proceedings of the American Philosophical Society, 33, 84-108, pls. 4-9.

Copley, H. (1941) A short account of the freshwater fishes of Kenya. Journal of the East Africa and Uganda Natural History Society, 16, 1-24.

Cornalia, A. (1849) Vertebratorum Synopsis in Museo Mediolanense Extantium quae per Novum Orbem Cajetanus Osculati Collegit ... (1849), 16 p., 1 pl. [Not seen]

Costa, W.J.E.M. (1987) Feeding habits of a fish community in a tropical coastal stream, rio Mato Grosso, Brazil. Studies on Neotropical Fauna and Environment, 22, 145-153. 
Costa, W.J.E.M. (1992) Description de huit nouvelles espèces du genre Trichomycterus (Siluriformes: Trichomycteridae), du Brésil oriental. Revue Française d'Aquariologie et Herpetologie, 18, for 1991, 101-110.

Costa, W.J.E.M. (1994) A new genus and species of Sarcoglanidinae (Siluriformes: Trichomycteridae) from the Araguaia basin, central Brazil, with notes on subfamilial phylogeny. Ichthyological Exploration of Freshwaters, 5, 207216.

Costa, W.J.E.M. \& Bockmann, F.A. (1993) Un nouveau genre néotropical de la famille des Trichomycteridae (Siluriformes: Loricarioidei). Revue Française d'Aquariologie et Herpetologie, 20, 43-46.

Costa, W.J.E.M. \& Bockmann, F.A. (1994a) A new genus and species of Sarcoglanidinae (Siluriformes: Trichomycteridae) from southeastern Brazil, with a re-examination of subfamilial phylogeny. Journal of Natural History, 28, 715-730.

Costa, W.J.E.M. \& Bockmann, F.A. (1994b) Typhlobelus macromycterus, a new blind glanapterygine fish (Siluriformes: Trichomycteridae) from the Rio Tocantins, Brazil. Tropical Zoology, 7, 67-72.

Costa, W.J.E.M., Lima, S.M.Q. \& Bizerril, C.R.S.F. (2004) Microcambeva ribeirae sp. n. (Teleostei: Siluriformes: Trichomycteridae): a new sarcoglanidine catfish from the Rio Ribeira do Iguape basin, southeastern Brazil. Zootaxa, 563, 1-10.

Crass, R.S. (1960) Notes on the freshwater fishes of Natal with descriptions of four new species. Annals of the Natal Museum, 14, 405-458.

Cui, G.-H. (1990) Bagridae (Siluriformes). In: Chu, X.-L. \& Chen, Y.-R. (Eds.), The Fishes of Yunnan, China. Part II Cyprinidae [sic, non-Cyprinidae], Science Press, Beijing, pp. 145-162. [In Chinese, with diagnoses of new taxa in English.]

Curran, D.J. (1989) Phylogenetic relationships among the catfish genera of the family Auchenipteridae (Teleostei: Siluroidea). Copeia, 1989, 408-419.

Cuvier, G. (1816) Le Règne Animal Distribué d'après son Organisation pour Servir de Base à l'Histoire Naturelle des Animaux et d'Introduction à l'Anatomie Comparée. Tome 2. Les Reptiles, les Poissons, les Mollusques et les Annélides, Deterville, Paris, xviii + 532 p. [pls. 9-10, in vol. 4]

Cuvier, G. (1829) Le Règne Animal Distribué d'après son Organisation pour Servir de Base à l'Histoire Naturelle des Animaux et d'Introduction à l'Anatomie Comparée. Nouvelle Éd., rev. et augm., Tome 2-Les Reptiles et les Poissons, Deterville, Paris, xviii $+532 \mathrm{p}$.

Cuvier, G. (1836) Le Règne Animal Distribué d'après son Organisation pour Servir de Base à l'Histoire Naturelle des Animaux et d'Introduction à l'Anatomie Comparée, Troisième Édition, Tome Premier, Louis Hauman et Comp e., Bruxelles, xxii +626 p., 14 pls.

Cuvier, G. \& Valenciennes, A. (1840a) Histoire Naturelle des Poissons. Tome Quatorzième. Suite du Livre Seizième. Labrö̈des. Livre Dix-septième. Des Malacoptérygiens, Ch. Pitois \& Ve Levrault, Paris; Strasbourg, xxii + $2+464+$ 4 p., pls. 389-420. [Vol. dated 1839]

Cuvier, G. \& Valenciennes, A. (1840b) Histoire Naturelle des Poissons. Tome Quinzième. Suite du Livre Dix-septième. Siluroïdes, Ch. Pitois \& V Levrault, Paris \& Strasbourg, xxxi + 540 p., pls. 421-455.

Cuvier, G. \& Valenciennes, A. (1846) Histoire Naturelle des Poissons. Tome Dix-huitième. Suite du Livre Dix-huitième. Cyprinö̈des. Livre Dix-neuvième. Des Ésoces ou Luciö̈des, Ch. Pitois, \& V Levrault, Paris \& Strasbourg, xix + $2+$ $505+2$ p., pls. 520-553.

Dabry de Thiersant, P. (1872) Nouvelles espèces de poissons de Chine. In: La pisciculture et la pêche en Chine. Masson, Paris, pp. 178-192, pls. 36-50.

Daget, J. (1948) La collection des poissons d'eau douce de l'I.F.A.N. Catologues et Documents (Institut Français d'Afrique Noire), 3, 1-59.

Daget, J. (1954) Les poissons du Niger Supérieur. Memoirs de l'Institut Français d'Afrique Noire, 36, 1-391.

Daget, J. (1959) Description de trois poissons nouveaux de la Gambie: Barbus niokoloensis n. sp., Chrysichthys johnelsi n. sp. et Amphilius rheophilus n. sp. Bulletin de l'Institut Français d'Afrique Noire (Série A), Sciences naturelles, 21, 689-694.

Daget, J. (1962) Les poissons du Fouta Dialon et de la basse Guinée. Memoirs de l'Institut Français d'Afrique Noire, 65, 1-210, pl. 1-13.

Daget, J. (1963) La Réserve naturelle intégrale du Mont Nimba. XXVII.- Poissons (deuxième note). Memoirs de l'Institut Français d'Afrique Noire, 66, 573-600.

Daget, J. (1965) Espèces nouvelles ou rares de Synodontis récoltés en Côte d'Ivoire. (Pisces, Siluriformes). Bulletin du Muséum National d'Histoire Naturelle (2érie), 36, for 1964, 472-476.

Daget, J. (1979) Description de Platyglanis depierrei n. gen., n. sp. (Pisces, Bagridae) du Sanaga (Sud Cameroun). Bulle- 
tin du Muséum National d'Histoire Naturelle (Série 4, section A), 1, for 1978, 821-825.

Daget, J. (1992) Ariidae. In: Lévêque, C., Paugy, D. \& Teugels, G.G. (Eds.), Faune des Poissons d'Eaux Douces et Saumâtres de l'Afrique de l'Ouest, Vol. 2, Musée Royal de l'Afrique Central, Tervuren, and Editions de l'ORSTOM, Paris, pp. 564-568.

Daget, J., Gosse, J.-P. \& Thys van den Audenaerde, D.F.E. (Eds.) (1986) Check-list of the Freshwater Fishes of Africa, Vol. 2, ISNB, Bruxelles; MRAC, Tervuren; ORSTOM, Paris.

Daget, J. \& Lévêque, C. (1981) Description d'un poisson nouveau de Côte d'Ivoire Synodontis comoensis n. sp. (Pisces, Mochocidae). Cybium (3e série), 5 (2), 45-52.

Daget, J. \& Planquette, P. (1967) Sur quelques poissons de Côte d'Ivoire avec la description d'une espèce nouvelle, Clarias lamottei n. sp. (Pisces, Siluriformes, Clariidae). Bulletin du Muséum National d'Histoire Naturelle (2e Série), 39 , 278-281.

Daget, J. \& Stauch, A. (1963) Poissons de la partie camerounaise du Bassin de la Bénoué. Memoirs de l'Institut Français d'Afrique Noire, 68, 85-107.

Dahl, G. (1942) Three new fishes of the family Loricariidae from the Magdalena system. Förhandlingar, Kungl. Fysiografiska Sällskapet i Lund, 11, 80-86.

Dahl, G. (1955) An ichthyological reconnaissance of the Sinu River. Revista Linneana, 1, 11-19.

Dahl, G. (1960a) New fresh-water fishes from western Colombia. Caldasia, 8, 451-484.

Dahl, G. (1960b) Nematognathous fishes collected during the Macarena Expedition 1959.— Part I. Novedades Colombianas, 1, 302-317.

Dahl, G. (1961) Nematognathous fishes collected during the Macarena Expedition 1959. — Part II: Pimelodidae, Callophysidae. Novedades Colombianas, 1, 483-514.

Dahl, G. \& Medem, F. (1964) Informe Sobre la Fauna Acuatica del Rio Sinu. I Parte. Los Peces y la Pesca del Rio Sinu, Corporacion Autonoma Regional de los Valles del Magdalena y del Sinu -CVM-. Departamento de Investigaciones Ictiologicas y Faunisticas, 109 p.

Dahl, G., Medem, F. \& Ramos Henao, A. (1964) "Bocachico," Contribución al Estudio de su Biología y de su Ambiente, El Departmento de Pesca de la Corporación Autónoma Regional de los Valles del Magdalena y del Sinú - C.V.M, $144 \mathrm{p}$.

Datovo, A. \& Landim, M.I. (2005) Ituglanis macunaima, a new catfish from rio Araguaia basin, Brazil (Siluriformes, Trichomycteridae). Neotropical Ichthyology, 3, 455-464.

Datta, A.K., Barman, R.P. \& Jayaram, K.C. (1987) On a new species of Kryptopterus (Pisces: Siluroidea, family: Siluridae) from Namdapha Wildlife Sanctuary, Arunachal Pradesh, India. Bulletin of the Zoological Survey of India, 8, 29-31.

Datta, A.K. \& Karmakar, A.K. (1980) Clupisoma bastari sp. nov. (Pisces: Schilbeidae) from Bastar, Madhya Pradesh, India. Bulletin of the Zoological Survey of India, 2, 193-196.

Datta Munshi, J.S. \& Srivastava, M.P. (1988) Natural History of Fishes and Systematics of Freshwater Fishes of India, Narendra Publ. House, Delhi, xviii +403 p.

David, A. (1962) Brief taxonomic account of the gangetic Pangasius pangasius (Hamilton) with a description of a new subspecies from the Godavary. Proceedings of the Indian Academy of Sciences, Section B, 56, 136-156.

David, L. (1935) Die Entwicklung der Clariiden und ihre Verbreitung. Eine anatomisch-systematische Untersuchung. Revue de Zoologie et de Botanique Africaines, 28, 77-147 + 5 foldout tables.

David, L. (1936a) Uegitglanis, silure aveugle de la Somalie italienne: Chainon entre Bagrides et Clariides. Revue de Zoologie et de Botanique Africaines, 28, 416-418.

David, L. (1936b) Deux espèces de Synodontis du Moyen-Congo. Revue de Zoologie et de Botanique Africaines, 28, 416-418.

David, L. \& Poll, M. (1937) Contribution à la faune ichthyologique du Congo Belge: Collections du Dr. H. Schouteden (1924-1926) et d'autres récolteurs. Annales du Musée du Congo Belge, Zoologie (Ser. 1), 3, 189-294, pl. 12.

Davis, J.W. (1887) The fossil fishes of the chalk of Mount Lebanon, in Syria. Transactions of the Royal Dublin Society, series 2, 3, 457-636, pls. 14-38.

Day, F. (1865a) The Fishes of Malabar, Bernard Quaritch, London, xxxii + 293 p., 20 pls.

Day, F. (1865b) On the fishes of Cochin, on the Malabar Coast of India. Part II.- Anacanthini. Proceedings of the Zoological Society of London, 1865, 286-318.

Day, F. (1867) On some new or imperfectly known fishes of Madras. Proceedings of the Zoological Society of London, $1867,558-565$.

Day, F. (1868) On some new or imperfectly known fishes of India. Proceedings of the Zoological Society of London, 
1868, 149-156.

Day, F. (1869) On the fishes of Orissa.- Part I. Proceedings of the Zoological Society of London, 1869, $296-310$.

Day, F. (1870a) Notes on the genus Hara. Journal of the Asiatic Society of Bengal, 39, 37-40, pl. 4.

Day, F. (1870b) Remarks on some of the Fishes in the Calcutta Museum.- Part I. Proceedings of the Zoological Society of London, 1869, 511-527.

Day, F. (1870c) On the freshwater fishes of Burma.- Part I. Proceedings of the Zoological Society of London, 1869 , 614-623.

Day, F. (1871) On the fishes of the Andaman Islands. Proceedings of the Zoological Society of London, 1870, 677-705.

Day, F. (1872) On the freshwater siluroids of India and Burmah. Proceedings of the Zoological Society of London, 1871, 703-721.

Day, F. (1873a) Report on the Fresh Water Fish and Fisheries of India and Burma, Office of the Superintendent of Government Printing, Calcutta, $118+$ cccvii p.

Day, F. (1873b) On some new fishes of India. The Journal of the Linnean Society of London. Zoology, 11, 524-530.

Day, F. (1873c) On new or imperfectly known fishes of India. Proceedings of the Zoological Society of London, 1873, 236-240.

Day, F. (1873d) Extracts from the late Dr. Buchanan's 'Fishes of Bengal,'with remarks. Proceedings of the Zoological Society of London, 1873, 743-748.

Day, F. (1875-78) The Fishes of India; being a Natural History of the Fishes Known to Inhabit the Seas and Fresh Waters of India, Burma, and Ceylon, Bernard Quaritch, London. [Issued in 5 parts, plus supplement (see Day, 1888). Dates of issuance of parts follow Menon \& Rama Rao (1974); all catfish names date to 1877.]

Day, F. (1876) On some of the fishes of the Deccan. The Journal of the Linnean Society of London. Zoology, 12, 565578.

Day, F. (1877a) Geographical distribution of Indian freshwater fishes. Part II.- the Siluridae. The Journal of the Linnean Society of London. Zoology, 13, 338-352.

Day, F. (1877b) On the fishes of Yarkand. Proceedings of the Zoological Society of London, 1876, 781-807.

Day, F. (1888) The Fishes of India; being a Natural History of the Fishes Known to Inhabit the Seas and Fresh Waters of India, Burma, and Ceylon, Supplement, Williams and Norgate, London and Edinburgh, pp. 779-816.

De Filippi, F. (1853) Nouvelles espèces de poissons. Revue et Magasin de Zoologie (Ser. 2), 5, 164-171.

De Kay, J.E. (1842) Fishes. In: Zoology of New-York; or the New-York Fauna; Comprising Detailed Descriptions of All the Animals Hitherto Observed Within the State of New-York, with Brief Notices of Those Occasionally Found Near its Borders, and Accompanied by Appropriate Illustrations, part 4, W. \& A. White, and J. Visscher, Albany, pp. 1415, pls. 1-79. [In 6 volumes, 1842-1844]

De Vis, C.W. (1884) Fishes from South Sea islands. Proceedings of the Linnean Society of New South Wales, 8, $445-457$.

De Vos, L. (1981) Description of Eutropius nyongensis spec. nov. from the Cameroons (Pisces, Schilbeidae). Revue de Zoologie Africaine, 95, 968-974.

De Vos, L. (1983) Note on the synonyms of Eutropius mandibularis Günther, 1867, and Eutropius multitaeniatus Pellegrin, 1913 (Pisces, Schilbeidae). Revue de Zoologie Africaine, 97, 268-287.

De Vos, L. (1984a) Note on the species of the genus Eutropius (Pisces, Schilbeidae) from the Quanza and Bengo rivers (Angola) with description of Eutropius angolensis spec. nov. Cybium (3e série), 8 (2), 3-18.

De Vos, L. (1984b) Preliminary data of a systematic revision of the African species of the family Schilbeidae (Pisces: Siluriformes). Revue de Zoologie Africaine, 98, 424-433.

De Vos, L. (1986) Schilbeidae. In: Daget, J., Gosse, J.-P. \& Thys van den Audenaerde, D.F.E. (Eds.), Check-list of the Freshwater Fishes of Africa, Vol. 2, ISNB, Bruxelles; MRAC, Tervuren; ORSTOM, Paris, pp. 36-53.

De Vos, L. (1992) Schilbeidae. In: Lévêque, C., Paugy, D. \& Teugels, G.G. (Eds.), Faune des Poissons d'Eaux Douces et Saumâtres de l'Afrique de l'Ouest, Vol. 2, Musée Royal de l'Afrique Central, Tervuren, and Editions de l'ORSTOM, Paris, pp. 432-450.

De Vos, L. (1993) Le genre Chiloglanis (Siluriformes, Mochokidae) dans le bassin de la Ruzizi: description de deux nouvelles espèces. Journal of African Zoology, 107, 153-168.

De Vos, L. (1995) A systematic revision of the African Schilbeidae (Teleostei, Siluriformes), with an annotated bibliography. Musée Royal de l'Afrique Centrale, Annales, Sciences Zoologiques, 271, 1-450.

De Vos, L. (2001a) Synodontis manni (Teleostei: Mochokidae), a new catfish from the Lower Tana River, Kenya. Ichthyological Exploration of Freshwaters, 12, 41-50.

De Vos, L. (2001b) Rediscovery of the giant catfish Pardiglanis tarabinii (Siluriformes: Claroteidae). Ichthyological Exploration of Freshwaters, 12, 212-214. 
De Vos, L. \& Lévêque, C. (1983) Etude systématique et morphologique du genre Eutropius (Pisces, Schilbeidae) en Afrique de l'Ouest. Revue de Zoologie Africaine, 97, 469-532.

De Vos, L. \& Skelton, P. (1990) Name changes for two common African catfishes. Rehabilitation of Schilbe intermedius Rüppell, 1832 (Siluriformes, Schilbeidae). Cybium (3e série), 14, 323-326.

De Vos, L. \& Thys van den Audenaerde, D.F.E. (1998) Le statut taxonomique de Synodontis serratus tanganyicae Borodin, 1936 du lac Tanganyika (Teleostei; Mochokidae): synonymie avec S. lacustricolus Poll, 1953. Journal of African Zoology, 112, 147-156.

Dean, B. (1916) A Bibliography of Fishes; Vol. 1, Publications Grouped Under the Names of Authors: A-K, American Museum of Natural History, New York.

Debouche, C., Marquet, J.-P. \& Teugels, G. (1979) Détermination de poissons du genre Clarias par une méthode généralisable aux poissons non écailleux. Bulletin de l'Institut fondamental d'Afrique noire. Série A, Sciences naturelles, 41, 844-862.

Delsman, N.C. (1941) Résultats scientifiques des croisières du Navire-École Belge "Mercator", vol. III, No. 3. Pisces. Mémoires du Musée Royal d'Histoire Naturelle de Belgique (Ser. 2), 21, 47-82.

Depéret, C. (1885) Description géologique du bassin tertiaire du Roussillon et description des vertébrés fossiles du terrain pliocène du Roussillon. Ann. Sci. géol. (Paris) 16, 1-272, pls. I-V, map. [not seen]

Deraniyagala, P.E.P. (1952) A Colored Atlas of Some Vertebrates from Ceylon, Vol. 1- Fishes, The Ceylon Government Press, Colombo, 149 p., 34 pls.

Deraniyagala, P.E.P. (1953) A new race of Wallago attu from Ceylon. Spolia Zeylanica, 27, 45.

Deraniyagala, P.E.P. (1958) Three new cyprinoids, a new cat fish and variation among some cyprinoids and an anabantoid of Ceylon. Spolia Zeylanica, 28, 129-138, pls. 1-2.

Derijst, E. (1991) Kataloog der ichthyologische publikaties en nieuwe taxa beschreven door: Dr. Max Poll. Aquarium Wereld, Special Publication 2, i-vii + 1-87. [Not seen, from Catalog of Fishes]

Derijst, E. (1996) Note on the type species of the mailed catfish genus Pareiorhaphis Miranda-Ribeiro, 1918 (Pisces: Siluriformes: Loricariidae), with the introduction of Isbrueckerichthys nom. nov. Aquarium Wereld, 49, 62-64.

Desoutter, M. (1975) Étude de quelques Bagridae (Siluriformes, Pisces) du Cambodge. Description d'une espèce nouvelle: Mystus aubentoni. Bulletin du Muséum National d'Histoire Naturelle, Zoologie (Sér. 3), 206, 441-462.

Desoutter, M. (1977) Révision du genre Hemipimelodus Bleeker, 1858 (Tachysuridae, Siluriformes, Pisces). Cybium (3e série), 1, 9-36.

Devaere, S., Adriaens, D., Teugels, G.G. \& Verraes, W. (2005) Morphology and spatial constraints in a dorso-ventrally flattened skull, with a revised species description of Platyallabes tihoni (Poll, 1944). Journal of Natural History, 39, 1653-1673.

Devaere, S., Adriaens, D., Teugels, G.G., Verraes, W., De Clerq, N. \& Postnov, A. (2005) Holotype skeletal morphology of Gymnallabes nops (Roberts \& Stewart, 1976), using micro CT-scanning. Cybium (3e série), 29, 281-293.

Devaere, S., Teugels, G.G., Adriaens, D., Huysentruyt, F. \& Verraes, W. (2004) Redescription of Dolichallabes microphthalmus (Poll, 1942) (Siluriformes, Clariidae). Copeia, 2004, 108-115.

Devincenzi, G.J. (1925) El primer ensayo sobre Ictiología del Uruguay. La clase "Peces" de la zoología de don Dámaso A. Larrañaga. Anales del Museo Nacional de Historia Natural de Montevideo (Ser. 2), 6, 295-323.

Devincenzi, G.J. (1933) Peces del Uruguay. Notas complementarias, II. Anales del Museo Nacional de Historia Natural de Montevideo (Ser. 2), 4, 1-11, pl. 1.

Devincenzi, G.J. (1943) El género Loricaria en la cuenca Platense; descripción de una nueva especie, Loricaria commersonoides n. sp. Comunicaciones Zoológicas del Museo de Historia Natural de Montevideo, 1, 1-6, 1 plate.

Devincenzi, G.J. \& Teague, G.W. (1942) Ictiofauna del Rio Uruguay medio. Anales del Museo Nacional de Historia Natural de Montevideo (Ser. 2), 5, 1-100 + index + i-viii, pls. 1-6.

Devincenzi, G.J. \& Vaz-Ferreira, R. (1939) Nota preliminar sobre un pygidido hematófago del Rio Uruguay. Archivos de la Sociedad de Biología de Montevideo, 9, 165-178.

Di Caporiacco, L. (1935) Spedizione Nello Beccari nella Guiana Britannica. Monitore Zoologico Italiano, 46, 55-70.

Di Caporiacco, L. (1948) Miscellanea ichthyologica. Bollettino di Pesca, Piscicoltura e Idrobiologia, 23 [= N.S., vol. 2], 193-205.

Ding, R.-H. (2003) [Pareuchiloglanis in western China and neighbouring countries]. Sichuan Journal of Zoology, 22, 27-28. [In Chinese.]

Ding, R.-H. \& Fang, S.-G. (1997) [Studies on the DNA fingerprinting in three species of the genus Pareuchiloglanis from China, with description of a new species.] Transactions of the Chinese Ichthyological Society, 6, 15-21. [In Chinese, with English abstract.] 
Ding, R.-H., Fu, T.-Y. \& Ye, M.-R. (1991) [Two new species of the genus Pareuchiloglanis from China (Siluriformes: Sisoridae)]. Acta Zootaxonomica Sinica, 16, 369-374. [In Chinese, with English abstract.]

Dinkelmeyer, J. (1995) Zwei neue Arten von Panzerwelsen der Gattung Corydoras Lacépède, 1803 aus Brasilien (Pisces, Siluriformes, Callichthyidae). Aquaristik Aktuell, 1995, 60-61.

Dinkelmeyer, J. (1996) Corydoras seussi n. sp., ein neuer Panzerwels aus Brasilien (Pisces, Siluriformes, Callichthyidae). Aquaristik Aktuell, 1996, 25-26.

Diogo, R. (2003a) Higher-level phylogeny of Siluriformes - an overview. In: Arratia, G., Kapoor, B.G., Chardon, M. \& Diogo, R. (Eds.), Catfishes, Science Publishers, Enfield, NH, USA, pp. 353-384.

Diogo, R. (2003b) Anatomy, phylogeny, and taxonomy of Amphiliidae. In: Arratia, G., Kapoor, B.G., Chardon, M. \& Diogo, R. (Eds.), Catfishes, Science Publishers, Enfield, NH, USA, pp. 401-438.

Diogo, R. (2004a) Phylogeny, origin and biogeography of catfishes: support for a Pangean origin of 'modern teleosts' and reexamination of some Mesozoic Pangean connections between the Gondwanan and Laurasian supercontinents. Animal Biology, 54, 331-351.

Diogo, R. (2004b) Morphological Evolution, Aptations, Homoplasies, Constraints, And Evolutionary Trends: Catfishes As A Case Study On General Phylogeny And Macroevolution, Science Publishers, Inc. Enfield, NH, USA, 487 p.

Diogo, R. \& Chardon, M. (2000) The structures associated with catfish (Teleostei: Siluriformes) mandibular barbels: origin, anatomy, function, taxonomic distribution, nomenclature and synonymy. Netherlands Journal of Zoology, 50, 455-478.

Diogo, R., Chardon, M. \& Vandewalle, P. (2001) Osteology and myology of the cephalic region and pectoral girdle of Bunocephalus knerii, and a discussion on the phylogenetic relationships of the Aspredinidae (Teleostei: Siluriformes). Netherlands Journal of Zoology, 51, 457-481.

Diogo, R., Chardon, M. \& Vandewalle, P. (2002) Osteology and myology of the cephalic region and pectoral girdle of Glyptothorax fukiensis, comparison with other sisorids, and discussion on the synapomorphies and phylogenetic relationships of the Sisoridae (Teleostei: Siluriformes). Belgian Journal of Zoology, 132, 95-103.

Diogo, R., Chardon, M. \& Vandewalle, P. (2003) Osteology and myology of the cephalic region and pectoral girdle of Centromochlus heckelii, comparison with other auchenipterids, and comments on the synapomorphies and phylogenetic relationships of the Auchenipteridae (Teleostei: Siluriformes). Animal Biology, 53, 397-416.

Diogo, R., Chardon, M. \& Vandewalle, P. (2003a) Osteology and myology of the cephalic region and pectoral girdle of Heteropneustes fossilis (Teleostei: Siluriformes), with comments on the phylogenetic relationships between Heteropneustes and the clariid catfishes. Animal Biology, 53, 379-396.

Diogo, R., Chardon, M. \& Vandewalle, P. (2003b) Osteology and myology of the cephalic region and pectoral girdle of Erethistes pusillus, comparison with other erethistids, and comments on the synapomorphies and phylogenetic relationships of the Erethistidae (Teleostei: Siluriformes). Journal of Fish Biology, 63, 1160-1176.

Diogo, R., Chardon, M. \& Vandewalle, P. (2004) Osteology and myology of the cephalic region and pectoral girdle of Schilbe mystus, comparison with other schilbids, and discussion on the monophyly and phylogenetic relationships of the Schilbidae (Teleostei: Siluriformes). Animal Biology, 54, 91-110.

Diogo, R., Oliveira, C. \& Chardon, M. (2000) The origin and transformation of catfish palatine-maxillary system: an example of macroevolution. Netherlands Journal of Zoology, 50, 373-389.

Diogo, R., Oliveira, C. \& Chardon, M. (2001) On the homologies of the skeletal components of catfish (Teleostei: Siluriformes) suspensorium. Belgian Journal of Zoology, 131, 93-109.

Diogo, R., Vandewalle, P. \& Chardon, M. (1999) Morphological description of the cephalic region of Bagrus docmac, with a reflection on Bagridae (Teleostei: Siluriformes) autapomorphies. Netherlands Journal of Zoology, 49, 207232.

Dixon, F. \& Egerton, P. de M.G. (1850) Description of fishes (from the Eocene of Bracklesham Bay and Selsey). In: Dixon, F. (Ed.), The Geology and Fossils of the Tertiary and Cretaceous Formations of Sussex, Brighton, pp. 195205, pls. 11-12.

Dmitrenko, E.M. (1974) [Arius of Day-Arius dayi sp. n. (Cypriniformes, Ariidae) from the Arabian Sea]. Vestnik Zoologii, 1974, 37-41. [In Russian.]

Do Nascimiento, C. \& Lundberg, J.G. (2005) Myoglanis aspredinoides (Siluriformes: Heptapteridae), a new catfish from the Río Ventuari, Venezuela. Zootaxa, 1009, 37-49.

Do Nascimiento, C., Provenzano, F. \& Lundberg, J.G. (2004) Rhamdia guasarensis (Siluriformes, Heptapteridae) a new species of cave catfish from the Sierra de Perijá, northwestern Venezuela. Proceedings of the Biological Society of Washington, 117, 564-574.

Do Nascimiento, C., Villarreal, O. \& Provenzano, F. (2001) Descripción de una nueva especie de bagre anoftalmo del 
género Trichomycterus (Siluriformes, Trichomycteridae), de una cueva de la Sierra de Perijá, Venezuela. Boletín de la Sociedad Venezolana de Espelelogía, 35, 20-26.

Dolgopol de Saez, M. (1945) Noticias sobre peces fósiles Argentinos: Siluroideos terciarios del Chabut. Notes del Museo de la Plata, 6, 451-457.

Douglas, N.H. (1972) Noturus taylori, a new species of madtom (Pisces, Ictaluridae) from the Caddo River, southwest Arkansas. Copeia, 1972, 785-789.

Driver, C.S. (1919) On the Luciopimelodinae, a new subfamily of the South American Siluridae. Proceedings of the American Philosophical Society, 58, 448-456, pls. 2-3.

Duméril, A.M.C. (1856) Ichthyologie analytique, ou classification des poissons, suivant la méthode naturelle, à l'aide de tableaux synoptiques. Mémoires de l'Académie des Sciences de l'Institut impérial de France, 27, 1-507.

Duncker, G. (1904) Die Fische der malayischen Halbinsel. Mitteilungen aus dem Naturhistorischen Museum in Hamburg, 21, 133-207, pls. 1-2.

Durand, J. (1940) Notes sur quelques poissons d'espèces nouvelles ou peu connues des eaux douces cambodgiennes. Station Maritime de Cauda; Institut Océanographique de l'Indochine, 34, 1-40, table, pls. 1-8.

Durand, J. (1968) Étude des poissons récoltés dans la grotte de Umayalanta (Bolivie), Trichomycterus chaberti sp. $\mathrm{n}$. Annales de Spéléologie, 23, 343-353.

Dutt, S. \& Sharma, S.V. (1979) Provisional key to the common freshwater catfishes of central coastal Andhra Pradesh. Mem. Soc. Zool. Guntur, 1, 109-119. [Not seen]

Dutt, S., Sharma, S.V. \& Desoutter, M. (1982) On the taxonomic position of Mystus cavasius (Hamilton-Buchanan) visa-vis M. nigriceps (Valenciennes, 1839) and M. keletius (Valenciennes, 1839). Cybium (3e série), 6 (4), 27-30.

Dybowski, B.I. (1872) Zur Kenntniss der Fischfauna des Amurgebietes. Verhandlungen der Kaiserlich Zoologisch-Botanischen Gesellshaft in Wien, 22, 209-222.

Eastman, C.R. (1917) Fossil fishes in the collection of the United States National Museum. Proceedings of the United States National Museum, 52, 235-304.

Eigenmann, C.H. (1890) The evolution of catfishes. Zoe, 1, 10-15.

Eigenmann, C.H. (1905) The mailed catfishes of South America. Science (n. s.), 21, 792-795.

Eigenmann, C.H. (1907) On a collection of fishes from Buenos Aires. Proceedings of the Washington Academy of Science, 8, 449-458, pls. 21-23.

Eigenmann, C.H. (1909a) Reports on the expedition to British Guiana of the Indiana University and the Carnegie Museum, 1908. Report no. 1.- Some new genera and species of fishes from British Guiana. Annals of the Carnegie Museum, 6, 4-54.

Eigenmann, C.H. (1909b) The fresh-water fishes of Patagonia and an examination of the Archiplata-Archhelenis theory. Reports of the Princeton University expeditions to Patagonia 1896-1899, Zoology, vol. 3, pt. 1, Princeton University, pp. 225-374, pls. 30-37.

Eigenmann, C.H. (1910) Catalogue of the fresh-water fishes of tropical and south temperate America. Reports of the Princeton University Expeditions to Patagonia 1896-1899, Zoology, vol. 3, pt. 2, Princeton University pp. $375-511$.

Eigenmann, C.H. (1911) Description of a new species of Pygidium. Annals of the Carnegie Museum, 7, 214 , pl. 32.

Eigenmann, C.H. (1912a) Some results from an ichthyological reconnaissance of Colombia, South America. Part I. Indiana University Studies, 16, [sic. 8], 1-27.

Eigenmann, C.H. (1912b) The freshwater fishes of British Guiana, including a study of the ecological grouping of species, and the relation of the fauna of the plateau to that of the lowlands. Memoirs of the Carnegie Museum, 5, i-xxii $+1-578$, pls. $1-103$.

Eigenmann, C.H. (1913) On two new species of fishes collected by Miss Lola Vance in Peru. Annals of the Carnegie Museum, 8, 421-422.

Eigenmann, C.H. (1914a) Some results from studies of South American fishes. IV.- New genera and species of South American fishes. Indiana University Studies, 20, 44-48.

Eigenmann, C.H. (1914b) On new species of fishes from the Rio Meta Basin of eastern Colombia and on albino or blind fishes from near Bogotá. Indiana University Studies, 23, 229-230.

Eigenmann, C.H. (1916) New and rare fishes from South American rivers. Annals of the Carnegie Museum, 10, 77-86, pls. 13-16.

Eigenmann, C.H. (1917a) New and rare species of South American Siluridae in the Carnegie Museum. Annals of the Carnegie Museum, 11, 398-404, pls. 39-41.

Eigenmann, C.H. (1917b) Pimelodella and Typhlobagrus. Memoirs of the Carnegie Museum, 7, 229-258, pls. 29-35.

Eigenmann, C.H. (1918a) The Pygidiidae, a family of South American catfishes. Memoirs of the Carnegie Museum, 7 , 
259-398, pls. 36-56.

Eigenmann, C.H. (1918b) Eighteen new species of fishes from northwestern South America. Proceedings of the American Philosophical Society, 56, for 1917, 673-689.

Eigenmann, C.H. (1918c) Descriptions of sixteen new species of Pygidiidae. Proceedings of the American Philosophical Society, 56, for 1917, 690-703.

Eigenmann, C.H. (1919a) Peces Colombianos de las cordilleras y de los llanos al oriente de Bogotá. Boletin de la Sociedad Colombiana de Ciencias Naturales, 7, 126-136.

Eigenmann, C.H. (1919b) Trogloglanis pattersoni a new blind fish from San Antonio, Texas. Proceedings of the American Philosophical Society, 58, 397-400.

Eigenmann, C.H. (1920a) The fishes of Lake Valencia, Caracas, and of the Rio Tuy at El Concejo, Venezuela. Indiana University Studies, 7, 1-13.

Eigenmann, C.H. (1920b) South America West of the Maracaibo, Orinoco, Amazon, and Titicaca basins, and the horizontal distribution of its fresh-water fishes. Indiana University Studies, 7, 1-24.

Eigenmann, C.H. (1920c) On a new species of Hatcheria and a new species of Pygidium. Revista Chilena de Historia Natural, Valparaiso, 23, 53-54.

Eigenmann, C.H. (1920d) Limits of the genera Vandellia and Urinophilus. Science (n. s.), 51 (no. 1322), 441.

Eigenmann, C.H. (1922a) On a new genus and two new species of Pygidiidae, a family of South American nematognaths. Bijdragen tot de Dierkunde, 22, 113-114, pls. 3-4.

Eigenmann, C.H. (1922b) The fishes of western South America, Part I.— The fresh-water fishes of northwestern South America, including Colombia, Panama, and the Pacific slopes of Ecuador and Peru, together with an appendix upon the fishes of the Rio Meta in Colombia. Memoirs of the Carnegie Museum, 9, 1-346, pls. 1-38.

Eigenmann, C.H. (1925) A review of the Doradidae, a family of South American Nematognathi, or catfishes. Transactions of the American Philosophical Society (N. S.), 22, 280-365, pls. 1-27.

Eigenmann, C.H. (1927) The fresh-water fishes of Chile. Memoirs of the National Academy of Sciences, 22, 1-63, pls. 116.

Eigenmann, C.H. \& Allen, W.R. (1942) Fishes of Western South America. I.- The Intercordilleran and Amazonian Lowlands of Peru. II.-The High Pampas of Peru, Bolivia, and Northern Chile; with a Revision of the Peruvian Gymnotidae, and of the Genus Orestias, University of Kentucky, xv + 494 p., 22 pl.

Eigenmann, C.H. \& Bean, B.A. (1907) An account of Amazon River fishes collected by J. B. Steere; with a note on Pimelodus clarias. Proceedings of the United States National Museum, 31, 659-668.

Eigenmann, C.H. \& Eigenmann, R.S. (1888a) American Nematognathi. American Naturalist, 22, 647-649.

Eigenmann, C.H. \& Eigenmann, R.S. (1888b) Preliminary notes on South American Nematognathi, I. Proceedings of the California Academy of Sciences, Series 2, 1, 119-172.

Eigenmann, C.H. \& Eigenmann, R.S. (1889a) Description of new nematognathoid fishes from Brazil. West American Scientist, 6, 8-10.

Eigenmann, C.H. \& Eigenmann, R.S. (1889b) Preliminary notes on South American Nematognathi, II. Proceedings of the California Academy of Sciences, Series 2, 2, 28-56.

Eigenmann, C.H. \& Eigenmann, R.S. (1890) A revision of the South American Nematognathi or cat-fishes. Occasional Papers of the California Academy of Sciences, 1, 1-508 + errata and map.

Eigenmann, C.H. \& Eigenmann, R.S. (1891) A catalogue of the fresh-water fishes of South America. Proceedings of the United States National Museum, 14, 1-81.

Eigenmann, C.H. \& Eigenmann, R.S. (1919) Steindachneridion. Science (n. s.), 50, 525-526.

Eigenmann, C.H. \& Fisher, H.G. (1917) On some species of Rhamdia, a genus of South American Siluridae, in the Carnegie Museum. Annals of the Carnegie Museum, 11, 394-397, pl. 38.

Eigenmann, C.H., Henn, A.W. \& Wilson, C. (1914) New fishes from western Colombia, Ecuador, and Peru. Indiana University Studies, 19, 1-15.

Eigenmann, C.H. \& Kennedy, C.H. (1903) On a collection of fishes from Paraguay, with a synopsis of the American genera of cichlids. Proceedings of the Academy of Natural Sciences, Philadelphia, 55, 497-537.

Eigenmann, C.H., McAtee, W.L. \& Ward, D.P. (1907) On further collections of fishes from Paraguay. Annals of the Carnegie Museum, 4, 110-157, pls. 31-45.

Eigenmann, C.H. \& Norris, A.A. (1900) Sobre alguns peixes de S. Paulo, Brazil. Revista do Museu Paulista, 4, 349-362.

Eigenmann, C.H. \& Norris, A.A. (1901) Bergiaria. Comunicaciones del Museo Nacional de Buenos Aires, 1, 272.

Eigenmann, C.H. \& Vance, L. (1917) Some species of Farlowella. Annals of the Carnegie Museum, 11, 297-303, pls. $29-31$. 
Ellis, M.D. (1913) The plated nematognaths. Annals of the Carnegie Museum, 8, 384-413, pls. 25-31.

Elwin, M.G. (1939) Corydoras arcuatus sp. n., an Amazonian catfish. Annals and Magazine of Natural History (Ser. 11), 3, 126-128, pl. 3.

Emmens, C.W. \& Axelrod, H.R. (1968) Catfish. TFH Publications, Neptune City, NJ, 96 p.

Eschmeyer, W.N. (1990) Catalog of the Genera of Recent Fishes, California Academy of Sciences, San Francisco, v + $697 \mathrm{p}$.

Eschmeyer, W.N. (Ed.) (1998) Catalog of Fishes, California Academy of Sciences, San Francisco, 2905 p.

Eschmeyer, W.N., Ferraris, C.J., Jr., Hoang, M. \& Long, D. (1998) Species of fishes. In: Eschmeyer, W.N. (Ed.), Catalog of Fishes, California Academy of Sciences, San Francisco, pp. 25-1820.

Espinosa Pérez, H., Gaspar Dillanes, M.T. \& Fuentes Mata, P. (1993) Listados Faunísticos de México. III.—Los Peces Dulceacuícolas Mexicanos. Universidad Nacional Autónoma de México, 98 p.

Etnier, D.A. \& Jenkins, R.E. (1980) Noturus stanauli, a new madtom catfish (Ictaluridae) from the Clinch and Duck rivers, Tennessee. Bulletin of the Alabama Museum of Natural History, 5, 17-22.

Evermann, B.W. \& Goldsborough, E.L. (1902) A report on fishes collected in Mexico and Central America, with notes and descriptions of five new species. Bulletin of the U. S. Fish Commission, 21, for 1901, 137-159.

Evermann, B.W. \& Kendall, W.C. (1894) The fishes of Texas and the Rio Grande basin, considered chiefly with reference to their geographic distribution. Bulletin of the U. S. Fish Commission, 12, for 1892, 57-126, pls. 10-50.

Evermann, B.W. \& Kendall, W.C. (1898) Descriptions of new or little-known genera and species of fishes from the United States. Bulletin of the U. S. Fish Commission, 17, for 1897, 125-133, pls. 6-9.

Fang, S.-M., Xu, T.-Q. \& Cui, G.-H. (1984) [A new species of the catfish genus Pareuchiloglanis (Pisces: Sisoridae) from China]. Acta Zootaxonomica Sinica, 9, 209-211. [In Chinese, with English abstract.]

Fernández, L.A. (1999) Revisión Taxonómica del Género Trichomycterus (Pisces: Trichomycteridae) de la República Argentina. Unpublished Ph.D. Dissertation, Universidad Tucumán, Argentina.

Fernández, L.A. (2000) A new species of Trichomycterus from northwestern Argentina (Ostariophysi: Trichomycteridae). Ichthyological Exploration of Freshwaters, 11, 349-354.

Fernández, L.A. (2000) Redescription of the teleost Trichomycterus barbouri (Eigenmann, 1911), occurrence in Argentina and comparison with related species (Ostariophysi: Siluriformes: Trichomycteridae). Studies on Neotropical Fauna and Environment, 35 27-33.

Fernández, L.A. \& Bichuette, M.E. (2002) A new cave dwelling species of Ituglanis from the São Domingos karst, central Brazil (Siluriformes: Trichomycteridae). Ichthyological Exploration of Freshwaters, 13, 273-278.

Fernández, L.A. \& Pinna, M.C.C., de. (2005) Phreatic catfish of the genus Silvinichthys from southern South America (Teleostei, Siluriformes, Trichomycteridae). Copeia, 2005, 100-108.

Fernández, L.A. \& Schaefer, S.A. (2003) Trichomycterus yuska, a new species from high elevations of Argentina (Siluriformes: Trichomycteridae). Ichthyological Exploration of Freshwaters, 14, 353-360.

Fernández, L.A. \& Schaefer, S.A. (2005) New Trichomycterus (Siluriformes: Trichomycteridae) from an offshore island of Colombia. Copeia, 2005, 68-76.

Fernández, L.A. \& Vari, R.P. (2000) New species of Trichomycterus (Teleostei: Siluriformes: Trichomycteridae) lacking a pelvic fin and girdle from the Andes of Argentina. Copeia, 2000, 990-996.

Fernández, L.A. \& Vari, R.P. (2002) New species of Trichomycterus from the Andes of Argentina with a redescription of Trichomycterus alterus (Siluriformes: Trichomycteridae). Copeia, 2002, 739-747.

Fernández, L.A. \& Vari, R.P. (2004) New species of Trichomycterus from midelevation localities of northwestern Argentina (Siluriformes: Trichomycteridae). Copeia, 2004, 876-882.

Fernández-Yépez, A. (1945) Un nuevo loricarido para Venezuela. Descripción de un ejemplar de Loricariidae, colectado en el Río Encantado, afluente del Río Tuy por medio del Río Grande. Memoria, Sociedad de Ciencias Naturales La Salle, 5, 27-34.

Fernández-Yépez, A. (1950a) Algunos peces del Rio Autana. Novedades Científicas; Contribuciones ocasionales del Museo de Historia Natural La Salle (Serie Zoológica), 2, 1-18, pls. 1-3.

Fernández-Yépez, A. (1950b) Notas sobre la fauna ictiológica de Venezuela. Memoria, Sociedad de Ciencias Naturales La Salle, 10, 113-118.

Fernández-Yépez, A. (1950c) Un nuevo pez de la familia Doradidae. Memoria, Sociedad de Ciencias Naturales La Salle, 10, 195-198.

Fernández-Yépez, A. (1951) Ginesia cunaguaro, nuevo pez para la Ciencia colectado en el Río Apure, Venezuela. Evencias, [1-4].

Fernández-Yépez, A. (1953) Algunas notas sobre los peces Asprediformes con descripción de Ernstichthys anduzei, 
nuevo e interesante bunocephalido. Novedades Científicas; Contribuciones ocasionales del Museo de Historia Natural La Salle (Serie Zoológica), 11, 1-6, 1 pl.

Fernández-Yépez, A. (1965) Contribución al conocimiento de los peces de Venezuela. Dieciséis especies nuevas para Venezuela. Evencias, 1-12.

Fernández-Yépez, A. (1967) Resultados zoologicos de la expedicion de la Universidad Central de Venezuela a la region del Auyantepui en la Guayana Venezolana, abril de 1956. 6.- Primera contribucion al conocimiento de los peces, con descripcion de dos especies y una subespecie nuevas. Acta Biologica Venezuelica, 5, 159-177.

Fernández-Yépez, A. (1968) Contribución al conocimiento de la familia Doradidae en Venezuela. Boletín del Instituto Oceanográfio. Universitad de Oriente, Cumaná, 7, 7-72.

Fernández-Yépez, A. (1970) Análisis Ictiológico del Complejo Hidrográfico (07) Río Unare, Dirección de Obras Hidráulicas, Ministerio de Obras Públicas, Republica de Venezuela, 20 p., 41 pl.

Fernández-Yépez, A. (1972a) Análisis Ictiológico del Complejo Hidrográfico (04) Río Yaracuy, Dirección de Obras Hidraulicas, Ministerio de Obras Públicas, Republica de Venezuela, 25 pp., 41 pl.

Fernández-Yépez, A. (1972b) El género Hemicetopis Blecker [sic, Bleeker], 1863 (Cetopsidae) en Venezuela. Lagena, 30, 19-21.

Fernández-Yépez, A. (1973) Contribución al conocimiento de Auchenipteridae. Evencias, 29, [1-7].

Fernández-Yépez, A. \& Martín Salazar, F. (1952) Notas sobre la fauna ictiológica de la region Baruta-El Hatillo. Memoria, Sociedad de Ciencias Naturales La Salle, 12, 31-45.

Fernández-Yépez, A. \& Martín Salazar, F. (1953) Apuntes sobre la ictiologia de Perija. Memoria, Sociedad de Ciencias Naturales La Salle, 13, 227-243.

Fernholm, B. \& Wheeler, A. (1983) Linnaean fish specimens in the Swedish Museum of Natural History, Stockholm. Zoological Journal of the Linnean Society, 78, 199-286.

Ferraris, C.J., Jr. (1988) The Auchenipteridae: Putative Monophyly and Systematics, with a Classification of the Neotropical Doradoid Catfishes (Ostariophysi: Siluriformes) Doctoral Dissertation, City University of New York, New York.

Ferraris, C.J., Jr. (1988) Relationships of the Neotropical catfish genus Nemuroglanis, with a description of a new species (Osteichthyes: Siluriformes: Pimelodidae). Proceedings of the Biological Society of Washington, 101, 509-516.

Ferraris, C.J., Jr. (1991) On the type species of Bunocephalus (Siluriformes: Aspredinidae). Copeia, 1991, $224-225$.

Ferraris, C.J., Jr. (1996) Denticetopsis, a new genus of South American whale catfish (Siluriformes: Cetopsidae, Cetopsinae), with two new species. Proceedings of the California Academy of Sciences, Series 4, 49, 161-170.

Ferraris, C.J., Jr. (1999a) Plotosidae. In: Carpenter, K.E. \& Niem, V.H. (Eds.), FAO Species Identification Guides for Fishery Purposes: The living Marine Resources of the Western Central Pacific Vol. 3: Batoid fishes, chimeras and bony fishes, part 1 (Elopidae to Linophrynidae), FAO, Rome, pp. 1880-1883.

Ferraris, C.J., Jr. (1999b) Rita sacerdotum, a valid species of catfish from Myanmar (Pisces, Bagridae). Bulletin of the Natural History Museum, London, (Zoology), 65, 15-21.

Ferraris, C.J., Jr. (2003a) Diplomystidae. In: Reis, R.E., Kullander, S.O. \& Ferraris, C.J., Jr. (Eds.), Check list of the Freshwater Fishes of South and Central America Edipucrs, Porto Alegre, Brazil, pp. 255-256.

Ferraris, C.J., Jr. (2003b) Neoplecostominae. In: Reis, R.E., Kullander, S.O. \& Ferraris, C.J., Jr. (Eds.), Check list of the Freshwater Fishes of South and Central America. Edipucrs, Porto Alegre, Brazil, pp. 319-320.

Ferraris, C.J., Jr. (2003c) Loricariinae. In: Reis, R.E., Kullander, S.O. \& Ferraris, C.J., Jr. (Eds.), Check list of the Freshwater Fishes of South and Central America Edipucrs, Porto Alegre, Brazil, pp. 330-350.

Ferraris, C.J., Jr. (2003d) Auchenipteridae. In: Reis, R.E., Kullander, S.O. \& Ferraris, C.J., Jr. (Eds.), Check list of the Freshwater Fishes of South and Central America Edipucrs, Porto Alegre, Brazil, pp. 470-482.

Ferraris, C.J., Jr. (2004) A new species of the Asian schilbid catfish genus Clupisoma from Myanmar, with a redescription of Clupisoma prateri Hora (Osteichthyes: Siluriformes: Schilbidae). Zootaxa, 439, 1-10.

Ferraris, C.J., Jr. \& Britz, R. (2005) A diminutive new species of Glyptothorax (Siluriformes: Sisoridae) from the upper Irrawaddy River basin, Myanmar, with comments on sisorid and erethistid phylogenetic relationships. Ichthyological Exploration of Freshwaters, 16, 375-383.

Ferraris, C.J., Jr. \& Brown, B.A. (1991) A new species of Pseudocetopsis from the Río Negro drainage of Venezuela (Siluriformes: Cetopsidae). Copeia, 1991, 161-165.

Ferraris, C.J., Jr. \& Fernandez, J. (1987) Trachelyopterichthys anduzei, a new species of auchenipterid catfish from the upper Río Orinoco of Venezuela with notes on T. taeniatus (Kner). Proceedings of the Biological Society of Washington, 100, 257-261.

Ferraris, C.J., Jr., Isbrücker, I.J.H. \& Nijssen, H. (1986) Neblinichthys pilosus, a new genus and species of mailed catfish 
from the Rio Baria system, southern Venezuela (Pisces, Siluriformes, Loricariidae). Revue Française d'Aquariologie et Herpetologie, 13, 69-72.

Ferraris, C.J., Jr. \& Mago-Leccia, F. (1989) A new genus and species of pimelodid catfish from the Río Negro and Río Orinoco drainages of Venezuela (Siluriformes: Pimelodidae). Copeia, 1989, 166-171.

Ferraris, C.J., Jr. \& Pinna, M.C.C., de. (1999) Higher level names for catfishes (Ostariophysi: Siluriformes). Proceedings of the California Academy of Sciences, Series 4, 51, 1-17.

Ferraris, C.J., Jr. \& Runge, K.E. (1999) Revision of the south Asian bagrid catfish genus Sperata, with the description of a new species from Myanmar. Proceedings of the California Academy of Sciences, Series 4, 51, 397-424.

Ferraris, C.J., Jr. \& Vari, R.P. (1992) Catalog of type specimens of Recent fishes in the National Museum of Natural History, Smithsonian Institution, 4: Gonorynchiformes, Gymnotiformes, and Siluriformes (Teleostei: Ostariophysi). Smithsonian Contributions to Zoology, 535, 1-52.

Ferraris, C.J., Jr. \& Vari, R.P. (1999) The South American catfish genus Auchenipterus Valenciennes, 1840 (Ostariophysi: Siluriformes: Auchenipteridae): monophyly and relationships, with a revisionary study. Zoological Journal of the Linnean Society, 126, 387-450.

Ferraris, C.J., Jr. \& Vari, R.P. (2000) The deep-water South American catfish genus Pseudepapterus (Ostariophysi: Auchenipteridae). Ichthyological Exploration of Freshwaters, 11, 97-112.

Ferraris, C.J., Jr., Vari, R.P. \& Raredon, S.J. (2005) Catfishes of the genus Auchenipterichthys (Osteichthyes: Siluriformes: Auchenipteridae), a revisionary study. Neotropical Ichthyology, 3, 81-98.

Figueiredo, F.J. \& Costa-Carvalho, B.C.M. (1999) Steindachneridion silvasantosi sp. nov. (Teleostei, Siluriformes, Pimelodidae) from the Tertiary of Taubaté Basin, São Paulo, Brazil. Anais da Academia Brasileira de Ciências, 71, 683-695.

Fisch-Muller, S. (1999) Systématique du Genre Ancistrus Kner (Teleostei, Loricariidae): Approches Morphologique et Génétique, Thèse de Doctorat, Université de Genève.

Fisch-Muller, S. (2003) Ancistrinae. In: Reis, R.E., Kullander, S.O. \& Ferraris, C.J., Jr. (Eds.), Check list of the Freshwater Fishes of South and Central America. Edipucrs, Porto Alegre, Brazil, pp. 373-400.

Fisch-Muller, S., Cardoso, A.R., da Silva, J.F.P. \& Bertaco, V.A. (2005a) Two new Amazonian species of armored catfishes (Siluriformes: Loricariidae): Ancistrus verecundus and Ancistrus parecis. Neotropical Ichthyology, 3, 525532.

Fisch-Muller, S., Cardoso, A.R., da Silva, J.F.P. \& Bertaco, V.A. (2005b) Three new species of Ancistrus Kner (Teleostei: Siluriformes: Loricariidae) from the upper Tapajós and Tocantins rivers. Revue suisse de Zoologie, Annales de la Société zoologique suisse et du Muséum d'Histoire naturelle de Genève, 112, 559-572.

Fisch-Muller, S., Mazzoni, R. \& Weber, C. (2001) Genetic and morphological evidences for two new sibling species of Ancistrus (Siluriformes: Loricariidae) in upper rio Tocantins drainage, Brazil. Ichthyological Exploration of Freshwaters, 12, 289-304.

Fisher, H.G. (1917) A list of the Hypophthalmidae, the Diplomystidae and of some unrecorded species of Siluridae in the collections of the Carnegie Museum. Annals of the Carnegie Museum, 11, 405-427, pl. 42.

Forey, P.L. \& Young, S.V.T. (1999) Late Miocene fishes of the Emirate of Abu Dhabi, United Arab Emirates. In: Whybrow, P.J. \& Hill, A. (Eds.), Fossil Vertebrates of Arabia, Yale University Press, London and New Haven, pp. 120135.

Forsskål, P. (1775) Descriptiones Animalium Avium, Amphibiorum, Piscium, Insectorum, Vermium; quae in Itinere Orientali Observavit... Post Mortem Auctoris Edidit Carsten Niebuhr, Hauniae, 20 + xxxiv + 164 p., map.

Fowler, H.W. (1904) A collection of fishes from Sumatra. Journal of the Academy of Natural Sciences of Philadelphia (Ser. 2), 12, 495-560, pls. 7-28.

Fowler, H.W. (1905) Some fishes from Borneo. Proceedings of the Academy of Natural Sciences, Philadelphia, 57, 455523.

Fowler, H.W. (1911) Some fishes from Venezuela. Proceedings of the Academy of Natural Sciences, Philadelphia, 63, 419-437.

Fowler, H.W. (1913) Fishes from the Madeira River, Brazil. Proceedings of the Academy of Natural Sciences, Philadelphia, 65, 517-579.

Fowler, H.W. (1914) Fishes from the Rupununi River, British Guiana. Proceedings of the Academy of Natural Sciences, Philadelphia, 66, 229-284.

Fowler, H.W. (1915a) Notes on nematognathous fishes. Proceedings of the Academy of Natural Sciences, Philadelphia, 67, 203-243.

Fowler, H.W. (1915b) Cold-blooded vertebrates from Florida, the West Indies, Costa Rica, and eastern Brazil. Proceed- 
ings of the Academy of Natural Sciences, Philadelphia, 67, 244-269.

Fowler, H.W. (1919a) A new siluroid fish of the genus Cyclopium from Colombia. Proceedings of the Academy of Natural Sciences, Philadelphia, 71, 125-127, pl. 8.

Fowler, H.W. (1919b) The fishes of the United States Eclipse Expedition to West Africa. Proceedings of the United States National Museum, 56, 195-292.

Fowler, H.W. (1930) The freshwater fishes obtained by the Gray African Expedition - 1929. With notes on other species in the Academy collection. Proceedings of the Academy of Natural Sciences, Philadelphia, 82, 27-83.

Fowler, H.W. (1931) Fishes obtained by the Barber Asphalt Company in Trinidad and Venezuela in 1930. Proceedings of the Academy of Natural Sciences, Philadelphia, 83, 391-410.

Fowler, H.W. (1932a) Zoological results of the Matto Grosso Expedition to Brazil in 1931, I.— Fresh water fishes. Proceedings of the Academy of Natural Sciences, Philadelphia, 84, 343-377.

Fowler, H.W. (1932b) Notes on fresh water fishes from Central America. Proceedings of the Academy of Natural Sciences, Philadelphia, 84, 379-385.

Fowler, H.W. (1934a) Zoological results of the third De Schauensee Siamese Expedition, Part I.- Fishes. Proceedings of the Academy of Natural Sciences, Philadelphia, 86, 67-163, pl. 12.

Fowler, H.W. (1934b) Zoological results of the third De Schauensee Siamese Expedition, Part V.- Additional fishes. Proceedings of the Academy of Natural Sciences, Philadelphia, 86, 335-352.

Fowler, H.W. (1935a) Scientific results of the Vernay-Lang Kalahari expedition, March to September, 1930. Fresh-water fishes. Annals of the Transvaal Museum, 16, 251-293, pls. 6-9.

Fowler, H.W. (1935b) Zoological results of the third De Schauensee Siamese Expedition, Part VI.- Fishes obtained in 1934. Proceedings of the Academy of Natural Sciences, Philadelphia, 87, 89-163.

Fowler, H.W. (1936a) Fresh-water fishes obtained in Guatemala by Mr. Rodolphe Meyer de Schauensee in 1935. Proceedings of the Academy of Natural Sciences, Philadelphia, 87, for 1935, 515-531.

Fowler, H.W. (1936b) Zoological results of the George Vanderbilt African Expedition of 1934. Part III.- The fresh water fishes. Proceedings of the Academy of Natural Sciences, Philadelphia, 88, 243-335.

Fowler, H.W. (1937) Zoological results of the third De Schauensee Siamese Expedition. Part VIII.- Fishes obtained in 1936. Proceedings of the Academy of Natural Sciences, Philadelphia, 89, 125-264.

Fowler, H.W. (1939) Zoological results of the third De Schauensee Siamese Expedition. Part IX.- Additional fishes obtained in 1936. Proceedings of the Academy of Natural Sciences, Philadelphia, 91, 39-76.

Fowler, H.W. (1940a) A collection of fishes obtained by Mr. William C. Morrow in the Ucayali River Basin, Peru. Proceedings of the Academy of Natural Sciences, Philadelphia, 91, for 1939, 219-289.

Fowler, H.W. (1940b) Fishes obtained in Chile by Mr. D. S. Bullock. Proceedings of the Academy of Natural Sciences, Philadelphia, 92, 171-190.

Fowler, H.W. (1940c) Zoological results of the second Bolivian expedition for the Academy of Natural Sciences of Philadelphia, 1936-1937. Part I.- The fishes. Proceedings of the Academy of Natural Sciences, Philadelphia, 92, 43103.

Fowler, H.W. (1941a) A collection of fresh-water fishes obtained in eastern Brazil by Dr. Rodolpho von Ihering. Proceedings of the Academy of Natural Sciences, Philadelphia, 93, 123-199.

Fowler, H.W. (1941b) Los peces del Peru. Catálogo systemático de los peces que habitan en aquas peruanas (Continuación). Boletín del Museo de Historia Natural “Javier Prado”, 5, 466-487.

Fowler, H.W. (1941c) Notes on Colombian fresh-water fishes with descriptions of four new species. Notulae Naturae (Philadelphia), 73, 1-10.

Fowler, H.W. (1942) A new cat-fish from Brazil (Plecostomus gomesi, new species). The Fish Culturist, 21, unpaginated.

Fowler, H.W. (1943a) A collection of fresh-water fishes from Colombia, obtained chiefly by Brother Nicéforo Maria. Proceedings of the Academy of Natural Sciences, Philadelphia, 95, 223-266.

Fowler, H.W. (1943b) Notes and descriptions of new or little known fishes from Uruguay. Proceedings of the Academy of Natural Sciences, Philadelphia, 95, 311-334.

Fowler, H.W. (1943c) Zoological results of the second Bolivian expedition for the Academy of Natural Sciences of Philadelphia, 1936-1937. Part II.- Additional new fishes. Notulae Naturae (Philadelphia), 120, 1-7.

Fowler, H.W. (1944a) A new glass catfish from Borneo. The Fish Culturist, 24, 1-2.

Fowler, H.W. (1944b) Fresh-water fishes from northwestern Colombia. Proceedings of the Academy of Natural Sciences, Philadelphia, 96, 227-248.

Fowler, H.W. (1944c) Results of the fifth George Vanderbilt expedition (1941) (Bahamas, Caribbean Sea, Panama, 
Galápagos Archipelago and Mexican Pacific islands).- The Fishes. Monograph. Academy of Natural Sciences, Philadelphia, 6, 57-529, pls. 1-20.

Fowler, H.W. (1945a) Colombian zoological survey. Pt. I.- The freshwater fishes obtained in 1945. Proceedings of the Academy of Natural Sciences, Philadelphia, 97, 93-135.

Fowler, H.W. (1945b) Descriptions of two new fresh-water fishes from Colombia. Notulae Naturae (Philadelphia), 158, $1-11$.

Fowler, H.W. (1945c) Descriptions of seven new fresh-water fishes from Peru. Notulae Naturae (Philadelphia), 159, 111.

Fowler, H.W. (1945d) A study of the fishes of the southern Piedmont and coastal plain. Monograph. Academy of Natural Sciences, Philadelphia, 7, i-vi + 1-408, 313 figs. on 73 unnum. pls.

Fowler, H.W. (1946) Notes on a collection of fishes from Trinidad. Notulae Naturae (Philadelphia), 165, 1-11.

Fowler, H.W. (1949) Results of the two Carpenter African expeditions, 1946-1948. Pt. II.- The fishes. Proceedings of the Academy of Natural Sciences, Philadelphia, 101, 233-275.

Fowler, H.W. (1951a) Os peixes de água doce do Brasil, 3. entrega. Arquivos de Zoologia do Estado de São Paulo, 6, 405-624.

Fowler, H.W. (1951b) Some new or emended names of fish-like vertebrates. The Fish Culturist, 39, 1-4.

Fowler, H.W. (1954) Os peixes de água doce do Brasil. 4. ${ }^{a}$ entrega. Arquivos de Zoologia do Estado de São Paulo, 9, 1400.

Fowler, H.W. (1958) Some new taxonomic names of fishlike vertebrates. Notulae Naturae (Philadelphia), 310, 1-16.

França, C. (1922) Doutor Alexandre Rodrigues Ferreira. História de uma missão scientifica ao Brasil no século XVIII. Boletim da Sociedade Broteriana, 1, 65-123, figs.

Franz, D. (2001) Schwielenwelse. Morphologische Untersuchung der Gattung Hoplosternum Gill, 1858 und Beschreibung der Gattung Diasternum gen. nov. (Actinopterygii: Callichthyidae). Das Aquarium, 389, 17-23.

Fraser-Brunner, A. (1938) Debutantes of the month. Aquarist and Pondkeeper, 8, 207-208.

Fraser-Brunner, A. (1947) New fishes of the genus Corydoras. Proceedings of the Zoological Society of London, 117, 241-246, pl. 1.

Freycinet, L. (Ed.) (1824-1825) Voyage Autour du Monde, Enterpris par Ordre du Roi, sous le Ministère et Conformemént aux Instructions de S. Exc. M. le Vicomte du Bouchage, Secrétaire d'État au Département de la Marine, Exécuté sur les Corvettes de S. M. l'Uranie et la Physicienne, Pendant les Années 1817, 1818, 1819 et 1820, Pillet Ainé, Imprimeur-Libraire, Paris, [p.1-328 in 1824; 329-616 in 1825].

Friel, J.P. (1994) A Phylogenetic Study of the Neotropical Banjo Catfishes (Teleostei: Siluriformes: Aspredinidae), Unpublished Ph.D dissertation, Duke University, Durham, NC.

Friel, J.P. (1995) Acanthobunocephalus nicoi, a new genus and species of miniature banjo-catfish from the upper Orinoco and Casiquiare rivers, Venezuela (Siluriformes: Aspredinidae). Ichthyological Exploration of Freshwaters, 6, 89-95.

Friel, J.P. (2003) Aspredinidae. In: Reis, R.E., Kullander, S.O. \& Ferraris, C.J., Jr. (Eds.), Check list of the Freshwater Fishes of South and Central America, Edipucrs, Porto Alegre, Brazil, pp. 261-267.

Friel, J.P. \& Lundberg, J.G. (1996) Micromyzon akamai, gen. et sp. nov., a small and eyeless banjo catfish (Siluriformes: Aspredinidae) from the river channels of the lower Amazon basin. Copeia, 1996, 641-648.

Frizzell, D.L. (1965) Otoliths of new fish (Vorhisia vulpes, n. gen., n. sp. Siluroidei?) from Upper Cretaceous of South Dakota. Copeia, 1965, 178-181.

Frizzell, D.L. \& Dante, J.H. (1965) Otoliths of some early Cenozoic fishes of the Gulf Coast. Journal of Paleontology, 39, 687-718.

Frizzell, D.L. \& Koenig, J.W. (1973) Upper Cretaceous ostariophysine (Vorhisia) redescribed from unique association of utricular and lagenal otoliths (lapillus and asteriscus). Copeia, 1973, 692-698.

Frost, G.A. (1925) Description of fish otoliths from the Tertiary formations of Atcheen, northern Sumatra. Wetenschappelijke Mededeelingen // Dienst van den Mijnbouw in Nederlandsch-Oost Indië, 2, 1-28. [Not seen, Journal title not verified]

Frost, G.A. (1926) Appendix.- Teleost otoliths. Pp. 83-86, pl. 18, figs. 7-12, In: E.I. White, Eocene fishes from Nigeria. Bulletin, Geological Survey of Nigeria, 10, 1-86, pls. 1-18.

Frost, G.A. (1934) Otoliths of fishes from the lower Tertiary formations of southern England. V.- Anacanthini, Heterosomata, Ostariophysi. Annals and Magazine of Natural History (Ser. 10), 14, 500-505, pl. 14.

Fu, T.-S. (1935) Study of the fishes of Sin-Yang. Bulletin of the Fan Memorial Institute of Biology, Peiping (Zoological series), 6, 178-181. 
Fuhrmann, O. (1906) Scleropages formosum und über Phreatobius cisternarum. Verhandlungen der Schweizerischen Naturforschenden Gesellschaft in Luzern, 88, 50-51.

Fumihito, A. (1989) Morphological comparison of the Mekong giant catfish, Pangasianodon gigas, with other pangasiid species. Japanese Journal of Ichthyology, 36, 113-119.

Galvis, G., Mojica, J.I. \& Camargo, M. (1997) Peces del Catatumbo, Asociación Cravo Norte, Ecopetrol, DXY \& Shell, Bogotá, 118 p.

Ganguly, D.N. \& Datta, N.C. (1975) A new cat fish of the genus Mystus Scopoli (family: Bagridae) from the vicinity of the Hundru Falls, Bihar, India with comment on the genus Mystus. In: Tiwari, K.K. \& Srivastava, C.B. (Eds.), Dr. B.S. Chauhan Commemoration Volume, Zoological Society of India, Orissa, pp. 293-298.

Ganguly, D.N., Datta, N.C. \& Sen, S. (1972) Two new catfishes of the genus Glyptothorax Blyth (family: Sisoridae) from Subarnarekha River, Bihar, India. Copeia, 1972, 340-344.

Garavello, J.C. (1976) Systematics and geographical distribution of the genus Parotocinclus Eigenmann \& Eigenmann, 1889 (Ostariophysi, Loricariidae). Arquivos de Zoologia (São Paulo), 28, 1-37.

Garavello, J.C. (1988) Three new species of Parotocinclus Eigenmann \& Eigenmann, 1889 with comments on their geographical distribution (Pisces, Loricariidae). Naturalia (São Paulo), 13, 117-128.

Garavello, J.C. (2005) Revision of genus Steindachneridion Eigenmann \& Eigenmann, 1919 (Ostariophysi, Siluriformes, Pimelodidae). Neotropical Ichthyology, 3, 607-623.

Garavello, J.C. \& Britski, H.A. (2003) Parotocinclus planicauda, a new species of the subfamily Hypoptopomatinae from southeastern Brazil (Ostariophysi: Loricariidae). Brazilian Journal of Biology, 63, 253-260.

Garavello, J.C., Britski, H.A. \& Schaefer, S.A. (1998) Systematics of the genus Otothyris Myers 1927, with comments on geographic distribution (Siluriformes: Loricariidae: Hypoptopomatinae). American Museum Novitates, 3222, 119.

Garman, S. (1890) Silurus (Parasilurus) aristotelis. Bulletin of the Essex Institute, 22, 56-59.

Garman, S. (1912) Pisces. In: Some Chinese vertebrates. Memoirs of the Museum of Comparative Zoology, 40, 111-123.

Gauger, M.F.W. \& Buckup, P.A. (2005) Two new species of Hypoptopomatinae from the rio Paraíba do Sul basin, with comments on the monophyly of Parotocinclus and the Otothyrini (Siluriformes: Loricariidae). Neotropical Ichthyology, 3, 509-518.

Gayet, M. (1988) Le plus ancien crane de siluriforme: Andinichthys bolivianensis nov. gen., nov. sp. (Andinichthyidae nov. fam.) du Maastrichtien de Tiupampa (Bolivie). Comptes Rendus de l'Academie des Sciences, Serie II: Mecanique, Physique-Chimie Sciences de l'Univers Sciences de la Terre, 307, 833-836.

Gayet, M. (1990) Nouveaux Silurformes du Maastrichtien de Tiupampa (Bolivie). Comptes Rendus de l'Academie des Sciences, Serie II: Mecanique, Physique-Chimie Sciences de l'Univers Sciences de la Terre, 310, 867-872.

Gayet, M. (1991) Holostean and teleostean fossils from Bolivia. In: R. Suárez-Sourco (Ed.), Fósiles y Facies de Bolivia. Revista Tech. YPFB Cochabamba, 12, 453-494.

Gayet, M. \& Meunier, F.J. (2003) Palaeontology and palaeobiogeography of catfishes. In: Arratia, G., Kapoor, B.G., Chardon, M. \& Diogo, R. (Eds.), Catfishes, Science Publishers, Inc., Enfield, NH, USA, pp. 491-522.

Geerinckx, T., Adriaens, D., Teugels, G.G. \& Verraes, W. (2003) Taxonomic evaluation and redescription of Anaspidoglanis akiri (Risch, 1987) (Siluriformes: Claroteidae). Cybium (3e série), 27, 17-25.

Geerinckx, T., Adriaens, D., Teugels, G.G. \& Verraes, W. (2004) A systematic revision of the African catfish genus Parauchenoglanis Boulenger, 1911 (Siluriformes: Claroteidae). Journal of Natural History, 38, $775-803$.

Geisler, R. (1969) Corydoras baderi, ein neuer Panzerwels, und sein Lebensraum im Grenzgefiet Brasilien-Surinam (Pisces, Teleostei, Callichthyidae). Senckenbergiana Biologica, 50, 353-357.

Geoffroy Saint-Hilaire, E. (1809) Poissons du Nil, de la mer Rouge et de la Méditerranée. In: Savigny, J. (Ed.), Description de l'Egypte... . Histoire Naturelle, vol. 1, part 1. Paris, pp. 1-52, poissons pls. 1-17.

Geoffroy Saint-Hilaire, I. (1827) Suite de l'histoire des poissons du Nil. In: Savigny, J. (Ed.), Description de l'Egypte... . Histoire Naturelle, vol. 1, part 1. Paris, pp. 265-310.

Ghazzi, M.S. (2005) Sturisoma kneri, new species, a name for an old yet poorly-known catfish (Siluriformes: Loricariidae). Copeia, 2005, 559-565.

Gianferrari, L. (1923) Uegitglanis zammaronoi un nuovo siluride cieco africano. Atti della Società Italiana di Scienze Naturali, Milano, 62, 1-3, pl. 1.

Gianferrari, L. (1932) Pesci raccolti in Africa dalla spedizione Baragiola-Durini. Atti della Società Italiana di Scienze Naturali, Milano, 71, 138-144.

Giebel, C.G.A. (1871) Trachypoma marmoratum, ein neuer Wels aus dem Amazonenstrome. Zeitschrift für Gesammten Naturwissenschaften, Berlin, 37 [= n.f., 3], 97. 
Gilbert, C.H. (1884) Notes on the fishes from Kansas. Bulletin of the Washburn Laboratory of Natutral History, 1, 1016.

Gilbert, C.H. (1885) Description of three new fishes from Kansas. Proceedings of the United States National Museum, 7 , 512-514.

Gilbert, C.H. (1891) Report of explorations made in Alabama during 1889, with notes on the fishes of the Tennessee, Alabama, and Escambia rivers. Bulletin of the U. S. Fish Commission, 9, for 1889, 143-159, pl. 43.

Gilbert, C.H. (1897) Descriptions of twenty-two new species of fishes collected by the steamer Albatross, of the United States Fish Commission. Proceedings of the United States National Museum, 19, 437-457, pls. 49-55.

Gilbert, C.H. (1904) Notes on fishes from the Pacific coast of North America. Proceedings of the California Academy of Sciences, Series 3, 3, 255-271, pls. 25-29.

Gilbert, C.H. \& Starks, E.C. (1904) The fishes of Panama Bay. Memoirs of the California Academy of Sciences, 4, 1304, pls.

Gilbert, C.R. (1998) Type catalog of Recent and fossil North American Freshwater fishes: families Cyprinidae, Catostomidae, Ictaluridae, Centrarchidae and Elassomatidae. Florida Museum of Natural History, Special Publication, 1, ii $+284 \mathrm{p}$.

Gilchrist, J.D.F. \& Thompson, W.W. (1913) The freshwater fishes of South Africa. Annals of the South African Museum, 11, 321-463.

Gilchrist, J.D.F. \& Thompson, W.W. (1916) Description of four new S. African fishes. Mar. Biol. Rep. So. Afr, 19141918, 56-61.

Gilchrist, J.D.F. \& Thompson, W.W. (1917) The freshwater fishes of South Africa (Continued). Annals of the South African Museum, 11, 465-575, pl. 3.

Gill, T.N. (1858) Synopsis of the fresh water fishes of the western portion of the island of Trinidad, W. I. Annals of the Lyceum of Natural History, New York, 6, 363-430.

Gill, T.N. (1859a) Description of a new genus of Pimelodinae from Canada. Annals of the Lyceum of Natural History, New York, 7, 39-42.

Gill, T.N. (1859b) Description of new South American type of siluroids, allied to Callophysus. Proceedings of the Academy of Natural Sciences, Philadelphia, 11, 196-197.

Gill, T.N. (1861a) Catalogue of the fishes of the eastern coast of North America, from Greenland to Georgia. Proceedings of the Academy of Natural Sciences, Philadelphia, 13, Supplement, 1-63.

Gill, T.N. (1861b) Descriptions of new species of Pimelodinae (abridged from the forthcoming report of Captain J. H. Simpson). Proceedings of the Boston Society of Natural History, 8, for 1861-1862, 42-46.

Gill, T.N. (1861c) Synopsis of the genera of the sub-family of Pimelodinae. Proceedings of the Boston Society of Natural History, 8, for 1861-1862, 46-55.

Gill, T.N. (1862) On the West African genus Hemichromis and descriptions of new species in the museums of the Academy and Smithsonian Institution. Proceedings of the Academy of Natural Sciences, Philadelphia, 14, 134-139.

Gill, T.N. (1863) Descriptive enumeration of a collection of fishes from the western coast of Central America, presented to the Smithsonian Institution by Captain John M. Dow. Proceedings of the Academy of Natural Sciences, Philadelphia, 15, 162-174.

Gill, T.N. (1870) On some new species of fishes obtained by Prof. Orton from the Maranon, or Upper Amazon, and Napo Rivers. Proceedings of the Academy of Natural Sciences, Philadelphia, 22, 92-96.

Gill, T.N. (1872) Arrangement of the families of fishes, or classes Pisces, Marsipobranchii, and Leptocardii. Smithsonian Miscellaneous Collection, 247, 1-49.

Gill, T.N. (1877) Notes on fishes from the Isthmus of Panama, collected by Dr. J. F. Bransford, U. S. N. Proceedings of the Academy of Natural Sciences, Philadelphia, 28, for 1876, 335-339.

Gill, T.N. (1888) Glyptocephalus not identical with Bucklandium. American Naturalist, 22, 925-926.

Gill, T.N. (1891a) Note on the Aspredinidae. Proceedings of the United States National Museum, 13, 347-352.

Gill, T.N. (1891b) Note on the genus Felichthys of Swainson. Proceedings of the United States National Museum, 13, 353-354.

Gill, T.N. (1893) Families and subfamilies of fishes. Memoirs of the National Academy of Sciences, Washington, 6, 125138.

Giltay, L. (1930a) Notes ichthyologiques, IV.- Description d'une espèce nouvelle de Bagridae (Auchenoglanis Wittei, nov. sp.). Revue de Zoologie et de Botanique Africaines, 19, 91-94.

Giltay, L. (1930b) Notes ichthyologiques, V.- Description d'une sous-espèce nouvelle de Ansorgia vitatta Blgr., provenant du Bas-Uélé. VI._- Ichthyoborus besse congolensis, nov. subsp., de Nyonga (Congo Belge). Revue de Zoologie 
et de Botanique Africaines, 19, 393-396.

Giltay, L. (1935) Notes ichthyologiques, X.- Description d'une espèce nouvelle de Trichomycteridae. Bulletin du Musée Royal d'Histoire Naturelle de Belgique, 11, 1-3.

Giltay, L. (1936) Notes Ichthyologiques, XI.- Revision du genre Hemipsilichthys (Loricariidae). Bulletin du Musée Royal d'Histoire Naturelle de Belgique, 12, 1-7.

Girard, C.F. (1855) Contributions to the Fauna of Chile. Report to Lieut. James M. Gilliss, U. S. N., Upon the Fishes Collected by the U. S. Naval Astronomical Expedition to the Southern Hemisphere During the Years 1849-50-51-52, Washington.

Girard, C.F. (1858a) Notes upon various new genera and new species of fishes, in the museum of the Smithsonian Institution, and collected in connection with the United States and Mexican boundary survey: Major William Emory, Commissioner. Proceedings of the Academy of Natural Sciences, Philadelphia, 10, 167-171.

Girard, C.F. (1858b) Fishes. In: General Report upon Zoology of the Several Pacific Railroad Routes, 1857, U. S. Senate, Miscellaneous Document, no. 78 (33rd. Congress, 2nd. Session), pp. i-xiv + 1-400, 21 pls.

Girard, C.F. (1859a) Ichthyology of the boundary. In: United States and Mexican Boundary Survey, Under the Order of Lieut. Col. W. H. Emory, Major First Cavalry and United States Commissioner. Vol. 2.-Zoology of the Boundary, Department of the Interior, Washington, pp. 1-85, fishes pls. 1-41.

Girard, C.F. (1859b) Ichthyological notices. Proceedings of the Academy of Natural Sciences, Philadelphia, 11, 157161.

Gistel, J. (1848) Naturgeschichte des Thierreichs, für Höhere Schulen. Stuttgart, xvi + 216 p., 32 pl.

Glaw, F. \& Vences, M. (1994) A Field Guide to the Amphibians and Reptiles of Madagascar. Second Edition, Including Mammals and Freshwater Fish, Privately published, Köln, Germany, 480 pp.

Glodek, G.S. (1976) Rhynchodoras woodsi, a new catfish from eastern Ecuador (Siluriformes: Doradidae) with a redefinition of Rhynchodoras. Copeia, 1976, 43-46.

Glodek, G.S. \& Carter, H.J. (1978) A new helogeneid catfish from eastern Ecuador (Pisces, Siluriformes, Helogeneidae). Fieldiana, Zoology, 72, 75-82.

Glodek, G.S., Whitmire, G.L. \& Orcés, G. (1976) Rhinodoras boehlkei, a new catfish from eastern Ecuador (Osteichthyes, Siluroidei, Doradidae). Fieldiana, Zoology, 70, 1-11.

Gmelin, J.F. (1788-1793) Caroli a Linné... Systema Naturae per Regna Tria Naturae, Secundum Classes, Ordines, Genera, Species; cum Characteribus, Differentiis, Synonymis, Locis. Editio Decimo Tertia, Aucta, Reformata, Lipsiae [Fishes in vol. 1, pt 3 (dated [1789]), pp 1033-1516.]

Godoy, M.P. (1969) Nova espécie de "Plecostomus" Gronovius, 1763 (Pisces, Loricariidae, Nematognathi. Revista Brasileira de Biologia, 29, 175-180.

Goeldi, E.A. (1898) Primeira contribuição para o conhecimento dos peixes do valle do Amazonas e das Guyanas. Estudos ichthyologicos dos annos 1894-1898. Boletim do Museu Paraense de Historia Natural e Ethnographia, Pará, 2, 443-488, 1 pl.

Goeldi, E.A. (1905) Nova zoologica aus der Amazonas-region. Neue Wirbeltiere. In: Bedot, M. (Ed.), Compte Rendu des Séances du Sixième Congrès International de Zoologie. Imprimerie W. Kündig \& Fils, Genève, pp. 542-549.

Golani, D. (2002) The Indo-Pacific striped eel catfish, Plotosus lineatus (Thunberg, 1787), (Osteichthyes: Siluriformes) a new record from the Mediterranean. Scientia Marina, 66, 321-323.

Gomes, A.L. (1946) A review of Microglanis, a genus of South American catfishes, with notes on related genera. Occasional Papers of the Museum of Zoology, University of Michigan, 494, 1-19, pl. 1.

Gomes, A.L. (1955) Descrição de Pseudotocinclus ribeiroi, nova espécie de cascudinho (Loricariidae, Hypoptopomatinae) da bacia do rio Itanhaem, São Paulo. Arquivos do Museu Nacional do Rio de Janeiro, 42, 221-227, pls. 1-2.

Gomes, A.L. (1956) Descrição de uma nova espécie de "Luciopimelodinae" do Rio Mogi Guaçu, Estado de São Paulo, (Pisces, Nematognathi, Pimelodidae). Revista Brasileira de Biologia, 16, 403-413.

Gomes, A.L. \& Schubart, O. (1958) Descrição de "Chasmocranus brachynema" sp. n., novo "Luciopimelodinae" da Bacia do Rio Mogi Guaçu, estado de São Paulo (Pisces, Nematognathi, Pimelodidae). Revista Brasileira de Biologia, 18, 413-416.

Gomez, S.E., Lopez, H.L. \& Toresani, N.I. (1990) Hypostomus derbyi (Haseman) e Hypostomus myersi (Gosline), descripcion complementaria y primeros registros para Argentina (Pisces, Loricariidae). Studies on Neotropical Fauna and Environment, 25, 139-152.

Gomon, J.R. \& Taylor, W.R. (1982) Plotosus nkunga, a new species of catfish from South Africa, with a redescription of Plotosus limbatus Valenciennes and key to the species of Plotosus (Siluriformes: Plotosidae). J. L. B. Smith Institute of Ichthyology, Special Publication, 22, 1-16. 
Gosline, W.A. (1940a) Rediscovery and redescription of Pariolius armillatus, a genus and species of pimelodid catfishes described by E. D. Cope from the Peruvian Amazon in 1872. Copeia, 1940, 78-80.

Gosline, W.A. (1940b) A revision of the neotropical catfishes of the family Callichthyidae. Stanford Ichthyological Bulletin, 2, 1-29.

Gosline, W.A. (1941) Synopsis of the genera of pimelodid catfishes without a free orbital rim. Stanford Ichthyological Bulletin, 2, 83-88.

Gosline, W.A. (1942) Notes on South American catfishes (Nematognathi). Copeia, 1942, 39-41.

Gosline, W.A. (1945) Catálogo dos nematognatos de água-doce da América do Sul e Central. Boletim do Museu Nacional, Nova série, Zoologia, 33, 1-138.

Gosline, W.A. (1947) Contributions to the classification of the loricariid catfishes. Arquivos do Museu Nacional do Rio de Janeiro, 41, 79-134, pls. 1-9.

Gosse, J.-P. (1982) Mutanda ichthyologica: Synodontis polli nom. nov. et Synodontis ornatissimus nom. nov. Cybium (3e série), $6,48$.

Gosse, J.-P. (1986a) Malapteruridae. In: Daget, J., Gosse, J.-P. \& Thys van den Audenaerde, D.F.E. (Eds.), Check-list of the Freshwater Fishes of Africa, Vol. 2, ISNB Bruxelles, MRAC Tervuren, ORSTOM, Paris, pp. $102-104$.

Gosse, J.-P. (1986b) Mochokidae. In: Daget, J., Gosse, J.-P. \& Thys van den Audenaerde, D.F.E. (Eds.), Check-list of the Freshwater Fishes of Africa, Vol. 2, ISNB Bruxelles, MRAC Tervuren, ORSTOM, Paris, pp. 105-152.

Grady, J.M. (1987) Biochemical Systematics and Evolution of the Ictalurid Catfish genus Noturus (Pisces, Siluriformes), Unpublished Ph.D. Dissertation, Southern Illinois University at Carbondale, Carbondale, IL.

Grady, J.M. \& LeGrande, W.H. (1992) Phylogenetic relationships, modes of speciation, and historical biogeography of the madtom catfishes, genus Noturus Rafinesque (Siluriformes: Ictaluridae). In: Mayden, R.L. (Ed.), Systematics, Historical Ecology, \& North American Freshwater Fishes, Stanford University Press, Stanford, California, pp. 747777.

Grande, L. (1987) Redescription of $\dagger$ Hypsidoris farsonensis (Teleostei: Siluriformes), with a reassessment of its phylogenetic relationships. Journal of Vertebrate Paleontology, 7, 24-54.

Grande, L. \& Lundberg, J.G. (1988) Revision and redescription of the genus $\uparrow$ Astephus (Siluriformes: Ictaluridae) with a discussion of its phylogenetic relationships. Journal of Vertebrate Paleontology, 8, 139-171.

Grande, L. \& Pinna, M., de. (1998) Description of a second species of the catfish $\dagger$ Hypsidoris and a reevaluation of the genus and the family $\dagger$ Hyspidoridae. Journal of Vertebrate Paleontology, 18, 451-474.

Grant, S. (1997) Descriptions of two new species of Corydoras, Lacepede, 1803 (Pisces, Siluriformes, Callichthyidae). Aquarist and Pondkeeper, 62, 41-45.

Grant, S. (1999) A replacement name (nomen novum) and neotype designation for Hara malabarica Day, 1865, with notes on related species (Siluriformes). Aqua, Journal of Ichthyology and Aquatic Biology, 3, 169-174.

Gras, R. (1961) Contribution à l'étude des poissons du Bas-Dahomey. Description de quatre espèces nouvelles. Bulletin du Muséum National d'Histoire Naturelle (2 Série), 32, for 1960, 401-410.

Gratzianov, V.J. (1907) [A synoptic essay of the fishes of the Russian Empire]. Trudy Otdela Ichtiol. Russ. Obsc. Akklimat. Zhiv. Moskva, 4, i-xxx + 1-567. [In Russian.]

Gray, J.E. (1830-1835) Illustrations of Indian Zoology; Chiefly Selected from the Collection of Major-General Hardwicke, Treuttel, Jun. and Richter, Paris; Grande Rue, Strasburg; Parbury Allen and Co., London. 20 parts in 2 vols., 202 pls. [Catfish illustrated in Vol. 1, plates 84 and 85, and dated 1830, following Sawyer (1953). See Eschmeyer (1998: 2627) for more information.]

Gray, J.E. (1831) Description of twelve new genera of fish, discovered by Gen. Hardwicke, in India, the greater part in the British Museum. Zoological Miscellany, 1831, 7-9.

Gray, J.E. (1854) Catalogue of Fish Collected and Described by Laurence Theodore Gronow, now in the British Museum, British Museum, London, vii + 196 p.

Greenfield, D.W. \& Glodek, G.S. (1977) Trachelyichthys exilis, a new species of catfish (Pisces: Auchenipteridae) from Peru. Fieldiana, Zoology, 72, 47-58.

Greenfield, D.W., Greenfield, T.A. \& Woods, R.L. (1982) A new subspecies of cave-dwelling pimelodid catfish, Rhamdia laticauda typhla from Belize, Central America. Brenesia, 19/20, 563-576.

Greenfield, D.W. \& Thomerson, J.E. (1997) Fishes of the Continental Waters of Belize, University of Florida Press, Gainesville, xxii +311 p.

Greenwood, P.H. (1956) A new species of Clariallabes (Pisces, Clariidae), from the Nile. Proceedings of the Zoological Society of London, 127, 555-564.

Greenwood, P.H. (1958) A new genus and species of cat-fish (Pisces, Clariidae) from the deeper waters of Lake Victoria. 
Annals and Magazine of Natural History (Ser. 13), 1, 321-325.

Greenwood, P.H. (1961) A revision of the genus Dinotopterus Blgr. (Pisces, Clariidae) with notes on the comparative anatomy of the suprabranchial organs in the Clariidae. Bulletin of the British Museum (Natural History), Zoology, 7 , 215-241.

Greenwood, P.H. (1963) A collection of fishes from the Aswa river drainage system, Uganda. Proceedings of the Zoological Society of London, 140, 61-74.

Gromov, I.A. (1970) [A new species of bagrid catfish (Mystus mica Gromov, sp. n. (Pisces, Bagridae)) in the Amur Basin]. Voprosy Ikhtiologii, 10, 400-405. [In Russian; translated into English in Journal of Ichthyology, 10 (3), $277-$ 281.]

Gronovius, L.T. (1754) Museum Ichthyologicum, Sistens Piscium Indigenorum \& Nonnullorum Exoticorum, quorum Maximum pars in Museo Laurenti Theodori Gronovii, J. U. D. Adservantar, Descriptiones, Ordine Systematico; Accedunt Nonnullorum Exoticorum Piscium Icones, Aeri Incisae, Theodorum Haak, Lugduni-Batavorum, 70 p. 4 pls.

Gronovius, L.T. (1756) Museum Ichthyologicum, Tomus Secundus, Sistens Piscium Indigenorum \& Nonnullorum Exoticorum, quorum Maximum pars in Museo Laurenti Theodori Gronovii, J. U. D. Adservantar, Descriptiones, Ordine Systematico; Accedunt Nonnullorum Exoticorum Piscium Icones, Aeri Incisae, Theodorum Haak, Lugduni-Batavorum, 88 p., pls. 5-7.

Gronovius, L.T. (1763) Zoophylacii Gronoviani, Fasciculus Primus, Exhibens Animalia Quadrupeda, Amphibia atque Pisces, quae in Museo suo Adservat, Rite Examinavit, Systematice Disposuit, Descripsit atque Iconibus Illustravit Laur. Theod. Gronovius, J.U.D. Theodorum Haak et Socium, et Samuelem et Johannem Luchtmans, Lugduni Batavorum, 136 p., 14 pls.

Guazzelli, G.M. (1997) Revisão das Espécies de Pimelodella Eigenmann \& Eigenmann, 1888 (Teleostei: Siluriformes: Pimelodidae) dos Sistemas Costeiros do Sul e Sudeste Brasileiro, Unpublished Master Dissertation, Pontifíca Universidade Católica do Rio Grande do Sul, Porto Alegre, 150 p.

Guichenot, A. (1848) Fauna Chilena. Peces. In: Gay, C. (Ed.), Historia Física y Politica de Chile, Zoología. Vol. 2, En casa del Autor, Paris; Museo de Historia Natural de Santiago, Chile, pp. 137-370.

Guichenot, A. (1860) Notice sur un nouveau poisson du genre des Trichomyctères. Revue et Magasin de Zoologie (Ser. 2), 12, 525-527.

Guimarães, A.R.P. (1884a) Description d'un nouveau poisson de l'intérieur d'Angola. Jornal do Sciências Mathemáticas, Physicas e Naturaes, Lisboa, 9, for 1882, 85-87, 1 pl. [apparently not published until 1884.]

Guimarães, A.R.P. (1884b) Diagnoses de trois nouveaux poissons d'Angola. Jornal do Sciências Mathemáticas, Physicas e Naturaes, Lisboa, 10, 1-10, pls. 1-2.

Guimarães, J.R.A. (1935) Contribuição para o conhecimento de uma nova especie de peixe hematophago, ectoparasita de "Characidae," encontrado em São Paulo (Rio Tietê). Vandellia hematophaga sp. n. Revistade Industria Animal, 2 , 300-304.

Güntert, H. (1938) Beschreibung einiger unbestimmter Clariiden mit drei neuen Arten aus dem Naturhistorischen Museum in Wien. Zoologischer Anzeiger, 124, 289-303.

Güntert, H. (1939) Ein neuer Clariide aus Kamerun. Anzeiger der Mathematisch- Naturwissenschaftlichen Classe der Kaiserlichen Akademie der Wissenschaften in Wien, 75, 25-26.

Güntert, H. (1942) Beschreibung einiger zum Teil noch unbekannter südamerikanischer Siluriden aus dem Naturhistorischen Museum in Basel. Zoologischer Anzeiger, 138, 27-40.

Günther, A. (1859) List of the cold-blooded vertebrata collected by Mr. Fraser in the Andes of western Ecuador. Proceedings of the Zoological Society of London, 27, 89-93.

Günther, A. (1860) Third list of cold-blooded Vertebrata collected by Mr. Fraser in Ecuador. Proceedings of the Zoological Society of London, 28, 233-240, pl. 10.

Günther, A. (1863) On new species of fishes from the Essequibo. Annals and Magazine of Natural History (Ser. 3), 12, 441-443.

Günther, A. (1864) Catalogue of the Fishes in the British Museum, vol. 5.-Catalogue of the Physostomi, Containing the Families Siluridae, Characinidae, Haplochitonidae, Sternoptychidae, Scopelidae, Stomiatidae in the Collection of the British Museum, Trustees, London, xxii +455 p.

Günther, A. (1865a) A contribution to the ichthyology of West Africa. Annals and Magazine of Natural History (Ser. 3), $15,452-453$.

Günther, A. (1865b) Pisces. Zoological Record, for 1864, 133-188.

Günther, A. (1866) Description of a new siluroid fish from Ceylon. Annals and Magazine of Natural History (Ser. 3), 18, 
473-474, pl. 15.

Günther, A. (1867a) Additions to the knowledge of Australian reptiles and fishes. Annals and Magazine of Natural History (Ser. 3), 20, 45-68.

Günther, A. (1867b) New fishes from the Gaboon and Gold Coast. Annals and Magazine of Natural History (Ser. 3), 20, 110-117, pls. 2-3.

Günther, A. (1867c) On the fishes of the states of Central America, founded upon specimens collected in fresh and marine waters of various parts of that country by Messrs. Salvin and Godman and Capt. J. M. Dow. Proceedings of the Zoological Society of London, 1866, 600-604.

Günther, A. (1867d) Description of some new or little-known species of fishes in the collection of the British Museum. Proceedings of the Zoological Society of London, 1867, 99-104, pl. 10.

Günther, A. (1868a) Diagnoses of some new freshwater fishes from Surinam and Brazil, in the collection of the British Museum. Annals and Magazine of Natural History (Ser. 4), 1, 475-481.

Günther, A. (1868b) Descriptions of freshwater fishes from Surinam and Brazil. Proceedings of the Zoological Society of London, 1868, 229-247, pls. 20-22.

Günther, A. (1868c) An account of the fishes of the states of Central America, based on collections made by Capt. J. M. Dow, F. Godman, Esq. and O. Salvin, Esq. Transactions of the Zoological Society of London, 6, 377-494, pls. 6387.

Günther, A. (1869) Descriptions of some species of fishes from the Peruvian Amazons. Proceedings of the Zoological Society of London, 1869, 423-429.

Günther, A. (1872) Notice of a large siluroid from the upper Amazons. Annals and Magazine of Natural History (Ser. 4), 10, 449-450.

Günther, A. (1873a) New fishes from Angola. Annals and Magazine of Natural History (Ser. 4), 12, $142-144$.

Günther, A. (1873b) Report on a collection of fishes from China. Annals and Magazine of Natural History (Ser. 4), 12, 239-250.

Günther, A. (1874) A contribution to the fauna of the river Tigris. Annals and Magazine of Natural History (Ser. 4), 14, 36-38, pls. 8-9.

Günther, A. (1876) Contributions to our knowledge of the fish-fauna of the Tertiary deposits of the highlands of Padang, Sumatra. The Geological Magazine, Series 2, 3, 443-440. [Also in 1878: Jaarb. Mijnw. Ned. Oost-Indië, 7: 171184.]

Günther, A. (1880a) A contribution to the knowledge of the fish fauna of the Rio de la Plata. Annals and Magazine of Natural History (Ser. 5), 6, 7-13, pl. 2.

Günther, A. (1880b) Report on the shore fishes procured during the voyage of H. M. S. Challenger in the years $1873-$ 1876. Report on the Scientific Results of the Voyage of H. M. S. Challenger During the Years 1873-76. Zoology Vol. 1 , pt. 6, pp. 1-82, pls. 1-32.

Günther, A. (1883) Notes on some Indian fishes in the collection of the British Museum. Annals and Magazine of Natural History (Ser. 5), 11, 137-140.

Günther, A. (1889) On some fishes from Kilima-Njaro District. Proceedings of the Zoological Society of London, 1889 , 70-72, pl. 8 .

Günther, A. (1892) List of the species of reptiles and fishes collected by Mr. A. E. Pratt on the upper Yang-tze-kiang and in the province Sze-Chuen, with description of the new species. Appendix II. In: Pratt, A.E. (Ed.), To the Snows of Tibet Through China, Longmans, Green and Co., London and New York, pp. 238-250, pls. 1-4.

Günther, A. (1894) Second report on the reptiles, batrachians, and fishes transmitted by Mr. H. H. Johnston, C. B., from British Central Africa. Proceedings of the Zoological Society of London, 1893, 616-628, pls. 53-57.

Günther, A. (1896a) Report on a collection of reptiles and fishes made by Miss. M. H. Kingsley during her travels on the Ogowe River and in Old Calabar. Annals and Magazine of Natural History (Ser. 6), 17, 261-285, pls. 13-15.

Günther, A. (1896b) Report on a collection of fishes made by Dr. A. Donaldson Smith during his expedition to Lake Rudolf. Proceedings of the Zoological Society of London, 1896, 217-224, pl. 9.

Günther, A. (1899) An account of a collection of fishes made by Mr. R. B. N. Walker, C. M. Z. S., on the Gold Coast. Proceedings of the Zoological Society of London, 1899, 716-729, pls. 41-45.

Günther, A. (1903) Last account of fishes collected by Mr. R. B. N. Walker, C. M. Z. S., on the Gold Coast. Proceedings of the Zoological Society of London, 1902, pt 2, 330-339, pls. 30-33.

Guo, X.-G., He, S.-P. \& Zhang, Y.-G. (2005) Phylogeny and biogeography of Chinese sisorid catfishes re-examined using mitochondrial cytochrome b and 16S rRNA gene sequences. Molecular Phylogenetics and Evolution, 35, 344-362.

Gupta, S.K. (1982) Redescription and distribution of a sisorid catfish Laguvia ribeiroi kapuri Tilak and Hussain, from 
Banda district (UP). Indian Journal of Physical and Natural Sciences, Section A, 2, 36-39.

Gupta, S.K., Jayaram, K.C. \& Hajela, K.P. (1981) On a new silurid cat-fish from Uttar Pradesh, India. Journal of the Bombay Natural History Society, 77, 290-291.

Gustiano, R., Teugels, G.G. \& Pouyaud, L. (2003) Revision of the Pangasius kunyit catfish complex, with description of two new species from South-east Asia (Siluriformes: Pangasiidae). Journal of Natural History, 37, 357-376.

Gustiano, R., Teugels, G.G. \& Pouyaud, L. (2004) Pangasius bedado Roberts, 1999: a junior synonym of Pangasius djambal Bleeker, 1846 (Siluriformes, Pangasiidae). Cybium (3e série), 28, 13-18.

Haig, J. (1952) Studies on the classification of the catfishes of the Oriental and Palaearctic family Siluridae. Records of the Indian Museum, 48, for 1950, 59-116.

Hamilton, F. (1822) An Account of the Fishes Found in the River Ganges and its Branches, Edinburgh \& London, vii + 405 p., $39 \mathrm{pl}$.

Hancock, J. (1828) Notes on some species of fishes and reptiles, from Demerara, presented to the Zoological Society by John Hancock, Esq., corr. memb. Zool. Soc. In a letter addressed to the secretary of the Society. Zoological Journal, London, 4, 240-247.

Hardenberg, J.D.F. (1936a) On a collection of fishes from the estuary and the lower and middle course of the River Kapuas (W. Borneo). Treubia, 15, 225-254.

Hardenberg, J.D.F. (1936b) Some new or rare fishes of the Indo-Australian Archipelago. — V. Treubia, 15, $367-378$.

Hardenberg, J.D.F. (1938) Some new or rare fishes of the Indo-Australian Archipelago. - VI. Treubia, 16, 311-320.

Hardenberg, J.D.F. (1941) Fishes of New Guinea. Treubia, 18, 217-231.

Hardenberg, J.D.F. (1948) Some new or rare fishes of the Indo-Australian Archipelago. - 8. Treubia, 19, 407-415.

Hardman, M. (2002) Phylogenetic Relationships Among Species of Ictaluridae (Otophysi: Siluriformes) and of the Family to Other Catfishes, Unpublished Ph.D. dissertation, University of Illinois, Urbana-Champaign.

Hardman, M. (2004) The phylogenetic relationships among Noturus catfishes (Siluriformes: Ictaluridae) as inferred from mitochondrial gene cytochrome $b$ and nuclear recombination activating gene 2. Molecular Phylogenetics and Evolution, 30, 395-408.

Hardman, M. (2005) The phylogenetic relationships among non-diplomystid catfishes as inferred from mitochondrial cytochrome b sequences; the search for the ictalurid sister taxon (Otophysi: Siluriformes). Molecular Phylogenetics and Evolution, 37, 700-720.

Hardman, M. \& Page, L.M. (2003) Phylogenetic relationships among bullhead catfishes of the genus Ameiurus (Siluriformes: Ictaluridae). Copeia, 2003, 20-33.

Hardy, M.E., Grady, J.M. \& Routman, E.J. (2002) Intraspecific phylogeography of the slender madtom: The complex evolutionary history of the Central Highlands of the United States. Molecular Ecology, 11, 2393-2403.

Harka, A. \& Pinter, K. (1990) Systematic status of Hungarian bullhead pout: Ictalurus nebulosus pannonicus ssp. n. Tiscia (Szeged), 25, 65-73.

Harry, R.R. (1947) Platysomatos, a neglected name for a genus of aspredinid catfishes. Copeia, 1947, 194.

Harry, R.R. (1953) A contribution to the classification of the African catfishes of the family Amphiliidae, with description of collections from Cameroon. Revue de Zoologie et de Botanique Africaines, 47, 177-200; 201-232.

Hase, A. (1914) Die Fische der Deutschen Grenzexpedition 1910 in das Kaiser-Wilhelms-Land, Neu Guinea. Jenaische Zeitschrift fur Naturwissenschaft, 51, 525-548.

Haseman, J.D. (1911a) Descriptions of some new species of fishes and miscellaneous notes on others obtained during the expedition of the Carnegie Museum to central South America. Annals of the Carnegie Museum, 7, 315-328, pls. 4652.

Haseman, J.D. (1911b) Some new species of fishes from the Rio Iguassú. Annals of the Carnegie Museum, 7, 374-387, pls. 50, 58, 73-83.

Hay, O.P. (1924) Description of some fossil vertebrates from the Upper Miocene of Texas. Proceedings of the Biological Society of Washington, 37, 1-19, pls. i-vi.

He, M.-J. \& Chen, Y.-R. (1981) [Two new catfishes of the genus Akysis from China]. Zoological Research, 2, $209-214$. [In Chinese]

He, S.-P. (1995) The analysis of historical biogeography for the glyptosternoid fishes (Teleostei: Siluriformes: Sisoridae). Biogeographica, 71, 145-160.

He, S.-P. (1996a) [A new species of the genus Gagata (Pisces: Sisoridae)]. Acta Zootaxonomica Sinica, 21, 380-382. [In Chinese and English]

He, S.-P. (1996b) The phylogeny of the glyptosternoid fishes (Teleostei: Siluriformes, Sisoridae). Cybium (3e série), 20 , $115-159$. 
He, S.-P., Gayet, M. \& Meunier, F.J. (1999) Phylogeny of the Amphiliidae (Teleostei: Siluriformes). Annales des sciences naturelles. Zoologie et biologie animale (Sér. 13), 20, 117-146.

He, S.-P. \& Meunier, F. (1998) The biogeography of Amphiliidae and Sisoridae (Teleostei: Siluriformes). Acta Zootaxonomica Sinica, 23 (Supplement), 1-9.

Heckel, J.J. (1838) Fische aus Caschmir Gesammelt und Herausgegeben von Carl Freiherrn v. Hügel, Beschrieben von Joh. Jacob Heckel, Gedrunckt bei den P. P. Mechitaristen, Wien, 112 p., 13 pls.

Heckel, J.J. (1843) Ichthyologie [von Syrien]. In: J. von Russegger, 1841-1849, Reisen in Europa, Asien und Afrika, mit besonderer Rücksicht auf die naturwissenschaftlichen Verhältnisse der betreffenden Länder, unternommen in den Jahren 1835 bis 1841, Vol. 1, part 2, E. Schweizerbart, Stuttgart, pp. 990-1099.

Heckel, J.J. (1849) Über eine neue fossile Fishgattung, Chirocentrites, und die ersten Ueberreste eines Siluroiden aus der Vorwelt. Sitzungsberichte der Kaiserlichen Akademie der Wissenschaften, Mathematisch- Naturwissenschaftlichen Classe, Wien, 2, 16-19.

Heckel, J.J. (1850) Beiträge zur Kenntniss der fossilen Fische Oesterreichs. Denkschriften der Mathematisch- Naturwissenschaftlichen Classe der Kaiserlichen Akademie der Wissenschaften in Wien, 1, 201-242, 13 pls.

Heilprin, A. (1887) Explorations on the west coast of Florida and in the Okeechobee wilderness, with special reference to the geology and zoology of the Floridian peninsula. A narrative of researches undertaken under the auspices of the Wagner Free Institute of Science. Transactions of the Wagner Free Institute of Science of Philadelphia, 1, 1-134, pls. 1-19.

Heitmans, W.R.B., Nijssen, H. \& Isbrücker, I.J.H. (1983) The mailed catfish genus Lasiancistrus Regan, 1904, from French Guiana and Surinam, with descriptions of two new species (Pisces, Siluriformes, Loricariidae). Bijdragen tot de Dierkunde, 53, 33-48.

Hemming, F. (Ed.) (1953) Copenhagen Decisions on Zoological Nomenclature: Additions to, and Modifications of, the Règles Internationales de la Nomenclature Zoologique. Approved and Adopted by the Fourteenth International Congress of Zoology, Copenhagen, August, 1953, International Trust for Zoological Nomenclature, London.

Henn, A.W. (1928) List of types of fishes in the collection of the Carnegie Museum on September 1, 1928. Annals of the Carnegie Museum, 19, 51-99.

Hensel, R.F. (1868) Beiträge zur Kenntniss der Wirbelthiere Südbrasiliens. Archiv für Naturgeschichte, 34, 323-375.

Hensel, R.F. (1870) Beiträge zur Kenntniss der Wirbelthiere Südbrasiliens (Fortsetzung). Archiv für Naturgeschichte, 36, $50-91$.

Hermann, J. (1804) Observationes Zoologicae, Quibus Novae Complures, Aliaeque Animalium Species Describuntur et Illustrantur. Opus Posthumum Edidit Fridericus Ludovicus Hammer, Argentorati, viii + 332 p. [Pisces on pp. 290328]

Herre, A.W.C.T. (1924a) Distribution of the true fresh-water fishes in the Philippines, II.- The Philippine Labyrinthici, Clariidae, and Siluridae. Philippine Journal of Science, 24, 683-709, pls. 1-2.

Herre, A.W.C.T. (1924b) The distribution of true fresh-water fishes in the Philippines and its significance. Proceedings of the Pan-Pacific Science Congress, 2 (Australia). pp. 1561-1570.

Herre, A.W.C.T. (1926) A summary of the Philippine catfishes, order Nematognathi. Philippine Journal of Science, 31, $385-411$, pl. 1.

Herre, A.W.C.T. (1932) Fishes from Kwangtung Province and Hainan Island, China. Lingnan Science Journal, 11, 423443.

Herre, A.W.C.T. (1933) Herklotsella anomala.— A new fresh water cat-fish from Hong Kong. Hong Kong Naturalist, 4, 179-180.

Herre, A.W.C.T. (1934) Notes on new or little known fishes from southeastern China. Lingnan Science Journal, 13, 285296.

Herre, A.W.C.T. (1935) New fishes obtained by the Crane Pacific expedition. Publication, Zoological Series, Field Museum of Natural History, 18, 383-438.

Herre, A.W.C.T. (1940) New species of fishes from the Malay Peninsula and Borneo. Bulletin of the Raffles Museum, 16, 5-26, pls. 1-20.

Herre, A.W.C.T. (1942) Glyptothorax housei, a new sisorid catfish from south India. Stanford Ichthyological Bulletin, 2, $117-119$.

Herre, A.W.C.T. \& Myers, G.S. (1937) A contribution to the ichthyology of the Malay Peninsula. Bulletin of the Raffles Museum, 13, 5-75, pls. 1-7.

Herzenstein, S.M. (1889a) Über einen russischen Wels (Exostoma Oschanini Herz.). Mélanges Biologiques, tirés du Bulletin physico-mathématique de l'Académie Impériale des Sciences de St. Pétersbourg, 13, 69-73. 
Herzenstein, S.M. (1889b) Über einen russischen Wels (Exostoma Oschanini Herz.). Bulletin de l'Académie Impériale des Sciences de St. Pétersbourg (n. s.), 1, 119-123.

Hieronimus, H. (1991) Corydoras similis spec. nov., ein neuer Panzerwels aus Brasilien (Pisces: Siluriformes: Callichthyidae). Zeitschrift für Fischkunde, 1, 39-46.

Hieronimus, H. (1995) Corydoras lacerdai spec. nov., ein neuer Panzerwels aus Brasilien (Teleostei: Siluriformes: Callichthyidae). Zeitschrift für Fischkunde, 3, 107-116.

Higuchi, H. (1992) A Phylogeny of the South American Thorny Catfishes (Osteichthyes; Siluriformes, Doradidae), Unpublished Ph.D. dissertation, Harvard University, Cambridge, Massachusetts.

Higuchi, H., Britski, H.A. \& Garavello, J.C. (1990) Kalyptodoras bahiensis, a new genus and species of thorny catfish from northeastern Brazil (Siluriformes: Doradidae). Ichthyological Exploration of Freshwaters, 1, 219-225.

Higuchi, H., Reis, E.G. \& Araújo, F.G. (1982) Uma nova espécie de bagre marinho do litoral do Rio Grande do sul e considerações sobre o género nominal Netuma Bleeker, 1858 no Atlántico sul ocidental (Siluriformes, Ariidae). Atlantica, Rio Grande, 5, 1-15.

Hildebrand, S.F. (1925) Fishes of the Republic of El Salvador, Central America. Bulletin of the Bureau of Fisheries, 41, 237-287.

Hildebrand, S.F. (1938) A new catalogue of the fresh-water fishes of Panama. Zoological Series, Field Museum of Natural History, 22, 217-259.

Hilgendorf, F.M. (1878) Einige neue japanische Fischgattungen. Sitzungsberichte der Gesellschaft Naturforschender Freunde zu Berlin, 1878, 404-457.

Hilgendorf, F.M. (1888) Fische aus dem Victoria-Nyanza (Ukerewe-See), gesammelt von dem verstorbenen Dr. G. A. Fischer. Sitzungsberichte der Gesellschaft Naturforschender Freunde zu Berlin, 1888, 75-79.

Hilgendorf, F.M. (1905) Fische von Deutsch und Englisch Ost-Africa. Zoologische Jahrbücher, Abteilung für Systematik, Geographie und Biologie der Tiere (Jena), 22, 405-420.

Hilgendorf, F.M. \& Pappenheim, P. (1903) Über die Fischfauna des Rukwa-Sees. Sitzungsberichte der Gesellschaft Naturforschender Freunde zu Berlin, 1903, 259-271.

Hoedeman, J.J. (1949) Onderorde Siluroidea "Meervallen". Encyclopaedie voor de aquariumhouder. pp. X.30.X.1-6.

Hoedeman, J.J. (1952a) Encyclopaedie voor de Aquariumhouder. De Regenboog, Amsterdam, 17 p.

Hoedeman, J.J. (1952b) Notes on the ichthyology of Surinam (Dutch Guiana). The catfish genera Hoplosternum and Callichthys, with key to the genera and groups of the family Callichthyidae. Beaufortia, 12, 1-11.

Hoedeman, J.J. (1961) Notes on the ichthyology of Surinam and other Guianas. 8.- Additional records of siluriform fishes (2). Bulletin of Aquatic Biology, 2, 129-139.

Hoedeman, J.J. (1965) Elseviers Pocketboek voor de Aquariumliefhebber, Elsevierpocket (A) 108, Elsevier, Amsterdam/ Brussels, $176 \mathrm{p}$.

Holbrook, J.E. (1855) An account of several species of fish observed in Florida, Georgia, etc. Journal of the Academy of Natural Sciences of Philadelphia (Ser. 2), 3, 47-58, pls. 5-6.

Hollanda Carvalho, P. \& Weber, C. (2004) Five new species of the Hypostomus cochliodon group (Siluriformes: Loricariidae) from the middle and lower Amazon System. Revue suisse de Zoologie, Annales de la Société zoologique suisse et du Muséum d'Histoire naturelle de Genève, 111, 953-978.

Holly, M. (1926) Zwei neue Siluriden und ein neuer Charcinide aus Kamerun. Anzeiger der Mathematisch- Naturwissenschaftlichen Classe der Kaiserlichen Akademie der Wissenschaften in Wien, 62, 157-159.

Holly, M. (1927a) Eine neue Siluriden-Subspezies aus Kamerun. Anzeiger der Mathematisch-Naturwissenschaftlichen Classe der Kaiserlichen Akademie der Wissenschaften in Wien, 64, 8-9.

Holly, M. (1927b) Siluriden, Cyprinodontiden, Acanthopterygien und Mastacembeliden aus Kamerun. Sitzungsberichte der Kaiserlichen Akademie der Wissenschaften, Mathematisch-Naturwissenschaftlichen Classe, Wien, Abt. 1, Botanik, Zoologie, Anatomie, Geologie und Paläontologie, 136, 195-230.

Holly, M. (1927c) Weitere Fische aus Kamerun. Sitzungsberichte der Kaiserlichen Akademie der Wissenschaften, Mathematisch- Naturwissenschaftlichen Classe, Wien, Abt. 1, Botanik, Zoologie, Anatomie, Geologie und Paläontologie, 136, 421-427.

Holly, M. (1929) Einige neue Fischformen aus Brasilien. Anzeiger der Mathematisch- Naturwissenschaftlichen Classe der Kaiserlichen Akademie der Wissenschaften in Wien, 66, 117-120.

Holly, M. (1930) Synopsis der Süsswasserfische Kameruns. Sitzungsberichte der Kaiserlichen Akademie der Wissenschaften, Mathematisch- Naturwissenschaftlichen Classe, Wien, Abt. 1, Botanik, Zoologie, Anatomie, Geologie und Paläontologie, 139, 195-281, pls. 1-2.

Holly, M. (1939) Zur Nomenklatur der Siluridengattung Macrones, C. Duméril. Zoologischer Anzeiger, $125,143$. 
Holly, M. (1940) Vier noch nicht beschriebene Corydoras Arten. Anzeiger der Mathematisch- Naturwissenschaftlichen Classe der Kaiserlichen Akademie der Wissenschaften in Wien, 77, 107-112.

Holmberg, E.L. (1893a) Nombres vulgares de peces Argentinos con sus equivalencias científicas. Revista del Jardin Zoológico de Buenos Ayres, 1, 85-96.

Holmberg, E.L. (1893b) Dos peces argentinos. Revista del Jardin Zoológico de Buenos Ayres, 1, $353-354$.

Holota, K. (1990) Pflege und Zucht von Ancistrus cf. hoplogenys. Die Aquarien- und Terrarien-Zeitschrift, 43, 147-148.

Hora, S.L. (1921a) Fish and fisheries of Manipur with some observations on those of the Naga Hills. Records of the Indian Museum, 22, 165-214, pls. 9-12.

Hora, S.L. (1921b) On some new or rare species of fish from the eastern Himalayas. Records of the Indian Museum, 22, 731-744, pl. 29.

Hora, S.L. (1923a) On a collection of fish from Siam. Journal of the Natural History Society of Siam, 6, 143-184, pls. 10-12.

Hora, S.L. (1923b) Notes on fishes in the Indian Museum, V.- On the composite genus Glyptosternon M'Clelland. Records of the Indian Museum, 25, 1-44, pls. 1-4.

Hora, S.L. (1929) An aid to the study of Hamilton Buchanan's "Gangetic Fishes". Memoirs of the Indian Museum, 9, 169-192, pl. 13-23.

Hora, S.L. (1931) On two small collections of fishes from Burma. Records of the Indian Museum, 33, 1-2.

Hora, S.L. (1932) Glyptosternum reticulatum, a siluroid fish from Afghanistan. Annals and Magazine of Natural History (Ser. 10), 10, 176-179.

Hora, S.L. (1933) Siluroid fishes of India, Burma and Ceylon. I.- Loach-like fishes of the genus Amblyceps Blyth. Records of the Indian Museum, 35, 607-621.

Hora, S.L. (1934) The fish of Chitral. Records of the Indian Museum, 36, 279-319, 2 pls.

Hora, S.L. (1936a) Siluroid fishes of India, Burma and Ceylon. II.— Fishes of the genus Akysis Bleeker. III.- Fishes of the genus Olyra M'Clelland. IV.- On the use of the generic name Wallago Bleeker. V.- Fishes of the genus Heteropneustes Müller. Records of the Indian Museum, 38, 199-209.

Hora, S.L. (1936b) Siluroid fishes of India, Burma and Ceylon. VI.- Fishes of the genus Clarias Gronovius. VII.Fishes of the genus Silurus Linnaeus. VIII.- Fishes of the genus Callichrous Hamilton. Records of the Indian Museum, 38, 347-361.

Hora, S.L. (1937a) Geogographical distribution of Indian freshwater fishes and its bearing on the probable land connections between India and the adjacent countries. Current Science, 5, 351-356.

Hora, S.L. (1937b) The game fishes of India. III.- Garua bachcha or Gaurchcha. Journal of the Bombay Natural History Society, 39, 659-678, pl. 1.

Hora, S.L. (1937c) A new genus of Siamese catfishes. Journal of the Siam Society, Natural History Supplement, 11, 3946, pl. 2.

Hora, S.L. (1937d) Notes on fishes in the Indian Museum, XXX.- On the systematic position of Cyprinus cosuatis Hamilton. XXXI._ On a small collection of fish from Sandoway, lower Burma. XXXII._ On a small collection of fish from the upper Chindwin drainage. XXXIII.- On a collection of fish from the Kumaon Himalayas. XXXIV.On a new catfish from Kwangsi, China. XXXV.- A further note on Hamilton's Cyprinus (Garra) lamta. XXXVI.- - On a new genus of Chinese catfishes allied to Pseudecheneis. Records of the Indian Museum, 39, 321350 .

Hora, S.L. (1937e) The game fishes of India. II.- The Bachhwa or Butchwa. Journal of the Bombay Natural History Society, 39, 431-446, 1 plate.

Hora, S.L. (1938a) The game fishes of India. IV.— The silonid catfish, Silonia silondia. Journal of the Bombay Natural History Society, 40, 137-147, plate.

Hora, S.L. (1938b) The game fishes of India. V.- The pungas catfish, Pangasius pangasius (Hamilton). Journal of the Bombay Natural History Society, 40, 355-366, plate.

Hora, S.L. (1938c) A new name for Silurus sinensis Hora. Records of the Indian Museum, 40, 243.

Hora, S.L. (1938d) Notes on fishes in the Indian Museum, XXXVIII. - On the systematic position of Bagrus lonah Sykes, with descriptions of and remarks on other glyptosternoid fishes from the Deccan. Records of the Indian Museum, 40, 363-375, pl. 7.

Hora, S.L. (1939a) The game fishes of India. VI.- The goonch, Bagarius bagarius (Hamilton). Journal of the Bombay Natural History Society, 40, 583-593, plate.

Hora, S.L. (1939b) The game fishes of India. VII.- The mulley of boali, Wallagonia attu (Bloch and Schneider). Journal of the Bombay Natural History Society, 41, 64-71, plate. 
Hora, S.L. (1941) Siluroid fishes of India, Burma and Ceylon. XI.- Fishes of the schilbeid genera Silonopangasius Hora, Pseudeutropius Bleeker, Proeutropiichthys Hora, and Ailia Gray. XII.- A further note on fishes of the genus Clarias Gronovius. Records of the Indian Museum, 43, 97-115.

Hora, S.L. (1949a) Systematic position of three glyptosternoid fishes described by Hamilton. Records of the Indian Museum, 46, for 1948, 55-62, pl. 2.

Hora, S.L. (1949b) Systematic position of siluroids in Hamilton's Gangetic fishes. Records of the Indian Museum, 46, for 1948, 63-72.

Hora, S.L. (1950) Siluroid fishes of India, Burma and Ceylon. XIII.- Fishes of the genera Erethistes Müller and Troschel, Hara Blyth and of two new allied genera. Records of the Indian Museum, 47, 183-202, pls. 1-2.

Hora, S.L. (1952) Parallel evolution of Pseudecheneis Blyth and similar fishes of south east Asia. Journal of the Asiatic Society. Science, 18, 183-202.

Hora, S.L. \& Chabanaud, P. (1930) The siluroid fish Pseudecheneis and an allied new genus. Records of the Indian Museum, 32, 215-221.

Hora, S.L. \& Gupta, J.C. (1941) Notes on Malayan fishes in the collection of the Raffles Museum, Singapore. I.- Catfishes of the families Siluridae, Bagridae, Amblycepidae, Akysidae, Sisoridae, Chacidae, Schilbeidae, and Clariidae. Bulletin of the Raffles Museum, 17, 12-43, pls. 2-4.

Hora, S.L. \& Law, N.C. (1941) Siluroid fishes of India, Burma and Ceylon. IX.- Fishes of the genera Gagata Bleeker and Nangra Day. X.- Fishes of the genus Batasio Blyth. Records of the Indian Museum (Calcutta), 43, 9-42, 2 pls.

Hora, S.L. \& Silas, E.G. (1952a) Notes on fishes in the Indian Museum, XLVII.- Revision of the glyptosternoid fishes of the family Sisoridae, with descriptions of new genera and species. Records of the Indian Museum, 49, for 1951, 5-29, pl. 1.

Hora, S.L. \& Silas, E.G. (1952b) Evolution and distribution of glyptosternoid fishes of the family Sisoridae (Order: Siluroidea). Proceedings of the National Institute of Sciences of India, 18, 309-322.

Hough, F.B. (1852) Catalogue of reptiles and fishes. Fishes. In: Fifth annual report of the Regents of the University, on the condition of the state cabinet of natural history, and the historical and antiquarian collection annexed thereto, Report no. 122, University of the State of New York, Albany, 24-28.

Houttuyn, M. (1782) Beschrijving van eenige Japanse visschen en andere zee-schepzelen. Verhandelingen der Hollandsche Maatschappij der Wetenschappen, Haarlem, 20, 311-350.

Howes, G.J. (1980) A new catfish from Sierra Leone. Bulletin of the British Museum (Natural History), Zoology, 38, $165-170$.

Howes, G.J. (1983a) Problems in catfish anatomy and phylogeny exemplified by the Neotropical Hypophthalmidae (Teleostei: Siluroidei). Bulletin of the British Museum (Natural History), Zoology, 45, 1-39.

Howes, G.J. (1983b) The cranial muscles of the loricarioid catfishes, their homologies and value as taxonomic characters (Teleostei: Siluroidei). Bulletin of the British Museum (Natural History), Zoology, 45, 309-345.

Howes, G.J. (1985) The phylogenetic relationships of the electric catfish family Malapteruridae (Teleostei: Siluroidei). Journal of Natural History, 19, 37-67.

Howes, G.J. \& Fumihito, A. (1991) Cranial anatomy and phylogeny of the south east Asian catfish genus Belodontichthys. Bulletin of the British Museum (Natural History), Zoology, 57, 133-160.

Hu, X.-Y., Lan, J.-H. \& Zhang, C.-G. (2004) A new species of the genus Duanensis [sic, Silurus] (Siluriformes, Siluridae) from Guangxi, China. Acta Zootaxonomica Sinica, 29, 586-590.

Huang, S.-Y. (1981) On two new species of the catfish genus Platytropius Hora from Yunnan, China. Acta Zootaxonomica Sinica, 6, 437-440. [In Chinese, with new species description repeated in English.]

Hubbs, C.L. (1935) Fresh-water fishes collected in British Honduras and Guatemala. Miscellaneous Publications, Museum of Zoology, University of Michigan, 28, 1-22, pls. 1-4.

Hubbs, C.L. (1936) Fishes of the Yucatan Peninsula. Carnegie Institution of Washington Publication, 157-287, pls. 115.

Hubbs, C.L. \& Bailey, R.M. (1947) Blind catfishes from artesian waters of Texas. Occasional Papers of the Museum of Zoology, University of Michigan, 499, 1-15, pl. 1.

Hubbs, C.L. \& Hibbard, C.W. (1951) Ictalurus lambda, a new catfish, based on a pectoral spine from the lower Pliocene of Kansas. Copeia, 1951, 8-14.

Hubbs, C.L. \& Miller, R.R. (1960) Potamarius, a new genus of ariid catfishes from the fresh waters of Middle America. Copeia, 1960, 101-112, pl. 1.

Hubbs, C.L. \& Raney, E.C. (1944) Systematic notes on North American siluroid fishes of the genus Schilbeodes. Occasional Papers of the Museum of Zoology, University of Michigan, 487, 1-36, 1 pl. 
Hubrecht, A.A.W. (1881) On a collection of fishes from the St. Paul's River, Liberia, with description of three new species. Notes from the Leyden Museum, 3, 66-71.

Humboldt, F.H.A.von. (1805a) Mémoire sur l'Eremophilus et Astroblepus, deux nouveaux genres de l'ordre des apodes. In: Voyage aux Régions Équinoxiales du Nouveau Continent fait en 1799 et 1800, par A. de Humboldt et A. Bonpland. Deuxième Partie: Observations de Zoologie et d'Anatomie Comparée, Paris, pp. 17-20, pls. 6-7.

Humboldt, F.H.A.von. (1805b) Mémoire sur une nouvelle espèce de pimelode, jetée par les volcans du Royaume de Quito. In: Voyage aux Régions Équinoxiales du Nouveau Continent fait en 1799 et 1800, par A. de Humboldt et A. Bonpland. Deuxième Partie: Observations de Zoologie et d'Anatomie Comparée, Paris, pp. 21-25, pl. 7.

Humboldt, F.H.A.von \& Valenciennes, A. (1821) Recherches sur les poissons fluviatiles de l'Amérique Équinoxiale. In: Voyage aux Régions Équinoxiales du Nouveau Continent fait en 1799 et 1800, par A. de Humboldt et A. Bonpland. Deuxième Partie: Observations de Zoologie et d'Anatomie Comparée, Paris, pp. 145-216, pls. 45-52.

Husain, A. \& Tilak, R. (1978) On the redescription and distribution of Hara jerdoni Day (Sisoridae: Siluriformes). Journal of the Inland Fishery Society of India, 9, 92-97.

Hussakof, L. (1932) The fossil fishes collected by the Central Asiatic Expedition. American Museum Novitates, 553, 119.

Hyrtl, C.J. (1859) Anatomische Untersuchung des Clarotes (Gonocephalus) heuglini Kner. Mit einer Abbildung und einer osteologischen Tabelle der Siluroiden. Denkschriften der Mathematisch-Naturwissenschaftlichen Classe der Kaiserlichen Akademie der Wissenschaften in Wien, 16, 1-18, 1 pl.

Ihering, H. von. (1898) Description of a new fish from Sao Paulo. Pp. 108-109, appended to: Contributions to the herpetology of Sao Paulo.- 1. Proceedings of the Academy of Natural Sciences, Philadelphia, 50, 101-109.

Ihering, R. von. (1905) Descriptions of four new loricariid fishes of the genus Plecostomus from Brazil. Annals and Magazine of Natural History (Ser. 7), 15, 558-561.

Ihering, R. von. (1907) Diversas especies novas de peixes nemathognathas do Brazil. (Several new species of Brazilian nematognath fishes.) Notas preliminares. Revista do Museu Paulista (N. S.), 1, 13-39.

Ihering, R. von. (1911) Algunas especies novas de peixes d'agua doce (Nematognatha) (Corydoras, Plecostomus, Hemipsilichthys). Revista do Museu Paulista, 8, for 1910, 380-404.

Ihering, R. von. (1928a) Uma nova especie de Otocinclus (Pisces. Nematognatha) "cascudinho" de S. Paulo. Boletím Biológico, São Paulo, 11, 1-3.

Ihering, R. von. (1928b) Glanidium cesarpintoi n. sp. de Peixe de couro (fam. Siluridae sub-fam. Auchenipterinae). Boletím Biológico, São Paulo, 12, 46-49.

Ihering, R. von. (1930) Notas ecologicas referentes a peixes d'agua doce do Estado de S. Paulo e descrição de 4 especies novas. Archivos do Instituto Biológico, São Paulo, 3, 93-103, pl. 13.

Ihering, R. von. (1968) Dicionário dos Animais do Brasil. Editora da Universidade de Brasília, São Paulo, Brazil, 790 p.

Inger, R.F. (1956) Notes on a collection of fishes from southeastern Venezuela. Fieldiana, Zoology, 34, 425-440.

Inger, R.F. \& Chin, P.K. (1959) New species of fresh-water catfishes from North Borneo. Fieldiana, Zoology, 39, 279296.

Innes, W.T. \& Myers, G.S. (1950) The "Imitator catfish," which mimics a Corydoras. The Aquarium, 19, 222-223.

International Commission on Zoological Nomenclature [ICZN] (1961) International Code of Zoological Nomenclature, Second Edition, International Trust for Zoological Nomenclature, London and Berkeley.

International Commission on Zoological Nomenclature [ICZN] (1985) International Code of Zoological Nomenclature, Third Edition, International Trust for Zoological Nomenclature, London and Berkeley.

International Commission on Zoological Nomenclature [ICZN] (1999) International Code of Zoological Nomenclature, Fourth Edition, International Trust for Zoological Nomenclature, London, 306 p.

International Commission on Zoological Nomenclature [ICZN] (2002) Opinion 2011. Hemibagrus Bleeker, 1862 (Osteichthyes, Siluriformes), Bagrus nemurus Valenciennes in Cuvier \& Valenciennes, 1840, B. planiceps Valenciennes, 1840, B. flavus Bleeker, 1846 and B. sieboldii Bleeker, 1846: Previous fixations of type specimens not to be set aside. Bulletin of Zoological Nomenclature, 59, 220-221.

Isbrücker, I.J.H. (1969) Over de namen van enkele Corydoras-soorten. Het Aquarium, 39, 202-203.

Isbrücker, I.J.H. (1971a) A redescription of the South American catfish Loricariichthys maculatus (Bloch, 1794), with designation of the lectotype and restriction of its type locality (Pisces, Siluriformes, Loricariidae). Bijdragen tot de Dierkunde, 41, 10-18.

Isbrücker, I.J.H. (1971b) Pseudohemiodon (Planiloricaria) cryptodon, a new species and subgenus from Peru (Pisces, Siluriformes, Loricariidae). Bonner Zoologische Beiträge, 21, for 1970, 274-283, 7 pls.

Isbrücker, I.J.H. (1972) The identity of the South American catfish Loricaria cataphracta Linnaeus, 1758, with descrip- 
tions of the original type specimens of four other nominal Loricaria species (Pisces, Siluriformes, Loricariidae). Beaufortia, 19, 163-191.

Isbrücker, I.J.H. (1973) Status of the primary homonymous South American catfish Loricaria cirrhosa Perugia, 1897, with remarks on some other loricariids (Pisces, Siluriformes, Loricariidae). Annali del Museo Civico de Storia Naturale di Genova, 79, 172-191.

Isbrücker, I.J.H. (1975a) Pseudohemiodon thorectes, a new species of mailed catfish from the Rio Mamoré system, Bolivia (Pisces, Siluriformes, Loricariidae). Beaufortia, 23, 85-92.

Isbrücker, I.J.H. (1975b) Metaloricaria paucidens, a new species and genus of mailed catfish from French Guiana (Pisces, Siluriformes, Loricariidae). Bulletin, Institut royal des Sciences naturelles de Belgique, Biologie, 50, 1-9, pls. 1-3.

Isbrücker, I.J.H. (1979a) Description préliminaire de nouveaux taxa de la famille des Loricariidae, poissons-chats cuirassés néotropicaux, avec un catalogue critique de la sous-famille nominale (Pisces, Siluriformes). Revue Française d'Aquariologie et Herpetologie, 5, for 1978, 86-116.

Isbrücker, I.J.H. (1979b) Les poissons de la famille des Loricariidés ou poissons-chats cuirassés. Revue Française d'Aquariologie et Herpetologie, 6, 109-124.

Isbrücker, I.J.H. (1980) Classification and catalogue of the mailed Loricariidae (Pisces, Siluriformes). Verslagen en Technische Gegevens, Instituut voor Taxonomische Zoölogie, Universiteit van Amsterdam, 22, 1-181.

Isbrücker, I.J.H. (1981) Revision of Loricaria Linnaeus, 1758 (Pisces, Siluriformes, Loricariidae). Beaufortia, 31, 5196.

Isbrücker, I.J.H. (1986) Trichomycteridae, mysterieuze meervallen. Het Aquarium, 56, 274-279.

Isbrücker, I.J.H. (1992) Überblick über die güttigen (Unter-) Gattungsnamen der Harnischwelse (Loricariidae) und ihre Synonyme. Die Aquarien- und Terrarien-Zeitschrift, 1992, 71-72.

Isbrücker, I.J.H. (1992) Ein fish findet nach 138 Jahren sein Zuhause. Der verborgene Fundort von Hemiancistrus medians (Kner, 1854). In: Stawikowski, R. (Ed.), Harnischwelse, Die Aquarien- und Terrarien- Zeitschrift, Sonderheft, Eugen Ulmer, Stuttgart, pp. 56-57.

Isbrücker, I.J.H. (1999) Corydoras geoffroy und Cataphractus punctatus. Revision der beiden zuerst entdeckten arten der Unterfamilie Corydoradinae (Actinopterygii, Siluriformes, Callichthyidae). Teil 1. Die Aquarien- und TerrarienZeitschrift, 52, 38-43.

Isbrücker, I.J.H. (1999b) Corydoras bolivianus und Corydoras geryi. Die Aquarien- und Terrarien- Zeitschrift, 52, 5859.

Isbrücker, I.J.H. (2000) Corydoras geoffroy and Cataphractus punctatus. History of both earliest discovered species of the subfamily Corydoradinae (Actinopterygii, Siluriformes, Callichthyidae). Cat Chat, 1, 11-18.

Isbrücker, I.J.H. (2001a) Nomenklator der Gattungen und Arten der Harnischwelse, Familie Loricariidae Rafinesque, 1815 (Teleostei, Ostariophysi). In: Stawikowski, R. (Ed.), Harnischwelse 2, Die Aquarien- und TerrarienZeitschrift, Eugen Ulmer, Stuttgart, pp. 25-32.

Isbrücker, I.J.H. (2001b) Catalogue of genera and species of Corydoradinae Hoedeman, 1952 (Teleostei, Ostariophysi, Callichthyidae), including type localities, type specimens, and etymology. In: Fuller, I.A.M. (Ed.), Breeding Corydoradine Catfishes, Ian Fuller Enterprises, Kidderminster, pp. 213-247.

Isbrücker, I.J.H., Britski, H.A., Nijssen, H. \& Ortega, H. (1983) Aposturisoma myriodon, une espèce et un genre nouveaux de poisson-chat cuirassé, tribu Farlowellini Fowler, 1958 du bassin du Rio Ucayali, Pérou (Pisces, Siluriformes, Loricariidae). Revue Française d'Aquariologie et Herpetologie, 10, 33-42.

Isbrücker, I.J.H. \& Nijssen, H. (1973) Two new species of the callichthyid catfish genus Corydoras from Brazil (Pisces, Siluriformes, Callichthyidae). Beaufortia, 21, 1-7.

Isbrücker, I.J.H. \& Nijssen, H. (1974a) Rhadinoloricaria gen. nov. and Planiloricaria, two genera of South American mailed catfishes (Pisces, Siluriformes, Loricariidae). Beaufortia, 22, 67-81.

Isbrücker, I.J.H. \& Nijssen, H. (1974b) On Hemiodontichthys acipenserinus and Reganella depressa, two remarkable mailed catfishes from South America (Pisces, Siluriformes, Loricariidae). Beaufortia, 22, 193-222.

Isbrücker, I.J.H. \& Nijssen, H. (1976a) The South American mailed catfishes of the genus Pseudoloricaria Bleeker, 1862 (Pisces, Siluriformes, Loricariidae). Beaufortia, 25, 107-129.

Isbrücker, I.J.H. \& Nijssen, H. (1976b) Rineloricaria heteroptera, a new species of mailed catfish from Rio Amazonas near Manaus, Brazil (Pisces, Siluriformes, Loricariidae). Zoologischer Anzeiger, 196, 109-124.

Isbrücker, I.J.H. \& Nijssen, H. (1978a) Two new species and a new genus of neotropical mailed catfishes of the subfamily Loricariinae Swainson, 1838 (Pisces, Siluriformes, Loricariidae). Beaufortia, 27, 177-206.

Isbrücker, I.J.H. \& Nijssen, H. (1978b) The neotropical mailed catfishes of the genera Lamontichthys P. de Miranda- 
Ribeiro, 1939 and Pterosturisoma n. gen., including the description of Lamontichthys stibaros n. sp. from Ecuador (Pisces, Siluriformes, Loricariidae). Bijdragen tot de Dierkunde, 48, 57-80.

Isbrücker, I.J.H. \& Nijssen, H. (1979) Three new South American mailed catfishes of the genera Rineloricaria and Loricariichthys (Pisces, Siluriformes, Loricariidae). Bijdragen tot de Dierkunde, 48, 191-211.

Isbrücker, I.J.H. \& Nijssen, H. (1982) New data on Metaloricaria paucidens from French Guiana and Surinam (Pisces, Siluriformes, Loricariidae). Bijdragen tot de Dierkunde, 52, 155-168.

Isbrücker, I.J.H. \& Nijssen, H. (1983a) Aphanotorulus frankei, une espèce et un genre nouveaux de poissons-chats cuirassés du bassin du Rio Ucayali au Pérou (Pisces, Siluriformes, Loricariidae). Revue Française d'Aquariologie et Herpetologie, 9, for 1982, 105-110.

Isbrücker, I.J.H. \& Nijssen, H. (1983b) Crossoloricaria rhami n. sp., un nouveau poisson-chat cuirassé du Rio Huacamayo, Pérou (Pisces, Siluriformes, Loricariidae). Revue Française d'Aquariologie et Herpetologie, 10, 9-12.

Isbrücker, I.J.H. \& Nijssen, H. (1984a) Rineloricaria castroi, a new species of mailed catfish from Rio Trombetas, Brazil (Pisces, Siluriformes, Loricariidae). Beaufortia, 34, 93-99.

Isbrücker, I.J.H. \& Nijssen, H. (1984b) Pyxiloricaria menezesi, a new genus and species of mailed catfish from Rio Miranda and Rio Cuiabá, Brazil (Pisces, Siluriformes, Loricariidae). Bijdragen tot de Dierkunde, 54, 163-168.

Isbrücker, I.J.H. \& Nijssen, H. (1984c) Hypostomus nematopterus, a new species of mailed catfish from the Oyapock River system, French Guiana (Pisces, Siluriformes, Loricariidae). Bulletin Zoölogisch Museum, Universiteit van Amsterdam, 10, 9-13.

Isbrücker, I.J.H. \& Nijssen, H. (1985) Exastilithoxus hoedemani, a new species of mailed catfish from Rio Marauiá, Est. Amazonas, Brazil (Pisces, Siluriformes, Loricariidae). Spixiana, 8, 221-229.

Isbrücker, I.J.H. \& Nijssen, H. (1986a) New records of the mailed catfish Planiloricaria cryptodon from the Upper Amazon in Peru, Brazil and Bolivia, with a key to the genera of the Planiloricariina. Bijdragen tot de Dierkunde, 56, 3946.

Isbrücker, I.J.H. \& Nijssen, H. (1986b) Apistoloricaria condei, nouveau genre et nouvelle espèce de poisson-chat cuirassé, tribu Loricariini Bonaparte, 1831, du bassin du Rio Napo, haute Amazone, Equateur (Pisces, Siluriformes, Loricariidae). Revue Française d'Aquariologie et Herpetologie, 12, for 1985, 103-108.

Isbrücker, I.J.H. \& Nijssen, H. (1988) Acanthicus adonis, ein neuer Harnischwels aus dem Rio Tocantins, Brasilien (Pisces, Siluriformes, Loricariidae). Die Aquarien- und Terrarien-Zeitschrift, 41, 164-167.

Isbrücker, I.J.H. \& Nijssen, H. (1989) Diagnose dreier neuer Harnischwelsgattungen mit fünf neuen Arten aus Brasilien (Pisces, Siluriformes, Loricariidae). Die Aquarien- und Terrarien-Zeitschrift, 42, 541-547.

Isbrücker, I.J.H. \& Nijssen, H. (1991) Hypancistrus zebra, a new genus and species of uniquely pigmented ancistrine loricariid fish from the Rio Xingu, Brazil (Pisces: Siluriformes: Loricariidae). Ichthyological Exploration of Freshwaters, 1, 345-350.

Isbrücker, I.J.H. \& Nijssen, H. (1992) Corydoras breei, a new species of callichthyid catfish from the Corantijn River basin in Surinam (Pisces, Siluriformes, Callichthyidae). Beaufortia, 43, 9-14.

Isbrücker, I.J.H. \& Nijssen, H. (1992) Sexualdimorphismus bei Harnischwelsen (Loricariidae). Odontoden, Zähne, Lippen, Tentakel, Genitalpapillen und Flossen. In: Stawikowski, R. (Ed.), Harnischwelse. Die Aquarien- und TerrarienZeitschrift Sonderheft, pp. 19-33.

Isbrücker, I.J.H., Nijssen, H. \& Cala, P. (1988) Lithoxancistrus orinoco, nouveau genre et espèce de poisson-chat cuirassé du Rio Orinoco en Colombie (Pisces, Siluriformes, Loricariidae). Revue Française d'Aquariologie et Herpetologie, 15, 13-16.

Isbrücker, I.J.H., Nijssen, H. \& Nico, L.G. (1992) Ein neuer Rüsselzahnwels aus oberen Orinoco-Zuflüssen in Venezuela und Kolumbien: Leporacanthicus triactis n. sp. (Pisces, Siluriformes, Loricariidae). Die Aquarien- und TerrarienZeitschrift, 46, 1-5.

Isbrücker, I.J.H., Seidel, I., Michels, J.P., Schraml, E. \& Werner, A. (2001) Diagnose vierzehn neuer Gatungen der Familie Loricariidae Rafinesque, 1815 (Teleostei, Ostariophysi). In: Stawikowski, R. (Ed.), Harnischwelse 2, Die Aquarien- und Terrarien- Zeitschrift, Sonderheft, Eugen Ulmer, Stuttgart, pp. 17-24.

Jackson, P.B.N. (1955) A new fish of the genus Clarias Gronov. from Lake Nyasa, with notes on the distribution of the Clariidae and other catfishes in the lake. Proceedings of the Zoological Society of London, 125, 681-684.

Jackson, P.B.N. (1959) Revision of the clariid catfishes of Nyasaland, with a description of a new genus and seven new species. Proceedings of the Zoological Society of London, 132, 109-128.

Jacquemont, V. (1835-1844) Voyage dans l'Inde Pendant les Années 1828 à 1832, Publié sous les Auspices de M. Guizot, Ministre de l'instruction Publique. Journal. 4 vols. Text, 2 vols, atlas, Paris. [Fish plates in vol. 2: pls. 13-18; dated to 1840 .] 
Jansen, G., Devaere, S., Weekers, P.H.H. \& Adriaens, D. (2005) Phylogenetic relationships and divergence time estimate of African anguilliform catfish (Siluriformes: Clariidae) inferred from ribosomal gene and spacer sequences. Molecular Phylogenetics and Evolution, 38, for 2006, 65-78.

Jayaram, K.C. (1952) Taxonomic notes on the fish Pseudobagrus chryseus Day, 1865. Annals and Magazine of Natural History (Ser. 12), 5, 980-983.

Jayaram, K.C. (1954) Siluroid fishes of India, Burma and Ceylon: XIV.- Fishes of the genus Mystus Scopoli. Records of the Indian Museum, 51, for 1953, 527-558, pl. 19.

Jayaram, K.C. (1955a) A preliminary review of the genera of the family Bagridae (Pisces: Siluroidea). Proceedings of the National Institute of Sciences of India, 21 B, 120-128.

Jayaram, K.C. (1955b) The Palaearctic element in the fish fauna of peninsular India. Bulletin of the National Institute of Sciences of India, 7, 260-263.

Jayaram, K.C. (1956a) Nomenclatural status of the names Bagre Cuvier (Oken), Bagrus Valenciennes, and Porcus, Geoffroy St. Hilaire. Copeia, 1956, 248-249.

Jayaram, K.C. (1956b) Taxonomic status of the Chinese catfish family Cranoglanididae Myers, 1931. Proceedings of the National Institute of Sciences of India, 21B, for 1955, 256-263, pl. 18.

Jayaram, K.C. (1959) Systematic position of fishes described under Bagrus by Valenciennes, 1839. Records of the Indian Museum, 54, for 1956, 53-59.

Jayaram, K.C. (1960) Racial analysis of Rita chrysea Day inhabiting the Mahanadi River. Journal of the Zoological Society of India, 12, for 1959, 85-103.

Jayaram, K.C. (1962) The nomenclatural status of Mystus, Macrones, Aoria and other names for a genus of Asiatic siluroid fishes. Proceedings of the First All India Congress of Zoology, 1959. Part 2.- Scientific papers. Jabalpur, pp. 632-635.

Jayaram, K.C. (1963) Systematic status of Ailichthys punctata bay [sic, Day] and its relationship with Ailia coila (Hamilton) (Siluroidea: Schilbeidae). Journal of the Zoological Society of India, 14, for 1962, 244-248.

Jayaram, K.C. (1965a) On a new species of fish of the family Bagridae. Bulletin of Systematic Zoology, Calcutta, 1, 910.

Jayaram, K.C. (1965b) Systematic position of the genus Liobagrus Hilgendorf (Pisces: Siluroidea). Science and Culture, 31, 436-437.

Jayaram, K.C. (1966a) Contributions to the study of bagrid fishes (Siluroidea: Bagridae): 5.- On a new species of the genus Coreobagrus Mori. Bulletin of the Biogeographical Society of Japan, 24, 29-32.

Jayaram, K.C. (1966b) Contributions to the study of the fishes of the family Bagridae: 2.- A systematic account of the African genera with a new classification of the family. Bulletin de l'Institut fondamental d'Afrique noire. Série A, Sciences naturelles, 28, 1064-1139.

Jayaram, K.C. (1966c) Contributions to the study of bagrid fishes (Siluroidea: Bagridae): 1.— A systematic account of the genera Rita Bleeker, Rama Bleeker, Mystus Scopoli, and Horabagrus Jayaram. Internationale Revue der Gesamten Hydrobiologie, 51, 433-450.

Jayaram, K.C. (1966d) A new species of sisorid fish from the Kameng Frontier Division, N.E.F.A. Journal of the Zoological Society of India, 15, for 1963, 85-87.

Jayaram, K.C. (1968a) Contributions to the study of bagrid fishes. 6.- Nomenclatural status and systematic position of the fishes of the subgenus Osteobagrus Jayaram (Siluroidea: Bagridae). Proceedings of the Indian Sci. Congress, 3 , 370 .

Jayaram, K.C. (1968b) Contributions to the study of bagrid fishes (Siluroidea: Bagridae): 3. - A systematic account of the Japanese, Chinese, Malayan and Indonesian genera. Treubia, Museum Zoologicum Borgoriense, 27, $287-386$.

Jayaram, K.C. (1971b) Contributions to the study of bagrid fishes: 4.- Nomenclatural status and systematic position of the fishes of the subgenus Osteobagrus Jayaram (Siluroidea: Bagridae). Records of the Zoological Survey of India, $63,185-194$.

Jayaram, K.C. (1972a) Siluroid fishes of India, Burma and Ceylon. 18.- Resurrection of the genus Nangra Day and its systematic position. Journal of the Zoological Society of India, 23, for 1971, 171-174.

Jayaram, K.C. (1972b) Siluroid fishes of India, Burma and Ceylon. 19._ Fishes of the genus Ketengus Bleeker (Family Ariidae). Proceedings of the Zoological Society of Calcutta, 25, 135-139.

Jayaram, K.C. (1973a) Contributions to the study of bagrid fishes (Siluroidea: Bagridae): 10.— Systematic position of Pimelodus chandramara Hamilton with description of a new genus. Internationale Revue der Gesamten Hydrobiologie, 57, for 1972, 815-820.

Jayaram, K.C. (1973b) Contributions to the study of bagrid fishes: 9.- Generic status of Aorichthys Wu (Siluroidea: 
Bagridae). Proceedings of the Zoological Society of Calcutta, 24, for 1971, 149-156.

Jayaram, K.C. (1973c) Siluroid fishes of India, Burma and Ceylon. 19.- Systematic position of the genus Laguvia Hora and its relationships. Records of the Zoological Survey of India, 67, 385-389.

Jayaram, K.C. (1974) Contributions to the study of bagrid fishes: 11.— Designation of a lectotype for Macrones argentivittatus Regan with notes on some bagrid species in the collections of the Natural History Museum, Geneva. Revue suisse de Zoologie, Annales de la Société zoologique suisse et du Muséum d'Histoire naturelle de Genève, 81, 785790.

Jayaram, K.C. (1976) Contributions to the study of bagrid fishes: 13.- Interrelationships of Indo-African catfishes of the family Bagridae. Matsya, 2, 47-53.

Jayaram, K.C. (1977a) Year of publication and type-locality of Ompok bimaculatus (Bloch) (Pisces: Siluroidea). Newsletter of the Zoological Survey of India, 3, 50-51.

Jayaram, K.C. (1977b) Contributions to the study of bagrid fishes: 12.- The correct family position of Batasio Blyth (Siluroidea). Newsletter of the Zoological Survey of India, 3, 242-245.

Jayaram, K.C. (1977c) Aid to identification of siluroid fishes of India, Burma, Sri Lanka, Pakistan and Bangladesh: 1.— Bagridae. Records of the Zoological Survey of India, Miscellaneous Publication, Occasional Paper, 8, 1-41.

Jayaram, K.C. (1977d) Aid to identification of siluroid fishes of India, Burma, Sri Lanka, Pakistan and Bangladesh: 2.Siluridae, Schilbeidae, Pangasiidae, Amblycipitidae, Akysidae. Records of the Zoological Survey of India, Miscellaneous Publication, Occasional Paper, 10, 1-33.

Jayaram, K.C. (1978a) The catfishes of India. Zoologiana (Calcutta), 1, 9-17.

Jayaram, K.C. (1978b) Contributions to the study of bagrid fishes: 14.- The systematic position of the species of Mystus Scopoli known from China. Proceedings of the Indian Academy of Science, 87B, 221-228.

Jayaram, K.C. (1979) Aid to identification of siluroid fishes of India, Burma, Sri Lanka, Pakistan and Bangladesh: 3.Sisoridae. Records of the Zoological Survey of India, Miscellaneous Publication, Occasional Paper, 11, 1-62.

Jayaram, K.C. (1980) Aid to identification of siluroid fishes of India, Burma, Sri Lanka, Pakistan and Bangladesh: 4.Clariidae, Heteropneustidae, Chacidae, and Olyridae. Records of the Zoological Survey of India, Miscellaneous Publication, Occasional Paper, 23, 1-23.

Jayaram, K.C. (1981) The Freshwater Fishes of India, Pakistan, Bangladesh, Burma, and Sri Lanka - a Handbook, Zoological Survey of India, Calcutta, 475 p., 12 pl.

Jayaram, K.C. (1982) Aid to identification of siluroid fishes of India, Burma, Sri Lanka, Pakistan and Bangladesh: 5.— Ariidae and Plotosidae. Records of the Zoological Survey of India, Miscellaneous Publication, Occasional Paper, $37,1-41$.

Jayaram, K.C. (1983) Ariidae. FAO Species Identification Sheets for Western Indian Ocean, Fishing Area 51, FAO, Rome, [48] p.

Jayaram, K.C. (1999) The Freshwater Fishes of the Indian Region, Narendra Publishing House, Delhi, xxvii + 551 p., 18 pl.

Jayaram, K.C. \& Anuradha, C. (1984) Contributions to the study of bagrid fishes: 17.- The history and usage of the name "Mystus". Bulletin of the Zoological Survey of India, 6, 289-293.

Jayaram, K.C. \& Anuradha, S. (2003) A taxonomic revision of the fishes of the genus Mystus Scopoli (Family: Bagridae). Records of the Zoological Survey of India, Miscellaneous Publication, Occasional Paper, 207, 1-136, pls. $1-5$.

Jayaram, K.C. \& Boeseman, M. (1976) The systematic position of the Chinese fish Macrones sinensis Bleeker (Siluroidea). Zoologische Mededelingen (Leiden), 50, 117-119, pl. 1.

Jayaram, K.C. \& Dhanze, J.R. (1978) Siluroid fishes of India, Burma, and Ceylon: 21.— A note on the systematic position of Tachysurus serratus (Day) (Ariidae). Bulletin of the Zoological Survey of India, 1, 203-205.

Jayaram, K.C. \& Dhanze, J.R. (1979) Siluroid fishes of India, Burma and Ceylon: 22.- - A preliminary review of the genera of the family Ariidae (Pisces: Siluroidea). Matsya, 4, for 1978, 42-51.

Jayaram, K.C. \& Dhanze, J.R. (1981a) Siluroid fishes of India, Burma, and Ceylon: 23.- The specific status of Tachysurus malabaricus (Day) (Ariidae: Siluriformes). Bulletin of the Zoological Survey of India, 4, 121-123.

Jayaram, K.C. \& Dhanze, J.R. (1981b) Siluroid fishes of India, Burma, and Ceylon 24.- The systematic status of Arius satparanus Chaudhuri (Ariidae: Siluriformes). Bulletin of the Zoological Survey of India, 4, 395-398.

Jayaram, K.C. \& Dhanze, J.R. (1985) Evolution and biogeography of the Indian genera of the family Ariidae. Proceedings of the Indian Academy of Sciences (Animal Sciences), 95, 279-288.

Jayaram, K.C. \& Majumdar, N. (1964) Siluroid fishes of India, Burma and Ceylon. 16.- Fishes of the genus Chaca Gray, 1831. Proceedings of the Zoological Society of Calcutta, 17, 177-181. 
Jayaram, K.C. \& Singh, R. (1982) Contributions to the study of bagrid fishes: 15.- A comparative account of the cranial musculature in four bagrid genera with a note on their phylogeny. Records of the Zoological Survey of India, 80, 231-250.

Jenkins, J.T. (1910) Notes on fish from India and Persia, with descriptions of new species. Records of the Indian Museum, 5, 123-140, pl. 6.

Jennet, A. (1928) Les poissons fossiles originaux conservés a l'institut de Géologie de l'université de Neuchatel. Bulletin de la société Neuchateloise des Sciences Naturelles, nouvelle série, 1, for 1927, 102-124.

Jenyns, L. (1840-1842) Fish. In: The Zoology of the Voyage of H. M. S. Beagle, Under the Command of Captain FitzRoy, R. N., During the years 1832 to 1836 [part 4: xvi + 172 p, 29 pl.], Smith, Elder, and Co, Cornhill.

Jerdon, T.C. (1849) On the fresh-water fishes of southern India. (Continued from p. 149). Madras Journal of Literature and Science, 15, 302-346.

Joannis, L. de. (1835) Observations sur les poissons du Nil, et description de plusieurs espèces nouvelles. Magasin de zoologie, 5, [1-53], pls. 1-15. [Each species account with a unique header code that links text to a plate]

Johnsen, S. (1926) On a collection of fishes from the White Nile and the Yei River. (With notes on the skull of Allabenchelys and Clarias). Bergens Museums Aarbok, 10, 1-22.

Jordan, D.S. (1877a) A partial synopsis of the fishes of upper Georgia; with supplementary papers on fishes of Tennessee, Kentucky, and Indiana. Annals of the Lyceum of Natural History, New York, 11, 307-377.

Jordan, D.S. (1877b) Contributions to North American Ichthyology based primarily on the collections of the United States National Museum. I.- Review of Rafinesque's memoirs on North American fishes. Bulletin of the U. S. National Museum, 9, 1-53.

Jordan, D.S. (1877c) Contributions to North American ichthyology based primarily on the collections of the United States National Museum. No. 2. B.- Synopsis of the Siluridae of the fresh waters of North America. Bulletin of the U. S. National Museum, 10, 69-103, pls. 1-45.

Jordan, D.S. (1885) A catalogue of the fishes known to inhabit the waters of North America, north of the Tropic of Cancer, with notes on species discovered in 1883 and 1884. Report of the U. S. Fish Commission, 13, for 1885, 789-973.

Jordan, D.S. (1889) Descriptions of fourteen species of fresh-water fishes collected by the U. S. Fish Commission in the summer of 1888. Proceedings of the United States National Museum, 11, 351-362, pls. 43-45.

Jordan, D.S. (1895) The fishes of Sinaloa. Proceedings of the California Academy of Sciences, Series 2, 5, 377-514, pls. 26-55.

Jordan, D.S. (1917) The genera of fishes, from Linnaeus to Cuvier, 1758-1833, seventy-five years, with the accepted type of each. A contribution to the stability of scientific nomenclature. (Assisted by Barton Warren Evermann.). Leland Stanford Jr. University Publications, University Series, 27, 1-161.

Jordan, D.S. (1919a) The genera of fishes, part II, from Agassiz to Bleeker, 1833-1858, twenty-six years, with the accepted type of each. A contribution to the stability of scientific nomenclature. Leland Stanford Jr. University Publications, University Series, 36, i-ix, 155-284, i-xiii.

Jordan, D.S. (1919b) The genera of fishes, part III, from Guenther to Gill, 1859-1880, twenty-two years, with the accepted type of each. A contribution to the stability of scientific nomenclature. Leland Stanford Jr. University Publications, University Series, 39, 285-410, i-xv.

Jordan, D.S. (1920) The genera of fishes, part IV, from 1881 to 1920, thirty-nine years, with the accepted type of each. A contribution to the stability of scientific nomenclature. Leland Stanford Jr. University Publications, University Series, 43, 411-576 + i-xviii.

Jordan, D.S. (1923) A classification of fishes including families and genera as far as known. Stanford University Publications, University Series, Biological Sciences, 3, 77-243 + i-x.

Jordan, D.S. \& Evermann, B.W. (1896) The fishes of North and Middle America: a descriptive catalogue of the species of fish-like vertebrates found in the waters of North America, north of the Isthmus of Panama. Part I. Bulletin of the U. S. National Museum, 47, i-1x + 1-1240.

Jordan, D.S. \& Evermann, B.W. (1898) The fishes of North and Middle America: a descriptive catalogue of the species of fish-like vertebrates found in the waters of North America north of the Isthmus of Panama. Part III. Bulletin of the U. S. National Museum, 47, i-xxiv + 2183a-3136.

Jordan, D.S. \& Evermann, B.W. (1900) The fishes of North and Middle America: a descriptive catalogue of the species of fish-like vertebrates found in the waters of North America, north of the Isthmus of Panama. Part IV. Bulletin of the U. S. National Museum, 47, i-ci + 3137-3313, pls. 1-392.

Jordan, D.S. \& Evermann, B.W. (1917) The genera of fishes, from Linnaeus to Cuvier, 1758-1833, seventy-five years, with the accepted type of each. A contribution to the stability of scientific nomenclature. Leland Stanford Jr. Univer- 
sity Publications, University Series, 27, 1-161.

Jordan, D.S. \& Fowler, H.W. (1903) A review of the siluroid fishes or catfishes of Japan. Proceedings of the United States National Museum, 26, 897-911.

Jordan, D.S. \& Gilbert, C.H. (1877) On the genera of North American freshwater fishes. Proceedings of the Academy of Natural Sciences, Philadelphia, 29, 83-104.

Jordan, D.S. \& Gilbert, C.H. (1882) A review of the siluroid fishes found on the Pacific coast of tropical America, with descriptions of three new species. Bulletin of the U. S. Fish Commission, 2, 34-54. [Bulletin issued in dated signatures; much of Vol. 2 issued in 1883, but this article issued in September and October, 1882.]

Jordan, D.S. \& Gilbert, C.H. (1886) List of fishes collected in Arkansas, Indian Territory, and Texas, in September, 1884, with notes and descriptions. Proceedings of the U. S. National Museum, 9, 1-25.

Jordan, D.S. \& Hubbs, C.L. (1925) Record of fishes obtained by David Starr Jordan in Japan, 1922. Memoirs of the Carnegie Museum, 10, 93-346, pls. 5-12.

Jordan, D.S. \& Snyder, J.O. (1899) Notes on a collection of fishes from the rivers of Mexico, with description of twenty new species. Bulletin of the U.S. Fish Commission, 19, 115-147.

Jubb, R.A. (1965) A new species of Clariallabes (Pisces, Clariidae) from the Upper Zambesi River. Annals and Magazine of Natural History (Ser. 13), 7, for 1964, 393-395.

Jubb, R.A. \& Le Roux, P. (1969) Revision of the Chiloglanis (Pisces: Mochokidae) of southern Africa and descriptions of two new species. Annals of the Cape Provincial Museums. Natural History, 8, 13-23.

Kailola, P.J. (1983) Arius graeffei and Arius armiger: Valid names for two common species of Australo-Papuan forktailed catfishes (Pisces: Ariidae). Transactions of the Royal Society of South Australia, 107, 187-196.

Kailola, P.J. (1986a) Ariidae systematics: Comparison of the giant sea catfishes Arius thalassinus and A. bilineatus of the Indo-Pacific. In: Uyeno, T., Arai, R., Taniuchi, T. \& Matsuura, K. (Eds.), Indo-Pacific Fish Biology. Proceedings of the Second International Conference on Indo-Pacific Fishes, Ichthyological Society of Japan, pp. 540-549.

Kailola, P.J. (1986b) Arius peronii: A distinct species of fork-tailed catfish (Pisces: Ariidae). Journal of Zoology (London), 210, 243-250.

Kailola, P.J. (1990) A review of the freshwater fork-tailed catfishes (Pisces: Ariidae) of northern New Guinea, with descriptions of two new species. Records of the Western Australian Museum, Supplement, 34, 1-30.

Kailola, P.J. (1999) Ariidae. In: Carpenter, K.E. \& Niem, V.H. (Eds.), FAO Species Identification Guides for Fishery Purposes: The Living Marine Resources of the Western Central Pacific. Vol. 3: Batoid fishes, chimeras and bony fishes, part 1 (Elopidae to Linophrynidae), FAO, Rome, pp. 1827-1879.

Kailola, P.J. (2000) Six new species of fork-tailed catfishes (Pisces, Teleostei, Ariidae) from Australia and New Guinea. The Beagle, Records of the Museums and Art Galleries of the Northern Territory, 16, 127-144.

Kailola, P.J. (2004) A phylogenetic exploration of the catfish family Ariidae (Otophysi; Siluriformes). The Beagle, Records of the Museums and Art Galleries of the Northern Territory, 20, 87-166.

Kailola, P.J. \& Bussing, W.A. (1995) Ariidae. In: Fischer, W., Krupp, F., Schneider, W., Sommer, C., Carpenter, K.E. \& Niem, V.H. (Eds.), Guía FAO para la Identificación para los Fines de la Pesca. Pacifico Centro-oriental. Vol. II. Vertebrados - Parte 1, FAO, Rome, pp. 860-886.

Kailola, P.J. \& Pierce, B.E. (1988) A new freshwater catfish (Pisces: Ariidae) from northern Australia. Records of the Western Australian Museum, 14, 73-89.

Kanazawa, R.H. (1958) A new species of catfish, family Loricariidae, from Ecuador. Copeia, 1958, 327-328.

Kastenberger, G. (1977) Der trommelapparat der Doradidae (Siluriformes, Pisces). Zoologische Jahrbücher. Abteilung für Allgemeine Zoologie und Physiologie der Tiere, 81, 281-309.

Keilhack, L. (1908) Bemerkungen zur Fishfauna des nördlichen Njassa-Gebietes: einige neue Arten aus den Gattungen Barbus und Synodontis und Beiträge zur Systematik der Gattung Clarias. Sitzungsberichte der Gesellschaft Naturforschender Freunde zu Berlin, 1908, 164-169.

Keilhack, L. (1910) Über einige von Herrn Dr. H. Monke in Duala (Kamerun) gesammelte Fische. Mitteilungen aus dem Zoologischen Museum in Berlin, 5, 117-124.

Keith, P. \& Merona, B. de (2000) Présence de Lithodoras dorsalis (Valenciennes in Cuvier et Valenciennes, 1840) (Siluriformes, Doradidae) en Guyane française. Cybium (3e série), 24, 101-103.

Kessler, K.T. (1872) [Ichthyological Fauna of Turkestan]. Izvestiia Imperatorskago Obshchestva Liubitelei Estestvozaniia, Antropologii i Etnografii, 10, 47-76, pls. 6-12. [In Russian.]

Khin-Thant. (1966) Silurus burmanensis, a new species of fish from the Inlé Lake, Southern Shan State, Burma. Journal of the Burma Research Society, 49, 219-221, pls. 1-3.

Kimura, S. (1934) Description of the fishes collected from the Yangtze-kiang, China, by the late Dr. K. Kishinouye and 
his party in 1927-1929. Journal of the Shanghai Science Institute. Section 3, Systematic and morphological biology and pharmacognosy, 1, 11-247, pls. 1-6.

Kindle, E.M. (1895) The South American cat-fishes belonging to Cornell University. Annals of the New York Academy of Sciences, 7, for 1894, 249-256.

Klausewitz, W. \& Rössel, F. (1961) Rhynchodoras xingui, ein bemerkenswerter neuer Wels aus Brasilien (Pisces, Siluroidea, Doradidae). Senckenbergiana Biologica, 42, 45-48.

Klein, J.T. (1775) Neuer Schauplatz der Natur, nach den Richtigsten Beobachtungen und Versuchen, in Alphabetischer Ordnung, Vorgestellt durch eine Gesellschaft von Gelehrten, Weidmann, Leipzig, xiv + 1044 p.

Kleynhans, C.J. \& James, N.P.E. (1995) Threatened fishes of the world: Chiloglanis bifurcus Jubb \& le Roux, 1969 (Mochokidae). Environmental Biology of Fishes, 43, 120.

Klunzinger, C.B. (1880) Die von Müller'sche Sammlung australischer Fische in Stuttgart. Sitzungsberichte der Kaiserlichen Akademie der Wissenschaften, Mathematisch- Naturwissenschaftlichen Classe, Wien, Abt. 1., Botanik, Zoologie, Anatomie, Geologie und Paläontologie, 80, 325-430, pls. 1-9.

Knaack, J. (1961) Ein neuer Panzerwels aus Brasilien (Corydoras guapore) (Pisces, Teleostei, Callichthyidae). Senckenbergiana Biologica, 42, 135-138.

Knaack, J. (1962) Zwei neue Panzerwelse, Corydoras haraldschultzi und Corydoras sterbai (Pisces, Teleostei, Callichthyidae). Senckenbergiana Biologica, 43, 129-135, pls. 11-12.

Knaack, J. (1966a) Ein Zwergpanzerwels - Corydoras pygmaeus. Die Aquarien- und Terrarien- Zeitschrift, 19, 168169.

Knaack, J. (1966b) Ein Zwergpanzerwels aus Brasilien: Corydoras pygmaeus. Neubeschreibung (Pisces, Teleostei, Callichthyidae). Aquarien-Terrar. Mschr. Ornith. Vivar, 13, 364-365.

Knaack, J. (1999a) A new species of bristlemouth catfish of the genus Ancistrus Kner, 1854 from the Mato Grosso, Brazil (Pisces, Siluriformes, Loricariidae). Tropical Fish Hobbyist, 47, 70-76.

Knaack, J. (1999b) A new species of suckermouth catfish (Hypostomus Lacépède, 1803) from the Mato Grosso, Brazil (Pisces, Siluriformes, Loricariidae). Tropical Fish Hobbyist, 47, 102-108.

Knaack, J. (1999c) Erstbeschreibung Corydoras spectabilis n. sp. VDA-aktuell, 1999, 74-79.

Knaack, J. (1999d) New Ancistrus species from the Rio Cuiba System, Brazil (Pisces, Siluriformes, Loricariidae). Tropical Fish Hobbyist, 47, 150-155.

Knaack, J. (1999e) Eine weitere neue Art der Gattung Corydoras Lacépède, 1803 aus dem Mato Grosso (Pisces, Siluriformes, Callichthyidae). Aquaristik Aktuell, 2000, 74-79. [Actually published in Dec. 1999.]

Knaack, J. (2000a) Ein weiterer neuer Harnischwels aus dem Rio Guaporé: Lasiancistrus guapore n. sp. Aquaristik Aktuell, 2000, 56-61.

Knaack, J. (2000b) Eine weitere neue Art der Gattung Corydoras La Cepède, 1803 aus dem Mato Grosso (Pisces, Siluriformes, Callichthyidae). VDA-aktuell, 2000, 45-56.

Knaack, J. (2001) Ein weiterer neuer Panzerwels aus Bolivien: Corydoras pantanalensis n. sp. (Pisces, Siluriformes, Callichthyidae). Aquaristik Aktuell, 2001, 58-63.

Knaack, J. (2002a) Corydoras bilineatus n. sp. (Pisces, Siluriformes, Callichthyidae). Aquaristik Aktuell, 10, 50-56.

Knaack, J. (2002b) Ein neuer Prachthexenwels aus Paraguay: Hemiloricaria aurata n. sp.(Pisces, Siluriformes, Loricariidae). Aquaristik aktuell, 11, for 2003, 56-61.

Knaack, J. (2002c) Ein weiterer neuer Panzerwels aus Bolivien: Corydoras cruziensis n. sp. (Pisces, Siluriformes, Callichthyidae). VDA-aktuell, 2002, 60-69.

Knaack, J. (2002d) Ein weiterer neuer Panzerwels aus Bolivien: Corydoras mamore n. sp. (Pisces, Siluriformes, Callichthyidae). VDA-aktuell, 2003, 16-24.

Knaack, J. (2004) Beschreibung von sechs neuen Arten der Gattung Corydoras La Cépède, 1803 (Teleostei: Siluriformes: Callichthyidae). Zoologische Abhandlungen; Staatliches Museums für Tierkunde in Dresden, 54, 55-105.

Kner, R. (1853a) Die Panzerwelse des K.K. Hof-naturalien-Cabinetes zu Wien. I Abtheilung: Loricarinae. Denkschriften der Mathematisch- Naturwissenschaftlichen Classe der Kaiserlichen Akademie der Wissenschaften in Wien, 6, for $1854,1-34$, pls. 1-8. [separate issued before volume]

Kner, R. (1853b) Über die Hypostomiden, oder die zweite Hauptgruppe der Panzerfische. Sitzungsberichte der Kaiserlichen Akademie der Wissenschaften, Mathematisch- Naturwissenschaftlichen Classe, Wien, 10, 278-282.

Kner, R. (1853c) Ueber einige Sexual-Unterschiede bei der Gattung Callichthys und die Schwimmblase bei Doras C. Val. Sitzungsberichte der Kaiserlichen Akademie der Wissenschaften, Mathematisch- Naturwissenschaftlichen Classe, Wien, 11, 138-146, 1 pl.

Kner, R. (1854) Die Hypostomiden. Zweite Hauptgruppe der Familie der Panzerfische. (Loricata vel Goniodontes). Den- 
kschriften der Mathematisch- Naturwissenschaftlichen Classe der Kaiserlichen Akademie der Wissenschaften in Wien, 7, 251-286, pls. 1-5.

Kner, R. (1855a) Ichthyologische Beiträge. Sitzungsberichte der Kaiserlichen Akademie der Wissenschaften, Mathematisch- Naturwissenschaftlichen Classe, Wien, 17, 92-162, pls. 1-6.

Kner, R. (1855b) Über ein neues genus aus der familie der Welse, Siluroidei. Sitzungsberichte der Kaiserlichen Akademie der Wissenschaften, Mathematisch-Naturwissenschaftichen Classe, Wien, 17, 313-316, pls. 1-2.

Kner, R. (1857) Ichthyologische Beiträge. II Abtheilung. Sitzungsberichte der Kaiserlichen Akademie der Wissenschaften, Mathematisch- Naturwissenschaftlichen Classe, Wien, 26, 373-448, pls. 1-9.

Kner, R. (1858) Kritische Bemerkungen über Castelnau's Siluroiden. Archiv für Naturgeschichte, 24, 344-350.

Kner, R. (1859) Zur Familie der Characinen. III.- Folge der Ichthyologischen Beiträge. Denkschriften der Mathematisch- Naturwissenschaftlichen Classe der Kaiserlichen Akademie der Wissenschaften in Wien, 17, 137-182, pls. 19.

Kner, R. (1863) Eine Uebersicht der ichthyologischen Ausbeute des Herrn Professors Dr. Mor. Wagner in CentralAmerika. Sitzungsberichte der Koningl. Bayerischen Akademie der Wissenschaften, München, 2, 220-230.

Kner, R. (1865) Fische. In: Reise der Österreichischen Fregatte "Novara" um die Erde in den Jahren 1857-59, Unter den Befehlen des Commodore B. von Wüllerstorf-Urbain. Zoologischer Theil, Wien, pp. 1-109, pls. 1-5.

Kner, R. (1866) Specielles Verzeichniss der während der Reise der kaiserlichen Fregatte "Novara" gesammelten Fische. III., und Schlussabtheilung. Sitzungsberichte der Kaiserlichen Akademie der Wissenschaften, Mathematisch- Naturwissenschaftlichen Classe, Wien, Abt. 1, Botanik, Zoologie, Anatomie, Geologie und Paläontologie, 53, 543-550.

Kner, R. (1867) Fische [continued]. In: Reise der Österreichischen Fregatte "Novara" um die Erde in den Jahren 185759, Unter den Befehlen des Commodore B. von Wüllerstorf-Urbain. Zoologischer Theil, Wien, pp. 275-433, pls. 1216.

Kner, R. \& Steindachner, F. (1864) Neue Gattungen und Arten von Fischen aus Central-Amerika; gesammelt von Prof. Moritz Wagner. Abhandlungen der Mathematisch-physikalischen Classe der Königlich Bayerischen Akademie der Wissenschaften, 10, 1-61, pls. 1-6.

Kner, R. \& Steindachner, F. (1867) Neue Fische aus dem Museum der Herren Joh. Ces. Godeffroy \& Sohn in Hamburg. Sitzungsberichte der Kaiserlichen Akademie der Wissenschaften, Mathematisch- Naturwissenschaftlichen Classe, Wien, Abth. 1, Botanik, Zoologie, Anatomie, Geologie und Paläontologie, 54, 356-395, pls. 1-5. [Separate issued with different pagination.]

Kobayakawa, M. (1989) Systematic revision of the catfish genus Silurus, with description of a new species from Thailand and Burma. Japanese Journal of Ichthyology, 36, 155-186.

Kobayakawa, M. \& Okuyama, S. (1994) Fossils of Silurus biwaensis (Siluridae) from the Ueno Formation, ancient Lake Biwa, Japan. Japanese Journal of Ichthyology, 40, 500-503.

Koch, W.R. (2002) Revisão taxonômica do gênero Homodiaetus (Teleostei, Siluriformes, Trichomycteridae). Iheringia, Série Zoologia, 92, 33-46.

Koch, W.R. \& Reis, R.E. (1996) Tatia boemia, a new species of authenipterid catfish (Teleostei: Siluriformes) from the rio Uruguai drainage, southern Brazil. Ichthyological Exploration of Freshwaters, 7, 85-90.

Kochetov. (1998) [A new species of Synodontis]. Aquarium, 2, 44-45. [In Russian; not seen]

Koken, E. (1884) Ueber Fish-Otolithen, insbesondere über diejenigen der nord-deutschen Oligocän-Ablagerungen. Zeitschrift der Deutschen Geologischen Gesellschaft, 36, 500-565, pls. 9-12.

Koken, E. (1885) Otolithen. In: A.V. Koenen, Uber eine palaeözane Fauna von Kopenhagen. Abhandlungen der Königlichen Gesellschaft der Wissenschaften zu Göttingen. Physikalische Klasse, 32, 113-116.

Koken, E. (1888) Neue Untersuchungen am tertiären Fisch-Otolithen, I. Zeitschrift der Deutschen Geologischen Gesellschaft, 40, 274-305, pls. 17-19.

Koken, E. (1891) Neue Untersuchungen am tertiären Fisch-Otolithen, II. Zeitschrift der Deutschen Geologischen Gesellschaft, 43, 77-170, pls. 1-10.

Koller, O. (1926) Einige neue Fischformen von der Insel Hainan. Anzeiger der Mathematisch- Naturwissenschaftlichen Classe der Kaiserlichen Akademie der Wissenschaften in Wien, 63, 74-77.

Koller, O. (1927) Fische von der Insel Hai-nan. Annalen des Naturhistorischen Museums in Wien, 41, 25-49, pl. 1.

König, C.D.E. (1825) Icones. Fossilium Sectiles. Centuria Prima, Fol, London, 4 p., 19 pls.

Kosygin, L. \& Vishwanath, W. (2005) Validity and redescription of Glyptothorax manipurensis Menon and record of Glyptothorax sinense (Regan) from India. Journal of the Bombay Natural History Society, 102, 61-65.

Kottelat, M. (1983) A new species of Erethistes Müller \& Troschel from Thailand and Burma (Osteichthyes: Siluriformes: Sisoridae). Hydrobiologia, 107, 71-74. 
Kottelat, M. (1984a) Catalogue des types du Musée d'Historie Naturelle de Neuchâtel. I.— Pisces. Bulletin de la Societe Neuchâteloise des Sciences Naturelles, 107, 143-153.

Kottelat, M. (1984b) A review of the species of Indochinese fresh-water fishes described by H. E. Sauvage. Bulletin du Muséum National d'Histoire Naturelle (série 4, section A), 6, 791-822.

Kottelat, M. (1985) Fresh-water fishes of Kampuchea. A provisory annotated check-list. Hydrobiologia, 121, $249-279$.

Kottelat, M. (1988) Authorship, dates of publication, status and types of Spix and Agassiz's Brazilian fishes. Spixiana, 11, 69-93.

Kottelat, M. (1989a) Zoogeography of the fishes from Indochinese inland waters with an annotated check-list. Bulletin Zoölogisch Museum, Universiteit van Amsterdam, 12, 1-56.

Kottelat, M. (1989b) On the validity of Phractocephalus Agassiz, 1829, vs. Pirarara Agassiz, 1829 (Osteichthyes: Pimelodidae). Spixiana, 12, 321.

Kottelat, M. (2000a) Diagnoses of a new genus and 64 new species of fishes from Laos (Teleostei: Cyprinidae, Balitoridae, Bagridae, Syngnathidae, Chaudhuriidae, and Tetraodontidae). Journal of South Asian Natural History, 5, 3782.

Kottelat, M. (2000b) Notes on taxonomy, nomenclature and distribution of some fishes of Laos. Journal of South Asian Natural History, 5, 83-90.

Kottelat, M. (2001a) Freshwater Fishes of Northern Vietnam. A Preliminary Check-list of the Fishes Known or Expected to Occur in Northern Vietnam with Comments on Systematics and Nomenclature, The World Bank, Environment and Social Development Unit, East Asia and Pacific Region, Washington, D.C., iii + $123+18$ p., 15 color pls.

Kottelat, M. (2001b) Fishes of Laos, WHT Publications, Colombo, Sri Lanka, 198 p.

Kottelat, M. \& Lim, K.K.P. (1995) Hemibagrus hoevenii, a valid species of Sundaic catfish (Teleostei: Bagridae). Malayan Nature Journal, 49, 41-47.

Kottelat, M., Lim, K.K.P. \& Ng, P.K.L. (1994) Bagrus hoevenii Bleeker, 1846 (currently Hemibagrus hoevenii; Osteichthyes, Siluriformes): proposed designation of a neotype. Bulletin of Zoological Nomenclature, 51, 320-322.

Kottelat, M. \& Ng, H.H. (1999) Belodontichthys truncatus, a new species of silurid catfish from Indochina (Teleostei: Siluridae). Ichthyological Exploration of Freshwaters, 10, 387-391.

Kottelat, M., Ng, H.H. \& Ng, P.K.L. (1998) Notes on the identity of Hemibagrus elongatus (Günther, 1864) and other east Asian species allied to H. guttatus (La Cepède, 1803) (Teleostei, Bagridae). The Raffles Bulletin of Zoology, 46, $565-572$.

Kottelat, M. \& Sutter, E. (1988) Catalogue des types de poissons du Musée d'histoire naturelle de Bâle (Naturhistorisches Museum Basel). Verhandlungen der Naturforschenden Gesellshaft in Basil, 98, 51-57.

Kottelat, M., Whitten, A.J., Kartikasari, S.N. \& Wirjoatmodjo, S. (1993) Freshwater Fishes of Western Indonesia and Sulawesi, Periplus Editions, Hong Kong, 259 p., 84 pl.

Kowarsky, J. (1976) Clarification of the name and distribution of the plotosid catfish Cnidoglanis macrocephalus. Copeia, 1976, 593-594.

Kulkarni, C. (1952) A new genus of schilbeid catfishes from the Deccan. Records of the Indian Museum, 49, 231-238.

Kullander, S.O., Fang, F., Delling, B. \& Åhlander, E. (1999) The fishes of the Kashmir Valley. In: Nyman, L. (Ed.), River Jhelum, Kashmir Valley: Impacts of the Aquatic Environment, SWEDMAR, pp. 99-167.

La Cepède, B.G.E. (1803) Histoire Naturelle des Poissons, Vol. 5, Plassan, Paris, lxviii + 803 p. + index, 21 pls.

La Monte, F. (1929) Two new fishes from Mt. Duida, Venezuela. American Museum Novitates, 373, 1-4.

La Monte, F. (1933a) A new subgenus of Plecostomus from Brazil. American Museum Novitates, 591, 1-2.

La Monte, F. (1933b) Pimelodus valenciennis Kr. the type of a new genus. Copeia, 1933, 226.

La Monte, F. (1935) Fishes from Rio Jurua and Rio Purus, Brazilian Amazonas. American Museum Novitates, $784,1-8$.

La Monte, F. (1939) Tridentopsis tocantinsi, a new pygidiid fish from Brazil. American Museum Novitates, $1024,1-2$.

La Monte, F. (1941) A new Corydoras from Brazil. Zoologica (New York), 26, 5-6.

Lacerda, M.T.C. \& Evers, H.-G. (1996) Parotocinclus-Arten aud Brasilien. Die Aquarien- und Terrarien-Zeitschrift, 49 , 88-95.

Ladiges, W. \& Voelker, J. (1961) Untersuchungen über die Fischfauna in Gebirgsgewässern des Wasserscheidenhochlands in Angola. Mitteilungen aus dem Hamburgischen Zoologischen Museum und Institut, 59, 117-140, pls. 3-7.

Ladiges, W., Wahlert, G. von \& Mohr, E. (1958) Die Typen und Typoide der Fischsammlung des Hamburgischen Zoologischen Staatsinstituts und Zoologischen Museums. Mitteilungen aus dem Zoologischen Museum in Berlin, 56, 155167.

Lambert, J.G. (1958) Poissons Siluriformes et Cyprinodontiformes récoltés en Guinée Française, avec la description d'une nouvelle espèce de Microsynodontis. Revue de Zoologie et de Botanique Africaines, 57, 39-56. 
Landim, M.I. \& Costa, W.J.E.M. (2002) Listrura tetraradiata (Siluriformes: Trichomycteridae): a new glanapterygine catfish from the southeastern Brazilian coastal plains. Copeia, 2002, 152-156.

Langeani, F. (1990) Revisão do gênero Neoplecostomus, com a descrição de quatro espécies novas do sudeste brasileiro (Ostariophysi, Siluriformes, Loricariidae). Comunicações do Museu de Ciências da PUCRS, Série Zoologia, Porto Alegre, 3, 3-31.

Langeani, F. \& Araujo, R.B. (1994) O gênero Rineloricaria Bleeker, 1862 (Ostariophysi, Siluriformes) na bacia do Rio Paraná superior: Rineloricaria pentamaculata sp. n. e Rineloricaria latirostris (Boulenger, 1900). Comunicações do Museu de Ciências da PUCRS, Série Zoologia, Porto Alegre, 7, 151-166.

Langeani, F., Oyakawa, O.T. \& Montoya-Burgos, J.I. (2001) New species of Harttia (Loricariidae, Loricariinae) from the Rio São Francisco basin. Copeia, 2001, 136-142.

Larrañaga, D.A. (1923) Escritos de Don Dámaso Antonio Larrañaga. Los Publica el Instituto Histórico y Geográphico del Uruguay, Edición Nacional, 512 p.

Lasso, C.A. (1990) Los peces de la Gran Sabana, Alto Caroni, Venezuela. Memoria, Sociedad de Ciencias Naturales La Salle, 49/50, 209-285.

Lasso, C.A. \& Provenzano, F. (1998) Chaetostoma vasquezi, nueva especie de corroncho del Escudo de Guayana, Estado Bolívar, Venezuela (Siluroidei: Loricariidae): descripción y consideraciones biogeográphicas. Memoria, Sociedad de Ciencias Naturales La Salle, 57, for 1997, 53-65.

Lasso, C.A. \& Provenzano, F. (2003) Dos nuevas especies de bagres del género Trichomycterus (Siluriformes: Trichomycteridae) de la Gran Sabana, Escudo de las Guayanas, Venezuela. Revista de Biología Tropical, 50, 1139-1149.

Latreille, P.A. (1825) Famillies Naturelles du Règne Animal Exposées Succinctement et dans un Ordre Analytique, avec l'Indication de leurs Genres, $2^{e}$ ed., Paris, 570 p.

Le Bail, P.-Y., Keith, P. \& Planquette, P. (2000) Atlas des Poissons d'Eau Douce de Guyane. Tome 2, Fascicule II, Siluriformes, Publications scientifiques du MNHN, Service du Patrimoine Naturel, Paris, 307 p.

Le Grande, W.H. (1981) Chromosomal evolution in North American catfishes (Siluriformes: Ictaluridae) with particular emphasis on the madtoms. Copeia, 1981, 33-52.

Lee, C.L. \& Kim, I.S. (1990) A taxonomic revision of the family Bagridae (Pisces: Siluriformes) from Korea. Korean Journal of Ichthyology, 2, 117-137.

Leege, C.O. (1922) Der Rumpfpanzer der Panzerwelse und seine Skelettbeziehungen (Plecostomus angipinnatus n. sp., Callichthys callichthys L. und Corydoras paleatus Jen.). Jenaische Zeitschrift fur Naturwissenschaft, 58, 145-270, pls. 9-10.

Lehmann A., P. \& Reis, R.E. (2004) Callichthys serralabium: a new species of neotropical catfish from the upper Orinoco and Negro rivers (Siluriformes: Callichthyidae). Copeia, 2004, 336-343.

Leidenfrost, G. (1925) Die fossilen Siluriden-Ungarns. Mitteilungen aus dem Jahrbuch der Kgl. Ungarischen Geologischen Anstalt, 24, 115-123, pls. 4-7.

Leidy, J. (1873a) Notice of remains of fishes in the Bridger Tertiary Formation of Wyoming. Proceedings of the Academy of Natural Sciences, Philadelphia, 1873, 97-99.

Leidy, J. (1873b) Contributions to the extinct vertebrate fauna of the western territories. Annual Report, United States Geological and Geographic Survey of the Territories, 1, for 1867, 1-358, $38 \mathrm{pl}$.

Leidy, J. (1889) Notice and description of fossils in caves and crevices of the limestone rocks of Pennsylvania. Annual Report, Geological Survey of Pennsylvania, for 1887, 1-20.

Lelek, A. (1987) The Freshwater Fishes of Europe. Vol. 9: Threatened Fishes of Europe, AULA-Verlag, Wiesbaden, 343 p.

Leriche, M. (1900) Faune ichthyologique des Sables à unios et térédines des environs d'Épernay (Marne). Annales de la Société Géologique du Nord, 29, 173-200.

Leriche, M. (1901) Contribution à l'étude des Siluridés fossiles. Annales de la Société Géologique du Nord, 30, 165-175.

Lesson, R.P. (1826-1831) Poissons. In: Duperrey, L.I. (Ed.), Voyage Autour du Monde, ..., sur la Corvette de La Majesté La Coquille, Pendant les Années 1822, 1823, 1824 et 1825... Vol. 5: Zoologie, Tome 2, Part 1, A. Bertrand, Paris, pp. 66-238, atlas: pls. 1-38. [Atlas to Vol. 5 apparently issued in 1826, text in 1831. See AMNH online catalog for details.]

Lesueur, C.A. (1819) Notice de quelques poissons découverts dans les lacs du Haut-Canada, durant l'été de 1816. Mémoires du Museum d'Histoire Naturelle, 5, 148-161, 2 pls.

Lévêque, C., Paugy, D. \& Teugels, G.G. (Eds.) (1992) Faune des Poissons d'Eaux Douces et Saumâtres de l'Afrique de l'Ouest. Vol. 2, Musée Royal de l'Afrique Central, Tervuren, and Editions de l'ORSTOM, Paris.

Lévêque, C., Paugy, D., Teugels, G.G. \& Romand, R. (1989) Inventaire taxonomique et distribution des poissons d'eau 
douce des bassins côtiers de Guinée et de Guinée Bissau. Revue d'Hydrobiologie Tropicale, 22, 107-127.

Leviton, A.E., Gibbs, J., R. H., Heal, E. \& Dawson, C.E. (1985) Standards in herpetology and ichthyology: Part I.-Standard symbolic codes for institutional resource collections in herpetology and ichthyology. Copeia, 1985, 802-832.

Leybold, F. (1859) Descripción de una nueva especie de pez, descubierto por don Federico Leybold en el Río Seco de los baños de Colina. Anales de la Universidad de Chile, 16, 1083-1085.

Li, G. \& Wang, J. (1979) [The taxonomy and description of Early Tertiary fossil fishes from Sanshui Basin and the adjacent districts of Guangdong]. Zhongshandaxue Xuebao, Zi Ran ke xue ban [=Acta Scientiarum Naturalium Universitatis Sunyatseni], 1979, 41-50. [In Chinese, with title and very brief abstract in English.]

Li, J., Chen, X. \& Chan, B.P.L. (2005) A new species of Pseudobagrus (Teleostei: Siluriformes: Bagridae) from southern China. Zootaxa, 1067, 49-57.

Li, S.-S. (1984a) [A study of infraspecies classification about Glyptothorax fukiensis (Rendahl) (comb. nov.)]. Journal of Yunnan University, 1984, 63-72. [In Chinese, with English summary]

Li, S.-S. (1984b) [A study of the classification of the striped chest sisorids (Glyptothorax Blyth) in China]. Journal of Yunnan University, 1984, 75-89. [In Chinese, with English summary]

Li, S.-S. (1986) Systematics, distribution and evolution of Glyptothorax (Siluriformes: Sisoridae). In: Uyeno, T., Arai, R., Taniuchi, T. \& Matsuura, K. (Eds.), Indo-Pacific Fish Biology. Proceedings of the Second International Conference on Indo-Pacific Fishes, Ichthyological Society of Japan, pp. 521-528.

Lichtenstein, M.H.C. (1819) Ueber einige neue Arten von Fishen aus der Gattung Silurus. Zoologisches Magazin (Wiedemann), 1, 57-63.

Lichtenstein, M.H.C. (1823) Verzeichniss der Doubletten des Zoologischen Museums der Königl. Universitat zu Berlin, nebst Beschreibung Vieler Bisher Unbekannter Arten von Säugethieren, Vögeln, Amphibien und Fischen, In Commission bei T. Trautwein, Berlin, $\mathrm{x}+118$ p., pl. 1.

Lilyestrom, C.G. (1984) Consideraciones sobre la taxonomia de las especies del genero Cochliodon Heckel en Venezuela (Pisces, Loricariidae). Revista UNELLEZ de Ciencia y Tecnologia, 2, 41-53.

Lim, K.K.P. \& Ng, H.H. (1999) Clarias batu, a new species of catfish (Teleostei: Clariidae) from Pulau Tioman, Peninsular Malaysia. The Raffles Bulletin of Zoology, Supplement, 6, 157-167.

Lima, F.C.T. \& Britto, M.R. (2001) New catfish of the genus Aspidoras (Siluriformes: Callichthyidae) from the upper Rio Paraguai system in Brazil. Copeia, 2001, 1010-1016.

Lima, S.M.Q. \& Costa, W.J.E.M. (2004) Trichomycterus giganteus (Siluriformes: Loricarioidea: Trichomycteridae): A new catfish from the Rio Guandu basin, southeastern Brazil. Zootaxa, 761, 1-6.

Lin, S.-Y. (1934) Three new fresh-water fishes of Kwangtung Province. Lingnan Science Journal, 13, $225-230$.

Lin, Y.-H. (2003) [A new species of the genus Glyptothorax Blyth from Guangdong, China (Siluriformes, Sisoridae)]. Acta Zootaxonomica Sinica, 28, 159-162. [In Chinese, with English abstract]

Linck, H.F. (1790) Versuch einer Eintheilung der Fische nach den Zähnen. Magazin für das Neueste aus der Physik und Naturgeschichte, Gotha, 6, 28-38.

Linder, R.S. \& Ng, H.H. (2002) Anmerkungen zu den Stachelwelsen der Gattung Bagrichthys (Teleostei, Bagridae). BSSW Report, 4, 4-9.

Linnaeus, C. (1754) Hans Maj:ts Adolf Frideriks vàr Allernàdigste Konungs Naturalie Samling Innehàllande Sällsynte och Främmande Djur, som Bevaras pà Kongl. Lust-slottet Ulriksdahl Beskrefne och Afritade Samt pà Nàdig Befallning Utgifne af Carl Linnaeus, Holmiae, $\mathrm{xxx}+96+7 \mathrm{p}$.

Linnaeus, C. (1758) Systema Naturae Sive Regna Tria Naturae, Secundum Classes, Ordines, Genera, Species, cum Characteribus, Differentiis, Synonymis, Locis.Tomus I. Editio decima, reformata, Holmiae, ii + 824 p.

Linnaeus, C. (1764) Museum S:ae R:ae M:tis Adolphi Friderici Regis Svecorum, Gothorum, Vandalorumque \&c. \&c. \&c. in quo Animalia Rariora Imprimis \& Exotica: Aves, Amphibia, Piscis Describuntur. Tomi Secundi Prodromus, Holmiae, 110 p.

Linnaeus, C. (1766) Systema Naturae Sive Regna Tria Naturae, Secundum Classes, Ordines, Genera, Species, cum Characteribus, Differentiis, Synonymis, Locis. 12th ed. Vol. 1, pt. 1, Laurentii Salvii, Holmiae, 532 p.

Littmann, M.W. (1998) Revision of the South American Shovelnose Catfishes of the Genus Sorubim (Siluriformes: Pimelodidae), with Descriptions of Two New Species. Unpublished M.Sc. thesis, Southern Illinois University, Carbondale.

Littmann, M.W., Burr, B.M. \& Buitrago-Suarez, U.A. (2001) A new cryptic species of Sorubim Cuvier (Siluriformes: Pimelodidae) from the upper and middle Amazon basin. Proceedings of the Academy of Natural Sciences, Philadelphia, 151, 87-93.

Littmann, M.W., Burr, B.M. \& Nass, P. (2000) Sorubim cuspicaudus, a new long-whiskered catfish from northwestern 
South America (Siluriformes: Pimelodidae). Proceedings of the Biological Society of Washington, 113, 900-917.

Littmann, M.W., Burr, B.M., Schmidt, R.E. \& Isern, E.R. (2001) Sorubim elongatus, a new species of catfish (Siluriformes: Pimelodidae) from tropical South America syntopic with S. lima. Ichthyological Exploration of Freshwaters, 12, 1-16.

Liu, C.-X., Peng, Z.-G. \& He, S.-P. (2005) Studies in species classification for genus Cranoglanis Peters with the method of morphometrics. Acta Hydrobiologia Sinica, 29, 507-512. [In Chinese, with English abstract]

Liu, H.T. \& Su, T.T. (1962) [Pliocene fishes from Yushe Basin, Shansi]. Vertebrate Palasiatica (Beijing), 6, 1-47. [In Chinese, with English summary.]

Lohberger, K. (1930) Fische aus dem Wamifluss. Zoologischer Anzeiger, 89, 88-94.

Lönnberg, E. (1895) Notes on fishes collected in the Cameroons by Mr. Y. Sjöstedt. Öfversigt af Kongl. Vetenskapsakademiens Forhandlingar, 1895, 179-195.

Lönnberg, E. (1924) Two new silurids from lower Congo. Annals and Magazine of Natural History (Ser. 9), 14, 619-621.

Lönnberg, E. \& Rendahl, H. (1920) On some freshwater fishes from Lower Congo. Annals and Magazine of Natural History (Ser. 9), 6, 167-176.

Lönnberg, E. \& Rendahl, H. (1922) Some new silurids from the Congo. Annals and Magazine of Natural History (Ser. 9), 10, 122-127.

Lönnberg, E. \& Rendahl, H. (1930) Eine neue Art der Gattung Corydoras. Arkiv för Zoologi, 22 A, 1-6.

López, H.L. \& Miquelarena, A.M. (1991a) Los Hypostominae (Pisces: Loricariidae) de Argentina. Fauna de Agua Dulce de la República Argentina, 40, Pisces, 1-64.

López, H.L. \& Miquelarena, A.M. (1991b) Peces loricaridos de la Cuenca de Plata, Argentina. Parte I.— El genero Cochliodon Heckel, 1854 (Pisces: Siluriformes). Gayana Zoologica, 55, 3-11.

Lopez-Fernandez, H. \& Winemiller, K.O. (2000) A review of Venezuelan species of Hypophthalmus (Siluriformes: Pimelodidae). Ichthyological Exploration of Freshwaters, 11, 35-46.

Loubens, G. \& Panfili, J. (2000) Biologie de Pseudoplatystoma fasciatum et P. tigrinum (Teleostei: Pimelodidae) dans le bassin du Mamoré (Amazonie Bolivienne). Ichthyological Exploration of Freshwaters, 11, 13-34.

Lowe-McConnell, R.H. (1964) The fishes of the Rupununi savanna district of British Guiana, South America. Part 1.Ecological groupings of fish species and effects of the seasonal cycle on the fish. The Journal of the Linnean Society of London. Zoology, 45, 103-144.

Lucena, C.A.S. de \& da Silva, J.F.P. (1991) Descrição de uma nova espécie do gênero Rhamdella Eigenmann \& Eigenmann, 1888 (Siluriformes: Pimelodidae) para o médio rio Uruguai, sul do Brasil. Comunicações do Museu de Ciências da PUCRS, Série Zoologia, Porto Alegre, 4, 28-47.

Lucena, C.A.S. de, Malabarba, L.R. \& Reis, R.E. (1992) Resurrection of the Neotropical pimelodid catfish Parapimelodus nigribarbis (Boulenger), with a phylogenetic diagnosis of the genus Parapimelodus (Teleostei: Siluriformes). Copeia, 1992, 138-146.

Lucena, Z.M.S. \& Lucena, C.A.S. de. (1990) Sobre a localidade-tipo das espécies de peixes descritas por Steindachner (1907). Comunicações do Museu de Ciências da PUCRS, Série Zoologia, Porto Alegre, 3, 99-101.

Lundberg, J.G. (1975) The fossil catfishes of North America. Claude W. Hibbard Memorial, Volume 2. University of Michigan, Papers on Paleontology, 11, i-iv, 1-51.

Lundberg, J.G. (1982) The comparative anatomy of the toothless blindcat, Trogloglanis pattersoni Eigenmann, with a phylogenetic analysis of the ictalurid catfishes. Miscellaneous Publications, Museum of Zoology, University of Michigan, 163, 1-85.

Lundberg, J.G. (1992) The phylogeny of ictalurid catfishes: A synthesis of recent work. In: Mayden, R.L. (Ed.), Systematics, Historical Ecology, \& North American Freshwater Fishes, Stanford Univ. Press, Stanford, CA, pp. $392-420$.

Lundberg, J.G. (1997) Fishes of the La Venta fauna: additional taxa, biotic and paleoenvironmental implications, In: Kay, R.F., et al. (Eds.), Vertebrate Paleontology in the Neotropics: The Miocene Fauna of La Venta, Colombia, Smithsonian Institution Press, Washington, pp. 67-91.

Lundberg, J.G. (1998) The temporal context for diversification of Neotropical fishes. In: Malabarba, L.R., Reis, R.E., Vari, R.P., Lucena, Z.M.S. \& Lucena, C.A.S. (Eds.), Phylogeny and Classification of Neotropical Fishes, Edipucrs, Porto Alegre, pp. 49-68.

Lundberg, J.G. (2005) Brachyplatystoma promagdalena n. sp., a fossil goliath catfish (Siluriformes: Pimelodidae) from the Miocene of Colombia, South America. Neotropical Ichthyology, 3, 597-605.

Lundberg, J.G. \& Aguilera, O. (2003) The late Miocene Phractocephalus catfish (Siluriformes: Pimelodidae) from Urumaco, Venezuela: additional specimens and reinterpretation as a distinct species. Neotropical Ichthyology, 1, 97109. 
Lundberg, J.G. \& Akama, A. (2005) Brachyplatystoma capapretum: a new species of goliath catfish from the Amazon basin, with a reclassification of allied catfishes (Siluriformes: Pimelodidae). Copeia, 2005, 492-516.

Lundberg, J.G. \& Baskin, J.N. (1969) The caudal skeleton of the catfishes, order Siluriformes. American Museum Novitates, 2398, 1-49.

Lundberg, J.G., Bornbusch, A.H. \& Mago-Leccia, F. (1991) Gladioglanis conquistador n. sp. from Ecuador with diagnoses of the subfamilies Rhamdiinae Bleeker and Pseudopimelodinae n. subf. (Siluriformes: Pimelodidae). Copeia, 1991, 190-209.

Lundberg, J.G. \& Case, G.R. (1970) A new catfish from the Eocene Green River Formation, Wyoming. Journal of Paleontology, 44, 451-457.

Lundberg, J.G., Linares, O., Nass, P. \& Antonio, M.E. (1988) Phractocephalus hemioliopterus (Pimelodidae: Siluriformes) from the Late Miocene Urumaco formation, Venezuela: A further case of evolutionary stasis and local extinction among South American fishes. Journal of Vertebrate Paleontology, 8, 131-138.

Lundberg, J.G. \& Littmann, M.W. (2003) Pimelodidae. In: Reis, R.E., Kullander, S.O. \& Ferraris, C.J., Jr. (Eds.), Check list of the Freshwater Fishes of South and Central America, Edipucrs, Porto Alegre, Brazil, pp. 432-446.

Lundberg, J.G., Mago-Leccia, F. \& Nass, P. (1991) Exallodontus aguanai, a new genus and species of Pimelodidae (Pisces: Siluriformes) from deep river channels of South America, and delimitation of the subfamily Pimelodinae. Proceedings of the Biological Society of Washington, 104, 840-869.

Lundberg, J.G. \& McDade, L.A. (1986) On the South American catfish Brachyrhamdia imitator Myers (Siluriformes, Pimelodidae), with phylogenetic evidence for a large intrafamilial lineage. Notulae Naturae (Philadelphia), 463, 124.

Lundberg, J.G., Nass, P. \& Mago-Leccia, F. (1989) Pteroglanis manni Eigenmann and Pearson, a juvenile of Sorubimichthys planiceps (Agassiz), with a review of the nominal species of Sorubimichthys (Pisces: Pimelodidae). Copeia, 1989 ,

Lundberg, J.G. \& Parisi, B.M. (2002) Propimelodus, new genus, and a description of Pimelodus eigenmanni van der Stigchel, 1946, a long recognized yet poorly-known South American catfish (Pimelodidae: Siluriformes). Proceedings of the Academy of Natural Sciences, Philadelphia, 152, 75-88.

Lundberg, J.G. \& Rapp Py-Daniel, L. (1994) Bathycetopsis oliveirai, gen. et sp. nov., a blind and depigmented catfish (Siluriformes: Cetopsidae) from the Brazilian Amazon. Copeia, 1994, 381-390.

Lütken, C.F. (1874a) Ichthyographiske bidrag. I.- Nogle nye eller mindre fuldstaendigt kjendte Pandsermaller, isaer fra det nordlige Sydamerica. Videnskabelige Meddelelser fra den Naturhistorisk Forening i Kjфbenhavn, 13/14, for 1873, 202-220, pl. 4.

Lütken, C.F. (1874b) Ichthyographiske Bidrag. II.- Nye eller mindre vel kjendte Malleformer fra forskjellige Verdensdele. Videnskabelige Meddelelser fra den Naturhistorisk Forening i Kjфbenhavn, for 1874, 190-220.

Lütken, C.F. (1874c) Siluridae novae Brasiliae centralis a clarissimo J. Reinhardt in provincia Minas-geraës circa oppidulum Lagoa Santa, praecipue in flumine Rio das Velhas et affluentibus collectae, secundum caracteres essentiales, breviter descriptae. Oversigt over Selskabets verksomhed, Kongelige Danske Videnskabernes selskab., Kjobenhavn, $1873,29-36$.

Lütken, C.F. (1875) Velhas-Flodens Fiske. Et Bidrag til Brasiliens Ichthyologi; efter Professor J. Reinhardts Indsamlinger og Optegnelser. Det Kongelige Danske Videnskabernes Selskabs Skrifter, Raekke 5, 12, 121-253, + 2 unnum., $+\mathrm{i}-\mathrm{xxi}$, pls. $1-5$.

Lütken, C.F. (1892) Om en med stegophiler og tricomycterer beslaegtet sydamerikansk mallefisk (Acanthopoma annectens Ltk. n. g. \& sp.?). Videnskabelige Meddelelser fra den Naturhistorisk Foreningi Kjфbenhavn, for 1891, 53-60.

Lydekker, R. (1886) Indian Tertiary and post-Tertiary vertebrata: Tertiary Fishes. Memoirs of the Geological Society of India, Palaeontologia Indica, Series 10, 3, 241-258, pls. 35-37.

Lynn, W.G. \& Melland, A.M. (1939) A fossil catfish (Felichthys stauroforus) from the Maryland Miocene. Journal of the Washington Academy of Sciences, 29, 14-20.

MacDonagh, E.J. (1931) Notas zoológicas de una excursión entre Patagones y San Blas. Notas Preliminares del Museo de La Plata, 1, 63-86.

MacDonagh, E.J. (1937) Sobre el Manguruyú (gênero Paulicea, Silurideos). Revista del Museo de La Plata (Nueva serie), 1, 3-30.

MacDonagh, E.J. (1938) Contribución a la sistemática y etología de los peces fluviatiles Argentinos. Revista del Museo de La Plata, Seccion Zoologia, 1, 119-208, pls. 1-5.

Mack. (1861) Trav. Ussuri (?= Puteschestvie po dolinge ryeki Usuri), 1, 195-198. [Not seen]

Macleay, W. (1881) A descriptive catalogue of Australian fishes.- Part IV. Proceedings of the Linnean Society of New 
South Wales, 6, 202-387.

Macleay, W. (1883) Contribution to a knowledge of the fishes of New Guinea.- - No. 4. Proceedings of the Linnean Society of New South Wales, 8, 252-280.

Magalhães, A.C. (1931) Monographia Brazileira de Peixes Fluviaes. Graphicars, São Paulo, 255 p.

Mago-Leccia, F. (1984) Entomocorus gameroi, una nueva especie de bagre auquenipterido (Teleostei, Siluriformes) de Venezuela, incluyendo la descripcion de su dimorfismo sexual secundario. Acta Biologica Venezuelica, 11, $215-236$.

Mai, D.Y. (1978) [Identification of the Fresh-water Fishes of North Viet Nam], Scientific \& Technology Publisher, Ha Noi, 340 p., 48 pl. [In Vietnamese. Selected parts translated into English in Kottelat (2001:74 et seq.).]

Malabarba, L.R. \& Mahler, J.K.F., Jr. (1998) Review of the genus Microglanis in the rio Uruguay and coastal drainages of southern Brazil (Ostariophysi: Pimelodidae). Ichthyological Exploration of Freshwaters, 9, 243-254.

Maldonado-Ocampo, J.A., Ortega-Lara, A., Usma Oveido, J.S., Galvis Vergara, G., Villa-Navarro, F.A., Vásquez Gamboa, L., Prada-Pedreros, S. \& Ardila-Rodríguez, C.A. (2005) Peces de los Andes de Colombia: Guía de Campo. Instituto de Investigación de Recursos Biológicos Alexander von Humboldt, Bogotá, Colombia, 346 pp.

Manimekalan, A. \& Arunachalam, M. (2002) Rediscovery of critically endangered air breathing cat fish Clarias dayi Hora (Pisces: Claridae) from Mudumalai Wildlife Sanctuary, Tamil Nadu. Journal of the Bombay Natural History Society, 99, 129-131.

Manimekalan, A. \& Das, H.S. (1998) Glyptothorax davissinghi (Pisces: Sisoridae) a new cat fish from Nilambur in the Nilgiri Biosphere Reserve, South India. Journal of the Bombay Natural History Society, 95, 87-91.

Marceniuk, A.P. (2003) Relações Filogenéticas e Revisão dos Gêneros da Família Ariidae (Ostariophysi, Siluriformes). Ph. D. Dissertation, Universidade de São Paulo, São Paulo.

Marceniuk, A.P. (2005a) Chave para identificação des espécies de bagres marinhos (Siluriformes, Ariidae) da costa Brasileira. Boletim do Instituto do Pesca, São Paulo, 31, 89-101.

Marceniuk, A.P. (2005b) Redescrição de Genidens barbus (Lacépède, 1803) e Genidens machadoi (Miranda-Ribeiro, 1918), bagres marinhos (Siluriformes, Ariidae) do Atlântico sul ocidental. Papéis Avulsos de Zoologia, 45, 111-125.

Marceniuk, A.P. \& Ferraris, C.J., Jr. (2003) Ariidae. In: Reis, R.E., Kullander, S.O. \& Ferraris, C.J., Jr. (Eds.), Check list of the Freshwater Fishes of South and Central America, Edipucrs, Porto Alegre, Brazil, pp. 447-455.

Marcgravius, G. (1648) Historia Naturalis Brasiliae, Auspicio et Beneficio Illustriss. I. Mauritii Com. Nassau Illius Provincjae et Maris Summi Praefecti Adornata in qua non Tantum Plantae et Animalia, sed et Indigenarum Morbi, Ingenia et Mores Describuntur et Iconibus Supra Quingentas Illustrantur, Lugduni Batavorum \& Amstellodami, $300 \mathrm{p}$.

Marck, W. von der. (1876a) Fossile fische von Sumatra. In: H.B. Geinitz \& W. von der Marck, Zur Geologie von Sumatra. Palaeontographica, 22, 405-414, 2 pl. [Also: 1878, Jaarb. Mijnw. Ned. Oost-Indië, 7, 138-155]

Marck, W. von der. (1876b) Fossile fische von Sumatra. Mitteilungen aus dem Königlichen Mineralogisch-Geologischen und Prähistorischen Museum in Dresden, 2, 7-16, 2 pls.

Marck, W. von der \& Schlüter, C. (1868) Neue Fische und Krebse aus der Kreide von Westphalen. Palaeontographica, 15, 269-305, pls. 41-44.

Marini, T.L., Nichols, J.T. \& La Monte, F.R. (1933) Six new eastern South American fishes examined in the American Museum of Natural History. American Museum Novitates, 618, 1-7.

Markle, D.F. (1997) Audubon's hoax: Ohio River fishes described by Rafinesque. Archives of Natural History, 24, 439447.

Marschall, A. (1873) Nomenclator Zoologicus. Continens Nomina Systematica Generum Animalium tam Viventium quam Fossilium, Secondum Ordinem Alphabeticum Disposita, Vindobonae, iv + 482 p.

Martín Salazar, F.J. (1964) Las especies del genero Farlowella de Venezuela (Pisces — Nematognathi — Loricariidae) Con descripcion de 5 especies y 1 sub-especies nuevas. Memoria, Sociedad de Ciencias Naturales La Salle, 24, 242-261.

Martín Salazar, F.J., Isbrücker, I.J.H. \& Nijssen, H. (1982) Dentectus barbarmatus, a new genus and species of mailed catfish from the Orinoco Basin of Venezuela (Pisces, Siluriformes, Loricariidae). Beaufortia, 32, $125-137$.

Matthes, H. (1959a) Poissons nouveaux du Ruanda. Folia Scientifica Africae Centralis, 5, 62.

Matthes, H. (1959b) Poissons nouveaux du Lac Tanganika. Descriptions préliminaires. Folia Scientifica Africae Centralis, 5, 77-78.

Matthes, H. (1962) Poissons nouveaux ou intéressants du lac Tanganyika et du Ruanda. Annales du Musée Royal de l'Afrique Centrale, Series in $8^{\circ}$, Sciences Zoologiques, 111, 27-88, pls. 1-4.

Matthes, H. (1964) Les poissons du lac Tumba et de la region d'Ikela. Étude systématique et écologique. Annales du Musée Royal de l'Afrique Centrale, Series in 8o, Sciences Zoologiques, 126, 1-204, map, table, pls. 1-6. 
Matthes, H. (1965) Quelques poissons nouveaux du Congo. Revue de Zoologie et de Botanique Africaines, 71, $177-193$.

Mazzoni, R., Caramaschi, U. \& Weber, C. (1994) Taxonomical revision of the species of Hypostomus Lacépède, 1803 (Siluriformes, Loricariidae) from the lower rio Paraiba do Sul, state of Rio de Janeiro, Brazil. Revue suisse de Zoologie, Annales de la Société zoologique suisse et du Muséum d'Histoire naturelle de Genève, 101, 3-18.

Mbega, J.D. \& Teugels, G.G. (1998) Révision systématique du genre Anaspidoglanis (Siluriformes: Claroteidae) de l'Afrique Centrale. Cybium (3e série), 22, 223-236.

M'Clelland, J. (1842) On the fresh-water fishes collected by William Griffith, Esq., F. L. S. Madras Medical Service, during his travels under the orders of the Supreme Government of India, from 1835 to 1842. Calcutta Journal of Natural History, and Miscellany of the Arts and Sciences in India, 2, 560-589, pls. 6, 15, 18, $20,21$.

M'Clelland, J. (1844a) Description of a collection of fishes made at Chusan and Ningpo in China, by Dr. G. R. Playfair, Surgeon of the Phlegethon, war steamer, during the late military operations in that country. Calcutta Journal of Natural History, and Miscellany of the Arts and Sciences in India, 4, for 1843, 390-413, pls. 21-25.

M'Clelland, J. (1844b) Apodal fishes of Bengal. Calcutta Journal of Natural History, and Miscellany of the Arts and Sciences in India, 5, 151-226, pls. 5-14.

Meek, S.E. (1902) A contribution to the ichthyology of Mexico. Field Columbian Museum, Zoological Series, 3, 63-128, pls. 14-31.

Meek, S.E. (1904) The fresh-water fishes of Mexico north of the isthmus of Tehuantepec. Field Columbian Museum, Zoological Series, 5, i-xiii + 1-252, pls. 1-17.

Meek, S.E. (1905) Two new species of fishes from Brazil. Proceedings of the Biological Society of Washington, 18, 241242.

Meek, S.E. (1906) Description of three new species of fishes from Middle America. Field Columbian Museum, Zoological Series, 7, 91-95.

Meek, S.E. (1907a) Synopsis of the fishes of the great lakes of Nicaragua. Field Columbian Museum, Zoological Series, 7, 97-132.

Meek, S.E. (1907b) Notes on fresh-water fishes from Mexico and Central America. Field Columbian Museum, Zoological Series, 7, 133-157.

Meek, S.E. (1909) New species of fishes from tropical America. Field Columbian Museum, Zoological Series, 7, 207211.

Meek, S.E. \& Hildebrand, S.F. (1913) New species of fishes from Panama. Publication. Zoological Series, Field Museum of Natural History, 10, 77-91.

Meek, S.E. \& Hildebrand, S.F. (1916) The fishes of the fresh waters of Panama. Publication. Zoological Series, Field Museum of Natural History, 10, 217-374, pls. 6-32.

Meek, S.E. \& Hildebrand, S.F. (1923) The marine fishes of Panama.- Part I. Publication. Zoological Series, Field Museum of Natural History, 15, i-xi + 1-330, pls. 1-24.

Mees, G.F. (1967) Freshwater fishes of Suriname: the genus Heptapterus (Pimelodidae). Zoologische Mededelingen (Leiden), 42, 215-229.

Mees, G.F. (1974) The Auchenipteridae and Pimelodidae of Suriname (Pisces, Nematognathi). Zoologische Verhandelingen (Leiden), 132, 1-256, pls. 1-15.

Mees, G.F. (1978a) On the identity of Arius oncinus R. H. Schomburgk (Pisces, Nematognathi, Auchenipteridae). Zoologische Mededelingen (Leiden), 52, 267-276.

Mees, G.F. (1978b) Two new species of Pimelodidae from northwestern South America (Pisces, Nematognathi). Zoologische Mededelingen (Leiden), 53, 253-261, pls. 1-3.

Mees, G.F. (1983) Naked catfishes from French Guiana (Pisces, Nematognathi). Zoologische Mededelingen (Leiden), 57, 43-58.

Mees, G.F. (1984) A note on the genus Tocantinsia (Pisces, Nematognathi, Auchenipteridae). Amazoniana, 9, 31-34.

Mees, G.F. (1985) Further records of Auchenipteridae and Pimelodidae from Suriname (Pisces: Nematognathi). Zoologische Mededelingen (Leiden), 59, 239-249.

Mees, G.F. (1986) Records of Auchenipteridae and Pimelodidae from French Guiana (Pisces, Nematognathi). Proceedings of the Koninklijke Nederlandse Akademie van Wetenschappen (Series C), 89, 311-325.

Mees, G.F. (1987a) A new species of Heptapterus from Venezuela (Pisces, Nematognathi, Pimelodidae). Proceedings of the Koninklijke Nederlandse Akademie van Wetenschappen (Series C), 90, 451-456.

Mees, G.F. (1987b) The members of the subfamily Aspredininae, family Aspredinidae in Suriname (Pisces, Nematognathi). Proceedings of the Koninklijke Nederlandse Akademie van Wetenschappen (Series C), 90, 173-192.

Mees, G.F. (1988a) Notes on the genus Tatia (Pisces, Nematognathi, Auchenipteridae). Proceedings of the Koninklijke 
Nederlandse Akademie van Wetenschappen (Series C), 91, 405-414.

Mees, G.F. (1988b) The genera of the subfamily Bunocephalinae (Pisces, Nematognathi, Aspredinidae). Proceedings of the Koninklijke Nederlandse Akademie van Wetenschappen (Series C), 91, 85-102.

Mees, G.F. (1989) Notes on the genus Dysichthys, subfamily Bunocephalidae, family Aspredinidae (Pisces, Nematognathi). Proceedings of the Koninklijke Nederlandse Akademie van Wetenschappen (Series C), 92, 189-250.

Mees, G.F. (1997) The type species of the genera Bunocephalus Kner, 1855, and Bunocephalichthys Bleeker, 1858 (Pisces, Aspredinidae, Bunocephalinae). Proceedings of the Koninklijke Nederlandse Akademie van Wetenschappen (Series C), 99, 225-228.

Mees, G.F. \& Cala, P. (1989) Two new species of Imparfinis from northern South America (Pisces, Nematognathi, Pimelodidae). Proceedings of the Koninklijke Nederlandse Akademie van Wetenschappen (Series C), 92, 379-394.

Meinken, H. (1937) Beiträge zur Fischfauna des mittleren Paranà — III. Blätter für Aquarien- und Terrarien-Kunde, 48, 73-80.

Meinken, H. (1957) Mitteilungen der Fischbestimmungsstelle des VDA. XXIV.— Über zwei der Liebhaberei bislang unbekannte Corydoras-Neuheiten (Callichthyidae — Ostariophysi). Die Aquarien- und Terrarien- Zeitschrift, 10, 47.

Menon, A.G.K. (1950) On a remarkable blind siluroid fish of the family Clariidae from Kerala (India). Records of the Indian Museum, 48, 59-66, pl. 1.

Menon, A.G.K. (1951) On certain features in the anatomy of Horaglanis Menon. Journal of the Zoological Society of India, 3, 249-253.

Menon, A.G.K. (1955) Further observations on the fish fauna of the Manipur State. Records of the Indian Museum, 52, for 1954, 21-26.

Menon, A.G.K. (1999) Check list, freshwater fishes of India. Records of the Zoological Survey of India, Miscellaneous Publication, Occasional Paper, 175, i-xxviiii + 1-366.

Menon, A.G.K. \& Rama Rao, K.V. (1974) On the dates of the parts of Day's 'Fishes of India'. Journal for the Society for the Bibliography of Natural History, 7, 143.

Menon, A.G.K. \& Yazdani, G.M. (1968) Catalogue of type-specimens in the Zoological Survey of India, Part 2.- Fishes. Records of the Zoological Survey of India, 61, for 1963, 91-190.

Menon, M.A.S. (1955) Notes on fishes of the genus Glyptothorax Blyth. Records of the Indian Museum, 52, for 1954, 27-54.

Meuschen, F.C. (1778) Museum Gronovianum. Sive, Index Rerum Naturalium, tam Mammalium, Amphibiorum, Piscium, Insectorum, ... , T. Haak, J. Meerburg, Lugundi Batavorum.

Meyen, F.J.F. (1834-1835) Reise um die Erde Ausgeführt auf dem Königlich Preussischen Seehandlungs-schiffe Prinzess Louise, Commandirt von Captain W. Wendt, in den Jahren 1830, 1831 und 1832; Historischer Hericht, Sander'sche Buchhandlung, Berlin.

Miao, C.P. (1934) Notes on the fresh-water fishes of the southern part of Kiangsu, I.- Chinkiang. Contributions from the Biological Laboratory of the Science Society of China, Zoological Series, 10, 111-244.

Miles, C. (1942) Descripción sistematica del "pez graso" del Lago de Tota (Boyacá). Caldasia, 5, 55-58.

Miles, C. (1943a) Estudio Economico y Ecologico de Los Peces de Agua Dulce del Valle de Cauca, Departamento del Valle de Cauca, Cali, Colombia, 97 p.

Miles, C. (1943b) On three recently described species and a new genus of pygidiid fishes from Colombia. Revista de la Academia Colombiana de Ciencias Exactas, Fisicas y Naturales, 5, 367-369.

Miles, C. (1945) Some newly recorded fishes from the Magdalena River system. Caldasia, 3, 453-464.

Miles, C. (1947) Los Peces del Río Magdalena, Ministerio de la Economia Nacional; seccion de piscicultura, pesca y caza, Bogota, 214 p., appendices.

Miller, G.S., Jr. (1905) Notes on the generic names Pteronotus and Dermonotus. Proceedings of the Biological Society of Washington, 18, 223.

Miller, R.R. (1984) Rhamdia reddelli, new species, the first blind pimelodid catfish from Middle America, with a key to the Mexican species. Transactions of the San Diego Society of Natural History, 20, 135-144.

Miller, R.R., Minckley, W.L. \& Norris, S.M. (2005) Freshwater Fishes of México, University of Chicago Press, Chicago, XXv +490 p., 99 pl.

Miller, R.R. \& Smith, G.R. (1967) New fossil fishes from Plio-Pleistocene Lake Idaho. Occasional Papers of the Museum of Zoology, University of Michigan, 654, 1-24.

Minding, J. (1832) Lehrbuch der Naturgeschichte der Fische, Berlin, xii + 132 p., 6 pls.

Miquelarena, A.M. \& Menni, R.C. (1999) Rhamdella aymarae, a new species from the Itiyuro River, northern Argentina 
(Siluriformes: Pimelodidae). Ichthyological Exploration of Freshwaters, 10, 201-210.

Miranda Ribeiro, A. (1906) Vertebrados do Itatiaya (Peixes, Serpentes, Saurios, Aves e Mammiferos). Resultados de excursões do Sr. Carlos Moreira, Assistente de Secção de Zoologia do Museu Nacional. Arquivos do Museu Nacional do Rio de Janeiro, 13, for 1905, 165-190, pls. 1-3.

Miranda Ribeiro, A. (1907a) Peixes do Iporanga - S. Paulo. Resultados de excursões do Sr. Ricardo Krone, membro correspondente do Museu Nacional do Rio de Janeiro. Boletim Sociedade Nacional de Agricultura, Rio de Janeiro [Lavoura], 11, 185-190.

Miranda Ribeiro, A. (1907b) Uma novidade ichthyologica. - Typhlobagrus kronei. Kosmos, Rio de Janeiro, 4, [1-3].

Miranda Ribeiro, A. (1908a) On fishes from the Iporanga River. S. Paulo-Brasil. Arkiv för Zoologi, 4, for 1907, 1-5, pl. 1.

Miranda Ribeiro, A. (1908b) Peixes da Ribeira. Resultados de excursão do Sr. Ricardo Krone, membro correspondente do Museu Nacional do Rio de Janeiro. Kosmos, Rio de Janeiro, 5, [1-5].

Miranda Ribeiro, A. (1911) Fauna brasiliense. Peixes. Tomo IV (A) [Eleutherobranchios Aspirophoros]. Arquivos do Museu Nacional do Rio de Janeiro, 16, 1-504, pls. 22-54.

Miranda Ribeiro, A. (1912) Loricariidae, Callichthyidae, Doradidae e Trichomycteridae. In: Commissão de Linhas Telegraphicas Estrategicas de Matto-Grosso ao Amazonas, Matto-Grosso, Annexo no. 5 (Historia Natural: Zoologia). 1-31 p., 1 pl.

Miranda Ribeiro, A. (1914) Pimelodidae, Trachycorystidae, Cetopsidae, Bunocephalidae, Auchenipteridae, e Hypophthalmidae. In: Commissão de Linhas Telegraphicas Estrategicas de Matto-Grosso ao Amazonas, Matto-Grosso, Annexo no. 5 (Historia Natural: Zoologia). 1-13 p., pls. 1-2.

Miranda Ribeiro, A. (1917) De scleracanthis._- Fluvio "Solimões" anno MCMVIII a cl. F. Machado da Silva duce brasiliense inventis et in Museu Urbis "Rio de Janeiro" servatis. Revista da Sociedade Brasileira de Sciências, 1, 4952.

Miranda Ribeiro, A. (1918a) Hemipsilichthys, Eignm. \& Eignm., e generos alliados. Revista da Sociedade Brasileira de Sciências, 2, 101-107, pls. 1-7.

Miranda Ribeiro, A. (1918b) Nova chave para a determinação das espécies do gênero Tachysurus. Revista da Sociedade Brasileira de Sciências, 2, 108-111.

Miranda Ribeiro, A. (1918c) Ancistrus. Revista da Sociedade Brasileira de Sciências, 2, 112-114.

Miranda Ribeiro, A. (1918d) Tres generos e dezesete especies novas de peixes Brasilieros determinados nas colleções do Museu Paulista. Revista do Museu Paulista, 10, 631-646, 1 pl.

Miranda Ribeiro, A. (1918e) Lista dos peixes Brasileiros do Museu Paulista, Primeira parte and Terceira parte. Revista do Museu Paulista, 10, 705-736, 759-783.

Miranda Ribeiro, A. (1918f) Considerações sôbre o gênero Brachyplatystoma e Platystomatichthys de Bleeker. Revista do Museu Paulista, 10, 247-283.

Miranda Ribeiro, A. (1920) Peixes (excl. Characinidae). Commissão de Linhas Telegraphicas Estrategicas de MattoGrosso ao Amazonas, Annexo no. 5 (Historia Natural: Zoologia), 58, 1-15, 17 unnum. pls.

Miranda Ribeiro, A. (1924) Ainda "Hemipsilichthys" e generos alliados. Boletim do Museu Nacional do Rio de Janeiro, Nova Série, zoologia, 1, 365-366.

Miranda Ribeiro, A. (1937) Sobre uma collecção de vertebrados do nordeste brasileiro. Primeira parte: Peixes e batrachios. O Campo, Rio de Janeiro, 1, 54-56.

Miranda Ribeiro, A. (1939) Alguns novos dados ictiológicos da nossa fauna. Boletim Biológico, São Paulo (Nova Série), 4, 358-363.

Miranda Ribeiro, P. (1939a) Sobre o gênero Harttia, Steind. (Peixes: Loricariidae). Boletim Biológico, São Paulo (Nova Série), 4, 11-13, pl. 2.

Miranda Ribeiro, P. (1939b) Um Paraotocinclus do Nordeste Brasileiro (Peixes — Larocaridae [sic, Loricariidae] — Hypoptopomatinae). Boletim Biológico, São Paulo (Nova Série), 4, 364-365.

Miranda Ribeiro, P. (1940) Alguns peixes do sul de Mato Grosso. O Campo, Rio de Janeiro, 60, 1.

Miranda Ribeiro, P. (1942) Um novo "Corydoras" do Rio Javarí, Amazonas, Brasil (Pisces, Callichthyidae). Revista Brasileira de Biologia, 2, 427-428.

Miranda Ribeiro, P. (1943) Dois novos pigidídeos Brasileiros (Pisces - Pygidiidae). Boletim do Museu Nacional do Rio de Janeiro, Nova Série, Zoologia, 9, 1-3.

Miranda Ribeiro, P. (1944a) Uma nova espécie para o gênero Bunocephalus Kner, 1855 (Pisces - Aspredinidae). Boletim do Museu Nacional do Rio de Janeiro, Nova Série, Zoologia, 13, 1-3.

Miranda Ribeiro, P. (1944b) Um pigidídeo do Alto Amazonas (Pisces - Pygidiidae). Boletim do Museu Nacional do Rio 
de Janeiro, Nova Série, Zoologia, 19, 1-3.

Miranda Ribeiro, P. (1944c) Nova espécie para o gênero Stegophilus Reinhardt, 1858 (Pisces — Pygidiidae — Stegophilinae). Boletim do Museu Nacional do Rio de Janeiro, Nova Série, Zoologia, 20, 1-3.

Miranda Ribeiro, P. (1946) Notas para o estudo dos Pygidiidae Brasileiros (Pisces — Pygidiidae — Stegophilinae). - I. Boletim do Museu Nacional do Rio de Janeiro, Nova Série, Zoologia, 58, 1-20, foldout table, pls. 1-7.

Miranda Ribeiro, P. (1947) Notas para o estudo dos Pygidiidae Brasileiros (Pisces — Pygidiidae — Vandelliinae). - II. Boletim do Museu Nacional do Rio de Janeiro, Nova Série, Zoologia, 78, 1-8, pls. 1-2.

Miranda Ribeiro, P. (1949a) Duas novas espécies de peixes na coleção ictiológica do Museu Nacional (Pisces, Callichthyidae et Pygidiidae). Revista Brasileira de Biologia, 9, 143-145.

Miranda Ribeiro, P. (1949b) Notas para o estudo dos Pygidiidae Brasileiros (Pisces — Pygidiidae — Pygidiinae.).— III. Boletim do Museu Nacional do Rio de Janeiro, Nova Série, Zoologia, 88, 1-3, 2 pls.

Miranda Ribeiro, P. (1951a) Sôbre Oxyropsis Eigenmann \& Eigenmann, 1889 (Pisces — Nematognathi — Loricariidae). Boletim do Museu Nacional do Rio de Janeiro, Nova Série, Zoologia, 104, 1-3, pls. 1-4.

Miranda Ribeiro, P. (1951b) Notas para o estudo dos Pygidiidae Brasileiros (Pisces — Pygidiidae — Stegophilinae).— IV. Boletim do Museu Nacional do Rio de Janeiro, Nova Série, Zoologia, 106, 1-16, table, pls. 1-3.

Miranda Ribeiro, P. (1951c) Reedição de algumas publicações de Alipio de Miranda-Ribeiro. Arquivos do Museu Nacional do Rio de Janeiro, 42, xxxvii-1xxx.

Miranda Ribeiro, P. (1953) Tipos das espéces e subespécies do Prof. Alipio de Míranda Ribeiro depositados no Museu Nacional. Arquivos do Museu Nacional do Rio de Janeiro, 42, 389-417.

Miranda Ribeiro, P. (1954) Catálogo dos Peixes do Museum Nacional. I.- Pygidiidae Eigenmann \& Eigenmann, 1888. Publicações Avulsas do Museu Nacional (Rio de Janeiro), 15, 1-17.

Miranda Ribeiro, P. (1956) Notas para o estudo dos Pygidiidae Brasileiros (Pisces — Pygidiidae). - V. Boletim do Museu Nacional do Rio de Janeiro, Nova Série, Zoologia, 142, 1-6.

Miranda Ribeiro, P. (1957) Notas para o estudo dos Pygidiidae Brasileiros (Pisces — Pygidiidae). - VI. Publicações Avulsas do Museu Nacional (Rio de Janeiro), 13, 71-73.

Miranda Ribeiro, P. (1959) Considerações sôbre Callichthyidae Gill, 1872 (Nematognathi). Boletim do Museu Nacional do Rio de Janeiro, Nova Série, Zoologia, 206, 1-9.

Miranda Ribeiro, P. (1959) Catálogo dos Peixes do Museu Nacional. III.-Callichthyidae Gill, 1872. Publicações Avulsas do Museu Nacional (Rio de Janeiro), 27, 1-16.

Miranda Ribeiro, P. (1962a) Apontamentos ictiológicos - I. Boletim do Museu Nacional do Rio de Janeiro, Nova Série, Zoologia, 240, 1-6.

Miranda Ribeiro, P. (1962b) Sôbre os gêneros Heptapterus Bleeker, 1858, e Acentronichthys Eigenmann \& Eigenmann, 1889 (Pisces - Pimelodidae). Boletim do Museu Nacional do Rio de Janeiro, Nova Série, Zoologia, 236, 1-11.

Miranda Ribeiro, P. (1964) Rhamdella schultzi sp. nov. (Pisces - Siluriformes - Pimelodidae). Boletim do Museu Nacional do Rio de Janeiro, Nova Série, Zoologia, 248, 1-4.

Miranda Ribeiro, P. (1968) Apontamentos ictiológicos - IV. Boletim do Museu Nacional do Rio de Janeiro, Nova Série, Zoologia, 262, 1-7.

Mirza, M.R. (1980) The systematics and zoogeography of the freshwater fishes of Pakistan and Azad Kashmir. Proceeding of the first Pakistan Congress of Zoology, 1-41.

Mirza, M.R. (2003) Checklist of freshwater fishes of Pakistan. Pakistan Journal of Zoology, Supplement Series, 3, 1-30.

Mirza, M.R. \& Awan, M.I. (1973) Two new catfishes (Pisces, Siluriformes) from Pakistan. Biologia (Lahore), 19, 145159.

Mirza, M.R. \& Hameed, K. (1974) Sisorid fishes (Osteichthyes: Sisoridae) of Pakistan and Azad Kashmir. Biologia (Lahore), 20, 83-89.

Mirza, M.R. \& Jan, M.A. (1989) Batasio pakistanicus new species, a new catfish (Pisces, Bagridae) from Pakistan. Scientific Khyber, 2, 283-286.

Mirza, M.R. \& Kashmiri, K.M. (1971) A note on the fishes of the genus Glyptothorax Blyth (Osteichthyes, Sisoridae) from West Pakistan with the description of a new subspecies. Biologia (Lahore), 17, 87-93.

Mirza, M.R. \& Naik, I.U. (1969) Fishes of Zhob District with the description of a new species. Pakistan Journal of Science, $21,121-125$.

Mirza, M.R., Nawaz, H. \& Javed, M.N. (1992) A note on the fishes of genus Aorichthys Wu with the description of a new subspecies from Pakistan. Pakistan Journal of Zoology, 24, 211-213.

Mirza, M.R. \& Nijssen, H. (1978) Glyptothorax stocki, a new sisorid catfish from Pakistan \& Azad Kashmir (Siluriformes, Sisoridae). Bulletin Zoölogisch Museum, Universiteit van Amsterdam, 6, 79-85. 
Mirza, M.R., Parveen, A. \& Javed, M.N. (1999) Gagata pakistanica, a new catfish from Pakistan (Pisces: Sisoridae). Punjab University, Journal of Zoology, 14, 1-4.

Misra, K.S. (1976) Teleostomi: Cypriniformes; Siluri. The Fauna of India and the Adjacent Countries (Pisces, 2nd Edition), Vol. 3, Zoological Survey of India, Calcutta, xxi +367 p., 5 pl.

Mitchell, T.L. (1838) Three Expeditions into the Interior of Eastern Australia, with Descriptions of Recently Explored Regions of Australia Felix, and of the Present Colony of New South Wales, Vol. 1, T. \& W. Boone, London, 351 p. + 51 pls.

Mitchill, S.L. (1815) The fishes of New York described and arranged. Transactions of the Literary and Philosophical Society of New-York, 1, 355-492, pls. 1-6.

Mitchill, S.L. (1817) [Report on the ichthyology of the Wallkill, from specimens of fishes presented to the society (Lyceum of Natural History)....by Dr. B. Akerly... .]. American Monthly Magazine and Critical Review, 1, $289-290$.

Mo, T. (1991) Anatomy, Relationships and Systematics of the Bagridae (Teleostei: Siluroidei) with a Hypothesis of Siluroid Phylogeny (Theses Zoologicae, 17), Koeltz, Koenigstein, 216 p., 63 figs.

Mo, T.-P. \& Chu, X.-L. (1986) [A revision of the sisorid catfish genus Glyptothorax from China]. Zoological Research, 7, 339-350. [In Chinese]

Mohsin, A.K.M. \& Ambak, M.A. (1983) Freshwater Fishes of Peninsular Malaysia, Penerbit Universiti Pertanian Malaysia, $284 \mathrm{p}$.

Mojica, J.I., Castellanos-C., C. \& Polanco-F, A. (2000) Redescubrimiento de Dupouyichthys sapito (Pisces: Siluriformes: Aspredinidae) en la cuenca del Río Magdalena (Colombia). Caldasia, 22, 353-355.

Molina, G.I. (1782) Saggio Sulla Storia Naturale del Chile, del Signor Abate Giovanni Ignazio Molina, Bologna, v +306 p. + errata + map.

Montoya-Burgos, J.-I. (2003) Historical biogeography of the catfish genus Hypostomus (Siluriformes: Loricariidae), with implications on the diversification of Neotropical ichthyofauna. Molecular Ecology, 12, 1855-1867.

Montoya-Burgos, J.-I., Muller, S., Weber, C. \& Pawlowski, J. (1997) Phylogenetic relationships between Hypostominae and Ancistrinae (Siluroidei: Loricariidae): first results from mitochondrial 12S and 16S rRNA gene sequences. Revue suisse de Zoologie, Annales de la Société zoologique suisse et du Muséum d'Histoire naturelle de Genève, 104, 185-198.

Montoya-Burgos, J.-I., Muller, S., Weber, C. \& Pawlowski, J. (1998) Phylogenetic relationships of the Loricariidae (Siluriformes) based on mitochondrial rRNA gene sequences. In: Malabarba, L.R., Reis, R.E., Vari, R.P., Lucena, Z.M.S.\& Lucena, C.A.S. (Eds.), Phylogeny and Classification of Neotropical Fishes, Edipucrs, Porto Alegre, pp. 363-374.

Montoya-Burgos, J.-I., Weber, C. \& Le Bail, P.-Y. (2002) Phylogenetic relationships within Hypostomus (Siluriformes: Loricariidae) and related genera based on mitochondrial D-loop sequences. Revue suisse de Zoologie, Annales de la Société zoologique suisse et du Muséum d'Histoire naturelle de Genève, 109, 369-382.

Mori, T. (1933) Second addition to the fish fauna of Tsi-nan, China, with descriptions of three new species. Japanese Journal of Zoology, 5, 165-169.

Mori, T. (1936) [Descriptions of one new genus and three new species of Siluroidea from Chosen]. Dobutsugaku Zasshi, 48, 671-675, pl. 24. [In Japanese and English].

Mori, T. (1952) Check list of the fishes of Korea. Memoirs of the Hyogo University of Agriculture, 1, 1-228.

Moyer, G.R., Burr, B.M. \& Krajewski, C. (2004) Phylogenetic relationships of thorny catfishes (Siluriformes: Doradidae) inferred from molecular and morphological data. Zoological Journal of the Linnean Society, 140, 551-575.

Mukerji, D.D. (1932) On a collection of fish from lower Burma. Records of the Indian Museum, 34, 281-286.

Müller, J. (1840) Vergleichende Anatomie der Myxinoiden, der Cyclostomen mit durchhohrten Gaumen. IV — Über das Gefäss-system. Abhandlungen der Deutschen Akademie der Wissenschaften zu Berlin, 1839, 175-304, pls. 1-5. [Abstracts apparently published in Berlin Akad. Wissenschaften, Berlin, 1839: 184-186 (see Dean, 1917), Archiv für Naturgeschichte (see Jordan, 1919: 196), and Archiv für Anatomie, Physiologie und wissenschaftliche Medizin (see Eschmeyer, 1998). Availability of names from abstracts, and priority of publication, not established.]

Müller, J. (1842a) Über die Schwimmblase der Fische, mit Bezug auf eínige neue Fishgattungen. Königlichen Preussische Akademie des Wissenschaften zu Berlin, 1842, 202-210.

Müller, J. (1842b) Beobachtungen über die Schwimmblase der Fische, mit Bezug auf einige neue Fischgattungen. Archiv für Anatomie, Physiologie und wissenschaftliche Medicin (Müller), Jahr, 1842, 307-329.

Müller, J. (1843) Beiträge zur Kenntniss der natürlichen Familien der Fische. Archiv für Naturgeschichte, 9, $292-330$.

Müller, J. (1865) Reisen in den Vereinigten Staaten, Canada und Mexico, 3 vols [1864-65], F.A. Brockhaus, Leipzig.

Müller, J. \& Troschel, F.H. (1848) Fische. In: Reisen in Britisch-Guiana in den Jahren 1840-44. Im Auftrag Sr. Mäjestat 
des Königs von Preussen ausgeführt von Richard Schomburgk. [Versuch einer Fauna und Flora von Britisch-Guiana.] Vol. 3, Berlin, pp. 618-644.

Müller, J. \& Troschel, F.H. (1849) Horae Ichthyologicae: Beschreibung und Abbildung neuer Fische, Drittes Heft, Verlag von Veit \& Co, Berlin, 28 p., 5 pls.

Muller, S. (1989) Description de deux nouvelles espèces paraguayennes du genre Ancistrus Kner, 1854 (Pisces, Siluriformes, Loricariidae). Revue suisse de Zoologie, Annales de la Société zoologique suisse et du Muséum d'Histoire naturelle de Genève, 96, 885-904.

Muller, S. (1990) Étude méristique et morphométrique d'Ancistrus piriformis Muller et Ancistrus pirareta Muller. Revue suisse de Zoologie, Annales de la Société zoologique suisse et du Muséum d'Histoire naturelle de Genève, 97, 153168.

Muller, S. \& Isbrücker, I.J.H. (1993) Lithoxus boujardi (Siluriformes, Loricariidae), une espèce nouvelle du Bassin de l'Approuague, Guyane Française. Cybium (3e série), 17, 71-76.

Muller, S., Rapp Py-Daniel, L.H. \& Zuanon, J. (1994) Ancistrus ranunculus, a new species of loricariid fish (Siluriformes: Loricariidae) from the Xingú and Tocantins rivers, Brazil. Ichthyological Exploration of Freshwaters, 5, 289-296.

Muller, S. \& Weber, C. (1992) Les dents des sous-familles Hypostominae et Ancistrinae (Pisces, Siluriformes, Loricariidae) et leur valeur taxonomique. Revue suisse de Zoologie, Annales de la Société zoologique suisse et du Muséum d'Histoire naturelle de Genève, 99, 747-754.

Muriel-Cunha, J. \& Pinna, M., de (2005) New data on cistern catfish, Phreatobius cisternarum, from subterranean waters at the mouth of the Amazon River (Siluriformes, Incertae sedis). Papéis Avulsos de Zoologia, 45, 327-339.

Murray, A. (1855) On electrical fishes; with a description of a new species of Malapterurus from Old Calabar, received from the Rev. Hope M. Waddell, missionary there. Proceedings of the Royal Physical Society of Edinburgh, 1, 20$21,1 \mathrm{pl}$.

Murray, A.M. \& Budney, L.A. (2003) A new species of catfish (Claroteidae, Chrysichthys) from an Eocene crater lake in East Africa. Canadian Journal of Earth Science, 40, 983-993.

Musikasinthorn, P., Utsugi, K. \& Watanabe, K. (1998) Rediscovery of the pangasid catfish Helicophagus typus in Borneo. Natural History Bulletin of the Siam Society, 46, 197-201.

Musyl, M.K. \& Keenan, C.P. (1996) Evidence for cryptic speciation in Australian freshwater eel-tailed catfish, Tandanus tandanus (Teleostei: Plotosidae). Copeia, 1996, 526-534.

Myers, G.S. (1925a) Description of a new catfish from Abyssinia. Copeia, no. 139, 12-13.

Myers, G.S. (1925b) Tridentopsis pearsoni a new pygidiid catfish from Bolivia. Copeia, no. 148, 83-86.

Myers, G.S. (1926) Descriptions of a new characin fish and a new pygidiid catfish from the Amazon Basin. Copeia, no. $156,150-152$.

Myers, G.S. (1927) Descriptions of new South American fresh-water fishes collected by Dr. Carl Ternetz. Bulletin of the Museum of Comparative Zoology, 68, 107-135.

Myers, G.S. (1928) New fresh-water fishes from Peru, Venezuela, and Brazil. Annals and Magazine of Natural History (Ser. 10), 2, 83-90.

Myers, G.S. (1931) On the fishes described by Koller from Hainan in 1926 and 1927. Lingnan Science Journal, 10, 255262.

Myers, G.S. (1932) Notes on Colombian fresh-water fishes, with description of a new Astroblepus. Copeia, 1932, 137138.

Myers, G.S. (1933) New importations: Leopard Corydoras. Aquarium, Philadelphia, 2, 188-189.

Myers, G.S. (1938) Notes on Ansorgia, Clarisilurus, Wallago, and Ceratoglanis, four genera of African and IndoMalayan catfishes. Copeia, 1938, 98.

Myers, G.S. (1941) A new name for Taenionema, a genus of Amazonian siluroid fishes. Stanford Ichthyological Bulletin, $2,88$.

Myers, G.S. (1942) Studies on South American fresh-water fishes.- I. Stanford Ichthyological Bulletin, 2, 89-114.

Myers, G.S. (1944) Two extraordinary new blind nematognath fishes from the Rio Negro, representing a new subfamily of Pygidiidae, with a rearrangement of the genera of the family, and illustrations of some previously described genera and species from Venezuela and Brazil. Proceedings of the California Academy of Sciences, Series 4, 23, 591602, pls. 52-56.

Myers, G.S. (1948) Note on two generic names of Indo-Malayan silurid fishes, Wallago and Wallagonia. Proceedings of the California Zoological Club, 1, 19-20.

Myers, G.S. (1953) A note on the habits and classification of Corydoras hastatus. The Aquarium Journal, 24, $268-270$. 
Myers, G.S. (1960) The genera and ecological geography of the South American banjo catfishes, family Aspredinidae. Stanford Ichthyological Bulletin, 7, 132-139.

Myers, G.S. \& Weitzman, S.H. (1954) Another new Corydoras from Brazil. The Aquarium Journal, $25,93-94$.

Myers, G.S. \& Weitzman, S.H. (1956) Two new Brazilian fresh water fishes. Stanford Ichthyological Bulletin, 7, 1-4.

Myers, G.S. \& Weitzman, S.H. (1960) Two new fishes collected by General Thomas D. White in eastern Colombia. Stanford Ichthyological Bulletin, 7, 98-109.

Myers, G.S. \& Weitzman, S.H. (1966) Two remarkable new trichomycterid catfishes from the Amazon basin in Brazil and Colombia. Journal of Zoology (London), 149, 277-287.

Nani, A. \& Fuster, M.L. (1947) Hypophthalmus oremaculatus una nueva especie del orden "Nematognathi" (Pisces, Hypophthal.). Comunicaciones del Museo Argentino de Ciencias Naturales "Bernardino Rivadavia", Serie Ciencias Zoológicas, 2, 1-9.

Naseka, A.M. \& Bogutskaya, N.G. (2004) Contribution to taxonomy and nomenclature of freshwater fishes of the Amur drainage area and the Far East (Pisces, Osteichthyes). Zoosystematica-Rossica, 12, 279-290.

Nass, P. (1991) Anatomía Comparada del Bagre Cunagaro Brachyplatystoma juruense (Boulenger, 1898), Incluyendo un Analisis Filogenético de la Familia Pimelodidae, Unpublished Ph.D. dissertation, Universidad Central de Venezuela, Caracas.

Nath, P. \& Dey, S.C. (1989) Two new fish species of the genus Amblyceps Blyth from Arunachal Pradesh, India. Journal of the Assam Science Society, 32, 1-6.

Neave, S.A. (1940) Nomenclator Zoologicus, Vol. 3, The Zoological Society of London, London.

Nelson, E.W. (1876) A partial catalogue of the fishes of Illinois. Bulletin of the Illinois Museum of Natural History, 1, 33-52.

Nelson, J.S. (2006) Fishes of the World, Fourth Edition, John Wiley and Sons, New York, 601 p.

Newton, E.T. (1889) A contribution to the history of Eocene siluroid fishes. Proceedings of the Zoological Society of London, 1889, 201-207, 1 pl.

Ng, H.H. (1996) Akysis heterurus, a new species of catfish (Teleostei: Akysidae) from eastern Sumatra, with notes on Akysis hendricksoni Alfred. The Raffles Bulletin of Zoology, 44, 3-10.

Ng, H.H. (1999a) Pterocryptis inusitata, a new species of silurid catfish from Laos (Teleostei: Siluriformes). Ichthyological Exploration of Freshwaters, 10, 371-374.

Ng, H.H. (1999b) Laides longibarbis, a valid species of schilbeid catfish from Indochina (Teleostei: Siluriformes). Ichthyological Exploration of Freshwaters, 10, 381-385.

Ng, H.H. (1999c) A review of the southeast Asian catfish genus Ceratoglanis (Siluriformes: Siluridae), with the description of a new species from Thailand. Proceedings of the California Academy of Sciences, Series 4, 51, 385-395.

Ng, H.H. (1999d) Two new species of catfishes of the genus Clarias from Borneo (Teleostei: Clariidae). The Raffles Bulletin of Zoology, 47, 17-32.

Ng, H.H. (1999e) The Akysis of Myanmar: a review (Teleostei: Akysidae). The Raffles Bulletin of Zoology, 47, 541-548.

Ng, H.H. (1999f) Bagrichthys obscurus, a new species of bagrid catfish from Indochina (Teleostei: Bagridae). Revista de Biología Tropical, 47, 545-552.

Ng, H.H. (2000) Bagrichthys vaillantii (Popta, 1906), a valid species of bagrid catfish from eastern Borneo (Teleostei: Siluriformes). Zoologische Mededelingen (Leiden), 73, 327-332.

Ng, H.H. (2001a) Kryptopterus dissitus, a new silurid catfish from Indochina (Teleostei, Siluridae). Folia Zoologica, 50, 197-200.

Ng, H.H. (2001b) Amblyceps macropterus, a new species of amblycipitid catfish (Osteichthyes: Amblycipitidae) from Pakistan. Ichthyological Exploration of Freshwaters, 12, 201-204.

Ng, H.H. (2001c) Clarias microstomus, a new species of clariid catfish from eastern Borneo (Teleostei: Siluriformes). Zoological Studies, 40, 158-162.

Ng, H.H. (2002a) Bagrichthys majusculus, a new catfish from Indochina (Teleostei, Bagridae). Folia Zoologica, 51, 4954.

Ng, H.H. (2002b) Ompok binotatus and Ompok pluriradiatus, two new species of silurid catfish from western Borneo (Teleostei: Siluriformes). Ichthyological Exploration of Freshwaters, 13, 25-32.

Ng, H.H. (2002c) Descriptions of two new species of Kryptopterus from Thailand and Borneo in the K. limpok species group (Teleostei: Siluridae). Ichthyological Exploration of Freshwaters, 13, 69-74.

Ng, H.H. (2002d) The identity of Mystus nigriceps (Valenciennes in Cuvier \& Valenciennes, 1840), with the description of a new bagrid catfish (Teleostei: Siluriformes) from Southeast Asia. The Raffles Bulletin of Zoology, 50, 161-168.

Ng, H.H. (2003a) Mystus impluviatus: a new species of bagrid catfish (Teleostei: Bagridae) from eastern Borneo. Copeia, 
2003, 373-378.

Ng, H.H. (2003b) A review of the Ompok hypophthalmus group of silurid catfishes with the description of a new species from South-east Asia. Journal of Fish Biology, 62, 1296-1311.

Ng, H.H. (2003c) A revision of the south Asian sisorid catfish genus Sisor (Teleostei: Siluriformes). Journal of Natural History, 37, 2871-2883.

Ng, H.H. (2003d) Kryptopterus paraschilbeides, a new species of catfish (Teleostei: Siluridae) from mainland Southeast Asia. The Natural History Journal of the Chulalongkorn University, 3, 1-8.

Ng, H.H. (2003e) Arius verrucosus, a new species of freshwater ariid catfish (Teleostei: Ariidae) from the Mekong River. Occasional Papers of the Museum of Zoology, University of Michigan, 734, 1-14.

Ng, H.H. (2003f) Ompok pinnatus, a new species of silurid catfish (Teleostei: Siluriformes: Siluridae) from mainland Southeast Asia. Proceedings of the Biological Society of Washington, 116, 47-51.

Ng, H.H. (2003g) Clarias nigricans, a new species of clariid catfish (Teleostei: Siluriformes) from eastern Borneo. The Raffles Bulletin of Zoology, 51, 393-398.

Ng, H.H. (2003h) Clarias insolitus, a new species of clariid catfish (Teleostei: Siluriformes) from southern Borneo. Zootaxa, 284, 1-8.

Ng, H.H. (2003i) Kryptopterus geminus, a new species of silurid catfish (Teleostei: Siluridae) from mainland Southeast Asia. Zootaxa, 305, 1-11.

Ng, H.H. (2003j) Phylogeny and systematics of Bagridae. In: Arratia, G., Kapoor, B.G., Chardon, M. \& Diogo, R. (Eds.), Catfishes, Vol. 1, Science Publishers, Enfield, NH, USA, pp. 439-464.

Ng, H.H. (2004a) Wallago micropogon: a new species of silurid catfish (Teleostei: Siluridae) from mainland Southeast Asia. Copeia, 2004, 92-97.

Ng, H.H. (2004b) New estuarine species of Mystus (Teleostei: Bagridae) from the Malay Peninsula. Copeia, 2004, 883887.

Ng, H.H. (2004c) Batasio elongatus, a new species of bagrid catfish from southwest Myanmar (Siluriformes: Bagridae). Ichthyological Exploration of Freshwaters, 15, 67-70.

Ng, H.H. (2004d) Clarias sulcatus, a new walking catfish (Teleostei: Clariidae) from Pulau Redang. Ichthyological Exploration of Freshwaters, 15, 289-294.

Ng, H.H. (2004e) Oreoglanis macronemus, a new species of glyptosternine catfish (Teleostei: Siluriformes: Sisoridae) from northern Laos. The Raffles Bulletin of Zoology, 52, 209-213.

Ng, H.H. (2004f) Kryptopterus platypogon, a new silurid catfish (Teleostei: Siluridae) from Borneo. Zootaxa, 398, 1-8.

Ng, H.H. (2004g) Two new glyptosternine catfishes (Teleostei: Sisoridae) from Vietnam and China. Zootaxa, 428, 1-12.

Ng, H.H. (2004h) The Microsynodontis (Teleostei: Siluriformes: Mochokidae) of the lower Guinea region, west central Africa, with the description of eight new species. Zootaxa, 531, 1-52.

Ng, H.H. (2004i) Rita macracanthus, a new riverine catfish (Teleostei: Bagridae) from South Asia. Zootaxa, 568, 1-12.

Ng, H.H. (2005a) Conta pectinata, a new erethistid catfish (Teleostei: Erethistidae) from northeast India. Ichthyological Exploration of Freshwaters, 16, 23-28.

Ng, H.H. (2005b) Pseudolaguvia foveolata, a new catfish (Teleostei: Erethistidae) from northeast India. Ichthyological Exploration of Freshwaters, 16, 173-178.

Ng, H.H. (2005c) Gogangra laevis, a new species of riverine catfish from Bangladesh (Teleostei: Sisoridae). Ichthyological Exploration of Freshwaters, 16, 279-286.

Ng, H.H. (2005d) Amblyceps carinatum, a new species of hillstream catfish from Myanmar (Teleostei: Amblycipitidae). The Raffles Bulletin of Zoology, 53, 243-249.

Ng, H.H. (2005e) Glyptothorax botius (Hamilton, 1822), a valid species of catfish (Teleostei: Sisoridae) from northeast India. Zootaxa, 930, 1-19.

Ng, H.H. (2005f) Erethistoides sicula, a new catfish (Teleostei: Erethistidae) from India. Zootaxa, 1021, 1-12.

Ng, H.H. (2005g) Two new species of Pseudolaguvia (Teleostei: Erethistidae) from Bangladesh. Zootaxa, $1044,35-47$.

Ng, H.H. \& Chan, B.P.-L. (2005) Revalidation and redescription of Pterocryptis anomala (Herre, 1933), a catfish (Teleostei: Siluridae) from southern China. Zootaxa, 1060, 51-64.

Ng, H.H. \& Dodson, J.J. (1999) Morphological and genetic descriptions of a new species of catfish, Hemibagrus chrysops, from Sarawak, east Malaysia, with an assessment of phylogenetic relationships (Teleostei: Bagridae). The Raffles Bulletin of Zoology, 47, 45-57.

Ng, H.H. \& Edds, D.R. (2004) Batasio macronotus, a new species of bagrid catfish from Nepal (Teleostei: Bagridae). Ichthyological Exploration of Freshwaters, 15, 295-300.

Ng, H.H. \& Edds, D.R. (2005a) Two new species of Erethistoides (Teleostei: Erethistidae) from Nepal. Ichthyological 
Exploration of Freshwaters, 16, 239-248.

Ng, H.H. \& Edds, D.R. (2005b) Two new species of Pseudecheneis, rheophilic catfishes (Teleostei: Sisoridae) from Nepal. Zootaxa, 1047, 1-19.

Ng, H.H. \& Ferraris, C.J., Jr. (2000) A review of the genus Hemibagrus in southern Asia, with descriptions of two new species. Proceedings of the California Academy of Sciences, Series 4, 52, 125-142.

Ng, H.H. \& Freyhof, J. (2001a) A review of the catfish genus Pterocryptis (Siluridae) in Vietnam, with the description of two new species. Journal of Fish Biology, 59, 624-644.

Ng, H.H. \& Freyhof, J. (2001b) Oreoglanis infulatus, a new species of glyptosternine catfish (Siluriformes: Sisoridae) form central Vietnam. Journal of Fish Biology, 59, 1164-1169.

Ng, H.H. \& Freyhof, J. (2003) Akysis clavulus, a new species of catfish (Teleostei: Akysidae) from central Vietnam. Ichthyological Exploration of Freshwaters, 14, 311-316.

Ng, H.H. \& Freyhof, J. (2005) A new species of Pseudomystus (Teleostei: Bagridae) from Central Vietnam. Copeia, 2005, 745-750.

Ng, H.H. \& Hadiaty, R.K. (2005) Two new bagrid catfishes (Teleostei: Bagridae) from the Alas River drainage, northern Sumatra. Ichthyological Exploration of Freshwaters, 16, 83-92.

Ng, H.H. \& Kottelat, M. (1996) Akysis fuscus, a new species of catfish (Teleostei: Akysidae) from the Kapuas basin, Borneo. Ichthyological Exploration of Freshwaters, 7, 19-26.

Ng, H.H. \& Kottelat, M. (1997) Silurichthys citatus, a new species of silurid catfish from western Borneo (Teleostei: Siluriformes). Ichthyological Exploration of Freshwaters, 7, 203-208.

Ng, H.H. \& Kottelat, M. (1998a) Hyalobagrus, a new genus of miniature bagrid catfish from southeast Asia (Teleostei: Siluriformes). Ichthyological Exploration of Freshwaters, 9, 335-346.

Ng, H.H. \& Kottelat, M. (1998b) Pterocryptis buccata, a new species of catfish from western Thailand (Teleostei: Siluridae) with epigean and hypogean populations. Ichthyological Research, 45, 393-399.

Ng, H.H. \& Kottelat, M. (1998c) The catfish genus Akysis Bleeker (Teleostei: Akysidae) in Indochina, with descriptions of six new species. Journal of Natural History, 32, 1057-1097.

Ng, H.H. \& Kottelat, M. (1999) Oreoglanis hypsiurus, a new species of glyptosternine catfish (Teleostei: Sisoridae). Ichthyological Exploration of Freshwaters, 10, 375-380.

Ng, H.H. \& Kottelat, M. (2000a) A review of the genus Amblyceps (Osteichthyes: Amblycipitidae) in Indochina, with descriptions of five new species. Ichthyological Exploration of Freshwaters, 11, 335-348.

Ng, H.H. \& Kottelat, M. (2000b) Description of three new species of catfishes (Teleostei: Akysidae and Sisoridae) from Laos and Vietnam. Journal of South Asian Natural History, 5, 7-15.

Ng, H.H. \& Kottelat, M. (2000c) Helicophagus leptorhynchus, a new species of molluscivorous catfish from Indochina (Teleostei: Pangasiidae). The Raffles Bulletin of Zoology, 48, 55-58.

Ng, H.H. \& Kottelat, M. (2000d) Cranoglanis henrici (Vaillant, 1893) a valid species of cranoglanidid catfish from Indochina (Teleostei: Cranoglanididae). Zoosystema, 22, 847-852.

Ng, H.H. \& Kottelat, M. (2001) A review of the genus Batasio (Teleostei: Bagridae) in Indochina, with the description of B. tigrinus sp. n. from Thailand. Revue suisse de Zoologie, Annales de la Société zoologique suisse et du Muséum d'Histoire naturelle de Genève, 108, 495-511.

Ng, H.H. \& Kottelat, M. (2003) Parakysis notialis, a new species of akysid catfish from Borneo (Siluriformes: Akysidae). Ichthyological Research, 50, 48-51.

Ng, H.H. \& Kottelat, M. (2004) Akysis vespa, a new species of catfish (Siluriformes: Akysidae) from the Ataran River drainage (Myanmar). Ichthyological Exploration of Freshwaters, 25, 193-200.

Ng, H.H. \& Kottelat, M. (2005) Caelatoglanis zontatus, a new genus and species of the Erethistidae (Teleostei: Siluriformes) from Myanmar, with comments on the nomenclature of Laguvia and Hara species. Ichthyological Exploration of Freshwaters, 16, 13-22.

Ng, H.H. \& Lim, K.K.P. (1995) A revision of the southeast Asian catfish genus Parakysis (Teleostei: Akysidae), with descriptions of two new species. Ichthyological Exploration of Freshwaters, 6, 255-266.

$\mathrm{Ng}$, H.H. \& Lim, K.K.P. (2000) Hemileiocassis panjang, a new genus and new species of bagrid catfish from Java (Teleostei, Siluriformes). Beaufortia, 50, 191-195.

Ng, H.H. \& Lim, K.K.P. (2005) The identity of Pseudomystus moeschii (Boulenger, 1890), with the description of two new species of bagrid catfishes from Southeast Asia (Teleostei: Bagridae). Zootaxa, 851, 1-18.

Ng, H.H., Martin-Smith, K.M. \& Ng, P.K.L. (2000) Hemibagrus furcatus, a new species of bagrid catfish (Teleostei: Siluriformes) from Sabah, east Malaysia. The Raffles Bulletin of Zoology, 48, 65-69.

Ng, H.H. \& Ng, P.K.L. (1998) A revision of the South-east Asian catfish genus Silurichthys. Journal of Fish Biology, 52 , 
291-333.

Ng, H.H. \& Ng, P.K.L. (2001) A revision of the akysid catfish genus Acrochordonichthys Bleeker. Journal of Fish Biology, 58, 386-418.

Ng, H.H. \& Rachmatika, I. (1999) The catfishes (Teleostei: Siluriformes) of Bentuang Karimun National Park, West Kalimantan, Indonesia. The Raffles Bulletin of Zoology, 47, 167-183.

Ng, H.H. \& Rachmatika, I. (2005) Glyptothorax exodon, a new species of rheophilic catfish from Borneo (Teleostei: Sisoridae). The Raffles Bulletin of Zoology, 53, 251-255.

Ng, H.H. \& Rainboth, W.J. (1999) The bagrid catfish genus Hemibagrus (Teleostei: Siluriformes) in central Indochina with a new species from the Mekong River. The Raffles Bulletin of Zoology, 47, 555-576.

Ng, H.H. \& Rainboth, W.J. (2001) A review of the sisorid catfish genus Oreoglanis (Siluriformes: Sisoridae) with descriptions of four new species. Occasional Papers of the Museum of Zoology, University of Michigan, 732, 1-34.

Ng, H.H. \& Rainboth, W.J. (2005) Four new species of Akysis (Teleostei: Siluriformes: Akysidae) from mainland Southeast Asia, with comments on A. similis. The Raffles Bulletin of Zoology, Supplement 13, 33-42.

Ng, H.H. \& Sabaj, M.H. (2005) Akysis hardmani (Siluriformes: Akysidae), a new species of catfish from Thailand. Ichthyological Exploration of Freshwaters, 16, 215-222.

Ng, H.H. \& Siebert, D.J. (1998) A revision of the akysid catfish genus Breitensteinia Steindachner with descriptions of two new species. Journal of Fish Biology, 53, 645-657.

Ng, H.H. \& Siebert, D.J. (2002) Rediscovery and redescription of Ompok weberi (Hardenberg, 1936), a poorly known species of silurid catfish (Teleostei: Siluriformes) from Borneo. The Raffles Bulletin of Zoology, 50, 169-173.

Ng, H.H. \& Siebert, D.J. (2004) A new species of the catfish genus Akysis (Siluriformes: Akysidae) from southern Borneo. Zootaxa, 733, 1-8.

Ng, H.H. \& Siebert, D.J. (2005) Pseudomystus stenogrammus, a new species of bagrid catfish from Borneo (Teleostei, Bagridae). Zootaxa, 813, 1-7.

Ng, H.H. \& Sparks, J.S. (2002) Plotosus fisadoha, a new species of marine catfish (Teleostei: Siluriformes: Plotosidae) from Madagascar. Proceedings of the Biological Society of Washington, 115, 80-85.

Ng, H.H. \& Sparks, J.S. (2003) The ariid catfishes (Teleostei: Siluriformes: Ariidae) of Madagascar, with the description of two new species. Occasional Papers of the Museum of Zoology, University of Michigan, 735, 1-21.

Ng, H.H. \& Sparks, J.S. (2005) Revision of the endemic Malagasy catfish family Anchariidae (Teleostei: Siluriformes), with descriptions of a new genus and three new species. Ichthyological Exploration of Freshwaters, 16, 303-323.

Ng, H.H. \& Tan, H.H. (1999) The fishes of the Endau drainage, Peninsular Malaysia with descriptions of two new species of catfishes (Teleostei: Akysidae, Bagridae). Zoological Studies, 38, 350-366.

Ng, H.H. \& Tan, H.H. (2000) A new species of Encheloclarias (Siluriformes: Clariidae) from Sumatra. Journal of Fish Biology, 57, 536-539.

Ng, H.H. \& Tan, H.H. (2002) Redescription of Acrochordonichthys ischnosoma Bleeker, 1858, a poorly known species of akysid catfish (Teleostei: Siluriformes) from Sumatra and Java. The Raffles Bulletin of Zoology, 50, 449-452.

Ng, H.H. \& Tan, H.H. (2004) Ompok platyrhynchus, a new silurid catfish (Teleostei: Siluridae) from Borneo. Zootaxa, $580,1-11$.

Ng, H.H., Wirjoatmodjo, S. \& Hadiaty, R.K. (2001a) Mystus punctifer, a new species of bagrid catfish (Teleostei: Siluriformes) from northern Sumatra. The Raffles Bulletin of Zoology, 49, 355-358.

Ng, H.H., Wirjoatmodjo, S. \& Hadiaty, R.K. (2001b) Hemibagrus caveatus, a new species of bagrid catfish (Teleostei: Siluriformes) from northern Sumatra. The Raffles Bulletin of Zoology, 49, 359-361.

Ng, H.H., Wirjoatmodjo, S. \& Hadiaty, R.K. (2004) Kryptopterus piperatus, a new species of silurid catfish (Teleostei: Siluridae) from northern Sumatra. Ichthyological Exploration of Freshwaters, 15, 91-95.

Ng, P.K.L. \& Lim, K.K.P. (1993) The Southeast Asian catfish genus Encheloclarias (Teleostei: Clariidae), with descriptions of four new species. Ichthyological Exploration of Freshwaters, 4, 21-37.

Ng, P.K.L. \& Ng, H.H. (1995) Hemibagrus gracilis, a new species of large riverine catfish (Teleostei: Bagridae) from Peninsular Malaysia. The Raffles Bulletin of Zoology, 43, 133-142.

Nguyen, H.D. \& Nguyen, V.H. (2001) [Two newly found fish species of Pareuchiloglanis genus (Sisoridae, Siluriformes) in Vietnam]. Tap chí Sinh Hoc [Journal of Biology], 23, 66-71. [In Vietnamese, with English title and summary; English title only roughly agrees with Vietnamese title]

Nichols, J.T. (1919a) "Cascudos" brazileiros do genero Plecostomus do Museu Paulista (Brasilian catfishes of the genus Plecostomus from the Museu Paulista). Revista do Museu Paulista, 11, 409-426.

Nichols, J.T. (1919b) Um novo genero de cascudos da familia Loricariidae. Revista do Museu Paulista, 11, 533-535, 539-540. 
Nichols, J.T. (1925) Some Chinese fresh-water fishes. X. - Subgenera of bagrin catfishes. XI.- Certain apparently undescribed carps from Fukien. XII.- A small goby from the central Yangtze. XIII.- A new minnow referred to Leucogobio. XIV.- Two apparently undescribed fishes from Yunnan. American Museum Novitates, 185, 1-7.

Nichols, J.T. (1926a) Some Chinese fresh-water fishes. XV.- Two apparently undescribed catfishes from Fukien. XVI.- Concerning gudgeons related to Pseudogobio, and two new species of it. XVII.- Two new rhodeins. American Museum Novitates, 214, 1-7.

Nichols, J.T. (1926b) Some Chinese fresh-water fishes. XVIII.— New species in recent and earlier Fukien collections. American Museum Novitates, 224, 1-7.

Nichols, J.T. (1930) Some Chinese fresh-water fishes. XXVI.— Two new species of Pseudogobio. XXVII.-A new catfish from northeastern Kiangsi. American Museum Novitates, 440, 1-5.

Nichols, J.T. (1931) Some Chinese fresh-water fishes. XXVIII.- A collection from Chungan Hsien, northwestern Fukien. American Museum Novitates, 449, 1-3.

Nichols, J.T. (1940) Results of the Archbold expeditions. No. 30. - New catfishes from northern New Guinea. American Museum Novitates, 1093, 1-3.

Nichols, J.T. (1941) Four new fishes from western China. American Museum Novitates, 1107, 1-3.

Nichols, J.T. (1943) The Fresh-water Fishes of China, Natural History of Central Asia, Vol. 9, American Museum of Natural History, New York, 322 p., 10 pl.

Nichols, J.T. (1956) A new pygidiin catfish from Argentina. American Museum Novitates, 1760, 1-3.

Nichols, J.T. \& Griscom, L. (1917) Fresh-water fishes of the Congo basin obtained by the American Museum Congo expedition, 1909-1915. Bulletin of the American Museum of Natural History, 37, 653-756, pls. 64-83.

Nichols, J.T. \& La Monte, F.R. (1933a) A new catfish, Amphilius pictus, and a discussion of a small Liberian collection of fishes. American Museum Novitates, 626, 1-3.

Nichols, J.T. \& La Monte, F.R. (1933b) New fishes from the Kasai district of the Belgian Congo. American Museum Novitates, 656, 1-6.

Nichols, J.T. \& La Monte, F.R. (1934) More new fishes from the Kasai district of the Belgian Congo. American Museum Novitates, 723, 1-6.

Nichols, J.T. \& La Monte, F.R. (1953) Two new African catfishes from eastern French Equatorial Africa. American Museum Novitates, 1648, 1-4.

Nichols, J.T. \& Pope, C.H. (1927) The fishes of Hainan. Bulletin of the American Museum of Natural History, 54, 321394, pl. 26.

Nico, L.G. \& Pinna, M.C.C., de. (1996) Confirmation of Glanapteryx anguilla (Siluriformes, Trichomycteridae) in the Orinoco River basin, with notes on the distribution and habits of the Glanapteryginae. Ichthyological Exploration of Freshwaters, 7, 27-32.

Nielsen, J.G. (1974) Fish Types in the Zoological Museum of Copenhagen, Copenhagen, 115 p.

Nijssen, H. (1970) Revision of the Surinam catfishes of the genus Corydoras La Cepède, 1803 (Pisces, Siluriformes, Callichthyidae). Beaufortia, 18, 1-75.

Nijssen, H. (1971) Two new species and one new subspecies of the South American catfish genus Corydoras (Pisces, Siluriformes, Callichthyidae). Beaufortia, 19, 89-98.

Nijssen, H. (1972) Records of the catfish genus Corydoras from Brazil and French Guiana with descriptions of eight new species (Pisces, Siluriformes, Callichthyidae). Netherlands Journal of Zoology, 21, 412-433, pls. 1-3.

Nijssen, H. \& Isbrücker, I.J.H. (1967) Notes on the Guiana species of Corydoras La Cepède, 1803, with descriptions of seven new species and designation of a neotype for Corydoras punctatus (Bloch, 1794) -(Pisces, Cypriniformes, Callichthyidae). Zoologische Mededelingen (Leiden), 42, 21-50, pls. 1-5.

Nijssen, H. \& Isbrücker, I.J.H. (1970) The South American catfish genus Brochis Cope, 1872 (Pisces, Siluriformes, Callichthyidae). Beaufortia, 18, 151-168.

Nijssen, H. \& Isbrücker, I.J.H. (1971) Two new species of the catfish genus Corydoras from Brazil and Peru (Pisces, Siluriformes, Callichthyidae). Beaufortia, 18, 183-189.

Nijssen, H. \& Isbrücker, I.J.H. (1975) Cataphractus punctatus Bloch, 1794 (Pisces, Siluriformes, Callichthyidae): request for invalidation of neotype and validation of a rediscovered syntype as lectotype. Bulletin of Zoological Nomenclature, 32, 63-64, pl. 1.

Nijssen, H. \& Isbrücker, I.J.H. (1976a) The South American plated catfish genus Aspidoras R. von Ihering, 1907, with descriptions of nine new species from Brazil (Pisces, Siluriformes, Callichthyidae). Bijdragen tot de Dierkunde, 46, 107-131.

Nijssen, H. \& Isbrücker, I.J.H. (1976b) Corydoras ornatus, a new species of callichthyid catfish from the Rio Tapajós 
drainage, Brazil (Pisces, Siluriformes, Callichthyidae). Bulletin Zoölogisch Museum, Universiteit van Amsterdam, 5, 125-129.

Nijssen, H. \& Isbrücker, I.J.H. (1976c) A new callichthyid catfish, Corydoras gracilis, from Brazil. Tropical Fish Hobbyist, 25, 90-98.

Nijssen, H. \& Isbrücker, I.J.H. (1979) Chronological enumeration of nominal species and subspecies of Corydoras (Pisces, Siluriformes, Callichthyidae). Bulletin Zoölogisch Museum, Universiteit van Amsterdam, 6, 129-135.

Nijssen, H. \& Isbrücker, I.J.H. (1980a) On the identity of Corydoras nattereri Steindachner 1877 with the description of a new species Corydoras prionotos (Pisces, Siluriformes, Callichthyidae). Beaufortia, 30, 1-9.

Nijssen, H. \& Isbrücker, I.J.H. (1980b) A review of the genus Corydoras La Cepède, 1803 (Pisces, Siluriformes, Callichthyidae). Bijdragen tot de Dierkunde, 50, 190-220.

Nijssen, H. \& Isbrücker, I.J.H. (1980c) Aspidoras virgulatus n. sp., a plated catfish from Espírto Santo, Brazil (Pisces, Siluriformes, Callichthyidae). Bulletin Zoölogisch Museum, Universiteit van Amsterdam, 7, 133-139.

Nijssen, H. \& Isbrücker, I.J.H. (1980d) Three new Corydoras species from French Guiana and Brazil (Pisces, Siluriformes, Callichthyidae). Netherlands Journal of Zoology, 30, 494-503.

Nijssen, H. \& Isbrücker, I.J.H. (1982) Corydoras boehlkei, a new catfish from the Rio Caura system in Venezuela (Pisces, Siluriformes, Callichthyidae). Proceedings of the Academy of Natural Sciences, Philadelphia, 134, 139142.

Nijssen, H. \& Isbrücker, I.J.H. (1983a) Brochis britskii, a new species of plated catfish from the upper Rio Paraguai system, Brazil (Pisces, Siluriformes, Callichthyidae). Bulletin Zoölogisch Museum, Universiteit van Amsterdam, 9, 177-186.

Nijssen, H. \& Isbrücker, I.J.H. (1983b) Review of the genus Corydoras from Colombia, with descriptions of two new species (Pisces, Siluriformes, Callichthyidae). Beaufortia, 33, 53-71.

Nijssen, H. \& Isbrücker, I.J.H. (1983c) Sept espèces nouvelles de poissons-chats cuirassés du genre Corydoras La Cepède, 1803, de Guyane française, de Bolivie, d'Argentine, du Surinam et du Brésil (Pisces, Siluriformes, Callichthyidae). Revue Française d'Aquariologie et Herpetologie, 10, 73-84.

Nijssen, H. \& Isbrücker, I.J.H. (1985) Lasiancistrus scolymus, a new species of mailed catfish from Rio Aripuanã, Est. Mato Grosso do Sul, Brazil (Pisces, Siluriformes, Loricariidae). Bijdragen tot de Dierkunde, 55, $242-248$.

Nijssen, H. \& Isbrücker, I.J.H. (1986a) Cinq espèces nouvelles de poissons-chats cuirassés du genre Corydoras Lacepède, 1803, du Pérou et de l'Equateur (Pisces, Siluriformes, Callichthyidae). Revue Française d'Aquariologie et Herpetologie, 12, for 1985, 65-76.

Nijssen, H. \& Isbrücker, I.J.H. (1986b) Review of the genus Corydoras from Peru and Ecuador (Pisces, Siluriformes, Callichthyidae). Studies on Neotropical Fauna and Environment, 21, 1-68.

Nijssen, H. \& Isbrücker, I.J.H. (1987) Spectracanthicus murinus, nouveaux genre et espèce de poisson-chat cuirassé du Rio Tapajós, Est. Pará, Brésil, avec des remarques sur d'autres genres de Loricariidés (Pisces, Siluriformes, Loricariidae). Revue Française d'Aquariologie et Herpetologie, 13, for 1986, 93-98.

Nijssen, H. \& Isbrücker, I.J.H. (1988) Trois nouvelles espèces du genre Apistoloricaria de Colombie et du Pérou, avec illustration du dimorphisme sexuel secondaire des lèvres de A. condei (Pisces, Siluriformes, Loricariidae). Revue Française d'Aquariologie et Herpetologie, 15, 33-38.

Nijssen, H. \& Isbrücker, I.J.H. (1990) Lithoxus stocki, a species new to science of ancistrin loricariid catfish from the Maroni River drainage, with a comparison of the primary type specimens of the six species of Lithoxus (syn. Paralithoxus) (Pisces, Siluriformes, Loricariidae). Bijdragen tot de Dierkunde, 60, 327-333.

Nikolsky, G. \& Soin, S.G. (1948) [On catfishes (family Siluridae) in the Amur basin]. Doklady Akademii nauk SSSR [ Comptes Rendus de l'Académie des Sciences de l'URSS], Moscow, 59, 1357-1360. [In Russian]

Nolf, D. (1981) Revision des types d'otolithes de poissons fossiles decrits par R. Schubert. Verhandlungen der Geologischen Bundesanstalt, 1981, 133-183.

Norman, J.R. (1922) Four new fishes from Tanganyika Territory. Annals and Magazine of Natural History (Ser. 9), 9, 686-688.

Norman, J.R. (1923a) Three new fishes from Yunnan, collected by Prof. J. W. Gregory, F.R.S. Annals and Magazine of Natural History (Ser. 9), 11, 561-563.

Norman, J.R. (1923b) Two new siluroid fishes from the Ivory Coast, collected by Mr. Willoughby P. Lowe. Annals and Magazine of Natural History (Ser. 9), 11, 582-583.

Norman, J.R. (1923c) A new cyprinoid fish from Tanganyika Territory, and two new fishes from Angola. Annals and Magazine of Natural History (Ser. 9), 12, 694-696.

Norman, J.R. (1925a) A new siluroid fish of the genus Clarias from southwestern Uganda. Occasional Papers of the 
Boston Society of Natural History, 5, 189-190, pl. 11.

Norman, J.R. (1925b) Two new fishes from Tonkin, with notes on the siluroid genera Glyptosternum, Exostoma, etc. Annals and Magazine of Natural History (Ser. 9), 15, 570-575.

Norman, J.R. (1926a) A new catfish of the genus Cetopsis from the Rio das Velhas, Brazil. Annals and Magazine of Natural History (Ser. 9), 17, 116.

Norman, J.R. (1926b) Descriptions of nine new freshwater fishes from French Guiana and Brazil. Annals and Magazine of Natural History (Ser. 9), 18, 91-97.

Norman, J.R. (1926c) A new blind catfish from Trinidad, with a list of the blind cave-fishes. Annals and Magazine of Natural History (Ser. 9), 18, 324-331.

Norman, J.R. (1928a) Two new fishes from Lake Victoria. Annals and Magazine of Natural History (Ser. 10), 2, 104106.

Norman, J.R. (1928b) A new siluroid fish of the genus Chrysichthys from Nigeria. Annals and Magazine of Natural History (Ser. 10), 2, 385.

Norman, J.R. (1932) A collection of fishes from Sierra Leone. Annals and Magazine of Natural History (Ser. 10), 10, 180-185.

Norman, J.R. (1935a) A collection of fishes from the Ashanti Forest, Gold Coast. Annals and Magazine of Natural History (Ser. 10), 15, 215-221.

Norman, J.R. (1935b) Description of a new loricariid catfish from Ecuador. Annals and Magazine of Natural History (Ser. 10), 15, 627-629.

Norris, S.M. (2002) A revision of the African electric catfishes, family Malapteruridae (Teleostei, Siluriformes), with erection of a new genus and descriptions of fourteen new species, and an annotated bibliography. Musée Royal de l'Afrique Centrale, Annales, Sciences Zoologiques, 289, 1-155.

Ogilby, J.D. (1898a) New genera and species of fishes. Proceedings of the Linnean Society of New South Wales, 23, 3241.

Ogilby, J.D. (1898b) New genera and species of fishes [continued from p. 41]. Proceedings of the Linnean Society of New South Wales, 23, 280-299.

Ogilby, J.D. (1899) Contribution to Australian ichthyology. Proceedings of the Linnean Society of New South Wales, 24, $154-186$.

Ogilby, J.D. (1908) New or little known fishes in the Queensland Museum. Annals of the Queensland Museum, 9, 1-41.

Ogilby, J.D. (1910) On new or insufficiently described fishes. Proceedings of the Royal Society of Queensland, 23, 1-55.

Okada, Y. \& Kubota, S.S. (1957) Description of a new fresh-water catfish, Coreobagrus ichikawai, with an emendation of the genus Coreobagrus Mori. Japanese Journal of Ichthyology, 5, 143-145.

Oken, L. (1817) Cuviers und Okens Zoologien neben einalder gestellt. Isis (Oken), 8, col.1145-1184 [incl.1779-1782, sic. 1179-1182].

Olazarri, J., Mones, A., Ximénez, A. \& Philippi, M.E. (1970) Lista de los ejemplares-tipo depositados en el Museo Nacional de Historia Natural de Montevideo, Uruguay. Comunicaciones Zoológicas del Museo de Historia Natural de Montevideo, 10, 1-12.

Oliveira, J.C. de. (1997) Redescrição de Hemipsilichthys garbei Ihering, 1911, com designação de lectótipo e commentários sobre a sua distribução e da de Hemipsilichthys gobio (Lütken, 1874) (Teleostei: Siluriformes: Loricariidae). Papeis Avulsos de Zoologia, São Paulo, 40, 113-126.

Oliveira, J.C. de, \& Britski, H.A. (2000) Redescrição de Taunayia bifasciata (Eigenmann \& Norris, 1900), comb. nova, um bagre enigmático do Estado de São Paulo (Siluriformes, Pimelodidae, Heptapterinae). Papeis Avulsos de Zoologia, São Paulo, 41, 119-133.

Oliveira, J.C. de, \& de Moraes, D.F., Jr. (1997) Presença de Hoplosternum Gill, 1858 (Teleostei, Siluriformes, Callichthyidae) nas bacias do rios são Francisco, Paraíba do Sul e Alto Paraná: primeiro registro e comentários. Boletim do Museu Nacional do Rio de Janeiro, Nova Série, zoologia, 383, 1-8.

Oliveira, J.C. de, \& de Moraes, D.F., Jr. (1997) Dados adicionais à descrição de Steindachneridion parahybae (Steindachner, 1876) (Teleostei, Siluroidei, Pimelodidae). Boletim do Museu Nacional do Rio de Janeiro, Nova Série, zoologia, 384, 1-11.

Oliveira, J.C. de, \& Oyakawa, O.T. (1999) Two new species of Hemipsilichthys (Teleostei: Loricariidae) from Serra do Espinhaço, Minas Gerais, Brazil. Ichthyological Exploration of Freshwaters, 10, 73-80.

Oliviera, J.C. de, Vari, R.P. \& Ferraris, C.J., Jr. (2001) A new species of "whale catfish" (Siluriformes, Cetopsidae) from the western portions of the Amazon basin. Proceedings of the Biological Society of Washington, 114, 574-578.

Orcés V., G. (1960) Peces ecuatorianos de la familia Callichthyidae, con la descripción de una especie nueva. Ciencia y 
Naturaleza (Quito), 3, 2-6, 1 pl.

Orcés V., G. (1961) Hallazgo de peces de los géneros Xiliphius y Hoplomyzon en el sistema del Amazonas. Descripción de una nueva especie. Ciencia y Naturaleza (Quito), 4, 3-6.

Orcés V., G. (1962) Dos nuevos peces del género Xyliphius. Ciencia y Naturaleza (Quito), 5, 50-54, 1 pl.

Orcés V., G. (1977) Contribuciones al conocimiento de los peces del Ecuador. 1.— Especies de la subfamilia Sorubiminae. Politécnica, Revista de Información Técnico-científica, Quito, 3, for 1976-1977, 76-91.

Orton, J. (1871) Contributions to the Natural History of the Valley of Quito. McCalla \& Stavely, Philadelphia.

Oshima, M. (1919) Contributions to the study of the fresh water fishes of the island of Formosa. Annals of the Carnegie Museum, 12, 169-328, pls. 48-53.

Oshima, M. (1926) Notes on a collection of fishes from Hainan, obtained by Prof. S. F. Light. Annotationes Zoologicae Japonenses, 11, 1-25.

Osório, B. (1909) Peixes d'agua doce da Guiné Portugueza. Memorias do Museum Bocage, Lisboa, 1, 95-107, pl. 1.

Ovchynnyk, M.M. (1971) Unrecorded and new species of fishes from fresh waters of Ecuador. Zoologischer Anzeiger, $187,82-122$.

Owen, R. (1853) Descriptive Catalogue of the Osteological Series Contained in the Museum of the Royal College of Surgeons of England, Vol. 1.- Pisces, Reptilia, Aves, Marsupialia, London, 350 p.

Oyakawa, O.T. (1993) Cinco espécies novas de Harttia Steindachner, 1876 da região sudeste do Brasil, de comentários sobre o gênero (Teleostei, Siluriformes, Loricariidae). Comunicações do Museu de Ciências da PUCRS, Série Zoologia, Porto Alegre, 6, 3-27.

Oyakawa, O.T., Akama, A. \& Zanata, A.M. (2005) Review of the genus Hypostomus Lacépède, 1803 from rio Ribeira de Iguape basin, with description of a new species (Pisces, Siluriformes, Loricariidae). Zootaxa, 921, 1-27.

Paepke, H.-J. (1999) Bloch's Fish Collection in the Museum für Naturkunde der Humboldt Universität zu Berlin: an Illustrated Catalog and Historical Account [Theses Zoologicae, 32], A. R. G. Gantner Verlag, KG, Ruggell, Liechtenstein, 216 p., 32 pl.

Page, L.M., Armbruster, J.W. \& Sabaj, M.H. (1996) Redescription of Glyptoperichthys scrophus, a loricariid catfish from Peru. Ichthyological Exploration of Freshwaters, 7, 185-191.

Page, L.M. \& Burr, B.M. (1991) A field guide to freshwater fishes: North America north of Mexico. The Peterson Field Guide Series Vol. 42, Houghton Mifflin Co., Boston, xii + 432 p.

Pallas, P.S. (1787) Piscium novae species descriptae. Nova Acta Academiae Scientiarum Imperialis Petropolitanae, 1, for 1783, 347-360, pls. 9-11. [Cover of journal lists date of publication as 1787; Agassiz (1854), lists date as 1783, Dean (1916) says 1788, AMNH library says vol. 1 published in 1783]

Pappenheim, P. (1911) Zoologische Ergebnisse der Expedition des Herrn G. Tessmann nach Süd-Kamerun und SpanischGuinea.- Fische. Mitteilungen aus dem Zoologischen Museum in Berlin, 5, 505-528.

Pappenheim, P. \& Boulenger, G.A. (1914) Fische. In: Schubotz, H. (Ed.), Wissenschaftliche Ergebnisse der Deutschen Zentral-Afrika Expedition, 1907-1908. Vol. 5 (Zoologie III), Klinkhardt \& Biermann, Leipzig, pp. 225-260, pls. 110. [In 8 vols. 1912-1914.]

Parameswaran, S., Selvaraj, C. \& Radhakrishnam, S. (1967) A review of the Indian freshwater fishes of the genus Ompok Lacépède. Journal of the Zoological Society of India, 19, 89-98.

Parisi, B.M. (2003) Contribution à L'Etude de la Taxonomie du Groupe de Poissons-chats Néotropicaux CalophysusPimelodus (Pimelodidae, Siluriformes) et Hypothèses Phylogénétiques, Doctoral dissertation, Muséum National d'Histoire Naturelle, Paris.

Patterson, C. (1993) Osteichthyes: Teleostei. In: Benton, M.J. (Ed.), The Fossil Record, Vol. 2, Chapman \& Hall, London.

Paugy, D. (1987) Description de deux nouvelles espèces de Synodontis du bassin du Konkouré (Guinée), S. dekimpei et S. levequei (Pisces, Mochokidae). Cybium (3e série), 11, 357-364.

Paugy, D. \& Bénech, V. (1989) Les poissons d'eau douce des bassins côtier du Togo (Afrique de l'Ouest). Revue d'Hydrobiologie Tropicale, 22, 295-316.

Paugy, D. \& Roberts, T.R. (1992) Mochokidae. In: Lévêque, C., Paugy, D. \& Teugels, G.G. (Eds.), Faune des Poissons d'Eaux Douces et Saumâtres de l'Afrique de l'Ouest Vol. 2, Musée Royal de l'Afrique Central, Tervuren, and Editions de l'ORSTOM, Paris, pp. 500-563.

Paxton, J.R., Hoese, D.F., Allen, G.R. \& Hanley, J.E. (1989) Zoological Catalogue of Australia. Volume 7.— Pisces. Petromyzontidae to Carangidae, Australian Government Publishing Service, Canberra, xii + 665 p.

Pearson, N.E. (1924) The fishes of the eastern slope of the Andes. I.- The fishes of the Rio Beni basin, Bolivia, collected by the Mulford expedition. Indiana University Studies, 11, 1-83, pls. 1-12. 
Pearson, N.E. (1937) The fishes of the Atlantic and Pacific slopes near Cajamarca, Peru. Proceedings of the California Academy of Sciences, Series 4, 23, 87-98, pls. 12-13.

Pellegrin, J. (1900) Poissons nouveaux ou rares du Congo français. Bulletin du Muséum d'Histoire Naturelle, Paris, 6, $177-182$.

Pellegrin, J. (1901) Poissons nouveaux ou rares du Congo français. Bulletin du Muséum d'Histoire Naturelle, Paris, 7 , $328-332$.

Pellegrin, J. (1906a) Collections recueillies par M. E. Haug dans l'Ogôoué (Poissons). Bulletin du Muséum d'Histoire Naturelle, Paris, 12, 467-471.

Pellegrin, J. (1906b) Poissons nouveaux du Soudan. Bulletin du Muséum d'Histoire Naturelle, Paris, 12, $472-474$.

Pellegrin, J. (1907a) Siluridé nouveau du Fouta-Djalon. Bulletin du Muséum National d'Histoire Naturelle, 13, $23-25$.

Pellegrin, J. (1907b) Poissons du sud Cameroun, recueillis par la mission Cottes. Bulletin du Muséum National d'Histoire Naturelle, 13, 319-322.

Pellegrin, J. (1908) Description de deux poissons nouveaux de l'Amérique du Sud, de la famille des Loricariidés. Bulletin de la Société Zoologique de France, 33, 125-127.

Pellegrin, J. (1909a) Collections recueillies par M. E. Haug dans l'Ogôoué (Poissons). Bulletin du Muséum National d'Histoire Naturelle, 15, 66-68.

Pellegrin, J. (1909b) Poissons de la Komadougou et du lac Tchad récoltés par la mission Tilho-Gaillard. Bulletin du Muséum National d'Histoire Naturelle, 15, 240-245.

Pellegrin, J. (1909c) Mission géodésique de l'Équateur. Collections recueillies par M. le Dr. Rivet. Description de deux poissons nouveaux de la famille des Loricariidae. Bulletin du Muséum National d'Histoire Naturelle, 15, $517-519$.

Pellegrin, J. (1909d) Note compléméntaire sur une seconde collections de poissons recueillie par M. E. Haug, à Ngomo (Ogôoué). Bulletin de la Société Philomathique (Ser. 10), 1, 45-53.

Pellegrin, J. (1909e) Les poissons du genre Vandellia C. V. Bulletin de la Société Philomathique (Ser. 10), 1, $197-204$.

Pellegrin, J. (1911) Poissons de l'Equateur recueillis par M. le Dr. Rivet. In: Mission du Service Géographique de l'Armée pour la Mesure d'un Arc de Méridien Équatorial en Amérique du Sud sous le Contrôle Scientifique de l'Académie des Sciences, 1899-1906, Tome 9 (Zoologie), Fasc. 2 (Reptiles, Poissons, Batraciens), Ministère de l'Instruction Publique, Paris, pp. 1-15, pl. 1.

Pellegrin, J. (1912) Description d'un poisson nouveau de l'Orénoque appartenant au genre Xenocara. Bulletin de la Société Zoologique de France, 37, 271-272.

Pellegrin, J. (1913a) Poissons nouveaux de Guinée française recueillis par M. Pobéguin. Bulletin de la Société Zoologique de France, 38, 236-241.

Pellegrin, J. (1913b) Poissons nouveaux de l'Ogôoué recueillis par Mr. Earnest Haug. Bulletin de la Société Zoologique de France, 38, 272-275.

Pellegrin, J. (1914) Poissons nouveaux du haut Zambèze recueillis par M. V. Ellenberger. Bulletin de la Société Zoologique de France, 39, 24-28.

Pellegrin, J. (1919a) Poissons nouveaux du Mozambique. Bulletin de la Société Zoologique de France, 44, $397-401$.

Pellegrin, J. (1919b) Poissons du Gribingui recueillis par M. Baudon. Description de sept espèces nouvelles. Bulletin de la Société Zoologique de France, 44, 201-214.

Pellegrin, J. (1920) Poissons des lagunes de la Côte d'Ivoire. Description de deux espèces nouvelles. Bulletin de la Société Zoologique de France, 45, 115-121.

Pellegrin, J. (1922a) Poissons de l'Oubanghi-Chari recueillis par M. Baudon. Description d'un genre, de cinq espèces et d'une variété. Bulletin de la Société Zoologique de France, 47, 64-76.

Pellegrin, J. (1922b) Poissons du Gribingui recueillis par M. Baudon. Description d'un Mormyridé et d'un Characinidé nouveaux. Bulletin de la Société Zoologique de France, 47, 220-223.

Pellegrin, J. (1922c) Poissons nouveaux de l'Afrique orientale. Bulletin du Muséum National d'Histoire Naturelle, 28, 349-351.

Pellegrin, J. (1922d) Poissons nouveaux ou rares du musée du Congo. Revue de Zoologie Africaine, 10, $272-280$.

Pellegrin, J. (1923a) Présentation d'un crâne de Clarias géant du Niger. Bulletin du Muséum National d'Histoire Naturelle, 29, 211-213.

Pellegrin, J. (1923b) Les Poissons des Eaux Douces de l'Afrique Occidentale (du Sénégal au Niger). Publications du Comité d'Etudes Historiques et Scientifiques, Emile Larose, Paris, 373 p.

Pellegrin, J. (1924a) Poissons du Niger recueillis par M. Jean Thomas. Description de deux espèces nouvelles. Bulletin du Muséum National d'Histoire Naturelle, 30, 457-463.

Pellegrin, J. (1924b) Description d'un poisson nouveau du Gabon appartenant au genre Synodontis. Bulletin de la Société 
Zoologique de France, 49, 320-322.

Pellegrin, J. (1924c) Description d'un Siluridé nouveau récolté au Congo Belge. Revue de Zoologie Africaine, 12, 487489.

Pellegrin, J. (1925) Poissons du Nord du Gabon et de la Sangha recueillis par M. Baudon. Description de deux espèces et d'une variété nouvelles. Bulletin de la Société Zoologique de France, 50, 97-106.

Pellegrin, J. (1926) Description de Siluridés, d'un Cyprinodontidé et d'un Tétrodontidé récoltés au Congo Belge par le Dr. Schouteden. Revue de Zoologie Africaine, 14, 201-208.

Pellegrin, J. (1927) Description d'un Siluridé nouveau du Cameroun appartenant au genre Synodontis. Bulletin de la Société Zoologique de France, 52, 365-366.

Pellegrin, J. (1928a) Sur une collection de poissons du Cameroun recueillie par M. Chamaulte. Annales. Société des Sciences Naturelles de al Charente-Inférieure (nouv. sér), 1, 1-11.

Pellegrin, J. (1928b) Poisson du Kasai (Congo Belge). Description d'un genre nouveau et de quatre espèces nouvelles. Bulletin de la Société Zoologique de France, 53, 103-113.

Pellegrin, J. (1928c) Description d'un poisson nouveau du Gabon appartenant au genre Amphilius. Bulletin de la Société Zoologique de France, 53, 453-454.

Pellegrin, J. (1929a) Mission Saharienne Augiéras-Draper, 1927-1928. Poissons. Bulletin du Muséum National d'Histoire Naturelle (2e Série), 1, 134-139.

Pellegrin, J. (1929b) Siluridés, Cyprinodontidés, Acanthoptérygiens du Cameroun recueillis par M. Th. Monod; description de cinq espèces et deux variétés nouvelles. Bulletin de la Société Zoologique de France, 54, 358-369.

Pellegrin, J. (1929c) Siluridé et Cyprinodontidé nouveaux du Gabon recueillis par M. A. Bandon. Bulletin de la Société Zoologique de France, 54, 640-643.

Pellegrin, J. (1930) Poissons de l'Ogôoué, du Kouilou, de l'Alima et de la Sangha recueillis par M. A. Baudon; description de cinq espèces et cinq variétés nouvelles. Bulletin de la Société Zoologique de France, 55, 196-210.

Pellegrin, J. (1931a) Poissons du Kouilou et de la Nyanga recueillis par M. A. Baudon. Bulletin de la Société Zoologique de France, 56, 205-211.

Pellegrin, J. (1931b) Description d'un poisson nouveau de l'Equateur appartenant à la famille des Loricariidés. Revue suisse de Zoologie, Annales de la Société zoologique suisse et du Muséum d'Histoire naturelle de Genève, 38, 113115.

Pellegrin, J. (1932) Description d'un Chrysichthys géant du Congo. Bulletin du Muséum National d'Histoire Naturelle (2e Série), 4, 165-168.

Pellegrin, J. (1933a) Voyage de Ch. Alluaud et P. A. Chappuis en Afrique occidentale Française (Dec. 1930 -Mars 1931). IV.- Poissons. Archiv für Hydrobiologie, 26, 101-120.

Pellegrin, J. (1933b) Poissons de la région du Kivu adressés par M. Guy Babault. Bulletin de la Société Zoologique de France, 58, 169-175.

Pellegrin, J. (1935) Poissons de Guinée Française recueillis par M. Waterlot; description d'une espèce et de deux variétés nouvelles. Bulletin de la Société Zoologique de France, 60, 462-466.

Pellegrin, J. (1936a) Contribution à l'Ichtyologie de l'Angola. Arquivos do Museu Bocage, Lisboa, 7, 45-62.

Pellegrin, J. (1936b) Poissons nouveaux du haut-Laos et de l'Annam. Bulletin de la Société Zoologique de France, 61, 243-248.

Pellegrin, J. (1938a) Poissons de l'Afrique équatoriale Française de Jean Thomas. Bulletin de la Société Zoologique de France, 63, 369-378.

Pellegrin, J. (1938b) Descrizione d'un Siluride nuovo del Giuba, appartenente al genere Clarotes. Bollettino di Pesca, Piscicoltura e Idrobiologia, 14, 218-222.

Pellegrin, J. \& Chevey, P. (1937) Poissons d'Indochine recueillis par MM. J. Delacour et Lowe; description d'une espèce nouvelle. Bulletin de la Société Zoologique de France, 62, 313-318.

Pellegrin, J. \& Fang, P.W. (1940) Poissons de Chine de M. Ho, description de deux espèces nouvelles. Bulletin de la Société Zoologique de France, 64, 338-343.

Peng, Z.G., He, S.P. \& Zhang, Y.G. (2002) Mitochondrial cytochrome b sequence variation and phylogeny of the East Asian bagrid catfishes. Progress in Natural Science, 12, 421-425.

Peng, Z.G., He, S.P. \& Zhang, Y.G. (2004) Phylogenetic relationships of glyptosternoid fishes (Siluriformes: Sisoridae) inferred from mitochondrial cytochrome $b$ gene sequences. Molecular Phylogenetics and Evolution, 31, 979-987.

Peng, Z.G., Zhang, Y.G., He, S.P. \& Chen, Y.Y. (2005) Phylogeny of Chinese catfishes inferred from mitochondrial cytochrome b sequences. Acta Genetica Sinica, 32, 145-154.

Perdices, A., Bermingham, E., Montilla, A. \& Doadrio, I. (2002) Evolutionary history of the genus Rhamdia (Teleostei: 
Pimelodidae) in Central America. Molecular Phylogenetics and Evolution, 25, 172-189.

Pereira, E.H.L. (2005) Resurrection of Pareiorhaphis Miranda Ribeiro, 1918 (Teleostei: Siluriformes: Loricariidae), and description of a new species from the rio Iguaçu basin, Brazil. Neotropical Ichthyology, 3, 271-276.

Pereira, E.H.L., Oliveira, J.C. \& Oyakawa, O.T. (2000) Hemipsilichthys papillatus, a new species of loricariid catfish (Teleostei: Siluriformes) from Minas Gerais, Brazil. Ichthyological Exploration of Freshwaters, 11, 377-383.

Pereira, E.H.L. \& Oyakawa, O.T. (2003) Isbrueckerichthys epakmos, a new species of loricariid catfish from the rio Ribeira de Iguape basin, Brazil (Teleostei, Siluriformes). Neotropical Ichthyology, 1, 3-9.

Pereira, E.H.L. \& Reis, R.E. (1992) Hemipsilichthys vestigipinnis sp. n. (Teleostei, Siluriformes) a new loricariid catfish from the rio Uruguay basin, southern Brazil. Revue Française d'Aquariologie et Herpetologie, 18, for 1991, 111116.

Pereira, E.H.L. \& Reis, R.E. (2002) Revision of the loricariid genera Hemipsilichthys and Isbrueckerichthys (Teleostei: Siluriformes), with descriptions of five new species of Hemipsilichthys. Ichthyological Exploration of Freshwaters, 13, 97-146.

Pereira, E.H.L., Reis, R.E., Souza, P.F.M. \& Lazzarotto, H. (2003) A new species of the loricariid catfish genus Hemipsilichthys from southern Rio de Janeiro coastal rivers, southeastern Brazil (Teleostei: Siluriformes). Zootaxa, 285, 110.

Pereira, S.M. (1984) Siluriformes (Osteichthyes, Teleostei) del Terciario tardio de los alrededores de Paraná (Entre Rios). Jorn. Argent. Paleontol. Vertebr., Res, 1, 2.

Pérez, A. \& Provenzano R, F. (1996) Cordylancistrus perijae, a new species of armored catfish (Siluroidei: Loricariidae) from the Maracaibo basin, Venezuela. Studies on Neotropical Fauna and Environment, 31, 27-34.

Pérez, A. \& Viloria, A. (1994) Ancistrus galani n. sp. (Siluriformes: Loricariidae), with comments on biospeleological explorations in western Venezuela. Mémoires de Biospéologie, 21, 103-107.

Perugia, A. (1891) Appunti sopra alcuni pesci sud-americani conservati nel Museo Civico di Storia Naturale di Genova. Annali del Museo Civico de Storia Naturale di Genova (Ser. 2a), 10, 605-657.

Perugia, A. (1892) Intorno ad alcuni pesci raccolti al Congo dal Capitano Giacomo Bove. Annali del Museo Civico de Storia Naturale di Genova (Ser. 2a), 10, 967-977.

Perugia, A. (1894) Viaggio di Lamberto Loria nella Papuasia orientale. XIII.- Pesci d'acqua dolce. Annali del Museo Civico de Storia Naturale di Genova (Ser. 2a), 14, 546-553.

Perugia, A. (1897) Di alcuni pesci raccolti in Bolivia dal Prof. Luigi Balzan. Annali del Museo Civico de Storia Naturale di Genova (Ser. 2a), 18, 16-27.

Peters, W. (1852) Diagnosen von neuen Flussfischen aus Mossambique. Monatsberichte der Königlichen Preussische Akademie des Wissenschaften zu Berlin, 1852, 275-276, 681-685.

Peters, W. (1861) Über zwei neue Gattungen von Fischen aus dem Ganges. Monatsberichte der Königlichen Preussische Akademie des Wissenschaften zu Berlin, 1861, 712-713.

Peters, W. (1868a) Über eine neue Nagergattung, Chiropodomys penicillatus, so wie über einige neue oder weniger bekannte Amphibien und Fische. Monatsberichte der Königlichen Preussische Akademie des Wissenschaften zu Berlin, 1868, 448-460, pl. 2.

Peters, W. (1868b) Ueber eine von dem Baron Carl von der Decken entdeckte neue Gattung von Welsen, Chiloglanis deckenii, und einige andere Süsswasserfische aus Ostafrika. Monatsberichte der Königlichen Preussische Akademie des Wissenschaften zu Berlin, 1868, 598-602, pl. 2.

Peters, W. (1868c) Flussfische. Naturwissenschaftliche Reise nach Mossambique auf befehl Seiner Mäjestat des Königs Friedrich Wilhelm IV, in den Jahren 1842 bis 1848 Ausgeführt. Zoologie, Vol. IV, G. Reimer, Berlin, xii + 116 p., 20 pls.

Peters, W. (1877) Über die von Dr. C. Sachs in Venezuela gesammelten Fische. Monatsberichte der Königlichen Preussische Akademie des Wissenschaften zu Berlin, 1877, 469-473.

Peters, W. (1881a) Über vier neue Fische. Sitzungsberichte der Gesellschaft Naturforschender Freunde zu Berlin, 1881, 17-19.

Peters, W. (1881b) Über eine Sammlung von Fischen, welche Hr. Dr. Gerlach in Hongkong gesandt hat. Monatsberichte der Königlichen Preussische Akademie des Wissenschaften zu Berlin, 1880, 1029-1037, 1 pl.

Peters, W. (1882) Über drei neue Arten von Mormyrus aus Ost- und Westafrika, und eine Art von Clarias aus Westafrika. Sitzungsberichte der Gesellschaft Naturforschender Freunde zu Berlin, 1882, 72-74.

Pethiyagoda, R. \& Bahir, M.M. (1998) Heteropneustes microps, a junior synonym of $H$. fossilis (Osteichthyes: Heteropneustidae). Journal of South Asian Natural History, 3, 113-114.

Pethiyagoda, R. \& Kottelat, M. (1994) Three new species of fishes of the genera Osteochilichthys (Cyprinidae), Travan- 
coria (Balitoridae) and Horabagrus (Bagridae) from the Chalakudy River, Kerala, India. Journal of South Asian Natural History, 1, 97-116.

Pethon, P. (1969) List of type specimens of fishes, amphibians and reptiles in the Zoological Museum, University of Oslo. Rhizocrinus, Occasional Papers of the Zoological Museum, University of Oslo, 1, 1-17.

Peyer, B. (1928) Ergebnisse der Forschungsreisen Prof. E. Stromers in den Wüsten Ägyptens. V.- Tertiäre Wirbeltiere. 2. Die Welse des ägyptischen Alttertiärs nebst einer kritischen Übersicht über alle fossilen Welse. Abhandlungen der Bayerischen Akademie der Wissenschaften, Mathematisch-Naturwissenschaftliche Abteilung, 32, 1-62, pls.1-6.

Pfaff, J.R. (1933) Report on the fishes collected by Mr. Harry Madsen during Professor O. Olufsen's Expedition to French Sudan in the years 1927-28. Videnskabelige Meddelelser fra Dansk Naturhistorisk Forening i Kjøbenhavn, 94, 273-315, pl. 6.

Pfeffer, G.J. (1889) Übersicht der von Herrn Dr. Franz Stuhlmann in Ägypten, auf Sanzibar und dem gegenüberliegenden Festlande gesammelten Reptilien, Amphibien, Fische, Mollusken und Krebse. Jahrbuch der Hamburgischen Wissenschaflichen Anstalten, 6, 1-36.

Pfeffer, G.J. (1896) Die Fische Ost-Afrikas. In: Möbius, K. (Ed.), Die Thierwelt Ost-Afrikas und der Nachbargebiete, Vol. 1, Lief. V, D. Reimer, Berlin, pp. xviii +72 p.

Pfeiffer, W. \& Eisenberg, J.F. (1965) Die lauterzeugung der dornwelse (Doradidae) und der fiederbartwelse (Mochokidae). Zeitschrift für Morphologie und Ökologie der Tiere, 54, 669-679.

Philippi, R.A. (1866) Bemerkungen über die chilienischen Flussfische. Monatsberichte der Königlichen Preussische Akademie des Wissenschaften zu Berlin, 1866, 708-717.

Pietschmann, V. (1913a) Eine neue Glyptosternum-Art aus dem Tigris. Anzeiger der Mathematisch-Naturwissenschaftlichen Classe der Kaiserlichen Akademie der Wissenschaften in Wien, 1913, 93-95.

Pietschmann, V. (1913b) Fische des Wiesbadener Museums. Jahrbücher des Nassauischen Vereins für Naturkunde, Wiesbaden, 66, 170-201, pls. 1-2.

Pietschmann, V. (1932) Ein neuer Wels aus dem Nil. Zoologischer Anzeiger, 100, 92-94.

Pietschmann, V. (1939) Matériali zoologici dell' Eritrea roccolti da G. Müller duranti la spedizione dell' istituto sieroterapico milanese e convservati al museo di Trieste. Parte VI.- Pisces. Atti del Museo Civico di Storia Naturale di Trieste, 14, 179-186.

Pinna, M.C.C. de. (1988) A new genus of trichomycterid catfish (Siluroidei, Glanapteryginae), with comments on its phylogenetic relationships. Revue suisse de Zoologie, Annales de la Société zoologique suisse et du Muséum d'Histoire naturelle de Genève, 95, 113-128.

Pinna, M.C.C. de. (1989a) A new sarcoglanidine catfish, phylogeny of its subfamily, and an appraisal of the phyletic status of the Trichomycterinae (Teleostei, Trichomycteridae). American Museum Novitates, 2950, 1-39.

Pinna, M.C.C. de. (1989b) Redescription of Glanapteryx anguilla, with notes on the phylogeny of Glanapteryginae (Siluriformes, Trichomycteridae). Proceedings of the Academy of Natural Sciences, Philadelphia, 141, 361-374.

Pinna, M.C.C. de. (1992a) Trichomycterus castroi, a new species of trichomycterid catfish from the Rio Iguaçu of Southeastern Brazil (Teleostei: Siluriformes). Ichthyological Exploration of Freshwaters, 3, 89-95.

Pinna, M.C.C. de. (1992b) A new subfamily of Trichomycteridae (Teleostei, Siluriformes), lower loricarioid relationships and a discussion on the impact of additional taxa for phylogenetic analysis. Zoological Journal of the Linnean Society, 106, 175-229.

Pinna, M.C.C. de. (1993) Higher-level Phylogeny of Siluriformes (Teleostei: Ostariophysi), with a New Classification of the Order, Unpublished Ph.D. dissertation, City University of New York, New York.

Pinna, M.C.C. de. (1996) A phylogenetic analysis of the Asian catfish families Sisoridae, Akysidae, and Amblycipitidae, with a hypothesis on the relationships of the neotropical Aspredinidae (Teleostei, Ostariophysi). Fieldiana, Zoology (New Series), 84, i-iv + 1-83.

Pinna, M.C.C. de. (1998a) Phylogenetic relationships of Neotropical Siluriformes (Teleostei: Ostariophysi): Historical overview and synthesis of hypotheses. In: Malabarba, L.R., Reis, R.E., Vari, R.P., Lucena, Z.M. \& Lucena, C.A.S. (Eds.), Phylogeny and Classification of Neotropical Fishes, Edipucrs, Porto Alegre, pp. 279-330.

Pinna, M.C.C. de. (1998b) A new species of the catfish genus Glanapteryx (Siluriformes: Trichomycteridae). Proceedings of the Biological Society of Washington, 111, 35-42.

Pinna, M.C.C. de. (2003) Nematogenyidae. In: Reis, R.E., Kullander, S.O. \& Ferraris, C.J., Jr. (Eds.), Check list of the Freshwater Fishes of South and Central America, Edipucrs, Porto Alegre, Brazil, pp. 268-269.

Pinna, M.C.C. de \& Britski , H.A. (1991) Megalocentor, a new genus of parasitic catfish from the Amazon basin: the sister group of Apomatoceros (Trichomycteridae: Stegophilinae). Ichthyological Exploration of Freshwaters, 2, 113128. 
Pinna, M.C.C. de \& Keith, P. (2003) A new species of the catfish genus Ituglanis from French Guyana (Osteichthyes; Siluriformes: Trichomycteridae). Proceedings of the Biological Society of Washington, 116, 873-882.

Pinna, M.C.C. de \& Ng, H.H. (2004) The second ural centrum in Siluriformes and its implication for the monophyly of superfamily Sisoroidea (Teleostei, Ostariophysi). American Museum Novitates, 3437, 1-23.

Pinna, M.C.C. de \& Starnes, W.C. (1990) A new genus and species of Sarcoglanidinae from the Río Mamoré, Amazon Basin, with comments on subfamilial phylogeny (Teleostei, Trichomycteridae). Journal of Zoology (London), 222, 75-88.

Pinna, M.C.C., de \& Vari, R.P. (1995) Monophyly and phylogenetic diagnosis of the family Cetopsidae, with synonymization of the Helogenidae (Teleostei: Siluriformes). Smithsonian Contributions to Zoology, 571, i-iii + 1-26.

Pinna, M.C.C. de \& Winemiller, K.O. (2000) A new species of Ammoglanis (Siluriformes: Trichomycteridae) from Venezuela. Ichthyological Exploration of Freshwaters, 11, 255-264.

Pinna, M.C.C. de \& Wosiacki, W. (2002) A new interstitial catfish of the genus Listrura from southern Brazil (Siluriformes: Trichomycteridae: Glanapteryginae). Proceedings of the Biological Society of Washington, 114, 720-726.

Pinna, M.C.C. de \& Wosiacki, W. (2003) Trichomycteridae. In: Reis, R.E., Kullander, S.O. \& Ferraris, C.J., Jr. (Eds.), Check list of the Freshwater Fishes of South and Central America, Edipucrs, Porto Alegre, Brazil, pp. 270-290.

Pinto, S.Y. \& Marzulo, D. (1975) Estudos morfológicos. VI.- Sobre Lophiosilurus alexandri Steindachner, 1876. (Actinopterygii, Cypriniformes, Pimelodidae). Boletim do Museu de História Natural da Universidade Federal de Minas Gerais, Zoologia, 21, 1-9, 4 pl.

Piorski, N.M. (1999) Diferenciação morfométrica entre as espécies Platydoras costatus (Linnaeus, 1766) e P. armatulus (Cuvier \& Valenciennes, 1840) (Pisces; Siluriformes; Doradidae). Comunicações do Museu de Ciências da PUCRS, Série Zoologia, Porto Alegre, 12, 19-30.

Playfair, R.L. (1867) On the fishes of Cachar. Proceedings of the Zoological Society of London, 1867, 14-17, pl. 3.

Playfair, R.L. \& Günther, A. (1867) The Fishes of Zanzibar, with a List of the Fishes of the Whole East Coast of Africa, Van Voorst, London, xix +153 p., 21 pl.

Poll, M. (1933) Contribution à la faune ichthyologique du Katanga. Annales du Musée du Congo Belge, Zoologie (Ser. 1), 3, 101-152.

Poll, M. (1938) Poissons du Katanga (bassin du Congo) récoltés par le professeur Paul Brien. Revue de Zoologie et de Botanique Africaines, 30, 389-423.

Poll, M. (1941a) Étude systématique et morphologique d'une collection de poissons de l'Uele (Congo Belge), comprenant trois espèces nouvelles. Bulletin du Musée Royal d'Histoire Naturelle de Belgique, 17, 1-18.

Poll, M. (1941b) Poissons nouveaux de la Côte d'Ivoire. Revue de Zoologie et de Botanique Africaines, 34, $133-143$.

Poll, M. (1942a) Les poissons du Lac Tumba, Congo belge. Bulletin du Musée Royal d'Histoire Naturelle de Belgique, $18,1-25$.

Poll, M. (1942b) Description d'un genre nouveau de Bagridae du lac Tanganika. Revue de Zoologie et de Botanique Africaines, 35, 318-322.

Poll, M. (1942c) Description d'un genre nouveau de Clariidae originaire du Congo belge. Revue de Zoologie et de Botanique Africaines, 36, 94-100.

Poll, M. (1943a) Description du Tanganikallabes mortiauxi, gen. nov., sp. n., de la famille des Clariidae. Revue de Zoologie et de Botanique Africaines, 37, 126-133.

Poll, M. (1943b) Descriptions de poissons nouveaux du Lac Tanganika, appartenant aux familles des Clariidae et Cichlidae. Revue de Zoologie et de Botanique Africaines, 37, 305-318.

Poll, M. (1944a) Descriptions de poissons nouveaux recueillis dans la région d'Albertville (Congo belge) par le Dr. G. Pojer. Bulletin du Musée Royal d'Histoire Naturelle de Belgique, 20, 1-12.

Poll, M. (1944b) Description préliminaire d'un Clariidae nouveau observé à Léopoldville par M. L. Tihon. Revue de Zoologie et de Botanique Africaines, 38, 79-82, 1 pl.

Poll, M. (1946) Révision de la faune ichthyologique du Lac Tanganika. Annales du Musée du Congo Belge, Zoologie (Ser. 1), 4, 146-364, pls. 1-3 + map.

Poll, M. (1948) Poissons recueillis au Katanga par H. J. Bredo. Bulletin du Musée Royal d'Histoire Naturelle de Belgique, 24, 1-24.

Poll, M. (1949) Résultats scientifiques des croisières du Navire-École Belge "Mercator" IV.— Poissons. Mémoires, Institut Royal des Sciences Naturelles de Belgique, série 2, 33, 173-269.

Poll, M. (1952) Poissons de rivières de la région des lacs Tanganika et Kivu recuellis par G. Marlier. Revue de Zoologie et de Botanique Africaines, 46, 221-236.

Poll, M. (1953) Poissons non Cichlidae. Exploration Hydrobiologique du Lac Tanganika (1946-1947), Vol. 3 (part 5a), 
Institut Royal des Sciences Naturelles de Belgique, Bruxelles, pp. 251 p., 11 pls.

Poll, M. (1954) Poissons de forêt des environs de Yangambi (Stanleyville) recueillis par A. Hulot. Annales du Musée royal du Congo Belge, (Série in $8^{\circ}$ ), Sciences Zoologiques, 1, 56-68.

Poll, M. (1957) Redescription du Gymnallabes tihoni Poll, 1944, Clariidae microphthalme du Stanley-Pool. Revue de Zoologie et de Botanique Africaines, 55, 237-248.

Poll, M. (1959) Recherches sur la faune ichthyologique de la région du Stanley Pool. Annales du Musée royal du Congo Belge, (Série in $8^{\circ}$ ), Sciences Zoologiques, 71, 75-174, pls. 12-26.

Poll, M. (1966) Genre et espèce nouveaux de Bagridae du fleuve Congo en région de Léopoldville. Revue de Zoologie et de Botanique Africaines, 74, 425-428.

Poll, M. (1967) Contribution à la faune ichthyologique de l'Angola. Publicações. Culturais, Companhia de Diamantes de Angola, 75, 1-381, pls. 1-20.

Poll, M. (1971) Révision des Synodontis Africains (Famille Mochocidae). Annales du Musée Royal de l'Afrique Centrale, Series in $8^{\circ}$, Sciences Zoologiques, 191, 1-497.

Poll, M. (1974) Un nouveau Synodontis (Pisces Mochocidae) de la rivière Lukenie (République du Zaïre). Revue de Zoologie Africaine, 88, 441-444.

Poll, M. (1976) Poissons. Exploration Parc National Upemba Mission G. F. de Witte, 73, 1-127, 43 pls.

Poll, M. (1977) Les genres nouveaux Platyallabes et Platyclarias comparés au genre Gymnallabes Gthr. Synopsis nouveau des genres de Clariidae. Bulletin de la Classe des Sciences. Académie Royale de Belgique (Ser. 5), 63, 122149.

Poll, M. \& Gosse, J.-P. (1963) Contribution à l'étude systématique de la faune ichthyologique du Congo Central. Annales du Musée Royal de l'Afrique Centrale, Series in 8*, Sciences Zoologiques, 116, 43-111, pls. 1-4.

Poll, M. \& Gosse, J.-P. (1969) Révision des Malapteruridae (Pisces, Siluriformes) et description d'une deuxième espèce de silure électrique: Malapterurus microstoma sp. n. Bulletin, Institut royal des Sciences naturelles de Belgique, 45, $1-12$, pls. 1-3.

Poll, M. \& Gosse, J.-P. (1994) Genera des poissons d'eau douce de l'Afrique. Mémoire de la Classe des Sciences, Académie royale de Belgique, 9, 1-324.

Poll, M. \& Lambert, J. (1958) Un Cyprinodontide et un Clariide nouveaux de la grande forêt congolaise. Revue de Zoologie et de Botanique Africaines, 58, 328-339.

Poll, M., Lanza, B. \& Romoli Sassi, A. (1972) Genre nouveau extraordinaire de Bagridae du fleuve Juba: Pardiglanis tarabinii gen. n. sp. n. (Pisces Siluriformes). Monitore Zoologico Italiano, N. S., suppl, 4, 327-345.

Poll, M. \& Roberts, T.R. (1968) Description d'une espèce nouvelle de Synodontis du Bassin du Congo. Revue de Zoologie et de Botanique Africaines, 77, 296-302.

Poll, M. \& Roman, B. (1967) Poissons nouveaux de la Haute Comoé. Revue de Zoologie et de Botanique Africaines, 75, 179-187.

Poll, M. \& Stewart, D.J. (1975) Un Mochocidae et un Kneriidae nouveaux de la rivière Luongo (Zambia), affluent du bassin du Congo (Pisces). Revue de Zoologie et de Botanique Africaines, 89, 151-158.

Popta, C.M.L. (1900) A new species of Arius. Notes from the Leyden Museum, 22, 71-74.

Popta, C.M.L. (1904) Descriptions préliminaires des nouvelles espèces de poissons recueillies au Bornéo central par M. le Dr. A. W. Nieuwenhuis en 1898 et en 1900. Notes from the Leyden Museum, 24, for 1902-04, 179-202.

Popta, C.M.L. (1906) Résultats ichthyologiques des voyages scientifiques de Monsieur le Professeur Dr. A. W. Nieuwenhuis dans le centre e Bornéo (1898 et 1900). Notes from the Leyden Museum, 27, 1-304, 10 pls.

Popta, C.M.L. (1911) Ueber Fische von Wladiwostok und von Blagoweschtensk a. Amur, gesammelt von Herrn Dr. P. v. Wittenburg. Jahresheshefte des Vereins für Vaterländische Naturkunde in Württemberg, 75, 333-353.

Popta, C.M.L. (1913) Auchenoglanis büttikoferi n. sp. from West Africa. Notes from the Leyden Museum, 35, 237-240, pl. 10 .

Popta, C.M.L. (1919) Description of Clarias nigeriae n. sp. from the Wari, mouth of the Niger, West Africa. Zoologische Mededelingen (Leiden), 5, 4.

Posada, A. (1909) Los peces. In: Estudios cientificos del doctor Andres Posada con algunos otros escritos suyos sobre diversos temas, Medellin, Colombia, pp. 285-322.

Pouyaud, L., Gustiano, R. \& Teugels, G.G. (2004) Contribution to the phylogeny of the Pangasiidae based on mitochondrial 12S RDNA. Indonesian Journal of Agricultural Science, 5, 45-62.

Pouyaud, L. \& Teugels, G.G. (2000) Description of a new pangasiid catfish from east Kalimantan, Indonesia (Siluriformes: Pangasiidae). Ichthyological Exploration of Freshwaters, 11, 193-200.

Pouyaud, L., Teugels, G.G., Gustiano, R. \& Legendre, M. (2000) Contributions to the phylogeny of pangasiid catfishes 
based on allozymes and mitochondrial DNA. Journal of Fish Biology, 56, 1509-1538.

Pouyaud, L., Teugels, G.G. \& Legendre, M. (1999) Description of a new pangasiid catfish from South-east Asia (Siluriformes). Cybium (3e série), 23, 247-258.

Prashad, B. \& Mukerji, D.D. (1929) The fish of the Indawgyi Lake and the streams of the Myitkyina District (Upper Burma). Records of the Indian Museum, 31, 161-223, pls. 7-10.

Priem, F. (1904) Sur les poissons du Bartonien et les siluridés et acipenséridés de l'Éocène du bassin de Paris. Bulletin de la Société Géologique de France, 4e serie, 4, 42-47.

Priem, F. (1906) Sur les Otolithes des Poissons éocènes du Bassin parisien. Bulletin de la Société Géologique de France, 4e serie, 6, 265-280

Priem, F. (1908) Étude des Poissons Fossiles du Bassin Parisien. Publications des Annales de Paléontologie, Paris 144 p., 74 figs., 5 pls.

Priem, F. (1914) Sur des poissons fossils et en particulier des Siluridés du Tertiaire supérieur et des couches récentes d'Afrique. Mémoires de la Société géologique de France, 21, 1-13, pls. 1-5.

Provenzano R., F. (1995) Leporacanthicus galaxias Isbrücker y Nijssen (1989) (Pisces: Siluriformes: Loricariidae), nueva cita de un bagre loricarido para Venezuela. Acta Biologica Venezuelica, 15, 97-98.

Provenzano R., F. (1997) Gelanoglanis stroudi new record of a catfish (Siluroidei: Auchenipteridae) for the Venezuelan continental ichthyofauna. Acta Biologica Venezuelica, 17, 79-81.

Provenzano R., F., Lasso, C. \& Ponte, V. (1995) Neblinichthys roraima, a new species of armored catfish (Siluroidei: Loricariidae) from río Kukenan, Venezuela, with considerations about the biogeography of the Guyana Shield. Ichthyological Exploration of Freshwaters, 6, 243-254.

Provenzano R., F., Machado-Allison, A., Chernoff, B., Willink, P. \& Petry, P. (2005) Harttia merevari a new species of catfish (Siluriformes: Loricariidae) from Venezuela. Neotropical Ichthyology, 3, 519-524.

Provenzano R., F., Schaefer, S.A., Baskin, J.N. \& Royero-Leon, R. (2003) New, possibly extinct lithogenine loricariid (Siluriformes, Loricariidae) from northern Venezuela. Copeia, 2003, 562-575.

Puyo, J. (1936) Contribution à l'étude ichthyologique de la Guyane française. Pêches et pêcheries. Bulletin de la Société d'Histoire Naturelle de Toulouse, 70, 5-258.

Quevedo, R. \& Reis, R. (2002) Pogonopoma obscurum: a new species of loricariid catfish (Siluriformes: Loricariidae) from southern Brazil, with comments on the genus Pogonopoma. Copeia, 2002, 402-410.

Quoy, J.R.C. \& Gaimard, J.P. (1824-1825) Description des Poissons, chapter IX. In: Freycinet, L., de (Ed.), Voyage Autour du Monde...Exécuté sur les Corvettes de L. M. L’Uranie et La Physicienne, Pendant les Années 1817, 1818, 1819 et 1820, Chez Pillet aîné, Paris, pp. 192-401, pls. 43-65. [Pp.192-328 issued in 1824; 329-401 in 1825]

Rafinesque, C.S. (1815) Analyse de la Nature, ou Tableau de l'Univers et des Corps Organisés, Palerme, 224 p.

Rafinesque, C.S. (1818a) Discoveries in natural history, made during a journey through the western region of the United States. American Monthly Magazine and Critical Review, 3, 354-356.

Rafinesque, C.S. (1818b) Further account of discoveries in natural history, in the western states, made during a journey through the western region of the United States. American Monthly Magazine and Critical Review, 4, 39-42.

Rafinesque, C.S. (1819) Prodrome de 70 nouveaux genres d'animaux découverts dans l'intérieur des États-Unis d'Amérique, durant l'année 1818. Journal de Physique, de Chimie et d'Histoire Naturelle, 88, 417-429.

Rafinesque, C.S. (1820a) Ichthyologia Ohiensis, or Natural History of the Fishes Inhabiting the River Ohio and its Tributary Streams, Preceded by a Physical Description of the Ohio and its Branches, Privately printed, Lexington, Kentucky, 90 p.

Rafinesque, C.S. (1820b) Description of the Silures or catfishes of the River Ohio. Quarterly Journal of Science, Literature and the Arts, 9, 48-52.

Rafinesque, C.S. (1820c) Ichthyologia Ohiensis [Part 7]. Western Review and Miscellaneous Magazine, 2, $355-363$.

Rafinesque, C.S. (1832) Extracts from a second series of zoological letters written to Baron Cuvier of Paris, by Prof. Rafinesque in 1831. Atlantic Journal and Friend of Knowledge, 1, 19-22.

Rainboth, W.J. (1996) Fishes of the Cambodian Mekong. FAO Species Identification Field Guide for Fishery Purposes, FAO, Rome, 265 p.

Ramakrishniah, M. (1988) A new bagrid fish of the genus Mystus (Scopoli) from Krishna River system. Matsya, 12/13, 139-143.

Ramsay, E.P. \& Ogilby, J.D. (1886) A contribution to the knowledge of the fish-fauna of New Guinea. Proceedings of the Linnean Society of New South Wales (Ser. 2), 1, 8-20.

Ranzani, C. (1841) [De nonnullis novis speciebus Piscium. Opusculum tertium]. Nuovi Annali delle Scienze Naturali (Bologna), 3, 60-66. 
Ranzani, C. (1842) De nonnullis novis speciebus piscium. Opusculum tertium. Novi Commentarii Academiae Scientiarum Instituti Bononiensis, 5, 307-338, pls. 23-28.

Rao, V.R. (1956) The skull of an Eocene siluroid fish from Western Kutch, India. Journal of the Paleontological Society of India, 1, 181-185, pl. 28.

Rapp Py-Daniel, L.H. (1981) Furcodontichthys novaesi n. gen., n. sp. (Osteichthyes, Siluriformes; Loricariidae) na bacia Amazônia, Brasil. Boletim do Museu Paraense Emilio Goeldi, Nova Serie, Zoologia, 105, 1-17.

Rapp Py-Daniel, L.H. (1985) Dekeyseria amazonica, novo gênero e nova espécie na região amazônica, Brasil, e Dekeyseria scaphirhyncha (Kner, 1854) nova combinação (Loricariidae: Siluriformes). Amazoniana, 9, 177-191.

Rapp Py-Daniel, L.H. (1988) Hypostomus hoplonites sp. n. da bacia amazônica, Brasil (Pisces, Siluroidea, Loricariidae). Iheringia, Série Zoologia, 68, 13-23.

Rapp Py-Daniel, L.H. (1989) Redescription of Parancistrus aurantiacus (Castelnau, 1855) and preliminary establishment of two new genera: Baryancistrus and Oligancistrus (Siluriformes, Loricariidae). Cybium (3e série), 13, 235246.

Rapp Py-Daniel, L.H. (1991) Chaetostoma jegui, a new mailed catfish from Rio Uraricoera, Brazil (Osteichthyes: Loricariidae). Ichthyological Exploration of Freshwaters, 2, 239-246.

Rapp Py-Daniel, L.H. \& Oliveira, E.C. (2001) Seven new species of Harttia from the Amazonian-Guyana region (Siluriformes: Loricariidae). Ichthyological Exploration of Freshwaters, 12, 79-96.

Rapp Py-Daniel, L.H. \& Zuanon, J. (2005) Description of a new species of Parancistrus (Siluriformes: Loricariidae) from the rio Xingu, Brazil. Neotropical Ichthyology, 3, 571-577.

Rashida, M., Mirza, M.R. \& Saleem, M. (1996) A contribution to the systematics and biology of Glyptothorax kashmirensis Hora (Pisces: Sisoridae) from Pakistan and Azad Kashmir. Biologia (Lahore), 42, 59-60.

Regan, C.T. (1903a) Description of a new fish of the genus Chaetostomus from Venezuela. Annals and Magazine of Natural History (Ser. 7), 11, 599.

Regan, C.T. (1903b) Descriptions of new South-American fishes in the collection of the British Museum. Annals and Magazine of Natural History (Ser. 7), 12, 621-630.

Regan, C.T. (1904a) On a collection of fishes made by Mr. John Graham at Yunnan Fu. Annals and Magazine of Natural History (Ser. 7), 13, 190-194.

Regan, C.T. (1904b) A monograph of the fishes of the family Loricariidae. Transactions of the Zoological Society of London, 17, 191-350, pls. 9-21.

Regan, C.T. (1905a) A synopsis of the species of the silurid genera Parexostoma, Chimarrhichthys, and Exostoma. Annals and Magazine of Natural History (Ser. 7), 15, 182-185.

Regan, C.T. (1905b) Description of a new loricariid fish of the genus Xenocara from Venezuela. Novitates Zoologicae (Tring), 12, 242.

Regan, C.T. (1905c) Description de six poissons nouveaux faisant partie de la collection du Musée d'Histoire Naturelle de Genève. Revue suisse de Zoologie, Annales de la Société zoologique suisse et du Muséum d'Histoire naturelle de Genève, 13, 389-393, pls. 5-6.

Regan, C.T. (1906-1908) Pisces. In: Godman, F.D. \& Salvin, O. (Eds.), Biologia Central-Americana, Part 193, London, pp. 1-203, 25 pls. [signatures dated to month and year]

Regan, C.T. (1906a) Notes on some loricariid fishes, with descriptions of two new species. Annals and Magazine of Natural History (Ser. 7), 17, 94-98.

Regan, C.T. (1906b) Descriptions of five new freshwater fishes from Sarawak, Borneo, collected by Dr. D. Hose. Annals and Magazine of Natural History (Ser. 7), 18, 66-68.

Regan, C.T. (1906c) On the fresh-water fishes of the island of Trinidad, based on the collection, notes, and sketches, made by Mr. Lechmere Guppy, Junr. Proceedings of the Zoological Society of London, 1906, pt 2, 378-393, pls. 2125.

Regan, C.T. (1907a) Descriptions of three new fishes from Yunnan, collected by Mr. J. Graham. Annals and Magazine of Natural History (Ser. 7), 19, 63-64.

Regan, C.T. (1907b) Descriptions of six new freshwater fishes from Mexico and Central America. Annals and Magazine of Natural History (Ser. 7), 19, 258-260.

Regan, C.T. (1907c) Fishes. Pp. 157-158, in: Reports on a collection of Batrachia, reptiles and fish from Nepal and the western Himalayas. Records of the Indian Museum, 1, 149-158, pl. 6.

Regan, C.T. (1908a) Descriptions of three new freshwater fishes from China. Annals and Magazine of Natural History (Ser. 8), 1, 109-111, pl. 4.

Regan, C.T. (1908b) Descriptions of new freshwater fishes from China and Japan. Annals and Magazine of Natural His- 
tory (Ser. 8), 1, 149-153.

Regan, C.T. (1908c) Descriptions of four new freshwater fishes from British New Guinea. Annals and Magazine of Natural History (Ser. 8), 1, 153-156.

Regan, C.T. (1908d) Description of a new loricariid fish of the genus Plecostomus from Argentina. Annals and Magazine of Natural History (Ser. 8), 2, 358.

Regan, C.T. (1908e) Description of new fishes from Lake Candidius, Formosa, collected by Dr. A. Moltrecht. Annals and Magazine of Natural History (Ser. 8), 2, 358-360.

Regan, C.T. (1908f) Descriptions of new loricariid fishes from South America. Proceedings of the Zoological Society of London, 1907, 795-800, pls. 47-49.

Regan, C.T. (1908g) The Duke of Bedford's Zoological Exploration in eastern Asia.- VIII. A collection of freshwater fishes from Corea. Proceedings of the Zoological Society of London, 1908, 59-63, pls. 2-3.

Regan, C.T. (1909) Descriptions of three new freshwater fishes from South America, presented to the British Museum by Herr J. Paul Arnold. Annals and Magazine of Natural History (Ser. 8), 3, 234-235.

Regan, C.T. (1911) The classification of the teleostean fishes of the order Ostariophysi. 2. Siluroidea. Annals and Magazine of Natural History (Ser. 8), 8, 553-577.

Regan, C.T. (1912a) A revision of the South-American siluroid fishes of the genus Corydoras, with a list of the specimens in the British Museum (Natural History). Annals and Magazine of Natural History (Ser. 8), 10, $209-220$.

Regan, C.T. (1912b) Descriptions of new fishes of the family Loricariidae in the British Museum Collection. Proceedings of the Zoological Society of London, 1912, 666-670, pls. 75-77.

Regan, C.T. (1913a) Descriptions of two new fishes from Paranagua, Brazil, presented to the British Museum by Herr A. Rachow. Annals and Magazine of Natural History (Ser. 8), 11, 231-232.

Regan, C.T. (1913b) A synopsis of the siluroid fishes of the genus Liocassis, with descriptions of new species. Annals and Magazine of Natural History (Ser. 8), 11, 547-554.

Regan, C.T. (1913c) Fishes from the River Ucayali, Peru, collected by W. Mounsey. Annals and Magazine of Natural History (Ser. 8), 12, 281-283.

Regan, C.T. (1913d) The fishes of the San Juan River, Colombia. Annals and Magazine of Natural History (Ser. 8), 12 , $462-473$.

Regan, C.T. (1913e) Description of a new loricariid fish of the genus Plecostomus from Rio Janeiro. Annals and Magazine of Natural History (Ser. 8), 12, 555.

Regan, C.T. (1916) A new loricariid fish of the genus Cyclopium from Ecuador. Annals and Magazine of Natural History (Ser. 8), 18, 80.

Regan, C.T. (1920a) Three new fishes from the Tanganyika Territory. Annals and Magazine of Natural History (Ser. 9), 6, 104-105.

Regan, C.T. (1920b) Pisces. Zoological Record, 55, for 1918, 1-19.

Regan, C.T. (1923) Note on the siluroid fishes of the genera Glyptosternum and Exostoma. Annals and Magazine of Natural History (Ser. 9), 11, 608-610.

Reichel, M. (1927) Étude anatomique du Phreatobius cisternarum Goeldi, silure aveugle du Brésil. Revue suisse de Zoologie, Annales de la Société zoologique suisse et du Muséum d'Histoire naturelle de Genève, 34, 285-403, pl. 26.

Reinhardt, J.T. (1859) Stegophilus insidiosus, en ny Mallefish fra Brasilien of dens Levemaade. Videnskabelige Meddelelser fra den Naturhistorisk Forening i Kjфbenhavn, Aaret, for 1858, 79-97, pl. 2.

Reis, R.E. (1983) Rineloricaria longicauda e Rineloricaria quadrensis, duas novas especies de Loricariinae do sul do Brasil (Pisces, Siluriformes, Loricariidae). Iheringia, Série Zoologia, 62, 61-80.

Reis, R.E. (1987) Ancistrus cryptophthalmus sp. n., a blind mailed catfish from the Tocantins River basin, Brazil (Pisces, Siluriformes, Loricariidae). Revue Française d'Aquariologie et Herpetologie, 14, 81-84.

Reis, R.E. (1992) Wir Fingen den blinden Antennenwels, Ancistrus cryptophthalmus. In: Stawikowski, R. (Ed.), Harnischwelse, Die Aquarien- und Terrarien Zeitschrift, Sonderheft, Eugen Ulmer, Stuttgart, pp. 54-56.

Reis, R.E. (1997) Revision of the neotropical catfish genus Hoplosternum (Ostariophysi: Siluriformes: Callichthyidae), with the description of two new genera and three new species. Ichthyological Exploration of Freshwaters, 7, 299326.

Reis, R.E. (1998a) Anatomy and phylogenetic analysis of the Neotropical callichthyid catfishes (Ostariophysi, Siluriformes). Zoological Journal of the Linnean Society, 124, 105-168.

Reis, R.E. (1998b) Systematics, biogeography, and the fossil record of the Callichthyidae: A review of the available data. In: Malabarba, L.R., Reis, R.E., Vari, R.P., Lucena, Z.M. \& Lucena, C.A.S. (Eds.), Phylogeny and Classification of 
Neotropical Fishes, Edipucrs, Porto Alegre, pp. 351-362.

Reis, R.E. (2003) Callichthyidae. In: Reis, R.E., Kullander, S.O. \& Ferraris, C.J., Jr. (Eds.), Check list of the Freshwater Fishes of South and Central America, Edipucrs, Porto Alegre, Brazil, pp. 291-309.

Reis, R.E. (2004) Otocinclus cocama, a new uniquely colored loricariid catfish from Peru (Teleostei: Siluriformes), with comments on the impact of taxonomic revisions to the discovery of new taxa. Neotropical Ichthyology, 2, 109-115.

Reis, R.E. \& Cardoso, A.R. (2001) Two new species of Rineloricaria from southern Santa Catarina and northeastern Rio Grande do Sul, Brazil (Teleostei: Loricariidae). Ichthyological Exploration of Freshwaters, 12, 319-332.

Reis, R.E. \& Kaefer, C.C. (2005) Two new species of the Neotropical catfish genus Lepthoplosternum (Ostariophysi: Siluriformes: Callichthyidae). Copeia, 2005, 724-731.

Reis, R.E., Kullander, S.O. \& Ferraris, C.J., Jr. (Eds.) (2003) Check List of the Freshwater Fishes of South and Central America, Edipucrs, Porto Alegre, Brazil, 729 p.

Reis, R.E., Le Bail, P.-Y. \& Mol, J.H.A. (2005) New arrangement in the synonymy of Megalechis Reis, 1997 (Siluriformes: Callichthyidae). Copeia, 2005, 678-682.

Reis, R.E. \& Pereira, E.H.L. (1999) Hemipsilichthys nudulus, a new, uniquely-plated species of loricariid catfish from the rio Araranguá basin, Brazil (Teleostei: Siluriformes). Ichthyological Exploration of Freshwaters, 10, 45-51.

Reis, R.E. \& Pereira, E.H.L. (2000) Three new species of the loricariid catfish genus Loricariichthys (Teleostei: Siluriformes) from southern South America. Copeia, 2000, 1029-1047.

Reis, R.E. \& Schaefer, S.A. (1992) Eurycheilus pantherinus (Siluroidei: Loricariidae), a new genus and species of Hypoptopomatinae from southern Brazil. Copeia, 1992, 215-223.

Reis, R.E. \& Schaefer, S.A. (1993) Eurycheilichthys nom. nov., a substitute name for Eurycheilus Reis and Schaefer, 1992 (Siluroidei: Loricariidae). Copeia, 1993, 894.

Reis, R.E. \& Schaefer, S.A. (1998) New cascudinhos from southern Brazil: Systematics, endemism, and relationships (Siluriformes, Loricariidae, Hypoptopomatinae). American Museum Novitates, 3254, 1-25.

Reis, R.E., Weber, C. \& Malabarba, L.R. (1990) Review of the genus Hypostomus Lacepéde, 1803 from southern Brazil, with descriptions of three new species (Pisces: Siluriformes: Loricariidae). Revue suisse de Zoologie, Annales de la Société zoologique suisse et du Muséum d'Histoire naturelle de Genève, 97, 729-766.

Reizer, C., Mattei, X. \& De Vos, L. (1980) Contribution à l'étude de la faune ichtyologique du bassin du fleuve Sénégal; V.- Schilbeidae. Bulletin de l'Institut fondamental d'Afrique noire. Série A, Sciences naturelles, 42, 181-200.

Rema Devi, K. \& Emilyamma, K.G. (1997) On the specific identity of Ompok bimaculatus (Siluriformes, Siluridae). Journal of the Bombay Natural History Society, 94, 421-422, 1 pl.

Rema Devi, K. \& Raghunathan, M.B. (1999) Heteropneustes longipectoralis (Siluriformes: Heteropneustidae) a new species from the Anamalai Hills, in the western Ghats. Records of the Zoological Survey of India, 97, 109-115, pls. $1-2$.

Rendahl, H. (1922) A contribution to the ichthyology of north-west Australia. Nyt Magazin for Naturvidenskaberne, Kristiania, 60, 163-197.

Rendahl, H. (1925) Eine neue Art der Gattung Glyptosternum aus China. Zoologischer Anzeiger, 64, 307-308.

Rendahl, H. (1928) Beiträge zur Kenntnis der Chinesischen süsswasserfische. I.—Systematischer teil. Arkiv för Zoologi, 20 A, 1-194.

Rendahl, H. (1932) Die Fischfauna der chinesischen Provinz Szetschwan. Arkiv för Zoologi, 24 A, 1-134.

Rendahl, H. (1937) Einige Fische aus Ecuador und Bolivia. Arkiv för Zoologi, 29 A, 1-11.

Rendahl, H. (1941) Fische aus dem pazifischen Abflussgebiet Kolumbiens. Arkiv för Zoologi, 33 A, 1-15.

Rendahl, H. \& Vestergren, G. (1941) Eine neue Art der Gattung Glyptosternon s. str. aus dem nordöstlichen Birma. Zoologischer Anzeiger, 133, 213-214.

Retzer, M.E. (2005) Description of a new species of Acestridium (Siluriformes: Loricariidae) from Colombia. Zootaxa, 972, 1-6.

Retzer, M.E., Nico, L.G. \& Provenzano R, F. (1999) Two new species of Acestridium (Siluriformes: Loricariidae) from southern Venezuela, with observations on camouflage and color change. Ichthyological Exploration of Freshwaters, 10, 313-326.

Retzer, M.E. \& Page, L.M. (1997) Systematics of the stick catfishes, Farlowella Eigenmann \& Eigenmann (Pisces, Loricariidae). Proceedings of the Academy of Natural Sciences, Philadelphia, 147, 33-88.

Ribeiro, A.C., Carvalho, M. \& Melo, A.L.A. (2005) Description and relationships of Otothyropsis marapoama, a new genus and species of hypoptopomatine catfish (Siluriformes: Loricariidae) from rio Tietê basin, southeastern Brazil. Neotropical Ichthyology, 3, 489-498.

Ribeiro, A.C., Melo, A.L.A. \& Pereira, E.H.L. (2002) A new species of Parotocinclus (Siluriformes: Loricariidae) from 
the rio São Francisco basin, southeastern Brazil. Ichthyological Exploration of Freshwaters, 13, 217-224.

Ricardo-Bertram, C.K. (1943) The fishes of the Bangweulu region. The Journal of the Linnean Society of London. Zoology, 41, 183-217.

Richardson, J. (1836) The Fish. In: Fauna Boreali-Americana; or the Zoology of the Northern Parts of British America: Containing Descriptions of the Objects of Natural History Collected on the Late Northern Land Expeditions, Under the Command of Sir John Franklin, R.N., Part 3, John Murray, Albemarle-Street; R. Bentley, London, pp. i-xv + 1327, pls. 74-97.

Richardson, J. (1844-1848) Ichthyology of the voyage of H. M. S. Erebus \& Terror. In: Richardson, J. \& Gray, J. E. (Eds.), The Zoology of the Voyage of H. M. S. Erebus \& Terror, Under the Command of Captain Sir J. C. Ross ... During ... 1839-43, Vol. 2, no. 2, London, pp. i-viii + 1-139, pls. 1-60. [Issued over several years, as follows: 1844: 1-16; 1845: 17-52; 1846: 53-74; 1848: i-viii + 75-139.]

Richardson, J. (1845) Ichthyology.- Part 3. In: Hinds, R.B. (Ed.), The Zoology of the Voyage of H. M. S. Sulphur, Under the Command of Captain Sir Edward Belcher, R. N., C. B., F. R. G. S., etc., During the Years 1836-42, No. 10, Smith, Elder \& Co, London, pp. 99-150, pls. 55-64.

Richardson, J. (1846) Report on the ichthyology of the seas of China and Japan. Report of the British Association for the Advancement of Science, 15th meeting, 1845, 187-320.

Richter. (1928). Abhandlungen und Berichte der Pommerschen Naturforschenden Gesellschaft, 9 [not seen; citation incomplete]

Ringuelet, R.A. (1965) Diferenciacion geografica de "otuno", Diplomystes viedmensis MacDonagh, 1931 (Pisces Siluriformes). Physis (Buenos Aires), 25, 89-92.

Ringuelet, R.A. (1982) Una nueva subespecie del bagre patagonico Diplomystes viedmensis Mac Donagh, 1931 en el Rio Senguer (Chubut, Argentina). Limnobios, 2, 349-351.

Ringuelet, R.A., Arámburu, R.H. \& Alonso de Arámburu, A. (1967) Los Peces Argentinos de Agua Dulce. Comision de Investegacion Cientifica, La Plata, $602 \mathrm{p}$.

Risch, L. (1981) The systematic status of Gephyroglanis longipinnis Boulenger, 1899, Chrysichthys magnus Pellegrin, 1922, and Gephyroglanis gigas Pellegrin, 1922 (Pisces, Bagridae). Revue de Zoologie Africaine, 95, 508-524.

Risch, L. (1983) Note on the synonyms of Eutropius mandibularis Günther, 1867, and Eutropius multitaeniatus Pellegrin, 1913 (Pisces, Schilbeidae). Revue de Zoologie Africaine, 97, 268-287.

Risch, L. (1984) Preliminary data of a systematic revision of the African species of the family Schilbeidae (Pisces: Siluriformes). Revue de Zoologie Africaine, 98, 424-433.

Risch, L. (1985a) Redécouverte de l'espèce Gephyroglanis ogooensis Pellegrin, 1900 (Pisces, Bagridae) das l'Ogôoué. Revue de Zoologie Africaine, 99, 97-104.

Risch, L. (1985b) Description of two new species in the genus Chrysichthys Bleeker, 1858 (Pisces, Bagridae). Revue de Zoologie Africaine, 99, 185-193.

Risch, L. (1986) Bagridae. In: Daget, J., Gosse, J.-P. \& Thys van den Audenaerde, D.F.E. (Eds.), Check-list of the Freshwater Fishes of Africa, Vol. 2, ISNB Bruxelles; MRAC Tervuren; ORSTOM, Paris, pp. 2-35.

Risch, L. (1987) Description of four new bagrid catfishes from Africa (Siluriformes: Bagridae). Cybium (3e série), 11, 20-38.

Risch, L. (1988) Description d'une espèce nouvelle de Chrysichthys (Pisces, Bagridae), provenant de la rivière Konkouré (République de Guinée). Cybium (3e série), 12, 3-7.

Risch, L. (1992a) Description de Chrysichthys dageti sp. n. (Teleostei, Bagridae) du bassin du Kouilou (République du Congo). Cybium (3e série), 16, 151-157.

Risch, L. (1992b) Bagridae. In: Lévêque, C., Paugy, D. \& Teugels, G.G. (Eds.), Faune des Poissons d'Eaux Douces et Saumâtres de l'Afrique de l'Ouest, Vol. 2, Musée Royal de l'Afrique Central, Tervuren, and Editions de l'ORSTOM, Paris, pp. 394-431.

Risch, L. \& Thys van den Audenaerde, D.F.E. (1981) Note on the systematical status of Gephyroglanis velifer Thys, 1965 (Pisces, Bagridae). Revue de Zoologie Africaine, 95, 245-251.

Risch, L. \& Thys van den Audenaerde, D.F.E. (1985a) Nouvelle description, distribution zoogéographique et affinités de Chrysichthys johnelsi Daget, 1959 (Pisces, Bagridae). Cybium (3e série), 9, 243-254.

Risch, L. \& Thys van den Audenaerde, D.F.E. (1985b) Note sur la présence de Chrysichthys longidorsalis (Pisces, Bagridae) et de Sanagia velifera (Pisces, Cyprinidae) dans le Nyong (Cameroun). Revue de Zoologie Africaine, 99, 87-96.

Risso, E.N.P. de \& Morra, M.I. (1964) Parapterodoras paranensis: nuevo género, nueva especie de Doradidae (Pisces Nematognathi). Notas del Museo de Ciencias Naturales del Chaco, Resistencia, 1, 1-5, pl. 1. 
Risso, F.J.J. \& de Risso, E.N.P. (1962) Epapterus chaquensis, nueva especie de Auchenipteridae (Pisces, Nematognathi). Notas Biologicas de la Facultad de Ciencias Exactas, Fisicas y Naturales, Corrientes, Zoologia, 3, 1-8.

Risso, F.J.J. \& de Risso, E.N.P. (1964a) Hallazgo de una neuva especie de Xyliphius en el Paraná (Pisces — Aspredinidae). Notas del Museo de Ciencias Naturales del Chaco, Resistencia, 1, 11-16.

Risso, F.J.J. \& de Risso, E.N.P. (1964b) Los Siluriformes conocidos como "Manduré" y otras especies afines (Pisces: Auchenipteridae, Hypophthalmidae, y Pimelodidae). Notas del Museo de Ciencias Naturales del Chaco, Resistencia, 1, 1-31.

Rivière, E. (1886) Exposition de la mission Brazza au Muséum. Revue Scientifique, 3e ser., 38, 13-23.

Roberts, T.R. (1967) Rheoglanis dendrophorus and Zaireichthys zonatus, bagrid catfishes from the lower rapids of the Congo River. Ichthyologia, The Aquarium Journal, 39, 119-131.

Roberts, T.R. (1978) An ichthyological survey of the Fly River in Papua New Guinea with descriptions of new species. Smithsonian Contributions to Zoology, 281, 1-72.

Roberts, T.R. (1982a) A revision of the south and southeastern Asian angler-catfishes (Chacidae). Copeia, 1982, 895901.

Roberts, T.R. (1982b) Systematics and geographical distribution of the Asian silurid catfish genus Wallago, with a key to the species. Copeia, 1982, 890-894.

Roberts, T.R. (1983) Revision of the south and southeast Asian sisorid catfish genus Bagarius, with description of a new species from the Mekong. Copeia, 1983, 435-445.

Roberts, T.R. (1989a) The freshwater fishes of western Borneo (Kalimantan Barat, Indonesia). Memoirs of the California Academy of Sciences, 14, i-xii + 1-210.

Roberts, T.R. (1989b) Systematic revision and description of new species of suckermouth catfishes (Chiloglanis, Mochokidae) from Cameroun. Proceedings of the California Academy of Sciences, Series 4, 46, 151-178.

Roberts, T.R. (1992) Revision of the striped catfishes of Thailand misidentified as Mystus vittatus, with descriptions of two new species (Pisces: Bagridae). Ichthyological Exploration of Freshwaters, 3, 77-88.

Roberts, T.R. (1993) The freshwater fishes of Java, as observed by Kuhl and van Hasselt in 1820-23. Zoologische Verhandelingen (Leiden), 285, 1-94.

Roberts, T.R. (1994) Systematic revision of Asian bagrid catfishes of the genus Mystus sensu stricto, with a new species from Thailand and Cambodia. Ichthyological Exploration of Freshwaters, 5, 241-256.

Roberts, T.R. (1998) Pseudecheneis sympelvicus, a new species of rheophilic sisorid catfish from Laos (Mekong basin). The Raffles Bulletin of Zoology, 46, 289-292.

Roberts, T.R. (1999) Pangasius bedado, a new species of molluscivorous catfish from Sumatra (Pisces, Siluriformes, Pangasiidae). Natural History Bulletin of the Siam Society, 47, 109-115.

Roberts, T.R. (2000) A review of the African electric catfish family Malapteruridae, with descriptions of new species. Occasional Papers in Ichthyology, 1, 1-15.

Roberts, T.R. (2001) Ayarnangra estuarius, a new genus and species of sisorid catfish from the Ayeyarwaddy basin, Myanmar. Natural History Bulletin of the Siam Society, 49, 81-87.

Roberts, T.R. (2003) Systematics and osteology of Leptoglaninae, a new subfamily of the African catfish family Amphiliidae, with descriptions of three new genera and six new species. Proceedings of the California Academy of Sciences, Series 4, 54, 81-132.

Roberts, T.R. \& Ferraris, C.J., Jr. (1998) Review of South Asian sisorid catfish genera Gagata and Nangra, with descriptions of a new genus and five new species. Proceedings of the California Academy of Sciences, Series 4, 50, 315345 .

Roberts, T.R. \& Jumnongthai, J. (1999) Miocene fishes from Lake Phetchabun in north central Thailand, with descriptions of new taxa of Cyprinidae, Pangasiidae, and Chandidae. Natural History Bulletin of the Siam Society, 47, 153189.

Roberts, T.R. \& Stewart, D.J. (1976) An ecological and systematic survey of fishes in the rapids of the lower Zaire or Congo River. Bulletin of the Museum of Comparative Zoology, 147, 239-317, pls. 1-14.

Roberts, T.R. \& Vidthayanon, C. (1991) Systematic revision of the Asian catfish family Pangasiidae, with biological observations and descriptions of three new species. Proceedings of the Academy of Natural Sciences, Philadelphia, $143,97-144$.

Rochebrune, A.T., de. (1885) Vertebratorum novorum vel minus cognitorum orae Africae occidentalis incolarum. Diagnoses. Bulletin de la Société Philomathique (Ser. 7), 9, 86-99.

Rodiles-Hernández, R., Hendrickson, D.A., Lundberg, J.G. \& Humphries, J.M. (2005) Lacantunia enigmatica (Teleostei: Siluriformes) a new and phylogenetically puzzling freshwater fish from Mesoamerica. Zootaxa, 1000, 1-24. 
Rodríguez, M.S. \& Miquelarena, A.M. (2005) A new species of Rineloricaria (Siluriformes: Loricariidae) from the Paraná and Uruguay river basins, Misiones, Argentina. Zootaxa, 945, 1-15.

Roedel, H. (1930) Fischotolithen aus Paläozängeschieben. Zeitschrift für Geschiebeforschung, 6, 49-77, 1 pl.

Röhl, E. (1942) Fauna Descriptiva de Venezuela. Tipografia Americana, Caracas, 432 p.

Roman, B. (1966) Les poissons des Haut-Bassins de la Volta. Annales du Musée Royal de l'Afrique Centrale, Series in 8, Sciences Zoologiques, 150, 1-191, pls. 1-7.

Roman, B. (1970) Nuevas especies de peces de Río Muni (Guinea Ecuatorial). Publicaciones del Instituto de Biología Aplicada, Barcelona, 49, 5-23.

Roman, B. (1975) Une nouvelle espèce de Synodontis (poissons, Mochocides) dans la Bougouriba, affluent de la Volta Noire: Synodontis voltae. Notes Docum. Volta, 8, 45-52.

Román-Valencia, C. (1998) Redescripcion de Branchioica phaneronema Miles, 1943 (Pisces, Trichomycteridae) de la cuene del rio Magdalena, Colombia. Revista de la Academia Colombiana de Ciencias Exactas, Fisicas y Naturales, 22, 299-303.

Román-Valencia, C., Lehmann A, P. \& Muñoz, A. (1999) Presencia del genero Callichthys (Siluriformes: Callichthyidae) en Colombia y descripcion de una nueva especie para el alto Rio Cauca. Dahlia - Revista de la Asociacion Colombiana de Ictiologos, 3, 53-62.

Römer, W. (1990) Ancistrus, deine Kinder! Nach Beobachtungen von Ewald Kuß. Die Aquarien- und TerrarienZeitschrift, 43, 146-147.

Rössel, F. (1961) Corydoras caudimaculatus ein neuer panzerwels aus Brasilien. (Pisces, Teleostei, Callichthyidae). Senckenbergiana Biologica, 42, 49-50.

Rössel, F. (1962a) Centromochlus schultzi, ein neues Wels aus Brasilien (Pisces, Teleostei, Auchenipteridae). Senckenbergiana Biologica, 43, 27-30.

Rössel, F. (1962b) Corydoras cervinus, ein neuer panzerwels aus Brasilien (Pisces, Teleostei, Callichthyidae). Senckenbergiana Biologica, 43, 31-33.

Rössel, F. (1962c) Corydoras axelrodi, ein neuer Panzerwels aus Columbien (Pisces, Teleostei, Callichthyidae). Senckenbergiana Biologica, 43, 335-337.

Rössel, F. (1963) Neue und seltene Corydoras-Arten aus Brasilien (Pisces, Teleostei, Callichthyidae). Senckenbergiana Biologica, 44, 359-363.

Rössel, F. (1964) Welse (Siluroidea) gesammelt von der deutschen Indien-Expedition 1955/58. Mitteilungen aus dem Hamburgischen Zoologischen Museum und Institut, 61, 145-158.

Royero, R. (1987) Morfología de la aleta dorsal en los bagres (Teleostei: Siluriformes), con especial referencia a las familias americanas. Escuela de Biología, Undergraduate thesis, Universidad Central de Venezuela, Faculdad de Ciencias, Caracas.

Royero, R. (1992) Tatia musaica, una neuva especie de bagre auquenipterido (Siluriformes — Auchenipteridae) de la cuenca del Rio Orinoco, Territorio Federal Amazonas, Venezuela. Acta Cientifica Venezuelica, 43, $300-306$.

Royero, R. (1999) Studies on the systematics and phylogeny of the catfish family Auchenipteridae (Teleostei: Siluriformes), Doctoral dissertation, University of Bristol, Bristol, U. K.

Royero, R. \& Hureau, J.-C. (1996) The type specimens of auchenipterid catfishes (Siluriformes: Auchenipteridae) in the Muséum National d'Histoire Naturelle, Paris. Cybium (3e série), 20, 369-377.

Ruiz, V.H. \& Berra, T.M. (1994) Fishes of the High Biobio River of south-central Chile with notes on diet and speculations on the origin of the ichthyofauna. Ichthyological Exploration of Freshwaters, 5, 5-18.

Rüppell, W.P.E.S. (1829) Beschreibung und Abbildung Mehrerer Neuer Fische im Nil Entdeckt, Brönner, Frankfurt-amMain, 12 p., 3 pls. [Issued in 3 memoirs: in 1829, 1832, and 1835].

Rüppell, W.P.E.S. (1832) Fortsetzung der Beschreibung und Abbildung Mehrerer Neuer Fische, im Nil Entdeckt, Brönner, Frankfurt-am-Main, 14 p., 3 pls. [Issued in 3 parts, in 1829, 1832, and 1835]

Rüppell, W.P.E.S. (1835-1838) Neue Wirbelthiere zu der Fauna von Abyssinien Gehörig. Fische des Rothen Meeres, Frankfurt-am-Main, 148 p., 33 pls. [1835:1-28, pls. 1-7; 1836:29-52, pls. 8-14; 1837:53-80, pls. 15-21; 1838:81148, pls. 22-33.]

Russell, A. (1794) Natural History of Aleppo, Containing a Description of the City, and the Principal Natural Productions in its Neighbourhood. Together with an Account of the Climate, Inhabitants, and Diseases, Particularly of the Plague; Second Edition, Revised, Enlarged, and Illustrated by P. Russell, Vol. 2, G.G. and J. Robinson, London, vii + $430+$ xxxiv p. +26 p. index, 16 pl.

Russell, P. (1803) Descriptions and Figures of Two Hundred Fishes Collected at Vizagapatam on the Coast of Coromandel, East India Company, London, vii + 162 p., 197 pl. [In two volumes]. 
Rutter, C.M. (1896) Notes on fresh water fishes of the Pacific slope of North America. II._- The fishes of Rio Yaqui, Sonora, with the description of a new genus of Siluridae. Proceedings of the California Academy of Sciences, Series 2, 6, 255-262.

Sabaj, M.H. (2002) Taxonomy of the Neotropical Thorny Catfishes (Siluriformes: Doradidae) and Revision of Genus Leptodoras, Unpublished Ph.D. dissertation, University of Illinois at Urbana-Champaign.

Sabaj, M.H. (2005) Taxonomic assessment of Leptodoras (Siluriformes: Doradidae) with descriptions of three new species. Neotropical Ichthyology, 3, 637-678.

Sabaj, M.H. \& Ferraris, C.J., Jr. (2003) Doradidae. In: Reis, R.E., Kullander, S.O. \& Ferraris, C.J., Jr. (Eds.), Check list of the Freshwater Fishes of South and Central America, Edipucrs, Porto Alegre, Brazil, pp. 456-469.

Sabino, J. \& Trajano, E. (1997) A new species of blind armoured catfish, genus Ancistrus, from caves of Bodoquena region, Mato Grosso do Sul, southwestern Brazil (Siluriformes, Loricariidae, Ancistrinae). Revue Française d'Aquariologie et Herpetologie, 24, 73-78.

Sagua, O. (1987) On a new species of electric catfish from Kainji, Nigeria, with some observations on its biology. Journal of Fish Biology, 30, 75-89.

Sahni, A. \& Mishra, V.P. (1975) Lower Tertiary vertebrates from western India. Monographs of the Palaeontological Society of India, 3, 1-48.

Sanders, M. (1934) Die fossilen Fische der Alttertiären Süsswasserablagerungen aus Mittel-Sumatra. Verhandelingen van het Geologisch Mijnbouwkundig Genootschap voor Nederland en Koloniën. Geologische serie, 11, 1-144, 9 pls. [Also as published Dissertation, University of Amsterdam. ]

Sands, D. (1990) A new species of callichthyid catfish, Corydoras araguaiaensis (Siluriformes, Callichthyidae) from the Rio Araguaia, Brazil. D. Sands, Catfishes of the World, Suppl., Self published, pp. [1-2].

Sands, D.D. (1995) Four new Corydoras (Callichthyidae) species from upper Negro River tributaries and a range extension, together with a discussion of $C$. bicolor Nijssen \& Isbrücker. Freshwater and Marine Aquarium, 18, 8-12, 14, 16, 18.

Sands, D.D. \& Black, B.K. (1985) Two new species of Brachyrhamdia, Myers, 1927, from Brazil and Peru, together with a redefinition of the genus. In: Sands, D. (Ed.), Catfishes of the World, Vol. 3, Suppl (first set), Self published, pp. 58(1)-58(8).

Santos, R.S. (1973) Steindachneridion iheringi (Woodward), um siluriforme da bacia do Paraíba, Estado de São Paulo. Anais da Academia Brasiliera de Ciências, 43, 667.

Sarmento-Soares, L.M. \& Buckup, P.A. (2005) A new Glanidium from the Rio São Francisco basin, Brazil (Siluriformes: Auchenipteridae: Centromochlinae). Copeia, 2005, 846-853.

Sarmento-Soares, L.M., Martins-Pinheiro, R.F., Aranda, A.T. \& Chamon, C.C. (2005) Trichomycterus pradensis, a new catfish from southern Bahia coastal rivers, northeastern Brazil (Siluriformes: Trichomycteridae). Ichthyological Exploration of Freshwaters, 16, 289-302.

Sauvage, H.E. (1874) Notices ichthyologiques. Revue et Magasin de Zoologie (Ser. 3), 2, 332-340.

Sauvage, H.E. (1878) Note sur quelques poissons d'espèces nouvelles provenant des eaux douces de l'Indo-Chine. Bulletin de la Société Philomathique (Ser. 7), 2, 233-242.

Sauvage, H.E. (1879) Notice sur la faune ichthyologique de l'Ogôoué. Bulletin de la Société Philomathique (Ser. 7), 3, 90-103.

Sauvage, H.E. (1880a) Description de quelques poissons de la collection du Muséum d'histoire naturelle. Bulletin de la Société Philomathique (Ser. 7), 4, 220-228.

Sauvage, H.E. (1880b) Notice sur quelques poissons de l'île Campbell et de l'Indo-Chine. Bulletin de la Société Philomathique (Ser. 7), 4, 228-233.

Sauvage, H.E. (1880c) Étude sur la faune ichthyologique de l'Ogôoué. Nouvelles Archives du Muséum d'Histoire Naturelle, Paris (Ser. 2), 3, 5-56, pls. 1-3.

Sauvage, H.E. (1881) Recherches sur la faune ichthyologique de l'Asie et description d'espèces nouvelles de l'IndoChine. Nouvelles Archives du Muséum d'Histoire Naturelle, Paris (Ser. 2), 4, 123-194, pls 5-8.

Sauvage, H.E. (1882) Catalogue des poissons recueillis par M. E. Chantre pendant son voyage en Syrie, Haute-Mésopotamie, Kurdistan et Caucase. Bulletin de la Société Philomathique (Ser. 7), 6, 163-168.

Sauvage, H.E. (1883a) Sur une collection de poissons recueillie dans le lac Biwako (Japon) par M. F. Steenackers. Bulletin de la Société Philomathique (Ser. 7), 7, 144-150.

Sauvage, H.E. (1883b) Sur une collection de poissons recueillie dans Mé-Nam (Siam) par M. Harmand. Bulletin de la Société Philomathique (Ser. 7), 7, 150-155.

Sauvage, H.E. (1883c) Descriptions de quelques poissons de la collection du Muséum d'histoire naturelle. Bulletin de la 
Société Philomathique (Ser. 7), 7, 156-161.

Sauvage, H.E. (1884a) Sur un siluroïde de la Réunion. Bulletin de la Société Philomathique (Ser. 7), 8, 147.

Sauvage, H.E. (1884b) Notice sur la Faune ichthyologique d' l'oust de l'Asie et plus particulièrement sur les poissons recueillis par M. Chantre pendant son voyage dans cette région. Nouvelles Archives du Muséum d'Histoire Naturelle, Paris (Ser. 2), 7, 1-41, pls. 1-3.

Sauvage, H.E. \& Dabry deThiersant, P. (1874) Notes sur les poissons des eaux douces de la Chine. Annales des sciences naturelles. Zoologie et biologie animale (Sér. 6), 1, 1-18.

Saville-Kent, W. (1889) Preliminary Report on the Food-fishes of Queensland, Government Printer, Brisbane, 16 p., 16 pls.

Saville-Kent, W. (1893) The Great Barrier Reef of Australia; its Products and Potentialities, W.H. Allen, London, xiii + 387 p., 48 pl.; 16 color plates.

Sawyer, F.C. (1953) The dates of issue of J. E. Gray's "Illustrations of Indian Zoology" (London, 1830-1835). Journal of the Society for the Bibliography of Natural History, 3, 48-55.

Schaefer, S.A. (1986) Historical Biology of the Loricariid Catfishes: Phylogenetics and Functional Morphology, Ph. D. Thesis, University of Chicago.

Schaefer, S.A. (1987) Osteology of Hypostomus plecostomus (Linnaeus), with a phylogenetic analysis of the loricariid subfamilies (Pisces: Siluriformes). Contributions in Science, Los Angeles County Museum, 394, 1-31.

Schaefer, S.A. (1988) A new species of the loricariid genus Parotocinclus from southern Venezuela (Pisces: Siluroidei). Copeia, 1988, 182-188.

Schaefer, S.A. (1990) Anatomy and relationships of the scoloplacid catfishes. Proceedings of the Academy of Natural Sciences, Philadelphia, 142, 167-210.

Schaefer, S.A. (1991) Phylogenetic analysis of the loricariid subfamily Hypoptopomatinae (Pisces: Siluroidei: Loricariidae), with comments on generic diagnoses and geographic distribution. Zoological Journal of the Linnean Society, 102, 1-41.

Schaefer, S.A. (1996a) Nannoptopoma, a new genus of loricariid catfishes (Siluriformes: Loricariidae) from the Amazon and Orinoco River Basins. Copeia, 1996, 913-926.

Schaefer, S.A. (1996b) Type designations for some Steindachner loricariid material (Siluriformes: Loricariidae) in the Natural History Museum, Vienna. Copeia, 1996, 1031-1035.

Schaefer, S.A. (1997) The neotropical cascudinhos: Systematics and biogeography of the Otocinclus catfishes (Siluriformes: Loricariidae). Proceedings of the Academy of Natural Sciences, Philadelphia, 148, 1-120.

Schaefer, S.A. (1998) Conflict and resolution: Impact of new taxa on phylogenetic studies of the neotropical cascudinhos (Siluroidei: Loricariidae). In: Malabarba, L.R., Reis, R.E., Vari, R.P., Lucena, Z.M. \& Lucena, C.A.S. (Eds.), Phylogeny and Classification of Neotropical Fishes, Edipucrs, Porto Alegre, pp. 375-400.

Schaefer, S.A. (2003a) Scoloplacidae. In: Reis, R.E., Kullander, S.O. \& Ferraris, C.J., Jr. (Eds.), Check list of the Freshwater Fishes of South and Central America, Edipucrs, Porto Alegre, Brazil, pp. 310-311.

Schaefer, S.A. (2003b) Astroblepidae. In: Reis, R.E., Kullander, S.O. \& Ferraris, C.J., Jr. (Eds.), Check list of the Freshwater Fishes of South and Central America, Edipucrs, Porto Alegre, Brazil, pp. 312-317.

Schaefer, S.A. (2003c) Hypoptopomatinae. In: Reis, R.E., Kullander, S.O. \& Ferraris, C.J., Jr. (Eds.), Check list of the Freshwater Fishes of South and Central America, Edipucrs, Porto Alegre, Brazil, pp. 321-329.

Schaefer, S.A. (2003d) Relationships of Lithogenes villosus Eigenmann, 1909 (Siluriformes, Loricariidae): Evidence from high-resolution computed microtomography. American Museum Novitates, 3402, 1-55.

Schaefer, S.A. \& Provenzano, R.F. (1998) Niobichthys ferrarisi, a new genus and species of armored catfish from southern Venezuela (Siluriformes: Loricariidae). Ichthyological Exploration of Freshwaters, 8, 221-230.

Schaefer, S.A. \& Provenzano, R.F. (1993) The Guyana Shield Parotocinclus: Systematics, biogeography, and description of a new Venezuelan species (Siluroidei: Loricariidae). Ichthyological Exploration of Freshwaters, 4, 39-56.

Schaefer, S.A., Provenzano, R.F., Pinna, M., de \& Baskin, J.N. (2005) Noteworthy Venezuelan glanapterygine catfishes (Siluriformes, Trichomycteridae), with discussion of their biogeography and psammophily. American Museum Novitates, 3496, 1-27.

Schaefer, S.A. \& Stewart, D.J. (1993) Systematics of the Panaque dentex species group (Siluriformes: Loricariidae), wood-eating armored catfishes from tropical South America. Ichthyological Exploration of Freshwaters, 4, 309342.

Schaefer, S.A., Weitzman, S.H. \& Britski, H.A. (1989) Review of the Neotropical catfish genus Scoloplax (Pisces: Loricarioidea: Scoloplacidae) with comments on reductive characters in phylogenetic analysis. Proceedings of the Academy of Natural Sciences, Philadelphia, 141, 181-211. 
Schilthuis, L. (1891) On a collection of fishes from the Congo; with description of some new species. Tijdschrift der Nederlandscfhe Dierkundige Vereeniging, Ser. 2, 3, 83-92, pl. 6.

Schindler, I. (1996) Ergänzende Beschreibung von Lithoxus bovallii (Regan, 1906) aus Guyana (Siluriformes, Loricariidae). Zeitschrift für Fischkunde, 3, 223-233.

Schindler, O. (1959) Loricariichthys melini nov. spec. Arkiv för Zoologi, 12, 387-389.

Schmidt, P. (1928) On three rare cat-fishes of the Magdalena River (South America, Columbia [sic]). Comptes Rendus de l'Académie des Sciences de 'URSS, [1928], 9-13.

Schmidt, R.E. (1985) New distribution records and complementary description of Haemomaster venezuelae (Siluriformes: Trichmycteridae), a rare and poorly known fish from northern South America. Studies on Neotropical Fauna and Environment, 20, 93-96.

Schmidt, R.E. (1987) Redescription of Vandellia beccarii (Siluriformes: Trichomycteridae) from Guyana. Copeia, 1987, 234-237.

Schmidt, R.E. (1993) Relationships and notes on the biology of Paracanthopoma parva (Pisces: Trichomycteridae). Ichthyological Exploration of Freshwaters, 4, 185-191.

Schmidt, R.E. \& Ferraris, C.J., Jr. (1985) A new species of Parotocinclus (Pisces: Loricariidae) from Guyana. Proceedings of the Biological Society of Washington, 98, 341-346.

Schomburgk, R.H. (1841) The Natural History of Fishes of Guiana.- Part I. In: Jardine, W. (Ed.), The Naturalists' Library, Vol. 3, W.H. Lizars, Edinburgh, 263 p., pls. 1-30.

Schomburgk, R.H. (1848) Reisen in Britisch-Guiana in den Jahren 1840-1844. Im Auftrag Sr. Mäjestat des Königs von Preussen Ausgeführt von Richard Schomburgk. Versuch einer Fauna und Flora von Britisch-Guiana, Nach Vorlagen von Johannes Muller, Ehrenberg, Erichson, Klotzch, Troschel, Cabanis und Andern, Leipzig.

Schubart, O. (1964a) Duas novas espécies de peixe da família Pimelodidae do Rio Mogi Guaçu (Pisces, Nematognathi). Boletim do Museu Nacional do Rio de Janeiro, Nova Série, zoologia, 244, 1-22.

Schubart, O. (1964b) Sôbre algumas Loricariidae da bacia do Rio Mogi Guaçu. Boletim do Museu Nacional do Rio de Janeiro, Nova Série, zoologia, 251, 1-19.

Schubart, O. \& Gomes, A.L. (1959) Descrição de Cetopsorhamdia iheringi sp. n. (Pisces, Nematognathi, Pimelodidae, Luciopimelodinae). Revista Brasileira de Biologia, 19, 1-7.

Schubert, R. (1908) Die Fischotolithen des Pausramer Mergels.- 2. Zeitschrift des Mährischen Landesmuseums, 8, $102-120$.

Schubert, R. (1916) Obereocäne Otolithen von Barton Cliff bei Christchurch (Hampshire). Jahrbuch der Kaiserlich Königlichen Geologischen Reichs-Anstalt, 65, 277-288.

Schultz, L.P. (1942) The fresh-water fishes of Liberia. Proceedings of the United States National Museum, 92, 301-348, pls. 35-36.

Schultz, L.P. (1944a) A new loricariid catfish from the Rio Truando, Colombia. Copeia, 1944, 155-156.

Schultz, L.P. (1944b) A new genus and species of pimelodid catfish from Colombia. Journal of the Washington Academy of Sciences, 34, 93-95.

Schultz, L.P. (1944c) The catfishes of Venezuela, with descriptions of thirty-eight new forms. Proceedings of the United States National Museum, 94, 173-338, pls. 1-14.

Schultz, L.P. (1944d) Two new species of fishes (Gymnotidae, Loricariidae) from Caripito, Venezuela. Zoologica (New York), 29, 39-44, pl. 1.

Schultz, L.P. (1945) Pygidium mondolfi, a new catfish from Venezuela. Journal of the Washington Academy of Sciences, 35, 29-31.

Scopoli, G.A. (1777) Introductio ad Historiam Naturalem, Sistens Genera Lapidum, Plantarum et Animalium Hactenus Detecta, Caracteribus Essentialibus Donata, in Tribus Divisa, Subinde ad Leges Naturae, Prague, x + 506 p.

Seba, A. (1734-1765) Locupletissimi Rerum Naturalium Thesauri Accurata Descriptio, et Iconibus Artificiosissimis Expressio, per Universam Physices Historiam. Opus, cui, in hoc Rerum Genere, Nullum par Exstitit. Ex Toto Terrarum Orbe Collegit, Digessit, Descripsit, et de Pingendum Curavit Albertus Seba, 4 vols, 449 pls, J. Wetstenium \& J. Gul. Smith et Janssonio-Waesbergios [etc], Amstelaedami. [Fish in vol. 3, published in 1758.]

Seegers, L. (1989) Leptoglanis wamiensis spec. nov. (Bagridae, Siluriformes) aus dem Wami- Einzug in Tanzania. Die Aquarien- und Terrarien- Zeitschrift, 42, 284-287.

Seegers, L. (1996) The fishes of the Lake Rukwa drainage. Musée Royal de l'Afrique Centrale, Annales, Sciences Zoologiques, 278, 1-407.

Seegers, L. (2003) Atopochilus vogti: Ein Afrika-Saugwels aus dem Wami-River, Tanzania. Aquarium (Bornheim), 405, $8-14$. 
Sen, N. \& Biswas, B.K. (1994) On a new species of Nangra Day (Pisces: Siluriformes: Sisoridae) from Assam, north east India with a note on comparative studies of other known species. Records of the Zoological Survey of India, 94, 441-446.

Serpa, A.T. (1967) Pesces del Oriente Peruano. Algunas especies de Loricariidae, con referencia especial de la "Caramacha"Pterygoplichthys multiradiatus (Hancock). Ecologia y Utilidade. Biota, Lima, 50, 201-259.

Shaw, G. (1804) General Zoology or Systematic Natural History. Vol. 5._- Pisces, in Two Parts, G. Kearsley, London. [(pt 1): i-v + 1-250, pls. 93-132, 43+, 65+, 6+, 74+ and (pt 2): i-vi + 251-463, pls. 132-182, 158+.]

Shaw, G.E. \& Shebbeare, E.O. (1936) Description of a new species of fish from northern Bengal. Journal of the Bombay Natural History Society, 39, 188-189, 1 pl.

Shaw, T.-H. (1930) Notes on some fishes from Ka-Shing and Shing-Tsong, Chekiang Province. Bulletin of the Fan Memorial Institute of Biology, Peiping, 1, 109-121.

Shepherd, C.E. (1916) Fossil otoliths. Knowledge, 39 (new series, 13), 177-184, 203-205, figs. $154-157$.

Sherborn, C.D. \& Griffin, F.J. (1934) On the dates of publication of the natural history portions of Alcide d'Orbigny's "Voyage Amerique meridionale". Annals and Magazine of Natural History (Ser. 10), 13, 130-134.

Shibatta, O.A. (1998) Sistemática e Evolução da Família Pseudopimelodidae (Ostariophysi, Siluriformes), com a Revisão Taxonômica de Gênero Pseudopimelodus, Ph.D. dissertation, Univ. Fed. São Carlos, São Paulo, Brazil.

Shibatta, O.A. (2003a) Pseudopimelodidae. In: Reis, R.E., Kullander, S.O. \& Ferraris, C.J., Jr. (Eds.), Check list of the Freshwater Fishes of South and Central America, Edipucrs, Porto Alegre, Brazil, pp. 401-405.

Shibatta, O.A. (2003b) Phylogeny and classification of "Pimelodidae". In: Arratia, G., Kapoor, B.G., Chardon, M. \& Diogo, R. (Eds.), Catfishes, Science Publishers, Enfield, NH, USA, pp. 385-400.

Shibatta, O.A. \& Benine, R.C. (2005) A new species of Microglanis Eigenmann, 1912 (Siluriformes: Pseudopimelodidae) from upper rio Paraná basin, Brazil. Neotropical Ichthyology, 3, 579-585.

Shibatta, O.A. \& Pavanelli, C.S. (2005) Description of a new Batrochoglanis species (Siluriformes, Pseudopimelodidae) from the rio Paraguai basin, State of Mato Grosso, Brazil. Zootaxa, 1092, 21-30.

Shimabukuro-Dias, C.K., Oliveira, C., Reis, R.E. \& Foresti, F. (2004) Molecular phylogeny of the armored catfish family Callichthyidae (Ostariophysi, Siluriformes). Molecular Phylogenetics and Evolution, 32, 152-163.

Shrestha, J. (1980) Fishes of Nepal. Curriculum Development Centre, Tribhuvan University, Kathmandu.

Sielfeld-K., W.H. (1979) Nuevo registro de Netuma barbus (La Cepède, 1803) para águas chilenas (Pisces: Siluriformes). Anales del Instituto de la Patagônia, 10, 189-192.

Silas, E.G. (1951a) On a collection of fish from the Anamalai and Nelliampathy Hill ranges, Western Ghats, with notes on their zoogeographic significance. Journal of the Bombay Natural History Society, 49, 679-681.

Silas, E.G. (1951b) Notes on fishes of the genus Glyptothorax Blyth from Peninsular India, with description of a new species. Journal of the Bombay Natural History Society, 50, 367-370.

Silfvergrip, A.M.C. (1992) Zungaro, a senior synonym of Paulicea (Teleostei: Pimelodidae). Ichthyological Exploration of Freshwaters, 3, 305-310.

Silfvergrip, A.M.C. (1996) A Systematic Revision of the Neotropical Catfish Genus Rhamdia (Teleostei, Pimelodidae), Stockholm University, Stockholm, 156 p., 8 pl.

Silfvergrip, A.M.C. \& Paepke, H.-J. (1997) Kritischer Katalog der Typen der Fischsammlung des Zoologischen Museums Berlin. Teil 7: Pimelodidae (Siluriformes). [Critical Catalogue of the Types of the Fish Collection of the Zoological Museum of Berlin. Part 7: Pimelodidae (Siluriformes)]. Mitteilungen aus dem Zoologischen Museum in Berlin, 73, 165-173.

Skelton, P.H. (1981) The description and osteology of a new species of Gephyroglanis (Siluriformes, Bagridae) from the Olifants River, South West Cape, South Africa. Annals of the Cape Provincial Museums. Natural History, 13, 217249.

Skelton, P.H. (1984) A systematic revision of species of the catfish genus Amphilius (Siluroidei, Amphiliidae) from east and southern Africa. Annals of the Cape Provincial Museums. Natural History, 16, 41-71.

Skelton, P.H. (1986) Two new Amphilius (Pisces, Siluroidei, Amphiliidae) from the Zaïre River system, Africa. Revue de Zoologie Africaine, 99, 263-291.

Skelton, P.H. (1989) Descriptions of two new species of West African amphiliid catfishes (Siluroidei: Amphiliidae). J. $L$. B. Smith Institute of Ichthyology, Special Publication, 48, 1-13.

Skelton, P.H. (1992) Amphiliidae. In: Lévêque, C., Paugy, D. \& Teugels, G.G. (Eds.), Faune des Poissons d'Eaux Douces et Saumâtres de l'Afrique de l'Ouest, Vol. 2, Musée Royal de l'Afrique Central, Tervuren, and Editions de l'ORSTOM, Paris, pp. 451-467.

Skelton, P.H. (1993) A Complete Guide to the Freshwater Fishes of Southern Africa, Southern Book Publishers, Ltd., 
Halfway House, South Africa, xiii +388 p.

Skelton, P.H. (2001) A Complete Guide to the Freshwater Fishes of Southern Africa, Second Edition, Struik Publishers, Cape Town, $x i v+395$ p.

Skelton, P.H., Bruton, M.N. \& al. (1985) The fishes of the Okavango drainage system in Angola, South West Africa and Botswana: Taxonomy and distribution. Ichthyological Bulletin of the J. L. B. Smith Institute of Ichthyology, 50, 121.

Skelton, P.H., Risch, L. \& De Vos, L. (1984) On the generic identity of the Gephyroglanis catfishes from southern Africa (Pisces, Siluroidei, Bagridae). Revue de Zoologie Africaine, 98, 337-372.

Skelton, P.H. \& Teugels, G.G. (1986) Amphiliidae. In: Daget, J., Gosse, J.-P. \& Thys van den Audenaerde, D.F.E. (Eds.), Check-list of the Freshwater Fishes of Africa, Vol. 2, ISNB, Bruxelles; MRAC, Tervuren; and ORSTOM, Paris, pp. $54-65$.

Skelton, P.H. \& Teugels, G.G. (1991) A review of the clariid catfishes (Siluroidei, Clariidae) occurring in southern Africa. Revue d'Hydrobiologie Tropicale, 24, 241-260.

Skelton, P.H. \& Teugels, G.G. (1992) Neotype description for the African catfish Clarias gariepinus (Burchell, 1822) (Pisces: Siluroidei: Clariidae). Ichthyological Bulletin of the J. L. B. Smith Institute of Ichthyology, 56, 1-7.

Skelton, P.H. \& White, P.N. (1990) Two new species of Synodontis (Pisces: Siluroidei: Mochokidae) from southern Africa. Ichthyological Exploration of Freshwaters, 1, 277-287.

Smith, A. (1838-1849) Pisces. In: Illustrations of the Zoology of South Africa; Consisting Chiefly of Figures and Descriptions of the Objects of Natural History Collected During an Expedition into the Interior of South Africa in 1834, 1835, and 1836, Fitted Out by the Cape of Good Hope Association for Exploring Central Africa, Vol. 4, Smith, Elder, London, pp. 1-77, 31 pl. In 5 vols.

Smith, C.L. (1962) Some Pliocene fishes from Kansas, Oklahoma, and Nebraska. Copeia, 1962, 505-520.

Smith, G.R., Morgan, N. \& Gustafson, E. (2000) Fishes of the Mio-Pliocene Ringold Formation, Washington: Pliocene capture of the Snake River by the Columbia River. University of Michigan Papers on Paleontology, 32, 1-47.

Smith, H.M. (1931) Descriptions of new genera and species of Siamese fishes. Proceedings of the United States National Museum, 79, 1-48, pl. 1.

Smith, H.M. (1933) Contributions to the ichthyology of Siam [II-VI]. Journal of the Siam Society, Natural History Supplement, 9, 53-87, pls. 1-3.

Smith, H.M. (1934) Contributions to the ichthyology of Siam: IX-XIX. Journal of the Siam Society, Natural History Supplement, 9, 287-325, pls. 10-14.

Smith, H.M. (1939) A new genus of clariid catfishes. Copeia, 1939, 236.

Smith, H.M. (1941) The proper name for the oriental catfish usually called Plotosus anguillaris (Bloch). Proceedings of the Biological Society of Washington, 54, 15-16.

Smith, H.M. (1945) The fresh-water fishes of Siam, or Thailand. United States National Museum, Bulletin, 188, i-xi + 1622 , pls. $1-9$.

Smith, H.M. \& Seale, A. (1906) Notes on a collection of fishes from the island of Mindanao, Philippine Archipelago, with descriptions of new genera and species. Proceedings of the Biological Society of Washington, 19, 73-82.

Smith, J.A. (1875) Notice of new fishes from West Africa. I._Ophiocephalus obscurus, Günther. II._- Synodontis robbianus nov. spec. mihi. Proceedings of the Royal Physical Society of Edinburgh, 8, 89-95.

Smith, M.L. (1987) Osteology and systematics of the fossil catfishes (genus Ictalurus) of central Mexico. Journal of Paleontology, 61, 380-387.

Soares-Porto, L.M. (1994) Auchenipterichthys dantei, a new species of catfish from the Amazon basin (Siluriformes: Auchenipteridae). Ichthyological Exploration of Freshwaters, 5, 281-287.

Soares-Porto, L.M. (1995) A new species of Tatia from the Amazon basin (Siluriformes: Auchenipteridae). Ichthyological Exploration of Freshwaters, 6, 201-206.

Soares-Porto, L.M. (1998) Monophyly and interrelationships of the Centromochlinae (Siluriformes: Auchenipteridae). In: Malabarba, L.R., Reis, R.E., Vari, R.P., Lucena, Z.M. \& Lucena, C.A.S. (Eds.), Phylogeny and Classification of Neotropical Fishes, Edipucrs, Porto Alegre, pp. 331-350.

Soares-Porto, L.M. (2000) A new species of Centromochlus (Siluriformes: Auchenipteridae) from the rio Negro drainage, Amazon basin, Brazil, with comments on its relationships. Ichthyological Exploration of Freshwaters, 11, 279287.

Soares-Porto, L.M., Walsh, S.J., Nico, L.G. \& Netto, J.M. (1999) A new species of Gelanoglanis from the Orinoco and Amazon river basins, with comments on miniaturization within the genus (Siluriformes: Auchenipteridae: Centromochlinae). Ichthyological Exploration of Freshwaters, 10, 63-72. 
Son, Y.-M., Kim, I.-S. \& Choo, I.-Y. (1987) A new species of torrent catfish, Liobagrus obesus from Korea. Korean Journal of Limnology, 20, 21-29.

Sonnini, C.S. ([1799]) Voyage dans la Haute et Basse Egypte, fait par Ordre de l'Ancien Gouvernement, F. Buisson, Paris.

Soriano-Señorans, J. (1950) Nota preliminar sobre Loricaria (Loricaria) devicenzii [sic] n. sp. de la Cuenca del Río Uruguay. Revista de la Facultad de Humanidides y Ciencias, Montevideo, 4, 265-266.

Sousa, L.M., de \& Rapp Py-Daniel, L.H. (2005) Description of two new species of Physopyxis Cope, 1871 and redescription of P. lyra (Siluriformes: Doradidae). Neotropical Ichthyology, 3, 625-636.

Sowerby, A. de C. (1921) On a new silurid fish from the Yalu River, South Manchuria. Proceedings of the United States National Museum, 60, 1-2.

Speirs, J.M. (1952) Nomenclature of the channel catfish and the burbot of North America. Copeia, 1952, 99-103.

Spix, J.B., von \& Agassiz, L. (1829-1831) Selecta Genera et Species Piscium quos in Itinere per Brasiliam Annos MDCCCXVII-MDCCCXX Jussu et Auspiciis Maximiliani Josephi I.... Colleget et Pingendso Curavit Dr J. B. de Spix, Typis C. Wolf, Monachii. [Issued in two parts: part 1: i-xvi + i-ii + 1-82, pls. 1-48, in 1829; part 2: 83-138, pls. 49101 , in 1831]

Srivastava, G.J. (1968) Fishes of Eastern Uttar Pradesh [India], Vishwavidyalaya Prakashan, Varanasi, xxii + 163 p.

Srivastava, M.P. (1989) A new hill-stream sisorid fish of the genus Hara (Blyth) 1860 from Kosi Belt, North Bihar, India. Journal of Freshwater Biology, 1, 121-125.

Stansch, K. (1914) Die Exotischen Zierfische in Wort und Bild. Part 8. G. Wenzel \& Sohn, Braunschweig, 349 p.

Starks, E.C. (1906) On a collection of fishes made by P. O. Simons in Ecuador and Peru. Proceedings of the United States National Museum, 30, 761-800, pls. 65-66.

Starks, E.C. (1913) The Fishes of the Stanford Expedition to Brazil, Stanford University Publication, University Series, 77 p., $15 \mathrm{pl}$.

Stauffer, J. (1869) [Fishes]. In: Mombert, J.I. (Ed.), An Authentic History of Lancaster County, in the State of Pennsylvania, J.E. Barr \& Co, Lancaster [PA], pp. 576-579.

Steindachner, F. (1864a) Ichthyologische Notizen. Anzeiger der Mathematisch-Naturwissenschaftlichen Classe der Kaiserlichen Akademie der Wissenschaften in Wien, 1, 37-38.

Steindachner, F. (1864b) Ichthyologische Notizen. Sitzungsberichte der Kaiserlichen Akademie der Wissenschaften, Mathematisch- Naturwissenschaftlichen Classe, Wien, Abt. 1, Botanik, Zoologie, Anatomie, Geologie und Paläontologie, 49, 200-214, pls. 1-2.

Steindachner, F. (1866) Ichthyologische Mittheilungen, IX. Verhandlungen der Kaiserlich Zoologisch-Botanischen Gesellshaft in Wien, 16, 761-796, pls. 13-18.

Steindachner, F. (1867a) Über einige Fische aus dem Fitzroy-Flusse bei Rockhampton in Ost-Australien. Anzeiger der Mathematisch- Naturwissenschaftlichen Classe der Kaiserlichen Akademie der Wissenschaften in Wien, 4, 7.

Steindachner, F. (1867b) Ichthyologische Notizen, vierte Folge. Anzeiger der Mathematisch- Naturwissenschaftlichen Classe der Kaiserlichen Akademie der Wissenschaften in Wien, 4, 63-64.

Steindachner, F. (1867c) Ichthyologische Notizen (5. Folge). Anzeiger der Mathematisch- Naturwissenschaftlichen Classe der Kaiserlichen Akademie der Wissenschaften in Wien, 4, 119-120.

Steindachner, F. (1867d) Über einige Fische aus dem Fitzroy-Flusse bei Rockhampton in Ost-Australien. Sitzungsberichte der Kaiserlichen Akademie der Wissenschaften, Mathematisch-Naturwissenschaftlichen Classe, Wien, Abt. 1, Botanik, Zoologie, Anatomie, Geologie und Paläontologie, 55, 9-16, pl. 1.

Steindachner, F. (1867e) Ichthyologische Notizen, IV. Sitzungsberichte der Kaiserlichen Akademie der Wissenschaften, Mathematisch- Naturwissenschaftlichen Classe, Wien, Abt. 1, Botanik, Zoologie, Anatomie, Geologie und Paläontologie, 55, 517-534, pls. 1-6.

Steindachner, F. (1867f) Ichthyologische Notizen, V. Sitzungsberichte der Kaiserlichen Akademie der Wissenschaften, Mathematisch- Naturwissenschaftlichen Classe, Wien, Abt. 1, Botanik, Zoologie, Anatomie, Geologie und Paläontologie, 55, 701-717, pls. 1-3.

Steindachner, F. (1867g) Characters of new fishes. Annals and Magazine of Natural History (Ser. 3), 19, 441-442.

Steindachner, F. (1870) Zur Fischfauna des Senegel, Zweite Abtheilung. Sitzungsberichte der Kaiserlichen Akademie der Wissenschaften, Mathematisch- Naturwissenschaftlichen Classe, Wien, Abt. 1, Botanik, Zoologie, Anatomie, Geologie und Paläontologie, 60, 945-995, pls. 1-8.

Steindachner, F. (1875) Über einige neue brasilienische Siluroiden aus der Gruppe der Doradinen. Sitzungsberichte der Kaiserlichen Akademie der Wissenschaften, Mathematisch- Naturwissenschaftlichen Classe, Wien, Abt. 1, Botanik, Zoologie, Anatomie, Geologie und Paläontologie, 71, 138-151, pls. 1-4. 
Steindachner, F. (1876) Ichthyologische Beiträge, IV. Sitzungsberichte der Kaiserlichen Akademie der Wissenschaften, Mathematisch- Naturwissenschaftlichen Classe, Wien, Abt. 1, Botanik, Zoologie, Anatomie, Geologie und Paläontologie, 72, 551-616, pls. 1-13.

Steindachner, F. (1877a) Ichthyologische Beiträge, V. Sitzungsberichte der Kaiserlichen Akademie der Wissenschaften, Mathematisch- Naturwissenschaftlichen Classe, Wien, Abt. 1, Botanik, Zoologie, Anatomie, Geologie und Paläontologie, 74, 49-240, pls. 1-15.

Steindachner, F. (1877b) Die Süsswasserfische des südöstlichen Brasilien, III. Sitzungsberichte der Kaiserlichen Akademie der Wissenschaften, Mathematisch-Naturwissenschaftlichen Classe, Wien, Abt. 1, Botanik, Zoologie, Anatomie, Geologie und Paläontologie, 74, 559-694, pls. 1-13.

Steindachner, F. (1877c) Die Süsswasserfische des südöstlichen Brasilien, IV. Sitzungsberichte der Kaiserlichen Akademie der Wissenschaften, Mathematisch-Naturwissenschaftlichen Classe, Wien, Abt. 1, Botanik, Zoologie, Anatomie, Geologie und Paläontologie, 76, 217-230, pls. 1-2.

Steindachner, F. (1878a) Zur Fischfauna des Magdalenen-Stromes. Anzeiger der Mathematisch-Naturwissenschaftlichen Classe der Kaiserlichen Akademie der Wissenschaften in Wien, 15, 88-91.

Steindachner, F. (1878b) Ichthyologische Beiträge, VI. Sitzungsberichte der Kaiserlichen Akademie der Wissenschaften, Mathematisch- Naturwissenschaftlichen Classe, Wien, Abt. 1, Botanik, Zoologie, Anatomie, Geologie und Paläontologie, 77, 379-392, pls. 1-3.

Steindachner, F. (1878c) Ichthyologische Beiträge, VII. Sitzungsberichte der Kaiserlichen Akademie der Wissenschaften, Mathematisch- Naturwissenschaftlichen Classe, Wien, Abt. 1, Botanik, Zoologie, Anatomie, Geologie und Paläontologie, 78, for 1879 [separate apparently released in 1878], 377-400.

Steindachner, F. (1879a) Über einige neue und seltene Fischarten aus den zoologischen Museum zu Wien, Stuttgart und Warschau. Anzeiger der Kaiserlichen Akademie der Wissenschaften, Mathematisch- Naturwissenschaftlichen Classe, 16, 29-34.

Steindachner, F. (1879b) Über die Fauna des Orinoco bei Ciudad Bolivar, des Mamoni-Flusses bei Chepo im Isthmus von Panama und einiger Flüsses Peru's; Beiträge zur Kenntniss der Süsswasserfische Südmaerikas. Anzeiger der Kaiserlichen Akademie der Wissenschaften, Mathematisch- Naturwissenschaftichen Classe, 16, 149-152.

Steindachner, F. (1879c) Ichthyologische Beiträge, VIII. Anzeiger der Kaiserlichen Akademie der Wissenschaften, Mathematisch- Naturwissenschaftlichen Classe, 16, 194-195.

Steindachner, F. (1879d) Zur Fischfauna des Magdalenen-Stromes. Denkschriften der Mathematisch- Naturwissenschaftlichen Classe der Kaiserlichen Akademie der Wissenschaften in Wien, 39, 19-78, pls. 1-15.

Steindachner, F. (1879e) Über einige neue und seltene Fisch-Arten aus den k. k. zoologischen Museum zu Wien, Stuttgart, und Warschau. Denkschriften der Mathematisch- Naturwissenschaftlichen Classe der Kaiserlichen Akademie der Wissenschaften in Wien, 41, 1-52, pls. 1-9.

Steindachner, F. (1879f) Beiträge zur Kenntniss der Flussfische Südamerika's. Denkschriften der Mathematisch- Naturwissenschaftlichen Classe der Kaiserlichen Akademie der Wissenschaften in Wien, 41, 151-172, pls. 1-4.

Steindachner, F. (1879g) Ichthyologische Beiträge, VIII. Sitzungsberichte der Kaiserlichen Akademie der Wissenschaften, Mathematisch- Naturwissenschaftlichen Classe, Wien, Abt. 1, Botanik, Zoologie, Anatomie, Geologie und Paläontologie, 80, 119-191, pls. 1-3.

Steindachner, F. (1880a) Beitäge zur Kenntniss der Flussfische Südamerikas, II, und Ichthyologische Beiträge, IX. Anzeiger der Kaiserlichen Akademie der Wissenschaften, Mathematisch- Naturwissenschaftlichen Classe, 17, 157159.

Steindachner, F. (1880b) Zur Fisch-fauna des Cauca und der Flüsse bei Guayaquil. Denkschriften der MathematischNaturwissenschaftlichen Classe der Kaiserlichen Akademie der Wissenschaften in Wien, 42, 55-104, pls. 1-9.

Steindachner, F. (1880c) Ichthyologische Beiträge, IX. Sitzungsberichte der Kaiserlichen Akademie der Wissenschaften, Mathematisch- Naturwissenschaftlichen Classe, Wien, Abt. 1, Botanik, Zoologie, Anatomie, Geologie und Paläontologie, 82, 238-266, pls. 1-6.

Steindachner, F. (1881a) Ichthyologische Beiträge, X. Anzeiger der Kaiserlichen Akademie der Wissenschaften, Mathematisch- Naturwissenschaftlichen Classe, 18, 45-46.

Steindachner, F. (1881b) Beiträge zur Kenntniss der Flussfische Südamerika's, III, und Ichthyologische Beiträge, XI. Anzeiger der Kaiserlichen Akademie der Wissenschaften, Mathematisch- Naturwissenschaftlichen Classe, 18, 97100 .

Steindachner, F. (1881c) Beiträge zur Kenntniss der Flussfische Südamerika's, II. Denkschriften der MathematischNaturwissenschaftlichen Classe der Kaiserlichen Akademie der Wissenschaften in Wien, 43, 103-146, pls. 1-7.

Steindachner, F. (1881d) Beiträge zur Kenntniss der Flussfische Südamerika's, III. Denkschriften der Mathematisch- 
Naturwissenschaftlichen Classe der Kaiserlichen Akademie der Wissenschaften in Wien, 44, for 1882, 1-18, pls. 15.

Steindachner, F. (1881e) Ichthyologische Beiträge, X. Sitzungsberichte der Kaiserlichen Akademie der Wissenschaften, Mathematisch- Naturwissenschaftlichen Classe, Wien, Abt. 1, Botanik, Zoologie, Anatomie, Geologie und Paläontologie, 83, 179-219, pls. 1-8.

Steindachner, F. (1881f) Ichthyologische Beiträge, XI. Sitzungsberichte der Kaiserlichen Akademie der Wissenschaften, Mathematisch- Naturwissenschaftlichen Classe, Wien, Abt. 1, Botanik, Zoologie, Anatomie, Geologie und Paläontologie, 83, 393-408, pl. 1.

Steindachner, F. (1882a) Ichthyologische Beiträge, XII. Anzeiger der Kaiserlichen Akademie der Wissenschaften, Mathematisch- Naturwissenschaftichen Classe, 19, 142-143.

Steindachner, F. (1882b) Beiträge zur Kenntniss der Flussfische Südamerika's, IV. Anzeiger der Kaiserlichen Akademie der Wissenschaften, Mathematisch-Naturwissenschaftlichen Classe, 19, 175-180.

Steindachner, F. (1882c) Beiträge zur Kenntniss der Flussfische Südamerikas, IV. Denkschriften der MathematischNaturwissenschaftlichen Classe der Kaiserlichen Akademie der Wissenschaften in Wien, 46, for 1883, 1-44, pls. 17.

Steindachner, F. (1882d) Ichthyologische Beiträge, XII. Sitzungsberichte der Kaiserlichen Akademie der Wissenschaften, Mathematisch- Naturwissenschaftlichen Classe, Wien, Abt. 1, Botanik, Zoologie, Anatomie, Geologie und Paläontologie, 86, 61-82, pls. 1-5.

Steindachner, F. (1883) Ichthyologische Beiträge, XIII. Anzeiger der Kaiserlichen Akademie der Wissenschaften, Mathematisch- Naturwissenschaftlichen Classe, 20, 194-197.

Steindachner, F. (1884) Ichthyologische Beiträge, XIII. Sitzungsberichte der Kaiserlichen Akademie der Wissenschaften, Mathematisch- Naturwissenschaftlichen Classe, Wien, Abt. 1, Botanik, Zoologie, Anatomie, Geologie und Paläontologie, 88, 1065-1114, pls. 1-8.

Steindachner, F. (1894) Die Fische Liberia's. Notes from the Leyden Museum, 16, 1-96, pls. 1-4.

Steindachner, F. (1900) Erstattungen eines vorlaüfigen Berichtes über einige von Ihrer königlichen Hoheit Frau Prinzessin Therese von Bayeren während einer Reise nach Südamerika 1898 gesammelte neue Fischarten. Anzeiger der Kaiserlichen Akademie der Wissenschaften, Mathematisch- Naturwissenschaftlichen Classe, 37, $206-208$.

Steindachner, F. (1901) Kükenthal's Ergebnisse einer zoologischen Forschungreise in den Molukken und Borneo. Fische. Abhandlungen Senckenbergische Naturforschende Gesellschaft, 25, 409-464, pls. 17-18.

Steindachner, F. (1902) Herpetologische und ichthyologische Ergebnisse einer Reise nach Südamerika, mit einer Einleitung con Therese Prinzessin von Bayern. Denkschriften der Mathematisch-Naturwissenschaftlichen Classe der Kaiserlichen Akademie der Wissenschaften in Wien, 72, 89-148, pls. 1-6.

Steindachner, F. (1906) Ueber zwei neue Corydoras-Arten aus dem Parnahyba und Parahimflusse im Staate Piauhy. Anzeiger der Kaiserlichen Akademie der Wissenschaften, Mathematisch- Naturwissenschaftlichen Classe, 1906, 478-480.

Steindachner, F. (1907a) Über eine neue Psilichthys-Art, Ps. cameroni aus dem Flusse Cubatão im Staate S. Catharina, Brasilien. Anzeiger der Kaiserlichen Akademie der Wissenschaften, Mathematisch- Naturwissenschaftlichen Classe, $44,82-85$.

Steindachner, F. (1907b) Über zwei neue Arten von Süsswasserfischen aus dem Stromgebiete des Parnahyba. Anzeiger der Kaiserlichen Akademie der Wissenschaften, Mathematisch- Naturwissenschaftlichen Classe, 44, $152-155$.

Steindachner, F. (1907c) Ueber eine neue Arges-Art aus den Hohen Anden von Cayendelet. Anzeiger der Kaiserlichen Akademie der Wissenschaften, Mathematisch- Naturwissenschaftlichen Classe, 44, 228-229.

Steindachner, F. (1907d) Über eine neue Coridoras [sic, Corydoras]-Art aus dem Rio Preto, einem sekundären Nebenflusse des Rio San Francisco, und eine Xenocara-Art aus dem Parnahyba bei Victoria und Sa. Filomena. Anzeiger der Kaiserlichen Akademie der Wissenschaften, Mathematisch- Naturwissenschaftlichen Classe, 44, $290-293$.

Steindachner, F. (1907e) Ueber einige Fischarten aus dem Flusse Cubatão im Staate Santa Catharina bei Theresopolis (Brasilien). Sitzungsberichte der Kaiserlichen Akademie der Wissenschaften, Mathematisch- Naturwissenschaftlichen Classe, Wien, Abt. 1, Botanik, Zoologie, Anatomie, Geologie und Paläontologie, 116, 475-492, pls. 1-2.

Steindachner, F. (1908a) Über eine im Rio Juraguá bei Joinville im Staate S. Catharina (Brasilien) vorkommende noch unbeschriebene Pseudochalceus-Art, Ch. affinis, sowie über eine neue Characinengattung und -art, Joinvillea rosae, von gleichem Fundorte. Anzeiger der Kaiserlichen Akademie der Wissenschaften, Mathematisch- Naturwissenschaftlichen Classe, 45, 28-31.

Steindachner, F. (1908b) Über drei neue Characinen und drei Siluroiden aus dem Stromgebiete des Amazonas innerhalb Brasilien. Anzeiger der Kaiserlichen Akademie der Wissenschaften, Mathematisch- Naturwissenschaftlichen Classe, 
45, 61-69.

Steindachner, F. (1908c) Über drei neue Arten von Süsswasserfischen aus dem Amazonasgebiet und aus dem See Candidius auf der Insel Formosa, ferner über die vorgerückte Altersform von Loricaria acuta C. V. Anzeiger der Kaiserlichen Akademie der Wissenschaften, Mathematisch- Naturwissenschaftlichen Classe, 45, 82-87.

Steindachner, F. (1908d) Ueber eine noch unbekannte Art der Gattung Bergiella Eig. aus dem La Plata. Anzeiger der Kaiserlichen Akademie der Wissenschaften, Mathematisch- Naturwissenschaftlichen Classe, 45, 110-113.

Steindachner, F. (1908e) Über eine während der brasilianischen Expedition entdeckte Brachyplatystoma-Art aus dem Rio Parnahyba und über eine dicht gefleckte und gestrichelte Varietät von Giton fasciatus aus den Gewässern von Santos (Staate Sao Paulo). Anzeiger der Kaiserlichen Akademie der Wissenschaften, Mathematisch- Naturwissenschaftlichen Classe, 45, 126-130.

Steindachner, F. (1908f) Über zwei neue Siluroiden und zwei Curimatus-Arten, sowie über eine Varietät von Ancistrus vittalus [sic, vittatus] aus dem Amazonasgebiete innerhalb Brasiliens. Anzeiger der Kaiserlichen Akademie der Wissenschaften, Mathematisch- Naturwissenschaftlichen Classe, 45, 163-168.

Steindachner, F. (1909a) Eine neue Brachyplatystoma und eine neue Loricaria-Art. Anzeiger der Kaiserlichen Akademie der Wissenschaften, Mathematisch- Naturwissenschaftlichen Classe, 46, 195-197.

Steindachner, F. (1909b) Über eine Ageneiosus (Pseudageneiosus)-Art im Rio Parnahyba und Rio Puty bei Therezina. Anzeiger der Kaiserlichen Akademie der Wissenschaften, Mathematisch- Naturwissenschaftlichen Classe, 46, 341342.

Steindachner, F. (1910a) Über eine neue Loricaria-Art aus dem Flußgebiete des Jaraguá und der Ribeira im Staate S. Paulo und Sa. Catharina, über eine mit Ancistrus aculeatus (Perugia) = Ancistrus gigas (Blgr.) Reg. sehr nahe verwandte Ancistrus-Art aus dem Rio S. Francisco bei Barra, über eine neue Corydoras-Art aus dem Jaraguá und über die äußeren Geschlechtunterschiede von Corydoras kronei, Ribeira [sic]. Anzeiger der Kaiserlichen Akademie der Wissenschaften, Mathematisch- Naturwissenschaftlichen Classe, 47, 57-62.

Steindachner, F. (1910b) Über eine noch unbeschriebene Oxyloricaria (= Sturisoma)-Art aus dem Rio Meta im Venezuela und über die relativen Längenmasse bei O. rostrata (Sp.). Anzeiger der Kaiserlichen Akademie der Wissenschaften, Mathematisch- Naturwissenschaftlichen Classe, 47, 410-411.

Steindachner, F. (1910c) Über einige Ageneiosus- und Farlowella-Arten, etc. Annalen des K. K. Naturhistorischen Hofmuseums, Wien, 24, 399-408.

Steindachner, F. (1910d) Die Fische des Itapocu und seiner Zuflüsse im Staate $S^{\text {a }}$ Catharina (Brasilien). Annalen des K. K. Naturhistorischen Hofmuseums, Wien, 24, 419-433, pl. 5.

Steindachner, F. (1911a) Über vier neue Siluroiden und Characinen aus dem Amazonasgebiete und von Ceará aus der Sammlung des Museums Göldi in Pará. Anzeiger der Kaiserlichen Akademie der Wissenschaften, MathematischNaturwissenschaftlichen Classe, 48, 324-331.

Steindachner, F. (1911b) Über einige neue und seltene südamerikanische Süsswasserfische. Anzeiger der Kaiserlichen Akademie der Wissenschaften, Mathematisch- Naturwissenschaftlichen Classe, 48, 369-376.

Steindachner, F. (1911c) Beiträge zur Kenntniss der Fischfauna des Tanganyikasees und des Kongogebietes. Anzeiger der Kaiserlichen Akademie der Wissenschaften, Mathematisch- Naturwissenschaftlichen Classe, 48, 528-530.

Steindachner, F. (1911d) Über einige neue und seltene afrikanische Süsswasserfische. Anzeiger der Kaiserlichen Akademie der Wissenschaften, Mathematisch- Naturwissenschaftlichen Classe, 48, 531-535.

Steindachner, F. (1911e) Beiträge zur Kenntniss der Fischfauna des Tanganyikasees und des Kongogebietes. Sitzungsberichte der Kaiserlichen Akademie der Wissenschaften, Mathematisch-Naturwissenschaftlichen Classe, Wien, Abt. 1, Botanik, Zoologie, Anatomie, Geologie und Paläontologie, 120, 1171-1186, pls. 1-3.

Steindachner, F. (1912) Zur Fischfauna des Dscha, eines sekundären Nebenflusses des Congo im Bezirke Molundu des südlichen Kamerun. Anzeiger der Kaiserlichen Akademie der Wissenschaften, Mathematisch- Naturwissenschaftlichen Classe, 49, 443-449.

Steindachner, F. (1913) Zur Fischfauna des Dscha, eines sekundären Nebenflusses des Kongo, im Bezirke Molundu, Kamerun. Denkschriften der Mathematisch- Naturwissenschaftlichen Classe der Kaiserlichen Akademie der Wissenschaften in Wien, 89, 1-64, pls. 1-9.

Steindachner, F. (1914) Bericht über die ichthyologischen Aufsammlungen der Brüder Adolf und Albin Horn wärend einer im Sommer 1913 ausgeführten Reise nach Deutsch-Ostafrika. Anzeiger der Kaiserlichen Akademie der Wissenschaften, Mathematisch- Naturwissenschaftlichen Classe, 51, 536-538.

Steindachner, F. (1915a) Vorlaüfigen bericht über einige neue Süsswasserfische aus Südamerika. Anzeiger der Kaiserlichen Akademie der Wissenschaften, Mathematisch- Naturwissenschaftlichen Classe, 52, 199-202.

Steindachner, F. (1915b) Beiträge zur Kenntnis der Flussfische Südamerikas, V. Anzeiger der Kaiserlichen Akademie der 
Wissenschaften, Mathematisch- Naturwissenschaftlichen Classe, 52, 217-219.

Steindachner, F. (1915c) Ichthyologische Beiträge, XVIII. Anzeiger der Kaiserlichen Akademie der Wissenschaften, Mathematisch- Naturwissenschaftlichen Classe, 52, 346-349.

Steindachner, F. (1915d) Bericht über die ichthyologischen Aufsammlungen der Brüder Adolf und Albin Horn während einer im Sommer 1913 ausgeführten Reise nach Deutsch-Ostafrika. Denkschriften der Mathematisch-Naturwissenschaftlichen Classe der Kaiserlichen Akademie der Wissenschaften in Wien, 92, 59-86, pls. 1-5.

Steindachner, F. (1915e) Beiträge zur Kenntniss der Flussfische Südamerikas, V. Denkschriften der MathematischNaturwissenschaftlichen Classe der Kaiserlichen Akademie der Wissenschaften in Wien, 93, for 1917, 15-106, pls. 1-13. [Issued first as a separate in 1915, with dual pagination.]

Steindachner, F. \& Döderlein, L. (1887) Beiträge zur Kenntniss der Fische Japan's, IV. Denkschriften der MathematischNaturwissenschaftlichen Classe der Kaiserlichen Akademie der Wissenschaften in Wien, 53, 257-296, pls. 1-4.

Steinitz, H. (1961) Notes on some glyptosternoid fishes (Sisoridae, Siluroidea). Mitteilungen aus dem Hamburgischen Zoologischen Museum und Institut, 59, 105-115.

Stewart, D.J. (1985a) A new species of Cetopsorhamdia (Pisces: Pimelodidae) from the Río Napo basin of eastern Ecuador. Copeia, 1985, 339-344.

Stewart, D.J. (1985b) A review of the South American catfish tribe Hoplomyzontini (Pisces, Aspredinidae), with descriptions of new species from Ecuador. Fieldiana, Zoology (New Series), 25, i-iii + 1-19.

Stewart, D.J. (1986a) Revision of Pimelodina and description of a new genus and species from the Peruvian Amazon (Pisces: Pimelodidae). Copeia, 1986, 653-672.

Stewart, D.J. (1986b) A new pimelodid catfish from the deep-river channel of the Río Napo, eastern Ecuador (Pisces: Pimelodidae). Proceedings of the Academy of Natural Sciences, Philadelphia, 138, 46-52.

Stewart, D.J. \& Pavlik, M.J. (1985) Revision of Cheirocerus (Pisces: Pimelodidae) from tropical freshwaters of South America. Copeia, 1985, 356-367.

Steyskal, G.C. (1980) The grammar of family-group names as exemplified by those of fishes. Proceedings of the Biological Society of Washington, 93, 168-177.

Stinton, F.C. (1962) Teleostean otoliths from the upper Tertiary strata of Sarawak, Brunei, and North Borneo. British Borneo Geological Survey Department, Annual Report for 1962, 75-92, figs. 1-18.

Stinton, F.C. (1966) Fish otoliths from the London Clay. In: Casier, E. (Ed.), Faune Icthyologique du London Clay, British Museum (Natural History), London, pp. 404-478, tables 1-4.

Stinton, F.C. (1977) Fish otoliths from the English Eocene. Palaeontographical Society Monographs, Part 2, for 1976, 57-26, pls. 4-8.

Stromer, E. (1904) Nematognathi aus de Fajûm und dem Natronthale in Äegypten. Neues Jahrbuch für Mineralogie, Geologie und Palaeontologie, 1, 1-7, pl. 1.

Subhash Babu, K.K. \& Nayar, C.K.G. (2004) A new species of the blind fish Horaglanis Menon (Siluroidea: Clariidae) from Parappukara (Trichur District) and a new report of Horaglanis krishnai Menon from Ettumanur (Kottayam district) Kerala. Journal of the Bombay Natural History Society, 101, 296-298.

Sudarto, Teugel, G.G. \& Pouyaud, L. (2003) Description of two new Clarias species from Borneo (Siluriformes, Clariidae). Cybium (3e série), 27 153-161.

Sudarto, Teugels, G.G. \& Pouyaud, L. (2004) Description of a new clariid catfish, Clarias pseudonieuhofii from west Borneo (Siluriformes, Clariidae). Zoological Studies, 43, 8-19.

Sufi, S.M.K. (1963) Checklist of the fishes of the genus Glyptothorax Blyth of W. Pakistan with record of Glyptothorax platypogonoides [sic] (Bleeker) from Peshwar district (U. P. ). Biologia (Lahore), 9, 23-27.

Suttkus, R.D. \& Taylor, W.R. (1965) Noturus munitus, a new species of madtom, family Ictaluridae, from southern United States. Proceedings of the Biological Society of Washington, 78, 169-178.

Svensson, G.S.O. (1933) Fresh water fishes from the Gambia River (British West Africa). Results of the Swedish Expedition 1931. Kungl. Svenska Vetenskapsakademiens Handlingar, 12, 1-102, pls. 1-8.

Svetovidova, A.A. (1978) [List of holotypes, syntypes and paratypes kept in the Department of Ichthyology of the Moscow State University Museum]. Sbornik Trudov Zoologicheskogo Muzeua Mgu, 16, 256-263. [In Russian.]

Swain, J. (1882) A review of Swainson's genera of fishes. Proceedings of the Academy of Natural Sciences, Philadelphia, 1882, 272-284.

Swain, J. \& Kalb, G.B. (1883) A review of the genus Noturus with a description of one new species. Proceedings of the United States National Museum, 5, 638-644.

Swainson, W. (1838) The Natural History and Classification of Fishes, Amphibians, \& Reptiles, or Monocardian Animals, vol. 1, Longman, Orme, Brown, Green and Longmans, and John Taylor, London, vi + 368 p. 
Swainson, W. (1839) The Natural History and Classification of Fishes, Amphibians, \& Reptiles, or Monocardian Animals, vol. 2, Vol. 2, Longman, Orme, Brown, Green and Longmans, and John Taylor, London, vi + 448 p.

Sydenham, D.H.J. (1978) Redescriptions of the type specimens of six clariid species (Pisces) from western Africa. Zoological Journal of the Linnean Society, 64, 347-371.

Sydenham, D.H.J. (1980) New species of Clarias from West Africa (Pisces, Clariidae). Revue de Zoologie Africaine, 94, 659-677.

Sydenham, D.H.J. \& Olawoye, O.F. (1981) A new species of Clarias from Nigeria (Pisces, Clariidae). Revue de Zoologie Africaine, 95, 234-244.

Sykes, W.H. (1839) On the fishes of the Deccan. Proceedings of the Zoological Society of London, 1838, $157-165$.

Sykes, W.H. (1840) An account of the fishes of Dukhun. Annals and Magazine of Natural History (n. s.), 4, 54-62.

Sykes, W.H. (1841) On the fishes of the Dukhun. Transactions of the Zoological Society of London, 2, 349-378, pls. 6067.

Sytchevskaya, E.K. (1986) [Palaeogene freshwater fish fauna of the USSR and Mongolia]. Sovmestnaya Sovetsko Mongol'skaya Paleontologicheskaya Ekspeditsiya Trudy, 29, 1-157. [In Russian.]

Sytchevskaya, E.K. (1989) [Neogene freshwater fish fauna of Mongolia]. The joint Soviet-Mongolian Paleontological Expedition; Transactions, 39, 8-142 [In Russian] [not seen]

Takako, A.K., Oliveira, C. \& Oyakawa, O.T. (2005) Revision of the genus Pseudotocinclus (Loricariidae: Hypoptopomatinae), with the description of two new species. Neotropical Ichthyology, 3, 499-508.

Talwar, P.K. (1976) A contribution to the systematics of Arius tenuispinis Day, 1877 (Pisces: Ariidae). Records of the Zoological Survey of India, 69, 291-294.

Talwar, P.K. \& Jhingran, A.G. (1991) Inland Fishes of India and Adjacent Countries, Oxford \& IBH Publishing Co, New Delhi, Bombay, Calcutta, xvii + [36] + 1158 p., 1 map.

Tamang, P. (1993) A Preliminary Annotated List of Fish Expected to Occur in Bhutanese River Systems. National Environmental Commission, Thimphu, Bhutan, $17 \mathrm{p}$.

Tan, H.H. \& Ng, H.H. (2000) The catfishes (Teleostei: Siluriformes) of central Sumatra. Journal of Natural History, 34, 267-303.

Tan, T.H.T. \& Ng, P.K.L. (1996) Catfishes of the Ompok leiacanthus (Bleeker, 1853) species group (Teleostei: Siluridae) from southeast Asia, with description of a new species. The Raffles Bulletin of Zoology, 44, 531-542.

Taphorn, D., Royero, R., Machado-Allison, A. \& Mago-Leccia, F. (1997) Lista actualizada de los peces de agua dulce de Venezuela. In: La Marca, E. (Ed.), Vertebrados Actuales y Fósiles de Venezuela, Museo de Ciencia y Technología de Mérida, Mérida, Venezuela, pp. 55-100.

Taphorn, D.C. \& Lilyestrom, C.G. (1983) Un nuevo pez del genero Xiliphius (Aspredinidae) de Venezuela. Revista UNELLEZ de Ciencia y Tecnologia, 1, 43-44, unnumbered plate.

Taphorn, D.C. \& Lilyestrom, C.G. (1984a) Rhinodoras thomersoni: un bagre sierra nuevo en Venezuela (Pisces, Doradidae). Revista UNELLEZ de Ciencia y Tecnologia, 2, 87-92.

Taphorn, D.C. \& Lilyestrom, C.G. (1984b) Lamontichthys maracaibero y L. llanero dos especies nuevas para Venezuela (Pisces, Loricariidae). Revista UNELLEZ de Ciencia y Tecnologia, 2, 93-100.

Taphorn, D.C. \& Marrero, C. (1990) Hoplomyzon sexpapilostoma, a new species of Venezuelan catfish (Pisces: Aspredinidae), with comments on the Hoplomyzontini. Fieldiana, Zoology (New Series), 61, 1-9.

Taverne, L. \& Aloulou-Triki, A. (1974) Étude anatomique, myologique et ostéologique du genre Synodontis Cuvier (Pisces: Siluriformes, Mochocidae). Annales du Musée Royal de l'Afrique Centrale, Series in $8^{\circ}$, Sciences Zoologiques, 210, pl. 1-2.

Taverne, L. \& Vos, L. de (1998) Clariallabes mutsindoziensis (Teleostei: Clariidae), nouveau silure du bassin de la Malagarasi (bassin du lac Tanganyika), Burundi. Ichthyological Exploration of Freshwaters, 8, 211-220.

Taylor, W.R. (1969) A revision of the catfish genus Noturus Rafinesque with an analysis of higher groups in the Ictaluridae. Bulletin of the U. S. National Museum, 282, i-vi +1-315, pls. 1-21.

Taylor, W.R. (1986a) Ariidae. In: Daget, J., Gosse, J.-P. \& Thys van den Audenaerde, D.F.E. (Eds.), Check-list of the Freshwater Fishes of Africa, Vol. 2, ISNB Bruxelles, MRAC Tervuren, ORSTOM, Paris, pp. 153-159.

Taylor, W.R. (1986b) Ariidae. In: Smith, M.M. \& Heemstra, P.C. (Eds.), Smiths' Sea Fishes, Macmillan South Africa, Johannesburg, pp. 211-213.

Taylor, W.R. (1990) Ariidae. In: Quéro, J.-C., Hureau, J.-C., Karrer, C., Post, A. \& Saldanha, L. (Eds.), Check-list of the Fishes of the Eastern Tropical Atlantic, UNESCO, Paris, pp. 230-234.

Taylor, W.R. \& Gomon, J.R. (1986) Plotosidae. In: Daget, J., Gosse, J.-P. \& Thys van den Audenaerde, D.F.E. (Eds.), Check-list of the Freshwater Fishes of Africa, Vol. 2, ISNB Bruxelles; MRAC Tervuren; ORSTOM, Paris, pp. 160- 
162.

Taylor, W.R. \& Menezes, N.A. (1978) Family Ariidae. In: Fischer, W. (Ed.), FAO Species Identification Sheets Fishery Purposes. Western Central Atlantic (Fishing Area 31), FAO, Rome.

Tchang, T.-L. (1934) Notes on a new catfish from Kaifeng. Bulletin of the Fan Memorial Institute of Biology, Peiping (Zoological series), 5, 41-43.

Tchang, T.-L. (1935a) A new catfish from Yunnan. Bulletin of the Fan Memorial Institute of Biology, Peiping (Zoological series), 6, 95-97.

Tchang, T.-L. (1935b) Two new catfishes from south China. Bulletin of the Fan Memorial Institute of Biology, Peiping (Zoological series), 6, 174-177.

Tchang, T.-L. (1936) Study on some Chinese catfishes. Bulletin of the Fan Memorial Institute of Biology, Peiping (Zoological series), 7,35 .

Tchang, T.-L. (1960) The Siluriform Fishes of China, People Education Press. [In Chinese, with English summary]

Tchang, T.-L. \& Shih, H.-J. (1934) Notes on the fishes of the valley of lower Kialingkiang. Lingnan Science Journal, 13, 431-435.

Tchernavin, V.V. (1944) A revision of some Trichomycterinae based on material preserved in the British Museum (Natural History). Proceedings of the Zoological Society of London, 114, 234-275.

Temminck, C.J. \& Schlegel, H. (1843-1850) Pisces. In: Fauna Japonica, sive Descriptio Animalium quae in Itinere per Japoniam Suscepto Annis 1823-30 Collegit, Notis Observationibus et Adumbrationibus Illustravit P. F. de Siebold, [Issued in parts: Parts 1-4: 1-72 (1843); parts 5-6, 73-112 (1844); parts 7-9, 113-172 and pls. 1-143 and pl. A (1845); parts 10-14, 173-269 (1846); part 15, 270-324 (1850)]

Terofal, F. (1983) Die Fischausbeute der Brasilien-Expedition 1817-1820 con J. B. Spix und C. F. Ph. V. Martius. Spixiana, 9, 313-317.

Teugels, G.G. (1980) Notes on the status and the synonyms of Clarias pachynema Boulenger, 1903 (Pisces, Clariidae). Revue de Zoologie Africaine, 94, 678-692.

Teugels, G.G. (1981) Notes on the status and the synonyms of Clarias laeviceps Gill, 1862 (Pisces, Clariidae). Revue de Zoologie Africaine, 95, 359-370.

Teugels, G.G. (1982a) A systematic outline of the African species of the genus Clarias (Pisces; Clariidae), with an annotated bibliography. Musée Royal de l'Afrique Centrale, Annales, Sciences Zoologiques, 236, 1-249.

Teugels, G.G. (1982b) On the rehabilitation of Clarias gabonensis Günther, 1867, with a redescription and notes on its systematical status (Pisces, Clariidae). Revue de Zoologie Africaine, 96, 45-60.

Teugels, G.G. (1982c) Preliminary data of a systematic outline of the African species of the genus Clarias (Pisces, Clariidae). Revue de Zoologie Africaine, 96, 731-748.

Teugels, G.G. (1983) Notes on the status of Clarias ngamensis Castelnau, 1861, C. mellandi Boulenger, 1905, C. prentissgrayi (Fowler, 1930) and C. lamottei Daget and Planquette, 1967 (Pisces, Clariidae) with the rehabilitation of Dinotopteroides Fowler, 1930, as a subgenus of Clarias. Cybium (3e série), 7 (1), 15-28.

Teugels, G.G. (1986a) A systematic revision of the African species of the genus Clarias (Pisces; Clariidae). Musée Royal de l'Afrique Centrale, Annales, Sciences Zoologiques, 247, 1-199.

Teugels, G.G. (1986b) Clariidae. In: Daget, J., Gosse, J.-P. \& Thys van den Audenaerde, D.F.E. (Eds.), Check-list of the Freshwater Fishes of Africa, Vol. 2, ISNB Bruxelles; MRAC Tervuren; ORSTOM, Paris, pp. 66-101.

Teugels, G.G. (1992a) Clariidae. In: Lévêque, C., Paugy, D. \& Teugels, G.G. (Eds.), Faune des Poissons d'Eaux Douces et Saumâtres de l'Afrique de l'Ouest, Vol. 2, Musée Royal de l'Afrique Central, Tervuren, and Editions de l'ORSTOM, Paris, pp. 468-495.

Teugels, G.G. (1992b) Malapteruridae. In: Lévêque, C., Paugy, D. \& Teugels, G.G. (Eds.), Faune des Poissons d'Eaux Douces et Saumâtres de l'Afrique de l'Ouest, Vol. 2, Musée Royal de l'Afrique Central, Tervuren, and Editions de l'ORSTOM, Paris, pp. 496-499.

Teugels, G.G. (1996) Taxonomy, phylogeny and biogeography of catfishes (Ostariophysi, Siluroidei): an overview. Aquatic Living Resources, 9, 9-34.

Teugels, G.G. (2003a) State of the art of Recent siluriform systematics. In: Arratia, G., Kapoor, B.G., Chardon, M. \& Diogo, R. (Eds.), Catfishes, Science Publishers, Enfield, NH, USA, pp. 317-352.

Teugels, G.G. (2003b) Clarias lamottei (Siluriformes: Clariidae), a natural intergeneric hybrid from West Africa. Cybium (3e série), 27, 11-15.

Teugels, G.G. \& Adriens, D. (2003) Taxonomy and phylogeny of Clariidae: an overview. In: Arratia, G., Kapoor, B.G., Chardon, M. \& Diogo, R. (Eds.), Catfishes, Science Publishers, Enfield, NH, USA, pp. 465-487.

Teugels, G.G., Denayer, B. \& Legendre, M. (1990) A systematic revision of the African catfish genus Heterobranchus 
Geoffroy-Saint-Hilaire, 1809 (Pisces: Clariidae). Zoological Journal of the Linnean Society, 98, 237-257.

Teugels, G.G., Diego, R.C., Pouyaud, L. \& Legendre, M. (1999) Redescription of Clarias macrocephalus (Siluriformes: Clariidae) from South-East Asia. Cybium (3e série), 23, 285-295.

Teugels, G.G., Risch, L., De Vos, L. \& Thys van den Audenaerde, D.F.E. (1991) Generic review of the African bagrid genera Auchenoglanis and Parauchenoglanis, with description of a new genus. Journal of Natural History, 25, 499517.

Teugels, G.G. \& Roberts, T.R. (1987) Silurus anguillaris Linnaeus, 1758: designation as type species of Clarias Scopoli, 1777 and rediscovery of holotype (Pisces: Clariidae). Zoological Journal of the Linnean Society, 90, 95-98.

Teugels, G.G., Skelton, P.H. \& Lévêque, C. (1987) A new species of Amphilius (Pisces, Amphiliidae) from the Konkoure Basin, Guinea, West Africa. Cybium (3e série), 11, 93-101.

Teugels, G.G., Sudarto \& Pouyaud, L. (2001) Description of a new Clarias species from Southeast Asia based on morphological and genetical evidence (Siluriformes, Clariidae). Cybium (3e série), 25, 81-92.

Teugels, G.G. \& Thys van den Audenaerde, D.F.E. (1981) On the synonymy of the West African species Clarias ebriensis and Clarias dahomeyensis (Pisces, Clariidae). Revue de Zoologie Africaine, 95, 11-28.

Thenius, E. (1952) Welsreste aus dem Unter-Pliozän des Wiener Beckens. Neues Jahrbuch für Geologie und Paläontologie. Monatshefte, 1952, 80-94.

Thomas, M.R. \& Burr, B.M. (2004) Noturus gladiator, a new species of madtom (Siluriformes: Ictaluridae) from coastal streams of Tennessee and Mississippi. Ichthyological Exploration of Freshwaters, 15, 351-368.

Thominot, A. (1886) Sur quelques poissons nouveaux appartenant à la collection du Muséum d'Histoire Naturelle. Bulletin de la Société Philomathique (Ser. 7), 10, 161-168.

Thompson, Z. (1842) History of Vermont, Natural, Civil, and Statistical, in Three Parts, with a New Map of the State, and 200 Engravings, Published for the author, Burlington.

Thunberg, C.P. (1787-1821) Museum Naturalium Academiae Upsaliensis ... Praesidae. C. P. Thunberg, etc., 33 parts, Upsaliae. [New species of fishes are in part 1, published in 1787.]

Thunberg, C.P. (1791) Tvånne utlåndska fiskar. Kongliga Svenska Vetenskaps Academiens Handlingar, Stockholm, 12 , 190-192, pl. 6.

Thunberg, C.P. (1792) Tvånne Japanske fiskar beskrifne. Kongliga Svenska Vetenskaps Academiens Handlingar, Stockholm, 13, 29-32, pl. 1.

Thys van den Audenaerde, D.F.E. (1964) Revision of the genus Eutropiellus Nichols and Lamonte (Pisces, Schilbeidae) with a description of a new species from Lower Nigeria Eutropiellus vandeweyeri sp. nov. Revue de Zoologie et de Botanique Africaines, 69, 214-230.

Thys van den Audenaerde, D.F.E. (1965a) Description d'une nouvelle espèce de Gephyroglanis (Pisces, Bagridae) de la rivière Sanaga (Cameroun). Revue de Zoologie et de Botanique Africaines, 71, 264-273.

Thys van den Audenaerde, D.F.E. (1965b) Description de Parauchenoglanis boutchangai sp. n. (Pisces, Bagridae) du bassin de la rivière Ngounié (Gabon). Revue de Zoologie et de Botanique Africaines, 72, 346-352.

Thys van den Audenaerde, D.F.E. \& De Vos, L.D.G. (1982) Description of Eutropius djeremi spec. nov. from the Cameroons (Pisces, Schilbeidae). Revue de Zoologie Africaine, 96, 179-184.

Tilak, R. (1967) Studies of the osteocranium and the Weberian apparatus of the fishes of the genus Batasio Blyth, 1860 (Pisces: Siluroidei) with remarks on the systematic position of the genus. Anatomischer Anzeiger, 121, 415-434.

Tilak, R. (1969) Descriptions of two new sisorids and a hybrid carp from Pauri Garhwal (Kamaon Hills) Uttar Pradesh. Journal of the Inland Fishery Society of India, 1, 37-48.

Tilak, R. (1970a) Siluroid fishes of India, Pakistan, Burma, and Ceylon: Family Plotosidae. Annals of Zoology (Warsaw), 27, 261-273.

Tilak, R. (1970b) A new sisorid catfish of the genus Gagata Bleeker from India. Zoologische Mededelingen (Leiden), 44, 207-215.

Tilak, R. (1978) Redescription of Hara hara (Hamilton) and Hara horai Misra with a key to the species of Hara Blyth (Pisces: Sisoridae). Bulletin of the Zoological Survey of India, 1, 295-301.

Tilak, R. (1982) Description of a new schilbeid fish, Proeutropiichthys taakree burmanicus from Burma. (Schilbeidae: Siluriformes). Uttar Pradesh Journal of Zoology, 1, for 1981, 34-39.

Tilak, R. (1987) Studies on the fish fauna of Uttar Pradesh Terai. I.- On the extension of range of distribution of Conta conta (Hamilton) and Chandramara chandramara (Hamilton) (Sisoridae: Bagridae: Siluriformes). Matsya, 12/13, 84-92.

Tilak, R. \& Husain, A. (1975) A new sisorid catfish, Laguvia ribeiroi kapuri (Sisoridae: Siluriformes) from Uttar Pradesh. Journal of the Inland Fishery Society of India, 6, 1-5. 
Tilak, R. \& Husain, A. (1976) Description of a new species of the genus Glyptothorax Blyth from River Yamuna, India (Pisces, Siluriformes: Sisoridae). Annals of Zoology (Warsaw), 33, 229-234.

Tilak, R. \& Husain, A. (1978) Redescription of Glyptothorax saisii (Jenkins) (Sisoridae: Siluriformes) with remarks on its discontinuous distribution. Annals of Zoology (Agra), 14, 33-40.

Tilak, R. \& Talwar, P.K. (1976) A taxonomic reassessment of Hara horai Misra (Pisces: Sisoridae) with a designation of neotype. Newsletter of the Zoological Survey of India, 2, 245-247.

Tomoda, Y. (1961) Two new catfishes of the genus Parasilurus found in Lake Biwa-ko. Memoirs of the College of Science, Kyoto University (Ser. B), 28, 347-354.

Tortonese, E. (1942) Descrizione di una nuova specie ecuadoriana del genere Pygidium (Teleostei: Nematognathi). Bollettino dei Musei di Zoologia ed Anatomia Comparata della Università di Torino (Ser. 4), 49, 1-3, pl. 1.

Toula, F. (1889) Untersuchungen im Centralen Balkan. Denkschriften der Kaiserlichen Akademie der Wissenschaften, Mathematisch-Naturwissenschaftliche Classe, Wien, 55, 1-108, pls. 1-9, + map.

Traill, T.S. (1832) Description of a Silurus, known in Demerara by the name of Gilbacke, more properly Geelbuik. Memoirs of the Wernerian Natural History Society, Edinburgh, 6, 377-380, pl. 6.

Trajano, E. \& Britski, H.A. (1992) Pimelodella kronei (Ribeiro, 1907) e seu sinônimo Caecorhamdella brasiliensis Borodin, 1927: Morfologia externa, taxonomia e evolução (Teleostomi, Siluriformes). Boletim de Zoologia, Universidade de São Paulo, 12, 53-89.

Trajano, E. \& Pinna, M.C.C., de. (1996) A new cave species of Trichomycterus from eastern Brazil (Siluriformes, Trichomycteridae). Revue Française d'Aquariologie et Herpetologie, 23, 85-90.

Trajano, E., Reis, R.E. \& Bichuette, M.E. (2004) Pimelodella spelaea: a new cave catfish from central Brazil, with data on ecology and evolutionary considerations (Siluriformes: Heptapteridae). Copeia, 2004, 315-325.

Trewavas, E. (1936) Dr. Karl Jordan's expedition to South-West Africa and Angola: The fresh-water fishes. Novitates Zoologicae (Tring), 40, 63-74, pls. 1-2.

Trewavas, E. (1943) New schilbeid fishes from the Gold Coast, with a synopsis of the African genera. Proceedings of the Zoological Society of London (Series B), 113, 164-171.

Trewavas, E. (1962) Fishes of the crater lakes of the northwestern Cameroons. Bonner Zoologische Beiträge, 13, 146192.

Trewavas, E. (1964) A new species of Irvineia, an African genus of schilbeid fishes. Annali del Museo Civico de Storia Naturale di Genova 'Giacomo Doria', 74, 388-396.

Trewavas, E. (1974) The freshwater fishes of rivers Mungo and Meme and Lakes Kotto, Mboandong and Soden, west Cameroon. Bulletin of the British Museum (Natural History), Zoology, 26, 331-419, pls. 1-5.

Triques, M.L. \& Vono, V. (2004) Three new species of Trichomycterus (Teleostei: Siluriformes: Trichomycteridae) from the Rio Jequitinhonha basin, Minas Gerais, Brazil. Ichthyological Exploration of Freshwaters, 15, 161-172.

Tschudi, J.J.von. (1846) Ichthyologie. In: Untersuchungen über die Fauna Peruana, Scheitlin \& Zollikofer, St. Gallen, pp. ii-Xxx + 1-35, pls. 1-6. [In 12 parts, 1844-46]

Tuckey, J.K. (1818) A general notice of the animals taken by Mr. John Cranch, during the expedition to explore the source of the River Zaire. Appendix 4. In: Narrative of an Expedition to Explore the River Zaire, Usually Called the Congo, in South Africa, in 1816, John Murray, London, pp. 407-419.

Turner, B.J., Diffoot, N. \& Rasch, E.M. (1992) The callichthyid catfish Corydoras aeneus is an unresolved diploid-tetraploid sibling species complex. Ichthyological Exploration of Freshwaters, 3, 17-23.

Turner, W. (1867) On a remarkable mode of gestation in an undescribed species of Arius (A. boakeii). Journal of Anatomy and Physiology, 1, 78-82.

Ünlü, E. \& Bozkurt, R. (1996) Notes on the catfish, Silurus triostegus (Siluridae) from the Euphrates River in Turkey. Cybium (3e série), 20, 315-317.

Unmack, P.J. (2001) Corrected identifications and lectotype designation for Porochilus argenteus (Zietz) (Osteichthyes: Siluriformes: Plotosidae). Records of the South Australian Museum, 34, 57-59.

Vaillant, L.L. (1880a) Note sur le genre Otocinclus et description d'une espèce nouvelle. Bulletin de la Société Philomathique (Ser. 7), 4, 145-148.

Vaillant, L.L. (1880b) Synopsis des espèces de Siluridae recueillies par M. le Dr. Jobert, à Caldéron (Haute-Amazone). Bulletin de la Société Philomathique (Ser. 7), 4, 150-159.

Vaillant, L.L. (1891) Note sur un nouveau genre de Siluroïdes (Diastatomycter) de Bornéo. Bulletin de la Société Philomathique (Ser. 8), 3, 181-182.

Vaillant, L.L. (1892a) Sur quelques poissons rapportés du haut-Tonkin, par M. Pavie. Bulletin de la Société Philomathique (Ser. 8), 4, 125-127. 
Vaillant, L.L. (1892b) [Poissons de l'Oubanghi rapportés par M. Dybowski]. Bulletin de la Société Philomathique (Ser. 8), $4,2$.

Vaillant, L.L. (1893a) Notes ichthyologiques. Bulletin de la Société Philomathique (Ser. 8), 5, 13-17.

Vaillant, L.L. (1893b) Sur les poissons provenant du voyage de M. Bonvalot et du Prince Henri d'Orléans. Bulletin de la Société Philomathique (Ser. 8), 5, 197-204.

Vaillant, L.L. (1893c) Sur une collection de poissons recueillie par M. Chaper, a Bornéo. Bulletin de la Société Zoologique de France, 18, 55-62.

Vaillant, L.L. (1894a) Note sur les poissons de la famille des Siluridées appartement à la Faune Madécasse et description d'une espèce nouvelle. Bulletin de la Société Philomathique (Ser. 8), 6, 75-80.

Vaillant, L.L. (1894b) Contribution à l'étude de la faune ichthyologique de Bornéo. Nouvelles Archives du Muséum d'Histoire Naturelle, Paris (Ser. 3), 5, 23-114, pls. 1-2.

Vaillant, L.L. (1895a) Sur la dentition des Synodontis et diagnose d'espèces nouvelles. Comptes Rendus de la Société Philomathique de Paris, 16, 47-48.

Vaillant, L.L. (1895b) Essai monographique sur les silures du genre Synodontis. Nouvelles Archives du Muséum d'Histoire Naturelle, Paris (Ser. 3), 7, 223-284, pls. 9-14.

Vaillant, L.L. (1896a) Note sur l'oeuvre ichthyologique de C. A. Lesueur. Bulletin de la Société Philomathique (Ser. 8), 8, 15-32, pls. 1-35.

Vaillant, L.L. (1896b) Essai monographique sur les silures du genre Synodontis, suite et fin. Nouvelles Archives du Muséum d'Histoire Naturelle, Paris (Ser. 3), 8, 87-178.

Vaillant, L.L. (1897) Siluroïde nouveau de l'Afrique orientale (Chimarrhoglanis leroyi). Bulletin du Muséum d'Histoire Naturelle, Paris, 3, 81-84.

Vaillant, L.L. (1899) Note préliminaire sur les collections ichthyologiques recueillies par M. Geay en 1897 et 1898 dans la Guyane française et le Contesté franco-brésilien. Bulletin du Muséum d'Histoire Naturelle, Paris, 5, 154-156.

Vaillant, L.L. (1900) Contribution à l'étude de la faune ichthyologique de la Guyane française et du contesté Franco-Brésilien. Nouvelles Archives du Muséum d'Histoire Naturelle, Paris (Ser. 4), 4, 126-136.

Vaillant, L.L. (1902) Résultats zoologiques de l'expédition scientifique Néerlandaise au Bornéo central. Poissons. Notes from the Leyden Museum, 24, 1-166, pls. 1-2.

Vaillant, L.L. (1904) Poissons Recueillis par M.A. Pavie en Indo-Chine. In: Pavie, A. (Ed.), Mission Pavie Indo-Chine, 1879-1895; Vol. 3, Recherches sur l'Histoire Naturelle de l'Indo-Chine Orientale, E. Leroux, Paris, pp. 459-470.

Valenciennes, A. (1832a) Nouvelles observations sur le capitan de Bogota, Eremophilus mutisii. In: Voyage de Humboldt et Bonpland, Deuxième Partie: Observations de Zoologie et d'Anatomie Comparée, Paris, pp. 341-348.

Valenciennes, A. (1832b) Poissons. In: Voyage aux Indes-Orientales, Pendant les Années 1825-1829, Charles Bélanger, Paris, pp. 339-399, poissons pls. 1-4.

Valenciennes, A. (1835-1847) Poissons; Catalogue des principales espèces de poissons, rapportées de l'Amérique méridionale. In: d'Orbigny, A. (Ed.), Voyage dans L’Amérique Méridionale (le Brésil, la République Orientale de l'Uruguay, la République Argentine, la Patagonie, la République du Chili, la République de Bolivia, la République du Pérou), Exécuté Pendant les Années 1826, 1827, 1828, 1829, 1830, 1832 et 1833. Vol. 5 (pt. 2), Bertrand et Levrault, Paris. [16 plates in poissons series issued between 1835 and 1842, plates 1-3 issued in 1835; 4-7 issued in 1836; text issued in 1847.]

Van der Hoeven, J. (1849) Handboek der Dierkunde; Tweede Verbeterde Uitgave; met Bijvoegsels en Aanmerkingen door Leuckart, Edition 2, Amsterdam.

Van der Horst, C.J. (1931) Some South African siluroid fishes. Annals of the Transvaal Museum, 14, $246-250$.

Van der Stigchel, J.W.R. (1946) The South American Nematognathi of the Museums at Leiden and Amsterdam, E.J. Brill, Leiden, 204 p., 3 tabs. [Also published in 1947, with the same title and pagination, in Zoologische Mededelingen (Leiden), 27:1-204.]

Van der Stigchel, J.W.R. (1964) A new species of pimelodid catfish from eastern Brazil, Pimelodella boschmai nov. spec. Zoologische Mededelingen (Leiden), 39, 327-330.

Van Neer, W. (1994) Cenozoic fish fossils from the Albertine Rift Valley in Uganda. In: Senut, B. \& Pickford, M. (Eds.), Geology and Palaeobioiology of the Albertine Rift Valley, Uganda-Zaire; vol. 2, Palaeobiology-paléobiologie, Occasional Publication, International Center for Training and Exchanges in the Geosciences, Orléans, France, pp. 89-128.

Vanni, S. (1991) Cataloghi del Museo Zoologico "La Specola" dell'Università di Firenze. VIII._- Osteichthyes: Tipi. Atti della Società Toscana di Scienze Naturali, Pisa (Serie B), 96, 219-229.

Vari, R.P. \& Ferraris, C.J., Jr. (1998) The neotropical catfish genus Epapterus Cope (Siluriformes: Auchenipteridae): a 
reappraisal. Proceedings of the Biological Society of Washington, 111, 992-1007.

Vari, R.P. \& Ferraris, C.J., Jr. (2003) Cetopsidae. In: Reis, R.E., Kullander, S.O. \& Ferraris, C.J., Jr. (Eds.), Check list of the Freshwater Fishes of South and Central America, Edipucrs, Porto Alegre, Brazil, pp. 257-260.

Vari, R.P., Ferraris, C.J., Jr. \& Keith, P. (2003) A new Pseudocetopsis species (Siluriformes: Cetopsidae) from Suriname and French Guiana. Proceedings of the Biological Society of Washington, 116, 692-698.

Vari, R.P., Ferraris, C.J., Jr. \& Pinna, M.C.C., de. (2005) The Neotropical whale catfishes (Siluriformes: Cetopsidae: Cetopsinae), a revisionary study. Neotropical Ichthyology, 3, 127-238.

Vari, R.P., Jewett, S.L., Taphorn, D.C. \& Gilbert, C.R. (1984) A new catfish of the genus Epapterus (Siluriformes: Auchenipteridae) from the Orinoco River basin. Proceedings of the Biological Society of Washington, 97, 462-472.

Vari, R.P. \& Ortega, H. (1986) The catfishes of the Neotropical family Helogenidae (Ostariophysi: Siluroidei). Smithsonian Contributions to Zoology, 442, i-iii + 1-20.

Vélez-Espino, L.A. (2004) Taxonomic revision, ecology, and endangerment categorization of the Andean catfish Astroblepus ubidiai (Teleostei: Astroblepidae). Reviews in Fish Biology and Fisheries, 13 (for 2003), 367-378.

Vidal, E.S. \& de Lucena, C.A.S. (1999) Pimelodus atrobrunneus, um nova espéce de pimelodídeo do rio Uruguai superior (Teleostei, Siluriformes, Pimelodidae). Biociências, Porto Alegre, 7, 121-134.

Vidthayanon, C. \& Ng, H.H. (2003) Acrochordonichthys gyrinus, a new species of akysid catfish (Teleostei: Siluriformes) from Thailand. Zootaxa, 183, 1-7.

Vidthayanon, C. \& Roongthongbaisuree, S. (1993) [Taxonomy of Thai Riverine Catfishes Family Schilbeidae and Pangasiidae], National Inland Fisheries Institute. Department of Fisheries, Bangkok, Technical Paper, 150, 1-57. [In Thai with English summary]

Villa, J. (1977) A new species of pimelodid catfish of the genus Rhamdia from Nicaragua, Central America. Brenesia, 133-142.

Vinciguerra, D. (1881) Appunti ittiologici sulle collezioni del Museo Civico di Genova. IV.- Prima contribuzione alla Fauna Ittiologica dell' isola di Borneo. Siluroidei raccolti durante il viaggio dei Signori Mse G. Doria e Dr. O. Beccari. Annali del Museo Civico de Storia Naturale di Genova, 16, 161-182.

Vinciguerra, D. (1890) Viaggio di Leonardo Fea in Birmania e regioni vicine. XXIV.— Pesci. Annali del Museo Civico de Storia Naturale di Genova (Ser. 2a), 9, 129-362, pls. 7-11.

Vinciguerra, D. (1893) Di alcuni pesci raccolti nel paese dei Somali dall' Ing,r. L. Brichetti-Robecchi. Annali del Museo Civico de Storia Naturale di Genova (Ser. 2a), 13, 448-455.

Vinciguerra, D. (1895) Esplorazione del Giuba e dei suoi affluenti compiuta dal Cap. V. Bottego durante gli anni 189293 sotto gli auspicii della Società geografica Italiana. III.- Pesci. Annali del Museo Civico de Storia Naturale di Genova (Ser. 2a), 15, 21-60, pl. 5.

Vinciguerra, D. (1897) Pesci raccolti dal Cap. V. Bottego durante la sua seconda spedizione nelle regioni dei Somali e dei Galla. Annali del Museo Civico de Storia Naturale di Genova (Ser. 2a), 17, 343-364.

Vinciguerra, D. (1898) I pesci dell'ultima spedizione del Cap. Bottego. Annali del Museo Civico de Storia Naturale di Genova (Ser. 2a), 19, 240-261.

Vinciguerra, D. (1928) Pesci raccolti dal March. ${ }^{\text {se }}$ Saverio Patrizi nel bacino del Congo. Annali del Museo Civico de Storia Naturale di Genova 'Giacomo Doria', 53, for 1928-29, 5-29, pl. 1.

Vishwanath, W. \& Darshan, A. (2005) A new catfish species of the genus Sisor Hamilton (Teleostei: Siluriformes) from Manipur, India. Zoos'Print Journal, 20, 1952-1954.

Vishwanath, W. \& Kosygin, L. (1999) A new sisorid catfish of the genus Myersglanis Hora \& Silas, 1951, from Manipur, India. Journal of the Bombay Natural History Society, 96, 291-296, pl. 1.

Vishwanath, W. \& Kosygin, L. (2000) On a new species of the genus Hara Blyth from Manipur, India. Indian Journal of Fisheries, 47, 143-147.

Vishwanath, W. \& Linthoingambi, I. (2005) A new sisorid catfish of the genus Glyptothorax Blyth from Manipur, India. Journal of the Bombay Natural History Society, 102, 201-203.

Voigt, E. (1926) Über ein bemerkenswertes Vorkommen neuer Fischotholithen in einem Senongeschiebe von Cöthen in Anhalt. Zeitschrift für Geschiebeforschung, 2, 172-187, pl. 2.

Volz, W. (1903a) Neue Fische aus Sumatra. Zoologischer Anzeiger, 26, 553-559.

Volz, W. (1903b) Fische von Sumatra. Zoologische Jahrbücher. Abteilung für Systematik, Geographie und Biologie der Tiere (Jena), 19, 347-419, pls. 25-26.

Volz, W. (1904) Fische von Sumatra, gesammelt von Herrn G. Schneider. Revue suisse de Zoologie, Annales de la Société zoologique suisse et du Muséum d'Histoire naturelle de Genève, 12, 451-493.

Walbaum, J.J. (1792) Petri Artedi Sueci Genera Piscium. In Quibus Systema Totum Ichthyologiae Proponitur cum Clas- 
sibus, Ordinibus, Generum Characteribus, Specierum Differentiis, Observationibus Plurimis. Redactis Speciebus 242 ad Genera 52. Ichthyologiae, pars iii, Grypeswaldiae, impensis A. F. Röse, 723 p., 3 pl.

Walschaerts, L. (1987) Catalogue des types de poissons recents de l'Institut Royal des Sciences naturelles de Belgique. Documents de Travail, 40, 1-67.

Walsh, S.J. (1990) A Systematic Revision of the Neotropical Catfish Family Ageneiosidae (Teleostei: Ostariophysi: Siluriformes), Doctoral dissertation, University of Florida, Gainesville.

Walsh, S.J., Chapman, L.J., Rosenberger, A.E. \& Chapman, C.A. (2000) Redescription of Amphilius jacksonii (Siluriformes: Amphiliidae) with habitat and life-history notes. Ichthyological Exploration of Freshwaters, 11, $163-174$.

Walsh, S.J. \& Gilbert, C.R. (1995) New species of troglobitic catfish of the genus Prietella (Siluriformes: Ictaluridae) from northeastern México. Copeia, 1995, 850-861.

Wandolleck, B. (1916) Arges stübeli n. sp. Zoologischer Anzeiger, 47, 158-162.

Wang, D., Zhao, Y.-H. \& Zhang, C.-G. (2005) Revision of giant sea-catfish Arius thalassinus in China and a new Chinese record of the species A. bilineatus (Siluriformes: Ariidae). Acta Zoologica Sinica, 51, 423-430.

Wang, D., Zhao, Y.-H. \& Zhang, C.-G. (2005) Revision of Arius arius (formerly Arius sinensis) in China and its sexual differences (Siluriformes: Ariidae). Acta Zoologica Sinica, 51, 431-439. [In Chinese, with English abstract]

Wang, J., Li, G. \& Wang, J. (1981) [The Early Tertiary fossil fishes from Sanshui and its adjacent basin, Guangdong]. Zhongguo Gushengwu Zhi Xin Bing Zhong [= Palaeontologia Sinica], Series C, 22, 1-90. [In Chinese.]

Watanabe, K. (1995) Pseudobagrus pratti (Günther, 1892), a senior synonym of P. emarginatus (Regan, 1913) (Siluriformes: Bagridae). Japanese Journal of Ichthyology, 42, 321-324.

Watanabe, K. (1998) Meristic variation in the endangered bagrid catfish, Pseudobagrus ichikawai. Ichthyological Research, 45, 99-104.

Watanabe, K. \& Ito, S. (1999) Population size and distribution of Pseudobagrus ichikawai, an endangered bagrid catfish in the Kawaura River. Japanese Journal of Ichthyology, 46, 15-30.

Watanabe, K. \& Maeda, H. (1995) Redescription of two ambiguous Japanese bagrids, Pseudobagrus aurantiacus (Temminck and Schlegel) and P. tokiensis Döderlein. Japanese Journal of Ichthyology, 41, 409-420.

Watanabe, K., Mori, S., Nagoshi, M., Jeon, S.-R. \& Shimizu, Y. (1992) Morphological differences between two bagrid catfishes, Coreobagrus ichikawai and C. brevicorpus. Japanese Journal of Ichthyology, 39, 157-162.

Watanabe, K. \& Okuyama, S. (1994) [Fossil remains of a bagrid catfish from the Lower Pliocene Ueno Formation of the Kobiwako Group at Ohyamada-mura, Mie Prefecture, Japan]. Bulletin of the Mizunami Fossil Museum, 21, 57-61, pl. 5. [In Japanese, with English abstract.]

Watanabe, K. \& Uyeno, T. (1999) Fossil bagrid catfishes from Japan and their zoogeography, with description of a new species, Pseudobagrus ikiensis. Ichthyological Research, 46, 397-412.

Watanabe, K., Zhang, C.-G. \& Zhao, Y.-H. (2002) Redescription of the East Asian bagrid catfish Pseudobagrus kyphus Mai, 1978, with a new record from China. Ichthyological Research, 49, 384-388.

Weber, A., Allegrucci, G. \& Sbordoni, V. (2003) Rhamdia laluchensis, a new species of troglobitic catfish (Siluriformes: Pimelodidae) from Chiapas, Mexico. Ichthyological Exploration of Freshwaters, 14, 273-280.

Weber, A. \& Wilkens, H. (1998) Rhamdia macuspanensis: a new species of troglobitic pimelodid catfish (Siluriformes; Pimelodidae) from a cave in Tabasco, Mexico. Copeia, 1998, 998-1004.

Weber, C. (1985) Hypostomus dlouhyi nouvelle espèce de poisson-chat cuirassé du Paraguay (Pisces, Siluriformes, Loricariidae). Revue suisse de Zoologie, Annales de la Société zoologique suisse et du Muséum d'Histoire naturelle de Genève, 92, 955-968.

Weber, C. (1986) Révision de Hypostomus boulengeri (Eigenmann \& Kennedy), et deux espèces nouvelles de poissonschats du Paraguay (Pisces, Siluriformes, Loricariidae). Revue suisse de Zoologie, Annales de la Société zoologique suisse et du Muséum d'Histoire naturelle de Genève, 93, 979-1007.

Weber, C. (1987) Hypostomus microstomus sp. nov. et autres poissons-chats cuirassés du Rio Parana (Pisces, Siluriformes, Loricariidae). Archives des Sciences (Geneva), 40, 273-284.

Weber, C. (1991) Nouveaux taxa dans Pterygoplichthys sensu lato (Pisces, Siluriformes, Loricariidae). Revue suisse de Zoologie, Annales de la Société zoologique suisse et du Muséum d'Histoire naturelle de Genève, 98, 637-643.

Weber, C. (1992) Révision du genre Pterygoplichthys sensu lato (Pisces, Siluriformes, Loricariidae). Revue Française d'Aquariologie et Herpetologie, 19, 1-36.

Weber, C. (2003) Hypostominae. In: Reis, R.E., Kullander, S.O. \& Ferraris, C.J., Jr. (Eds.), Check list of the Freshwater Fishes of South and Central America, Edipucrs, Porto Alegre, Brazil, pp. 351-372.

Weber, C. \& Montoya-Burgos, J.-I. (2002) Hypostomus fonchii n. sp. (Siluriformes: Loricariidae) from Peru, a key species suggesting the synonymy of Cochliodon with Hypostomus. Revue suisse de Zoologie, Annales de la Société 
zoologique suisse et du Muséum d'Histoire naturelle de Genève, 109, 355-368.

Weber, C., Muller, S. \& Mahnert, V. (1992) Harnischwelse Paraguays. In: Stawikowski, R. (Ed.), Harnischwelse. Die Aquarien- und Terrarien- Zeitschrift Sonderheft. Verlag Eugen Ulmer, Stuttgart, pp. 10-13.

Weber, M. (1897) Beiträge zur Kenntniss der Fauna von Süd-Afrika. I.—Zur Kenntniss der Süsswasser-Fauna von SüdAfrika. Zoologische Jahrbücher. Abteilung für Systematik, Geographie und Biologie der Tiere, Suppl. (Jena), 10, 135-199.

Weber, M. (1907) Süsswasserfische von Neu-Guinea ein Beitrag zur Frage nach dem früheren Zusammenhang von NeuGuinea und Australien. In: Nova Guinea. Résultats de l'expédition scientifique Néerlandaise à la Nouvelle-Guinée Vol. 5 (Zool.) pt 2, E. J. Brill, Leiden, pp. 201-267, pls. 11-13.

Weber, M. (1910) Neue Fische aus Niederländisch Süd-Neu-Guinea. Notes from the Leyden Museum, 32, 225-240, pl. 3.

Weber, M. (1913) Süsswasserfische aus Niederländisch Süd- und Nord-Neu-Guinea. In: Nova Guinea. Résultats de l'expédition scientifique Néerlandaise à la Nouvelle-Guinée, 9, Zoologie, livr. 4, E. J. Brill, Leiden, pp. 513-613, pls. 12-14.

Weber, M. \& Beaufort, L.F. de. (1912) Der Fische. In: Maass, A. (Ed.), Durch Zentral-Sumatra, Vol. 2, W. Süsserott, Berlin, pp. 522-541, pls. 11-12.

Weber, M. \& Beaufort, L.F. de. (1913) The Fishes of the Indo-Australian Archipelago. II. Malacopterygii, Myctophoidea, Ostariophysi: I Siluroidea, E. J. Brill, Leiden, xx + 404 p.

Weber, S.I.F. (1999) Étude Comparative de Deux Genres de Pimelodidae (Actionopterygii, Siluriformes) du Paraguay: Phenacorhamdia Dahl et Imparfinis Eigenmann \& Norris, Graduate thesis, Museum d'histoire naturelle de la Ville de Genève, Genève.

Weiler, W. (1935) Ergebnisse der Forschungsreisen Prof. E. Stromers in den Wüsten Ägyptens. II.- Wirbeltierreste der Baharîje-Stufe (unterstes Cenoman). 16. Neue Untersuchungen an den Fischresten. Abhandlungen der Bayerischen Akademie der Wissenschaften, Mathematisch-Naturwissenschaftliche Abteilung, (n. f.), 32, 1-57, 3 pls.

Weiler, W. (1956) Über eine neue Gattung der Welse (Fam. Siluridae) aus dem Pliozän von Wellershausen. Paläontologische Zeitschrift, 30, 180-189.

Weiler, W. (1968) Otolithi Piscium (Neubearbeitung). In: Westphal, F. (Ed.), Fossilium Catalogus, Vol. 1.—Animalia; Pars 117, Dr. W. Junk, N.V., Gravenhage.

Weitzman, S.H. (1956) A description, supplementary notes and a figure of Corydoras cochui Myers and Weitzman, a Brazilian catfish. Stanford Ichthyological Bulletin, 7, 14-18.

Weitzman, S.H. (1960a) Figures and description of four South American catfishes of the genus Corydoras, including two new species. Stanford Ichthyological Bulletin, 7, 140-154.

Weitzman, S.H. (1960b) Figures and description of a South American catfish, Corydoras reticulatus Fraser-Brunner. Stanford Ichthyological Bulletin, 7, 155-161.

Weitzman, S.H. (1961) A new catfish, Corydoras concolor (Callichthyidae) from Venezuela. Proceedings of the Biological Society of Washington, 74, 105-110.

Weitzman, S.H. (1963) A new catfish, Corydoras pastazensis (Callichthyidae) from Ecuador. Proceedings of the Biological Society of Washington, 76, 59-64.

Weitzman, S.H. (1964) One new species and two redescriptions of catfishes of the South American callichthyid genus Corydoras. Proceedings of the United States National Museum, 116, 115-126.

Weitzman, S.H. \& Nijssen, H. (1970) Four new species and one new subspecies of the catfish genus Corydoras from Ecuador, Colombia and Brazil (Pisces, Siluriformes, Callichthyidae). Beaufortia, 18, 119-133.

Weitzman, S.H. \& Vari, R.P. (1988) Miniaturization in South American freshwater fishes; an overview and discussion. Proceedings of the Biological Society of Washington, 101, 444-465.

Werneke, D.C., Armbruster, J.W., Lujan, N.K. \& Taphorn, D.C. (2005) Hemiancistrus guahiborum, a new suckermouth armored catfish from Southern Venezuela (Siluriformes: Loricariidae). Neotropical Ichthyology, 3, 543-548.

Werneke, D.C., Sabaj, M.H., Lujan, N.K. \& Armbruster, J.W. (2005) Baryancistrus demantoides and Hemiancistrus subviridis, two new uniquely colored species of loricariids from Venezuela (Siluriformes: Loricariidae). Neotropical Ichthyology, 3, 533-542.

Werner, F. (1906) Ergebnisse der mit Subvention aus der Erbschaft Treitl unternommenen zoologischen Forschungsreise Dr. Franz Werner's in den ägyptischen Sudan und nach Nord-Uganda. V.- Beiträge zur kenntnis der Fischfauna des Nils. Anzeiger der Kaiserlichen Akademie der Wissenschaften, Mathematisch- Naturwissenschaftlichen Classe, 43, 325-327, pls. 1-4.

Weyenbergh, H. (1877) Algunos nuevos pescados del Museo Nacional, y algunas noticias ictiológicas. Actas de la Academia Nacional de Ciencias Exactas, Buenos Aires, 3, 1-21, pls. 1-4. 
Wheeler, A. (1985) The Linnaean fish collection in the Linnean Society of London. Zoological Journal of the Linnean Society, 84, 1-76.

Wheeler, A. \& Baddokwaya, A. (1981) The generic nomenclature of the marine catfishes usually referred to the genus Arius (Osteichthyes - Siluriformes). Journal of Natural History, 15, 769-773.

White, E.I. (1926) Eocene fishes from Nigeria. Bulletin, Geological Survey of Nigeria, 10, 1-82, pls. 1-18.

White, E.I. (1934) Fossil fishes of Sokoto Province. Bulletin, Geological Survey of Nigeria, 14, 1-78, 15 figs., 10 pls.

White, E.I. (1937) The name of a fossil cat-fish. The Geological Magazine, 74, 144.

White, E.I. \& Moy-Thomas, J.A. (1940) Notes on the nomenclature of fossil fishes. Part I.- Homonyms A-C. Annals and Magazine of Natural History (Ser. 11), 5, 502-507.

White, E.I. \& Moy-Thomas, J.A. (1941) Notes on the nomenclature of fossil fishes. Part III.- Homonyms M-Z. Annals and Magazine of Natural History (Ser. 11), 7, 395-400.

Whitehead, P.J.P. (1958) A new species of Chiloglanis (Pisces, Mochocidae) in Kenya. Annals and Magazine of Natural History (Ser. 13), 1, 197-208.

Whitehead, P.J.P. (1962a) Two new river fishes from eastern Kenya. Annali del Museo Civico de Storia Naturale di Genova 'Giacomo Doria', 73, 98-108.

Whitehead, P.J.P. (1962b) A new species of Synodontis (Pisces: Mochocidae) and notes on a mormyrid fish from the eastern rivers of Kenya. Revue de Zoologie et de Botanique Africaines, 65, 97-120.

Whitehead, P.J.P. (1970) The Reeves Collection of Chinese fish drawings. Bulletin of the British Museum (Natural History), Historical Series, 3, for 1969, 191-233, pls. 1-29.

Whitley, G.P. (1928) Studies in ichthyology, no. 2. Records of the Australian Museum, 16, 211-239, pls. 16-18.

Whitley, G.P. (1933) Studies in ichthyology, no. 7. Records of the Australian Museum, 19, 60-112, pls. 11-15.

Whitley, G.P. (1935) Studies in ichthyology, no. 9. Records of the Australian Museum, 19, 215-250, pl. 18.

Whitley, G.P. (1938) Descriptions of some New Guinea fishes. Records of the Australian Museum, 20, $223-233$.

Whitley, G.P. (1939) Ichthyological genotypes: Desmarest's designations, 1874. Australian Zoologist, 9, $222-226$.

Whitley, G.P. (1940a) The Nomenclator Zoologicus and some new fish names. Australian Naturalist, 10, $241-243$.

Whitley, G.P. (1940b) Illustrations of some Australian fishes. Australian Zoologist, 9, 397-428, pls. 30-31.

Whitley, G.P. (1941a) The catfish and its kittens. Australian Museum Magazine, 7, 306-313.

Whitley, G.P. (1941b) Ichthyological notes and illustrations. Australian Zoologist, 10, 1-50, pls. 1-2.

Whitley, G.P. (1944) New sharks and fishes from Western Australia. Australian Zoologist, 10, 252-273.

Whitley, G.P. (1947) New sharks and fishes from Western Australia._- Part 3. Australian Zoologist, 11, 129-150, pl. 11.

Whitley, G.P. (1956a) List of the native freshwater fishes of Australia. Proceedings of the Royal Zoological Society of New South Wales, 1954-55, 39-47.

Whitley, G.P. (1956b) A new catfish from New Guinea. Proceedings of the Royal Zoological Society of New South Wales, 1954-55, 68.

Wilkens, H. (1993) A new species of Rhamdia (Pisces: Pimelodidae) from a cave in the Sierra de Zongolica (Veracruz, Mexico). Mitteilungen aus dem Hamburgischen Zoologischen Museum und Institut, 90, 375-378.

Willoughby, N.G. (1994) The taxonomy of the genus Synodontis (Pisces: Siluroidea) in Lake Kainji, Nigeria. The African Journal of Tropical Hydrobiology and Fisheries, 5, 25-30.

Woodward, A.S. (1887) On some remains of siluroid fishes from British Eocene formations. The Geological Magazine, new series, decade 3, 4, 303-307.

Woodward, A.S. (1889a) Catalog of the Fossil Fishes in the British Museum Natural History, Vol. 1, Trustees, London, xlvii + 474 p., 17 pl.

Woodward, A.S. (1889b) Note on Bucklandium diluvii König, a siluroid fish from the London Clay of Sheppey. Proceedings of the Zoological Society of London, 1889, 208-210, pl. 22.

Woodward, A.S. (1899) Considerações sobre alguns peixes Terciarios dos schistos de Taubaté, Estado de S. Paulo, Brazil. Revista do Museu Paulista, 3, for 1898, 63-70, pls. 2-4.

Woodward, A.S. (1901) Catalogue of the Fossil Fishes in the British Museum. Part IV.-Containing the Actinopterygian Teleostomi of the Suborders Isospondyli (in part), Ostariophysi, Apodes, Percesoces, Hemibranchii, Acanthopterygii, and Anacanthini, Trustees, London, xxxviii + 636 p., 19 pls., 22 tables.

Worthington, E.B. (1933a) The fishes (other than Cichlidae) of Lake Bangweulu and adjoining regions, including descriptions of three new species. Annals and Magazine of Natural History (Ser. 10), 12, 34-52.

Worthington, E.B. (1933b) The fishes of Lake Nyasa (other than Cichlidae). Proceedings of the Zoological Society of London, 1933, 285-316.

Worthington, E.B. \& Ricardo, C.K. (1937) The fish of Lake Tanganyika (other than Cichlidae). Proceedings of the Zoo- 
logical Society of London, 1936, 1061-1112.

Wosiacki, W.B. (2004) New species of the catfish genus Trichomycterus (Siluriformes: Trichomycteridae) from the headwaters of the rio São Francisco basin, Brazil. Zootaxa, 592, 1-12.

Wosiacki, W.B. (2005) A new species of Trichomycterus (Siluriformes: Trichomycteridae) from south Brazil and redescription of T. iheringi (Eigenmann). Zootaxa, 1040, 49-64.

Wosiacki, W.B. \& Garavello, J.C. (2004) Five new species of Trichomycterus from the rio Iguaçu (rio Paraná Basin), southern Brazil (Siluriformes: Trichomycteridae). Ichthyological Exploration of Freshwaters, 15, 1-16.

Wosiacki, W.B. \& Oyakawa, O.T. (2005) Two new species of catfish genus Trichomycterus (Siluriformes — Trichomycteridae) from the rio Ribeira de Iguape Basin, Southeastern Brazil. Neotropical Ichthyology, 3, 465-472.

Wu, H.-W. (1930a) Description de poissons nouveaux de Chine. Bulletin du Muséum National d'Histoire Naturelle (2e Série), 2, 255-259.

Wu, H.-W. (1930b) Notes on some fishes collected by the Biological Laboratory Science Society of China. Contributions from the Biological Laboratory of the Science Society of China, Zoological Series, 6, 45-57.

Wu, H.-W. (1930c) On some fishes collected from the upper Yangtse Valley. Sinensia, 1, 65-86.

Wu, H.-W. (1939) On the fishes of Li-Kiang. Sinensia, 10, 92-142, pls. 1-3.

Wu, X.-W., He, M.J. \& Chu, S.-L. (1981) [On the fishes of Sisoridae from the region of Xizang]. Oceanologia et Limnologia Sinica, 12, 74-79. [In Chinese.]

Wu, Y.-F. \& Chen, Y. (1979) [Notes on fishes from Golog and Yushu region of Qinghai Province, China.]. Acta Zootaxonomica Sinica, 4, 287-296. [In Chinese.]

Wulff, J.C. (1765) Ichthyologia, cum Amphibiis Regni Borsussici. Methodo Linnaeana Disposita. Regiomonti, xii + 60 p.

Xie, Z., Zhang, E. \& He, S.-P. (2001) Study on species validation for Glytothorax sinense [sic] (Regan) and G. fukiensis [sic] (Rendahl) with the method of morphometrics. Journal of Huazhong Agricultural University, 20, 169-172. [In Chinese, with English abstract]

Yerger, R.W. \& Relyea, K. (1968) The flat-headed bullheads (Pisces: Ictaluridae) of the southeastern United States, and a new species of Ictalurus from the Gulf Coast. Copeia, 1968, 361-384.

Young, T.S. \& Laerm, J. (1993) A Late Pleistocene vertebrate assemblage from the St. Marks River, Wakulla County, Florida. Brimleyana, 18, 15-57.

Zakaria-Ismail, M. (1992) Notes on the catfish Laides sinensis (Pisces: Pangasiidae) from Peninsular Malaysia. Malayan Nature Journal, 46, 111-114.

Zarske, A. (1998) Phenacorhamdia nigrolineata spec. nov., ein neuer Antennenwels aus dem Einzugsgebiet des Rio Ucayali in Peru (Teleostei: Siluriformes: Pimelodidae). Zoologische Abhandlungen; Staatliches Museums für Tierkunde in Dresden, 50, 27-31.

Zarske, A. (2003) Wiederbeschreibung von Rhamdia marthae (Sands \& Black, 1985) (Teleostei, Siluriformes, Pimelodidae). Redescription of Rhamdia marthae (Sands \& Black, 1985) (Teleostei, Siluriformes, Pimelodidae). Zoologische Abhandlungen; Staatliches Museums für Tierkunde in Dresden, 53, 47-55.

Zhang, Y. \& Wang, D. (1996) Studies on the taxonomical position of Pseudobagrus brevicaudatus (Bagridae, Siluriformes). Acta Hydrobiologica Sinica, 20, 379-382. [In Chinese.]

Zhao, Y., Lan, J. \& Zhang, C. (2004) A new species of amblycipitid catfish, Xiurenbagrus gigas (Teleostei: Siluriformes), from Guangxi, China. Ichthyological Research, 51, 228-232.

Zheng, C.-Y. (1979) [On a new catfish of the genus Leiocassis from Guangdong Province, China]. Acta Zootaxonomica Sinica, 4, 182-184. [In Chinese.]

Zheng, P.-S. (Ed.) (1981) [Freshwater Fishes of Guangxi Province], Guangxi People's Publishers, 257 p. [In Chinese.]

Zhou, W. \& Chu, X.-I. (1992) [A new species of Pseudecheneis with comments on osteological differentiations at species level (Siluriformes: Sisoridae)]. Acta Zootaxonomica Sinica, 17, 110-115. [In Chinese, with English abstract.]

Zhou, W. \& Zhou, Y.-W. (2005) Phylogeny of the genus Pseudecheneis (Sisoridae) with an explanation of its distribution pattern. Zoological Studies, 44, 417-433.

Zietz, A.H.C. (1896) Description of an additional new species of fish from the Finke and Barcoo rivers. In: Spencer, B. (Ed.), Report on the Work of the Horn Scientific Expedition to Central Australia; Part 2.-Zoology, Dulau and Co., London, and Melville, Mullen and Slade, Melbourne, Australia, pp. 410-411, pl. 16.

Zolezzi, G. (1939) Descrizione di tre nuovi pesci del Giuba raccolti dalla Missione Ittiologica in A.O.I. Bollettino di Pesca, Piscicoltura e Idrobiologia, 15, 168-173.

Zuanon, J.A.S., Rapp Py-Daniel, L.H. \& Jégu, M. (1993) Two new species of Aguarunichthys from the Amazon basin (Siluroidei: Pimelodidae). Ichthyological Exploration of Freshwaters, 4, 251-260. 


\section{INDEX}

Names in bold indicate valid taxa; page numbers in italics indicate primary entry for the name.

5-tentaculatus, Heterobranchus, 197

6-tentaculatus, Heterobranchus, 197, 200

7-radiatus, Silurus, 122

9-radiatus, Silurus, 201

11-radiatus, Silurus, 335

12-radiatus, Silurus, 177

16-radiatus, Silurus, 44

38-radiatus, Silurus, 70

\section{A}

aaldereni, Hemipimelodus, 32

abbreviatus, Bagrus, 95

abbreviatus, Clarias, 148

abbreviatus, Plotosus, 351

aboinensis, Clarias (Clarioides), 144

Abron, 342

absconditus, Pimelodus, 336

abuelo, Cheirocerus, 332

abuelo, Sovichthys, 332

aburrense, Chaetostoma, 227

aburrensis, Hypostomus, 227

Acanthicini, 217

Acanthicus, 217, 218

acanthicus, Rinelepis, 218

Acanthicus adonis, 218

Acanthicus canensis, 266

Acanthicus hystrix, 218

Acanthobunocephalus, 57

Acanthobunocephalus nicoi, 57

acanthochiroides, Batrochoglanis, 352

acanthochiroides, Pseudopimelodus, 352

acanthochirus, Pseudopimelodus, 353

Acanthocleithron, 303

Acanthocleithron chapini, 303

Acanthodemus, 278

Acanthodoras, 167

Acanthodoras cataphractus, 167

Acanthodoras depressus, 167

Acanthodoras spinosissimus, 168

acanthomias, Synodontis, 311

Acanthonotus, 356

Acanthonotus Hardwickii, 356

acanthoperca, Synodontis, 441

Acanthopoma, 403

Acanthopoma annectens, 403

Acanthopoma bondi, 413

accipenser, Loricaria, 270

Acentronichthys, 180

Acentronichthys leptos, 180

Acestra, 217, 237

Acestra acus, 237

Acestra amazonum, 237

Acestra gladiolus, 237
Acestra gladius, 239

Acestra Knerii, 238

Acestra oxyrrhyncha, 239

Acestra oxyrryncha, 239

acestrichthys, Farlowella, 238

Acestridiinae, 217

Acestridium, 217, 218

Acestridium colombiense, 218

Acestridium colombiensis, 218

Acestridium dichromum, 218

Acestridium discus, 218

Acestridium martini, 218

Acestrini, 217

acicularis, Sperata,106

Acipenser plecostomus, 258, 259

acipenserinus, Hemiodon, 247

acipenserinus, Hemiodontichthys, 247

acipenserinus, Leptodoras, 172

acipenserinus, Oxydoras, 172

acrensis, Corydoras, 112

acrocephalus, Arius, 47

Acrochordonichthyini, 12

Acrochordonichthys, 12

Acrochordonichthys Büttikoferi, 13

Acrochordonichthys chamaeleon, 12

Acrochordonichthys falcifer, 12

Acrochordonichthys guttatus, 12

Acrochordonichthys gyrinus, 12

Acrochordonichthys ischnosoma, 12

Acrochordonichthys mahakamensis, 12

Acrochordonichthys melanogaster, 13

Acrochordonichthys obscurus, 13

Acrochordonichthys pachyderma, 13

Acrochordonichthys platycephalus, 13

Acrochordonichthys rugosus, 13

Acrochordonichthys septentrionalis, 13

Acrochordonichthys strigosus, 13

Acrochordonichthys varius, 13

Acrochordonichthys zonatus, 13

aculeatus, Chaetostomus, 272

aculeatus, Leiocassis, 92

aculeatus, Plecostomus, 224

aculeatus, Pterygoplichthys, 272

acus, Acestra, 237

acus, Farlowella, 237

acus venezuelensis, Farlowella, 239

acuta, Loricaria, 269

acuticeps, Auchenoglanis, 154

acutirostris, Oxyropsis, 276

acutirostris, Arius, 31

acutirostris, Chrysichthys, 158

acutirostris, Neotropius, 359

acutirostris, Oxyropsis, 276 acutirostris, Pseudeutropius, 359

acutivelis, Arius, 155, 158

acutus, Arius, 51

acutus, Corydoras, 112

acutus, Loricariichthys, 269

Adansonii, Bagrus, 365

Adelopeltis, 107

Adelopeltis angusticeps, 107

Adelopeltis laticeps, 107

adiposalis, Pseudobagrus, 101

adolfoi, Corydoras, 112

adonis, Acanthicus, 218

adspersus, Callichthys, 127

aegyptiaca, Eopeyeria, 428

aegyptiacus, Ariopsis, 428

Aelurichthys, 37

Aelurichthys isthmensis, 38

Alurichthys longispinis, 38

Elurichthys nuchalis, 38

Aelurichthys panamensis, 37, 38

Aelurichthys pinnimaculatus, 38

Aelurichthys scutatus, 38

aeneum, Hoplosoma, 112

aeneus, Ariodes, 49

aeneus, Corydoras, 112

aeneus, Pimelodus, 215

aequalicuspis, Rineloricaria, 293

aequibarbis, Arius, 55

aequilabialis, Pangasius, 327

aequinoctiale, Chaetostoma, 227

aequinoctialis, Chaetostomus, 227

aequus, Otolithus (Arius), 435

affine, Platystoma, 330

affinis, Ailia, 356

affinis, Amblydoras, 168

affinis, Auchenipterus

(Pseudauchenipterus), 76

affinis, Bagrus, 107

affinis, Batasio, 85

affinis, Callichthys, 111

affinis, Doras, 168

affinis, Hassar, 171

affinis, Hypostomus, 251

affinis, Macrotocinclus, 271

affinis, Malapterurus, 301

affinis, Otocinclus, 271

affinis, Oxydoras, 171

affinis, Pimelodus, 208

affinis, Plecostomus, 251

affinis, Pseudauchenipterus, 76

affinis, Silurus (Callichrus), 374

afghana, Silurus, 376

africana, Arius, 31

africana, Arius falcarius, 31

africanus, Otolithus (Arius), 435 
afrofischeri, Synodontis, 311

Afro-fischeri, Synodontis, 311

Agamyxis, 168

Agamyxis albomaculatus, 168

Agamyxis pectinifrons, 168

Agassizi, Pseudopimelodus, 353

agassizii, Cathorops, 39

agassizii, Corydoras, 112

Agassizii, Pinirampus, 339

Agassizii, Rhinelepis, 289

agassizii, Tachisurus, 39

agastor, Paraloricaria, 278

agboyiensis, Clarias, 139

agboyiensis, Clarias (Clarioides), 139

Agenciosus polystictus, 70

Ageneiosi, 68

Ageneiosus, 68

Ageneiosus (Pseudageneiosus)

therezinae, 69

Ageneiosus armatus, 68, 69

Ageneiosus atronasus, 68

Ageneiosus barranquerensis, 69

Ageneiosus brevifilis, 68, 69

Ageneiosus brevis, 69

Ageneiosus caucanus, 70

Ageneiosus childreni, 366

Ageneiosus dentatus, 70

Ageneiosus freiei, 70

Ageneiosus gabardinii, 69

Ageneiosus guianensis, 70

Ageneiosus inermis, 69

Ageneiosus madeirensis, 69

Ageneiosus magoi, 69

Ageneiosus marmoratus, 69

Ageneiosus marquesi, 70

Ageneiosus melanopogon, 68

Ageneiosus militaris, 68, 69

Ageneiosus mino, 38

Ageneiosus ogilviei, 69

Ageneiosus pardalis, 70

Ageneiosus parnaguensis, 70

Ageneiosus piperatus, 70

Ageneiosus polystictus, 70

Ageneiosus quadrifilis, 78

Ageneiosus rondoni, 69

Ageneiosus sebae, 69

Ageneiosus ucayalensis, 70

Ageneiosus uruguayensis, 70

Ageneiosus valenciennesi, 69

Ageneiosus virgo, 70

Ageneiosus vittatus, 70

Ageneisus polystictus, 70

Ageniosus (Silonia) diaphina, 86

Ageniosus (Silonia) lurida, 366

Ageniosus axillaris, 69

Ageniosus militaris, 69

Ageniosus porphyreus, 70

ageneiosus, Batrachocephalus, 38

Agenius, 68
Aglyptosternon, 387

Agmus, 58

Agmus lyriformis, 60

agna, Hypostomus, 251

ãgnã, Plecostomus, 251

agricolus, Bagrus, 107

aguaboensis, Ancistrus, 219

aguadulce, Cathorops, 39

aguadulce, Galeichthys, 39

aguanai, Exallodontus, 333

aguarague, Trichomycterus, 441

Aguarunichthys, 329

Aguarunichthys inpai, 329

Aguarunichthys tocantinsensis, 329

Aguarunichthys torosus, 329

aguilerae, Silvaichthys, 76

agustini, Farlowella, 240

ahli, Auchenoglanis, 162

ahli, Parauchenoglanis, 162

Ailia, 356

Ailia affinis, 356

Ailia coila, 356

Ailia occidentalis, 360

Ailia punctata, 356

Ailia somalensis, 360

Ailichthyoidei, 356

Ailiichthys, 356

Ailiichthys punctata, 356

Ailurichthys, 37

ailurus, Pimelodus, 205

akamai, Micromyzon, 61

akhtari, Glyptosternon, 387

akhtari, Glyptosternum, 387

akiri, Anaspidoglanis, 154

akiri, Auchenoglanis, 154

aky, Epactionotus, 236

Akyses, 12

Akysidae, 12

Akysis, 12

Akysis alfredi, 16

Akysis armatus, 98

Akysis baramensis, 16

Akysis brachybarbatus, 13

Akysis clavulus, 14

Akysis clinatus, 14

Akysis ephippifer, 14

Akysis filifer, 16

Akysis fuliginatus, 14

Akysis fuscus, 16

Akysis hendricksoni, 14

Akysis heterurus, 14

Akysis inermis, 16

Akysis kurzii, 18

Akysis leucorhynchus, 16

Akysis longifilis, 442

Akysis macronema, 16, 17

Akysis maculipinnis, 14

Akysis major, 391

Akysis meridionalis, 17
Akysis microps, 14

Akysis nitidus, 17

Akysis pictus, 14

Akysis prashadi, 14

Akysis pseudobagarius, 16, 17

Akysis recavus, 14

Akysis similis, 17

Akysis sinensis, 17

Akysis sinesis, 17

Akysis variegatus, 15

Akysis variegatus variegatus, 14

Akysis varius, 15

Akysis vespa, 15

alaknandi, Glyptothorax, 388

alaknandi, Glyptothorax brevipinnis, 388

alasensis, Mystus, 94

alatus, Arius, 53

alatus, Hypostomus, 251

albater, Aspidoras, 108

albater, Noturus, 211, 441

alberti, Synodontis, 311

albescens, Glanidium, 75

albicans, Arius, 337

albicans, Bagrus, 54

albicans, Pimelodus, 337

albicollaris, Leiocassis, 104

albicrux, Trachelyopterus, 79

albicrux, Trachycorystes, 79

albidus, Arius, 337

albidus, Callichthys, 128

albidus, Pimelodus, 40, 204

albifasciatus, Bunocephalus, 62

albilabris, Bagrus, 95

albilabris, Paraplotosus, 349

albilabris, Plotosus, 349

albinotatus, Trichomycterus, 414

albocinctus, Hemiancistrus, 223

albofasciatus, Pimelodus, 337

albolineata, Synodontis, 311

albolineatus, Corydoras, 112

albolineatus, Mystus, 94

albolineatus, Synodontis, 311

albomaculatus, Agamyxis, 168

albomaculatus, Doras, 168

albomaculatus, Panaqolus, 277

albomaculatus, Panaque, 277

albomarginatus, Cephalosilurus, 353

albomarginatus, Leiocassis, 101

albomarginatus, Pseudobagrus, 101

albomarginatus, Pseudopimelodus,

353

albopunctatus, Clarias, 139

albopunctatus, Hypostomus, 251

albopunctatus, Plecostomus, 251

aleppensis, Macrones, 97

aleuropsis, Bunocephalus, 58

alexandri, Lophiosilurus, 353

alfaroi, Rhamdia, 199 
alfredi, Akysis, 16

alfredi, Pseudobagarius, 16

alga, Chaetostomus, 221

alikunhii, Horaglanis, 152

alipionis, Hemipsilichthys, 262

alipionis, Isbrueckerichthys, 262

Allabenchelys, 137

Allabenchelys attemsi, 137

Allabenchelys brevior, 137

Allabenchelys dhonti, 142

Allabenchelys engelseni, 142

Allabenchelys laticeps, 137

Allabenchelys longicauda, 137

Allabenchelys manyangae, 137

Allabenchelys pietschmanni, 138

alleni, Apomatoceros, 403

alluaudi, Clarias, 139

aloikae, Bunocephalus amaurus, 58

alta, Tympanopleura, 69

altae, Centromochlus, 73

altamazonicum, Lepthoplosternum, 128

alternatum, Pygidium, 414

alternatus, Trichomycterus, 414

alternifasciatum, Chaetostoma, 227

alternus, Ochmacanthus, 409

alterum, Pygidium, 415

alterus, Trichomycterus, 415

altifrons, Pangasius, 325

altipinnis, Auchenoglanis, 162

altipinnis, Eutropius, 365

altipinnis, Hemiloricaria, 245

altipinnis, Liposarcus, 291

altipinnis, Loricaria, 245

altipinnis, Parauchenoglanis, 162

altipinnis, Pimelodella, 189

altipinnis, Pimelodus, 189

altissimus, Pimelodus, 337

altocorpus, Farlowella, 444

altus, Silurus, 381

alula, Doumea, 24

aluuensis, Chrysichthys, 155

alvarezi, Gymnallabes, 150

amandajanea, Corydoras, 112

amapaensis, Corydoras, 113

Amaralia, 57

Amaralia hypsiura, 57

Amarginops, 153

Amarginops hildae, 153

Amarginops platus, 153

amatitlanensis, Rhamdia, 198

amaurus, Bunocephalus 58

amazona, Farlowella, 237

amazonae, Helogenes, 134

amazonensis, Parotocinclus, 281

amazonica, Dekeyseria, 118

amazonica, Loricaria, 270

amazonicus, Bunocephalus, 59

amazonicus, Dysichthys, 59 amazonicus, Ituglanis, 406

amazonicus, Trichomycterus, 406

amazonum, Acesta, 237

amazonum, Loricaria apeltogaster, 288

amazonum, Rhinodoras, 170

amazonus, Pseudohemiodon, 288

ambiacus, Corydoras, 113

amblops, Felichthys, 79

amblops, Trachelyopterus, 79

Amblycepinae, 17

Amblyceps, 17, 98

Amblyceps apangi, 17

Amblyceps arnunachalensis, 17

Amblyceps caecutiens, 17, 18

Amblyceps cacutiens, 18

Amblyceps carinatum, 18

Amblyceps deyi, 17

Amblyceps foratum, 18

Amblyceps horae, 99

Amblyceps inermis, 17

Amblyceps laticeps, 18

Amblyceps macropterus, 18

Amblyceps mangois, 18

Amblyceps marginatoides, 19

Amblyceps marginatus, 19

Amblyceps mucronatum, 18

Amblyceps murraystuarti, 18

Amblyceps murray-stuarti, 18

Amblyceps platycephalus, 18

Amblyceps serratum, 18

Amblyceps tenuispinis, 18

Amblyceps variegatum, 19

Amblycipitidae, 17

Amblydoras, 168, 179

Amblydoras affinis, 168

Amblydoras bolivarensis, 168

Amblydoras gonzalezi, 168

Amblydoras monitor, 169, 169

Amblydoras nauticus, 169

Amblydoras truncatus, 168

amblyurum, Steindachneridion, 343

amblyurus, Steindachneria, 343

amboinensis, Cryptopterus, 369

ambrosettii, Liposarcus, 291

ambrosettii, Pterygoplichthys, 291

ambyiacus, Auchenipterus, 71

Ameiurina, 203

Ameiurus, 203, 204

Ameiurus brunneus, 204

Ameiurus cancellatus, 211

Ameiurus catus, 204

Ameiurus hazenensis, 204

Ameiurus lavetti, 204

Ameiurus leidyi, 204

Ameiurus macgrewi, 204

Ameiurus maconnellii, 211

Ameiurus melas, 205

Ameiurus natalis, 205
Ameiurus nebulosus, 205

Ameiurus pectinatus, 206

Ameiurus platycephalus, 206

Ameiurus primaevus, 207

Ameiurus reticulatus, 206

Ameiurus sawrockensis, 206

Ameiurus serracanthus, 206

Ameiurus vespertinus, 206

amekiensis, Otolithus (Arius), 435

amemiyae, Aoria, 91

amemiyai, Aoria, 91

americanus, Cataphractus, 167

Amissidens, 32

Amissidens hainesi, 32

Amiurus, 204

Amiurus australis, 208

Amiurus bolli, 205

Amiurus brachyacanthus, 205

Amiurus brunneus, 204

Amiurus cragini, 205

Amiurus decorus, 210

Amiurus dugèsii, 208

Amiurus erebennus, 205

Amiurus lophius, 204

Amiurus meeki, 209

Amiurus meridionalis, 208

Amiurus mexicanus, 209

Amiurus mispilliensis, 206

Amiurus natalis analis, 205

Amiurus niveiventris, 204

Amiurus obesus, 205

Amiurus prosthistius, 205

Ammoglanis, 403

Ammoglanis diaphanus, 403

Ammoglanis pulex, 403

ammophilus, Aphanotorulus, 225

amphibelus, Corydoras, 113

Amphiliidae, 20

Amphilius, 20, 21

Amphilius atesuensis, 21

Amphilius baudoni, 21

Amphilius baudoni uniformis, 21

Amphilius baudoni var. uniformis., 21

Amphilius brevidorsalis, 23

Amphilius brevis, 21

Amphilius cryptobullatus, 21

Amphilius grammatophorus, 22, 23

Amphilius grammatophorus

brevipinna, 22

Amphilius grammatophorus

inaequalis, 23

Amphilius grammatophorus

marmoratus, 23

Amphilius grammatophorus var.

brevipinna, 23

Amphilius grammatophorus var. inaequalis, 23

Amphilius grandis, 23

Amphilius hargeri, 23 
Amphilius jacksonii, 21

Amphilius kakrimensis, 21

Amphilius kivuensis, 21

Amphilius krefftii, 23

Amphilius lamani, 22

Amphilius lampei, 22

Amphilius laticaudatus, 22

Amphilius lentiginosus, 22

Amphilius longirostris, 22

Amphilius maesii, 22

Amphilius natalensis, 22

Amphilius nigricaudatus, 21

Amphilius nigricaudatus

multipunctata, 21

Amphilius nigricaudatus var. multipunctata, 21

Amphilius notatus, 27

Amphilius opisthophthalmus, 22

Amphilius oxyrhinus, 23

Amphilius pictus, 21

Amphilius platychir, 22

Amphilius platychir cubangoensis, 23

Amphilius platychir var. cubangoensis, 23

Amphilius pulcher, 23

Amphilius pulcher ephippiata, 23

Amphilius rheophilus, 23

Amphilius transvaaliensis, 23

Amphilius uranoscopus, 23

Amphilius zairensis, 23

amphiloxa, Cetopsis, 131

amphiloxus, Hemicetopsis, 131

amplexicauda, Clarias, 148

Anacanthus, 301

Anadoras, 169, 179

Anadoras grypus, 169

Anadoras regani, 169

Anadoras weddellii, 169

anale, Chaetostoma, 227

analis, Amiurus natalis, 205

analis, Hypocolpterus, 227

analis, Leiocassis (Dermocassis), 101

analis, Pseudobagrus, 101

analis, Trachycorystes, 80

anamalaiensis, Glyptothorax, 388

ananas, Physopyxis, 176

Anaspidoglanis, 153

Anaspidoglanis akiri, 154

Anaspidoglanis boutchangai, 154

Anaspidoglanis macrostomus, 154

Anchariidae, 29

Ancharius, 29

Ancharius brevibarbis, 29

Ancharius fuscus, 29

Ancharius griseus, 29

Ancistomus, 283

Ancistri, 216

ancistroides, Hypostomus, 251

ancistroides, Plecostomus, 251
Ancistrus, 216, 219

Ancistrus (Hemiancistrus) yaravi, 273

Ancistrus (Pseudancistrus) coquenani, 287

Ancistrus aguaboensis, 219

Ancistrus annectens, 242

Ancistrus barrae, 272

Ancistrus baudensis, 220

Ancistrus bodenhameri, 219

Ancistrus bolivianus, 219

Ancistrus Bovallii, 267

Ancistrus brachyurus, 234

Ancistrus brevifilis, 219

Ancistrus brevifilis bodenhameri, 219

Ancistrus brevipinnis, 219

Ancistrus bufonius, 219

Ancistrus caucanus, 219

Ancistrus centrolepis, 219

Ancistrus chagresi, 220, 222

Ancistrus cirrhosus, 220

Ancistrus cirrhosus dubius, 221

Ancistrus claro, 220

Ancistrus clementinae, 220

Ancistrus cryptophthalmus, 220

Ancistrus cuiabae, 220

Ancistrus damasceni, 220

Ancistrus dolichopterus, 220

Ancistrus dubius, 221

Ancistrus erinaceus, 221

Ancistrus eustictus, 221

Ancistrus formoso, 221

Ancistrus fulvus, 221

Ancistrus füsslii, 235

Ancistrus galani, 221

Ancistrus gibbiceps, 291

Ancistrus guacharote, 225

Ancistrus guentheri, 287

Ancistrus gymnorhynchus, 221

Ancistrus heterorhynchus, 221

Ancistrus hoplogenys, 221

Ancistrus jataiensis, 222

Ancistrus jelskii, 222

Ancistrus latifrons, 222

Ancistrus leucostictus, 222

Ancistrus lineolatus, 222

Ancistrus lithurgicus, 222

Ancistrus lituratus, 290, 291

Ancistrus longimanus, 291

Ancistrus macrophthalmus, 222

Ancistrus maculatus, 222

Ancistrus malacops, 222

Ancistrus maracasae, 223

Ancistrus martini, 223

Ancistrus mattogrossensis, 300

Ancistrus mattogrossenssis, 300

Ancistrus medians, 242, 243

Ancistrus megalostomus, 223

Ancistrus melas, 220

Ancistrus minutus, 223
Ancistrus montanus, 223

Ancistrus multiradiatus alternans, 291

Ancistrus multispinis, 223

Ancistrus multispinis, 265

Ancistrus mystacinus, 265

Ancistrus nationi, 223

Ancistrus nudiceps, 223

Ancistrus occidentalis, 223

Ancistrus occloi, 223

Ancistrus parecis, 224

Ancistrus pictus, 234, 265

Ancistrus pirareta, 224

Ancistrus piriformis, 224

Ancistrus planiceps, 264

Ancistrus ranunculus, 224

Ancistrus reisi, 224

Ancistrus salgadae, 300

Ancistrus scaphirhynchus, 234

Ancistrus snethlageae, 283, 285

Ancistrus spinosus, 224

Ancistrus stigmaticus, 224

Ancistrus tamboensis, 224

Ancistrus taunayi, 224

Ancistrus temminckii, 224

Ancistrus tombador, 225

Ancistrus trinitatis, 225

Ancistrus triradiatus, 225

Ancistrus triradiatus martini, 223

Ancistrus variolus, 225

Ancistrus verecundus, 225

Ancistrus vittatus var. vermiculata, 285

Ancistrus vittatus vermiculata, 285

andamanensis, Arius, 56

andersoni, Liobagrus, 19

andersoni, Corymbophanes, 232

Andersonia, 23

Andersonia brevior, 24

Andersonia leptura, 24

Andersonia pellegrini, 24

andersonii, Exostoma, 386

andersonii, Glaridoglanis, 386

Andinichthyidae, 30

Andinichthys, 30

Andinichthys bolivianensis, 30

Anduzedoras, 169

Anduzedoras arleoi, 169

Anduzedoras copei, 172

Anduzedoras oxyrhynchus, 169

anduzei, Ernstichthys, 60

anduzei, Trachelyopterichthys, 78

Aneistrus (Hemiancistrus) pulcher, 234

Anemanotus, 37

anfractus, Clarias, 139

angeli, Pterodoras, 177

angelica, Synodontis, 311

angelicus, Otolithus (Arius), 434

angelicus zonatus, Synodontis, 311

angelis, Ptedoras, 178 
angipinnatus, Hypostomus, 251

angipinnatus, Plecostomus, 251

angius, Pimelodus, 359

angolensis, Chiloglanis, 304

angolensis, Clarias, 139

angolensis, Doumea, 25

angolensis, Eutropius, 363

angolensis, Schilbe, 363

angolensis macronema, Chlarias, 141

angosturae, Farlowella, 240

anguilla, Ictalurus, 210

anguilla, Glanapteryx, 404

anguillaris, Clarias, 140, 144

anguillaris, Platystacus, 350, 351

anguillaris, Silurus, 138, 140, 143

anguillaris nigeriensis, Clarias, 140

anguillicauda, Liobagrus, 19

Anguilloclarias, 139

angulatus, Arius, 55

angulatus, Otolithus (Arius), 435

angulicauda, Plecostomus, 235

anguilicauda, Delturus, 234, 444

angusticeps, Adelopeltis, 107

angustifrons, Anoplopterus, 27

angustifrons, Tetracamphilius, 27

angustirostre, Scleronema, 413

angustirostris, Pygidium, 413

anisitsi, Liposarcus, 291

anisitsi, Pterygoplichthys, 291

anisitsi, Homodiaetus, 406

anisura, Phenacorhamdia, 188

anisurus, Bagrus, 90

anisurus, Heptapterus, 188

anisurus, Pimelodus, 98

annae, Chaetostomus, 297

annae, Plecostomus, 297

annae, Squaliforma, 297

annandalei, Glyptothorax, 388

annectens, Acanthopoma, 403

annectens, Ancistrus, 242

annectens, Hemiancistrus, 242

annectens, Synodontis, 312

Anodontiglanis, 345

Anodontiglanis dahli, 345

anomala, Herklotsella, 375

anomala, Pterocryptis, 375

anomala sovichthys, Chaetostoma, 230

anomalopteryx, Parakysis, 15

anomalum, Chaetostoma, 227

anomalus, Chastostomus, 227

Anopleutropius, 165

Anopleutropius henrici, 165

Anoplopterus, 21

Anoplopterus angustifrons, 27

Anoplopterus longirostris, 22

Anoplopterus uranoscopus, 21, 23

anostomus, Silurus, 374

anoterus, Chiloglanis, 304

Ansorgia, 360
Ansorgia, vitata, 360

Ansorgia vittata, 360, 361

Ansorgia vittata bistriata, 361

ansorgii, Chrysichthys, 155

ansorgii, Eutropius, 363

ansorgii, Parauchenoglanis, 154

ansorgii, Phractura, 26

ansorgii, Physalia, 360

ansorgii, Synodontis, 312

Ansorgiichthys, 360

anteanalis, Pareuchiloglanis, 398

anthrax, Lasiancistrus, 288

anthrax, Pseudolithoxus, 288

antiquus, Astephus, 207

antiquus, Pimelodus, 207

antoniensis, Pimelodus, 205

anus, Loricaria, 269

anus, Loricariichthys, 269

Anyperistius, 347

Anyperistius perugiae, 347

aor, Pimelodus, 106

aor, Sperata, 106

aor sarwari, Aorichthys, 107

aorella, Sperata, 107

aorellus, Bagrus, 107

Aoria, 106

Aoria amemiyae, 91

Aoria amemiyai, 91

Aoria henryi, 100

Aoria hoi, 107

Aoria lacus, 108

Aoria rendahli, 108

Aoria virgatus, 101

Aorichthys, 106

Aorichthys aor sarwari, 107

aorides, Bagrus, 106

aorinus, Bagrus, 107

apangi, Amblyceps, 17

apeltogaster, Loricaria, 268

apeltogaster amazonum, Loricaria, 288

apeltogaster var. amazonum,

Loricaria, 288

Aphanotorulus, 225

Aphanotorulus ammophilus, 225

Aphanotorulus frankei, 225, 226

Aphanotorulus unicolor, 225

Apistoloricaria, 226

Apistoloricaria condei, 226

Apistoloricaria laani, 226

Apistoloricaria listrorhinos, 226

Apistoloricaria ommation, 226

apithanos, Pseudohemiodon, 288

Apodoglanis, 375

Apodoglanis furnessi, 375, 376

apogon, Phalacronotus, 374

apogon, Silurus, 374

Apomatoceros, 403

Apomatoceros alleni, 403
Aposturisoma, 226

Aposturisoma myriodon, 226

approuaguensis, Corydoras, 113

Apuredoras, 177

Apuredoras rivasi, 177

apurensis, Cephalosilurus, 353

apurensis, Pseudopimelodus, 353

apurensis, Sachsdoras, 177, 178

apus, Channallabes, 137

apus, Gymnallabes, 137

aquilus, Lophiobagrus, 161

arab, Plotosus, 351

arabi, Synodontis, 321

arafurensis, Arius, 49

araguaiaensis, Corydoras, 113

araguayensis, Galeichthys, 339

aralensis, Silurus glanis, 379

arcana, Cetopsis, 131

arcifer, Callichthys, 111

arcuatus, Corydoras, 113

arcuatus, Gogo, 29

arcuatus, Rhineastes, 207

areio, Corydoras, 113

arekaima, Pimelodus, 334

arenaria, Peckoltia, 284

arenarius, Arius, 34

arenarius, Bagrus (Ariodes), 34

arenarius, Hemiancistrus, 284

arenatus, Arius, 39

arenatus, Cathorops, 39

areolatus, Trichomycterus, 415

Argeini, 63

argentata, Silurus (Clupisoma), 357

argenteus, Arius, 56

argenteus, Neosilurus, 345

argenteus, Pimelodus, 53

argenteus, Plotosus, 345

argenteus, Porochilus, 345

argentina, Corydoras punctatus, 122

argentina, Megalonema, 335

argentina, Perugia, 335

argentinus, Arius, 428

argentinus, Silurus, 209

argentivittatus, Macrones, 100

argentivittatus, Pelteobagrus, 100

Arges, 63

Arges boulengeri, 63

Arges brachycephalus, 63

Arges chotae, 63

Arges cirratus, 64

Arges eigenmanni, 64

Arges festae, 64

Arges fissidens, 64

Arges heterodon, 64

Arges homodon, 64

Arges longifilis, 65

Arges marmoratus, 65

Arges orientalis, 65

Arges peruanus, 66 
Arges regani, 66

Arges retropinna, 66

Arges sabalo, 63, 67

Arges simonsii, 67

Arges stübeli, 67

Arges taczanowskii, 67

Arges theresiae, 67

Arges vaillanti, 67

Arges whymperi, 68

argus, Hypostomus, 251

argus, Plecostomus, 251

argyropleuron, Arius, 51

argyropleuron, Plicofollis, 51

argyrus, Pimelodus, 209

Arii, 30

Ariidae, 30

Ariodes, 34

Ariodes aeneus, 49

Ariodes macrocephalus, 51

Arius tonggol, 52

Arioida, 429, 432

arioides, Bagrus, 40, 54

Ariopsis, 32

Ariopsis, 428

Ariopsis aegyptiacus, 428

Ariopsis assimilis, 33

Ariopsis bonillai, 33

Ariopsis felis, 33

Ariopsis guatemalensis, 33

Ariopsis lentiginosa, 33

Ariopsis robertsi, 48

Ariopsis seemanni, 33

Ariopsis peyeria, 432

aripuanensis, Parotocinclus, 281

Aristommata, 249

Aristommata inexpectata, 249, 250

Aristotelis, Glanis, 378

aristotelis, Silurus, 378

aristotelis, Silurus (Parasilurus), 378

Arius, 30, 34

Arius (?) bartonensis, 56

Arius (Hemiarius) carinatus, 42

Arius (Hemiarius) danielsi, 42

Arius (Hemiarius) nudidens, 56

Arius acrocephalus, 47

Arius acutirostris, 31

Arius acutivelis, 155, 158

Arius acutus, 51

Arius aequibarbis, 55

Arius africana, 31

Arius alatus, 53

Arius albicans, 337

Arius albidus, 337

Arius andamanensis, 56

Arius angulatus, 55

Arius arafurensis, 49

Arius arenarius, 34

Arius arenatus, 39

Arius argenteus, 56
Arius argentinus, 428

Arius argyropleuron, 51

Arius arius, 34

Arius armiger, 46

Arius assimilis, 33, 434

Arius augustus, 46

Arius australis, 47

Arius baroni, 434

Arius Belangerii, 54

Arius bicolor, 34

Arius bleekeri, 46

Arius boakeii, 34

Arius bonneti, 36, 56

Arius borneënsis, 56

Arius Brandtii, 54

Arius brevirostris, 31

Arius brunellii, 31

Arius Buchanani, 34

Arius burmanicus, 42

Arius cacharioides, 166

Arius caelatoides, 55

Arius calatus, 46

Arius Capellonis, 32

Arius cavatus, 435

Arius carulescens, 33

Arius chondropterygioides, 55

Arius chondropterygius, 55

Arius clavispinosus, 36

Arius cleptolepis, 47

Arius clijpeaster, 55

Arius clypeastroides, 55

Arius coatesi, 47

Arius cochinchinensis, 34

Arius cookei, 50

Arius cous, 31

Arius crassus, 435

Arius crossocheilos, 52

Arius cruciger, 106

Arius curtisii, 47

Arius dasycephalus, 39

Arius dayi, 49

Arius despaxi, 36

Arius Dieperinki, 32

Arius digulensis, 47

Arius dioctes, 44

Arius dispar, 34

Arius doriae, 41

Arius dussumieri, 52

Arius Dutemplei, 56

Arius egertoni, 56

Arius egertoni belgicus, 56

Arius elatturus, 50

Arius equestris, 33

Arius falcarius, 34

Arius falcarius africana, 31

Arius falcarius var. africana, 31

Arius fangi, 34

Arius festae, 56

Arius festinus, 34
Arius fissus, 39

Arius Fraasi, 56

Arius froggatti, 42

Arius Fürthii, 39

Arius gagora, 34

Arius gagoroides, 35

Arius gigas, 31, 56

Arius goniaspis, 52

Arius graeffei, 47

Arius grandicassis, 49, 50

Arius grandoculis, 53

Arius granducolis, 53

Arius granosus, 54

Arius granulatus, 32

Arius guatemalensis, 33

Arius hainesi, 32

Arius hamiltonis, 51

Arius hardenbergi, 36

Arius hastatus, 105

Arius Heckelii, 55

Arius heudelotii, 31

Arius heward-belli, 56

Arius hypophthalmus, 39, 40

Arius iheringi, 343

Arius insculptus, 50

Arius insidiator, 45

Arius jella, 36

Arius kanganamanensis, 39

Arius Kessleri, 50

Arius kirkii, 52

Arius kitsoni, 56

Arius kutchensis, 56

Arius laeviceps, 55

Arius lagoensis, 31

Arius laticeps, 41

Arius latirostris, 47

Arius latiscutatus, 31

Arius layardi, 51

Arius leiotetocephalus, 52

Arius lemoinei, 432

Arius leptonotacanthus, 34

Arius longibarbis, 334

Arius luniscutis, 36

Arius macracanthus, 36

Arius macrocephalus, 51

Arius macronotacanthus, 47

Arius macruropterygius, 55

Arius maculatus, 34, 35

Arius madagascariensis, 35

Arius magatensis, 52

Arius malabaricus, 35

Arius manillensis, 35

Arius manjong, 55

Arius mastersi, 45

Arius melanochir, 41

Arius melanopterygius, 56

Arius melanopus, 40

Arius mercatoris, 31

Arius microcephalus, 35 
Arius microgastropterygius, 55

Arius micronotacanthus, 55

Arius micropterus, 166

Arius microstomus, 39

Arius micruropterygius, 36

Arius midgleyi, 47

Arius militaris, 51

Arius molliceps, 55

Arius multiradiatus, 40

Arius nasutus, 49

Arius neogranatensis, 50

Arius nigricans, 54

Arius nox, 38, 39

Arius nuchalis, 41

Arius nucleus, 57

Arius obesus, 81

Arius oetik, 35

Arius oncina, 75

Arius oncinus, 75

Arius osculus, 50

Arius papillosus, 165

Arius parkii, 32

Arius parmocassis, 50

Arius parvipinnis, 47

Arius paucus, 48

Arius pavimentatus, 105

Arius pectoralis, 48

Arius phrygiatus, 32

Arius physacanthus, 36

Arius pidada, 55

Arius planiceps, 50

Arius platypogon, 54

Arius platystomus, 52

Arius pleurops, 39

Arius polystaphylodon, 52

Arius proximus, 48, 49

Arius pumilus, 386

Arius puncticulatus, 41

Arius quadriscutis, 37

Arius ritoides, 106

Arius robertsi, 48

Arius rostratus, 55

Arius rugispinis, 32

Arius russi, 57

Arius rutschi, 435

Arius sagoroides, 45

Arius satparanus, 56

Arius schlegeli, 56

Arius sciurus, 35

Arius seemanni, 33

Arius serratus, 49

Arius similis, 434

Arius sinensis, 55

Arius solidus, 39

Arius spatula, 42, 43

Arius squalus, 166

Arius stauroforus, 32

Arius stirlingi, 46

Arius stricticassis, 50
Arius subrostratus, 35

Arius sumatranus, 36

Arius synodon, 166

Arius taylori, 40, 48

Arius tenuispinis, 52, 56

Arius tonggol, 52

Arius truncatus, 43

Arius tuyra, 40

Arius uncinatus, 36

Arius utarus, 48

Arius utik, 35

Arius vandeli, 50

Arius variolosus, 41

Arius venosus, 36

Arius verrucosus, 45

Arius villosus, 166

Arius viviparus, 55

arleoi, Anduzedoras, 169

arleoi, Pygidium, 415

arleoi, Trichomycterus, 415

armatulus, Doras, 176

armatulus, Platydoras, 176

armatus, Ageneiosus, 68, 69

armatus, Akysis, 98

armatus, Callichthys, 113

armatus, Corydoras, 113

armatus, Macrones, 94

armatus, Microsynodontis, 309

armatus, Mystus, 94, 98

armatus, Nanobagrus, 98

armatus, Silurus, 177

armbrusteri, Notarius, 440

armeniacum, Glyptosternum, 388

armeniacus, Glyptothorax, 388

armiger, Arius, 46

armiger, Mystus, 94

armiger, Nemapteryx, 46

armillatus, Pariolius, 188

arnoldi, Otocinclus, 271

arnoulti, Synodontis, 312

arnunachalensis, Amblyceps, 17

artedii, Platystoma, 341

ascita, Erethistoides, 384

ascita, Mystus, 177

ascita, Silurus, 54

asopos, Mastiglanis, 187

asotus, Parasilurus, 379

asotus, Silurus, 378, 381

asotus longus, Parasilurus, 379

asper, Callichthys, 110, 167

aspera, Rhinelepis, 293

asperatus, Hypostomus, 251

asperispinis, Lophiobagrus, 161

asperus, Pimelodus, 395

Aspidobagrus, 93

aspidolepis, Chaetostomus, 242

aspidolepis, Hemiancistrus, 242

Aspidoradidi, 108

Aspidoras, 108
Aspidoras albater, 108

Aspidoras belenos, 108

Aspidoras brunneus, 109

Aspidoras carvalhoi, 109

Aspidoras depinnai, 109

Aspidoras eurycephalus, 109

Aspidoras fuscoguttatus, 109

Aspidoras lakoi, 109

Aspidoras maculosus, 109

Aspidoras menezesi, 109

Aspidoras microgalaeus, 109

Aspidoras pauciradiatus, 109

Aspidoras poecilus, 109

Aspidoras psammatides, 110

Aspidoras raimundi, 110

Aspidoras rochai, 110

Aspidoras spilotus, 110

Aspidoras taurus, 110

Aspidoras velites, 110

Aspidoras virgulatus, 110

aspilogaster, Hypostomus, 251

aspilogaster, Plecostomus, 251

Aspistor, 36

Aspistor hardenbergi, 36

Aspistor luniscutis, 36

Aspistor parkeri, 36

Aspistor quadriscutis, 37

Aspredinichthys, 57

Aspredinichthys filamentosus, 57

Aspredinichthys tibicen, 58

Aspredinidae, 57

aspredinoides, Myoglanis, 187

Aspredo, 57, 58

Aspredo aspredo, 58

Aspredo batrachus, 58

Aspredo filamentosus, 57

Aspredo gronovii, 58, 60

Aspredo sexcirrhis, 61

Aspredo sicuephorus, 58

Aspredo sicyephorus, 58

Aspredo spectrum, 61

Aspredo tibicen, 57, 58

Aspredo verrucosa, 60

aspredo, Aspredo, 58

aspredo, Silurus, 58

assamensis, Clarias, 148

assamensis, Nangra, 396

assimilis, Ariopsis, 33

assimilis, Arius, 33

astatodon, Atopochilus, 308

astatodon, Euchilichthys, 308

Astemomycterus, 411

Astephinae, 203

Astephus, 203, 207

Astephus antiquus, 207

Astephus resimus, 207

asterifrons, Astrodoras, 170

asterifrons, Doras, 169, 170

Asterophysi, 68 
Asterophysus, 68, 70

Asterophysus batrachus, 70

Astroblepidae, 63

Astroblepiformes, 63

Astroblepus, 63

Astroblepus boulengeri, 63

Astroblepus brachycephalus, 63

Astroblepus caquetae, 63

Astroblepus chapmani, 63

Astroblepus chimborazoi, 63

Astroblepus chotae, 63

Astroblepus cirratus, 64

Astroblepus cyclopus, 64

Astroblepus cyclopus santanderensis, 67

Astroblepus eigenmanni, 64

Astroblepus festae, 64

Astroblepus fissidens, 64

Astroblepus formosus, 64

Astroblepus frenatus, 64

Astroblepus grixalvii, 64

Astroblepus grixalvii micrescens, 65

Astroblepus guentheri, 64

Astroblepus heterodon, 64

Astroblepus homodon, 64

Astroblepus jurubidae, 65

Astroblepus labialis, 65

Astroblepus latidens, 65

Astroblepus longiceps, 65

Astroblepus longifilis, 65

Astroblepus mancoi, 65

Astroblepus mariae, 65

Astroblepus marmoratus, 65

Astroblepus micrescens, 65

Astroblepus mindoensis, 65

Astroblepus nicefori, 65

Astroblepus nicéfori, 65

Astroblepus orientalis, 65

Astroblepus peruanus, 66

Astroblepus phelpsi, 66

Astroblepus pholeter, 66

Astroblepus pirrensis, 66

Astroblepus praeliorum, 66

Astroblepus prenadillus, 66

Astroblepus regani, 66

Astroblepus rengifoi, 66

Astroblepus retropinnus, 66

Astroblepus riberae, 66

Astroblepus rosei, 66

Astroblepus sabalo, 67

Astroblepus santanderensis, 67

Astroblepus simonsii, 67

Astroblepus stuebeli, 67

Astroblepus supramollis, 67

Astroblepus taczanowskii, 67

Astroblepus theresiae, 67

Astroblepus trifasciatus, 67

Astroblepus ubidiai, 67

Astroblepus unifasciatus, 67
Astroblepus vaillanti, 67

Astroblepus vanceae, 67

Astroblepus ventralis, 68

Astroblepus whymperi, 68

Astrodoras, 169

Astrodoras asterifrons, 170

Astrophysi, 68

Astrophysus, 70

asymetricaudalis, Chiloglanis, 304

atahualpa, Paracetopsis, 135

atavus, Silurus glanis, 382

ater, Cossyphus, 139, 148

ater, Galeichthys, 43

ater, Microglanis, 354

ater, Neosilurus, 347

ater sepikensis, Lambertichthys, 347

aterrima, Synodontis, 312

aterrimus, Synodontis, 312

atesuensis, Amphilius, 21

atherinoides, Neotropius, 359

atherinoides, Silurus, 359

atherinoides walkeri, Pseudeutropius, 359

athiensis, Chiloglanis, 304

athu, Silurus, 380

atochae, Pygidium, 423

Atopochilus, 303

Atopochilus astatodon, 308

Atopochilus chabanaudi, 303

Atopochilus christyi, 303

Atopochilus güntheri, 309

Atopochilus macrocephalus, 303

Atopochilus mandevillei, 304

Atopochilus pachychilus, 304

Atopochilus savorgnani, 304

Atopochilus vogti, 304

atra, Lambertia, 347

atrarius, Pimelodus, 205

atratoensis, Dolichancistrus, 235

atratoensis, Spatuloricaria, 296

atratoênsis, Pseudancistrus, 235

atribranchus, Dinotopterus, 148

atrifasciatus, Mystus, 94

atripes, Trachydoras, 179

atripinnis, Hemipimelodus, 34

atrizona, Hoplomyzon, 61

atrobrunneus, Pimelodus, 337

atronasus, Ageneiosus, 68

atropersonatus, Corydoras, 113

atropinnis, Hypostomus, 251

atroplumbeus, Tachysurus, 32

atrorus, Schilbeodes marginatus, 213

attemsi, Allabenchelys, 137

attemsi, Clariallabes, 137

attu, Silurus, 380

attu, Wallago, 380

attu, Wallagonia, 381

attu valeya, Wallago, 381

aubentoni, Mystus, 91
Auchenauglanis macrostom, 154

Auchenaspis, 154

Auchenipterichthys, 71

Auchenipterichthys coracoideus, 71

Auchenipterichthys dantei, 71

Auchenipterichthys longimanus, 71

Auchenipterichthys punctatus, 71

Auchenipterichthys thoracatus, 71

Auchenipteridae, 68

Auchenipterini, 68

Auchenipterus, 68, 71

Auchenipterus (Pseudauchenipterus) affinis, 76

Auchenipterus (Pseudauchenipterus) Jequitinhonhae, 76

Auchenipterus (Pseudauchenipterus) striatulus, 80

Auchenipterus (Pseudepapterus)

hasemani, 76

Auchenipterus ambyiacus, 71

Auchenipterus brachyurus, 72

Auchenipterus brevibarbis, 80

Auchenipterus brevior, 72

Auchenipterus britskii, 72

Auchenipterus ceratophysus, 79

Auchenipterus demerarae, 72

Auchenipterus dentatus, 72

Auchenipterus fordicei, 72

Auchenipterus furcatus, 76

Auchenipterus glaber, 80

Auchenipterus Heckelii, 73

Auchenipterus immaculatus, 80

Auchenipterus insignis, 79

Auchenipterus isacanthus, 80

Auchenipterus lacustris, 79

Auchenipterus longimanus, 71

Auchenipterus maculosus, 79

Auchenipterus Magdalenae, 79

Auchenipterus menezesi, 72

Auchenipterus nigripinnis, 72

Auchenipterus nuchalis, 72

Auchenipterus obscurus, 81

Auchenipterus osteomystax, 72

Auchenipterus paysanduanus, 72

Auchenipterus punctatus, 71

Auchenipterus robustus, 80

Auchenipterus thoracatus, 71

Auchenipterus thoracicus, 71

Auchenipterus trachycorystes, 80, 81

Auchenoglanidinae, 153

Auchenoglanis, 153, 154

Auchenoglanis acuticeps, 154

Auchenoglanis ahli, 162

Auchenoglanis akiri, 154

Auchenoglanis altipinnis, 162

Auchenoglanis ballayi, 162

Auchenoglanis ballayi gravoti, 162

Auchenoglanis ballayi var. gravoti, 162 
Auchenoglanis biscutatus, 154 Auchenoglanis büttikoferi, 163 Auchenoglanis fasciatus, 163 Auchenoglanis grandis, 163 Auchenoglanis iturii, 163 Auchenoglanis longiceps, 163 Auchenoglanis macrostoma, 154 Auchenoglanis maculosus, 163 Auchenoglanis monkei, 163 Auchenoglanis ngamensis, 163 Auchenoglanis occidentalis, 154 Auchenoglanis occidentalis tanganicanus, 154

Auchenoglanis occidentalis tchadiensis, 154

Auchenoglanis occidentalis var. tanganicanus, 154

Auchenoglanis occidentalis var. tchadiensis, 154

Auchenoglanis pantherinus, 163

Auchenoglanis pietschmanni, 162

Auchenoglanis pulcher, 162

Auchenoglanis punctatus, 163

Auchenoglanis ubangensis, 162

Auchenoglanis Wittei, 154

Auchenopterus, 71

augierasi, Synodontis, 314

augusta, Nemapteryx, 46

augustus, Arius, 46

aulometopon, Netuma, 56

aulopygia, Tatia, 77

aulopygius, Centromochlus, 77

aurantiacus, Bagrus, 101

aurantiacus, Hypostomus, 278

aurantiacus, Parancistrus, 278

aurantiacus, Pseudobagrus, 101, 108

aurata, Hemiloricaria, 245

auratus, Bagrus, 155

auratus, Chrysichthys, 155, 158, 160

auratus, Pimelodus, 155

auratus, Porcus, 155

auratus, Schilbe, 364

aurea, Loricaria, 298

aureatus, Scobinancistrus, 296

aureum, Sturisoma, 298

auritus, Siluranodon, 366

auritus, Silurus, 366

aurofrenatus, Corydoras, 113

auroguttatus, Hypostomus, 251

auroguttatus, Trichomycterus, 415

australe, Corydoras, 60

australe, Dysichthys, 118

australis, Amiurus, 208

australis, Arius, 47

australis, Ictalurus, 208

australis, Pimelodella, 189

australis, Neosilurus, 347, 348

austriacus, Heterobranchus, 382

Austroglanididae, 81
Austroglanis, 81

Austroglanis barnardi, 81

Austroglanis gilli, 81

Austroglanis sclateri, 81

Autanadoras, 178

Autanadoras milesi, 178, 179

avanhandavae, Pimelodella, 189

axelrodi, Corydoras, 113

axillaris, Ageniosus, 69

Ayarnangra, 382

Ayarnangra estuarius, 382

aymarae, Rhamdella, 196

azureus, Galeichthys, 33

azygia, Farlowella, 238

azygolechis, Hemipsilichthys, 279

azygolechis, Pareiorhaphis, 279

B

bachi, Chaetostomus, 284

bachi, Oxydoras, 174

bachi, Peckoltia, 284

Bachmannia, 428

Bachmannia chabutensis, 428

baculum, Encheloclarias, 149

badeli, Trachycorystes insignis, 80

baderi, Corydoras, 113

Bagarina, 382

Bagarius, 382, 383

Bagarius bagarius, 383

Bagarius Buchanani, 383

Bagarius gigas, 383

Bagarius lica, 383

Bagarius Nieuwenhuisii, 383

Bagarius rutilus, 383

Bagarius suchus, 383

Bagarius yarrelli, 383

bagarius, Bagarius, 383

bagarius, Pimelodus, 383

Bagre, 30, 37

Bagre bagre, 37, 97

Bagre marinus, 37

Bagre panamensis, 38

Bagre pinnimaculatus, 38

bagre, Bagre, 37, 97

bagre, Silurus, 37, 38

bagre, Stearopterus, 37

Bagreidae, 30

Bagrichthyes, 81

Bagrichthyoidei, 81

Bagrichthys, 81, 82

Bagrichthys hypselopterus, 82

Bagrichthys macracanthus, 82

Bagrichthys macropterus, 82

Bagrichthys majusculus, 83

Bagrichthys micranodus, 83

Bagrichthys obscurus, 83

Bagrichthys vaillantii, 83

Bagridae, 81

Bagroides, 82, 83

Bagroïdes, 83
Bagroïdes macracanthus, 82

Bagroides macropterus, 82, 83

Bagroides melanopterus, 83

Bagroides melapterus, 83

Bagroïdes melapterus, 83

Bagroides Vaillantii, 83

Bagroidinae, 82

Bagropsis, 329

Bagropsis reinhardti, 329

Bagrus, 81, 83

Bagrus (Ariodes) arenarius, 34

Bagrus (Ariodes) Meyenii, 52

Bagrus (Bagrus) ramentosus, 108

Bagrus (Sciades) emphysetus, 53, 54

Bagrus (Sciades) pictus, 334

Bagrus abbreviatus, 95

Bagrus Adansonii, 365

Bagrus affinis, 85, 107

Bagrus agricolus, 107

Bagrus albicans, 54

Bagrus albilabris, 95

Bagrus anisurus, 90

Bagrus aorellus, 107

Bagrus aorides, 106

Bagrus aorinus, 107

Bagrus arioides, 40, 54

Bagrus aurantiacus, 101

Bagrus auratus, 155

Bagrus bajad, 83

Bagrus barbatus, 44

Bagrus bayad macropterus, 83

Bagrus bayad var. macropterus, 84

Bagrus bilineatus, 49

Bagrus Birmannus, 95

Bagrus bouderius, 165

Bagrus buchanani, 361

Bagrus caeruleus, 84

Bagrus capensis, 43

Bagrus capito, 155

Bagrus carchariorhijnchos, 49

Bagrus chinta, 431

Bagrus corsula, 89

Bagrus couma, 53

Bagrus crinalis, 55

Bagrus degeni, 84

Bagrus depressirostris, 364

Bagrus docmac niger, 84

Bagrus docmak, 84, 313

Bagrus doroides, 45

Bagrus exodon, 359

Bagrus filamentosus, 84

Bagrus flavicans, 344

Bagrus flavus, 90

Bagrus fuscus, 95

Bagrus gagorides, 35

Bagrus genidens, 43, 44

Bagrus goliath, 331

Bagrus gulioides, 95

Bagrus Halepensis, 93, 94, 96, 97 
Bagrus heterurus, 97

Bagrus hoevenii, 88

Bagrus hypselopterus, 82

Bagrus Javensis, 45

Bagrus Keletius, 98

Bagrus koenigi, 84

Bagrus laevigatus, 49

Bagrus Lamarrii, 106, 107

Bagrus leucophasis, 96

Bagrus limbatus, 107

Bagrus lonah, 391

Bagrus lubosicus, 84

Bagrus macronemus, 37, 97

Bagrus macronemus, 93, 97

Bagrus Malabaricus, 96

Bagrus maurus, 158

Bagrus melas, 95

Bagrus meridionalis, 84

Bagrus mesops, 53

Bagrus micracanthus, 96

Bagrus micropogon, 92, 93

Bagrus montanus, 96

Bagrus nemurus, 87, 90

Bagrus netuma, 48, 49

Bagrus nigriceps, 96

Bagrus nigrita, 160

Bagrus nigropunctatus, 340

Bagrus oculatus, 96

Bagrus orientalis, 84

Bagrus passany, 54

Bagrus pemecus, 53

Bagrus planiceps, 90

Bagrus poecilopterus, 93

Bagrus proops, 54

Bagrus punctatus, 91

Bagrus punctulatus, 331, 339, 340

Bagrus reticulatus, 330, 331

Bagrus rhodonotus, 49

Bagrus rhodopterygius, 95

Bagrus rousseauxii, 331

Bagrus schilbeides, 362, 365

Bagrus Schlegelii, 36, 95

Bagrus shuwaiensis, 84

Bagrus Sieboldii, 90

Bagrus sinensis, 108

Bagrus singaringan, 97

Bagrus sondaicus, 45

Bagrus stenomus, 103, 104

Bagrus sumatranus, 36

Bagrus temminckianus, 55

Bagrus Temminckii, 55

Bagrus thalassinus, 49

Bagrus trachacanthus, 89

Bagrus trachipomus, 55

Bagrus ubangensis, 84

Bagrus urostigma, 84

Bagrus ussuriensis, 92, 100

Bagrus vachellii, 101

Bagrus venaticus, 55
Bagrus vertagus, 55

Bagrus wolffii, 98

Bagrus Wyckii, 91

Bagrus Yarrelli, 383

bahianus, Corymbophanes, 279

bahianus, Pareiorhaphis, 279

bahianus, Pimelodus, 195

bahianus, Trichomycterus, 415

bahiensis, Galeichthys, 38

bahiensis, Kalyptodoras, 172

bahiensis, Microlepidogaster, 281

bahiensis, Parotocinclus, 281

bahuaja, Crossoloricaria, 232

baileyi, Noturus, 211

bajad, Bagrus, 83

bajad, Silurus, 83

balayi, Parauchenoglanis, 162

balayi, Pimelodus, 162

ballayi, Auchenoglanis, 162

ballayi gravoti, Auchenoglanis, 162

ballayi var. gravoti, Auchenoglanis, 162

balsanus, Ictalurus, 208

balsanus, Istlarius, 208

balsanus occidentalis, Istlarius, 208

Balzanii, Vandellia, 427

bambui, Ituglanis, 406

banguela, Homodiaetus, 406

banguelensis, Eutropius, 363

banguelensis, Schilbe, 363

bankae, Chaca, 135

bankanensis, Chaca, 135

banneaui, Pygidium, 415

banneaui, Trichomycterus, 415

banneaui maracaiboensis, Pygidium, 420

barakensis, Pterocryptis, 441

barakensis, Sisor, 402

baramensis, Akysis, 16

baramensis, Hemibagrus, 87

baramensis, Kryptopterus, 369

baramensis, Liocassis, 93

baramensis, Macrones, 87

baramensis, Pseudobagarius, 16

barbancho, Pimelodus, 339

barbarmatus, Dentectus, 235

barbata, Loricaria, 298, 299

barbata, Microcambeva, 409

barbata, Rhamdia, 201

barbatula, Trichomycterus, 422

barbatum, Sturisoma, 299

barbatus, Bagrus, 44

barbatus, Callichthys, 129

barbatus, Hypostomus, 286, 287

barbatus, Malapterurus, 301

barbatus, Platysilurus, 340

barbatus, Pseudancistrus, 287

barbatus, Scleromystax, 129

barbatus, Xyliphius, 62 barbonica, Laimumena, 431, 432

barbouri, Pygidium, 415

barbouri, Trichomycterus, 415

barbus, Genidens, 44

barbus, Pimelodus, 44

barnardi, Austroglanis, 81

barnardi, Gephyroglanis, 81

baroni, Arius, 434

Baronis Mülleri, Pimelodus

(Rhamdia), 201

barrae, Ancistrus, 272

barrae, Megalancistrus, 272

barranquerensis, Ageneiosus, 69

bartonensis, Arius bartonensis, 56

bartonensis, Otolithus (Arius) crassus, 434

bartonensis, Otolithus (Arius) danicus, 434

bartoni, Neosilurus, 347

Baryancistrus, 226

Baryancistrus demantoides, 226

Baryancistrus longipinnis, 227

Baryancistrus niveatus, 227

basilewskii, Silurichthys, 381

bastari, Clupisoma, 357

bastiani, Synodontis, 312

batarensis, Pseudeutropius murius, 357

Batasinae, 81

Batasio, 81, 84

Batasio affinis, 85

Batasio batasio, 85, 86

Batasio buchanani, 84, 85

Batasio dayi, 85

Batasio elongatus, 85

Batasio fasciolatus, 442

Batasio havmolleri, 85

Batasio macronotus, 85

Batasio merianiensis, 85

Batasio niger, 445

Batasio pakistanicus, 85

Batasio sharavatiensis, 86

Batasio spilurus, 442

Batasio tengana, 86, 442

Batasio tigrinus, 86

Batasio travancoria, 86

batasio, Batasio, 85, 86

batasio, Pimelodus, 85

batasius, Pimelodus, 85, 98

batensoda, Brachysynodontis, 304

batensoda, Synodontis, 304

Batesi longispinis, Synodontis, 316

Batesi var. longispinis, Synodontis, 316

batesii, Chiloglanis, 304

batesii, Microsynodontis, 309

batesii, Synodontis, 312

Bathophilus, 412

Bathybagrus, 154

Bathybagrus tetranema, 154, 155 
Bathycetopsis, 131

Bathycetopsis oliveirai, 131, 132

Bathyclarias, 136, 148

Bathyclarias euryodon, 136

Bathyclarias filicibarbis, 148

Bathyclarias gigas, 149

Bathyclarias ilesi, 136

Bathyclarias longibarbis, 136

Bathyclarias loweae, 149

Bathyclarias rotundifrons, 136

Bathyclarias worthingtoni, 136

Bathypygidium, 412

bathyurus, Pimelodus, 201

Batrachocephalinae, 30

Batrachocephalus, 30, 38

Batrachocephalus ageneiosus, 38

Batrachocephalus micropogon, 38

Batrachocephalus mino, 38

Batrachoglanis, 352

batrachostoma, Gyrinurus, 409

batrachostoma, Ochmacanthus, 409

batrachus, Aspredo, 58

batrachus, Asterophysus, 70

batrachus, Clarias, 140

batrachus, Silurus, 140

Batrochoglanis, 352

Batrochoglanis acanthochiroides, 352

Batrochoglanis melanurus, 353

Batrochoglanis raninus, 353

Batrochoglanis transmontanus, 353

Batrochoglanis villosus, 353

batu, Clarias, 140

baudensis, Ancistrus, 220

baudoensis, Cetopsis, 131

baudoênsis, Pseudocetopsis, 131

baudoni, Amphilius, 21

baudoni uniformis, Amphilius, 21

baudoni var. uniformis, Amphilius, 21

bayad, Porcus, 83

bayad macropterus, Porcus, 83

bayad var. macropterus, Porcus, 84

Beadlei, Synechoglanis, 207, 210

beani, Pangasius, 328

beccarii, Vandellia, 427

bedado, Pangasius, 325

bedfordi, Silurus, 379

Belangerii, Arius, 54

beldti, Cryptopterella, 369, 371

belenos, Aspidoras, 108

belensis, Trichomycterus, 415

belgicus, Arius egertoni, 56

Belodontichthys, 368

Belodontichthys dinema, 368

Belodontichthys javanensis, 381

Belodontichthys macrochir, 368

Belodontichthys truncatus, 368

Belonoglanis, 24

Belonoglanis brieni, 24

Belonoglanis curvirostris, 24
Belonoglanis nudipectus, 24

Belonoglanis tenuis, 24

benderensis, Ictalurus, 206

benedettii, Nannorhamdia, 198

beninensis, Malapterurus, 301

bengalensis, Malapterus (Ailia), 356

beni, Hemiloricaria, 245

beni, Lepthoplosternum, 128

beni, Loricaria, 245

benjamini, Entomocorus, 74

benuensis, Chiloglanis, 305

berdmorei, Exostoma, 385

berdmorei, Pterocryptis, 376, 377

Berdmorei, Silurichthys, 376

Bergiaria, 329

Bergiaria platana, 330

Bergiaria westermanni, 330

Bergiella, 329

Bergiella platana, 330

berneyi, Neoarius, 47

berneyi, Tachysurus (Pararius), 47

bernhardi, Hemipimelodus, 39

bertoni, Branchioica, 410

bertoni, Corydoras, 130

bertonii, Branchioica, 410

bicarinatus, Chaenothorax, 111, 125

bicirrhis, Kryptopterus, 369

bicirrhis, Silurus, 369

bicirrosus, Plecostomus, 259

bicolor, Arius, 34

bicolor, Bunocephalus, 59

bicolor, Corydoras, 114

bicolor, Hemipimelodus, 34

bicolor, Leiocassis, 104

bicolor, Phractocephalus, 336

bicolor, Pirarara, 336

bidentatus, Parotocinclus, 282

bidorsalis, Clarotes, 160

bidorsalis, Heterobranchus, 150

bifasciata, Taunayia, 203

bifasciatus, Corydoras, 114

bifasciatus, Nannoglanis, 203

biffi, Notarius, 49

bifidus, Bunocephalus, 59

bifurcus, Chiloglanis, 305

bilineata, Netuma, 49

bilineatus, Bagrus, 49

bilineatus, Corydoras, 114

bilineatus, Epactionotus, 236

bilineatus, Pimelodus, 107

bilobatum, Hypoptopoma, 250

bimaculatus, Macrones, 94

bimaculatus, Mystus, 94

bimaculatus, Ompok, 372

bimaculatus, Silurus, 372

binotata, Synodontis notatus, 318

binotatus, Ompok, 372

Birmannus, Bagrus, 95

biscutatus, Auchenoglanis, 154 biscutatus, Pimelodus, 154

biseriata, Squaliforma, 297

biseriatus, Plecostomus, 297

bistriata, Ansorgia vittata, 361

biwaensis, Parasilurus, 379

biwaensis, Silurus, 379

bleekeri, Arius, 46

bleekeri, Cryptopterus, 374

bleekeri, Heptapterus, 184

bleekeri, Macrones, 89, 94

bleekeri, Micronema, 374

bleekeri, Mystus, 94

bleekeri, Nemapteryx, 46

bleekeri, Paracetopsis, 134

bleekeri, Phalacronotus, 374

blochi, Corydoras, 114

blochi vittatus, Corydoras, 126

Blochii, Cotylephorus, 61

blochii, Doras, 167

Blochii, Galeichthys, 38

Blochii, Osteogeneiosus, 51

blochii, Pimelodus, 336, 337

blohmi, Epapterus, 74

Blythii, Exostoma, 396

Blythii, Macrones, 85

blythii, Myersglanis, 396

bo, Macrones, 88

boakeii, Arius, 34

boalis, Silurus, 380

bocagii, Chrysichthys, 155

Bocagii, Eutropius, 363

bocagii, Schilbe, 363

bockmanni, Glanidium, 75

bocourti, Heterobagrus, 94

bocourti, Mystus, 94

bocourti, Pangasius, 325

Bocourti, Pangasius

(Pseudopangasius), 325

bodenhameri, Ancistrus, 219

boehlkei, Corydoras, 114

boehlkei, Rhinodoras, 178

boemia, Tatia, 77

boesemani, Corydoras, 114

bogotense, Pygidium, 415

bogotensis, Trichomycterus, 415

bokorensis, Penesilurus, 376

bokorensis, Pterocryptis, 376

bolivarensis, Amblydoras, 168

bolivarensis, Hildadoras, 168

boliviana, Farlowella, 238

boliviana, Phenacorhamdia, 188

boliviana, Pimelodella, 189

boliviana, Xenocara, 219

bolivianensis, Andinichthys, 30

bolivianus, Ancistrus, 219

bolivianus, Corydoras, 117

bolivianus, Hypostomus, 252

bolivianus, Imparfinis, 188

bolivianus, Plecostomus, 252 
bolli, Amiurus, 205

bolteni, Callichthys callichthys, 111

bomae, Eutropius, 363

bomboides, Pseudomystus, 103

bomboizanum, Pygidium, 416

bomboizanus, Trichomycterus, 416

bondi, Acanthopoma, 413

bondi, Corydoras, 114

bondi, Schultzichthys, 413

bondi coppenamensis, Corydoras, 115

bongan, Hemibagrus, 87

bongan, Macrones, 87

bonillai, Ariopsis, 33

bonillai, Galeichthys, 33

bonneti, Arius, 36, 56

boquillae, Cetopsorhamdia, 181

borealis, Silurus (Pimelodus), 210

borellii, Hypostomus, 252

borellii, Plecostomus, 252

borellii, Trichomycterus, 416

borneensis, Callichrous (Silurodes), 372

borneensis, Cephalocassis, 41, 42

borneensis, Ompok, 372

borneёnsis, Arius, 56

borneёnsis, Pimelodus, 41

borodini, Imparfinis, 185

bororo, Otocinclus, 274

börressoni, Chrysichthys, 156

bortayro, Silvinichthys, 413

boschmai, Pimelodella, 190

bostocki, Tandanus, 352

bostockii, Cnidoglanis, 346

boticario, Listrura, 408

botius, Glyptothorax, 388

botius, Pimelodus, 388

Boucardi, Pimelodus, 201

bouderius, Bagrus, 165

bouderius, Cranoglanis, 165

bouilloni, Leptoglanis, 164

boujardi, Lithoxus, 267

boulengeri, Arges, 63,

boulengeri, Astroblepus, 63

boulengeri, Euchilichthys, 309

boulengeri, Hemidoras, 175

boulengeri, Hemidoras (Leptodoras), 175

boulengeri, Heterobranchus, 151

boulengeri, Hypostomus, 252

boulengeri, Opsodoras, 172, 175

boulengeri, Plecostomus, 252

bourguyi, Microlepidogaster, 300

boutchangai, Anaspidoglanis, 154

boutchangai, Parauchenoglanis, 154

bouvieri, Schilbe, 364

bovallii, Ancistrus, 267

bovallii, Lithoxus, 267

bovei, Peltura, 26

bovei, Phractura, 26 boylei, Pygidium, 416

boylei, Trichomycterus, 416

brachiatus, Centrodoras, 170

brachiatus, Doras, 170

Brachspondylus cretaceus, 432

brachyacanthus, Amiurus, 205

brachybarbatus, Akysis, 13

brachycephalus, Arges, 63

brachycephalus, Astroblepus, 63

brachycephalus, Pimelodus, 198

Brachyglanis, 180

Brachyglanis frenata, 180

Brachyglanis frenatus, 180

Brachyglanis magoi, 180

Brachyglanis melas, 181

Brachyglanis microphthalmus, 181

Brachyglanis nocturnus, 181

Brachyglanis phalacra, 181

Brachymystus, 87

brachynema, Chrysichthys, 155

brachynema, Chasmocranus, 182

brachynemus, Chasmocranus, 182

Brachyplatystoma, 329, 330

Brachyplatystoma capapretum, 330

Brachyplatystoma filamentosum, 330

Brachyplatystoma goeldii, 330

Brachyplatystoma juruense, 331

Brachyplatystoma paraense, 331

Brachyplatystoma parnahybae, 331

Brachyplatystoma platynema, 331

Brachyplatystoma platynemum, 331

Brachyplatystoma promagdalena, 331

Brachyplatystoma rousseauxii, 331

Brachyplatystoma tigrinum, 331

Brachyplatystoma vaillantii, 331

Brachyplatystomatini, 329

brachypopterus, Eutropius, 362

brachypopterus, Pseudeutropius, 362

brachypterus, Pimelodus (Rhamdia), 198

Brachyrhamdia, 181

Brachyrhamdia heteropleura, 181

Brachyrhamdia imitator, 181

Brachyrhamdia marthae, 181

Brachyrhamdia meesi, 181

Brachyrhamdia rambarrani, 181

brachyrhyncha, Pareiorhina, 281

brachysoma, Clarias, 140

brachysoma, Horabagrus, 429

brachysoma, Pseudobagrus, 429

brachysoma, Pseudexostoma, 401

Brachyspondylus, 432

Brachyspondylus indicus, 326

Brachyspondylus saropterix, 82

Brachystacus, 135

Brachysynodontis, 304

Brachysynodontis batensoda, 304

brachyura, Dekeyseria, 234

brachyurus, Ancistrus, 234 brachyurus, Auchenipterus, 72

brachyurus, Euanemus, 72

Branchioica, 410

Branchioica bertoni, 410

Branchioica bertonii, 410

Branchioica magdalenae, 411

Branchioica phaneronema, 411

Branchiosteus, 17

Brandtii, Arius, 54

branickii, Chaetostoma, 228

branickii, Chaetostomus, 228

branneri, Rhamdia, 201

branneri voulezi, Rhamdia, 201

bransfordi, Loricaria, 240

bransfordii, Rhamdia, 201

brashnikowi, Leiocassis, 100

brashnikowi, Macrones (Leiocassis), 100

brashnikowi, Pelteobagrus, 100

brasiliensis, Caecorhamdella, 189, 190

brasiliensis, Pimelodella, 190, 192

brasiliensis, Pimelodus

(Pseudorhamdia), 192

brasiliensis, Plecostomus, 259

brasiliensis, Trichomycterus, 416

brasiliensis itatiayae, Trichomycterus, 419

brasiliensis tristis, Trichomycterus, 416

braueri, Hemiancistrus, 284

braueri, Peckoltia, 284

breei, Corydoras, 114

Breitensteinia, 15

Breitensteinia cessator, 15

Breitensteinia hypselurus, 15

Breitensteinia insignis, 15

breve, Chaetostoma, 228

brevianalis, Eutropius, 363

brevianalis, Pseudobagrus, 101

brevianalis, Schilbe, 363

brevibarbis, Ancharius, 29

brevibarbis, Auchenipterus, 80

brevibarbis, Chiloglanis, 305

brevibarbis, Chrysichthys, 156

brevibarbis, Chrysobagrus, 155, 156

brevibarbis, Clariallabes, 137

brevibarbis, Gogo, 29

brevibarbus, Plotosus, 351

brevicauda, Hypostomus, 252

brevicauda, Phractura, 26

brevicauda, Plecostomus, 252

brevicaudatus, Leiocassis, 102

brevicaudatus, Pseudobagrus, 102

Brevicephaloides, 139

Breviceps, 37

breviceps, Clarias, 141

breviceps, Liocassis, 103

breviceps, Pimelodella, 190

breviceps, Pimelodus, 190 
breviceps, Pseudomystus, 103

brevicorpus, Coreobagrus, 86

brevidorsalis, Amphilius, 23

brevidorsalis, Copidoglanis, 347

brevidorsalis, Neosilurus, 347

brevifilis, Ageneiosus, 68, 69

brevifilis, Ancistrus, 219

brevifilis bodenhameri, Ancistrus, 219

Breviglanis, 180

brevilabiatum, Chaetostoma, 228

brevilabiatus, Chaetostomus, 228

brevinuchalis, Clarias, 140

brevior, Allabenchelys, 137

brevior, Andersonia, 24

brevior, Auchenipterus, 72

brevior, Chasmocranus, 182

brevipinna, Amphilius

grammatophorus, 22

brevipinna, Amphilius

grammatophorus var., 23

brevipinnis, Ancistrus, 219

brevipinnis, Glyptothorax, 388

brevipinnis, Xenocara, 219

brevipinnis alaknandi, Glyptothorax, 388

brevirostre, Sturisoma, 299

brevirostris, Arius, 31

brevirostris, Corydoras, 114

brevirostris, Loricaria, 299

brevirostris, Loricaria (Rineloricaria), 298

brevis, Ageneiosus, 69

brevis, Amphilius, 21

brevis, Chaetostomus, 228

brevis, Delturus, 443

brevis, Doras (Corydoras), 179

brevis, Hemiancistrus, 284

brevis, Hypostomus, 252

brevis, Leptoglanis, 28

brevis, Mochocus, 310

brevis, Mochokus, 310

brevis, Peckoltia, 284

brevis, Pimelodus, 337

brevis, Plecostomus, 252

brevis, Trachydoras, 179

brevis, Tridens, 426

brevis, Tridensimilis, 426

brevis, Zaireichthys, 28

brevispinis, Lasiancistrus, 287

brevispinis, Lophiobagrus, 161

brevispinis, Pseudancistrus, 287

brevitentaculatus, Hypostomus, 291

brichardi, Phyllonemus, 164

brichardi, Synodontis, 312

brieni, Belonoglanis, 24

britskii, Auchenipterus, 72

britskii, Brochis, 114

britskii, Corydoras, 114

britskii, Parotocinclus, 282 broadbenti, Tachysurus, 51

Brochiloricaria, 227

Brochiloricaria chauliodon, 227

Brochiloricaria macrodon, 227

Brochis, 111

Brochis britskii, 114

Brochis coeruleus, 111, 125

Brochis dipterus, 111, 125

Brochis multiradiatus, 120

Brochis splendens, 125

Brontes, 63

Brontes prenadilla, 63, 66

brunellii, Arius, 31

brunnea, Loricaria, 269

brunnea, Tatia, 77

brunnescens, Doras, 168

brunneus, Ameiurus, 204

brunneus, Amiurus, 204

brunneus, Aspidoras, 109

brunneus, Loricariichthys, 269

Brustiarius, 38

Brustiarius nox, 38

Brustiarius solidus, 39

buccata, Pterocryptis, 376

bucculenta, Nangra, 396

Buchanani, Arius, 34

Buchanani, Bagarius, 383

buchanani, Bagrus, 361

Buchanani, Batasio, 84, 85

buchanani, Chaca, 136

buchanani, Glyptothorax, 388

buchanani, Hara, 395

Buchanani, Nangra, 397

Buchanani, Pangasius, 325, 327

buchanani, Proeutropiichthys, 361

buchanani, Rama, 105

Buchanani, Rita, 105, 106

Bucklandium, 432

Bucklandium diluvii, 432, 433

buckleyi, Pimelodella, 190

buckleyi, Pimelodus, 190

budgetti, Clarias, 140

budgetti, Synodontis, 312

buettikoferi, Clarias, 140

buettikoferi, Parauchenoglanis, 163

buffei, Eutropiellus, 361

buffei, Pareutropius 360

bufonia, Xenocara, 219

bufonius, Ancistrus, 219

bufonius, Pimelodus, 355

bufonius, Pseudopimelodus, 355

bufonius, Xenocara, 219

bullocki, Hatcheria, 404

Bullockia, 403

Bullockia maldonadoi, 404

bulumae, Clarias, 140

Bunocephalichthys, 58

Bunocephalichthys gronovii, 60

Bunocephalini, 57
Bunocephalus, 57, 58

Bunocephalus albifasciatus, 62

Bunocephalus aleuropsis, 58

Bunocephalus amaurus, 58

Bunocephalus amaurus aloikae, 58

Bunocephalus amaurus sipaliwini, 58

Bunocephalus amazonicus, 59

Bunocephalus bicolor, 59

Bunocephalus bifidus, 59

Bunocephalus carvalhoi, 59

Bunocephalus chamaizelus, 59

Bunocephalus colombianus, 59

Bunocephalus coracoideus, 59

Bunocephalus depressus, 62

Bunocephalus dolichurus, 62

Bunocephalus doriae, 59

Bunocephalus haggini, 59

Bunocephalus hypsiurus, 57

Bunocephalus iheringii, 59

Bunocephalus knerii, 59

Bunocephalus larai, 59

Bunocephalus melas, 58

Bunocephalus minutus, 59

Bunocephalus quadriradiatus, 60

Bunocephalus retropinnis, 59

Bunocephalus rugosus, 60

Bunocephalus salathei, 59

Bunocephalus scabriceps, 58, 60

Bunocephalus verrucosus, 60

burgessi, Corydoras, 114

burgini, Pangasius, 326

burmanensis, Pterocryptis, 376

burmanensis, Silurus, 376

burmanica, Cochlefelis, 42

burmanica, Olyra, 99

burmanicus, Arius, 42

burmanicus, Glyptothorax, 388

burmanicus, Proeutropiichthys

taakree, 362

burmannicus, Eutropiichthys, 358

Burmeisteri, Pygidium, 405

burmensis, Chaca, 135

butantanis, Plecostomus margaritifer, 256

butcheri, Pseudopimelodus villosus, 352

buthupogon, Clarias, 141

butleri, Paraplotosus, 349

Büttikoferi, Acrochordonichthys, 13

Büttikoferi, Auchenoglanis, 163

Büttikoferi, Clarias, 141

Büttikoferi, Chrysichthys, 158

C

cabrerae, Rhamdia, 198

cacerensis, Hemiloricaria, 245

cacerensis, Loricaria, 245

cacharioides, Arius, 166

cadeae, Loricaria, 294

cadeae, Rineloricaria, 293 
Caecorhamdella, 189

Caecorhamdella brasiliensis, 189, 192

Caecorhamdia, 197

Caecorhamdia urichi, 197, 202

caecutiens, Amblyceps, 17, 18

Caelatoglanis, 382, 383

Caelatoglanis zonatus, 383

caelata, Nemapteryx, 46

calatus, Arius, 46

caelatoides, Arius, 55

caerulescens, Pimelodus, 81

caeruleus, Bagrus, 84

caerulorostris, Platypogon, 339, 340

caesius, Plotosus, 350

caesius, Propimelodus, 443

cahuali, Tridentopsis, 426

cahyensis, Ituglanis, 444

caiapo, Cetopsis, 131

Cainosilurus, 347

calamita, Hypostomus, 219

Calichthys, 110

caliense, Pygidium, 416

caliensis, Trichomycterus, 416

callarias, Silurus, 313

Callichrous, 371

Callichrous (Silurodes) borneensis, 372

Callichrous ceylonensis, 372

Callichrous egertonii, 374

Callichrous eugeneiatus, 369

Callichrous nigrescens, 374

Callichrous notatus, 374

Callichrous Sindensis, 373

Callichrous weberi, 373

Callichthini, 108

Callichthyidae, 108

Callichthys, 108, 110

Callichthys adspersus, 127

Callichthys affinis, 111

Callichthys albidus, 128

Callichthys arcifer, 111

Callichthys armatus, 113

Callichthys asper, 110, 167

Callichthys barbatus, 129

Callichthys callichthys, 110

Callichthys callichthys bolteni, 111

Callichthys callichthys demararae, 111

Callichthys chiquitos, 128

Callichthys exaratus, 129

Callichthys fabricioi, 111

Callichthys hemiphractus, 111

Callichthys kneri, 111

Callichthys laevigatus, 127

Callichthys littoralis, 127

Callichthys longifilis, 129

Callichthys loricatus, 111

Callichthys melampterus, 127, 128

Callichthys oibaensis, 111

Callichthys paleatus, 122
Callichthys pectoralis, 128

Callichthys pictus, 129

Callichthys serralabium, 111

Callichthys splendens, 125

Callichthys sulcatus, 129

Callichthys taiosh, 125

Callichthys tamoata, 111

Callichthys thoracatus, 129

callichthys, Callichthys, 110

callichthys bolteni, Callichthys, 111

callichthys demararae, Callichthys, 111

Callomystax, 385

Callomystax Schmidti, 392

callopterus, Glyptothorax, 388

calmoni, Hemipsilichthys, 279

Calophysinae, 329

Calophysus, 329, 332

Calophysus macropterus, 332

calvarius, Silurus, 99, 100

calvus, Isbrueckerichthys, 441

calvus, Rhineastes, 207

camaronensis, Chrysichthys, 158

camelopardalis, Synodontis, 312

cameronensis, Chiloglanis, 305

cameronensis, Chrysichthys, 158

cameronensis, Clarias, 144

cameronensis, Eutropius, 365

cameroni, Hemipsilichthys, 279

cameroni, Pareiorhaphis, 279

cameroni, Psilichthys, 247, 279

camerunensis, Clarias, 139, 141, 144

camerunensis, Leptoglanis, 28

camerunensis, Zaireichthys, 28

camposensis, Diplomystes, 165

camposi, Eremophilus, 408

camposi, Listrura, 408

canaliferus, Otothyris, 275, 276

canarensis, Mystus, 96

cancellatus, Ameiurus, 211

candidus, Eremophilus, 416

candidus, Trichomycterus, 416

candiru, Cetopsis, 131

candombe, Hisonotus, 440

canensis, Acanthicus, 266

canensis, Leptoancistrus, 266

canio, Silurus, 373, 374

canius, Plotosus, 350

Canthopomus, 289

Canthopomus montebelloi, 263

Cantonensis, Pimelodus, 431

cantoris, Osteogeneiosus, 51

capapretum, Brachyplatystoma, 330

caparary, Sorubim, 341

Capellonis, Arius, 32

capensis, Bagrus, 43

capensis, Clarias, 143

capetensis, Dasyloricaria, 233

capetensis, Loricaria, 233 capito, Bagrus, 155

capitulum, Macrones fortis, 88

caquetae, Astroblepus, 63

caquetae, Corydoras, 119

caquetae, Euacanthagenys, 296

caquetae, Harttia, 300

caquetae, Hemiancistrus, 265

caquetae, Loricaria, 296

caquetae, Spatuloricaria, 296

caquetae, Sturisomatichthys, 300

caracasensis, Hemiloricaria, 244, 245

caracassensis, Hemiloricaria, 245

carachama, Monistiancistrus, 289

carcharhinoides, Nangra, 396

carchariorhijnchos, Bagrus, 49

carcio, Pimelodus, 85, 98

carinata, Farlowella, 237

carinata, Loricaria, 268

carinata, Oxyropsis, 276

carinata, Trewavasia, 433

carinatum, Amblyceps, 18

carinatum, Hypoptopoma, 276

carinatus, Arius (Hemiarius), 42

carinatus, Cinetodus, 42

carinatus, Doras, 171, 173

carinatus, Hypostomus, 252

carinatus, Plecostomus, 252

carinatus, Silurus, 170, 171

carinatus, Xenopholis, 433

Carinotus, 234

carinotus, Delturus, 235

carinotus, Plecostomus (Carinotus), 234, 235

carlae, Corydoras, 115

Carnaticus, Pimelodus, 383

carnegiei, Dolichancistrus, 235

carnegiei, Pseudancistrus, 235

carnosus, Chiloglanis, 305

carnosus, Pseudomystus, 103

carolinensis, Mystus, 37

carrancas, Pareiorhina, 281

carrioni, Lipopterichthys, 266, 267

carsonii, Clarias, 145

carvalhoi, Aspidoras, 109

carvalhoi, Bunocephalus, 59

carvalhoi, Harttia, 240

carvalhoi, Hypostomus, 252

carvalhoi, Plecostomus, 252

Cascadura, 108, 127

Cascadura maculocephala, 127, 128

Cascaduridi, 108

cashibo, Loricaria, 270

cashibo, Loricariichthys, 270

castanea, Loricaria, 270

castaneoüdes, Plotosus, 351

castaneo-ventris, Doras, 168

castaneus, Helogenes, 134

castaneus, Leyvaichthys, 134, 407

castaneus, Loricariichthys, 270 
castaneus, Mystus, 94

castaneus, Plotosus, 351

castelnaui, Hemiancistrus, 265

castroi, Hemiloricaria, 245

castroi, Rineloricaria, 245

castroi, Trichomycterus, 416

catamarcensis, Loricaria, 294

catamarcensis, Rineloricaria, 294

catamarcensis, Trichomycterus, 416

cataniai, Leptodoras, 172

cataphracta, Loricaria, 268, 270

Cataphractops, 127

Cataphractus, 110, 167

Cataphractus americanus, 167

Cataphractus callichthys, 111

Cataphractus depressus, 111

Cataphractus punctatus, 111, 123

cataphractus, Plecostomus, 270

cataphractus, Silurus, 167, 168

cataractus, Clarias, 141

cataractus, Phagorus, 141

Catastoma, 48

catharinensis, Glanidium, 75

Cathorops, 39

Cathorops agassizii, 39

Cathorops aguadulce, 39

Cathorops arenatus, 39

Cathorops dasycephalus, 39

Cathorops fuerthii, 39

Cathorops hypophthalmus, 40

Cathorops mapale, 40

Cathorops melanopus, 40

Cathorops multiradiatus, 40

Cathorops spixii, 40

Cathorops steindachneri, 40

Cathorops tuyra, 40

catulus, Pimelodus, 205

catus, Ameiurus, 204

catus, Silurus, 204

caucanus, Ageneiosus, 70

caucanus, Ancistrus, 219

caucanus, Lasiancistrus, 264

caudalis, Synodontis, 312

cauda-furcatus, Pimelodus, 209

caudimaculatus, Corydoras, 115

caudofasciatus, Trichomycterus, 416

caudivittatus, Paradoxoglanis, 302

caudovittata, Synodontis, 312

caudovittatus, Synodontis, 313

cavalliensis, Malapterurus, 301

cavasius, Pimelodus, 95

cavasius, Mystus, 95

cavatica, Peckoltia, 284

cavatura, Erethistoides, 384

cavatus, Arius, 435

caveatus, Hemibagrus, 87

cavernicola, Clarias, 141

cavia, Glyptothorax, 388

cavia, Pimelodus, 388 caxarari, Otocinclus, 274

cayennae, Hoplosternum thoracatum, 128

cacutiens, Amblyceps, 18

cacutiens, Silurus, 131

calatus, Arius, 46

calatus, Callichthys, 111

carulescens, Arius, 33

carulescens, Pimelodus, 81

cearensis, Parotocinclus, 282

celsae, Trichomycterus, 416

cenia, Gagata, 386

cenia, Pimelodus, 386

centralis, Clariallabes, 137

centralis, Clarias, 137

centralis, Synodontis, 313

centralus, Hemibagrus, 87

Centranodon japonicus, 379

Centrochir, 170

Centrochir crocodili, 170

Centrochirinae, 166

Centrodoras, 170

Centrodoras brachiatus, 170

Centrodoras hasemani, 170

centrolepis, Ancistrus, 219

Centromochli, 68

Centromochlus, 68, 73

Centromochlus (Gephyromochlus)

leopardus, 75

Centromochlus altae, 73

Centromochlus aulopygius, 77

Centromochlus concolor, 73

Centromochlus creutzbergi, 77

Centromochlus dunni, 77

Centromochlus existimatus, 73

Centromochlus gyrinus, 77

Centromochlus heckelii, 73

Centromochlus intermedius, 77

Centromochlus macracanthus, 73

Centromochlus megalops, 73

Centromochlus perugiae, 73

Centromochlus punctatus, 73

Centromochlus reticulatus, 73

Centromochlus romani, 73

Centromochlus schultzi, 73

Centromochlus Steindachneri, 73

Centrophorus, 411

cephalacanthus, Otocinclus, 276

cephalaspis, Crossoloricaria, 232

Cephalocassis, 41

Cephalocassis borneensis, 41, 42

Cephalocassis jatia, 41

Cephalocassis manillensis, 41

Cephalocassis melanochir, 41

Cephalocassis Stormii, 44, 45

Cephalosilurus, 353

Cephalosilurus albomarginatus, 353

Cephalosilurus apurensis, 353

Cephalosilurus fowleri, 353
Cephalosilurus nigricaudus, 353

Ceratocheilus, 71

Ceratocheilus osteomystax, 71, 72

Ceratoglanis, 368

Ceratoglanis pachynema, 368

Ceratoglanis pachynemus, 368

Ceratoglanis scleronemus, 368

ceratophysus, Auchenipterus, 79

ceratophysus, Trachelyopterus, 79

Ceratorhynchus, 68

cerosus, Hemipsilichthys, 279

cerosus, Pareiorhaphis, 279

Cerulescens, Pimelodus, 211

Cerulescens, Silurus, 207, 208, 211

cerulescens melanurus, Silurus, 209

cerulescens var. melanurus, Silurus, 209

cervinus, Corydoras, 115

cesarpintoi, Glanidium, 75

cesarpintoi, Paraotocinclus, 282

cesarpintoi, Parotocinclus, 282

cessator, Breitensteinia, 15

Cetopagansius, 324

Cetopangasius, 324

Cetopangasius chaetobranchus, 324

Cetopsidae, 130

Cetopsidium, 130

Cetopsidium ferreirai, 130

Cetopsidium minutum, 130

Cetopsidium morenoi, 130

Cetopsidium orientale, 130

Cetopsidium pemon, 131

Cetopsidium roae, 131

Cetopsini, 130

Cetopsis, 130, 131

Cetopsis amphiloxa, 131

Cetopsis arcana, 131

Cetopsis baudoensis, 131

Cetopsis caiapo, 131

Cetopsis candiru, 131

Cetopsis chalmersi, 132

Cetopsis coecutiens, 131

Cetopsis fimbriata, 132

Cetopsis gobioides, 131, 132

Cetopsis jurubidae, 132

Cetopsis macroteronema, 133

Cetopsis montana, 132

Cetopsis motatanensis, 132

Cetopsis occidentalis, 134, 135

Cetopsis oliveirai, 132

Cetopsis orinoco, 132

Cetopsis othonops, 132

Cetopsis parma, 132

Cetopsis pearsoni, 132

Cetopsis plumbea, 133

Cetopsis plumbeus, 133

Cetopsis sandrae, 133

Cetopsis sarcodes, 133

Cetopsis Spixii, 131 
Cetopsis starnesi, 133

Cetopsis umbrosa, 133

Cetopsis ventralis, 135

Cetopsogiton, 135

Cetopsorhamdia, 181

Cetopsorhamdia boquillae, 181

Cetopsorhamdia filamentosa, 182

Cetopsorhamdia iheringi, 182

Cetopsorhamdia insidiosa, 182

Cetopsorhamdia molinae, 182

Cetopsorhamdia nasus, 181, 182

Cetopsorhamdia orinoco, 182

Cetopsorhamdia phantasia, 182

Cetopsorhamdia picklei, 182

Cetopsorhamdia pijpersi, 186

Cetopsorhamdia shermani, 182

ceylonensis, Callichrous, 372

chabanaudi, Atopochilus, 303

chaberti, Trichomycterus, 417

chabutensis, Bachmannia, 428

Chaca, 135

Chaca bankae, 135

Chaca bankanensis, 135

Chaca burmensis, 135

Chaca chaca, 136

Chaca hamiltonii, 135, 136

Chaca lophioides, 135, 136

chaca, Chaca, 136

chaca, Platystacus, 135, 136

Chacidae, 135

Chacini, 135

Chaenothorax, 111

Chaenothorax bicarinatus, 111, 125

Chaenothorax eigenmanni, 125

Chaenothorax multiradiatus, 120

chaetobranchus, Cetopangasius, 324

Chaetostoma, 227

Chaetostoma aburrense, 227

Chaetostoma aequinoctiale, 227

Chaetostoma alternifasciatum, 227

Chaetostoma anale, 227

Chaetostoma anomala sovichthys, 230

Chaetostoma anomalum, 227

Chaetostoma branickii, 228

Chaetostoma breve, 228

Chaetostoma brevilabiatum, 228

Chaetostoma changae, 444

Chaetostoma daidalmatos, 444

Chaetostoma dermorhynchum, 228

Chaetostoma dorsale, 228

Chaetostoma dupouii, 228

Chaetostoma fischeri, 228

Chaetostoma greeni, 228

Chaetostoma guairense, 228

Chaetostoma jegui, 228

Chaetostoma lepturum, 229

Chaetostoma leucomelas, 229

Chaetostoma lineopunctata, 229

Chaetostoma lineopunctatum, 229
Chaetostoma loborhynchos, 227, 229

Chaetostoma machiquense, 229

Chaetostoma machiquensis, 229

Chaetostoma marcapatae, 229

Chaetostoma marginatum, 229

Chaetostoma marmorescens, 229

Chaetostoma microps, 229

Chaetostoma milesi, 229

Chaetostoma mollinasum, 230

Chaetostoma niveum, 230

Chaetostoma nudirostre, 230

Chaetostoma palmeri, 230

Chaetostoma patiae, 230

Chaetostoma paucispinis, 230

Chaetostoma pearsei, 230

Chaetostoma sericeum, 230

Chaetostoma sovichthys, 230

Chaetostoma stannii, 230

Chaetostoma stroumpoulos, 444

Chaetostoma tachiraense, 230

Chaetostoma tachiraensis, 230

Chaetostoma taczanowskii, 231

Chaetostoma thomsoni, 231

Chaetostoma vagum, 231

Chaetostoma vasquezi, 231

Chaetostoma venezuelae, 231

Chaetostoma yurubiense, 231

Chaetostomidi, 217

Chaetostomus, 217

Chaetostomus (Ancistrus) cirrhosus maculatus, 222

Chaetostomus (Ancistrus) cirrhosus punctata, 222

Chaetostomus (Ancistrus) cirrhosus var. maculatus, 222

Chaetostomus (Ancistrus) cirrhosus var. punctata, 222

Chaetostomus aculeatus, 272

Chaetostomus aequinoctialis, 227

Chaetostomus alga, 221

Chaetostomus annae, 297

Chaetostomus anomalus, 227

Chaetostomus aspidolepis, 242

Chaetostomus bachi, 284

Chaetostomus branickii, 228

Chaetostomus brevilabiatus, 228

Chaetostomus brevis, 228

Chaetostomus cirrhosus var. maculata, 228

Chaetostomus cochliodon, 278

Chaetostomus dentex, 277

Chaetostomus depressus, 287

Chaetostomus dermorhynchus, 228

Chaetostomus dorsalis, 228

Chaetostomus eptingi, 253

Chaetostomus Fischeri, 228

Chaetostomus Fordii, 286

Chaetostomus furcatus, 284

Chaetostomus gibbosus, 278
Chaetostomus gigas, 272

Chaetostomus guairensis, 228

Chaetostomus heteracanthus, 264, 265

Chatostomus hoplogenys, 221

Chaetostomus Jelskii, 222

Chaetostomus latifrons, 219, 222

Chaetostomus lepturus, 229

Chaetostomus leucomelas, 229

Chaetostomus leucostictus, 222

Chaetostomus macrops, 243

Chaetostomus maculatus, 228

Chaetostomus malacops, 222

Chaetostomus marcapatae, 229

Chaetostomus marginatus, 229

Chaetostomus megacephalus, 244

Chaetostomus microps, 229

Chaetostomus milesi, 229

Chaetostomus mollinasus, 230

Chaetostomus nigrolineatus, 277, 278

Chaetostomus nudirostris, 230

Chaetostomus oligospilus, 284

Chaetostomus palmeri, 230

Chaetostomus paucispinis, 230

Chaetostomus pearsei, 230

Chaetostomus platycephalus, 231

Chaetostomus punctatissimus, 274

Chaetostomus schomburgkii, 265

Chaetostomus sericeus, 230

Chaetostomus setosus, 235

Chaetostomus Stannii, 230

Chaetostomus Taczanowskii, 231

Chaetostomus tectirostris, 221

Chaetostomus thomsoni, 231

Chaetostomus trinitatis, 225

Chaetostomus undecimalis, 292

Chaetostomus vagus, 231

Chaetostomus variolus, 225

Chaetostomus vittatus, 283, 285

chagresi, Ancistrus, 220, 222

chagresi, Pimelodella, 190

Chagresi, Pimelodus

(Pseudorhamdia), 190

chagresi odynea, Pimelodella, 193

chalmersi, Cetopsis, 132

chamaeleon, Acrochordonichthys, 12

chamaeleon, Sosia, 12

Chamaigenes, 57

chamaizelus, Bunocephalus, 59

Chandramara, 86

Chandramara chandramara, 86, 105

chandramara, Chandramara, 86, 105

chandramara, Pimelodus, 86

chandramara, Rama, 86

changae, Chaetostoma, 444

changae, Panaqolus, 277

changae, Panaque, 277

changi, Pseudobagrus, 103

chanjoo, Loricariichthys, 270

chanjoo, Parahemiodon, 270 
Channallabes, 137

Channallabes apus, 137

chantrei, Silurus, 379

chaparae, Pimelodella, 190

chaparae, Plecostomus, 226

Chaperi, Diastatomycter, 368

chapini, Acanthocleithron, 303

chapmani, Astroblepus, 63

chapmani, Cyclopium, 63

chapmani, Pygidium, 417

chapmani, Trichomycterus, 417

chappuisi, Doumea, 25

chaquensis, Epapterus, 74

charmuth, Macropteronotus, 138, 148

charrua, Hisonotus, 440

charus, Pimelodus, 355

charus, Pseudopimelodus, 355

chaseni, Leiocassis, 93

Chasmocephalus, 182

Chasmocranes rosae, 183

Chasmocranus, 182

Chasmocranus brachynema, 182

Chasmocranus brachynemus, 182

Chasmocranus brevior, 182

Chasmocranus chimantanus, 183

Chasmocranus longior, 182, 183

Chasmocranus lopezi, 183

Chasmocranus peruanus, 183

Chasmocranus quadrizonatus, 183

Chasmocranus rosae, 183

Chasmocranus surinamensis, 183

Chasmocranus truncatorostris, 183

chaudhurii, Glyptosternum, 385

chauliodon, Brochiloricaria, 227

chechra, Silurus, 374

Cheiridodus, 250

Cheirocerus, 332

Cheirocerus abuelo, 332

Cheirocerus eques, 332

Cheirocerus goeldii, 332

chennuah, Sisor, 402

Cheveyi, Cryptopterus, 371

cheveyi, Micronema, 371

childreni, Ageneiosus, 366

childreni, Silonia, 366

chilensis, Diplomystes, 165

chilensis, Silurus, 165

Chiloglanidinae, 303

Chiloglanis, 303, 304

Chiloglanis angolensis, 304

Chiloglanis anoterus, 304

Chiloglanis asymetricaudalis, 304

Chiloglanis athiensis, 304

Chiloglanis batesii, 304

Chiloglanis benuensis, 305

Chiloglanis bifurcus, 305

Chiloglanis brevibarbis, 305

Chiloglanis cameronensis, 305

Chiloglanis carnosus, 305
Chiloglanis congicus, 305

Chiloglanis deckenii, 304, 305

Chiloglanis disneyi, 305

Chiloglanis dybowskii, 309

Chiloglanis elisabethianus, 305

Chiloglanis emarginatus, 305

Chiloglanis engiops, 308

Chiloglanis fasciatus, 306

Chiloglanis harbinger, 306

Chiloglanis kalambo, 306

Chiloglanis lamottei, 306

Chiloglanis lufirae, 306

Chiloglanis lukugae, 306

Chiloglanis macropterus, 306

Chiloglanis marlieri, 306

Chiloglanis mbozi, 306

Chiloglanis micropogon, 305

Chiloglanis microps, 306

Chiloglanis modjensis, 306

Chiloglanis neumanni, 307

Chiloglanis niger, 307

Chiloglanis niloticus, 307

Chiloglanis niloticus waterloti, 307

Chiloglanis normani, 307

Chiloglanis occidentalis, 307

Chiloglanis paratus, 307

Chiloglanis pojeri, 307

Chiloglanis polyodon, 307

Chiloglanis polypogon, 307

Chiloglanis pretoriae, 307

Chiloglanis productus, 443

Chiloglanis pumilus, 307

Chiloglanis reticulatus, 307

Chiloglanis rukwaensis, 308

Chiloglanis ruziziensis, 308

Chiloglanis sanagaensis, 308

Chiloglanis sardinhai, 308

Chiloglanis somereni, 308

Chiloglanis swierstrai, 308

Chiloglanis trilobatus, 308

Chiloglanis voltae, 308

chiltoni, Pygidium, 417

chiltoni, Trichomycterus, 417

chimantanus, Chasmocranus, 183

Chimarrhoglanis, 21

Chimarrhoglanis Leroyi, 21, 23

Chimarrichthys, 385

Chimarrichthys Davidi, 385

chimborazoi, Astroblepus, 63

chimborazoi, Cyclopium, 63

chinensis, Macrones, 88

chinensis, Pseudobagrus, 101

chinensis, Tachisurus, 431

chinta, Bagrus, 431

chiquitos, Callichthys, 128

Chlarias, 138

Chlarias angolensis macronema, 141

Chlarias olivaceus, 146

chlorostictus, Hemiancistrus, 243
Choeroplotosus, 346

Choeroplotosus decemfilis, 346

Choeroplotosus limbatus, 346

chondropterygioides, Arius, 55

chondropterygius, Arius, 55

chotae, Arges, 63

chotae, Astroblepus, 63

christyi, Atopochilus, 303

Christyi, Microsynodontis, 309

chrysea, Rita, 105

chryseus, Pseudobagrus, 429

Chrysichthyinae, 153

Chrysichthys, 153, 155

Chrysichthys acutirostris, 158

Chrysichthys aluuensis, 155

Chrysichthys ansorgii, 155

Chrysichthys auratus, 155, 158, 160

Chrysichthys bocagii, 155

Chrysichthys börressoni, 156

Chrysichthys brachynema, 155

Chrysichthys brevibarbis, 156

Chrysichthys büttikoferi, 158

Chrysichthys camaronensis, 158

Chrysichthys cameronensis, 158

Chrysichthys coriscanus, 160

Chrysichthys cranchii, 156

Chrysichthys cyclurus, 161

Chrysichthys dageti, 156

Chrysichthys delhezi, 156

Chrysichthys delhezi thomasi, 156

Chrysichthys duttoni, 156

Chrysichthys eaglesomei, 160

Chrysichthys filamentosus, 155

Chrysichthys furcatus, 156

Chrysichthys grandis, 156

Chrysichthys graueri, 156

Chrysichthys habereri, 156

Chrysichthys helicophagus, 156

Chrysichthys hildae, 153

Chrysichthys johnelsi, 156

Chrysichthys kingsleyae, 160

Chrysichthys lagoensis, 160

Chrysichthys laticeps, 157

Chrysichthys levequei, 157

Chrysichthys longibarbis, 157

Chrysichthys longidorsalis, 157

Chrysichthys longidorsalis nyongensis, 158

Chrysichthys longifilis, 155

Chrysichthys longipinnis, 157

Chrysichthys mabusi, 157

Chrysichthys macropogon, 160

Chrysichthys macrops, 155

Chrysichthys macropterus, 157

Chrysichthys macrotis, 157

Chrysichthys magnus, 157

Chrysichthys mahengeensis, 157

Chrysichthys maurus, 157, 158, 160

Chrysichthys myriodon, 155 
Chrysichthys nigrodigitatus, 158, 160

Chrysichthys nyongensis, 158

Chrysichthys ogooensis, 158

Chrysichthys ogowensis, 158

Chrysichthys okae, 158

Chrysichthys ornatus, 158

Chrysichthys persimilis, 158

Chrysichthys pitmani, 160

Chrysichthys platycephalus, 158

Chrysichthys polli, 159

Chrysichthys punctatus, 159

Chrysichthys rueppelli, 159

Chrysichthys sharpii, 159

Chrysichthys sianenna, 159

Chrysichthys stappersii, 159

Chrysichthys teugelsi, 159

Chrysichthys theobaldi, 105

Chrysichthys thonneri, 159

Chrysichthys thysi, 159

Chrysichthys uniformis, 159

Chrysichthys velifer, 157, 158

Chrysichthys wagenaari, 159

Chrysichthys walkeri, 159, 160

Chrysobagrus, 155

Chrysobagrus brevibarbis, 155, 156

Chrysobagrus longibarbis, 157

chrysops, Hemibagrus, 87

chungaraensis, Trichomycterus, 417

cibelae, Microglanis, 354

cinerascens, Pimelodella, 201

cinerascens, Pimelodus, 200

cinereus, Silurus, 379

Cinetodus, 42

Cinetodus carinatus, 42

Cinetodus conorhynchus, 42

Cinetodus crassilabris, 42

Cinetodus froggatti, 42

cirratus, Arges, 64

cirratus, Astroblepus, 64

cirrhosa, Loricaria, 268, 296

cirrhosa, Vandellia, 427

cirrhosus, Ancistrus, 220

cirrhosus, Hypostomus, 219, 220

cirrhosus dubius, Ancistrus, 221

cirrhosus maculatus, Chaetostomus

(Ancistrus), 222

cirrhosus punctata, Chaetostomus

(Ancistrus), 222

cirrhosus var. maculata,

Chaetostomus, 228

cirrhosus var. maculatus,

Chaetostomus (Ancistrus), 222

cirrhosus var. punctata, Chaetostomus

(Ancistrus), 222

cisternarum, Phreatobius, 429, 430

citatus, Silurichthys, 377

Citernii, Synodontis, 314

citurensis, Oxyloricaria, 300

citurensis, Sturisomatichthys, 300
Claibornichthys, 434

Claibornichthys troelli, 434, 435

clandestinus, Tetracamphilius, 27

Clariallabes, 137

Clariallabes attemsi, 137

Clariallabes brevibarbis, 137

Clariallabes centralis, 137

Clariallabes dumerili, 138

Clariallabes heterocephalus, 137

Clariallabes laticeps, 137

Clariallabes longicauda, 137

Clariallabes longicaudatus, 150

Clariallabes manyangae, 137

Clariallabes melas, 137

Clariallabes melas lembesseensis, 137

Clariallabes mutsindoziensis, 137

Clariallabes petricola, 138

Clariallabes pietschmanni, 138

Clariallabes platyprosopos, 138

Clariallabes simeonsi, 138

Clariallabes teugelsi, 138

Clariallabes uelensis, 138

Clariallabes variabilis, 138

Clarias, 136, 138

Clarias (Allabenchelys) dumerili longibarbis, 138

Clarias (Allabenchelys) submarginatus thysvillensis, 141

Clarias (Allabenchelys) uelensis, 138

Clarias (Clarioides) aboinensis, 144

Clarias (Clarioides) agboyiensis, 139

Clarias (Clarioides) curtus, 140

Clarias (Clarioides) isheriensis, 139

Clarias abbreviatus, 148

Clarias agboyiensis, 139

Clarias albopunctatus, 139

Clarias alluaudi, 139

Clarias amplexicauda, 148

Clarias anfractus, 139

Clarias angolensis, 139

Clarias anguillaris, 140, 144, 148

Clarias anguillaris nigeriensis, 140

Clarias Assamensis, 148

Clarias batrachus, 140

Clarias batu, 140

Clarias brachysoma, 140

Clarias breviceps, 141

Clarias brevinuchalis, 140

Clarias budgetti, 140

Clarias buettikoferi, 140

Clarias bulumae, 140

Clarias buthupogon, 141

Clarias büttikoferi, 141

Clarias cameronensis, 144

Clarias camerunensis, 139, 141, 144

Clarias capensis, 143

Clarias carsonii, 145

Clarias cataractus, 141

Clarias cavernicola, 141
Clarias centralis, 137

Clarias confluentus, 140

Clarias congicus, 142

Clarias dahomeyensis, 142

Clarias dayi, 141

Clarias depressus, 143

Clarias dhonti, 142

Clarias dialonensis, 142

Clarias dolloi, 142

Clarias dorsimarmoratus, 141

Clarias duchaillui, 141

Clarias dumerilii, 142

Clarias dussumieri, 142

Clarias ebriensis, 142

Clarias ekibondoi, 147

Clarias engelseni, 142

Clarias esamesae, 146

Clarias eupogon, 153

Clarias falconeri, 142

Clarias fouloni, 148

Clarias foveolatus, 149

Clarias fuscus, 142

Clarias gabonensis, 142

Clarias gariepinus, 140, 143, 148

Clarias gilli, 146

Clarias gracilis, 144

Clarias guentheri, 143

Clarias guineensis, 141

Clarias hasselquistii, 140

Clarias hilgendorfi, 139

Clarias hilli, 144

Clarias hollyi, 144, 147

Clarias insolitus, 144

Clarias intermedius, 144

Clarias jaensis, 144

Clarias kapuasensis, 144

Clarias kingsleyae, 144

Clarias laeviceps, 142, 144

Clarias laeviceps dialonensis, 142

Clarias lamottei, 144

Clarias lazera, 143

Clarias leiacanthus, 145

Clarias liacanthus, 145

Clarias liberiensis, 141

Clarias lindicus, 141

Clarias liocephalus, 145

Clarias loangwensis, 151

Clarias longibarbis, 136, 138

Clarias longiceps, 143

Clarias longior, 145

Clarias lualae, 142

Clarias maclareni, 145

Clarias macracanthus, 143

Clarias macrocephalus, 145

Clarias macromystax, 145

Clarias macrurus, 148

Clarias malaris, 143

Clarias marpus, 148

Clarias megapogon, 141 
Clarias meladerma, 145

Clarias melanoderma, 146

Clarias melanopogon, 145

Clarias melas, 137

Clarias melasoma, 146

Clarias Mellandi, 146

Clarias microphthalmus, 143

Clarias microstomus, 146

Clarias monkei, 141

Clarias moorii, 143

Clarias mossambicus, 143

Clarias mülleri, 143

Clarias N'gamensis, 146

Clarias nebulosus, 148

Clarias neumanni, 145

Clarias ngamensis, 146

Clarias ngola, 142

Clarias nieuhofii, 139, 146

Clarias nigeriae, 141

Clarias nigricans, 146

Clarias nigromarmoratus, 146

Clarias notozygurus, 143

Clarias nyasensis, 149

Clarias obscurus, 141

Clarias olivaceus, 146

Clarias ornatus, 145

Clarias orontis, 143

Clarias oxycephalus, 146

Clarias pachynema, 146

Clarias parvimanus, 140

Clarias pentapterus, 146

Clarias phillipsi, 145

Clarias planiceps, 147

Clarias platycephalus, 139, 147

Clarias pliocaenicus, 432

Clarias poensis, 141

Clarias pseudoleiacanthus, 147

Clarias pseudonieuhofii, 147

Clarias pulcher, 147

Clarias pulicaris, 142

Clarias punctatus, 140

Clarias robecchii, 143

Clarias salae, 147

Clarias sauteri, 148

Clarias Senegalensis, 140

Clarias smithii, 143

Clarias stappersii, 147

Clarias submarginatus, 147

Clarias sulcatus, 147

Clarias Syriacus, 143

Clarias teijsmanni, 147

Clarias tenuis, 148

Clarias theodorae, 139, 147

Clarias thienemanni, 148

Clarias tsanensis, 143

Clarias vandenhoutei, 144

Clarias varispinis, 147

Clarias vinciguerrae, 143

Clarias walkeri, 141
Clarias werneri, 148

Clarias xenodon, 143

Clarias youngicus, 145

Clarias zygouron, 141

clarias, Silurus, 311, 313, 321, 336, 337

clarias, Pimelodus, 320

clarias, Pseudariodes, 336

clarias, Synodontis, 313

clarias coprophagus, Pimelodus, 337

Clariidae, 136

Clariini, 136

Clarioides, 139

Clarisilurus, 151

Clarisilurus kemratensis, 151, 152

claro, Ancistrus, 220

Claroteidae, 153

Claroteini, 153

Clarotes, 153, 160

Clarotes bidorsalis, 160

Clarotes heuglini, 160

Clarotes laticeps, 160

Clarotes macrocephalus, 160

clauseni, Phractura, 26

clavipinna, Loricaria, 268

clavispinosus, Arius, 36

clavulus, Akysis, 14

clementinae, Ancistrus, 220

cleptolepis, Arius, 47

clijpeaster, Arius, 55

clinatus, Akysis, 14, 357

Clupisoma bastari, 357

Clupisoma garua, 357

Clupisoma longianalis, 357

Clupisoma montana, 357

Clupisoma naziri, 357

Clupisoma nujiangense, 357

Clupisoma prateri, 357

Clupisoma roosae, 357

Clupisoma sinense, 358

clypeastroides, Arius, 55

Cnidoglanis, 345

Cnidoglanis bostockii, 346

Cnidoglanis lepturus, 346, 442

Cnidoglanis macrocephalus, 346

Cnidoglanis microcephalus, 346

Cnidoglanis mülleri, 349

Cnidoglanis nudiceps, 346

coatesi, Arius, 47

coatesi, Neoarius, 47

coatesi, Neosilurus, 348

coatesi, Tandanus, 348

Cobitiglanis, 405

cobrensis, Dolichancistrus, 235

cobrensis, Pseudancistrus pediculatus, 235

cocama, Otocinclus, 274

cochabambae, Imparfinis, 185

cochabambae, Pimelodella, 185 cochinchinensis, Arius, 34

cochinchinensis, Pterocryptis, 376

cochinchinensis, Silurus, 376

cochlearis, Hemipimelodus, 43

Cochlefelis, 42

Cochlefelis burmanica, 42

Cochlefelis danielsi, 42

Cochlefelis spatula, 43

Cochliodon, 250

Cochliodon hypostomus, 252

Cochliodon plecostomoides, 258

Cochliodon pospisili, 254

Cochliodon pyrineusi, 259

Cochliodon taphorni, 261

cochliodon, Chaetostomus, 278

cochliodon, Hypostomus, 250, 252,

253-262

cochliodon, Panaque, 277

cochui, Corydoras, 115

coecutiens, Cetopsis, 131

coecutiens, Silurus, 131

coelestinus, Bagrus (Bagrus), 53

coerulescens, Pimelodus, 81

coeruleus, Brochus, 111, 125

coheni, Glyptothorax, 388, 389

coila, Ailia, 356

coila, Malapterurus, 356

colcloughi, Hemipimelodus, 51

Collettii, Heptapterus, 99

collinsae, Parotocinclus, 282

collinus, Leiocassis, 443

colombianus, Bunocephalus, 59

colombiense, Acestridium, 218

colombiensis, Acestridium, 218

colombiensis, Farlowella, 237

colvillii, Macrones, 97

colyeri, Synodontis, 318

colymbetes, Euanemus, 71, 72

commersoni, Hypostomus, 252

Commersonii, Pimelodus, 43, 44

commersonii scabriceps, Plecostomus, 260

commersonoides, Loricaria, 278

commersonoides, Paraloricaria, 278

commersonoides, Plecostomus, 255

comoensis, Synodontis, 313

conchophilus, Pangasius, 325

concolor, Centromochlus, 73

concolor, Corydoras, 115

concolor, Tatia, 73

concolor, Trichomycterus, 417

condei, Apistoloricaria, 226

condiscipulus, Corydoras, 115

confinis, Pimelodus, 205

confluentus, Clarias, 140

congensis, Schilbe, 363

congensis, Silurus, 362, 363

congica, Parailia, 360

congica, Synodontis, 313 
congicus, Chiloglanis, 305

congicus, Clarias, 142

congicus, Gephyroglanis, 160, 161

congicus, Synodontis, 313

congolensis, Eutropius, 363

congolensis, Schilbe, 364

conirostre, Glyptosternum, 389

conirostre poonaensis, Glyptothorax, 395

conirostris, Conorhynchos, 428

conirostris, Glyptothorax, 389, 395

conirostris, Pimelodus, 428

conirostris punjabensis, Glyptothorax, 393

Conorhynchichthys, 428

Conorhynchos, 53, 428

Conorhynchos conirostris, 428

Conorhynchos nelsoni, 53

Conorhynchus, 428

conorhynchus, Cinetodus, 42

conorhynchus, Tetranesodon, 42

Conorhynchus glaber, 428

Conostome, 428

conquetaensis, Pimelodella, 190 conquistador, Gladioglanis, 183

conradi, Pygidium, 417

conradi, Trichomycterus, 417

Conta, 382, 383

Conta conta, 384

Conta pectinata, 384

conta, Conta, 384

conta, Pimelodus, 383, 384

Continae, 382

contracta, Synodontis, 313

contractus, Synodontis, 313

cookei, Arius, 50

cookei, Notarius, 50

cooperensis, Neosiluroides, 347

copei, Anduzedoras, 172

copei, Corydoras, 115

copei, Leptodoras, 172

copei, Pimelodella, 190

Copidoglanis, 351

Copidoglanis brevidorsalis, 347

Copidoglanis curtus, 345

Copidoglanis equinus, 348

Copidoglanis gjellerupi, 348

Copidoglanis glencoensis, 348

Copidoglanis idenburgi, 348

Copidoglanis labiosus, 345

Copidoglanis labrosus, 345

Copidoglanis levis, 345

Copidoglanis longifilis, 349

Copidoglanis meraukensis, 351

Copidoglanis novae-guineae niger, 349

Copidoglanis obscurus, 345, 350

Copidoglanis papuensis, 348

Copidoglanis rendahli, 345
Copionodon, 403, 404

Copionodon lianae, 404

Copionodon orthiocarinatus, 404

Copionodon pecten, 404

Copionodontinae, 403

coppenamensis, Corydoras, 115

coppenamensis, Corydoras bondi, 115

coppenamensis, Hypostomus, 253

coprophagus, Pimelodus, 337

coprophagus, Pimelodus clarias, 337

coquenani, Ancistrus (Pseudancistrus), 287

coquenani, Pseudancistrus, 287

coracoideus, Auchenipterichthys, 71

coracoideus, Bunocephalus, 59

coracoideus, Dysichthys, 58, 59

coracoideus, Trachycorystes, 71

Coraglanis, 385

Coraglanis kishinouyei, 385

corantijni, Hypostomus, 253

cordobensis, Leptoancistrus, 266

Cordorinus, 111

cordovae, Plecostomus, 258

corduvensis, Trichomycterus, 417

Cordylancistrus, 231, 443

Cordylancistrus daguae, 231

Cordylancistrus nephelion, 443

Cordylancistrus perijae, 231

Cordylancistrus platycephalus, 231

Cordylancistrus platyrhynchus, 232

Cordylancistrus torbesensis, 232

Coreobagrus, 86, 100

Coreobagrus brevicorpus, 86

Coreobagrus ichikawai, 86

Coreobagrus okadai, 86

coriaceus, Trachelyopterus, 79

coriaceus maculosus, Trachelyopterus, 79

coriatae, Corydoras, 115

coriscanus, Chrysichthys, 160

corruscans, Platystoma, 341

corruscans, Pseudoplatystoma, 341

corsula, Bagrus, 89

corsula, Macrones, 90

corsula, Mugil, 89

corsula, Pimelodus, 89

cortesi, Corydoras, 124

Corumbataia, 232

Corumbataia cuestae, 232

Corumbataia tocantinensis, 232

coruscans, Pimelodes, 37

coruscans, Platystoma, 341

Corydoradinae, 108

Corydoras, 108, 111

Corydoras acrensis, 112

Corydoras acutus, 112

Corydoras adolfoi, 112

Corydoras aeneus, 112

Corydoras agassizii, 112
Corydoras albolineatus, 112

Corydoras amandajanea, 112

Corydoras amapaensis, 113

Corydoras ambiacus, 113

Corydoras amphibelus, 113

Corydoras approuaguensis, 113

Corydoras araguaiaensis, 113

Corydoras arcuatus, 113

Corydoras areio, 113

Corydoras armatus, 113

Corydoras atropersonatus, 113

Corydoras aurofrenatus, 113

Corydoras australe, 118

Corydoras axelrodi, 113

Corydoras baderi, 113

Corydoras bertoni, 130

Corydoras bicolor, 114

Corydoras bifasciatus, 114

Corydoras bilineatus, 114

Corydoras blochi, 114

Corydoras blochi vittatus, 126

Corydoras boehlkei, 114

Corydoras boesemani, 114

Corydoras bolivianus, 117

Corydoras bondi, 114

Corydoras bondi coppenamensis, 115

Corydoras breei, 114

Corydoras brevirostris, 114

Corydoras britskii, 114

Corydoras burgessi, 114

Corydoras caquetae, 119

Corydoras carlae, 115

Corydoras caudimaculatus, 115

Corydoras cervinus, 115

Corydoras cochui, 115

Corydoras concolor, 115

Corydoras condiscipulus, 115

Corydoras copei, 115

Corydoras coppenamensis, 115

Corydoras coriatae, 115

Corydoras cortesi, 124

Corydoras crimmeni, 115

Corydoras cruziensis, 115

Corydoras crypticus, 115

Corydoras davidsandsi, 116

Corydoras delphax, 116

Corydoras difluviatilis, 116

Corydoras diphyes, 116

Corydoras dubius, 126

Corydoras duplicareus, 116

Corydoras edentatus, 176

Corydoras ehrhardti, 116

Corydoras eigenmanni, 129

Corydoras elegans, 111, 116

Corydoras elegans nijsseni, 121

Corydoras ellisae, 116

Corydoras ephippifer, 116

Corydoras episcopi, 126

Corydoras eques, 112, 116 
Corydoras esperanzae, 117

Corydoras evelynae, 117

Corydoras filamentosus, 117

Corydoras flaveolus, 117

Corydoras fowleri, 117

Corydoras funnelli, 119

Corydoras garbei, 117

Corydoras geoffroy, 111, 117

Corydoras geryi, 117

Corydoras gomezi, 117

Corydoras gossei, 118

Corydoras gracilis, 118

Corydoras grafi, 113

Corydoras griseus, 118

Corydoras guapore, 118

Corydoras guianensis, 118

Corydoras habrosus, 118

Corydoras haraldschultzi, 118

Corydoras hastatus, 112, 118

Corydoras heteromorphus, 118

Corydoras imitator, 118

Corydoras incolicana, 118

Corydoras isbrueckeri, 119

Corydoras julii, 119

Corydoras juquiaae, 121

Corydoras juquiae, 121

Corydoras kanei, 119

Corydoras kronei, 129

Corydoras lacerdai, 119

Corydoras lamberti, 119

Corydoras latus, 119

Corydoras leopardus, 119

Corydoras leucomelas, 119

Corydoras loretoensis, 119

Corydoras loxozonus, 119

Corydoras macropterus, 130

Corydoras macrosteus, 112

Corydoras maculatus, 122

Corydoras maculifer, 119

Corydoras mamore, 120

Corydoras mamoré, 120

Corydoras marmoratus, 122

Corydoras melanistius, 120

Corydoras melanistius brevirostris, 114

Corydoras melanistius longirostris, 113

Corydoras melanotaenia, 120

Corydoras melini, 120

Corydoras meridionalis, 116

Corydoras metae, 120

Corydoras micracanthus, 120

Corydoras microcephalus, 122

Corydoras microps, 112

Corydoras multimaculatus, 120

Corydoras multiradiatus, 120

Corydoras myersi, 123

Corydoras nanus, 120

Corydoras napoensis, 120
Corydoras narcissus, 120

Corydoras nattereri, 121

Corydoras nattereri triseriatus, 121

Corydoras negro, 121

Corydoras nijsseni, 121

Corydoras noelkempffi, 121

Corydoras octocirrus, 117

Corydoras oelemariensis, 114

Corydoras oiapoquensis, 121

Corydoras ornatus, 121

Corydoras orphnopterus, 121

Corydoras osteocarus, 121

Corydoras ourastigma, 121

Corydoras oxyrhynchus, 121

Corydoras paleatus, 122

Corydoras panda, 122

Corydoras pantanalensis, 122

Corydoras paragua, 122

Corydoras parallelus, 122

Corydoras pastazensis, 122

Corydoras pastazensis orcesi, 122

Corydoras paucerna, 122

Corydoras pauciradiatus, 109

Corydoras pestai, 116

Corydoras pinheiroi, 122

Corydoras polystictus, 123

Corydoras potaroensis, 123

Corydoras prionotos, 130

Corydoras pulcher, 123

Corydoras punctatus, 123

Corydoras punctatus argentina, 122

Corydoras punctatus sipaliwini, 125

Corydoras pygmaeus, 123

Corydoras rabauti, 123

Corydoras raimundi, 110

Corydoras reticulatus, 123

Corydoras revelatus, 123

Corydoras reynoldsi, 123

Corydoras robineae, 124

Corydoras robustus, 124

Corydoras sanchesi, 124

Corydoras saramaccensis, 124

Corydoras sarareensis, 124

Corydoras schultzei, 112

Corydoras schwartzi, 124

Corydoras schwartzi surinamensis, 126

Corydoras semiaquilus, 124

Corydoras semiscutatus, 125

Corydoras septentrionalis, 124

Corydoras serratus, 124

Corydoras seussi, 124

Corydoras similis, 124

Corydoras simulatus, 124

Corydoras sipaliwini, 125

Corydoras sodalis, 125

Corydoras solox, 125

Corydoras spectabilis, 125

Corydoras spilurus, 125
Corydoras splendens, 125

Corydoras steindachneri, 125

Corydoras stenocephalus, 125

Corydoras sterbai, 125

Corydoras surinamensis, 126

Corydoras sychri, 126

Corydoras treitlii, 126

Corydoras trilineatus, 126

Corydoras tukano, 126

Corydoras undulatus, 126

Corydoras venezuelanus, 112

Corydoras virescens, 123

Corydoras virginiae, 126

Corydoras vittatus, 126

Corydoras weitzmani, 126

Corydoras wotroi, 120

Corydoras xinguensis, 126

Corydoras zygatus, 126

Corymbophanes, 217, 232

Corymbophanes andersoni, 232

Corymbophanes bahianus, 279

Corymbophanes kaiei, 232

Corymbophanes venezuelae, 231

Cossyphus, 139

Cossyphus ater, 139, 148

costatus, Platydoras, 176

costatus, Silurus, 176

cottoides, Microglanis, 354

cottoides, Pimelodus

(Pseudopimelodus), 354

Cotylephorus, 61

Cotylephorus blochii, 61

cotylephorus, Platystacus, 61

couma, Bagrus, 53

couma, Sciades, 53

courteti, Synodontis, 313

cous, Arius, 31

cous, Glyptothorax, 31, 389

cous, Silurus, 31, 387, 389

cragini, Amiurus, 205

cranchii, Chrysichthys, 156

cranchii, Pimelodus, 156

Cranoglanididae, 164

Cranoglanidae, 164

Cranoglanis, 164

Cranoglanis bouderius, 165

Cranoglanis henrici, 165

Cranoglanis multiradiatus, 165

Cranoglanis sinensis, 164, 165

crassicauda, Harttia, 242

crassicauda, Harttiella, 242

crassicauda, Hypostomus, 253

crassicauda, Pseudecheneis, 400

crassilabris, Cinetodus, 42

crassilabris, Hemipimelodus, 42

crassilabris, Leiocassis, 92

crassilabris, Liocassis, 92

crassilabris macrops, Leiocassis, 92

crassirostris, Leiocassis. 92 
crassirostris, Liocassis, 92

crassus, Arius, 435

crassus, Otolithus cf., 434

crassus, Otolithus (incertae sedis), 434

crassus, Tachysurus, 434, 435

cratensis, Trachycorystes, 80

crenula, Pterocryptis, 376

cretaceus, Brachyspondylus, 432

creutzbergi, Centromochlus, 77

creutzbergi, Tatia, 77

crimmeni, Corydoras, 115

crinalis, Bagrus, 55

cristata, Pimelodella, 190

cristata, Physopyxis, 176

cristatus, Parotocinclus, 282

cristatus, Pimelodus, 189, 190

crocodili, Centrochir, 170

Crocodili, Doras, 170

crossocheilos, Arius, 52

Crossoloricaria, 232

Crossoloricaria bahuaja, 232

Crossoloricaria cephalaspis, 232

Crossoloricaria rhami, 232

Crossoloricaria variegata, 233

Crossoloricaria venezuelae, 233

cruciger, Arius, 106

Cruciglanis, 443

Cruciglanis pacifici, 443

cruxenti, Pimelodella, 190

cruziensis, Corydoras, 115

Cryptarius, 43

Cryptarius daugueti, 43

Cryptarius truncatus, 43

crypticus, Corydoras, 115

crypticus, Noturus, 211, 442

cryptobullatus, Amphilius, 21

cryptodon, Planiloricaria, 285

cryptodon, Pseudohemiodon

(Planiloricaria), 285

cryptophthalmus, Ancistrus, 220

Cryptopterella, 369

Cryptopterella beldti, 369, 371

Cryptopterus, 369

Cryptopterus amboinensis, 369

Cryptopterus bleekeri, 374

Cryptopterus cheveyi, 371

Cryptopterus latovittatus, 374

Cryptopterus lumholtzi, 370

Cryptopterus urbaini, 373

cryptopterus, Kryptopterus, 369

cryptopterus, Silurus, 369

cryptus, Paradoxoglanis, 303

Cteniloricaria, 233

Cteniloricaria fowleri, 233

Cteniloricaria maculata, 233

Cteniloricaria platystoma, 233

ctenodus, Pimelodus, 332

cuangoana, Synodontis, 313

cuangoanus, Synodontis, 313 cuao, Pygidianops, 412

cubangoensis, Amphilius platychir, 23

cubataonis, Loricaria, 294

cubataonis, Rineloricaria, 294

cubataonis, Trichomycterus, 425

cucphuongensis, Pterocryptis, 376

cucphuongensis, Silurus, 376

cucuhyensis, Pseudepapterus, 76

cuestae, Corumbataia, 232

cuiabae, Ancistrus, 220

cuivi, Nematogenys, 324

cultratus, Pangasius, 328

cunaguaro, Ginesia, 330, 331

cunningtoni, Dinotopterus, 148

cupido, Trachymochlus, 431, 432

cuprea, Pimelodus (Rhamdia) Queleni, 201

cupreoides, Pimelodus, 205

cupreus, Silurus, 215

curtirostra, Farlowella, 237

curtisii, Arius, 47

curtisoma, Encheloclarias, 149

curtus, Clarias (Clarioides), 140

curtus, Copidoglanis, 345

curvirostris, Belonoglanis, 24

curvispina, Loricaria, 296

curvispina, Spatuloricaria, 296

cuspicaudus, Sorubim, 342

cuvieri, Genidens, 44

Cuvieri, Silurus (Acanthonotus), 356

Cuyabae, Pimelodus (Rhamdia), 201

cuyanus, Diplomystes viedmensis, 166

cuyanus, Olivaichthys, 166

cyanochloros, Pimelodus, 392

cyanostigma, Pimelodella, 190

cyanostigma, Rhamdia, 190

Cyclopidae, 63

Cyclopium, 63

Cyclopium chapmani, 63

Cyclopium chimborazoi, 63

Cyclopium humboldtii, 63, 64

Cyclopium mariae, 65

Cyclopium mindoense, 65

Cyclopium pirrense, 66

Cyclopium trifasciatum, 67

Cyclopium ubidiai, 67

Cyclopium unifasciatum, 67

Cyclopium vanceae, 67

Cyclopium ventrale, 68

cyclopium, Pimelodus, 64

cyclopum, Pimelodus, 64

cyclopus, Astroblepus, 64

cyclopus santanderensis, Astroblepus, 67

cyclurus, Chrysichthys, 161

cyclurus, Lophiobagrus, 161

D

dageti, Chrysichthys, 156

dageti, Synodontis, 312 daguae, Cordylancistrus, 231

daguae, Hemiancistrus, 231

dahli, Anodontiglanis, 345

dahomeyensis, Clarias, 142

daidalmatos, Chaetostoma, 444

daillyi, Hoplosternum littorale, 128

dakpathari, Glyptothorax, 389

dalungshanensis, Mystus, 95

damasceni, Ancistrus, 220

damasceni, Xenocara, 220

danicus, Otolithus (Arius), 434

danielsi, Arius (Hemiarius), 42

danielsi, Cochlefelis, 42

dantei, Auchenipterichthys, 71

dariense, Sturisoma, 299

dariensis, Oxyloricaria, 299

dasycephalus, Arius, 39

dasycephalus, Cathorops, 39

Dasyloricaria, 233

Dasyloricaria capetensis, 233

Dasyloricaria filaentosa, 233

Dasyloricaria latiura, 233

Dasyloricaria seminuda, 234

Dasyloricaria tuyrensis, 234

daugueti, Cryptarius, 43

Daugueti, Hemipimelodus, 43

dauuricus, Silurus, 379

Davalla, 68

Davalla Schomburgkii, 68, 69

davalla, Hypothalmus, 69

Davidi, Chimarrichthys, 385

davidi, Euchiloglanis, 385

davidi, Synodontis, 313

davidsandsi, Corydoras, 116

davisi, Pygidium, 417

davisi, Trichomycterus, 417

davissinghi, Glyptothorax, 389

dawalla, Hypothalmus, 69

dayi, Arius, 49

dayi, Batasio, 85

dayi, Clarias, 141

dayi, Hemipimelodus, 46

Dayi, Macrones, 85

dayi, Nedystoma, 46

de Zwaani, Pangasius, 328

debauwi, Eutropius, 361

debauwi, Pareutropius, 361

Decapogon, 127

Decapogon urostriatum, 127

Decapogon urotriatum, 127

Decapogon verissimi, 127

decaradiatus, Trachelyichthys, 78

decemfilis, Trachelyichthys, 346

decipiens, Otolithus (Sciaenidarum), 434

deckenii, Chiloglanis, 304, 305

decolor, Rhamdia guatemalensis, 202

decora, Synodontis, 314

decorus, Amiurus, 210 
decorus, Synodontis, 314

degeni, Bagrus, 84

deignani, Kryptopterus, 375

dekayi, Pimelodus, 206

Dekeyseria, 234

Dekeyseria amazonica, 234

Dekeyseria brachyura, 234

Dekeyseria niveata, 234

Dekeyseria picta, 234

Dekeyseria pulchra, 234

Dekeyseria scaphirhyncha, 234

dekimpei, Synodontis, 314

dekkanense, Glyptosternum, 391

delacouri, Oreoglanis, 397

delacouri, Paroreoglanis, 397

delhezi, Chrysichthys, 156

delhezi thomasi, Chrysichthys, 156

delicata, Psammphiletria, 27

delicatissimus, Pangasius, 328

delphax, Corydoras, 116

Deltadoras, 173

Deltadoras guayoensis, 173, 174

Delturinae, 444

Delturus, 234, 444

Delturus angulicauda, 234, 444

Delturus brevis, 443

Delturus carinotus, 235

Delturus parahybae, 234, 235, 444

demantoides, Baryancistrus, 226

demararae, Callichthys callichthys, 111

demerarae, Auchenipterus, 72

dendrophorus, Rheoglanis, 164

Dentata, Loricaria, 268

dentatus, Ageneiosus, 70

dentatus, Auchenipterus, 72

dentatus, Doras, 177

Dentectus, 235

Dentectus barbarmatus, 235

dentex, Chaetostomus, 277

dentex, Panaqolus, 277

dentex, Panaque, 277

Denticetopsis, 133

Denticetopsis epa, 133

Denticetopsis iwokrama, 133

Denticetopsis macilenta, 133

Denticetopsis praecox, 133

Denticetopsis royeroi, 133

Denticetopsis sauli, 133, 134

Denticetopsis seducta, 134

depauwi, Synodontis, 314

depierrei, Platyglanis, 164

depinnai, Aspidoras, 109

Deportator, 350

Deppei, Pimelodus, 200

depressa, Harttia, 240

depressa, Hemiodon, 293

depressa, Reganella, 293

depressa, Rhamdia, 201 depressa, Tocantinsia, 78

depressicauda, Hisonotus, 248

depressicauda, Otocinclus, 248

depressinotus, Hisonotus, 248

depressinotus, Microlepidogaster, 248

depressirostris, Bagrus, 364

depressirostris, Eutropius, 364

depressus, Acanthodoras, 167

depressus, Bunocephalus, 62

depressus, Cataphractus, 111

depressus, Chaetostomus, 287

depressus, Clarias, 143

depressus, Doras (Rhinodoras), 167

depressus, Gnathobagrus, 161

depressus, Hemiodon, 292, 293

depressus, Pseudancistrus, 287

depressus, Pterobunocephalus, 62

deqinensis, Glyptothorax, 389

derbyi, Hypostomus, 253

derbyi, Loricariichthys, 270

derbyi, Plecostomus, 253

Dermocassis, 92

dermorhynchum, Chaetostoma, 228

dermorhynchus, Chaetostomus, 228

despaxi, Arius, 36

devall, Hypophthalmus, 334

devincenzii, Loricaria, 288

devincenzii, Pseudohemiodon, 288

deyi, Amblyceps, 17

dhonti, Allabenchelys, 142

dhonti, Clarias, 142

dhonti, Synodontis, 314

diabolicus, Plectrochilus, 411

diabolicus, Urinophilus, 411

diabolus, Trichomycterus, 417

dialonensis, Clarias, 142

dialonensis, Clarias laeviceps, 142

Dianema, 108, 127

Dianema longibarbis, 127

Dianema urostriatum, 127

Dianemidi, 108

Diapeltoplites, 249

diaphanus, Ammoglanis, 403

diaphina, Ageniosus (Silonia), 86

Diastatomycter, 368

Diastatomycter Chaperi, 368

Diasternum, 127

dibrugarensis, Macrones montanus, 96

dichromum, Acestridium, 218

dicra, Scoloplax, 367

Dieperinki, Arius, 32

difluviatilis, Corydoras, 116

digulensis, Arius, 47

diluvii, Bucklandium, 432, 433

dinema, Belodontichthys, 368

dinema, Wallago, 368

Dinotopteroides, 139

Dinotopteroides prentissgrayi, 139, 146
Dinotopterus, 148

Dinotopterus atribranchus, 148

Dinotopterus cunningtoni, 148

Dinotopterus euryodon, 136

Dinotopterus filicibarbis, 148

Dinotopterus foveolatus, 149

Dinotopterus gigas, 149

Dinotopterus ilesi, 136

Dinotopterus jacksoni, 149

Dinotopterus jallae, 146

Dinotopterus longibarbis, 136

Dinotopterus loweae, 149

Dinotopterus nyasensis, 149

Dinotopterus rotundifrons, 136

Dinotopterus worthingtoni, 136

dioctes, Arius, 44

dioctes, Hemiarius, 44

diphyes, Corydoras, 116

Diplomystax, 165

Diplomyste, 165

Diplomystes, 165

Diplomystes camposensis, 165

Diplomystes chilensis, 165

Diplomystes mesembrinus, 166

Diplomystes nahuelbutaensis, 166

Diplomystes rudis, 435

Diplomystes viedmensis, 166

Diplomystes viedmensis cuyanus, 166

Diplomystes viedmensis mesembrinus, 166

Diplomystidae, 165

dipterus, Brochus, 111, 125

discus, Acestridium, 218

disjunctivus, Liposarcus, 291

disjunctivus, Pterygoplichthys, 291

disneyi, Chiloglanis, 305

dispar, Arius, 34

dispar, Pygidium, 417

dispar, Trichomycterus, 417

dispilurus, Epapterus, 74

dissidens, Harttia, 241

dissitus, Kryptopterus, 369

distolothrix, Scoloplax, 367

djambal, Pangasius 325

djemeri, Eutropius, 363

djemeri, Schilbe, 363

dlouhyi, Hypostomus, 253

doceana, Steindachneria, 343

doceana, Steindachneridion, 343

doceanus, Microlepidogaster, 282

doceanus, Parotocinclus, 282

docmac niger, Bagrus, 84

docmak, Bagrus, 84, 313

docmak, Silurus, 84

Doiichthyidae, 30

Doiichthys, 30, 46

Doiichthys novaeguineae, 46

Doiichthys novae-guineae, 46

Dolichallabes, 149 
Dolichallabes microphthalmus, 149

Dolichamphilius, 24

Dolichamphilius brieni, 24

Dolichamphilius longiceps, 24

Dolichancistrus, 235

Dolichancistrus atratoensis, 235

Dolichancistrus carnegiei, 235

Dolichancistrus cobrensis, 235

Dolichancistrus fuesslii, 235

Dolichancistrus pediculatus, 235

Dolichancistrus setosus, 235

dolicholophia, Scoloplax, 367

dolichopterus, Ancistrus, 220

dolichurus, Bunocephalus, 62

dolichurus, Pterobunocephalus, 62

dolloi, Clarias, 142

Doradidae, 166

Doradini, 166

dorae, Leptoglanis, 28

dorae, Zaireichthys, 28

Doraops, 170

Doraops zuloagai, 170

Doras, 166, 170

Doras (Agamyxis) flavopictus, 168

Doras (Corydoras) brevis, 179

Doras (Corydoras) ophthalmus, 169

Doras (Corydoras) punctatus, 167

Doras (Oxydoras) d'Orbignyi, 178

Doras (Oxydoras) lipophthalmus, 169

Doras (Oxydoras) niger, 175

Doras (Oxydoras) stenopeltis, 171, 172

Doras (Rhinodoras) depressus, 167

Doras affinis, 168

Doras albomaculatus, 168

Doras armatulus, 176

Doras asterifrons, 169, 170

Doras Blochii, 167

Doras brachiatus, 170

Doras brunnescens, 168

Doras Calderonensis, 179

Doras carinatus, 171, 173

Doras castaneo-ventris, 168

Doras cataphractus, 168

Doras Crocodili, 170

Doras dentatus, 177

Doras dorsalis, 173

Doras fimbriatus, 167

Doras granulosus, 177

Doras grypus, 169

Doras Hancockii, 177

Doras Heckelii, 178

Doras helicophilus, 177

Doras humboldti, 176

Doras humeralis, 174

Doras insculptus, 179

Doras laevigatulus, 177

Doras lentiginosus, 177

Doras libertatis, 174

Doras lithogaster, 173
Doras longispinis, 170

Doras loricatus, 167

Doras maculatus, 177

Doras marmoratus, 171

Doras micropoeus, 171

Doras murica, 177

Doras nebulosus, 178

Doras niger, 175

Doras Oxyrhynchus, 169

Doras papilionatus, 173

Doras pectinifrons, 168

Doras polygramma, 168

Doras punctatus, 167

Doras regani, 169

Doras spinosissimus, 168

Doras uranoscopus, 173, 174

Doras weddellii, 169

d'Orbigny, Doras (Oxydoras), 178

d'Orbignyi, Doras (Oxydoras), 178

dorbignyi, Doras (Oxydoras), 178

dorbigny, Rhinodoras, 178

dorbignyi, Rhinodoras, 178

doriae, Arius, 41

doriae, Bunocephalus, 59

doriae, Liocassis, 93

doroides, Bagrus, 45

dorsale, Chaetostoma, 228

dorsalis, Chaetostomus, 228

dorsalis, Doras, 173

dorsalis, Glyptothorax, 389

dorsalis, Lithodoras, 173

dorsalis, Rhamdia, 201

dorseyi, Pimelodella, 190

dorsimarmoratus, Clarias, 141

dorsomaculata, Synodontis, 314

dorsomaculatus, Synodontis, 314

dorsostriatum, Pygidium, 417

dorsotriatum, Pygidium, 417

dorsotriatus, Trichomycterus, 417

Doumea, 20, 24

Doumea alula, 24

Doumea angolensis, 25

Doumea chappuisi, 25

Doumea scaphyrhynchura, 27

Doumea thysi, 25

Doumea typica, 24, 25

Doumeinae, 20

dowii, Leptarius, 53

dowii, Sciades, 53

Duanensis, 378

duanensis, Silurus, 379

dubia, Netuma, 54

dubius, Ancistrus, 221

dubius, Corydoras, 126

duchaillui, Clarias, 141

duda, Silurus, 374

duellmani, Trichomycterus, 418

dugesii, Ictalurus, 208, 209

dugèsii, Amiurus, 208 dukai, Silurus, 377

dumerili, Clariallabes, 138

dumerili, Leiocassis, 93

dumerili, Rhinobagrus, 92, 93

dumerili longibarbis, Clarias

(Allabenchelys), 138

dumerilii, Clarias, 142

dumus, Lasiancistrus, 289

dumus, Pseudolithoxus, 289

dunni, Centromochlus, 77

dunni, Tatia, 77

duodecimalis, Hypostomus, 290, 291

Duopalatinus, 332

Duopalatinus emarginatus, 332

Duopalatinus olallae, 340

Duopalatinus peruanus, 332

Duoplatinus goeldii, 340

duplicareus, Corydoras, 116

dupouii, Chaetostoma, 228

Dupouyichthys, 60

Dupouyichthys sapito, 60

duquei, Rhamdia, 202

dura, Loricaria, 268

duriceps, Ostophycephalus, 346

durinii, Eutropius, 363

durinii, Schilbe, 363

duriventris, Harttia, 241

duseni, Hemipsilichthys, 262, 279

duseni, Isbrueckerichthys, 262

Dussumieri, Arius, 52

dussumieri, Clarias, 142

dussumieri, Plicofollis, 52

Dutemplei, Arius, 56

duttoni, Chrysichthys, 156

dybowskii, Chiloglanis, 309

dybowskii, Euchilichthys, 309

Dysichthys, 58

Dysichthys amazonicus, 59

Dysichthys australe, 60

Dysichthys coracoideus, 58, 59

Dysichthys quadriradiatus, 60

E

eaglesomei, Eaglesomia, 160

Eaglesomia, 160

Eaglesomia eaglesomei, 160

ebriensis, Clarias, 142

eburneensis, Synodontis, 312

echinatus, Ictalurus, 208

echthrus, Megalocentor, 409

eclipsis, Eutropius, 363

eddsi, Pseudecheneis, 442

edentatus, Corydoras, 176

edentatus, Hypophthalmus, 333

edentatus, Loricariichthys, 270

egertoni, Arius, 56

Egertoni, Silurus, 56

egertoni belgicus, Arius, 56

egertonii, Callichrous, 374

ehrhardti, Corydoras, 116 
eichorniarum, Ituglanis, 407

eichorniarum, Trichomycterus, 407

Eidouxii, Galeichthys, 38

eigenmanni, Arges, 64

eigenmanni, Astroblepus, 64

eigenmanni, Chaenothorax, 125

eigenmanni, Corydoras, 129

eigenmanni, Galeichthys, 33

eigenmanni, Heptapterus, 184

eigenmanni, Hemiloricaria, 245

Eigenmanni, Loricaria, 245

eigenmanni, Orinocodoras, 175

eigenmanni, Oxydoras, 167

eigenmanni, Pimelodella, 191, 340

eigenmanni, Pimelodella, 193

eigenmanni, Pimelodus, 340

eigenmanni, Pimelodus (Pimelodella), 191

eigenmanni, Propimelodus, 340

eigenmanni, Pygidianops, 412

eigenmanni, Trichomycterus, 423

eigenmanniorum, Pimelodella, 191

eigenmanniorum, Rhamdia, 191

ekibondoi, Clarias, 147

elassochir, Noturus, 212

elatturus, Arius, 50

electricus, Malapterurus, 301

electricus, Silurus, 301

electricus ogooensis, Malapterurus, 302

electricus oguensis, Malapterurus, 302

elegans, Corydoras, 111, 116

elegans, Noturus, 212

elegans nijsseni, Corydoras, 121

eleutherus, Noturus, 212

elisabethianus, Chiloglanis, 305

Ellenriederii, Leiocassis, 104

Elliops, 207

ellisae, Corydoras, 116

Ellisichthys, 127

elongata, Eumeda, 347, 348

elongata, Hara, 384

elongata, Olyra, 99

elongata, Pimelodella, 191

elongatus, Batasio, 73

elongatus, Macrones, 89

elongatus, Nemadoras, 174

elongatus, Oxydoras, 174

elongatus, Pangasius, 326

elongatus, Pimelodus, 191

elongatus, Plotosus, 346

elongatus, Sorubim, 342

elongatus hongus, Hemibagrus, 89

emanueli, Trichomycterus, 418

emanueli emanueli, Pygidium, 418

emanueli motatanensis, Pygidium, 421

emarginata, Microsynodontis, 309

emarginata, Squaliforma, 297

emarginatum, Platystoma, 332 emarginatus, Chiloglanis, 305

emarginatus, Duopalatinus, 332

emarginatus, Hypostomus, 297

emarginatus, Liocassis, 102

emarginatus, Microsynodontis, 309

emarginatus, Pseudobagrus, 100

Emini, Schilbe, 364

emmelane, Tachysurus, 40

emphysetus, Bagrus (Sciades), 53, 54

empousa, Scoloplax, 367

Encheloclarias, 149

Encheloclarias baculum, 149

Encheloclarias curtisoma, 149

Encheloclarias kelioides, 149

Encheloclarias prolatus, 150

Encheloclarias tapeinopterus, 150

Encheloclarias velatus, 150

Endorrhis, 349

enfurnada, Rhamdia, 197

engelseni, Allabenchelys, 142

engelseni, Clarias, 142

engiops, Chiloglanis, 308

enigmatica, Lacantunia, 216

enochi, Pimelodella, 191

Entomocorus, 74

Entomocorus benjamini, 74

Entomocorus gameroi, 74

Entomocorus melaphareus, 74

Entomocorus radiosus, 443

Eobagrus, 87

Eobagrus hoerdzanicus, 87

Eomacrones, 87

Eomacrones wilsoni, 87

Eopeyeria, 428

Eopeyeria aegyptiaca, 428

epa, Denticetopsis, 133

Epactionotus, 236

Epactionotus aky, 236

Epactionotus bilineatus, 236

Epactionotus gracilis, 236

Epactionotus itaimbezinho, 236

Epactionotus yasi, 236

epakmos, Isbrueckerichthys, 262

Epapterus, 74

Epapterus blohmi, 74

Epapterus chaquensis, 74

Epapterus dispilurus, 74

ephippiata, Amphilius pulcher, 23

ephippifer, Akysis, 14

ephippifer, Corydoras, 116

epikarsticus, Ituglanis, 407

episcopi, Corydoras, 126

eppleyi, Parotocinclus, 282

eptingi, Chaetostomus, 253

eptingi, Hypostomus, 253

equatorialis, Tachysurus, 40

eques, Cheirocerus, 332

eques, Corydoras, 112, 116

eques, Goeldiella, 184 eques, Pimelodus, 184

equestris, Arius, 33

equinus, Copidoglanis, 348

equinus, Neosilurus, 348

erebennus, Amiurus, 205

Eremophilini, 403

Eremophilus, 403, 404

Eremophilus camposi, 408

Eremophilus candidus, 416

Eremophilus mutisii, 404

Erethistes, 382, 384, 444

Erethistes maesotensis, 384

Erethistes pusillus, 384

Erethistes pussilus, 384

Erethistidae, 382

Erethistides, 382

Erethistoides, 382

Erethistoides ascita, 384

Erethistoides cavatura, 384

Erethistoides infuscatus, 442

Erethistoides montana, 384

Erethistoides montana pipri, 384

Erethistoides pipri, 384

Erethistoides sicula, 384

eriarcha, Rhamdella, 196

eriarcha, Rhamdia, 196

ericae, Hypostomus, 253

ericius, Hypostomus, 253

erinaceus, Ancistrus, 221

erinaceus, Hypostomus, 221

Ernstichthys, 60

Ernstichthys anduzei, 60

Ernstichthys intonsus, 60

Ernstichthys megistus, 60

erythrogaster, Silurus (Callichrus), 373

Erythroptera, Silurus maculatus, 209

erythrurus, Urinophilus, 427

esamesae, Clarias, 146

esmeraldas, Paracetopsis, 135

esperanzae, Corydoras, 117

espiritosantensis, Neoplecostomus, 273

essequibensis, Leptoglanis, 187

essequibensis, Leptorhamdia, 187

estuarius, Ayarnangra, 382

etentaculatum, Hypostoma, 291

etentaculatus, Pterygoplichthys, 291

Euacanthagenys, 296

Euacanthagenys caquetae, 296

euacanthagenys, Spatuloricaria, 296

Euanemini, 68

Euanemus, 68, 71

Euanemus brachyurus, 72

Euanemus colymbetes, 71, 72

Euanemus longipinnis, 74

Euanemus nigripinnis, 72

Euchilichthys, 308

Euchilichthys astatodon, 308 
Euchilichthys boulengeri, 309

Euchilichthys dybowskii, 309

Euchilichthys guentheri, 309

Euchilichthys habereri, 309

Euchilichthys royauxi, 309

Euchiloglanis, 384

Euchiloglanis davidi, 385

Euchiloglanis gracilicaudata, 398

Euchiloglanis hodgarti, 398

Euchiloglanis kamengensis, 399

Euchiloglanis kishinouyei, 385

Euchiloglanis longicauda, 399

Euchiloglanis macrotrema, 399

Euchiloglanis myzostoma, 399

Euchiloglanis sinensis, 399

Euclyptosternum, 387

eugeneiatus, Callichrous, 369

eugeneiatus, Kryptopterus, 369

Euglyptosternum lineatum, 388

Eumeda, 347

Eumeda elongata, 347, 348

eupogoides, Pseudobagrus, 107

eupogon, Clarias, 153

eupogon, Pelteobagrus, 100

eupogon, Pseudobagrus, 100

eupogon, Xenoclarias, 153

euptera, Synodontis, 314

eupterus, Synodontis, 314

Euristhmus, 346

Euristhmus lepturus, 346

Euristhmus microceps, 346

Euristhmus microphthalmus, 442

Euristhmus nudiceps, 346

Euristhmus sandrae, 442

eurycephalus, Aspidoras, 109

eurycephalus, Hemipsilichthys, 279

eurycephalus, Pareiorhaphis, 279

Eurycheilichthys, 236

Eurycheilichthys limulus, 236

Eurycheilichthys pantherinus, 236

Eurycheilus, 236

Eurycheilus pantherinus, 236

euryodon, Bathyclarias, 136

euryodon, Dinotopterus, 136

eurystoma, Microglanis, 354

eurystomus, Satan, 216

eurystomus, Synodontis, 305, 319

eustictus, Ancistrus, 221

eustictus, Pristiancistrus, 219, 221

eutaenia, Pimelodella, 191

Eutropiellus, 360

Eutropiellus buffei, 361

Eutropiellus kasai, 360, 361

Eutropiellus vandeweyeri, 361

Eutropiichthys, 358

Eutropiichthys burmannicus, 358

Eutropiichthys murius, 358

Eutropiichthys vacha, 358

Eutropius, 362
Eutropius altipinnis, 365

Eutropius angolensis, 363

Eutropius ansorgii, 363

Eutropius banguelensis, 363

Eutropius bocagii, 363

Eutropius bomae, 363

Eutropius brachypopterus, 362

Eutropius brevianalis, 363

Eutropius cameronensis, 365

Eutropius congolensis, 363, 364

Eutropius debauwi, 361

Eutropius depressirostris, 364

Eutropius djemeri, 363

Eutropius durinii, 363

Eutropius eclipsis, 363

Eutropius gastratus, 363

Eutropius grenfelli, 363

Eutropius laticeps, 364

Eutropius lemairii, 364

Eutropius liberiensis, 364

Eutropius longifilis, 361

Eutropius macropthalmos, 361, 362

Eutropius mandibularis, 364

Eutropius mentalis, 364

Eutropius micropogon, 364

Eutropius möbiusii, 364, 365

Eutropius moebii, 365

Eutropius multilineatus, 365

Eutropius multitaeniatus, 365

Eutropius nasalis, 363

Eutropius nyongensis, 365

Eutropius obtusirostris, 365

Eutropius seraoi, 363

Eutropius tumbanus, 365

Eutropius yangambianus, 365

Evansii, Loricaria, 296

evansii, Spatuloricaria, 296

evelynae, Corydoras, 117

evermanni, Tachysurus, 40

Exallodontus, 333

Exallodontus aguanai, 333

exaratus, Callichthys, 129

Exastilithoxus, 236

Exastilithoxus fimbriatus, 237

Exastilithoxus hoedemani, 237

Exilichthys, 346

exilis, Noturus, 212

exilis, Trachelyichthys, 78

existimatus, Centromochlus, 73

exodon, Bagrus, 359

exodon, Glyptothorax, 389

Exostoma, 382, 385

Exostoma andersonii, 386

Exostoma berdmorei, 385

Exostoma Blythii, 396

Exostoma Feae, 398

Exostoma gracile, 387

Exostoma labiatum, 385

Exostoma labrax, 387
Exostoma macropterum, 398

Exostoma Oschanini, 402

Exostoma stoliczkae, 387

Exostoma Vinciguerrae, 385

Exostomatina, 382

exsudans, Pimelodus, 196

exsudans, Rhamdella, 196

Eydouxii, Galeichthys, 38

F

fabricioi, Callichthys, 111

Fajumia, 428

Fajumia menoni, 429

Fajumia misrai, 429

Fajumia schweinfurthi, 428, 429

Fajumia stromeri, 429

falcarius, Arius, 34

falcarius, Mystus, 95

falcarius africana, Arius, 31

falcarius var. africana, Arius, 31

falcifer, Acrochordonichthys, 12

falconeri, Clarias, 143

fallax, Hemiloricaria, 245

fallax, Loricaria (Loricariichthys), 245

fangi, Arius, 34

fangi, Pseudobagrus, 101

Farlowella, 217, 237

Farlowella acestrichthys, 238

Farlowella acus, 237

Farlowella acus venezuelensis, 239

Farlowella agustini, 240

Farlowella altocorpus, 444

Farlowella amazona, 237

Farlowella amazonum, 237

Farlowella angosturae, 240

Farlowella azygia, 238

Farlowella boliviana, 238

Farlowella carinata, 237

Farlowella colombiensis, 237

Farlowella curtirostra, 237

Farlowella gladiolus, 237

Farlowella gracilis, 237

Farlowella guaricensis, 240

Farlowella hahni, 238

Farlowella hargreavesi, 238

Farlowella hasemani, 238

Farlowella henriquei, 238

Farlowella isbruckeri, 238

Farlowella jauruensis, 238

Farlowella jauruënsis, 238

Farlowella knerii, 238

Farlowella latisoma, 239

Farlowella mariaelenae, 238

Farlowella martini, 238

Farlowella nattereri, 238

Farlowella odontotumulus, 238

Farlowella oliveirae, 237

Farlowella oxyrryncha, 239

Farlowella paraguayensis, 239

Farlowella paranaënse, 237 
Farlowella parvicarinata, 239

Farlowella platorynchus, 239

Farlowella pleurotaenia, 237

Farlowella pseudogladiolus, 237

Farlowella reticulata, 239

Farlowella roncallii, 240

Farlowella rugosa, 239

Farlowella schreitmuelleri, 239

Farlowella schreitmülleri, 239

Farlowella smithi, 239

Farlowella taphorni, 239

Farlowella venezuelensis, 239

Farlowella vittata, 239

Farlowellidi, 217

farsonensis, Hypsidoris, 203

fasciata, Phractura, 26

fasciata, Schilbe senegalensis, 364

fasciatum, Pseudoplatystoma, 341

fasciatum brevefile, Pseudoplatystoma, 341

fasciatum intermedium,

Pseudoplatystoma, 341

fasciatum nigricans,

Pseudoplatystoma, 341

fasciatum reticulatum,

Pseudoplatystoma, 341

fasciatus, Auchenoglanis, 163

fasciatus, Chiloglanis, 306

fasciatus, Nannoglanis, 187, 188

fasciatus, Noturus, 212

fasciatus, Silurus, 340, 341

fasciolatus, Batasio, 442

fascipinna, Synodontis, 314

fassli, Pygidium, 418

fassli, Trichomycterus, 418

fasslii, Pygidium, 418

feae, Exostoma, 398

feae, Pareuchiloglanis, 398

feliceps, Galeichthys, 43

Felichthys, 37

Felichthys amblops, 79

Felichthys filamentosus, 37

Felichthys flavescens, 76

Felichthys stauroforus, 32

felinus, Pimelodus, 205

felipponei, Loricaria, 294

felipponei, Rineloricaria, 294

felis, Ariopsis, 33

felis, Pimelodus, 206

felis, Silurus, 33

ferox, Silurus, 431

ferrarisi, Niobichthys, 274

ferreirai, Cetopsidium, 130

ferula, Pseudolaguvia, 442

festae, Arges, 64

festae, Arius, 56

festae, Astroblepus, 64

festae, Plecostomus, 263

festinus, Arius, 34 festivum, Sturisoma, 299

figueroai, Pimelodella, 191

filamentissima, Harttia, 264

filamentosa, Cetopsorhamdia, 182

filamentosa, Dasyloricaria, 233

filamentosa, Hara, 395

filamentosa, Harttia, 263, 264

filamentosa, Loricaria, 233

filamentosa, Synodontis, 314

filamentosa latiura, Loricaria, 233

filamentosa seminuda, Loricaria, 234

filamentosum, Brachyplatystoma, 330

filamentosus, Aspredinichthys, 57

filamentosus, Aspredo, 57

filamentosus, Bagrus, 84

filamentosus, Chrysichthys, 155

filamentosus, Corydoras, 117

filamentosus, Felichthys, 37

filamentosus, Lamontichthys, 263

filamentosus, Pimelodes, 330

filamentosus, Synodontis, 314

filamentus, Hemibagrus, 88

filamentus, Macrones (Hemibagrus),

88

filicaudata, Peckoltia, 284

filicaudatus, Peckoltichthys, 283, 284

filicibarbis, Bathyclarias, 148

filicibarbis, Dinotopterus, 148

filifer, Akysis, 16

filifer, Pseudobagarius, 16

filinemus, Phyllonemus, 164

fimbriata, Cetopsis, 132

fimbriata, Loricaria, 297

fimbriata, Spatuloricaria, 297

fimbriatus, Doras, 167

fimbriatus, Exastilithoxus, 237

fimbriatus, Hypophthalmus, 333

fimbriatus, Otocinclus, 271

fimbriatus, Pseudacanthicus

(Lithoxus), 236, 237

fimbriatus, Pseudocanthicus

(Lithoxus), 237

firestonei, Paramphilius, 25

fisadoha, Plotosus, 350

fischeri, Chaetostoma, 228

Fischeri, Chaetostomus, 228

fisheri, Trachelyopterus, 79

fisheri, Trachycorystes, 79

fissidens, Arges, 64

fissidens, Astroblepus, 64

fissipinnis, Heptapterus, 184

fissus, Arius, 39

flabelliferus, Ochmacanthus, 409

flagellaris, Plecostomus, 268

flava, Loricaria, 259

flavater, Noturus, 212

flaveolus, Corydoras, 117

flavescens, Bagrus, 54

flavescens, Felichthys, 76 flavescens, Pseudauchenipterus, 76

flavicans, Bagrus, 344

flavipinnis, Noturus, 212

flavipinnis, Pimelodina, 336

flavipinnis, Pimelodus (Pimelodina), 336

flavipinnis, Pseudomystus, 103

flavitaeniata, Synodontis, 314

flavitaeniatus, Synodontis, 314

flavolineatus, Plotosus, 345

flavomaculatus, Leptoglanis, 28

flavomaculatus, Zaireichthys, 28

flavopictus, Doras (Agamyxis), 168

flavus, Bagrus, 90

flavus, Hyalobagrus, 92

flavus, Noturus, 211, 212

flexilis, Macrotocinclus, 271

flexilis, Otocinclus, 271, 272

florense, Pygidium, 414

fluviatilis, Hypostomus, 253

fluviatilis, Leiocassis, 85

fluviatilis, Mystus, 164

fluviatilis, Plecostomus, 253

Fluvidraco, 101

foina, Pimelodus, 197

foina, Rhamdia, 197

fokiensis, Glyptosternum, 389

fokiensis, Glyptothorax, 389

fonchii, Hypostomus, 253

Fonchiiichthys, 240

Fonchiiichthys rupestris, 240

Fonchiiichthys uracanthus, 240

foratum, Amblyceps, 18

fordicei, Auchenipterus, 72

Fordii, Chaetostomus, 286

fordii, Pseudacanthicus, 286

forficulatus, Hypodoras, 172

formosa, Hemiloricaria, 245

formosa, Rineloricaria, 245

formosanus, Liobagrus, 19

formoso, Ancistrus, 221

formosus, Astroblepus, 64

fortis, Hemibagrus, 88

fortis, Macrones, 88

fortis capitulum, Macrones, 88

fortis var. capitulum, Macrones, 88

fossilis, Heteropneustes, 151

fossilis, Silurus, 151

fossor, Pimelodes, 431

Fouloni, Clarias, 148

foveolata, Pseudolaguvia, 401

foveolatus, Clarias, 149

foveolatus, Dinotopterus, 149

fowleri, Cephalosilurus, 353

fowleri, Corydoras, 117

fowleri, Cteniloricaria, 233

Fowleri, Oxyloricaria, 233

fowleri, Pangasius, 328

Fraasi, Arius, 56 
francirochai, Hisonotus, 248

francirochai, Otocinclus, 248

francisci, Hypostomus, 254

francisci, Plecostomus, 254

Franciscodoras, 171

Franciscodoras marmoratus, 171

franciscoensis, Neoplecostomus, 273

frankei, Aphanotorulus, 225, 226

freiei, Ageneiosus, 70

frenata, Brachyglanis, 180

frenata, Loricaria, 299

frenatum, Sturisoma, 299

frenatus, Astroblepus, 64

frenatus, Brachyglanis, 180

frenatus, Oreoglanis, 397

frenatus, Rhamdioglanis, 202

froggatti, Arius, 42

froggatti, Cinetodus, 42

frontosa, Synodontis, 314

frontosus, Synodontis, 314

fuelleborni, Synodontis, 315

fuerthii, Cathorops, 39

fuesslii, Dolichancistrus, 235

fugleri, Hemiancistrus, 243

fui, Pseudobagrus, 107

fukiensis, Glyptothorax, 389

fukiensis fukiensis, Glyptothorax, 389

fukiensis hainanensis, Glyptothorax, 390

fukiensis honghensis, Glyptothorax, 390

fukiensis honghenensis, Glyptothorax, 390

fuliginatus, Akysis, 14

fuliginosus, Hemiancistrus, 243

fulva, Xenocara, 221

fulvidraco, Pelteobagrus, 100

fulvi-draco, Pimelodus, 100

fulvus, Ancistrus, 221

fumidus, Ompok, 372

fumosus, Pseudomystus, 103

funebris, Noturus, 212

funnelli, Corydoras, 119

fur, Pimelodus, 337

fur, Pseudorhamdia, 337

furcata, Peckoltia, 284

furcatus, Auchenipterus, 76

furcatus, Chaetostomus, 284

furcatus, Chrysichthys, 156

furcatus, Hemibagrus, 88

furcatus, Ictalurus, 208, 211

furcatus, Nemasiluroides, 361

furcatus, Pimelodus, 208, 209

furcifer, Pimelodus, 210

Furcodontichthys, 240

Furcodontichthys novaesi, 240

furiosus, Noturus, 211, 212

furnessi, Apodoglanis, 375, 376

furnessi, Pterocryptis, 376
Fürthii, Arius, 39

Fuscatus, Silurus lividus, 205

fuscoguttatus, Aspidoras, 109

fuscum, Pygidium, 427

fuscus, Akysis, 16

fuscus, Ancharius, 29

fuscus, Bagrus, 95

fuscus, Clarias, 142

fuscus, Glyptothorax, 389

fuscus, Leiocassis, 103

fuscus, Macropteronotus, 142

fuscus, Neobagrus, 19, 20

fuscus, Pseudobagarius, 16

fuscus, Pseudomystus, 103

Fusiloricaria, 268

füsslii, Ancistrus, 235

G

gabardinii, Ageneiosus, 69

gabonensis, Clarias, 142

gabrieli, Pygidium, 418

gabrieli, Trichomycterus, 418

gadense, Nigerium, 98

Gagata, 385

Gagata cenia, 386

Gagata dolichonema, 386

Gagata gagata, 386

Gagata gasawyuh, 386

Gagata itchkeea, 386

Gagata melanoptera, 386

Gagata melanopterus, 386

Gagata pakistanica, 386

Gagata sexualis, 386

Gagata typus, 386

Gagata youssoufi, 386

gagata, Gagata, 385, 386

gagora, Arius, 34

gagora, Pimelodus, 34

gagorides, Bagrus, 35

gagoroides, Arius, 35

galani, Ancistrus, 221

galaxias, Leporacanthicus, 265

galaxias, Tatia, 77

galeatus, Silurus, 79

galeatus, Trachelyopterus, 79

Galeichthys, 43

Galeichthys aequus, 435

Galeichthys aguadulce, 39

Galeichthys angelicus, 434

Galeichthys araguayensis, 339

Galeichthys ater, 43

Galeichthys azureus, 33

Galeichthys bahiensis, 38

Galeichthys Blochii, 38

Galeichthys bonillai, 33

Galeichthys eigenmanni, 33

Galeichthys, Eidouxii, 38

Galeichthys Eydouxii, 38

Galeichthys feliceps, 43

Galeichthys gilberti, 33
Galeichthys Gronovii, 37

Galeichthys guentheri, 33

Galeichthys ocellatus, 43

Galeichthys Parrae, 38

Galeichthys peruvianus, 43

Galeichthys simonsi, 33

Galeichthys stanneus, 49

Galeichthys xenauchen, 33

galinae, Synodontis, 323

gallowayi, Schilbeodes, 212

gambensis, Pimelodus, 54

gambiensis, Synodontis, 315

gambiensis latifrons, Synodontis, 315

gameroi, Entomocorus, 74

gangelica, Pterocryptis, 375, 376, 377

gangetica, Silundia, 366

Gangra, 395

garavelloi, Harttia, 241

garavelloi, Microglanis, 354

garbei, Corydoras, 117

garbei, Hemipsilichthys, 279

garbei, Pareiorhaphis, 279

garbei, Pimelodela, 189

garciabarrigai, Pimelodus, 337

garcia-barrigai, Pimelodus, 337

garhwali, Glyptothorax, 389

gariepinus, Clarias, 140, 143, 148

gariepinus, Silurus (Heterobranchus), 143

garmani, Hypostomus, 254

garmani, Plecostomus, 254

garua, Clupisoma, 357

garua, Silurus, 357

gasawyuh, Gagata, 386

gastratus, Eutropius, 363

Gastrodermus, 111

gaudryi, Silurus, 432

Gelanoglanis, 73, 74

Gelanoglanis nanonocticolus, 74

Gelanoglanis stroudi, 74

gelatinosus, Malacoglanis, 408

geledensis, Synodontis, 315

geminus, Kryptopterus, 369

genibarbis, Pseudorinelepis, 289

genibarbis, Rinelepis, 289

Genidens, 32, 43

Genidens barbus, 44

Genidens cuvieri, 44

Genidens genidens, 44

Genidens granulosus, 44

Genidens machadoi, 44

Genidens planifrons, 44

Genidens valenciennesii, 44

genidens, Genidens, 44

genidens, Pimelodus, 43, 44

genisetiger, Pseudancistrus, 287

geoffroy, Corydoras, 111, 117

Geoffroyi, Heterobranchus, 150

Gephyroglanidini, 153 
Gephyroglanis, 153, 160

Gephyroglanis barnardi, 81

Gephyroglanis congicus, 160, 161

Gephyroglanis gigas, 157

Gephyroglanis gilli, 81

Gephyroglanis gymnorhynchus, 161

Gephyroglanis habereri, 161

Gephyroglanis longipinnis, 157

Gephyroglanis lowei, 158

Gephyroglanis ogoensis, 158

Gephyroglanis ogooensis, 158

Gephyroglanis rotundiceps, 28

Gephyroglanis sclateri, 81

Gephyroglanis tilhoi, 155

Gephyroglanis velifer, 157

Gephyromochlus, 75

germanicus, Otolithus (Arius), 434

geryi, Corydoras, 117

geryi, Pimelodella, 191

gibbiceps, Ancistrus, 291

gibbiceps, Pterygoplichthys, 291

gibbiceps, Silurichthys, 377

gibbosa, Lampiella, 264

gibbosus, Chaetostomus, 278

gibbosus, Otocinclus, 264

gigantea, Vandellia, 427

giganteus, Trichomycterus, 418

gigas, Arius, 31, 56

gigas, Bagarius, 383

gigas, Bathyclarias, 149

gigas, Chaetostomus, 272

gigas, Dinotopterus, 149

gigas, Gephyroglanis, 157

gigas, Pangasianodon, 325

gigas, Platystoma, 330

gigas, Xiurenbagrus, 20

gilberti, Galeichthys, 33

gilberti, Noturus, 213

gilberti, Silurus, 376

gilli, Austroglanis, 81

gilli, Clarias, 146

gilli, Gephyroglanis, 81

gilli, Rhamdella, 196

gilli, Rhamdia, 196

Ginesia, 330

Ginesia cunaguaro, 330, 331 gjellerupi, Copidoglanis, 348 gjellerupi, Neosilurus, 348 glaber, Auchenipterus, 80

glaber, Conorhynchus, 428

glaber, Otolithus (Arius ?), 435

gladiator, Noturus, 213

Gladioglanis, 183

Gladioglanis conquistador, 183

Gladioglanis machadoi, 183

gladiolus, Acestra, 237

gladius, Acestra, 239

gladysae, Phractura, 26

Glanapteryginae, 403
Glanapteryx, 403, 404

Glanapteryx anguilla, 404

Glanapteryx niobium, 405

Glanidium, 75

Glanidium albescens, 75

Glanidium bockmanni, 75

Glanidium catharinensis, 75

Glanidium cesarpintoi, 75

Glanidium leopardum, 75

Glanidium melanopterum, 75

Glanidium neivai, 77

Glanidium piresi, 78

Glanidium ribeiroi, 75

Glanis, 37, 378

Glanis Aristotelis, 378

glanis aralensis, Silurus, 379

glanis, Silurus, 378, 379

Glaphyropoma, 405

Glaphyropoma rodriguesi, 405

Glaridoglanis, 386

Glaridoglanis andersonii, 386

glencoensis, Copidoglanis, 348

gloveri, Neosilurus, 348

Glyptocephalus, 432

Glyptocephalus radiatus, 432, 433

Glyptoperichthys, 290

Glyptoperichthys joselimaianus, 291

Glyptoperichthys parnaibae, 292

Glyptoperichthys xinguensis, 292

Glyptosterni, 382

Glyptosternon, 382, 387

Glyptosternon akhtari, 387

Glyptosternon hainanensis, 390

Glyptosternon interspinalum, 390

Glyptosternon labiatus, 385

Glyptosternon maculatum, 387

Glyptosternon malaisei, 387

Glyptosternon minutum, 393

Glyptosternon Nieuwenhuisi, 392

Glyptosternon pectinopterus, 392

Glyptosternon punctatum, 389

Glyptosternon quadriocellatum, 393

Glyptosternon reticulatum, 387

Glyptosternon reticulatus, 387

Glyptosternon retuculatus, 387

Glyptosternon striatus, 387, 394

Glyptosternon sulcatus, 400

Glyptosternum, 387

Glyptosternum akhtari, 387

Glyptosternum armeniacum, 388

Glyptosternum chaudhurii, 385

Glyptosternum conirostre, 389

Glyptosternum dekkanense, 391

Glyptosternum fokiensis, 389

Glyptosternum gracile, 390

Glyptosternum hodgarti, 398

Glyptosternum kükenthali, 391

Glyptosternum kurdistanicum, 390

Glyptosternum laak, 395
Glyptosternum madraspatanum, 391

Glyptosternum modestum, 389

Glyptosternum pallozonum, 388, 392

Glyptosternum saisii, 393

Glyptosternum sinense, 393

Glyptosternum Stoličkae, 393

Glyptosternum steindachneri, 393

Glyptosternum stuarti, 385

Glyptosternum sykesi, 394

Glyptosternum tiong, 395

Glyptosternum yunnanensis, 401

Glyptothoracini, 382

Glyptothorax, 382, 387

Glyptothorax alaknandi, 388

Glyptothorax anamalaiensis, 388

Glyptothorax annandalei, 388

Glyptothorax armeniacus, 388

Glyptothorax botius, 388

Glyptothorax brevipinnis, 388

Glyptothorax brevipinnis alaknandi, 388

Glyptothorax buchanani, 388

Glyptothorax burmanicus, 388

Glyptothorax callopterus, 388

Glyptothorax cavia, 388

Glyptothorax coheni, 388, 389

Glyptothorax conirostre poonaensis, 395

Glyptothorax conirostris, 389, 395

Glyptothorax conirostris punjabensis, 393

Glyptothorax cous, 31, 389

Glyptothorax dakpathari, 389

Glyptothorax davissinghi, 389

Glyptothorax deqinensis, 389

Glyptothorax dorsalis, 389

Glyptothorax exodon, 389

Glyptothorax fokiensis, 389

Glyptothorax fukiensis fukiensis, 389

Glyptothorax fukiensis hainanensis, 390

Glyptothorax fukiensis honghensis, 390

Glyptothorax fukiensis honghenensis, 390

Glyptothorax fuscus, 389

Glyptothorax garhwali, 389

Glyptothorax gracilis, 389, 390

Glyptothorax hainanensis, 390

Glyptothorax honghensis, 390

Glyptothorax horai, 390

Glyptothorax housei, 390

Glyptothorax indicus, 390

Glyptothorax interspinalus, 390

Glyptothorax jalalensis, 390

Glyptothorax kashmirensis, 390, 393

Glyptothorax kurdistanicus, 390

Glyptothorax lampris, 391

Glyptothorax laosensis, 391 
Glyptothorax lonah, 391, 394

Glyptothorax longicauda, 391

Glyptothorax longinema, 394

Glyptothorax longjiangensis, 391

Glyptothorax macromaculatus, 391

Glyptothorax madraspatanus, 391

Glyptothorax major, 391

Glyptothorax manipurensis, 391

Glyptothorax merus, 390

Glyptothorax minimaculatus, 391

Glyptothorax minutus, 391, 392, 393

Glyptothorax naziri, 392

Glyptothorax nelsoni, 392

Glyptothorax nieuwenhuisi, 392

Glyptothorax obscura, 392

Glyptothorax obscurus, 392

Glyptothorax pallozonus, 392

Glyptothorax panda, 392

Glyptothorax pectinopterus, 392

Glyptothorax platypogon, 392

Glyptothorax platypogonides, 392

Glyptothorax platypogonoides, 392

Glyptothorax prashadi, 393

Glyptothorax punjabensis, 393

Glyptothorax quadriocellatus, 393

Glyptothorax rubermentus, 394

Glyptothorax saisii, 393

Glyptothorax siamensis, 393

Glyptothorax silviae, 393

Glyptothorax sinensis, 393

Glyptothorax spectrum, 393

Glyptothorax steindachneri, 393

Glyptothorax stocki, 393

Glyptothorax stolickae, 393

Glyptothorax striatus, 394

Glyptothorax sufii, 394

Glyptothorax sykesi, 394

Glyptothorax telchitta, 394

Glyptothorax telchitta sufii, 394

Glyptothorax trewavasae, 394

Glyptothorax trilineatoides, 394

Glyptothorax trilineatus, 394

Glyptothorax tuberculatus, 401, 402

Glyptothorax ventrolineatus, 394

Glyptothorax zainaensis, 394

Glyptothorax zanaensis, 394

Glyptothorax zhujiangensis, 394

Gnathobagrus, 161

Gnathobagrus depressus, 161

gnomus, Panaqolus, 277

gnomus, Panaque, 277

goae, Silurus, 374

Gobibagrus, 87

Gobibagrus hoerdzanicus, 87

gobio, Hemipsilichthys, 247

gobio, Xenomystus, 247

gobioides, Cetopsis, 131, 132

gobroni, Synodontis, 315

godavarii, Pangasius pangasius, 327 godfreyi, Tachysurus (Pararius), 45

godmanni, Pimelodus, 200

Goeldiella, 184

Goeldiella eques, 184

goeldii, Brachyplatystoma, 330

goeldii, Cheirocerus, 332

goeldii, Duoplatinus, 340

goeldii, Pimelodina, 332

goeldii, Trichomycterus, 418

Gogangra, 395

Gogangra laevis, 395

Gogangra viridescens, 395

Gogo, 29

Gogo arcuatus, 29

Gogo brevibarbis, 29

Gogo ornatus, 29

gogra, Phractocephalus, 105

gogra, Rita, 105

Gogrius, 105

Gogrius sykesii, 105

Goliath, Bagrus, 331

gomesi, Plecostomus, 298

gomesi, Squaliforma, 298

gomezi, Corydoras, 117

gongshanensis, Pareuchiloglanis, 398

goniaspis, Arius, 52

Gonocephalus, 160

gonzalezi, Amblydoras, 168

gonzalezi, Zathorax, 168

goodi, Paramphilius, 25

goongwaree, Hypophthalmus, 362

goongwaree, Proeutropiichthys, 362

gorgona, Trichomycterus, 418

Goslinia, 330

gossei, Corydoras, 118

gossei, Malapterurus, 302

gouldingi, Helogenes, 134

gouldingi, Stauroglanis, 413

goyazensis, Hypostomus, 254

goyazensis, Plecostomus, 254

gracile, Exostoma, 387

gracile, Glyptosternum, 390

gracilicaudata, Euchiloglanis, 398

gracilicaudatus, Pareuchiloglanis, 398

graciliformis, Schmidelia, 432

gracilior, Ituglanis, 407

gracilior, Pygidium, 407

gracilis, Clarias, 144

gracilis, Corydoras, 118

gracilis, Epactionotus, 236

gracilis, Farlowella, 237

gracilis, Glyptothorax, 389, 390

gracilis, Harttia, 241

gracilis, Hemibagrus, 88

gracilis, Pimelodella, 191

gracilis, Pimelodus, 191, 196, 210

gracilis, Osteogeneiosus, 51

gracilis, Pseudepapterus, 76 gracilis, Pseudobagrus, 102

gracilis, Schultzichthys, 413

gracilis, Trichomycterus, 422

graciosa, Homodiaetus, 406

graciosus, Pimelodus, 210

Graeffei, Arius, 47

graeffei, Neoarius, 47

grafi, Corydoras, 113

grahami, Silurus, 379

grammatophorus, Amphilius, 22, 23

grammatophorus brevipinna,

Amphilius, 22

grammatophorus inaequalis,

Amphilius, 23

grammatophorus marmoratus,

Amphilius, 23

grandicassis, Arius, 49, 50

grandicassis, Notarius, 50

grandiops, Synodontis, 445

grandis, Amphilius, 23

grandis, Auchenoglanis, 163

grandis, Chrysichthys, 156

grandis, Parakysis 15

grandis, Socnopaea, 430

grandiscutata, Rita, 105

grandoculis, Arius, 53

grandoculis, Potamarius, 53

granducolis, Arius, 53

grangeri, Rhineastes, 432

granosus, Arius, 54

granosus, Hypostomus, 273

granosus, Neoplecostomus, 273

granulosa, Synodontis, 315

granulatus, Arius, 32

granulosus, Doras, 177

granulosus, Genidens 44

granulosus, Pterodoras, 177

granulosus, Synodontis, 315

graueri, Chrysichthys, 156

gravoti, Auchenoglanis ballayi, 162

greeni, Chaetostoma, 228

grenfelli, Eutropius, 363

grenfelli, Schilbe, 363

greshoffi, Synodontis, 315

griffini, Pimelodella, 191

grisea, Pimelodella, 191

griseus, Ancharius, 29

griseus, Corydoras, 118

griseus, Limatulichthys, 266

griseus, Loricaria, 266

griseus, Pimelodus (Pimelodella), 191

grixalvii, Astroblepus, 63, 64

grixalvii micrescens, Astroblepus, 65

Gronias, 204

Gronias nigrilabris, 204, 206

Gronovii, Galeichthys, 37

gronovii, Aspredo, 58, 60

grosskopfii, Pimelodus, 338 
grosskopfii, Pimelodus (Pimelodus), 338

grosskopfii navarroi, Pimelodus, 338 grunniens, Pimelodus, 336

grypus, Anadoras, 169

grypus, Doras, 169

guacamaya, Typhlobelus, 426

guacari, Hypostomus, 250, 258

guacharote, Ancistrus, 225

guacharote, Hypostomus, 265

guacharote, Lasiancistrus, 265

guahiborum, Hemiancistrus, 243

guairense, Chaetostoma, 228

guairensis, Chaetostomus, 228

guairensis, Rhamdia, 198

guapore, Corydoras, 118

guapore, Lasiancistrus, 265

guaraquessaba, Trichomycterus, 418

guaricensis, Farlowella, 240

guasarensis, Rhamdia, 197

guatemalensis, Ariopsis, 33

guatemalensis, Arius, 33

guatemalensis, Pimelodus, 200

guatemalensis decolor, Rhamdia, 202

guatemalensis muriei, Rhamdia, 202

guatemalensis stygaea, Rhamdia, 202

guayaberensis, Medemichthys, 188

guayaberensis, Ituglanis, 407

guayaberensis, Pygidium metae, 407

guayoensis, Deltadoras, 173

guayoensis, Megalodoras, 173

guentheri, Ancistrus, 287

guentheri, Astroblepus, 64

guentheri, Atopochilus, 308

guentheri, Clarias, 143

guentheri, Euchilichthys, 309

guentheri, Galeichthys, 33

guentheri, Hypoptopoma, 250

guentheri, Microlepidogaster, 296

guentheri, Pseudancistrus, 287

guentheri, Oxyloricaria, 299

guentheri, Sturisoma, 299

guentheri, Stygogenes, 64

Guentheri, Synodontis, 309

guianense, Hypoptopoma, 250

guianense, Pygidium, 418

guianensis, Ageneiosus 70

guianensis, Corydoras, 118

guianensis, Harttia, 241

guianensis, Pygidium, 418

guianensis, Trichomycterus, 418

guineensis, Clarias, 141

Guirali, Pimelodus, 162

Guiritinga, 43

gulare, Hypoptopoma, 249, 250

gulio, Bagrus, 93

gulio, Mystus, 95

gulio, Pimelodus, 93, 95

gulioides, Bagrus, 95 gulosus, Tachisurus, 40

guntheri, Microlepidogaster, 295

guntheri, Schizolecis, 295

güntheri, Atopochilus, 309

güntheri, Ancistrus, 287

güntheri, Microlepidogaster, 296

guppyi, Pseudauchenipterus, 76

gurgu, Silurus, 431

guttata, Synodontis, 315

guttatus, Acrochordonichthys, 12

guttatus, Hemibagrus, 88

guttatus, Hypostomus, 287

guttatus, Imparfinis, 185

guttatus, Nannorhamdia, 185

guttatus, Parauchenoglanis, 163

guttatus, Pimelodus, 88, 162, 163

guttatus, Synodontis, 315

Guyanancistrus, 287

Gymnallabes, 137, 150

Gymnallabes alvarezi, 150

Gymnallabes apus, 137

Gymnallabes nops, 150

Gymnallabes tihoni, 152

Gymnallabes typus, 150

Gymnallabes typus forma

heterocercalis, 150

Gymnallabes typus heterocercalis, 150

gymnogaster, Loricaria, 297

gymnogaster, Spatuloricaria, 297

gymnogaster lagoichthys, Loricaria, 297

gymnorhynchus, Ancistrus, 221

gymnorhynchus, Gephyroglanis, 161

gymnorhynchus, Hypostomus, 254

gymnorhynchus, Plecostomus, 254

gymnorhynchus occidentalis,

Hypostomus, 257

gymnorhynchus tapanahoniensis,

Hypostomus, 261

gyrina, Tatia, 77

Gyrinurus, 409

Gyrinurus batrachostoma, 409

gyrinus, Acrochordonichthys 12

gyrinus, Centromochlus, 77

gyrinus, Noturus, 213

gyrinus, Silurus, 211, 213, 214

H

habereri, Chrysichthys, 156

habereri, Euchilichthys, 309

habereri, Gephyroglanis, 161

habrosus, Corydoras, 118

Haemomaster, 405

Haemomaster venezuelae, 405

haemomyzon, Homodiaetus, 412

haemomyzon, Pseudostegophilus, 412

haggini, Bunocephalus, 59

hahni, Farlowella, 238

hainanensis, Glyptosternon, 390

hainanensis, Glyptothorax, 390 hainanensis, Glyptothorax fukiensis, 390

hainanensis, Hemibagrus, 88

hainanensis, Leiocassis, 88

hainesi, Amissidens, 32

hainesi, Arius, 32

Halepensis, Bagrus, 93, 94, 96, 97

hamiltonii, Chaca, 135, 136

hamiltonii, Plotosus (Clarias), 140

hamiltonis, Arius, 51

hammarlundi, Hemiancistrus, 243

Hammondii, Pimelodus, 210

Hancockii, Doras, 177

Haplodoras, 173

Hara, 382, 395, 444

Hara buchanani, 395

Hara elongata, 384

Hara filamentosa, 395

Hara hara, 395, 396

Hara horai, 396

Hara jerdoni, 396

Hara Malabarica, 96

Hara saharsai, 396

Hara serrata, 396

Hara serratus, 396

hara, Hara, 396

hara, Pimelodus, 395, 396

haraldschultzi, Corydoras, 118

harbinger, Chiloglanis, 306

hardenbergi, Arius, 36

hardenbergi, Aspistor, 36

Hardwickii, Acanthonotus, 356

Hargeri, Amphilius, 23

hargreavesi, Farlowella, 238

Harmandi, Hemiarius, 31

haroldoi, Parotocinclus, 282

hartii, Pimelodella, 191

Hartii, Pimelodus (Pseudorhamdia), 191

Harttia, 217, 240, 440

Hartia caquetae, 300

Harttia carvalhoi, 240

Harttia crassicauda, 242

Harttia depressa, 240

Harttia dissidens, 241

Harttia duriventris, 241

Harttia filamentissima, 264

Hartia filamentosa, 263, 264

Harttia garavelloi, 241

Harttia gracilis, 241

Harttia guianensis, 241

Harttia kronei, 241

Harttia leiopleura, 240, 241

Harttia longipinna, 241

Harttia loricariformis, 240, 241

Harttia merevari, 241

Harttia microps, 290

Harttia nijsseni, 272

Harttia novalimensis, 241 
Harttia punctata, 241

Harttia rhombocephala, 242

Harttia surinamensis, 242

Harttia torrenticola, 242

Harttia trombetensis, 242

Harttia uatumensis, 242

Harttiella, 242

Harttiella crassicauda, 242

Harttiinae, 217

hartwelli, Pimelodella, 192

hasemani, Auchenipterus

(Pseudepapterus), 76

hasemani, Centrodoras, 170

hasemani, Farlowella, 238

hasemani, Hemidoras, 172

hasemani, Hemiloricaria, 245

hasemani, Imparfinis, 185

hasemani, Leptodoras, 172

hasemani, Otocinclus, 274

hasemani, Oxydoras (Rhinodoras) amazonum, 170

hasemani, Pimelodella, 192

hasemani, Pseudepapterus 76

hasemani, Pygidium, 418

hasemani, Rineloricaria, 245

hasemani, Trichomycterus, 418

hasemani, Vandellia, 411

Hassar, 171

Hassar affinis, 171

Hassar iheringi, 171

Hassar orestis, 171

Hassar praelongus, 173

Hassar ucayalensis, 171

Hassar wilderi, 171

Hassar woodi, 171

Hasselquistii, Clarias, 140

Hasselquistii, Schilbe, 365

hasseltii, Silurichthys, 377

hassleriana, Netuma, 56

hastatus, Arius, 105

hastatus, Corydoras, 112, 118

Hatcheria, 405

Hatcheria bullocki, 404

Hatcheria macraei, 405

Hatcheria Maldonadoi, 403, 404

Hatcheria patagoniensis, 405

Hatcheria pique, 405

Hatcheria titcombi, 405

haugi, Synodontis, 315

Haustor, 208

Haustor ochoterenai, 209

hauxwelli, Loricariichthys, 270

havmolleri, Batasio, 85

havmolleri, Mystus, 85

hazenensis, Ameiurus, 204

hazenensis, Ictalurus (Amiurus), 204

Heckelii, Arius, 55

heckelii, Auchenipterus, 73

heckelii, Centromochlus, 73
Heckelii, Doras, 178

heckelii, Scorpiodoras, 178

Helicophagus, 324

Helicophagus hypophthalmus, 325

Helicophagus leptorhynchus, 324

Helicophagus typus, 324, 325

Helicophagus waandersii, 325

helicophagus, Chrysichthys 156

helicophilus, Doras, 177

Helogenes, 130, 134

Helogenes amazonae, 134

Helogenes castaneus, 134

Helogenes gouldingi, 134

Helogenes marmoratus, 134

Helogenes marmoratus uruyensis, 134

Helogenes unidorsalis, 134

Helogenes uruyensis, 134

Helogenidae, 130

hematophaga, Vandellia, 410

Hemiancistrus, 218, 242

Hemiancistrus albocinctus, 223

Hemiancistrus annectens, 242

Hemiancistrus arenarius, 284

Hemiancistrus aspidolepis, 242

Hemiancistrus braueri, 284

Hemiancistrus brevis, 284

Hemiancistrus caquetae, 265

Hemiancistrus castelnaui, 265

Hemiancistrus chlorostictus, 243

Hemiancistrus daguae, 231

Hemiancistrus fugleri, 243

Hemiancistrus fuliginosus, 243

Hemiancistrus guahiborum, 243

Hemiancistrus hammarlundi, 243

Hemiancistrus holostictus, 243

Hemiancistrus landoni, 243

Hemiancistrus longipinnis, 227

Hemiancistrus macrops, 243

Hemiancistrus maracaiboensis, 243

Hemiancistrus mayoloi, 264

Hemiancistrus medians, 243

Hemiancistrus megacephalus, 244

Hemiancistrus megalopteryx, 244

Hemiancistrus meizospilos, 244

Hemiancistrus micrommatos, 244

Hemiancistrus niceforoi, 257

Hemiancistrus niger, 287

Hemiancistrus platyrhynchus, 232

Hemiancistrus punctulatus, 244

Hemiancistrus spilomma, 244

Hemiancistrus spinosissimus, 244

Hemiancistrus subviridis, 244

Hemiancistrus ucayalensis, 283, 285

Hemiancistrus votouro, 244

Hemiancistrus wilsoni, 244

Hemiarius, 44

Hemiarius dioctes, 44

Hemiarius harmandi, 31

Hemiarius insidiator, 44
Hemiarius sona, 44

Hemiarius stormi, 45

Hemiarius stormii, 45

Hemiarius verrucosus, 45

Hemibagrus, 87

Hemibagrus baramensis, 87

Hemibagrus bongan, 87

Hemibagrus caveatus, 87

Hemibagrus centralus, 87

Hemibagrus chrysops, 87

Hemibagrus elongatus hongus, 89

Hemibagrus filamentus, 88

Hemibagrus fortis, 88

Hemibagrus furcatus, 88

Hemibagrus gracilis, 88

Hemibagrus guttatus, 88

Hemibagrus hainanensis, 88

Hemibagrus hoevenii, 88

Hemibagrus hongus, 89

Hemibagrus imbrifer, 89

Hemibagrus johorensis, 89

Hemibagrus macropterus, 87, 89, 91

Hemibagrus major, 89

Hemibagrus maydelli, 89

Hemibagrus menoda, 89

Hemibagrus microphthalmus, 90

Hemibagrus nemurus, 90

Hemibagrus olyroides, 90

Hemibagrus peguensis, 90

Hemibagrus planiceps, 90

Hemibagrus pluriradiatus, 90

Hemibagrus punctatus, 91

Hemibagrus sabanus, 91

Hemibagrus spilopterus, 91

Hemibagrus taphrophilus, 108

Hemibagrus variegatus, 91

Hemibagrus velox, 91

Hemibagrus vietnamicus, 91

Hemibagrus vietnammicus, 91

Hemibagrus wyckii, 91

Hemibagrus wyckioides, 91

Hemicetopsis, 131

Hemicetopsis amphiloxus, 131

Hemicetopsis macilentus, 133

Hemicetopsis minutus, 130

Hemicetopsis morenoi, 130

Hemicetopsis othonops, 132

hemicochliodon, Hypostomus, 254

Hemidoradinae, 166

Hemidoras, 171

Hemidoras (Leptodoras) boulengeri, 175

Hemidoras boulengeri, 175

Hemidoras hasemani, 172

Hemidoras leporhinus, 174

Hemidoras micropoeus, 171

Hemidoras microstomus, 179

Hemidoras morrisi, 172

Hemidoras notospilus, 171 
Hemidoras paraguayensis, 179

Hemidoras stenopeltis, 172

Hemileiocassis, 91

Hemileiocassis panjang, 91

Hemiloricaria, 244

Hemiloricaria altipinnis, 245

Hemiloricaria aurata, 245

Hemiloricaria beni, 245

Hemiloricaria cacerensis, 245

Hemiloricaria caracasensis, 244, 245

Hemiloricaria caracassensis, 245

Hemiloricaria castroi, 245

Hemiloricaria eigenmanni, 245

Hemiloricaria fallax, 245

Hemiloricaria formosa, 245

Hemiloricaria hasemani, 245

Hemiloricaria hoehnei, 245

Hemiloricaria jubata, 246

Hemiloricaria konopickyi, 246

Hemiloricaria lanceolata, 246

Hemiloricaria magdalenae, 246

Hemiloricaria melini, 246

Hemiloricaria morrowi, 246

Hemiloricaria nigricauda, 246

Hemiloricaria parva, 246

Hemiloricaria phoxocephala, 246

Hemiloricaria platyura, 246

Hemiloricaria sneiderni, 246

Hemiloricaria stewarti, 246

Hemiloricaria teffeana, 247

Hemiloricaria wolfei, 247

Hemiodon, 292

Hemiodon acipenserinus, 247

Hemiodon depressus, 292, 293

Hemiodon platycephalus, 288

Hemiodontichthyina, 217

Hemiodontichthys, 217, 247

Hemiodontichthys acipenserinus, 247

hemioliopterus, Phractocephalus, 336

hemioliopterus, Silurus, 336

hemipeltis, Nemadoras, 174

hemipeltis, Opsodoras, 174

hemiphractus, Callichthys, 111

Hemipimelodinae, 30

Hemipimelodus, 30, 41

Hemipimelodus aaldereni, 32

Hemipimelodus atripinnis, 34

Hemipimelodus bernhardi, 39

Hemipimelodus bicolor, 34

Hemipimelodus cochlearis, 43

Hemipimelodus colcloughi, 51

Hemipimelodus crassilabris, 42

Hemipimelodus daugueti, 43

Hemipimelodus dayi, 46

Hemipimelodus intermedius, 42

Hemipimelodus macrocephalus, 41

Hemipimelodus macrorhynchus, 32

Hemipimelodus papillifer, 48

Hemipimelodus siamensis, 41
Hemipimelodus sundanensis, 56

Hemipimelodus taylori, 48

Hemipimelodus velutinus, 48

Hemiplatystoma, 341

Hemipsilichthys, 247, 444

Hemipsilichthys azygolechis, 279

Hemipsilichthys calmoni, 279

Hemipsilichthys cameroni, 279

Hemipsilichthys cerosus, 279

Hemipsilichthys duseni, 262, 279

Hemipsilichthys eurycephalus, 279

Hemipsilichthys garbei, 279

Hemipsilichthys gobio, 247

Hemipsilichthys hypselurus, 280

Hemipsilichthys hystrix, 280

Hemipsilichthys mutuca, 280

Hemipsilichthys nimius, 247, 444

Hemipsilichthys nudulus, 280

Hemipsilichthys papillatus, 248, 444

Hemipsilichthys regani, 280

Hemipsilichthys splendens, 280

Hemipsilichthys steindachneri, 280

Hemipsilichthys stephanus, 280

Hemipsilichthys stomias, 281

Hemipsilichthys vestigipinnis, 281

Hemisilurus, 368

Hemisilurus heterorhynchus, 368

Hemisilurus mekongensis, 368

Hemisilurus moolenburghi, 369

Hemisilurus schilbeides, 371

Hemisilurus scleronema, 368

Hemisorubim, 333

Hemisorubim platyrhynchos, 333

Hemisynodontis, 309

Hemisynodontis membranacea, 309

hemiurus, Hypostomus, 254

hemiurus, Plecostomus, 254

hendricksoni, Akysis, 14

henni, Hexanematichthys, 56

Henonemus, 405

Henonemus intermedius, 405

Henonemus macrops, 406

Henonemus panzeri, 414

Henonemus punctatus, 406

Henonemus taxistigma, 406

Henonemus triacanthopomus, 440

henrici, Anopleutropius, 165

henrici, Cranoglanis, 165

henriquei, Farlowella, 238

henryi, Aoria, 100

henselii, Loricaria, 294

henselii, Rineloricaria, 294

Heptapteridae, 180

Heptapterinae, 180

Heptapterus, 180, 184

Heptapterus anisurus, 188

Heptapterus bleekeri, 184

Heptapterus collettii, 99

Heptapterus eigenmanni, 184
Heptapterus fissipinnis, 184

Heptapterus multiradiatus, 184

Heptapterus mustelinus, 184

Heptapterus ñssipinnis, 184

Heptapterus ornaticeps, 185

Heptapterus somnians, 189

Heptapterus stewarti, 184

Heptapterus surinamensis, 183

Heptapterus sympterygium, 184

Heptapterus tapanahoniensis, 184

Heptapterus tenuis, 184

heraldoi, Pimelodus, 338

herberti, Ituglanis, 407

Herberti, Trichomycterus, 407

Herklotsella, 375

Herklotsella anomala, 375

hermanni, Hypostomus, 254

Hermanni, Plecostomus, 254

herzbergii, Sciades, 53

Herzbergii, Silurus, 53

herzensteini, Leiocassis, 92

herzensteini, Macrones, 92

hesperius, Kryptopterus, 370

heteracantha, Rhamdia, 201

heteracanthus, Chaetostomus, 264, 265

heteracanthus, Lasiancistrus, 265

Heterobagrus, 94

Heterobagrus bocourti, 94

Heterobranchoides, 139

Heterobranchus, 136, 150

Heterobranchus 5-tentaculatus, 197

Heterobranchus 6-tentaculatus, 197, 200

Heterobranchus austriacus, 382

Heterobranchus bidorsalis, 150

Heterobranchus boulengeri, 151

Heterobranchus Geoffroyi, 150

Heterobranchus intermedius, 150

Heterobranchus isopterus, 151

Heterobranchus laticeps, 151

Heterobranchus longifilis, 151

Heterobranchus macronema, 151

Heterobranchus palceindicus, 151

Heterobranchus platycephalus, 151

Heterobranchus Senegalensis, 150

Heterobranchus sextentaculatus, 197, 200

Heterobranchus tapeinopterus, 149, 150

heterocephalus, Clariallabes, 137

heterocercalis, Gymnallabes typus, 150

heterodon, Arges, 64

heterodon, Astroblepus, 64

heterodon, Leporacanthicus, 266

heterodontum, Pygidium, 418

heterodontus, Trichomycterus, 418

heteromorphus, Corydoras, 118 
heteropleura, Brachyrhamdia, 181

heteropleurus, Pimelodus, 181

Heteropneustes, 136, 151

Heteropneustes fossilis, 151

Heteropneustes kemratensis, 152

Heteropneustes longipectoralis, 152

Heteropneustidae, 136

heteroptera, Rineloricaria, 293, 294

heterorhynchus, Ancistrus, 221

heterorhynchus, Hemisilurus, 368

heterorhynchus, Wallago, 368

heterorhynchus, Xenocara, 221

heterurus, Akysis, 14

heterurus, Bagrus, 97

heterurus, Zaireichthys, 28

Heudelotii, Arius, 31

heuglini, Clarotes, 160

heward-belli, Arius, 56

hexacicinnus, Macropteronotus, 148

hexadactylus, Silurus, 61

hexanema, Laides, 358

hexanema, Pangasius, 358

hexanema, Silurodon, 380, 381

Hexanematichthys, 45

Hexanematichthys henni, 56

Hexanematichthys hymenorrhinos, 54

Hexanematichthys leptaspis, 47

Hexanematichthys leptocassis, 45

Hexanematichthys mastersi, 45

Hexanematichthys sagor, 45

Hexanematichthys sundaicus, 45

Hexanematichthys surinamensis, 33

hexapterus, Micronema, 371

hexapterus, Silurus 371

heylandi, Kronichthys, 263

heylandi, Plecostomus, 263

Hilarii, Pimelodus, 200

Hildadoras, 175

Hildadoras bolivarensis, 168

Hildadoras orinocensis, 175, 176

hildae, Amarginops, 153

hildae, Chrysichthys, 153

hildebrandi, Noturus, 213

hildebrandi, Schilbeodes, 213

hildebrandi lautus, Noturus, 213

hilgendorfi, Clarias, 139

hilli, Clarias, 144

hirsuta, Microsynodontis, 310

hirsutus, Liocassus, 107

hirsutus, Microsynodontis, 310

Hisonotus, 248

Hisonotus candombe, 440

Hisonotus charrua, 440

Hisonotus depressicauda, 248

Hisonotus depressinotus, 248

Hisonotus francirochai, 248

Hisonotus insperatus, 248

Hisonotus laevior, 248

Hisonotus leptochilus, 248
Hisonotus leucofrenatus, 248

Hisonotus maculipinnis, 248

Hisonotus nigricauda, 248

Hisonotus notatus, 248

Hisonotus paulinus, 249

Hisonotus ringueleti, 249

Hisonotus taimensis, 249

histrix, Pseudacanthicus, 286

histrix, Rinelepis, 218, 286

Hito, 375

Hito taytayensis, 375, 377

Hitoichthys, 375

Hitoichthys taytayensis, 375, 377

hodgarti, Euchiloglanis, 398

hodgarti, Glyptosternum, 398

hodgarti, Parachiloglanis, 398

hoedemani, Exastilithoxus, 237

hoehnei, Hemiloricaria, 245

hoehnei, Loricaria, 245

hoehnei, Nannoglanis, 188

hoehnei, Phenacorhamdia, 188

hoeksi, Pangasius, 328

hoerdzanicus, Eobagrus. 87

hoerdzanicus, Gobibagrus, 87

hoevenii, Bagrus, 88

hoevenii, Hemibagrus, 88

Hoffstetterichthys pucai, 30

hoi, Aoria, 107

holdeni, Oxydoras, 176

hollandi, Imparfinis, 185

Hollyi, Clarias, 144

hollyi, Clarias, 147

Hollyi, Synodontis, 323

Hollyi ntemensis, Synodontis, 322

holobranchus, Xenoclarias, 153

holomelas, Pimelodus, 199

holomelas rupununi, Rhamdia, 197

holopercnus, Synodontis, 315

holostictus, Hemiancistrus, 243

Homodiaetus, 406

Homodiaetus anisitsi, 406

Homodiaetus banguela, 406

Homodiaetus graciosa, 406

Homodiaetus haemomyzon, 412

Homodiaetus passarellii, 406

Homodiaetus vazferreirai, 406

homodon, Arges, 64

homodon, Astroblepus, 64

hondae, Hypostomus, 254

hondae, Plecostomus, 250, 254

honghenensis, Glyptothorax fukiensis, 390

honghensis, Glyptothorax, 390

honghensis, Glyptothorax fukiensis, 390

hongus, Hemibagrus, 89

hongus, Hemibagrus elongatus, 89

Hopladelus, 215

Hopliancistrini, 217
Hopliancistrus, 217, 249

Hopliancistrus tricornis, 249

Hoplisoma, 111

Hoplodaros, 173

Hoplodoras, 173

Hoplodoras ramirezi, 174

hoplogenys, Ancistrus, 221

hoplogenys, Chatostomus, 221

Hoplomizoninae, 57

Hoplomyzon, 57, 61

Hoplomyzon atrizona, 61

Hoplomyzon atrizona petroleus, 61

Hoplomyzon megistus, 60

Hoplomyzon papillatus, 61

Hoplomyzon sexpapilostoma, 61

Hoplomyzontinae, 57

hoplonites, Hypostomus, 254

Hoplosoma aeneum, 112

Hoplosterninae, 108

Hoplosternum, 108, 127

Hoplosternum littorale, 127

Hoplosternum littorale daillyi, 128

Hoplosternum magdalenae, 128

Hoplosternum oronocoi, 129

Hoplosternum punctatum, 127, 128

Hoplosternum schreineri, 128

Hoplosternum shirui, 128

Hoplosternum stevardii, 128

Hoplosternum thoracatum cayennae, 128

Hoplosternum thoracatum

surinamensis, 129

hoppei, Otocinclus, 275

Horabagrinae, 441

Horabagrus, 356, 429, 441

Horabagrus brachysoma, 429

Horabagrus nigricollaris, 429

horae, Amblyceps, 99

horae, Olyra, 99

Horaglanidinae, 441

Horaglanis, 152, 441

Horaglanis alikunhii, 152

Horaglanis krishnai, 152

horai, Glyptothorax, 390

horai, Glyptothorax, 390

horai, Hara, 396

horai, Mystus, 95

horai, Mystus (Mystus) vittatus, 95

horai, Olyra, 99

horai, Pteroglanis, 387, 390

horai, Socnopaea, 431

Horiomyzon, 185

Horiomyzon retropinnatus, 185

horrida, Squaliforma, 298

horridus, Hypostomus, 297, 298

horridus, Plotosus, 350

Hosii, Liocassis, 93

houghi, Pimelodus, 210

housei, Glyptothorax, 390 
howesi, Pimelodella, 192

howong, Macrones, 88

hoyi, Pimelodus, 206

huaorani, Otocinclus, 275

huberi, Oxydoras (Rhinodoras), 174

humboldti, Doras, 176

Humboldtii, Cyclopium, 63, 64

humboldtii, Zungaro, 344

humeralis, Doras, 174

humeralis, Nemadoras, 174

humeralis, Pangasius, 326

humeratus, Synodontis, 321

humilis, Pimelodus, 198

humilis, Rhamdia, 198

hwanghoensis, Leiocassis, 107

Hyalobagrus, 92

Hyalobagrus flavus, 92

Hyalobagrus leiacanthus, 92

Hyalobagrus ornatus, 92

hydrostaticus, Pleurophysus, 410

hymenorrhinos, Hexanematichthys, 54

Hypancistrus, 249

Hypancistrus inspector, 249

Hypancistrus zebra, 249

Hypocolpterus, 227

Hypocolpterus analis, 227

Hypodoras, 172

Hypodoras forficulatus, 172

Hypophthalmini, 329

Hypophthalmus, 329, 333

Hypophthalmus devall, 334

Hypophthalmus edentatus, 333

Hypophthalmus fimbriatus, 333

Hypophthalmus goongwaree, 362

Hypophthalmus longifilis, 334

Hypophthalmus marginatus, 333

Hypophthalmus niloticus, 362, 365

Hypophthalmus nuchalis, 71, 72

Hypophthalmus oremaculatus, 333

Hypophthalmus perporosus, 334

Hypophthalmus Spixii, 333

Hypophthalmus taakree, 362

hypophthalmus, Helicophagus, 325

hypophthalmus, Arius, 39, 40

hypophthalmus, Cathorops, 40

hypophthalmus, Pangasianodon, 325

hypophthalmus, Ompok, 371, 372

hypophthalmus, Silurus, 372

Hypoptopoma, 217, 249

Hypoptopoma bilobatum, 250

Hypoptopoma carinatum, 276

Hypoptopoma guentheri, 250

Hypoptopoma guianense, 250

Hypoptopoma gulare, 249, 250

Hypoptopoma inexpectata, 250

Hypoptopoma inexspectatum, 250

Hypoptopoma joberti, 250

Hypoptopoma psilogaster, 250

Hypoptopoma steindachneri, 250
Hypoptopoma thoracatum, 249, 250

Hypoptopomatinae, 217

Hypoptopominae, 217

Hypostoma etentaculatum, 291

Hypostoma punctatum, 220

Hypostoma squalinum, 298

Hypostomiden, 216

Hypostomus, 216, 217, 250

Hypostomus aburrensis, 227

Hypostomus affinis, 251

Hypostomus agna, 251

Hypostomus alatus, 251

Hypostomus albopunctatus, 251

Hypostomus ancistroides, 251

Hypostomus angipinnatus, 251

Hypostomus argus, 251

Hypostomus asperatus, 251

Hypostomus aspilogaster, 251

Hypostomus atropinnis, 251

Hypostomus aurantiacus, 278

Hypostomus auroguttatus, 251

Hypostomus barbatus, 286, 287

Hypostomus bolivianus, 252

Hypostomus borellii, 252

Hypostomus boulengeri, 252

Hypostomus brevicauda, 252

Hypostomus brevis, 252

Hypostomus brevitentaculatus, 291

Hypostomus bufonius, 219

Hypostomus calamita, 219

Hypostomus carinatus, 252

Hypostomus carvalhoi, 252

Hypostomus cirrhosus, 219, 220

Hypostomus cochliodon, 250, 252,

253-262

Hypostomus commersoni, 252

Hypostomus coppenamensis, 253

Hypostomus corantijni, 253

Hypostomus crassicauda, 253

Hypostomus derbyi, 253

Hypostomus dlouhyi, 253

Hypostomus duodecimalis, 290, 291

Hypostomus emarginatus, 297

Hypostomus eptingi, 253

Hypostomus ericae, 253

Hypostomus ericius, 253

Hypostomus erinaceus, 221

Hypostomus fluviatilis, 253

Hypostomus fonchii, 253

Hypostomus francisci, 254

Hypostomus garmani, 254

Hypostomus goyazensis, 254

Hypostomus granosus, 273

Hypostomus guacari, 250, 258, 259

Hypostomus guacharote, 265

Hypostomus guttatus, 287

Hypostomus gymnorhynchus, 254

Hypostomus gymnorhynchus

occidentalis, 257
Hypostomus gymnorhynchus tapanahoniensis, 261

Hypostomus hemicochliodon, 254

Hypostomus hemiurus, 254

Hypostomus hermanni, 254

Hypostomus hondae, 254

Hypostomus hoplonites, 254

Hypostomus horridus, 297, 298

Hypostomus iheringii, 255

Hypostomus interruptus, 255

Hypostomus isbrueckeri, 255

Hypostomus itacua, 217

Hypostomus jaguribensis, 255

Hypostomus johnii, 255

Hypostomus laplatae, 255

Hypostomus latifrons, 255

Hypostomus latirostris, 255

Hypostomus levis, 255

Hypostomus lexi, 255

Hypostomus lima, 256

Hypostomus longiradiatus, 256

Hypostomus luteomaculatus, 256

Hypostomus luteus, 256

Hypostomus macrophthalmus, 256

Hypostomus macrops, 256

Hypostomus macushi, 256

Hypostomus margaritifer, 256

Hypostomus meleagris, 256

Hypostomus micromaculatus, 256

Hypostomus microstomus, 250, 257

Hypostomus multiradiatus, 290, 291

Hypostomus mutucae, 257

Hypostomus myersi, 257

Hypostomus nematopterus, 257

Hypostomus niceforoi, 257

Hypostomus nickeriensis, 257

Hypostomus niger, 257

Hypostomus nigricans, 278

Hypostomus nigromaculatus, 257

Hypostomus niveatus, 226, 227

Hypostomus nudiceps, 223

Hypostomus nudiventris, 257

Hypostomus occidentalis, 257

Hypostomus oculeus, 257

Hypostomus pagei, 257

Hypostomus pantherinus, 258

Hypostomus papariae, 258

Hypostomus paranensis, 258

Hypostomus pardalis, 292

Hypostomus paucimaculatus, 258

Hypostomus paucipunctatus, 258

Hypostomus paulinus, 258

Hypostomus pictus, 265

Hypostomus piratatu, 258

Hypostomus plecostomoides, 258

Hypostomus plecostomus, 258

Hypostomus pseudohemiurus, 259

Hypostomus pseudohemiurus

macrophthalmus, 256 
Hypostomus punctatus, 220, 259

Hypostomus pusarum, 259

Hypostomus pyrineusi, 259

Hypostomus regani, 259

Hypostomus robinii, 259

Hypostomus rondoni, 259

Hypostomus roseopunctatus, 259

Hypostomus saramaccensis, 259

Hypostomus scabriceps, 260

Hypostomus scaphyceps, 260

Hypostomus sculpodon, 260

Hypostomus seminudus, 260

Hypostomus serratus, 286

Hypostomus simios, 260

Hypostomus sipaliwinii, 260

Hypostomus soniae, 260

Hypostomus spinosus, 286

Hypostomus strigaticeps, 260

Hypostomus subcarinatus, 260

Hypostomus surinamensis, 260

Hypostomus tapanahoniensis, 260

Hypostomus taphorni, 261

Hypostomus tapijara, 261

Hypostomus Temminckii, 224

Hypostomus tenuis, 298

Hypostomus ternetzi, 261

Hypostomus tietensis, 261

Hypostomus topavae, 261

Hypostomus unae, 261

Hypostomus uruguayensis, 261

Hypostomus vaillanti, 261

Hypostomus variipictus, 261

Hypostomus varimaculosus, 261

Hypostomus variostictus, 261

Hypostomus ventromaculatus, 262

Hypostomus vermicularis, 262

Hypostomus verres, 262

Hypostomus vicinus, 278

Hypostomus waiampi, 262

Hypostomus watwata, 262

Hypostomus winzi, 262

Hypostomus wuchereri, 262

Hypothalmus dawalla, 69

Hypselobagrus, 93

Hypselobagrus macronema, 97

hypselopterus, Bagrichthys, 82

hypselopterus, Bagrus, 82

hypselurus, Breitensteinia, 15

hypselurus, Hemipsilichthys, 280

hypselurus, Pareiorhaphis, 280

hypselurus, Pimelodus, 198

Hypsidoridae, 203

Hypsidoris, 203

Hypsidoris farsonensis, 203

Hypsidoris oregonensis, 203

hypsiura, Amaralia, 57

hypsiurus, Bunocephalus, 57

hypsiurus, Oreoglanis, 397

hyrtlii, Neosilurus, 347, 348 hystrix, Acanthicus, 218

hystrix, Hemipsilichthys, 280

hystrix, Pareiorhaphis, 280

I

ichikawai, Coreobagrus, 86

ichikawai, Pseudobagrus, 86

ichneumon, Coreobagrus, 431

Ichthaelurinae, 203

Ichthaelurus, 203, 208

Ichthaelurus Kevinskii, 204

Ichthaelurus McCaskei, 204

Ichthaelurus robustus, 210

Ichthyaelurus, 208

Ictaluri, 203

Ictaluridae, 203

Ictalurus, 203, 207

Ictalurus (Amiurus) hazenensis, 204

Ictalurus anguilla, 210

Ictalurus australis, 208

Ictalurus balsanus, 208

Ictalurus benderensis, 206

Ictalurus dugesii, 208, 209

Ictalurus echinatus, 208

Ictalurus furcatus, 208, 211

Ictalurus lambda, 209

Ictalurus lavetti, 204

Ictalurus leidyi, 204

Ictalurus lupus, 209, 211

Ictalurus macgrewi, 205

Ictalurus mexicanus, 209

Ictalurus nebulosus pannonicus, 206

Ictalurus ochoterenai, 209

Ictalurus okeechobeensis, 210

Ictalurus pectinatus, 206

Ictalurus peregrinus, 207

Ictalurus pricei, 209

Ictalurus punctatus, 208, 209, 211

Ictalurus rhaeas, 210

Ictalurus sawrockensis, 206

Ictalurus serracanthus, 206

Ictalurus simpsonii, 210

Ictalurus spodius, 211

Ictalurus vespertinus, 206

idenburgi, Copidoglanis, 348

idenburgi, Neosilurus, 348

ignobilis, Rhamdella, 196

iheringi, Arius, 343

iheringi, Cetopsorhamdia, 182

iheringi, Hassar, 171

iheringi, Microglanis, 354

iheringi, Plecostomus, 298

iheringi, Pygidium, 419

iheringi, Steindachneridion, 343

iheringi, Trichomycterus, 419

Iheringichthys, 334

Iheringichthys labrosus, 334

Iheringichthys megalops, 334

iheringii, Bunocephalus, 59

iheringii, Hypostomus, 255 iheringii, Plecostomus, 255, 298

ikapor, Plotoseus, 350

ikiensis, Pseudobagrus, 102

ilebrevis, Synodontis, 445

ilesi, Bathyclarias, 136

ilesi, Dinotopterus, 136

Ilictis, 215

imberbis, Silurus, 70, 379

imbrifer, Hemibagrus, 89

imitator, Brachyrhamdia, 181

imitator, Corydoras, 118

immaculata, Pseudecheneis, 400

immaculatum, Pygidium, 419

immaculatus, Auchenipterus, 80

immaculatus, Pseudecheneis, 400

immaculatus, Silurus (Callichrus), 374

immaculatus, Trichomycterus, 419

Imparales, 180, 188

Imparales mariai, 188

Imparales panamensis, 180

Imparfinis, 185

Imparfinis bolivianus, 188

Imparfinis borodini, 185

Imparfinis cochabambae, 185

Imparfinis guttatus, 185

Imparfinis hasemani, 185

Imparfinis hollandi, 185

Imparfinis insidiosus, 182

Imparfinis lineatus, 185

Imparfinis longicauda, 185

Imparfinis longicauda, 185

Imparfinis microps, 186

Imparfinis minutus, 186

Imparfinis mirini, 186

Imparfinis nemacheir, 186

Imparfinis pijpersi, 186

Imparfinis piperatus, 185, 186

Imparfinis pristos, 186

Imparfinis pseudonemacheir, 186

Imparfinis schubarti, 186

Imparfinis spurrellii, 186

Imparfinis stictonotus, 186

Imparfinis tenebrosus, 189

impluviatus, Mystus, 95

inaequalis, Amphilius

grammatophorus, 23

incae, Trichomycterus, 423

Incaichthys suarezi, 30

incolicana, Corydoras, 118

indicus, Brachyspondylus, 326

indicus, Glyptothorax, 390

indicus, Kryptopterus, 367

indicus, Pangasius, 326

indicus, Pimelodus, 19

indicus, Silurus, 374

indragiriensis, Silurichthys, 378

ineac, Phractura, 28

ineac, Trachyglanis, 28

inermis, Ageneiosus, 69 
inermis, Akysis, 16

inermis, Amblyceps, 17

inermis, Nematogenys, 324

inermis, Pseudobagarius, 16

inermis, Silurus, 69, 379

inermis, Trichomycterus, 324

inexpectata, Aristommata, 249, 250

inexpectata, Hypoptopoma, 250

inexspectatum, Hypoptopoma, 250

infraoculare, Sorubim, 342

infulatus, Oreoglanis, 397

infuscatus, Erethistoides, 442

ingluvies, Osteogeneiosus, 51

inornata, Pseudolaguvia, 401

inornatus, Liocassis, 103

inornatus, Pseudomystus, 103

inpai, Aguarunichthys, 329

insculptus, Arius, 50

insculptus, Doras, 179

insculptus, Notarius, 50

insidiator, Arius, 45

insidiator, Hemiarius, 44

insidiosa, Cetopsorhamdia, 182

insidiosus, Imparfinis, 182

insidiosus, Stegophilus, 414

insignarius, Pimelodon, 211, 213

insigne, Pimelodus, 213

insignis, Auchenipterus, 79

insignis, Breitensteinia, 15

insignis, Noturus, 213

insignis, Oreoglanis, 397

insignis, Pimelodella, 190

insignis, Pimelodus, 339

insignis, Trachelyopterus, 79

insignis badeli, Trachycorystes, 80

insignis peloichthys, Trachycorystes, 80

insolitus, Clarias, 144

inspector, Hypancistrus, 249

insperatus, Hisonotus, 248

insularum, Netuma, 50

intermedia, Phractura, 26

intermedia, Tatia, 77

intermedium, Pseudoplatystoma

fasciatum, 341

intermedius, Centromochlus, 77

intermedius, Clarias, 144

intermedius, Hemipimelodus, 42

intermedius, Henonemus, 405

intermedius, Heterobranchus, 150

intermedius, Pelteobagrus, 100

intermedius, Pseudecheneis, 400

intermedius, Pseudobagrus, 100

intermedius, Pseudotocinclus, 290

intermedius, Schilbe, 363

intermedius, Stegophilus, 405

intermedius, Trachyglanis, 28

interruptus, Hypostomus, 255

interruptus, Plecostomus, 255 interspinalum, Glyptosternon, 390

interspinalus, Glyptothorax, 390

intonsus, Ernstichthys, 60

inusitata, Pterocryptis, 377

irsacae, Synodontis, 314

Irvineia, 358

Irvineia orientalis, 358

Irvineia voltae, 358

irwini, Megalodoras, 173, 174

isacanthus, Auchenipterus, 80

isbrueckeri, Farlowella, 238

isbrueckeri, Corydoras, 119

isbrueckeri, Farlowella, 238

isbrueckeri, Hypostomus, 255

Isbrueckerichthys, 262, 441

Isbrueckerichthys alipionis, 262

Isbrueckerichthys calvus, 441

Isbrueckerichthys duseni, 262

Isbrueckerichthys epakmos, 262

Isbrueckerichthys saxicola, 441

ischnosoma, Acrochordonichthys, 12

isheriensis, Clarias (Clarioides), 139

Isidori, Schilbe, 365

isopterus, Heterobranchus, 151

Isorineloricaria, 263

Isorineloricaria spinosissima, 263

isthmensis, Aelurichthys, 38

Istlarius, 208

Istlarius balsanus, 208

Istlarius balsanus occidentalis, 208

itacaiunas, Rhamdia, 198

itacambirussu, Trichomycterus, 419

itacarambiensis, Trichomycterus, 419

itacua, Hypostomus, 217

itaimbezinho, Epactionotus, 236

itapicuruensis, Pimelodella, 192

itatiayae, Trichomycterus, 419

itchkeea, Gagata, 386

itchkeea, Phractocephalus, 386

Ituglanis, 406

Ituglanis amazonicus, 406

Ituglanis bambui, 406

Ituglanis cahyensis, 444

Ituglanis eichorniarum, 407

Ituglanis epikarsticus, 407

Ituglanis gracilior, 407

Ituglanis guayaberensis, 407

Ituglanis herberti, 407

Ituglanis laticeps, 407

Ituglanis macunaima, 407

Ituglanis metae, 407

Ituglanis nebulosus, 407

Ituglanis parahybae, 407

Ituglanis parkoi, 407

Ituglanis passensis, 408

Ituglanis proops, 408

Ituglanis ramiroi, 408

ituriensis, Synodontis, 315

iturii, Auchenoglanis, 163 iturii, Synodontis, 315

iwokrama, Denticetopsis, 133

Ixinandria, 263

Ixinandria montebelloi, 263

Ixinandria steinbachi, 263

izabalensis, Potamarius, 53

J

jacksonensis, Netuma thalassina, 49 jacksoni, Dinotopterus, 149

jacksonii, Amphilius, 21

jacupiranga, Trichomycterus, 419

jaekeli, Otolithus (Arius), 435

jaensis, Clarias, 144

jagur, Macropteronotus, 140

jaguribensis, Hypostomus, 255

jaguribensis, Plecostomus, 255

jahu, Paulicea, 344

jahu, Zungaro, 344

jalalensis, Glyptothorax, 390

jallae, Dinotopterus, 146

jallae, Synodontis, 316

jandia, Sorubim, 342, 343

janeirensis, Pseudotothyris, 290

japonicus, Centranodon, 379

japonicus, Silurus, 378, 379

jaraguensis, Loricaria, 294

jaraguensis, Rineloricaria, 294

jataiensis, Ancistrus, 222

jatia, Cephalocassis, 41

jatius, Pimelodus, 41

jauruensis, Farlowella, 238

jauruënsis, Farlowella, 238

javanensis, Belodontichthys, 381

javanica, Rhamdia, 431

Javensis, Bagrus, 45

javus, Pimelodus, 431

jayarami, Myersglanis, 396

jaynei, Ompok, 372

jeanesianus, Liposarcus, 292

jegui, Chaetostoma, 228

jella, Arius, 36

jelskii, Ancistrus, 222

Jelskii, Chaetostomus, 222

jenynsii, Pimelodus, 196

jenynsii, Rhamdella, 196

jequitinhonha, Rhamdia, 198

Jequitinhonhae, Auchenipterus

(Pseudauchenipterus), 76

jequitinhonhae, Pseudauchenipterus,

76

jequitinhonhae, Trichomycterus, 419

jerdoni, Hara, 396

jimi, Parotocinclus, 282

jivaro, Pimelodus, 338

joberti, Hypoptopoma, 250

joberti, Otocinclus, 250

johannae, Septobranchus, 42

johnelsi, Chrysichthys, 156

johnii, Hypostomus, 255 
Johnii, Plecostomus, 255

johnsoni, Pygidium, 419

johnsoni, Trichomycterus, 419

johorensis, Hemibagrus, 89

johorensis, Mystus, 89

jokeannae, Trachycorystes, 80

jordani, Tachisurus, 33

joselimai, Leporacanthicus, 266

joselimaianus, Glyptoperichthys, 291

joselimaianus, Pterygoplichthys, 291

juaro, Pangasius, 327

jubata, Hemiloricaria, 246

jubata, Loricaria, 246

julii, Corydoras, 119

jumbo, Parotocinclus, 282

juquiaae, Corydoras, 121

juquiae, Corydoras, 121

juquiae, Otothyris, 276

juquiae, Pseudotocinclus, 290

jurubidae, Astroblepus, 65

jurubidae, Cetopsis, 132

jurubidae, Pseudocetopsis, 132

juruense, Brachyplatystoma, 331

juruense, Platystoma, 331

juruensis, Leptodoras, 172

juvens, Pterygoplichthys, 291

$\mathbf{K}$

kaiei, Corymbophanes, 232

kaifenensis, Leiocassis, 107

kajan, Macrones, 88

kakrimensis, Amphilius, 21

kalambo, Chiloglanis, 306

Kalyptodoras, 172

Kalyptodoras bahiensis, 172

kamengensis, Euchiloglanis, 399

kamengensis, Pareuchiloglanis, 399

kanei, Corydoras, 119

kanganamanensis, Arius, 39

kanpurensis, Pinniwallago, 375

kapuasensis, Clarias, 144

kapuri, Laguvia ribeiroi, 401

kapuri, Pseudolaguvia, 401

kasai, Eutropiellus, 360, 361

kashmirensis, Glyptothorax, 390, 393

katangae, Synodontis, 315

Keletius, Bagrus, 98

kelioides, Encheloclarias, 149

kempi, Olyra, 99

kemratensis, Clarisilurus, 151, 152

kemratensis, Heteropneustes, 152

kessleri, Arius, 50

kessleri, Notarius, 50

Ketengus, 45

Ketengus typus, 45

Kevinskii, Ichthaelurus, 204

khartoumensis, Synodontis, 316

khavalchor, Neotropius, 359

kinabatanganensis, Pangasius, 326

kingi, Liobagrus, 19 kingsleyae, Clarias, 144

kingsleyae, Chrysichthys, 160

kirkii, Arius, 52

kishinouyei, Coraglanis, 385

kishinouyei, Euchiloglanis, 385

kitsoni, Arius 56

kivuensis, Amphilius, 21

kneri, Callichthys, 111

kneri, Loricaria, 299

kneri, Oxydoras, 175

kneri, Sturisoma, 299

Knerii, Acestra, 238

knerii, Bunocephalus, 59

knerii, Farlowella, 238

knerii, Pimelodus (Rhamdia), 199

knerii, Trichomycterus, 419

koenigi, Bagrus, 84

koensis, Synodontis, 316

koepckei, Myoglanis, 187

konopickyi, Hemiloricaria, 246

konopickyi, Loricaria, 246

koreanus, Pseudobagrus, 102

krattensis, Wallago, 374

krefftii, Amphilius, 23

krempfi, Pangasius, 326

krishnai, Horaglanis, 152

krishnensis, Mystus, 89

kronei, Corydoras, 129

kronei, Harttia, 241

kronei, Loricaria, 294

kronei, Pimelodella, 192

kronei, Rineloricaria, 294

kronei, Typhlobagrus, 189, 192

Kronichthys, 263

Kronichthys heylandi, 263

Kronichthys lacerta, 263

Kronichthys subteres, 263

Kryptopterichthys, 369

Kryptopterichthys macrocephalus, 370

Kryptopterini, 367

Kryptopterus, 367, 369

Kryptopterus baramensis, 369

Kryptopterus bicirrhis, 369

Kryptopterus cheveyi, 371

Kryptopterus cryptopterus, 369

Kryptopterus deignani, 375

Kryptopterus dissitus, 369

Kryptopterus eugeneiatus, 369

Kryptopterus geminus, 369

Kryptopterus hesperius, 370

Kryptopterus indicus, 367

Kryptopterus lais, 370

Kryptopterus limpok, 370

Kryptopterus lumholtzi, 370

Kryptopterus macrocephalus, 370

Kryptopterus micropus, 369

Kryptopterus minimus, 370

Kryptopterus minor, 370

Kryptopterus mononema, 370
Kryptopterus moorei, 371

Kryptopterus palembangensis, 370

Kryptopterus paraschilbeides, 370

Kryptopterus parvanalis, 375

Kryptopterus piperatus, 370

Kryptopterus platypogon, 371

Kryptopterus sabanus, 370

Kryptopterus schilbeides, 371

kryptos, Xiliphius, 62

kuhlmanni, Peckoltia, 284

kuhlmanni, Peckoltichthys, 284

kükenthali, Glyptosternum, 391

kunyit, Pangasius, 326

kurdistanicum, Glyptosternum, 390

kurdistanicus, Glyptothorax, 390

kurzii, Akysis, 18

kutchensis, Arius, 56

kuturnee, Phractocephalus, 105

kuturnee, Rita, 105

kyphus, Pelteobagrus, 82

kyphus, Pseudobagrus, 82

$\mathbf{L}$

laak, Glyptosternum, 395

laani, Apistoloricaria, 226

labeo, Synodontis, 323

labialis, Astroblepus, 65

labialis, Loricaria, 270

labialis, Loricariichthys, 270

labiatum, Exostoma, 385

labiatus, Glyptosternon, 385

labiosus, Copidoglanis, 345

labrax, Exostoma, 387

labrosus, Copidoglanis, 345

labrosus, Iheringichthys, 334

labrosus, Pimelodus, 334

labrosus, Xyliphius, 62

Lacantunia, 216

Lacantunia enigmatica, 216

Lacantuniidae, 216

lacerdai, Corydoras, 119

lacerta, Kronichthys, 263

lacerta, Plecostomus, 263

lachneri, Noturus, 213

lacus, Aoria, 108

lacustricolus, Synodontis, 322

lacustris, Auchenipterus, 79

lacustris, Trachelyopterus, 79

lacustris, Gadus, 208

laessoei, Synodontis, 316

laeviceps, Arius, 55

laeviceps, Clarias, 144

laeviceps dialonensis, Clarias, 142

laevigata, Microsynodontis, 310

laevigatulus, Doras, 177

laevigatus, Bagrus, 49

laevigatus, Callichthys, 127

laevigatus, Microsynodontis, 310

laevior, Hisonotus, 248

laevis, Gogangra, 395 
laevis, Platystacus, 58

laeviuscula, Loricaria, 289

laeviuscula, Pseudoloricaria, 289

lagoensis, Arius, 31

lagoensis, Chrysichthys, 160

lagoichthys, Loricaria gymnogaster, 297

lagoichthys, Spatuloricaria, 297

Laguvia, 382, 395

Laguvia manipurensis, 382

Laguvia ribeiroi, 401

Laguvia ribeiroi kapuri, 401

Laguvia shawi, 395, 402

Laguviini, 382

Laides, 358

Laides hexanema, 358

Laides longibarbis, 359

Laimumena, 431

Laimumena barbonica, 431, 432

Lais, 358

lais, Kryptopterus, 370

Laïs, 358

laïs, Silurus, 370

lakoi, Aspidoras, 109

laluchensis, Rhamdia, 198

lamani, Amphilius, 22

Lamarrii, Bagrus, 106, 107

lambda, Ictalurus, 209

lamberti, Corydoras, 119

lamberti, Microsynodontis, 310

Lambertia, 347

Lambertia atra, 347

Lambertichthys, 347

Lambertichthys ater sepikensis, 347

lamghur, Silurus, 373

lamina, Loricaria, 288

laminus, Pseudohemiodon, 288

Lamontichthys, 263

Lamontichthys filamentosus, 263

Lamontichthys llanero, 264

Lamontichthys maracaibero, 264

Lamontichthys stibaros, 264

lamottei, Chiloglanis, 306

lamottei, Clarias, 144

lampei, Amphilius, 22

Lampiella, 217, 264

Lampiella gibbosa, 264

Lampiellini, 217

lampris, Glyptothorax, 391

lanceolata, Hemiloricaria, 246

lanceolata, Loricaria, 246

lanceolata, Rineloricaria, 246

lanceolatus, Nemuroglanis, 188

landanensis, Tachysurus, 57

landinga, Trichomycterus, 419

landoni, Hemiancistrus, 243

lanzhouensis, Silurus, 380

laosensis, Glyptothorax, 391

laplatae, Hypostomus, 255 laplatae, Plecostomus, 255

larai, Bunocephalus, 59

larnaudii, Pangasius, 326

Lasiancistrus, 264

Lasiancistrus anthrax, 288

Lasiancistrus brevispinis, 287

Lasiancistrus caucanus, 264

Lasiancistrus dumus, 289

Lasiancistrus guacharote, 265

Lasiancistrus guapore, 265

Lasiancistrus heteracanthus, 265

Lasiancistrus longispinis, 287

Lasiancistrus maracaiboensis, 265

Lasiancistrus mayoloi, 265

Lasiancistrus nationi, 223

Lasiancistrus nicoi, 289

Lasiancistrus saetiger, 265

Lasiancistrus schomburgkii, 265

Lasiancistrus scolymus, 265

Lasiancistrus tentaculatus, 265

Lasiancistrus tigris, 288, 289

Lasiancistrus volcanensis, 264

lata, Loricaria, 268

lateralis, Pimeletropis, 332

Lateralis, Pimelodus pallidus var., 210

Lateralis, Silurus pallidus, 210

lateristriga, Pimelodella, 192

lateristrigus, Pimelodes, 192

lateristrigus, Pimelodus, 192

laticauda, Rhamdia, 198, 199, 202

laticauda typhla, Rhamdia, 198

laticaudatus, Amphilius, 22

laticaudus, Pimelodus, 198

laticeps, Adelopeltis, 107

laticeps, Allabenchelys, 137

laticeps, Amblyceps, 17

laticeps, Arius, 41

laticeps, Chrysichthys, 157

laticeps, Clariallabes, 137

laticeps, Clarotes, 160

laticeps, Eutropius, 364

laticeps, Heterobranchus, 151

laticeps, Ituglanis, 407

laticeps, Loricaria, 288

laticeps, Olyra, 17, 18

laticeps, Pimelodella, 192

laticeps, Pimelodus, 160

laticeps, Plotosus, 345

laticeps, Pseudohemiodon, 288

laticeps, Schilbe, 364

laticeps, Silurus, 151

laticeps, Trichomycterus, 407

laticeps australis, Pimelodella, 189

latidens, Astroblepus, 65

latidens, Pygidium, 420

latidens, Trichomycterus, 419

latifrons, Ancistrus, 222

latifrons, Chaetostomus, 219, 222

latifrons, Hypostomus, 255 latifrons, Noturus, 211

latifrons, Synodontis gambiensis, 315

latirostris, Arius, 47

latirostris, Hypostomus, 255

latirostris, Loricaria, 294

latirostris, Neoarius, 47

latirostris, Plecostomus, 255

latirostris, Rineloricaria, 294

latirostris, Sorubim, 342

latiscutatus, Arius, 31

latisoma, Farlowella, 239

latistriatum, Pygidium, 420

latistriatus, Trichomycterus, 420

latiura, Dasyloricaria, 233

latiura, Loricaria filamentosa, 233

latovittatus, Cryptopterus, 374

latus, Corydoras, 119

laucaensis, Trichomycterus, 420

laukidi, Rhamdia, 199

laurenti, Pimelodella, 192

lautus, Noturus hildebrandi, 213

lavetti, Ameiurus, 204

lavetti, Ictalurus, 204

layardi, Arius, 51

lazera, Clarias, 143

laviuscula, Loricaria, 289

leerii, Wallago, 380, 381

lehmanni, Rhamdia, 202

leiacanthus, Clarias, 145

leiacanthus, Hyalobagrus, 92

leiacanthus, Leiocassis, 104

leiacanthus, Ompok, 371, 372

leiacanthus, Pseudomystus, 104

leiacanthus, Wallago, 371, 372

Leiarius, 334

Leiarius marmoratus, 334

Leiarius pictus, 334

leidyi, Ameiurus, 204

leidyi, Ictalurus, 204

leightoni, Oxyloricaria, 300

leightoni, Sturisomatichthys, 300

Leiocassis, 92

Leiocassis (Dermocassis) analis, 101

Leiocassis aculeatus, 92

Leiocassis albicollaris, 104

Leiocassis albomarginatus, 101

Leiocassis bicolor, 104

Leiocassis brashnikowi, 100

Leiocassis brevicaudatus, 102

Leiocassis chaseni, 93

Leiocassis collinus, 443

Leiocassis crassilabris, 92

Leiocassis crassilabris macrops, 92

Leiocassis crassirostris, 92

Leiocassis ellenriederii, 104

Leiocassis fluviatilis, 85

Leiocassis fuscus, 103

Leiocassis hainanensis, 88

Leiocassis herzensteini, 92 
Leiocassis hwanghoensis, 107

Leiocassis kaifenensis, 107

Leiocassis leiacanthus, 104

Leiocassis longibarbus, 92

Leiocassis longirostris, 93

Leiocassis lui, 102

Leiocassis macropterus, 83

Leiocassis mahakamensis, 104

Leiocassis micropogon, 93

Leiocassis microps, 100

Leiocassis moeschii, 105

Leiocassis myersi, 104

Leiocassis nitidus, 82

Leiocassis omeihensis, 102

Leiocassis poecilopterus, 93

Leiocassis regani, 93

Leiocassis robustus, 104

Leiocassis saravacensis, 93

Leiocassis similis, 108

Leiocassis sinyanensis, 108

Leiocassis tenebricus, 443

Leiocassis tenuifurcatus, 93

Leiocassis trilineatus, 103

Leiocassis ussuriensis, 101

Leiocassis virgatus, 101

leiopleura, Harttia, 240, 241

Leiosynodontis, 311

leiotetocephalus, Arius, 52

Leliella, 293, 294

lemai, Rhamdella, 196

lemairii, Eutropius, 364

lembesseensis, Clariallabes melas, 137

lemniscatus, Pimelodus, 213

lemoinei, Arius, 432

lentiginosa, Ariopsis, 33

lentiginosa, Loricaria prolixa, 286

lentiginosa, Proloricaria, 286

lentiginosus, Amphilius, 22

lentiginosus, Doras, 177

lentiginosus, Tachisurus, 33

leonensis, Malapterurus, 301

leoparda, Synodontis, 316

leopardina, Synodontis, 316

leopardinus, Synodontis, 316

leopardinus, Trachelyopterus, 80

leopardinus, Trachycorystes, 80

leopardum, Glanidium, 75

leopardus, Centromochlus

(Gephyromochlus), 75

leopardus, Corydoras, 119

leopardus, Pseudacanthicus, 286

leopardus, Stoneiella, 286

leopardus, Synodontis, 316

Leporacanthicus, 265

Leporacanthicus galaxias, 265

Leporacanthicus heterodon, 266

Leporacanthicus joselimai, 266

Leporacanthicus triactis, 266

leporhinus, Hemidoras, 174 leporhinus, Nemadoras, 174

leptacanthus, Noturus, 213

Leptarius, 53

Leptarius dowii, 53

leptaspis, Hexanematichthys, 47

leptaspis, Neoarius, 47

Lepthoplosternum, 128

Lepthoplosternum altamazonicum, 128

Lepthoplosternum beni, 128

Lepthoplosternum pectorale, 128

Lepthoplosternum stellatum, 128

Lepthoplosternum tordilho, 129

Lepthoplosternum ucamara, 129

Leptoancistrus, 266

Leptoancistrus canensis, 266

Leptoancistrus cordobensis, 266

leptocassis, Hexanematichthys, 45

leptochilus, Hisonotus, 248

Leptodoras, 172

Leptodoras acipenserinus, 172

Leptodoras cataniai, 172

Leptodoras copei, 172

Leptodoras hasemani, 172

Leptodoras juruensis, 172

Leptodoras linnelli, 173

Leptodoras myersi, 173

Leptodoras nelsoni, 173

Leptodoras praelongus, 173

Leptodoras rogersae, 173

Leptodoras trimaculatus, 174

Leptoglanidinae, 20

Leptoglaninae, 20

Leptoglanis, 20, 25

Leptoglanis, 187

Leptoglanis bouilloni, 164

Leptoglanis brevis, 28

Leptoglanis brieni, 24

Leptoglanis camerunensis, 28

Leptoglanis dorae, 28

Leptoglanis essequibensis, 187

Leptoglanis flavomaculatus, 28

Leptoglanis mandevillei, 28

Leptoglanis wamiensis, 29

Leptoglanis xenognathus, 25

leptonema, Silurus, 374

leptonotacanthus, Arius, 34

Leptops, 215

Leptorhamdia, 187

Leptorhamdia essequibensis, 187

Leptorhamdia marmorata, 187

Leptorhamdia schultzi, 187

leptorhynchus, Helicophagus, 324

leptos, Acentronichthys, 180

leptosoma, Rhamdella, 196

leptostriatus, Microglanis, 441

leptura, Andersonia, 24

lepturum, Chaetostoma, 229

lepturus, Chaetostomus, 229 lepturus, Cnidoglanis, 346, 442

lepturus, Euristhmus, 346

lepturus, Oreoglanis, 397

lepturus, Xyliphius, 62

leptus, Pimelodus, 332

Lerichei, Otolithus (Arius), 434

leroyi, Chimarrhoglanis, 21, 23

lestradei, Lophiobagrus, 161, 162

leucofrenatus, Hisonotus, 248

leucofrenatus, Otocinclus, 248

leucomelas, Chaetostoma, 229

leucomelas, Chaetostomus, 229

leucomelas, Corydoras, 119

leucophasis, Bagrus, 96

leucophasis, Mystus, 96

leucopodus, Silurichthys, 378

Leucoptera, Pimelodus pallidus, 210

Leucoptera, Silurus pallidus, 210

leucorhynchus, Akysis, 16

leucorhynchus, Pseudobagarius, 16

leucostictus, Ancistrus, 222

leucostictus, Chaetostomus, 222

levequei, Chrysichthys, 157

levequei, Synodontis, 316

levis, Copidoglanis, 345

levis, Hypostomus, 255

levis, Rhinelepis, 255

lewi, Trichomycterus, 420

lexi, Hypostomus, 255

lexi, Plecostomus, 255

Leyvaichths, 134

Leyvaichthys, 134

Leyvaichthys castaneus, 134, 407

liacanthus, Clarias, 145

lianae, Copionodon, 404

Liauchenoglanis, 161

Liauchenoglanis maculatus, 161

liberiensis, Clarias, 141

liberiensis, Eutropius, 364

libertatis, Doras, 174

lica, Bagarius, 383

lima, Hypostomus, 256

lima, Loricaria, 294

lima, Plecostomus, 256

lima, Rineloricaria, 294

lima, Silurus, 342

lima, Sorubim, 342

lima atropinnis, Plecostomus, 251

lima microlepidota, Loricaria, 295

lima var. microlepidota, Loricaria, 295

Limatulichthys, 266

Limatulichthys griseus, 266

Limatulichthys petleyi, 266

limbatus, Bagrus, 107

limbatus, Choeroplotosus, 346

limbatus, Plotosus, 108, 346, 350

limosus, Pimelodus, 215

limosus, Plecostomus, 253

limosus, Pylodictis, 215 
limosus, Silurus, 215

limpok, Kryptopterus, 370

limpok, Silurus, 370

limulus, Eurycheilichthys, 236

linami, Pimelodella, 192

lindica, Phractura, 26

lindicus, Clarias, 141

lineata, Nannorhamdia, 185

lineatum, Euglyptosternum, 388

lineatus, Imparfinis, 185

lineatus, Plotosus, 350

lineatus, Plotosus, 351, 352

lineatus, Silurus, 350

lineolatus, Ancistrus, 222

lineopunctata, Chaetostoma, 229

lineopunctatum, Chaetostoma, 229

linnelli, Leptodoras 173

Liobagrus, 19

Liobagrus andersoni, 19

Liobagrus anguillicauda, 19

Liobagrus formosanus, 19

Liobagrus kingi, 19

Liobagrus marginatoides, 19

Liobagrus marginatus, 19

Liobagrus mediadiposalis, 19

Liobagrus nantoensis, 19

Liobagrus nantoënsis, 19

Liobagrus nigricauda, 20

Liobagrus obesus, 20

Liobagrus reinii, 19, 20

Liobagrus styani, 20

Liobagrus sugubii, 20

Liobagrus xiurenensis, 20

Liocassis, 92

Liocassis baramensis, 93

Liocassis breviceps, 103

Liocassis crassilabris, 92

Liocassis crassirostris, 92

Liocassis doriae, 93

Liocassis emarginatus, 102

Liocassis hosii, 93

Liocassis inornatus, 103

Liocassis longirostris, 92, 93

Liocassis merabensis, 93

Liocassis moeschii, 104

Liocassis naso, 108

Liocassis rugosus, 104

Liocassis saravacensis, 93

Liocassis siamensis, 104

Liocassis torosilabris, 108

Liocassis truncatus, 103

Liocassis vaillanti, 105

Liocassus hirsutus, 107

liocephalus, Clarias, 145

Liosomadoras, 75

Liosomadoras morrowi, 75

Liosomadoras oncinus, 75

lipophthalmus, Doras (Oxydoras), 169

Lipopterichthys, 266
Lipopterichthys carrioni, 266, 267

Liposarcus, 290

Liposarcus altipinnis, 291

Liposarcus ambrosettii, 291

Liposarcus anisitsi, 291

Liposarcus disjunctivus, 291

Liposarcus jeanesianus, 292

Liposarcus scrophus, 292

Liposarcus varius, 292

liropus, Tachysurus, 41

listrorhinos, Apistoloricaria, 226

Listrura, 408

Listrura boticario, 408

Listrura camposi, 408

Listrura nematopteryx, 408

Listrura picinguabae, 444

Listrura tetraradiata, 408

Lithodoradinae, 166

Lithodoras, 173

Lithodoras dorsalis, 173

lithogaster, Doras, 173

Lithogeneinae, 217

Lithogenes, 217, 267

Lithogenes valencia, 267

Lithogenes villosus, 267

lithoides, Lithoxus, 267

lithophilus, Parasilurus, 380

lithophilus, Silurus, 380

lithostoma, Pangasius, 326

Lithoxancistrus, 286

Lithoxancistrus orinoco, 286, 287

Lithoxina, 217

Lithoxus, 217, 267

Lithoxus (Paralithoxus)

pallidimaculatus, 267

Lithoxus (Paralithoxus) planquettei, 267

Lithoxus (Paralithoxus) surinamensis, 268

Lithoxus boujardi, 267

Lithoxus bovallii, 267

Lithoxus lithoides, 267

Lithoxus pallidimaculatus, 267

Lithoxus planquettei, 267

Lithoxus stocki, 268

Lithoxus surinamensis, 268

lithurgicus, Ancistrus, 222

littorale, Hoplosternum, 127

littorale daillyi, Hoplosternum, 128

littoralis, Callichthys, 127

lituratus, Ancistrus, 290, 291

lituratus, Pterygoplichthys, 291

lividus, Silurus, 204, 205

lividus Fuscatus, Silurus, 205

lividus var. Fuscatus, Silurus, 205

livrée, Pimélode, 213

llanero, Lamontichthys, 264

loangwensis, Clarias, 151

loborhynchos, Chaetostoma, 227, 229 lombarderoi, Xyliphius, 62

lonah, Bagrus, 391

lonah, Glyptothorax, 391, 394

longianalis, Clupisoma, 357

longianalis, Platytropius, 357

longibarbatus, Trichomycterus, 420

longibarbis, Arius, 334

longibarbis, Bathyclarias, 136

longibarbis, Chrysichthys, 157

longibarbis, Chrysobagrus, 157

longibarbis, Clarias, 136, 138

longibarbis, Clarias (Allabenchelys)

dumerili, 138

longibarbis, Dianema, 127

longibarbis, Dinotopterus, 136

longibarbis, Laides, 359

longibarbis, Pangasius, 359

longibarbus, Leiocassis, 92

longicauda, Allabenchelys, 137

longicauda, Clariallabes, 137

longicauda, Euchiloglanis, 399

longicauda, Glyptothorax, 391

longicauda, Imparfinis, 185

longicauda, Pareuchiloglanis, 399

longicauda, Phractura, 26

longicauda, Pimelodus (Rhamdia), 185

longicauda, Rineloricaria, 294

longicaudata, Olyra, 99

longicaudatus, Olyra, 99

longicaudatus, Clariallabes, 150

longicehpalus, Tachisurus, 39

longicephalus, Tachisurus, 39

longiceps, Astroblepus, 65

longiceps, Auchenoglanis, 163

longiceps, Clarias, 143

longiceps, Dolichamphilius, 24

longiceps, Osteogeneiosus, 51

longiceps, Parauchenoglanis, 163

longidorsalis, Chrysichthys, 157

longidorsalis nyongensis,

Chrysichthys, 158

longifilis, Akysis, 442

longifilis, Arges, 65

longifilis, Astroblepus, 65

longifilis, Callichthys, 129

longifilis, Chrysichthys, 155

longifilis, Copidoglanis, 349

longifilis, Eutropius, 361

longifilis, Heterobranchus, 151

longifilis, Hypophthalmus, 334

longifilis, Parailia, 360

longifilis, Pareutropius, 361

longifilis, Pimelodus, 338

longimanus, Ancistrus, 291

longimanus, Auchenipterichthys, 71

longimanus, Auchenipterus, 71

longimanus, Pseudeutropius, 362

longinema, Glyptothorax, 394

longior, Chasmocranus, 182, 183 
longior, Clarias, 145

longipectoralis, Heteropneustes, 152

longipinna, Harttia, 241

longipinnis, Bariancistrus, 227

longipinnis, Chrysichthys, 157

longipinnis, Euanemus, 74

longipinnis, Gephyroglanis, 157

longipinnis, Hemiancistrus, 227

longipinnis, Rhamdella, 196

longipinnis, Trichogenes, 414

longiradiatus, Hypostomus, 256

longiradiatus, Plecostomus, 256

longirostris, Amphilius, 22

longirostris, Anoplopterus, 22

longirostris, Corydoras melanistius, 113

longirostris, Leiocassis, 93

longirostris, Liocassis, 92, 93

longirostris, Parakysis, 16

longirostris, Parotocinclus, 282

longirostris, Synodontis, 316

longispinis, Alurichthys, 38

longispinis, Doras, 170

longispinis, Lasiancistrus, 287

longispinis, Pseudancistrus, 287

longispinis, Synodontis, 316

longispinis, Synodontis Batesi, 316

longisrostris, Nkondobagrus, 99

longiuscula, Rhamdella, 196

longjiangensis, Glyptothorax, 391

longus, Parasilurus asotus, 379

lopezi, Chasmocranus, 183

Lophiobagrus, 161

Lophiobagrus aquilus, 161

Lophiobagrus asperispinis, 161

Lophiobagrus brevispinis, 161

Lophiobagrus cyclurus, 161

Lophiobagrus lestradei, 161, 162

lophioides, Chaca, 135, 136

Lophiosilurus, 353

Lophiosilurus alexandri, 353

lophius, Amiurus, 204

lophophanes, Otothyris, 276

lophophanes, Rhinelepis, 276

loppei, Synodontis, 318

loretoensis, Corydoras, 119

Loricaria, 216, 268

Loricaria (Loricariichthys) fallax, 245

Loricaria accipenser, 270

Loricaria acuta, 269

Loricaria altipinnis, 245

Loricaria amazonica, 270

Loricaria anus, 269

Loricaria apeltogaster, 268

Loricaria apeltogaster amazonum, 288

Loricaria apeltogaster var. amazonum, 288

Loricaria aurea, 298

Loricaria barbata, 298, 299
Loricaria beni, 245

Loricaria bransfordi, 240

Loricaria brevirostris, 299

Loricaria brunnea, 269

Loricaria cacerensis, 245

Loricaria cadeae, 294

Loricaria capetensis, 233

Loricaria caquetae, 296

Loricaria carinata, 268

Loricaria cashibo, 270

Loricaria castanea, 270

Loricaria catamarcensis, 294

Loricaria cataphracta, 268, 270

Loricaria cirrhosa, 268, 296

Loricaria clavipinna, 268

Loricaria commersonoides, 278

Loricaria cubataonis, 294

Loricaria curvispina, 296

Loricaria Dentata, 268

Loricaria devincenzii, 288

Loricaria dura, 268

Loricaria Eigenmanni, 245

Loricaria Evansii, 296

Loricaria felipponei, 294

Loricaria filamentosa, 233, 234

Loricaria filamentosa latiura, 233

Loricaria filamentosa seminuda, 234

Loricaria fimbriata, 297

Loricaria flava, 259

Loricaria frenata, 299

Loricaria griseus, 266

Loricaria gymnogaster, 297

Loricaria gymnogaster lagoichthys, 297

Loricaria henselii, 294

Loricaria hoehnei, 245

Loricaria jaraguensis, 294

Loricaria jubata, 246

Loricaria kneri, 299

Loricaria konopickyi, 246

Loricaria kronei, 294

Loricaria labialis, 270

Loricaria laeviuscula, 289

Loricaria laviuscula, 289

Loricaria lamina, 288

Loricaria lanceolata, 246

Loricaria lata, 268

Loricaria laticeps, 288

Loricaria latirostris, 294

Loricaria lima, 293, 294

Loricaria lima microlepidota, 295

Loricaria lima var. microlepidota, 295

Loricaria macrodon, 227

Loricaria macromystax, 293

Loricaria macrops, 293

Loricaria maculata, 269, 270

Loricaria magdalenae, 246

Loricaria melanoptera, 252

Loricaria microdon, 271
Loricaria microlepidogaster, 295

Loricaria nickeriensis, 269

Loricaria nigricauda, 246

Loricaria nudirostris, 271

Loricaria nudiventris, 297

Loricaria panamensis, 299

Loricaria parahemiodon, 270

Loricaria pareiacantha, 295

Loricaria parnahybae, 269

Loricaria parva, 246

Loricaria paulina, 294

Loricaria phoxocephala, 246

Loricaria piauhiae, 269

Loricaria piracicabae, 269

Loricaria platystoma, 233

Loricaria platyura, 246

Loricaria prolixa, 286

Loricaria prolixa lentiginosa, 286

Loricaria puganensis, 297

Loricaria punctata, 266, 292

Loricaria rostrata, 245, 298, 300

Loricaria scolopacina, 237

Loricaria setifera, 268

Loricaria simillima, 269

Loricaria sneiderni, 246

Loricaria spinosae, 286

Loricaria Spixii, 270

Loricaria Steinbachi, 263

Loricaria steindachneri, 295

Loricaria stewarti, 247

Loricaria strigilata, 295

Loricaria Stübelii, 271

Loricaria submarginatus, 246

Loricaria teffeana, 247

Loricaria thrissoceps, 295

Loricaria tucumanensis, 269

Loricaria tuyrensis, 234

Loricaria uracantha, 240

Loricaria uracantha rupestre, 240

Loricaria Valenciennesii, 247

Loricaria variegata, 232, 233

Loricaria variegata venezuelae, 233

Loricaria vetula, 278

loricariformis, Harttia, 240, 241

Loricariichthyina, 217

Loricariichthys, 217, 269

Loricariichthys acutus, 269

Loricariichthys anus, 269

Loricariichthys brunneus, 269

Loricariichthys cashibo, 270

Loricariichthys castaneus, 270

Loricariichthys chanjoo, 270

Loricariichthys derbyi, 270

Loricariichthys edentatus, 270

Loricariichthys hauxwelli, 270

Loricariichthys labialis, 270

Loricariichthys maculatus, 270

Loricariichthys melanocheilus, 271

Loricariichthys melini, 246 
Loricariichthys microdon, 271

Loricariichthys nudirostris, 271

Loricariichthys parnahybae, 266

Loricariichthys platymetopon, 271

Loricariichthys rostratus, 271

Loricariichthys stuebelii, 271

Loricariichthys ucayalensis, 271

Loricariidae, 216

loricatus, Callichthys, 111

loricatus, Doras, 167

loweae, Bathyclarias, 149

loweae, Dinotopterus, 149

lowei, Gephyroglanis, 158

loxozonus, Corydoras, 119

lualae, Clarias, 142

lubosicus, Bagrus, 84

lucenai, Trachelyopterus, 80

Luceri, Platystoma, 342

Luciopimelodinae, 329

Luciopimelodus, 329, 334

Luciopimelodus pati, 334

lucipinnis, Synodontis, 445

luderwaldti, Pseudancistrus, 280

lufirae, Chiloglanis, 306

lufirae, Synodontis, 316

lui, Leiocassis, 102

luigiana, Rhamdia, 199

lukugae, Chiloglanis, 306

lukugae, Phractura, 26

lumholtzi, Cryptopterus, 370

lumholtzi, Kryptopterus, 370

lundbergi, Prietella, 215

lundbergi, Typhlobelus, 426

luniscutis, Arius, 36

luniscutis, Aspistor, 36

lupus, Ictalurus, 209, 211

lupus, Pimelodus, 209

lurida, Ageniosus (Silonia), 366

luridus, Macrones, 90

luridus, Pachypterus, 327

luteomaculatus, Hypostomus, 256

luteomaculatus, Plecostomus, 256

lutescens, Pimelodus, 215

luteus, Hypostomus, 256

luteus, Noturus, 212

luteus, Oloplotosus, 349

luteus, Plecostomus, 256

Lütkeni, Platystoma, 344

Lütkeni, Plecostomus, 251

luvur, Silurus, 431

lynx, Pimelodus, 204

lyra, Oxyloricaria, 299

lyra, Physopyxis, 176

lyra, Sturisoma, 299

lyriformis, Agmus, 60

M

mabusi, Chrysichthys, 157

macarenensis, Phenacorhamdia, 188, 189 maccus, Panaqolus, 277

maccus, Panaque, 277

macgrewi, Ameiurus, 204

macgrewi, Ictalurus, 205

machadoi, Genidens, 44

machadoi, Gladioglanis, 183

machadoi, Platyclarias, 152

machadoi, Plectrochilus, 411

machadoi, Tachysurus, 44

machiquense, Chaetostoma, 229

machiquensis, Chaetostoma, 229

macilenta, Denticetopsis, 133

macilentus, Hemicetopsis, 133

maclareni, Clarias, 145

maconnellii, Ameiurus, 211

macracanthus, Arius, 36

macracanthus, Bagrichthys, 82

macracanthus, Bagroïdes, 82

macracanthus, Centromochlus, 73

macracanthus, Clarias, 143

macracanthus, Pseudobagrichthys, 82

macracanthus, Rita, 106

macraei, Hatcheria, 405

macraei, Thrichomycterus, 405

macrepipterus, Synodontis, 314

macrocephala, Nannorhamdia, 192

macrocephala, Pimelodella, 192

macrocephalus, Ariodes, 51

macrocephalus, Arius, 51

macrocephalus, Atopochilus, 303

macrocephalus, Clarias, 145

macrocephalus, Clarotes, 160

macrocephalus, Cnidoglanis, 346

macrocephalus, Hemipimelodus, 41

macrocephalus, Kryptopterichthys, 370

macrocephalus, Kryptopterus, 370

macrocephalus, Osteogeneiosus, 51

macrocephalus, Plotosus, 346

macrocephalus, Silurus, 341

macrochir, Belodontichthys, 368

macrodon, Brochiloricaria, 227

macrodon, Loricaria, 227

macrodon, Synodontis, 313

macromaculatus, Glyptothorax, 391

macromycterus, Typhlobelus, 426

macromystax, Clarias, 145

macromystax, Loricaria, 293

macromystax, Rhadinoloricaria, 293

macronema, Akysis, 16

macronema, Chlarias angolensis, 141

macronema, Heterobranchus, 151

macronema, Hypselobagrus, 97

macronema, Pangasius, 327

macronema, Pseudorhamdia, 337

macronema, Silurodes, 372

macronema, Silurus, 371, 372

macronemus, Bagrus, 37, 97

macronemus, Bagrus, 93, 97 macronemus, Oreoglanis, 397

macronemus, Pseudobagarius, 16

Macrones, 85, 106

Macrones (Hemibagrus) filamentus, 88

Macrones (Hemibagrus) wyckioides, 91

Macrones (Leiocassis) brashnikowi, 100

Macrones (Liocassis) taeniatus, 102

Macrones (Pseudobagrus) tenuis, 103

Macrones aleppensis, 97

Macrones argentivittatus, 100

Macrones armatus, 94

Macrones baramensis, 87

Macrones bimaculatus, 94

Macrones bleekeri, 89, 94

Macrones Blythii, 85

Macrones bo, 88

Macrones bongan, 87

Macrones chinensis, 88

Macrones colvillii, 97

Macrones corsula, 90

Macrones Dayi, 85

Macrones elongatus, 88

Macrones fortis, 88

Macrones fortis capitulum, 88

Macrones fortis var. capitulum, 88

Macrones herzensteini, 92

Macrones howong, 88

Macrones kajan, 88

Macrones luridus, 90

Macrones medianalis, 102

Macrones merianiensis, 85

Macrones microphthalmus, 90

Macrones montanus dibrugarensis, 96

Macrones montanus var.

dibrugarensis, 96

Macrones peguensis, 90

Macrones pluriradiatus, 90

Macrones pratti, 102

Macrones pulcher, 97

Macrones rufescens, 94, 97

Macrones sinensis, 165

Macrones vittata, 106

Macronichthys, 106

Macronoides, 85, 87

Macronoides wilsoni, 87

macronotacanthus, Arius, 47

macronotus, Batasio, 85

macrophthalma, Synodontis, 316

macrophthalma, Xenocara, 222

macrophthalmus, Ancistrus, 222

macrophthalmus, Hypostomus, 256

macrophthalmus, Hypostomus

pseudohemiurus, 256

macrophthalmus, Plotosus, 349

macrophthalmus, Synodontis, 316

macropogon, Chrysichthys, 160

macrops, Chaetostomus, 243 
macrops, Chrysichthys, 155

macrops, Hemiancistrus, 243

macrops, Henonemus, 406

macrops, Hypostomus, 256

macrops, Leiocassis crassilabris, 92

macrops, Loricaria, 293

macrops, Plecostomus, 256

macrops, Ricola, 293

macrops, Stegophilus, 406

macrops, Synodontis, 316

Macropterobagrus, 87

Macropteronotus, 138

Macropteronotus charmuth, 138, 148

Macropteronotus fuscus, 142

Macropteronotus hexacicinnus, 148

Macropteronotus jagur, 140

Macropteronotus magur, 140

macropterum, Exostoma, 398

macropterus, Amblyceps, 18

macropterus, Bagrichthys, 82

macropterus, Bagroides, 82, 83

macropterus, Bagroïdes, 82

macropterus, Bagrus bayad, 83

macropterus, Calophysus, 332

macropterus, Chiloglanis, 306

macropterus, Chrysichthys, 157

macropterus, Corydoras, 130

macropterus, Hemibagrus, 87, 89, 91

macropterus, Leiocassis, 83

macropterus, Pareuchiloglanis, 399

macropterus, Pimelodes, 332

macropterus, Porcus bayad, 83

macropterus, Oreoglanis, 398

macropterus, Scleromystax, 130

macropthalmos, Eutropius, 361, 362

macropthalmos, Proeutropiichthys, 362

macropthalmos, Pseudosilurus, 374

macrorhynchus, Hemipimelodus, 32

macrospila, Piramutana, 339

macrospilus, Otocinclus, 275

macrosteus, Corydoras, 112

macrostigma, Synodontis, 317

macrostoma, Auchenoglanis, 154

macrostoma, Synodontis, 317

macrostomus, Anaspidoglanis, 154

macrostomus, Silurus (Callichrus), 381

macroteronema, Cetopsis, 133

macrotis, Chrysichthys, 157

Macrotocinclus, 271

Macrotocinclus affinis, 271

Macrotocinclus flexilis, 271

macrotrema, Euchiloglanis, 399

macrotremus, Pareuchiloglanis, 399

macrura, Phractura, 27

macruropterygius, Arius, 55

macrurus, Clarias, 148

macturki, Pimelodella, 192

maculata, Cteniloricaria, 233 maculata, Loricaria, 269, 270

maculata, Parasturisoma, 233

maculata, Wertheimeria, 179

maculatum, Glyptosternon, 387

maculatum, Parexostoma, 387

maculatus, Ancistrus, 222

maculatus, Arius, 34, 35

maculatus, Chaetostomus, 228

maculatus, Chaetostomus (Ancistrus)

cirrhosus, 222, 228

maculatus, Chaetostomus (Ancistrus)

cirrhosus var., 222, 228

maculatus, Corydoras, 122

maculatus, Doras, 177

maculatus, Liauchenoglanis, 161

maculatus, Loricariichthys, 270

maculatus, Nothoglanidium, 161

maculatus, Parastegophilus, 410

maculatus, Pimelodus, 336, 338, 345

maculatus, Pimelodus, 209

maculatus, Pimelodus (Bagrus), 345

maculatus, Silurus, 35, 207, 209

maculatus, Stegophilus, 410

maculatus, Synodontis, 318

maculatus, Trichomycterus, 415

maculatus, Wallago, 381

maculatus Erythroptera, Silurus, 209

maculatus var. Erythroptera, Silurus, 209

maculicauda, Otocinclus, 281, 283

maculicauda, Parotocinclus, 283

maculifer, Corydoras, 119

maculipinna, Synodontis, 320

maculipinnis, Akysis, 14

maculipinnis, Hisonotus, 248

maculipinnis, Otocinclus, 248

maculocephala, Cascadura, 127, 128

maculosus, Aspidoras, 109

maculosus, Auchenipterus, 79

maculosus, Auchenoglanis, 163

maculosus, Synodontis, 311, 318, 321

maculosus, Trachelyopterus coriaceus, 79

macunaima, Ituglanis, 407

macushi, Hypostomus, 256

macuspanensis, Rhamdia, 199

madagascariensis, Arius, 35

madeira, Plecostomus, 225

madeirensis, Ageneiosus, 69

madraspatanum, Glyptosternum, 391

madraspatanus, Glyptothorax, 388,

391

maesii, Amphilius, 22

maesotensis, Erethistes, 384

magatensis, Arius, 52

Magdalenae, Auchenipterus, 79

magdalenae, Branchioica, 411

magdalenae, Hemiloricaria, 246

magdalenae, Hoplosternum, 128
Magdalenae, Loricaria, 246

magdalenae, Trachycorystes, 79

magdalenae, Xyliphius, 62

magnus, Chrysichthys, 157

magoi, Ageneiosus, 69

magoi, Brachyglanis, 180

magoi, Pygidianops, 412

magur, Macropteronotus, 140

mahakamensis, Acrochordonichthys, 12

mahakamensis, Leiocassis, 104

mahakamensis, Pangasius, 327

mahakamensis, Pseudomystus, 104

mahengeensis, Chrysichthys, 157

major, Akysis, 391

major, Glyptothorax, 391

major, Hemibagrus, 89

majusculus, Bagrichthys, 83

malabarbai, Microglanis, 354

Malabarica, Hara, 96

malabaricus, Arius, 35

Malabaricus, Bagrus, 96

malabaricus, Mystus, 96

malabaricus, Ompok, 372

malabaricus, Silurus, 372

Malacobagrus, 330

Malacoglanis, 408

Malacoglanis gelatinosus, 408

malacops, Ancistrus, 222

malacops, Chaetostomus, 222

Malacopterurus, 301

malaisei, Glyptosternon, 387

Malapteruridae, 300

Malapterurini, 300

Malapterurus, 300, 301

Malapterurus affinis, 301

Malapterurus barbatus, 301

Malapterurus beninensis, 301

Malapterurus cavalliensis, 301

Malapterurus coila, 356

Malapterurus electricus, 301

Malapterurus electricus oguensis, 302

Malapterurus electricus, var.

ogooensis, 302

Malapterurus electricus var. oguensis, 302

Malapterurus gossei, 302

Malapterurus leonensis, 301

Malapterurus melanochir, 301

Malapterurus microstoma, 301

Malapterurus microstomus, 301

Malapterurus minjiriya, 302

Malapterurus monsembeensis, 302

Malapterurus murrayi, 302

Malapterurus occidentalis, 302

Malapterurus oguensis, 302

Malapterurus polli, 302

Malapterurus punctatus, 302

Malapterurus shirensis, 302 
Malapterurus stiassnyae, 301

Malapterurus tanganyikaensis, 302

Malapterurus tanoensis, 302

Malapterurus teugelsi, 302

Malapterurus thysi, 301

Malapterurus zambeziensis, 302

Malapterus, 301

Malapterus, 301, 356

Malapterus (Ailia) bengalensis, 356

Malapturus, 301

malaris, Clarias, 143

malarmo, Platysilurus, 340

maldonadoi, Bullockia, 404

Maldonadoi, Hatcheria, 403, 404

malignus, Plotosus, 351

Malopterurus, 301

mamore, Corydoras, 120

mamoré, Corydoras, 120

managuensis, Pimelodus, 199

mancoi, Astroblepus, 65

mandevillei, Atopochilus, 304

mandevillei, Leptoglanis, 28

mandevillei, Pareutropius, 361

mandevillei, Zaireichthys, 28

mandibularis, Eutropius, 364

mandibularis, Schilbe, 364

mangois, Amblyceps, 18

mangois, Pimelodus, 18

mangrullo, Silurus, 341

mangurus, Pimelodus, 355

mangurus, Pseudopimelodus, 355

manillensis, Arius, 35

manillensis, Cephalocassis, 41

Manillensis, Pimelodus, 41

manipurensis, Glyptothorax, 391

manipurensis, Laguvia, 382

maniradii, Sorubim, 342

manjong, Arius, 55

manni, Pteroglanis, 343

manni, Synodontis, 317

manyangae, Allabenchelys, 137

manyangae, Clariallabes, 137

mapale, Cathorops, 40

maquinensis, Rineloricaria, 294

maracaibero, Lamontichthys, 264

maracaiboensis, Hemiancistrus, 243

maracaiboensis, Lasiancistrus, 265

maracaiboensis, Pygidium banneaui, 420

maracaiboensis, Trichomycterus, 420

maracasae, Ancistrus, 223

marapoama, Otothyropsis, 276

marcapatae, Chaetostoma, 229

marcapatae, Chaetostomus, 229

margaritifer, Hypostomus, 256

margaritifer, Plecostomus, 256

margaritifer butantanis, Plecostomus, 256 marginata, Pimelodus pallidus var., 210

marginata, Taunayia, 203

marginatoides, Amblyceps, 19

marginatoides, Liobagrus, 19

marginatum, Chaetostoma, 229

marginatus, Amblyceps, 19

marginatus, Chaetostomus, 229

marginatus, Hypophthalmus, 333

marginatus, Liobagrus, 19

marginatus, Noturus, 213

marginatus, Pimelodus, 206

marginatus, Plotosus, 350

marginatus, Silurus pallidus, 210

marginatus, Silurus pallidus var., 210

marginatus atrorus, Schilbeodes, 213

mariae, Astroblepus, 65

mariae, Cyclopium, 65

mariae, Oloplotosus, 349

mariae, Otocinclus, 275

mariaelenae, Farlowella, 238

mariai, Imparales, 188

mariai, Nemuroglanis, 188

marinus, Bagre, 37

marinus, Silurus, 37, 38, 44

marlieri, Chiloglanis, 306

marmorata, Leptorhamdia, 187

marmorata, Synodontis, 317

marmoratum, Trachypoma, 404

marmoratus, Ageneiosus, 69

marmoratus, Amphilius

grammatophorus, 23

marmoratus, Arges, 65

marmoratus, Astroblepus, 65

marmoratus, Corydoras, 122

marmoratus, Helogenes, 134

marmoratus, Doras, 171

marmoratus, Franciscodoras, 171

marmoratus, Leiarius, 334

marmoratus, Pimelodus, 206

marmoratus, Schilbe, 364

marmoratus, Sciades, 334

marmoratus, Silurichthys, 378

marmoratus, Synodontis, 317

marmoratus, Trichomycterus, 415

marmoratus truncatus, Synodontis, 317

marmorescens, Chaetostoma, 229

marpus, Clarias, 148

marquesi, Ageneiosus, 70

marthae, Brachyrhamdia, 181

marthae, Rhamdia, 181

martinezi, Pimelodella, 193

martini, Acestridium, 218

martini, Ancistrus, 223

martini, Ancistrus triradiatus, 223

martini, Farlowella, 238

Martyi, Rhamdia sebae, 202

mastersi, Arius, 45

mastersi, Hexanematichthys, 45
Mastiglanis, 187

Mastiglanis asopos, 187

mathisoni, Zungaro, 356

matthesi, Synodontis, 317

mattogrossensis, Ancistrus, 300

mattogrossenssis, Ancistrus, 300

maurus, Bagrus, 158

maurus, Chrysichthys, 157, 158, 160

maydelli, Hemibagrus, 89

maydelli, Mystus (Mystus), 89

maydeni, Noturus, 441

mayoloi, Hemiancistrus, 264

mayoloi, Lasiancistrus, 265

mazatlana, Netuma, 54

mboycy, Trichomycterus, 420

mbozi, Chiloglanis, 306

McCaskei, Ichthaelurus, 204

Medemichthys, 188

Medemichthys guayaberensis, 188 mediadiposalis, Liobagrus, 19 medianalis, Macrones, 102 medianalis, Pseudobagrus, 102

medians, Ancistrus, 242, 243

medians, Hemincistrus, 243

mediobarbis, Neosilurus, 347

meeki, Amiurus, 209

meeki, Pimelodella, 193

meesi, Brachyrhamdia, 181

megacephalus, Chaetostomus, 244

megacephalus, Hemiancistrus, 244

Megalancistrus, 272

Megalancistrus barrae, 272

Megalancistrus parananus, 272

Megalechis, 129

Megalechis picta, 129

Megalechis thoracata, 129

Megalocentor, 409

Megalocentor echthrus, 409

Megalodoras, 173

Megalodoras guayoensis, 173

Megalodoras irwini, 173, 174

Megalodoras paucisquamatus, 173

Megalodoras uranoscopus, 174

Megalonema, 335

Megalonema argentina, 335

Megalonema pauciradiatum, 335

Megalonema platanum, 335

Megalonema platycephalum, 335

Megalonema platycephalum

psammium, 335

Megalonema psammium, 335

Megalonema punctatum, 339

Megalonema rhabdostigma, 338

Megalonema robustum, 339

Megalonema xanthum, 335

megalops, Centromochlus, 73

megalops, Iheringichthys, 334

megalops, Pimelodella, 193

megalops, Pimelodus, 210 
megalops, Pseudeutropius, 362

megalopteryx, Hemiancistrus, 244

megalostomus, Ancistrus, 223

megalura, Pimelodella, 193

megapogon, Clarias, 141

megastomus, Plotosus, 345, 346

megistus, Ernstichthys, 60

megistus, Hoplomyzon, 60

meizospilos, Hemiancistrus, 244

mekongensis, Hemisilurus, 368

mekongensis, Pangasius, 327

meladerma, Clarias, 145

melampterus, Callichthys, 127, 128

melanistius, Corydoras, 120

melanistius brevirostris, Corydoras,

114

melanistius longirostris,

Corydoras, 113

melanocheilus, Loricariichthys, 271

melanochir, Arius, 41

melanochir, Cephalocassis, 41

melanochir, Malapterurus, 301

Melanodactylus, 155

melanoderma, Clarias, 146

melanodermatum, Steindachneridion, 343

melanogaster, Acrochordonichthys, 13

melanogaster, Pimelodus, 12, 13

melanopogon, Ageneiosus, 68

melanopogon, Clarias, 145

melanops, Tridens, 426

melanoptera, Gagata, 386

melanoptera, Loricaria, 252

melanoptera, Synodontis, 317

melanopterum, Glanidium, 75

melanopterus, Bagroides, 83

melanopterus, Gagata, 386

melanopterus, Synodontis, 317

melanopterus, Xyliphius, 62

melanopterygius, Arius, 56

melanopus, Arius, 40

melanopus, Cathorops, 40

melanostictus, Synodontis, 318

melanostictus iturii, Synodontis, 315

melanostictus var. iturii, Synodontis, 315

melanotaenia, Corydoras, 120

melanurus, Batrochoglanis, 353

melanurus, Pachypterus, 358

melanurus, Silurus cerulescens, 209

melanurus, Silurus cerulescens, var., 209

melaphareus, Entomocorus, 74

melapterus, Bagroides, 83

melapterus, Bagroïdes, 83

melas, Ameiurus, 205

melas, Ancistrus, 220

melas, Bagrus, 95

melas, Brachyglanis, 181 melas, Bunocephalus, 58

melas, Clariallabes, 137

melas, Clarias, 137

melas, Pimelodus, 205

melas, Silurus, 205

melas lembesseensis, Clarias, 137

melasoma, Clarias, 146

meleagris, Hypostomus, 256

meleagris, Plecostomus, 256

melini, Corydoras, 120

melini, Hemiloricaria, 246

melini, Loricariichthys, 246

Mellandi, Clarias, 146

membranacea, Hemisynodontis, 309

membranaceus, Pimelodus, 309

mendozensis, Silvinichthys, 413

mendozensis, Trichomycterus, 413

menezesi, Aspidoras, 109

menezesi, Auchenipterus, 72

menezesi, Pyxiloricaria, 292

menoda, Hemibagrus, 89

menoda, Pimelodus, 89

menoni, Fajumia, 429

mentalis, Eutropius, 364

mento, Silurus, 380

merabensis, Liocassis, 93

meraukensis, Copidoglanis, 351

meraukensis, Porochilus, 351

mercatoris, Arius, 31

merevari, Harttia, 241

merianiensis, Batasio, 85

merianiensis, Macrones, 85

meridae, Trichomycterus, 420

meridionalis, Akysis, 17

meridionalis, Amiurus, 208

meridionalis, Bagrus, 84

meridionalis, Corydoras, 116

meridionalis, Pseudobagarius, 17

meridionalis, Silurus, 380

meridionalis, Silurus soldatovi, 380

Merodontotus, 330

Merodontotus tigrinus, 330, 331

merus, Glyptothorax, 390

mesembrinus, Diplomystes, 166

mesembrinus, Diplomystes viedmensis, 166

mesembrinus, Olivaichthys, 166

mesops, Bagrus, 53

metae, Corydoras, 120

metae, Ituglanis, 407

metae, Pimelodella, 193

metae, Pygidium, 407

metae guayaberensis, Pygidium, 407

Metaloricaria, 217, 272

Metaloricaria nijsseni, 272

Metaloricaria paucidens, 272

Metaloricariina, 217

mexicanus, Amiurus, 209

mexicanus, Ictalurus, 209 meyenii, Bagrus (Ariodes), 52

mica, Mystus, 100

mica, Pelteobagrus, 100

micayi, Rhamdia, 197

micracanthus, Bagrus, 96

micracanthus, Corydoras, 120

micranodus, Bagrichthys, 83

micrescens, Astroblepus, 65

micrescens, Astroblepus grixalvii, 65

micristius, Pareutropius, 360, 361

Microcambeva, 409

Microcambeva barbata, 409

Microcambeva ribeirae, 409

microcephala, Rhamdia, 202

microcephala, Rhamdiopsis, 202

microcephalus, Arius, 35

microcephalus, Cnidoglanis, 346

microcephalus, Corydoras, 122

microcephalus, Pseudarius, 35

microcephalus, Saccobranchus, 151

microcephalus, Silurus, 374

microceps, Euristhmus, 346

microceps, Plotosus, 346

Microcorydoras, 112

microdon, Loricaria, 271

microdon, Loricariichthys, 271

microdorsalis, Parasilurus, 380

microdorsalis, Silurus, 380

microgalaeus, Aspidoras, 109

microgastropterygius, Arius, 55

Microglanis, 354

Microglanis ater, 354

Microglanis cibelae, 354

Microglanis cottoides, 354

Microglanis eurystoma, 354

Microglanis garavelloi, 354

Microglanis iheringi, 354

Microglanis leptostriatus, 441

Microglanis malabarbai, 354

Microglanis nigripinnis, 354

Microglanis parahybae, 354

Microglanis pataxo, 444

Microglanis pellopterygius, 354

Microglanis poecilus, 354

Microglanis secundus, 355

Microglanis variegatus, 355

Microglanis zonatus, 355

Microlepidogaster, 272

Microlepidogaster bahiensis, 281

Microlepidogaster bourguyi, 300

Microlepidogaster depressinotus, 248

Microlepidogaster doceanus, 282

Microlepidogaster guentheri, 296

Microlepidogaster guntheri, 295

Microlepidogaster güntheri, 296

Microlepidogaster perforated, 272

Microlepidogaster perforatus, 272

Microlepidogaster taimensis, 249

microlepidogaster, Loricaria, 295 
microlepidogaster, Rineloricaria, 295 microlepidota, Loricaria lima, 295 microlepidota, Rineloricaria, 295 micromaculatus, Hypostomus, 256 micrommatos, Hemiancistrus, 244 Micromyzon, 61

Micromyzon akamai, 61

Micronema, 371

Micronema bleekeri, 374

Micronema cheveyi, 371

Micronema hexapterus, 371

Micronema moorei, 371

Micronema platypogon, 371

Micronema typus, 375

micronemus, Pangasius, 328

micronemus, Phalacronotus, 375

micronemus, Pseudolais, 328

micronemus, Silurus, 375

micronotacanthus, Arius, 55 microphthalmus, Brachyglanis, 181 microphthalmus, Clarias, 143 microphthalmus, Dolichallabes, 149 microphthalmus, Euristhmus, 442 microphthalmus, Hemibagrus, 90 microphthalmus, Macrones, 90 micropoeus, Doras, 171 micropoeus, Hemidoras, 171 micropogon, Bagrus, 92, 93 micropogon, Batrachocephalus, 38 micropogon, Chiloglanis, 305 micropogon, Eutropius, 364 micropogon, Leiocassis, 93 micropogon, Schilbe, 364 micropogon, Silurus, 374 micropogon, Wallago, 381 microps, Akysis, 14 microps, Chaetostoma, 229 microps, Chaetostomus, 229 microps, Chiloglanis, 306 microps, Corydoras, 112 microps, Harttia, 290 microps, Imparfinis, 186 microps, Leiocassis, 100 microps, Neoplecostomus, 273 microps, Pareiodon, 411 microps, Pelteobagrus, 100 microps, Plecostomus, 273, 281 microps, Plecostomus (Rhinelepis), 281

microps, Pterosturisoma, 290 microps, Rhamdia, 201 microps, Saccobranchus, 151 micropterus, Arius, 166 micropterus, Pimelodus, 200 micropunctatus, Plecostomus, 225 micropus, Kryptopterus, 369 microstoma, Malapterurus, 301 microstoma, Pimelodus, 338 microstomus, Arius, 39 microstomus, Clarias, 146 microstomus, Hemidoras, 179 microstomus, Hypostomus, 250, 257 microstomus, Malapterurus, 301 microstomus, Trachydoras, 179 Microsynodontis, 309

Microsynodontis armatus, 309

Microsynodontis batesii, 309

Microsynodontis Christyi, 309 Microsynodontis emarginata, 309

Microsynodontis emarginatus, 309

Microsynodontis hirsuta, 310

Microsynodontis hirsutus, 310

Microsynodontis laevigata, 310

Microsynodontis laevigatus, 310

Microsynodontis lamberti, 310

Microsynodontis nannoculus, 310

Microsynodontis nasutus, 310

Microsynodontis notata, 310

Microsynodontis notatus, 310

Microsynodontis polli, 310

Microsynodontis vigilis, 310

micruropterus, Phalacronotus, 375

micruropterygius, Arius, 36

midgleyi, Arius, 47

midgleyi, Neoarius, 47

migrans, Pygidium, 420

migrans, Trichomycterus, 420

milesi, Autanadoras, 178, 179

milesi, Chaetostoma, 229

milesi, Chaetostomus, 229

militaris, Ageneiosus, 68, 69

militaris, Ageniosus, 68

militaris, Arius, 51

militaris, Osteogeneiosus, 51

militaris, Silurus, 51, 68, 69

mimonha, Trichomycterus, 420

mimulus, Otocinclus, 275

mindoense, Cyclopium, 65

mindoensis, Astroblepus, 65

minimaculatus, Glyptothorax, 391

minimus, Kryptopterus, 370

minjiriya, Malapterurus, 302

mino, Ageneiosus, 38

mino, Batrachocephalus, 38

minor, Kryptopterus, 370

minuta, Rhamdia, 186

minutum, Cetopsidium, 130

minutum, Glyptosternon, 393

minutum, Scleronema, 413

minutus, Ancistrus, 223

minutus, Bunocephalus, 59

minutus, Hemicetopsis, 130

minutus, [Hisonotus], 249

minutus, Imparfinis, 186

minutus, Glyptothorax, 391, 392, 393

minutus, Parotocinclus, 283

minutus, Trachyglanis, 28

minutus, Silurus, 431 minutus, Trichomycterus, 413

miostoma, Wallago, 372

miostomus, Ompok, 372

mirini, Imparfinis, 186

mirissumba, Trichomycterus, 420

misionera, Rineloricaria, 295

mispilliensis, Amiurus, 206

misrai, Fajumia, 429

misrai, Mystus, 97

mitchelli, Pseudeutropius, 362

Miuroglanis, 409

Miuroglanis platycephalus, 409

miurus, Noturus, 214

möbiusii, Eutropius, 364

Mochocus, 310

Mochocus brevis, 310

Mochokidae, 303

Mochokiella, 310

Mochokiella paynei, 310

Mochokus, 303, 310

Mochokus brevis, 310

Mochokus niloticus, 310, 311

modesta, Pimelodella, 193

modestum, Glyptosternum, 389

modestus, Pimelodus, 193

modjensis, Chiloglanis, 306

moebii, Eutropius, 365

moebiusii, Schilbe, 364

moeschii, Leiocassis, 105

moeschii, Liocassis, 104

moeschii, Pseudomystus 104

molinae, Cetopsorhamdia, 182

mollespiculum, Neosilurus, 348

molliceps, Arius, 55

mollinasum, Chaetostoma, 230

mollinasus, Chaetostomus, 230

mollis, Silurus, 214

mondolfi, Pygidium, 421

mondolfi, Trichomycterus, 421

mong, Pimelodus, 55

mongallensis, Slatinia, 24

Monistiancistrus, 289

Monistiancistrus carachama, 289

monitor, Amblydoras, 169

monitor, Zathorax, 168, 169

monkei, Auchenoglanis, 163

monkei, Clarias, 141

monkei, Parauchenoglanis, 163

mononema, Kryptopterus, 370

mononema, Silurus, 370

monopelte, Sturisoma, 299

monsembeensis, Malapterurus, 302

montana, Cetopsis, 132

montana, Clupisoma, 357

montana, Erethistoides, 384

montana, Pimelodella, 193

montana, Rhamdella, 196

montana, Xenocara, 223

montana pipri, Erethistoides, 384 
montanus, Ancistrus, 223

montanus, Bagrus, 96

montanus, Mystus, 96

montanus dibrugarensis, Macrones, 96

montebelloi, Canthopomus, 263

montebelloi, Ixinandria, 263

moolenburghae, Pseudeutropius, 362

moolenburghi, Hemisilurus, 369

moorei, Kryptopterus, 371

moorei, Micronema, 371

moorii, Clarias, 143

moravicus, Otolithus (Arius ?), 434

morehensis, Silurus, 376

morei, Opsodoras, 175

Morei, Oxydoras, 175

moreirai, Rhamdiopsis, 202, 203

morenoi, Cetopsidium, 130

morenoi, Hemicetopsis, 130

Mormyrostoma, 170

morrisi, Hemidoras, 172

morrowi, Hemiloricaria, 246

morrowi, Liosomadoras, 75

morrowi, Rhineloricaria, 246

mortiauxi, Tanganikallabes, 152

mortoni, Neosilurus, 348

mossambicus, Clarias, 143

motaguensis, Pimelodus, 198

motatanensis, Cetopsis, 132

motatanensis, Pseudocetopsis

plumbeus, 132

motatanensis, Pygidium emanueli, 421

motatanensis, Trichomycterus, 421

mounseyi, Rhamdia, 201

mucosa, Pimelodella, 193

mucosa, Platystoma, 340

mucosus, Platysilurus, 340

mucronatum, Amblyceps, 18

muelleri, Paraplotosus, 349

muelleri, Rhamdia, 199

Mugil corsula, 89

mukherjii, Mystus, 95

mülleri, Clarias, 143

mülleri, Cnidoglanis, 349

mülleri, Pimelodus, 199

Mülleri, Silurus, 380, 381

multilineatus, Eutropius, 365

multimaculata, Synodontis, 317

multimaculatus, Corydoras, 120

multimaculatus, Synodontis, 317

multimaculatus, Zungaropsis, 344

multipunctata, Amphilius

nigricaudatus, 21

multipunctata, Synodontis, 317

multipunctatus, Synodontis, 317

multiradiatus, Arius, 40

multiradiatus, Brochis, 120

multiradiatus, Cathorops, 40

multiradiatus, Chaenothorax, 120

multiradiatus, Corydoras, 120 multiradiatus, Cranoglanis, 165

multiradiatus, Heptapterus, 184

multiradiatus, Hypostomus, 290, 291

multiradiatus, Mystus, 96

multiradiatus, Pimelodus, 334

multiradiatus, Plotosus, 350

multiradiatus, Pseudeutropichthys, 165

multiradiatus, Pterygoplichthys, 291

multiradiatus alternans, Ancistrus, 291

multispinis, Ancistrus, 223

multispinis, Ancistrus, 265

multispinis, Xenocara, 223

multitaeniatus, Eutropius, 365

multitaeniatus, Schilbe, 365

munitus, Noturus, 214

mura, Otocinclus, 275

muriaensis, Parotocinclus, 283

murica, Doras, 177

muricata, Pseudolaguvia, 401

muriei, Rhamdia guatemalensis, 202

murinus, Spectracanthicus, 297

murius, Eutropiichthys, 357

murius, Pimelodus, 357

murius batarensis, Pseudeutropius, 357

murrayi, Malapterurus, 302

murraystuarti, Amblyceps, 18

murray-stuarti, Amblyceps, 18

musaica, Tatia, 77

musculus, Pimelodus, 200

mustelinus, Heptapterus, 184

mustelinus, Pimelodus, 184

muticus, Silurus, 337

mutisii, Eremophilus, 404

mutsindoziensis, Clariallabes, 137

mutuca, Hemipsilichthys, 280

mutuca, Pareiorhaphis, 280

mutucae, Hypostomus, 257

myanmar, Pangasius, 327

Myersglanis, 396

Myersglanis blythii, 396

Myersglanis jayarami, 396

myersi, Corydoras, 123

myersi, Hypostomus, 257

myersi, Leiocassis, 104

myersi, Leptodoras, 173

myersi, Plecostomus, 257

myersi, Pseudomystus, 104

Myoglanis, 187

Myoglanis aspredinoides, 187

Myoglanis koepckei, 187

Myoglanis potaroensis, 187

Myoglanis potaroënsis, 187

myriodon, Aposturisoma, 226

myriodon, Chrysichthys, 155

Mysoricus, Silurus, 374

mystacinus, Ancistrus, 265

mysteriosus, Pimelodus, 338

mysticetus, Mystus, 96
Mystus, 93

Mystus (Mystus) maydelli, 89

Mystus (Mystus) vittatus horai, 95

Mystus alasensis, 94

Mystus albolineatus, 94

Mystus armatus, 94, 98

Mystus armiger, 94

Mystus ascita, 177

Mystus atrifasciatus, 94

Mystus aubentoni, 91

Mystus bimaculatus, 94

Mystus bleekeri, 94

Mystus bocourti, 94

Mystus canarensis, 96

Mystus carolinensis, 37

Mystus castaneus, 94

Mystus cavasius, 95

Mystus dalungshanensis, 95

Mystus falcarius, 95

Mystus fluviatilis, 164

Mystus gulio, 95

Mystus havmolleri, 85

Mystus horai, 95

Mystus impluviatus, 95

Mystus johorensis, 89

Mystus krishnensis, 89

Mystus leucophasis, 96

Mystus malabaricus, 96

Mystus mica, 100

Mystus misrai, 97

Mystus montanus, 96

Mystus mukherjii, 95

Mystus multiradiatus, 96

Mystus mysticetus, 96

Mystus nigriceps, 96

Mystus oculatus, 96

Mystus olyroides, 90

Mystus pahangensis, 90

Mystus pelusius, 96

Mystus pulcher, 97

Mystus punctifer, 97

Mystus rhegma, 97

Mystus rufescens, 94, 97

Mystus sabanus, 91

Mystus seengtee, 97

Mystus singaringan, 97

Mystus spinipectoralis, 97

Mystus stigmaturus, 85

Mystus tengara, 97, 98

Mystus vittatus, 98

Mystus wolffii, 98

mystus, Schilbe, 365

mystus, Silurus, 362, 365

myzostoma, Euchiloglanis, 399

myzostomus, Pareuchiloglanis, 399

$\mathbf{N}$

N'gamensis, Ciarias, 146

$N^{\prime}$ gamensis, Clarias, 146

nahuelbutaensis, Diplomystes, 166 
naipi, Trichomycterus, 421

namdia, Pimelodus, 200

Nangra, 396

Nangra assamensis, 396

Nangra bucculenta, 396

Nangra Buchanani, 397

Nangra carcharhinoides, 396

Nangra nangra, 397

Nangra ornata, 397

Nangra punctata, 395

Nangra robusta, 397

nangra, Nangra, 397

nangra, Pimelodus, 396, 397

nannoculus, Microsynodontis, 310

Nannoglanis, 187

Nannoglanis bifasciatus, 203

Nannoglanis fasciatus, 187, 188

Nannoglanis hoehnei, 188

Nannoptopoma, 272

Nannoptopoma spectabile, 272

Nannoptopoma sternoptychum, 273

Nannorhamdia, 180, 185

Nannorhamdia benedettii, 198

Nannorhamdia guttatus, 185

Nannorhamdia lineata, 185

Nannorhamdia macrocephala, 192

Nannorhamdia nemacheir, 186

Nannorhamdia schubarti, 186

Nannorhamdia spurrellii, 185, 186

Nannorhamdia stictonotus, 186

Nanobagrus, 98

Nanobagrus armatus, 98

Nanobagrus nebulosus, 98

Nanobagrus stellatus, 98

nanonocticolus, Gelanoglanis, 74

nantoensis, Liobagrus, 19

nantoënsis, Liobagrus 19

nanus, Corydoras, 120

napoensis, Corydoras, 120

narcissus, Corydoras, 120

nasalis, Eutropius, 363

naso, Liocassis, 108

Nasocassis, 92

nassi, Phractocephalus, 336

nasus, Cetopsorhamdia, 181, 182

nasus, Pimelodina, 336

nasuta, Psammphiletria, 27

nasuta, Rhamdia, 201

nasutus, Arius, 49

nasutus, Microsynodontis, 310

nasutus, Pangasius, 327

nasutus, Pseudopangasius, 327

natalensis, Amphilius, 22

natalis, Ameiurus, 205

natalis, Pimelodus, 205

natalis analis, Amiurus, 205

nationi, Ancistrus, 223

nationi, Lasiancistrus, 223

nattereri, Corydoras, 121 nattereri, Farlowella, 238

Nattereri, Oxydoras, 179

nattereri, Trachydoras, 179

nattereri triseriatus, Corydoras, 121

nauticus, Amblydoras, 169

nauticus, Zathorax, 169

navarroi, Pimelodus, 338

navarroi, Pimelodus grosskopfii, 338

naziri, Clupisoma, 357

naziri, Glyptothorax, 392

Neblinichthys, 273

Neblinichthys pilosus, 273

Neblinichthys roraima, 273

Neblinichthys yaravi, 273

nebulifer, Pareuchiloglanis, 399

nebulosa, Synodontis, 317

nebulosus, Ameiurus, 205

nebulosus, Clarias, 148

nebulosus, Doras, 178

nebulosus, Ituglanis, 407

nebulosus, Nanobagrus, 98

nebulosus, Pimelodus, 205, 215

nebulosus, Silurus, 215

nebulosus, Silurus (Callichrus), 374

nebulosus, Synodontis, 317

nebulosus, Wallago, 381

nebulosus pannonicus, Ictalurus, 206

Nedystoma, 46

Nedystoma dayi, 46

Nedystoma novaeguineae, 46

negro, Corydoras, 121

neivai, Glanidium, 77

neivai, Tatia, 77

nella, Pimelodus, 52

nella, Plicofollis, 52

nelsoni, Conorhynchos, 53

nelsoni, Glyptothorax, 392

nelsoni, Leptodoras, 173

nelsoni, Potamarius, 53

nemacheir, Imparfinis, 186

nemacheir, Nannorhamdia, 186

Nemadoras, 174

Nemadoras elongatus, 174

Nemadoras hemipeltis, 174

Nemadoras humeralis, 174

Nemadoras leporhinus, 174

Nemadoras trimaculatus, 174

Nemapteryx, 46

Nemapteryx armiger, 46

Nemapteryx augusta, 46

Nemapteryx bleekeri, 46

Nemapteryx caelata, 46

Nemapteryx macronotacantha, 46

Nemapteryx nenga, 46, 47

Nemasiluroides, 361

Nemasiluroides furcatus, 361

Nematogenyidae, 323

Nematogenyini, 323

Nematogenys, 323, 324
Nematogenys cuivi, 324

Nematogenys inermis, 324

Nematogenys nigricans, 324

Nematogenys pallidus, 324

nematophorus, Platystacus, 61

nematopterus, Hypostomus, 257

nematopteryx, Listrura, 408

Nemuroglanis, 180, 188

Nemuroglanis lanceolatus, 188

Nemuroglanis mariai, 188

Nemuroglanis pauciradiatus, 188

nemurus, Bagrus, 87, 90

nemurus, Hemibagrus, 90

nemurus, Pseudostegophilus, 412

nemurus, Stegophilus, 412

nenga, Nemapteryx, 46, 47

nenga, Pimelodus, 47

Neoarius, 33, 47

Neoarius berneyi, 47

Neoarius coatesi, 47

Neoarius graeffei, 47

Neoarius latirostris, 47

Neoarius leptaspis, 47

Neoarius midgleyi, 47

Neoarius paucus, 48

Neoarius pectoralis, 48

Neoarius taylori, 48

Neoarius utarus, 48

Neoarius velutinus, 48

Neobagrus, 19

Neobagrus fuscus, 19, 20

neogranatensis, Arius, 50

neogranatensis, Notarius, 50

Neopangasius, 325

Neopangasius Nieuwenhuisii, 325, 327

Neoplecostominae, 217

Neoplecostomus, 217, 273

Neoplecostomus espiritosantensis, 273

Neoplecostomus franciscoensis, 273

Neoplecostomus granosus, 273

Neoplecostomus microps, 273

Neoplecostomus paranensis, 273

Neoplecostomus ribeirensis, 274

Neoplecostomus variipictus, 274

Neoplotosus, 346

Neoplotosus waterhousii, 346

Neosiluroides, 347

Neosiluroides cooperensis, 347

Neosilurus, 347

Neosilurus ater, 347

Neosilurus argenteus, 345

Neosilurus australis, 347, 348

Neosilurus bartoni, 347

Neosilurus brevidorsalis, 347

Neosilurus coatesi, 348

Neosilurus equinus, 348

Neosilurus gjellerupi, 348

Neosilurus gloveri, 348 
Neosilurus hyrtlii, 347, 348

Neosilurus idenburgi, 348

Neosilurus mediobarbis, 347

Neosilurus mollespiculum, 348

Neosilurus mortoni, 348

Neosilurus novaeguineae, 348

Neosilurus pseudospinosus, 349

Neosilurus rendahli, 345

Neosilurus robustus, 348

Neotropius, 356, 359

Neotropius acutirostris, 359

Neotropius atherinoides, 359

Neotropius khavalchor, 359

nephelion, Cordylancistrus, 443

Netuma, 48

Netuma aulometopon, 56

Netuma bilineata, 49

Netuma dubia, 54

Netuma hassleriana, 56

Netuma insularum, 50

Netuma mazatlana, 54

Netuma osakae, 49

Netuma planifrons, 44

Netuma proxima, 48

Netuma radiata, 435

Netuma thalassina, 48

Netuma thalassina jacksonensis, 49

netuma, Bagrus, 49

neumanni, Chiloglanis, 307

neumanni, Clarias, 145

newtoni, Otolithus (Arius), 435

ngamensis, Clarias, 146

ngamensis, Auchenoglanis, 163

ngamensis, Parauchenoglanis, 163

ngola, Clarias, 142

nicaraguensis, Pimelodus, 199

nicaraguensis, Rhamdia, 199

nicefori, Astroblepus, 65

nicéfori, Astroblepus, 65

niceforoi, Hemiancistrus, 257

niceforoi, Hypostomus, 257

nickeriensis, Hypostomus, 257

nickeriensis, Loricaria, 269

nicoi, Acanthobunocephalus, 57

nicoi, Lasiancistrus, 289

nicoi, Pseudolithoxus, 289

nieuhofii, Clarias, 139, 146

Nieuwenhuisi, Glyptosternon, 392

nieuwenhuisi, Glyptothorax, 392

Nieuwenhuisii, Bagarius, 383

Nieuwenhuisii, Neopangasius, 325, 327

nieuwenhuisii, Pangasius, 327

niger, Bagrus docmac, 84

niger, Batasio, 445

niger, Chiloglanis, 307

niger, Copidoglanis novae-guineae, 349

niger, Doras, 175 niger, Doras (Oxydoras), 175

niger, Hemiancistrus, 287

niger, Hypostomus, 257

niger, Oxydoras, 175

niger, Plecostomus, 257

niger, Pseudancistrus, 287

nigeriae, Clarias, 141

nigeriensis, Clarias anguillaris, 140

Nigerium, 98

Nigerium gadense, 98

Nigerium wurnoense, 98, 99

nigrescens, Callichrous, 374

nigrescens, Pseudancistrus, 287

nigrescens, Silurus (Pimelodus), 210

nigribarbis, Parapimelodus, 335

nigribarbis, Pimelodus

(Pseudorhamdia), 335

nigricans, Arius, 54

nigricans, Clarias, 146

nigricans, Hypostomus, 278

nigricans, Nematogenys, 324

nigricans, Pimelodus, 208, 209, 210

nigricans, Plotosus, 352

nigricans, Pseudoplatystoma

fasciatum, 341

nigricans, Trichomycterus, 414, 421

nigricauda, Hemiloricaria, 246

nigricauda, Hisonotus, 248

nigricauda, Liobagrus, 20

nigricauda, Loricaria, 246

nigricauda, Otocinclus, 248

nigricauda, Pseudopimelodus, 353

nigricaudatus, Amphilius, 21

nigricaudatus multipunctata,

Amphilius, 21

nigricaudus, Cephalosilurus, 353

nigriceps, Bagrus, 96

nigriceps, Mystus, 96

nigricollaris, Horabagrus, 429

nigricollis, Tympanopleura, 68

nigrilabris, Gronias, 204, 206

nigripinnis, Auchenipterus, 72

nigripinnis, Euanemus, 72

nigripinnis, Microglanis, 354

nigrirostrum, Sturiosoma, 299

nigrita, Bagrus, 160

nigrita, Synodontis, 317

nigriventris, Synodontis, 318

nigrodigitatus, Chrysichthys, 158, 160

nigrodigitatus, Pimelodus, 158

nigrofasciata, Pimelodella, 193

nigrofasciatus, Pimelodus, 193

nigrolineata, Phenacorhamdia, 189

nigrolineatus, Chaetostomus, 277, 278

nigrolineatus, Panaque, 278

nigrolineatus, Pseudauchenipterus, 76

nigromaculata, Synodontis, 318

nigromaculatus, Hypostomus, 257

nigromaculatus, Plecostomus, 257 nigromaculatus, Synodontis, 318

nigromaculatus, Trichomycterus, 421 nigromarmoratus, Clarias, 146

nigropunctatus, Bagrus, 340

nijsseni, Corydoras, 121

nijsseni, Corydoras elegans, 121

nijsseni, Harttia, 272

nijsseni, Metaloricaria, 272

niloticus, Chiloglanis, 307

niloticus, Hypophthalmus, 362, 365

niloticus, Mochokus, 310, 311

niloticus, Silurus schilbe, 431

niloticus waterloti, Chiloglanis, 307

nimius, Hemipsilichthys, 247, 444

Niobichthys, 274

Niobichthys ferrarisi, 274

niobium, Glanapteryx, 405

nitidus, Akysis, 17, 82

nitidus, Leiocassis, 82

nitidus, Pelteobagrus, 82

nitidus, Pseudobagarius, 17

nitidus, Pseudobagrus, 82

niveata, Dekeyseria, 234

niveatus, Baryancistrus, 227

niveatus, Hypostomus, 226, 227

niveatus, Plecostomus, 234

niveiventris, Amiurus, 204

niveum, Chaetostoma, 230

njassae, Synodontis, 318

Nkondobabrus, 99

Nkondobagrus longisrostris, 99

nkunga, Plotosus, 351

nocturnus, Brachyglanis, 181

nocturnus, Noturus, 214

nocturnus, Panaqolus, 277

nocturnus, Panaque, 277

nodosus, Pseudauchenipterus, 76

nodosus, Silurus, 75, 76

noelkempffi, Corydoras, 121

nops, Gymnallabes, 150

normani, Chiloglanis, 307

Notarius, 49, 440

Notarius armbrusteri, 440

Notarius biffi, 49

Notarius cookei, 50

Notarius grandicassis, 50

Notarius insculptus, 50

Notarius kessleri, 50

Notarius neogranatensis, 50

Notarius osculus, 50

Notarius planiceps, 50

notata, Microsynodontis, 310

notata, Synodontis, 318

notatus, Amphilius, 27

notatus, Callichrous, 374

notatus, Hisonotus, 248

notatus, Microsynodontis, 310

notatus, Pimelodus, 210, 339

notatus, Platynematichthys, 339 
notatus, Synodontis, 318

notatus, Tetracamphilius, 27

notatus binotata, Synodontis, 318

notatus ocellatus, Synodontis, 318

notatus var. binotata, Synodontis, 318

notatus var. ocellatus, Synodontis, 318

notialis, Parakysis, 16

Notoglanidium, 161, 162

Notoglandium maculatus, 161

Notoglanidium pallidum, 162

Notoglanidium thomasi, 162

Notoglanidium walkeri, 162

notomelas, Pimelodella, 193

Notophthalmus, 333

notospilus, Hemidoras, 171

notozygurus, Clarias, 143

Noturus, 211

Noturus albater, 211

Noturus baileyi, 211

Noturus crypticus, 211, 442

Noturus elassochir, 212

Noturus elegans, 212

Noturus eleutherus, 212

Noturus exilis, 212

Noturus fasciatus, 212

Noturus flavater, 212

Noturus flavipinnis, 212

Noturus flavus, 211, 212

Noturus funebris, 212

Noturus furiosus, 211, 212

Noturus gilberti, 213

Noturus gladiator, 213

Noturus gyrinus, 213

Noturus hildebrandi, 213

Noturus hildebrandi lautus, 213

Noturus insignis, 213

Noturus lachneri, 213

Noturus latifrons, 211

Noturus leptacanthus, 213

Noturus luteus, 212

Noturus marginatus, 213

Noturus maydeni, 441

Noturus miurus, 214

Noturus munitus, 214

Noturus nocturnus, 214

Noturus occidentalis, 212

Noturus phaeus, 214

Noturus placidus, 214

Noturus platycephalus, 212

Noturus sialis, 213

Noturus stanauli, 214, 442

Noturus stigmosus, 214

Noturus taylori, 214

Noturus trautmani, 214

novaeguineae, Nedystoma, 46

novaeguineae, Neosilurus, 348

novaesi, Furcodontichthys, 240

novae-guineae, Copidoglanis, 348

novae-guineae, Doiichthys, 46 novae-guineae niger, Copidoglanis, 349

novalimensis, Harttia, 241

nox, Arius, 38, 39

nox, Brustiarius, 38

ntemensis, Synodontis Hollyi, 322

nuchalis, Alurichthys, 38

nuchalis, Arius, 41

nuchalis, Auchenipterus, 72

nuchalis, Hypophthalmus, 71, 72

nucleus, Arius, 57

nudiceps, Ancistrus, 223

nudiceps, Cnidoglanis, 346

nudiceps, Euristhmus, 346

nudiceps, Hypostomus, 223

nudiceps, Pelteobagrus, 100

nudiceps, Pseudobagrus, 100

nudidens, Arius (Hemiarius), 56

nudipectus, Belonoglanis, 24

nudirostre, Chaetostoma, 230

nudirostris, Chaetostomus, 230

nudiventris, Hypostomus, 257

nudirostris, Loricaria, 271

nudirostris, Loricariichthys, 271

nudiventris, Plecostomus, 257

nudiventris, Loricaria, 297

nudiventris, Parancistrus, 279

nudiventris, Spatuloricaria, 297

nudulus, Hemipsilichthys, 280

nudulus, Pareiorhaphis, 280

nujiangense, Clupisoma, 357

nummifer, Synodontis, 318

nyasensis, Clarias, 149

nyasensis, Dinotopterus, 149

nyongensis, Chrysichthys, 158

nyongensis, Chrysichthys

longidorsalis, 158

nyongensis, Eutropius, 365

nyongensis, Schilbe, 365

ñssipinnis, Heptapterus, 184

$\mathbf{O}$

oaxacae, Rhamdia, 201

obbesi, Porochilus, 351

obesa, Rhamdia, 199

obesus, Amiurus, 205

obesus, Arius, 81

obesus, Liobagrus, 20

obesus, Synodontis, 318, 323

oblongus, Tachysurus, 435

obscura, Glyptothorax, 392

obscurum, Pogonopoma, 285

obscurus, Acrochordonichthys 13

obscurus, Auchenipterus, 81

obscurus, Bagrichthys, 83

obscurus, Clarias, 141

obscurus, Copidoglanis, 345, 350

obscurus, Glyptothorax, 392

obtusa, Pseudotothyris, 290

obtusirostris, Eutropius, 365 obtusirostris, Plecostomus, 218

obtusos, Otocinclus, 290

obtusus, Otocinclus, 290

occidentalis, Ailia, 360

occidentalis, Ancistrus, 223

occidentalis, Auchenoglanis, 154

occidentalis, Cetopsis, 134, 135

occidentalis, Chiloglanis, 307

occidentalis, Hypostomus, 257

occidentalis, Hypostomus

gymnorhynchus, 257

occidentalis, Istlarius balsanus, 208

occidentalis, Malapterurus, 302

occidentalis, Noturus, 212

occidentalis, Parailia, 360

occidentalis, Pimelodus, 154

occidentalis, Xenocara, 223

occidentalis tanganicanus,

Auchenoglanis, 154

occidentalis tchadiensis,

Auchenoglanis, 154

occidentalis var. tanganicanus,

Auchenoglanis, 154

occidentalis var. tchadiensis,

Auchenoglanis, 154

occloi, Ancistrus, 223

ocellatus, Galeichthys, 43

ocellatus, Silurus, 35

ocellatus, Synodontis notatus, 318

ocellifer, Synodontis, 318

Ochmacanthus, 409

Ochmacanthus alternus, 409

Ochmacanthus batrachostoma, 409

Ochmacanthus flabelliferus, 409

Ochmacanthus orinoco, 409

Ochmacanthus reinhardtii, 410

Ochmacanthus taxistigma, 405, 406

ochoterenai, Haustor, 209

ochoterenai, Ictalurus, 209

octocirrhus, Pimelodus, 431

octocirrus, Corydoras, 117

Octonematichthys, 160

oculatus, Bagrus, 96

oculatus, Mystus, 96

oculeus, Hypostomus, 257

oculeus, Panaque, 257

odontotumulus, Farlowella, 238

odynea, Pimelodella, 193

odynea, Pimelodella chagresi, 193

oelemariensis, Corydoras, 114

oetik, Arius, 35

ogilviei, Ageneiosus, 69

ogilviei, Opsodoras, 174, 175

ogoensis, Gephyroglanis, 158

ogooensis, Chrysichthys, 158

ogooensis, Gephyroglanis, 158

ogooensis, Malapterurus electricus,

var., 302

ogowensis, Chrysichthys, 158 
oguensis, Malapterurus, 302

oguensis, Malapterurus electricus, 302

oguensis, Malapterurus electricus var., 302

oiapoquensis, Corydoras, 121

oibaensis, Callichthys, 111

okadai, Coreobagrus, 86

okae, Chrysichthys, 158

okeechobeensis, Ictalurus, 210

olallae, Duopalatinus, 340

olallae, Platysilurus, 340

Oligancistrus, 274

Oligancistrus punctatissimus, 274

oligospila, Peckoltia, 284

oligospilus, Chaetostomus, 284

olivaceus, Chlarias, 146

olivaceus, Clarias, 146

olivaceus, Pimelodus, 210

Olivaichthys, 166

Olivaichthys cuyanus, 166

Olivaichthys mesembrinus, 166

Olivaichthys viedmensis, 166

olivaris, Pylodictis, 215

olivaris, Silurus, 215

oliveirae, Farlowella, 237

oliveirai, Bathycetopsis, 131, 132

oliveirai, Cetopsis, 132

Oloplotosus, 349

Oloplotosus luteus, 349

Oloplotosus mariae, 349

Oloplotosus torobo, 349

Olyra, 81, 99

Olyra burmanica, 99

Olyra elongata, 99

Olyra horae, 99

Olyra horai, 99

Olyra kempi, 99

Olyra laticeps, 17, 18

Olyra longicaudata, 99

Olyra longicaudatus, 99

Olyrinae, 81

olyroides, Hemibagrus, 90

olyroides, Mystus, 90

omeihensis, Leiocassis, 102

omeihensis, Pseudobagrus, 102

omias, Synodontis, 319

ommation, Apistoloricaria, 226

Ompok, 371

Ompok bimaculatus, 372

Ompok binotatus, 372

Ompok borneensis, 372

Ompok fumidus, 372

Ompok hypophthalmus, 371, 372

Ompok jaynei, 372

Ompok leiacanthus, 371, 372

Ompok malabaricus, 372

Ompok miostomus, 372

Ompok pabda, 372

Ompok pabo, 373
Ompok pinnatus, 373

Ompok platyrhynchus, 373

Ompok pluriradiatus, 373

Ompok rhadinurus, 373

Ompok sabanus, 371

Ompok siluroides, 371

Ompok siluroïdes, 373

Ompok sindensis, 373

Ompok urbaini, 373

Ompok weberi, 373

oncina, Arius, 75

oncinus, Arius, 75

oncinus, Liosomadoras, 75

ondon, Pseudobagrus, 102

operculatum, Scleronema, 413

ophthalmica, Pimelodella, 193

ophthalmicus, Pimelodus, 194

ophthalmus, Doras (Corydoras), 169

opisthophthalmus, Amphilius, 22

Opladelus, 215

Opsodoras, 175

Opsodoras boulengeri, 172, 175

Opsodoras hemipeltis, 174

Opsodoras morei, 175

Opsodoras ogilviei, 174, 175

Opsodoras orthacanthus, 175

Opsodoras parallelus, 174

Opsodoras steindachneri, 169

Opsodoras stuebelii, 175

Opsodoras ternetzi, 175

Opsodoras trimaculatus, 174

orbignianus, Platystoma, 341

orbignyanum, Platystoma, 341

orcesi, Corydoras pastazensis, 122

oregonensis, Hypsidoris, 203

oremaculatus, Hypophthalmus, 333

Oreoglanis, 397

Oreoglanis delacouri, 397

Oreoglanis frenatus, 397

Oreoglanis hypsiurus, 397

Oreoglanis infulatus, 397

Oreoglanis insignis, 397

Oreoglanis lepturus, 397

Oreoglanis macronemus, 397

Oreoglanis macropterus, 398

Oreoglanis setiger, 398

Oreoglanis siamensis, 397, 398

orestes, Oxydoras, 171

Orestis, Oxydoras, 171

orientale, Cetopsidium, 130

orientale, Pseudocetopsis, 130

orientalis, Arges, 65

orientalis, Astroblepus, 65

orientalis, Bagrus, 84

orientalis, Irvineia, 358

orinocensis, Hildadoras, 175, 176

orinoco, Cetopsis, 132

orinoco, Cetopsorhamdia, 182

orinoco, Lithoxancistrus, 286, 287 orinoco, Ochmacanthus, 409

orinoco, Pseudancistrus, 287

orinoco, Pseudocetopsis plumbeus, 132

Orinocodoras, 175

Orinocodoras eigenmanni, 175

ornata, Nangra, 397

ornaticeps, Heptapterus, 185

ornatipinnis, Synodontis, 319

ornatissima, Synodontis, 319

ornatissimus, Synodontis, 319

ornatus, Chrysichthys, 158

ornatus, Clarias, 145

ornatus, Corydoras, 121

ornatus, Gogo, 29

ornatus, Hyalobagrus, 92

ornatus, Pimelodus, 338

ornatus, Pseudobagrus, 92

ornatus, Pseudomystus, 104

ornatus, Synodontis, 317, 319

oronocoi, Hoplosternum, 129

orontis, Clarias, 143

oroyae, Pygidium, 423

orphnopterus, Corydoras, 121

orthacanthus, Opsodoras, 175

orthiocarinatus, Copionodon, 404

ortmanni, Pimelodus, 338

ortoni, Rhamdia, 201

ortoni, Sorubimichthys, 343

osakae, Netuma, 49

Oschanini, Exostoma, 402

osculus, Arius, 50

osculus, Notarius, 50

Osteobagrus, 106

osteocarus, Corydoras, 121

Osteogaster, 112

Osteogeneiosus, 51

Osteogeneiosus blochii, 51

Osteogeneiosus cantoris, 51

Osteogeneiosus gracilis, 51

Osteogeneiosus ingluvies, 51

Osteogeneiosus longiceps, 51

Osteogeneiosus macrocephalus, 51

Osteogeneiosus militaris, 51

Osteogeneiosus valenciennesi, 51

Osteogeniosus sthenocephalus, 51

Osteogeniosus stenocephalus, 51

Osteomystax, 71

osteomystax, Auchenipterus, 72

osteomystax, Ceratocheilus, 71, 72

Ostophycephalus, 346

Ostophycephalus duriceps, 346

othonops, Cetopsis, 132

othonops, Hemicetopsis, 132

Otocinclini, 217

Otocinclus, 217, 264, 271, 274

Otocinclus (Microlepidogaster)

tietensis, 290

Otocinclus affinis, 271 
Otocinclus arnoldi, 271

Otocinclus bororo, 274

Otocinclus caxarari, 274

Otocinclus cephalacanthus, 276

Otocinclus cocama, 274

Otocinclus depressicauda, 248

Otocinclus fimbriatus, 271

Otocinclus flexilis, 271, 272

Otocinclus francirochai, 248

Otocinclus gibbosus, 264

Otocinclus hasemani, 274

Otocinclus hoppei, 275

Otocinclus huaorani, 275

Otocinclus joberti, 250

Otocinclus leucofrenatus, 248

Otocinclus macrospilus, 275

Otocinclus maculicauda, 281, 283

Otocinclus maculipinnis, 248

Otocinclus mariae, 275

Otocinclus mimulus, 275

Otocinclus mura, 275

Otocinclus nigricauda, 248

Otocinclus obtusos, 290

Otocinclus obtusus, 290

Otocinclus paulinus, 249

Otocinclus spectabilis, 273

Otocinclus tapirape, 275

Otocinclus vestitus, 274, 275

Otocinclus vittatus, 275

Otocinclus xakriaba, 275

Otolithus (Arius) aequus, 435

Otolithus (Arius) africanus, 435

Otolithus (Arius) amekiensis, 435

Otolithus (Arius) angelicus, 434

Otolithus (Arius) angulatus, 435

Otolithus (Arius) crassus bartonensis, 434

Otolithus (Arius) danicus, 434

Otolithus (Arius) danicus bartonensis, 434

Otolithus (Arius) decipiens, 434

Otolithus (Arius) germanicus, 434

Otolithus (Arius ?) glaber, 435

Otolithus (Arius) jaekeli, 435

Otolithus (Arius) Lerichei, 434

Otolithus (Arius ?) moravicus, 434

Otolithus (Arius) newtoni, 435

Otolithus (Arius ?) parvus, 435

Otolithus (Arius) planus, 435

Otolithus (Arius) rotundatus, 435

Otolithus (Arius) tenius, 435

Otolithus (Arius) tenuis, 435

Otolithus (Arius) vanigonis, 434

Otolithus (incertae sedis) crassus, 434

Otolithus (Sciaenidarum) decipiens, 434

Otolithus (Siluridarum ?) incertus, 434

Otothyrini, 217, 440

Otothyris, 217, 275
Otothyris canaliferus, 275, 276

Otothyris juquiae, 276

Otothyris lophophanes, 276

Otothyris rostrata, 276

Otothyris travassosi, 276

Otothyropsis, 276

Otothyropsis marapoama, 276

ourastigma, Corydoras, 121

ovidius, Synodontis, 316

oxycephalus, Clarias, 146

Oxydoras, 175

Oxydoras (Rhinodoras) amazonum hasemani, 170

Oxydoras (Rhinodoras) huberi, 174

Oxydoras acipenserinus, 172

Oxydoras affinis, 171

Oxydoras bachi, 174

Oxydoras eigenmanni, 167

Oxydoras elongatus, 174

Oxydoras holdeni, 176

Oxydoras kneri, 175

Oxydoras Morei, 175

Oxydoras Nattereri, 179

Oxydoras niger, 175

Oxydoras orestes, 171

Oxydoras Orestis, 171

Oxydoras sifontesi, 176

Oxydoras steindachneri, 179

Oxydoras Stübelii, 175

Oxydoras trachyparia, 179

Oxydoras trimaculatus, 174

Oxyglanis, 154

Oxyglanis sacchii, 154

Oxyloricaria, 298

Oxyloricaria citurensis, 300

Oxyloricaria dariensis, 299

Oxyloricaria Fowleri, 233

Oxyloricaria guentheri, 299

Oxyloricaria leightoni, 300

Oxyloricaria lyra, 299

Oxyloricaria robusta, 299

Oxyloricaria tamanae, 300

Oxyloricaria tenuirostris, 300

oxyptera, Paravandellia, 410

oxyrhinus, Amphilius, 23

oxyrhynchus, Anduzedoras, 169

oxyrhynchus, Corydoras, 121

Oxyrhynchus, Doras, 169

Oxyropsis, 276

Oxyropsis acutirostris, 276

Oxyropsis carinata, 276

Oxyropsis wrightiana, 276

Oxyropsis wrightii, 276

oxyrrhyncha, Acestra, 239

oxyrryncha, Acestra, 239

oxyrryncha, Farlowella, 239

$\mathbf{P}$

pabda, Ompok, 372

pabda, Silurus, 371, 372 pabo, Ompok, 373

pabo, Silurus, 373

pachychilus, Atopochilus, 304

pachyderma, Acrochordonichthys, 13

pachynema, Ceratoglanis, 368

pachynemus, Ceratoglanis, 368

pachynema, Clarias, 146

Pachypterus, 359

Pachypterus luridus, 327

Pachypterus melanurus, 358

Pachypterus punctatus, 358

Pachypterus trifasciatus, 359

Pachyula, 42

pacifici, Cruciglanis, 443

pagei, Hypostomus, 257

pahangensis, Mystus, 90

pakistanica, Gagata, 386

pakistanicus, Batasio, 85

palavanensis, Penesilurus, 375, 377

palceindicus, Heterobranchus, 151

paleatus, Callichthys, 122

paleatus, Corydoras, 122

palembangensis, Kryptopterus, 370

palembangensis, Silurus, 369, 370

palleus, Trichomycterus, 415

pallida, Pimelodella, 194

pallida, Sosia chamaeleon, 13

pallidimaculatus, Lithoxus, 267

pallidimaculatus, Lithoxus

(Paralithoxus), 267

pallidum, Notoglanidium, 162

pallidus, Nematogenys, 324

pallidus, Silurus, 210

pallidus Lateralis, Silurus, 210

pallidus Leucoptera, 210

pallidus Marginatus, Silurus, 210

pallidus var. Lateralis, Pimelodus, 210

pallidus var. Lateralis, Silurus, 210

pallidus var. Leucoptera, Pimelodus,

210

pallidus var. Leucoptera, Silurus, 210 pallidus var. Marginata, Silurus, 210

pallidus var. Marginatus, Pimelodus,

$$
210
$$

pallidus var. Marginatus, Silurus, 210 pallozonum, Glyptosternum, 388, 392 pallozonus, Glyptothorax, 392

palmeri, Chaetostoma, 230

palmeri, Chaetostomus 230

palmeri, Schilbe, 364

panamense, Sturisoma, 299

panamensis, Aelurichthys, 37, 38

panamensis, Bagre, 38

panamensis, Imparales, 180

panamensis, Loricaria, 299

panamensis, Plecostomus plecostomus, 243

Panaqolus, 277

Panaqolus albomaculatus, 277 
Panaqolus changae, 277

Panaqolus dentex, 277

Panaqolus gnomus, 277

Panaqolus maccus, 277

Panaqolus nocturnus, 277

Panaqolus purusiensis, 277

Panaque, 277

Panaque albomaculatus, 277

Panaque changae, 277

Panaque cochliodon, 277

Panaque dentex, 277

Panaque gnomus, 277

Panaque maccus, 277

Panaque nigrolineatus, 278

Panaque nocturnus, 277

Panaque oculeus, 257

Panaque purusiensis, 277

Panaque suttoni, 278

Panaque suttonorum, 278

panda, Corydoras, 122

panda, Glyptothorax, 392

Pangasianodon, 324

Pangasianodon gigas, 325

Pangasianodon hypophthalmus, 325

Pangasianodonidi, 324

Pangasiidae, 324

Pangasini, 324

Pangasius, 324, 325

Pangasius (Pseudopangasius) bocourti, 325

Pangasius aequilabialis, 327

Pangasius altifrons, 325

Pangasius beani, 328

Pangasius bedado, 325

Pangasius bocourti, 325

Pangasius Buchanani, 325, 327

Pangasius burgini, 326

Pangasius conchophilus, 325

Pangasius cultratus, 328

Pangasius de Zwaani, 328

Pangasius delicatissimus, 328

Pangasius djambal, 325, 326

Pangasius elongatus, 326

Pangasius fowleri, 328

Pangasius hexanema, 358

Pangasius hoeksi, 328

Pangasius humeralis, 326

Pangasius indicus, 326

Pangasius juaro, 327

Pangasius kinabatanganensis, 326

Pangasius krempfi, 326

Pangasius kunyit, 326

Pangasius larnaudii, 326

Pangasius lithostoma, 326

Pangasius longibarbis, 359

Pangasius macronema, 327

Pangasius mahakamensis, 327

Pangasius mekongensis, 327

Pangasius micronemus, 328
Pangasius myanmar, 327

Pangasius nasutus, 327

Pangasius nieuwenhuisii, 327

Pangasius pangasius, 327

Pangasius pangasius godavarii, 327

Pangasius pangasius upiensis, 327

Pangasius paucidens, 325

Pangasius pleurotaenia, 328

Pangasius polyuranodon, 325, 327

Pangasius ponderosus, 327

Pangasius rheophilus, 328

Pangasius rios, 328

Pangasius sabahensis, 328

Pangasius sanitwongsei, 328

Pangasius siamensis, 327

Pangasius sutchi, 325

Pangasius taeniura, 326

Pangasius tubbi, 328

panjang, Hemileiocassis, 91

pannonicus, Ictalurus nebulosus, 206

pantanalensis, Corydoras, 122

panthale, Platystoma, 341, 342

pantherinus, Auchenoglanis, 163

pantherinus, Eurycheilichthys, 236

pantherinus, Eurycheilus, 236

pantherinus, Hypostomus, 258

pantherinus, Parauchenoglanis, 163

pantherinus, Pseudariodes, 339

pantherinus, Synodontis, 315

pantherinus, Trichomycterus, 421

panzeri, Henonemus, 414

panzeri, Stegophilus, 414

paolence, Pygidium, 421

paolencis, Trichomycterus, 421

papariae, Hypostomus, 258

papariae, Plecostomus plecostomus, 258

papariae, Pseudancistrus, 287

papariae, Rhamdella, 197

papilionatus, Doras, 173

papillatus, Hemipsilichthys, 248, 444

papillatus, Hoplomyzon, 61

papillifer, Hemipimelodus, 48

papilliferus, Trichomycterus, 421

papillosus, Arius, 165

pappenheimi, Pimelodella, 194

papuensis, Copidoglanis, 348

papuensis, Plotosus, 351

paquequerense, Pygidium, 421

paquequerensis, Trichomycterus, 421

Parabranchioica, 410

Parabranchioica teaguei, 411

Paracanthopoma, 410

Paracanthopoma parva, 410

Paracetopsis, 134

Paracetopsis atahualpa, 135

Paracetopsis bleekeri, 134, 135

Paracetopsis esmeraldas, 135

Parachiloglanis, 398
Parachiloglanis hodgarti, 398

Paradiplomystes, 37

Paradoxoglanis, 302

Paradoxoglanis caudivittatus, 302

Paradoxoglanis cryptus, 303

Paradoxoglanis parvus, 303

paraense, Brachyplatystoma, 331

Paraglyptothorax, 388

paragua, Corydoras, 122

paraguayensis, Farlowella, 239

paraguayensis, Hemidoras, 179

paraguayensis, Trachydoras, 179

paraguensis, Rhinolepis, 275

Parahemiodon, 269

Parahemiodon chanjoo, 270

Parahemiodon typus, 269, 270

parahemiodon, Loricaria, 270

parahybae, Delturus, 234, 235, 444

parahybae, Ituglanis, 407

parahybae, Microglanis, 354

parahybae, Pimelodus (Rhamdia), 201

Parahybae, Platystoma, 344

parahybae, Pogonopoma, 285

parahybae, Pseudopimelodus, 354

parahybae, Pseudotocinclus, 290

parahybae, Pygidium proops, 406, 407

Parahybae, Rhinelepis, 285

parahybae, Steindachneridion, 344

Parailia, 360

Parailia congica, 360

Parailia longifilis, 360

Parailia occidentalis, 360

Parailia pellucida, 360

Parailia somalensis, 360

Parailia spiniserrata, 360

Parakysidae, 12

Parakysis, 12, 15

Parakysis anomalopteryx, 15

Parakysis grandis, 15

Parakysis longirostris, 16

Parakysis notialis, 16

Parakysis verrucosa, 15, 16

Parakysis verrucosus, 16

Paralithoides, 267

Paralithoxus, 267

parallelus, Corydoras, 122

parallelus, Opsodoras, 174

Paraloricaria, 278

Paraloricaria agastor, 278

Paraloricaria commersonoides, 278

Paraloricaria vetula, 278

Paramphilius, 25

Paramphilius firestonei, 25

Paramphilius goodi, 25

Paramphilius teugelsi, 25

Paramphilius trichomycteroides, 25

paranaënse, Farlowella, 237

paranaensis, Pimelodus, 338

parananus, Megalancistrus, 272 
parananus, Pterygoplichthys

(Ancistrus), 272

Parancistrus, 278

Parancistrus aurantiacus, 278

Parancistrus nudiventris, 279

paranensis, Hypostomus, 258

paranensis, Neoplecostomus, 273

paranensis, Parapterodoras, 177

Paraotocinclus cesarpintoi, 282

Paraphractura, 26

Paraphractura tenuicauda, 26, 27

Parapimelodus, 335

Parapimelodus nigribarbis, 335

Parapimelodus valenciennis, 335

Paraplotosus, 349

Paraplotosus albilabris, 349

Paraplotosus butleri, 349

Paraplotosus muelleri, 349

Parapseudecheneis, 400

Parapterodoras, 177

Parapterodoras paranensis, 177

Pararius, 48

paraschilbeides, Kryptopterus, 370

Parasilurus, 378

Parasilurus asotus longus, 379

Parasilurus biwaensis, 379

Parasilurus lithophilus, 380

Parasilurus microdorsalis, 380

Parastegophilus, 410

Parastegophilus maculatus, 410

Parastegophilus paulensis, 410

Parasturisoma, 298

Parasturisoma maculata, 233

paratus, Chiloglanis, 307

Parauchenipterus, 79

Parauchenipterus paseae, 79

Parauchenoglanis, 162

Parauchenoglanis ahli, 162

Parauchenoglanis altipinnis, 162

Parauchenoglanis ansorgii, 154

Parauchenoglanis balayi, 162

Parauchenoglanis boutchangai, 154

Parauchenoglanis buettikoferi, 163

Parauchenoglanis longiceps, 163

Parauchenoglanis monkei, 163

Parauchenoglanis ngamensis, 163

Parauchenoglanis pantherinus, 163

Parauchenoglanis punctatus, 163

Paravandellia, 410

Paravandellia oxyptera, 410

Paravandellia phaneronema, 411

pardale, Platystoma, 341

pardalis, Ageneiosus, 70

pardalis, Hypostomus, 292

pardalis, Platystoma, 341, 342

pardalis, Pterygoplichthys, 292

pardalis, Synodontis, 319

Pardiglanis, 163

Pardiglanis tarabinii, 163 pardus, Trichomycterus, 423

parecis, Ancistrus, 224

pareiacantha, Loricaria, 295

pareiacantha, Rineloricaria, 295

Pareiodon, 403, 411

Pareiodon microps, 411

Pareiodontinae, 403

Pareiorhaphis, 247, 279

Pareiorhaphis alipionis, 262

Pareiorhaphis azygolechis, 279

Pareiorhaphis bahianus, 279

Pareiorhaphis cameroni, 279

Pareiorhaphis cerosus, 279

Pareiorhaphis eurycephalus, 279

Pareiorhaphis garbei, 279

Pareiorhaphis hypselurus, 280

Pareiorhaphis hystrix, 280

Pareiorhaphis mutuca, 280

Pareiorhaphis nudulus, 280

Pareiorhaphis parmula, 280

Pareiorhaphis regani, 280

Pareiorhaphis splendens, 280

Pareiorhaphis steindachneri, 280

Pareiorhaphis stephanus, 280

Pareiorhaphis stomias, 280

Pareiorhaphis vestigipinnis, 281

Pareiorhina, 281

Pareiorhina brachyrhyncha, 281

Pareiorhina carrancas, 281

Pareiorhina rudolphi, 281

Pareuchiloglanis, 398

Pareuchiloglanis anteanalis, 398

Pareuchiloglanis feae, 398

Pareuchiloglanis gongshanensis, 398

Pareuchiloglanis gracilicaudatus, 398

Pareuchiloglanis kamengensis, 399

Pareuchiloglanis longicauda, 399

Pareuchiloglanis macropterus, 399

Pareuchiloglanis macrotremus, 399

Pareuchiloglanis myzostomus, 399

Pareuchiloglanis nebulifer, 399

Pareuchiloglanis poilanei, 398, 399

Pareuchiloglanis rhabdurus, 399

Pareuchiloglanis robusta, 399

Pareuchiloglanis robustus, 399

Pareuchiloglanis sichuanensis, 399

Pareuchiloglanis sinensis, 399

Pareuchiloglanis songdaensis, 400

Pareuchiloglanis songmaensis, 400

Pareuchiloglanis tianquanensis, 400

Pareutropius, 360

Pareutropius buffei, 360

Pareutropius debauwi, 361

Pareutropius longifilis, 361

Pareutropius mandevillei, 361

Pareutropius micristius, 360, 361

Parexostoma, 387

Parexostoma maculatum, 387

pariolispos, Scobinancistrus, 296
Pariolius, 188

Pariolius armillatus, 188

parkeri, Aspistor, 36

parkeri, Silurus, 36

parkii, Arius, 32

parkoi, Ituglanis, 407

parkoi, Pygidium, 407

parma, Cetopsis, 132

parmocassis, Arius, 50

parnaguensis, Ageneiosus, 70

parnahybae, Brachyplatystoma, 331

parnahybae, Loricaria, 269

parnahybae, Loricariichthys, 266

parnahybae, Pimelodella, 194

parnaibae, Glyptoperichthys, 292

parnaibae, Pterygoplichthys, 292

Paroreoglanis, 397

Paroreoglanis delacouri, 397

Parotocinclus, 281

Parotocinclus amazonensis, 281

Parotocinclus aripuanensis, 281

Parotocinclus bahiensis, 281

Parotocinclus bidentatus, 282

Parotocinclus britskii, 282

Parotocinclus cearensis, 282

Parotocinclus cesarpintoi, 282

Parotocinclus collinsae, 282

Parotocinclus cristatus, 282

Parotocinclus doceanus, 282

Parotocinclus eppleyi, 282

Parotocinclus haroldoi, 282

Parotocinclus jimi, 282

Parotocinclus jumbo, 282

Parotocinclus longirostris, 282

Parotocinclus maculicauda, 283

Parotocinclus minutus, 283

Parotocinclus muriaensis, 283

Parotocinclus planicauda, 283

Parotocinclus polyochrus, 283

Parotocinclus prata, 283

Parotocinclus spilosoma, 283

Parotocinclus spilurus, 283

Parotocinclus steindachneri, 283

Parrae, Galeichthys, 38

parryi, Rhamdia, 199

parva, Hemiloricaria, 246

parva, Loricaria, 246

parva, Paracanthopoma, 410

parva, Pimelodella, 194

parva, Pseudotatia, 77

parvanalis, Kryptopterus, 375

parvanalis, Phalacronotus, 375

parvicarinata, Farlowella, 239

parvimanus, Clarias, 140

parvipinnis, Arius, 47

parvus, Otolithus (Arius ?), 435

parvus, Paradoxoglanis, 303

parvus, Pimelodus (Rhamdia), 180

paseae, Parauchenipterus, 79 
passany, Bagrus, 54

passany, Sciades, 54

passarellii, Homodiaetus, 406

passarellii, Stegophilus, 406

passensis, Ituglanis, 408

pastazensis, Corydoras, 122

pastazensis orcesi, Corydoras, 122

patagoniensis, Hatcheria, 405

pataxo, Microglanis, 444

pati, Luciopimelodus, 334

pati, Pimelodus, 334

pati, Silurus, 335

patiae, Chaetostoma, 230

pattersoni, Trogloglanis, 216

paucerna, Corydoras, 122

paucidens, Metaloricaria, 272

paucidens, Pangasius, 325

paucimaculatus, Hypostomus, 258

paucipunctatus, Hypostomus, 258

pauciradiatum, Megalonema, 335

pauciradiatus, Aspidoras, 109

pauciradiatus, Corydoras, 109

pauciradiatus, Nemuroglanis, 188

pauciradiatus, Trichomycterus, 440

paucispinis, Chaetostoma, 230

paucispinis, Chaetostomus, 230

paucisquamatus, Megalodoras, 173

paucus, Arius, 48

paucus, Neoarius, 48

paulensis, Parastegophilus, 410

paulensis, Pseudostegophilus, 410

Paulicea, 344

Paulicea jahu, 344

paulina, Loricaria, 294

paulinus, Hisonotus, 249

paulinus, Hypostomus, 258

paulinus, Otocinclus, 249

paulinus, Plecostomus, 258

paviei, Pseudecheneis, 400

pavimentatus, Arius, 105

paynei, Mochokiella, 310

paysanduanus, Auchenipterus, 72

pearsei, Chaetostoma, 230

pearsei, Chaetostomus, 230

pearsoni, Cetopsis, 132

pearsoni, Tridentopsis, 426

Peckoltia, 283

Peckoltia arenaria, 284

Peckoltia bachi, 284

Peckoltia braueri, 284

Peckoltia brevis, 284

Peckoltia cavatica, 284

Peckoltia filicaudata, 284

Peckoltia furcata, 284

Peckoltia kuhlmanni, 284

Peckoltia oligospila, 284

Peckoltia sabaji, 284

Peckoltia snethlageae, 285

Peckoltia ucayalensis, 285
Peckoltia vermiculata, 285

Peckoltia vittata, 285

Peckoltichthys, 283

Peckoltichthys filicaudatus, 283, 284

Peckoltichthys kuhlmanni, 284

pecten, Copionodon, 404

pectinata, Conta, 384

pectinatus, Ameiurus, 206

pectinatus, Ictalurus, 206

pectinatus, Tetracamphilius, 27

pectinidens, Pimelodus, 46

pectinifer, Pimelodella, 194

pectinifrons, Agamyxis, 168

pectinifrons, Doras, 168

pectinopterus, Glyptosternon, 392

pectinopterus, Glyptothorax, 392

pectorale, Lepthoplosternum, 128

pectoralis, Arius, 48

pectoralis, Callichthys, 128

pectoralis, Neoarius, 48

pediculatus, Dolichancistrus, 235

pediculatus, Pseudancistrus, 235

pediculatus cobrensis, Pseudancistrus,

235

peguensis, Hemibagrus, 90

peguensis, Macrones, 90

pellegrini, Andersonia, 24

pellegrini, Plecostomus, 289

pellopterygius, Microglanis, 354

pellucida, Parailia, 360

pellucida, Physailia, 360

Pelodichthys, 215

peloichthys, Trachelyopterus, 80

peloichthys, Trachycorystes insignis, 80

peltatus, Rhineastes, 430

Pelteobagrini, 81

Pelteobagrus, 81, 99

Pelteobagrus argentivittatus, 100

Pelteobagrus brashnikowi, 100

Pelteobagrus eupogon, 100

Pelteobagrus fulvidraco, 100

Pelteobagrus intermedius, 100

Pelteobagrus kyphus, 82

Pelteobagrus mica, 100

Pelteobagrus microps, 100

Pelteobagrus nitidus, 82

Pelteobagrus nudiceps, 100

Pelteobagrus ramentosus, 108

Pelteobagrus ussuriensis, 100

Pelteobagrus vachellii, 101

Pelteobagrus virgatus, 101

Pelteobagrus virgatus vinhensis, 101

Peltura, 26

Peltura Bovei, 26

pelusius, Mystus, 96

pelusius, Silurus, 96

pemecus, Bagrus, 53

pemon, Cetopsidium, 131
Penesilurus, 375

Penesilurus bokorensis, 376

Penesilurus palavanensis, 375, 377

pentamaculata, Rineloricaria, 295

pentapterus, Clarias, 146

pentlandi, Trichomycterus, 423

Pentlandii, Pimelodus, 200

peregrinus, Ictalurus, 207

perforated, Microlepidogaster, 272

perforatus, Microlepidogaster, 272

perijae, Cordylancistrus, 231

perporosus, Hypophthalmus, 334

Perrunichthys, 335

Perrunichthys perruno, 335, 336

perruno, Perrunichthys, 335, 336

persimilis, Chrysichthys, 158

personatus, Callychthys, 129

peruana, Pimelodella, 194

peruanus, Arges, 66

peruanus, Astroblepus, 66

peruanus, Chasmocranus, 183

peruanus, Duopalatinus, 332

peruense, Pimelodella, 194

peruensis, Pimelodella, 194

Perugia, 339

Perugia argentina, 335

Perugia xanthus, 335

perugiae, Anyperistius, 347

perugiae, Centromochlus, 73

peruvianus, Galeichthys, 43

pestai, Corydoras, 116

Petacara, 62

petenensis, Pimelodus, 198

petleyi, Limatulichthys, 266

petleyi, Rhineloricaria, 266

petricola, Clariallabes, 138

petricola, Synodontis, 319

petroleus, Hoplomyzon atrizona, 61

Peyeria, 428

peyeria, Ariopsis, 432

pfefferi, Synodontis, 311

phaeus, Noturus, 214

Phagorus, 139

Phagorus cataractus, 141

phaiosoma, Silurichthys, 378

phaiosoma, Silurus, 377, 378

phalacra, Brachyglanis, 181

Phalacronotini, 367

Phalacronotus, 367, 374

Phalacronotus apogon, 374

Phalacronotus bleekeri, 374

Phalacronotus micronemus, 375

Phalacronotus micruropterus, 375

Phalacronotus parvanalis, 375

phalacronotus, Silurus, 374, 375

phaneronema, Branchioica, 411

phaneronema, Paravandellia, 411

phantasia, Cetopsorhamdia, 182

phelpsi, Astroblepus, 66 
phelpsi, Spatuloricaria, 296, 297

Phenacorhamdia, 188

Phenacorhamdia anisura, 188

Phenacorhamdia boliviana, 188

Phenacorhamdia hoehnei, 188

Phenacorhamdia macarenensis, 188, 189

Phenacorhamdia nigrolineata, 189

Phenacorhamdia somnians, 189

Phenacorhamdia tenebrosa, 189

Phenacorhamdia unifasciata, 189

philippinus, Pseudarius, 35

phillipsi, Clarias, 145

pholeter, Astroblepus, 66

phoxocephala, Hemiloricaria, 246

phoxocephala, Loricaria, 246

Phractocephalus, 336

Phractocephalus bicolor, 336

Phractocephalus gogra, 105

Phractocephalus hemioliopterus, 336

Phractocephalus itchkeea, 386

Phractocephalus kuturnee, 105

Phractocephalus nassi, 336

Phractura, 26

Phractura ansorgii, 26

Phractura bovei, 26

Phractura brevicauda, 26

Phractura clauseni, 26

Phractura fasciata, 26

Phractura gladysae, 26

Phractura ineac, 28

Phractura intermedia, 26

Phractura lindica, 26

Phractura longicauda, 26

Phractura lukugae, 26

Phractura macrura, 27

Phractura scaphyrhynchura, 27

Phractura tenuicauda, 27

Phreatobinae, 429

Phreatobius, 429

Phreatobius cisternarum, 429, 430

phreatophila, Prietella, 215

phrixosoma, Plecostomus, 298

phrixosoma, Squaliforma, 298

phrygiatus, Arius, 32

Phyllonemus, 164

Phyllonemus brichardi, 164

Phyllonemus filinemus, 164

Phyllonemus typus, 164

physacanthus, Arius, 36

Physailia, 360

Physailia ansorgii, 360

Physailia pellucida, 360

Physailia somalensis tanensis, 360

Physailia villiersi, 360

Physopyxis, 176

Physopyxis ananas, 176

Physopyxis cristata, 176

Physopyxis lyra, 176 piauhiae, Loricaria, 269

picinguabae, Listrura, 444

picklei, Cetopsorhamdia, 182

picta, Dekeyseria, 234

picta, Megalechis, 129

pictus, Akysis, 14

pictus, Amphilius, 21

pictus, Ancistrus, 234, 265

pictus, Bagrus (Sciades), 334

pictus, Callichthys, 129

pictus, Hypostomus, 265

pictus, Leiarius, 334

pictus, Pimelodus, 338

pictus, Trichomycterus, 423

pidada, Arius, 55

pietschmanni, Allabenchelys, 138

pietschmanni, Auchenoglanis, 162

pietschmanni, Clariallabes, 138

pijpersi, Cetopsorhamdia, 186

pijpersi, Imparfinis, 186

pilosus, Neblinichthys, 273

Pimelenotus, 197

Pimelenotus Vilsoni, 197, 200

Pimeletropis, 332

Pimeletropis lateralis, 332

Pimelode livrée, 213

Pimelodela garbei, 189

Pimelodella, 189

Pimelodella altipinnis, 189

Pimelodella australis, 189

Pimelodella avanhandavae, 189

Pimelodella boliviana, 189

Pimelodella boschmai, 190

Pimelodella brasiliensis, 190, 192

Pimelodella breviceps, 190

Pimelodella buckleyi, 190

Pimelodella chagresi, 190

Pimelodella chagresi odynea, 193

Pimelodella chaparae, 190

Pimelodella cinerascens, 201

Pimelodella cochabambae, 185

Pimelodella conquetaensis, 190

Pimelodella copei, 190

Pimelodella cristata, 190

Pimelodella cruxenti, 190

Pimelodella cyanostigma, 190

Pimelodella dorseyi, 190

Pimelodella eigenmanni, 191, 193, 340

Pimelodella eigenmanniorum, 191

Pimelodella elongata, 191

Pimelodella enochi, 191

Pimelodella eutaenia, 191

Pimelodella figueroai, 191

Pimelodella garbei, 189

Pimelodella geryi, 191

Pimelodella gracilis, 191

Pimelodella griffini, 191

Pimelodella grisea, 191
Pimelodella hartii, 191

Pimelodella hartwelli, 192

Pimelodella hasemani, 192

Pimelodella howesi, 192

Pimelodella insignis, 190

Pimelodella itapicuruensis, 192

Pimelodella kronei, 192

Pimelodella lateristriga, 192

Pimelodella laticeps, 192

Pimelodella laticeps australis, 189

Pimelodella laurenti, 192

Pimelodella linami, 192

Pimelodella macrocephala, 192

Pimelodella macturki, 192

Pimelodella martinezi, 193

Pimelodella meeki, 193

Pimelodella megalops, 193

Pimelodella megalura, 193

Pimelodella metae, 193

Pimelodella modesta, 193

Pimelodella montana, 193

Pimelodella mucosa, 193

Pimelodella nigrofasciata, 193

Pimelodella notomelas, 193

Pimelodella odynea, 193

Pimelodella ophthalmica, 193

Pimelodella pallida, 194

Pimelodella pappenheimi, 194

Pimelodella parnahybae, 194

Pimelodella parva, 194

Pimelodella pectinifer, 194

Pimelodella peruana, 194

Pimelodella peruense, 194

Pimelodella peruensis, 194

Pimelodella procera, 194

Pimelodella rambarrani, 181

Pimelodella rendahli, 196

Pimelodella reyesi, 194

Pimelodella roccae, 194

Pimelodella rudolphi, 194

Pimelodella serrata, 194

Pimelodella spelaea, 195

Pimelodella steindachneri, 195

Pimelodella taeniophora, 195

Pimelodella taenioptera, 195

Pimelodella tapatapae, 195

Pimelodella transitoria, 195

Pimelodella vittata, 195

Pimelodella wesselii, 195

Pimelodella witmeri, 195

Pimelodella yuncensis, 195

Pimelodes filamentosus, 330

Pimelodes fossor, 431

Pimelodes lateristrigus, 192

Pimelodes macropterus, 332

Pimelodidae, 329

Pimelodina, 336

Pimelodina flavipinnis, 336

Pimelodina goeldii, 332 
Pimelodina nasus, 336

Pimelodinae, 329

Pimelodini, 329

Pimelodon, 211

Pimelodon insignarius, 211, 213

Pimelodus, 336

Pimelodus (Bagrus) maculatus, 345

Pimelodus (Pimelodella) eigenmanni, 191, 193

Pimelodus (Pimelodella) griseus, 191

Pimelodus (Pimelodella) taeniophorus, 195

Pimelodus (Pimelodina) flavipinnis, 336

Pimelodus (Pimelodus) grosskopfii, 338

Pimelodus (Pseudopimelodus) cottoides, 354

Pimelodus (Pseudopimelodus) pulcher, 355

Pimelodus (Pseudorhamdia)

brasiliensis, 190

Pimelodus (Pseudorhamdia) Chagresi, 190

Pimelodus (Pseudorhamdia) Hartii, 191

Pimelodus (Pseudorhamdia) nigribarbis, 335

Pimelodus (Pseudorhamdia) Wesselii, 195

Pimelodus (Rhamdia) Baronis Mülleri, 201

Pimelodus (Rhamdia) brachypterus, 198

Pimelodus (Rhamdia) Cuyabae, 201

Pimelodus (Rhamdia) Knerii, 199

Pimelodus (Rhamdia) longicauda, 185

Pimelodus (Rhamdia) Parahybae, 201

Pimelodus (Rhamdia) parvus, 180

Pimelodus (Rhamdia) Queleni cuprea, 201

Pimelodus absconditus, 336

Pimelodus aeneus, 215

Pimelodus affinis, 208

Pimelodus ailurus, 205

Pimelodus albicans, 337

Pimelodus albidus, 40, 204

Pimelodus albofasciatus, 337

Pimelodus altipinnis, 189

Pimelodus altissimus, 337

Pimelodus angius, 359

Pimelodus anisurus, 98

Pimelodus antiquus, 207

Pimelodus antoniensis, 205

Pimelodus aor, 106

Pimelodus arekaima, 334

Pimelodus argenteus, 53

Pimelodus argyrus, 209

Pimelodus arius, 34
Pimelodus asperus, 395

Pimelodus atrarius, 205

Pimelodus atrobrunneus, 337

Pimelodus auratus, 155

Pimelodus bagarius, 383

Pimelodus bahianus, 195

Pimelodus balayi, 162

Pimelodus barbancho, 339

Pimelodus barbus, 44

Pimelodus batasio, 84, 85

Pimelodus Batasius, 85, 98

Pimelodus bathyurus, 201

Pimelodus bilineatus, 107

Pimelodus biscutatus, 154

Pimelodus blochii, 336, 337

Pimelodus borneënsis, 41

Pimelodus botius, 388

Pimelodus Boucardi, 201

Pimelodus brachycephalus, 198

Pimelodus breviceps, 190

Pimelodus brevis, 337

Pimelodus buckleyi, 190

Pimelodus Bufonius, 355

Pimelodus caerulescens, 81

Pimelodus carulescens, 81

Pimelodus Cantonensis, 431

Pimelodus carcio, 85, 98

Pimelodus Carnaticus, 383

Pimelodus catulus, 205

Pimelodus cauda-furcatus, 209

Pimelodus cavasius, 95

Pimelodus cavia, 388

Pimelodus cenia, 386

Pimelodus chandramara, 86

Pimelodus charus, 355

Pimelodus cinerascens, 200

Pimelodus clarias, 320

Pimelodus clarias coprophagus, 337

Pimelodus coerulescens, 81

Pimelodus Commersonii, 43, 44

Pimelodus confinis, 205

Pimelodus conirostris, 428

Pimelodus conta, 383, 384

Pimelodus coprophagus, 337

Pimelodus cranchii, 156

Pimelodus cristatus, 189, 190

Pimelodus ctenodus, 332

Pimelodus cupreoides, 205

Pimelodus cupreus, 215

Pimelodus cyanochloros, 392

Pimelodus cyclopum, 64

Pimelodus dekayi, 206

Pimelodus Deppei, 200

Pimelodus eigenmanni, 340

Pimelodus elongatus, 191

Pimelodus eques, 184

Pimelodus exsudans, 196

Pimelodus felinus, 205

Pimelodus felis, 206
Pimelodus foina, 197

Pimelodus fur, 337

Pimelodus furcatus, 208, 209

Pimelodus furcifer, 210

Pimelodus gagata, 385, 386

Pimelodus gagora, 34

Pimelodus gambensis, 54

Pimelodus garciabarrigai, 337

Pimelodus garcia-barrigai, 337

Pimelodus gaudryi, 432

Pimelodus genidens, 43, 44

Pimelodus godmanni, 200

Pimelodus gracilis, 191, 196, 210

Pimelodus graciosus, 210

Pimelodus grosskopfii, 338

Pimelodus grosskopfii navarroi, 338

Pimelodus grunniens, 336

Pimelodus guatemalensis, 200

Pimelodus guirali, 162

Pimelodus gulio, 93, 95

Pimelodus guttatus, 88, 162, 163

Pimelodus Hammondii, 210

Pimelodus hara, 395, 396

Pimelodus heraldoi, 338

Pimelodus heteropleurus, 181

Pimelodus Hilarii, 200

Pimelodus holomelas, 199

Pimelodus houghi, 210

Pimelodus hoyi, 206

Pimelodus humilis, 198

Pimelodus hypselurus, 198

Pimelodus indicus, 19

Pimelodus insigne, 213

Pimelodus insignis, 339

Pimelodus jatius, 41

Pimelodus javus, 431

Pimelodus jenynsii, 196

Pimelodus jivaro, 338

Pimelodus labrosus, 334

Pimelodus lateristrigus, 192

Pimelodus laticaudus, 198

Pimelodus laticeps, 160

Pimelodus lemniscatus, 213

Pimelodus leptus, 332

Pimelodus limosus, 215

Pimelodus lividus, 205

Pimelodus longifilis, 338

Pimelodus lupus, 209

Pimelodus lutescens, 215

Pimelodus lynx, 204

Pimelodus maculatus, 209, 336, 338, 345

Pimelodus managuensis, 199

Pimelodus mangois, 18

Pimelodus mangurus, 355

Pimelodus Manillensis, 41

Pimelodus marginatus, 206

Pimelodus marmoratus, 206

Pimelodus megalops, 210 
Pimelodus melanogaster, 12, 13

Pimelodus melas, 205

Pimelodus membranaceus, 309

Pimelodus menoda, 89

Pimelodus micropterus, 200

Pimelodus microstoma, 338

Pimelodus modestus, 193

Pimelodus mong, 55

Pimelodus motaguensis, 198

Pimelodus mülleri, 199

Pimelodus multiradiatus, 334

Pimelodus murius, 358

Pimelodus musculus, 200

Pimelodus mustelinus, 184

Pimelodus mysteriosus, 338

Pimelodus namdia, 200

Pimelodus nangra, 396, 397

Pimelodus natalis, 205

Pimelodus navarroi, 338

Pimelodus nebulosus, 205, 215

Pimelodus nella, 52

Pimelodus nenga, 47

Pimelodus nicaraguensis, 199

Pimelodus nigricans, 208, 209, 210

Pimelodus nigrodigitatus, 158

Pimelodus nigrofasciatus, 193

Pimelodus notatus, 210, 339

Pimelodus occidentalis, 154

Pimelodus octocirrhus, 431

Pimelodus olivaceus, 210

Pimelodus ophthalmicus, 194

Pimelodus ornatus, 338

Pimelodus ortmanni, 338

Pimelodus pallidus var. Lateralis, 210

Pimelodus pallidus var. Leucoptera, 210

Pimelodus pallidus var. Marginata, 210

Pimelodus pangasius, 325, 327

Pimelodus paranaensis, 338

Pimelodus pati, 334

Pimelodus pectinidens, 46

Pimelodus Pentlandii, 200

Pimelodus petenensis, 198

Pimelodus pictus, 338

Pimelodus pinirampus, 339

Pimelodus pirinampu, 339

Pimelodus platanus, 335

Pimelodus platespogon, 383

Pimelodus platicirris, 339

Pimelodus platycephalus, 206

Pimelodus platychir, 21, 22

Pimelodus platypogon, 392

Pimelodus platypogonides, 392

Pimelodus pleurostigma, 13

Pimelodus polycaulus, 198

Pimelodus pullus, 205

Pimelodus puma, 205

Pimelodus punctatus, 339
Pimelodus punctulatus, 216

Pimelodus pusillus, 431

Pimelodus quelen, 200

Pimelodus rama, 105

Pimelodus raninus, 352, 353

Pimelodus rigidus, 339

Pimelodus rita, 106

Pimelodus Rogersi, 198

Pimelodus rugosus, 13

Pimelodus Sadleri, 431

Pimelodus sagor, 45

Pimelodus salvini, 198

Pimelodus sapo, 200

Pimelodus sebae, 197, 200

Pimelodus seengtee, 97

Pimelodus Sellonis, 200

Pimelodus silondia, 366

Pimelodus sona, 45

Pimelodus Spegazzinii, 335

Pimelodus spixii, 40

Pimelodus Stegelichii, 200

Pimelodus synodontis, 313

Pimelodus tachisurus, 431

Pimelodus telchita, 394

Pimelodus telchitta, 394

Pimelodus tengana, 86

Pimelodus tengara, 97

Pimelodus thunberg, 35

Pimelodus urua, 359

Pimelodus vacha, 358

Pimelodus valenciennis, 335

Pimelodus variegatus, 13, 14, 15

Pimelodus velifer, 203

Pimelodus versicolor, 44

Pimelodus viridescens, 395

Pimelodus viscosus, 215

Pimelodus vulgaris, 206

Pimelodus vulpeculus, 206

Pimelodus vulpes, 211

Pimelodus wagneri, 201

Pimelodus Westermanni, 329, 330

Pimelodus wuchereri, 200

Pimelodus Xanthocephalus, 207

Pimelodus zonatus, 13

Pimelodus zungaro, 344

Pimelotus, 197

pinheiroi, Corydoras, 122

Pinirampidae, 329

Pinirampus, 329, 339

Pinirampus pirinampu, 339

Pinirampus typus, 339

pinirampus, Pimelodus, 339

pinnatus, Ompok, 373

pinnimaculatus, Aelurichthys, 38

pinnimaculatus, Bagre, 38

Pinniwallago, 375

Pinniwallago kanpurensis, 375

piperata, Tympanopleura, 68, 70

piperatus, Ageneiosus, 70 piperatus, Imparfinis, 185, 186

piperatus Kryptopterus, 370

pipri, Erethistoides, 384

pipri, Erethistoides montana, 384

pique, Hatcheria, 405

piracicabae, Loricaria, 269

Piramutana, 330

Piramutana macrospila, 339

Pirarara, 336

Pirarara bicolor, 336

pirareta, Ancistrus, 224

piratatu, Hypostomus, 258

Piratinga, 330

Piratinga pirá-aïba, 330

Pirauáca, Sorubim, 343

pirá-aïba, Piratinga, 330

piresi, Glanidium, 78

piresi, Tocantinsia, 78

piriformis, Ancistrus, 224

pirinampu, Pimelodus, 339

pirinampu, Pinirampus, 339

Pirinampus, 339

Pirinampus agassizii, 339

pirrense, Cyclopium, 66

pirrensis, Astroblepus, 66

piscatrix, Pseudorhamdia, 337

pitmani, Chrysichthys, 160

piurae, Pygidium punctulatum, 421

piurae, Trichomycterus, 421

placidus, Noturus, 214

planicauda, Parotocinclus, 283

planiceps, Ancistrus, 264

planiceps, Arius, 50

planiceps, Bagrus, 90

planiceps, Clarias, 147

planiceps, Hemibagrus, 90

planiceps, Notarius, 50

planiceps, Platystoma, 343

planiceps, Sorubimichthys, 343

planifrons, Genidens, 44

planifrons, Netuma, 44

Planiloricaria, 217, 285

Planiloricaria cryptodon, 285

Planiloricariina, 217

planquettei, Lithoxus, 267

planquettei, Lithoxus (Paralithoxus), 267

planus, Otolithus (Arius), 435

planus, Tachysurus, 434, 435

platana, Bergiaria, 330

platana, Bergiella, 330

platanum, Megalonema, 335

platanus, Pimelodus, 335

platespogon, Pimelodus, 383

platicirris, Pimelodus, 339

platorynchus, Farlowella, 239

platus, Amarginops, 153

Platyallabes, 152

Platyallabes tihoni, 152 
Platycephaloides, 139

platycephalum, Megalonema, 335

platycephalum psammium,

Megalonema, 335

platycephalus, Acrochordonichthys,

12, 13

platycephalus, Amblyceps, 18

platycephalus, Ameiurus, 206

platycephalus, Chaetostomus, 231

platycephalus, Chrysichthys, 158

platycephalus, Clarias, 139, 147

platycephalus, Cordylancistrus, 23

platycephalus, Hemiodon, 288

platycephalus, Heterobranchus, 151

platycephalus, Miuroglanis, 409

platycephalus, Noturus, 212

platycephalus, Pimelodus, 206

platycephalus, Pseudohemiodon, 288

platychir, Amphilius, 22

platychir, Pimelodus, 21, 22

platychir cubangoensis, Amphilius, 23

Platyclarias, 152

Platyclarias machadoi, 152

Platydoras, 176

Platydoras armatulus, 176

Platydoras costatus, 176

Platyglanis, 164

Platyglanis depierrei, 164

platymetopon, Loricariichthys, 271

platynema, Brachyplatystoma, 331

Platynematichthys, 339

Platynematichthys notatus, 339

platynemum, Brachyplatystoma, 331

Platypogon, 339

Platypogon caerulorostris, 339, 340

platypogon, Arius, 54

platypogon, Glyptothorax, 392

platypogon, Kryptopterus, 371

platypogon, Micropogon, 371

platypogon, Pimelodus, 392

platypogon, Sciades, 54

platypogonides, Glyptothorax, 392

platypogonides, Pimelodus, 392

platypogonoides, Glyptothorax, 392

platyprosopos, Clariallabes, 138

platyrhynchos, Hemisorubim, 333

platyrhynchos, Platystoma, 333

platyrhynchus, Cordylancistrus, 232

platyrhynchus, Hemiancistrus, 232

platyrhynchus, Ompok, 373

Platysilurus, 340

Platysilurus barbatus, 340

Platysilurus malarmo, 340

Platysilurus mucosus, 340

Platysilurus olallae, 340

Platysomatos, 61

Platystacinae, 57

Platystacus, 57, 61

Platystacus anguillaris, 350, 351
Platystacus chaca, 135, 136

Platystacus cotylephorus, 61

Platystacus laevis, 58

Platystacus nematophorus, 61

Platystacus verrucosus, 58, 60

Platystoma, 342

Platystoma affine, 330

Platystoma artedii, 341

Platystoma corruscans, 341

Platystoma coruscans, 341

Platystoma emarginatum, 332

Platystoma juruense, 331

Platystoma Luceri, 342

Platystoma lütkeni, 344

Platystoma mucosa, 340

Platystoma orbignianus, 341

Platystoma orbignyanum, 341

Platystoma panthale, 341, 342

Platystoma parahybae, 344

Platystoma pardale, 341

Platystoma pardalis, 341, 342

Platystoma planiceps, 343

Platystoma platyrhynchos, 333

Platystoma punctifer, 342

Platystoma seenghala, 107

Platystoma spatula, 343

Platystoma sturio, 340

Platystoma tigrinum, 341

Platystoma truncatum, 341

Platystoma Vaillantii, 331

platystoma, Cteniloricaria, 233

platystoma, Loricaria, 233

Platystomatichthys, 340

Platystomatichthys sturio, 340

platystomus, Arius, 52

platystomus, Plicofollis, 52

Platytropius, 361

Platytropius longianalis, 357

Platytropius siamensis, 361

Platytropius sinensis, 358

platyura, Hemiloricaria, 246

platyura, Loricaria, 246

plazaii, Vandellia, 427

plecostomoides, Cochliodon, 258

plecostomoides, Hypostomus, 258

Plecostomus, 250

Plecostomus (Carinotus) carinotus,

234, 235

Plecostomus (Rhinelepis) microps, 281

Plecostomus aculeatus, 224

Plecostomus affinis, 251

Plecostomus ãgnã, 251

Plecostomus albopunctatus, 251

Plecostomus ancistroides, 251

Plecostomus angipinnatus, 251

Plecostomus angulicauda, 235

Plecostomus annae, 297

Plecostomus argus, 251

Plecostomus aspilogaster, 251
Plecostomus bicirrosus, 259

Plecostomus biseriatus, 297

Plecostomus bolivianus, 252

Plecostomus borellii, 252

Plecostomus boulengeri, 252

Plecostomus brasiliensis, 259

Plecostomus brevicauda, 252

Plecostomus brevis, 252

Plecostomus carinatus, 252

Plecostomus carvalhoi, 252

Plecostomus chaparae, 226

Plecostomus commersonii scabriceps, 260

Plecostomus commersonoides, 255

Plecostomus cordovae, 258

Plecostomus derbyi, 253

Plecostomus festae, 263

Plecostomus flagellaris, 268

Plecostomus fluviatilis, 253

Plecostomus francisci, 254

Plecostomus garmani, 254

Plecostomus gomesi, 298

Plecostomus goyazensis, 254

Plecostomus gymnorhynchus, 254

Plecostomus hemiurus, 254

Plecostomus Hermanni, 254

Plecostomus heylandi, 263

Plecostomus hondae, 250, 254

Plecostomus iheringi, 298

Plecostomus iheringii, 255, 298

Plecostomus interruptus, 255

Plecostomus jaguribensis, 255

Plecostomus johnii, 255

Plecostomus lacerta, 263

Plecostomus laplatae, 255

Plecostomus latirostris, 255

Plecostomus lexi, 255

Plecostomus lima, 256

Plecostomus lima atropinnis, 251

Plecostomus limosus, 253

Plecostomus longiradiatus, 256

Plecostomus luteomaculatus, 256

Plecostomus luteus, 256

Plecostomus Lütkeni, 251

Plecostomus macrops, 256

Plecostomus madeirce, 225

Plecostomus margaritifer, 256

Plecostomus margaritifer butantanis, 256

Plecostomus meleagris, 256

Plecostomus microps, 273, 281

Plecostomus micropunctatus, 225

Plecostomus myersi, 257

Plecostomus niger, 257

Plecostomus nigromaculatus, 257

Plecostomus niveatus, 234

Plecostomus nudiventris, 257

Plecostomus obtusirostris, 218

Plecostomus paulinus, 258 
Plecostomus pellegrini, 289

Plecostomus phrixosoma, 298

Plecostomus plecostomus panamensis, 243

Plecostomus plecostomus papariae, 258

Plecostomus popoi, 225

Plecostomus pusarum, 259

Plecostomus rachovii, 255

Plecostomus regani, 259

Plecostomus Robinii, 261

Plecostomus rondini, 259

Plecostomus rondoni, 259

Plecostomus scaphyceps, 260

Plecostomus scaplyceps, 260

Plecostomus scopularius, 298

Plecostomus seminudus, 260

Plecostomus spilosoma, 283

Plecostomus spilurus, 283

Plecostomus spiniger, 252

Plecostomus spinosissimus, 263

Plecostomus strigaticeps, 260

Plecostomus tenuicauda, 298

Plecostomus ternetzi, 261

Plecostomus tietensis, 261

Plecostomus topavae, 261

Plecostomus Unae, 261

Plecostomus unicolor, 225

Plecostomus vaillanti, 261

Plecostomus variipictus, 261

Plecostomus varimaculosus, 261

Plecostomus variostictus, 261

Plecostomus vermicularis, 262

Plecostomus villarsi, 298

Plecostomus virescens, 298

Plecostomus Wertheimeri, 285

Plecostomus winzi, 262

Plecostomus wuchereri, 262

plecostomus, Acipencer, 258

plecostomus, Hypostomus, 258

plecostomus, Plecostomus, 243

plecostomus panamensis, Plecostomus, 243

plecostomus papariae, Plecostomus, 258

Plectrochilus, 411

Plectrochilus diabolicus, 411

Plectrochilus machadoi, 411

Plectrochilus wieneri, 411

Pleurophysus, 410

Pleurophysus hydrostaticus, 410

pleurops, Arius, 39

pleurops, Synodontis, 319

pleurostigma, Pimelodus, 13

pleurotaenia, Farlowella, 237

pleurotaenia, Pangasius, 328

pleurotaenia, Pseudolais, 328

Plicofollis, 32, 51

Plicofollis argyropleuron, 51
Plicofollis crossocheilos, 52

Plicofollis dussumieri, 52

Plicofollis nella, 52

Plicofollis platystomus, 52

Plicofollis polystaphylodon, 52

Plicofollis tenuispinis, 52

Plicofollis tonggol, 52

pliocaenicus, Silurus, 432

Pliosilurus, 430

Pliosilurus primus, 430

Plotoseus, 350

Plotoseus ikapor, 350

Plotosichthyoidei, 345

Plotosidae, 345

Plotosis, 350

Plotosius, 350

Plotosus, 345, 350

Plotosus (Clarias) hamiltonii, 140

Plotosus (Tandanus) tandanus, 351, 352

Plotosus abbreviatus, 351

Plotosus albilabris, 349

Plotosus argenteus, 345

Plotosus brevibarbus, 351

Plotosus caesius, 350

Plotosus canius, 350

Plotosus castaneoüdes, 351

Plotosus castaneus, 351

Plotosus elongatus, 346

Plotosus fisadoha, 350

Plotosus flavolineatus, 345

Plotosus horridus, 350

Plotosus laticeps, 345

Plotosus limbatus, 108, 346, 350

Plotosus lineatus, 350

Plotosus lineatus, 351, 352

Plotosus macrocephalus, 346

Plotosus macrophthalmus, 349

Plotosus malignus, 351

Plotosus marginatus, 350

Plotosus megastomus, 345, 346

Plotosus microceps, 346

Plotosus multiradiatus, 350

Plotosus nigricans, 352

Plotosus nkunga, 351

Plotosus papuensis, 351

Plotosus thunbergianus, 350

Plotosus unicolor, 350, 352

Plotosus vittatus, 350

Plotosus viviparus, 350

plumbea, Cetopsis, 133

plumbeus, Cetopsis, 132, 133

plumbeus, Trichomycterus, 421

plumbeus motatanensis,

Pseudocetopsis, 132

plumbeus orinoco, Pseudocetopsis, 132

pluriradiatus, Hemibagrus, 90

pluriradiatus, Macrones, 90 pluriradiatus, Ompok, 373

poecilopterus, Bagrus, 93

poecilopterus, Leiocassis, 93

poecilus, Aspidoras, 109

poecilus, Microglanis, 354

poensis, Clarias, 141

poeyanus, Trichomycterus, 423

poeyi, Rhamdia, 199

Pogonopoma, 285

Pogonopoma obscurum, 285

Pogonopoma parahybae, 285

Pogonopoma wertheimeri, 285

Pogonopomoides, 285

poilanei, Pareuchiloglanis, 398, 399

pojeri, Chiloglanis, 307

polli, Chrysichthys, 159

polli, Malapterurus, 302

polli, Microsynodontis, 310

polli, Synodontis, 319

polycaulus, Pimelodus, 198

polygramma, Doras, 168

polyochrus, Parotocinclus, 283

polyodon, Chiloglanis, 307

polyodon, Synodontis, 319

polypogon, Chiloglanis, 307

polystaphylodon, Arius, 52

polystaphylodon, Plicofollis, 52

polystictus, Agenciosus, 70

polystictus, Ageneisus, 70

polystictus, Ageneiosus, 70

polystictus, Corydoras, 123

polystigma, Synodontis, 319

polyuranodon, Pangasius, 325, 327

ponderosus, Pangasius, 327

poonaensis, Glyptothorax conirostre, 395

popoi, Plecostomus, 185

Porcinae, 81

Porcus, 81, 83

Porcus auratus, 155

Porcus bayad, 83

Porcus bayad macropterus, 83

Porochilus, 351

Porochilus meraukensis, 351

Porochilus obbesi, 351

Porochilus rendahli, 345

porosus, Silurus, 95

porosus, Trachycorystes, 81

porphyreus, Ageniosus, 70

pospisili, Cochliodon, 254

Potamarius, 53

Potamarius grandoculis, 53

Potamarius izabalensis, 53

Potamarius nelsoni, 53

potaroensis, Corydoras, 123

potaroensis, Myoglanis, 187

potaroënsis, Myoglanis, 187

potschi, Trichomycterus, 421

pradensis, Trichomycterus, 422 
praecox, Pseudocetopsis, 133 praecox, Denticetopsis, 133 praeliorum, Astroblepus, 66 praelongus, Hassar, 173 praelongus, Leptodoras, 173 Prajadhipokia, 94 Prajadhipokia rex, 94 prashadi, Akysis, 14 prashadi, Glyptothorax, 393 prata, Parotocinclus, 283 prateri, Clupisoma, 357 pratti, Macrones, 102 pratti, Pseudobagrus, 102 prenadilla, Brontes, 63, 66 prenadillus, Astroblepus, 66 prentissgrayi, Dinotopteroides, 139, 146 pretoriae, Chiloglanis, 307 prianomus, Rhinodoras, 176 pricei, Ictalurus, 209 pricei, Villarius, 208, 209 Prietella, 215

Prietella lundbergi, 215

Prietella phreatophila, 215

primaevus, Ameiurus, 207

primaevus, Propygidium, 433

primus, Pliosilurus, 430

prionotos, Corydoras, 130

prionotos, Scleromystax, 130

Pristiancistrus, 219

Pristiancistrus eustictus, 219, 221

pristos, Imparfinis, 186

procera, Pimelodella, 194

productus, Chiloglanis, 443

Proeutropiichthys, 361

Proeutropiichthys buchanani, 361

Proeutropiichthys goongwaree, 362

Proeutropiichthys macropthalmos, 362

Proeutropiichthys taakree, 362

Proeutropiichthys taakree burmanicus, 362

Proeutropius, 362

prolatus, Encheloclarias, 150

prolixa, Loricaria, 286

prolixa, Proloricaria, 286

prolixa lentiginosa, Loricaria, 286

Proloricaria, 286

Proloricaria lentiginosa, 286

Proloricaria prolixa, 286

promagdalena, Brachyplatystoma, 331

proops, Bagrus, 54

proops, Ituglanis, 408

proops, Sciades, 54

proöps, Trichomycterus, 408

proops parahybae, Pygidium, 406, 407

Prophagorus, 139

Propimelodus, 340
Propimelodus caesius, 443

Propimelodus eigenmanni, 340

Propseudecheneis, 400

Propseudecheneis tchangi, 400, 401

Propygidium, 403, 433

Propygidium primaevus, 433

prosthistius, Amiurus, 205

proxima, Netuma, 49

proximus, Arius, 48, 49

psammatides, Aspidoras, 110

psammium, Megalonema, 335

psammium, Megalonema

platycephalum, 335

Psammphiletria, 27

Psammphiletria delicata, 27

Psammphiletria nasuta, 27

Pseudacanthicini, 217

Pseudacanthicus, 217, 286

Pseudacanthicus (Lithoxus) fimbriatus, 237

Pseudacanthicus fimbriatus, 236

Pseudacanthicus fordii, 286

Pseudacanthicus histrix, 286

Pseudacanthicus leopardus, 286

Pseudacanthicus serratus, 286

Pseudacanthicus spinosus, 286

Pseudageneiosus, 68

Pseudancistrus, 286

Pseudancistrus atratoênsis, 235

Pseudancistrus barbatus, 287

Pseudancistrus brevispinis, 287

Pseudancistrus carnegiei, 235

Pseudancistrus coquenani, 287

Pseudancistrus depressus, 287

Pseudancistrus genisetiger, 287

Pseudancistrus guentheri, 287

Pseudancistrus longispinis, 287

Pseudancistrus luderwaldti, 280

Pseudancistrus niger, 287

Pseudancistrus nigrescens, 287

Pseudancistrus orinoco, 287

Pseudancistrus papariae, 287

Pseudancistrus pediculatus, 235

Pseudancistrus pediculatus cobrensis, 235

Pseudancistrus sidereus, 288

Pseudancistrus torbesensis, 231, 232

Pseudariodes, 336

Pseudariodes pantherinus, 339

Pseudarius, 34

Pseudarius microcephalus, 35

Pseudarius philippinus, 35

Pseudauchenipterini, 68

Pseudauchenipterus, 68, 75

Pseudauchenipterus affinis, 76

Pseudauchenipterus flavescens, 76

Pseudauchenipterus guppyi, 76

Pseudauchenipterus jequitinhonhae, 76
Pseudauchenipterus nigrolineatus, 76

Pseudauchenipterus nodosus, 76

Pseudecheneidina, 382

Pseudecheneis, 382, 400

Pseudecheneis crassicauda, 400

Pseudecheneis eddsi, 442

Pseudecheneis immaculata, 400

Pseudecheneis immaculatus, 400

Pseudecheneis intermedius, 400

Pseudecheneis paviei, 400

Pseudecheneis serracula, 400

Pseudecheneis stenura, 442

Pseudecheneis sulcata, 400, 442

Pseudecheneis sulcatoides, 400

Pseudecheneis suppaetula, 442

Pseudecheneis sympelvica, 401

Pseudecheneis sympelvicus, 401

Pseudecheneis tchangi, 401

Pseudepapterus, 76

Pseudepapterus cucuhyensis, 76

Pseudepapterus gracilis, 76

Pseudepapterus hasemani, 76

Pseudeutropichthys, 165

Pseudeutropichthys multiradiatus, 165

Pseudeutropius, 356, 357, 362

Pseudeutropius acutirostris, 359

Pseudeutropius atherinoides, 359

Pseudeutropius atherinoides walkeri, 359

Pseudeutropius brachypopterus, 362

Pseudeutropius longimanus, 362

Pseudeutropius megalops, 362

Pseudeutropius mitchelli, 362

Pseudeutropius moolenburghae, 362

Pseudeutropius murius batarensis, 357

Pseudeutropius siamensis, 361

Pseudeutropius verbeekii, 326

Pseudexostoma, 401

Pseudexostoma brachysoma, 401

Pseudexostoma yunnanense, 401

Pseudexostoma yunnanensis

brachysoma, 401

Pseudocanthicus(Lithoxus) fimbriatus, 237

Pseudobagarius, 16

Pseudobagarius alfredi, 16

Pseudobagarius baramensis, 16

Pseudobagarius filifer, 16

Pseudobagarius fuscus, 16

Pseudobagarius hardmani, 16

Pseudobagarius inermis, 16

Pseudobagarius leucorhynchus, 16

Pseudobagarius macronemus, 16

Pseudobagarius meridionalis, 17

Pseudobagarius nitidus, 17

Pseudobagarius pseudobagarius, 17

Pseudobagarius similis, 17

Pseudobagarius sinensis, 17

pseudobagarius, Akysis, 16, 17 
pseudobagarius, Pseudobagarius, 17

Pseudobagrichthys, 82

Pseudobagrichthys macracanthus, 82

Pseudobagrus, 86, 100, 101

Pseudobagrus adiposalis, 101

Pseudobagrus albomarginatus, 101

Pseudobagrus analis, 101

Pseudobagrus aurantiacus, 101, 108

Pseudobagrus brachysoma, 429

Pseudobagrus brevianalis, 101

Pseudobagrus brevicaudatus, 102

Pseudobagrus changi, 103

Pseudobagrus chinensis, 101

Pseudobagrus chryseus, 429

Pseudobagrus emarginatus, 100

Pseudobagrus eupogoides, 107

Pseudobagrus eupogon, 100

Pseudobagrus fangi, 101

Pseudobagrus fui, 107

Pseudobagrus gracilis, 102

Pseudobagrus henryi, 100

Pseudobagrus ichikawai, 86

Pseudobagrus ikiensis, 102

Pseudobagrus intermedius, 100

Pseudobagrus koreanus, 102

Pseudobagrus kyphus, 82

Pseudobagrus medianalis, 102

Pseudobagrus nitidus, 82

Pseudobagrus nudiceps, 100

Pseudobagrus omeihensis, 102

Pseudobagrus ondon, 102

Pseudobagrus ornatus, 92

Pseudobagrus pratti, 102

Pseudobagrus taeniatus, 102

Pseudobagrus taiwanensis, 102

Pseudobagrus tenuis, 102

Pseudobagrus tokiensis, 103

Pseudobagrus trilineatus, 103

Pseudobagrus truncatus, 103

Pseudobagrus ussuriensis, 101

Pseudobagrus virgatus, 101

Pseudobagrus wangi, 108

Pseudobagrus wittenburgii, 108

Pseudobagrus wui, 101

Pseudocallophysus, 332

Pseudocanthicus (Lithoxus) fimbriatus, 237

Pseudocetopsis, 131

Pseudocetopsis baudoênsis, 131

Pseudocetopsis jurubidae, 132

Pseudocetopsis orientale, 130

Pseudocetopsis plumbeus

motatanensis, 132

Pseudocetopsis plumbeus orinoco, 132

Pseudocetopsis praecox, 133

Pseudodoras, 175

pseudogladiolus, Farlowella, 237

Pseudohemiodon, 288
Pseudohemiodon (Planiloricaria) cryptodon, 285

Pseudohemiodon amazonus, 288

Pseudohemiodon apithanos, 288

Pseudohemiodon devincenzii, 288

Pseudohemiodon laminus, 288

Pseudohemiodon laticeps, 288

Pseudohemiodon platycephalus, 288

Pseudohemiodon thorectes, 288

pseudohemiurus, Hypostomus, 259

pseudohemiurus macrophthalmus,

Hypostomus, 256

Pseudohypophthalmus, 333

Pseudolaguvia, 382, 401

Pseudolaguvia ferula, 442

Pseudolaguvia foveolata, 401

Pseudolaguvia inornata, 401

Pseudolaguvia kapuri, 401

Pseudolaguvia muricata, 401

Pseudolaguvia ribeiroi, 401

Pseudolaguvia shawi, 401, 444

Pseudolaguvia tenebricosa, 402

Pseudolaguvia tuberculata, 402

Pseudolais, 328

Pseudolais micronemus, 328

Pseudolais pleurotaenia, 328

Pseudolais tetranema, 328

pseudoleiacanthus, Clarias, 147

Pseudolithoxus, 288

Pseudolithoxus anthrax, 288

Pseudolithoxus dumus, 289

Pseudolithoxus nicoi, 289

Pseudolithoxus tigris, 289

Pseudoloricaria, 289

Pseudoloricaria laeviuscula, 289

Pseudomystus, 103

Pseudomystus bomboides, 103

Pseudomystus breviceps, 103

Pseudomystus carnosus, 103

Pseudomystus flavipinnis, 103

Pseudomystus fumosus, 103

Pseudomystus fuscus, 103

Pseudomystus inornatus, 103

Pseudomystus leiacanthus, 104

Pseudomystus mahakamensis, 104

Pseudomystus moeschii, 104

Pseudomystus myersi, 104

Pseudomystus ornatus, 104

Pseudomystus robustus, 104

Pseudomystus rugosus, 104

Pseudomystus siamensis, 104

Pseudomystus sobrinus, 104

Pseudomystus stenogrammus, 104

Pseudomystus stenomus, 104

Pseudomystus vaillanti, 105

pseudonemacheir, Imparfinis, 186

pseudonieuhofii, Clarias, 147

Pseudopangasius, 325

Pseudopangasius nasutus, 327
Pseudopimelodidae, 352

Pseudopimelodinae, 352

Pseudopimelodus, 352, 355

Pseudopimelodus acanthochirus, 353

Pseudopimelodus Agassizi, 353

Pseudopimelodus albomarginatus, 353

Pseudopimelodus apurensis, 353

Pseudopimelodus bufonius, 355

Pseudopimelodus charus, 355

Pseudopimelodus mangurus, 355

Pseudopimelodus nigricauda, 353

Pseudopimelodus parahybae, 354

Pseudopimelodus pulcher, 355

Pseudopimelodus roosevelti, 355

Pseudopimelodus schultzi, 355

Pseudopimelodus transmontanus, 353

Pseudopimelodus variolosus, 355

Pseudopimelodus villosus, 353

Pseudopimelodus villosus butcheri, 352

Pseudoplatystoma, 340

Pseudoplatystoma corruscans, 341

Pseudoplatystoma fasciatum, 341

Pseudoplatystoma fasciatum

intermedium, 341

Pseudoplatystoma fasciatum nigricans, 341

Pseudoplatystoma fasciatum

reticulatum, 341

Pseudoplatystoma tigrinum, 341

Pseudorhamdia, 336

Pseudorhamdia fur, 337

Pseudorhamdia macronema, 337

Pseudorhamdia piscatrix, 337

Pseudorhamdia vittatus, 195

Pseudorinelepis, 289

Pseudorinelepis genibarbis, 289

Pseudosilurus, 371

Pseudosilurus macropthalmos, 374

pseudosilvinichthys, Trichomycterus, 422

pseudospinosus, Neosilurus, 349

Pseudostegophilus, 411

Pseudostegophilus haemomyzon, 412

Pseudostegophilus nemurus, 412

Pseudostegophilus paulensis, 410

Pseudostegophilus scarificator, 410

Pseudosynodontis, 311

Pseudotatia, 77

Pseudotatia parva, 77

Pseudotocinclus, 289

Pseudotocinclus intermedius, 290

Pseudotocinclus juquiae, 290

Pseudotocinclus parahybae, 290

Pseudotocinclus ribeiroi, 295

Pseudotocinclus tietensis, 290

Pseudotothyris, 290

Pseudotothyris janeirensis, 290

Pseudotothyris obtusa, 290 
Psilichthys, 247

Psilichthys cameroni, 247, 279

psilogaster, Hypoptopoma, 250

Ptedoras angelis, 178

Pterobunocephalus, 62

Pterobunocephalus depressus, 62

Pterobunocephalus dolichurus, 62

Pterocryptis, 367, 375

Pterocryptis anomala, 375

Pterocryptis barakensis, 441

Pterocryptis berdmorei, 376, 377

Pterocryptis bokorensis, 376

Pterocryptis buccata, 376

Pterocryptis burmanensis, 376

Pterocryptis cochinchinensis, 376

Pterocryptis crenula, 376

Pterocryptis cucphuongensis, 376

Pterocryptis furnessi, 376

Pterocryptis gangelica, 375, 376

Pterocryptis inusitata, 377

Pterocryptis taytayensis, 377

Pterocryptis torrentis, 377

Pterocryptis verecunda, 377

Pterocryptis wynaadensis, 377

Pterodoras, 177

Pterodoras angeli, 177, 178

Pterodoras granulosus, 177

Pterodoras rivasi, 177

Pteroglanis, 343, 387

Pteroglanis horai, 387, 390

Pteroglanis manni, 343

Pteronotidae, 180

Pteronotus, 180, 197

Pteropangasius, 328

Pteropsoglanis, 387

Pterosturisoma, 290

Pterosturisoma microps, 290

Pterygophlichtys, 290

Pterygoplichthys, 217, 290

Pterygoplichthys (Ancistrus) parananus, 272

Pterygoplichthys aculeatus, 272

Pterygoplichthys ambrosettii, 291

Pterygoplichthys disjunctivus, 291

Pterygoplichthys etentaculatus, 291

Pterygoplichthys gibbiceps, 291

Pterygoplichthys joselimaianus, 291

Pterygoplichthys juvens, 291

Pterygoplichthys lituratus, 291

Pterygoplichthys multiradiatus, 291

Pterygoplichthys pardalis, 292

Pterygoplichthys parnaibae, 292

Pterygoplichthys punctatus, 292

Pterygoplichthys scrophus, 292

Pterygoplichthys undecimalis, 292

Pterygoplichthys xinguensis, 292

Pterygoplichthys zuliaensis, 292

pubescens, Rhamdia, 201

pucai, Hoffstetterichthys, 30 puganensis, Loricaria, 297

puganensis, Spatuloricaria, 297

pulcher, Amphilius, 23

pulcher, Aneistrus (Hemiancistrus), 234

pulcher, Auchenoglanis, 162

pulcher, Corydoras, 123

pulcher, Clarias, 147

pulchra, Dekeyseria, 234

pulcher, Macrones, 97

pulcher, Mystus, 97

pulcher, Pimelodus

(Pseudopimelodus), 355

pulcher, Pseudopimelodus, 355

pulcher, Synodontis, 319

pulcher ephippiata, Amphilius, 23

pulex, Ammoglanis, 403

pulicaris, Clarias, 142

pullus, Pimelodus, 205

puma, Pimelodus, 205

pumilus, Arius, 386

pumilus, Chiloglanis, 30

punctata, Ailia, 356

punctata, Ailiichthys, 356

punctata, Chaetostomus (Ancistrus)

cirrhosus, 222

punctata, Harttia, 241

punctata, Loricaria, 266, 292

punctata, Steindachneria scripta, 344

punctata, Nangra, 395

punctata, Tatia, 73

punctatissimus, Chaetostomus, 274

punctatissimus, Oligancistrus, 274

punctatissimus, Trichomycterus, 422

punctatum, Glyptosternon, 389

punctatum, Hoplosternum, 127, 128

punctatum, Hypostoma, 220

punctatum, Megalonema, 339

punctatum, Steindachneridion, 344

punctatus, Doras (Corydoras), 167

punctatus, Auchenipterichthys, 71

punctatus, Auchenipterus, 71

punctatus, Auchenoglanis, 163

punctatus, Bagrus, 91

punctatus, Cataphractus, 111, 123

punctatus, Centromochlus, 73

punctatus, Chrysichthys, 159

punctatus, Clarias, 140

punctatus, Corydoras, 123

punctatus, Doras, 167

punctatus, Hemibagrus, 91

punctatus, Henonemus, 406

punctatus, Hypostomus, 220, 259

punctatus, Ictalurus, 208, 209, 211

punctatus, Malapterurus, 302

punctatus, Pachypterus, 358

punctatus, Parauchenoglanis, 163

punctatus, Pimelodus, 339

punctatus, Pterygoplichthys, 266, 292 punctatus, Silurus, 209, 377, 379

punctatus, Stegophilus, 406

punctatus argentina, Corydoras, 122

punctatus sipaliwini, Corydoras, 125

puncticulatus, Arius, 41

punctifer, Mystus, 97

punctifer, Platystoma, 342

punctifer, Synodontis, 319

punctulata, Synodontis, 320

punctulatum piurae, Pygidium, 421

punctulatus, Bagrus, 331, 339, 340

punctulatus, Hemiancistrus, 244

punctulatus, Pimelodus, 216

punctulatus, Synodontis, 320

punctulatus, Trichomycterus, 422

punjabensis, Glyptothorax, 393

punjabensis, Glyptothorax conirostris, 393

purusiensis, Panaqolus, 277

purusiensis, Panaque, 277

pusarum, Hypostomus, 259

pusarum, Plecostomus, 259

Pusichthys, 362

pusillus, Erethistes, 384

pusillus, Pimelodus, 431

pusillus, Trichomycterus, 411

pussilus, Erethistes, 384

Pygidianops, 412

Pygidianops cuao, 412

Pygidianops eigenmanni, 412

Pygidianops magoi, 412

Pygidiidae, 427

Pygidium, 427

Pygidium alternatum, 414

Pygidium alterum, 415

Pygidium angustirostris, 413

Pygidium arleoi, 415

Pygidium atochae, 423

Pygidium banneaui, 415, 420

Pygidium banneaui maracaiboensis, 420

Pygidium barbouri, 415

Pygidium bogotense, 415

Pygidium bomboizanum, 416

Pygidium boylei, 416

Pygidium Burmeisteri, 405

Pygidium caliense, 416

Pygidium chapmani, 417

Pygidium chiltoni, 417

Pygidium conradi, 417

Pygidium davisi, 417

Pygidium dispar, 417

Pygidium dorsostriatum, 417

Pygidium dorsotriatum, 417

Pygidium emanueli emanueli, 418

Pygidium emanueli motatanensis, 421

Pygidium fassli, 418

Pygidium fasslii, 418

Pygidium florense, 414 
Pygidium fuscum, 427

Pygidium gabrieli, 418

Pygidium gracilior, 407

Pygidium guianense, 418

Pygidium guianensis, 418

Pygidium hasemani, 418

Pygidium heterodontum, 418

Pygidium iheringi, 419

Pygidium immaculatum, 419

Pygidium johnsoni, 419

Pygidium latidens, 420

Pygidium latistriatum, 420

Pygidium metae, 407

Pygidium metae guayaberensis, 407

Pygidium migrans, 420

Pygidium mondolfi, 421

Pygidium oroyae, 423

Pygidium paolence, 421

Pygidium paquequerense, 421

Pygidium parkoi, 407

Pygidium proops parahybae, 406, 407

Pygidium punctulatum piurae, 421

Pygidium quechuorum, 423

Pygidium regani, 422

Pygidium reinhardti, 422

Pygidium riojanum, 422

Pygidium romeroi, 423

Pygidium santae-ritae, 423

Pygidium Schmidti, 416

Pygidium septentrionale, 424

Pygidium Spegazzinii, 423

Pygidium spilosoma, 423

Pygidium stawiarski, 423

Pygidium stellatum, 424

Pygidium stramineum, 424

Pygidium straminium, 424

Pygidium striatum, 424

Pygidium taenia transandianum, 424

Pygidium tenue, 424

Pygidium tiraquae, 423

Pygidium totae, 412

Pygidium travassosi, 414

Pygidium triguttatum, 425

Pygidium unicolor, 425

Pygidium venulosum, 425

Pygidium vermiculatum, 425

Pygidium weyrauchi, 425

Pygidium zonatum, 425

pygmaeus, Corydoras, 123

pygmaeus, Silurus, 132

Pylodictis, 215

Pylodictis limosus, 215

Pylodictis olivaris, 215

pyrineusi, Cochliodon, 259

pyrineusi, Hypostomus, 259

Pyxiloricaria, 292

Pyxiloricaria menezesi, 292

Q

quadrensis, Rineloricaria, 295 quadricostatus, Silurus, 122

quadrifilis, Ageneiosus, 78

quadrifilis, Tetranematichthys, 78

quadrimaculatus, Silurus, 200

quadriocellatum, Glyptosternon, 393

quadriocellatus, Glyptothorax, 393

quadriradiatus, Bunocephalus, 60

quadriradiatus, Dysichthys, 60

quadriscutis, Arius, 37

quadriscutis, Aspistor, 37

quadrizonatus, Chasmocranus, 183

quechuorum, Pygidium, 423

quelen, Pimelodus, 200

quelen, Rhamdia, 199

Queleni cuprea, Pimelodus (Rhamdia), 201

Quiritixys, 240

$\mathbf{R}$

rabauti, Corydoras, 123

rabdophorus, Sisor, 402

Rabida, 211

rachovii, Plecostomus, 255

radiata, Netuma, 435

radiatus, Glyptocephalus, 432, 433

radiosus, Entomocorus, 443

radulus, Rhineastes, 430

raimundi, Aspidoras, 110

raimundi, Corydoras, 110

Raja similis, 434

Rama, 105

Rama buchanani, 105

Rama chandramara, 86

Rama rama, 105

rama, Pimelodus, 105

rama, Rama, 105

rambarrani, Brachyrhamdia, 181

rambarrani, Pimelodella, 181

ramentosus, Bagrus (Bagrus), 108

ramentosus, Pelteobagrus, 108

ramirezi, Hoplodoras, 174

ramiroi, Ituglanis, 408

ramosus, Trichomycterus, 422

raninus, Batrochoglanis, 353

raninus, Pimelodus, 352, 353

ranunculus, Ancistrus, 224

rebeli, Synodontis, 320, 323

recavus, Akysis, 14

reddelli, Rhamdia, 202

Reganella, 217, 292

Reganella depressa, 293

Reganellina, 217

regani, Anadoras, 169

regani, Arges, 66

regani, Astroblepus, 66

regani, Doras, 169

regani, Hypostomus, 259

regani, Hemipsilichthys, 280

regani, Leiocassis, 93

regani, Pareiorhaphis, 280 regani, Plecostomus, 259

regani, Pygidium, 422

regani, Rhamdia, 198

regani, Trichomycterus, 422

reinhardti, Bagropsis, 329

reinhardti, Pygidium, 422

reinhardti, Trichomycterus, 422

reinhardtii, Ochmacanthus, 410

Reinhardtii, Stegophilus, 410

reinii, Liobagrus, 19, 20

reisi, Ancistrus, 224

rendahli, Aoria, 108

rendahli, Copidoglanis, 345

rendahli, Neosilurus, 345

rendahli, Pimelodella, 196

rendahli, Porochilus, 345

rengifoi, Astroblepus, 66

resimus, Astephus, 207

resupinata, Synodontis, 320

resupinatus, Synodontis, 320

reticulata, Farlowella, 239

reticulata, Tatia, 73

reticulatum, Glyptosternon, 387

reticulatum, Pseudoplatystoma

fasciatum, 341

reticulatus, Ameiurus, 206

reticulatus, Bagrus, 330, 331

reticulatus, Centromochlus, 73

reticulatus, Chiloglanis, 307

reticulatus, Corydoras, 123

reticulatus, Glyptosternon, 387

retropinna, Arges, 66

retropinnatus, Horiomyzon, 185

retropinnis, Bunocephalus, 59

retropinnis, Trichomycterus, 422

retropinnus, Astroblepus, 66

retuculatus, Glyptosternon, 387

revelatus, Corydoras, 123

rex, Prajadhipokia, 94

reyesi, Pimelodella, 194

reynoldsi, Corydoras, 123

rhabdophorus, Sisor, 402

rhabdostigma, Megalonema, 338

rhabdurus, Pareuchiloglanis, 399

Rhadinoloricaria, 293

Rhadinoloricaria macromystax, 293

rhadinurus, Ompok, 373

rhaeas, Ictalurus, 210

rhaeas, Rhineastes, 210

Rhamdella, 196

Rhamdella aymarae, 196

Rhamdella eriarcha, 196

Rhamdella exsudans, 196

Rhamdella gilli, 196

Rhamdella ignobilis, 196

Rhamdella jenynsii, 196

Rhamdella lemai, 196

Rhamdella leptosoma, 196

Rhamdella longipinnis, 196 
Rhamdella longiuscula, 196

Rhamdella montana, 196

Rhamdella papariae, 197

Rhamdella robinsoni, 197

Rhamdella rusbyi, 197

Rhamdella schultzi, 187

Rhamdella straminea, 196

Rhamdella wolfi, 197

Rhamdia, 197

Rhamdia alfaroi, 199

Rhamdia amatitlanensis, 198

Rhamdia barbata, 201

Rhamdia branneri, 201

Rhamdia branneri voulezi, 201

Rhamdia bransfordii, 201

Rhamdia cabrerae, 198

Rhamdia cyanostigma, 190

Rhamdia depressa, 201

Rhamdia dorsalis, 201

Rhamdia duquei, 202

Rhamdia eigenmanniorum, 191

Rhamdia enfurnada, 197

Rhamdia eriarcha, 196

Rhamdia foina, 197

Rhamdia gilli, 196

Rhamdia guairensis, 198

Rhamdia guasarensis, 197

Rhamdia guatemalensis decolor, 202

Rhamdia guatemalensis muriei, 202

Rhamdia guatemalensis stygaea, 202

Rhamdia heteracantha, 201

Rhamdia holomelas rupununi, 197

Rhamdia humilis, 198

Rhamdia itacaiunas, 198

Rhamdia javanica, 431

Rhamdia jequitinhonha, 198

Rhamdia laluchensis, 198

Rhamdia laticauda, 198, 199, 202

Rhamdia laticauda typhla, 198

Rhamdia laukidi, 199

Rhamdia lehmanni, 202

Rhamdia luigiana, 199

Rhamdia macuspanensis, 199

Rhamdia marthae, 181

Rhamdia micayi, 197

Rhamdia microcephala, 202

Rhamdia microps, 201

Rhamdia minuta, 186

Rhamdia mounseyi, 201

Rhamdia muelleri, 199

Rhamdia nasuta, 201

Rhamdia nicaraguensis, 199

Rhamdia oaxacae, 201

Rhamdia obesa, 199

Rhamdia ortoni, 201

Rhamdia parryi, 199

Rhamdia poeyi, 199

Rhamdia pubescens, 201

Rhamdia quelen, 199
Rhamdia reddelli, 202

Rhamdia regani, 198

Rhamdia riojae, 201

Rhamdia sacrificii, 198

Rhamdia saijaensis, 202

Rhamdia sebae Martyi, 202

Rhamdia tenella, 199

Rhamdia underwoodi, 198

Rhamdia xetequepeque, 202

Rhamdia zongolicensis, 199

Rhamdiae, 180

Rhamdioglanis, 202

Rhamdioglanis frenatus, 202

Rhamdioglanis transfasciatus, 202

Rhamdiopsis, 202

Rhamdiopsis microcephala, 202

Rhamdiopsis moreirai, 202, 203

rhami, Crossoloricaria, 232

rhegma, Mystus, 97

Rheoglanis, 164

Rheoglanis dendrophorus, 164

rheophilus, Amphilius, 23

rheophilus, Pangasius, 328

rheophilus, Sisor, 402

Rhineastes, 207, 430

Rhineastes arcuatus, 207

Rhineastes calvus, 207

Rhineastes grangeri, 432

Rhineastes peltatus, 430

Rhineastes radulus, 430

Rhineastes rhaeas, 210

Rhineastes smithii, 430

Rhinelepis, 217, 293

Rhinelepis Agassizii, 289

Rhinelepis aspera, 293

Rhinelepis levis, 255

Rhinelepis lophophanes, 276

Rhinelepis parahybae, 285

Rhinelepis rudolphi, 281

Rhinelepis strigosa, 293

Rhineloricaria morrowi, 246

Rhineloricaria petleyi, 266

Rhineloricaria wolfei, 247

Rhinobagrus, 92

Rhinobagrus dumerili, 92, 93

Rhinodoras, 178

Rhinodoras amazonum, 170

Rhinodoras boehlkei, 178

Rhinodoras dorbignyi, 178

Rhinodoras prianomus, 176

Rhinodoras teffeanus, 176

Rhinodoras thomersoni, 178

Rhinoglanina, 303

Rhinoglanis, 303, 310

Rhinoglanis typus, 310, 311

Rhinoglanis Vannutellii, 311

Rhinolepis paraguensis, 275

Rhizosomichthys, 412

Rhizosomichthys totae, 412 rhodonotus, Bagrus, 49

rhodopterygius, Bagrus, 95

rhombocephala, Harttia, 242

Rhynchodoras, 178

Rhynchodoras woodsi, 178

Rhynchodoras xingui, 178

ribeirae, Microcambeva, 409

ribeirensis, Neoplecostomus, 274

ribeiroi, Glanidium, 75

ribeiroi, Laguvia, 401

ribeiroi, Pseudolaguvia, 401

ribeiroi, Pseudotocinclus, 295

ribeiroi kapuri, Laguvia, 401

riberae, Astroblepus, 66

ribularis, Silurus, 201

ricardoae, Synodontis, 320

Ricola, 217, 293

Ricola macrops, 293

Ricolina, 217

rigidus, Pimelodus, 339

Rinelepis acanthicus, 218

Rinelepis genibarbis, 289

Rinelepis histrix, 218, 286

Rineloricaria, 217, 244, 293

Rineloricaria aequalicuspis, 293

Rineloricaria cadeae, 293

Rineloricaria castroi, 245

Rineloricaria catamarcensis, 294

Rineloricaria cubataonis, 294

Rineloricaria felipponei, 294

Rineloricaria formosa, 245

Rineloricaria hasemani, 245

Rineloricaria henselii, 294

Rineloricaria heteroptera, 293, 294

Rineloricaria jaraguensis, 294

Rineloricaria kronei, 294

Rineloricaria lanceolata, 246

Rineloricaria latirostris, 294

Rineloricaria lima, 294

Rineloricaria longicauda, 294

Rineloricaria maquinensis, 294

Rineloricaria microlepidogaster, 295

Rineloricaria microlepidota, 295

Rineloricaria misionera, 295

Rineloricaria pareiacantha, 295

Rineloricaria pentamaculata, 295

Rineloricaria quadrensis, 295

Rineloricaria steindachneri, 295

Rineloricaria strigilata, 295

Rineloricaria thrissoceps, 295

Rineloricaria uracantha, 240

Rineloricariina, 217

ringueleti, Hisonotus, 249

riojae, Rhamdia, 201

riojanum, Pygidium, 422

riojanus, Trichomycterus, 422

rios, Pangasius, 328

Rita, 81, 105

Rita Buchanani, 105, 106 
Rita chrysea, 105

Rita gogra, 105

Rita grandiscutata, 105

Rita kuturnee, 105

Rita macracanthus, 106

Rita rita, 106

Rita sacerdotum, 106

rita, Pimelodus, 106

rita, Rita, 106

Ritae, 81

ritoides, Arius, 106

rivasi, Apuredoras, 177

rivasi, Pterodoras, 177

rivularis, Silurus, 201

rivulatus, Trichomycterus, 422

roae, Cetopsidium, 131

robbianus, Synodontis, 320

robecchii, Clarias, 143

robertsi, Ariopsis, 48

robertsi, Arius, 48

robertsi, Synodontis, 320

robineae, Corydoras, 124

robinii, Hypostomus, 259

Robinii, Plecostomus, 261

robinsoni, Rhamdella, 197

robusta, Nangra, 397

robusta, Oxyloricaria, 299

robusta, Pareuchiloglanis, 399

robusta, Sundagagata, 387, 392

robustum, Megalonema, 339

robustum, Sturisoma, 299

robustus, Auchenipterus, 80

robustus, Corydoras, 124

robustus, Ichthaelurus, 210

robustus, Leiocassis, 104

robustus, Neosilurus, 348

robustus, Pareuchiloglanis, 399

robustus, Pseudomystus, 104

roccae, Pimelodella, 194

rochai, Aspidoras, 108, 110

rodriguesi, Glaphyropoma, 405

rogersae, Leptodoras, 173

Rogersi, Pimelodus, 198

roigi, Trichomycterus, 423

romani, Centromochlus, 73

romani, Tatia, 73

romeroi, Pygidium, 423

romeroi, Trichomycterus, 423

roncallii, Farlowella, 240

rondoni, Ageneiosus, 69

rondini, Hypostomus, 259

rondoni, Plecostomus, 259

roosae, Clupisoma, 357

roosevelti, Pseudopimelodus, 355

roraima, Neblinichthys, 273

rosae, Chasmocranus, 183

rosei, Astroblepus, 66

roseopunctatus, Hypostomus, 259

rostrata, Loricaria, 245, 298, 300 rostrata, Otothyris, 276

rostratum, Sturisoma, 300

rostratus, Arius, 55

rostratus, Loricariichthys, 271

rothschildi, Xenocara, 221

rotundatus, Otolithus (Arius), 435

rotundiceps, Gephyroglanis, 28

rotundiceps, Zaireichthys, 28

rotundifrons, Bathyclarias, 136

rotundifrons, Dinotopterus, 136

rousseauxii, Bagrus, 331

rousseauxii, Brachyplatystoma, 331

royauxi, Euchilichthys, 309

royeroi, Denticetopsis, 133

ruandae, Synodontis, 320

rubermentus, Glyptothorax, 394

rudis, Diplomystes, 435

rudolphi, Pareiorhina, 281

rudolphi, Pimelodella, 194

rudolphi, Rhinelepis, 281

rueppelli, Chrysichthys, 159

rufescens, Macrones, 97

rufescens, Mystus, 94, 97

rufigiensis, Synodontis, 320

rugispinis, Arius, 32

rugosa, Farlowella, 239

rugosus, Acrochordonichthys, 13

rugosus, Bunocephalus, 60

rugosus, Liocassis, 104

rugosus, Pimelodus, 13

rugosus, Pseudomystus, 104

rukwaensis, Chiloglanis, 308

rukwaensis, Synodontis, 320

rukwaensis, Synodontis zambezensis, 320

rupestre, Loricaria uracantha, 240

rupestris, Fonchiiichthys, 240

rupununi, Rhamdia holomelas, 197

rusbyi, Rhamdella, 197

Russellii, Wallago, 381

russi, Arius, 57

rutilus, Bagarius, 383

rutschi, Arius, 435

ruziziensis, Chiloglanis, 308

$\mathbf{S}$

sabahensis, Pangasius, 328

sabaji, Peckoltia, 284

sabalo, Arges, 63, 67

sabalo, Astroblepus, 67

sabanus, Hemibagrus, 91

sabanus, Kryptopterus, 370

sabanus, Mystus, 91

sabanus, Ompok, 371

Sacchii, Oxyglanis, 154

Saccobranchus, 151

Saccobranchus microcephalus, 151

Saccobranchus microps, 151

sacerdotum, Rita, 106

Sachsdoras, 177
Sachsdoras apurensis, 177, 178

sacrificii, Rhamdia, 198

Sadleri, Pimelodus, 431

saetiger, Lasiancistrus, 265

sagor, Hexanematichthys, 45

sagor, Pimelodus, 45

sagoroides, Arius, 45

saharsai, Hara, 396

saijaensis, Rhamdia, 202

saisii, Glyptosternum, 393

saisii, Glyptothorax, 393

salae, Clarias, 147

salathei, Bunocephalus, 59

salgadae, Ancistrus, 300

salmacis, Scleromystax, 130

salvini, Pimelodus, 198

sanagaensis, Chiloglanis, 308

sanchesi, Corydoras, 124

sandrae, Cetopsis, 133

sandrae, Euristhmus, 442

sanghensis, Trachyglanis, 28

sanguinea, Vandellia, 427

sanguineus, Silurichthys, 378

sanitwongsei, Pangasius, 328

santaeritae, Trichomycterus, 423

santae-ritae, Pygidium, 423

santanderensis, Astroblepus, 67

santanderensis, Astroblepus cyclopus, 67

sapito, Dupouyichthys, 60

sapo, Pimelodus, 200

saramaccensis, Corydoras, 124

saramaccensis, Hypostomus 259

sarareensis, Corydoras, 124

saravacensis, Leiocassis, 93

saravacensis, Liocassis, 93

sarcodes, Cetopsis, 133

Sarcogenys, 48

Sarcoglanidinae, 403

Sarcoglanis, 403, 412

Sarcoglanis simplex, 412

sardinhai, Chiloglanis, 308

sarmientoi, Stenolicmus, 414

saropterix, Brachyspondylus, 82, 432

Satan, 216

Satan eurystomus, 216

satparanus, Arius, 56

sauli, Denticetopsis, 133, 134

sauteri, Clarias, 148

savorgnani, Atopochilus, 303, 304

sawrockensis, Ameiurus, 206

sawrockensis, Ictalurus, 206

saxicola, Isbrueckerichthys, 441

scabriceps, Bunocephalus, 58, 60

scabriceps, Hypostomus, 260

scabriceps, Plecostomus commersonii, 260

scaphirhyncha, Dekeyseria, 234

scaphirhynchus, Ancistrus, 234 
scaphyceps, Hypostomus, 260 scaphyceps, Plecostomus, 260 scaphyrhynchura, Doumea, 27 scaphyrhynchura, Phractura, 27 scaplyceps, Plecostomus, 260 scarificator, Pseudostegophilus, 410 schall, Silurus, 320

schall, Synodontis, 315, 320

Schilbe, 356, 362

Schilbe angolensis, 363

Schilbe auratus, 364

Schilbe banguelensis, 363

Schilbe bocagii, 363

Schilbe bouvieri, 364

Schilbe brevianalis, 363

Schilbe congensis, 363

Schilbe congolensis, 364

Schilbe djemeri, 363

Schilbe durinii, 363

Schilbe emini, 364

Schilbe grenfelli, 363

Schilbe Hasselquistii, 365

Schilbe intermedius, 363

Schilbe Isidori, 365

Schilbe laticeps, 364

Schilbe mandibularis, 364

Schilbe marmoratus, 364

Schilbe micropogon, 364

Schilbe moebiusii, 364

Schilbe multitaeniatus, 365

Schilbe mystus, 365

Schilbe nyongensis, 365

Schilbe palmeri, 364

Schilbe senegalensis, 364

Schilbe senegalensis fasciata, 364

Schilbe Senegallus, 364

Schilbe steindachneri, 364

Schilbe tumbanus, 365

Schilbe uranoscopus, 362, 365

Schilbe yangambianus, 365

Schilbe zairensis, 366

Schilbeichthys, 357

Schilbeidae, 356

schilbeides, Bagrus, 362, 365

schilbeides, Hemisilurus, 371

schilbeides, Kryptopterus, 371

Schilbeini, 356

Schilbeodes, 211

Schilbeodes gallowayi, 212

Schilbeodes hildebrandi, 213

Schilbeodes marginatus atrorus, 213

Schilbidae, 324, 356, 429

schilboides, Bagrus, 362

schilby, Silurus, 431

Schillee Sykesii, 362

Schizolecis, 295

Schizolecis guntheri, 295

schlegeli, Arius, 56

Schlegelii, Bagrus, 36, 95
Schmidelia graciliformis, 432

Schmidti, Callomystax, 392

Schmidti, Pygidium, 416

schneideri, Silurichthys, 378

Schomburgkii, Davalla, 68, 69

schomburgkii, Chaetostomus, 265

schomburgkii, Lasiancistrus, 265

schoutedeni, Synodontis, 321

schreineri, Hoplosternum, 128

schreitmuelleri, Farlowella, 239

schreitmülleri, Farlowella, 239

schubarti, Imparfinis, 186

schubarti, Nannorhamdia, 186

schultzei, Corydoras, 112

schultzi, Centromochlus, 73

schultzi, Leptorhamdia, 187

schultzi, Pseudopimelodus, 355

schultzi, Rhamdella, 187

schultzi, Zungaro zungaro, 355

Schultzichthys, 413

Schultzichthys bondi, 413

Schultzichthys gracilis, 413

schwartzi, Corydoras, 124

schwartzi surinamensis, Corydoras, 126

schweinfurthi, Fajumia, 428, 429

Sciadeichthys, 53

Sciadeichthys (Selenaspis) walrechti, 53

Sciadeoides, 334

Sciadeops, 53

Sciades, 53

Sciades couma, 53

Sciades dowii, 53

Sciades herzbergii, 53

Sciades marmoratus, 334

Sciades passany, 54

Sciades platypogon, 54

Sciades proops, 54

Sciades troschelii, 53, 54

sciurus, Arius, 35

sclateri, Austroglanis, 81

sclateri, Gephyroglanis, 81

Scleromystax, 112, 129

Scleromystax barbatus, 129

Scleromystax macropterus, 130

Scleromystax prionotos, 130

Scleromystax salmacis, 130

Scleronema, 413

Scleronema angustirostre, 413

Scleronema minutum, 413

Scleronema operculatum, 413

scleronema, Hemisilurus, 368

scleronemus, Ceratoglanis, 368

Scobinancistrus, 296

Scobinancistrus aureatus, 296

Scobinancistrus pariolispos, 296

scolopacina, Loricaria, 237

Scoloplacidae, 366
Scoloplacinae, 366

Scoloplax, 366, 367

Scoloplax dicra, 367

Scoloplax distolothrix, 367

Scoloplax dolicholophia, 367

Scoloplax empousa, 367

scolymus, Lasiancistrus, 265

scopularia, Squaliforma, 298

scopularius, Plecostomus, 298

Scorpiodoras, 178

Scorpiodoras heckelii, 178

scripta, Steindachneria, 344

scripta punctata, Steindachneria, 344

scriptum, Steindachneridion, 344

scrophus, Liposarcus, 292

scrophus, Pterygoplichthys, 292

sculpodon, Hypostomus, 260

scutatus, Aelurichthys, 38

sebae, Ageneiosus, 69

sebae, Pimelodus, 197, 200

sebae Martyi, Rhamdia, 202

secundus, Microglanis, 355

seducta, Denticetopsis, 134

seemanni, Ariopsis, 33

seemanni, Arius, 33

seenghala, Platystoma, 107

seenghala, Sperata, 107

seengtee, Mystus, 97

Seengtee, Pimelodus, 97

Selenaspis, 53

Sellonis, Pimelodus, 200

semiaquilus, Corydoras, 124

semicultratus, Sinopangasius, 325, 326

seminuda, Dasyloricaria, 234

seminuda, Loricaria filamentosa, 234

seminudus, Hypostomus, 260

seminudus, Plecostomus, 260

semiscutatus, Corydoras, 125

Senegalensis, Clarias, 140

Senegalensis, Heterobranchus, 150

senegalensis, Schilbe, 364

senegalensis fasciata, Schilbe, 364

Senegallus, Schilbe, 364

sepikensis, Lambertichthys ater, 347

septentrionale, Pygidium, 424

septentrionalis, Acrochordonichthys,

13

septentrionalis, Corydoras, 124

septentrionalis, Stegophilus, 414

Septobranchus, 42

Septobranchus johannae, 42

seraoi, Eutropius, 363

Serdicensis, Silurus, 432

sericeum, Chaetostoma, 230

sericeus, Chaetostomus, 230

serpentis, Synodontis, 321

serracanthus, Ameiurus, 206

serracanthus, Ictalurus, 206

serracula, Pseudecheneis, 400 
serralabium, Callichthys, 111

serrata, Hara, 396

serrata, Pimelodella, 194

serrata, Synodontis, 321

serratum, Amblyceps, 18

serratus, Arius, 49

serratus, Corydoras, 124

serratus, Hara, 396

serratus, Hypostomus, 286

serratus, Pseudacanthicus, 286

serratus, Synodontis, 311, 321

serratus tanganaicae, Synodontis, 322

setifera, Loricaria, 268

setiger, Oreoglanis, 398

setosus, Chaetostomus, 235

setosus, Dolichancistrus, 235

seussi, Corydoras, 124

sexcirrhis, Aspredo, 61

sexpapilostoma, Hoplomyzon, 61

sextentaculatus, Heterobranchus, 197, 200

sexualis, Gagata, 386

sharavatiensis, Batasio, 86

sharpii, Chrysichthys, 159

shawi, Laguvia, 395, 402

shawi, Pseudolaguvia, 401, 444

shermani, Cetopsorhamdia, 182

shirensis, Malapterurus, 302

shirui, Hoplosternum, 128

shuwaiensis, Bagrus, 84

sialis, Noturus, 213

siamensis, Glyptothorax, 393

siamensis, Hemipimelodus, 41

siamensis, Liocassis, 104

siamensis, Oreoglanis, 397, 398

siamensis, Pangasius, 327

siamensis, Platytropius, 361

siamensis, Pseudeutropius, 361

siamensis, Pseudomystus, 104

sianenna, Chrysichthys, 159

sichuanensis, Pareuchiloglanis, 399

sicuephorus, Aspredo, 58

sicula, Erethistoides, 384

sicyephorus, Aspredo, 58

Sieboldii, Bagrus, 90

sifontesi, Oxydoras, 176

Silondia, 366

silondia, Silonia, 366

Silonia, 366

Silonia childreni, 366

Silonia silondia, 366

Silonopangasius, 366

Silundia, 366

Silundia gangetica, 366

Silundia Sykesii, 366

Siluranodon, 356, 366

Siluranodon auritus, 366

Siluranodontinae, 356

Silurichthys, 377
Silurichthys australis, 348

Silurichthys basilewskii, 381

Silurichthys berdmorei, 376

Silurichthys citatus, 377

Silurichthys gibbiceps, 377

Silurichthys hasseltii, 377

Silurichthys indragiriensis, 378

Silurichthys leucopodus, 378

Silurichthys marmoratus, 378

Silurichthys phaiosoma, 378

Silurichthys sanguineus, 378

Silurichthys schneideri, 378

Siluridae, 367

Silurodes, 371

Silurodes macronema, 372

Silurodon, 380

Silurodon hexanema, 380, 381

siluroides, Ompok, 371

Siluroïdes, 367

silurö̈des, Ompok, 373

Silurus, 375, 378

Silurus (?)gaudryi, 432

Silurus (Acanthonotus) cuvieri, 356

Silurus (Callichrus) affinis, 374

Silurus (Callichrus) erythrogaster, 373

Silurus (Callichrus) immaculatus, 374

Silurus (Callichrus) macrostomus, 381

Silurus (Callichrus) nebulosus, 374

Silurus (Callichrus) vittatus, 372

Silurus (Clupisoma) argentata, 357

Silurus (Heterobranchus) gariepinus, 143

Silurus (Parasilurus) aristotelis, 378

Silurus (Pimelodus) nigrescens, 210

Silurus 11-radiatus, 335

Silurus 12-radiatus, 177

Silurus 16-radiatus, 44

Silurus 38-radiatus, 70

Silurus 7-radiatus, 122

Silurus 9-radiatus, 201

Silurus afghana, 376

Silurus altus, 381

Silurus anguillaris, 138, 140, 143

Silurus anostomus, 374

Silurus apogon, 374

Silurus arab, 351

Silurus argentinus, 209

Silurus aristotelis, 378

Silurus armatus, 177

Silurus ascita, 54

Silurus asotus, 378, 381

Silurus aspredo, 58

Silurus Atherinoides, 359

Silurus athu, 380

Silurus attu, 380

Silurus auritus, 366

Silurus bagre, 37

Silurus bajad, 83

Silurus batrachus, 140
Silurus bedfordi, 379

Silurus bicirrhis, 369

Silurus bimaculatus, 372

Silurus biserratus, 151

Silurus biwaensis, 379

Silurus boalis, 380

Silurus burmanensis, 376

Silurus callarias, 313

Silurus callichthys, 110

Silurus calvarius, 99, 100

Silurus canio, 373, 374

Silurus carinatus, 170, 171

Silurus cataphractus, 167

Silurus catus, 204

Silurus cacutiens, 131

Silurus Cerulescens, 207, 208, 211

Silurus cerulescens melanurus, 209

Silurus cerulescens var. melanurus, 209

Silurus chantrei, 379

Silurus chechra, 374

Silurus chilensis, 165

Silurus cinereus, 379

Silurus clarias, 311, 313, 321, 336, 337

Silurus Cochinchinensis, 376

Silurus coecutiens, 131, 132

Silurus congensis, 362, 363

Silurus costatus, 176

Silurus cous, 31, 387, 389

Silurus cryptopterus, 369

Silurus cucphuongensis, 376

Silurus cupreus, 215

Silurus dauuricus, 379

Silurus docmak, 84

Silurus duanensis, 379

Silurus duda, 374

Silurus dukai, 377

Silurus Egertoni, 56

Silurus electricus, 301

Silurus fasciatus, 340, 341

Silurus felis, 33

Silurus ferox, 431

Silurus fossilis, 151

Silurus galeatus, 79

Silurus garua, 357

Silurus gilberti, 375, 376

Silurus glanis, 378, 379

Silurus glanis aralensis, 379

Silurus glanis atavus, 382

Silurus goae, 374

Silurus grahami, 379

Silurus gurgu, 431

Silurus gyrinus, 211, 213, 214

Silurus hemioliopterus, 336

Silurus Herzbergii, 53

Silurus hexadactylus, 61

Silurus hexapterus, 371

Silurus hypophthalmus, 372

Silurus ichneumon, 431 
Silurus imberbis, 70, 379

Silurus indicus, 374

Silurus inermis, 69, 379

Silurus japonicus, 378, 379

Silurus laïs, 370

Silurus lamghur, 373

Silurus lanzhouensis, 380

Silurus laticeps, 151

Silurus leptonema, 374

Silurus lima, 342

Silurus limosus, 215

Silurus limpok, 370

Silurus lineatus, 350, 351

Silurus lithophilus, 380

Silurus lividus, 204, 205

Silurus lividus Fuscatus, 205

Silurus lividus var. Fuscatus, 205

Silurus luvur, 431

Silurus macrocephalus, 341

Silurus macronema, 371, 372

Silurus maculatus, 35, 207, 209

Silurus maculatus Erythroptera, 209

Silurus maculatus var. Erythroptera, 209

Silurus Malabaricus, 372

Silurus mangrullo, 341

Silurus marinus, 37, 38, 44

Silurus melas, 205

Silurus mento, 380

Silurus meridionalis, 380

Silurus microcephalus, 374

Silurus microdorsalis, 380

Silurus micronemus, 375

Silurus micropogon, 374

Silurus militaris, 51, 68, 69

Silurus minutus, 431

Silurus mollis, 214

Silurus mononema, 370

Silurus morehensis, 376

Silurus Mülleri, 380, 381

Silurus muticus, 337

Silurus Mysoricus, 374

Silurus mystus, 362, 365

Silurus nebulosus, 215

Silurus nodosus, 75, 76

Silurus ocellatus, 35

Silurus olivaris, 215

Silurus pabda, 371, 372

Silurus pabo, 373

Silurus palembangensis, 369, 370

Silurus pallidus, 210

Silurus pallidus Lateralis, 210

Silurus pallidus Leucoptera, 210

Silurus pallidus Marginatus, 210

Silurus pallidus var. Lateralis, 210

Silurus pallidus var. Leucoptera, 210

Silurus pallidus var. Marginatus, 210

Silurus parkeri, 36

Silurus pati, 335
Silurus pelusius, 96

Silurus phaiosoma, 377, 378

Silurus phalacronotus, 374, 375

Silurus pliocaenicus, 432

Silurus porosus, 95

Silurus punctatus, 209, 377, 379

Silurus pygmaeus, 132

Silurus quadricostatus, 122

Silurus quadrimaculatus, 200

Silurus ribularis, 201

Silurus rivularis, 201

Silurus schall, 320

Silurus schilbe niloticus, 431

Silurus schilby, 431

Silurus Serdicensis, 432

Silurus silurus, 379

Silurus sinensis, 375, 376, 381

Silurus singio, 151

Silurus soldatovi, 380

Silurus soldatovi meridionalis, 380

Silurus stenocephalus, 432

Silurus torrentis, 377

Silurus triostegus, 380

Silurus undecimalis, 431

Silurus unitius, 350

Silurus Vaillantii, 331

Silurus verrucosus, 60

Silurus viscosus, 215

Silurus vittatus, 98

Silurus wallagoo, 381

Silurus wynaadensis, 375, 377

Silurus Xanthocephalus, 207

Silurus xanthosteus, 379

silurus, Silurus, 379

Silvaichthys, 76

Silvaichthys aguilerae, 76

silvasantosi, Steindachneridion, 344

silviae, Glyptothorax, 393

Silvinichthys, 413

Silvinichthys bortayro, 413

Silvinichthys mendozensis, 413

simeonsi, Clariallabes, 138

similis, Akysis, 17

similis, Corydoras, 124

similis, Leiocassis, 108

similis, Pseudobagarius, 17

similis, Raja, 434

simillima, Loricaria, 269

simios, Hypostomus, 260

simonsi, Galeichthys, 33

simonsii, Arges, 67

simonsii, Astroblepus, 67

simplex, Sarcoglanis, 412

simplex, Tatia, 78

simpsonii, Ictalurus, 210

simulatus, Corydoras, 124

Simuldentinae, 303

Sindensis, Callichrous, 373

sindensis, Ompok, 373 sinesis, Akysis, 17

sinense, Clupisoma, 358

sinense, Glyptosternum, 393

sinensis, Akysis, 17

sinensis, Arius, 55

sinensis, Bagrus, 108

sinensis, Cranoglanis, 164, 165

sinensis, Euchiloglanis, 399

sinensis, Glyptothorax, 393

sinensis, Macrones, 165

sinensis, Pareuchiloglanis, 399

sinensis, Platytropius, 358

sinensis, Pseudobagarius, 17

sinensis, Silurus, 375, 376, 381

sinensis, Tachysurus, 431

singaringan, Bagrus, 97

singaringan, Mystus, 97

Sinopangasius, 325

Sinopangasius semicultratus, 325, 326

sinyanensis, Leiocassis, 108

sipaliwini, Bunocephalus amaurus, 58

sipaliwini, Corydoras, 125

sipaliwini, Corydoras punctatus, 125

sipaliwini, Hypostomus, 260

Sisor, 382, 402

Sisor barakensis, 402

Sisor chennuah, 402

Sisor rabdophorus, 402

Sisor rhabdophorus, 402

Sisor rheophilus, 402

Sisor tortosus, 402

Sisorichthyoidei, 382

Sisoridae, 382

Slatinia, 24

Slatinia mongallensis, 24

smithi, Farlowella, 239

smithii, Clarias, 143

smithii, Rhineastes, 430

smithii, Synodontis, 321

smiti, Synodontis, 321

sneiderni, Hemiloricaria, 246

sneiderni, Loricaria, 246

snethlageae, Ancistrus, 283, 285

snethlageae, Peckoltia, 285

sobrinus, Pseudomystus, 104

Socnopaea, 430

Socnopaea grandis, 430

Socnopaea horai, 431

sodalis, Corydoras, 125

soldatovi, Silurus, 380

soldatovi meridionalis, Silurus, 380

solidus, Arius, 39

solidus, Brustiarius, 39

soloni, Synodontis, 321

solox, Corydoras, 125

somalensis, Ailia, 360

somalensis, Parailia, 360

somalensis tanensis, Physailia, 360

somereni, Chiloglanis, 308 
somnians, Heptapterus, 189

somnians, Phenacorhamdia, 189

sona, Hemiarius, 45

sona, Pimelodus, 45

sondaicus, Bagrus, 45

songdaensis, Pareuchiloglanis, 400

songmaensis, Pareuchiloglanis, 400

soniae, Hypostomus, 260

Sophiancistrus, 283

sorex, Synodontis, 321

Sorubim, 281, 342

Sorubim caparary, 341

Sorubim cuspicaudus, 342

Sorubim elongatus, 342

Sorubim infraoculare, 342

Sorubim jandia, 342, 343

Sorubim latirostris, 342

Sorubim lima, 342

Sorubim maniradii, 342

Sorubim Pirauáca, 343

Sorubim trigonocephalus, 342

Sorubimichthys, 342

Sorubimichthys ortoni, 343

Sorubimichthys planiceps, 343

Sorubinae, 329

Sorubium, 329, 342

Sosia, 12

Sosia chamaeleon, 12, 13

Sosia chamaeleon pallida, 13

Sosia chamaeleon var. pallida, 13

Sovichthys, 332

Sovichthys abuelo, 332

sovichthys, Chaetostoma, 230

sovichthys, Chaetostoma anomala, 230

spatula, Arius, 42, 43

spatula, Cochlefelis, 43

spatula, Platystoma, 343

Spatuloricaria, 296

Spatuloricaria atratoensis, 296

Spatuloricaria caquetae, 296

Spatuloricaria curvispina, 296

Spatuloricaria euacanthagenys, 296

Spatuloricaria evansii, 296

Spatuloricaria fimbriata, 297

Spatuloricaria gymnogaster, 297

Spatuloricaria lagoichthys, 297

Spatuloricaria nudiventris, 297

Spatuloricaria phelpsi, 296, 297

Spatuloricaria puganensis, 297

spectabile, Nannoptopoma, 272

spectabilis, Corydoras. 125

spectabilis, Otocinclus, 272, 273

Spectracanthicina, 217

Spectracanthicus, 217, 297

Spectracanthicus murinus, 297

spectrum, Aspredo, 61

spectrum, Glyptothorax, 393

Spegazzinii, Pimelodus, 335

Spegazzinii, Pygidium, 423 spegazzinii, Trichomycterus, 423

spelaea, Pimelodella, 195

spelaeus, Trichomycterus, 423

Sperata, 106

Sperata acicularis, 106

Sperata aor, 106

Sperata aorella, 107

Sperata sarwari, 107

Sperata seenghala, 107

spilomma, Hemiancistrus, 244

spilopterus, Hemibagrus, 91

spilosoma, Parotocinclus, 283

spilosoma, Plecostomus, 283

spilosoma, Pygidium, 423

spilosoma, Trichomycterus, 423

spilotus, Aspidoras, 110

spilurus, Batasio, 442

spilurus, Corydoras, 125

spilurus, Parotocinclus, 283

spilurus, Plecostomus, 283

spiniger, Plecostomus, 252

spinipectoralis, Mystus, 97

spiniserrata, Parailia, 360

spinosae, Loricaria, 286

spinosissimus, Acanthodoras, 168

spinosissimus, Doras, 168

spinosissimus, Hemiancistrus, 244

spinosissima, Isorineloricaria, 263

spinosissimus, Plecostomus, 263

spinosus, Ancistrus, 224

spinosus, Hypostomus, 286

spinosus, Pseudacanthicus, 286

spixii, Cathorops, 40

Spixii, Cetopsis, 131

Spixii, Hypophthalmus, 333

Spixii, Loricaria, 270

spixii, Pimelodus, 40

splendens, Brochis, 125

splendens, Callichthys, 125

splendens, Corydoras, 125

splendens, Hemipsilichthys, 280

splendens, Pareiorhaphis, 280

spodius, Ictalurus, 211

spurrellii, Imparfinis, 186

spurrellii, Nannorhamdia, 185, 186

Squaliforma, 297

Squaliforma annae, 297

Squaliforma biseriata, 297

Squaliforma emarginata, 297

Squaliforma gomesi, 298

Squaliforma horrida, 298

Squaliforma phrixosoma, 298

Squaliforma scopularia, 298

Squaliforma squalina, 298

Squaliforma tenuicauda, 298

Squaliforma tenuis, 298

Squaliforma villarsi, 298

Squaliforma virescens, 298

squalina, Squaliforma, 298 squalinum, Hypostoma, 298

squalus, Arius, 166

stanauli, Noturus, 214, 442

stanneus, Galeichthys, 49

stannii, Chaetostoma, 230

Stannii, Chaetostomus, 230

stappersii, Chrysichthys, 159

stappersii, Clarias, 147

starnesi, Cetopsis, 133

stauroforus, Arius, 32

stauroforus, Felichthys, 32

Stauroglanis, 413

Stauroglanis gouldingi, 413

stawiarski, Pygidium, 423

stawiarski, Trichomycterus, 423

Stearopterus, 37

Stearopterus bagre, 37

steerei, Taenionema, 330, 331

Stegelichii, Pimelodus, 200

Stegophilina, 403

Stegophilus, 403, 414

Stegophilus insidiosus, 414

Stegophilus intermedius, 405

Stegophilus macrops, 406

Stegophilus maculatus, 410

Stegophilus nemurus, 412

Stegophilus panzeri, 414

Stegophilus passarellii, 406

Stegophilus punctatus, 406

Stegophilus Reinhardtii, 410

Stegophilus septentrionalis, 414

steinbachi, Ixinandria, 263

Steinbachi, Loricaria, 263

steindachneri, Cathorops, 40, 48

Steindachneri, Centromochlus, 73

steindachneri, Corydoras, 125

steindachneri, Glyptosternum, 393

steindachneri, Glyptothorax, 393

steindachneri, Hemipsilichthys, 280

steindachneri, Hypoptopoma, 250

steindachneri, Loricaria, 295

Steindachneri, Oxydoras, 179

steindachneri, Opsodoras, 169

steindachneri, Pareiorhaphis, 280

steindachneri, Parotocinclus, 283

steindachneri, Pimelodella, 195

steindachneri, Rineloricaria, 295

steindachneri, Schilbe, 364

steindachneri, Synodontis, 321

steindachneri, Tachysurus, 40

steindachneri, Trachydoras, 169

Steindachneria, 343

Steindachneria amblyurus, 343

Steindachneria doceana, 343

Steindachneria scripta, 344

Steindachneria scripta punctata, 344

Steindachneridion, 343

Steindachneridion amblyurum, 343

Steindachneridion doceana, 343 
Steindachneridion iheringi, 343

Steindachneridion melanodermatum, 343

Steindachneridion parahybae, 344

Steindachneridion punctatum, 344

Steindachneridion scriptum, 344

Steindachneridion silvasantosi, 344

stellatum, Lepthoplosternum, 128

stellatum, Pygidium, 424

stellatus, Nanobagrus, 98

stellatus, Trichomycterus, 424

stenocephalus, Corydoras, 125

stenocephalus, Osteogeniosus, 51

stenocephalus, Silurus, 432

stenogrammus, Pseudomystus, 104

Stenolicmus, 414

Stenolicmus sarmientoi, 414

stenomus, Bagrus, 103, 104

stenomus, Pseudomystus, 104

stenopeltis, Doras (Oxydoras), 171, 172

stenopeltis, Hemidoras, 172

stenura, Pseudecheneis, 442

stephanus, Pareiorhaphis, 280

sterbai, Corydoras, 125

sternoptychum, Nannoptopoma, 273

stevardii, Hoplosternum, 128

stewarti, Hemiloricaria, 246

stewarti, Heptapterus, 184

stewarti, Loricaria, 247

sthenocephalus, Osteogeniosus, 51

stiassnyae, Malapterurus, 301

stibaros, Lamontichthys, 264

stictonotus, Imparfinis, 186

stictonotus, Nannorhamdia, 186

stigmaticus, Ancistrus, 224

stigmaturus, Mystus, 85

stigmosus, Noturus, 214

stirlingi, Arius, 46

stocki, Glyptothorax, 393

stocki, Lithoxus, 268

Stoličkae, Glyptosternum, 393

stolickae, Glyptothorax, 393

stoliczkae, Exostoma, 387

stomias, Hemipsilichthys, 281

stomias, Pareiorhaphis, 280

Stoneiella, 286

Stoneiella leopardus, 286

Stormi, Hemiarius, 45

Stormii, Cephalocassis, 44, 45

stormii, Hemiarius, 45

straminea, Rhamdella, 196

stramineum, Pygidium, 424

straminium, Pygidium, 424

straminius, Trichomycterus, 424

Strephon, 63

striatulus, Auchenipterus

(Pseudauchenipterus), 80

striatulus, Trachelyopterus, 80 striatum, Pygidium, 424

Striatus, Glyptosternon, 387, 394

striatus, Glyptothorax, 394

striatus, Trichomycterus, 424

stricticassis, Arius, 50

strigata, Tatia, 78

strigaticeps, Hypostomus, 260

strigaticeps, Plecostomus, 260

strigilata, Loricaria, 295

strigilata, Rineloricaria, 295

strigosa, Rhinelepis, 293

strigosus, Acrochordonichthys, 13

stromeri, Fajumia, 429

stroudi, Gelanoglanis, 74

stroumpoulos, Chaetostoma, 444

stuarti, Glyptosternum, 385

stübeli, Arges, 67

Stübelii, Loricaria, 271

Stübelii, Oxydoras, 175

stuebeli, Astroblepus, 67

stuebelii, Loricariichthys, 271

stuebelii, Opsodoras, 175

sturio, Platystoma, 340

sturio, Platystomatichthys, 340

Sturiosoma nigrirostrum, 299

Sturisoma, 298

Sturisoma aureum, 298

Sturisoma barbatum, 299

Sturisoma brevirostre, 299

Sturisoma dariense, 299

Sturisoma festivum, 299

Sturisoma frenatum, 299

Sturisoma guentheri, 299

Sturisoma kneri, 299

Sturisoma lyra, 299

Sturisoma monopelte, 299

Sturisoma nigrirostrum, 299

Sturisoma panamense, 299

Sturisoma robustum, 299

Sturisoma rostratum, 300

Sturisoma tenuirostre, 300

Sturisomatichthys, 300

Sturisomatichthys caquetae, 300

Sturisomatichthys citurensis, 300

Sturisomatichthys leightoni, 300

Sturisomatichthys tamanae, 300

styani, Liobagrus, 20

stygaea, Rhamdia guatemalensis, 202

Stygogenes, 63

Stygogenes guentheri, 64

Stygogenes humboldtii, 64

Suarezi, Incaichthys, 30

subcarinatus, Hypostomus, 260

submarginatus, Clarias, 147

submarginatus, Loricaria, 246

submarginatus thysvillensis, Clarias

(Allabenchelys), 141

subrostratus, Arius, 35

subteres, Kronichthys, 263 subviridis, Hemiancistrus, 244

suchus, Bagarius, 383

sufii, Glyptothorax, 394

sufii, Glyptothorax telchitta, 394

sugubii, Liobagrus, 20

sulcata, Pseudecheneis, 400

sulcatoides, Pseudecheneis, 400

sulcatus, Callichthys, 129

sulcatus, Clarias, 147

sulcatus, Glyptosternon, 400

sumatranus, Arius, 36

sumatranus, Bagrus, 36

Sundagagata, 387

Sundagagata robusta, 387, 392

sundaicus, Hexanematichthys, 45

sundanensis, Hemipimelodus, 56

Superglyptothorax, 388

suppaetula, Pseudecheneis, 442

supramollis, Astroblepus, 67

surinamensis, Chasmocranus, 183

surinamensis, Corydoras, 126

surinamensis, Corydoras schwartzi,

126

surinamensis, Harttia, 242

surinamensis, Heptapterus, 183

surinamensis, Hexanematichthys, 33

surinamensis, Hoplosternum

thoracatum, 129

surinamensis, Hypostomus, 260

surinamensis, Lithoxus, 268

surinamensis, Lithoxus (Paralithoxus), 268

sutchi, Pangasius, 325

suttoni, Panaque, 278

suttonorum, Panaque, 278

swierstrai, Chiloglanis, 308

sychri, Corydoras, 126

sykesi, Glyptosternum, 394

sykesi, Glyptothorax, 394

sykesii, Gogrius, 105

Sykesii, Schillee, 362

Sykesii, Silundia, 366

sympelvica, Pseudecheneis, 401

sympelvicus, Pseudecheneis, 401

sympterygium, Heptapterus, 184

Synechoglanis, 207

Synechoglanis Beadlei, 207, 210

synodon, Arius, 166

Synodontes, 311

Synodontini, 303

Synodontis, 303, 311

Synodontis acanthomias, 311

Synodontis acanthoperca, 441

Synodontis afrofischeri, 311

Synodontis Afro-fischeri, 311

Synodontis alberti, 311

Synodontis albolineata, 311

Synodontis albolineatus, 311

Synodontis angelica, 311 
Synodontis angelicus, 311

Synodontis angelicus var. zonatus, 311

Synodontis angelicus zonatus, 311

Synodontis annectens, 312

Synodontis ansorgii, 312

Synodontis arabi, 321

Synodontis arnoulti, 312

Synodontis aterrima, 312

Synodontis aterrimus, 312

Synodontis augierasi, 314

Synodontis bastiani, 312

Synodontis batensoda, 304

Synodontis batesi longispinis, 316

Synodontis batesi var. longispinis, 316

Synodontis batesii, 312

Synodontis brichardi, 312

Synodontis budgetti, 312

Synodontis camelopardalis, 312

Synodontis caudalis, 312

Synodontis caudovittata, 312

Synodontis caudovittatus, 313

Synodontis centralis, 313

Synodontis citernii, 314

Synodontis clarias, 313, 320

Synodontis colyeri, 318

Synodontis comoensis, 313

Synodontis congica, 313

Synodontis congicus, 313

Synodontis contracta, 313

Synodontis contractus, 313

Synodontis courteti, 313

Synodontis cuangoana, 313

Synodontis cuangoanus, 313

Synodontis dageti, 312

Synodontis davidi, 313

Synodontis decora, 314

Synodontis decorus, 314

Synodontis dekimpei, 314

Synodontis depauwi, 314

Synodontis dhonti, 314

Synodontis dorsomaculata, 314

Synodontis dorsomaculatus, 314

Synodontis eburneensis, 312

Synodontis euptera, 314

Synodontis eupterus, 314

Synodontis eurystomus, 305, 319

Synodontis fascipinna, 314

Synodontis filamentosa, 314

Synodontis filamentosus, 314

Synodontis flavitaeniata, 314

Synodontis flavitaeniatus, 314

Synodontis frontosa, 314

Synodontis frontosus, 314

Synodontis fuelleborni, 315

Synodontis galinae, 323

Synodontis gambiensis, 315

Synodontis gambiensis latifrons, 315

Synodontis geledensis, 315

Synodontis gobroni, 315
Synodontis grandiops, 445

Synodontis granulosa, 315

Synodontis granulosus, 315

Synodontis greshoffi, 315

Synodontis guentheri, 309

Synodontis guttata, 315

Synodontis guttatus, 315

Synodontis haugi, 315

Synodontis Hollyi, 323

Synodontis Hollyi ntemensis, 322

Synodontis holopercnus, 315

Synodontis humeratus, 321

Synodontis ilebrevis, 445

Synodontis irsacae, 314

Synodontis ituriensis, 315

Synodontis iturii, 315

Synodontis jallae, 316

Synodontis katangae, 315

Synodontis khartoumensis, 316

Synodontis koensis, 316

Synodontis labeo, 323

Synodontis lacustricolus, 322

Synodontis laessoei, 316

Synodontis leopardina, 316

Synodontis leopardinus, 316

Synodontis leoparda, 316

Synodontis leopardus, 316

Synodontis levequei, 316

Synodontis longirostris, 316

Synodontis longispinis, 316

Synodontis loppei, 318

Synodontis lucipinnis, 445

Synodontis lufirae, 316

Synodontis macrepipterus, 314

Synodontis macrodon, 313

Synodontis macrophthalma, 316

Synodontis macrophthalmus, 316

Synodontis macrops, 316

Synodontis macrostigma, 317

Synodontis macrostoma, 317

Synodontis maculatus, 318

Synodontis maculipinna, 320

Synodontis maculosus, 311, 318, 321

Synodontis manni, 317

Synodontis marmorata, 317

Synodontis marmoratus, 317

Synodontis marmoratus truncatus, 317

Synodontis matthesi, 317

Synodontis melanoptera, 317

Synodontis melanopterus, 317

Synodontis melanostictus, 318

Synodontis melanostictus iturii, 315

Synodontis melanostictus var. iturii, 315

Synodontis multimaculata, 317

Synodontis multimaculatus, 317

Synodontis multipunctata, 317

Synodontis multipunctatus, 317

Synodontis nebulosa, 317
Synodontis nebulosus, 317

Synodontis nigrita, 317

Synodontis nigriventris, 318

Synodontis nigromaculata, 318

Synodontis nigromaculatus, 318

Synodontis njassae, 318

Synodontis notata, 318

Synodontis notatus, 318

Synodontis notatus binotata, 318

Synodontis notatus ocellatus, 318

Synodontis notatus var. binotata, 318

Synodontis notatus var. ocellatus, 318

Synodontis nummifer, 318

Synodontis obesus, 318, 323

Synodontis ocellifer, 318

Synodontis omias, 319

Synodontis ornatipinnis, 319

Synodontis ornatissima, 319

Synodontis ornatissimus, 319

Synodontis ornatus, 317, 319

Synodontis ovidius, 316

Synodontis pantherinus, 315

Synodontis pardalis, 319

Synodontis petricola, 319

Synodontis pfefferi, 311

Synodontis pleurops, 319

Synodontis polli, 319

Synodontis polyodon, 319

Synodontis polystigma, 319

Synodontis pulcher, 319

Synodontis punctifer, 319

Synodontis punctulata, 320

Synodontis punctulatus, 320

Synodontis rebeli, 320, 323

Synodontis resupinata, 320

Synodontis resupinatus, 320

Synodontis ricardoae, 320

Synodontis robbianus, 320

Synodontis robertsi, 320

Synodontis ruandae, 320

Synodontis rufigiensis, 320

Synodontis rukwaensis, 320

Synodontis schall, 315, 320

Synodontis schoutedeni, 321

Synodontis serpentis, 321

Synodontis serrata, 321

Synodontis serratus, 311, 321

Synodontis serratus tanganaicae, 322

Synodontis smithii, 321

Synodontis smiti, 321

Synodontis soloni, 321

Synodontis sorex, 321

Synodontis steindachneri, 321

Synodontis tanganaicae, 321

Synodontis tanganyicae, 322

Synodontis tenuis, 321

Synodontis tessmanni, 322

Synodontis thamalakanensis, 322

Synodontis tholloni, 311 
Synodontis thomasi, 313

Synodontis thysi, 322

Synodontis tourei, 322

Synodontis unicolor, 322

Synodontis vaillanti, 323

Synodontis vanderwaali, 322

Synodontis velifer, 322

Synodontis vermiculata, 322

Synodontis vermiculatus, 322

Synodontis victoriae, 322

Synodontis violacea, 322

Synodontis violaceus, 322

Synodontis vittatus, 314

Synodontis voltae, 323

Synodontis wamiensis, 320

Synodontis waterloti, 323

Synodontis werneri, 311

Synodontis woosnami, 323

Synodontis xiphias, 323

Synodontis zambesensis, 323

Synodontis zambezensis, 323

Synodontis zambezensis rukwaensis, 320

Synodontis zanzibarica, 323

Synodontis zanzibaricus, 316, 320, 323

synodontis, Pimelodus, 313

Syriacus, Clarias, 143

$\mathbf{T}$

taakree, Hypophthalmus, 362

taakree, Proeutropiichthys, 362

taakree burmanicus,

Proeutropiichthys, 362

tachiraense, Chaetostoma, 230

tachiraensis, Chaetostoma, 230

Tachisurus agassizii, 39

Tachisurus gulosus, 40

Tachisurus jordani, 33

Tachisurus lentiginosus, 33

Tachisurus longicehpalus, 39

Tachisurus longicephalus, 39

Tachisurus upsulonophorus, 44 tachisurus, Pimelodus, 431

Tachysurus, 48, 431

Tachysurus (Pararius) berneyi, 47

Tachysurus (Pararius) godfreyi, 45

Tachysurus atroplumbeus, 32

Tachysurus broadbenti, 51

Tachysurus crassus, 434, 435

Tachysurus emmelane, 40

Tachysurus equatorialis, 40

Tachysurus evermanni, 40

Tachysurus landanensis, 57

Tachysurus liropus, 41

Tachysurus machadoi, 44

Tachysurus oblongus, 435

Tachysurus planus, 434, 435

Tachysurus sinensis, 431

Tachysurus steindachneri, 40

taczanowskii, Arges, 67 taczanowskii, Astroblepus, 67

taczanowskii, Chaetostoma, 231

Taczanowskii, Chaetostomus, 231

taczanowskii Trichomycterus, 424

taenia, Trichomycterus, 407, 424

taenia transandianum, Pygidium, 424

taeniatus, Macrones (Liocassis), 102

taeniatus, Plecostomus, 255

taeniatus, Pseudobagrus, 102

taeniatus, Trachelyopterichthys, 78

taeniatus, Trachelyopterus, 78

Taenionema, 330

Taenionema steerei, 330, 331

taeniophora, Pimelodella, 195

taeniophorus, Pimelodus

(Pimelodella), 195

taeniops, Trichomycterus, 424

taenioptera, Pimelodella, 195

taeniura, Pangasius, 326

taimensis, Hisonotus, 249

taimensis, Microlepidogaster, 249

taiosh, Callichthys, 125

taiwanensis, Pseudobagrus, 102

tamanae, Oxyloricaria, 300

tamanae, Sturisomatichthys, 300

tamboensis, Ancistrus, 224

tamoata, Callichthys, 111

Tandanus, 351

Tandanus bostocki, 352

Tandanus coatesi, 348

Tandanus tandanus, 352

tandanus, Tandanus, 352

tanensis, Physailia somalensis, 360

tanganaicae, Synodontis, 321

tanganaicae, Synodontis serratus, 322

tanganicanus, Auchenoglanis

occidentalis, 154

Tanganikallabes, 152

Tanganikallabes mortiauxi, 152

tanganyicae, Synodontis, 322

tanganyikaensis, Malapterurus, 302

tanoensis, Malapterurus, 302

tapanahoniensis, Heptapterus, 184

tapanahoniensis, Hypostomus, 260

tapanahoniensis, Hypostomus

gymnorhynchus, 261

tapatapae, Pimelodella, 195

tapeinopterus, Encheloclarias, 150

tapeinopterus, Heterobranchus, 149, 150

taphorni, Cochliodon, 261

taphorni, Farlowella, 239

taphorni, Hypostomus, 261

taphrophilus, Hemibagrus, 108

tapijara, Hypostomus, 261

tapirape, Otocinclus, 275

tarabinii, Pardiglanis, 163

taroba, Trichomycterus, 424

Tatia, 77
Tatia aulopygia, 77

Tatia boemia, 77

Tatia brunnea, 77

Tatia concolor, 73

Tatia creutzbergi, 77

Tatia dunni, 77

Tatia galaxias, 77

Tatia gyrina, 77

Tatia intermedia, 77

Tatia musaica, 77

Tatia neivai, 77

Tatia perugiae, 73

Tatia punctata, 73

Tatia reticulata, 73

Tatia romani, 73

Tatia simplex, 78

Tatia strigata, 78

taunayi, Ancistrus, 224

Taunayia, 203

Taunayia bifasciata, 203

Taunayia marginata, 203

taurus, Aspidoras, 110

taxistigma, Henonemus, 406

taxistigma, Ochmacanthus, 405, 406

taylori, Arius, 40, 48

taylori, Hemipimelodus, 48

taylori, Neoarius, 48

taylori, Noturus, 214

taytayensis, Hito, 375, 377

taytayensis, Hitoichthys, 375, 377

taytayensis, Pterocryptis, 377

tchadiensis, Auchenoglanis

occidentalis, 154

tchangi, Pseudecheneis, 401

teaguei, Parabranchioica, 410, 411

teaguei, Trachelyopterus, 80

teaguei, Trachycorystes, 80

tectirostris, Chaetostomus, 221

teffeana, Hemiloricaria, 247

teffeana, Loricaria, 247

teffeanus, Rhinodoras, 176

teijsmanni, Clarias, 147

telchita, Pimelodus, 394

telchitta, Glyptothorax, 394

telchitta, Pimelodus, 394

telchitta sufii, Glyptothorax, 394

temminckianus, Bagrus, 55

temminckii, Ancistrus, 224

Temminckii, Bagrus, 55

Temminckii, Hypostomus, 224

tenebricosa, Pseudolaguvia, 402

tenebricus, Leiocassis, 443

tenebrosa, Phenacorhamdia, 189

tenebrosus, Imparfinis, 189

tenella, Rhamdia, 199

tengana, Batasio, 86, 442

tengana, Pimelodus, 86

tengara, Mystus, 97, 98

tengara, Pimelodus, 97 
tenius, Otolithus (Arius), 435

tentaculatus, Lasiancistrus, 265

tenue, Pygidium, 424

tenuicauda, Paraphractura, 26, 27

tenuicauda, Phractura, 27

tenuicauda, Plecostomus, 298

tenuicauda, Squaliforma, 298

tenuifurcatus, Leiocassis, 93

tenuirostre, Sturisoma, 300

tenuirostris, Oxyloricaria, 300

tenuis, Belonoglanis, 24

tenuis, Clarias, 148

tenuis, Heptapterus, 184

tenuis, Hypostomus, 298

tenuis, Macrones (Pseudobagrus), 103

tenuis, Otolithus (Arius), 435

tenuis, Pseudobagrus, 102

tenuis, Squaliforma, 298

tenuis, Synodontis, 321

tenuis, Trichomycterus, 424

tenuispinis, Amblyceps, 18

tenuispinis, Arius, 52, 56

tenuispinis, Plicofollis, 52

ternetzi, Hypostomus, 261

ternetzi, Opsodoras, 175

ternetzi, Plecostomus, 261

ternetzi, Typhlobelus, 426, 427

tessmanni, Synodontis, 322

Tetracamphilius, 27

Tetracamphilius angustifrons, 27

Tetracamphilius clandestinus, 27

Tetracamphilius notatus, 27

Tetracamphilius pectinatus, 27

tetranema, Bathybagrus, 154, 155

tetranema, Pseudolais, 328

Tetranematichthys, 78

Tetranematichthys quadrifilis, 78

Tetranematichthys wallacei, 444

Tetranesodon, 42

Tetranesodon conorhynchus, 42

tetraradiata, Listrura, 408

teugelsi, Chrysichthys, 159

teugelsi, Clariallabes, 138

teugelsi, Malapterurus, 302

teugelsi, Paramphilius, 25

thalassina, Netuma, 49

thalassina jacksonensis, Netuma, 49

thalassinus, Bagrus, 49

thamalakanensis, Synodontis, 322

theobaldi, Chrysichthys, 105

theodorae, Clarias, 139, 147

theresiae, Arges, 67

theresiae, Astroblepus, 67

therezinae, Ageneiosus

(Pseudageneiosus), 69

thienemanni, Clarias, 148

tholloni, Synodontis, 311

thomasi, Chrysichthys delhezi, 156

thomasi, Notoglanidium, 162 thomasi, Synodontis, 313

thomersoni, Rhinodoras, 178

thomsoni, Chaetostoma, 231

thomsoni, Chaetostomus, 231

thonneri, Chrysichthys, 159

thoracata, Megalechis, 129

thoracatum, Hypoptopoma, 249, 250

thoracatum cayennae, Hoplosternum, 128

thoracatum surinamensis,

Hoplosternum, 129

thoracatus, Auchenipterichthys, 71

thoracatus, Auchenipterus, 71

thoracatus, Callichthys, 129

thoracicus, Auchenipterus, 71

thorectes, Pseudohemiodon, 288

Thrichomycterus, 404

Thrichomycterus macraei, 405

thrissoceps, Loricaria, 295

thrissoceps, Rineloricaria, 295

thunberg, Pimelodus, 35

thunbergianus, Plotosus, 350

Thysanocara, 219

thysi, Chrysichthys, 159

thysi, Doumea, 25

thysi, Malapterurus, 301

thysi, Synodontis, 322

thysvillensis, Clarias (Allabenchelys)

submarginatus, 141

tianquanensis, Pareuchiloglanis, 400

tibicen, Aspredinichthys, 58

tibicen, Aspredo, 57, 58

tietensis, Hypostomus, 261

tietensis, Otocinclus

(Microlepidogaster), 290

tietensis, Plecostomus, 261

tietensis, Pseudotocinclus, 290

tigrinum, Brachyplatystoma, 331

tigrinum, Pseudoplatystoma, 341

tigrinus, Batasio, 86

tigrinus, Merodontotus, 330, 331

tigrinus, Trichomycterus, 415

tigris, Lasiancistrus, 288, 289

tigris, Pseudolithoxus, 289

tihoni, Gymnallabes, 152

tihoni, Platyallabes, 152

tilhoi, Gephyroglanis, 155

tiong, Glyptosternum, 395

tiraquae, Pygidium, 423

titcombi, Hatcheria, 405

tocantinensis, Corumbataia, 232

tocantinsensis, Aguarunichthys, 329

tocantinsi, Tridentopsis, 426

Tocantinsia, 78

Tocantinsia depressa, 78

Tocantinsia piresi, 78

tokiensis, Pseudobagrus, 103

tombador, Ancistrus, 225

tonggol, Ariodes, 52 tonggol, Arius, 52

tonggol, Plicofollis, 52

topavae, Hypostomus, 261

topavae, Plecostomus, 261

torbesensis, Cordylancistrus, 232

torbesensis, Pseudancistrus, 231, 232

tordilho, Lepthoplosternum, 129

torobo, Oloplotosus, 349

torosilabris, Liocassis, 108

torosus, Aguarunichthys, 329

Torpedo, 301

torrenticola, Harttia, 242

torrentis, Pterocryptis, 377

torrentis, Silurus, 377

tortosus, Sisor, 402

totae, Pygidium, 412

totae, Rhizosomichthys, 412

tourei, Synodontis, 322

trachacanthus, Bagrus, 89

Trachelyichthys, 78

Trachelyichthys decaradiatus, 78

Trachelyichthys exilis, 78

Trachelyopterichthys, 78

Trachelyopterichthys anduzei, 78

Trachelyopterichthys taeniatus, 78

Trachelyopterini, 68

Trachelyopterus, 68, 79

Trachelyopterus albicrux, 79

Trachelyopterus amblops, 79

Trachelyopterus ceratophysus, 79

Trachelyopterus coriaceus, 79

Trachelyopterus coriaceus maculosus, 79

Trachelyopterus fisheri, 79

Trachelyopterus galeatus, 79

Trachelyopterus insignis, 79

Trachelyopterus lacustris, 79

Trachelyopterus leopardinus, 80

Trachelyopterus lucenai, 80

Trachelyopterus peloichthys, 80

Trachelyopterus striatulus, 80

Trachelyopterus taeniatus, 78

Trachelyopterus teaguei, 80

trachipomus, Bagrus, 55

Trachycorystes, 80

Trachycorystes albicrux, 79

Trachycorystes analis, 80

Trachycorystes coracoideus, 71

Trachycorystes cratensis, 80

Trachycorystes fisheri, 79

Trachycorystes insignis badeli, 80

Trachycorystes insignis peloichthys, 80

Trachycorystes jokeannae, 80

Trachycorystes leopardinus, 80

Trachycorystes magdalenae, 79

Trachycorystes porosus, 81

Trachycorystes teaguei, 80

Trachycorystes trachycorystes, 80

Trachycorystes typus, 81 
trachycorystes, Auchenipterus, 80

trachycorystes, Trachycorystes, 80

Trachydoras, 179

Trachydoras atripes, 179

Trachydoras brevis, 179

Trachydoras microstomus, 179

Trachydoras nattereri, 179

Trachydoras paraguayensis, 179

Trachydoras steindachneri, 179

Trachyglanis, 28

Trachyglanis ineac, 28

Trachyglanis intermedius, 28

Trachyglanis minutus, 28

Trachyglanis sanghensis, 28

Trachymochlus, 431

Trachymochlus cupido, 431, 432

trachyparia, Oxydoras, 179

Trachypoma, 404

Trachypoma marmoratum, 404

transandianum, Pygidium taenia, 424

transandianus, Trichomycterus, 424

transfasciatus, Rhamdioglanis, 202

transitoria, Pimelodella, 195

transmontanus, Batrochoglanis, 353

transmontanus, Pseudopimelodus, 353

transvaaliensis, Amphilius, 23

trautmani, Noturus, 214

travancoria, Batasio, 86

travassosi, Otothyris, 276

travassosi, Pygidium, 414

trefauti, Trichomycterus, 425

treitlii, Corydoras, 126

trewavasae, Glyptothorax, 394

Trewavasia, 433

Trewavasia carinata, 433

triacanthopomus, Henonemus, 440

triactis, Leporacanthicus, 266

Trichogenes, 403, 414

Trichogenes longipinnis, 414

Trichogeninae, 403

Trichomycteridae, 403

Trichomycterini, 403

trichomycteroides, Paramphilius, 25

Trichomycterus, 403, 413

Trichomycterus aguarague, 441

Trichomycterus albinotatus, 414

Trichomycterus alternatus, 414

Trichomycterus alterus, 415

Trichomycterus amazonicus, 406

Trichomycterus areolatus, 415

Trichomycterus arleoi, 415

Trichomycterus auroguttatus, 415

Trichomycterus bahianus, 415

Trichomycterus banneaui, 415

Trichomycterus barbatula, 422

Trichomycterus barbouri, 415

Trichomycterus belensis, 415

Trichomycterus bogotensis, 415

Trichomycterus bomboizanus, 416
Trichomycterus borellii, 416

Trichomycterus boylei, 416

Trichomycterus brasiliensis, 416

Trichomycterus brasiliensis itatiayae, 419

Trichomycterus brasiliensis tristis, 416

Trichomycterus caliensis, 416

Trichomycterus candidus, 416

Trichomycterus castroi, 416

Trichomycterus catamarcensis, 416

Trichomycterus caudofasciatus, 416

Trichomycterus celsae, 416

Trichomycterus chaberti, 417

Trichomycterus chapmani, 417

Trichomycterus chiltoni, 417

Trichomycterus chungaraensis, 417

Trichomycterus concolor, 417

Trichomycterus conradi, 417

Trichomycterus corduvensis, 417

Trichomycterus cubataonis, 425

Trichomycterus davisi, 417

Trichomycterus diabolus, 417

Trichomycterus dispar, 417

Trichomycterus dorsotriatus, 417

Trichomycterus duellmani, 418

Trichomycterus eichorniarum, 407

Trichomycterus eigenmanni, 423

Trichomycterus emanueli, 418

Trichomycterus fassli, 418

Trichomycterus gabrieli, 418

Trichomycterus giganteus, 418

Trichomycterus goeldii, 418

Trichomycterus gorgona, 418

Trichomycterus gracilis, 422

Trichomycterus guaraquessaba, 418

Trichomycterus guianensis, 418

Trichomycterus hasemani, 418

Trichomycterus herberti, 407

Trichomycterus heterodontus, 418

Trichomycterus iheringi, 419

Trichomycterus immaculatus, 419

Trichomycterus incae, 423

Trichomycterus inermis, 324

Trichomycterus itacambirussu, 419

Trichomycterus itacarambiensis, 419

Trichomycterus itatiayae, 419

Trichomycterus jacupiranga, 419

Trichomycterus jequitinhonhae, 419

Trichomycterus johnsoni, 419

Trichomycterus knerii, 419

Trichomycterus landinga, 419

Trichomycterus laticeps, 407

Trichomycterus latidens, 419

Trichomycterus latistriatus, 420

Trichomycterus laucaensis, 420

Trichomycterus lewi, 420

Trichomycterus longibarbatus, 420

Trichomycterus maculatus, 415

Trichomycterus maracaiboensis, 420
Trichomycterus marmoratus, 415

Trichomycterus mboycy, 420

Trichomycterus mendozensis, 413

Trichomycterus meridae, 420

Trichomycterus migrans, 420

Trichomycterus mimonha, 420

Trichomycterus minutus, 413

Trichomycterus mirissumba, 420

Trichomycterus mondolfi, 421

Trichomycterus motatanensis, 421

Trichomycterus naipi, 421

Trichomycterus nigricans, 414, 421

Trichomycterus nigromaculatus, 421

Trichomycterus palleus, 415

Trichomycterus pantherinus, 421

Trichomycterus paolencis, 421

Trichomycterus papilliferus, 421

Trichomycterus paquequerensis, 421

Trichomycterus pardus, 423

Trichomycterus pauciradiatus, 440

Trichomycterus pentlandi, 423

Trichomycterus pictus, 423

Trichomycterus piurae, 421

Trichomycterus plumbeus, 421

Trichomycterus poeyanus, 423

Trichomycterus potschi, 421

Trichomycterus pradensis, 422

Trichomycterus proöps, 408

Trichomycterus pseudosilvinichthys, 422

Trichomycterus punctatissimus, 422

Trichomycterus punctulatus, 422

Trichomycterus pusillus, 411

Trichomycterus ramosus, 422

Trichomycterus regani, 422

Trichomycterus reinhardti, 422

Trichomycterus retropinnis, 422

Trichomycterus riojanus, 422

Trichomycterus rivulatus, 422

Trichomycterus roigi, 423

Trichomycterus romeroi, 423

Trichomycterus santaeritae, 423

Trichomycterus spegazzinii, 423

Trichomycterus spelaeus, 423

Trichomycterus spilosoma, 423

Trichomycterus stawiarski, 423

Trichomycterus stellatus, 424

Trichomycterus straminius, 424

Trichomycterus striatus, 424

Trichomycterus taczanowskii, 424

Trichomycterus taenia, 407, 424

Trichomycterus taeniops, 424

Trichomycterus taroba, 424

Trichomycterus tenuis, 424

Trichomycterus tigrinus, 415

Trichomycterus transandianus, 424

Trichomycterus trefauti, 425

Trichomycterus triguttatus, 425

Trichomycterus tupinamba, 425 
Trichomycterus unicolor, 425

Trichomycterus variegatus, 425

Trichomycterus venulosus, 425

Trichomycterus vermiculatus, 425

Trichomycterus vittatus, 425

Trichomycterus weyrauchi, 425

Trichomycterus yuska, 425

Trichomycterus zonatus, 425

tricornis, Hopliancistrus, 249

Tridens, 403, 426

Tridens brevis, 426

Tridens melanops, 426

Tridensimilis, 426

Tridensimilis brevis, 426

Tridensimilis venezuelae, 426

Tridentinae, 403

Tridentopsis, 426

Tridentopsis cahuali, 426

Tridentopsis pearsoni, 426

Tridentopsis tocantinsi, 426

trifasciatum, Cyclopium, 67

trifasciatus, Astroblepus, 67

trifasciatus, Pachypterus, 359

trigonocephalus, Sorubim, 342

triguttatum, Pygidium, 425

triguttatus, Trichomycterus, 425

trilineatoides, Glyptothorax, 394

trilineatus, Corydoras, 126

trilineatus, Glyptothorax, 394

trilineatus, Leiocassis, 103

trilineatus, Pseudobagrus, 103

trilobatus, Chiloglanis, 308

trimaculatus, Leptodoras, 174

trimaculatus, Nemadoras, 174

trimaculatus, Opsodoras, 174

trimaculatus, Oxydoras, 174

trinitatis, Ancistrus, 225

trinitatis, Chaetostomus, 225

triostegus, Silurus, 380

triradiatus, Ancistrus, 225

triradiatus martini, Ancistrus, 223

triseriatus, Corydoras nattereri, 121

tristis, Trichomycterus brasiliensis,

416

troelli, Claibornichthys, 434, 435

Trogloglanis, 216

Trogloglanis pattersoni, 216

trombetensis, Harttia, 242

troschelii, Sciades, 53, 54

truncatorostris, Chasmocranus, 183

truncatum, Platystoma, 341

truncatus, Amblydoras, 168

truncatus, Arius, 43

truncatus, Belodontichthys, 368

truncatus, Cryptarius, 43

truncatus, Liocassis, 103

truncatus, Pseudobagrus, 103

truncatus, Synodontis marmoratus, 317

tsanensis, Clarias, 143 tubbi, Pangasius, 328

tucumanensis, Loricaria, 269

tukano, Corydoras, 126

tumbanus, Eutropius, 365

tumbanus, Schilbe, 365

tupinamba, Trichomycterus, 425

tuyra, Arius, 40

tuyra, Cathorops, 40

tuyrensis, Dasyloricaria, 234

tuyrensis, Loricaria, 234

tweediei, Wallagonia, 381

Tympanopleura, 68

Tympanopleura alta, 69

Tympanopleura nigricollis, 68

Tympanopleura piperata, 68, 70

typhla, Rhamdia laticauda, 198

Typhlobagrus, 189

Typhlobagrus kronei, 189, 192

Typhlobelus, 426

Typhlobelus guacamaya, 426

Typhlobelus lundbergi, 426

Typhlobelus macromycterus, 426

Typhlobelus ternetzi, 426

typica, Doumea, 24, 25

typus, Gagata, 386

typus, Gymnallabes, 159

typus, Helicophagus, 324, 325

typus, Ketengus, 45

typus, Micronema, 375

typus, Parahemiodon, 269, 270

typus, Phyllonemus, 164

typus, Pinirampus, 339

typus, Rhinoglanis, 310, 311

typus, Trachycorystes, 81

typus heterocercalis, Gymnallabes, 150

$\mathbf{U}$

uatumensis, Harttia, 242

ubangensis Auchenoglanis, 162

ubangensis, Bagrus, 84

ubidiai, Astroblepus, 67

ubidiai, Cyclopium, 67

ucamara, Lepthoplosternum, 129

ucayalensis, Ageneiosus, 70

ucayalensis, Hemiancistrus, 283, 285

ucayalensis, Hassar, 171

ucayalensis, Loricariichthys, 271

ucayalensis, Peckoltia, 285

Uegitglanis, 152

Uegitglanis zammaranoi, 152, 153

uelensis, Clariallabes, 138

uelensis, Clarias (Allabenchelys), 138

umbrosa, Cetopsis, 133

unae, Hypostomus, 261

Unae, Plecostomus, 261

uncinatus, Arius, 36

undecimalis, Chaetostomus, 292

undecimalis, Pterygoplichthys, 292

undecimalis, Silurus, 431 underwoodi, Rhamdia, 198

undulatus, Corydoras, 126

unicolor, Aphanotorulus, 225

unicolor, Plecostomus, 225

unicolor, Plotosus, 350, 352

unicolor, Pygidium, 425

unicolor, Synodontis, 322

unicolor, Trichomycterus, 425

unidorsalis, Helogenes, 134

unifasciata, Phenacorhamdia, 189

unifasciatum, Cyclopium, 67

unifasciatus, Astroblepus, 67

uniformis, Amphilius baudoni, 21

uniformis, Chrysichthys, 159

unitius, Silurus, 350

upiensis, Pangasius pangasius, 327

Upsilodus, 247

Upsilodus victori, 247

upsulonophorus, Tachisurus, 44

uracantha, Loricaria, 240

uracantha, Rineloricaria, 240

uracantha rupestre, Loricaria, 240

uracanthus, Fonchiiichthys, 240

uranoscopus, Amphilius, 23

uranoscopus, Anoplopterus, 21, 23

uranoscopus, Doras, 173, 174

uranoscopus, Megalodoras, 174

uranoscopus, Schilbe, 362, 365

urbaini, Cryptopterus, 373

urbaini, Ompok, 373

urichi, Caecorhamdia, 197, 202

Urinophilus, 427

Urinophilus diabolicus, 411

Urinophilus erythrurus, 427

urostigma, Bagrus, 84

urostriatum, Decapogon, 127

urostriatum, Dianema, 127

urotriatum, Decapogon, 127

urua, Pimelodus, 359

uruguayensis, Ageneiosus, 70

uruguayensis, Hypostomus, 261

uruyensis, Helogenes, 134

uruyensis, Helogenes marmoratus, 134

ussuriensis, Bagrus, 92, 100

ussuriensis, Leiocassis, 101

ussuriensis, Pelteobagrus, 100

ussuriensis, Pseudobagrus, 101

utarus, Arius, 48

utarus, Neoarius, 48

utik, Arius, 35

V

vacha, Eutropiichthys, 358

vacha, Pimelodus, 358

vachellii, Bagrus, 101

vachellii, Pelteobagrus, 101

vagum, Chaetostoma, 231

vagus, Chaetostomus, 231

vaillanti, Arges, 67

vaillanti, Astroblepus, 67 
vaillanti, Hypostomus, 261

vaillanti, Liocassis, 105

Vaillanti, Plecostomus, 261

vaillanti, Pseudomystus, 105

vaillanti, Synodontis, 323

vaillantii, Bagrichthys, 83

Vaillantii, Bagroides, 83

vaillanti, Brachyplatystoma, 331

Vaillanti, Platystoma, 330, 331

vaillantii, Silurus, 331

valencia, Lithogenes, 267

valenciennesi, Ageneiosus, 69

valenciennesi, Osteogeneiosus, 51

valenciennesii, Genidens, 44

Valenciennesii, Loricaria, 247

valenciennis, Parapimelodus, 335

valenciennis, Pimelodus, 335

valeya, Wallago attu, 381

vanceae, Astroblepus, 67

vanceae, Cyclopium, 67

vandeli, Arius, 50

Vandellia, 403, 427

Vandellia Balzanii, 427

Vandellia beccarii, 427

Vandellia cirrhosa, 427

Vandellia gigantea, 427

Vandellia hasemani, 411

Vandellia hematophaga, 410

Vandellia plazaii, 427

Vandellia sanguinea, 427

Vandellia wieneri, 411

Vandelliini, 403

vandenhoutei, Clarias, 144

vanderwaali, Synodontis, 322

vandeweyeri, Eutropiellus, 361

vanigonis, Otolithus (Arius), 434

Vannutellii, Rhinoglanis, 311

variabilis, Clariallabes, 138

variegata, Crossoloricaria, 233

variegata, Loricaria, 232, 233

variegata venezuelae, Loricaria, 233

variegatum, Amblyceps, 19

variegatus, Pimelodus, 13, 14, 15

variegatus, Akysis, 15

variegatus, Akysis variegatus, 14

variegatus, Hemibagrus, 91

variegatus, Microglanis, 355

variegatus, Trichomycterus, 425

variipictus, Hypostomus, 261

variipictus, Neoplecostomus, 274

variipictus, Plecostomus, 261

varimaculosus, Hypostomus, 261

varimaculosus, Plecostomus, 261

variolosus, Arius, 41

variolosus, Pseudopimelodus, 355

variolus, Ancistrus, 225

variolus, Chaetostomus, 225

variostictus, Hypostomus, 261

variostictus, Plecostomus, 261 varispinis, Clarias, 147

varius, Acrochordonichthys, 13

varius, Akysis, 15

varius, Liposarcus, 292

vasquezi, Chaetostoma, 231

vazferreirai, Homodiaetus, 406

velatus, Encheloclarias, 150

velifer, Chrysichthys, 157, 158

velifer, Gephyroglanis, 157

velifer, Pimelodus, 203

velifer, Synodontis, 322

velites, Aspidoras, 110

velox, Hemibagrus, 91

velutinus, Hemipimelodus, 48

velutinus, Neoarius, 48

venaticus, Bagrus, 55

venezuelae, Chaetostoma, 231

venezuelae, Corymbophanes, 231

venezuelae, Crossoloricaria, 233

venezuelae, Haemomaster, 405

venezuelae, Loricaria variegata, 233

venezuelae, Tridensimilis, 426

venezuelanus, Corydoras, 112

venezuelensis, Farlowella, 239

venezuelensis, Farlowella acus, 239

venosus, Arius, 36

ventrale, Cyclopium, 68

ventralis, Astroblepus, 68

ventralis, Cetopsis, 135

ventromaculatus, Hypostomus, 262

venulosum, Pygidium, 425

venulosus, Trichomycterus, 425

verbeekii, Pseudeutropius, 326

verecunda, Pterocryptis, 377

verecundus, Ancistrus, 225

verissimi, Decapogon, 127

vermicularis, Hypostomus, 262

vermicularis, Plecostomus, 262

vermiculata, Ancistrus vittatus, 285

vermiculata, Peckoltia, 285

vermiculata, Synodontis, 322

vermiculatum, Pygidium, 425

vermiculatus, Synodontis, 322

vermiculatus, Trichomycterus, 425

verres, Hypostomus, 262

verrucosa, Aspredo, 60

verrucosa, Parakysis, 15, 16

verrucosus, Arius, 45

verrucosus, Bunocephalus, 60

verrucosus, Hemiarius, 45

verrucosus, Parakysis, 16

verrucosus, Platystacus, 58, 60

verrucosus, Silurus, 60

versicolor, Pimelodus, 44

vertagus, Bagrus, 55

vespa, Akysis, 15

vespertinus, Ameiurus, 206

vespertinus, Ictalurus, 206

vestigipinnis, Hemipsilichthys, 281 vestigipinnis, Pareiorhaphis, 281

vestitus, Otocinclus, 274, 275

vetula, Loricaria, 278

vetula, Paraloricaria, 278

vicinus, Hypostomus, 278

victori, Upsilodus, 247

victoriae, Synodontis, 322

viedmensis, Diplomystes, 166

viedmensis, Olivaichthys, 166

viedmensis cuyanus, Diplomystes, 166

viedmensis mesembrinus, Diplomystes, 166

vietnamicus, Hemibagrus, 91

vietnammicus, Hemibagrus, 91

vigilis, Microsynodontis, 310

Villarius, 208

Villarius pricei, 208, 209

villarsi, Plecostomus, 298

villarsi, Squaliforma, 298

villiersi, Physailia, 360

villosus, Arius, 166

villosus, Batrochoglanis, 353

villosus, Lithogenes, 267

villosus, Pseudopimelodus, 353

villosus butcheri, Pseudopimelodus, 352

Vilsoni, Pimelenotus, 197, 200

vinciguerrae, Clarias, 143

Vinciguerrae, Exostoma, 385

vinhensis, Pelteobagrus virgatus, 101

violacea, Synodontis, 322

violaceus, Synodontis, 322

virescens, Corydoras, 123

virescens, Plecostomus, 298

virescens, Squaliforma, 298

virgatus, Aoria, 101

virgatus, Leiocassis, 101

virgatus, Pelteobagrus, 101

virgatus, Pseudobagrus, 101

virgatus vinhensis, Pelteobagrus, 101

virginiae, Corydoras, 126

virgo, Ageneiosus, 70

virgulatus, Aspidoras, 110

viridescens, Gogangra, 395

viridescens, Pimelodus, 395

viscosus, Pimelodus, 215

viscosus, Silurus, 215

vitata, Ansorgia, 361

vittata, Ansorgia, 360, 361

vittata, Farlowella, 239

vittata, Macrones, 106

vittata, Peckoltia, 285

vittata, Pimelodella, 195

vittata bistriata, Ansorgia, 361

vittatus, Ageneiosus, 70

vittatus, Chaetostomus, 283, 284, 285

vittatus, Corydoras, 126

vittatus, Corydoras blochi, 126

vittatus, Mystus, 98 
vittatus, Otocinclus, 275

vittatus, Plotosus, 350

vittatus, Pseudorhamdia, 195

vittatus, Silurus, 98

vittatus, Silurus (Callichrus), 372

vittatus, Synodontis, 314

vittatus, Trichomycterus, 425

vittatus horai, Mystus (Mystus), 95

vittatus vermiculata, Ancistrus, 285

viviparus, Arius, 55

viviparus, Plotosus, 350

vogti, Atopochilus, 304

volcanensis, Lasiancistrus, 264

voltae, Chiloglanis, 308

voltae, Irvineia, 358

voltae, Synodontis, 323

Vorhisia, 434

Vorhisia vulpes, 434, 435

Vorhisiidae, 434

votouro, Hemiancistrus, 244

voulezi, Rhamdia branneri, 201

vulgaris, Pimelodus, 206

vulpeculus, Pimelodus, 206

vulpes, Pimelodus, 211

vulpes, Vorhisia, 434, 435

W

waandersii, Helicophagus, 325

wagenaari, Chrysichthys, 159

wagneri, Pimelodus, 201

waiampi, Hypostomus, 262

walkeri, Chrysichthys, 159, 160

walkeri, Clarias, 141

walkeri, Notoglanidium, 162

walkeri, Pseudeutropius atherinoides, 359

wallacei, Tetranematichthys, 444

Wallago, 380

Wallago attu, 380

Wallago attu valeya, 381

Wallago dinema, 368

Wallago heterorhynchus, 368

Wallago krattensis, 374

Wallago leerii, 380, 381

Wallago leiacanthus, 371, 372

Wallago maculatus, 381

Wallago micropogon, 381

Wallago miostoma, 372

Wallago nebulosus, 381

Wallago Russellii, 381

Wallagonia, 380

Wallagonia tweediei, 381

wallagoo, Silurus, 381

walrechti, Sciadeichthys (Selenaspis), 53

wamiensis, Leptoglanis, 29

wamiensis, Synodontis, 320

wamiensis, Zaireichthys, 29

wangi, Pseudobagrus, 108

Watawata, 250 waterhousii, Neoplotosus, 346

waterloti, Chiloglanis niloticus, 307

waterloti, Synodontis, 323

watwata, Hypostomus, 262

weberi, Callichrous, 373

weberi, Ompok, 373

weddellii, Anadoras, 169

weddellii, Doras, 169

weitzmani, Corydoras, 126

werneri, Clarias, 148

werneri, Synodontis, 311

Wertheimeri, Plecostomus, 285

wertheimeri, Pogonopoma, 285

Wertheimeria, 179

Wertheimeria maculata, 179

wesselii, Pimelodella, 195

Wesselii, Pimelodus (Pseudorhamdia), 195

westermanni, Bergiaria, 330

Westermanni, Pimelodus, 329, 330

weyrauchi, Pygidium, 425

weyrauchi, Trichomycterus, 425

whymperi, Arges, 68

whymperi, Astroblepus, 68

wieneri, Plectrochilus, 411

wieneri, Vandellia, 411

wilderi, Hassar, 171

wilsoni, Eomacrones, 87

wilsoni, Hemiancistrus, 244

wilsoni, Macronoides, 87

winzi, Hypostomus, 262

winzi, Plecostomus, 262

witmeri, Pimelodella, 195

Wittei, Auchenoglanis, 154

wittenburgii, Pseudobagrus, 108

wolfei, Hemiloricaria, 247

wolfei, Rhineloricaria, 247

Wolffii, Bagrus, 98

wolffii, Mystus, 98

wolfi, Rhamdella, 197

woodi, Hassar, 171

woodsi, Rhynchodoras, 178

woosnami, Synodontis, 323

worthingtoni, Bathyclarias, 136

worthingtoni, Dinotopterus, 136

wotroi, Corydoras, 120

wrightiana, Oxyropsis, 276

wrightii, Oxyropsis, 276

wuchereri, Hypostomus, 262

wuchereri, Pimelodus, 200

wuchereri, Plecostomus, 262

wui, Pseudobagrus, 101

wurnoense, Nigerium, 98, 99

Wyckii, Bagrus, 91

wyckii, Hemibagrus, 91

wyckioides, Hemibagrus, 91

wyckioides, Macrones (Hemibagrus),

91

wynaadensis, Pterocryptis, 377 wynaadensis, Silurus, 375, 377

$\mathbf{X}$

xakriaba, Otocinclus, 275

Xanthocephalus, Pimelodus, 207

Xanthocephalus, Silurus, 207

xanthosteus, Silurus, 379

xanthum, Megalonema, 335

xanthus, Perugia, 335

xenauchen, Galeichthys, 33

Xenocara, 219

Xenocara boliviana, 219

Xenocara brevipinnis, 219

Xenocara bufonia, 219

Xenocara damasceni, 220

Xenocara fulva, 221

Xenocara heterorhynchus, 221

Xenocara macrophthalma, 222

Xenocara montana, 223

Xenocara multispinis, 223

Xenocara occidentalis, 223

Xenocara rothschildi, 221

Xenoclarias, 153

Xenoclarias eupogon, 153

Xenoclarias holobranchus, 153

xenodon, Clarias, 143

xenognathus, Leptoglanis, 25

Xenomystus, 247

Xenomystus gobio, 247

Xenopholis, 433

Xenopholis carinatus, 433

Xenopholoides, 433

xetequepeque, Rhamdia, 202

Xiliphius, 62

Xiliphius kryptos, 62

xinguensis, Corydoras, 126

xinguensis, Glyptoperichthys, 292

xinguensis, Pterygoplichthys, 292

xingui, Rhynchodoras, 178

xiphias, Synodontis, 323

Xiurenbagrus, 20

Xiurenbagrus gigas, 20

Xiurenbagrus xiurenensis, 20

xiurenensis, Xiurenbagrus, 20

Xyliphius, 62

Xyliphius barbatus, 62

Xyliphius kryptos, 62

Xyliphius labrosus, 62

Xyliphius lepturus, 62

Xyliphius lombarderoi, 62

Xyliphius magdalenae, 62

Xyliphius melanopterus, 62

Y

yangambianus, Eutropius, 365

yangambianus, Schilbe, 365

yaravi, Ancistrus (Hemiancistrus), 273

yaravi, Neblinichthys, 273

yarrelli, Bagarius, 383

Yarrelli, Bagrus, 383

yasi, Epactionotus, 236 
youngicus, Clarias, 145

youssoufi, Gagata, 386

yuncensis, Pimelodella, 195

yunnanense, Pseudexostoma, 401

yunnanensis, Glyptosternum, 401

yunnanensis brachysoma,

Pseudexostoma, 401

yurubiense, Chaetostoma, 231

yuska, Trichomycterus, 425

$\mathbf{Z}$

zainaensis, Glyptothorax, 394

Zaireichthys, 25, 28

Zaireichthys brevis, 28

Zaireichthys camerunensis, 28

Zaireichthys dorae, 28

Zaireichthys flavomaculatus, 28

Zaireichthys heterurus, 28

Zaireichthys mandevillei, 28

Zaireichthys rotundiceps, 28

Zaireichthys wamiensis, 29

Zaireichthys zonatus, 28, 29

zairensis, Amphilius, 23

zairensis, Schilbe, 366

zambesensis, Synodontis, 323

zambezensis, Synodontis, 323

zambezensis rukwaensis, Synodontis, 320

zambeziensis, Malapterurus, 302

zammaranoi, Uegitglanis, 152, 153

zanaensis, Glyptothorax, 394

zanzibarica, Synodontis, 323

zanzibaricus, Synodontis, 316, 320, 323

Zathorax, 168

Zathorax gonzalezi, 168

Zathorax monitor, 168, 169

Zathorax nauticus, 169

zebra, Hypancistrus, 249

zhujiangensis, Glyptothorax, 394

Zonancistrus, 234

zonatum, Pygidium, 425

zonatus, Acrochordonichthys, 13

zonatus, Caelatoglanis, 383

zonatus, Microglanis, 355

zonatus, Pimelodus, 13

zonatus, Synodontis angelicus, 311

zonatus, Trichomycterus, 425

zonatus, Zaireichthys, 28, 29

zongolicensis, Rhamdia, 199

zuliaensis, Pterygoplichthys, 292

zuloagai, Doraops, 170

Zungaro, 344

Zungaro humboldtii, 344

Zungaro jahu, 344

Zungaro mathisoni, 356

Zungaro zungaro, 344

Zungaro zungaro schultzi, 355

zungaro, Zungaro, 344

zungaro schultzi, Zungaro 355
Zungaropsis, 344

Zungaropsis multimaculatus, 344

zygatus, Corydoras, 126

zygouron, Clarias, 141 